\title{
Baseline Risk Assessment for the F-Area Burning/Rubble Pits and Rubble Pit
}

by

E. Palmer

Westinghouse Savannah River Company

Savannah River Site

Aiken, South Carolina 29808

RECEIVED

JUL 071998

. OSTI

This paper was prepared in connection with work done under the above contract number with the U.S.

Department of Energy. By acceptance of this paper, the publisher and/or recipient acknowledges the U.S. Government's right to retain a nonexclusive, royalty-free license in and to any copyright covering this paper, along with the right to reproduce and to authorize others to reproduce all or part of the copyrighted paper. 
BASELINE RISK ASSESSMENT

FOR THE

\section{F-AREA BURNING/RUBBLE PITS \\ AND RUBBLE PIT $(u)$}

\section{FINAL}

MARCH 1996

Prepared by:

Westinghouse Savannah River Company . Savannah River Site Aiken, South Carolina 29808

Prepared for the U.S. Department of Energy Under Contract DE-AC09-89-SR18035 


\section{Rev. 1.2 Baseline Risk Assessment For the F-Area Burning/Rubble Pits and Rubble Pit (231-F, 231-1F, And 231-2F) (U)}

\section{DISCLAIMER}

This report was prepared as an account of work sponsored by an agency of the United States Government. Neither the United States Government nor any agency thereof, nor any of their employees, makes any warranty, express or implied, or assumes any legal liability or responsibility for the accuracy, completeness, or usefulness of any information, apparatus, product, or process disclosed, or represents that its use would not infringe privately owned rights. Reference herein to any specific commercial product, process, or service by trade name, trademark, manufacturer, or otherwise does not necessarily constitute or imply its endorsement, recommendation, or favoring by the United States Government or any agency thereof. The views and opinions of authors expressed herein do not necessarily state or reflect those of the United States Government or any agency thereof.

This report has been reproduced directly from the best available copy.

Available to DOE and DOE contractors from the Office of Scientific and Technical Information, P.O. Box 62, Oak Ridge, TN 37831; prices available from (615) 576-8401.

Available to the public from the National Technical Information Service, U.S. Department of Commerce; 5285 Port Royal Road, Springfield, VA 22161.

Printed in the United States of America

Prepared for

U.S. Department of Energy and Westinghouse Savannah River Company

Prepared by

Parsons Engineering Science, Inc.

Aiken, South Carolina

U.S.A. 


\section{DISCLAIMER}

Portions of this document may be illegible electronic image products. Images are produced from the best available original document. 


\section{TABLE OF CONTENTS}

\section{EXECUTIVE SUMMARY}

\subsection{INTRODUCTION}

ES-1

1.1 Overview of the Site

1.2 Objective of the BRA

1.3 Unit Background

1.4 Scope of the BRA

1.5 Report Organization

2.0 DATA COLLECTION

2.1 Soil

2-2

2.1.1 Pit 231-F and Pit 231-1F

2.1.2 Pit 231-2F

2.1.3 Background

2.2 Groundwater

2.2.1 Quarterly Monitoring Data

2.2.2 Remedial Investigation Wells

2.2.3 Background

2.3 Surface Water

2.3.1 Unit-Related

2.3.2 Background

2.4 Sediment

2.4.1 Unit-Related

2.4.2 Background

\subsection{HUMAN HEALTH RISK ASSESSMENT}

3.1 Identification of Human Health Chemicals of Potential Concern

3.1.1 Rationale and Criteria for Selection of COPCs

3.1.2 Chemicals of Potential Concern

3.2 Exposure Assessment

3.2.1 Characterization of Exposure Setting 


\section{TABLE OF CONTENTS (Continued)}

3.2.1.1 Environmental Setting 3-8

3.2.1.2 Land Use and Demography · 3-14

3.2.2 Identification of Human Health Exposure Pathways 3-15

3.2.2.1 Contaminant Sources and Release Mechanisms 3-16

3.2.2.2 Fate and Transport Mechanisms 3-16

3.2.2.3 Exposure Points 3-17

3.2.2.4 Potential Receptors and Exposure Routes 3-18

$\begin{array}{ll}\text { 3.2.3 Exposure Point Concentrations } & 3-20\end{array}$

3.2.3.1 Soil 3-21

3.2.3.2 Groundwater 3-22

3.2.3.3 Surface Water 3-22

3.2.3.4 Sediment 3-22

3.2.3.5 Air 3-22

3.2.3.6 Homegrown Produce 3-23

3.2.4 Development of Contaminant Intakes and Doses 3-23

3.2.4.1 Intake Parameters 3-24

3.2.4.2 Equations for Exposure to Soil and Sediment 3-27

3.2.4.3 Equations for Exposure to Water 3-30

3.2.4.4 Equations for Exposure to Air 3-33

3.2.4.5 Equation for Exposure to Direct Radiation 3-35

3.2.4.6 Equation for Exposure to Homegrown Produce 3-35

3.3 Toxicity Assessment 3-36

3.3.1 Non-Radiological Toxicity _ 3-37

3.3.1.1 Carcinogens 3-37

3.3.1.2 Noncarcinogens 3-40

3.3.2 Radiation Toxicity $\quad 3-42$

3.3.2.1 Radiation Types 3-42

3.3.2.2 Radiation Effects 3-43 
TABLE OF CONTENTS (Continued)

3.4 Risk Characterization

3.4.1 Current Land Use 3-47

3.4.1.1 Nonradiological Carcinogenic Risk 3-48

3.4.1.2 Nonradiological Noncarcinogenic Hazards 3-48

3.4.1.3 Radiological Risks 3-48

$\begin{array}{lll}3.4 .2 & \text { Future Land Use 3-48 }\end{array}$

3.4.2.1 Nonradiological Carcinogenic Risk 3-49

3.4.2.2 Nonradiological Noncarcinogenic Hazards 3-51

3.4.2.3 Radiological Risks 3-53

3.4.3 Unçertainty 3-55

3.4.3.1 Chemicals of Potential Concern 3-55

3.4.3.2 Exposure Assessment 3-57

3.4.3.3 Toxicity Assessment 3-58

3.4.3.4 Risk Characterization 3-59

4.0 ECOLOGICAL RISK ASSESSMENT 4-1

4.1 Identification of Chemicals of Potential Concern 4-2

4.1.1 Exposure Point Concentrations 4-5

4.2 Environmental Setting 4-6

4.2.1 Characterization of Exposure Setting 4-6

4.2.1.1 Physical Setting 4-6

4.2.1.2 Ecological Setting 4-8

4.2.1.3 Conceptual Site Model 4-13

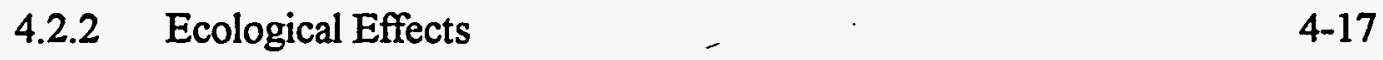

4.3 Endpoint Selection 4-17

4.4 Exposure Assessment 4-23

4.4.1 Chemical Distribution 4-23

4.4.2 Receptor Distribution 4-23

4.4.3 Quantification of Exposure 4-26 
BASELINE RISK ASSESSMENT

WSRC-TR-94-108

F-AREA BURNING/RUBBLE PITS AND RUBBLE PIT

REV. 1.2, MARCH 1996

TABLE OF CONTENTS (Continued)

4.5 Effects Assessment $\quad$ 4-29

4.5.1 Methodology 4-29

4.5.1.1 Nonradioactive Contaminants 4-30

4.5.1.2 Radioactive Contaminants 4-33

4.6 Risk Characterization 4-38

4.6.1 Risk Estimation 4-38

4.6.1.1 Current Land Usc $\quad 4-40$

4.6.1.2 Future Land Use 4-43

4.6.1.3 Uncertainty 4-44

4.6.2 Risk Description 4-46

4.6.2.1 Ecological Risk Summary 4-47

5.0 SUMMARY $5-1$

5.1 Human Health Risk Assessment $5-1$

5.1.1 Current Land Use · $\quad 5-2$

5.1.1.1 On-Unit Visitor Carcinogenic Risk 5-2

5.1.1.2 On-Unit visitor Noncarcinogenic Hazard 5-2

5.1.2 Future Land Use 5-3

5.1.2.1 Resident Carcinogenic Risks 5-3

5.1.2.2 Resident Noncarcinogenic Hazards 5-3

5.1.2.3 Commercial/Industrial Worker Carcinogenic Risks 5-3

5.1.2.4 Commercial/Industrial Worker Noncarcinogenic Hazards

$5-4$

5.1.3 Uncertainties 5-4

5.1.3.1 Uncertainties Leading to Potential Over

Estimation of Risk 5-4

5.1.3.2 Uncertainties Leading to Potential Under

Estimation of Risk $\quad 5-5$

5.2 Ecological Risk Assessment $5-6$

5.3 Remedial Goal Options $\quad 5-8$ 
TABLE OF CONTENTS (Continued)

\subsubsection{Human Health RGOs}

5-8

5.3.1.1 Groundwater

$5-8$

5.3.1.2 Soils

5-10 -

5.3.1.3 Derivation of RGO Values

5-11

5.3.2 Ecological Risk-Based RGOs

$5-12$

5.4 Conclusions

$5-12$

REFERENCES

REF-1 


\section{APPENDICES}

Appendix A Statistical Methods and Sample Calculations

Appendix B Predicted Soil COPC Concentrations in Groundwater

Appendix C Air Modeling - Particulates and VOCs

Appendix D D.1: Calculation of RME Carcinogenic Risk and Noncarcinogenic Hazards for Current and Future Receptors

D.2: Calculation of Radiological Risk for Current and Future Receptors

D.3: Input/Output for Lead Model

Appendix E Toxicity Profiles

Appendix F Calculation of Risk-Based Remedial Goal Options

Human Health and Ecological 


\section{LIST OF FIGURES}

Figure 1-1 Location of the Savannah-River Site . . 1-7

Figure 1-2 Location of F-Area Burning/Rubble Pits and Rubble Pit at the Savannah River Site $\quad 1-8$

Figure 1-3 Location of F-Area Burning/Rubble Pits and Rubble Pit in Relation to Other Savannah River Site Facilities

Figure 1-4 F-Area Burning/Rubble Pits and Rubble Pit Unit and Baseline Risk Assessment Study Area

Figure 2-1 F-Area Burning/Rubble Pits and Rubble Pit Unit Assessment

Sampling Locations for the Baseline Risk Assessment

Figure 3-1 Human/Ecological Data Evaluation COPC Identification

Figure 3-2 Surface Runoff Drainage Patterns for the F-Area Burning/

Rubble Pits

Figure 3-3 Human Health Conceptual Site Model for the F-Area Burning/Rubble Pits (231-F/231-1F)and Rubble Pit (231-2F)

Figure 3-4 Exposure Pathways for the F-Area Burning/Rubble Pits and Rubble Pit

Figure 4-1 Ecological Habitat Types at the F-Area Burning/Rubble Pits and Rubble Pit

Figure 4-2 Ecological Conceptual Site Model for the F-Area Burning/Rubble Pits and Rubble Pit

Figure 5-1 Summary of RME Carcinogenic Risk

Figure 5-2 Summary of RME Noncarcinogenic Hazard

Figure 5-3 Summary of RME Carcinogenic Risk

Figure 5-4 Summary of RME Noncarcinogenic Hazard

Figure 5-5 Summary of RME Carcinogenic Risk 
BASELINE RISK ASSESSMENT

WSRC-TR-94-108

F-AREA BURNING/RUBBLE PITS AND RUBBLE PIT

REV. 1.2, MARCH 1996

\section{LIST OF FIGURES (Continued)}

Figure 5-7 Summary of RME Carcinogenic Risk

$5-21$

Figure 5-8 Summary of RME Noncarcinogenic Hazard

$5-22$ 


\section{LIST OF TABLES}

Table 2.1 Soil Data Collection

Table 2.2 Mean Background Concentrations in Soil

Table 2.3 Mean Background Concentrations in Groundwater

Table 3.1 Chemicals Present in Unit Samples (CPUs), Surface Soil Summary (0-0.5 ft), Burning/Rubble Pits 231-F and 231-1F

Table 3.2 Chemicals Present in Unit Samples (CPUs), Surface Soil Summary (0-0.5 ft), Rubble Pit 231-2F

Table 3.3 Chemicals Present in Unit Samples (CPUs), Surface Soil Summary (0-2 ft), Burning/Rubble Pits 231-F and 231-1F

Table 3.4 Chemicals Present in Unit Samples (CPUs), Surface Soil Summary (0-2 ft), Rubble Pit 231-2F

Table 3.5 Chemicals Present in Unit Samples (CPUs), Soil Summary (0-4. ft), Burning/Rubble Pits 231-F and 231-1F

Table 3.6 Chemicals Present in Unit Samples (CPUs), Soil Summary (0-4 ft), Rubble Pit 231-2F

Table 3.7 Chemicals Present in Unit Samples (CPUs), Soil Summary ( $4 \mathrm{ft}$ to bottom of the pit), Burning/Rubble Pits 231-F and 231-1F

Table 3.8 Chemicals Present in Unit Samples (CPUs), Soil Summary ( $4 \mathrm{ft}$ to bottom of the pit), Rubble Pit 231-2F

Table 3.9 Chemicals Present in Unit Samples (CPUs), Groundwater Data, Burning/Rubble Pits 231-F, 231- 1F and 231-2F

Table 3.10 Chemicals Present in Unit Samples (CPUs), Surface Water

Data, Burning/Rubble Pits 231-F, 231-1F and 231-2F

Table 3.11 Chemicals Present in Unit Samples (CPUs), Sediment

Data, Burning/Rubble Pits 231-F, 231-1F and 231-2F

Table 3.12 Comparison of Soil Analytes to Background Concentration .Burning/Rubble Pits 231-F and 231-1F 


\section{LIST OF TABLES (Continued)}

Table 3.13 Comparison of Soil Analytes to Background Concentrations, Rubble Pit 231-2F

Table 3.14 Comparison of Groundwater Analytes to Background

Concentrations, Burning/Rubble Pits and Rubble Pit

Table 3.15 Comparison of Soil Analytes to Risk-Based Concentrations,

Burning/Rubble Pits 231-F and 231-1F (1)

Table 3.16 Comparison of Soil Analytes to Risk-Based Concentrations, Rubble Pit 231-2F

Table 3.17 Comparison of Groundwater Analytes to Risk-Based Concentrations, Burning/Rubble Pits and Rubble Pit

Table 3.18 Essential Nutrient Information, Burning/Rubble Pits and Rubble Pit

Table 3.19 COPC Selection in Groundwater, Essential Nutrient Information, Burning/Rubble Pits and Rubble Pit

Table 3.20 Frequency of Detection in Soil (1), Burning/Rubble Pits 231-F and $231-1 F$

Table 3.21 Groundwater Exposure Point Concentrations from Groundwater Modeling, Burning/Rubble Pits 231-F and 231-1F

Table 3.22 Chemicals of Potential Concern, Burning/Rubble Pits - F Area

Table 3.23 Modeled Exposure Point Concentrations of Soil Particulates in Air, Burning/Rubble Pits 231-F and 231-1F

Table 3.24 Modeled Exposure Point Concentrations of Soil Particulates in Air, Rubble Pit 231-2F

Table 3.25 Modeled Exposure Point Concentrations of Volatile Soil Contaminants in Air, Burning/Rubble Pits 231-F and 231-1F

Table 3.26 Modeled Exposure Point Concentrations of Volatile Soil Contaminants, Rubble Pit 231-2F

Table 3.27 RME Human Exposure Parameters, F-Area Burning/Rubble Pits 


\section{LIST OF TABLES (Continued)}

Table 3.28 Dermal Permeability Constants for COPCs in Surface Water, Burning/Rubble Pits 231-F, 231-1F and 231-2F

Table 3.29 Soil-to-Plant Transfer Factors (1), Burning/Rubble Pits 231-F 231-1F and 231-2F

Table 3.30 Chemical Specific Toxicity Values, Noncarcinogenic Effects from Ingestion and Dermal Absorption(1), F-Area Burning/Rubble Pits and Rubble Pit

Table 3.31 Chemical Specific Toxicity Values, Noncarcinogenic Effects from Inhalation(1), F-Area Burning/Rubble Pits and Rubble Pit

Table 3.32 Chemical Specific Toxicity Values, Carcinogenic Effects from Ingestion and Dermal Absorpíion(1), F-Area Burning/Rubble Pits and Rubble Pit

Table 3.33 Chemical Specific Toxicity Values, Carcinogenic Effects from Inhalation(1), F-Area Burning/Rubble Pits and Rubble Pit

Table 3.34 Radionuclide Carcinogenicity - Slope Factors(1), F-Area Burning/Rubble Pits and Rubble Pit

Table 3.35 Total Carcinogenic and Noncarcinogenic Risk by Medium, Burning/Rubble Pits 231-F and 231-1F

Table 3.36 Total Carcinogenic and Noncarcinogenic Risk by Medium, Rubble Pit 231-2F

Table 3.37 Carcinogenic and Noncarcinogenic Risk Summary by Receptor and Medium, Burning/Rubble Pits 231-F and 231-1F

Table 3.38 Carcinogenic and Noncarcinogenic Risk Summary by Receptor and Medium, Rubble Pit 231-2F

Table 3.39 Chemicals Contributing Significantly to Human Health Risk(1) Burning/Rubble Pits 231-F and 231-1F

Table 3.40 Chemicals Contributing Significantly to Human Health Risk(1) Rubble Pit 231-2F 


\section{LIST OF TABLES (Continued)}

Table 3.41 Radionuclide Carcinogenic Risk Summary by Receptor and Medium Burning/Rubble Pits 231-F and 231-1F

Table 4.1 Soil Analytes and Background Screening (0-0.6 m), BRPs 231F \&1F, Current Conditions

Table 4.2 Soil Analytes \& Background Screening (0-1.2 m), BRPs 231F \&1F, Future Conditions

Table 4.3 Soil Analytes \& Background Screening (0-0.6 m), RPs 231-2F, Current Conditions

Table 4.4 Soil Analytes \& Background Screening (0-1.2 m), RPs 231-2F, Future Conditions

Table 4.5 Toxicity Data for Ecological Screening, BRPs 231F, 1F \& RP 2F, Soil

Table 4.6 Soil Screening Against Ecological Toxicity Screening Benchmarks (0-0.6 M) BRP 231F \& 1F, Current Conditions, Non-radioactive Analytes 4-68

Table 4.7 Soil Screening Against Ecological Toxicity Screening Benchmarks, (0-0.6 m), BRPs 231-F \& 1F, Current Conditions, Radionuclides

Table 4.8 Soil Screening Against Ecological Toxicity Screening Benchmarks, (0-1.2 m), BRPs 231-F \& 1F, Future Conditions, Non-Radioactive Analytes

Table 4.9 Soil Screening Against Ecological Toxicity Screening Benchmarks, (0-1.2 m), BRPs 231-F \& 1F, Future Conditions, Radionuclides

Table 4.10 Soil Screening Against Ecological Toxicity Screening Benchmarks, (0-0.6 m), RP 231-2F, Current Conditions,-Non-Radioactive Analytes

Table 4.11 Soil Screening Against Ecological Toxicity Screening Benchmarks, (0-0.6 m), RP 231-2F, Current Conditions, Radionuclides

Table 4.12 Soil Screening Against Ecological Toxicity Screening Benchmarks, (0-1.2 m), RP 231-2F, Future Conditions, Non-Radioactive Analytes 


\section{LIST OF TABLES (Continued)}

Table 4.13 Soil Screening Against Ecological Toxicity Screening Benchmarks, (0-1.2 m), RP 232-2F, Future Conditions, Radionuclides

Table 4.14 Surface Water Screening Against Ecological Toxicity Screening Benchmarks, BRPs 231-F, IF \& RP 231-2F, Wetland, Current and Future Conditions, Non-Radioactive Analytes

Table 4.15 Surface Water Screening Against Ecological Toxicity Screening Benchmarks, BRPs 231-F/1F \& RP 231-2F, Wetland, Current and Future Conditions, Radionuclides

Table 4.16 Sediment Screening Against Toxicity Screening Benchmarks, BRPs 231-F/1F and RP 231-2F, Wetland, Current and Future Conditions, Non-Radioactive Analytes

Table 4.17 Sediment Screening Against Toxicity Screening Benchmarks, BRPs 231-F/1F and RP 231-2F, Wetland, Current and Future Conditions, Radionuclides

Table 4.18 Ecological COPCs in Each Medium, BRPs 231-F/1F and RP 231-2F, Non-Radioactive Analytes

Table 4.19 Ecological COPCs in Each Medium, BRPs 231-F/1F and RP 231-2F, Radionuclides

Table 4.20 Threatened, Endangered, and Sensitive Species that Potentially Could Occur in the Area of the F-Area BRP

Table 4.21 Principal Exposure Pathways Selected for Quantitative Evaluation of Risk to Ecological Receptors

Table 4.22 Evaluation of Potential Ecological Receptors as Assessment Endpoints BRPs 231-F/1F and RP 231-2F

Table 4.23 Comparison of Potential Receptor Ranges with Unit Area

Table 4.24 Oldfield Mouse Intake Rates

Table 4.25 Calculated Soil Receptor Exposure, (0-0..6 m), BRPs 231-F/1F 
BASELINE RISK ASSESSMENT

WSRC-TR-94-108

F-AREA BURNING/RUBBLE PITS AND RIIBBLE PIT

REV. 1.2, MARCH 1996

LIST OF TABLES (Continued)

Table 4.26 Calculated Soil Receptor Exposure, (0-1.2 m), BRPs 231-F/1F

Future Conditions

Table 4.27 Calculated Soil Receptor Exposure, (0-0.6 m), RP 231-2F, Current Conditions

Table 4.28 Calculated Soil Receptor Exposure (0-1.2 m), RP 231-2F,

Future Conditions

Table 4.29 Calculated Sediment Receptor Exposure, BRPs 231-F/1F and RP 231-2F, Wetland, Current and Future Conditions

Table 4.30 Toxicity Reference Values for Ecological COPCs, BRPs 231-F/1F and RP 231-2F

Table 4.31 Exposure to Radiation from Sediment, BRPs 231-F/1F and RP 231-2F, Current and Future Conditions

Table 4.32 Calculation of Soil Ecological Quotients, (0-0.6 m), BRPs 231-F/1F, Current Conditions

Table 4.33 Calculation of Soil Ecological Quotients, (0-1.2 m), BRPs 231-F/1F, Future Conditions

Table 4.34 Calculation of Soil Ecological Quotients, (0-0.6 m), RP 231-2F, Current Conditions

Table 4.35 Calculation of Soil Ecological Quotients, 0-1.2 m), RP 231-2F, Future Conditions

Table 4.36 Calculation of Surface Water Ecological Quotients, BRPs 231-F/1F and RP 231-2F, Wetland, Current and Future Conditions

Table 4.37 Calculation of Sediment Ecological Quotients, BRPs 231-F/1F and RP 231-2F, Wetland, Current and Future Conditions

Table 5.1 Summary of Carcinogenic Risk and Noncarcinogenic Hazards for Potential On-Unit Visitor Exposure Pathways for Burning Rubble Pits 231-F and 231-1F 


\section{LIST OF TABLES (Continued)}

Table 5.2 Summary of Carcinogenic Risk and Noncarcinogenic Hazards for Potential On-Unit Visitor Exposure Pathways for Rubble Pit 231-2F

Table 5.3 Summary of Carcinogenic Risk and Noncarcinogenic Hazards for

Potential Future On-Unit Resident Exposure Pathways for Burning Rubble Pits 231-F and 231-1F

Table 5.4 Summary of Carcinogenic Risk and Noncarcinogenic Hazards for Potential Future On-Unit Resident Exposure Pathways for Rubble Pit 231-2F

Table 5.5 Summary of Carcinogenic Risk and Noncarcinogenic Hazards for Potential Future On-Unit Commercial/Industrial Worker Exposure Pathways for Burning Rubble Pits 231-F and 231-1F

Table 5.6 Summary of Carcinogenic Risk and Noncarcinogenic Hazards for Potential Future On-Unit Commercial/Industrial Worker Exposure Pathways for Rubble Pit 231-2F

Table 5.7 Summary of Carcinogenic Risk and Noncarcinogenic Hazards for Soil, Current Visitors and Hypothetical Future Residents and Workers, Burning Rubble Pits 231-F and 231-1F

Table 5.8 Summary of Carcinogenic Risk and Noncarcinogenic Hazards for Current and Hypothetical Future Receptor Exposure Pathways to Soil for Rubble Pit 231-2F

Table 5.9 Summary of Carcinogenic Risk and Noncarcinogenic Hazards for Potential Future On-Unit Resident and Worker Exposure Pathways for Groundwater

Table 5.10 Summary of Carcinogenic Risk and Noncarcinogenic Hazards for Current and Hypothetical Future Receptors Ephemeral Wetland (Surface Water/Sediment) 


\section{LIST OF TABLES (Continued)}

Table 5.11 Applicable or Relevant and-Appropriate Requirements for Groundwater, Burning/Rubble Pit - F Area

Table 5.12 Remedial Goals for contaminants of Concern by Receptor and Medium Burning/Rubble Pits (231-F/1F) and Rubble Pit (231-2F)

Table 5.13 Summary of COCs and Maximum Detected Concentrations in Groundwater 
$\mathrm{AF}$

AQUIRE

ARARs

AWQC

$\mathrm{BaB}$

BAF

$\mathrm{BCF}$

BLS

BRA

BRP(s)

BW

CERCLA
Adherence Factor

Aquatic Information Retrieval

Applicable or Relevant and Appropriate Requirements

EPA Ambient Water Quality Criteria

Blanton Sands

Bioaccumulation Factor

Bioconcentration Factor

Below Land Surface

Baseline Risk Assessment

Burning/Rubble Pit(s)

Body Weight

Comprehensive Environmental Response, Compensation and Liability Act

CF Conversion Factor

$\mathrm{COC}$

Chemical of Concern

COPC Chemical of Potential Concern

CPUS Chemicals Present in Unit Samples

CSMs Conceptual Site Models

DOE U.S. Department of Energy

EC

ED

Environmental Concentration

EF

Exposure Duration

Exposure Frequency

EPA

U.S. Environmental Protection Agency

EEQ
Ecological Effects Quotient 
TB Toxicity Benchmark

TCL/TAL Target Compound List/Target Analyte List

TEF

Toxicity Equivalency Factors

TES

Threatened, Endangered, Sensitive

TRV

Toxicity Reference Value

TuE

Troup and Lucy sands

Ud

Udorthents

UF

Uncertainty Factors

UL95

95th percentile of the Upper Confidence limit

USDA

U.S. Department of Agriculture

USFS

U.S. Forest Service

UV

Ultra Violet

VF

Volatilization Factor

VOC

Volatile Organic Compounds

WSRC

Westinghouse Savannah River Company 


\section{LIST OF UNITS OF MEASURE}

ac

cm

${ }^{\circ} \mathrm{C}$

$\mathrm{cm}^{2}$

$\mathrm{cm}^{3} / \mathrm{g}$

DCF

$\mathrm{d} / \mathrm{yr}$

${ }^{\circ} \mathrm{F}$

$\mathrm{ft}$

ha

$\mathrm{hr} / \mathrm{d}$

in.

kg

$\mathrm{km}$

m

$\mathrm{mCi}$

$\mathrm{m} / \mathrm{sec}$

$\mathrm{m}^{3} / \mathrm{hr}$

$\mathrm{m}^{3 / \mathrm{sec}}$

$\mathrm{m}^{3} /$ workday

$\mathrm{mg} / \mathrm{cm}^{2}$

$\mathrm{mg} / \mathrm{d}$

$\mathrm{mg} / \mathrm{kg}$

$\mathrm{mg} / \mathrm{m}^{3}$

$\mathrm{ml} / \mathrm{g}$ acre

centimeter

degrees Celsius

square centimeter

cubic centimeters per gram

dose conversion factor

days per year

degrees Farenheit

feet

hectacre

hours per day

inch

kilogram

kilometer

meter

milliCuries

meters per second

cubic meters per hour

cubic meters per second

cubic meters per workday

milligrams per square centimeter

milligrams per day

milligrams per kilogram

milligrams per cubic meter

milliliters per gram 
from 3Q84 through 2Q92 indicated exceedances of maximum contaminant limits (MCLs) for carbon tetrachloride, iron, lead, manganese, nitrate, radium, and trichloroethylene. Groundwater data from 3Q84 through 2Q92 were excluded from the risk assessment because it did not meet data quality requirements for use in BRAs. There was no evidence that hazardous substances were contained in RP 231-2F. However, sidegradient groundwater data indicated that manganese, nitrate, radium, and trichloroethylene exceeded MCLs. Therefore, additional soil and groundwater sampling were conducted as part of the F-Area BRPs RFI/RI Unit Assessment completed in 1993. These data provide the basis for this baseline risk assessment (BRA).

The objective of the BRA was to assess potential risks to human and ecological receptors that could result from the release of and exposure to chemical and radionuclide contaminants under current conditions and hypothetical future land uses. Chemical and radionuclide cancer risks and health hazards in humans, and potential impacts to ecological receptors, associated with the FArea BRPs and RP were estimated for chemicals of potential concern (COPCs) based on U.S. Environmental Protection Agency (EPA) guidance.

Areas of suspected contamination included four areas of concern: F-Area BRPs 231-F/1F; RP 231-2F; an ephemeral wetland area located to the west of BRPs $231-\mathrm{F} / 1 \mathrm{~F}$; and general area groundwater. Potential unit risks were estimated for reasonable maximum exposure (RME) conditions. Two land use scenarios were considered: current land use (industrial) and future land use (residential and industrial). Potential human receptors that were addressed included on-unit visitors, hypothetical future on-unit residents, industrial workers, and off-SRS residents. Terrestrial and aquatic receptors were evaluated in the ecological assessment using current and hypothetical future use scenarios.

At the waste unit, the primary contaminant source considered was buried wastes in the pits. Secondary sources considered at the waste unit were surface and deep soils. Surface soils (0 to $0.6 \mathrm{~m}=0$ to $2 \mathrm{ft}$ ) were contaminated from the direct deposition of waste materials and/or ash from burning. Excavation and distribution of deeper contaminated soils ( 0 to $1.2 \mathrm{~m}=0$ to $4 \mathrm{ft}$ ) onto surface soils was considered to be a potential future source of contamination to surface soil. Percolation and/or infiltration of liquid wastes or contaminated leachate from deep soil to groundwater also were considered potential release mechanisms.

\section{Data Collection and Evaluation}

The four areas of suspected contamination at the BRP area included: soils at the BRP 231-F/1F area. soils at the RP $231-2 \mathrm{~F}$ area, surface water and sediment in the ephemeral wetland area, and 


\section{EXECUTIVE SUMMARY}

The Savannah River Site (SRS) is located principa!ly in Barnwell and Aiken Counties near the city of Aiken, South Carolina. SRS is owned by the U.S. Department of Energy (DOE). Management and operating services are provided by Westinghouse Savannah River Company (WSRC). SRS has historically produced tritium, plutonium, and other special nuclear materials for national defense. SRS also has provided nuclear materials for the space program and for medical, industrial, and research efforts.

The F-Area Burning/Rubble Pits Operable Unit is comprised of two burning/rubble pits (BRPs), BRPs 231-F/1F, and one rubble pit (RP), RP 231-2F, located to the west of the F-Area industrial complex. The BRPs were constructed in 1951 for burning of construction debris. During the operating period from 1951 to 1973 , waste was typically burned on a monthly basis. Waste materials burned included paper, plastics, wood, rubber, rags, cardboard, oil degreasers, and drummed solvents. Waste burning was discontinued by October 1973 and the pits were backfilled and used as rubble pits until 1978. Waste materials placed as rubble included concrete, bricks, tile, asphalt, plastic, metal, empty drums, wood products, and rubber. The pits reached capacity in 1978 and were taken out of service. The three pits were filled to capacity, backfilled with a layer of soil, graded, compacted, mounded, and covered with 5 to $8 \mathrm{~cm}$ (2 to 3 in) of clay.

SRS records indicate that concrete, lumber, cement, fence and telephone poles, rip rap, brick, tile, wallboard, paneling, metal scrap and shavings, drums, electrical conduit, furniture, and firehose may have been disposed in RP 231-2F. No known records at SRS indicate that any radionuclides or hazardous chemicals were disposed in this pit, but the possibility of such disposal in this pit cannot be eliminated. Management controls dictated no disposal of any suspected radioactive material in the BRPs. Rather, suspected radioactivity-contaminated material was disposed of in the SRS Radioactive Waste Burial Grounds.

\section{Unit Background}

The two BRPs, 231-F and 231-1F, are contiguous units separated by an undisturbed clay berm. Unit screening was completed in November 1989. Screening for BRPs 231-F/1F indicated the presence of metals which occur naturally in SRS soils (arsenic, cadmium, chromium, lead, mercury, nickel), semivolatile organic contaminants (components of waste oils or waste insecticides and phthalate ester plasticizers), and trace levels of indicators of radionuclides that occur naturally in SRS soils (gross alpha, nonvolatile beta, total radium), tritium, and one polychlorinated tiphenyl (PCB) species. Data from the SRS Groundwater Monitoring Program 
from 3 Q84 through $2 \mathrm{Q} 92$ indicated exceedances of maximum contaminant limits (MCLs) for carbon tetrachloride, iron, lead, manganese, nitrate, radium, and trichloroethylene. Groundwater data from 3Q84 through 2Q92 were excluded from the risk assessment because it did not meet data quality requirements for use in BRAs. There was no evidence that hazardous substances were contained in RP 231-2F. However, sidegradient groundwater data indicated that manganese, nitrate, radium, and trichloroethylene exceeded MCLs. Therefore, additional soil and groundwater sampling were conducted as part of the F-Area BRPs RFI/RI Unit Assessment completed in 1993. These data provide the basis for this baseline risk assessment (BRA).

The objective of the BRA was to assess potential risks to human and ecological receptors that could result from the release of and exposure to chemical and radionuclide contaminants under current conditions and hypothetical future land uses. Chemical and radionuclide cancer risks and health hazards in humans, and potential impacts to ecological receptors, associated with the FArea BRPs and RP were estimated for chemicals of potential concern (COPCs) based on U.S. Environmental Protection Agency (EPA) guidance.

Areas of suspected contamination included four areas of concern: F-Area BRPs 231-F/1F; RP 231-2F; an ephemeral wetland area located to the west of BRPs $231-\mathrm{F} / 1 \mathrm{~F}$; and general area groundwater. Potential unit risks were estimated for reasonable maximum exposure (RME) conditions. Two land use scenarios were considered: current land use (industrial) and future land use (residential and industrial). Potential human receptors that were addressed included on-unit visitors, hypothetical future on-unit residents, industrial workers, and off-SRS residents. Terrestrial and aquatic receptors were evaluated in the ecological assessment using current and hypothetical future use scenarios.

At the waste unit, the primary contaminant source considered was buried wastes in the pits. Secondary sources considered at the waste unit were surface and deep soils. Surface soils (0 to $0.6 \mathrm{~m}=0$ to $2 \mathrm{ft}$ ) were contaminated from the direct deposition of waste materials and/or ash from burning. Excavation and distribution of deeper contaminated soils ( 0 to $1.2 \mathrm{~m}=0$ to $4 \mathrm{ft}$ ) onto surface soils was considered to be a potential future source of contamination to surface soil. Percolation and/or infiltration of liquid wastes or contaminated leachate from deep soil to groundwater also were considered potential release mechanisms.

\section{Data Collection and Evaluation}

The four areas of suspected contamination at the BRP area included: soils at the BRP 231-F/1F area, soils at the RP 231-2F area, surface water and sediment in the ephemeral wetland area, and 
general area groundwater. Data were collected from these four areas. Data used for the BRA were collected during the period May 4, 1993, through December 1, 1993, as part of the F-Area BRP RFI/RI Unit Assessment. Additional data used were collected as part of the ongoing groundwater monitoring program at SRS during the period March 1993 through May 1993. It was determined that unit screening data and data obtained from the SRS Groundwater Monitoring Program from $3 \mathrm{Q} 84$ through $2 \mathrm{Q} 92$ did not meet data quality requirements and, therefore, were not used in the BRA. Unit-specific background data was utilized in the BRA as a reference baseline for soil background data.

Both radionuclides and nonradioactive analytes were evalıated as COPCs. After combining analytical data and eliminating those analytes that were not detected in any samples in a given medium, the analytical data were evaluated on the basis of quality witt. respect to sample quantitation limits, laboratory qualifiers and codes, and blanks.

Following the elimination of unreliable data, analyte concentrations were compared to appropriate background levels. Naturally-occurring nonradioactive inorganic and radionuclide analytes in soil and groundwater were eliminated as COPCs if the maximum detected concentration from the unit sample was less than twice the mean background concentration. Those chemicals exceeding background concentrations were then compared to appropriate human and ecological risk-based concentrations. Finally, a screen for essential human nutrients and frequency of detection was performed. The chemicals and radionuclides remaining following the screening process were considered COPCs.

\section{Human Health Risk}

Potential human exposure routes were identified for each medium based on a specific receptor and anticipated activity at an exposure point. Exposures to radionuclides and nonradioactive contaminants present or modeled in air (dust and vapor), soil, homegrown produce, surface water, sediments, and groundwater were evaluated. Radiological exposures also were evaluated for direct radiation.

Toxicity information used to derive risk estimates from estimated chemical intakes was first obtained from the Integrated Risk Information System (IRIS). If values were not available from IRIS, the Health Effects Assessment Summary Tables (HEAST) or the Superfund Health Risk Technical Support Center-Environmental Criteria and Assessment Office were consulted. The human cancer risk from exposure to contaminants was expressed as the increased probability of developing cancer over a 70-year lifetime. The potential for adverse non-carcinogenic human 
health effects was expressed as a hazard index, the sum of chemical-specific hazard quotients.

Exposure point concentrations were calculated or modeled, as appropriate, and used to estimate potential risks. Carcinogenic risks and noncarcinogenic hazard indices for the exposure scenarios and receptors described above were then calculated and compared to EPA risk guidelines. To interpret the risk characterization results, a lisk of less than one excess cancer in one million people $\left(1 \times 10^{-6}\right)$ is not considered a potential human health concern by EPA. Additional cases of cancer resulting from risk less than $1 \times 10^{-6}$ cannot be distinguished in an exposed population. The NCP states that acceptable exposure levels are generally concentration levels that represent an excess upper bound lifetime cancer risk between one in ten thousand $\left(1 \times 10^{-4}\right)$ to one in one million $\left(1 \times 10^{-6}\right)$. EPA Region IV and SCDHEC have generally indicated that risks falling within this range should be evaluated to determine if risk reduction is feasible. Risks greater than one excess cancer in ten thousand pcople $\left(1 \times 10^{-4}\right)$ typically represent risks that will require remedial action in risk management decisions (EPA 1991e). Accordingly, those COPCs contributing significantly to risk (risk greater than $1 \times 10^{-6}$ ) for a pathway with a total risk greater than $1 \times 10^{-4}$ ) are identified as chemicals of concern (COCs) (EPA, 1994b).

Noncarcinogenic effects were evaluated by comparing an estimated exposure level (dose) over a specified time period (e.g., lifetime) with a reference dose (RfD) derived for a similar exposure period. To evaluate the noncarcinogenic effects of exposure to contaminants, the hazard quotient (HQ) is calculated. The HQ is the ratio of the estimated dose to the RfD. An HQ was calculated for each contaminant. There is a level of exposure (i.e., the $\mathrm{RfD}$ ) below which it is unlikely for even sensitive populations to experience adverse health effects. If the estimated dose exceeds the $\mathrm{RfD}$ (i.e., if the HQ exceeds 1), there may be concern for potential noncarcinogenic adverse health effects. The HQs for individual chemicals are summed for each exposure pathway to create a pathway-specific hazard index (HI) for each exposure scenario. Generally, the more the HI exceeds 1 , the greater the concern for potential adverse health effects.

Four areas (soils at the BRP 231-F/1F area, soils at the RP 231-2F, media in the ephemeral wetland area and general area groundwater) were evaluated under current and future land use scenarios. These land use scenarios are discussed in the following paragraphs.

For current land use (a current on-unit visitor), risks and hazards were calculated for exposure to soil, air, surface water and sediment. The total risks and hazards from all media did not exceed the EPA targets for cancer risk of $10^{-4}$ or a hazard of 1.0, indicating that negligible adverse health effects are expected for the current on-unit visitor. 
Future hypothetical on-unit residents were assumed to be exposed to soil, air, groundwater, homegrown produce, and surface water and sediment in the ephemeral wetland area. For future residents at the BRP 231-F/1F area, a total risk of $2 \times 10^{-4}$ and a hazard index of 3.0 were calculated. These hazards and risks result primarily from exposure to groundwater. The chemicals and radionuclides resulting in the increased groundwater risks $\left(>10^{-6}\right)$ include carbon tetrachloride, dichloromethane, tetrachloroethylene, arsenic, iodine-129, radium-226, radium-228, strontium-90, technetium-99 and tritium. Iodine-129 and technetium- 99 were not detected in groundwater, and were included through modeling of future soil leachability to groundwater. The highest concentration of dichloromethane was detected in an upgradient well. The chemicals resulting in a hazard index greater than 1.0 inciude carbon tetrachloride, arsenic, cadmium and manganese. Potential exposure to soils in the BRP 231-F/1F area, media in the ephemeral wetland area and homegrown produce did not result in risks in excess of $1 \times 10^{-4}$ or hazards in excess of 1.0 for hypothetical future residents.

For hypothetical workers exposed to soil, air, and groundwater at both the BRP 231-F/1F and RP 231-2F units, neither the cancer risk nor the hazard index exceeded the EPA targets of $10^{-4}$ or 1.0 , respectively, for any media. Consequently, negligible carcinogenic risks nor noncancer hazards are likely to be associated with workers exposed to media at these units.

For future residents at the RP 231-2F, a total risk of $2 \times 10^{-4}$ and a hazard index of 5.0 were calculated. These hazards and risks result from exposure to groundwater and soil. The increased risks from groundwater are the same as for BRPs 231-F/1F because the groundwater contamination is an area-wide phenomenon. The increased hazard index from soil was associated with exposure to PCB-1254.

The only increased cancer risks and hazards were associated with hypothetical future on-unit residents. The risks and hazards derived for current on-unit visitors and hypothetical future workers did not exceed the EPA targets. According to the Savannah River Site Future Use Project Report (January 1996), "residential uses oi SRS land should be prohibited." Additionally, the report recommends that the F-Area BRPs and RP be located within the future use "Industrial (Nuclear) Zone." Consequently, the only realistic future receptors will be workers.

Of the four areas of concern associated with the F-Area (soils at BRP 231-F/1F, soils at RP 231$2 \mathrm{~F}$, groundwater, and surface water and sediment in the ephemeral wetland area), only two were associated with cancer risks in excess of $1 \times 10^{-4}$ or hazards exceeaing 1.0: soils at RP 231-2F and general area groundwater. As discussed above, the increased risks and hazards were only 
associated with future residents, which are unlikely to be present at SRS, given the future land use plans for SRS. The current visitor and future worker, the only realistic receptors, should not be adversely effected by exposure to media at the unit.

\section{Ecological Risk}

The purpose of the ecological risk assessment component of the BRA was to evaluate the likelihood that adverse ecological effects are occurring or may occur as a result of exposure of ecological components to unit-related chemicals. The methodology used in this assessment was based on and complies with the intent of the Draft Ecological Risk Assessors' Guide for Evaluation of Waste Units on the Savannah River Site (WSRC, 1993), the Framework for Ecological Risk Assessment (EPA, 1992f), and the Risk Assessment Guidance for Superfund, Volume II, Environmental Evaluation Manual (EPA, 1989b).

Four areas of concern are associated with the F-Area BRPs Operable Unit: soils at BRPs 231$F / 1 F$, soils at RP 231-2F, surface water and sediment associated with the ephemeral wetland area, and general area groundwater. For the ecological assessment, only soils, surface water and sediment were quantitatively evaluated: groundwater is not a primary media of concern for ecological receptors.

Ecological COPCs were identified following data evaluation and quality screening, screening of inorganics and naturally occurring radionuclides against unit-specific background levels, and screening against toxicological benchmarks. COPCs were present in soil from the BRPs 231F/1F, RP 231-2F, and the small ephemeral wetland (surface water) that receives limited runoff from the BRPs 231-F/1F. COPCs were identified in soil for both current and hypothetical future conditions, in which deeper soils may be excavated and spread on the surface.

An ecological survey of the unit and surrounding area was conducted in January 1994 for the purpose of characterizing existing habitats and mapping vegetative communities. Seven major vegetative community types were identified within the study area. Vegetative patterns were found to be dependent on topography, soil type, moisture, and disturbance activities. Observations of animal species inhabiting the F-Area BRPs and surrounding study area were made during the January 1994 ecological survey and in three previous surveys. During these surveys, animal species were recorded based on actual sightings, trapping, and other evidence, such as tracks, scats, nests, and burrows. Animal species not recorded but likely to inhabit the study area were inferred based on their geographical ranges and habitat requirements. 
The ecological setting of the unit is not characterized by uniqueness or significance. There are no known endangered, threatened, or special concern species in the vicinity. The species that inhabit the unit are not rare in the region, and are not generally considered to be of special societal value. The area of the unit is small and the habitat is relatively low in diversity and productivity.

Based on characterization of the environmental setting and identification of potential receptor organisms, a conceptual site model was developed. Assessment endpoints were selected to represent ecological values to be protected with regard to exposure to unit-related chemicals, and measurement endpoints were selected that could be measured and related to the assessment endpoints. The proximate assessment endpoints chosen were protection of small mammal populations at the unit, as represented by the native oldfield mouse population, and protection of populations of amphibians that may reproduce in the ephemeral wetland and be exposed to surface water during the limited exposure period of reproduction and surface water availability, as represented by the southern toads found on the unit. The ultimate assessment endpoint chosen was protection of the overall health and biodiversity of the community of species inhabiting the vicinity of the unit. Measurement endpoints were chosen that could be extrapolated to the assessment endpoints. The measurement endpoints were preferentially chosen to represent chronic exposures and effects likely to impact population levels, such as increased mortality or decreased fecundity of individuals.

Food chain intakes of COPCs (terrestrial) or concentrations of COPCs (aquatic) were derived for the receptors, and quotients were calculated to represent the ratio of estimated intake (terrestrial) or concentration (aquatic) to the intake or concentration at which toxicity is likely to occur. The threshold for ecological effects is generally considered to be a quotient of 1 , and greater quotients indicate greater likelihood of effects. Those COPCs with quotients exceeding 1 were further evaluated as part of the risk estimation in order to estimate the likelihood that they might pose risk to assessment endpoints.

The results of the risk estimation step of the risk characterization indicated that, although several metals in soil (barium, chromium, mercury, lead), and mercury in sediment, had quotients exceeding a value of 1 , only mercury actually posed more than minimal risk to assessment endpoints. All other COPCs for which toxicity data or Applicable or Relevant and Appropriate Requirements (ARARs) were available had quotients less than 1, indicating that they do not pose ecologically significant risk to assessment endpoints through direct exposure to RME concentrations in environmental media. 
Only mercury in soil at BRPs $231-\mathrm{F} / 1 \mathrm{~F}$ and in the wetland sediment was identified in the risk estimation step of the risk characterization as having the potential to pose risk to assessment endpoints for the F-Area BRP waste unit. Actual community-level ecological effects are highly unlikely given the small area of the unit, its limited biological productivity and diversity, and its minimal utilization as a food source for predators. As a result, the likelihood of unit-related chemicals causing an ecologically significant decline in populations of native mice at the unit was estimated to be low; the likelihood of ecologically significant effects on amphibian populations due to surface water contaminants at the ephemeral wetland was estimated to be negligible; and there was concluded to be no likelihood that ecologically significant impacts to the community of species in the vicinity of the unit will occur.

\section{Remedial Goal Options}

Chemical-specific remedial goal options (RGOs) are concentration goals for individual chemicals for specific media and land use scenarios at CERCLA sites. General sources of chemical-specific RGOs include: (1) concentrations based on ARARs, and (2) concentrations based on risk values produced from the risk assessment.

The COCs identified from the human health portion of the baseline risk assessment include several organics (carbon tetrachloride, dichloromethane, tetrachloroethylene), metals (arsenic, cadmium, manganese) and radionuclides (iodine-129, radium-226, radium-228, strontium-90, technetium-99, tritium) in groundwater and PCB-1254 in soil at RP 231-2F. These chemicals and radionuclides were evaluated to determine whether Remedial Goal Options (RGO) were appropriate. A comparison to Applicable and Relevant and Appropriate Requirements (ARARs). was completed to establish whether the risk drivers were present in concentrations that exceeded regulatory levels. Although a chemical or radionuclide may result in a calculated risk or hazard in the risk assessment, a comparison to ARARs is necessary to establish whether the concentrations exceed regulatory standards. Regulatory standards may be higher than risk-based standards because regulatory standards take into account the feasibility of achieving a risk-based goal. If a risk-based goal is unachievable, an achievable value is designated as the regulatory standard. RGOs were derived for those chemicals that exceeded the ARARs.

\section{Groundwater}

For groundwater, ARARs included Federal and State of South Carolina maximum contaminant levels (MCLs). Neither iodine-129 nor technetium-99 were detected in groundwater. Their inclusion as risk drivers in the BRA resulted from the modeling of soil leaching to groundwater. 
Given that they have not and are not detected in groundwater, a comparison to ARARs and derivation of an RGO are not appropriate.

The MCL for strontium-90 is $8 \mathrm{pCi} / \mathrm{L}$. The maximum concentration of $15.6 \mathrm{pCi} / \mathrm{L}$ of Sr-90 was . detected in the upgradient well, FBP-8D. Also, Sr-90 was only detected in one of three groundwater samples from that well out of 12 groundwater samples for all three rounds of samples from all wells. Given the low frequency of detection it is probable that the Sr-90 detected in groundwater may be a spurioius result. Alternatively, the Sr-90 concentration detected may be from an upgradient source since it was only detected in an upgradient well. Consequently, $\mathrm{Sr}-90$ is not considered a $\mathrm{COC}$ at this unit and derivation of an RGO is not appropriate.

For manganese, it should be noted that an MCL is not available, only a secondary MCL (SMCL). SMCLs account for organoleptic properties only, such as taste and smell, and do not reflect riskbased levels.

Following comparison of COCs to ARARs in groundwater, the following chemicals and radionuclides were considered COCs:

- carbon tetrachloride,

- dichlormethane,

- tetrachloroethylene, and

- manganese.

RGOs were derived for both residential and industrial exposure scenarios. Given that the risk drivers resulted in increased carcinogenic risk and hazards only in residents, residential RGOs were derived. However, given that the future land use at the BRP OU will likely be industrial (nuclear), industrial RGOs were derived to provide more realistic values for the unit. 
Soil

PCB-1254 was detected in only 1 of 8 samples in the 0 to $0.6 \mathrm{~m}(0$ to $2 \mathrm{ft})$ soil interval at $\mathrm{RP}$ 231-2F. The maximum detected value of PCB-1254 $(2.87 \mathrm{mg} / \mathrm{kg})$ was quantitatively evaluated for human health risk over the entire pit area, which likely overestimates the risk from PCBs at RP 231-2F. While an increased hazard was calculated for the hypothetical future on-unit residents, given the low frequency of detection and the low level of the maximum detected concentration, it is unlikely that PCBs are a unit-wide contaminant at levels that would cause concern for human health protection, but, rather represent an isolated area (or "hot spot") of contamination.

According to EPA ( $A$ Guide on Remedial Actions at Superfund Sites with PCB Contamination, August 1990), soil action levels for PCBs in soil are $1 \mathrm{mg} / \mathrm{kg}$ for residential land use and 10-25 $\mathrm{mg} / \mathrm{kg}$ for industrial land use. The $1 \mathrm{mg} / \mathrm{kg}$ action level for residential exposure was exceeded and, therefore, an RGO was derived for PCB-1254. According to the Savannah River Site Future Use Project Report (January, 1996), "residential uses of SRS land should be prohibited." Additionally, the report recommends that the F-Area BRPs and RP be located within the future. use "Industrial (Nuclear) Zone". Given that the most likely future land use will be industrial, however, the action level of $10-25 \mathrm{mg} / \mathrm{kg}$ for the industrial worker is more appropriate. The maximum detected concentration of PCB-1254 does not exceed the $10-25 \mathrm{mg} / \mathrm{kg}$ action level for industrial workers. Additionally, the PCB levels detected do not pose an unacceptable.hazard for future industrial workers.

\section{Ecological}

The only COC identified from the ecological portion of the baseline risk assessment was mercury in soil at BRPs 231-F/1F and in sediment at the ephemeral wetland. The results of the ecological assessment, however, indicated that although mercury in soil and sediment exceeded the effects threshold of 1.0, it did not pose an ecologically significant risk to assessment endpoint. An $\mathrm{RGO}$, therefore, was not estimated for mercury in the ecological-assessment. 
SECTION 1

INTRODUCTION 


\section{THIS PAGE INTENTIONALLY LEFT BLANK}




\subsection{INTRODUCTION}

This section provides a brief overview of the Savannah River Site (SRS) and a description of the F-Area Burning/Rubble Pits (BRPs) and Rubble Pit (RP) unit. This section also describes the objectives and scope of the baseline risk assessment (BRA) and provides an overview of the report organization. Additional site information can be obtained in the Resource Conservation and Recovery Act (RCRA) Facility Investigation/Remedial Investigation (RI) Work Plan for the unit (WSRC, 1992).

\subsection{Overview of the Site}

SRS is located principally in Barnwell and Aiken Counties near the city of Aiken, South Carolina (Figure 1-1). SRS is owned by the U.S. Department of Energy (DOE). Management and operating services are provided by Westinghouse Savannah River Company (WSRC). SRS historically produced tritium, plutonium, and other special nuclear materials for national defense. SRS also provided nuclear materials for the space program and for medical, industrial, and research efforts. Chemical and radioactive wastes are by-products of nuclear material production processes. Hazardous substances, as defined by the Comprehensive Environmental Response, Compensation, and Liability Act (CERCLA) and hazardous waste as defined by RCRA are currently present in the environment at SRS.

SRS manages waste materials that are regulated under RCRA. Certain SRS activities required operating or post-closure permits under RCRA. SRS received a RCRA hazardous waste permit from the South Carolina Department of Health and Environmental Control (SCDHEC) on September 30, 1987. On December 21, 1989, SRS was placed on the National Priorities List (NPL). In accordance with Section 120 of CERCLA, DOE entered into a Federal Facility Agreement (FFA) with the U.S. Environmental Protection Agency (EPA) - Region IV and SCDHEC to coordinate cleanup activities at SRS into one comprehensive strategy that fulfills RCRA Section 3004(u) and CERCLA assessment, investigation, and response action requirements. Appendix $\mathrm{C}$ of the FFA lists the F-Area BRPs as a RCRA/CERCLA unit.

Figures 1-2 and 1-3 show the location of the F-Area BRPs at SRS and their relative locations to other SRS facilities. The F-Area BRPs are comprised of two burning/rubble pits, BRPs 231-F/1F, and one rubble pit, RP 231-2F, located to the west of F Area (Figurc 1-3). These BRPs are permanently inactive RFI/RI units. The three inactive pits have been backfilled and covered with 5 to $8 \mathrm{~cm}(2$ to $3 \mathrm{in})$ of clay.

The F-Area Burning/Rubble Pits Operable Unit is comprised of two burning/rubble pits (BRPs), 
BRPs 231-F/1F, and one rubble pit (RP), RP 231-2F, located to the west of the F-Area industrial complex. The BRPs were constructed in 1951 for burning of construction debris. During the operating period from 1951 to 1973 , waste was typically burned on a monthly basis. Waste materials burned included paper, plastics, wood, rubber, rags, cardboard, oil degreasers, and drummed solvents. Waste burning was discontinued by October 1973 and the pits were backfilled and used as rubble pits until 1978. Waste materials placed as rubble included concrete, bricks, tile, asphalt, plastic, metal, empty drums, wood products, and rubber. The pits reached capacity in 1978 and were taken out of service. The three pits were filled to capacity, backfilled with a layer of soil, graded, compacted, mounded, and covered with 5 to $8 \mathrm{~cm}$ ( 2 to $3 \mathrm{in}$ ) of clay.

SRS records indicate that concrete, lumber, cement, fence and telephone poles, rip rap, brick, tile, wallboard, paneling, metal scrap and shavings, drums, electrical conduit, furniture, and firehose may have been disposed in RP 231-2F. No known records at SRS indicate that any radionuclides or hazardous chemicals were disposed in this pit, but the possibility of such disposal in this pit cannot be eliminated. Management controls dictated no disposal of any suspected radioactive material in the BRPs. Rather, suspected radioactivity-contaminated material was disposed of in the SRS Radioactive Waste Burial Grounds.

\subsection{Objective of the BRA}

This risk assessment provides information on baseline and future risks under the No Action Alternative to support the evaluation of closure options for the F-Area BRPs by determining potential risks to human health and ecological receptors. Specifically, the BRA assesses potential risk that may result from release of and exposure to radioactive and nonradioactive contaminants under reasonable maximum exposure (RME) conditions for possible current and hypothetical future land use scenarios and receptors. The RME represents the highest exposure that is reasonably expected to occur at the unit.

A potential exists for uncontrolled releases of contaminants to the environment from the BRPs. Contaminants could be released from these sources via infiltration and percolation through deep soils to groundwater, surface water runoff, vegetative uptake, and volatilization from soil or particulate emissions. Also, direct external radiation exposure or dermal exposure at the unit due to unit-related, geological, or site background radiation is possible.

This BRA identifies the primary sources/release mechanisms, environmental transport media, principal exposure points, and exposure routes/receptors at the F-Area BRPs. Potential human health risks and ecological impacts are documented for current and future land uses in the absence 
of remedial action, and preliminary remediation goals are evaluated. Information from the BRA will support identification of those areas where no further action, or selected remedial action, may be warranted. Figure 1-4 delineates the study area that was evaluated for human and ecological receptors.

\subsection{Unit Background}

The F-Area BRPs unit consists of two burning/rubble pits and one rubble pit (Figure 1-4). The two burning/rubble pits, BRP 231-F/1F, are contiguous units. An undisturbed clay berm, approximately $6.1 \mathrm{~m}(20 \mathrm{ft})$ wide, separated the two pits. Facility records indicate that the dimensions of BRP $231-\mathrm{F}$ are $83.8 \mathrm{~m}(275 \mathrm{ft})$ long by $18.9 \mathrm{~m}(62 \mathrm{ft})$ wide by $3.1 \mathrm{~m}(10 \mathrm{ft})$ deep. The dimensions of BRP 231-1F are $99 \mathrm{~m}(325 \mathrm{ft})$ long by $27.1 \mathrm{~m}(89 \mathrm{ft})$ wide by $3.1 \mathrm{~m}(10 \mathrm{ft})$ deep. The unit screening conducted in 1989 indicated that the waste disposal unit widths of each pit ranged from 12.2 to $15.2 \mathrm{~m}$ ( 40 to $50 \mathrm{ft}$ ), with waste depth ranges from 0.6 to $2.4 \mathrm{~m}$ ( 2 to 8 $\mathrm{ft})$. SRS records indicate that the dimensions of rubble pit RP $231-2 \mathrm{~F}$ are $154.1 \mathrm{~m}(508 \mathrm{ft})$ long by $76.2 \mathrm{~m}(250 \mathrm{ft})$ wide. No record of depth for the rubble pit was available. Unit reconnaissance conducted in January 1989 indicated that this pit is approximately $50 \mathrm{~m}$ (164 ft) long and $10.1 \mathrm{~m}$ $(33 \mathrm{ft})$ wide.

Unit screening was completed in November 1989. Screening data for BRPs 231-F/1F indicated the presence of metals (arsenic, cadmium, chromium, lead, mercury, nickel), semivolatile organic contaminants (components of waste oils or waste insecticides and phthalate ester plasticizers), and trace levels of radionuclide indicators (gross alpha, nonvolatile beta, total radium), tritium, and one polychlorinated biphenyl (PCB) species. Data from the SRS Groundwater Monitoring Program from 3Q84 through 2Q92 indicated exceedences of maximum contaminant levels (MCLs) for carbon tetrachloride, iron, lead, manganese, nitrate, radium, and trichloroethylene. Groundwater data from $3 \mathrm{Q} 84$ through $2 \mathrm{Q} 92$ were excluded from the risk assessment because it did not meet data quality requirements for use in BRAs. There was no evidence that hazardous substances were contained in RP 231-2F. However, sidegradient groundwater data indicated that manganese, nitrate, radium, and trichloroethylene exceeded MCLs. Therefore, additional soil and groundwater sampling were conducted as part of the F-Area BRPs RFI/RI Unit Assessment completed in 1993 . These data provide the basis for this BRA.

\subsection{Scope of the BRA}

The scope of this study is to develop risk information based on unit-specific data and to quantify associated risks to human and ecological receptors to support closure options for the F-Area 
BRPs. Data contained in previous reports were used to identify and screen chemicals of potential concern (COPCs) for the risk assessment. The COPC screening is focused primarily on the comparison of site concentrations to background levels and risk-based concentrations, as well as consideration of detection frequency. Exposure point concentrations are estimated for the COPCs and used to estimate potential exposure and risk for various receptors and exposure scenarios. These risks are then compared with EPA guidelines for exposure and risk. Chemicals of concern (COCs) were identified and discussed, and Remedial Goal Options (RGOs) were developed.

The "hypothetical future" in this assessment of risk is the immediate future (i.e., the next 30 years), as is typically evaluated. Not considered in this BRA is further dispersal of contaminants occurring over periods of time greater than 30 years. Contaminant concentrations currently present in the environmental media identified for this unit were assumed for each exposure scenario evaluated, including the future scenarios.

In accordance with EPA risk assessment guidance (EPA, 1989a), this BRA considers impacts that could occur if no remedial action is performed. It assesses impacts under both current and hypothetical future conditions. The BRA for the F-Area BRPs assumes that the current land use scenario includes the retention of existing institutional controls (e.g., access restrictions). The future land use scenario does not consider the existence of institutional controls.

The extent of measured chemical contamination at the unit is restricted to the areas sampled within and adjacent to BRPs 231-F/1F, RP 231-2F, the unit groundwater plume, and the small ephemeral wetland to the west of the BRPs. Unit-related contamination of soils located beyond the immediate vicinity of the pits or of surface water and sediment located in areas other than the ephemeral wetland has not been assessed. The combined area of the contiguous BRPs 231-F/1F is $0.4 \mathrm{ha}(1 \mathrm{ac})$, the area of RP $231-2 \mathrm{~F}$ is $0.05 \mathrm{ha}(0.1 \mathrm{ac})$, and the area of the ephemeral wetland is approximately $0.1 \mathrm{ha}(0.2 \mathrm{ac})$. Thus, the total unit area in which COPCs were detected is approximately 0.6 ha $(1.3 \mathrm{ac})$. Additional detail regarding exposure scenarios, contaminani sources, transport mechanisms, and exposure pathways is provided in Section 3.2.

\subsection{Report Organization}

The BRA was organized according to guidelines described in EPA's Risk Assessment Guidance for Superfund (EPA, 1989a).

- Section 2.0, "Data Collection," reviews data collected from the F-Area BRPs and surrounding areas, and describes data collection and analytical procedures. 
- Section 3.0, "Human Health Risk Assessment," has four subsections.

- Section 3.1 describes the chemicals of potential concern (COPC) selection criteria.

- Section 3.2 describes those physical features of the F-Area BRPs that affect the risk assessment, especially in terms of fate and transport of radionuclides and nonradioactive contaminants present at the unit. The section also summarizes site characteristics pertinent to the exposure assessment, identifies potentially exposed populations, defines primary exposure pathways, develops exposure point concentrations, and provides equations proposed for estimating intakes and doses.

- Section 3.3 discusses human health effects for each category of COPC, and presents quantitative toxicity values.

- Section 3.4 presents estimates of incremental risk to each receptor for each selected $\mathrm{COPC}$ and exposure pathway.

- Section 4.0, "Ecological Risk Assessment," has six subsections.

- Section 4.1 identifies COPCs at the unit.

- Section 4.2 describes the environmental setting, the potentially complete exposure pathways (based on a conceptual site model), and any ecological effects observed at the unit.

- Section 4.3 discusses the selection of endpoints related to the ecological values to be protected at the unit.

- Section 4.4 describes the spatial distribution of COPCs at the unit, the spatial and temporal distribution of ecological receptors, and the magnitude and patterns of exposure that may result from the overlap of these distributions.

- Section 4.5 includes an evaluation of the potential toxicity of each COPC in each medium.

- Section 4.6 integrates the results of the preceding elements of the assessment. It estimates risk with respect to the assessment endpoints, based on the predicted exposure to and toxicity of each COPC. 
- Section 4.6 integrates the results of the preceding elements of the assessment. It estimates risk with respect to the assessment endpoints, based on the predicted exposure to and toxicity of each COPC.

- Section 5.0, "Summary," has two subsections.

- Section 5.1 summarizes the human health and ecological risk assessments.

- Section 5.2 discusses the calculation of remedial goal options.

- The following appendices support information presented in the BRA:

- Appendix A discusses statistical methods used in the BRA.

- Appendix B discusses the soil leachability to groundwater.

- Appendix C discusses the modeling of soil COPC concentrations to derive air VOC and particulate concentrations.

- Appendix D contains the calculation tables for carcinogenic risk and noncarcinogenic hazards based on reasonable maximum exposure for current and future receptors.

- Appendix E contains toxicity profiles for COPCs.

- Appendix F illustrates the equations used for calculation of remedial goal options. 


\section{THIS PAGE INTENTIONALLY LEFT BLANK}




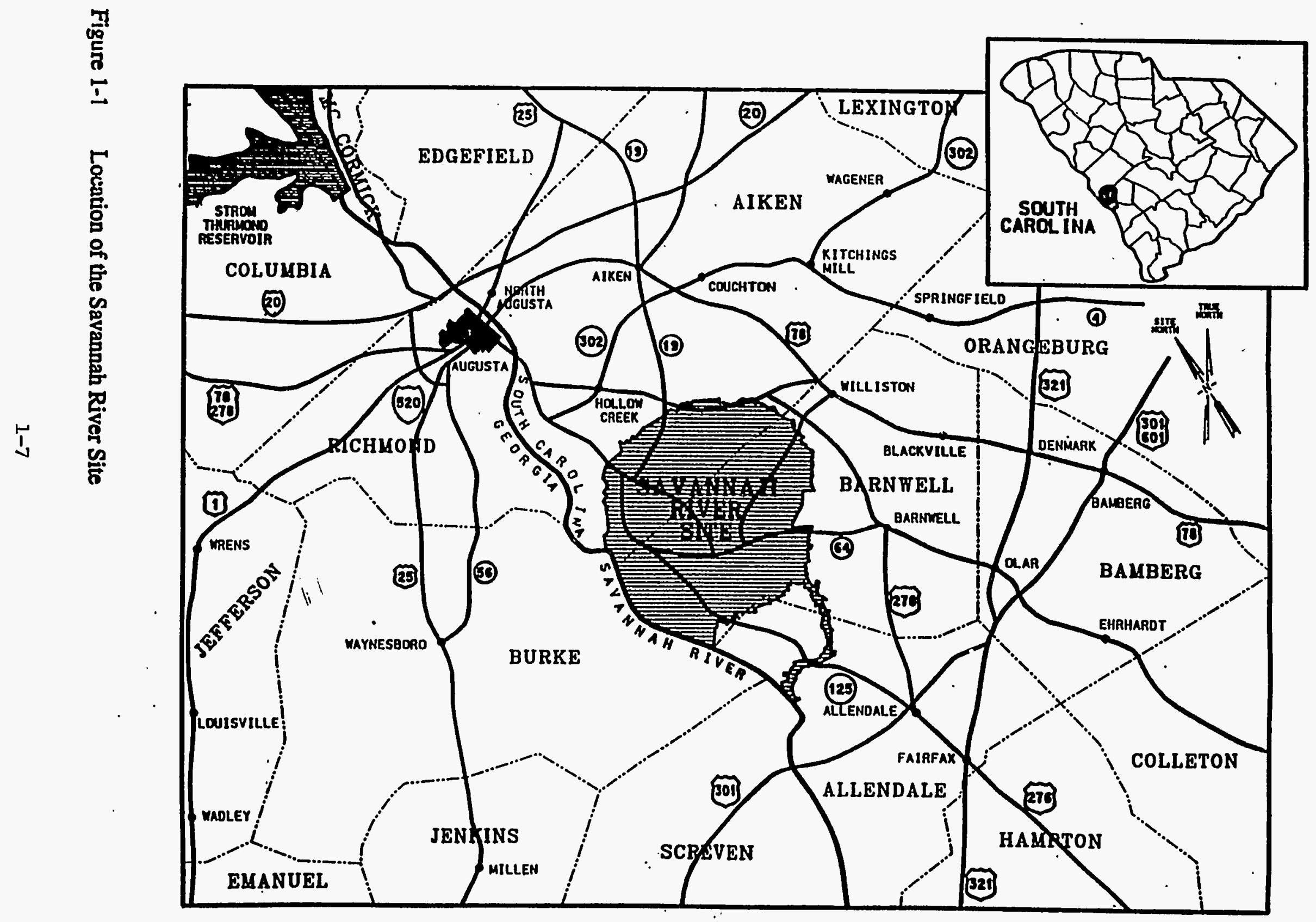

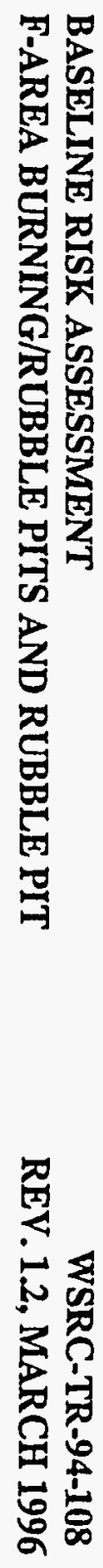




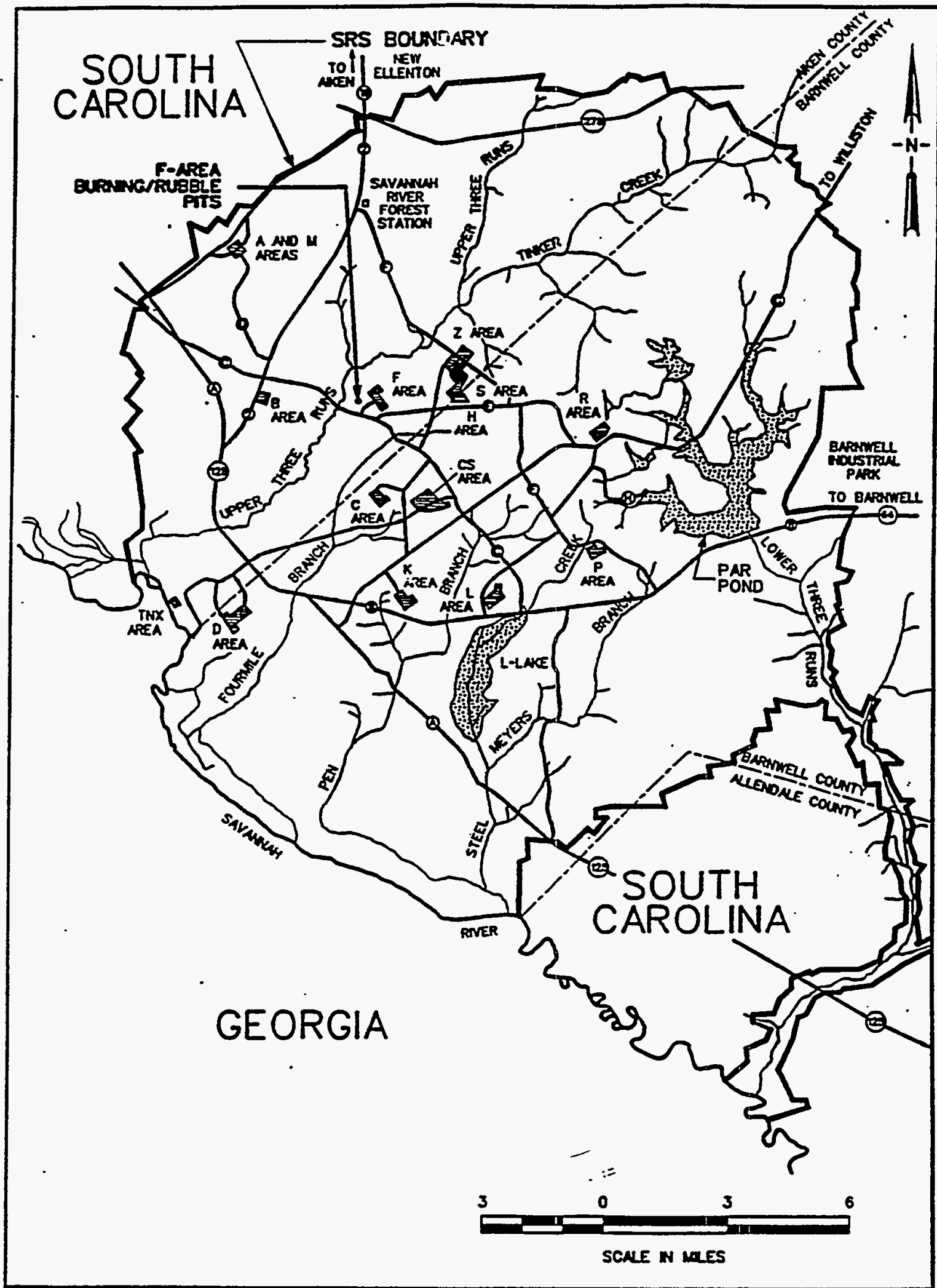

Figure 1-2 Location of F-Area Burning/Rubble Pits and Rubble Pit at the Savannah River Site 


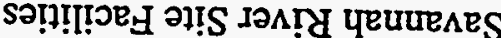

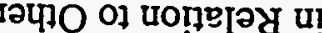

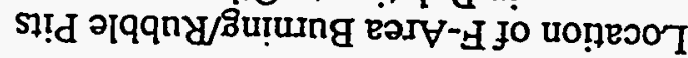

$\varepsilon-[$ әाnช!!]
6-I

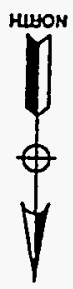

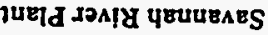

L86I SOSA :כวגnos

000'8b:1 गएक्ड

(1) -

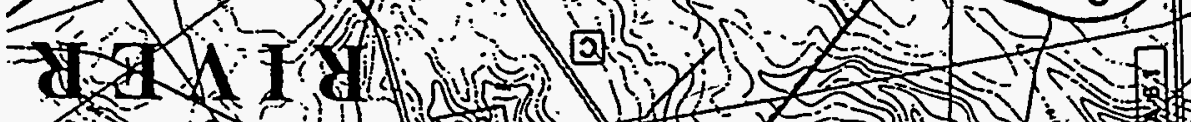
30146.

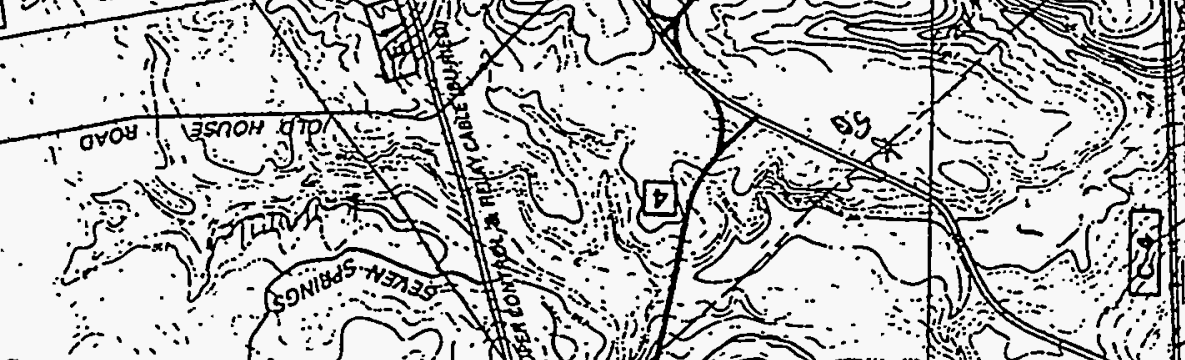

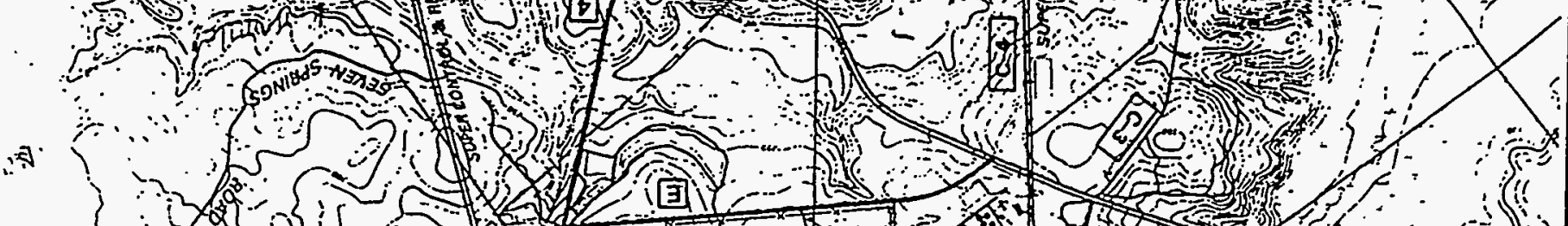

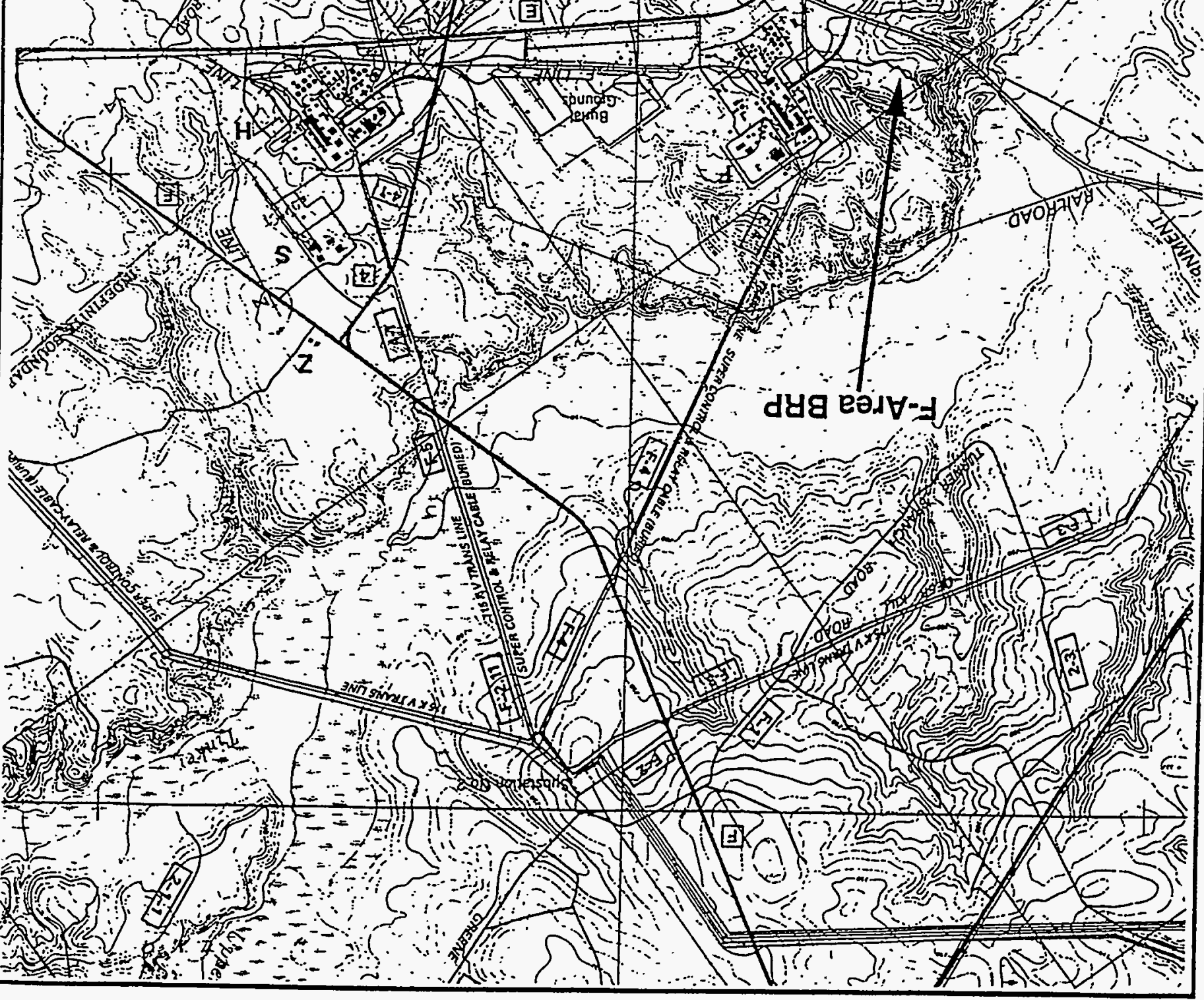


-... 
BASELINE RISK ASSESSMENT

F-AREA BURNING/RUBBLE PITS AND RUBBLE PIT
WSRC-TR-94-108

REV. 1.2, MARCH 1996

\section{THIS PAGE INTENTIONALLY LEFT BLANK}




\subsection{DATA COLLECTION}

Data used for the BRA were collected during the period May 4, 1993, through December 1, 1993, as part of the F-Area BRP RFI/RI Unit Assessment. Additional data were collected as part of the ongoing groundwater monitoring program at SRS during the period March 1993 through May 1993. Unit-specific background data, collected as part of the RFI/RI Unit Assessment, were used to screen Chemicals Present in Unit Samples (CPUSs). Unit screening data and data obtained from the SRS Groundwater Monitoring Program from 3Q84 through 2Q92 did not meet data quality requirements and, therefore, were not used in the BRA.

The objectives of the RFI/RI Assessment Program were to:

- Define the extent and magnitude of hazardous substance release, if any, from the RFI/RI units to other environmental media.

- Provide additional data for conducting a human health and ecological baseline risk assessment.

- Generate information necessary to conduct a Corrective Measures Study/Feasibility Study.

- Determine an adequate sample density sufficient for the needs of future studies, and determine if increased sampling density is necessary.

The Phase II RCRA Facility Investigation/Remedial Investigation Plan for the F-Area Burning/Rubble Pits (231-F/-1F) and Rubble Pit (231-2F) (U) (WSRC, 1992) describes the preliminary unit evaluation results, the unit screening results, and provides the unit assessment work plan, the quality assurance and quality control plan, and the data management plan. Locations of samples collected as part of the unit assessment and used in the BRA are identified in Figure 2-1.

Chemical analyses for soil, surface water, and sediment samples were performed by Roy F. Weston, Inc. Groundwater samples were analyzed by Roy F. Weston, Inc. and General Engineering Laboratories. All samples were analyzed in accordance with EPA-approved protocols. The detailed analytical results are contained in the F-Area Burning/Rubble Pits and Rubble Pit Data Summary Report (WSRC, 1994).

Validation and verification of the analytical data were performed as part of the RFI/RI data review process; therefore, the data are considered acceptable for this evaluation. Uncertainties associated with analytical data or the identification of chemicals of potential concern (COPCs) are 
discussed in Section 3.4.3. Field screening data are not considered appropriate for use in the quantification of risk by EPA, and therefore, were not included as part of the data set.

\section{$2.1 \quad$ Soil}

Soil sampling involved continuous sampling (i.e., no interval between samples) at 11 locations within the pit confines and through the bottom of the pit boundary (Figure 2-1). Two additional soil sample locations were selected adjacent to pit confines, including one background location. Soil sampling locations were chosen to adequately characterize and define the lateral and vertical extent of contaminant impact on soils from the F-Area BRPs. Soil analytes included semivolatile organic compounds (SVOCs) including polycyclic aromatic hydrocarbons (PAHs), volatile organic compounds (VOCs), total metals (arsenic, barium, cadmium, chromium, copper, manganese, nickel, silver, lead, mercury, selenium), radionuclide indicators (gross alpha, nonvolatile beta, total radium), total tritium, pesticides, polychlorinated biphenyls (PCBs), polychlorinated di-benzo dioxins and furans, radionuclides (potassium-40, iodine-129, plutonium238, plutonium-239, strontium-90, uranium-234, cesium-137, technetium-99, cobalt-60) and Target Compound List/Target Analyte List constituents (TCL/TAL).

For the BRA, soil data were aggregated into three groups: inside the two contiguous pits 231$\mathrm{F} / 1 \mathrm{~F}$, inside pit $231-2 \mathrm{~F}$, and background. This grouping of data was performed to provide relatively homogeneous areas of potential contaminant impact.

\subsubsection{Pit 231-F/1F}

Data for these two pits were aggregated due to the contiguous nature of the pits. Data from sample locations F2311-09, -10, -11, -12, -13, -14, -15, and -16 were included in the data analyses for this group. Samples were collected from the 0 to $0.15 \mathrm{~m}$ ( 0 to $0.5 \mathrm{ft}$ ) and 0.15 to 0.6

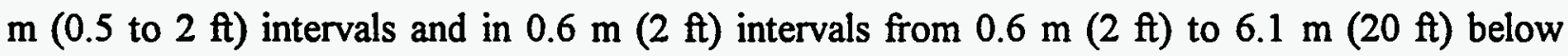
ground surface. Table 2-1 lists the sample identification numbers and sample collection dates for data from this group. Only those samples taken in the 0 to $0.15 \mathrm{~m}(0$ to $0.5 \mathrm{ft})$ and 0.15 to $0.6 \mathrm{~m}$ $(0.5$ to $2 \mathrm{ft})$ intervals were used to represent surface soil for the BRA. Samples from 0 to $1.2 \mathrm{~m}$ ( 0 to $4 \mathrm{ft}$ ) were used to evaluate a hypothetical future excavation scenario. Samples from $1.2 \mathrm{~m}$ $(4 \mathrm{ft})$ to the bottom of the pit were used to assess impacts to groundwater following leaching of contaminants from soil to groundwater. 


\subsubsection{Pit 231-2F}

Data from sample locations F2312-17, $-18,-19$, and -20 were included in the data analyses for this group. Samples were collected from the 0 to $0.15 \mathrm{~m}(0$ to $0.5 \mathrm{ft})$ and 0.15 to $0.6 \mathrm{~m}(0$ to $2 \mathrm{ft}$ )

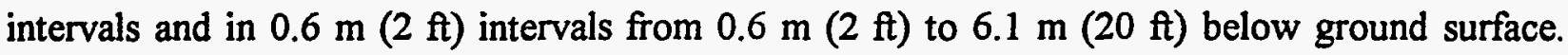
Table 2-1 lists the sample identification numbers and sample collection dates for data from this group. Only those samples collected in the 0 to $0.15 \mathrm{~m}(0$ to $0.5 \mathrm{ft})$ and 0.15 to $0.6 \mathrm{~m}(0.5$ to 2 ft) intervals were used to represent surface soil for the BRA. Samples from 0 to $1.2 \mathrm{~m}(0$ to $4 \mathrm{ft})$ were used to evaluate a future excavation scenario. Samples from $1.2 \mathrm{~m}(4 \mathrm{ft})$ to the bottom of the pit were used to assess impacts to groundwater following leaching of contaminants from soil to groundwater.

\subsubsection{Background}

Unit-specific soil background data were considered for use in this BRA. One sample location (F2312-23; Figure 2-1) was designated as a unit-specific background location, based on the criteria of its proximity to the units and its potential for not having been impacted by unit activities. The following intervals from sample F2312-23 were averaged to evaluate background conditions: $0-0.5$ foot, $0-2$ foot, 5-7 foot and 10-12 foot. These intervals were chosen to reflect those intervals evaluated in the risk assessment.

Table 2-2 provides information regarding background soil sample F2312-23: frequency of detection, mean background, and twice mean background, which was used in the evaluation of background for screening purposes.

\subsection{Groundwater}

Groundwater data were taken from two sources, the ongoing quarterly monitoring program and the unit assessment sampling effort. Analytes for groundwater samples included SVOCs including PAHs, VOCs, total metals (arsenic, barium, cadmium, chromium, copper, manganese, nickel, silver, lead, mercury, selenium), radionuclide indicators (gross alpha, nonvolatile beta, total radium), tritium, pesticides, PCBs, polychlorinated di-benzo dioxins and furans, radionuclides (potassium-40, iodine-129, plutonium-238, plutonium-239, strontium-90, uranium-234, uranium235 , uranium-238, cesium-137, technetium-99, cobalt-60) and TCL/TAL constituents. 


\subsubsection{Quarterly Monitoring Data}

A quarterly monitoring program is in place for the wells at the F-Area BRPs that were installed before the RFI/RI Unit Assessment. These wells were installed in 1984 and are screened in the water table aquifer. Data from the wells and collection dates listed below were used in the BRA. The locations of these wells are shown on Figure 2-1.

Well Collection Date

FBP-2A 3/20/93

FBP-4 5/22/93

\subsubsection{Remedial Investigation Wells}

Additional temporary groundwater monitoring wells were installed as part of the RFI/RI Unit Assessment. All wells are screened in the water table aquifer. The data from these wells were used in the BRA as listed below with collection dates. The locations of these wells are shown on Figure 2-1.

$\begin{array}{ll}\text { Well } & \text { Collection Date } \\ \text { FBP-5D } & 8 / 9 / 93 \\ & 9 / 14 / 93 \\ & 12 / 18 / 93 \\ \text { FBP-6D } & 8 / 11 / 93 \\ & 9 / 16 / 93 \\ & 12 / 19 / 93 \\ \text { FBP-8D } & 8 / 19 / 93 \\ & 9 / 16 / 93 \\ & 12 / 19 / 93 \\ \text { FBP-9D } & 8 / 10 / 93 \\ & 9 / 15 / 93 \\ & 12 / 26 / 93 \\ \text { FBP-12D } & 8 / 10 / 93 \\ & 9 / 14 / 93 \\ & 12 / 19 / 93 \\ \text { FBP-13D } & 8 / 10 / 93 \\ & 9 / 16 / 93\end{array}$




\subsection{3 . Background}

Wells FBP-4, FBP-8D, and FBP-12D are considered to be upgradient from the unit because groundwater flows to the west-northwest. Data from these wells were chosen to represent unitspecific background concentrations for groundwater. Table 2.3 provides information regarding the background groundwater samples: frequency of detection, mean background, and twice mean background, which was used in the evaluation of background for screening purposes.

\subsection{Surface Water}

Surface water sampling was conducted to cinnfirm the presence or absence of contaminants in the surface water near the pits: specifically, the wetland area to the west of the BRPs 231-F/1F. Analytes for surface water included SVOCs (including PAHs), VOCs, total metals, radionuclide indicators, pesticides/PCBs, and polychlorinated di-benzo dioxins and furans.

\subsubsection{Unit-Related}

Data from sample locations F2312-25, -26 , and -27 in the wetland area west of BRPs 231-F/1F were included in the analyses for surface water. These samples were collected on May 11, 1993.

\subsubsection{Background}

No unit-specific background samples were collected. No other representative data were available for surface water background.

\subsection{Sediment}

Sediment sampling was conducted to confirm the presence or absence of unit-related contaminants in the sediment of any surface water body near the pits: specifically, the wetland area to the west of the burning/rubble pits. Sediment sample analytes were the same as those for surface water with the addition of individual radionuclides.

\subsubsection{Unit-Related}

Data from sample locations F2312-25, -26 , and -27 in the wetland area west of BRPs 231-F/1F were included in the analyses for sediment (Figure 2-1). These samples were collected on May 11,1993 . Samples were taken in the 0 to $30.5 \mathrm{~cm}$ (0 to 12 inch) interval only. 


\subsubsection{Background}

No unit-specific sediment background samples were collected. However, based on evaluation of the soil type present in the wetland area and on available information (Rogers, 1994), the same background data set that was used with the soils was used as background for sediment. 


\section{FIGURES}




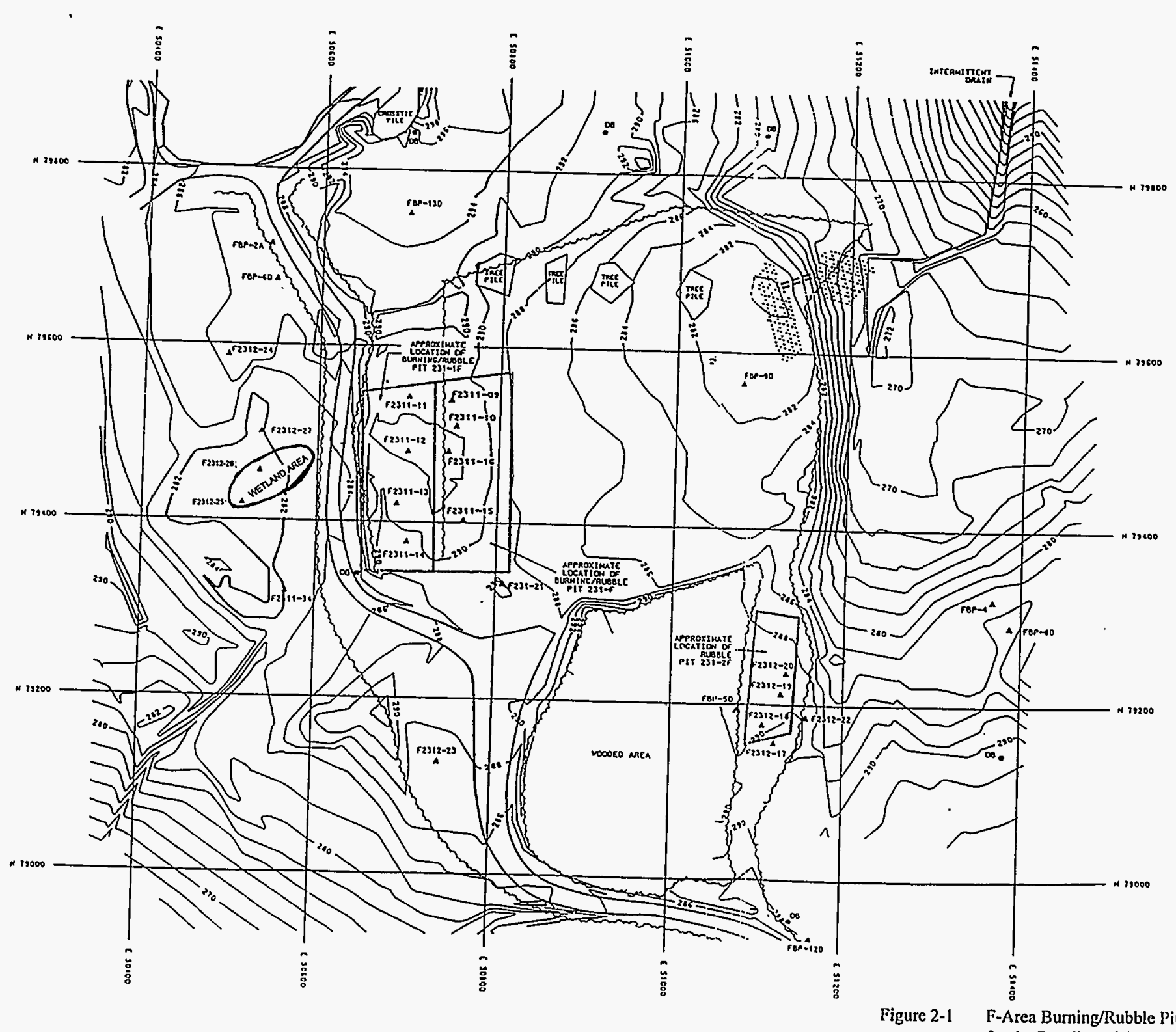


BASELINE RISK ASSESSMENT

- F-AREA BURNING/RUBBLE PITS AND RUBBBLE PIT
WSRC-TR-94-108

REV. 1.2, MARCH 1996

THIS PAGE INTENTIONALLY LEFT BLANK 
Table 2-1

Soil Data Collection

\begin{tabular}{|c|c|}
\hline $\begin{array}{c}\text { Sample Identification } \\
\text { Number }\end{array}$ & Collection Date \\
\hline $\begin{array}{l}\text { Pits 231F and 231-1F } \\
\text { F23109 } \\
\text { F231 09 } \\
\text { F231 10 } \\
\text { F231 10 } \\
\text { F231111 } \\
\text { F231111 } \\
\text { F231112 } \\
\text { F231112 } \\
\text { F231113 } \\
\text { F231113 } \\
\text { F231114 } \\
\text { F231114 } \\
\text { F231114 } \\
\text { F231115 } \\
\text { F231115 } \\
\text { F231116 } \\
\text { F231116 }\end{array}$ & $\begin{array}{l}5 / 11 / 93 \\
5 / 11 / 93 \\
5 / 12 / 93 \\
5 / 12 / 93 \\
5 / 17 / 93 \\
5 / 17 / 93 \\
5 / 14 / 93 \\
5 / 14 / 93 \\
5 / 17 / 93 \\
5 / 17 / 93 \\
5 / 12 / 93 \\
5 / 12 / 93 \\
5 / 13 / 93 \\
5 / 07 / 93 \\
5 / 07 / 93 \\
5 / 10 / 93 \\
5 / 10 / 93\end{array}$ \\
\hline $\begin{array}{l}\text { Pit 231-2F } \\
\text { F231217 } \\
\text { F231217 } \\
\text { F231218 } \\
\text { F231218 } \\
\text { F231219 } \\
\text { F231219 } \\
\text { F231220 } \\
\text { F231220 }\end{array}$ & $\begin{array}{l}5 / 19 / 93 \\
5 / 19 / 93 \\
5 / 18 / 93 \\
5 / 18 / 93 \\
5 / 19 / 93 \\
5 / 19 / 93 \\
5 / 20 / 93 \\
5 / 20 / 93\end{array}$ \\
\hline
\end{tabular}


Table 2.2

Mean Background Concentrations

in Soil

BRP-231F/F1 and RP 231-F2

Savannah River Site

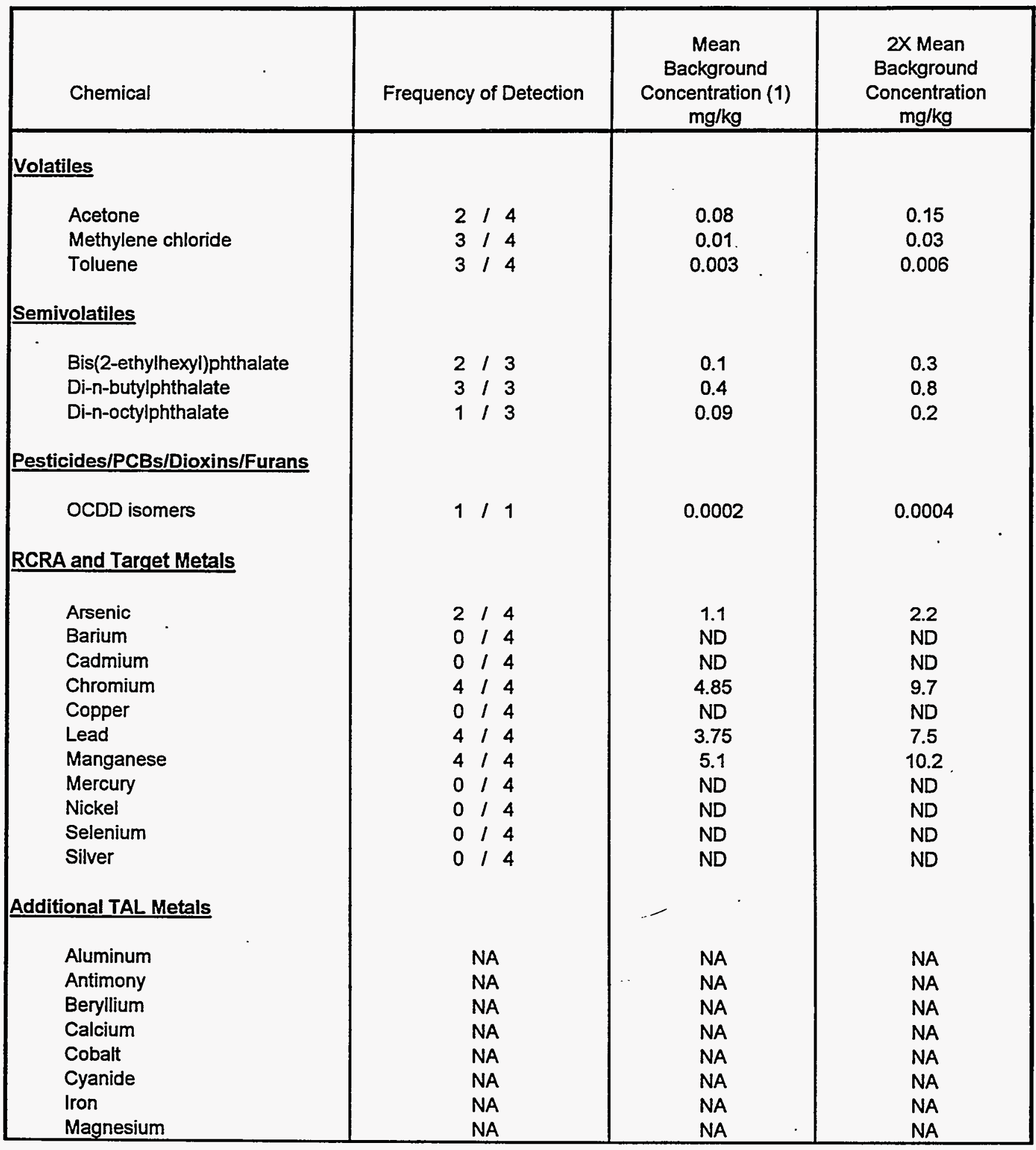


Table 2.2

Mean Background Concentrations

in Soil

BRP-231F/F1 and RP 231-F2

Savannah River Site

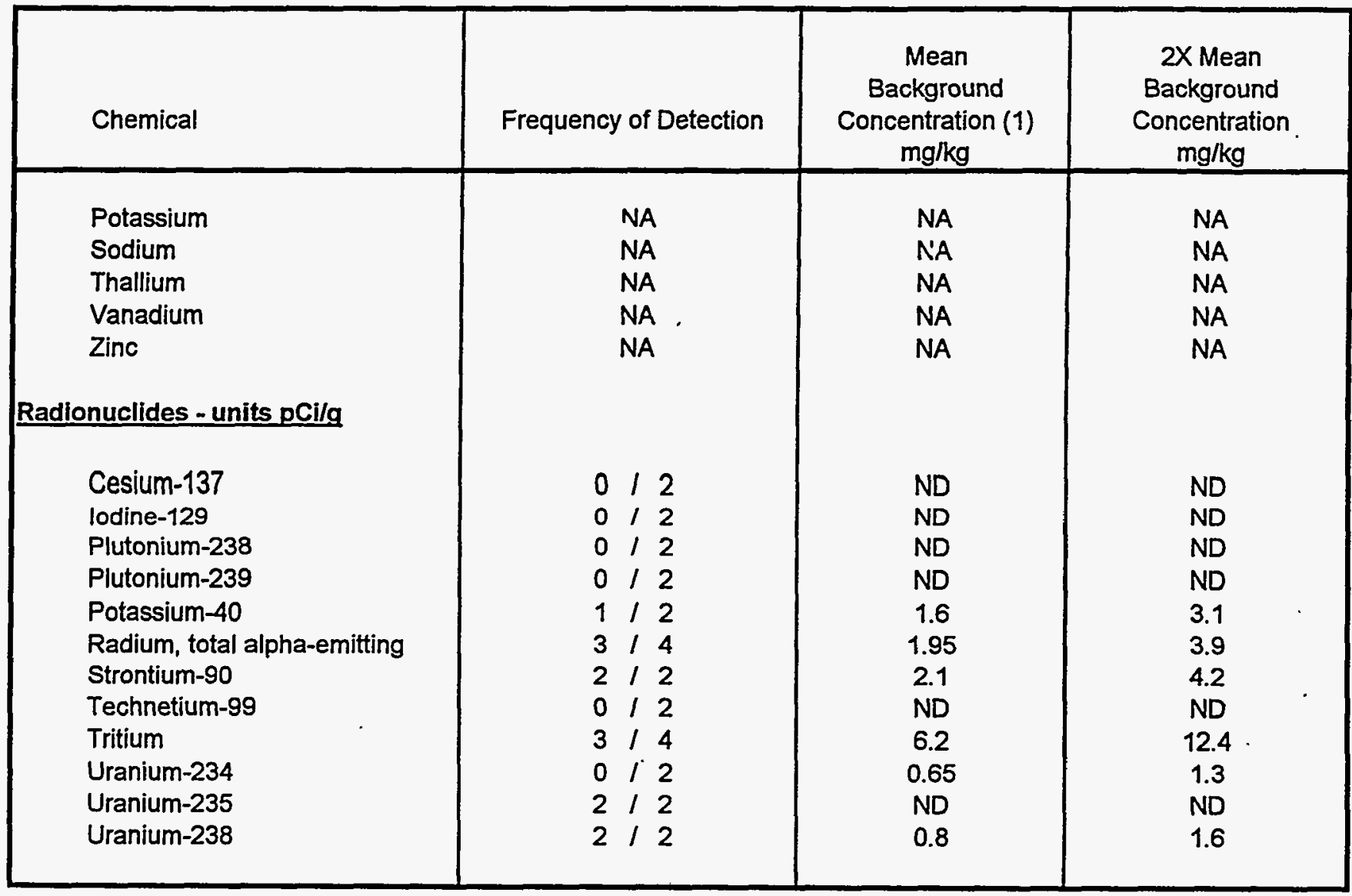

$N D=$ Not Detected $N A=$ Not Analyzed

(1) Mean background concentrations were determined by averaging the detected soil concentrations in sample F2312-23 at the following intervals: 0 to $0.5 \mathrm{ft}, 0.5$ to $2 \mathrm{ft}$, 5 to $7 \mathrm{ft}$ and 10 to $12 \mathrm{ft}$. Mean background concentrations were derived by averaging the detected concentrations. Nondetects were excluded from the average. 
Table 2.3

Mean Background Concentrations

in Groundwater

BRP-231-F/1F and RP 231-2F

Savannah River Site

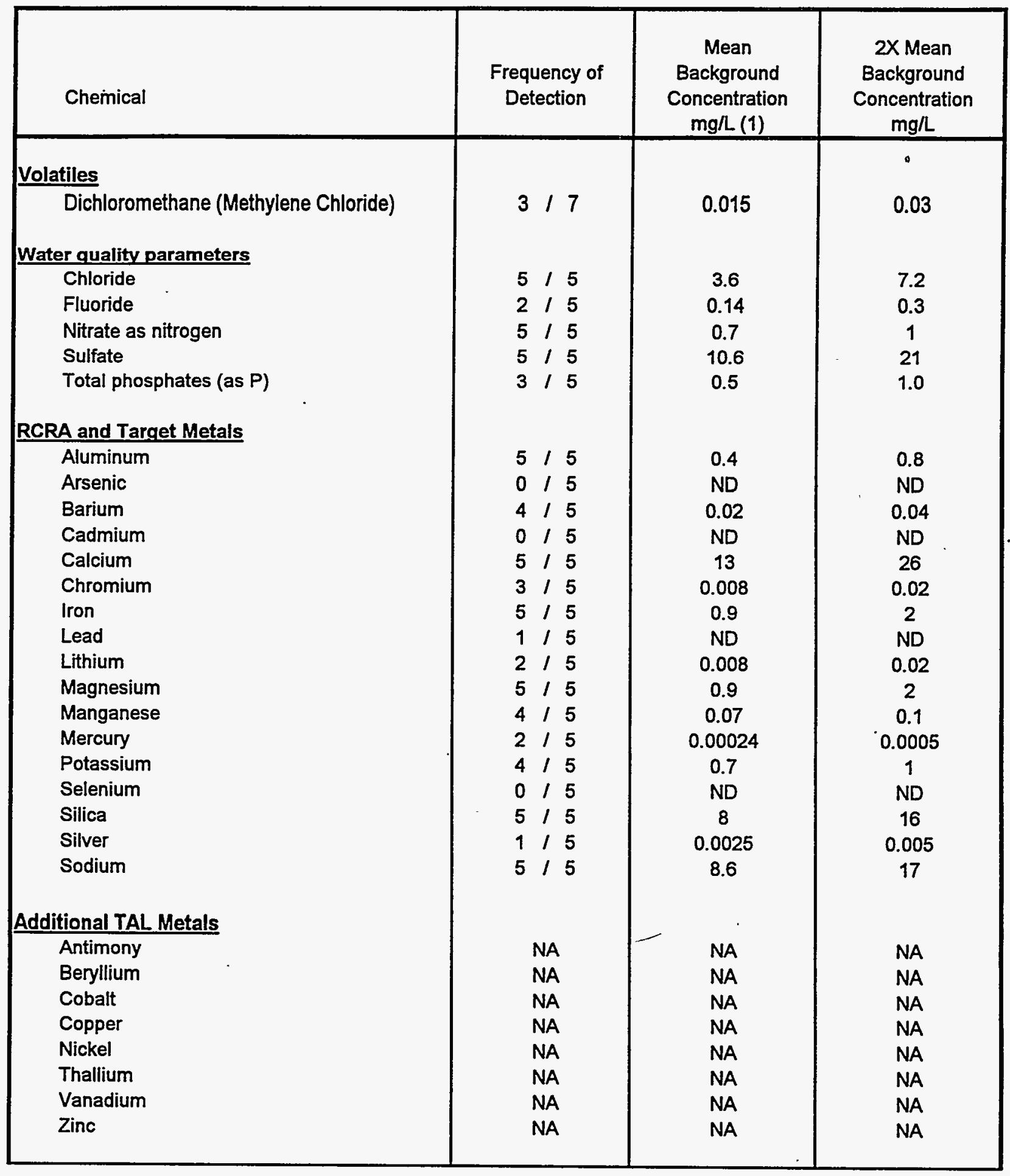


Table 2.3

Mean Background Concentrations

in Groundwater

BRP-231-F/1F and RP 231-2F

Savannah River Site

\begin{tabular}{|c|c|c|c|}
\hline Chemical & $\begin{array}{c}\text { Frequency of } \\
\text { Detection }\end{array}$ & $\begin{array}{c}\text { Mean } \\
\text { Background } \\
\text { Concentration } \\
m g / L(1)\end{array}$ & $\begin{array}{c}2 X \text { Mean } \\
\text { Background } \\
\text { Concentration } \\
\text { mg/L }\end{array}$ \\
\hline \multicolumn{4}{|c|}{ Radionuclides - units pCill } \\
\hline Radium-226 & $4 / 4$ & 0.7 & 1.5 \\
\hline Radium-228 & $4 / 4$ & 1.2 & 2.4 \\
\hline Strontium-90 & $1 / 4$ & 15.6 & 31.2 \\
\hline Uranium-233/234 & $1 / 4$ & 1.3 & 2.5 \\
\hline Tritium (pCi/ml) & $4 / 4$ & 4.5 & 9.0 \\
\hline
\end{tabular}

$\mathrm{ND}=$ Not Detected

$\mathrm{NA}=$ Not Analyzed

(1) Mean background concentrations were evaluated using the following background wells: FBP-4, FBP-8D and FBP-12D. Mean concentrations

were derived by averaging the detected concentrations. Nondetects were excluded from the average. 


\subsection{HUMAN HEALTH RISK ASSESSMENT}

\subsection{Identification of Human Health Chemicals of Potential Concern}

This section describes the methods and rationale for identifying human health COPCs at the unit. Both radionuclides and nonradioactive analytes were evaluated. Chemicals detected on-unit were evaluated against background concentrations, risk-based concentrations, nutrient essentiality, and frequency of detection. Uncertainties associated with the identification of human health COPCs are discussed in Section 3.4.3.

\subsubsection{Rationale and Criteria for Selection of COPCs}

Identified below are the locations where data were collected for soil, groundwater, surface water, and sediment within each area (see Figure 2-1). Soil data for RP 231-2F were evaluated independently of data for BRPs $231-\mathrm{F} / 1 \mathrm{~F}$, which were combined and evaluated.

$\underline{\text { Area }}$

BRPs $23^{\circ} 1-\mathrm{F} / 1 \mathrm{~F}$

RP 231-2F

General BRP Area

Wetland

Background

Background $\underline{\text { Media }}$

Soil

Soil

Groundwater

Surface Water

Sediment

Soil

Groundwater

\section{Sample Locations}

F2311-09, -10, -11, -12, -13, -14, $15,-16$

F2312-17, -18, -19, -20

FBP-5D, -6D, -8D, -9D, -12D, -13D

F23.12-25, -26, -27

F2312-25, -26, -27

F2312-23

FBP-4, -8D,-12D

Chemicals present in unit samples (CPUSs) were compiled for each media of concern and are presented in Table 3.1 through 3.11. Reported for each CPUS are frequency of detection (number of detects/number of analyses), range of sample quantitation limits, range of detection limits (range of laboratory reported detection limits) and the exposure point concentration used in the risk assessment. Gross alpha, nonvolatile beta, and total alpha emitting radium are indicator parameters and are not included as CPUSs. Statistical methods used to evaluate the data are presented in Appendix A and discussed further in Section 3.2.3.

CPUSs for surface soils in the BRP 231-F/1F and RP 231-2F areas were evaluated for the following intervals: 0 to $0.2 \mathrm{~m}(0$ to $0.5 \mathrm{ft})$ and $(0$ to $0.6 \mathrm{~m}$ ( 0 to $2 \mathrm{ft})$. Tables 3.1 and 3.2 present 
the statistical information for the 0 to $0.2 \mathrm{~m}(0$ to $0.5 \mathrm{ft})$ interval at BRP 231-F/1F and RP 231$2 \mathrm{~F}$, respectively, and Tables 3.3 and 3.4 present the statistical information for the 0 to $0.6 \mathrm{~m}(0$ to $2 \mathrm{ft}$ ) interval at BRP 231-F/1F and RP 231-2F, respectively.

As Tables 3.1 through 3.4 demonstrate, concentrations of contaminants detected in the 0 to $0.2 \mathrm{~m}$ ( 0 to $0.5 \mathrm{ft}$ ) intervals at both the BRP 231-F/1F and RP 231-2F units were within the same order of magnitude as those detected in the 0 to $0.6 \mathrm{~m}(0$ to $2 \mathrm{ft}$ ) interval. Consequently, because the 0 to $0.6 \mathrm{~m}(0$ to $2 \mathrm{ft})$ interval consists of a greater number of samples, this interval was screened and quantitatively evaluated in the risk assessment.

CPUSs for subsurface soils in the BRP 231-F/1F and RP 231-2F areas were evaluated for the 0 to $1.2 \mathrm{~m}(0$ to $4 \mathrm{ft})$ interval. This interval was used to account for potential excavation and redistribution of soils during hypothetical future development of the unit. Tables 3.5 and 3.6 present the statistical information for the 0 to $1.2 \mathrm{~m}(0$ to $4 \mathrm{ft})$ interval soils at BRP $231-\mathrm{F} / 1 \mathrm{~F}$ and RP 231-2F, respectively.

Soils located at a depth where direct human contact is unlikely (depths greater than $1.2 \mathrm{~m} \mathrm{(4 \textrm {ft } )}$ below ground surface) were evaluated for potential impacts on groundwater via leaching from soil to groundwater. The greatest concentration of contaminants is located within the confines of the pits, only soils located in the $1.2 \mathrm{~m}(4 \mathrm{ft})$ to bottom-of-the-pit interval were used to evaluate potential impacts to groundwater. The modeled groundwater concentrations were used in addition to the analytical groundwater data to evaluate potential impacts on future receptors exposed to groundwater. Tables 3.7 and 3.8 present the statistical information for the $1.2 \mathrm{~m}(4 \mathrm{ft})$ to bottom-of-the-pit interval for soils at BRP 231-F/1F and RP 231-2F, respectively. Table 3.9 presents the statistical information for the unit-wide groundwater.

Figure 3-1 shows the process for identification and selection of COPCs. After combining analytical data and eliminating those analytes not detected in any samples in a particular medium, the analytical data were evaluated on the basis of quality, with respect to sample quantitation limits, laboratory qualifiers and codes, and blanks. Data selected for use in the BRA included unqualified data and those data with qualifiers that indicated uncertainties in concentrations but not in compound identification. Analytical data with an " $R$ " (unreliable) qualifier were not selected for use in the BRA. Also not selected were data with qualifiers indicating that the analyte was detected in a laboratory blank at a level below the 10-times or 5-times rule for organics (for common laboratory contaminants and other compounds, respectively) or below the 5-times rule for pesticides and inorganics (EPA, 1989a). These data were indicated by a J/V result qualifier/analysis qualifier pair for all groundwater data and all soil, sediment, and surface water 
data, with the exception of the radionuclide analyses. The soil, sediment, and surface water radionuclide data were flagged with a $\mathrm{U} / \mathrm{V}$ result qualifier/analysis qualifier pair to indicate blank contamination below the appropriate rule.

For the human health evaluation,.. CPUSs in groundwater-and soils were screened against background concentrations, risk-based concentrations, nutrient essentiality, and frequency of detection. Given that background concentrations and human health risk-based concentrations were not available for sediment and surface water, a screening was not performed for these media. All contaminants detected in surface water and sediment were quantitatively evaluated in the human health risk assessment. Tables 3.10 and 3.11 present the statistical information for surface water and sediment, respectively.

The COPC screening was performed per SCDHEC (1995) direction. Although the COPC selection process differs from the new "Interim Supplemental Guidance to RAGS: Region 4 Bulletins, Human Health Risk Assessment" (EPA, 1995a) because background comparisons were conducted prior to risk-based concentration comparisons, the COPCs retained after these steps are the same, regardless of their order.

For soils and groundwater, concentrations were compared to appropriate background levels. Nonradioactive inorganic analytes and naturally occurring radionuclides in soil and groundwater were eliminated as COPCs if the maximum detected concentration was less than twice the mean background concentration (EPA, 1991d). Only naturally occurring radionuclides and nonradioactive analytes were eliminated as COPCs when screened against background for possible elimination. Naturally occurring radionuclides detected at the F-Area BRPs included: potassium-40, radium-226, radium-228, total radium, thorium-232, and uranium-234, -235 , and 238. Tables 3.12 and 3.13 present the background comparison for soil located in BRP 231-F/1F and in RP 231-2F, respectively, and Table 3.14 presents the background comparison for groundwater.

For chemicals in soils that were considered to be unit-related (i.e., were not considered to be indicative of background concentrations), a comparison to risk-based concentrations was performed. Tables 3.15 and 3.16 present a comparison of maximum detected soil concentrations in BRP 231-F/1F and RP 231-2F, respectively. A comparison of maximum concentrations was performed for exposure to soils following both ingestion and inhalation of contaminants in soils. Those chemicals that exceeded both criteria were retained as COPCs. Table 3.17 presents the comparable comparison for groundwater. 
Human nutrient essentiality was evaluated for both soil and groundwater for those inorganics that exceeded both the background and risk-based concentration screening. The essential nutrient screen was performed per SCDHEC (1995). For soils, manganese was evaluated at both BRP 231-F/1F and RP 231-2F (Table 3.18). To determine if manganese should be eliminated as a COPC, the maximum soil concentration was multiplied by an adjusted reasonable maximum exposure (RME) ingestion rate of $200 \mathrm{mg} / \mathrm{d}$ for the future on-unit resident child (EPA, 1991a). The adjusted ingestion rate is the product of the RME ingestion rate and the estimated fraction (1.0) assumed to be ingested from the contamination source. The estimated dose was compared to the recommended daily allowance (RDA) (National Research Council, 1989), Safe and Adequate Daily Intake (NAS, 1989) and the oral Reference Dose (RfD) (see Section 3.3). For nutrients with an $\mathrm{RfD}$, the nutrient was eliminated as a COPC if the estimated dose was below the maximum RDA/SADI and if the RDA/SADI was one-half or less of the oral RfD. If the estimated dose was below the RDA/SADI and the RDA/SADI was greater than one-half the RAD, the screening level was set at one-half the RfD (SCDHEC, 1995). For manganese, the screening level was set at one-half the oral $\mathrm{RfD}(0.17 \mathrm{mg} /$ day $)$.

For groundwater, the following inorganics were evaluated for human nutrient essentiality: calcium, iron, magnesium, manganese, potassium, sodium and total phosphates (as P) (Table 3.19). As detailed above for the screening process for soils, an estimated dose of the nutrients in groundwater was estimated and compared to the RDA, SADI and RfD values. The dose of nutrients in groundwater was estimated by multiplying the maximum groundwater concentration by a reasonable maximum exposure (RME) ingestion rate of $2 \mathrm{~L} / \mathrm{d}$ for the future on-unit resident (EPA, 1991a). The adjusted ingestion rate is the product of the RME ingestion rate and the estimated fraction (1.0) assumed to be ingested from the contamination source.

The final screening for COPCs was performed based on the frequency of detection of a chemical in a given media. Per Region IV guidance, only sample populations greater than 20 were considered for this screening step. Given that the only population that exceeded the 20-sample guidance was soils located in the 0 to $1.2 \mathrm{~m}(0$ to $4 \mathrm{ft})$ interval at BRP 231-F/1F, this was the only interval that was screened for frequency of detection (Table 3.20). Neither the soil other intervals at BRP 231-F/1F or RP 231-2F nor the groundwater had greater than 20 samples.

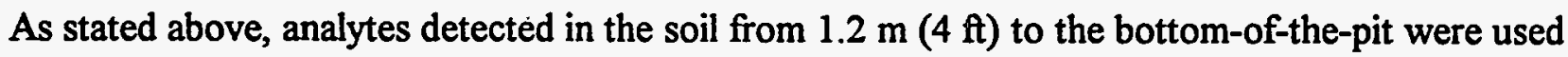
to model the soil leachability to groundwater pathway. The modeling approach consisted of a series of conservative screening steps and estimates (Appendix B). First, the RME soil concentrations were compared to generic soil screening levels with no dilution or attenuation. If the RME soil concentration was less than the generic screening level, the contaminant was 
eliminated from further consideration. For those contaminants that passed the generic screening, a conservative dilution attenuation estimate was made based on an approach that is consistent with the Simple Site-Specific Method of EPA's Soil Screening Guidance (EPA, 1994a). That is, parameters that are fairly well known and easily obtained were used in addition to reference values. Preliminary risks and hazard quotients were calculated for contaminants after this simple, site-specific screening estimate. For those contaminants that potentially posed a risk after the simple site-specific screening, less conservative, but more realistic, parameters were sometimes used to estimate a final groundwater exposure concentration. After the two screening steps, only 1,2-dichloroethylene, dichloromethane, tetrachloroethylene, delta-benzene hexachloride, heptachlor epoxide, cadmium, iodine-129, technetium-99, and tritium were considered for the soil leachability to groundwater pathway (Table 3.21). These chemicals were considered COPCs for groundwater.

Table 3.22 provides a list of the COPCs in each media at each unit (BRP 231F/1F and RP 2312F). COPCs for each area and medium are grouped by non-radiological and radiological classifications. These COPCs were quantitatively evaluated in the human health risk assessment.

\subsubsection{Chemicals of Potential Concern}

COPCs were identified following the methods and rationale described in Section 3.1.1. COPCs were identified independently for soils at the two areas: BRPs $231-\mathrm{F} / 1 \mathrm{~F}$ and RP 231-2F. COPCs for sediment and surface water were identified as all chemicals detected on-unit, given that no human health criteria or background information was available to perform a screening. Current groundwater pathways are not complete, the only completed groundwater pathways are for hypothetical future receptors. Soil data obtained from BRPs $231-\mathrm{F} / 1 \mathrm{~F}$ and RP 231-2F at depths

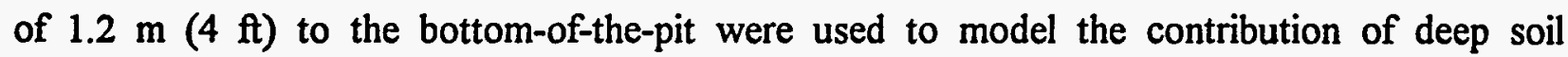
contaminants to groundwater. .The results of the modeling were used in conjunction with the analytical groundwater data to evaluate potential impacts to future groundwater users. A complete discussion of the methodology used to model the leaching of soil contaminants to groundwater is contained in Appendix B.

\section{Soils}

Sixty-six CPUSs were detected in the 0 to $0.6 \mathrm{~m}$ ( 0 to $2 \mathrm{ft}$ ) soil (Table 3.3) along with the three radionuclide indicators (gross alpha, nonvolatile beta, total alpha-emitting radium) at BRP 231F/1F. No COPCs were eliminated when screened against twice the mean background concentration (Table 3.12). Forty-three chemicals were eliminated as COPCs following the 
screening against risk-based concentrations: 35 nonradionuclides and 8 radionuclides (Table 3.15). Manganese, which exceeded both the background concentration and the risk-based concentration, was screened as an essential nutrient (Table 3.18). Manganese was retained as a COPC because it was present at a concentration that would result in a daily intake which exceeded the nutrient benchmark. A screening evaluation based on the frequency of detection was not performed since less than 20 samples were included in the 0 to $0.6 \mathrm{~m}(0$ to $2 \mathrm{ft})$ interval.

Sixty-eight CPUSs were detected (Table 3.5) along with the three radionuclide indicators (gross alpha, nonvolatile beta and total alpha-emitting radium) in the 0 to $1.2 \mathrm{~m}(0$ to $4 \mathrm{ft})$ interval at BRP 231-F/1F. No COPCs were eliminated when screened against twice the mean background concentration (Table 3.12).. Forty-four. chemicals were eliminated as COPCs following the screening against risk-based concentrations: 36 nonradionuclides and 8 radionuclides (Table 3.15). Manganese, which exceeded both the background concentration and the risk-based concentration, was screened as an essential nutrient (Table 3.18). Manganese was retained as a COPC because it was present at a concentration that would result in a daily intake which exceeded the nutrient benchmark. A screening evaluation based on the frequency of detection was performed for the 0 to $1.2 \mathrm{~m}(0$ to $4 \mathrm{ft}$ ) interval at BRP 231-F/1F because greater than 20 analyses were available for inorganic constituents in this soil interval (Table 3.20). No COPCs were eliminated based on the frequency of detection analysis.

Thirty-eight CPUSs were detected in the 0 to $0.6 \mathrm{~m}$ ( 0 to $.2 \mathrm{ft}$ ) soil (Table 3.4$)$ along with the three radionuclide indicators (gross alpha, nonvolatile beta and total alpha-emitting radium) at $\mathbf{R P}$ 231-2F. Arsenic was eliminated as a COPC when screened against twice the mean background concentration (Table 3.13). Twenty-five chemicals were eliminated as COPCs following the screening against risk-based concentrations: 19 nonradionuclides and 6 radionuclides (Table 3.16). Manganese, which exceeded both the background concentration and the risk-based concentration, was screened as an essential nutrient (Table 3.18). Manganese was eliminated as a COPC because it was present at a concentration that would not result in a daily intake which would exceed the nutrient benchmark. A screening evaluation based on the frequency of detection was not performed since less than 20 samples were.included in the 0 to $0.6 \mathrm{~m}$ ( 0 to $2 \mathrm{ft}$ ) interval.

Thirty-seven CPUSs were detected (Table 3.6) along with the three radionuclide indicators (gross alpha, nonvolatile beta and total alpha-emitting radium) at the 0 to $1.2 \mathrm{~m}(0$ to $4 \mathrm{ft})$ interval at $R P$ 231-2F. Arsenic was eliminated as a COPC when screened against twice the mean background concentration (Table 3.13). Twenty-four chemicals were eliminated as COPCs following the screening against risk-based concentrations: 19 nonradionuclides and 5 radionuclides (Table 
3.16). Manganese, which exceeded both the background concentration and the risk-based concentration, was screened as an essential nutrient (Table 3.18). Manganese was eliminated as a COPC because it was present at a concentration that would not result in a daily intake which would exceed the nutrient benchmark. A screening evaluation based on the frequency of detection was not performed since less than 20 samples were included in the 0 to $1.2 \mathrm{~m}$ ( 0 to $4 \mathrm{ft}$ ) interval.

\section{Groundwater}

Thirty-four analytes were detected in the groundwater beneath the F-Area BRPs (Table 3.9), along with two indicators of groundwater quality (silica and sulfate) and the three radionuclide indicators (gross alpha, nonvolatile beta and total alpha-emitting radium). Chloride, fluoride, lithium, mercury, silver, and U-233/234 were eliminated as COPCs when screened against twice the mean background concentrations (Table 3.14). Eight chemicals were eliminated as COPCs following the screening against risk-based concentrations: 8 nonradionuclides (Table 3.17). The following essential nutrients were eliminated based on the human nutrient essentiality screening: calcium, magnesium, potassium, sodium and total phosphates (as P) (Table 3.19). A screening evaluation based on the frequency of detection was not performed since less than 20 samples were available.

As discussed above, groundwater concentrations modeled from deep soils were also evaluated as COPCs.

\section{Surface Water}

Seven analytes were detected in the ephemeral wetland surface water along with two radionuclide indicators (gross alpha and nonvolatile beta) (Table 3.10). Neither background information nor risk-based concentrations for human health were available for surface water. Consequently, the COPC screening process was not performed and all detected contaminants were quantitatively evaluated in the human health risk assessment.

\section{Sediment}

Thirty-seven analytes were detected in the wetland sediment along with two radionuclide indicators (gross alpha and nonvolatile beta) (Table 3.11). Neither background information nor risk-based concentrations for human health were available for sediment. Consequently, the COPC screening process was not performed and all detected contaminants were quantitatively evaluated in the human health risk assessment. 


\subsection{Exposure Assessment}

This section addresses the environmental fate and transport of the COPCs identified in Section 3.1 and the potential pathways by which human populations (e.g., workers, visitors, and residents) could be exposed to radionuclides and nonradioactive analytes at or originating from the F-Area BRPs. In identifying primary pathways of exposure, current and hypothetical future land uses of the unit and surrounding area are considered. This section describes exposure scenarios, develops information on exposure pathways, estimates the concentration of the radioactive and nonradioactive COPCs at points of human exposure, and determines receptor intakes (doses). RME estimates are presented for radiation dose and chemical intakes within each scenario. Uncertainties of the exposure assessment are-discussed in Section 3.4.3.

\subsubsection{Characterization of Exposure Setting}

Exposure setting for the F-Area BRP is described briefly in terms of both the natural environment and local land use and demography. The setting is described in more detail in the RFI/RI Work Plan (WSRC, 1992). The following discussion provides information about the identification of exposure pathways and estimation of exposure factors for hypothetical human receptors who potentially could be exposed to contaminants.

\subsubsection{Environmental Setting}

Environmental factors that can affect human exposure to contaminated materials at the F-Area BRPs include topography, climate, ecological resources, geology, surface water, groundwater, and soil type.

\section{Topography}

The study area is located within the Upper Loam Hills Region of the Coastal Plain Province (Myers, Zahner, and Jones, 1986). The area is best described as a ridge which extends in a northwesterly direction toward Upper Three Runs Creek. Elevations range from $37 \mathrm{~m}$ (120 ft) mean sea level (msl) along Upper Three Runs Creek to $91 \mathrm{~m}$ (300 ft) msl near RP 231-2F.

The F-Area BRPs are located approximately $0.8 \mathrm{~km}(1 / 2$ mile) west of F Area and approximately $305 \mathrm{~m}$ (1000 ft) east of SRS Road C. The pits are surrounded by gravel access roads and flat, wooded terrain. BRPs $231-\mathrm{F} / 1 \mathrm{~F}$ and RP 231-2F are located at a ground surface elevation of approximately $88 \mathrm{~m}(290 \mathrm{ft}) \mathrm{msl}$ and are located on a gently sloping ridge between two unnamed 
tributaries of Upper Three Runs Creek. Surface water drains is both to the southwest and northeast.

\section{Climate}

The regional climate at SRS is characterized by warm, humid summers and mild winters. The average annual temperature is $18^{\circ} \mathrm{C}\left(64^{\circ} \mathrm{F}\right)$, with monthly average temperatures ranging from $7^{\circ} \mathrm{C}$ $\left(45^{\circ} \mathrm{F}\right)$ in January to $27^{\circ} \mathrm{C}\left(87^{\circ} \mathrm{F}\right)$ in July. The average annual precipitation at SRS is $122 \mathrm{~cm}$. Precipitation is distributed fairly evenly throughout the year, with the highest precipitation in summer $(36.1 \mathrm{~cm})$ and lowest in fall $(22.4 \mathrm{~cm})$. Snowfall is rare, occurring at an annual average of $3.0 \mathrm{~cm}$ (DOE, 1991).

Seventy-three percent of the average annual precipitation is lost to evapotranspiration (DOE, 1991). The remaining precipitation represents the amount available to surface streams for overland flow and to groundwater for recharge.

The annual average wind speed is $3.9 \mathrm{~m} / \mathrm{s}$ ( 8.7 miles/hour), and monthly average wind speeds range from $3.4 \mathrm{~m} / \mathrm{s}$ ( 7.6 miles/hour) from July through August to $4.6 \mathrm{~m} / \mathrm{s}$ (10.3 miles/hour) in February (DOE, 1991). The wind data measured at the 6-m level at SRS indicate that there is no predominant wind direction at SRS (DOE, 1991). Severe storms at SRS occasionally bring strong and gusty surface winds with speeds as high as $32 \mathrm{~m} / \mathrm{s}$ ( 72 miles/hour).

\section{Ecological Resources}

An ecological survey of the F-Area BRPs was conducted in January 1994 as part of the BRA. The survey was conducted to characterize and map existing terrestrial, aquatic, and wetland habitats. The characterization began with a delineation of vegetative cover types based on an interpretation of 1992 infrared aerial photographs. Within each cover type, dominant plant species were identified for three vegetative strata (overstory, sapling/shrub, and herbaceous). Scientific and common names of plant species used in the characterization are those described by Radford et al. (1968). Evidence of wildlife species (visual sightings, tracks, feces, etc.) present on the unit also were noted. A small isolated wetland approximately 0.1 hectare $(0.25$ acre $)$ in size was observed approximately $76 \mathrm{~m}(250 \mathrm{ft})$ west of the BRP.

The F-Area BRP provides habitat for numerous species of birds, reptiles, amphibians, and mammals. Federal and/or state-listed endangered species have been identified at SRS; however, none are known to occur at the F-Area BRP. 
A more detailed discussion of the ecological setting, including habitats, flora, and fauna, is contained in Section 4.1.1.2.

\section{Geology}

The F Area is located within the Upper Coastal Plain of the Aiken Plateau. SRS is in the process of updating the stratigraphy of the unit in relation to Coastal Plain sediments of the southeast; however, the regional geology for F Area described in this document follows that presented in detail in the RFI/RI Work Plan (WSRC, 1992) and in the Part B Permit Application (DOE, 1992).

The subsurface geology of the F Area is characterized by stratigraphic units commonly encountered in the Coastal Plain. The following is a list of those units from oldest to youngest:

Cape Fear Formation

Lumbee Group

- Middendorf Formation

- Black Creek Formation

- Peedee Formation

Black Mingo Group

- Ellenton Member of the Rhems Formation

- Snapp Member of the Williamsburg Formation

Orangeburg Group

- Congaree Formation

- McBean Member of the Santee Limestone Formation

Barnwell Group

- Tobacco Road Formation

- Dry Branch Formation

Upland Unit 


\section{Hydrogeology}

Groundwater in the F Area is controlled by a multilayer hydrologic system. The hydrogeology described below is detailed in the RFI/RI Work Plan (WSRC, 1992) and in Section E of the Part B Permit Application.(DOE, 1992). Geographic names for the hydrostratigraphic units have been proposed; however, the alpha-numeric hydrostratigraphic nomenclature will be adhered to in this document. The Cretaceous sediments of Aquifer Units 1A and 1B (Middendorf, Black Creek, and Peedee sands) constitute the most important regional aquifer (Aquifer System I), which is approximately $137 \mathrm{~m}(450 \mathrm{ft}$ ) thick. The top of the aquifer is approximately $23 \mathrm{~m}(75 \mathrm{ft})$ below MSL. Aquifer System I is separated from Aquifer System II by Confining System I-II (Peedee and Ellenton clays). Aquifer System II is composed of Aquifer units IIA (Congaree sands) and IIB (water table aquifer) separated by confining unit IIA-IIB ("Green Clay"). Aquifer Unit IIB includes the shallow water table aquifer at SRS, composed of sediments from the Tobacco Road, Dry Branch, and McBean Formations. The seüirients of Aquifer Unit IIB typically exhibit a wide variation in lithologic composition, both vertically and laterally.

There are four shallow water table (Aquifer IIB) groundwater monitoring wells in the FBP series that surround the burning/rubble pits and rubble pit. One of the four wells, FBP-4, is upgradient from the units. Well FBP-2A is downgradient from BRPs $231-\mathrm{F} / 1 \mathrm{~F}$, and wells FBP-3 and FBP-1 are sidegradient from BRPs 231-F/1F, and RP 231-2F, respectively. Potentiometric data from the four monitoring wells indicate that flow within the Water Table Aquifer (Aquifer Unit IIB) is to the west-northwest.

Groundwater flow in the deeper Aquifer Unit IIA is northwest towards Upper Three Runs Creek. Aquifer Unit IIA is separated from IIB by Confining Zone IIA-IIB (previously called the "Green Clay"). The regional aquifer (Aquifer Unit I) is separated hydraulically from Aquifer Unit IIA by Confining System I-II. Flow in this unit is toward the Savannah River.

A detailed description of the area hydrogeology is included in the RFI/RI Work Plan (WSRC, 1992).

\section{Surface Water}

Surface water runoff from BRP 231-F occurs in a west to southwesterly direction toward a gully located southwest of BRPs 231-F/-1F (Figure 3-2). Surface water runoff eventually discharges into Upper Three Runs Creek (UTRC). Runoff from RP 231-2F migrates in a northeasterly direction, also toward a gully. The gullies that drain the surface water runoff are characterized by a relatively steep gradient, and isolated, small pools. These drainage features empty into a small, 
unnamed tributary that receives much of its flow from F-Area National Pollutant Discharge Elimination System (NPDES) outfall F-001. Below the confluence with the gullies, flow in the unnamed tributary increases noticeably with distance downstream, possibly indicating groundwater discharge. The tributary is a rather small, shallow, gently-flowing stream with a sandy substrate. It flows through a forested ravine and empties into Upper Three Runs Creek at the north end of the study area. The unnamed tributary appears to be perennial, probably due to the NPDES discharge. Some of the surface runoff from BRP 231-1F flows in a westerly direction, eventually discharging into the ephemeral wetland area. Analytical data were available only for surface water and sediment in the ephemeral wetland area.

Upper Three Runs Creek is a relatively large, swiftly flowing stream with significant depth and discharge. It flows southwestward toward the Savannah River and is surrounded by riparian wetlands.

\section{Soils}

The F-Area BRPs are located within the Upper Loam Hills Region of the Coastal Plain Province (Myers et al., 1986). The area is best described as a ridge which extends in a northwesterly direction towards Upper Three Runs Creek. The ridge comprises approximately 81 hectares (200 acres) bounded to the west by Upper Three Runs Creek, to the south by Road C, to the east by $F$ Area, and to the north by an unnamed tributary of Upper Three Runs Creek.

Soil series present in the F Area include Troup and Lucy sands (TuE), Blanton sands (BaB), Udorthents (Ud), and Fuquay sands (FuB). The soil units range from sandy to sandy, clayey loam, with slopes ranging from 0 to 25 percent. Soils within these units are well to excessively drained. They have a low organic matter and nutrient content and a low available water capacity. The soils are considered fairly suitable for openland and woodland habitats and very poorly suited to wetland habitats.

Udorthents (Ud) are the predominant soil units in the area. They are well-drained sandy loam to sandy, clay loam soils with coarsely mottled shades of red, brown, yellow, and gray. Udorthents range in size from 2 to 8 hectares ( 5 to 20 acres) and have slopes of 0 to 10 percent. The surface layer consists of sandy clay loam to sandy loam, coarsely mottled shades of red, brown, yellow, and gray, $0.3 \mathrm{~m}(1 \mathrm{ft})$ to more than $1.8 \mathrm{~m}(6 \mathrm{ft})$ thick. The firm substratum below is sandy clay loam to loamy sand. The gray mottles increase with increasing depths. They are strongly acid to extremely acid. Permeability is slow to moderate. They have a low organic matter and nutrient 
content and are not suited to row crops, or timber production. The Udorthents are poorly suited to woodland wildlife and wetland. wildlife habitats. They are fairly suitable for openland wildlife.

Blanton Sands $(\mathrm{BaB})$ are somewhat excessively drained soils, occurring in broad upland swales and on low-lying ridges and side slopes of the Coastal Plain and Sand Hills. They are oblong, irregularly shaped soils, ranging in size from 4 to 81 hectares (10 to 200 acres). Slopes range from 0 to 6 percent. A dark grayish brown surface layer, approximately $17.8 \mathrm{~cm}(7 \mathrm{in})$ thick, overlays a subsurface layer, 17.8 to $121.9 \mathrm{~cm}$ ( 7 to 48 in) thick. A sandy loam subsoil extends to $203 \mathrm{~cm}$ (80 in) or more. The soil is moderately to strongly acid in the surface and subsurface layers. It is strongly or very strongly acid in the subsoil and moderately permeable. The Blanton sands are poorly suited for row crops, but are suitable for tir.tser production.

Fuquay sands (FuB) are well-drained grayish brown sands and sandy, clay loams. They are found on very broad ridges of the Upper Coastal Plain. Slopes range from 0 to 2 percent and are smooth and convex. These soils range in size from 2 to 20 hectares ( 5 to 50 acres). The surface layer is a grayish brown, approximately $20.3 \mathrm{~cm}$ ( 8 in) thick. A yellowish brown sand forms the subsurface layer that is 20.3 to $55.9 \mathrm{~cm}$ ( 8 to $22 \mathrm{in}$ ) deep. The subsoil layer is a yellowish brown sandy clay loam from 55.9 to $88.9 \mathrm{~cm}$ (22 to $35 \mathrm{in}$ ) and from 88.9 to $152.4 \mathrm{~cm}$ (35 to $60 \mathrm{in}$ ) it has mottled red, strong brown, and light gray colors with more than 5 percent of plinthite. Fuquay sands are moderately acid to very strongly acid, and permeability is low in the subsoil. The soil is suited for row crops and timber production.

Troup and Lucy sands (TuE) are well-drained soils located on the southeast bank of Upper Three Runs Creek. The areas are mostly long and narrow, approximately 2 to 12 hectares (5 to 30 acres) in size. The Troup surface and subsurface soil layer consists of a yellowish brown to light reddish brown sand that is about $137.2 \mathrm{~cm}$ (54 in) thick. The surface layer is moderately to very strongly acid. The subsoil is a yellowish red sandy clay loam about 137.2 to $205.8 \mathrm{~cm}$ (54 to 81 in) deep. It is strongly to very strongly acid and moderately permeable.

Lucy soils consist of a surface layer of brown sand, approximately $17.8 \mathrm{~cm}(7 \mathrm{in})$ thick with a subsurface layer of yellowish red loamy sand with a depth of 17.8 to $63.5 \mathrm{~cm}$ (7 to $25 \mathrm{in}$ ). A subsoil layer of red sandy loam 63.5 to $76.2 \mathrm{~cm}$ ( 25 to $30 \mathrm{in}$ ), or red sandy clay loam 76.2 to $157.5 \mathrm{~cm}$ (30 to $62 \mathrm{in}$ ) thick is also characteristic of Lucy soils. Soils in the surface and subsurface layer are moderately to very strongly acid and permeability is rapid. The subsoil is strongly or very strongly acid and permeability is moderate. 
Troup and Lucy soils are not suited to row crops because of the 15 to 25 percent slope. However, they are suited to timber production.

\subsubsection{Land Use and Demography}

\section{Land Use}

Less than 5 percent of the existing land in the area surrounding SRS is devoted to urban and developed uses (WSRC, 1991). Most of the urbanized development in the area has occurred in and around the cities of Augusta, Georgia, and Aiken, South Carolina. Agriculture accounts for 24 percent of total land use; forests, wetlands, water bodies, and unclassified land that is predominantly rural account for about 70 percent of the total land use. A projected 2 percent increase in the development of urban land surrounding SRS is expected by the year 2000.

Less than 5 percent of the total SRS land area is used by facilities engaged in the production of special nuclear materials. Reservoirs and ponds comprise approximately 13 square $\mathrm{km}(5$ square miles) of SRS. The remainder of the $777+$ square $\mathrm{km}(300+$ square mile) area is undeveloped.

The F-Area BRPs are located near a highly industrialized area within the SRS facility. They are surrounded by a gravel access road and flat, wooded terrain.

According to the Savannah River Site: Future Use Project Report (January, 1996), "residential uses of SRS land should be prohibited." Additionally, the report recommends that the F-Area BRPs and RP be located within the future use "Industrial (Nuclear) Zone." Consequently, future residential development of the unit is highly unlikely.

\section{Demography}

SRS is located approximately $40.2 \mathrm{~km}$ (25 miles) southeast of Augusta, Georgia, and $32.2 \mathrm{~km}$ (20 miles) south of Aiken, South Carolina. According to 1990 census data (Rand McNalley, 1992), the average population densities (in people per square mile) for the surrounding South Carolina counties are 111 for Aiken County, 36 for Barnwell County, and 28 for Allendale County, and for the surrounding Georgia counties are 228 for Columbia County, 524 for Richmond County, 25 for Burke County, and 21 for Screven County. The population within an $80.5 \mathrm{~km}$ (50-mile) radius of SRS is approximately 635,000 people.

The estimated population for the area in the year 2000 is projected to be 852,000 (WSRC, 1991). This estimate was calculated using the 1970 to 1980 growth rate of each county in an $80.5 \mathrm{~km}$ (50-mile) radius, assuming that the same growth rates would continue in the future. For counties 
that experienced a negative population growth rate between 1970 and 1980, the calculation assumed that no continued population decline would occur.

Additional demographic data is available for the six-county area that provides 90 percent of the SRS work force (DOE, 1994). These are Aiken, Allendale; Bamberg, and Barnwell counties in South Carolina, and Columbia and Richmond counties in Georgia. The population in those six counties increased 13 percent between 1980 and 1990, from 376,000 to 425,607, and is expected to increase to 474,820 by the year 2000 (DOE, 1992). A disproportionate share of the six-county population increase was concentrated in Columbia county, where the population increased more than 55 percent to 66,031 between 1980 and 1991 .

Currently, there are approximately 14,000 employees working within the SRS facility. Of these employees, approximately 2,000 work at the various facilities located in F Area.

\subsubsection{Identification of Human Health Exposure Pathways}

A complete exposure pathway consists of the following four elements:

(1) a source and mechanism of contaminant release to the environment;

(2) an environmental transport mechanism for the released contaminants;

(3) a point of contact with the contaminated medium; and

(4) a route of contaminant entry into the receptor at the exposure point.

An integration of contaminant sources and release mechanisms, fate and transport mechanisms, and exposure points and routes were evaluated to identify complete exposure pathways. If any of these elements was missing, the pathway was determined to be incomplete and was not considered further in the BRA.

A conceptual site model (CSM) was developed to illustrate potential contaminant sources, release and transport mechanisms, exposure pathways, and receptors at F-Area BRPs 231-F/1F, and RP 231-2F (Figure 3-3). Two land use scenarios were considered: current land use (industrial) and future land use (industrial and residential). Potential human receptors addressed are on-unit visitors, hypothetical future on-unit industrial workers and residents, and off-SRS residents.

A complete pathway exists when a receptor could be exposed to a contaminant source through one of the potential exposure routes. In the CSM diagram, open circle and closed circle 
designations were used to denote complete exposure pathways for qualitative and quantitative evaluation, respectively. A dash was used to denote incomplete pathways. Even though a complete exposure pathway may exist for a particular receptor, quantification of risk for the pathway may not be practicable due to the lack of toxicity factors or may not be warranted because of the expected minimal contribution to risk relative to other major pathways. The principal complete exposure pathways that were determined to be quantifiable are shown by a closed circle. An incomplete pathway occurs when any of the components of the pathway are missing. Figure 3-4 presents a chart identifying the exposure pathways for both human and ecological receptors by media of concern. Again, open and closed circle designations were used to denote complete exposure pathways for qualitative and quantitative evaluation, respectively.

\subsubsection{Contaminant Sources and Release Mechanisms}

At the F-Area BRPs, the primary contaminant source is waste buried in the burning/rubble and rubble pits. Based on available facility information, wastes which were disposed of in BRPs 231F/1F include paper, plastics, wood, rubber, rags, cardboard, oil, degreasers, drummed solvents, concrete, empty galvanized steel barrels, and cans (WSRC, 1992). Wastes were reported to have been burned in these pits on a monthly basis. Concrete, lumber, cement, fencing and telephone poles, rip rap, brick, tile, wallboard, paneling, metal scrap and shavings, drums, electrical conduit, furniture, and firehose material were reported to have been disposed in RP 231-2F.

Primary release mechanisms at the F-Area BRPs include (1) deposition of ash from waste burning activities (at BRPs 231-F/1F) and the inadvertent deposition of waste materials or wind-derived particulates onto surface soils outside the pits during disposal activities, and (2) infiltration and/or percolation of waste liquids (oil, degreasers, solvents) or contaminated leachate into deep soils.

Secondary contaminant sources at the F-Area BRPs are surface and deep soils. As noted earlier, surface soils were potentially contaminated from the direct deposition of waste materials or ash. Excavation and distribution of deeper contaminated soils onto surface soils also may result in the contamination of surface soils. Percolation or infiltration of liquid wastes or contaminated leachate from surface soil to deep soil and from deep soil to groundwater also are considered potential release mechanisms.

\subsubsection{Fate and Transport Mechanisms}

Following release from contaminant sources, contaminants may migrate in environmental media by any of several transport (secondary release) mechanisms, including: 
- Wind-blown suspension and dispersal of particulate contaminants or contaminants adsorbed to surface soil particles.

- Direct volatilization of volatile organic compounds from surface soil to air.

- Uptake of soil contaminants by terrestrial plants or uptake of contaminants in surface water and sediments by aquatic plants.

- Transport of soil contaminants by stormwater runoff to surface water and sediments.

- Discharge of groundwater into surface water and sediments.

In addition to the exposure pathways which result from the transport of contaminants by the above secondary release mechanisms, potential receptors also may become exposed directly to contaminants present in surface soils and groundwater.

\subsubsection{Exposure Points}

Exposure points are locations where receptors can potentially come into contact with contaminated media. If a probable route of intake into the receptor is available at the point of exposure, an exposure pathway is complete. As shown in Figure 3-3, there are six media (pathways) through which exposure to unit-related chemicals may occur: air (dust and vapor), soil, biota, groundwater, surface water, and sediments.

Surface soil or subsurface soils that have been excavated and redistributed onto the surface are potential exposure points for COPCs in soils. Exposure points for chemicals in groundwater and chemicals that may infiltrate/percolate from deep soils to groundwater exist during future household use of groundwater. Under current land use conditions, there is no direct exposure of receptors to groundwater.

Surface water and sediments become exposure points for chemicals in soil through erosion and transport by wind and water. Chemicals in groundwater also may migrate to surface water and sediments where they may be contacted by potential receptors.

COPCs present in surface soil, or in deep soils that have been excavated and redistributed onto the surface, around the F-Area BRPs could result in the airborne dispersion of particulate chemicals or chemicals adsorbed to surface soil particulates (windblown dust). Exposure points exist where chemicals are suspended in the air. Exposure points also exist at the F-Area BRPs for chemicals in air that have volatilized from surface soils and/or redistributed deep soils. 
Produce grown by hypothetical on-unit residents in the F-Area BRPs soils is a potential exposure point for chemicals in soil. Future excavation of soils below the surface soil depth of $0.6 \mathrm{~m}(2 \mathrm{ft})$ may result in greater chemical concentrations in surface soils, and potentially greater concentrations in produce tissue.

\subsubsection{Potential Receptors and Exposure Routes}

Potential receptors are those individuals that may come into contact with potentially contaminated media associated with the F-Area BRPs. The possible receptors under current land use conditions include on-unit visitors and off-unit residents. Possible receptors under hypothetical future land use conditions include an on-unit resident and on-unit indus'rial worker. Receptors are discussed in conjunction with potential exposure routes in the following paragraphs.

An exposure route is the means by which a receptor comes in contact with a contaminated medium, (e.g., by inhalation, ingestion, dermal contact, or external radiation). Probable exposure routes (i.e., potentially complete pathways) were identified for each receptor for each medium based on anticipated activities at the exposure point. Exposures to radionuclides and nonradioactive contaminants may occur through ingestion, inhalation, and dermal contact. Radiological exposure also may occur through external radiation.

Current populations that potentially could be receptors for exposure to chemicals associated with the F-Area BRPs include visitors to the unit and residents living outside the SRS boundaries. Visitors were considered to be individuals who visit the unit under current land use conditions on an infrequent or occasional basis, such as researchers associated with organizations that use SRS as an outdoor laboratory for scientific studies. Visitors are not expected to be involved in the excavation or collection of contaminated media. The potentially complete pathways by which an on-unit visitor could be exposed to chemicals in surface soil $(0$ to $0.6 \mathrm{~m}=0$ to $2 \mathrm{ft})$ are incidental ingestion, dermal contact, inhalation of dust or volatile organics in air, or through external radiation. Potentially complete pathways also exist for current on-unit visitors for exposure to chemicals as a result of incidental ingestion of wetlands sediment and dermal exposure to wetlands surface water and wetlands sediment. These pathways were quantitatively evaluated in the human health risk assessment.

Residents living outside SRS boundaries could potentially be exposed to unit-related chemicals through the inhalation of airborne chemicals in the form of vapor or dust (suspended particulates). However, the potential for transport of significant concentrations to residences beyond the SRS 
boundary is expected to be negligible considering their distances from the units [at least $6 \mathrm{~km} \mathrm{(4}$ $\mathrm{mi})$ ]. Therefore, exposure via the air pathway was not quantified for off-unit residents.

Incidental ingestion of or dermal contact with contaminants in surface water and sediments associated with Upper Three Runs 'Creek and the Savannah River also are potentially complete pathways for the off-unit resident, as are ingestion of, dermal contact with, and inhalation of VOCs from groundwater. In the event that contaminants do migrate to Upper Three Runs Creek, they could conceivably reach the Savannah River. Assuming an off-unit resident lives at a point nearest the confluence of the water bodies, the distance is approximately 8 miles. However, dilution and degradation of unit-related chemicals during transport, in conjunction with the travel time required for chemicals to migrate beyond SRS boundaries to a likely exposure point, would significantly reduce the potential for risk from these pathways relative to other principal pathways. Therefore, no pathways were quantitatively evaluated for off-unit residents.

According to the Savannah River Site: Future Use Project Report (January, 1996), "residential uses of SRS land should be prohibited." Additionally, the report recommends that the F-Area BRPs and RP be located within the future use "Industrial (Nuclear) Zone." Consequently, future residential development of the unit is highly unlikely. A hypothetical future residential exposure scenario, therefore, is presented for comparative purposes only: the more realistic future land use is industrial.

A hypothetical on-unit resident may potentially become exposed to chemicals in surface soil ( 0 to $0.6 \mathrm{~m}=0$ to $2 \mathrm{ft}$ ) through ingestion, dermal contact, inhalation of contaminated dust or volatile chemicals, or external radiation. Under hypothethical future land use conditions, an exposure pathway for an on-unit resident's exposure to chemicals in soil also may be complete as a result of the ingestion of homegrown produce, which has taken up contaminants from the soil. These pathways were quantitatively evaluated in the human health risk assessment.

Because institutional controls preventing the excavation of contaminated soils cannot be guaranteed for all time, the hypothetical future residential scenario also assumes the possible excavation of soils from depths of 0 to $1.2 \mathrm{~m}(0$ to $4 \mathrm{ft})$ and subsequent spreading of those soils on the surface as the result of construction activities. One and two-tenths meters is considered a reasonable depth for a residential contractor to excavate, for foundation footers, during typical home construction. A hypothetical future resident potentially could be exposed to either surface soils or the redistributed soils through the exposure routes described above. 
Exposure to contaminated surface water and sediment through dermal contact or incidental ingestion is a potential exposure pathway for the hypothetical future on-unit resident. The most likely receptor for this scenario was considered to be a child playing in the ephemeral wetland located near BRP 231-1F. Dermal contact with and ingestion of chemicals in groundwater and inhalation of volatile chemicals during showering with groundwater also are considered complete pathways for the hypothetical future on-unit resident (adult and child).

If future land use at the F-Area BRPs becomes commercial/industrial, workers could potentially be exposed to unit-related chemicals in surface soil $(0$ to $0.6 \mathrm{~m}=0$ to $2 \mathrm{ft}$ or 0 to $1.2 \mathrm{~m}=0$ to 4 $\mathrm{ft}$ ) thrcugh the inhalation of fugitive dust and volatile organics, accidental ingestion, dermal contact, and direct radiation. Exposure to unit-related contaminants also could occur from the ingestion of groundwater. Exposure to groundwater via dermal contact and inhalation of VOCs are not expected to be significant pathways of exposure for future workers. It is considered unlikely that the future workers will be involved in activities outside a controlled work area. Therefore, the future worker is not expected to come in contact with surface water and sediment.

\subsubsection{Exposure Point Concentrations}

To determine the estimated mean environmental concentration in media of concern, detected concentrations were evaluated along with nondetects at half the reported quantitation limit as a proxy concentration. Per EPA guidance (EPA, 1995a), soil and sediment data were assumed to be lognormally distributed and the data were transformed. For groundwater and surface water, data were evaluated for normality and the data were transformed if appropriate (i.e., if the data were considered to be lognormally distributed, the data were transformed). For both normally and lognormally distributed data, if the 95 percent upper confidence limit (UCL) on the arithmetic mean calculated for an analyte exceeded the maximum concentration detected, the maximum level was used instead of the 95 percent UCL. Appendix A discusses the statistical methodology used in the assessment of the data and the determination of appropriate exposure point concentrations

Exposure point concentrations were calculated based on the reasonable maximum exposure (an upper-bound conservative exposure case that is still within the range of possible exposures) for each complete pathway. Exposure point concentrations for the COPCs identified in Section 3.1 and in Appendix B were determined for surface soil, redistributed deep soils, groundwater (analytical and modeled values from deep soil leachability), and surface water and sediment in the ephemeral wetland (Tables 3.1 through 3.11 and Table 3.21). When analytical data were not available for a particular exposure point (inhalation of volatile organic compounds and airborne soil particulates), an appropriate modeling technique was used to estimate environmental 
each complete pathway. Exposure point concentrations for the COPCs identified in Section 3.1 and in Appendix B were determined for surface soil, redistributed deep soils, groundwater (analytical and modeled values from deep soil leachability), and surface water and sediment in the ephemeral wetland (Tables 3.1 through 3.11 and Table 3.21). When analytical data were not available for a particular exposure point (inhalation of volatile organic compounds and airborne soil particulates), an appropriate modeling technique was used to estimate environmental concentrations. Exposure point concentrations for particulates and VOCs in air (modeled values) associated with the F-Area BRPs are presented in Tables 3.23 through 3.26. The chemical concentrations (radionuclides only) present in homegrown produce were estimated using soil-toplant transfer factors (NRC, 1992).

Appendix A contains a detailed description of the statistical methods used in development of calculated concentrations. Appendix B contains predicted exposure point concentrations for groundwater derived from the modeling of deep soil contamination. Appendix $\mathrm{C}$ contains methodology for the modeling of volatile organic compounds and airborne particulates from soil.

\subsubsection{Soil}

As Tables 3.1 through 3.4 demonstrate, concentrations of contaminants detected in the 0 to $0.2 \mathrm{~m}$ ( 0 to $0.5 \mathrm{ft}$ ) intervals at both the BRP 231-F/1F and RP 231-2F units were within the same order of magnitude as those detected in the 0 to $0.6 \mathrm{~m}(0$ to $2 \mathrm{ft}$ ) interval. Consequently; because the 0 to $0.6 \mathrm{~m}(0$ to $2 \mathrm{ft})$ interval consists of a greater number of samples, this interval was screened and quantitatively evaluated in the risk assessment.

Soil sampling data were used to estimate COPC exposure point concentrations in surface soil for the current visitor and potential future industrial and residential scenarios at the F-Area BRPs. Data for BRPs 231-F/1F and for RP 231-2F included soil samples collected from depths of 0 to $0.6 \mathrm{~m}(0$ to $2 \mathrm{ft})$ and 0 to $1.2 \mathrm{~m}(0$ to $4 \mathrm{ft})$. Samples collected at depths of 0 to $1.2 \mathrm{~m}(0 \mathrm{to} 4 \mathrm{ft})$ were used to represent excavated soils for the future residential scenarios. To determine the contribution of soil contamination to future groundwater concentrations, data obtained from

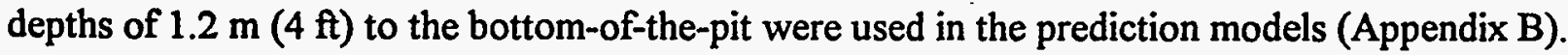

Tables 3.3 through 3.6 present the exposure point concentrations for the 0 to $0.6 \mathrm{~m}(0$ to $2 \mathrm{ft})$ and 0 to $1.2 \mathrm{~m}$ ( 0 to $4 \mathrm{ft}$ ) intervals at the BRP $231 \mathrm{~F} / 1 \mathrm{~F}$ and $\mathrm{RP} 231-2 \mathrm{~F}$, respectively. Tables 3.23 through 3.26 present the exposure point concentrations for volatiles and particulates in air modeled from soils at the two units (Appendix C). 


\subsubsection{Groundwater}

Groundwater data from the water table aquifer were obtained from sampling conducted for the unit assessment and from quarterly monitoring sampling of wells installed prior to the unit assessment (Table 3.9). As noted previously, the analytical data were used in conjunction with the exposure point concentrations generated using soil leachability to groundwater modeling.

The soil leachability analysis consisted of two distinct calculations. The first calculation estimated the leachability of contaminants from the source area. For the FBRP, the source of contaminants

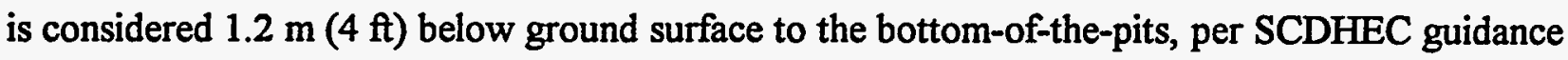
for the FBRP. The leachability analysis provides an estimate of the soil water contaminant concentration at the water table surface. This soil water concentration is used to calculate a groundwater concentration at the receptor by dividing by a groundwater dilution factor. The groundwater dilution factor accounts for mixing that occurs when the leached contaminants are transported into the "clean" groundwater. The modeled concentrations are presented in Table 3.21 and the derivation of these values is discussed in Appendix B.

\subsubsection{Surface Water}

Concentrations of the COPCs measured in standing water at the wetland area to the west of the BRPs were used to estimate exposure point concentrations (assuming a constant concentration over time) in surface water.

\subsubsection{Sediment}

Sediment exposure point concentrations were estimated from measured concentrations of COPCs in sediment at the ephemeral wetland area to the west of the BRPs.

\subsubsection{Air}

In accordance with EPA guidance (EPA, 1991a, 1994a), inhalation of volatiles and particulates released from soil was quantitatively evaluated for both current and future receptors. Simplified soil-to-air transmission relationships specified by EPA were used to determine air concentrations of contaminants generated from soils in the 0 to $0.6 \mathrm{~m}(0$ to $2 \mathrm{ft}$ ) interval (current visitors and future residents and workers) and the 0 to $1.2 \mathrm{~m} \mathrm{(0} \mathrm{to} 4 \mathrm{ft}$ ) interval (future residents and workers). These estimated air concentrations were then used in the exposure calculations for inhalation pathways. Assumptions and equations used in the modeling are provided in 
Appendix C. The results of the modeling used in the risk assessment are provided in Table 3.23 through 3.26.

EPA Region IV (EPA, 1995a) guidance was used to assess exposure to VOCs during showering. Dermal and inhalation exposure to VOCs and tritium in groundwater was assumed to be equal to the estimate of intake from ingestion of $2 \mathrm{~L} /$ day of water.

\subsubsection{Homegrown Produce}

Exposure point concentrations (radionuclides only) in homegrown produce were calculated using soil-to-plant transfer factors. The equations used to estimate radionuclide concentrations in homegrown produce were obtained from Nuclear Regulatory Commission Guidance (NRC, 1992). Equations used in the calculations are shown in Section 3.2.4.6.

\subsubsection{Development of Contaminant Intakes and Doses}

Chemical-specific intakes, or doses, were calculated for the receptors and exposure pathways identified for quantitative evaluation in Section 3.2.2. The development of chemical intakes was based on methodology provided in EPA risk assessment guidance (EPA, 1989a; 1991a). Under the current land use scenario, estimated intakes were calculated for an on-unit visitor exposed to contaminants in the following media:

(1) Soil, through ingestion, dermal contact, inhalation of particulates and volatile organics in air and direct radiation;

(2) surface water in the ephemeral wetland area, through dermal contact; and

(3) sediment in the ephemeral wetland area, through ingestion and dermal contact.

Under the future land use scenario, intakes resulting from the above pathways were estimated for the hypothetical future on-unit. In addition, intakes were estimated for the following:

(1) surface water in the ephemeral wetland area, through ingestion;

(2) groundwater, through ingestion, inhalation, and dermal contact; and

(3) homegrown produce ingestion (radionuclide intake only).

Estimated intakes were calculated for a future industrial worker exposed to contaminants in the following media: 
(1) Soil, through ingestion, dermal contact, inhalation of particulates and volatile organics in air and direct radiation; and

(2) groundwater, through ingestion.

RME estimates of intake were developed for each exposure pathway (EPA, 1992b). The RME estimate, the highest exposure that is reasonably expected to occur in a small but definable "highend" segment of the potentially exposed population, was derived using maximum or nearmaximum (UL95) values for one or a few of the most sensitive exposure parameters (e.g., chemical concentration, intake rate, exposure duration) and average values for the remaining parameters. The most sensitive parameters are determined by using parameters that represent the upper percentile or UL95 for a population or by using best professional judgment in the absence of unit-specific data (EPA, 1991a).

Chemical-specific intakes were developed for the complete exposure pathways (see Appendix D). The estimates of contaminant intake and dose are based on the exposure point concentrations for COPCs developed in Section 3.2.3 and the exposure assumptions described below.

\subsubsection{Intake Parameters}

The values assumed for the RME human exposure parameters, and the guidance they are based on, are presented in Table 3.27. Exposure time, frequency, and duration estimate the total time a receptor may be exposed to a contaminated source. Exposure time (ET) is the number of hours per day (\# hours / 24 hours) that a receptor is present at a specific exposure point.' Exposure frequency (EF) is the number of days per year that the exposure occurs, and exposure duration (ED) is the number of years over which exposure occurs.

Under current land use, the on-unit receptor is an individual who visits the unit on an infrequent or occasional basis, such as a researcher associated with an organization that uses SRS as an outdoor laboratory. The ED during which a visitor could be expected to come in contact with the unit was assumed to be 5 years, a reasonable maximum length for an individual to work on a scientific study. An EF of 6 days per year, or once every other month, was used in conjunction with an ET of 1 hour for contact with soil and 0.5 hour for contact with the wetland area.

For the future residential and industrial scenarios, an ED of 30 and 25 years was assumed, respectively. These values represent the national upper-bound (90th and 95th percentile) time spent at one residence/location (EPA, 1991a). Residential ED was apportioned between adult and child, with 24 years as an adult and 6 years as a child. An EF of 350 days/year was assumed 
for the resident, and 250 days/year was assumed for the worker. Exposure times assumed for inhalation of VOCs from soil was 15 hours/day (resident adult) and 18 hours/day (resident child) based on average time spent at home as reported in time use studies (EPA, 1990). An exposure time of 1 hour/day was assumed for the future worker based on the amount of time the worker was assumed to spend outdoors while at the job site. For the assessment of exposure to external radiation, future adult and child residents were assumed to spend 2 and 3 hours per day, respectively, outdoors (EPA, 1990).

Under future residential land use, an adolescent (7-16 years old) will be identified as the receptor for exposure to surface water and sediment (EPA, 1995a). The $\overline{E D}$, based on the residential scenario, was 10 years: For surface water, the EF of 50 days/year represents 3 days/week during the 13-week summer season plus 1 day/week for 11 weeks during spring (the portion of the year when standing water is probably present in the wetland area). For sediment, an EF of 39 days reflects the exposure of 3 days/week for the 13-week summer, but excludes the time that the standing water is present in the wetland area. An ET of 2 hours/day was assumed for an adolescent wading in the wetland area.

Incidental soil ingestion rates (IRs) were based on EPA guidance (EPA, 1991a). For the current on-unit visitor and future industrial worker, a soil IR of $50 \mathrm{mg} / \mathrm{d}$ was assumed. For the future onunit residential receptor, an upper bound soil $\mathbb{R}$ of $200 \mathrm{mg} / \mathrm{d}$ was assumed for children 1-6 years of age and $100 \mathrm{mg} /$ day for older children and adults (EPA, 1991a). For adolescents exposed to sediment, an IR of $20 \mathrm{mg} /$ day was assumed. "This sediment ingestion rate was based on the approximate ratio of time spent outdoors as compared to indoors ( 2 hours outdoor/18 hours total). The sediment ingestion rate used for the on-unit visitor was $50 \mathrm{mg} / \mathrm{d}$.

An adult resident drinking water $\mathbb{R}$ of 2 liters/day (90th percentile) and a commercial/industrial worker IR of 1 liter/d was assumed (EPA, 1991a). The child IR used was 1 liter/day (EPA, 1990). An $\mathbb{R}$ of 0.01 liter/d was used for the incidental ingestion of surface water by the resident child. Chemical intakes (radionuclides only) from the ingestion of homegrown produce by the future resident adult were calculated using IRs of 113,202 ,-and $123 \mathrm{~g} / \mathrm{d}$ for leafy vegetables, tuberous vegetables, and fruits, respectively. IRs of 42,75 , and $45 \mathrm{~g} / \mathrm{d}$ for leafy vegetables, tuberous vegetables, and fruits were used for the future child. The ingestion rates for the adult are based on an average daily consumption of total fruits and vegetables from a 3-day dietary recall at the 95th percentile (EPA, 1990). Ingestion rates for the child were assumed to be approximately equivalent to 37 percent of the ingestion rate for the adult. 
An inhalation rate of $2.5 \mathrm{~m}^{3} / \mathrm{hr}$ was used for the current on-unit visitor and future industrial worker, based on a reasonable upper-bound inhalation rate of $20 \mathrm{~m}^{3} /$ workday (EPA, 1991a) for an adult male working at a moderate level of activity and an 8-hour workday (i.e., $20 \mathrm{~m}^{3} / \mathrm{d} \div 8$ hours $/ \mathrm{d}=2.5 \mathrm{~m}^{3} / \mathrm{hr}$ ).

An inhalation rate of $0.83 \mathrm{~m} 3 / \mathrm{hr}$ (i.e., $20 \mathrm{~m}^{3} / \mathrm{d} \div 24$ hours $/ \mathrm{d}$ ) was used for adult receptors in the future residential scenario (EPA, 1991a). This value represents the reasonable upper-bound inhalation rate over an entire day for indoor and outdoor activities, including periods of rest and light, moderate and heavy activity. The corresponding inhalation rate for children (used for the residential scenario) is $1.0 \mathrm{~m}^{3} / \mathrm{hr}$ (EPA, 1990) for the inhalation of soil particulates/VOCs and 0.6 $\mathrm{m}^{3} / \mathrm{hr}$ (EPA, 1990) for inhalation of volatiles generated from groundwater.

The values used for body weight (BW) were the average weights over the exposure period: 70 $\mathrm{kg}$ for adult and $15 \mathrm{~kg}$ for child (EPA, 1991a). In accordance with EPA guidance (EPA, 1991a), an adult body weight was used to calculate intake for older children and adults. Body weight for the wading child (45 kg) was derived, based on age, from EPA guidance (EPA, 1995a).

Skin surface areas (SA) used for resident exposure to soil were 50th percentile values for the body parts representing the RME: head, hands, forearms and lower legs. It was assumed that the resident adult or child wears a short sleeve shirt, shorts, and shoes while gardening, working, or playing outdoors at home, with about 25 percent of the total skin surface area exposed. This equates to an SA of $5000 \mathrm{~cm}^{2}$ for the adult and $1800 \mathrm{~cm}^{2}$ for the child (EPA, 1992c). The SA used for the on-unit visitor and industrial worker exposure to soil was the 50th percentile value for head, hands, and forearms: $3200 \mathrm{~cm}^{2}$ (EPA, 1990). The entire body surface area was used for exposure to groundwater while bathing (resident adult $=20,000 \mathrm{~cm}^{2}$, resident child $=7300$ $\left.\mathrm{cm}^{2} ; \mathrm{EPA}, 1992 \mathrm{c}\right)$.

The SA used for the child wading was $3100 \mathrm{~cm}^{2}$, which represents the 50th percentile value for feet, lower legs, hands, and lower arms (EPA, 1990). For the on-unit visitor, an SA of $5000 \mathrm{~cm}^{2}$ was used (EPA, 1990), based on the same body parts as the child wading.

For soil ingestion by the on-unit visitor, future on-unit resident and worker, the value assumed for fraction ingested (FI) from a contaminated source will be 1.0, a conservative estimate that all soil ingested was from the contaminated source. FI values used in the calculation of intakes from homegrown produce ingestion by the future adult were $0.042,0.119$, and 0.487 for leafy and tuberous vegetables, and fruits, respectively (EPA, 1990). The same FI values were used for the 
future child. The FI values represent the homegrown portion of all vegetables ingested by the future adult and child resident.

The adherence factor (AF) assumed for soil was $0.2 \mathrm{mg} / \mathrm{cm}^{2}$, which represents the lower end of the range believed to be typical for environmental exposures (EPA, 1992d). This value was selected because of the sandy nature of surface soil at the unit. An AF value of $0.6 \mathrm{mg} / \mathrm{cm}^{2}$ was used for sediment assuming a smaller grain size and greater adherence potential.

Different dermal absorption factors (ABS) for soil were used for organic and inorganic COPCs: 1.0 percent for organics and 0.1 percent for inorganics (EPA, 1995a). Dermal permeability constants (PC) used for COPCs in groundwater and surface water were obtained from the EPA guidance document on dermal exposure assessment (EPA, 1992c). Table 3.28 presents these constants and identifies whether they are published or predicted values from the open literature, or they were calculated based on the COPCs octannl/water partition coefficient.

\subsubsection{Equations for Exposure to Soil and Sediment}

Equation 1, used to calculate intake (absorbed dose) of chemicals from dermal contact with surface soil or sediment, was obtained from EPA risk assessment guidance (EPA, 1989a) as follows:

Absorbed Dose (mg/kg-day) $=\frac{C S \times C F \times S A \times A F \times A B S \times E F \times E D}{B W \times A T \times A D}$

Where:

$\mathrm{CS}=$ Chemical Concentration in Soil or Sediment $(\mathrm{mg} / \mathrm{kg})$

$\mathrm{CF}=$ Conversion Factor $\left(10^{-6} \mathrm{~kg} / \mathrm{mg}\right)$

$\mathrm{SA}=$ Skin Surface Area Available for Contact ( $\left.\mathrm{cm}^{2} / \mathrm{event}\right)$

$\mathrm{AF}=$ Soil to Skin Adherence Factor $\left(\mathrm{mg} / \mathrm{cm}^{2}\right)$

$\mathrm{ABS}=$ Absorption Factor (unitless)

$\mathrm{EF}=$ Exposure Frequency (events/year)

$\mathrm{ED}=$ Exposure Duration (years)

$\mathrm{BW}=$ Body Weight $(\mathrm{kg})$

AT $=$ Averaging Time (365 days/year) 
$\mathrm{AD}=$ Averaging Duration (years) (equal to ED for non-carcinogens and 70 years for carcinogens)

This equation was modified for the hypothetical future scenario to apportion intake between children and adults, based upon their different skin surface areas and exposure durations, shown in Equation 2.

For carcinogens, the two components of Equation 2 (adult and child) were summed to yield an age-adjusted intake value. For noncarcinogens, the intake values for the adult and child also were considered separately in order to evaluate the risk from noncarcinogens to young children (0-6 years old).

Absorbed Dose (mg/kg-day) $=\frac{\mathrm{CS} \times \mathrm{CF} \times \mathrm{SAa} \times \mathrm{AF} \times \mathrm{ABS} \times \mathrm{EFa} \times \mathrm{EDa}}{\mathrm{BWa} \times \mathrm{AT} \times \mathrm{AD}}+$

CS $\times$ CF $\times$ SAc $\times A F \times A B S \times E F c \times E D C$

$B W c \times A T \times A D$

Where:

$\mathrm{CS}=$ Chemical Concentration in Soil $(\mathrm{mg} / \mathrm{kg})$

$\mathrm{CF}=$ Conversion Factor $\left(10^{-6} \mathrm{~kg} / \mathrm{mg}\right)$

$\mathrm{SAa}=$ Adult Skin Surface Area Available for Contact ( $\mathrm{cm}^{2} /$ event)

$\mathrm{SAc}=$ Child Skin Surface Area Available for Contact ( $\mathrm{cm}^{2} /$ event)

$\mathrm{AF}=$ Soil to Skin Adherence Factor $\left(\mathrm{mg} / \mathrm{cm}^{2}\right)$

$\mathrm{ABS}=$ Absorption Factor (unitless)

$\mathrm{EFa}=$ Adult Exposure Frequency (events/year)

EFc $=$ Child Exposure Frequency (events/year)

$\mathrm{EDa}=$ Adult Exposure Duration (years)

EDc $=$ Child Exposure Duration (years)

$\mathrm{BWa}=\quad$ Adult Body Weight (kg)

$\mathrm{BWc}=$ Child Body Weight (kg)

AT = Averaging Time (365 days/year)

$\mathrm{AD}=$ Averaging Duration (years) (equal to ED for noncarcinogens and 70 years for carcinogens) 
Equation 3 was used to calculate intake for soil or sediment ingestion, as given in EPA risk assessment guidance (EPA, 1989a).

Intake $(\mathrm{mg} / \mathrm{kg}$-day $)=\frac{\mathrm{CSc} \times \mathrm{IR} 1 \times \mathrm{CFc} \times \mathrm{FI} \times \mathrm{EF} \times \mathrm{ED}}{\mathrm{BW} \times \mathrm{AT} \times \mathrm{AD}}$

The intake of radiological contaminants resulting from incidental ingestion of soil or sediment was calculated using Equation 4.

Intake $(\mathrm{pCi})=\operatorname{CSr} \times \mathrm{IR} 1 \times \mathrm{CFr} \times \mathrm{FI} \times \mathrm{EF} \times \mathrm{ED}$

Where:

CSc $=$ Chemical Concentration in Soil or Sediment $(\mathrm{mg} / \mathrm{kg})$

$\mathrm{CSr}=$ Radionuclide Concentration in Soil or Sediment $(\mathrm{pCi} / \mathrm{g})$

IR1 = Ingestion Rate (mg soil/day)

$\mathrm{CFc}=$ Conversion Factor $\left(10^{-6} \mathrm{~kg} / \mathrm{mg}\right)$

$\mathrm{CFr}=$ Conversion Factor $\left(10^{-3} \mathrm{~g} / \mathrm{mg}\right)$

FI = Fraction Ingested from Contaminated Source (unitless).

$\mathrm{EF}=$ Exposure Frequency (days/year)

$\mathrm{ED}=$ Exposure Duration (years)

BW $=$ Body Weight $(\mathrm{kg})$

$\mathrm{AT}=$ Averaging Time (365 days/year)

$\mathrm{AD}=$ Averaging Duration (years) (equal to $\mathrm{ED}$ for non-carcinogens and 70 years for carcinogens)

Equations 3 and 4 were modified as recommended by EPA (EPA, 1991a; EPA, 1989a) for the hypothetical future scenarios to apportion intake between young children (1 to 6 years, the period of highest soil ingestion), and older children and adults. The modified equations are shown in Equations 5 and 6, respectively. As described above for Equation 2, the two components of Equation 5 were summed to derive carcinogenic intake and considered separately for noncarcinogenic intake.

Intake $(\mathrm{mg} / \mathrm{kg}-$ day $)=\frac{\mathrm{CSc} \times \mathrm{AIR} 1 \times \mathrm{CFc} \times \mathrm{FI} \times \mathrm{EF} \times \mathrm{EDa}}{\mathrm{BWa} \times \mathrm{AT} \times \mathrm{AD}}+$ 
CSc $\times$ CIR $1 \times$ CFc $\times$ FI $\times$ EF $\times$ EDc

BWc $\times$ AT $\times$ AD

Intake $(\mathrm{pCi})=\operatorname{CSr} \times \mathrm{AIR} 1 \times \mathrm{CFr} \times \mathrm{FI} \times \mathrm{EF} \times \mathrm{EDa}+$

CSr $\times$ CIR $1 \times$ CFr $\times$ FI $\times$ EF $\times$ EDc

Where:

$\mathrm{CSc}=$ Chemical Concentration in Soil $(\mathrm{mg} / \mathrm{kg})$

$\mathrm{CSr}=$ Radionuclide Concentration in Soil $(\mathrm{pCi} / \mathrm{g})$

AIR1 = Adult Ingestion Rate (mg soil/day)

CIR1 = Child Ingestion Rate (mg soil/day)

$\mathrm{CFc}=$ Conversion Factor $\left(10^{-6} \mathrm{~kg} / \mathrm{mg}\right)$

- $\mathrm{CFr}=$ Conversion Factor $\left(10^{-3} \mathrm{~g} / \mathrm{mg}\right)$

FI $=$ Fraction Ingested from Contaminated Source (unitless)

$\mathrm{EF}=$ Exposure Frequency (days/year)

$\mathrm{EDa}=$ Adult Exposure Duration (years)

$\mathrm{EDc}=$ Child Exposure Duration (years)

$\mathrm{BWa}=\quad$ Adult Body Weight $(\mathrm{kg})$

$\mathrm{BWc}=$ Child Body Weight $(\mathrm{kg})$

AT $=$ Averaging Time (365 days/year)

$\mathrm{AD}=$ Averaging Duration (years) (equal to ED for noncarcinogens and 70 years for carcinogens)

\subsubsection{Equations for Exposure to Water}

Equation 7 was obtained from EPA risk assessment guidance (EPA, 1989a) for the calculation of chemical intake from dermal contact with chemicals in surface water.

Absorbed Dose $\left(\mathrm{mg} / \mathrm{kg}\right.$-day) $=\frac{\mathrm{CW} \times \mathrm{SA} \times \mathrm{PC} \times \mathrm{Et} \times \mathrm{EF} \times \mathrm{ED} \times \mathrm{CF}}{\mathrm{BW} \times \mathrm{AT} \times \mathrm{AD}}$

Where:

$\mathrm{CW}=$ Chemical Concentration in Water (mg/l) 


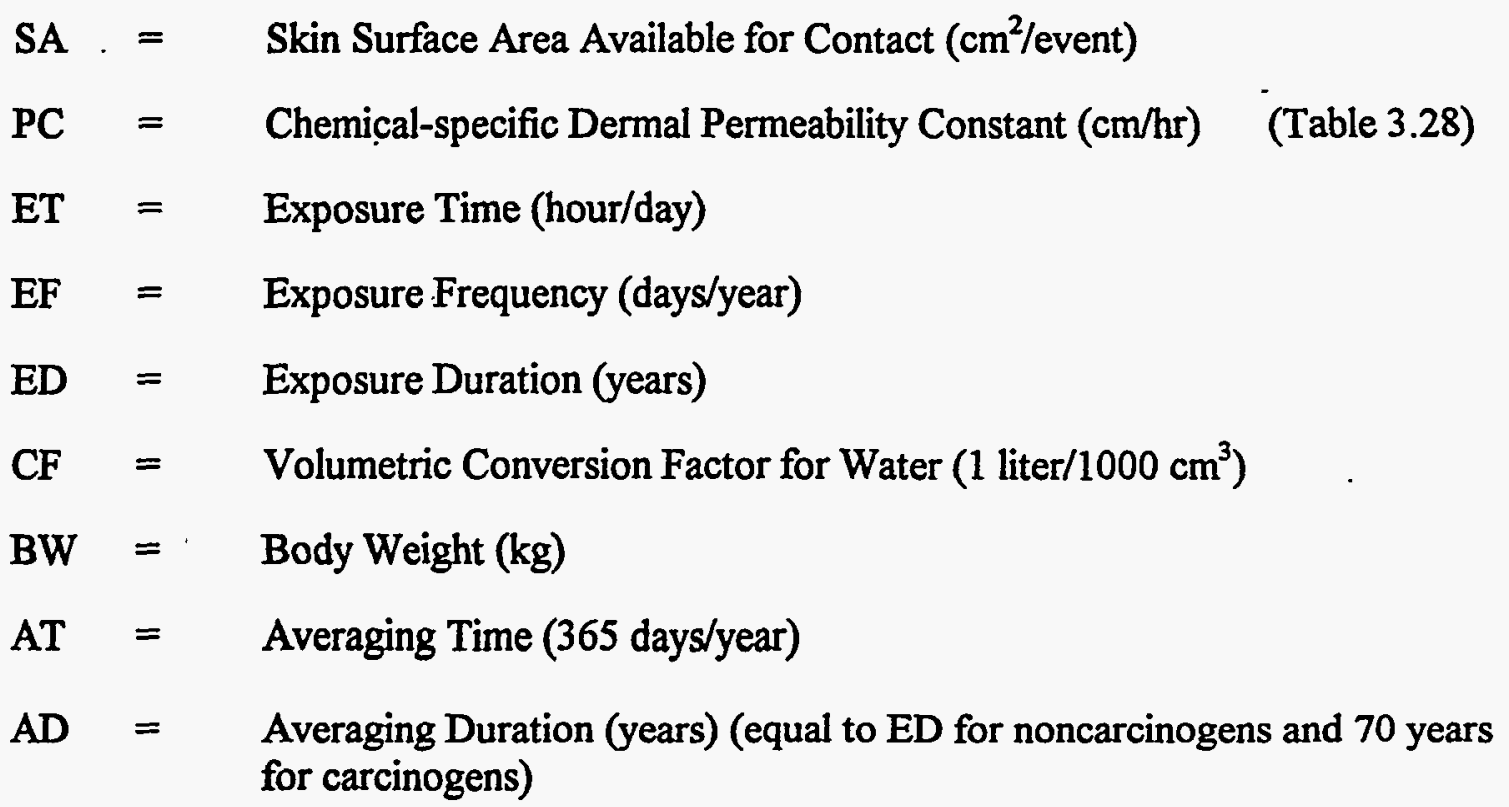

This equation was modified to estimate a weighted average of child and adult exposures to groundwater under the hypothetical future residential scenario, as shown in Equation 8. The two components of Equation 8 were summed to derive carcinogenic intake, and they were considered separately for non-carcinogenic intake.

Dermal and inhalation exposure to VOCs and tritium in groundwater was assumed to be equal to the estimate of intake from ingestion of 2 liters/day. Therefore, VOC intake from dermal contact with groundwater was not estimated using Equation 8.

$$
\begin{aligned}
\text { Absorbed Dose (mg/kg-day) }= & \frac{\mathrm{CW} \times \mathrm{SAa} \times \mathrm{PC} \times \mathrm{ET} \times \mathrm{EF} \times \mathrm{EDa} \times \mathrm{CF}}{\mathrm{BWa} \times \mathrm{AT} \times \mathrm{AD}}+ \\
& \frac{\mathrm{CW} \times \mathrm{SAc} \times \mathrm{PC} \times \mathrm{ET} \times \mathrm{EF} \times \mathrm{EDC} \times \mathrm{CF}}{\mathrm{BWc} \times \mathrm{AT} \times \mathrm{AD}}
\end{aligned}
$$

Where:
$\mathrm{CW}=$ Chemical Concentration in Water (mg/l)
$\mathrm{SAa}=$ Adult Skin Surface Area Available for Contact $\left(\mathrm{cm}^{2} /\right.$ event $)$
SAc = Child Skin Surface Area Available for Contact ( $\left.\mathrm{cm}^{2} / \mathrm{event}\right)$
PC $=$ Chemical-specific Dermal Permeability Constant $(\mathrm{cm} / \mathrm{hr})$
(Table 3.28)
ET $=$ Exposure Time (hours/day)
$\mathrm{EF}=$ Exposure Frequency (days/year) 


$\mathrm{EDa}=$ Adult Exposure Duration (years)
$\mathrm{EDc}=$ Child Exposure Duration (years)
$\mathrm{CF}=$ Volumetric Conversion Factor for Water $\left(1\right.$ liter $\left./ 1000 \mathrm{~cm}^{3}\right)$
$\mathrm{BWa}=$ Adult Body Weight $(\mathrm{kg})$
$\mathrm{BWc}=\quad$ Child Body Weight $(\mathrm{kg})$
$\mathrm{AT}=$ Averaging Time ( 365 days/year)
$\mathrm{AD}=\quad$ Averaging Duration (years) (equal to ED for noncarcinogens and 70 years

Equation 9 was obtained from EPA risk assessment guidance (EPA, 1989a) for the calculation of chemical intake from residential ingestion of groundwater used as a drinking water source. VOC intake from residential inhalation and dermal exposure during household use of groundwater also was assumed to be equivalent to the VOC intake from residential ingestion of groundwater. One hundred percent of the total drinking water ingested is considered to be from the aquifer below the unit. The equation has been modified to apportion the intake between children and adults. The two components of Equation 9 were summed to represent intake for carcinogens. They were considered separately for non-carcinogenic intake.

Intake $(\mathrm{mg} / \mathrm{kg}$-day $)=$

$$
\frac{\mathrm{CWc} \times \mathrm{AIR} 3 \times \mathrm{EF} \times \mathrm{EDa}}{\mathrm{BWa} \times \mathrm{AT} \times \mathrm{AD}}+\frac{\mathrm{CWc} \times \mathrm{CIR} 3 \times \mathrm{EF} \times \mathrm{EDc}}{\mathrm{BWc} \times \mathrm{AT} \times \mathrm{AD}}
$$

The intake of radiological contaminants resulting from residential ingestion of groundwater was calculated using Equation 10. This equation was also used to calculate the intake of tritium resulting from inhalation of tritium during household use of groundwater.

Intake $(\mathrm{pCi})=\mathrm{CWr} \times \mathrm{AIR} 3 \times \mathrm{EF} \times \mathrm{EDa}+\mathrm{CWr} \times \mathrm{CIR} 3 \times \mathrm{EF} \times \mathrm{EDc}$

Where:

$\mathrm{CWc}=$ Chemical Concentration in Water $(\mathrm{mg} / \mathrm{l})$

$\mathrm{CWr}=$ Radionuclide Concentration in Water $(\mathrm{pCi} / \mathrm{l})$

AIR3 = Adult Ingestion Rate (liters/day)

CIR3 = Child Ingestion Rate (liters/day) 
EF . = Exposure Frequency (days/year)

$\mathrm{EDa}=$ Adult Exposure Duration (years)

$\mathrm{EDc}=$ Child Exposure Duration (years)

$\mathrm{BWa}=$ Adult Body Weight $(\mathrm{kg})$

$\mathrm{BWc}=$ Child Body Weight $(\mathrm{kg})$

AT $=$ Averaging Time (365 days/year)

$\mathrm{AD}=$ Averaging Duration (years) (equal to $\mathrm{ED}$ for noncarcinogens and 70 years for carcinogens)

\subsubsection{Equations for Exposure to Air}

The equation used to calculate intakes from inhalation of contaminated airborne soil particulates or vapor were obtained from EPA risk assessment guidance (EPA, 1989a). Equation 11 was used for exposure to particulates or organic vapors derived from surface soil.

Intake $\left(\mathrm{mg} / \mathrm{kg}\right.$-day) $=\frac{\mathrm{CAc} \times \mathrm{IR} 2 \times \mathrm{ET} \times \mathrm{EF} \times \mathrm{ED}}{\mathrm{BW} \times \mathrm{AT} \times \mathrm{AD}}$

Radiological intake from the inhalation of airborne particulates was calculated using Equation 12.

Intake $(\mathrm{pCi})=\mathrm{CAr} \times \mathrm{R} 2 \times \mathrm{ET} \times \mathrm{EF} \times \mathrm{ED}$

Where:

$\mathrm{CAc}=$ Chemical Concentration in Air $\left(\mathrm{mg} / \mathrm{m}^{3}\right)$

$\mathrm{CAr}=$ Radionuclide Concentration in $\operatorname{Air}\left(\mathrm{pCi} / \mathrm{m}^{3}\right)$

IR2 = Inhalation Rate $\left(\mathrm{m}^{3} /\right.$ hour $)$

ET = Exposure Time (hours/day)

$\mathrm{EF}=$ Exposure Frequency (days/year)

ED = Exposure Duration (years)

$\mathrm{BW}=$ Body Weight $(\mathrm{kg})$

$\mathrm{AT}=$ Averaging Time (365 days/year)

$\mathrm{AD}=$ Averaging Duration (years) (equal to ED for noncarcinogens and 70 years for carcinogens) 
Equations 11 and 12 were modified for the future scenario to estimate chemical and radionuclide intakes for adults and children separately, based on their different inhalation rate, body weight, and exposure time as shown in Equations 13 and 14, respectively. These equations were used for exposure of hypothetical future on-unit residents to organic vapors from deep soils excavated and spread over the surface. The two components of Equation 13 were summed for carcinogens. For noncarcinogens, the first component was used for adult intake, while the second component was used to represent intake for the child.

Intake $(\mathrm{mg} / \mathrm{kg}$-day $)=$

$\frac{\mathrm{CAc} \times \mathrm{AIR} \times \mathrm{ETa} \times \mathrm{EF} \times \mathrm{EDa}}{\mathrm{BWa} \times \mathrm{AT} \times \mathrm{AD}}+\frac{\mathrm{CAc} \times \mathrm{CIR} 2 \times \mathrm{ETc} \times \mathrm{EF} \times \mathrm{EDc}}{\mathrm{BWc} \times \ldots \times \mathrm{AD}}$

Intake $(\mathrm{pCi})=\operatorname{CAr} \times \mathrm{AIR} 2 \times \mathrm{ETa} \times \mathrm{EF} \times \mathrm{EDa}+\mathrm{CAr} \times \mathrm{CIR} 2 \times \mathrm{ETc} \times \mathrm{EF} \times \mathrm{EDc}$

Where:

$\mathrm{CAc}=$ Chemical Concentration in Air $\left(\mathrm{mg} / \mathrm{m}^{3}\right)$

$\mathrm{CAr}=$ Radionuclide Concentration in $\mathrm{Air}\left(\mathrm{pCi} / \mathrm{m}^{3}\right)$

$\operatorname{AIR2}=\quad$ Adult Inhalation Rate $\left(\mathrm{m}^{3} / \mathrm{hr}\right)$

$\mathrm{CIR} 2=$ Child Inhalation Rate $\left(\mathrm{m}^{3} / \mathrm{hr}\right)$

$\mathrm{ETa}=$ Adult Exposure Time (hour/day)

ETc = Child Exposure Time (hour/day)

$\mathrm{EF}=$ Exposure Frequency (days/year)

$\mathrm{EDa}=$ Adult Exposure Duration (years)

EDc = Child Exposure Duration (years)

$\mathrm{BWa}=\quad$ Adult Body Weight $(\mathrm{kg})$

$\mathrm{BWc}=$ Child Body Weight $(\mathrm{kg})$

AT $=$ Averaging Time (365 days/year)

$\mathrm{AD}=$ Averaging Duration (years) (equal to ED for noncarcinogens and 70 years for carcinogens)

Intake from inhalation of VOCs and tritium released from groundwater during showering and other household uses was not estimated using Equations 13 and 14. Instead, the intakes from 
inhalation and dermal contact were assumed to be equivalent to the intake from ingestion of 2 liters/day of groundwater, in accordance with EPA guidance (EPA, 1995a).

\subsubsection{Equation for Exposure to Direct Radiation}

Exposures through direct radiation were estimated using Equation 15 below:

External exposure $(\mathrm{pCi}$ - year/g) $=\mathrm{CS} \times(\mathrm{EF} \times \mathrm{SF}) \times \mathrm{ED} \times \mathrm{CF}$

Where:

$\mathrm{CS}=$ Radionuclide Concentration in Soil (pCi/g)

$\mathrm{EF} \quad=\quad$ Exposure Frequency (days/year)

SF = Shielding Factor (unitless)

ED = Exposure Duration (years)

$\mathrm{CF}=$ Conversion Factor (1 year/365 days)

Because the external exposure slope factor is given in units of risk/year per $\mathrm{pCi} / \mathrm{g}$ soil, the conversion factor (CF) is necessary to convert the external exposure to $\mathrm{pCi}$ - year/g.

\subsubsection{Equation for Exposure to Homegrown Produce}

The equation used to calculate the intake of contaminated vegetation was obtained from EPA risk assessment guidance (EPA, 1989a). Equation 16 was used to estimate the intake of radionuclides from the edible portions of vegetation grown within a garden of a hypothetical future resident.

Intake $(\mathrm{pCi})=\mathrm{CV} \times \mathbb{R} \times \mathrm{FI} \times \mathrm{EF} \times \mathrm{ED}$

Where:

$\mathrm{CV}=$ Radionuclide Concentration in Vegetation (pCi/g)

IR = Ingestion Rate ( $/$ /day)

FI $=$ Fraction Ingested (unitless)

$\mathrm{EF}=$ Exposure Frequency (days/year)

$\mathrm{ED}=$ Exposure Duration (years)

The equation used to estimate plant uptake of radionuclides from soils (CV) was obtained from NRC guidance (NRC, 1992). Equation 17 was used for estimating the vegetative uptake of radioactive contaminants from soils. 


$$
\mathrm{CV}=\mathrm{CS} \times \mathrm{TF} \times \mathrm{CF}
$$

Where:

$\mathrm{CV}=$ Radionuclide Concentration in Vegetation ( $\mathrm{pCi} / \mathrm{g})$

$\mathrm{CS}=$ Radionuclide Concentration in Soil $(\mathrm{pCi} / \mathrm{g})$

$\mathrm{TF}=$ Soil-to-Plant Transfer Factor ( $\mathrm{pCi} / \mathrm{g}$ plant/pCi/g dry soil)

$\mathrm{CF}=$ Dry-to-Wet Weight Conversion Factor (unitless) $(0.2,0.25$, and 0.18 for leafy vegetables, tuberous vegetables, and fruits, respectively)

The soil-to-plant transfer factors for each radionuclide are shown in Table 3.29 for leafy vegetables, tuberous vegetables, and fruits.

\subsection{Toxicity Assessment}

The following section is an overview of the human health toxicity of COPCs identified in Section 3.1. The objective of the toxicity assessment is to weigh available evidence each COPCs potential to cause adverse health effects in exposed individuals and to provide, where possible, an estimate of the relationship between the extent of exposure and the severity of adverse effects (EPA, 1989a).

Toxicity information used in this evaluation was first obtained from the Integrated Risk Information System (IRIS; EPA, 1996a). If values were not available from IRIS, the Health Effects Assessment Summary Tables (HEAST; EPA, 1995b) or the Superfund Health Risk Technical Support Center-Environmental Criteria and Assessment Office (EPA, 1996b) were consulted. Toxicological data for the nonradioactive COPCs, including both carcinogens and noncarcinogens, are presented in Tables 3.30 through 3.33. Toxicity data for the radioactive COPCs are presented in Table 3.34.

To assess toxicity via the dermal absorption route of intake, oral toxicity factors based on administered doses were adjusted to express toxicity on the basis of absorbed dose. Oral absorption efficiencies (percent absorbed by the gastrointestinal tract following oral intake) were identified for each COPC and used to modify toxicity values as follows:

- for carcinogens, the oral slope factor was divided by the oral absorption efficiency to derive an adjusted slope factor; and 
- for noncarcinogens, the oral reference dose was multiplied by the oral absorption efficiency to derive an adjusted reference dose.

If an appropriate oral absorption efficiency value was not identified, the following default values recommended by EPA Region IV were used: . 80 percent for VOCs, 50 percent for SVOCs, and 20 percent for inorganics (EPA, 1995a). Tables 3.30 and 3.32 present the adjusted slope factors (SFs) and reference doses (RfDs) for all COPCs for which oral toxicity values were available.

In order to assess the toxicity of certain organic compounds for which specific data is insufficient, EPA adopted a methodology based on toxicity equivalency factors (TEFs). The TEF methodology was used to evaluate the carcinogenic and noncarcinogenic toxicity of the chlorinated dibenzo-p-dioxins and dibenzofurans (CDDs and CDFs), heptachlorodibenzo-p-dioxin (HpCDD), heptachlorodibenzofuran (HpCDF), hexachlorodibenzo-p-furan (HxCDF), and OCDD isomers. For CDDs and CDFs, TEFs are based on the toxicity of these compounds relative to the toxicity of 2,3,7,8-tetrachlorodibenzo-p-dioxin (2,3,7,8-TCDD), the most toxic isomer in this class of compounds. Thus, concentrations of $\mathrm{HpCDD}$ and $\mathrm{HpCDF}$ were multiplied by their established TEF of 0.01 , concentrations of HXCDF were multiplied by its established TEF of 0.1 , and concentrations of octachlorodibenzo-p-dioxin (OCDD) were multiplied by its established TEF of 0.001 (EPA, 1995a). The TEF adjustment expressed the concentrations in terms of 2,3,7,8TCDD equivalency.

A similar methodology was followed to evaluate the carcinogenic toxicity of certain polycyclic aromatic hydrocarbons (PAHs). Relative potency values have been established for PAHs classified as probable human carcinogens based on each compound's carcinogenic potency relative to that of benzo(a)pyrene. Concentrations of certain PAHs were multiplied by their respective relative potency factors (RPFs) in order to express the concentrations in terms of equivalency to benzo(a)pyrene. The RPFs available for the PAHs of potential concern at the unit were: 1.0 for benzo(a)pyrene and dibenz(a,h)anthracene; 0.1 for benzo(a)anthracene, benzo(b)fluoranthene, and indeno(1,2,3-cd)pyrene; 0.01 for benzo(k)fluoranthene; and 0.001 for chrysene (EPA, 1995a).

\subsubsection{Non-Radiological Toxicity}

\subsubsection{Carcinogens}

For risk assessment, EPA subscribes to the "non-threshold" theory of carcinogenesis, which proposes that there is essentially no level of exposure to a carcinogen that does not pose a finite probability of generating a carcinogenic response. This theory assumes that a small number of molecular events can evoke changes in a single cell that may lead to uncontrolled cellular 
proliferation and eventually to cancer (EPA, 1989a). Therefore, no dose is thought to be risk free and, in evaluating cancer risk, an effect threshold cannot be estimated. As a result, EPA takes a probabilistic approach to the evaluation of the carcinogenicity of chemicals. This two-step evaluation includes the assignment of a weight-of-evidence classification to each chemical based (1) on the strength of evidence that it is-a human carcinogen, and (2) the calculation of a slope factor for those chemicals that are possible, probable, or proven human carcinogens (EPA, 1989a).

The EPA weight-of-evidence classification system characterizes a chemical's carcinogenicity based on the availability of animal, human, and other supportive data. A chemical is assigned to one of the following classes, based on the strength of the evidence that a chemical produces carcinogenic effects in humans (EPA, 1989a):

- Group A - Human Carcinogen. This category indicates that there is sufficient evidence from epidemiological studies to demonstrate carcinogenicity in humans.

- Group B - Probable Human Carcinogen. This category is subdivided into Group B1 and Group B2:

Group B1 indicates limited data are available suggesting carcinogenicity in humans.

Group B2 indicates there is sufficient evidence of carcinogenicity in animals and inadequate or no evidence in humans.

- Group C - Possible Human Carcinogen. This category indicates that there is limited evidence of carcinogenicity in animals and inadequate or no evidence in humans.

- Group D - Not Classifiable. This category indicates that there is inadequate or no data by which to classify a chemical as a human carcinogen.

- Group E - Evidence of Human Noncarcinogenicity. This category indicates there was no evidence of carcinogenicity in an adequate number.of studies.

The slope factor (SF) is a plausible upper-bound estimate of the probability of a carcinogenic response per unit intake of a chemical over a lifetime. It is usually the upper 95th percent confidence limit of the slope of the dose-response curve and is expressed as the reciprocal of $\mathrm{mg}$ of chemical intake per $\mathrm{kg}$ of body weight per day $\left[(\mathrm{mg} / \mathrm{kg} \text {-day })^{-1}\right]$. The SF is used in risk assessments to estimate an upper-bound lifetime probability of an individual developing cancer as a result of expnsure to a particular level of a potential carcinogen. SFs are accompanied by the 
weight-of-evidence classification to indicate the strength of the evidence that the chemical is a human carcinogen (EPA, 1989a).

SFs are reported as "risk per unit dose" $\left[(\mathrm{mg} / \mathrm{kg} \text {-day })^{-1}\right]$ or as "unit risk". Unit risk expresses risk from a substance per concentration of that-substance in the medium where human contact occurs. For example, inhalation SFs are usually reported as risk per unit concentration in air $\left[\left(\mu \mathrm{g} / \mathrm{m}^{3}\right)^{-1}\right]$. Conversion of a unit risk concentration to a SF (dose) is not generally recommended by EPA due to the potential for inaccuracy.

Carcinogenic toxicity values calculated for the dermal route of intake are rare, therefore, EPA recommends that an oral slope factor for a chemical be modified based on its gastrointestinal absorption efficiency in order to derive a slope factor based on absorbed dose for use in evaluating dermal absorption (EPA, 1989a). Oral absorption efficiency factors and adjusted slope factors are listed in Tables 3.30 and 3.32 .

Seventeen COPCs have been classified as potential carcinogens and assigned a carcinogenicity weight-of-evidence by the EPA (Tables 3.32 and 3.33): two COPCs in Group A (human carcinogens), 16 COPCs in Group B (probable human carcinogens), and one COPC in Group C (possible human carcinogens).

Nineteen COPCs are categorized as Class D, signifying that they are not classifiable as to human carcinogenicity. The remaining $13 \mathrm{COPCs}$ were not classified due to the lack of available data.

COPCs classified as Group A carcinogens are arsenic and chromium. Exposure to these chemicals may result in tumors in the lungs, bronchial system, and the skin. The majority of Group B and C carcinogenic COPCs cause tumors in the liver or kidney.

To provide more complete toxicity data, surrogate slope factors were used for several COPCs. These include:

- Oral SFs for benzo(a)pyrene were used for all PAHs [benzo(a)anthracene, benzo(b)flouranthene, benzo(k)fluoranthene, chrysene, dibenzo(a)anthracene, and indeno(1,2,3-cd)pyrene].

- Oral and inhalation SFs of 2,3,7,8-tetrachlorodibenzo-p-dioxin isomers were used as surrogates for heptachlorodibenzo-p-dioxin, heptachlorodibenzo-p-furan, hexachlorodibenzo-p-furan, octachlorodibenzo-p-dioxin and octachlorodibenzo-p-furan isomers. 
- Oral SFs were not available for specific PCB congeners, therefore surrogate values for total PCBs were used.

Although the toxicity values for the PAHs and chlorinated dibenzo-p-dioxin (CDDs) and chlorinated dibenzo-p-furans (CDFs) are surrogates, adjustment of the PAH exposure point concentrations by RPFs and the adjustment of the CDD and CDF exposure point concentrations by TEFs is expected to account for the differences in toxicity between the surrogates and respective congeners. Hence, the toxicity of the TEF or RPF adjusted congeners is considered equivalent to the toxicity of the surrogate congener (EPA, 1989e, 1995a).

\subsubsection{Noncarcinogens}

For many noncarcinogenic toxicity effects, protective mechanisms may exist that must be overcome before an adverse effect is manifested. As a result, a range of exposures, from zero to some finite threshold value, may be tolerated by an organism without any expression of adverse effects. In developing toxicity values to evaluate noncarcinogenic effects, the EPA approach is to identify the upper bound of this tolerance range (i.e., the maximum subthreshold level). For most chemicals, this level can only be estimated, so uncertainty factors and modifying factors are applied to this estimated level in order to derive a reference dose (RfD) for evaluation of noncarcinogens (EPA, 1989a).

An $\mathrm{RfD}$ reported as an intake (in $\mathrm{mg} / \mathrm{kg}$-day) is the toxicity value used most often in evaluating noncarcinogenic effects. Reference concentrations (RfCs), reported as a concentration in air or water (in $\mathrm{mg} / \mathrm{m}^{3}$ or $\mathrm{mg} / \mathrm{l}$ ), are used to evaluate noncarcinogenic effects. Except for the estimation of risk from inhalation of VOCs in groundwater, derivation and/or conversion from an RfC (concentration) to an $\mathrm{RfD}$ (dose) was not employed because it is not generally recommended by EPA. Tables 3.30 and 3.31 presents the RfD and RfC, respectively, confidence level, routes of exposure, critical effects, and uncertainty and modifying factors for each of the COPCs.

RfDs are developed and verified by EPA and are defined as "an estimate of a daily exposure level for the human population, including sensitive subpopulations, that is likely to be without an appreciable risk of deleterious effects during a lifetime" (EPA, 1989a). RfDs are preferably based on the highest concentration of a chemical tested at which no adverse effects were demonstrated in animal experiments (the NOAEL, or no observed adverse effect level). To calculate an RfD, the NOAEL is divided by uncertainty and modifying factors. If a NOAEL is not available for a chemical, a LOAEL (lowest observed adverse effect level) may be divided by additional factors for use as an RfD (EPA, 1989a). 
RfDs/RfCs are reported with their uncertainty factors (UFs). UFs generally consist of multiples of 10 , with each factor representing a specific area of uncertainty inherent in the extrapolation from available data. The use of UFs helps to ensure that the potential for adverse noncarcinogenic effects is not underestimated, even for sensitive subpopulations, during the derivation of RfDs/RfCs. Because there are few, if any, noncarcinogenic toxicity values for dermal exposure, oral values are frequently used to assess risks from dermal exposure. The EPA recommends that oral $\mathrm{RfD}$ s based on administered dose be modified using an absorption factor so that they represent absorbed dose (EPA, 1989a). Oral absorption efficiency (percent absorbed) values were identified for each COPC and used to convert oral RfDs to dermal RfDs (absorbed dose).

The critical toxic effects most frequently listed in Tables 3.30 and 3.31 are renal and hepatic effects, followed by neural and hematological effects.

Lead was evaluated separately from the other chemicals. There are no toxicity values available for lead, so a hazard index or risk cannot be calculated. At the BRP 231-F/1F and RP 231-2F, concentrations in soil and groundwater did not exceed the EPA screening levels of $400 \mathrm{mg} / \mathrm{kg}$ in soil and $15 \mu \mathrm{g} / \mathrm{L}$ in groundwater (EPA, 1995a). To be conservative, however, lead was evaluated using the USEPA Integrated Exposure Lead Uptake Biokinetic (IEUBK) Model, Version 0.99d (EPA, 1994b). The results of the lead model are presented in Appendix D.

This model predicts blood lead concentrations in children exposed to lead through a variety of media. The model is designed to estimate blood lead levels, using a combination of default assumptions and exposure concentrations combined with site-specific information where available. The model contains two separate components: an uptake section that allows for the estimation of lead uptake from five media (air, drinking water, soil/dust, food and paint), and the biokinetic section, which uses the uptake data to estimate the blood lead levels. The default values used by the uptake program are based on nationwide surveys of lead distribution in the environment and studies of inhalation and ingestion for each biologic age modeled. A nonlinear method of gastrointestinal absorption of lead was utilized in the uptake-section, which allows for a more accurate estimate over a wider range of environmental lead levels.

For this assessment, blood lead levels for children from 0 to 7 years of age were modeled. Lowlevel exposure to lead during infancy and childhood has been associated with an increase in the risk of irreversible neurobehavioral deficits at blood levels as low as $10 \mathrm{mg} / \mathrm{dL}$. In this assessment, the criterion of a blood level of $10 \mathrm{mg} / \mathrm{dL}$ or less in 95 percent of children was used as a health guideline. The model output predicts a probability curve around the geometric mean 
blood lead concentration. From this curve, the 95th percentile of children's blood lead concentration can be determined. The model plots this blood curve, marks the $10 \mathrm{mg} / \mathrm{dL}$ level of concern and predicts the percentage of the population falling above the level of concern.

\subsubsection{Radiation Toxicity}

\subsubsection{Radiation Types}

EPA classifies all radionuclides as Group A (known human) carcinogens based on their emission of ionizing radiation and on the extensive weight of evidence provided by epidemiological studies of radiation-induced cancers in humans. Ionizing radiations have sufficient energy that their interactions with matter will produce an ejected electron and a positively charged ion (EPA, 1991b). These positively charged ions, known as free radicals, are highly reactive and may combine with other elements or compounds within a cell to produce toxins or otherwise disrupt the chemical balance. Ionizing radiations have been implicated in damage to the genetic material of cells, which result in mutations or other deleterious effects. Radionuclides are characterized by the type and energy level of the radiation emitted. Radiation emissions fall into two major categories: particles (alpha and beta), and electromagnetic radiation (gamma rays) (ATSDR, 1989k).

A radionuclide which emits alpha particles has a characteristic energy level for the alpha emissions. The alpha particle has a charge of +2 and a comparably large size. Alpha particles have a great ability to react with or ionize other molecules but have very little penetrating power. They cannot penetrate a piece of paper or human skin. If inhaled or ingested, however, alpha particles react quickly in localized areas of soft tissues, such as in the lungs or liver.

Beta emissions generally refer to beta negative particle emissions. Radionuclides with an excess of neutrons achieve stability by beta decay. Beta-emitting radionuclides can cause injury to the skin and superficial body tissue but are most destructive when inhaled or ingested. Many beta emitters are present in spent nuclear fuels and reprocessed wastes, and they are reactor fission products. These beta emitters are similar chemically to naturally occurring body elements and will, therefore, tend to accumulate in certain specific tissues. For example, strontium-90 resembles calcium and accumulates in the bones, where it causes continuous exposures. Accordingly, the health effects of beta particle emissions depend upon the target organ. For example, those beta particle emission seeking the bones may cause a prolonged exposure of the bone marrow and affect blood cell formation, possibly resulting in leukemia, other blood 
disorders, or bone cancers. Similarly, those seeking the liver may result in liver diseases or cancers, and those seeking the thyroid may produce thyroid and related metabolic disorders.

Gamma emissions are electromagnetic radiation (photons) released from transformations of the atomic nucleus. Gamma radiation can penetrate the body from the outside and does not require ingestion or inhalation to damage body tissue. Gamma radiation is not directly ionizing and does not directly cause tissue damage in the body, as do alpha and beta radiation. Gamma emissions release their energy in other reactions that yield another high energy particle, such as an electron. The electron then causes tissue damage.

Humans are constantly exposed to gamma radiation from naturally occurring radioactive decay processes occurring in minerals in the environment, from radiations naturally occurring inside our bodies, from the atmosphere as fallout from nuclear testing or explosions, and from cosmic sources. These contribute to backgre.nd exposire to radiation. Exposure to naturally occurring background levels may contribute to a slight increase in the risk of cancer (ATSDR, 1989k).

Radiation exposures can be both internal (as a result of ingestion, inhalation, or dermal absorption) and external. Internal exposures occur when radionuclides that have entered the body undergo radioactive decay, resulting in tissue damage dependent on the type and quantity of radiation emitted, as previously described. External exposures occur when radiation enters the body directly from sources located outside the body, such as from radionuclides in soil or water. In general, external exposures result from radionuclides that emit gamma radiation, which readily penetrates skin and clothing. Alpha and beta radiation from external sources are far less penetrating and deposit their energy primarily on the outer layer of skin or on clothing. Consequently, their contribution to the total dose of external radiation absorbed by an individual is negligible compared to that deposited by gamma rays (ATSDR, 1989k).

Many alpha-emitting radionuclides (e.g., all radium isotopes except radium-228) or their daughters also emit gamma rays (Turner, 1986). Therefore, slope factors for external radiation exposure have been calculated for alpha-emitting radium isotopes and their daughters, as shown in Table 3.34. Tritium, however, is a pure beta-emitter and does not emit gamma rays (Turner, 1986). Therefore, tritium does not have a slope factor for external radiation exposure since its radiation does not penetrate the body from external sources.

\subsubsection{Radiation Effects}

Exposure to a high dose of radiation (e.g., a thousand times the average annual background dose rate) during a short period of time (a few hours) produces detrimental effects in all the organs and 
systems of the body. However, such acute exposures are not expected at the F-Area BRPs. The only possible exposures are chronic, low-level exposures. Although lethal effects in human populations from chronic low-level exposures have never been documented, the effects have been projected from animal experiments (at high doses and dose rates). Studies assessing the difference between acute (short-term) and chronic (long-term) exposures show that radiation effects decrease dramatically as the period over which a given exposure is administered is extended (National Research Council, 1989). Thus, for units like the F-Area BRPs, where all exposures are longer-term and low-level, no immediate harmful effects are expected. Rather the statistical impacts of possible increases in cancer or genetic changes are the only credible potential radiation effects (National Research Council, 1989).

The general health effects of radiation can be divided into stochastic and non-stochastic effects. The risk of development of cancer from exposure to radiation is a stochastic effect. Examples of nonstochastic effects include acute radiation syndrome and cataract formation which occur only at high levels of exposures (Killough and Eckerman, 1983).

Radiation can damage cells in different ways. First, the radiation can cause damage to the strands of genetic material, DNA, in the cell. Damaged DNA continually attempts repair of its structure. However, the cell may not be able to recover from this type of damage, or the cell may live on but function abnormally. If the abnormally functioning cell divides and reproduces, a tumor or mutation in the tissue may develop. Rapidly dividing cells, such as those that line the intestines and the stomach, and the cells that make blood cells in the bone marrow, are particularly sensitive to radiation damage. Organ damage results from the damage caused to the individual cells and its tissues. This type of damage has been reported with doses of 10 to 500 rads. Acute radiation sickness is seen only after doses of greater than 50 rads. A dose of this magnitude is usually only received in a nuclear accident (ATSDR, 1989k).

When the cells damaged by radiation are reproductive cells, genetic damage can occur in the offspring of the person exposed. In addition, the developing fetus is especially sensitive to radiation. The types of malformations that may occur are related to the stage of fetal development and the cells that are differentiating at the time of exposure. Radiation damage to children exposed in the womb also is related to the magnitude of dose received by the pregnant mother. Mental retardation is one of many possible effects of fetal radiation exposure.

The risk of developing cancer as a result of internal or external exposure to radionuclides was evaluated using radionuclide slope factors calculated by EPA's Office of Radiation and Indoor Air (EPA, 1993a). Radionuclide ingestion and inhalation slope factors (Table 3.34) are best estimates 
(i.e., median or 50th percentile values) of the age-averaged, lifetime excess cancer incidence (fatal and nonfatal cancer) risk per unit of activity inhaled or ingested, expressed as risk/pCi. External exposure slope factors are best estimates of the lifetime excess cancer incidence risk for each year of exposure to external radiation from gamma-emitting radionuclides distributed uniformly in a thick layer of soil, and are expressed as risk/year per pCi/gram of soil (Table 3.34) (EPA, 1993a).

\subsection{Risk Characterization}

To characterize potential noncarcinogenic effects, comparisons are made between projected intakes of substances and toxicity values. To characterize potential carcinogenic effects, probabilities that an individual will develop cancer over a li ${ }^{3}$ time of exposure are estimated from projected intakes and chemical-specific dose-response information. Major assumptions, scientific judgments, and to the extent possible, estimates of the uncertainties embodied in the risk assessment are also presented in this section.

For each COPC having available toxicity values, cancer risk and hazard index (HI) estimates are presented. Appendix D presents the cancer risk and $\mathrm{HI}$ estimates derived for each receptor, pathway, and chemical. Tables 3.35 and 3.36 present carcinogenic and noncarcinogenic risk summaries by medium for BRP $231-\mathrm{F} / 1 \mathrm{~F}$ and RP $231-2 \mathrm{~F}$, respectively. Tables 3.37 and 3.38 provide a summary by receptor, medium and pathway for BRP 231-F/1F and RP 231-2F, respectively and Tables 3.39 and 3.40 provide a summary of the primary chemicals contributing to increased cancer and noncancer risks at BRP $231-\mathrm{F} / 1 \mathrm{~F}$ and RP 231-2F, respectively. Table 3.41 presents the radiological risks associated with $\mathrm{BRP} 231-\mathrm{F} / \mathrm{F}$ and $\mathrm{RP}$ 231-2F. Appendix $\mathrm{E}$ provides toxicity profiles describing information on fate and transport, human health effects, ecological effects and human health and environmental criteria for each COC.

\section{Carcinogenic Effects}

Carcinogenic risk is expressed as a probability of developing cancer as a result of lifetime exposure. For a given chemical and route of exposure, carcinogenic risk is calculated as follows:

$\begin{array}{lll}\text { Oral risk } & =\begin{array}{l}\text { exposure intake (administered dose) } \mathrm{x} \text { oral } \\ \text { slope factor (administered dose) }\end{array} \\ \text { Inhalation risk } \quad=\quad \begin{array}{l}\text { exposure intake (administered dose) } \mathrm{x} \text { inhalation } \\ \text { unit risk factor (administered dose) }\end{array}\end{array}$


Dermal risk $\quad=\quad$ intake (absorbed dose) $\mathrm{x}$ oral slope factor (absorbed dose)

For simultaneous exposure to several carcinogens, USEPA assumes that the risks are additive. That is to say:

RiskT $=$ Risk1 + Risk2 $+\ldots+$ Riski

Where:

RiskT $=$ the total cancer risk, expressed as a unitless probability, and

Riski $=$ the risk estimate for the ith substance

Addition of the carcinogenic risks is valid when the following assumptions are met:

- Doses are low;

- No synergistic or antagonistic interactions occur; and

- Similar endpoints are evaluated.

EPA's target range for carcinogenic risk associated with NPL sites is one-in-ten thousand (1E-04) to one-in-one million (1E-06). That is, the risks due to the site should not exceed this target range. Those COPCs that were identified during the risk characterization as contributing significantly to a pathway with a total cancer risk of greater than $1 \times 10^{-4}$ were identified as chemicals of concern (COCs).

\section{Noncarcinogenic Effects}

The potential for noncarcinogenic effects is evaluated by comparing an exposure level or intake (chronic daily intake or CDI) over a specified time period with a reference dose derived for a similar exposure period. This ratio termed the Hazard Quotient (HQ). In other words, the hazard quotient equals the intake divided by the reference value, or:

Oral HQ $\quad=\quad$ exposure intake (administered dose)/oral

RfD (administered dose)

Inhalation $\mathrm{HQ}=$ intake (administered dose)/inhalation

RfC (administered dose) 
Dermal HQ = intake (absorbed dose)/oral RfD (absorbed dose)

The HQ assumes that there is a level of exposure (i.e. $\mathrm{RfD}$ or $\mathrm{RfC}$ ) below which it is unlikely for even sensitive populations to experience adverse health effects. If the exposure level (E) exceeds the threshold (i.e., if $\mathrm{HQ}$ exceeds unity) there may be concern for potential noncancer effects.

To assess the overall potential for noncarcinogenic effects posed by more than one chemical, a hazard index (HI) approach has been developed by the EPA. This approach assumes that simultaneous subthreshold exposures to several chemicals could result in an adverse health effect. The HI is calculated as follows:

$$
\text { Hazard Index }(\mathrm{HI})=\mathrm{HQ} 1+\mathrm{HQ} 2+\ldots+\mathrm{HQi}
$$

Where:

\section{$\mathrm{HQi}=$ the hazard quotient for the ith toxicant}

It should be noted that exposure intake is taken to mean "chronic" exposure. Chronic exposure is defined as exposure that occurs over the majority of a life span.

Calculation of a $\mathrm{HI}$ in excess of 1 (one) indicates the potential for adverse health effects. Indices greater than 1 will be generated any time intake for any of the COPCs exceeds its RfD or RfC. However, if there are two or more chemicals involved, it is possible to generate a $\mathrm{HI}$ greater than 1 , even if none of the individual chemical intaks $s$ or concentrations exceed their respective RfDs or RfCs. If a particular COPC was determined to contribute significantly to a pathway ( $\mathrm{HI}$ of one or greater), it was identified as a COC.

\subsubsection{Current Land Use}

The only receptor quantitatively evaluated for current land use was the on-unit visitor. This receptor was assumed to be exposed to surface soils ( 0 to $0.6 \mathrm{~m}=0$ to $2 \mathrm{ft}$ ) via ingestion, dermal contact, and inhalation of volatiles and particulates generated from soil, as well as to surface water (dermal contact) and sediment (ingestion and dermal contact). Two receptor populations were evaluated: those exposed to soils at the BRP $231-\mathrm{F} / 1 \mathrm{~F}$ and those exposed to soils at RP 231-2F. Both populations were assumed to be exposed to surface water and sediment from the ephemeral wetland. The following sections report the derived cancer and noncancer risks derived for these receptors. 


\subsubsection{Nonradiological Carcinogenic Risk}

At BRPs 231-F/1F and RP 231-2F, the carcinogenic risks from all media to current visitors resulting from exposure to nonradiological carcinogens were $3 \times 10^{-7}$ and $5 \times 10^{-7}$, which were below the EPA target range of $10^{-6}$.to $10^{-4}$, indicating that an increased incidence of carcinogenic effects is not likely in these receptors (Tables 3.35 to 3.40 )

The cancer risks for inhalation of volatiles from soil were not calculated due to insufficient toxicity information for the COPCs present in soil.

\subsubsection{Nonradiological Noncarcinogenic Hazards}

At BRPs 231-F/1F and RP 231-2F, the hazard indices for all media for current visitors resulting from exposure to nonradiological noncarcinogens were 0.03 and 0.004 , respectively, which were well below the EPA threshold value of 1.0, indicating that adverse effects are not expected to occur in exposed receptors.

The HIs for inhalation of volatiles from soil were not calculated due to insufficient toxicity information for the COPCs present in soil.

\subsubsection{Radiological Risks}

At BRPs 231-F/1F and RP 231-2F, the cancer risks to current visitors resulting from exposure to radionuclides summed across all receptors and pathways were $3 \times 10^{-7}$ for both units, which were below the EPA target range of $10^{-6}$ to $10^{-4}$, indicating that an increased incidence of carcinogenic effects is not likely in these receptors (Tables 3.35 to 3.40 ).

The cancer risks for inhalation of volatiles from soil were not calculated due to insufficient toxicity information for the COPCs present in soil.

\subsubsection{Future Land Use}

Future receptors at the F-area units include both residents and workers. Both receptor populations were assumed to be exposed to surface soils ( 0 to $0.6 \mathrm{~m}=0$ to $2 \mathrm{ft}$ ) via ingestion, dermal contact, and inhalation of volatiles and particulates generated from soil. Both groups were also assumed to be exposed to groundwater via ingestion. In addition, future residents were assumed to be exposed to groundwater via dermal and inhalation exposure. Groundwater exposure was assessed using analytical concentrations as well as modeled soil-to-groundwater values (Appendix B). A future adolescent receptor was assumed to contact sediment and surface 
water at the unit. The risks and hazard indices derived for the future resident includes a summation of risks and hazards from all of the media of concern.

In addition to an evaluation of risks resulting from exposure to surface soils $(0$ to $0.6 \mathrm{~m}=0$ to 2 $\mathrm{ft}$ ), future receptors were also evaluated for exposure to soils located at the 0 to $1: 2 \mathrm{~m}(0$ to $4 \mathrm{ft}$ ) interval. Soils located in the 0 to $1.2 \mathrm{~m}(0$ to $4 \mathrm{ft}$ ) interval are assumed to be excavated and mixed with surface soils in the future as a result of development of the unit. The risks and hazards derived for these two soil intervals were nearly identical, given that the two data sets were comparable (Tables 3.35 through 3.38). Consequently, the total receptor risks and hazards presented in the following sections reflect the interval with the higher risk, if appropriate. The following sections report the derived cancer and noncancer risks for these receptors (Tables 3.35 to 3.40 ).

\subsubsection{Nonradiological Carcinogenic Risk}

\section{Residents}

At BRPs $231-\mathrm{F} / 1 \mathrm{~F}$ and $\mathrm{RP} 231-2 \mathrm{~F}$, the carcinogenic risk resulting from exposure to nonradiological carcinogens was $9 \times 10^{-5}$ for both units, which was within the EPA target range of $10^{-6}$ to $10^{-4}$, indicating that an increased incidence of carcinogenic effects may occur in the future residents. The increased cancer risks were due to potential exposure to groundwater and soil (Tables 3.35 to 3.40 ).

\section{Soil}

The carcinogenic risks resulting from exposure to soil at both units were $2 \times 10^{-5}$ for future residents, which is within the EPA target risk range. These risks were primarily the result of exposure to arsenic, PAHs, and dioxins at BRP 231-F/1F and PCB-1254 at RP 231-2F. 
The cancer risks for inhalation of volatiles from soil were not calculated due to insufficient toxicity information for the COPCs present in soil.

\section{Groundwater}

Future residents were assumed to be exposed to unit-wide groundwater. Analytical groundwater data was used in conjunction with modeled groundwater concentrations (Appendix B) to assess potential risks to future residents. If a chemical was present in soils at both the BRP 231-F/1F, a future concentration in groundwater was modeled for each unit.and the maximum value was used as the exposure point concentration (Table 3.21).

The carcinogenic risk derived for future residents was $7 \times 10^{-5}$. This risk was due primarily to dichloromethane, tetrachloroethylene, carbon tetrachloride, and arsenic in groundwater. Modeled as well as analytical values were available for dichloromethane and tetrachloroethylene, and the highest value (analytical) was used in the BRA. The modeled values were at least an order of magnitude lower than the analytical values as a result of the inclusion of biodegradation factors in the modeling (dichloromethane: analytical $=0.0302 \mathrm{mg} / \mathrm{L}$, modeled $=0.0038 \mathrm{mg} / \mathrm{L}$; tetrachloroethylene: analytical $=0.0328 \mathrm{mg} / \mathrm{L}$, modeled $=0.0007 \mathrm{mg} / \mathrm{L}$ ).

\section{Surface Water and Sediment in the Ephemeral Wetland}

For the exposure of the future adolescent resident to wetland surface water and sediment, the risk associated with sediment was $3 \times 10^{-6}$ and the risk associated with surface water was $7 \times 10^{-7}$. Although the risk from sediment falls within the EPA target range of $1 \times 10^{-4}$ to $1 \times 10^{-6}$, this risk resulted from a summation of risks that were below the EPA target range. The risk from surface water fell below the EPA target range.

\section{Workers}

At BRPs 231-F/1F and RP 231-2F, the carcinogenic risks to workers resulting from exposure to nonradiological carcinogens were $2 \times 10^{-5}$ for both units, which falls within the EPA target range of $10^{-6}$ to $10^{-4}$, indicating that an increased incidence of carcinogenic effects may occur in the workers (Tables 3.35 to 40 ).

\section{Soil}

For workers, the cancer risks were $6 \times 10^{-6}$ for the BRP 231-F/1F and $5 \times 10^{-6}$ for the RP 231-2F, which fall within the EPA target range. For workers, the increased cancer risks fesulted from exposure to arsenic, and HpCDD at BRP 231-F/1F and PCB-1254 at RP 231-2F. 
The cancer risks for inhalation of volatiles from soil were not calculated due to insufficient toxicity information for the COPCs present in soil.

\section{Groundwater}

Future workers were assumed to be exposed to unit-wide groundwater. Analytical groundwater data was used in conjunction with modeled groundwater concentrations (Appendix B) to assess potential risks to future workers. If a chemical was present in soils at both the BRP 231-F/1F, a future concentration in groundwater was modeled for each unit and the maximum value was used as the exposure point concentration (Table 3.21).

The carcinogenic risk derived for future residents was $2 \times 10^{-5}$. This risk was due primarily to tetrachloroethylene, carbon tetrachloride, and arsenic in groundwater. A modeled as well as analytical value were available for tetrachloroethylene, and the higher value (analytical) was used in the BRA. The modeled value $(0.0007 \mathrm{mg} / \mathrm{L})$ was almost two orders of magnitude lower than the analytical value $(0.0328 \mathrm{mg} / \mathrm{L})$ as a result of the inclusion of biodegradation factors in the modeling.

\subsubsection{Nonradiological Noncarcinogenic Hazards}

\section{Residents}

At BRPs 231-F/1F and RP 231-2F, the hazard indices resulting from exposure to noncarcinogens were 4 and 5 , respectively. The hazards derived for future residents at both units exceeded the EPA threshold of 1.0, indicating that adverse effects may occur in these receptors. The increase hazard indices resulted from potential exposure to groundwater and soil (Tables 3.35 to 3.40).

The results of the USEPA Lead Uptake Biokinetic Model indicated that lead in soil at the unit should not pose a threat to children at either the BRP 231-F/1F or RP 231-2F, as predicted blood lead concentrations for $>99 \%$ percent of the exposed population were expected to be below 10 $\mathrm{ug} / \mathrm{dL}$. The criterion of a blood level of $10 \mu \mathrm{g} / \mathrm{dL}$ or less in 95 percent of children was used a health guideline. The predicted blood lead concentration (geometric mean) of current/future children was $2.4 \mathrm{ug} / \mathrm{dL}$ for both the 0 to $0.6 \mathrm{~m}(0$ to $2 \mathrm{ft})$ and 0 to $1.2 \mathrm{~m}$ ( 0 to $4 \mathrm{ft})$ intervals at BRP 231-F/1F and $3.2 \mathrm{ug} / \mathrm{dL}$ ( 0 to $0.6 \mathrm{~m}=0$ to $2 \mathrm{ft}$ ) and $2.9 \mathrm{ug} / \mathrm{dL}$ ( 0 to $0.6 \mathrm{~m}=0$ to $2 \mathrm{ft}$ ) at $R P$ 231-2F. Appendix D contains the inputs and the outputs for the IEUBK Model. 


\section{Soil}

The hazard indices resulting from exposure to soil were 0.7 for BRP $231-F / 1 F$ and 2.0 for RP 231-2F. The hazard index of 0.7 for the BRP $231-/ 1 F$ was below the threshold of 1.0 , indicating that adverse health effects are not likely to occur in residents exposed to these soils. For the RP 231-2F, the increased hazard index was primarily due to exposure to PCB-1254.

The hazard indices for inhalation of volatiles from soil were not calculated due to insufficient toxicity information for the COPCs present in soil.

\section{Groundwater}

Future residents were assumed to be exposed to unit-wide groundwater. Analytical groundwater data was used in conjunction with modeled groundwater concentrations (Appendix B) to assess potential risks to future residents. If a chemical was present in soils at both the BRP 231-F/1F, a future concentration in groundwater was modeled for each unit and the maximum value was used as the exposure point concentration (Table 3.21).

The hazard index derived for future residents was 3. This risk was due primarily to arsenic, cadmium, carbon tetrachloride, and manganese in groundwater. Modeled as well as analytical values were available for cadmium, and the highest value (modeled) was used in the BRA. The modeled value was comparable to the analytical value, reflecting the fact that little biodegradation is expected to occur with cadmium over time (analytical $=0.002 \mathrm{mg} / \mathrm{L}$, modeled $=0.0048 \mathrm{mg} / \mathrm{L}$ ).

\section{Surface Water and Sediment in the Ephemeral Wetland}

For the exposure of the future adolescent resident to wetland surface water and sediment, the hazard index associated with sediment was 0.004 and the hazard index associated with surface water was 0.03 . These risks fall below the EPA threshold of 1.0, indicating the adverse effects are not likely to occur in future residents exposed to these media.

\section{Workers}

At BRPs 231-F/1F and RP 231-2F, the hazard indices resulting from exposure to nonradiological carcinogens were 0.4 and 0.5 , respectively. The hazards fall below the EPA threshold of 1.0, indicating that adverse effects are not likely to occur in the future workers (Tables 3.35 to 3.40 ). 
Soil

For workers, the hazard indices were 0.03 for the BRP 231-F/1F and 0.1 for the RP 231-2F. Both of these hazard indices were below the threshold of 1.0, indicating that adverse health effects are not likely to occur in workers exposed to unit soils.

The hazard indices for inhalation of volatiles from soil were not calculated due to insufficient toxicity information for the COPCs present in soil.

\section{Groundwater}

Future workers were assumed to be exposed to unit-wide groundwater. Analytical groundwater data was used in conjunction with modeled groundwater concentrations (Appendix B) to assess potential risks to future workers. If a chemical was present in soils at both the BRP 231-F/1F, a future concentration in groundwater was modeled for each unit and the maximum value was used as the exposure point concentration (Table 3.21).

The hazard index derived for future workers was 0.4, which falls below the EPA target of 1.0, indicating that adverse health effects are not likely to occur in future workers exposed to groundwater.

\subsubsection{Radiological Risks}

\section{Residents}

At BRPs 231-F/1F and RP 231-2F, the radiological risks resulting from exposure to radionuclides were $8 \times 10^{-5}$ for both units. This risk falls within the EPA target range of $10^{-6}$ to $10^{-4}$, indicating that an increased incidence of carcinogenic effects may occur in exposed residents. The increased risks resulted from potential exposure to soil and groundwater (Table 3.41).

\section{Soil}

The radiological risk resulting from exposure to radionuclides in soil was $2 \times 10^{-5}$ for both BRP 231-F/1F and RP 231-2F, which falls within the EPA target range of $10^{-6}$ to $10^{-4}$. The increased risks were due primarily to direct radiation from cesium-137 and ingestion of potassium-40 in homegrown produce at BRP 231-F/1F. For BRP 231-F/1F, the increased risks resulted from direct radiation from cesium- 137 and ingestion of potassium- 40 and strontium-90 in produce. 


\section{Groundwater}

The radiological risk derived for future residents exposed to groundwater was $6 \times 10^{-5}$, which was within the EPA target range of $10^{-6}$ to $10^{-4}$. The increased risk was primarily due to iodine-129, radium-226, radium-228, strontium-90, technetium-99 and tritium in groundwater. As discussed above in Section 3.4.2.1, analytical groundwater values were used in conjunction with modeled (soil to groundwater) values to assess potential exposure of future receptors. Although technetium-99 and iodine-129 were not detected in current groundwater, they were detected in deep soils and, therefore, were mocieled for potential impacts to future groundwater. Given that they were not historically present in groundwater at the unit, they are not expected to be present in the future at concentrations that will result in adverse health effects in groundwater users.

\section{Surface Water and Sediment}

The radiological risk values for the resident adolescent exposed to wetland surface water and sediment were $1 \times 10^{-9}$ and $2 \times 10^{-11}$, respectively. These risks fall well below the EPA target range of $10^{-6}$ to $10^{-4}$, indicating that increased incidences of carcinogenic effects are not expected in these receptors.

\section{Homegrown Produce}

The total radiological risk to the future resident via ingestion of produce grown at BRPs $231-\mathrm{F} / 1 \mathrm{~F}$ and RP 231-2F was $1 \times 10^{-5}$ at both units (Table 3.41), which falls within the EPA target risk range of $10^{-6}$ to $10^{-4}$, indicating that increased carcinogenic risks may result from the ingestion of produce. The risks were $6 \times 10^{-6}, 4 \times 10^{-6}$, and $3 \times 10^{-6}$ for the ingestion of leafy vegetables, tuberous vegetables, and fruits, respectively at BRP 231-F/1F (Appendix D). For the RP 231-2F, the risks were $5 \times 10^{-6}, 3 \times 10^{-6}$ and $2 \times 10^{-6}$ for the ingestion of leafy vegetables, tuberous vegetables and fruits, respectively (Appendix $D$ ). The increased risks resulting from the ingestion of homegrown produce was primarily the result of exposure to potassium- 40 present at both units.

\section{Workers}

At BRPs 231-F/1F and RP 231-2F, the radiological risks from all media to workers resulting from exposure to radionuclides were $2 \times 10^{-5}$ for both the BRP 231-F/1F and RP 231-2F), which falls within the EPA target risk range of $10^{-6}$ to $10^{-4}$, indicating that increased carcinogenic risks may result in future workers exposed to site media (Table 3.41). 


\section{Soil}

The radiological risk resulting from exposure to radionuclides in soil was $3 \times 10^{-6}$ for BRP 231$\mathrm{F} / 1 \mathrm{~F}$ and $2 \times 10^{-6}$ for RP $231-2 \mathrm{~F}$, which fall within the EPA target range of $10^{-6}$ to $10^{-4}$. The increased risks were due primarily to direct radiation from cesium- 137 and potassium- 40 at both units.

\section{Groundwater}

The radiological risk derived for future workers exposed to groundwater was $2 \times 10^{-5}$, which was within the EPA target range of $10^{-6}$ to $10^{-4}$. The increased risk was primarily due to iodine-129, radium-226, radium-228, strontium-90, technetium-99 and tritium in groundwater. As discussed above in Section 3.4.2.1, analytical groundwater values were used in conjunction with modeled (soil to groundwater) values to assess potential exposure of future receptors. Although technetium-99 and iodine-129 were not detected in current groundwater, they were detected in deep soils and, therefore, were modeled for potential impacts to future groundwater. Given that they were not historically present in groundwater at the unit, they are not expected to be present in the future at concentrations that will result in adverse health effects in groundwater users.

\subsubsection{Uncertainty}

The evaluation of chemical and radiological risks to human health presented in this baseline risk assessment was, by necessity, based on a number of assumptions. In addition, many uncertainties are inherent in the risk assessment process. This section provides a discussion of the uncertainties associated with key site-related variables and the major assumptions used in this assessment in order to address their potential impact on the results contained herein.

\subsubsection{Chemicals of Potential Concern}

Several potential sources of contamination located adjacent to the F-Area BRPs have documented soil and groundwater contamination associated with them. Contaminants from these sources may be inadvertently attributed to the BRPs, thereby overestimating the risk contribution from the unit. Sampling data may not completely represent the actual distribution of contamination at the F-Area BRPs and in offunit mobile environmental media (surface water and sediment), which may result in an over- or under-estimate of potential risk. 
The sampling data collected at any unit are inevitably a limited subset of the nearly unlimited quantity of data that potentially could be collected, and as such may result in an underestimation of risk. On the other hand, given that the objective of the RFI process it to define the nature and extent of contamination, samples were not collected randomly and may be biased toward overestimation of chemical concentrations representative of the unit. The lack of surface water and sediment data from the small, unnamed tributary located east of RP 231-2F adds to the uncertainty in assessing the extent and nature of contamination, and in quantifying total unitrelated risk.

Uncertainty also is inherent in the selection of COPCs. Uncertainty in contaminant identification is considered low because sampling protocol generally targets appropriate analytes based on historical information and guidance. Reasonable certainty also is assumed because of the sample data validation and quality assurance/quality control procedures applied to sample analysis and data evaluation.

In the COPC screening process used in this BRA, chemicals in groundwater and soil were eliminated following screening against background, risk-based concentrations, essential nutrients and frequency of detection. Limited background data resulted in a uncertainty in the background screening portion of the COPC process. Given that only radium (BRP 231-F/1F and RP 231-2F) and arsenic (RP 231-2F) were eliminated during the background screen in soil, the uncertainty regarding this step may not be significant. In groundwater, six COPCs were eliminated from consideration following the background screening, leading to increased uncertainty regarding the resultant COPCs. The majority of the chemicals in both soil and groundwater were eliminated following the screening against risk-based concentrations. Risk-based concentrations are dynamic and, therefore, uncertainty in these values cannot be eliminated. Eliminating contaminants in the COPC screening process can lead to lower estimates of potential health effects than inclusion of all analytes.

In particular to uranium isotopes, the levels appear to the naturally occurring because of their relative activities. The maximum detected concentrations of uranium-234, -235 , and -238 are $3.58 \mathrm{pCi} / \mathrm{g}, 6.14 \mathrm{pCi} / \mathrm{g}$, and $3.28 \mathrm{pCi} / \mathrm{g}$, respectively. If these activities normalized to uranium238 , the activity ratios of $234: 235: 238$ are $1.09,0.043$, and 1.00. Naturally occurring weight of ratios of 234:235:238 are 1.02, 0.48, and 1.00. From this comparison, it appears that the uranium isotopes in BRP soils are naturally occurring; however, when maximum concentrations of uranium are compared to twice the unit-specific background, the maximum detected uranium levels exceed background. This comparison requires inclusion of the uranium isotopes as COPCs. Additionally, the radium isotopes detected in F-Area BRP soils are most likely daughters of 
naturally occurring uranium and thorium. However, again in the comparison of maximum radium levels to that of twice background, radium isotopes were retained as COPCs. Therefore, with the inclusion of uranium and radium isotopes as COPCs, the risk from exposure is likely overestimated.

For this BRA, groundwater was assessed using analytical data along with modeled values. The values were modeled from soils located at a depth of $1.2 \mathrm{~m}(4 \mathrm{ft})$ to the bottom-of-the-pit. Several contaminants detected in groundwater during the sampling were not detected in soils and, therefore, were not quantified in the risk assessment. Given the fact that these contaminants were not found in soils indicates that the F-Area unit is not likely to be their source. Therefore, not including these in the risk assessment may be a realistic approach to assessing contamination originating in the F-Area. However, elimination of those chemicals detected in the groundwater may underestimate the risks associated with this medium. Also, high concentrations of PAHs were detected in soils located within the depths of soil used in the modeling. As discussed in the RFI, these high concentrations of PAHs were likely the result of sampling close to preserved wood or asphalt rubble, indicating that the concentrations were not indicative of contamination at the units. This data was included in the modeling and did not result in a significant groundwater risk.

\subsubsection{Exposure Assessment}

Factors that can contribute to uncertainty in the exposure assessment include identification of exposure pathways; assumptions for scenario development, intake parameters, and exposure pathways; and derivation of exposure point concentrations.

The identification of potential exposure pathways and receptors was based on site-specific reasonable current use and hypothetical future residential land use. Although it is not possible to substantiate statements regarding the future land use, it is believed that the probability is low that the F-Area BRPs will be developed for residential use. Therefore, the residential scenario may over-estimate potential risk for the future land use scenario. Unit-specific receptors were identified to the extent possible and exposure parameters tailored to these receptors to minimize uncertainty in the postulated scenarios and exposure assessment.

Values assumed for exposure parameters (e.g., inhalation rate and exposure frequency) used in calculations for intakes were based primarily on EPA guidance (EPA, 1990, 1991a and 1995a). These assumptions might result in underestimating or overestimating the intakes calculated for specific receptors, depending on the accuracy of the assumptions relative to actual site conditions 
and uses. In the case of dermal exposure, there is uncertainty associated with the conversion from an administered intake to an absorbed intake because of uncertainty associated with the conversion factors.

One potential pathway identified in the exposure assessment, the ingestion of homegrown produce by future on-unit residents, was only evaluated for radiological COPCs. The baseline risk assessment does not include an estimation of the exposure through this pathway for nonradiological contaminants because of the large degree of uncertainty in the soil-to-plant uptake factors necessary to calculate intake for this pathway. Risk from nonradiological COPCs through this pathway, however, is expected to be insignificant relative to other exposure pathways.

There are several sources of uncertainty associated with the estimation of leachability of contaminants from soil to groundwater. The major sources of uncertainty are associated with accurate delineation of the contaminants which forces conservative assumptions to describe the source. A second major uncertainty regards the distribution of contaminants between the soil and water phases. There is a complex chemistry associated with multiple analytes at a site. Conservative assumptions are required because the environmental chemistry is extremely difficult to accurately describe or predict.

\subsubsection{Toxicity Assessment}

Uncertainty is inherent in the toxicity values utilized in characterizing the carcinogenic and noncarcinogenic risks. Such uncertainty is chemical-specific and is incorporated into the toxicity value during its development. For example, an uncertainty factor may be applied for interspecies and intrahuman variability, for extrapolation from subchronic to chronic exposures, or for epidemiological data limitations.

A number of identified COPCs are currently under EPA review with the possibility of changed reference doses, slope factors, or carcinogenic weight-of-evidence. Interim and provisional toxicity values were used, where available, when values could not be obtained from IRIS. Because toxicity information is limited for many chemicals, toxicity numbers from similar or related chemicals are sometimes used. Toxicity values could not be obtained for some COPCs, thereby precluding their inclusion in the quantitative risk estimates. The resulting risk estimates do not include the incremental chemical-specific risks from these chemicals, and, therefore, may underestimate risk.

Because toxicity information is limited for many chemicals, toxicity numbers from similar or related chemicals are sometimes used. The use of surrogate toxicity values may underestimate or 
overestimate risk. For other chemicals, analytical results may not distinguish between different isomers or forms of a chemical although available toxicity information does, or vice versa. The absence of toxicity values for other chemicals may tend to underestimate risks.

Toxicity values for specific chlorodibenzo-p-dioxin and chlorodibenzo-p-furan isomers and specific PAHs were not available. Therefore, the toxicity value for the most toxic chemical of each of these respective chemical classes was used. The actual chemical concentration was then modified by a toxicity equivalency factor (TEF) for the chlorodibenzo-p-dioxins and furans or by a relative potency factor for PAHs in order to adjust the intake and resulting risk and hazard index values to more adequately reflect the actual toxicity of the chemical being characterized. The application of TEFs or relative potency factors provides only an approximation of relative toxicity and therefore may underestimate or overestimate actual risks.

Toxicity information was not available fnr derma! exposure; hence several assumptions were made which may overestimate or underestimate risk.

Toxicity information also was not available for nine chemicals, which will result in an underestimation of potential risks (Table 3.42). The lack of toxicity values for these chemicals will result in a potential underestimation of risks and hazards associated with the units Although lead has not toxicity values, it was addressed in the BRA using the Biokinetic Uptake. Model (EPA, 1994b).

\section{3:4.3.4 Risk Characterization}

Uncertainties in the toxicity assessment are compounded in the risk characterization under the assumption of dose additivity for multiple substance exposure. This assumption ignores possible synergisms and antagonisms among chemicals. The assumption that all the toxic and carcinogenic effects are additive could result in the underestimation of risk because concurrent exposure to several contaminants might have synergistic toxic effects (e.g., exposure to two metals concurrently might induce a greater toxic effect than indicated by simply adding the toxic effects from each metal). However, summing risks for chemicals having various weight-of-evidence classifications as well as toxic effects to different target organs and ignoring possible antagonistic effects of some chemicals may overestimate risks.

Some of the procedures used and uncertainties inherent in the human health assessment process may tend to underestimate potential risk. However, assumptions built into this BRA tend to overestimate rather than underestimate potential risks, including conservative assumptions for the 
exposure scenarios. For example, contamination is assumed to remain constant over time. Fate and transport mechanisms, which would result in the degradation and loss of some COPCs from the environment, were not considered in the exposure evaluation for future residents. Actual concentrations may change over time, which would influence the intake and related risk values. Thus, actual risks are likely to be lower than those presented in this assessment. 
BASELINE RISK ASSESSMENT

WSRC-TR-94-108

F-AREA BURNING/RUBBLE PITS AND RUBBLE PIT

REV. 1.2, MARCH 1996 
FIGURE 3-1.

HUMAN/ECOLOGICAL DATA EVALUATION

IDENTIFICATION OF CHEMICALS OH POTENTIAL CONCERN

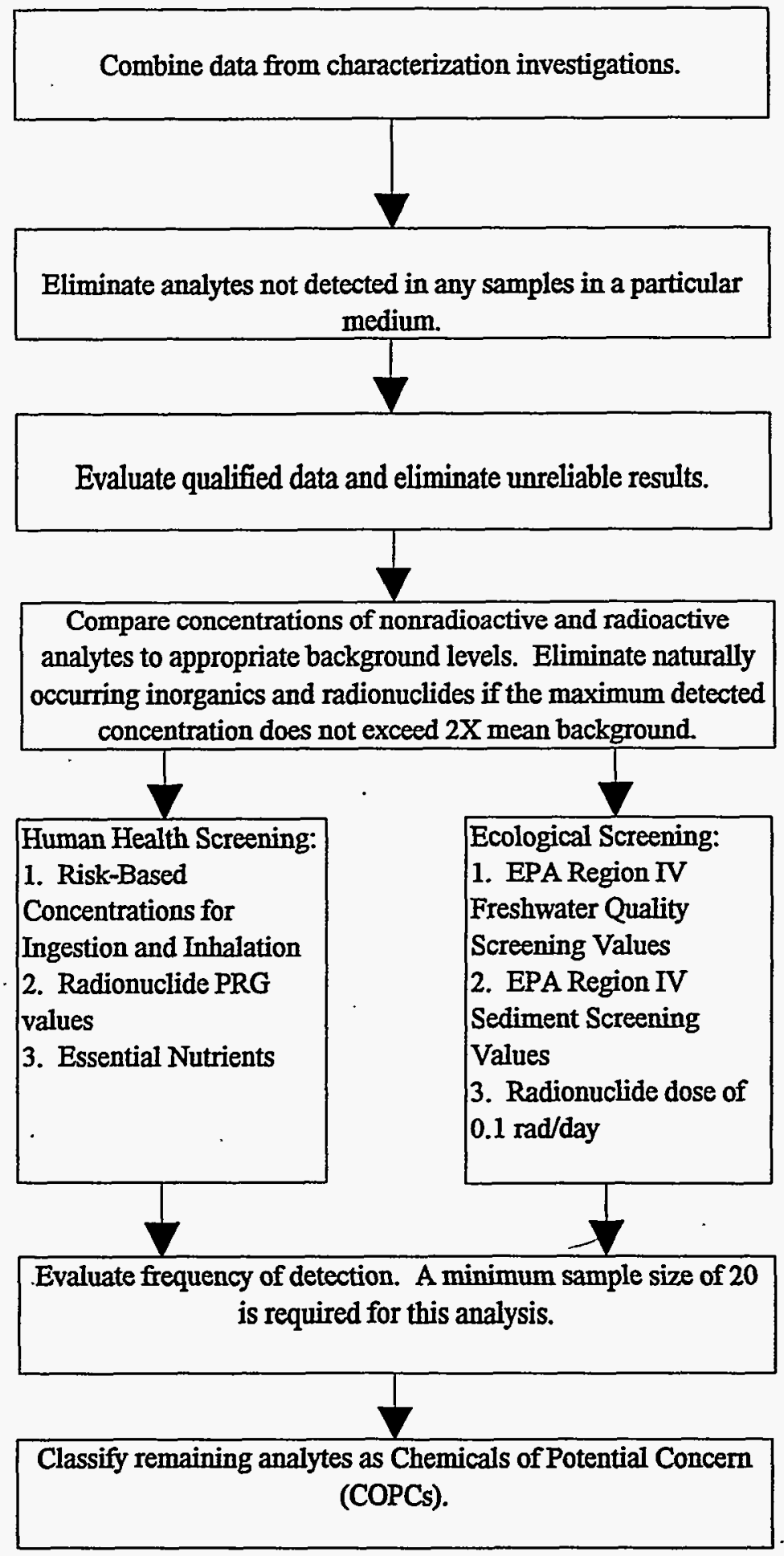




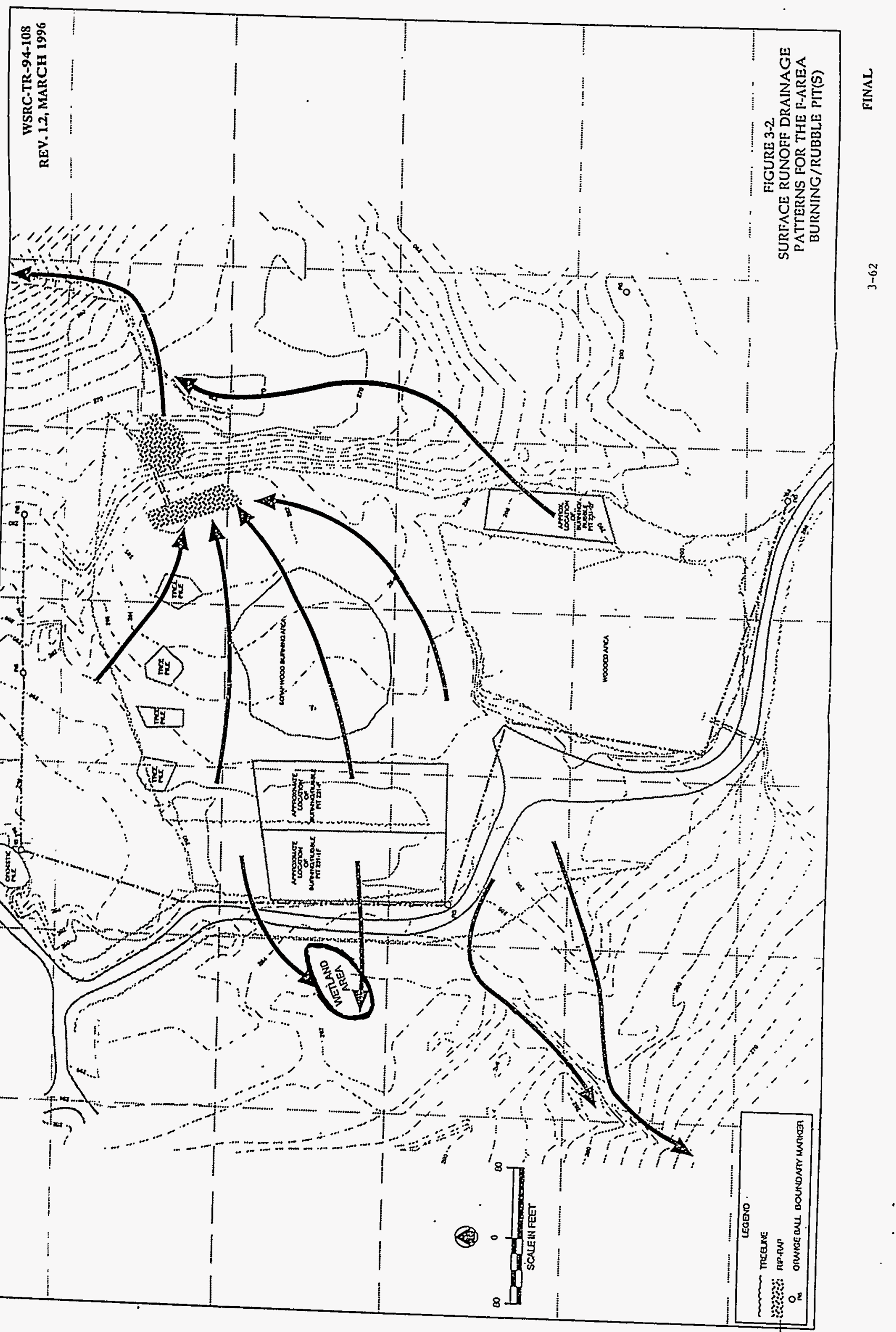




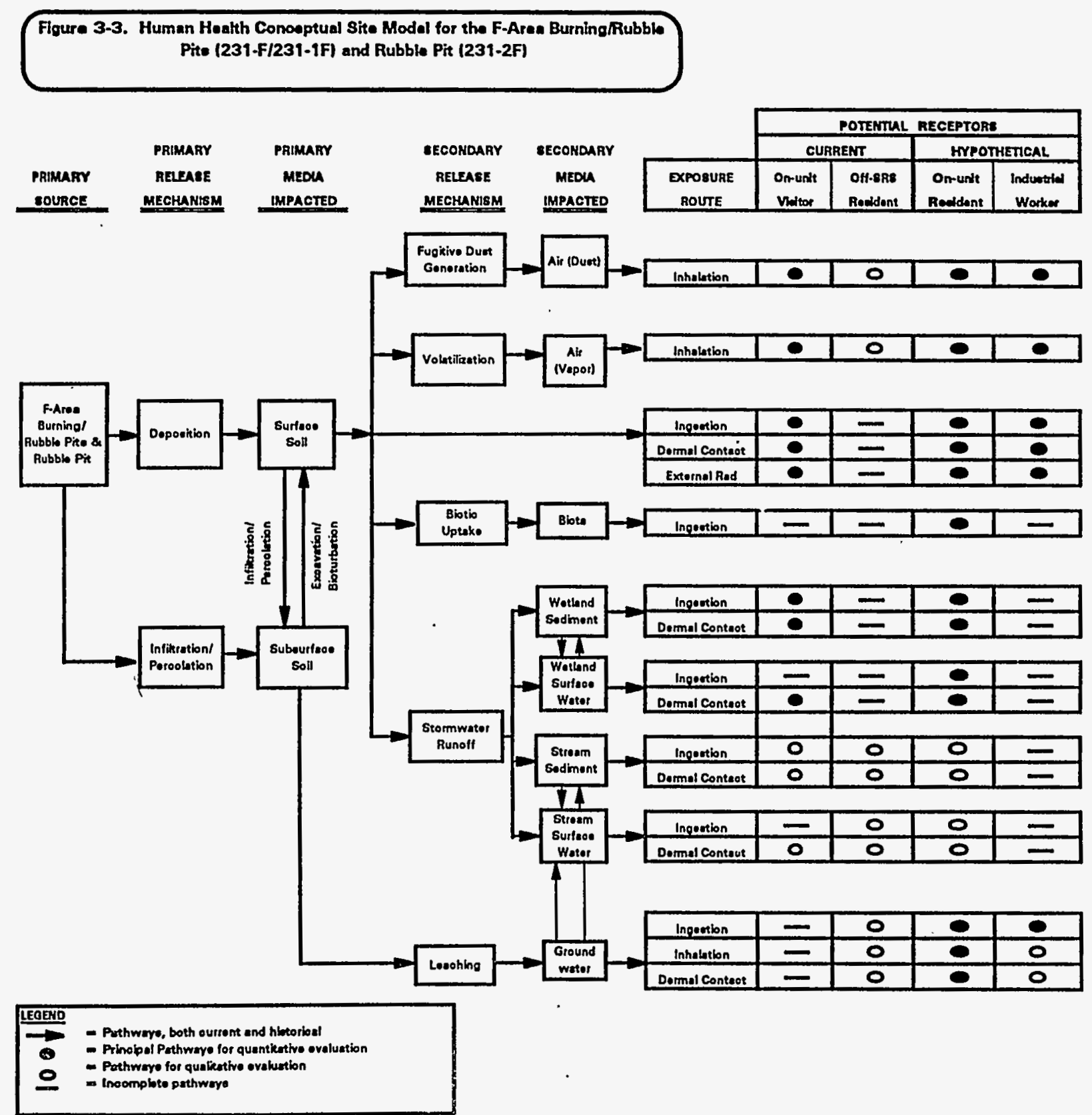




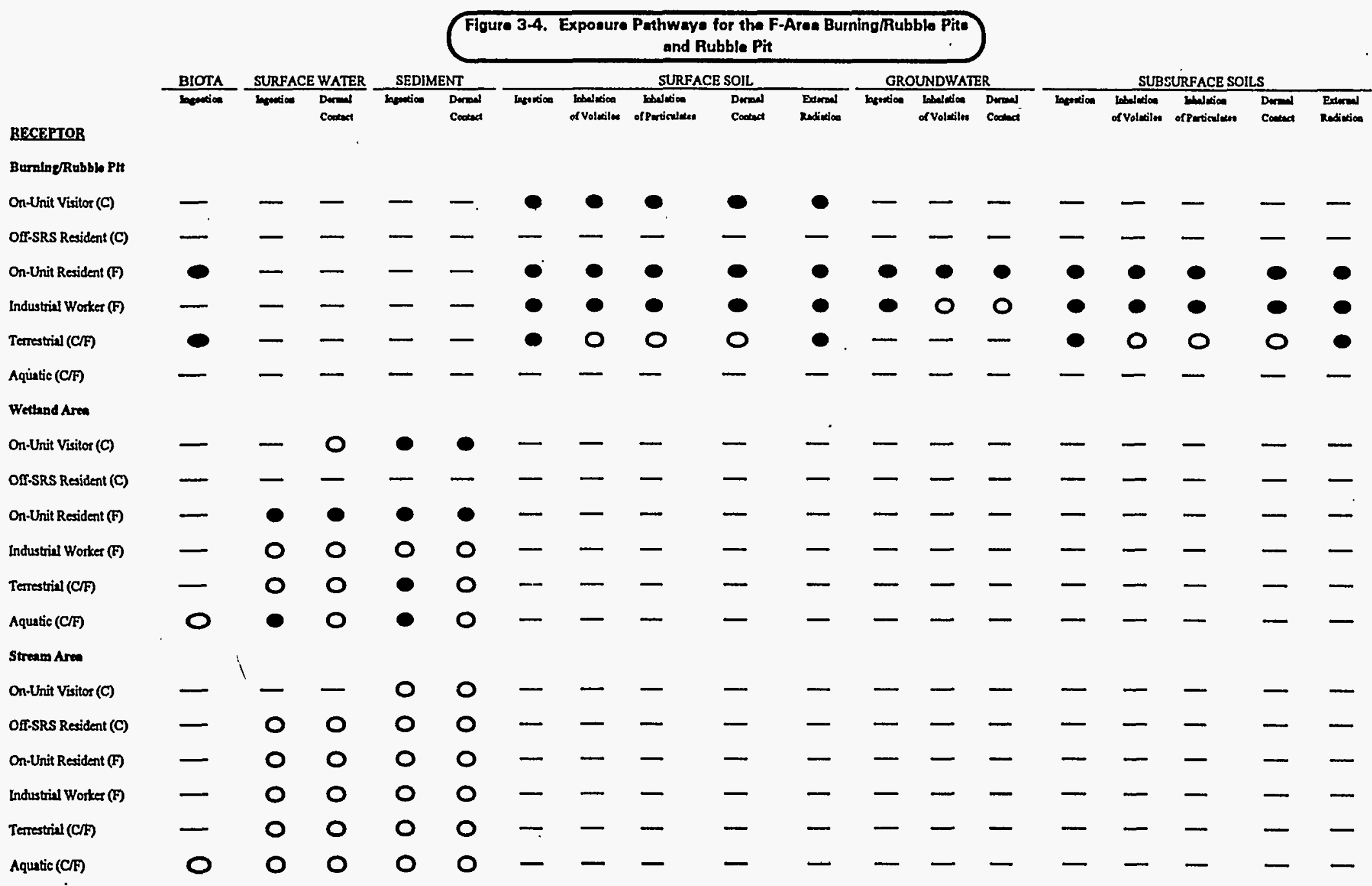


TABLE 3.1

SAVANNAH RIVER SITE

BURNING/RUBBLE PITS 231-F AND 231-1F

CHEMICALS PRESENT IN UNIT SAMPLES (CPUSS)

SURFACE SOIL SUMMARY (0-0.5 FEET)

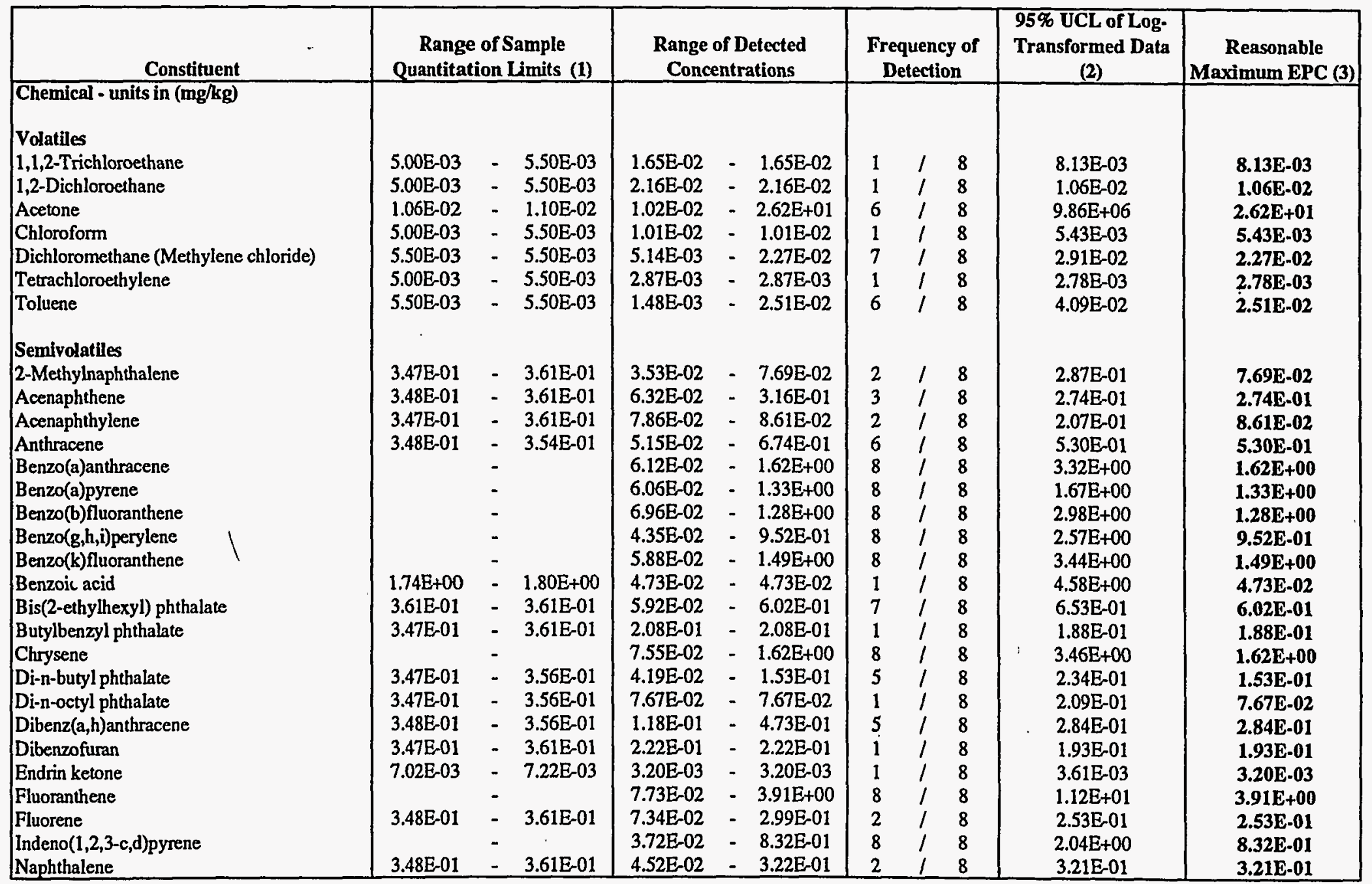


TABLE 3.1

SAVANNAH RIVER SITE

BURNING/RUBBLE PITS 231-F AND 231-1F

CHEMICALS PRESENT IN UNIT SAMPLES (CPUSS)

SURFACE SOIL SUMMARY (0-0.5 FEE'T)

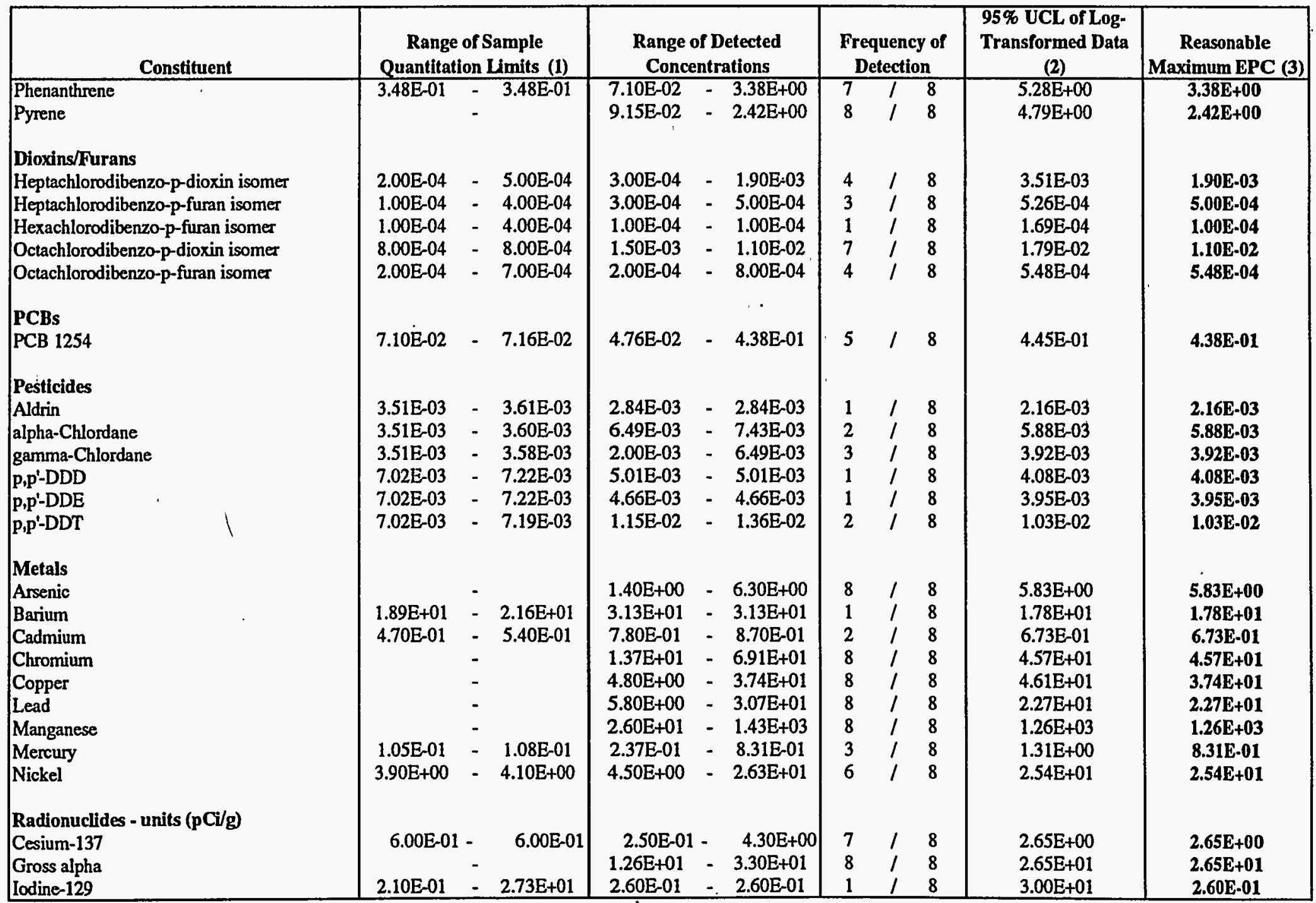


TABLE 3.1

SAVANNAH RIVER SITE

BURNING/RUBBLE PITS 231-F AND 231-1F

CHEMICALS PRESENT IN UNIT SAMPLES (CPUSS)

SURFACE SOIL SUMMARY (0-0.5 FEET)

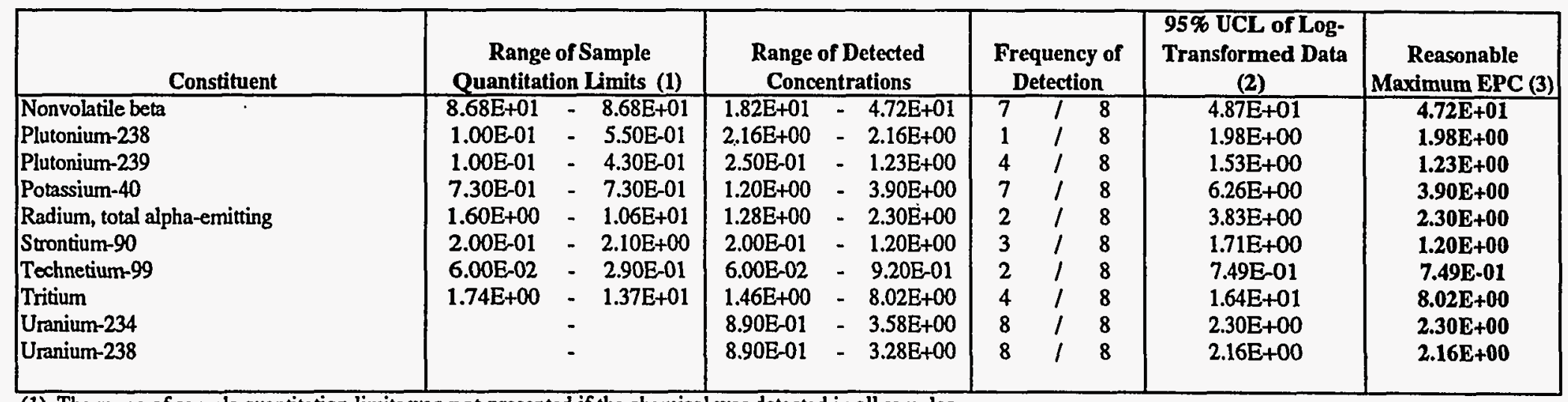

(1) The range of sample quantitation limits was not presented if the chemical was detected in all samples.

(2) The data were assumed to be lognomally distributed and, therefore, were transformed.

(3) The lesser of either the 95\% UCL of the transformed data or the maximum was used as the Exposure Point Concentration (F.PC). 
TABLE 3.2

SAVANNAH RIVER SITE

RUBBLE PIT 231-2F

CHEMICALS PRESENT IN UNIT SAMPLES (CPUSs)

SURFACE SOIL SUMMARY (0-0.5 FEET)

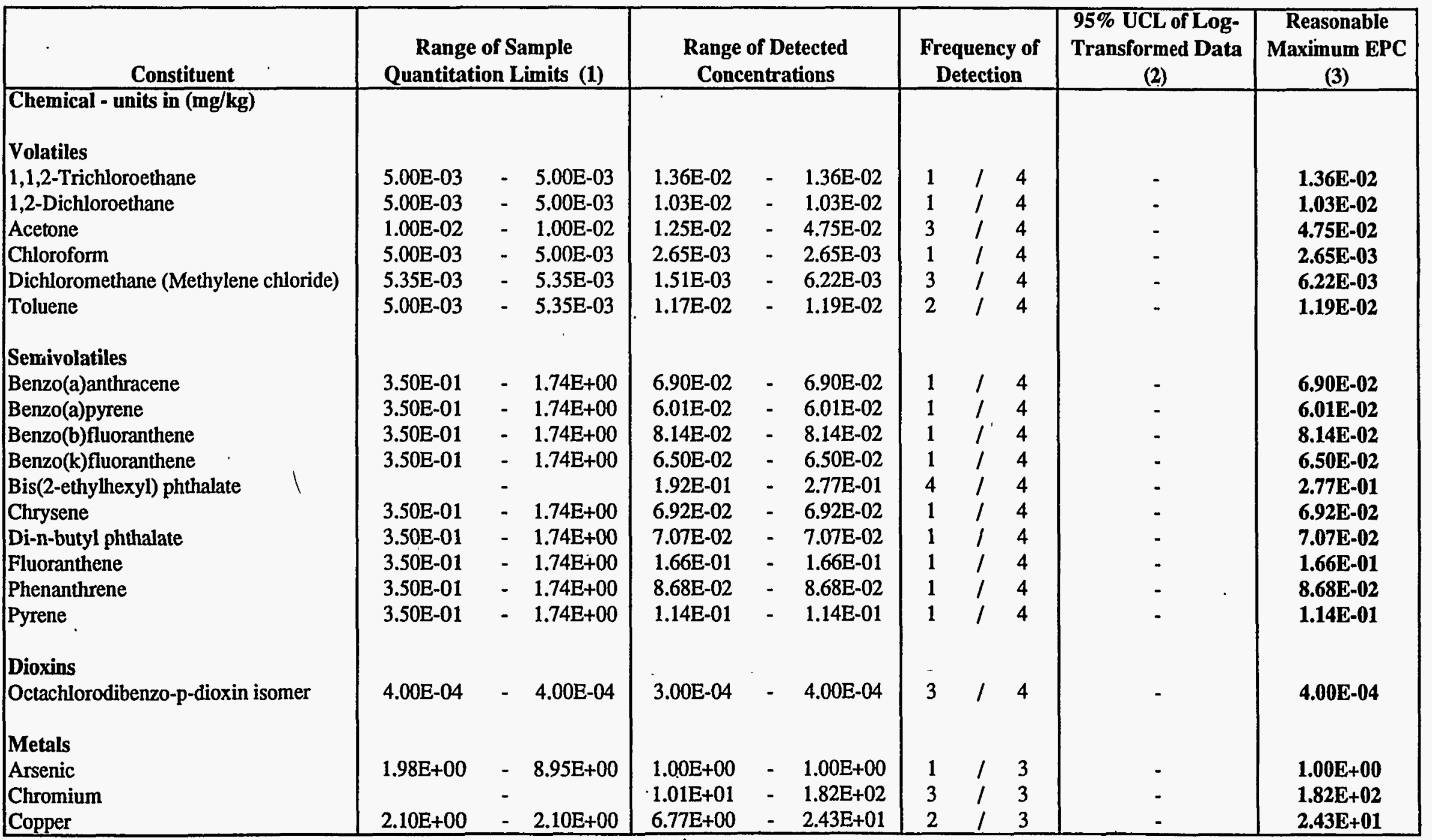


TABLE 3.2

SAVANNAH RIVER SITE

RUBBLE PIT 231-2F

CHEMICALS PRESENT IN UNIT SAMPLES (CPUSS) SURFACE SOIL SUMMARY (0-0.5 FEET)

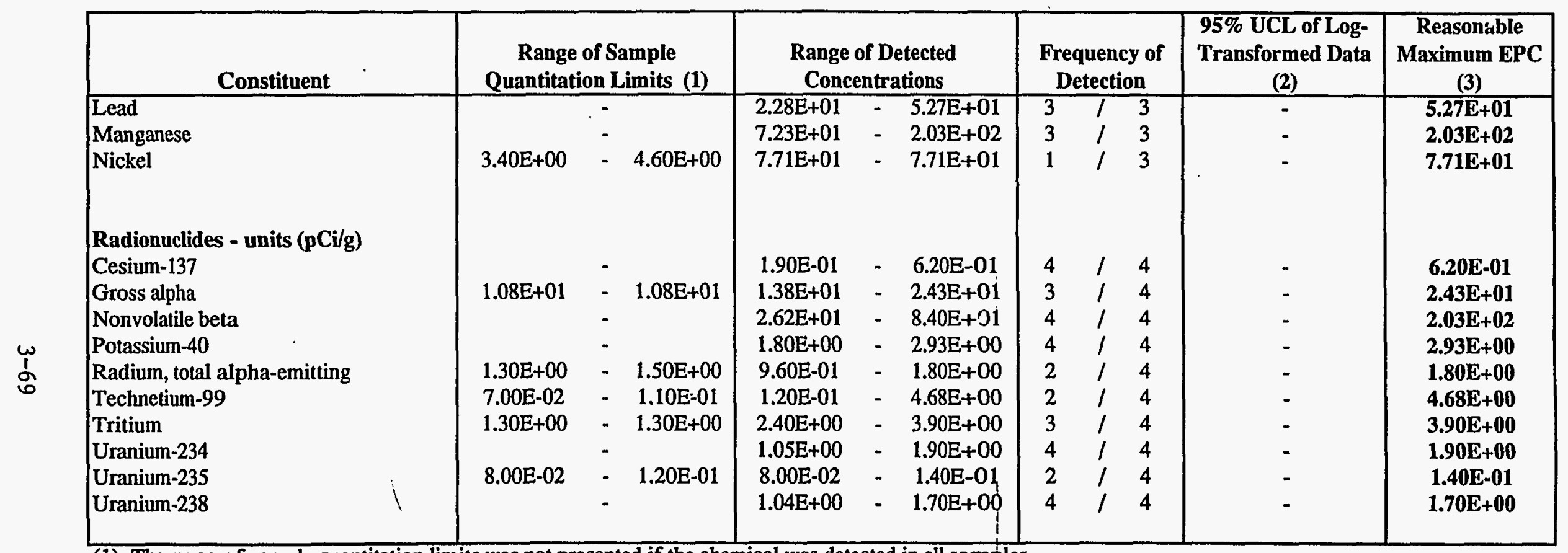

(1) The range of sample quantitation limits was not presented if the chemical was detected in all samples.

(2) The data were assumed to be lognormally distributed and, therefore, were transformed.

(3) The lesser of either the $95 \%$ UCL of the transformed data or the maximum was used as the Exposure Point Concentration (EPC). 
TABLE 3.3

SAVANNAH RIVER SITE

BURNING/RUBBLE PITS 231-F AND 231-1F

CHEMICALS PRESENT IN UNIT SAMPLES (CPUSS) SURFACE SOIL SUMMARY (0-2 FEET)

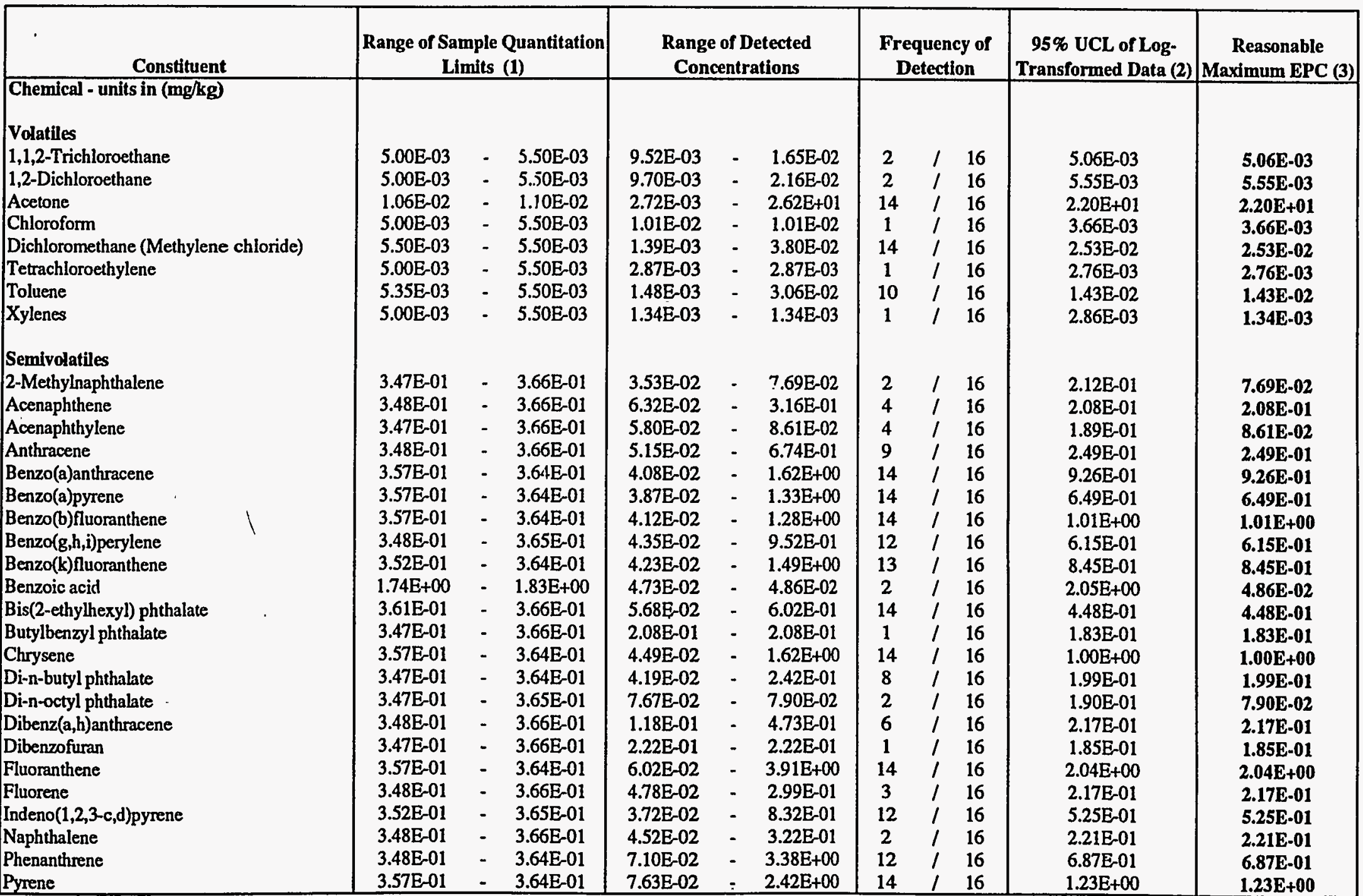


TABLE 3.3

SAVANNAH RIVER SITE

BURNING/RUBBLE PITS 231-F AND 231-1F

CHEMICALS PRESENT IN UNIT SAMPLES (CPUSS)

SURFACE SOIL SUMMARY (0-2 FEET)

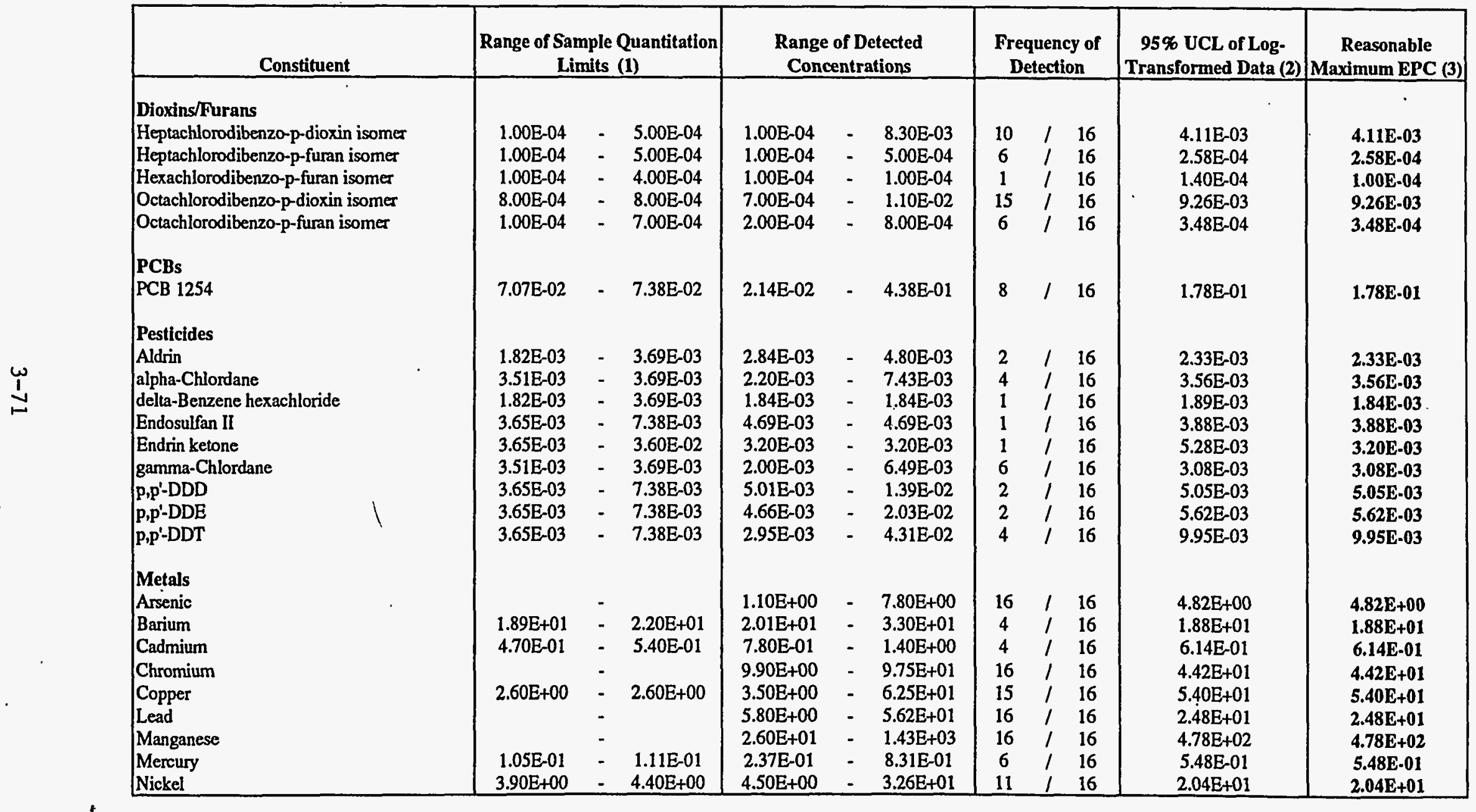


TABLE 3.3

SAVANNAH RIVER SITE

BURNING/RUBBLE PITS 231-F AND 231-1F CHEMICALS PRESENT IN UNIT SAMPLES (CPUSS)

SURFACE SOIL SUMMARY (0-2 FEET)

\begin{tabular}{|c|c|c|c|c|c|c|c|c|c|}
\hline Constituent & \multicolumn{2}{|c|}{$\begin{array}{c}\text { Range of Sample Quantitation } \\
\text { Limits (1) }\end{array}$} & \multicolumn{2}{|c|}{$\begin{array}{l}\text { Range of Detected } \\
\text { Concentrations }\end{array}$} & \multicolumn{3}{|c|}{$\begin{array}{c}\text { Frequency of } \\
\text { Detection }\end{array}$} & $\begin{array}{l}\text { 95\% UCL of Log. } \\
\text { Transformed Data (2) }\end{array}$ & $\begin{array}{c}\text { Reasonable } \\
\text { Maximum EPC (3) }\end{array}$ \\
\hline
\end{tabular}

(1) The range of sample quantitation limits was not presented if the chemical was detected in all samples.

(2) The data were assumed to be lognormally distributed and, therefore, were transformed.

(3) The lesser of either the 95\% UCL of the transformed data or the maximum was used as the Exposure Point Concentration (EPC). 
TABLE 3.4

SAVANNAH RIVER SITE

RUBBLE PIT 231-2F

CHEMICALS PRESENT IN UNIT SAMPLES (CPUSS)

SURFACE SOIL SUMMARY (0-2 FEET)

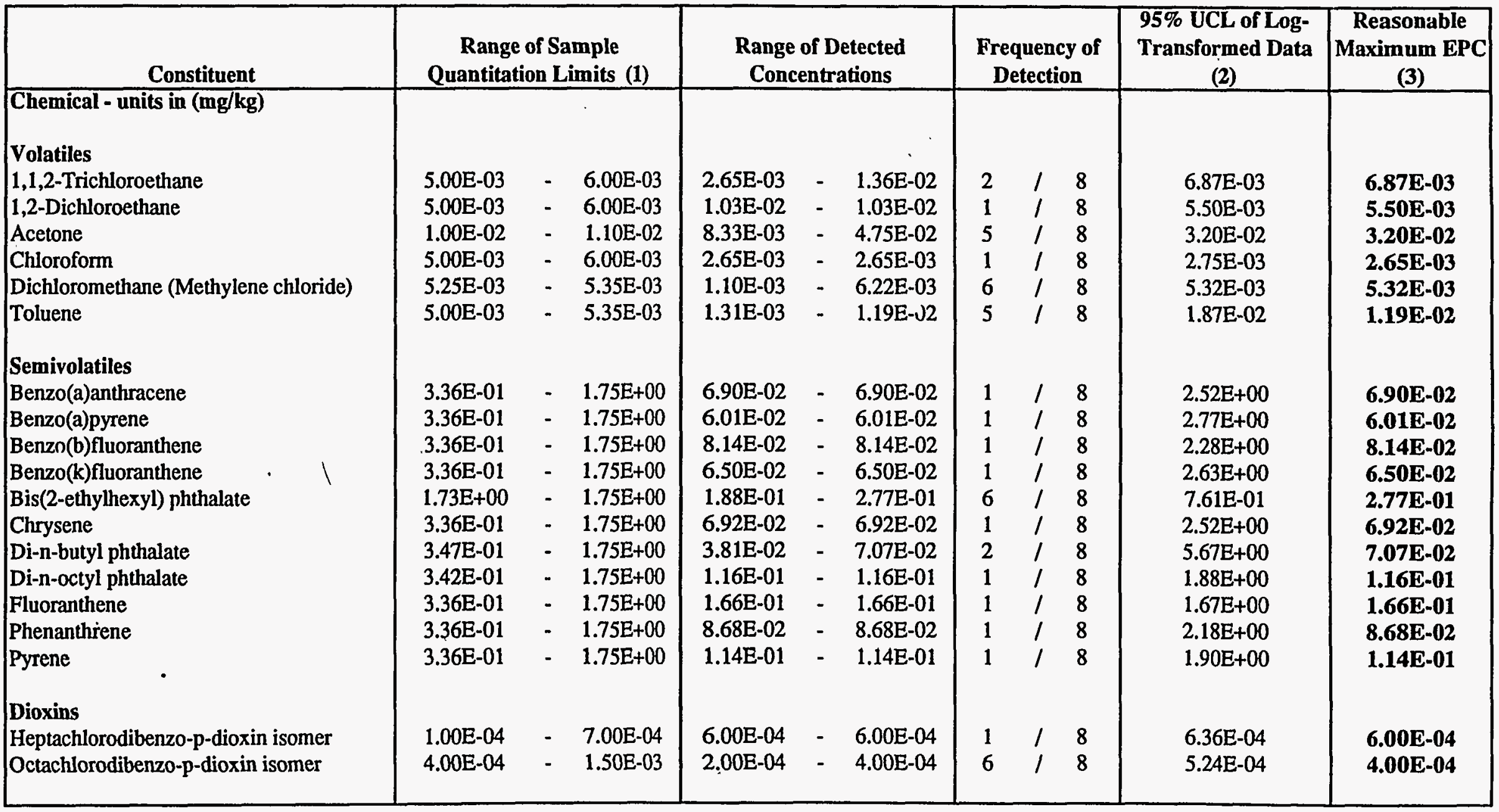


TABLE 3.4

SAVANNAH RIVER SITE

RUBBLE PIT 231-2F

CHEMICALS PRESENT IN UNIT SAMPLES (CPUSs)

SURFACE SOIL SUMMARY (0-2 FEET)

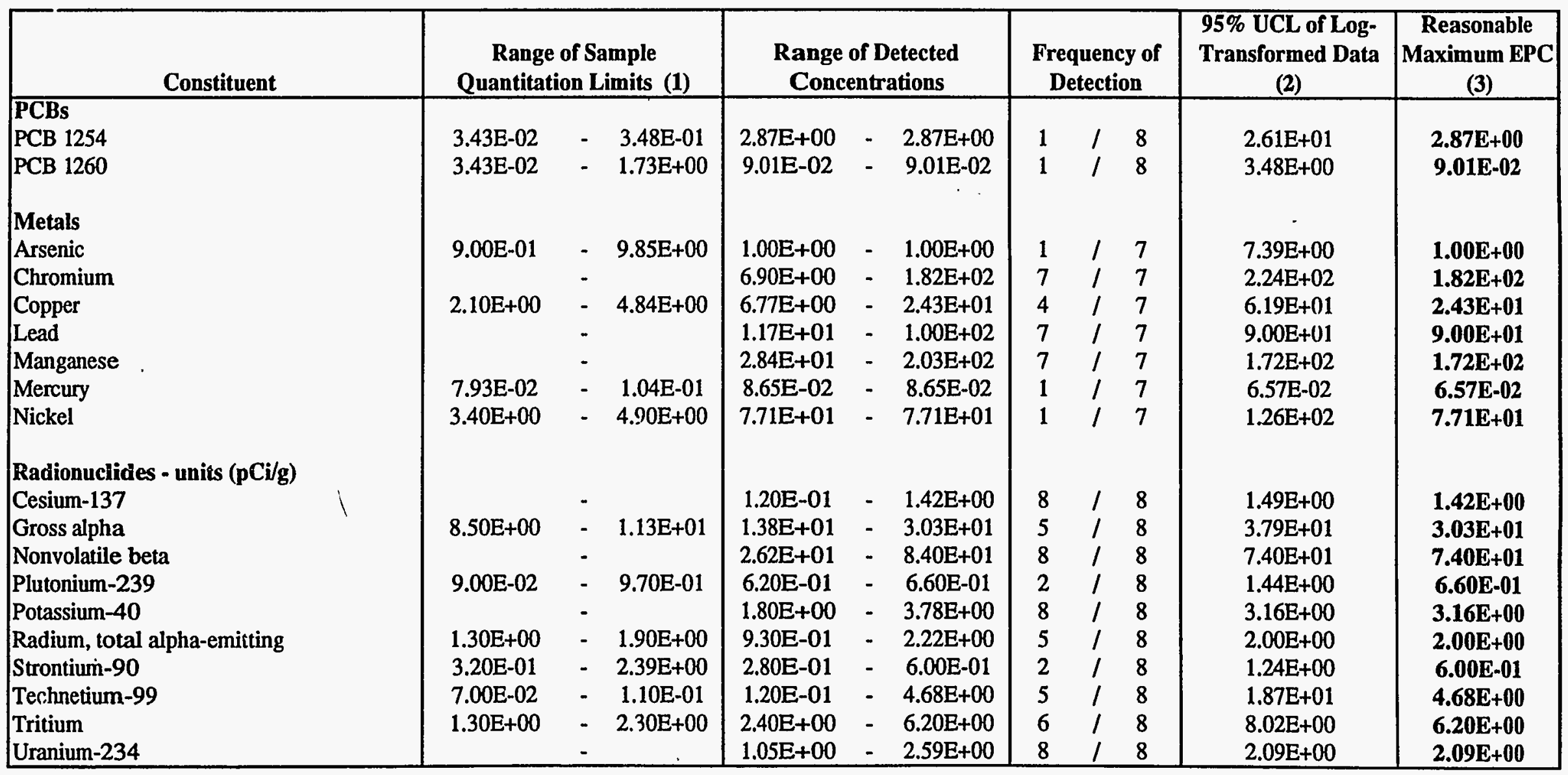


TABLE 3.4

SAVANNAH RIVER SITE

RUBBLE PIT 231-2F

CHEMICALS PRESENT IN UNIT SAMPLES (CPUSS) SURFACE SOIL SUMMARY (0-2 FEET)

\begin{tabular}{|c|c|c|c|c|c|}
\hline Constituent & $\begin{array}{c}\text { Range of Sample } \\
\text { Quantitation Limits (1) }\end{array}$ & $\begin{array}{c}\text { Range of Detected } \\
\text { Concentrations }\end{array}$ & $\begin{array}{l}\text { Frequency of } \\
\text { Detection }\end{array}$ & $\begin{array}{l}\text { 95\% UCL of Log- } \\
\text { Transformed Data } \\
\text { (2) }\end{array}$ & \begin{tabular}{|c|} 
Reasonable \\
Maximum EPC \\
(3)
\end{tabular} \\
\hline $\begin{array}{l}\text { Uranium-235 } \\
\text { Uranium-238 }\end{array}$ & $\begin{array}{ccc}7.00 \mathrm{E}-02 & - & 1.20 \mathrm{E}-01 \\
& - & \end{array}$ & $\begin{array}{ccc}8.00 \mathrm{E}-02 & - & 1.40 \mathrm{E}-01 \\
1.04 \mathrm{E}+00 & - & 2.00 \mathrm{E}+00\end{array}$ & $\begin{array}{lll}5 & 1 & 8 \\
8 & 1 & 8\end{array}$ & $\begin{array}{l}1.65 \mathrm{E}-01 \\
1.81 \mathrm{E}+00\end{array}$ & $\begin{array}{l}1.40 \mathrm{E}-01 \\
1.81 \mathrm{E}+00\end{array}$ \\
\hline
\end{tabular}

(1) The range of sample quantitation limits was not presented if the chemical was detected in all samples.

(2) The data were assumed to be lognormally distributed and, therefore, were transformed.

(3) The lesser of either the $95 \%$ UCL of the transformed data or the maximum was used as the Exposure Point Concentration (EPC). 
TABLE 3.5

SAVANNAH RIVER SITE

BURNING/RUBBLE PITS 231-F AND 231-1F

CHEMICALS PRESENT IN UNIT SAMPLES (CPUSS)

SOIL SUMMARY (0.4 FEET)

\begin{tabular}{|c|c|c|c|c|c|c|c|c|}
\hline Constituent & \multicolumn{2}{|c|}{$\begin{array}{c}\text { Range of Sample Quantitation } \\
\text { Limits (1) } \\
\end{array}$} & \multicolumn{2}{|c|}{$\begin{array}{c}\text { Range of Detected } \\
\text { Concentrations } \\
\end{array}$} & \multicolumn{2}{|c|}{$\begin{array}{c}\text { Frequency of } \\
\text { Detection }\end{array}$} & $\begin{array}{l}95 \% \text { UCL of Log- } \\
\text { Transformed Data } \\
\text { (2) }\end{array}$ & $\begin{array}{c}\text { Reasonable } \\
\text { Maximum EPC } \\
\text { (3) }\end{array}$ \\
\hline \multicolumn{9}{|l|}{ Volatiles } \\
\hline 1,1,2-Trichloroethane & 4.95E-03 & $6.50 \mathrm{E}-03$ & $9.52 \mathrm{E}-03$ & $1.65 \mathrm{E}-02$ & 2 & 24 & $4.13 \mathrm{E}-03$ & 4.13E-03 \\
\hline 1,2-Dichloroethane & 4.95E-03 & $6.50 \mathrm{E}-03$ & 9.70E-03 & - $\quad 2.16 \mathrm{E}-02$ & 2 & 24 & 4.37E-03 & 4.37E-03 \\
\hline Acetone & $1.05 \mathrm{E}-02$ & $1.20 \mathrm{E}-02$ & 2.72E-03 & - $\quad 2.62 \mathrm{E}+01$ & 20 & 24 & $1.64 \mathrm{E}+00$ & $1.64 \mathrm{E}+00$ \\
\hline Chloroform & 4.95E-03 & $6.50 \mathrm{E}-03$ & 1.01E-02 & $1.01 \mathrm{E}-02$ & 1 & 24 & $3.36 \mathrm{E}-03$ & $3.36 \mathrm{E}-03$ \\
\hline Dichloromethane (Methylene chloride) & 5.25E-03 & $6.50 \mathrm{E}-03$ & $1.39 \mathrm{E}-03$ & $3.80 \mathrm{E}-02$ & 20 & 24 & $1.91 \mathrm{E}-02$ & $1.91 \mathrm{E}-02$ \\
\hline Ethylbenzene & 4.95E-03 & $-\quad 6.50 \mathrm{E}-03$ & $1.29 \mathrm{E}-03$ & $-\quad 1.29 \mathrm{E}-03$ & 1 & 24 & $2.88 \mathrm{E}-03$ & $1.29 \mathrm{E}-03$ \\
\hline Tetrachloroethylene & $4.95 \mathrm{E}-03$ & $-\quad 6.50 \mathrm{E}-03$ & 2.87E-03 & $-\quad 2.87 \mathrm{E}-03$ & 1 & 24 & $2.84 \mathrm{E}-03$ & $2.84 \mathrm{E}-03$ \\
\hline Toluene & $5.25 \mathrm{E}-03$ & $-\quad 6.50 \mathrm{E}-03$ & $1.48 \mathrm{E}-03$ & $-\quad 3.06 \mathrm{E}-02$ & 16 & 24 & $1.18 \mathrm{E}-02$ & $1.18 \mathrm{E}-02$ \\
\hline Xylenes & 4.95E-03 & $-\quad 6.50 \mathrm{E}-03$ & $1.34 \mathrm{E}-03$ & $-\quad 1.34 \mathrm{E}-03$ & 1 & 24 & $2.88 \mathrm{E}-03$ & $1.34 \mathrm{E}-03$ \\
\hline \multicolumn{9}{|l|}{ Semivolatiles } \\
\hline 2-Methylnaphthalene & $3.47 \mathrm{E}-01$ & $3.66 \mathrm{E}-01$ & $3.53 \mathrm{E}-02$ & 7.69E-02 & 2 & 16 & 2.12E-01 & 7.69E-02 \\
\hline Acenaphthene & $3.48 \mathrm{E}-01$ & $3.66 \mathrm{E}-01$ & 6.32E-02 & $-\quad 3.16 \mathrm{E}-01$ & 4 & 16 & $2.08 \mathrm{E}-01$ & 2.08E-01 \\
\hline Acenaphthylene & 3.47E-01 & $3.66 \mathrm{E}-01$ & $5.80 \mathrm{E}-02$ & - $\quad 8.61 \mathrm{E}-02$ & 4 & 16 & $1.89 \mathrm{E}-01$ & 8.61E-02 \\
\hline Anthracene & $3.48 \mathrm{E}-01$ & $3.66 \mathrm{E}-01$ & $5.15 \mathrm{E}-02$ & $6.74 \mathrm{E}-01$ & 9 & 16 & 2.49E-01 & $2.49 \mathrm{E}-01$ \\
\hline Benzo(a)anthracene : & 3.57E-01 & $3.64 \mathrm{E}-01$ & $4.08 \mathrm{E}-02$ & $-\quad 1.62 E+00$ & 14 & 16 & $9.26 \mathrm{E}-01$ & $9.26 E-61$ \\
\hline Benzo(a)pyrene & 3.57E-01 & $3.64 \mathrm{E}-01$ & $3.87 \mathrm{E}-02$ & $-\quad 1.33 \mathrm{E}+00$ & 14 & 16 & $6.49 \mathrm{E}-01$ & $6.49 \mathrm{E}-01$ \\
\hline Benzo(b)fluoranthene & 3.57E-01 & $-\quad 3.64 \mathrm{E}-01$ & 4.12E-02 & $-\quad 1.28 \mathrm{E}+00$ & 14 & 16 & $1.01 E+00$ & $1.01 E+00$ \\
\hline Benzo(g,h,i)perylene & $3.48 \mathrm{E}-01$ & $-\quad 3.65 \mathrm{E}-01$ & 4.35E-02 & - $\quad 9.52 \mathrm{E}-01$ & 12 & 16 & $6.15 \mathrm{E}-01$ & $6.15 \mathrm{E}-01$ \\
\hline Benzo(k)fluoranthene & $3.52 \mathrm{E}-01$ & $-\quad 3.64 \mathrm{E}-01$ & $4.23 \mathrm{E}-02$ & $-\quad 1.49 \mathrm{E}+00$ & 13 & 16 & 8.45E-01 & $8.45 \mathrm{E}-01$ \\
\hline Benzoic acid & $1.74 \mathrm{E}+00$ & $-\quad 1.83 E+00$ & 4.73E-02 & - $\quad 4.86 \mathrm{E}-02$ & 2 & 16 & $2.05 \mathrm{E}+00$ & $4.86 \mathrm{E}-02$ \\
\hline Bis(2-ethylhexyl; phthalate & 3.61E-01 & $-\quad 3.66 \mathrm{E}-01$ & $5.68 \mathrm{E}-02$ & $-\quad 6.02 \mathrm{E}-01$ & 14 & 16 & $4.48 \mathrm{E}-01$ & $4.48 \mathrm{E}-01$ \\
\hline Butylbenzyl phthalate & 3.47E-01 & $3.66 \mathrm{E}-01$ & $2.08 \mathrm{E}-01$ & 2.08E-01 & 1 & 16 & $1.83 \mathrm{E}-01$ & $1.83 E-01$ \\
\hline Chrysene & 3.57E-01 & $3.64 \mathrm{E}-01$ & 4.49E-02 & $-\quad 1.62 E+00$ & 14 & 16 & $1.00 \mathrm{E}+00$ & $1.00 \mathrm{E}+00$ \\
\hline Di-n-butyl phthalate & $3.47 \mathrm{E}-01$ & $3.64 \mathrm{E}-01$ & 4.19E-02 & 2.42E-01 & 8 & 16 & $1.99 \mathrm{E}-01$ & $1.99 \mathrm{E}-01$ \\
\hline Di-n-octyl phthalate & 3.47E-01 & $=\quad 3.65 \mathrm{E}-01$ & $7.67 \mathrm{E}-02$ & $7.90 \mathrm{E}-02$ & 2 & 16 & $1.90 \mathrm{E}-01$ & $7.90 \mathrm{E}-02$ \\
\hline Dibenz(a,h)anthracene & $3.48 \mathrm{E}-01$ & $-\quad 3.66 \mathrm{E}-01$ & $1.18 \mathrm{E}-01$ & 4.73E-01 & 6 & 16 & 2.17E-01 & 2.17E-01 \\
\hline Dibenzofuran & $3.47 \mathrm{E}-01$ & $-\quad 3.66 \mathrm{E}-01$ & 2.22E-01 & $2.22 \mathrm{E}-01$ & 1 & 16 & $1.85 \mathrm{E}-01$ & $1.85 \mathrm{E}-01$ \\
\hline Fluoranthene & $3.57 \mathrm{E}-01$ & $3.64 \mathrm{E}-01$ & $6.02 \mathrm{E}-02$ & $3.91 E+00$ & 14 & 16 & $2.04 \mathrm{E}+00$ & $2.04 E+00$ \\
\hline Fluorene & $3.48 \mathrm{E}-01$ & $3.66 \mathrm{E}-01$ & $4.78 \mathrm{E}-02$ & $2.99 \mathrm{E}-01$ & 3 & 16 & 2.17E-01 & 2.17E-01 \\
\hline Indeno(1,2,3-c,d)pyrene & $3.52 \mathrm{E}-01$ & $3.65 \mathrm{E}-01$ & $3.72 \mathrm{E}-02$ & - $\quad 8.32 \mathrm{E}-01$ & 12 & 16 & $5.25 \mathrm{E}-01$ & $5.25 \mathrm{E}-01$ \\
\hline Naphthalene & $3.48 \mathrm{E}-01$ & $3.66 \mathrm{E}-01$ & $4.52 \mathrm{E}-02$ & $3.22 \mathrm{E}-01$ & 2 & 16 & $2.21 \mathrm{E}-01$ & $2.21 \mathrm{E} \cdot 01$ \\
\hline Phenanthrene & $3.48 \mathrm{E}-01$ & $-\quad 3.64 \mathrm{E}-01$ & $7.10 \mathrm{E}-02$ & $-\quad 3.38 E+00$ & 12 & 16 & $6.87 \mathrm{E}-01$ & $6.87 \mathrm{E}-01$ \\
\hline
\end{tabular}


TABLE 3.5

SAVANNAH RIVER SITE

BURNING/RUBBLE PITS 231-F AND 231-1F

CHEMICALS PRESENT IN UNIT SAMPLES (CPUSS)

SOIL SUMMARY (0-4 FEET)

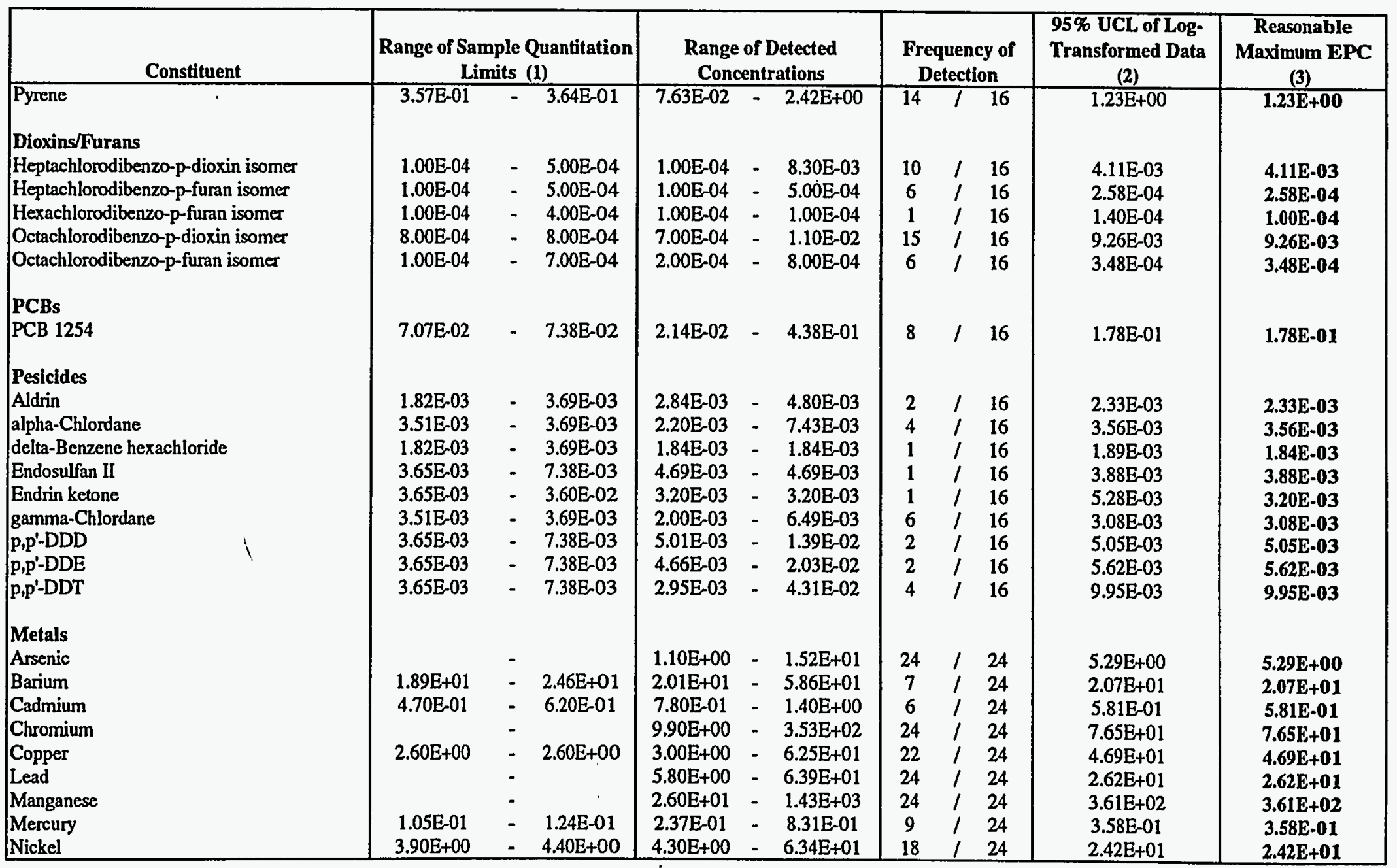


TABLE 3.5

SAVANNAH RIVER SITE

BURNING/RUBDLE PITS 231-F AND 231-1F

CHEMICALS PRESENT IN UNIT SAMPLES (CPUSs)

SOIL SUMMARY (0-4 FEET)

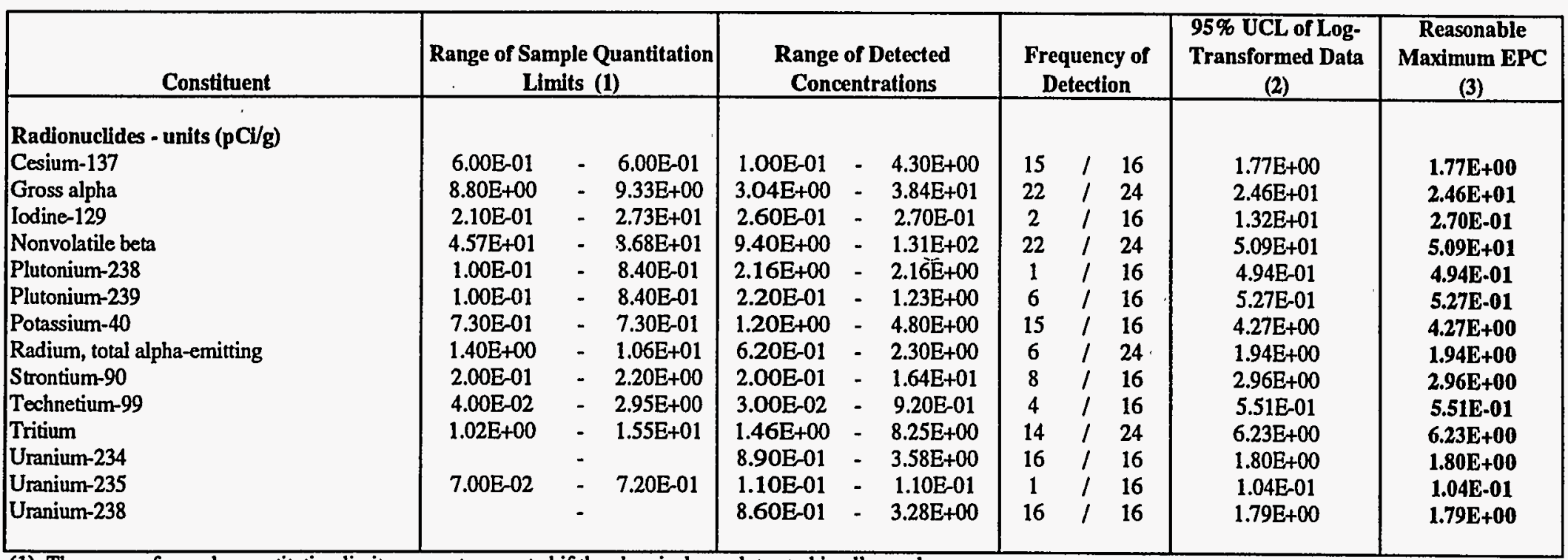

(1) The range of sample quantitation limits was not presented if the chemical was detected in all samples.

(2) The data were assumed to be lognormally distributed and, therefore, were transformed.

(3) The lesser of either the 95\% UCL of the transformed data or the maximum was used as the Exposure Point Concentration (EPC). 
TABLE 3.6

SAVANNAH RIVER SITE

RUBBLE PIT 231-2F

\section{CHEMICALS PRESENT IN UNIT SAMPLES (CPUSs)} SOIL SUMMARY (0-4 FEET)

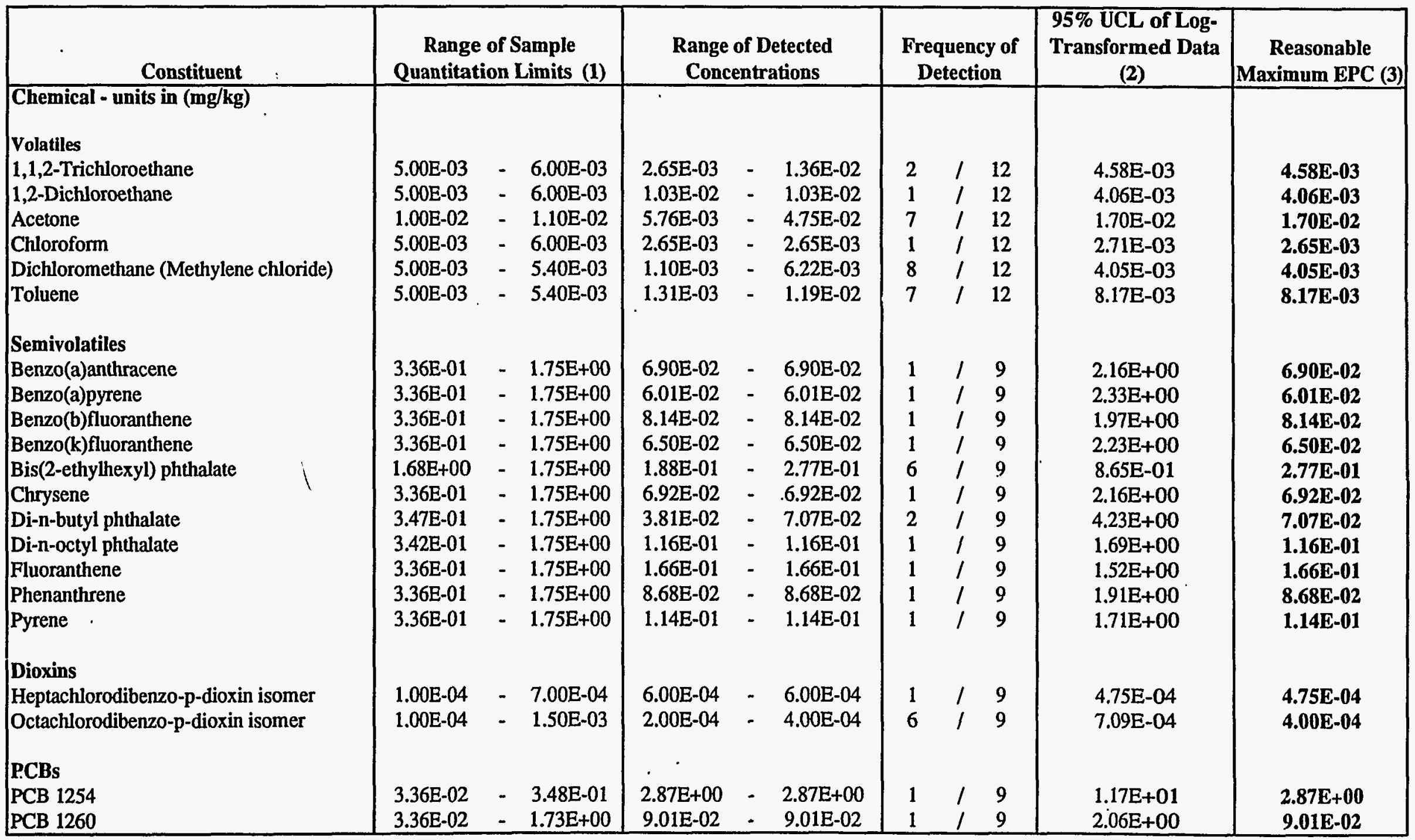


TABLE 3.6

SA VANNAH RIVER SITE

RUBBLE PIT 231-2F

\section{CHEMICALS PRESENT IN UNIT SAMPLES (CPUSS)} SOIL SUMMARY (0-4 FEET)

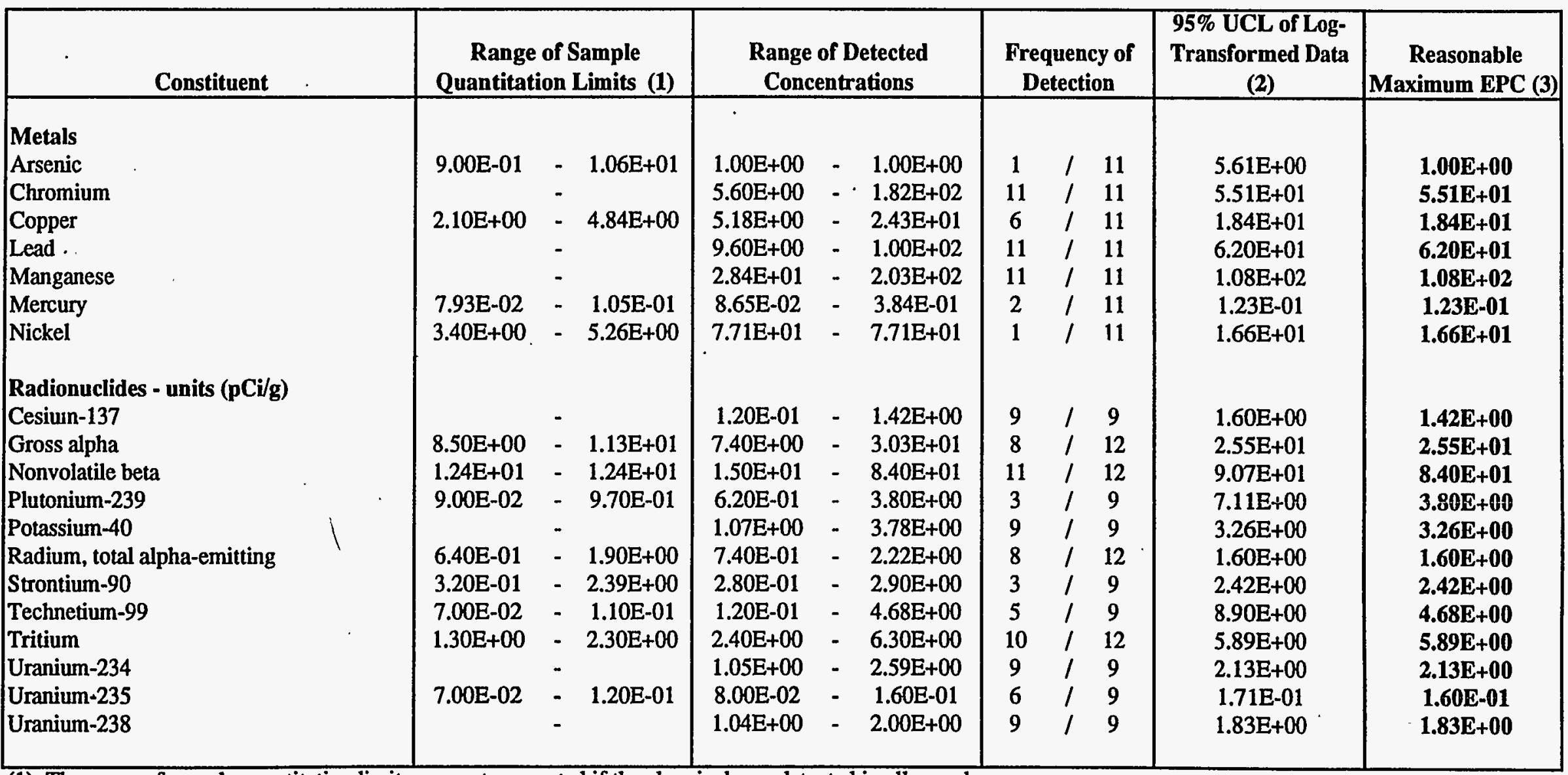

(1) The range of sample quantitation limits was not presented if the chemical was detected in all samples.

(2) The data were assumed to be lognormally distributed and, therefore, were transformed.

(3) The lesser of either the $95 \%$ UCL of the transformed data or the maximum was used as the Exposure Point Concentration (EPC). 
TABLE 3.7

SAVANNAH RIVER SITE

BURNING/RUBBLE PITS 231-F AND 231-1F

CHEMICALS PRESENT IN UNIT SAMPLES (CPUSS)

SOIL SUMMARY (4 FEET TO BOTTOM OF THE PIT)

\begin{tabular}{|c|c|c|c|c|c|c|c|c|c|}
\hline Constituent & \multicolumn{2}{|c|}{$\begin{array}{c}\text { Range of Sample } \\
\text { Quantitation Limits (1) }\end{array}$} & \multicolumn{2}{|c|}{$\begin{array}{l}\text { Range of Detected } \\
\text { Concentrations }\end{array}$} & \multicolumn{3}{|c|}{$\begin{array}{l}\text { Frequency of } \\
\text { Detection }\end{array}$} & $\begin{array}{l}\text { 95\% UCL of Log- } \\
\text { Transformed Data } \\
\text { (2) }\end{array}$ & $\begin{array}{c}\text { Reasonable } \\
\text { Maximum EPC } \\
\text { (3) }\end{array}$ \\
\hline \multicolumn{10}{|l|}{ Volatlles } \\
\hline 1,2-Dichloroethylene & $5.50 \mathrm{E}-03$ & 7.21E-01 & 3.65E-03 & $3.65 \mathrm{E}-03$ & 1 & 1 & 21 & $1.39 \mathrm{E}-02$ & 3.65E-03 \\
\hline Acetone & $1.10 \mathrm{E}-02$ & $1.44 E+00$ & $3.64 \mathrm{E}-03$ & $1.16 \mathrm{E}+00$ & 18 & I & 21 & $6.45 \mathrm{E}-01$ & $6.45 \mathrm{E}-01$ \\
\hline Carbon disulfide & $5.50 \mathrm{E}-03$ & $7.21 \mathrm{E}-01$ & $1.80 \mathrm{E}-02$ & $1.80 \mathrm{E}-02$ & 1 & I & 21 & $1.66 \mathrm{E}-02$ & $1.66 \mathrm{E} \cdot 02$ \\
\hline Chlorobenzene & $5.50 \mathrm{E}-03$ & $7.21 \mathrm{E}-01$ & $4.88 \mathrm{E}-03$ & $4.88 \mathrm{E}-03$ & 1 & 1 & 21 & $1.41 \mathrm{E}-02$ & 4.88E-03 \\
\hline Dichloromethane (Methylene chloride) & $5.50 \mathrm{E}-03$ & $2.90 \mathrm{E}-02$ & 2.02E-03 & $1.24 \mathrm{E}+01$ & 17 & i & 21 & $2.65 \mathrm{E}-01$ & $2.65 \mathrm{E}-01$ \\
\hline Ethylbenzene & $5.50 \mathrm{E}-03$ & $2.90 \mathrm{E}-02$ & $1.67 \mathrm{E}-02$ & $9.62 \mathrm{E}-01$ & 2 & 1 & 21 & $2.69 \mathrm{E}-02$ & $2.69 \mathrm{E}-02$ \\
\hline Methyl ethyl ketone & $1.10 \mathrm{E}-02$ & - $\quad 5.80 \mathrm{E}-02$ & $1.43 \mathrm{E}-02$ & $9.75 \mathrm{E}-01$ & 2 & 1 & 21 & $3.37 \mathrm{E}-02$ & 3.37E.02 \\
\hline Tetrachloroethylene & $5.50 \mathrm{E}-03$ & $6.95 \mathrm{E}-03$ & 2.82E-03 & $1.60 \mathrm{E}-01$ & 3 & 1 & 21 & $9.77 \mathrm{E}-03$ & $9.77 \mathrm{E} \cdot 03$ \\
\hline Toluene & $5.50 \mathrm{E}-03$ & $2.90 \mathrm{E}-02$ & $1.79 \mathrm{E}-03$ & $2.72 \mathrm{E}-01$ & 13 & 1 & 21 & $2.15 \mathrm{E}-02$ & 2.15E.02 \\
\hline Xylenes & $5.50 \mathrm{E}-03$ & $2.90 \mathrm{E}-02$ & $6.76 \mathrm{E}-03$ & $2.25 \mathrm{E}+00$ & 3 & I & 21 & $4.70 \mathrm{E}-02$ & 4.70E-02 \\
\hline \multicolumn{10}{|l|}{ Semivolatiles } \\
\hline 2-Methylnaphthalene & $3.49 \mathrm{E}-01$ & 5.07E-01 & $2.26 \mathrm{E}-01$ & $1.61 \mathrm{E}+00$ & 2 & I & 17 & $3.28 \mathrm{E}-01$ & 3.28E-01 \\
\hline Acenaphthene & 3.49E-01 & 7.62E-01 & $5.19 \mathrm{E}-02$ & $1.37 \mathrm{E}+01$ & 5 & 1 & 17 & $1.13 \mathrm{E}+00$ & $1.13 E+00$ \\
\hline Acenaphthylene & $3.55 \mathrm{E}-01$ & 7.62E-01 & $5.65 \mathrm{E}-02$ & $2.82 \mathrm{E}-01$ & 2 & I & 17 & $2.43 \mathrm{E}-01$ & 2.43E-01 \\
\hline Anthracene & $3.55 \mathrm{E}-01$ & $7.62 \mathrm{E}-01$ & $1.22 \mathrm{E}-01$ & $9.40 \mathrm{E}+00$ & 6 & 1 & 17 & $1.63 \mathrm{E}+00$ & $1.63 \mathrm{E}+00$ \\
\hline Benzo(a)anthracene & $3.55 \mathrm{E}-01$ & $5.07 \mathrm{E}-01$ & $7.82 \mathrm{E}-02$ & $6.46 \mathrm{E}+00$ & 8 & 1 & 17 & $7.46 \mathrm{E}-01$ & $7.46 \mathrm{E}-01$ \\
\hline Benzo(a)pyrene & 3.55E-01 & $7.62 \mathrm{E}-01$ & $9.59 \mathrm{E}-02$ & $2.37 \mathrm{E}+00$ & 4 & 1 & 17 & $4.28 \mathrm{E}-01$ & 4.28E-01 \\
\hline Benzo(b)fluoranthene & $3.55 \mathrm{E}-01$ & 7.62E-01 & $4.09 \mathrm{E}-02$ & $2.85 E+\infty$ & 5 & 1 & 17 & $5.38 \mathrm{E}-01$ & 5.38E-01 \\
\hline Benzo(g,h,i)perylene & $3.55 \mathrm{E}-01$ & 7.62E-01 & $2.76 \mathrm{E}-01$ & $9.12 \mathrm{E}-01$ & 2 & I & 17 & $2.98 \mathrm{E}-01$ & $2.98 \mathrm{E} .01$ \\
\hline Benzo(k)fluoranthene & $3.55 \mathrm{E}-01$ & $7.62 \mathrm{E}-01$ & $4.53 \mathrm{E}-02$ & $2.54 \mathrm{E}+00$ & 5 & 1 & 17 & $5.15 \mathrm{E}-01$ & $5.15 \mathrm{E}-01$ \\
\hline Bis(2-ethylhexyl) phthalate & 3.75E-01 & $3.86 \mathrm{E}-01$ & $9.13 \mathrm{E}-02$ & $7.33 \mathrm{E}+00$ & 15 & 1 & 17 & $2.39 \mathrm{E}+00$ & $2.39 \mathrm{E}+00$ \\
\hline Butylbenzyl phthalate & 3.49E-01 & 5.07E-01 & $2.66 \mathrm{E}-01$ & $2.66 \mathrm{E}-01$ & 1 & 1 & 17 & $2.06 \mathrm{E}-01$ & $2.06 \mathrm{E}-01$ \\
\hline Chrysene & $3.70 \mathrm{E}-01$ & $5.07 \mathrm{E}-01$ & $4.95 \mathrm{E}-02$ & $6.45 \mathrm{E}+00$ & 11 & 1 & 17 & $1.08 \mathrm{E}+00$ & $1.08 \mathrm{E}+00$ \\
\hline Di-n-butyl phthalate & $3.62 \mathrm{E}-01$ & 5.07E-01 & $4.00 \mathrm{E}-02$ & 7.99E-01 & 11 & 1 & 17 & $2.92 \mathrm{E}-01$ & $2.92 \mathrm{E}-01$ \\
\hline Di-n-octyl phthalate & $3.49 \mathrm{E}-01$ & $-\quad 5.07 \mathrm{E}-01$ & 2.13E-01 & $4.61 \mathrm{E}-01$ & 4 & 1 & 17 & $2.51 \mathrm{E}-01$ & 2.51E.01 \\
\hline Dibenz(a,h)anthracene & $3.55 \mathrm{E}-01$ & - 7.62E-01 & $3.94 \mathrm{E}-02$ & $2.43 \mathrm{E}-01$ & 2 & 1 & 17 & $2.52 \mathrm{E}-01$ & 2.43E-01 \\
\hline Dibenzofuran & $3.49 \mathrm{E}-01$ & 7.62E-01 & $9.70 \mathrm{E}-02$ & $7.71 \mathrm{E}+00$ & 4 & 1 & 17 & $7.21 \mathrm{E}-01$ & $7.21 \mathrm{E} .01$ \\
\hline Fluoranthene & 3.67E-01 & $5.07 \mathrm{E}-01$ & $4.04 \mathrm{E}-02$ & $3.19 E+01$ & 11 & 1 & 17 & $4.28 \mathrm{E}+00$ & $4.28 \mathrm{E}+00$ \\
\hline Fluorene & $3.49 \mathrm{E}-01$ & $7.62 \mathrm{E}-01$ & $5.17 \mathrm{E}-02$ & $1.29 E+01$ & 6 & 1 & 17 & $1.28 \mathrm{E}+00$ & $1.28 \mathrm{E}+00$ \\
\hline Indeno $(1,2,3-c, d)$ pyrene & $3.55 \mathrm{E}-01$ & 7.62E-01 & 2.75E-01 & $4.64 \mathrm{E}-01$ & 2 & 1 & 17 & $2.54 \mathrm{E}-01$ & $2.54 \mathrm{E}-01$ \\
\hline Naphthalene & $3.49 \mathrm{E}-01$ & 5.07E-01 & $1.01 \mathrm{E}-01$ & 8.32E-01 & 3 & 1 & 17 & 2.95E-01 & $2.95 \mathrm{E}-01$ \\
\hline
\end{tabular}


TABLE 3.7

SAVANNAH RIVER SITE

BURNING/RUBBLE PITS 231-F AND 231-1F

CHEMICALS PRESENT IN UNIT SAMPLES (CPUSS)

SOIL SUMMARY (4 FEET TO BOTTOM OF THE PIT)

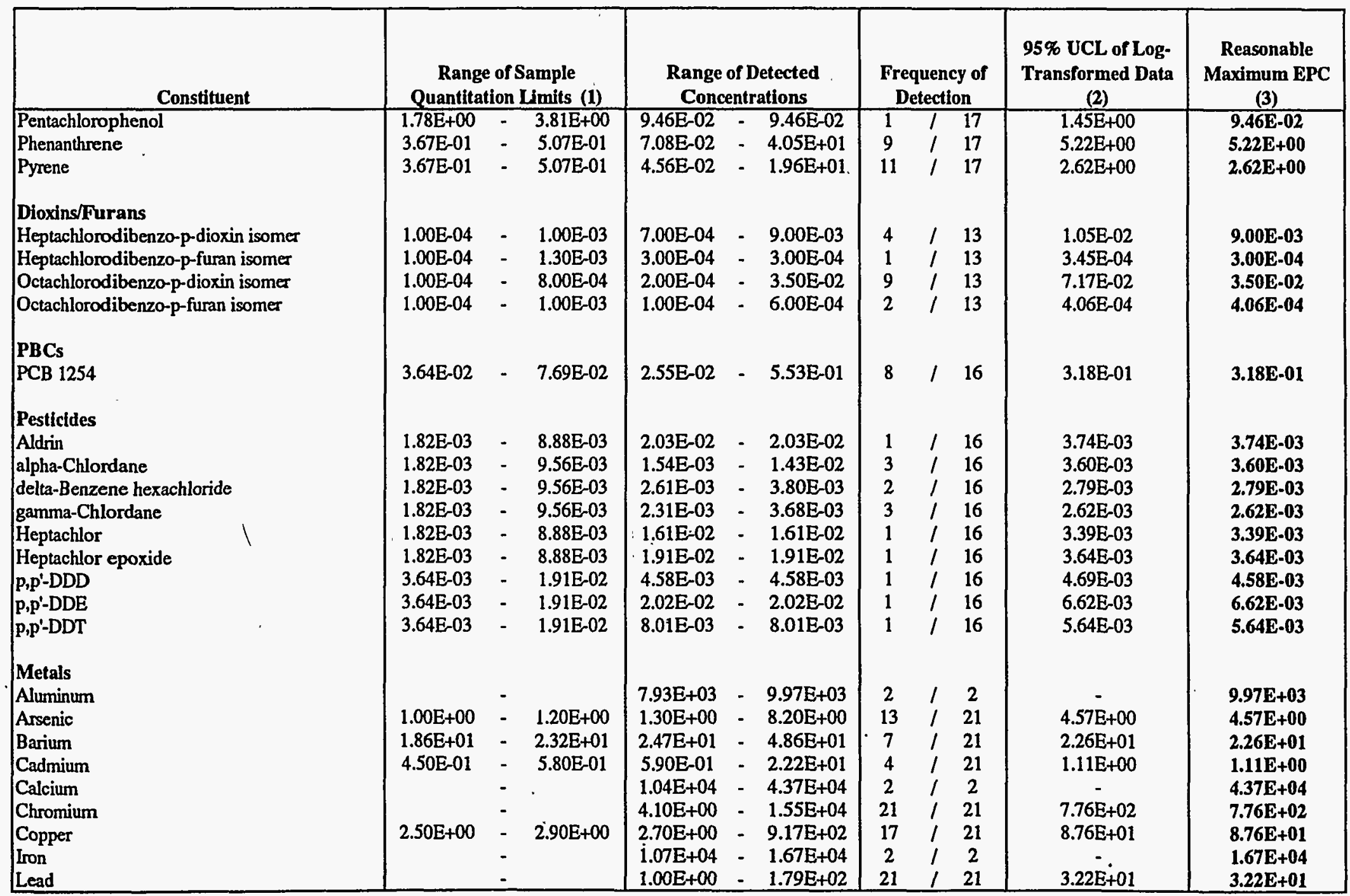


TABLE 3.7

SAVANNAH RIVER SITE

BURNING/RUBBLE PITS 231-F AND 231-1F

CHEMICALS PRESENT IN UNIT SAMPLES (CPUSS)

SOIL SUMMARY (4 FEET TO BOTTOM OF THE PIT)

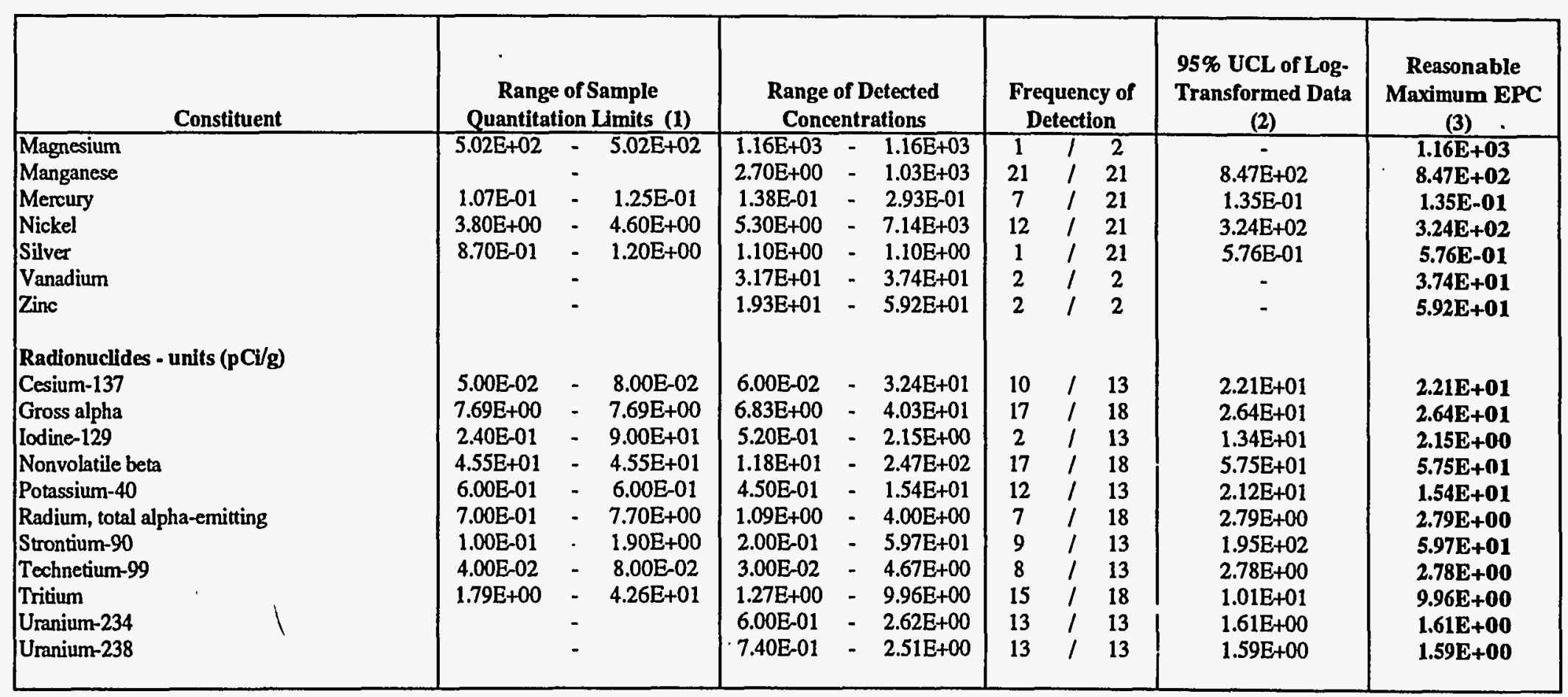

(1) The range of sample quantitation limits was not presented if the chemical was detected in all samples.

(2) The data were assumed to be lognomally distributed and, therefore, were transformed.

(3) The lesser of either the 95\% UCL of the transformed data or the maximum was used as the Exposure Point Concentration (EPC). 
TABLE 3.8

SAVANNAH RIVER SITE

RUBBLE PIT 231-2F

CHEMICALS PRESENT IN UNIT SAMPLES (CPUSS)

SOIL SUMMARY (4 FEET TO BOTTOM OF THE PIT)

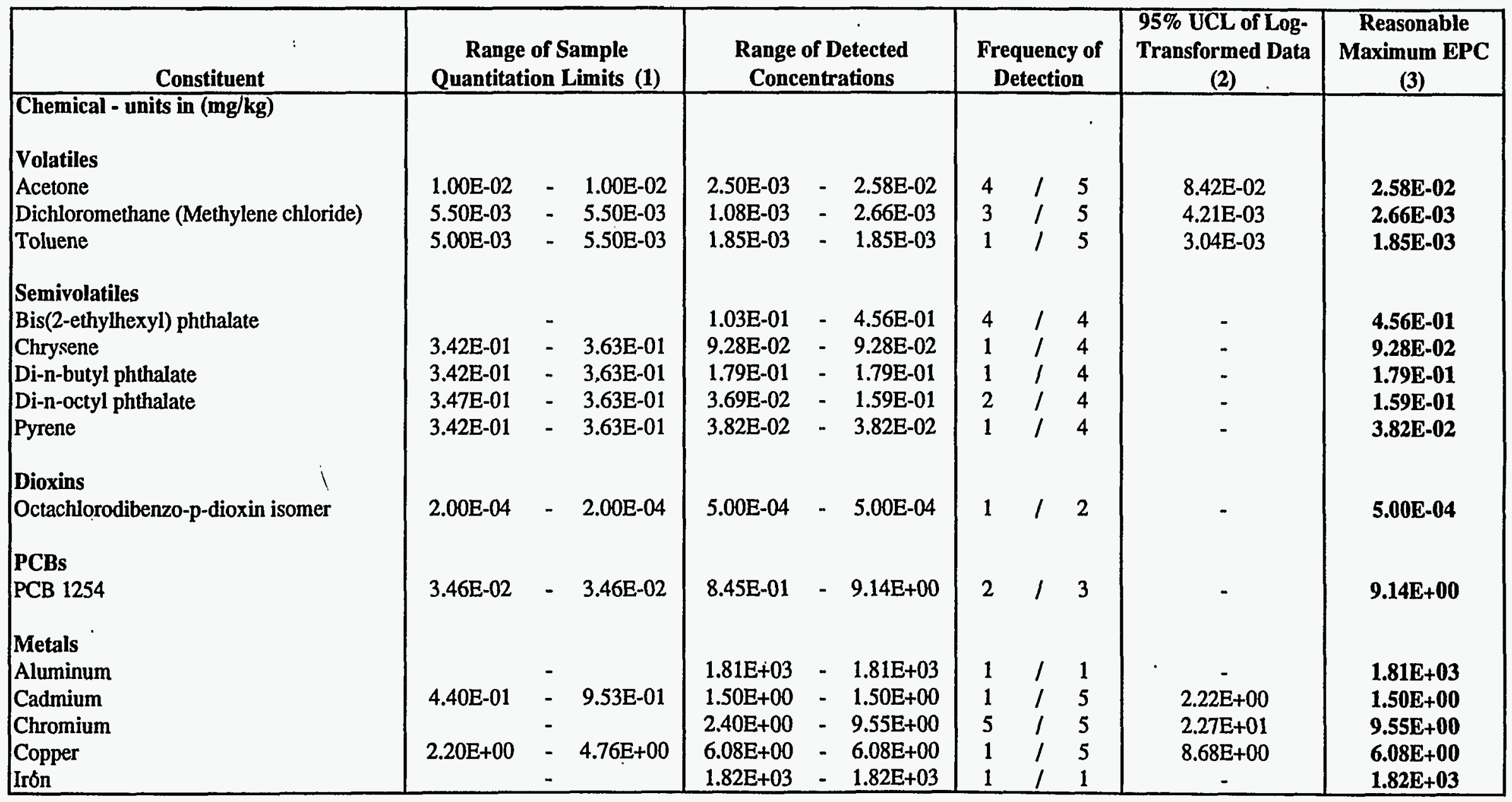


TABLE 3.8

SAVANNAH RIVER SITE

RUBBLE PIT 231-2F

CHEMICALS PRESENT IN UNIT SAMPLES (CPUSS)

SOIL SUMMARY (4 FEET TO BOTTOM OF THE PIT)

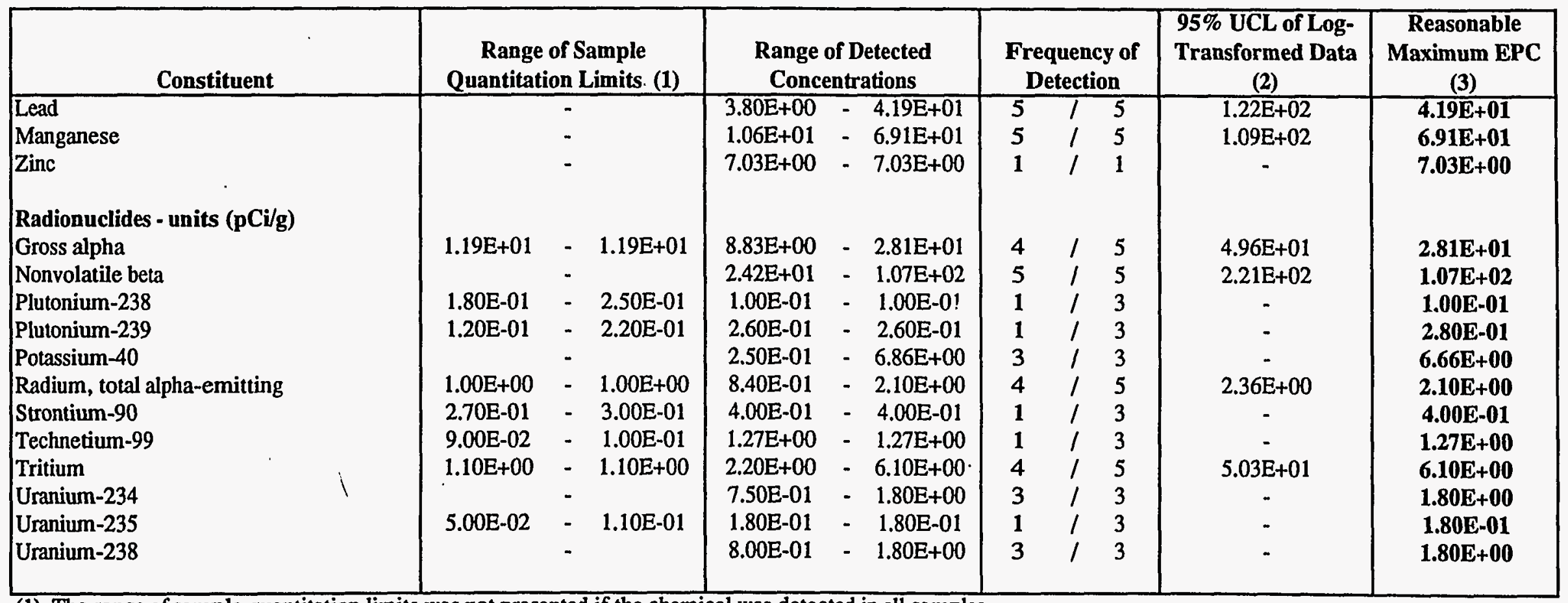

(1) The range of sample quantitation limits was not presented if the chemical was detected in all samples.

(2) The data were assumed to be lognormally distributed and, therefore, were transformed.

(3) The lesser of either the 95\% UCL of the transformed data or the maximum was used as the Exposure Point Concentration (EPC). 
TABLE 3.9

SAVANNAH RIVER SITE

BURNING/RUBBLE PITS 231F, 231-1F AND RUBBLE PIT 231-2F

CHEMICALS PRESENT IN UNIT SAMPLES (CPUSs) GROUNDWATER DATA

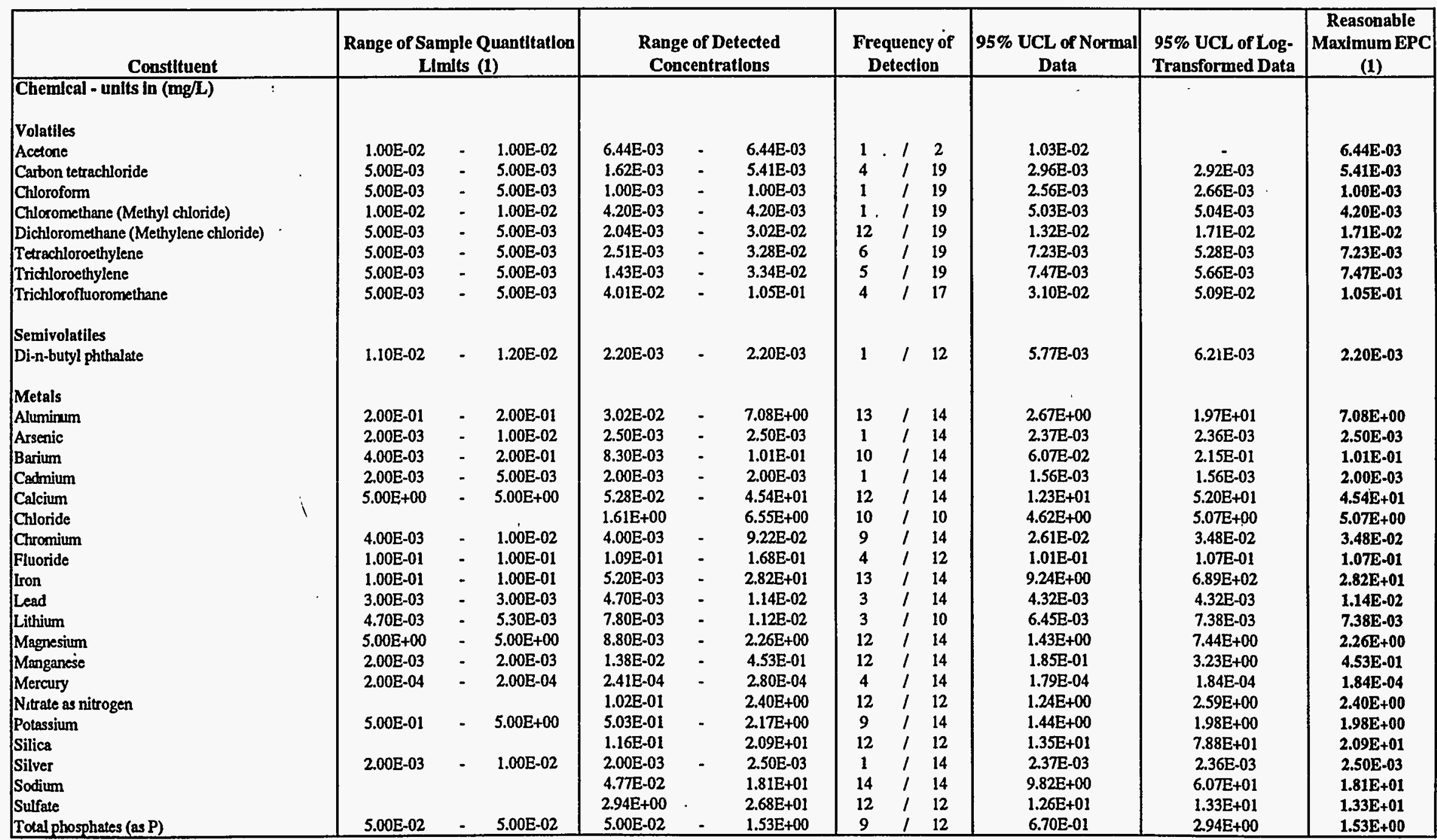


TABLE 3.9

SAVANNAH RIVER SITE

BURNING/RUBBLE PITS 231F, 231-1F AND RUBBLE PIT 231-2F

CHEMICALS PRESENT IN UNIT SAMPLES (CPUSs)

GROUNDWATER DATA

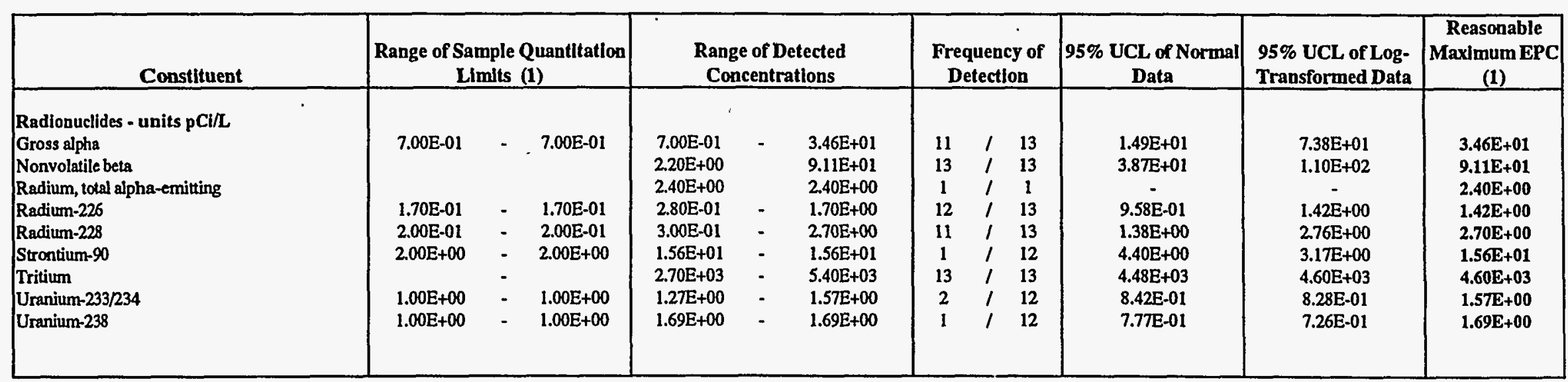

(2) For chemicals with $50 \%$ or greater detections, the lesser of log-transformed data $95 \%$ UCL versus maximum detected value was used.

For chemicals with $25 \%$ up to $50 \%$ detections, the maximum $95 \%$ UCL from both distribution types was compared to the maximum detected concentration, and the lesser value was used.

For chemicals with less than $25 \%$ detections, the maximum detected value was used.

"--": Denotes chemicals for which 95\% UCL is incalculable (e.g., sample size below 5, standard deviation of zero, etc.). 
TABLE 3.10

SAVANNAH RIVER SITE

BURNING/RUBBLE PITS 231-F, 231-1F AND RUBBLE PIT 231-2F

CHEMICALS PRESENT IN UNIT SAMPLES (CPUSs)

SURFACE WATER DATA

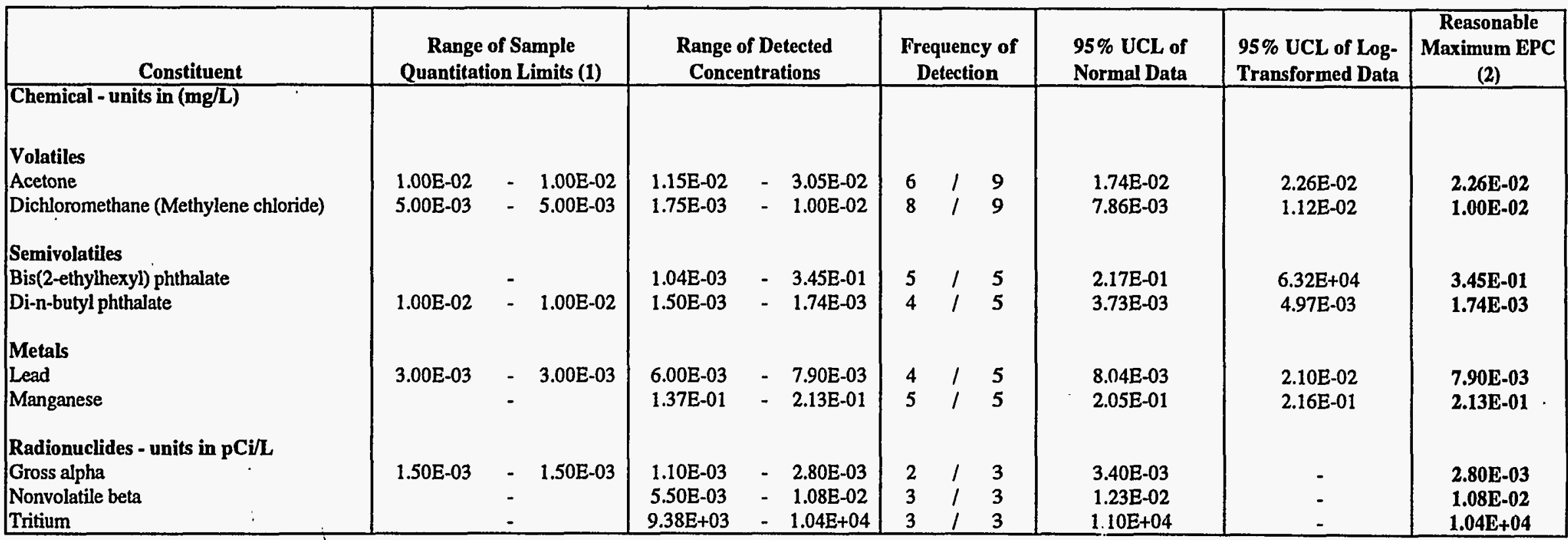

(1) If the chemical was detected in all samples, the range of sample quantitation limits was not reported.

(2) For chemicals with $50 \%$ or greater detections, the lesser of log-transformed data $95 \%$ UCL versus maximum detected valuc was used.

For chemicals with $25 \%$ up to $50 \%$ detections, the maximum $95 \%$ UCL from both distribution types was compared to maximum detectud concentration,

and the lesser value was used.

For chemicals with less than $25 \%$ detections, the maximum detected value was used.

"--": Denotes chemicals for which 95\%UCL is incalculable (e:g., sample size below 5, standard deviation of zero, etc.). 
TABLE 3.11

SAVANNAH RIVER SITE

BURNING/RUBBLE PITS 231-F, 231-1F AND RUBBLE PIT 231-2F

CHEMICALS PRESENT IN UNIT SAMPLES (CPUSs)

\section{SEDIMENT DATA}

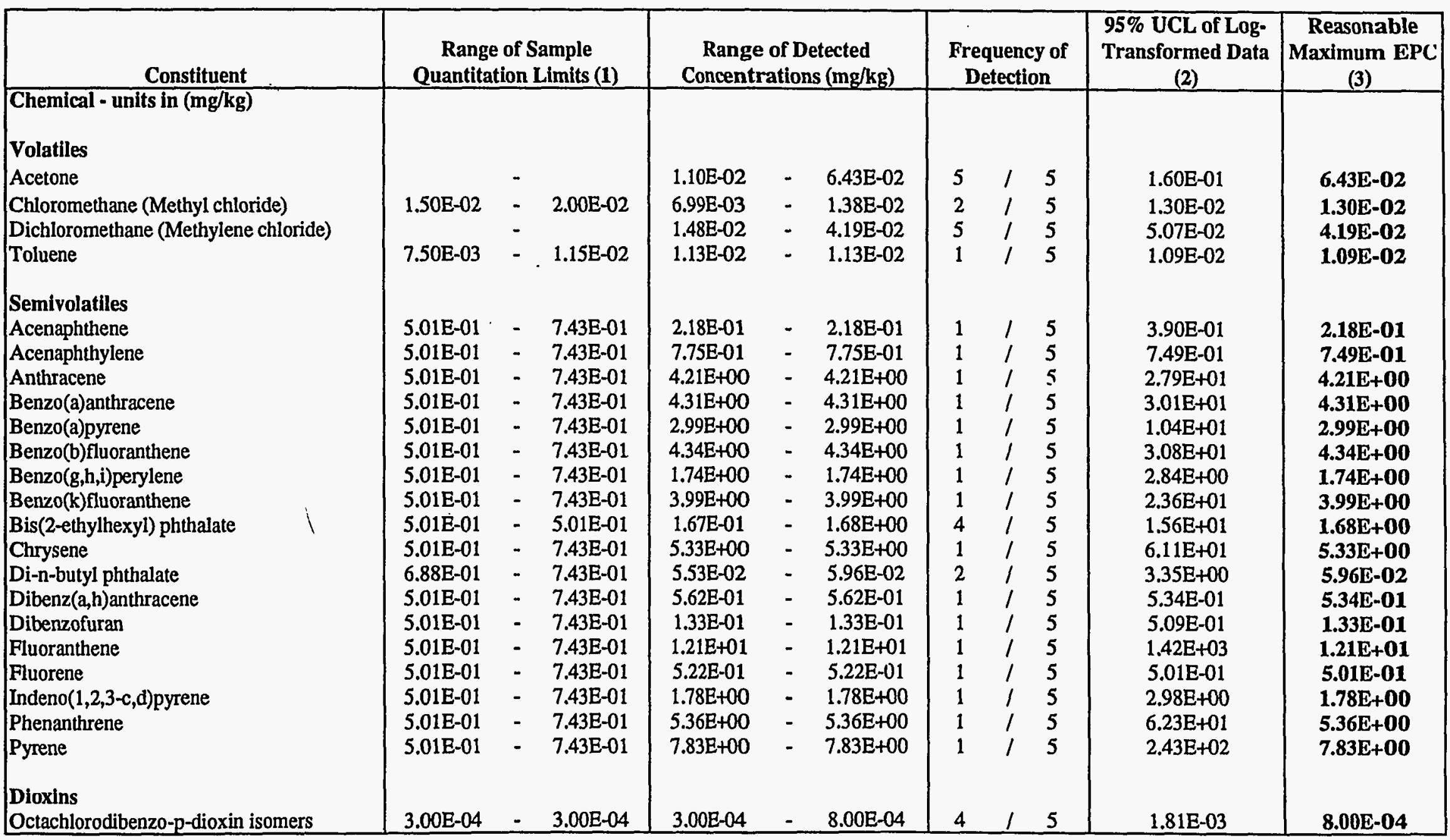


TABLE 3.11

SAVANNAH RIVER SITE

BURNING/RUBBLE PITS 231-F, 231-1F AND RUBBLE PIT 231-2F CHEMICALS PRESENT IN UNIT SAMPLES (CPUSS) SEDIMENT DATA

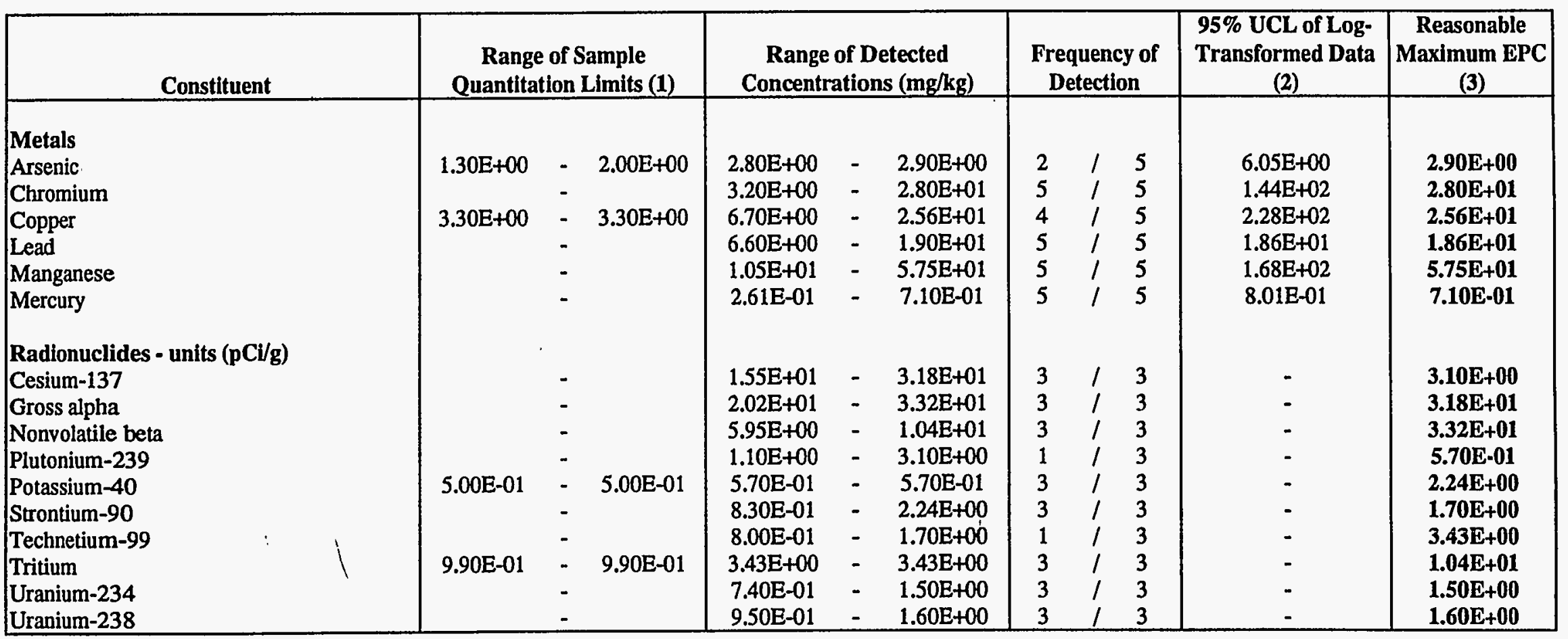

(1) If the chemical was detected in all samples, the range of sample quantitation limits was not reported.

(2) The sediment data were assumed to be lognormally distributed and, therefore, were transformed.

(3) The lesser of either the $95 \%$ UCL on the arithmetic mean for transformed data or the maximum detected values was considered the Exposure Point Concentration (EPC).

"--": Denotes chemicals for which 95\% UCL is incalculable (e.g., sample size below 5, standard deviation of zero, etc.). 
TABLE 3.12

COMPARISON OF SOIL ANALYTES TO BACKGROUND CONCENTRATIONS

SAVANNAH RIVER SITE

BURNING/RUBBLE PITS 231-F AND 231-1F

\begin{tabular}{|c|c|c|c|c|c|c|}
\hline \multirow[b]{3}{*}{ Constituents } & \multirow[b]{3}{*}{$\begin{array}{c}\text { Mean } \\
\text { Background (a) }\end{array}$} & \multirow[b]{3}{*}{$\begin{array}{l}\text { 2x Background } \\
\cdot \text { Mean }\end{array}$} & \multicolumn{4}{|c|}{ Pits 231-F and 231-1F } \\
\hline & & & \multicolumn{2}{|c|}{ 0-2 Feet } & \multicolumn{2}{|c|}{ 0-4 Feet } \\
\hline & & & $\begin{array}{c}\text { Maximum } \\
\text { Detected } \\
\text { Concentration } \\
\end{array}$ & $\begin{array}{c}\text { Retained as } \\
\text { COPC (b) } \\
\text { Y/N }\end{array}$ & $\begin{array}{c}\text { Maximum } \\
\text { Detected } \\
\text { Concentration }\end{array}$ & $\begin{array}{c}\text { Retained as } \\
\text { COPC (b) } \\
\text { Y/N }\end{array}$ \\
\hline \multicolumn{7}{|l|}{ Chemical - units in (mg/kg) } \\
\hline Volatiles & & & & & & \\
\hline 1,1,2-Trichloroethane & ND & ND & $1.65 \mathrm{E}-02$ & $\mathbf{Y}$ & $1.65 \mathrm{E}-02$ & $\mathbf{Y}$ \\
\hline 1,2-Dichloroethane & ND & ND & $2.16 \mathrm{E}-02$ & $\mathbf{Y}$ & $2.16 \mathrm{E}-02$ & $Y$ \\
\hline Acetone & 5.30E-02 & $1.06 \mathrm{E}-01$ & $2.62 \mathrm{E}+01$ & $Y(c)$ & $2.62 \mathrm{E}+01$ & $\mathrm{Y}(\mathrm{c})$ \\
\hline Chloroform & ND & ND & $1.01 \mathrm{E}-02$ & $\mathrm{Y}$ & $1.01 \mathrm{E}-02$ & $\mathrm{Y}$ \\
\hline Dichloromethane (Methylene chloride) & $7.00 \mathrm{E}-03$ & $1.40 \mathrm{E}-02$ & $3.80 \mathrm{E}-02$ & $Y(c)$ & 3.80E-02 & $Y(c)$ \\
\hline Ethylbenzene & ND & ND & ND & - & $1.29 \mathrm{E}-03$ & $\mathrm{Y}$ \\
\hline Tetrachloroethylene & ND & ND & 2.87E-03 & $\mathbf{Y}$ & 2.87E-03 & $\mathrm{Y}$ \\
\hline Toluene & $1.40 \mathrm{E}-02$ & $2.80 \mathrm{E}-02$ & $3.06 \mathrm{E}-02$ & $r(c)$ & $3.06 \mathrm{E}-02$ & $\mathrm{Y}(\mathrm{c})$ \\
\hline Xylenes & ND & ND & $1.34 \mathrm{E}-03$ & $\mathbf{Y}$ & $1.34 \mathrm{E}-03$ & $\mathrm{Y}$ \\
\hline \multicolumn{7}{|l|}{ Semivolatiles } \\
\hline 2-Methylnaphthalene & ND & ND & $7.69 \mathrm{E}-02$ & $\mathbf{Y}$ & $7.69 \mathrm{E}-02$ & $\mathrm{Y}$ \\
\hline Acenaphthene & ND & ND & $3.16 \mathrm{E}-01$ & $\mathrm{Y}$ & $3.16 \mathrm{E}-01$ & $\mathbf{Y}$ \\
\hline Acenaphthylene & ND & ND & $8.61 \mathrm{E}-02$ & $\mathrm{Y}$ & 8.61E-02 & $\mathrm{Y}$ \\
\hline Anthracene & ND & ND & $6.74 \mathrm{E}-01$ & $\mathbf{Y}$ & $6.74 \mathrm{E}-01$ & $Y$ \\
\hline Benzo(a)anthracene & ND & ND & $1.62 \mathrm{E}+00$ & $\mathbf{Y}$ & $1.62 \mathrm{E}+00$ & $\mathrm{Y}$ \\
\hline Benzo(a)pyrene & ND & ND & $1.33 E+00$ & $\mathbf{Y}$ & $1.33 \mathrm{E}+00$ & $\mathbf{Y}$ \\
\hline Benzo(b)fluoranthene & ND & ṆD & $1.28 \mathrm{E}+00$ & $\mathbf{Y}$ & $1.28 \mathrm{E}+00$ & $\mathrm{Y}$ \\
\hline Benzo(g,h,i)perylene & ND & ND & 9.52E-01 & $\mathrm{Y}$ & 9.52E-01 & $\mathrm{Y}$ \\
\hline Benzo(k)fluoranthene & ND & ND & $1.49 E+00$ & $Y$ & $1.49 \mathrm{E}+00$ & $\mathbf{Y}$ \\
\hline Benzoic acid & ND & ND & $4.86 \mathrm{E}-02$ & $\mathrm{Y}$ & $4.86 \mathrm{E}-02$ & $Y$ \\
\hline
\end{tabular}


TABLE 3.12

COMPARISON OF SOIL ANALYTES TO BACKGROUND CONCENTRATIONS

SAVANNAH RIVER SITE

BURNING/RUBBLE PITS 231-F AND 231-1F

\begin{tabular}{|c|c|c|c|c|c|c|}
\hline \multirow{2}{*}{ Constituents } & \multirow{2}{*}{$\begin{array}{c}\text { Mean } \\
\text { Background (a) }\end{array}$} & \multirow{2}{*}{$\begin{array}{l}\text { 2x Background } \\
\cdot \text { Mean }\end{array}$} & \multicolumn{4}{|c|}{ Pits 231-F and 231-1F } \\
\hline & & & \multicolumn{2}{|c|}{$0-2$ Feet } & \multicolumn{2}{|c|}{0.4 Feet } \\
\hline Bis(2-ethylhexyl) phthalate & $7.80 \mathrm{E}-01$ & $1.56 \mathrm{E}+00$ & $6.02 \mathrm{E}-01$ & $\mathrm{Y}(\mathrm{c})$ & $6.02 \mathrm{E}-01$ & $Y(c)$ \\
\hline Butylbenzyl phthalate & ND & ND & $2.08 \mathrm{E}-01$ & $\mathbf{Y}$ & $2.08 \mathrm{E}-01$ & $\mathrm{Y}$ \\
\hline Chrysene & ND & ND & $1.62 E+00$ & $\mathrm{Y}$ & $1.62 \mathrm{E}+00$ & $\mathrm{Y}$ \\
\hline Di-n-butyl phthalate & $6.30 \mathrm{E}-01$ & $1.26 \mathrm{E}+00$ & $2.42 \mathrm{E}-01$ & $\mathrm{Y}(\mathrm{c})$ & $2.42 \mathrm{E}-01$ & $\mathrm{Y}(\mathrm{c})$ \\
\hline Di-n-octyl phthalate & 8.50E-02 & $1.70 \mathrm{E}-01$ & $7.90 \mathrm{E}-02$ & $Y(c)$ & $7.90 \mathrm{E}-02$ & $\mathrm{Y}(\mathrm{c})$ \\
\hline Dibenz(a,h)anthracene & ND & ND & $4.73 \mathrm{E}-01$ & $\mathrm{Y}$ & 4.73E-01 & $\mathrm{Y}$ \\
\hline Dibenzofuran & ND & ND & $2.22 \mathrm{E}-01$ & $\mathbf{Y}$ & $2.22 \mathrm{E}-01$ & $\mathrm{Y}$ \\
\hline Fluoranthene & $5.30 \mathrm{E}-02$ & $1.06 \mathrm{E}-01$ & $3.91 \mathrm{E}+00$ & $Y(c)$ & $3.91 \mathrm{E}+00$ & $Y(c)$ \\
\hline Fluorene & ND & ND & $2.99 \mathrm{E}-01$ & $\mathrm{Y}$ & $2.99 \mathrm{E}-01$ & $\mathrm{Y}$ \\
\hline Indeno( $(1,2,3-c, d)$ pyrene & ND & ND & 8.32E-01 & $\mathrm{Y}$ & $8.32 \mathrm{E}-01$ & $\mathrm{Y}$ \\
\hline Naphthalene & ND & ND & $3.22 \mathrm{E}-01$ & Y & 3.22E-01 & $\mathrm{Y}$ \\
\hline Phenanthrene & ND & ND & $3.38 \mathrm{E}+00$ & $\mathrm{Y}$ & $3.38 \mathrm{E}+00$ & $\mathrm{Y}$ \\
\hline Pyrene & ND & ND & $2.42 \mathrm{E}+00$ & $\mathrm{Y}$ & $2.42 \mathrm{E}+00$ & $\mathrm{Y}$ \\
\hline \multicolumn{7}{|l|}{ Dioxins/Furans } \\
\hline Heptachlorodibenzo-p-dioxin isomers & ND & ND & $8.30 \mathrm{E}-03$ & $\mathrm{Y}$ & $8.30 \mathrm{E}-03$ & $\mathbf{Y}$ \\
\hline Heptachlorodibenzo-p-furan isomers & ND & ND & $5.00 \mathrm{E}-04$ & $\mathrm{Y}$ & $5.00 \mathrm{E}-04$ & $\mathrm{Y}$ \\
\hline Hexachlorodibenzo-p-furan isomers & ND & ND & $1.00 \mathrm{E}-04$ & $\mathrm{Y}$ & $1.00 \mathrm{E}-04$ & $\mathrm{Y}$ \\
\hline Octachlorodibenzo-p-dioxin isomers & 5.00E-04 & $1.00 \mathrm{E}-03$ & $1.10 \mathrm{E}-02$ & $\mathrm{Y}(\mathrm{c})$ & $1.10 \mathrm{E}-02$ & $Y(c)$ \\
\hline Octachlorodibenzo-p-furan isomers & ND & ND & $8.00 \mathrm{E}-04$ & $\mathrm{Y}$ & $8.00 \mathrm{E}-04$ & $\mathrm{Y}$ \\
\hline \multicolumn{7}{|l|}{ PCBs } \\
\hline PCB 1254 & ND & ND & 4.38E-01 & $\mathrm{Y}$ & 4.38E-01 & $\mathrm{Y}$ \\
\hline
\end{tabular}


TABLE 3.12

COMPARISON OF SOIL ANALYTES TO BACKGROLND CONCENTRATIONS

SAVANNAH RIVER SITE

BURNING/RUBBLE PITS 231-F AND 231-1F

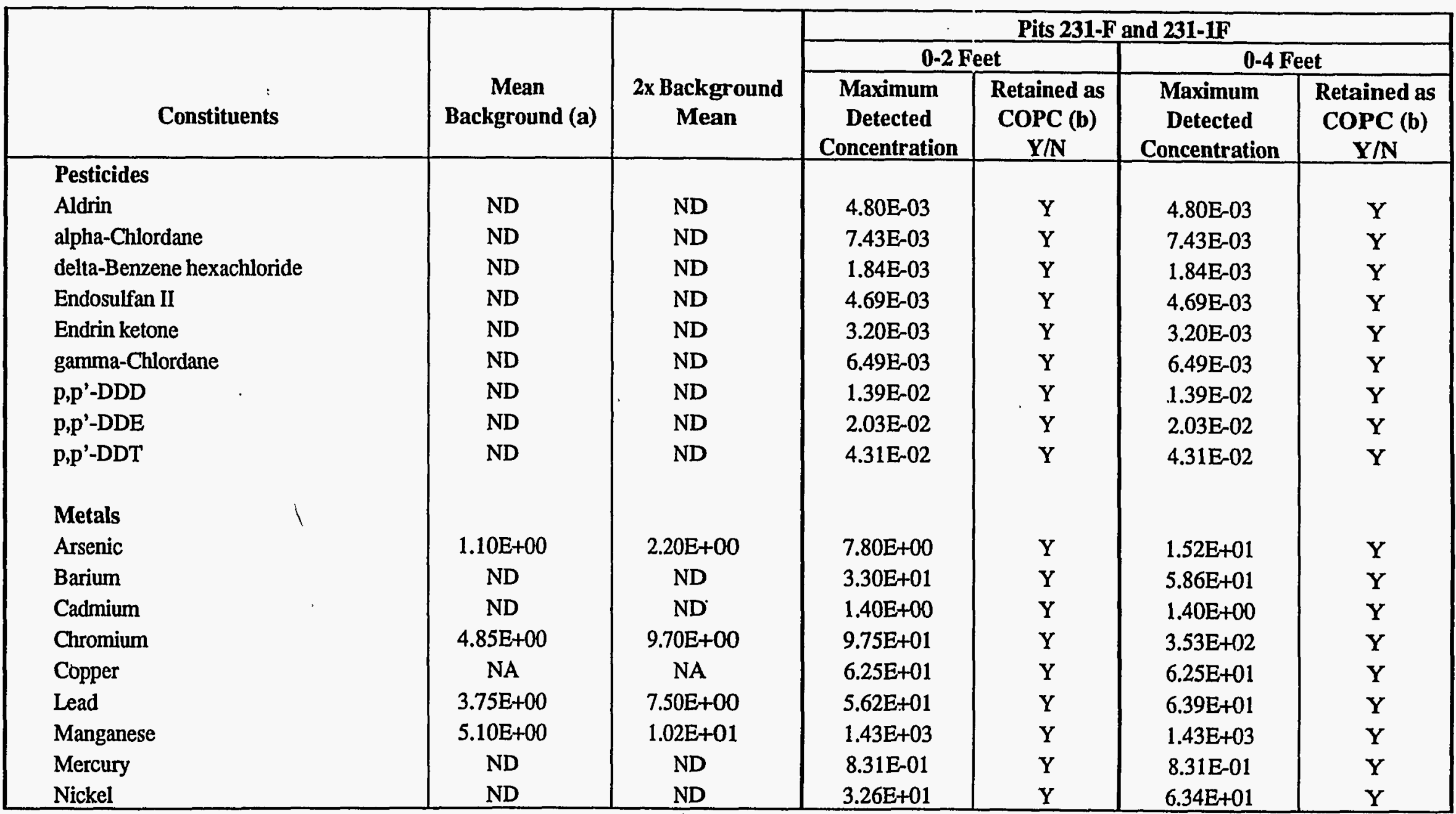


TABLE 3.12

COMPARISON OF SOIL ANALYTES TO BACKGROUND CONCENTRATIONS

SAVANNAH RIVER SITE

BURNING/RUBBLE PITS 231-F AND 231-1F

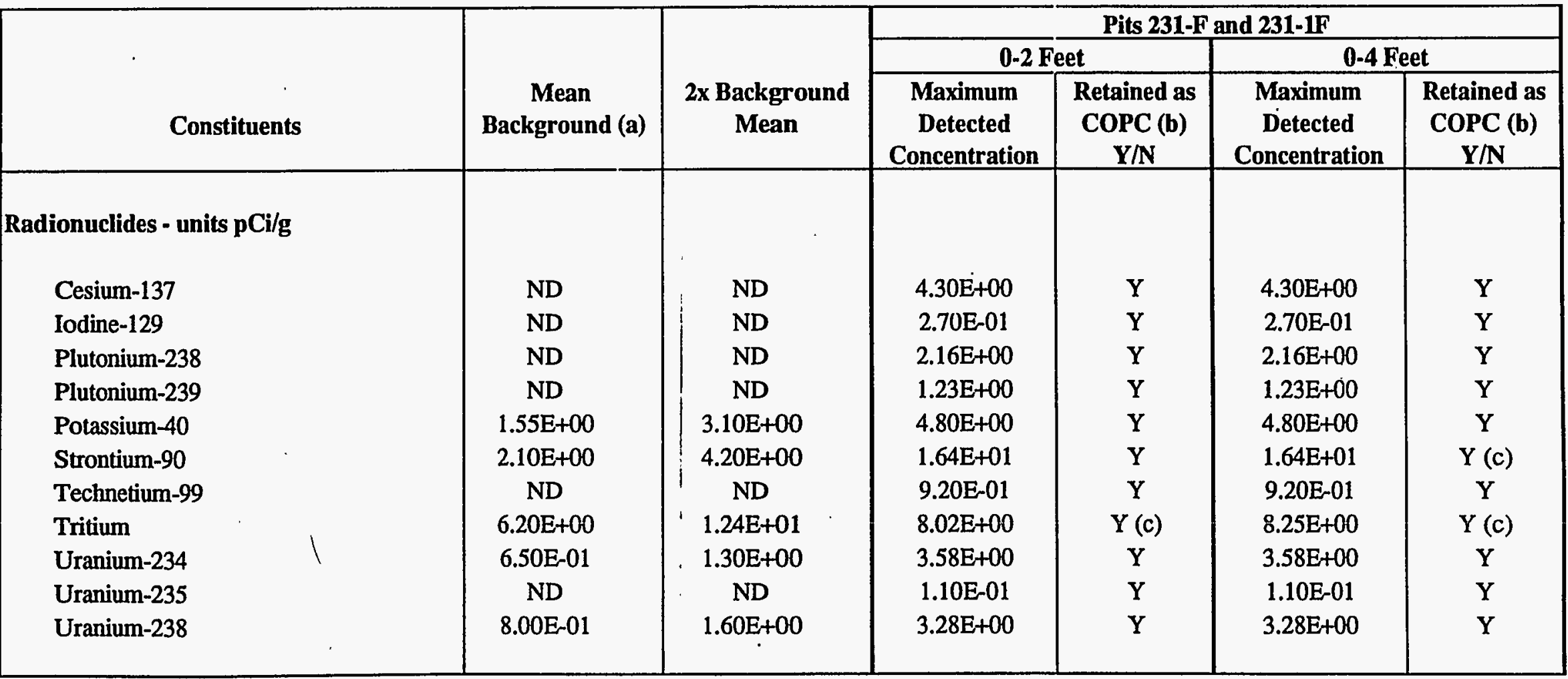

COPC $=$ Chemical of Potential Concern

(a) Mean background was determined by averaging the detected soil concentrations in sample $231-\mathrm{F}$ and $\mathrm{F} 1$ at the following intervals: $0-0.5 \mathrm{ft}, 0.5-2 \mathrm{ft}, 5-7 \mathrm{ft}$ and 10-12 ft. Mean background concentrations were derived by averaging the detected concentrations - nondetects were excluded from the average. ND $=$ Not Detected; NA $=$ Not Analyzed

(b) Maximum detected concentrations were compared to $2 x$ the background mean. The chemical was retained if the maximum concentration exceeded $2 x$ the background mean or if background data were not available.

(c) For organics and non-naturally occurring radionuclides, the $2 x$ background rule was used for comparative purposes only. Elimination of COPCs was only performed for naturally-occurring inorganics and radionuclides. 
TABLE 3.13

COMPARISON OF SOIL ANALYTES TO BACKGROUND CONCENTRATIONS

SAVANNAH RIVER SITE

RUBBLE PIT 231-2F

\begin{tabular}{|c|c|c|c|c|c|c|}
\hline \multirow[b]{3}{*}{ Constituent } & \multirow[b]{3}{*}{$\begin{array}{c}\text { Mean } \\
\text { Background (a) }\end{array}$} & \multirow[b]{3}{*}{$\begin{array}{c}\text { 2x Background } \\
\text { Mean }\end{array}$} & \multicolumn{4}{|c|}{ Pit 231-F2 } \\
\hline & & & \multicolumn{2}{|c|}{$0-2$ Feet } & \multicolumn{2}{|c|}{ 0-4 Feet } \\
\hline & & & $\begin{array}{c}\text { Maximum } \\
\text { Detected } \\
\text { Concentration' }\end{array}$ & $\begin{array}{l}\text { Retained as } \\
\text { COPC (b) } \\
\quad Y / N \\
\end{array}$ & $\begin{array}{c}\text { Maximum } \\
\text { Detected } \\
\text { Concentration }\end{array}$ & $\begin{array}{c}\text { Retained as } \\
\text { COPC (b) } \\
\text { Y/N }\end{array}$ \\
\hline \multicolumn{7}{|l|}{ Chemical - units in (mg/kg) } \\
\hline Volatiles & & & & & & \\
\hline 1,1,2-Trichloroethane & ND & ND & $1.36 \mathrm{E}-02$ & Y & $1.36 \mathrm{E}-02$ & Y \\
\hline 1,2-Dichloroethane & ND & ND & $1.03 \mathrm{E}-02$ & $\mathrm{Y}$ & $1.03 \mathrm{E}-02$ & $\mathrm{Y}$ \\
\hline Acetone & $5.30 \mathrm{E}-02$ & $1.06 \mathrm{E}-01$ & $4.75 \mathrm{E}-02$ & $Y(c)$ & 4.75E-02 & $\mathrm{Y}(\mathrm{c})$ \\
\hline Chloroform & ND & ND & $2.65 \mathrm{E}-03$ & $\mathrm{Y}$ & $2.65 \mathrm{E}-03$ & $\mathrm{Y}$ \\
\hline Dichloromethane (Methylene chloride) & $7.00 \mathrm{E}-03$ & $1.40 \mathrm{E}-02$ & $6.22 \mathrm{E}-03$ & $Y(c)$ & $6.22 \mathrm{E}-03$ & $Y(c)$ \\
\hline Toluene & $1.40 \mathrm{E}-02$ & $2.80 \mathrm{E}-02$ & $1.19 \mathrm{E}-02$ & $Y(c)$ & $1.19 \mathrm{E}-02$ & $\mathrm{Y}(\mathrm{c})$ \\
\hline \multicolumn{7}{|l|}{ Semivolatiles } \\
\hline Benzo(a)anthracene & ND & ND & $6.90 \mathrm{E}-02$ & $\mathrm{Y}$ & $6.90 \mathrm{E}-02$ & $\mathbf{Y}$ \\
\hline Benzo(a)pyrene & ND & ND & $6.01 \mathrm{E}-02$ & $\mathbf{Y}$ & $6.01 \mathrm{E}-02$ & Y \\
\hline Benzo(b)fluoranthene & ND & ND & $8.14 \mathrm{E}-02$ & $\mathrm{Y}$ & $8.14 \mathrm{E}-02$ & $\mathrm{Y}$ \\
\hline Benzo(k)fluoranthene & ND & ND & $6.50 \mathrm{E}-02$ & $\mathrm{Y}$ & $6.50 \mathrm{E}-02$ & $\mathrm{Y}$ \\
\hline Bis(2-ethylhexyl) phthalate & $7.80 \mathrm{E}-01$ & $1.56 \mathrm{E}+00$ & $2.77 \mathrm{E}-01$ & $Y(c)$ & 2.77E-01 & $Y(c)$ \\
\hline Chrysene & ND & ND & $6.92 \mathrm{E}-02$ & $\mathrm{Y}$ & $6.92 \mathrm{E}-02$ & $\mathrm{Y}$ \\
\hline Di-n-butyl phthalate & $6.30 \mathrm{E}-01$ & $1.26 \mathrm{E}+00$ & $7.07 \mathrm{E}-02$ & $Y(c)$ & $7.07 \mathrm{E}-02$ & $\mathrm{Y}(\mathrm{c})$ \\
\hline Di-n-octyl phthalate & $8.50 \mathrm{E}-02$ & $1.70 \mathrm{E}-01$ & $1.16 \mathrm{E}-01$ & $Y(c)$ & $1.16 \mathrm{E}-01$ & $Y(c)$ \\
\hline Fluoranthene & $5.30 \mathrm{E}-02$ & $1.06 \mathrm{E}-01$ & $1.66 \mathrm{E}-01$ & $Y(c)$ & $1.66 \mathrm{E}-01$ & $Y(c)$ \\
\hline Phenanthrene & ND & $\mathrm{ND}$ & $8.68 \mathrm{E}-02$ & $Y$ & $8.68 \mathrm{E}-02$ & $\mathrm{Y}$ \\
\hline Pyrene & ND & ND & $1.14 \mathrm{E}-01$ & $\mathbf{Y}$ & $1.14 \mathrm{E}-01$ & $\mathrm{Y}$ \\
\hline
\end{tabular}


TABLE 3.13

COMPARISON OF SOLL ANALYTES TO BACKGROUND CONCENTRATIONS

SAVANNAH RIVER SITE

RUBBLE PIT 231-2F

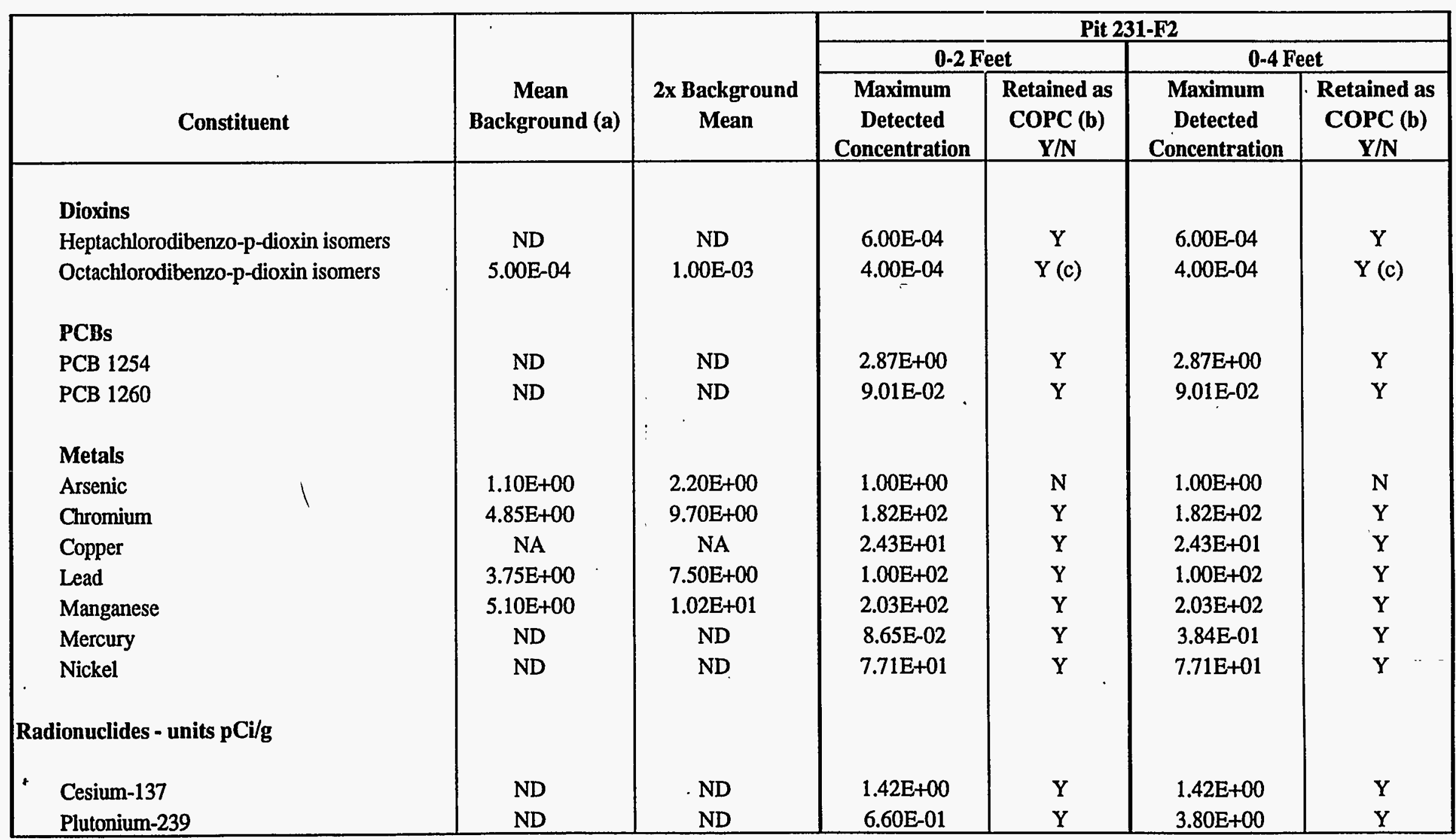


TABLE 3.13

COMPARISON OF SOIL ANALYTES TO BACKGROUND CONCENTRATIONS

SAVANNAH RIVER SITE

RUBBLE PIT 231-2F

\begin{tabular}{|c|c|c|c|c|c|c|}
\hline \multirow[b]{3}{*}{ Constituent } & \multirow[b]{3}{*}{$\begin{array}{c}\text { Mean } \\
\text { Background (a) }\end{array}$} & \multirow[b]{3}{*}{$\begin{array}{c}\text { 2x Background } \\
\text { Mean }\end{array}$} & \multicolumn{4}{|c|}{ Pit 231-F2 } \\
\hline & & & \multicolumn{2}{|c|}{ 0-2 Feet } & \multicolumn{2}{|c|}{ 0-4 Feet } \\
\hline & & & $\begin{array}{c}\text { Maximum } \\
\text { Detected } \\
\text { Concentration }\end{array}$ & $\begin{array}{c}\text { Retained as } \\
\text { COPC (b) } \\
\text { Y/N } \\
\end{array}$ & $\begin{array}{c}\text { Maximum } \\
\text { Detected } \\
\text { Concentration }\end{array}$ & $\begin{array}{c}\text { Retained as } \\
\text { COPC (b) } \\
\text { Y/N }\end{array}$ \\
\hline Potassium-40 & $1.55 E+00$ & $3.10 \mathrm{E}+00$ & $3.78 \mathrm{E}+00$ & $\mathrm{Y}$ & $3.78 \mathrm{E}+00$ & $\mathrm{Y}$ \\
\hline Strontium-90 & $2.10 \mathrm{E}+00$ & $4.20 \mathrm{E}+00$ & $6.00 \mathrm{E}-01$ & $Y(c)$ & $2.90 \mathrm{E}+00$ & $Y(c)$ \\
\hline Technetium-99 & ND & ND & $4.68 \mathrm{E}+00$ & $\mathrm{Y}$ & $4.68 \mathrm{E}+00$ & $\mathrm{Y}$ \\
\hline Tritium & $6.20 \mathrm{E}+00$ & $1.24 \mathrm{E}+01$ & $6.20 E+00$ & $Y(c)$ & $6.30 \mathrm{E}+00$ & $Y(c)$ \\
\hline $\begin{array}{l}\text { Uranium-234 } \\
\text { Uranium-235 }\end{array}$ & $\begin{array}{c}\text { 6.50E-01 } \\
\text { ND }\end{array}$ & $\begin{array}{c}1.30 \mathrm{E}+00 \\
\mathrm{ND}\end{array}$ & $\begin{array}{c}2.59 \mathrm{E}+00 \\
1.40 \mathrm{E}-01\end{array}$ & $\begin{array}{l}\mathrm{Y} \\
\mathrm{Y}\end{array}$ & $\begin{array}{c}2.59 \mathrm{E}+00 \\
1.60 \mathrm{E}-01\end{array}$ & $\begin{array}{l}\mathrm{Y} \\
\mathrm{Y}\end{array}$ \\
\hline Uranium-238 & $8.00 \mathrm{E}-01$ & $1.60 \mathrm{E}+00$ & $2.00 \mathrm{E}+00$ & $\mathrm{Y}$ & $2.00 \mathrm{E}+00$ & $\mathbf{Y}$ \\
\hline
\end{tabular}

COPC $=$ Chemical of Potential Concern

(a) Mean background was determined by averaging the detected soil concentrations in sample RP 231-2F at the following intervals: $0-0.5 \mathrm{ft}, 0.5-2 \mathrm{ft}, 5-7 \mathrm{ft}$ and 10-12 ft. Mean background concentrations were derived by averaging the detected concentrations - hondetects were excluded from the average. ND $=$ Not Detected; NA = Not Analyzed

(b) Maximum detected concentrations were compared to $2 x$ the background mean. The chemical was retained if the maximum concentration exceeded $2 x$ the background mean or if background data were not available.

(c) For organics and non-naturally occurring radionuclides, the $2 x$ background rule was used for comparative purposes only. Elimination of COPCs was only performed for naturally-occurring inorganics and radionuclides. 
TABLE 3.14

COMPARISON OF GROUNDWATER ANALYTES TO BACKGROUND CONCENTRATIONS SAVANNAB RIVER SITE

F-AREA BURNING/RUBBLE PISS AND RUBBLE PIT

\begin{tabular}{|c|c|c|c|}
\hline Constituent & $\begin{array}{c}\text { Maximum } \\
\text { Detected } \\
\text { Concentration }\end{array}$ & $\begin{array}{c}2 \times \text { Mean } \\
\text { Background (a) }\end{array}$ & $\begin{array}{c}\text { Retained as } \\
\text { COPC } \\
\text { Y/N (b) }\end{array}$ \\
\hline \multicolumn{4}{|l|}{ Chemical - units in $(\mathrm{mg} / \mathrm{L})$} \\
\hline \multicolumn{4}{|l|}{ Volatlles } \\
\hline Acetone & $6.44 \mathrm{E}-03$ & ND & $\mathbf{Y}$ \\
\hline Carbon tetrachloride & 5.41E-03 & ND & $\mathbf{Y}$ \\
\hline Chloroform & $1.00 \mathrm{E}-03$ & ND & $\mathbf{Y}$ \\
\hline Chloromethane (Methyl Chloride) & $4.20 \mathrm{E}-03$ & ND & $\mathbf{Y}$ \\
\hline Dichloromethane (Methylene chloride) & $3.02 \mathrm{E}-02$ & $3.00 \mathrm{E}-02$ & $\mathbf{Y}(\mathbf{c})$ \\
\hline Tetrachloroethylene & $3.28 \mathrm{E}-02$ & ND & $\mathbf{Y}$ \\
\hline Trichloroethylene & $3.34 \mathrm{E}-02$ & ND & $\mathbf{Y}$ \\
\hline Trichlorofluoromethane & $1.05 \mathrm{E}-01$ & ND & $\mathbf{Y}$ \\
\hline \multicolumn{4}{|l|}{ Semivolatiles } \\
\hline Di-n-butylphthalate & $2.20 \mathrm{E}-03$ & ND & $\mathbf{Y}$ \\
\hline \multicolumn{4}{|l|}{ Metals } \\
\hline Aluminum & $7.08 \mathrm{E}+00$ & $8.48 \mathrm{E}-01$ & $\mathbf{Y}$ \\
\hline Arsenic & $2.50 \mathrm{E}-03$ & $\mathrm{ND}$ & $\mathbf{Y}$ \\
\hline Barium & $1.01 \mathrm{E}-01$ & $4.16 \mathrm{E}-02$ & $\mathbf{Y}$ \\
\hline Cadmium & $2.00 \mathrm{E}-03$ & $\mathrm{ND}$ & $\mathbf{Y}$ \\
\hline Chloride & $6.55 \mathrm{E}+00$ & $7.21 \mathrm{E}+00$ & $\mathbf{N}$ \\
\hline Chromium & $9.22 \mathrm{E}-02$ & $1.52 \mathrm{E}-02$ & $\mathbf{Y}$ \\
\hline Fluoride & $1.68 \mathrm{E}-01$ & 2.77E-01 & $\mathbf{N}$ \\
\hline Iron & $2.82 E+01$ & $1.84 \mathrm{E}+00$ & $\mathbf{Y}$ \\
\hline Lead & $1.14 \mathrm{E}-02$ & ND & $\mathrm{Y}$ \\
\hline Lithium & $1.12 \mathrm{E}-02$ & $1.68 \mathrm{E}-02$ & $\mathrm{~N}$ \\
\hline Manganese & 4.53E-01 & $1.47 \mathrm{E}-01$ & $\mathbf{Y}$ \\
\hline Mercury & $2.80 \mathrm{E}-04$ & $4.80 \mathrm{E}-04$ & $\mathbf{N}$ \\
\hline Nitrate (as nitrogen) & $2.40 \mathrm{E}+00$ & $1.30 \mathrm{E}+00$ & $\mathbf{Y}$ \\
\hline Potassium & $2.17 \mathrm{E}+00$ & $1.36 \mathrm{E}+00$ & $\mathbf{Y}$ \\
\hline Silver & $2.50 \mathrm{E}-03$ & $5.00 \mathrm{E}-03$ & $\mathbf{N}$ \\
\hline \multicolumn{4}{|l|}{ Radionuclides- units in $\mathrm{pC} / \mathrm{L}$} \\
\hline Radium-226 & $1.70 \mathrm{E}+00$ & $1.48 \mathrm{E}+00$ & $\mathbf{Y}$ \\
\hline Radium-228 & $2.70 \mathrm{E}+00$ & $2.36 \mathrm{E}+00$ & $\mathrm{Y}$ \\
\hline Strontium-90 & $1.56 \mathrm{E}+01$ & $3.12 \mathrm{E}+01$ & $Y(c)$ \\
\hline Tritium & $5.40 \mathrm{E}+03$ & $9.00 \mathrm{E}+03$ & $Y(c)$ \\
\hline Uranium-233/234 & $1.57 \mathrm{E}+00$ & $2.54 \mathrm{E}+00$ & $\mathbf{N}$ \\
\hline Uranium-238 & $1.69 \mathrm{E}+00$ & ND & $\mathbf{Y}$ \\
\hline
\end{tabular}

$\mathrm{COPC}=$ Chemical of Potential Concern

ND $=$ Not Detected

(a) Background monitoring wells used were FBP-4, FBP-8D and FBP-12D. Mean background concentrations were derived by averaging the detected concentrations. Nondetects were excluded from the average.

(b) If background data was not available, the chemical was retained.

(c) For organics and non-naturally occurring radionuclides, the $2 \mathrm{X}$ background rule was used for comparative purposes only. Elimination of COPCs was only performed for naturally-occurring inorganics. 
COMPARISON OF SOIL ANALYTES TO RISK-BASED CONCENTRATIONS

SAVANNAH RIVER SITE

BURNING/RUBBLE PITS 231-F AND 231-1F (1)

\begin{tabular}{|c|c|c|c|c|c|c|}
\hline \multirow[b]{3}{*}{ Constituent } & \multirow{2}{*}{\multicolumn{2}{|c|}{ EPA Region III (2) }} & \multicolumn{4}{|c|}{ Pits 231-F and 231-F1 } \\
\hline & & & \multicolumn{2}{|c|}{$0-2$ Feet } & \multicolumn{2}{|c|}{0.4 Feet } \\
\hline & $\begin{array}{c}\text { Risk-Based } \\
\text { Concentration for } \\
\text { Soil - Residential } \\
\end{array}$ & $\begin{array}{l}\text { Soil Screening } \\
\text { Level - Transfer } \\
\text { from Soil to Air } \\
\end{array}$ & $\begin{array}{c}\text { Maximum } \\
\text { Detected } \\
\text { Concentration }\end{array}$ & $\begin{array}{c}\text { Retained as } \\
\text { COPC (3) } \\
Y / N \\
\end{array}$ & $\begin{array}{c}\text { Maximum } \\
\text { Detected } \\
\text { Concentration }\end{array}$ & \begin{tabular}{|c} 
Retained as \\
COPC (3) \\
$\mathbf{Y} / \mathbf{N}$ \\
\end{tabular} \\
\hline \multicolumn{6}{|l|}{ Chemical - units in (mg/kg) } & \\
\hline 1,1,2-Trichloroethane & $1.10 \mathrm{E}+01$ & $8.00 \mathrm{E}-01$ & $1.65 \mathrm{E}-02$ & $\mathbf{N}$ & $1.65 \mathrm{E}-02$ & $\mathbf{N}$ \\
\hline 1,2-Dichloroethane & $7.00 \mathrm{E}+00$ & $3.00 \mathrm{E}-01$ & $2.16 \mathrm{E}-02$ & $\mathbf{N}$ & $2.16 \mathrm{E}-02$ & $\mathbf{N}$ \\
\hline Acetone & $7.80 \mathrm{E}+02$ & $6.20 \mathrm{E}+03$ & $2.62 \mathrm{E}+01$ & $\mathbf{N}$ & $2.62 \mathrm{E}+01$ & $\mathbf{N}$ \\
\hline Chloroform & $1.00 \mathrm{E}+02$ & $2.00 \mathrm{E}-01$ & 1.01E-02 & $\mathbf{N}$ & $1.01 \mathrm{E}-02$ & $\mathbf{N}$ \\
\hline Dichloromethane (Methylene chloride) & $8.50 \mathrm{E}+01$ & $7.00 \mathrm{E}+00$ & $3.80 \mathrm{E}-02$ & $\mathbf{N}$ & $3.80 \mathrm{E}-02$ & $\mathbf{N}$ \\
\hline Ethylbenzene & $7.80 \mathrm{E}+02$ & $2.60 \mathrm{E}+01$ & ND & - & $1.29 \mathrm{E}-03$ & $\mathbf{N}$ \\
\hline Tetrachloroethylene & $1.20 \mathrm{E}+01$ & $1.10 \mathrm{E}+01$ & $2.87 \mathrm{E}-03$ & $\mathbf{N}$ & $2.87 \mathrm{E}-03$ & $\mathbf{N}$ \\
\hline Toluene & $1.60 \mathrm{E}+03$ & $5.20 \mathrm{E}+01$ & $3.06 \mathrm{E}-02$ & $\mathbf{N}$ & $3.06 \mathrm{E}-02$ & $\mathbf{N}$ \\
\hline Xylenes & $1.60 \mathrm{E}+04$ & $3.20 \mathrm{E}+01$ & $1.34 \mathrm{E}-03$ & $\mathbf{N}$ & $1.34 \mathrm{E}-03$ & $\mathbf{N}$ \\
\hline \multicolumn{7}{|l|}{ Semivolatiles } \\
\hline 2-Methylnaphthalene & NR & NR & $7.69 \mathrm{E}-02$ & $\mathbf{Y}$ & $7.69 \mathrm{E}-02$ & $\mathbf{Y}$ \\
\hline Acenaphthene & $4.70 \mathrm{E}+02$ & $1.20 \mathrm{E}+01$ & $3.16 \mathrm{E}-01$ & $\mathbf{N}$ & $3.16 \mathrm{E}-01$ & $\mathbf{N}$ \\
\hline Acenaphthylene & NR & NR & 8.61E-02 & $\mathbf{Y}$ & $8.61 \mathrm{E}-02$ & $\mathbf{Y}$ \\
\hline Anthracene & $2.30 \mathrm{E}+03$ & $6.80 \mathrm{E}-01$ & $6.74 \mathrm{E}-01$ & $\mathbf{N}$ & $6.74 \mathrm{E}-01$ & $\mathbf{N}$ \\
\hline Benzo(a)anthracene & $8.80 \mathrm{E}-01$ & $2.70 E+01$ & $1.62 \mathrm{E}+00$ & $\mathrm{Y}$ & $1.62 \mathrm{E}+00$ & $\mathbf{Y}$ \\
\hline Benzo(a)pyrene & $8.80 \mathrm{E}-02$ & $1.10 \mathrm{E}+01$ & $1.33 \mathrm{E}+00$ & $\mathbf{Y}$ & $1.33 E+00$ & $\mathbf{Y}$ \\
\hline Benzo(b)fluoranthene & $8.80 \mathrm{E}-01$ & $2.30 \mathrm{E}+01$ & $1.28 \mathrm{E}+00$ & $\mathrm{Y}$ & $1.28 \mathrm{E}+00$ & $\mathbf{Y}$ \\
\hline Benzo(g,h,i)perylene & NR & NR & $9.52 \mathrm{E}-01$ & $\mathrm{Y}$ & $9.52 \mathrm{E}-01$ & $\mathbf{Y}$ \\
\hline Benzo(k)fluoranthene & $8.80 \mathrm{E}+00$ & NR & $1.49 \mathrm{E}+00$ & $\mathbf{N}$ & $1.49 \mathrm{E}+00$ & $\mathbf{N}$ \\
\hline Benzoic acid & $3.10 \mathrm{E}+04$ & $3.20 \mathrm{E}+01$ & $4.86 \mathrm{E}-02$ & $\mathrm{~N}$ & $4.86 \mathrm{E}-02$ & $\mathbf{N}$ \\
\hline Bis(2-ethylhexyl) phthalate & $4.60 \mathrm{E}+01$ & $2.10 \mathrm{E}+02$ & $6.02 \mathrm{E}-01$ & $\mathbf{N}$ & $6.02 \mathrm{E}-01$ & $\mathbf{N}$ \\
\hline
\end{tabular}


TABLE 3.15

COMPARISON OF SOIL ANALYTES TO RISK-BASED CONCENTRATIONS SAVANNAH RIVER SITE

BURNING/RUBBLE PITS 231-F AND 231-1F (1)

\begin{tabular}{|c|c|c|c|c|c|c|}
\hline \multirow[b]{3}{*}{ Constituent } & \multirow{2}{*}{\multicolumn{2}{|c|}{ EPA Region III (2) }} & \multicolumn{4}{|c|}{ Pits 231-F and 231-F1 } \\
\hline & & & \multicolumn{2}{|c|}{ 0-2 Feet } & \multicolumn{2}{|c|}{ 0-4 Feet } \\
\hline & \begin{tabular}{l}
\multicolumn{1}{c}{ Risk-Based } \\
Concentration for \\
Soil - Residential \\
\end{tabular} & $\begin{array}{l}\text { Soil Screening } \\
\text { Level - Transfer } \\
\text { from Soil to Air }\end{array}$ & $\begin{array}{c}\text { Maximum } \\
\text { Detected } \\
\text { Concentration } \\
\end{array}$ & $\begin{array}{c}\text { Retained as } \\
\text { COPC (3) } \\
\mathbf{Y} / \mathrm{N} \\
\end{array}$ & $\begin{array}{c}\text { Maximum } \\
\text { Detected } \\
\text { Concentration } \\
\end{array}$ & $\begin{array}{c}\text { Retained as } \\
\text { COPC (3) } \\
\text { Y/N } \\
\end{array}$ \\
\hline Butylbenzyl phthalate & $1.60 \mathrm{E}+03$ & $5.30 \mathrm{E}+01$ & $2.08 \mathrm{E}-01$ & $\mathbf{N}$ & $2.08 \mathrm{E}-01$ & $\mathbf{N}$ \\
\hline Chrysene & $8.80 \mathrm{E}+01$ & $3.60 \mathrm{E}+00$ & $1.62 \mathrm{E}+00$ & $\mathbf{N}$ & $1.62 \mathrm{E}+00$ & $\mathbf{N}$ \\
\hline Di-n-butyl phthalate & $7.80 \mathrm{E}+02$ & $1.00 \mathrm{E}+01$ & $2.42 \mathrm{E}-01$ & $\mathbf{N}$ & 2.42E-01 & $\mathbf{N}$ \\
\hline Di-n-octyl phthalate & $1.60 \mathrm{E}+02$ & $1.00 \mathrm{E}+05$ & $7.90 \mathrm{E}-02$ & $\mathbf{N}$ & $7.90 \mathrm{E}-02$ & $\mathbf{N}$ \\
\hline Dibenz(a,h)anthracene & $8.80 \mathrm{E}-02$ & $7.20 \mathrm{E}+00$ & 4.73E-01 & $\mathbf{Y}$ & 4.73E-01 & $\mathbf{Y}$ \\
\hline Dibenzofuran & $3.10 \mathrm{E}+01$ & $1.20 \mathrm{E}+01$ & $2.22 \mathrm{E}-01$ & $\mathbf{N}$ & $2.22 \mathrm{E}-01$ & $\mathbf{N}$ \\
\hline Fluoranthene & $3.10 \mathrm{E}+02$ & $6.80 \mathrm{E}+00$ & $3.91 \mathrm{E}+00$ & $\mathbf{N}$ & $3.91 \mathrm{E}+00$ & $\mathbf{N}$ \\
\hline Fluorene & $3.10 \mathrm{E}+02$ & $8.90 \mathrm{E}+00$ & $2.99 \mathrm{E}-01$ & $\mathbf{N}$ & $2.99 \mathrm{E}-01$ & $\mathbf{N}$ \\
\hline Indeno(1,2,3-c,d)pyrene & $8.80 \mathrm{E}-01$ & $2.80 \mathrm{E}+02$ & 8.32E-01 & $\mathbf{N}$ & 8.32E-01 & $\mathbf{N}$ \\
\hline Naphthalene & $3.10 \mathrm{E}+02$ & $1.80 \mathrm{E}+01$ & $3.22 \mathrm{E}-01$ & $\mathbf{N}$ & $3.22 \mathrm{E}-01$ & $\mathbf{N}$ \\
\hline Phenanthrene & NR & NR & $3.38 \mathrm{E}+00$ & $\mathbf{Y}$ & $3.38 \mathrm{E}+00$ & $\mathrm{Y}$ \\
\hline Pyrene & $2.30 \mathrm{E}+02$ & $5.60 \mathrm{E}+00$ & $2.42 E+00$ & $N$ & $2.42 E+00$ & $\mathbf{N}$ \\
\hline \multicolumn{7}{|l|}{ Dioxins/Furans } \\
\hline Heptachlorodibenzo-p-dioxin isomers - & NR & NR & 8.30E-03 & $\mathbf{Y}$ & $8.30 \mathrm{E}-03$ & $\mathbf{Y}$ \\
\hline Heptachlorodibenzo-p-furan isomers & NR & NR & $5.00 \mathrm{E}-04$ & $\mathbf{Y}$ & $5.00 \mathrm{E}-04$ & $\mathbf{Y}$ \\
\hline Hexachlorodibenzo-p-furan isoniers & NR & NR & $1.00 \mathrm{E}-04$ & $\mathbf{Y}$ & $1.00 \mathrm{E}-04$ & $\mathbf{Y}$ \\
\hline Octachlorodibenzo-p-dioxin isomers & NR & NR & $1.10 \mathrm{E}-02$ & $\mathbf{Y}$ & $1.10 \mathrm{E}-02$ & $\mathrm{Y}$ \\
\hline Octachlorodibenzo-p-furan isomers & NR & NR & $8.00 \mathrm{E}-04$ & $\mathbf{Y}$ & $8.00 \mathrm{E}-04$ & $\mathrm{Y}$ \\
\hline \multicolumn{7}{|l|}{ PCBs } \\
\hline PCB 1254 & $1.60 \mathrm{E}-01$ & NR & $4.38 \mathrm{E}-01$ & $\mathrm{Y}$ & $4.38 \mathrm{E}-01$ & $Y$ \\
\hline \multicolumn{7}{|l|}{ Pesticides } \\
\hline Aldrin & $3.80 \mathrm{E}-02$ & $5.00 \mathrm{E}-01$ & $4.80 \mathrm{E}-03$ & $\mathbf{N}$ & $4.80 \mathrm{E}-03$ & $\mathrm{~N}$ \\
\hline
\end{tabular}


COMPARISON OF SOIL ANALYTES TO RISK-BASED CONCENTRATIONS

SAVANNAH RIVER SITE

BURNING/RUBBLE PITS 231-F AND 231-1F (1)

\begin{tabular}{|c|c|c|c|c|c|c|}
\hline \multirow[b]{3}{*}{ Constituent } & \multirow{2}{*}{\multicolumn{2}{|c|}{ EPA Region III (2) }} & \multicolumn{4}{|c|}{ Pits 231-F and 231-F1 } \\
\hline & & & \multicolumn{2}{|c|}{$0-2$ Feet } & \multicolumn{2}{|c|}{ 0-4 Feet } \\
\hline & \begin{tabular}{l}
\multicolumn{1}{c}{ Risk-Based } \\
Concentration for \\
Soil - Residential \\
\end{tabular} & $\begin{array}{l}\text { Soil Screening } \\
\text { Level - Transfer } \\
\text { from Soil to Air }\end{array}$ & $\begin{array}{c}\text { Maximum } \\
\text { Detected } \\
\text { Concentration } \\
\end{array}$ & $\begin{array}{c}\text { Retained as } \\
\text { COPC (3) } \\
\mathrm{Y} / \mathrm{N} \\
\end{array}$ & $\begin{array}{c}\text { Maximum } \\
\text { Detected } \\
\text { Concentration } \\
\end{array}$ & $\begin{array}{c}\text { Retained as } \\
\text { COPC (3) } \\
\text { Y/N } \\
\end{array}$ \\
\hline alpha-Chlordane & 4.90E-01 (a) & $1.00 \mathrm{E}+01 \quad$ (a) & 7.43E-03 & $\mathbf{N}$ & $7.43 \mathrm{E}-03$ & $\mathrm{~N}$ \\
\hline delta-Benzene hexachloride & NR & NR & $1.84 \mathrm{E}-03$ & $\mathbf{Y}$ & $1.84 \mathrm{E}-03$ & $\mathrm{Y}$ \\
\hline Endosulfan II & $4.70 \mathrm{E}+01 \quad$ (d) & $1.00 \mathrm{E}-01$ & $4.69 \mathrm{E}-03$ & $\mathbf{N}$ & $4.69 \mathrm{E}-03$ & $\mathrm{~N}$ \\
\hline Endrin ketone & NR & NR & $3.20 \mathrm{E}-03$ & $\mathbf{Y}$ & $3.20 \mathrm{E}-03$ & $\mathbf{Y}$ \\
\hline gamma-Chlordane & $4.90 \mathrm{E}-01$ & $1.00 \mathrm{E}+01$ & $6.49 \mathrm{E}-03$ & $N$ & $6.49 \mathrm{E}-03$ & $\mathbf{N}$ \\
\hline $\mathrm{p}, \mathrm{p}^{\prime}-\mathrm{DDD}$ & $2.70 \mathrm{E}+00$ & $3.70 \mathrm{E}+01$ & 1.39E-02 & $\mathbf{N}$ & $1.39 \mathrm{E}-02$ & $\mathbf{N}$ \\
\hline $\mathrm{p}, \mathrm{p}^{\prime}-\mathrm{DDE}$ & $1.90 \mathrm{E}+00$ & $1.00 \mathrm{E}+01$ & $2.03 \mathrm{E}-02$ & $\mathrm{~N}$ & 2.03E-02 & $\mathrm{N}$ \\
\hline $\mathrm{p}, \mathrm{p}^{\prime}-\mathrm{DDT}$ & $1.90 \mathrm{E}+00$ & $8.00 E+01$ & $4.31 \mathrm{E}-02$ & $\mathbf{N}$ & 4.31E-02 & $\mathbf{N}$ \\
\hline \multicolumn{7}{|l|}{ Metals } \\
\hline Arsenic & $4.30 \mathrm{E}-01 \quad$ (b) & $3.80 \mathrm{E}+02$ & $7.80 \mathrm{E}+00$ & $\mathbf{Y}$ & $1.52 \mathrm{E}+01$ & $\mathbf{Y}$ \\
\hline Barium & $5.50 \mathrm{E}+02$ & $3.50 \mathrm{E}+04$ & $3.30 \mathrm{E}+01$ & $\mathbf{N}$ & $5.86 \mathrm{E}+01$ & $\mathbf{N}$ \\
\hline Cadmium & $3.90 \mathrm{E}+00$ & $9.20 \mathrm{E}+01$ & $1.40 \mathrm{E}+00$ & $\mathbf{N}$ & $1.40 \mathrm{E}+00$ & $\mathbf{N}$ \\
\hline Chromium & $3.90 \mathrm{E}+01$ & $1.40 \mathrm{E}+01$ & $9.75 \mathrm{E}+01$ & $\mathrm{Y}$ & $3.53 \mathrm{E}+02$ & $\mathbf{Y}$ \\
\hline Copper & $3.10 \mathrm{E}+02$ & NR & $6.25 \mathrm{E}+01$ & $\mathbf{N}$ & $6.25 E+01$ & $\mathbf{N}$ \\
\hline Copper & $3.10 \mathrm{E}+02$ & NR & $6.25 \mathrm{E}+01$ & $\mathbf{N}$ & $6.25 \mathrm{E}+01$ & $\mathbf{N}$ \\
\hline Lead & NR & NR & $5.62 \mathrm{E}+01$ & $\mathbf{Y}$ & $6.39 E+01$ & $\mathbf{Y}$ \\
\hline Manganese & $3.90 \mathrm{E}+01$ & NR & $1.43 \mathrm{E}+03$ & $\mathrm{Y}$ & $1.43 E+03$ & $Y$ \\
\hline Mercury & $2.30 \mathrm{E}+00$ & $7.00 \mathrm{E}-01$ & 8.31E-01 & $\mathrm{Y}$ & 8.31E-01 & $\mathrm{Y}$ \\
\hline Nickel & $1.60 E+02$ & $6.90 \mathrm{E}+02$ & $3.26 \mathrm{E}+01$ & $\mathbf{N}$ & $6.34 \mathrm{E}+01$ & $\mathbf{N}$ \\
\hline \multicolumn{7}{|l|}{ Radionuclides - units pCi/g } \\
\hline Cesium-137 & $2.08 \mathrm{E}-02 \quad(\mathrm{e}, \mathrm{f})$ & - & $4.30 \mathrm{E}+00$ & $\mathrm{Y}$ & $4.30 \mathrm{E}+00$ & $\mathbf{Y}$ \\
\hline Iodine-129 & $2.90 \mathrm{E}+00$ & - & $2.70 \mathrm{E}-01$ & $\mathrm{~N}$ & $2.70 \mathrm{E}-01$ & $\mathbf{N}$ \\
\hline
\end{tabular}


TABLE 3.15

COMPARISON OF SOIL ANALYTES TO RISK-BASED CONCENTRATIONS

SAVANNAH RIVER SITE

BURNING/RUBBLE PITS 231-F AND 231-1F (1)

\begin{tabular}{|c|c|c|c|c|c|c|}
\hline \multirow[b]{3}{*}{ Constituent } & \multirow{2}{*}{\multicolumn{2}{|c|}{ EPA Region III (2) }} & \multicolumn{4}{|c|}{ Pits 231-F and 231-F1 } \\
\hline & & & \multicolumn{2}{|c|}{0.2 Feet } & \multicolumn{2}{|c|}{ 0-4 Feet } \\
\hline & \begin{tabular}{c|} 
Risk-Based \\
Concentration for \\
Soil - Residential \\
\end{tabular} & $\begin{array}{l}\text { Soil Screening } \\
\text { Level - Transfer } \\
\text { from Soil to Air } \\
\end{array}$ & $\begin{array}{c}\text { Maximum } \\
\text { Detected } \\
\text { Concentration } \\
\end{array}$ & $\begin{array}{c}\text { Retained as } \\
\text { COPC (3) } \\
\text { Y/N } \\
\end{array}$ & $\begin{array}{c}\text { Maximum } \\
\text { Detected } \\
\text { Concentration } \\
\end{array}$ & $\begin{array}{c}\text { Retained as } \\
\text { COPC (3) } \\
Y / N \\
\end{array}$ \\
\hline Plutonium-238 & $3.49 \mathrm{E}+00 \quad(\mathrm{e})$ & - & $2.16 \mathrm{E}+00$ & $\mathrm{~N}$ & $2.16 \mathrm{E}+00$ & $\mathbf{N}$ \\
\hline Plutonium-239 & $3.34 \mathrm{E}+00 \quad$ (e) & - & $1.23 E+00$ & $\mathbf{N}$ & $1.23 E+00$ & $\mathbf{N}$ \\
\hline Potassium-40 & $7.71 \mathrm{E}-02 \quad$ (e) & - & $4.80 \mathrm{E}+00$ & $\mathbf{Y}$ & $4.80 \mathrm{E}+00$ & $\mathbf{Y}$ \\
\hline Strontium-90 & $2.33 \mathrm{E}+01$ & - & $1.64 \mathrm{E}+01$ & $\mathbf{N}$ & $1.64 \mathrm{E}+01$ & $\mathbf{N}$ \\
\hline Technetium-99 & $5.87 \mathrm{E}+02$ & - & $9.20 \mathrm{E}-01$ & $\mathbf{N}$ & $9.20 \mathrm{E}-01$ & $\mathbf{N}$ \\
\hline Tritium & $1.42 \mathrm{E}+04$ & - & $8.02 \mathrm{E}+00$ & $\mathrm{~N}$ & $8.25 \mathrm{E}+00$ & $\mathbf{N}$ \\
\hline Uranium-234 & $4.65 \mathrm{E}+01$ & - & $3.58 \mathrm{E}+00$ & $\mathbf{N}$ & $3.58 \mathrm{E}+00$ & $\mathbf{N}$ \\
\hline Uranium-235 & $1.73 \mathrm{E}-01 \quad$ (e) & - & $1.10 \mathrm{E}-01$ & $\mathbf{N}$ & $1.10 \mathrm{E}-01$ & $\mathbf{N}$ \\
\hline Uranium-238 & $1.11 E+00 \quad(e, g)$ & - & $3.28 \mathrm{E}+00$ & $\mathrm{Y}$ & $3.28 \mathrm{E}+00$ & $\mathrm{Y}$ \\
\hline
\end{tabular}

COPC $=$ Chemical of Potential Concern

(1) Comparisons were performed using in the following criteria: cancer risk of $1 \mathrm{E}-06$ and Hazard Index of 0.1. NR = Not Reported

(2) EPA 1995, Risk-Based Concentrations Table, July-December.

(3) Chemical was retained if the maximum concentration exceeded the Risk-Based Concentration (RBC) or Soil Screening Level. If a RBC and a Soil Screening Level were not available, the chemical was retained.

(a) RBC for chlordane

(b) $\mathrm{RBC}$ for arsenic as a carcinogen

(c) RBC for chromium VI

(d) RBC for endosulfan

(e) Screening levels for radionuclides were derived using the Preliminary Remediation Goals (PRGs) equations for radionuclides in RAGS, Part $B$.

(f) Value for CS-137 and decay chain products

(g) Value for U-238 and decay chain products 
TABLE 3.16

COMPARISON OF SOIL ANALYTES TO RISK-BASED CONCENTRATIONS SAVANNAH RIVER SITE RUBBLE PIT 231-2F (1)

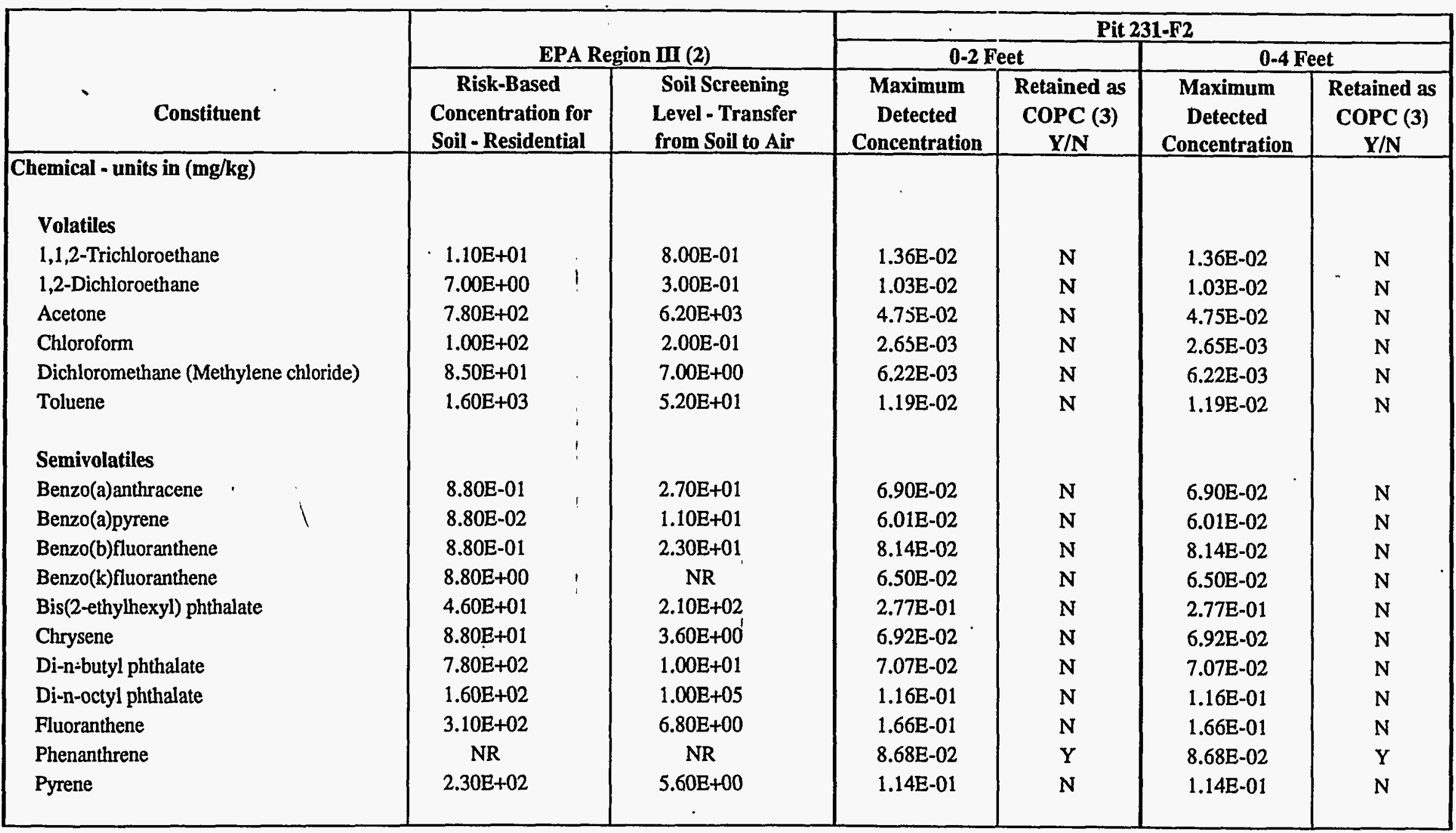


TABLE 3.16

COMPARISON OF SOIL ANALYTES TO RISK-BASED CONCENTRATIONS

SAVANNAH RIVER SITE

RUBBLE PIT 231-2F (1)

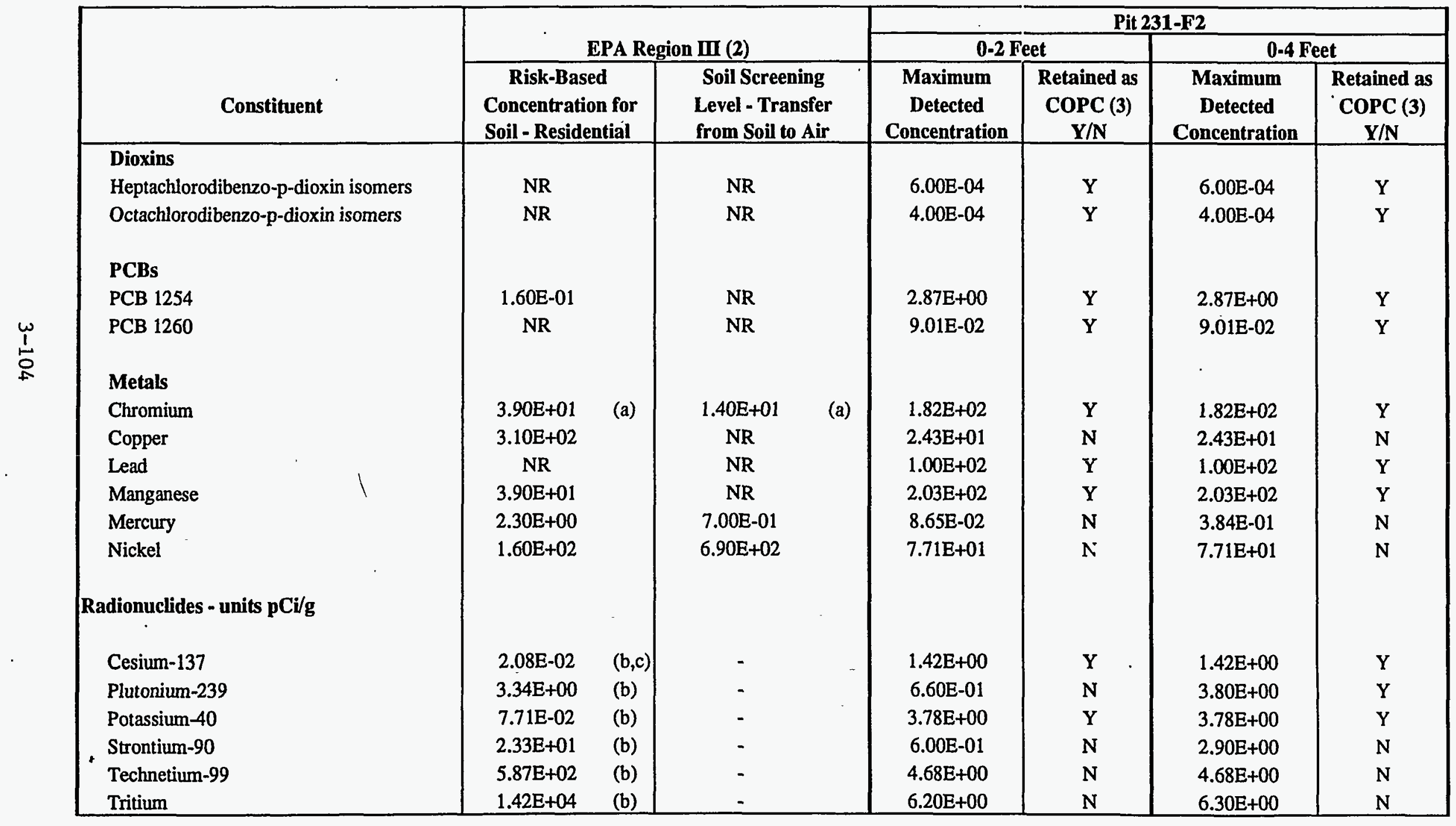


TABLE 3.16

COMPARISON OF SOIL ANALYTES TO RISK-BASED CONCENTRATIONS SAVANNAH RIVER SITE RUBBLE PIT 231-2F (1)

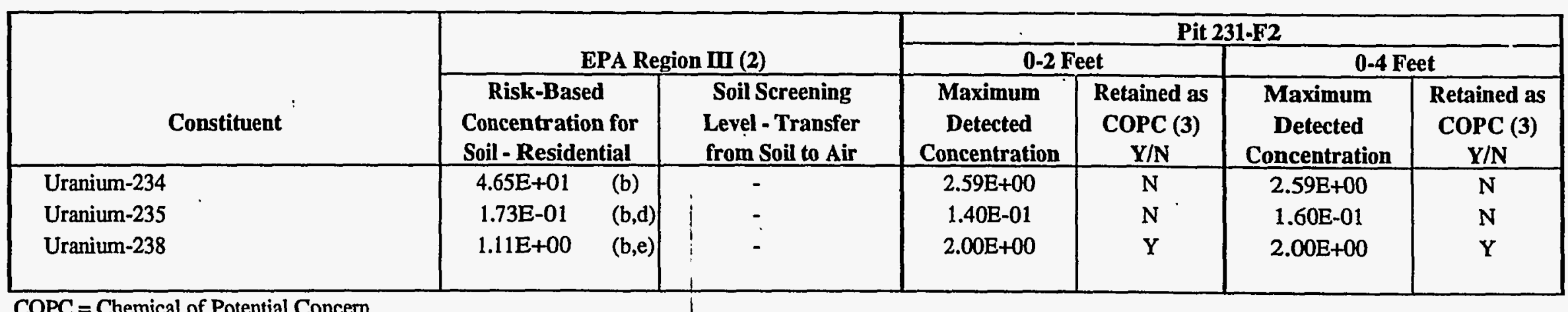

COPC $=$ Chemical of Potential Concern

(1) Comparisons were performed using in the following criteria: cancer risk of $1 E-06$ and Hazard Index of 0.1. NR = Not Reported

(2) EPA 1995, Risk-Based Concentration Table, July-December.

(3) Chemical was retained if the maximum concentration exceeded the Risk-Based Concentration (RBC) or Soil Screening Level. If a RBC and a Soil Screening Level were not available, the chemical was retained.

(a) RBC for chromium VI

(b) Screening levels for radionuclides were derived using the Preliminary Remediation Goals (PRGs) equations for radionuclides in RAGS, Part $B$.

(c) Value for CS-137 and decay chain products

(d) Value for U-235 and decay chain products

(e) Value for U-238 and decay chain products 
TABLE 3.17

COMPARISON OF GROUNDWATER ANALYTES TO RISK-BASED CONCENTRATIONS (1) SAVANNAH RIVER SITE F-AREA BURNING/RUBBLE PITS AND RUBBLE PIT

\begin{tabular}{|c|c|c|c|}
\hline Constituent & $\begin{array}{c}\text { Maximum } \\
\text { Detected } \\
\text { Concentration }\end{array}$ & $\begin{array}{c}\text { EPA Region III } \\
\text { Risk-Based } \\
\text { Concentration } \\
\text { for Tap Water }\end{array}$ & $\begin{array}{c}\text { Retained as } \\
\text { COPC } \\
\text { Y/N (a). }\end{array}$ \\
\hline \multicolumn{4}{|l|}{ Chemcial - units in (mg/L) } \\
\hline \multicolumn{4}{|l|}{ Volatiles } \\
\hline Acetone & $6.44 \mathrm{E}-03$ & $3.70 \mathrm{E}+00$. & $\mathbf{N}$ \\
\hline Carbon tetrachloride & 5.41E-03 & $1.60 \mathrm{E}-04$ & $\mathrm{Y}$ \\
\hline Chloroform & $1.00 \mathrm{E}-03$ & $1.50 \mathrm{E}-04$ & $\mathbf{Y}$ \\
\hline Chloromethane (Methyl Chloride). & $4.20 \mathrm{E}-03$ & $1.40 \mathrm{E}-03$ & $\mathrm{Y}$ \\
\hline Dichloromethane (Methylene chloride) & 3.02E-02 & $4.10 \mathrm{E}-03$ & $\mathrm{Y}$ \\
\hline Tetrachloroethylene & $3.28 \mathrm{E}-02$ & $1.10 \mathrm{E}-03$ & $\mathrm{Y}$ \\
\hline Trichloroethylene & 3.34E-02 & $1.60 \mathrm{E}-03$ & $\mathrm{Y}$ \\
\hline Trichlorofluoromethane & $1.05 \mathrm{E}-01$ & $1.30 \mathrm{E}+00$ & $\mathbf{N}$ \\
\hline \multicolumn{4}{|l|}{ Semivolatiles } \\
\hline Di-n-butyl phthalate & $2.20 \mathrm{E}-03$ & $3.70 \mathrm{E}+00$ & $\mathbf{N}$ \\
\hline \multicolumn{4}{|l|}{ Metals } \\
\hline Aluminum & $7.08 \mathrm{E}+00$ & $3.70 \mathrm{E}+01$ & $\mathbf{N}$ \\
\hline Arsenic & $2.50 \mathrm{E}-03$ & $4.50 \mathrm{E}-05$ & $\mathrm{Y}$ \\
\hline Barium & $1.01 \mathrm{E}-01$ & $2.60 \mathrm{E}+00$ & $\mathbf{N}$ \\
\hline Cadmium & $2.00 \mathrm{E}-03$ & $1.80 \mathrm{E}-02$ & $\mathbf{N}$ \\
\hline Chromium & $9.22 \mathrm{E}-02$ & $1.80 \mathrm{E}-01$ & $\mathbf{N}$ \\
\hline Iron & $2.82 \mathrm{E}+01$ & $1.10 E+01$ & $\mathrm{Y}$ \\
\hline Lead & $1.14 \mathrm{E}-02$ & NR & $\mathrm{Y}$ \\
\hline Manganese & 4.53E-01 & $1.80 \mathrm{E}-01$ & $\mathrm{Y}$ \\
\hline Nitrate (as nitrogen) & $2.40 \mathrm{E}+00$ & $5.80 \mathrm{E}+01$ & $\mathbf{N}$ \\
\hline \multicolumn{4}{|l|}{ Radionuclides - units in $\mathrm{pCi} / \mathrm{L}$} \\
\hline Radium-226 & $1.70 \mathrm{E}+00$ & 4.18E-03 (b) & $\mathbf{Y}$ \\
\hline Radium-228 & $2.70 \mathrm{E}+00$ & $1.84 \mathrm{E}-02(\mathrm{~b})$ & $\mathrm{Y}$ \\
\hline Strontium-90 & $1.56 \mathrm{E}+01$ & 1.77E-01 (b) & $\mathbf{Y}$ \\
\hline Tritium & $5.40 \mathrm{E}+03$ & $1.37 \mathrm{E}+01(\mathrm{~b})$ & $\mathbf{N}$ \\
\hline Uranium-238 & $1.69 \mathrm{E}+00$ & $2.44 \mathrm{E}-04(\mathrm{~b})$ & $\mathrm{Y}$ \\
\hline
\end{tabular}

COPC $=$ Chemical of Potential Concern

(1) Comparisons were performed using the following criteria: cancer risk of 1E-06 and Hazard Index of 0.1.

(a) If Risk-Based Concentration was unavailable, the chemical was retained.

(b) Value derived using USEPA guidance cited in RAGS, Part B for radionuclide PRGs. 
TABLE 3.18

ESSENTLAL NUTRIENT INFORMATION

SAVANNAH RIVER SITE

F-AREA - BURNING/RUBBLE PITS AND RUBBLE PIT

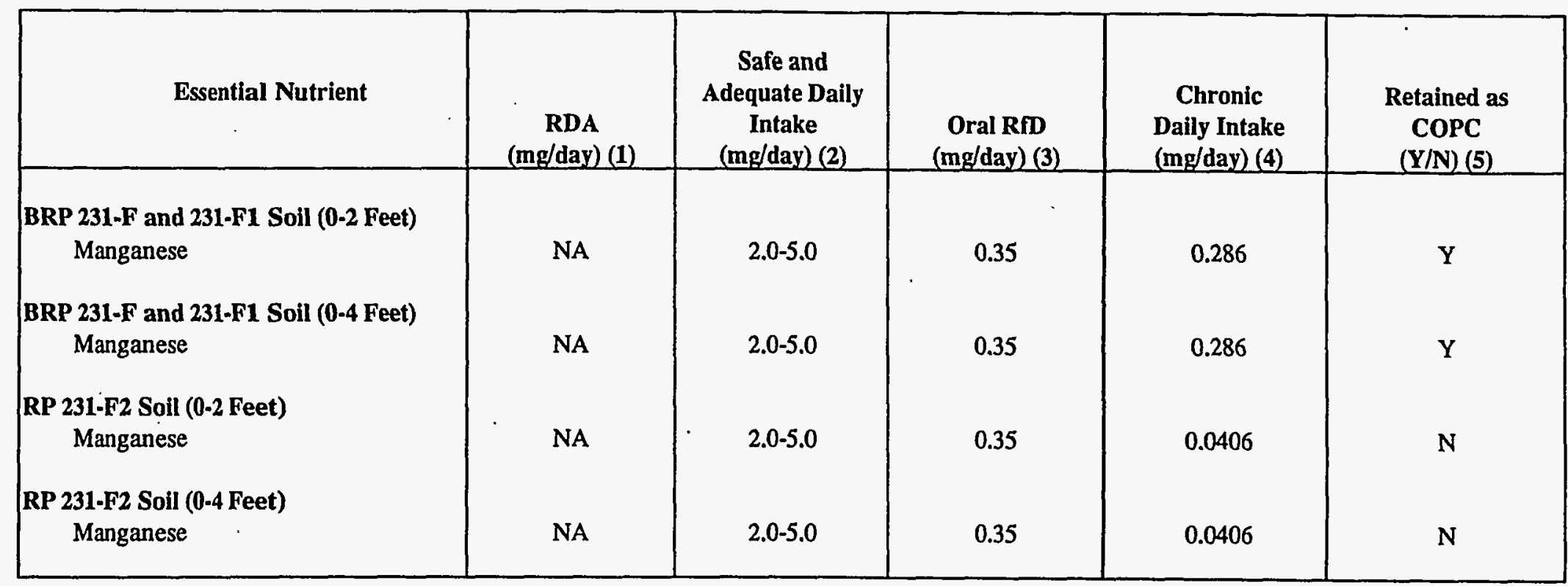

COPC $=$ Chemical of Potential Concern

BRP-Burning Rubble Pit

RP - Rubble Pit

NA - Not Available

(1) Recommended Daily Allowances (National Academy of Sciences [NAS], 1989)

(2) Safe and Adequate Daily Intake (NAS, 1989)

(3) Oral RfD reported in units of $\mathrm{mg} / \mathrm{kg}$-day from IRIS (EPA, 1995). Converted to a dose of $\mathrm{mg} /$ day.

(4) Chronic Daily Intake derived by multiplying the maximum concentration in soil ( $\mathrm{mg} / \mathrm{kg}$ ) by an intake value of $0.0002 \mathrm{~kg} /$ day $(200 \mathrm{mg} /$ day for a child).

(5) Nutrients with no available RfD were retained if the CDI was less than the RDA or SADI.

For nutrients with an RfD, the nutrient was eliminated if the CDI was below the maximum RDA or SADI and the RDA or SADI were one-half or less of the RfD. If the CDI was below the RDA or SADI and the RDA or SADI was greater than one-half the RfD, the screening level was set at one-half the RfD. 
TABLE 3.19

COPC SELECTION IN GROUNDWATER, ESSENTIAL NUTRIENT INFORMATION

SAVANNAH RIVER SITE

F-AREA BURNING/RUBBLE PITS AND RUBBLE PIT

\begin{tabular}{|c|c|c|c|c|c|}
\hline $\begin{array}{c}\text { Essential Nutrient } \\
:\end{array}$ & $\begin{array}{c}\text { RDA } \\
\text { (mg/day) (1) }\end{array}$ & $\begin{array}{c}\text { Safe and } \\
\text { Adequate Daily } \\
\text { Intake } \\
\text { (mg/day) (2) }\end{array}$ & $\begin{array}{c}\text { Oral RfD } \\
\text { (mg/day) (3) }\end{array}$ & $\begin{array}{c}\text { Chronic } \\
\text { Daily Intake } \\
\text { (mg/day) (4) }\end{array}$ & $\begin{array}{c}\text { Retained in } \\
\text { Risk Assessment } \\
(\mathbf{Y} / \mathbf{N})(5) \\
\end{array}$ \\
\hline $\begin{array}{l}\text { Calcium } \\
\text { Iron } \\
\text { Magnesium } \\
\text { Manganese } \\
\text { Potassium } \\
\text { Sodium } \\
\text { Total phosphates (as P) }\end{array}$ & $\begin{array}{c}800-1,200 \\
10-15 \\
280-400 \\
\text { NA } \\
\text { NA } \\
\text { NA } \\
\text { NA } \\
\end{array}$ & $\begin{array}{c}\text { NA } \\
\text { NA } \\
\text { NA } \\
2-5 \\
1,875-5,625 \\
1,100-3,300 \\
800-1,200 \\
\end{array}$ & $\begin{array}{l}\text { NA } \\
\text { NA } \\
\text { NA } \\
0.35 \\
\text { NA } \\
\text { NA } \\
\text { NA } \\
\end{array}$ & $\begin{array}{c}90.8 \\
56.4 \\
4.52 \\
0.906 \\
4.34 \\
36.2 \\
3.06 \\
\end{array}$ & $\begin{array}{l}\mathrm{N} \\
\mathrm{Y} \\
\mathrm{N} \\
\mathrm{Y} \\
\mathrm{N} \\
\mathrm{N} \\
\mathrm{N} \\
\end{array}$ \\
\hline
\end{tabular}

(1) Recommended Daily Allowances (National Academy of Sciences [NAS], 1989)

(2) Safe and Adequate Daily Intake (NAS, 1989)

(3) Oral RfD reported in units of $\mathrm{mg} / \mathrm{kg}$-day from IRIS (USEPA, 1995). Converted to a dose of $\mathrm{mg} /$ day.

(4) Chronic Daily Intake derived by multiplying the maximum concentration in groundwater $(\mathrm{mg} / \mathrm{L}$ ) by an intake value of $2 \mathrm{~L} /$ day.

(5) Nutrients with no available RfD were retained if the CDI was less than the RDA or SADI.

For nutrients with an RfD, the nutrient was eliminated if the CDI was below the maximum RDA or SADI and the RDA or SADI were one-half or less of the RfD. If the CDI was below the RDA or SADI and the RDA or SADI was greater than one-half the RfD, the screening level was set at one-half the RfD. 
TABLE 3.20

FREQUENCY OF DETECTION IN SOIL (1)

SAVANNAH RIVER SITE

BURNING/RUBBLE PITS 231-F AND 231-1F

\begin{tabular}{|c|c|c|}
\hline \multirow[b]{3}{*}{ Constituent } & \multicolumn{2}{|c|}{ Pits 231-F and 231-1F } \\
\hline & \multicolumn{2}{|c|}{ 0-4 Feet } \\
\hline & $\begin{array}{l}\text { Frequency } \\
\text { of Detection }\end{array}$ & $\begin{array}{c}\text { Analvte Frequency of } \\
\text { Dectection is More Than 5\% } \\
\text { Y/N }\end{array}$ \\
\hline \multicolumn{3}{|l|}{ Chemicals } \\
\hline \multicolumn{3}{|l|}{ Semivolatiles } \\
\hline 2-Methylnaphthalene & $2 / 16$ & $\mathbf{Y}$ \\
\hline Acenaphthylene & $4 / 16$ & $\mathbf{Y}$ \\
\hline Benzo(a)anthracene & $14 / 16$ & $\mathbf{Y}$ \\
\hline Benzo(a)pyrene & $14 / 16$ & $\mathbf{Y}$ \\
\hline Benzo(b)fluoranthene & $14 / 16$ & $\mathrm{Y}$ \\
\hline Benzo(g,h,i)perylene & $12 / 16$ & $Y$ \\
\hline Dibenz(a,h)anthracene & $6 / 16$ & $\mathbf{Y}$ \\
\hline Dibenzofuran & $1 / 16$ & $\mathbf{Y}$ \\
\hline Fluoranthene & $14 / 16$ & $\mathrm{Y}^{\circ}$ \\
\hline Phenanthrene & $12 / 16$ & $\hat{Y}$ \\
\hline \multicolumn{3}{|l|}{ Dioxins/Furans } \\
\hline Heptachlorodibenzo-p-dioxin isomers & $10 / 16$ & $\mathbf{Y}$ \\
\hline Heptachlorodibenzo-p-furan isomers & $6 / 16$ & $\mathbf{Y}$ \\
\hline Hexachlorodibenzo-p-furan isomers & $1 / 16$ & $\mathrm{Y}$ \\
\hline Octachlorodibenzo-p-dioxin isomers & $15 / 16$ & $\mathbf{Y}$ \\
\hline Octachlorodibenzo-p-furan isomers & $6 / 16$ & $\mathbf{Y}$ \\
\hline \multicolumn{3}{|l|}{ PCBs } \\
\hline РCB 1254 & $8 / 16$ & $\mathbf{Y}$ \\
\hline \multicolumn{3}{|l|}{ Pesticides } \\
\hline delta-Benzene hexachloride & $1 / 16$ & $\mathbf{Y}$ \\
\hline Endrin ketone & $1 / 16$ & $\mathbf{Y}$ \\
\hline \multicolumn{3}{|l|}{ Metals } \\
\hline Arsenic & $24 / 24$ & $\mathbf{Y}$ \\
\hline Chromium & $24 / 24$ & $\mathrm{Y}$ \\
\hline Lead & $24 / 24$ & $\mathbf{Y}$ \\
\hline Manganese & $24 / 24$ & $\mathbf{Y}$ \\
\hline Mercury & $9 / 24$ & $\mathrm{Y}$ \\
\hline Nickel & $16 / 24$ & $\mathbf{Y}$ \\
\hline \multicolumn{3}{|l|}{ Radionuclides } \\
\hline Cesium-137 & $15 / 16$ & $\mathbf{Y}$ \\
\hline Potassium- 40 & $15 / 16$ & $\mathrm{Y}$ \\
\hline Tritium & $14 / 24$ & $\mathbf{Y}$ \\
\hline Uranium-238 & $16 / 16$ & $\mathbf{Y}$ \\
\hline
\end{tabular}

(1) A minimum of 20 samples is needed to screen for frequency of detection. Consequently, the frequency of detection screening was only performed for 0-4 feet in Pits 231-F and 231-F1. 
TABLE 3.21

GROUNDWATER EXPOSURE POINT CONCENTRATIONS

FROM GROUNDWATER MODELING

SAVANNAH RIVER SITE

BURNING/RUBBLE PITS 231F-2311F and RUBBLE PIT 231-2F

\begin{tabular}{|l|c|}
\multicolumn{1}{|c|}{ Constituent } & \multicolumn{1}{|c|}{$\begin{array}{c}\text { Maximum } \\
\text { Exposure Point } \\
\text { Concentration (1) }\end{array}$} \\
\hline Chemical - units in (mg/L) & \\
Volatiles & \\
1,2-Dichloroethylene & $4.47 \mathrm{E}-06$ \\
Dichloromethane & $3.78 \mathrm{E}-03$ \\
Tetrachloroethylene & $7.06 \mathrm{E}-04$ \\
Pesticides & \\
delta-BHC & \\
Heptachlor epoxide & $2.36 \mathrm{E}-04$ \\
Metals & $1.85 \mathrm{E}-06$ \\
Cadmium & \\
& \\
Radionuclides - units in pCi/L & $4.83 \mathrm{E}-03$ \\
Iodine-129 & \\
Technetium-99 & \\
Tritium & $7.29 \mathrm{E}+00$ \\
& $2.36 \mathrm{E}+02$ \\
\hline
\end{tabular}

(1) See Appendix B for soil leachability to groundwater modeling details. 
TABLE 322

CHEMICALS OF POTENTUL CONCERN

SAVANNAH RIVER STTE

BURNING RUBBLE PITS - F AREA

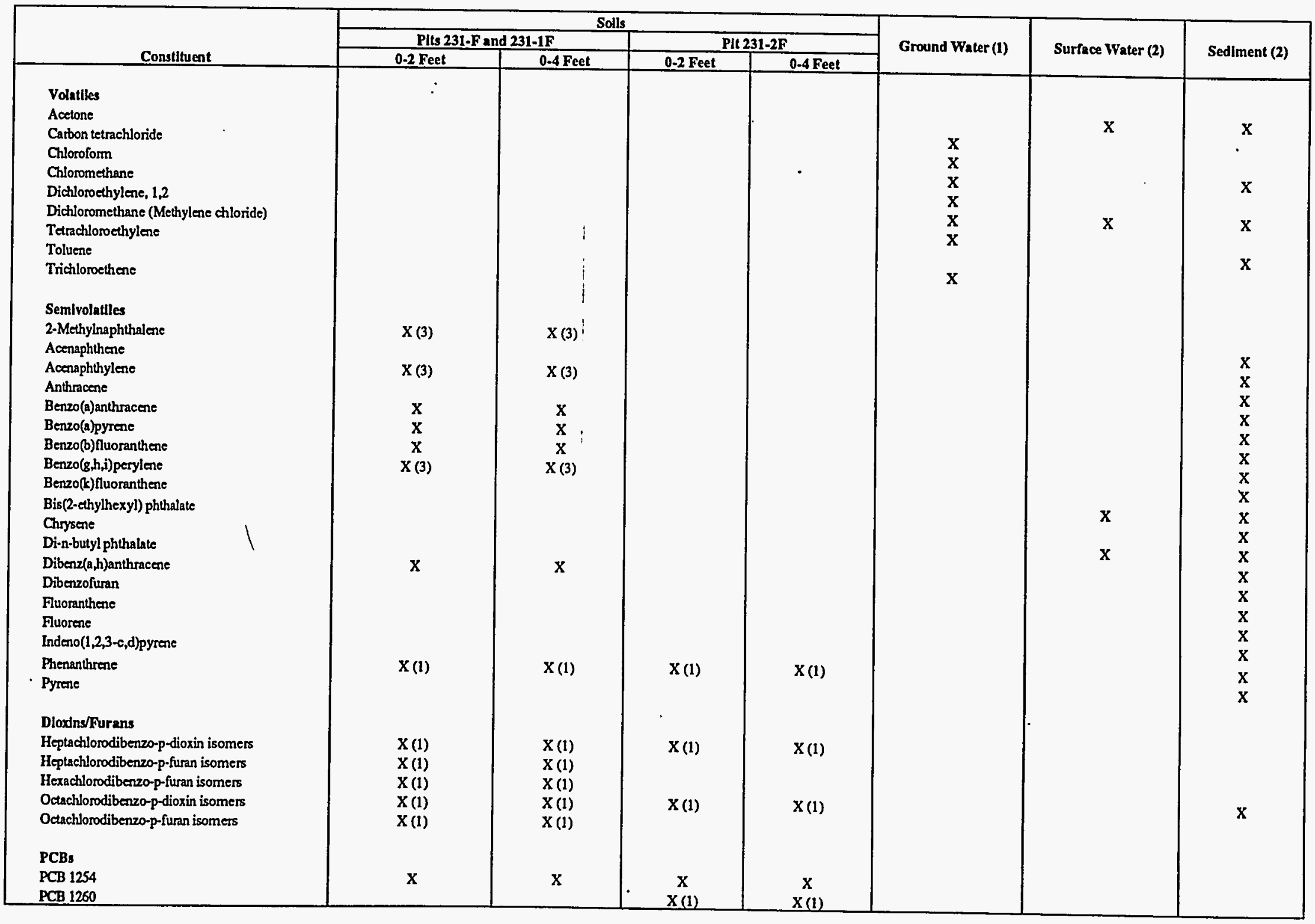


CHEMICALS OF POTENTIAL CONCERN

SAVANNAH RIVER STTE

BURNING RUBBLE PITS - F AREA

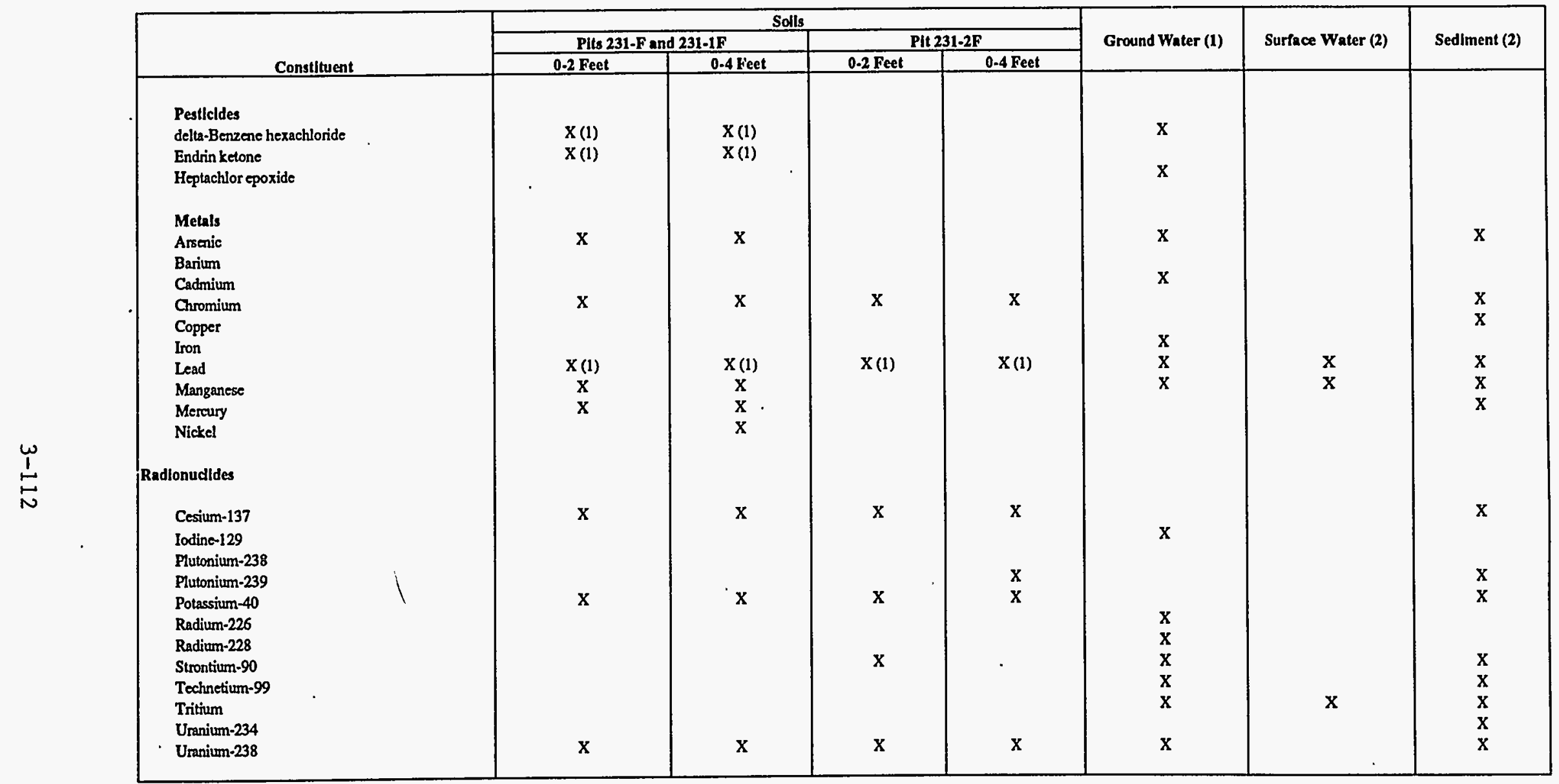

\section{COPC $=$ Chemical of Potential Concem}

(1) Risk-based screening was not performed. Risk assessment was based on modeled chemical concentrations.

(2) Human risk-based scroening level was not available for this chemical or media.

(3) No risk-based concentration was available. 
TABLE 3.23

MODELED EXPOSURE POINT CONCENTRATIONS

OF SOIL PARTICULATES IN AIR SAVANNAH RIVER SITE

BURNING/RUBBLE PITS 231-F AND 231-1F

\begin{tabular}{|c|c|c|c|c|c|c|}
\hline \multirow[b]{2}{*}{ CAS \# } & \multirow[b]{2}{*}{ Constituent } & \multicolumn{2}{|c|}{$\begin{array}{c}\text { EPC in Soil } \\
(\mathrm{mg} / \mathrm{kg})\end{array}$} & \multicolumn{3}{|c|}{$\begin{array}{c}\text { EPC in } \operatorname{Air}^{\star} \\
\left(\mathrm{mg} / \mathrm{m}^{3}\right)\end{array}$} \\
\hline & & $0-2^{\prime}$ bls & $0-4^{\prime}$ bls & $\begin{array}{l}\text { Current } \\
\text { Visitor } \\
\text { 0-2' bls } \\
\end{array}$ & $\begin{array}{c}\text { Future Worker } \\
\text { and Resident } \\
0-2^{\prime} \text { bls } \\
\end{array}$ & $\begin{array}{c}\text { Future Worker } \\
\text { and Resident } \\
0-4^{\prime} \text { bls } \\
\end{array}$ \\
\hline & Semivolatiles & & & & & \\
\hline $91-57-6$ & 2-Methylnaphthalene & $7.69 \mathrm{E}-02$ & $7.69 \mathrm{E}-02$ & $3.56 \mathrm{E}-11$ & 3.53E-11 & 3.53E-11 \\
\hline $208-96-8$ & Acenaphthalylene & 8.61E-02 & 8.61E-02 & $3.99 \mathrm{E}-11$ & $3.95 \mathrm{E}-11$ & $3.95 \mathrm{E}-11$ \\
\hline $56-55-3$ & Benzo(a)anthracene & $9.26 \mathrm{E}-01$ & $9.26 \mathrm{E}-01$ & $4.29 \mathrm{E}-10$ & $4.25 \mathrm{E}-10$ & $4.25 \mathrm{E}-10$ \\
\hline $50-32-8$ & Benzo(a)pyrene & $6.49 \mathrm{E}-01$ & $6.49 \mathrm{E}-01$ & $3.00 \mathrm{E}-10$ & $2.98 \mathrm{E}-10$ & $2.98 \mathrm{E}-10$ \\
\hline $205-99-2$ & Benzo(b)fluoranthene & $1.01 \mathrm{E}+00$ & $1.01 E+00$ & $4.68 \mathrm{E}-10$ & $4.63 \mathrm{E}-10$ & $4.63 E-10$ \\
\hline $191-24-2$ & Benzo $(\mathrm{g}, \mathrm{h}, \mathrm{i})$ perylene & $6.15 \mathrm{E}-01$ & $6.15 \mathrm{E}-01$ & $2.85 \mathrm{E}-10$ & $2.82 \mathrm{E}-10$ & $2.82 \mathrm{E}-10$ \\
\hline $53-70-3$ & Dibenz(a,h)anthrancene & 2.17E-01 & $2.17 \mathrm{E}-01$ & $1.00 \mathrm{E}-10$ & $9.95 \mathrm{E}-11$ & $9.95 \mathrm{E}-11$ \\
\hline $85-01-8$ & $\begin{array}{l}\text { Phenanthrene } \\
\vdots \\
\text { Dioxins/Furans }\end{array}$ & $6.87 \mathrm{E}-01$ & $6.87 \mathrm{E}-01$ & $3.18 \mathrm{E}-10$ & $3.15 \mathrm{E}-10$ & 3.15E-10 \\
\hline $37871-00-4$ & Heptachlorodibenzo-p-dioxin isomer & 4.11E-03 & $4.11 \mathrm{E}-03$ & $1.90 \mathrm{E}-12$ & $1.89 \mathrm{E}-12$ & $1.89 \mathrm{E}-12$ \\
\hline $38998-75-3$ & Heptachlorodibenzo-p-furan isomer & $2.58 \mathrm{E}-04$ & $2.58 \mathrm{E}-04$ & $1.19 \mathrm{E}-13$ & $1.18 \mathrm{E}-13$ & $1.18 \mathrm{E}-13$ \\
\hline $55684-94-1$ & Hexachlorodibenzo-p-furan isomer & $1.00 \mathrm{E}-04$ & $1.00 \mathrm{E}-04$ & 4.63E-14 & $4.59 \mathrm{E}-14$ & $4.59 \mathrm{E}-14$ \\
\hline $3268-87-9$ & Octachlorodibenzo-p-dioxin isomer & $9.26 \mathrm{E}-03$ & $9.26 \mathrm{E}-03$ & $4.29 \mathrm{E}-12$ & $4.25 \mathrm{E}-12$ & $4.25 \mathrm{E}-12$ \\
\hline $39001-02-0$ & $\begin{array}{l}\text { Octachlorodibenzo-p-furan isomer } \\
\text { PCBs }\end{array}$ & $3.48 \mathrm{E}-04$ & $3.48 \mathrm{E}-04$ & $1.61 \mathrm{E}-13$ & $1.60 \mathrm{E}-13$ & $1.60 \mathrm{E}-13$ \\
\hline $11097-69-1$ & PCB 1254 & $1.78 \mathrm{E}-01$ & $1.78 \mathrm{E}-01$ & $8.24 \mathrm{E}-11$ & $8.17 \mathrm{E}-11$ & 8.17E-11 \\
\hline
\end{tabular}


TABLE 3.23

MODELED EXPOSURE POINT CONCENTRATIONS

OF SOIL PARTICULATES IN AIR

SAVANNAH RIVER SITE

BURNING/RUBBLE PITS 231-F AND 231-1F

\begin{tabular}{|c|c|c|c|c|c|c|}
\hline \multirow[b]{2}{*}{ CAS \# } & \multirow[b]{2}{*}{ Constituent } & \multicolumn{2}{|c|}{$\begin{array}{c}\text { EPC in Soil } \\
(\mathrm{mg} / \mathrm{kg})\end{array}$} & \multicolumn{3}{|c|}{$\begin{array}{c}\text { EPC in Air } \\
\left(\mathrm{mg} / \mathrm{m}^{3}\right)\end{array}$} \\
\hline & & 0-2' bls & $0-4^{\prime}$ bls & $\begin{array}{l}\text { Current } \\
\text { Visitor } \\
\text { 0-2' bls } \\
\end{array}$ & $\begin{array}{c}\text { Future Worker } \\
\text { and Resident } \\
\text { 0-2' bls }\end{array}$ & $\begin{array}{c}\text { Future Worker } \\
\text { and Resident } \\
0-4^{\prime} \text { bls }\end{array}$ \\
\hline & Pesticides & & & & & \\
\hline $319-86-8$ & delta-BHC & $1.84 \mathrm{E}-03$ & $1.84 \mathrm{E}-03$ & $8.52 \mathrm{E}-13$ & $8.44 \mathrm{E}-13$ & $8.44 \mathrm{E}-13$ \\
\hline $53494-70-5$ & $\begin{array}{l}\text { Endrin Ketone } \\
\text { Metals }\end{array}$ & $3.20 \mathrm{E}-03$ & $3.20 \mathrm{E}-03$ & $1.48 \mathrm{E}-12$ & $1.47 \mathrm{E}-12$ & $1.47 \mathrm{E}-12$ \\
\hline $7440-38-2$ & $\overline{\text { Arsenic }}$ & $4.82 E+00$ & $5.29 \mathrm{E}+00$ & $2.23 \mathrm{E}-09$ & $2.21 \mathrm{E}-09$ & 2.43E-09 \\
\hline $7440-47-3$ & Chromium & $4.42 \mathrm{E}+01$ & $7.65 \mathrm{E}+01$ & $2.05 \mathrm{E}-08$ & $2.03 \mathrm{E}-08$ & 3.51E-08 \\
\hline $7439-92-1$ & Lead & $2.48 \mathrm{E}+01$ & $2.62 \mathrm{E}+01$ & $1.15 \mathrm{E}-08$ & $1.14 \mathrm{E}-08$ & $1.20 \mathrm{E}-08$ \\
\hline $7439-96-5$ & Manganese & $4.78 \mathrm{E}+02$ & $3.61 E+02$ & 2.21E-07 & 2.19E-07 & 1.66E-07 \\
\hline $7439-97-6$ & Mercury & $5.48 \mathrm{E}-01$ & $3.58 \mathrm{E}-01$ & $2.54 \mathrm{E}-10$ & $2.51 \mathrm{E}-10$ & $1.64 \mathrm{E}-10$ \\
\hline
\end{tabular}

* Exposure Point Concentration (EPC) in air was derived by dividing the EPC in soil by the following particulate emission factors: current visitor 0-2'bls $2.16 \mathrm{E}+09$ and future scenarios $0-2$ ' and $0-4 '$ bls $2.18 \mathrm{E}+09$. 
TABLE 3.24

MODELED EXPOSURE POINT CONCENTRATIONS

OF SOIL PARTICULATES IN AIR

SAVANNAB RIVER SITE

RUBBLE PIT 231-2F

\begin{tabular}{|c|c|c|c|c|c|c|}
\hline \multirow[b]{2}{*}{ CAS \# } & \multirow[b]{2}{*}{ Constituent } & \multicolumn{2}{|c|}{$\begin{array}{c}\text { EPC } \\
(\mathrm{mg} / \mathrm{kg})\end{array}$} & \multicolumn{3}{|c|}{$\begin{array}{c}\text { EPC in Air * } \\
\left(\mathbf{m g} / \mathbf{m}^{3}\right) \\
\end{array}$} \\
\hline & & $0-2^{\prime}$ bls & 0-4' bls & $\begin{array}{l}\text { Current } \\
0-2^{\prime} \text { bls } \\
\end{array}$ & $\begin{array}{l}\text { Future } \\
\text { 0-2' bls }\end{array}$ & $\begin{array}{l}\text { Future } \\
0-4^{\prime} \text { bls }\end{array}$ \\
\hline $85-01-8$ & $\begin{array}{l}\frac{\text { Semivolatiles }}{\text { Phenanthrene }} \\
\text { Dioxins }\end{array}$ & 8.68E-02 & 8.68E-02 & $2.70 \mathrm{E}-11$ & $1.28 \mathrm{E}-11$ & $1.28 \mathrm{E}-11$ \\
\hline $37871-00-4$ & Heptachlorodibenzo-p-dioxin isomer & $6.00 \mathrm{E}-04$ & 4.75E-04 & $1.86 \mathrm{E}-13$ & 7.03E-14 & $7.03 \mathrm{E}-14$ \\
\hline $3268-87-9$ & $\begin{array}{l}\text { Octachlorodibenzo-p-dioxin isomer } \\
\text { PCBs }\end{array}$ & $4.00 \mathrm{E}-04$ & $4.00 \mathrm{E}-04$ & $1.24 \mathrm{E}-13$ & $5.92 \mathrm{E}-14$ & $5.92 \mathrm{E}-14$ \\
\hline $11097-69-1$ & $\overline{\mathrm{PCB}} 1254$ & 2.87E+00 & $2.87 \mathrm{E}+00$ & 8.91E-10 & 4.25E-10 & $4.25 \mathrm{E}-10$ \\
\hline $11096-82-5$ & $\begin{array}{l}\text { PCB } 1260 \\
\text { Metals }\end{array}$ & $9.01 E-02$ & $9.01 \mathrm{E}-02$ & $2.80 \mathrm{E}-11$ & $1.33 \mathrm{E}-11$ & $1.33 \mathrm{E}-11$ \\
\hline $7440-47-3$ & $\overline{\text { Chromium }}$ & $1.82 E+02$ & $5.51 \mathrm{E}+01$ & $5.65 \mathrm{E}-08$ & 8.15E-09 & 8.15E-09 \\
\hline $743.9-92-1$ & Lead & $9.00 \mathrm{E}+01$ & $6.20 \mathrm{E}+01$ & $2.80 \mathrm{E}-08$ & $9.17 \mathrm{E}-09$ & 9.17E-09 \\
\hline $7439-97-6$ & Mercury & ND & $1.23 \mathrm{E}-01$ & - & - & $1.82 \mathrm{E}-11$ \\
\hline
\end{tabular}

ND - Not Detected

* Exposure Point Concentration (EPC) in air was derived by dividing the EPC

in soil by the following particulate emission factors: current visitor $0-2$ 'bls $3.22 \mathrm{E}+09$, and future scenarios $0-2^{\prime}$ bls and $0-4$ 'bls $6.76 \mathrm{E}+09$ 
TABLE 3.25

MODELED EXPOSURE POINT CONCENTRATIONS

OF VOLATILE SOIL CONTAMINANTS

SAVANNAH RIVER SITE

BURNING/RUBBLE PITS 231F AND 231-1F

\begin{tabular}{|c|c|c|c|c|c|c|c|c|c|}
\hline \multirow[b]{2}{*}{ CAS\# } & \multirow[b]{2}{*}{ Constltuent } & \multicolumn{2}{|c|}{$\begin{array}{c}\text { EPC In Soll } \\
(\mathrm{mg} / \mathrm{Kg})\end{array}$} & \multicolumn{3}{|c|}{ Volatillzation Factor } & \multicolumn{3}{|c|}{$\begin{array}{c}\text { EPC In Alr* } \\
\left(\mathrm{m} / \mathrm{m}^{3}\right)\end{array}$} \\
\hline & & $0-2^{\prime}$ bls & $0.4^{\prime}$ bls & $\begin{array}{l}\text { Current } \\
\text { VIsitor } \\
0-2 \text { bls } \\
\end{array}$ & $\begin{array}{c}\text { Future Worker } \\
\text { and Resident } \\
0-2^{\prime} \text { bls } \\
\end{array}$ & $\begin{array}{c}\text { Future Worker } \\
\text { and Resident } \\
0-4^{\prime} \text { bls } \\
\end{array}$ & $\begin{array}{l}\text { Current } \\
\text { Visitor } \\
0-2^{\prime} \text { bls } \\
\end{array}$ & $\begin{array}{c}\text { Future Worker } \\
\text { and Resldent } \\
0-2 \cdot \text { bls } \\
\end{array}$ & $\begin{array}{c}\text { Future Worker } \\
\text { and Resident } \\
0-4^{\prime} \text { bls } \\
\end{array}$ \\
\hline $\begin{array}{c}91-57-6 \\
208-96-8 \\
85-01-8\end{array}$ & $\begin{array}{l}\text { Semivolatlles } \\
\text { 2-Methylnaphthalene } \\
\text { Acenaphthylene } \\
\text { Phenanthrene }\end{array}$ & $\begin{array}{l}7.69 \mathrm{E}-02 \\
8.61 \mathrm{E}-02 \\
6.87 \mathrm{E}-01\end{array}$ & $\begin{array}{l}7.69 \mathrm{E}-02 \\
8.61 \mathrm{E}-02 \\
6.87 \mathrm{E}-01\end{array}$ & $\begin{array}{l}1.55 \mathrm{E}+04 \\
9.34 \mathrm{E}+03 \\
7.61 \mathrm{E}+04\end{array}$ & $\begin{array}{l}1.67 \mathrm{E}+00 \\
1.29 \mathrm{E}+05 \\
1.27 \mathrm{E}+06\end{array}$ & $\begin{array}{l}1.82 \mathrm{E}+00 \\
1.42 \mathrm{E}+05 \\
1.39 \mathrm{E}+06\end{array}$ & $\begin{array}{l}4.96 \mathrm{E}-06 \\
9.22 \mathrm{E}-06 \\
9.03 \mathrm{E}-06\end{array}$ & $\begin{array}{l}4.60 \mathrm{E}-02 \\
6.67 \mathrm{E}-07 \\
5.41 \mathrm{E}-07\end{array}$ & $\begin{array}{l}4.60 \mathrm{E}-02 \\
6.67 \mathrm{E}-07 \\
5.41 \mathrm{E}-07\end{array}$ \\
\hline
\end{tabular}

* Exposure Point Concentration in air (EPC) was derived by dividing the EPC

in soil by the volatilization factor. 
TABLE 3.26

MODELED EXPOSURE POINT CONCENTRATICNS

OF VOLATILE SOIL CONTAMINANTS

SAVANNAH RIVER SITE

RUBBLE PIT 231-2F

\begin{tabular}{|c|c|c|c|c|c|c|c|c|c|c|c|c|c|}
\hline \multirow[b]{2}{*}{ CAS \# } & \multirow[b]{2}{*}{ Constituent } & \multicolumn{2}{|c|}{$\begin{array}{c}\text { EPC in Soll } \\
(\mathrm{mg} / \mathrm{kg})\end{array}$} & \multicolumn{5}{|c|}{$\begin{array}{c}\begin{array}{c}\text { Volatilization Factor } \\
\left(\mathrm{m}^{3} / \mathrm{kg}\right)\end{array} \\
\end{array}$} & \multicolumn{5}{|c|}{$\begin{array}{c}\text { EPC In Air * } \\
\left(\mathrm{mg} / \mathrm{m}^{3}\right)\end{array}$} \\
\hline & & $0-2^{\prime}$ bls & $0.4^{\prime}$ bls & $\begin{array}{l}\text { Current } \\
\text { Vlsitor } \\
0.2^{\prime} \text { bls }\end{array}$ & $\begin{array}{l}\text { Future } \\
\text { Worker } \\
\text { 0-2' bls }\end{array}$ & $\begin{array}{l}\text { Future } \\
\text { Resident } \\
0-2^{\prime} \text { bls }\end{array}$ & $\begin{array}{l}\text { Future } \\
\text { Worker } \\
0.4^{\prime} \text { bis }\end{array}$ & $\begin{array}{l}\text { Future } \\
\text { Resident } \\
0-4^{\prime} \text { bls }\end{array}$ & $\begin{array}{l}\text { Current } \\
\text { Visitor } \\
0.2^{\prime} \text { bls }\end{array}$ & $\begin{array}{l}\text { Future } \\
\text { Worker } \\
0.21 \text { bls }\end{array}$ & $\begin{array}{c}\text { Future } \\
\text { Resident } \\
0-2^{\prime} \text { bls }\end{array}$ & $\begin{array}{l}\text { Future } \\
\text { Worker } \\
0-d^{\prime} \text { bls }\end{array}$ & $\begin{array}{c}\text { Future } \\
\text { Resident } \\
0-4^{\prime} \text { bls }\end{array}$ \\
\hline $85-01-8$ & $\begin{array}{l}\text { Semivolatille } \\
\text { Phenanthrene }\end{array}$ & $2.90 \mathrm{E}+00$ & $6.87 \mathrm{E}-01$ & $5.66 \mathrm{E}+04$ & $1.27 E+06$ & $3.93 E+06$ & $1.27 \mathrm{E}+06$ & $3.93 \mathrm{E}+06$ & 5.12E-05 & $2.28 \mathrm{E}-06$ & 7.38E-07 & 5.41E-07 & $1.75 \mathrm{E}-07$ \\
\hline
\end{tabular}

$\stackrel{\omega}{1}$

* Exposure Point Concentration in air (EPC) was derived by dividing the EPC

in soil by the volatilization factor. 
TABLE 3.27

RME HUMAN EXPOSURE PARAMETERS

SAVANNAH RIVER SITE

F AREA - BURNING/RUBBLE PITS

\begin{tabular}{|c|c|c|c|c|c|c|c|c|c|c|c|c|c|c|c|}
\hline \multirow[t]{2}{*}{$\begin{array}{l}\text { Fxposure } \\
\text { Assumptions }\end{array}$} & \multirow[t]{2}{*}{ Unit } & \multicolumn{3}{|c|}{$\begin{array}{l}\text { Current On-Unit } \\
\text { Visitor }\end{array}$} & \multicolumn{3}{|c|}{$\begin{array}{l}\text { Future On-Unit } \\
\text { Resident - Adult }\end{array}$} & \multicolumn{5}{|c|}{$\begin{array}{c}\text { Future On-Unit } \\
\text { Resident - Child (a) }\end{array}$} & \multicolumn{2}{|c|}{$\begin{array}{l}\text { Future On-Unit } \\
\text { Industrial Worker }\end{array}$} & \multirow[t]{2}{*}{ Reference } \\
\hline & & Soil & SW & Sed & Soll & GW & Veg & Soil & GW & Veg & SW & Sed & Soil & $\mathbf{G W}$ & \\
\hline $\begin{array}{l}\text { Body Weight } \\
\text { (BW) }\end{array}$ & $\mathrm{K}_{\mathrm{B}}$ & 70 & 70 & 70 & 70 & 70 & 70 & 15 & 15 & 15 & 45 & 45 & 70 & 70 & (2) \\
\hline $\begin{array}{l}\text { Exposure } \\
\text { Duration (ED) }\end{array}$ & $\mathrm{yr}$ & 5 & 5 & 5 & 24 & 24 & 24 & 6 & 6 & 6 & 10 & 10 & 25 & 25 & $\begin{array}{l}\text { Resident and } \\
\text { Worker }(1)(2) \\
\text { Visitor - Site- } \\
\text { Specific }\end{array}$ \\
\hline $\begin{array}{l}\text { Exposure } \\
\text { Frequency (EF) }\end{array}$ & $d / y r$ & 6 & 6 & 6 & 350 & 350 & 350 & 350 & 350 & 350 & 50 & 39 & 250 & 250 & $\begin{array}{l}\text { Resident and } \\
\text { Worker (3) } \\
\text { Visitor - Site- } \\
\text { Specific }\end{array}$ \\
\hline $\begin{array}{l}\text { Exposure Time } \\
\text { (ET) }\end{array}$ & $\mathrm{hr} / \mathrm{d}$ & . & 0.5 & NA & 15 & $0.20(\mathrm{~b})$ & NA & 18 & 0.20 (b) & NA & 2 & NA & 1 & 0.2 & $\begin{array}{l}\text { Resident (2) } \\
\text { Visitor and } \\
\text { Worker - Site- } \\
\text { Specific } \\
\end{array}$ \\
\hline $\begin{array}{l}\text { Soil to Skin } \\
\text { Adherence } \\
\text { Factor (AF) } \\
\end{array}$ & $\mathrm{mg} / \mathrm{cm}^{2}$ & 0.2 & NA & 0.6 & 0.2 & NA & NA & 0.2 & NA & NA & NA & 0.6 & 0.2 & $\mathrm{NA}$ & $(4)$ \\
\hline $\begin{array}{l}\text { Skin Surface } \\
\text { Area Available } \\
\text { for Contact (SA) }\end{array}$ & $\mathrm{cm} 2$ & 3,200 & 5,000 & 5,000 & 5,000 & 20,000 & NA & 1,800 & 7,300 & NA & 3,100 & 3,100 & 3,200 & $\mathbf{N A}$ & $\begin{array}{l}\text { Resident (2)(4) } \\
\text { Visitor and } \\
\text { Worker (2) } \\
\end{array}$ \\
\hline $\begin{array}{l}\text { Ingestion Rate } \\
\text { (IR) (c) }\end{array}$ & $/ \mathrm{d}$ & 50 & NA & 50 & 100 & 2 & $\begin{array}{l}113(\mathrm{e}) \\
202(\mathrm{f}) \\
123(\mathrm{~g}) \\
\end{array}$ & 200 & 1 & $\begin{array}{l}42(\mathrm{e}) \\
75(\mathrm{f}) \\
45(\mathrm{~g}) \\
\end{array}$ & 0.01 & 20 & 50 & 1 & $(2)(3)$ \\
\hline $\begin{array}{l}\text { Inhalation Rate } \\
\text { (IR) }\end{array}$ & $\mathrm{m}^{3} / \mathrm{hr}$ & 2.5 & NA & NA & 0.83 & 0.83 & NA & 1 & 0.6 & $\mathrm{NA}$ & NA & NA & 2.5 & $\mathrm{NA}$ & $\begin{array}{l}\text { Adult, Visitor, } \\
\text { and Worker (2) } \\
\text { Child (3) }\end{array}$ \\
\hline $\begin{array}{l}\text { Fraction } \\
\text { Ingestion from } \\
\text { Contaminated } \\
\text { Source (FD) } \\
\end{array}$ & unitless & 1 & NA & 1 & 1 & NA & $\begin{array}{l}0.042 \text { (d) } \\
0.119 \text { (e) } \\
0.487 \text { (f) }\end{array}$ & 1 & NA & $\begin{array}{l}0.042 \text { (d) } \\
0.119(\mathrm{e}) \\
0.487(\mathrm{f})\end{array}$ & NA & 1 & 1 & NA & $(2)(7)$ \\
\hline $\begin{array}{l}\text { Absorption } \\
\text { Factor (ABS) }\end{array}$ & unitless & $0.01 / 0.001$ & NA & $0.01 / 0.001$ & $0.01 / 0.001$ & NA & NA & $\begin{array}{c}0.01 / 0.001 \\
.\end{array}$ & NA & NA & NA & $0.01 / 0.001$ & $0.01 / 0.001$ & NA & (6) \\
\hline
\end{tabular}


TABLE 3.27

RME HUMAN EXPOSURE PARAMETERS

SAVANNAH RIVER SITE

F AREA - BURNING/RUBBLE PITS

\begin{tabular}{|c|c|c|c|c|c|c|c|c|c|c|c|c|c|c|c|}
\hline \multirow[t]{2}{*}{$\begin{array}{c}\text { Exposure } \\
\text { Assumptions }\end{array}$} & \multirow[t]{2}{*}{ Unit } & \multicolumn{3}{|c|}{$\begin{array}{l}\text { Current On-Unit } \\
\text { Visitor }\end{array}$} & \multicolumn{3}{|c|}{$\begin{array}{l}\text { Future On-Unit } \\
\text { Resident - Adult }\end{array}$} & \multicolumn{5}{|c|}{$\begin{array}{c}\text { Future On-Unit } \\
\text { Resident - Child (a) }\end{array}$} & \multicolumn{2}{|c|}{$\begin{array}{l}\text { Future On-Unit } \\
\text { Industrial Worker }\end{array}$} & \multirow[t]{2}{*}{ Reference } \\
\hline & & Soil & SW & Sed & Soil & GW & Veg & Soil & GW & Veg & Sw & Sed & Soil & $\overline{G W}$ & \\
\hline \begin{tabular}{|l|} 
Dermal \\
Permeability \\
Constant (PC)
\end{tabular} & $\mathrm{cm} / \mathrm{hr}$ & $\overline{\mathrm{NA}}$ & $\begin{array}{c}\text { see Table } \\
3.28\end{array}$ & NA & NA & \begin{tabular}{|c|} 
see Table \\
3.28
\end{tabular} & $\mathrm{NA}$ & $\overline{\mathrm{NA}}$ & \begin{tabular}{|c|} 
see Table \\
3.28
\end{tabular} & $\mathrm{NA}$ & \begin{tabular}{|c|} 
see Table \\
3.28
\end{tabular} & $\mathrm{NA}$ & NA & $\begin{array}{c}\text { see Table } \\
3.28\end{array}$ & $\begin{array}{l}\text { Chemical- } \\
\text { specific }\end{array}$ \\
\hline \begin{tabular}{l|} 
External Gamma \\
Shielding Factor
\end{tabular} & unitless & 0.7 & $\overline{\mathrm{NA}}$ & 0.7 & 0.7 & $\overline{\mathrm{NA}}$ & $\overline{\mathrm{NA}}$ & 0.7 & $\mathrm{NA}$ & NA & NA & 0.7 & 0.7 & NA & (5) \\
\hline
\end{tabular}

RME - Reasonable Maximum Exposure

SW - Surface Water

GW - Ground Water

References:

Veg - Vegetation
(1) EPA, 1989a
(2) EPA, 1990a
(6) EPA, 1992d
(7) EPA Region IV default value.

(3) EPA, 1991a

(4) EPA, 19920

(5) Gilbert et al., 1989

NA - Not Applicable

(a) 0-6 years old for soil and groundwater contact; 7-16 years old for surface water and sediment contact.

(b) For dermal contact while bathing.

(c) Ingestion rates for soil, sediment, and vegetation are expressed in mg/day; surface water and groundwater are expressed in L/day.

(d) Leaves (lettuce in tossed salad).

(c) Roots (white potatoes).

(f) Fruits (tomatoes).

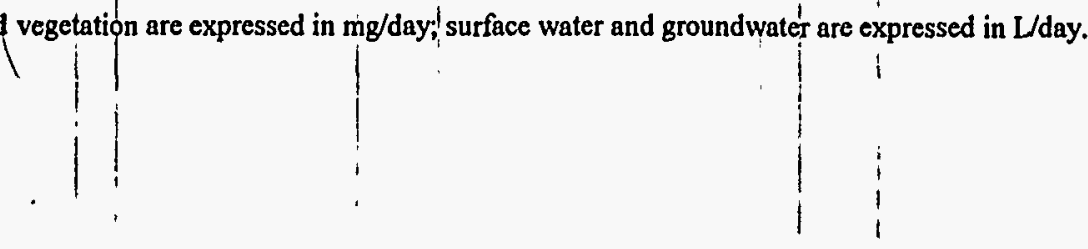


TABLE 3.28

DERMAL PERMEABILITY CONSTANTS

FOR COPCS IN WATER

SAVANNAH RIVER SITE

BURNING/RUBBLE PITS 231-F AND 231-1F AND RUBBLE PIT 231-2F

\begin{tabular}{|c|c|c|}
\hline CAS & Analyte & $\begin{array}{c}\text { Permeability Coefficient (1) } \\
\text { (cm/hour) }\end{array}$ \\
\hline & Volatiles & \\
\hline 67641 & $\overline{\text { Acetone }}$ & $3.10 \mathrm{E}-04 *$ \\
\hline 74873 & Chloromethane & $4.20 \mathrm{E}-03$ \\
\hline 540590 & 1,2-Dichloroethene (total) & $1.00 \mathrm{E}-02$ \\
\hline 100414 & Ethylbenzene & $7.40 \mathrm{E}-02$ \\
\hline 74873 & Methyl Chloride & $4.20 \mathrm{E}-03 *$ \\
\hline 75092 & Methylene Chloride & $4.50 \mathrm{E}-03$ \\
\hline 127184 & Tetrachloroethylene (PCE) & $4.80 \mathrm{E}-02$ \\
\hline \multirow[t]{2}{*}{108883} & Toluene & $4.50 \mathrm{E}-02$ \\
\hline & Semivolatiles & \\
\hline 91576 & 2-Methylnaphthalene & $6.90 \mathrm{E}-02 *$ \\
\hline 83329 & Acenaphthene & $1.50 \mathrm{E}-01 *$ \\
\hline 208968 & Acenaphthylene & $9.60 \mathrm{E}-02 *$ \\
\hline 120127 & Anthracene & $2.20 \mathrm{E}-01 *$ \\
\hline 56553 & Benzo(a)anthracene & $8.10 \mathrm{E}-01$ \\
\hline 50328 & Benzo(a)pyrene & $1.20 \mathrm{E}+00$ \\
\hline 205992 & Benzo(b)fluoranthene & $1.20 \mathrm{E}+00$ \\
\hline 191242 & Benzo(g,h,i)perylene & $1.60 \mathrm{E}+00 *$ \\
\hline 207089 & Benzo(k)fluoranthene & $1.10 \mathrm{E}+00 *$ \\
\hline 117817 & bis(2-Ethylhexyl)phthalate & $3.40 \mathrm{E}-02 *$ \\
\hline 218019 & Chrysene & $8.10 \mathrm{E}-01$ \\
\hline 84742 & Di-n-butylphthalate & $3.30 \mathrm{E}-02$ \\
\hline 53703 & Dibenz(a,h)anthracene & $2.70 \mathrm{E}+00$ \\
\hline 132649 & Dibenzofuran & $1.50 \mathrm{E}-01 *$ \\
\hline 84662 & Diethylphthalate & $4.80 \mathrm{E}-03$ \\
\hline 206440 & Fluoranthene & $3.60 \mathrm{E}-01$ \\
\hline 86737 & Fluorene & $1.70 \mathrm{E}-01 *$ \\
\hline 193395 & Indeno(1,2,3-cd)pyrene & $1.90 \mathrm{E}+00$ \\
\hline 85018 & Phenanthrene & $2.70 \mathrm{E}-01$ \\
\hline \multirow[t]{2}{*}{129000} & Pyrene & $3.40 \mathrm{E}-01 *$ \\
\hline & Pesticides/PCBs & \\
\hline 319868 & $\overline{\text { delta-BHC }}$ & $2.80 E-02 *$ \\
\hline 53494705 & Endrin ketone & $1.60 \mathrm{E}-02 *$ \\
\hline 1024573 & Heptachlor epoxide & $5.50 \mathrm{E}-02 *$ \\
\hline
\end{tabular}


TABLE 3.28

DERMAL PERMEABILITY CONSTANTS

FOR COPCS IN WATER

SAVANNAH RIVER SITE

BURNING/RUBBLE PITS 231-F AND 231-1F AND RUBBLE PIT 231-2F

\begin{tabular}{|c|c|c|}
\hline CAS & Analyte & $\begin{array}{c}\text { Permeability Coefficient (1) } \\
\text { (cm/hour) }\end{array}$ \\
\hline $\begin{array}{l}7440382 \\
7440393 \\
7440439 \\
7440473 \\
7440508 \\
7439921 \\
7439965 \\
7439976 \\
7440020 \\
7440666\end{array}$ & $\begin{array}{l}\text { Heptachlorodibenzo-p-dioxin } \\
\text { Heptachlorodibenzo-p-furan } \\
\text { Hexachlorodibenzo-p-furan } \\
\text { Octachlorodibenzo-p-dioxin } \\
\text { Octachlorodibenzo-p-furan } \\
\text { PCB } 1254 \\
\text { PCB } 1260 \\
\text { Inorganics } \\
\text { Arsenic } \\
\text { Barium } \\
\text { Cadmium } \\
\text { Chromium } \\
\text { Copper } \\
\text { Lead } \\
\text { Manganese } \\
\text { Mercury } \\
\text { Nickel } \\
\text { Zinc }\end{array}$ & $\begin{array}{l}1.00 \mathrm{E}-03 \\
1.00 \mathrm{E}-03 \\
1.00 \mathrm{E}-03 \\
1.00 \mathrm{E}-03 \\
1.00 \mathrm{E}-03 \\
1.00 \mathrm{E}-03 \\
1.00 \mathrm{E}-03 \\
1.00 \mathrm{E}-03 \\
1.00 \mathrm{E}-03 \\
1.00 \mathrm{E}-03\end{array}$ \\
\hline
\end{tabular}

(1) USEPA, 1992a. An asterisk ("*") indicates a value calculated using the following formula:

$\log =-2.72+0.71 \log$ Kow $-0.0061 \mathrm{MW}$

Where: $\mathrm{Kp}=$ permeability coefficient

MW $=$ molecular weight

Kow and MW obtained from Howard (1990 and 1991) and ATSDR (1989-1991) 
TABLE 3.29

SOIL-TO-PLANT TRANSFER FACTORS (1)

SAVANNAH RIVER SITE

BURNING/RUBBLE PITS 231-F AND 231-1F AND RUBBLE PIT 231-2F

\begin{tabular}{|l|c|c|c|}
\hline \multicolumn{1}{|c|}{ Constituent (2) } & Leafy Vegetables & Tuberous Vegetables & Fruits \\
\hline & $1.30 \mathrm{E}-01$ & & $2.20 \mathrm{E}-02$ \\
Cesium-137 & $3.40 \mathrm{E}-03$ & $4.90 \mathrm{E}-02$ & $5.00 \mathrm{E}-02$ \\
Iodine-129 & $3.90 \mathrm{E}-04$ & $5.00 \mathrm{E}-02$ & $4.50 \mathrm{E}-05$ \\
Plutonium-238-/239 & $1.00 \mathrm{E}+00$ & $2.00 \mathrm{E}-04$ & $5.50 \mathrm{E}-01$ \\
Potassium-40 & $7.50 \mathrm{E}-02$ & $5.50 \mathrm{E}-01$ & $6.10 \mathrm{E}-03$ \\
Radium & $1.60 \mathrm{E}+00$ & $3.20 \mathrm{E}-03$ & $1.70 \mathrm{E}-01$ \\
Strontium-90 & $4.40 \mathrm{E}+01$ & $8.10 \mathrm{E}-01$ & $1.50 \mathrm{E}+00$ \\
Technetium-99 & $1.70 \mathrm{E}-02$ & $1.10 \mathrm{E}+00$ & $.4 .00 \mathrm{E}-03$ \\
Uranium-234/235/238 & & $1.40 \mathrm{E}-02$ & \\
\hline
\end{tabular}

(1) NUREG/CR-5512 (NRC, 1992)

(2) Evaluation was not performed, because tritium contamination is expected to rapidly escape from soil or plants, assuming there is no additional tritium from air and water (NRC, 1992). 
TABLE 3.30

CHEMICAL SPECIFIC TOXICITY VALUES

NONCARCINOGENIC EFFECTS FROM INGESTION AND DERMAL ABSORPTION (1)

SAVANNAH RIVER SITE

F-AREA BURNING/RUBBLE PITS AND RUBBLE PIT

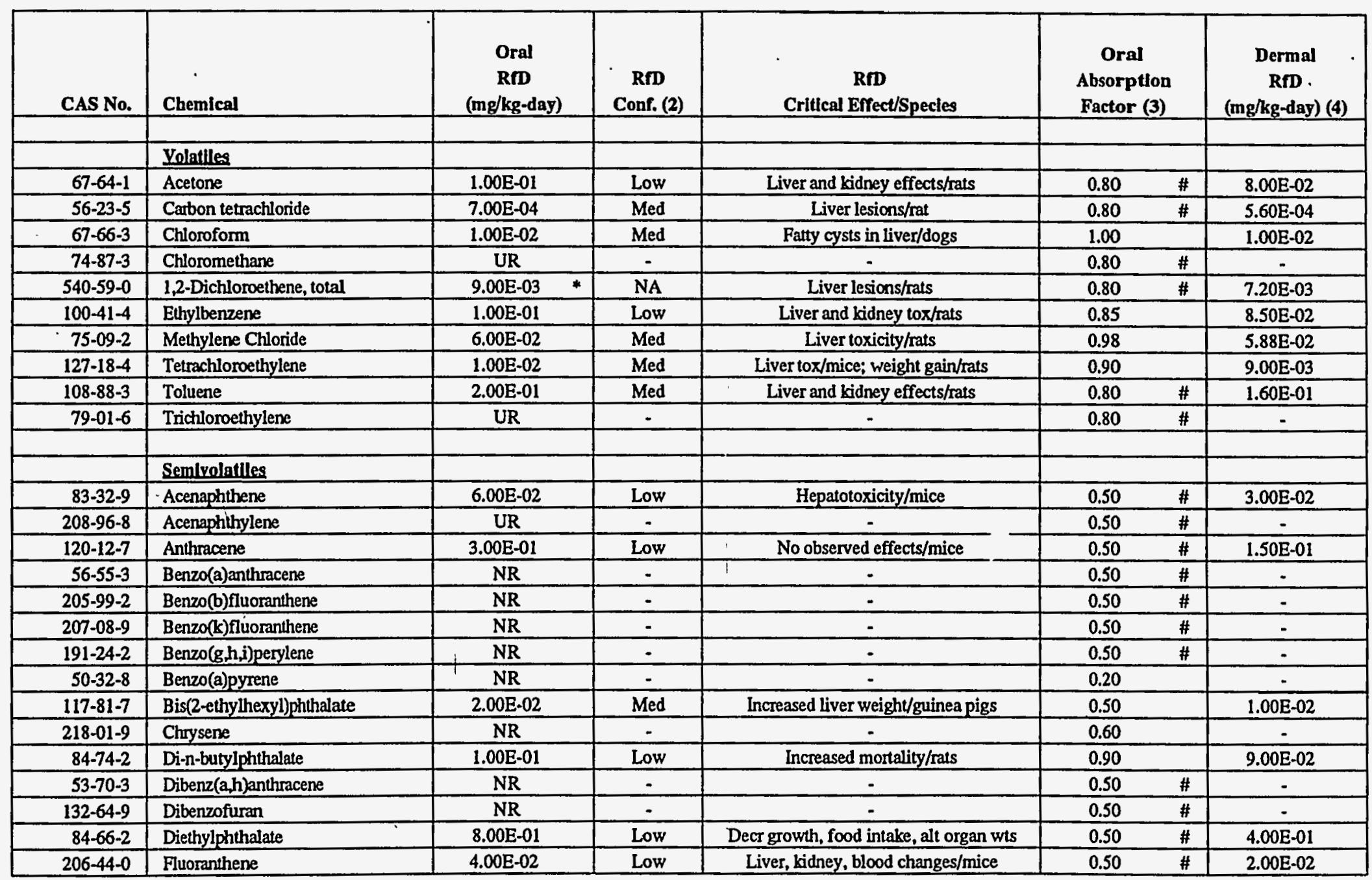




\section{TABLE 3.30 \\ CHEMICAL SPECIFIC TOXICITY VALUES \\ NONCARCINOGENIC EFFECTS FROM INGESTION AND DERMAL ABSORPTION (1) \\ SAVANNAH RIVER SITE \\ F-AREA BURNING/RUBBLE PITS AND RUBBLE PIT}

\begin{tabular}{|c|c|c|c|c|c|c|}
\hline CAS No. & Chemical & $\begin{array}{c}\text { Oral } \\
\text { RFD } \\
\text { (mg/kg-day) } \\
\end{array}$ & $\begin{array}{c}\text { RfD } \\
\text { Conf. (2) } \\
\end{array}$ & $\begin{array}{c}\text { RTD } \\
\text { Critical Errect/Species }\end{array}$ & $\begin{array}{c}\text { Oral } \\
\text { Absorptlon } \\
\text { Factor (3) } \\
\end{array}$ & $\begin{array}{c}\text { Dermal } \\
\text { RfD } \\
\text { (mg/kg-day) (4) }\end{array}$ \\
\hline 86-73-7 & Fluorene & $4.00 \mathrm{E}-02$ & Low & Hematological effects/mice & 0.50 & $2.00 \mathrm{E}-02$ \\
\hline $193-39-5$ & Indeno(1,2,3-cd)pyrene & NR & - & - & 0.50 & - \\
\hline $91-57-6$ & 2-Methylnaphthalene & NR & - & - & 0.50 & - \\
\hline $85-01-8$ & Phenanthrene & NR & - & - & 0.50 & - \\
\hline \multirow[t]{2}{*}{$129-00-0$} & Pyrene & $3.00 \mathrm{E}-02$ & Low & Kidney effects/mice & 0.50 & $1.50 \mathrm{E}-02$ \\
\hline & Dloxins/Furans & & ; & & & \\
\hline $37871-00-4$ & Heptachlorodibenzo-p-dioxin & NR & - & - & 0.87 & - \\
\hline $55684-94-1$ & Heptachlorodibenzo-p-furan & $\mathrm{NR}$ & - & - & 0.87 & - \\
\hline $3268-87-9$ & Hexachlorodibenzo-p-furan & $\mathrm{NR}$ & - & - & 0.87 & - \\
\hline $3268-87-9$ & Octachlorodibenzo-p-dioxin & NR & - & - & 0.87 & - \\
\hline \multirow[t]{2}{*}{$39001-02-0$} & Octachlorodibenzo-p-furan & NR & - & $\therefore$ & 0.87 & - \\
\hline & PCAs & & & & & \\
\hline $11097-69-1$ & Aroclor-1254 & $2.00 \mathrm{E}-05$ & Med & Various clinical effects/monkeys & 0.90 & $1.80 \mathrm{E}-05$ \\
\hline \multirow[t]{3}{*}{$11096-82-5$} & Aroclor-1260 (5) & $2.00 \mathrm{E}-05$ & Med & Various clinical effects/monkeys & 0.90 & $1.80 \mathrm{E}-05$ \\
\hline & & - & & & & \\
\hline & Pesticides: & & & & & \\
\hline $319-86-8$ & delta-BHC & NR & - & - & 0.92 & - \\
\hline $53494-70-5$ & Endrin ketone & NR & - & - & 0.50 & - \\
\hline \multirow[t]{3}{*}{ 1024-57-3 } & Heptachlor epoxide & 1.30E-05 & Low & Increased liver weight/dogs & 0.20 & $2.60 \mathrm{E}-06$ \\
\hline & & & & & & \\
\hline & Metals & & & & & \\
\hline $7440-38-2$ & Arsenic & $3.00 \mathrm{E}-04$ & Med & Skin and vascular effects/humans & 0.80 & 2.40E-04 \\
\hline $7440-39-3$ & Barium & $7.00 \mathrm{E}-02$ & Med & Increased blood pressure/humans & 0.05 & $3.50 \mathrm{E}-03$ \\
\hline $7440-43-9$ & Cadmium & $5.00 \mathrm{E}-04 \quad(7)$ & High & Significant proteinuria/humans & 0.02 & $1.00 \mathrm{E}-05$ \\
\hline $7440-47-3$ & Chromium VI & $5.00 \mathrm{E}-03$ & Low & No observed effects/rats & 0.02 & $1.00 \mathrm{E}-04$ \\
\hline $7440-50-8$ & Copper & $4.00 \mathrm{E}-02 *$ & NR & Gastrointestinal irritation/humans & 0.60 & $2.40 \mathrm{E}-02$ \\
\hline
\end{tabular}




\section{TABLE 3.30 \\ CHEMICAL SPECIFIC TOXICITY VALUES \\ NONCARCINOGENIC EFFECTS FROM INGESTION AND DERMAL ABSORPTION (1) \\ SAVANNAH RIVER SITE \\ F-AREA BURNING/RUBBLE PITS AND RUBBLE PIT}

\begin{tabular}{|c|c|c|c|c|c|c|}
\hline CAS No. & $\begin{array}{c}: \\
\text { Chemical }\end{array}$ & $\begin{array}{c}\text { Oral } \\
\text { RfD } \\
\text { (mg/kg-day) } \\
\end{array}$ & $\begin{array}{c}\text { RfD } \\
\text { Conf. (2) } \\
\end{array}$ & $\begin{array}{c}\text { RfD } \\
\text { Critical Errect/Species }\end{array}$ & $\begin{array}{c}\text { Oral } \\
\text { Absorption } \\
\text { Factor (3) } \\
\end{array}$ & $\begin{array}{c}\text { Dermal } \\
\text { RfD } \\
\text { (mg/kg-day) (4) }\end{array}$ \\
\hline $7439-92-1$ & Lead & NR & - & - & 0.15 & - \\
\hline $7439-96-5$ & Manganese & $5.00 \mathrm{E}-02 \quad(8)$ & Med/Low & CNS effects/humans & 0.03 & $1.50 \mathrm{E}-03$ \\
\hline $7439-97-6$ & Mercury & $3.00 \mathrm{E}-04 *$ & NA & Kidney effects/rats & 0.01 & $3.00 \mathrm{E}-06$ \\
\hline $7440-02-0$ & Nickel & $2.00 \mathrm{E}-02$ & Med & Decreased body and organ wt/rats & 0.04 & $8.00 \mathrm{E}-04$ \\
\hline $7440-66-6$ & Zinc & $3.00 \mathrm{E}-01$ & Med & RBC effects/humans & 0.25 & $7.50 \mathrm{E}-02$ \\
\hline
\end{tabular}

(1) From IRIS (EPA, 1996a). When IRIS values were unavailable, HFAST (EPA, 1995) was used. An asterisk $\left(^{*}\right)$ indicates values taken from HEAST. A double asterisk (**) indicates that value from USEPA (1996b - $\mu \cdot$ rsonal communication with the Superfund Technical Support Center).

$\mathrm{RfD}=$ reference dose, $\mathrm{NR}=$ not reported, $\mathrm{UR}=$ under review, $\mathrm{NI}=$ nonverifiable, $\mathrm{NA}=$ not applicable

CNS $=$ Central Nervous System, RBC $=$ Red Blood Cells, incr $=$ ircreased, $w t=$ weight

A dash (" $\bullet$ ) indicates that this item is not applicable, or that there is no entry in either IRIS or HEAST.

(2) EPA-assigned level of confidence in toxicity value. Med = medium.

(3) The absorption values were cited in the appropriate ATSDR Profiles. The pound sign (\#) indicates that the ATSDR Profiles were not available and default values were úsed as follows: 0.8 for volatiles, 0.5 for semi-volatiles and pesticides, and 0.20 for metals.

(4) Dermal-RfD $=$ Oral-RfD $x$ oral absorption factor.

(5) The RfD is based on the RfD for Aroclor-1254.

(6) Oral absorption factor based on 2,3,7,8-TCDD.

(7) Two RfD values are available for cadmium and manganese and the most conservative are presented.

(8) For dietary intake, an RfD of $0.14 \mathrm{mg} / \mathrm{kg}$-day was presented in IRIS. For non-dietary intake (groundwater/soil), apply a modifying factor of 3 was applied, nesulting in an RfD of $0.05 \mathrm{mg} / \mathrm{kg}$-day. 
TABLE 3.31

CHEMICAL SPECIFIC TOXICITY VALUES

NONCARCINOGENIC EFFECTS FROM INHALATION (1)

SAVANNAH RIVER SITE

F-AREA BURNING/RUBBLE PITS AND RUBBLE PIT

\begin{tabular}{|c|c|c|c|c|}
\hline CAS No. & Chemical & $\begin{array}{c}\text { Inhalation } \\
\mathrm{PfC} \\
\left(\mathrm{mg} / \mathrm{m}^{3}\right) \\
\end{array}$ & $\begin{array}{c}\text { RfC } \\
\text { Conf. (2) } \\
\end{array}$ & $\begin{array}{c}\text { RfC } \\
\text { Critical Effect/Species }\end{array}$ \\
\hline & Yolatiles & & & \\
\hline $67-64-1$ & Acetone & NR & - & - \\
\hline $56-23-5$ & Carbon Tetrachloride & NR & - & - \\
\hline $67-66-3$ & Chloroform & $\mathrm{UR}$ & - & - \\
\hline 74-87-3 & Chloromethane & UR. & - & - \\
\hline $540-59-0$ & 1,2-Dichloroethene, total & NR & - & - \\
\hline $100-41-4$ & Ethylbenzene & $1.00 \mathrm{E}+00$ & Low & Developmental tox/rats and rabbits \\
\hline $75-09-2$ & Methylene Chloride & $3.00 \mathrm{E}+00$ & NA & Liver toxicity/rats \\
\hline $127-18-4$ & Tetrachloroethylene & NR & - & - \\
\hline $108-88-3$ & Toluene & $4.00 \mathrm{E}-01$ & Med & CNS effects/human; nasal effects/rat \\
\hline \multirow[t]{2}{*}{ 79-01-6 } & Trichloroethylene & $\mathrm{UR}$ & - & - \\
\hline & Semivolatiles & & & \\
\hline $83-32-9$ & Acenaphthene & NR & - & - \\
\hline $208-96-8$ & Acenaphthylene & NR & $\because$ & - \\
\hline $120-12-7$ & Anthracene & $\mathbf{N R}$ & - & - \\
\hline $56-55-3$ & Benzo(a)anthracene & NR & - & - \\
\hline $205-99-2$ & Benzo(b)fluoranthene & NR & - & $=$ \\
\hline $207-08-9$ & Benzo(k)fluoranthene & $\mathbf{N R}$ & - & - \\
\hline $191-24-2$ & Benzo(g,h,i)perylene & NR & - & - \\
\hline $50-32-8$ & Benzo(a)pyrene & NR & - & - \\
\hline $117-81-7$ & Bis(2-ethylhexyl)phthalate & NR & - & - \\
\hline $218-01-9$ & Chrysene & NR & - & - \\
\hline $84-74-2$ & Di-n-butylphthalate & $\mathrm{NV}$ & - & - \\
\hline $53-70-3$ & Dibenz(a,h)anthracene & NR & - & - \\
\hline $132-64-9$ & Dibenzofuran & NR & - & - \\
\hline $84-66-2$ & Diethylphthalate & NR & - & - \\
\hline $206-44-0$ & Fluoranthene & NR & - & - \\
\hline $86-73-7$ & Fluorene & NR & - & - \\
\hline $193-39-5$ & Indeno(1,2,3-cd)pyrene & NR & - & - \\
\hline $91-57-6$ & 2-Methylnaphthalene & NR & - & - \\
\hline $85-01-8$ & Phenanthrene & NR & - & - \\
\hline \multirow[t]{3}{*}{$129-00-0$} & Pyrene & NV & - & - \\
\hline & & & - & \\
\hline & Dioxins/Furans & & & \\
\hline $37871-00-4$ & Heptachlorodibenzo-p-dioxin & NR & - & - \\
\hline 55684-94-1 & Heptachlorodibenzo-p-furan & NR & - & - \\
\hline $3268-87-9$ & Hexachlorodibenzo-p-furan & NR & - & - \\
\hline $3268-87-9$ & Octaclorodibenzo-p-dioxin & NR & - & - \\
\hline \multirow[t]{2}{*}{$39001-02-0$} & Octachlorodibenzo-p-furan & NR & - & - \\
\hline & PCBs & & & \\
\hline $11097-69-1$ & Aroclor-1254 & NR & - & - \\
\hline $11096-82-5$ & Aroclor-1260 (3) & NR & - & - \\
\hline
\end{tabular}


TABLE 3.31

CHEMICAL SPECIFIC TOXICITY VALUES

NONCARCINOGENIC EFFECTS FROM INHALATION (1)

SAVANNAH RIVER SITE

F-AREA BURNING/RUBBLE PITS AND RUBBLE PIT

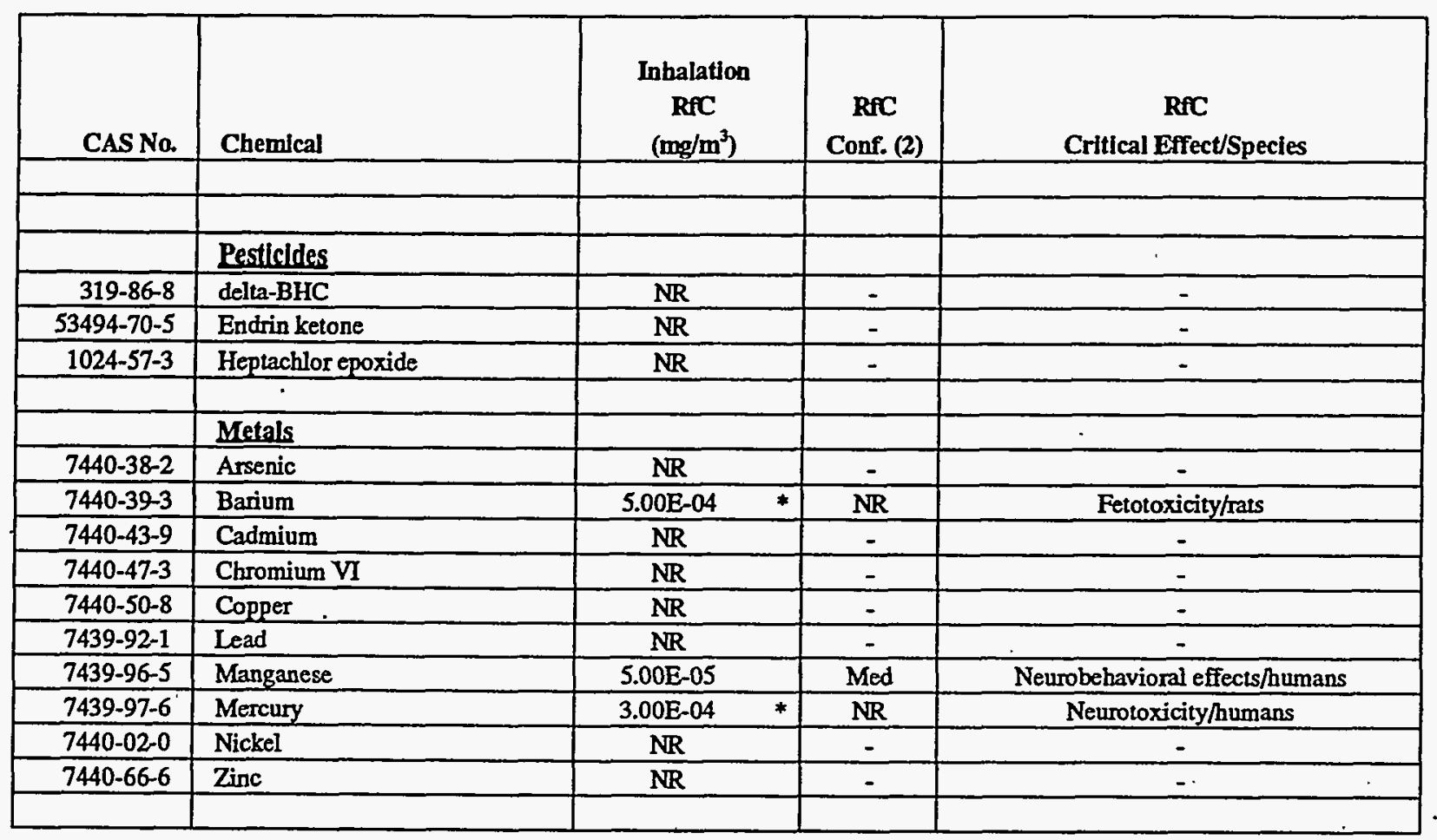

(1) From IRIS (EPA, 1996a). When IRIS values were unavailable, HEAST (EPA, 1995) was used.

An asterisk $\left(^{*}\right)$ indicates values taken from HEAST.

A double asterisk (**) indicates value from EPA (1996b - personal communication with the Superfund

Technical Support Center). $R f D=$ reference dose, $N R=$ not reported, $U R=$ under review, $N V=$ nonverifiable,

NA = not applicable. CNS = Central Nervous System, RBC $=$ Red Blood Cells, incr $=$ increased, wt $=$ weight

A dash ("-") indicates that this item is not applicable, or that there is no entry in either IRIS or HEAST.

(2) EPA-assigned level of confidence in toxicity value. Med = medium.

(3) The RfD is based on the RfD for Aroclor-1254. 
TABLE 3.32

CHEMICAL SPECIFIC TOXICITY VALUES

CARCINOGENIC EFFECTS FROM INGESTION AND DERMAL ABSORPTION (1)

SAVANNAH RIVER SITE

F-AREA BURNING/RUBBLE PITS AND RUBBLE PIT

\begin{tabular}{|c|c|c|c|c|c|c|}
\hline CAS No. & Chemical & $\begin{array}{c}\text { CAG } \\
\text { Group (2) } \\
\end{array}$ & $\begin{array}{c}\text { Oral } \\
\text { Slope Factor } \\
\text { (mg/kg-day) }^{-1}\end{array}$ & $\begin{array}{c}\text { Slope Factor } \\
\text { Target Organ (Tumor Type)/Species }\end{array}$ & $\begin{array}{c}\text { Oral } \\
\text { Absorption } \\
\text { Factor (3) }\end{array}$ & $\begin{array}{c}\text { Dermal } \\
\text { Slope Factor } \\
\text { (mg/kg-day) })^{-1}(\mathbf{\phi})\end{array}$ \\
\hline & Yolatlles & & & & & \\
\hline $67-64-1$ & Acetone & D & - & -1 & 0.80 & - \\
\hline $56-23-5$ & Carbon tetrachloride & $\mathbf{B 2}$ & $1.30 \mathrm{E}-01$ & Liver (carcinoma/heptatoma): rodents & 0.80 & $1.63 \mathrm{E}-01$ \\
\hline $67-66-3$ & Chloroform & B2 & $6.10 \mathrm{E}-03$ & Kidney (NR): rats & 1.00 & $6.10 \mathrm{E}-03$ \\
\hline $75-00-3$ & Chloroethane & NR & - & -1 & 0.80 & - \\
\hline $74-87-3$ & Chloromethane & $\mathbf{C}$ & $1.30 \mathrm{E}-02 *$ & Kidney (NR): mice & 0.80 & $1.63 \mathrm{E}-02$ \\
\hline $540-59-0$ & 1,2-Dichloroethene, total & D & - & $\begin{array}{ll}- & 1 \\
\end{array}$ & 0.80 & - \\
\hline $100-41-4$ & Ethylbenzene & D & $\cdot$ & $\cdot$ & 0.85 & - \\
\hline $75-09-2$ & Methylene Chloride & B2 & $7.50 \mathrm{E}-03$ & Liver (adenomas, carcinomas): mici & 0.98 & $7.65 \mathrm{~F}-03$ \\
\hline $127-18-4$ & Tetrachloroethylene & NR & $5.20 \mathrm{E}-02 \quad * *$ & NR & 0.90 & $5.78 \mathrm{E}-02$ \\
\hline $108-88-3$ & Toluene & $\mathrm{D}$ & - & $\cdot$ & 0.80 & - \\
\hline \multirow[t]{2}{*}{$79-01-6$} & Trichloroethylene & NR & $1.10 \mathrm{E}-02 \quad * *$ & NR & 0.80 & $1.38 \mathrm{E}-02$ \\
\hline & & & & & & \\
\hline $83-32-9$ & Acenaphthene & NR & - & - & 0.50 & - \\
\hline $208-96-8$ & Acenaphthylene & $\mathrm{D}$ & - & - & 0.50 & - \\
\hline $120-12-7$ & Anthracene & $\mathrm{D}$ & + & $\cdot$ & 0.50 & - \\
\hline $56-55-3$ & Benzo(a)anthracene & B2 & $7.30 \mathrm{E}-01 \quad(5)$ & Forestomach (carcinomas): mice & 0.50 & $1.46 \mathrm{E}+00$ \\
\hline $205-99-2$ & Benzo(b)fluoranthene & B2 & $7.30 \mathrm{E}-01 \quad(5)$ & Forestomach (carcinomas): mice & 0.50 & $1.46 \mathrm{E}+00$ \\
\hline $207-08-9$ & Benzo(k)fluoranthene & B2 & $7.30 \mathrm{E}-02 \quad(5)$ & Forestomach (carcinomas): mice & 0.50 & $1.46 \mathrm{E}-01$ \\
\hline 191-24-2 & Benzo $(g, h, i)$ perylene & $\mathrm{D}$ & - & - & 0.50 & - \\
\hline $50-32-8$ & Benzo(a)pyrene & B2 & $7.30 \mathrm{E}+00$ & Forestomach (carcinomas): mice & 0.20 & $3.65 \mathrm{E}+01$ \\
\hline $117-81-7$ & Bis(2-ethylhexyl)phthalate & B2 & $1.40 \mathrm{E}-02$ & Liver (adenoma and carcinoma): mice & 0.50 & $2.80 \mathrm{E}-02$ \\
\hline $218-01-9$ & Chrysene & B2 & $7.30 \mathrm{E}-03 \quad(5)$ & Forestomach (carcinomas): mice & 0.60 & $1.22 \mathrm{E}-02$ \\
\hline $84-74-2$ & Di-n-butylphthalate & D & - & - & 0.90 & - \\
\hline $53-70-3$ & Dibenz(ah)anthracene & B2 & $7.30 \mathrm{E}+00 \quad(5)$ & Forestomach (carcinomas): mice & 0.50 & $1.46 \mathrm{E}+01$ \\
\hline $132-64-9$ & Dibenzofuran & D & $-\quad \cdot$ & - & 0.50 & - \\
\hline $84-66-2$ & Diethylphthalate & $\mathbf{D}$ & - & - & 0.50 & - \\
\hline
\end{tabular}


TABLE 3.32

CHEMICAL SPECIFIC TOXICITY VALUES

CARCINOGENIC EFFECTS FROM INGESTION AND DERMAL ABSORPTION (1)

SAVANNAH RIVER SITE

F-AREA BURNING/RUBBLE PITS AND RUBBLE PIT

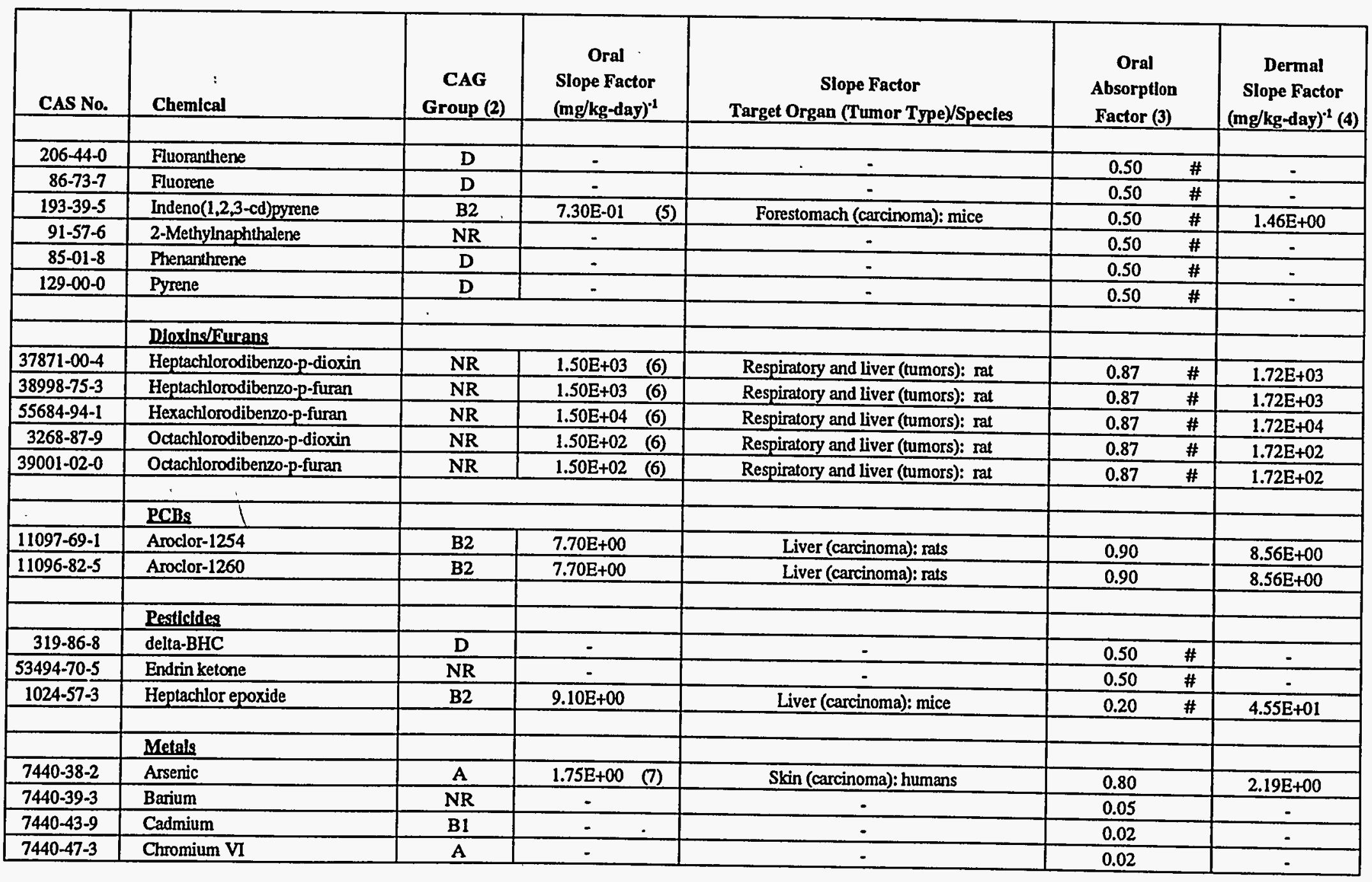




\section{TABLE 3.32 \\ CHEMICAL SPECIFIC TOXICITY VALUES \\ CARCINOGENIC EFFECTS FROM INGESTION AND DERMAL ABSORPTION (1) \\ SAVANNAH RIVER SITE \\ F-AREA BURNING/RUBBLE PITS AND RUBBLE PIT}

\begin{tabular}{|c|c|c|c|c|c|c|}
\hline CAS No. & Chemical & $\begin{array}{c}\text { CAG } \\
\text { Group (2) }\end{array}$ & $\begin{array}{c}\text { Oral } \\
\text { Slope Factor } \\
\text { (mg/kg-day) }\end{array}$ & $\begin{array}{c}\text { Slope Factor } \\
\text { Target Organ (Tumor Type)/Specles }\end{array}$ & $\begin{array}{c}\text { Oral } \\
\text { Absorption } \\
\text { Factor (3) }\end{array}$ & $\begin{array}{c}\text { Dermal } \\
\text { Slope Factor } \\
(\mathrm{mg} / \mathrm{kg} \text {-day })^{-1}(\mathbf{4})\end{array}$ \\
\hline \multicolumn{7}{|l|}{ 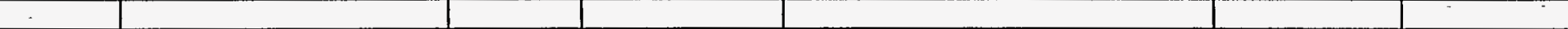 } \\
\hline $7440-50-8$ & Copper & D & - & - & 0.60 & - \\
\hline $7439-92-1$ & Lead & B2 & - & $=$ & 0.15 & - \\
\hline $7439-96-5$ & Manganese & D & - & - & 0.03 & - \\
\hline $7439-97-6$ & Mercury & $\mathrm{D}$ & - & - & 0.01 & - \\
\hline $7440-02-0$ & Nickel & NR & - & $=$ & 0.04 & - \\
\hline $7440-66-6$ & Zinc & $\mathrm{D}$ & - & - & 0.25 & - \\
\hline & & & & - & & \\
\hline
\end{tabular}

(1) From IRIS (EPA, 1996a). When IRIS values were unavailable, HEA ST (EPA, 1995) was used. An asterisk (*) indicates values taken from HEAST.

A double asterisk (**) indicates that value from USEPA (1996b - personal communication with the Superfund Technical Support Center).

$\mathrm{NR}=$ not reported, $\mathrm{UR}=$ under review

A dash ("-") indicates that this item is not applicable, or that there is no entry in either IRIS or HEAST.

(2) $\mathbf{C A G}=\mathrm{EPA}$ Carcinogen Assessment Group

(3) The absorption values were cited in the appropriate ATSDR Profiles. The pound sign (\#) indicates that the ATSDR Profiles were not available and default values were used as follows: 0.8 for volatiles, 0.5 for semi-volatiles and pesticides,

and 0.20 for metals.

(4) Dermal-SP = Oral-SF/oral absorption factor.

(5) The slope factors for the carcinogenic PAHs were derived by multiplying the slope factor for benzo(a)pyrene by the following

toxicity equivalency factors: 0.1 for benzo(a)anthracene, benzo(b)fluoranthene and indeno(1,2,3-cd)perylene, 0.01 for benzo(k)fluoranthene,

0.001 for chrysene and 1.0 for dibenz $(a, h)$ anthracene.

(6) Slope factors were derived by multiplying the slope factor for $2,3,7,8$ TCDD by the following toxicity equivalency factcrs:

0.01 for $\mathrm{HPCDD}$ and HpCDF, 0.1 for $\mathrm{HxCDF}$, and 0.001 for OCDD and OCDF. The oral absorption factor for 2,3,7,8 TCDD was also used.

(7) The slope factor for arsenic was derived from an oral unit risk of 5E-05 (L/ug). 
TABLE 3.33

CHEMICAL SPECIFIC TOXICITY VALUES

CARCINOGENIC EFFECTS FROM INHALATION (1)

SAVANNAH RIVER SITE

F-AREA BURNING/RUBBLE PITS AND RUBBLE PIT

\begin{tabular}{|c|c|c|c|c|}
\hline CAS No. & Chemical & $\begin{array}{c}\text { CAG } \\
\text { Group (2) }\end{array}$ & $\begin{array}{c}\text { Inhalation } \\
\text { Unit Risk } \\
\left(\mathrm{ug} / \mathrm{m}^{3}\right)^{-1} \\
\end{array}$ & $\begin{array}{c}\text { Unit Risk } \\
\text { Target Organ (Tumor Type)/Species }\end{array}$ \\
\hline & Yolatiles & & & \\
\hline $67-64-1$ & Acetone & D & - & - \\
\hline $56-23-5$ & Carbon Tetrachloride & B2 & 1.50E-05 & Liver (carcinoma/heptatoma): rodents \\
\hline $67-66-3$ & Chloroform & B2 & $2.30 \mathrm{E}-05$ & Liver (carcinoma): mice \\
\hline $75-00-3$ & Chloroethane & NR & - & - \\
\hline $74-87-3$ & Chloromethane & C & $1.80 \mathrm{E}-06 *$ & Kidney (NR): mice \\
\hline $540-59-0$ & 1,2-Dichloroethene, total & D & - & - \\
\hline $100-41-4$ & Ethylbenzene & D & - & - \\
\hline $75-09-2$ & Methylene Chloride & $\mathrm{B2}$ & 4.70E-07 & Liver/lung (adenoma, carcinoma): mice \\
\hline $127-18-4$ & Tetrachloroethylene & NR & $5.80 \mathrm{E}-07 \quad * *$ & NR \\
\hline $108-88-3$ & Toluene & $\mathrm{D}$ & - & - \\
\hline \multirow[t]{2}{*}{$79-01-6$} & Trichloroethylene & NR & $1.70 \mathrm{E}-06 \quad * *$ & NR \\
\hline & Semirolatiles & & & \\
\hline $83-32-9$ & Acenaphthene & NR & - & - \\
\hline $208-96-8$ & Acenaphthylene & $\mathrm{D}$ & - & - \\
\hline $120-12-7$ & Anthracene & D & - & - \\
\hline $56-55-3$ & Benzo(a)anthracene & B2 & - & - \\
\hline $205-99-2$ & Benzo(b)fluoranthene & B2 & - & - \\
\hline $207-08-9$ & Benzo(k)fluoranthene & $\mathrm{B2}$ & - & - \\
\hline 191-24-2 & Benzo(g,h,i)perylene & $\mathrm{D}$ & - & - \\
\hline $50-32-8$ & Benzo(a)pyrene & B2 & - & - \\
\hline $117-81-7$ & Bis(2-ethylhexyl)phthalate & B2 & - & - \\
\hline $218-01-9$ & Chrysene & B2 & - & $=$ \\
\hline 84-74-2 & Di-n-butylphthalate & D & - & - \\
\hline $53-70-3$ & Dibenz(a,h)anthracene & B2 & - & - \\
\hline $132-64-9$ & Dibenzofuran & $\mathrm{D}$ & - & - \\
\hline $84-66-2$ & Diethylphthalate & $\mathrm{D}$ & - & - \\
\hline $206-44-0$ & Fluoranthene & $\mathrm{D}$ & - & - \\
\hline $86-73-7$ & Fluorene & D & - & - \\
\hline $193-39-5$ & Indeno $(1,2,3-c d)$ pyrene & B2 & - & - \\
\hline $91-57-6$ & 2-Methylnaphthalene & $\mathrm{NR}$ & - & - \\
\hline $85-01-8$ & Phenanthrene & $\mathrm{D}$ & - & - \\
\hline \multirow[t]{2}{*}{$129-00-0$} & Pyrene & $\mathrm{D}$ & 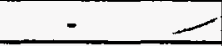 & - \\
\hline & Dioxins/Furans & & & \\
\hline $37871-00-4$ & Heptachlorodibenzo-p-dioxin & NR & $3.30 \mathrm{E}-01$ & Respiratory and liver (tumors): rat \\
\hline 38998-75-3 & Heptachlorodibenzo-p-furan & NR & $3.30 \mathrm{E}-01$ & Respiratory and liver (tumors): rat \\
\hline $55684-94-1$ & Hexachlorodibenzo-p-furan & NR & $3.30 \mathrm{E}+00$ & Respiratory and liver (urmors): rat \\
\hline $3268-87-9$ & Octachlorodibenzo-p-dioxin & NR & $3.30 \mathrm{E}-02 \quad(0)$ & Respiratory and liver (tumors): rat \\
\hline \multirow[t]{2}{*}{$39001-02-0$} & Octachlorodibenzo-p-furan & NR & $3.30 \mathrm{E}-02 \quad(6)$ & Respiratory and liver (tumors): rat \\
\hline & PCBs & & & . \\
\hline $11097-69-1$ & Aroclor- 1254 & B2 & - & - \\
\hline
\end{tabular}


TABLE 3.33

CHEMICAL SPECIFIC TOXICITY VALUES

CARCINOGENIC EFFECTS FROM INHALATION (1)

SAVANNAH RIVER SITE

F-AREA BURNING/RUBBLE PITS AND RUBBLE PIT

\begin{tabular}{|c|c|c|c|c|}
\hline CAS No. & Chemical & $\begin{array}{c}\text { CAG } \\
\text { Group (2) }\end{array}$ & $\begin{array}{l}\text { Inhalation } \\
\text { Unit Risk } \\
\left(\text { ug/m } \mathbf{m}^{\mathbf{3}}\right)^{-1}\end{array}$ & $\begin{array}{c}\text { Unit Risk } \\
\text { Target Organ (Tumor Type)/Species }\end{array}$ \\
\hline \multirow[t]{2}{*}{$11096-82-5$} & Aroclor- 1260 & B2 & - & - \\
\hline & Pesticides & & & \\
\hline $319-86-8$ & delta-BHC & D & - & - \\
\hline $53494-70-5$ & Endrin ketone & NR & - & - \\
\hline \multirow[t]{3}{*}{$1024-57-3$} & Heptachlor epoxide & B2 & $2.60 \mathrm{E}-03$ & Liver (carcinoma): mice \\
\hline & & & & . \\
\hline & Metals & & 1 & \\
\hline $7440-38-2$ & Arsenic & $\mathbf{A}$ & $4.30 \mathrm{E}-03$ & Respiratory system(carcinoma):human \\
\hline $7440-39-3$ & Barium & NR & - & - \\
\hline $7440-43-9$ & Cadmium & B1 & $1.80 \mathrm{E}-03$ & Lung (NR): humans \\
\hline $7440-47-3$ & Chromium VI & $\mathbf{A}$ & $1.20 \mathrm{E}-02$ & Lung (NR): humans \\
\hline $7440-50-8$ & Copper & D & - & - \\
\hline $7439-92-1$ & Lead & B2 & - & - \\
\hline $7439-96-5$ & Manganese & $\mathbf{D}$ & - & - \\
\hline $7439-97-6$ & Mercury & D & - & - \\
\hline $7440-02-0$ & Nickel & NR & - & - \\
\hline $7440-66-6$ & $\operatorname{Zinc}$ & D & - & - \\
\hline
\end{tabular}

(1) From IRIS (EPA, 1996a). When IRIS values were unavailable, HEAST (EPA, 1995) was used.

An asterisk $(*)$ indicates that values taken from HEAST.

A double asterisk (**) indicates that value from EPA (1996b - personal communication with the Superfund Technical Support Center).

NR = not reported, UR = under review

A dash ("-") indicates that this item is not applicable, or that there is no entry in either IRIS or HEAST.

(2) CAG = EPA Carcinogen Assessment Group

(3) Slope factors were derived by multiplying the slope factor for 2,3,7,8 TCDD by the following toxicity equivalency factors:

0.01 for HpCDD and HpCDF, 0.1 for HxCDF, and 0.001 for OCDD and OCDF.

The oral absorption factor for 2,3,7,8 TCDD was also used. 
TABLE 3.34

RADIONUCLIDE CARCINOGENICITY - SLOPE FACTORS (1)

SAVANNAH RIVER SITE

F-AREA BURNING/RUBBLE PITS AND RUBBLE PIT

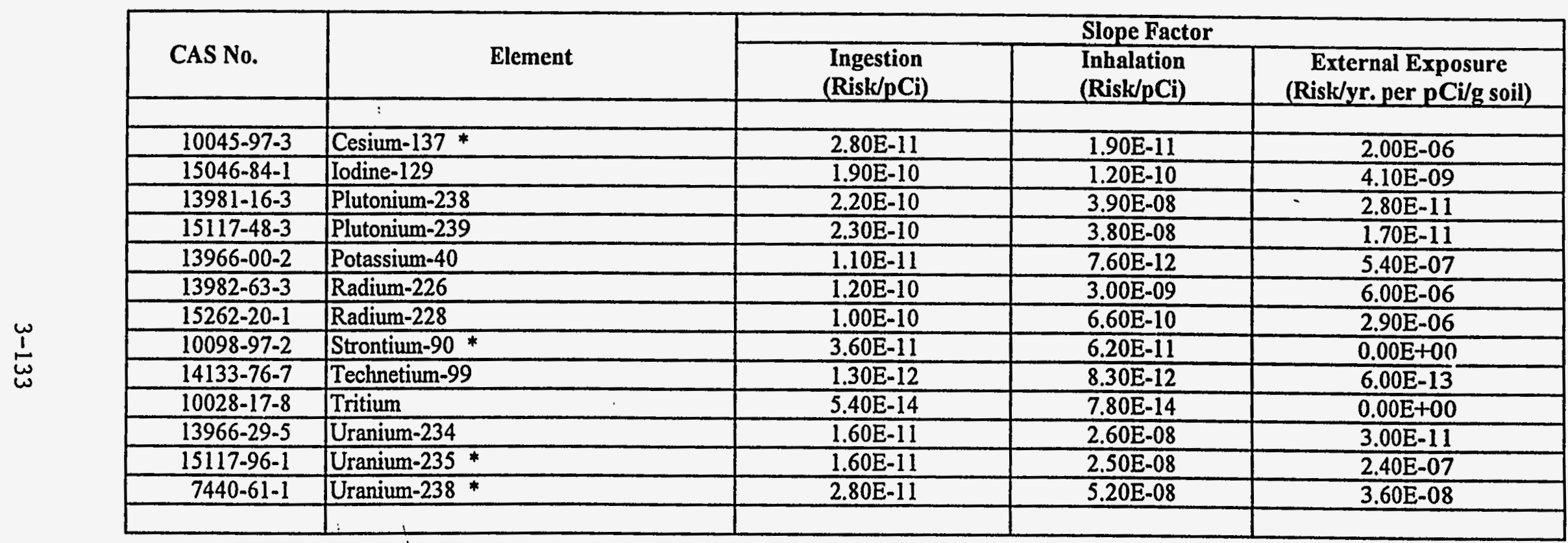

(1) HEAST (1993a)

(2) Values for Radium-226 (CAS No. 13982-63-3) and decay chain products.

* Values for isotope and decay chain products. 
TABLE 3.35

Savannah River Site

Burning/Rubble Pits (231-F, 231-1F)

Total Carcinogenic and Noncarcinogenic Risk by Medium

\begin{tabular}{|c|c|}
\hline Total RME Cancer Risk & Total RME Hazard Quotient \\
\hline
\end{tabular}

\section{Current Visitor ${ }^{(2)}$}

\begin{tabular}{lcc} 
Soil 0-2' bls & $3 \mathrm{E}-08$ & $2 \mathrm{E}-02$ \\
Soil 0-4'bls & - & - \\
Surface water & $1 \mathrm{E}-08$ & $8 \mathrm{E}-04$ \\
Sediment & $3 \mathrm{E}-07$ & $6 \mathrm{E}-04$ \\
Groundwater & - & - \\
\cline { 2 - 3 } \multicolumn{1}{r}{ Receptor Total } & $3 \mathrm{E}-07$ & $3 \mathrm{E}-02$
\end{tabular}

Future Worker $^{(3)}$

\begin{tabular}{lcc} 
Soil 0-2'bls & 5E-06 & $3 \mathrm{E}-02$ \\
Soil 0-4'bls & $6 \mathrm{E}-06$ & $3 \mathrm{E}-02$ \\
Surface water & - & - \\
Sediment & - & - \\
Groundwater & $2 \mathrm{E}-05$ & $4 \mathrm{E}-01$ \\
\cline { 2 - 3 } \multicolumn{1}{r}{ Receptor Total } & 2E-05 & $4 \mathrm{E}-01$
\end{tabular}

Future Resident $^{(4)}$

\begin{tabular}{lrr} 
Soil 0-2' bls & 2E-05 & $6 \mathrm{E}-01$ \\
Soil 0-4'bls & $2 \mathrm{E}-05$ & $7 \mathrm{E}-01$ \\
Surface water & TE-07 & $2 \mathrm{E}-02$ \\
Sediment & $3 \mathrm{E}-06$ & $4 \mathrm{E}-03$ \\
Groundwater & $\mathbf{7 E - 0 5}$ & $3 \mathrm{E}+00$ \\
\hline \multicolumn{1}{c}{ Receptor Total } & $\mathbf{9 E - 0 5}$ & $\mathbf{4 E + 0 0}$
\end{tabular}

Note:

1. RME - reasonable maximum exposure

2. Dermal exposure to surface water and ingestion and dermal exposure to sediment are considered complete pathways for the current visitor.

3. Surface water and sediment pathways are considered incomplete for the future worker due to site controls.

4. Combined actult/child future resident used except where child resident used for ingestion and dermal contact with surface water and sediment. Risk and hazards were calculated for both $0-2 \mathrm{ft}$ and $0-4 \mathrm{ft}$ soil intervals; receptor totals reflect the interval with the highest risk estimale.

-_ Indicates incomplete pathway for this receptor at this site and medium NC - not calculable due to lack of toxicity dats for COPC. BOLD -Receptor risk equals or exceeds USEPA target risk range of 1 E-06 to 1 E-04 or hazard quotient of 1. 
TABLE 3.36

Savannah River Site

Rubble Pit (231-2F)

Total Carcinogenic and Noncarcinogenic Risk by Medium

\begin{tabular}{|l|l|}
\hline Total RME Cancer Risk $^{(1)}$ & Total RME Hazard Quotient \\
\hline
\end{tabular}

Current Visitor ${ }^{(2)}$

\begin{tabular}{lcc} 
Soil 0-2' bls & $2 \mathrm{E}-07$ & $3 \mathrm{E}-03$ \\
Soil 0-4'bls & - & - \\
Surface water & $1 \mathrm{E}-08$ & $8 \mathrm{E}-04$ \\
Sediment & $3 \mathrm{E}-07$ & $6 \mathrm{E}-04$ \\
Groundwater & - & - \\
\cline { 2 - 3 } \multicolumn{1}{r}{ Receptor Total } & $5 \mathrm{E}-07$ & $4 \mathrm{E}-03$
\end{tabular}

Future Worker $^{(3)}$

\begin{tabular}{lcc} 
Soil 0-2' bls & 5E-E6 & 1E-01 \\
Soil 0-4'bls & 5E-06 & $9 \mathrm{E}-02$ \\
Surface water & - & - \\
Sediment & - & - \\
Groundwater & 2E-05 & $4 \mathrm{E}-01$ \\
\cline { 2 - 3 } \multicolumn{1}{l}{ Receptor Total } & 2E-05 & 5E-01
\end{tabular}

Future Resident ${ }^{\left({ }^{(t)}\right.}$

\begin{tabular}{lrc} 
Soil 0-2' bls & 2E-05 & 2E+00 \\
Soil 0-4'bls & 2E-05 & 2E+00 \\
Surface water & TE-07 & 2E-02 \\
Sediment & 3E-06 & $4 \mathrm{E}-03$ \\
Groundwater & 7E-05 & 3E+00 \\
\cline { 2 - 3 } \multicolumn{1}{c}{ Receptor Total } & 9E-05 & 5E+00
\end{tabular}

Note:

1. RME - ressonable maximum exposure

2. Dermal exposure to surface water and ingestion and dermal exposure to sediment are considered complete pathways for the current visitor.

3. Surface water and sediment pathways are considered incomplete for the future worker due to site controls.

4. Combined atulvchild future resident used except where child resident used for ingestion and dermal contact with surface water and sediment Risk and hazards were calculated for both $0-2 \mathrm{ft}$ and $0-4 \mathrm{ff}$ soil intervals; receptor totals reflect the interval with the higheat risk estimate.

- Indieates incomplete pathway for this receptor at this site and medium.

NC - not calculable due to lack of toxicity data for COPC. BOLD - Receptor risk equals or exceeds USEPA target risk range of 1 E-06 to 1 E-04 or hazard quotient of 1. 
TABLE 3.37

CARCINOGENIC AND NONCARCINOGENIC RISK SUMMARY BY RECEPTOR AND MEDIUM SAVANNAH RIVER SITE

BURNING/RUBBLE PITS 231-F AND 231-1F

\begin{tabular}{|c|c|c|c|c|c|c|c|c|c|c|c|}
\hline \multirow{2}{*}{$\begin{array}{c}\text { Receptor } \\
\text { Pathway }\end{array}$} & \multirow[b]{2}{*}{ Medium: (1) } & \multicolumn{5}{|c|}{ RME Cancer Risk ${ }^{(2)}$} & \multicolumn{5}{|c|}{ RME Hazard Quotient } \\
\hline & & SL 0-2' & SL 0.4' & SW & SD & GW & SL 0-2' & SL 0.4' & SW & SD & GW \\
\hline \multicolumn{12}{|l|}{ Current Visitor ${ }^{(3)}$} \\
\hline Ingestion & & $2 \mathrm{E}-08$ & -- & - & 1E-07 & -- & 5E-04 & -- & -- & 2E-04 & $-\infty$ \\
\hline Dermal Contact & & $5 E-09$ &.- & 1E-08 & 1E-07 & -- & $2 \mathrm{E}-02$ & -- & 8E-04 & 4E-04 & - \\
\hline Inhalation of Volatiles & & NC &.- & -- & -- & -- & $\mathrm{NC}$ & -- & -. & -. & -- \\
\hline Inhalation of Particulates & & $4 \mathrm{E}-11$ & - & - & -. & -- & $9 \mathrm{E}-06$ & - & -. & $\ldots$ & -- \\
\hline Medium Total & & $3 E-08$ & & $1 \mathrm{E}-08$ & $3 \mathrm{E}-07$ & & $2 \mathrm{E}-02$ & & $8 \mathrm{E}-04$ & $6 \mathrm{E}-04$ & \\
\hline Receptor Total & & & & & & & & & & & \\
\hline \multicolumn{12}{|l|}{ Euture Worker ${ }^{(4)}$} \\
\hline Ingestion & & 5E-06 & $5 E-06$ & -- & .- & 2E-05 & $2 \mathrm{E}-02$ & 2E-02 & -. & -- & 4E-01 \\
\hline Dermal Contact & & 1E-06 & $1 \mathrm{E}-06$ & -- & -- & -- & $7 \mathrm{E}-03$ & $8 \mathrm{E}-03$ & -- & -- & -- \\
\hline Inhalation of Volatiles & & $\mathrm{NC}$ & NC &.- & -. & - & $\mathrm{NC}$ & NC & - & -- & - \\
\hline Inhalation of Particulates & & $8 \mathrm{E}-09$ & $1 \mathrm{E}-08$ &.- & -- & - & NC & $3 E-04$ & $\ldots$ & -. & -. \\
\hline Medium Total & & $5 E-06$ & $6 \mathrm{E}-06$ & & & $2 \mathrm{E}-05$ & $3 \mathrm{E}-02$ & 3E-02 & & & $4 \mathrm{E}-01$ \\
\hline Receptor Total & & & & & & & & & & & \\
\hline \multicolumn{12}{|l|}{ Euture Resident ${ }^{(5)}$} \\
\hline Ingestion & & 2E-05 & $2 \mathrm{E}-05$ & $4 \mathrm{E}-08$ & 1E-05 & $5 \mathrm{E}-05$ & $6 \mathrm{E}-01$ & $6 \mathrm{E}-01$ & 1E-03 & 1E-02 & $2 \mathrm{E}+00$ \\
\hline Dermal Contact & & 3E-06 & 3E-06 & 9E-07 & 2E-06 & 2E-07 & $2 \mathrm{E}-02$ & $3 \mathrm{E}-02$ & $2 \mathrm{E}-02$ & $3 \mathrm{E}-03$ & $8 \mathrm{E}-02$ \\
\hline Inhalation of Volatiles & & $\mathrm{NC}$ & NC & - & - & 1E-05 & NC & NC & -. & -. & $6 \mathrm{E}-01$ \\
\hline Inhalation of Particulates & & $8 \mathrm{E}-08$ & 1E-07 & - & -- & - & $\mathrm{NC}$ & $1 \mathrm{E}-02$ & - & -- & -- \\
\hline Medium Total & & $2 \mathrm{E}-05$ & $2 \mathrm{E}-05$ & $9 \mathrm{E}-07$ & $2 \mathrm{E}-05$ & $7 \mathrm{E}-05$ & $6 \mathrm{E}-01$ & $7 \mathrm{E}-01$ & $3 \mathrm{E}-02$ & $2 E-02$ & $3 \mathrm{E}+00$ \\
\hline Receptor Total & & & & & & & & & & & \\
\hline
\end{tabular}

(1) SL .- soil from 0-2' bls, and 0-4' bls; SD - sodiment; SW -- surface water, GW - ground water.

Risk and hazaris were calculated for both $0-2 \mathrm{ft}$ and $0-4 \mathrm{f}$. soil intervals; .eceptor totals reflect the interval with the highest risk estimate.

"--"Indicates incomplete patinway for this receptor at this unit and modium.

NC - not calculable due to lack of toxicity data for COPC.

(2) RME - reasonable maximum exposure

(3) Demal exposure to surface wates and ingestion and demnal exposure to sediment are considered complete pathways for the current visitor.

(4) Surface water and sediment pathways are considered incomplete for the future worker due to site controls.

(5) The future resident totals for a combined adult/child resident except ingestion and demal contzet with surface water and sodiment totals for child resident

BOLD - Receptor risk equals or exceeds USEPA target risk range of 1 E-06 to 1 E-04 or hazard quotient of 1. 
TABLE 3.38

CARCINOGENIC AND NONCARCINOGENIC RISK SUMMARY BY RECEPTOR AND MEDIUM

SAVANNAH RIVER SITE

RUBBLE PIT 231-2F

\begin{tabular}{|c|c|c|c|c|c|c|c|c|c|c|c|}
\hline \multirow{2}{*}{$\begin{array}{c}\begin{array}{c}\text { Receptor } \\
\text { Pathway }\end{array} \\
\end{array}$} & \multirow[b]{2}{*}{ Medium: ${ }^{(1)}$} & \multicolumn{5}{|c|}{ RME Cancer Risk ${ }^{(2)}$} & \multicolumn{5}{|c|}{ RME Hazard Quotient } \\
\hline & & SL 0-2' & SL 0-4 & SW & SD & GW & SL 0-2' & SL 0-4' & SW & SD & GW \\
\hline \multicolumn{12}{|l|}{ Current Visitor ${ }^{(3)}$} \\
\hline Ingestion & & 2E-08 & -- & - & $1 \mathrm{E}-07$ & -- & 2E-03 & - & - & $2 \mathrm{E}-04$ & -- \\
\hline Dermal Contact & & $3 E-09$ & -- & 1E-08 & 1E-07 & -- & SE-04 & - & 8E-04 & 4E-04 & -- \\
\hline Inhalation of Volatiles & & NC & - & - & - & - & NC & - & - & -- & -- \\
\hline Inhalation of Particulates & & 2E-07 & - & - & -- & - & $3 \mathrm{E}-06$ & - & - & - & -- \\
\hline Medium Total & & $2 \mathrm{E}-07$ & & $1 \mathrm{E}-08$ & 3E-07 & & $3 \mathrm{E}-03$ & & $8 \mathrm{E}-04$ & 6E-04 & \\
\hline Receptor Total & & & . & & & & & & & & \\
\hline \multicolumn{12}{|l|}{ Euture Worker ${ }^{(4)}$} \\
\hline Ingestion & & $4 E-06$ & 4E-06 & -- & - & $2 \mathrm{E}-05$ & 9E-02 & $8 \mathrm{E}-02$ & - & -- & 4E-01 \\
\hline Dermal Contact & & $6 \mathrm{E}-07$ & $6 \mathrm{E}-07$ & - & - & -- & $2 \mathrm{E}-02$ & $1 \mathrm{E}-02$ & - & -- & -. \\
\hline Inhalation of Volatiles & & $\mathrm{NC}$ & NC & - & -- & - & NC & $\mathrm{NC}$ & -- & - & -- \\
\hline Inhalation of Particulates & & $1 \mathrm{E}-08$ & 3E-09 & - & - & -- & $\mathrm{NC}$ & 5E-09 & - & - & - \\
\hline Medium Total & & 5E-06 & 5E-06 & & & $2 E-05$ & $1 \mathrm{E}-01$ & $9 \mathrm{E}-02$ & & & 4E-01 \\
\hline Receptor Total & & & & & & & & & & & \\
\hline \multicolumn{12}{|l|}{ Euture Resident ${ }^{(5)}$} \\
\hline Ingestion & & 2E-05 & 2E-05 & 4E-08 & 1E-05 & 5E-05 & $2 \mathrm{E}+00$ & $2 \mathrm{E}+00$ & $1 \mathrm{E}-03$ & $1 \mathrm{E}-02$ & $2 \mathrm{E}+00$ \\
\hline Dermal Contact & & 2E-06 & 2E-06 & 9E-07 & $2 \mathrm{E}-06$ & 2E-07 & $8 \mathrm{E}-02$ & $5 \mathrm{E}-02$ & 2E-02 & 3E-03 & $8 \mathrm{E}-02$ \\
\hline Inhalation of Volatiles & & $\mathrm{NC}$ & NC & -- & -- & 1E-05 & $\mathrm{NC}$ & NC & -- & - & $6 \mathrm{E}-01$ \\
\hline Inhalation of Particulates & & $1 \mathrm{E}-07$ & 3E-08 & - & -- & -- & NC & 2E-07 & -- & - & -- \\
\hline Medium Tolal & & $2 \mathrm{E}-05$ & $2 \mathrm{E}-05$ & $9 \mathrm{E}-07$ & $2 \mathrm{E}-05$ & $7 \mathrm{E}-05$ & $2 \mathrm{E}+00$ & $2 \mathrm{E}+00$ & $3 \mathrm{E}-02$ & $2 \mathrm{E}-02$ & $3 \mathrm{E}+00$ \\
\hline Receptor Total & & & & & & & & & & & \\
\hline
\end{tabular}

(1) SL -. soil from 0-2bls, and O-A' bls; SD - sodiment; SW - surface water, GW - ground water.

Risk and hazards were calculated for both $0-2 \mathrm{ft}$, and $0-4 \mathrm{fin}$. soil intervals; receptor totals reflect the interval with the highest risk estimate.

"-" Indicates incomplete pathway for this receptor at this unit and modium.

NC - not calculable due to lack of toxicity data for COPC.

(2) RME - resongble maximum exposune

(3) Demal exposure to surface water and ingestion and dernal exposure to sodiment are considered complete pathways for the current visitor.

(4) Surface water and sodiment pathways are considered incomplete for the future worker due to site controls.

(5) The future resident totals for a combined adultchild resident except ingestion and dermal contact with surface water and sediment totals for child resident.

BOLD - Receptor risk equals or exceeds EPA target risk range of 1 E-06 to $1 \mathrm{E}-04$ or hazard quatient of 1. 
TABLE 3.39

CHEMICALS CONTRIBUTING SIGNIFICANTLY TO HUMAN HEALTH RISK (1)

SAVANNAH RIVER SITE

BURNING/RUBBLE PITS 231-F AND 231-1F

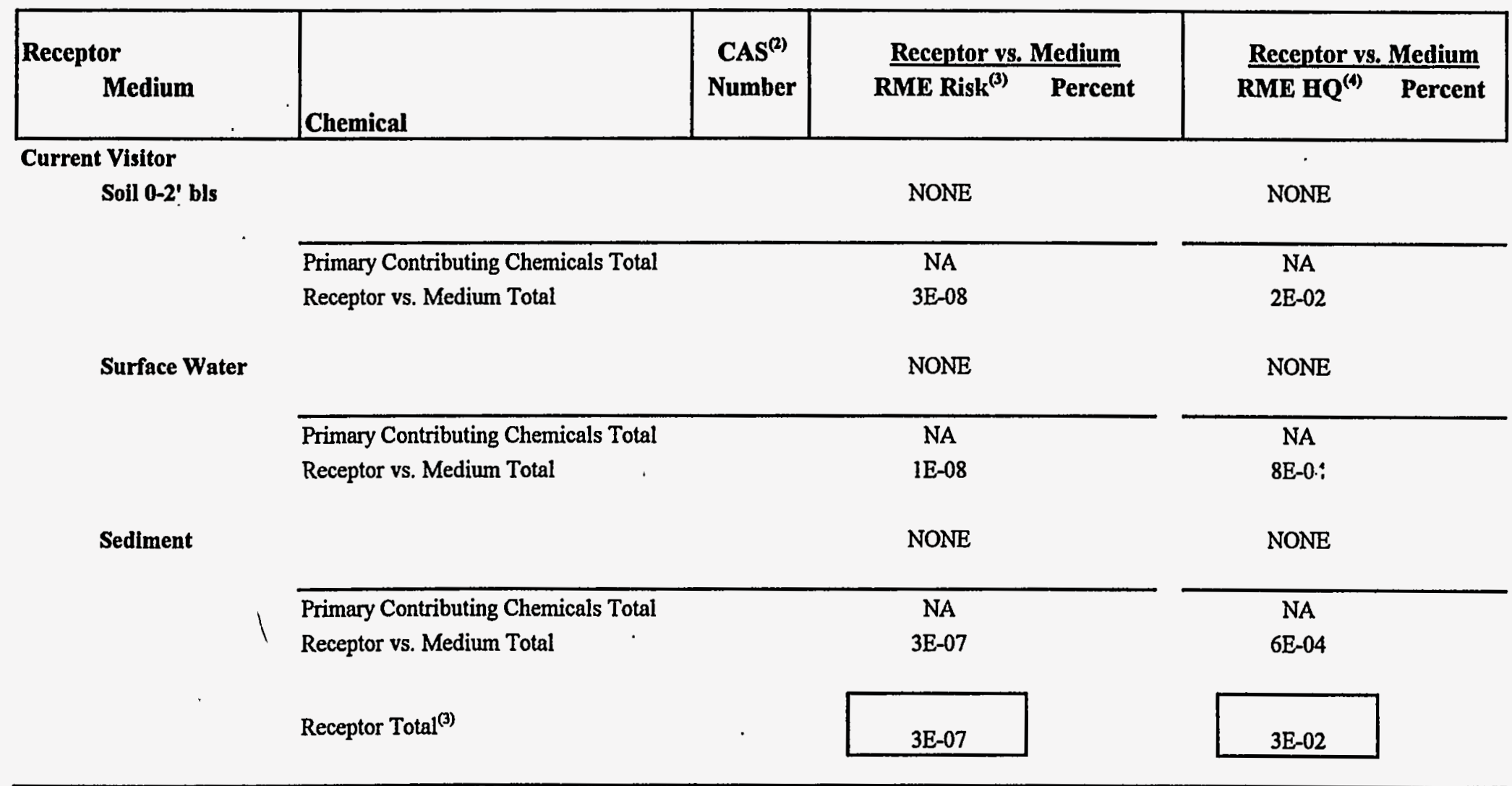


TABLE 3.39

CHEMICALS CONTRIBUTING SIGNIFICANTLY TO HUMAN HEALTH RISK (1)

SAVANNAH RIVER SITE

BURNING/RUBBLE PITS 231-F AND 231-1F

\begin{tabular}{|c|c|c|c|c|c|c|}
\hline \multirow{2}{*}{\begin{tabular}{|r}
$\begin{array}{r}\text { Receptor } \\
\text { Medium }\end{array}$ \\
Future Worker
\end{tabular}} & Chemical & $\begin{array}{c}\text { CAS }^{(2)} \\
\text { Number }\end{array}$ & $\frac{\text { Recentor v }}{\text { RME Risk }^{(3)}}$ & $\frac{\text { Ledium }}{\text { Percent }}$ & $\frac{\text { Receptor v }}{\text { RME HQ } \mathbf{Q}^{(4)}}$ & $\frac{\text { Medium }}{\text { Percent }}$ \\
\hline & & & & & & \\
\hline \multirow[t]{4}{*}{ Soil (0-2' bls) } & Arsenic & 7440382 & $1 \mathrm{E}-06$ & 23 & $8 \mathrm{E}-03$ & 27 \\
\hline & Heptachlorodibenzo-p-dioxin isomer & 37871004 & 1E-06 & 20 & & \\
\hline & Primary Contributing Chemicals Total & & $2 \mathrm{E}-06$ & 43 & 8E-03 & 27 \\
\hline & Receptor vs. Medium Total & & 6E-06 & & $3 \mathrm{E}-02$ & \\
\hline \multirow[t]{4}{*}{ Soll $\left(0-4^{\prime}\right.$ bls $)$} & Arsenic & 7440382 & 1E-06 & 25 & 9E-03 & 30 \\
\hline & Heptachlorodibenzo-p-dioxin isomer & 37871004 & 1E-06 & 20 & & \\
\hline & Primary Contributing Chemicals Total & - & $3 \mathrm{E}-06$ & 45 & 9E-03 & 30 \\
\hline & Receptor vs. Medium Total & & $6 \mathrm{E}-06$ & & $3 E-02$ & \\
\hline \multirow[t]{6}{*}{ Groundwater } & Arsenic & 7440382 & 1E-05 & 73 & 8E-02 & 23 \\
\hline & Carbon tetrachloride & 56235 & $3 \mathrm{E}-06$ & 14 & $8 \mathrm{E}-02$ & 22 \\
\hline & Tetrachloroethylene & 127184 & 1E-06 & 7 & 7E-03 & 2 \\
\hline & Primary Contributing Chemicals Total & & 2E-05 & 94 & 2E-01 & 47 \\
\hline & Receptor vs. Medium Total & & 2E-05 & & 4E-01 & \\
\hline & Receptor Total & & $2 \mathrm{E}-05$ & & $4 \mathrm{E}-01$ & \\
\hline
\end{tabular}


TABLE 3.39

CHEMICALS CONTRIBUTING SIGNIFICANTLY TO HUMAN HEALTH RISK (1)

SAVANNAH RIVER SITE

BURNING/RUBBLE PITS 231-F AND 231-1F

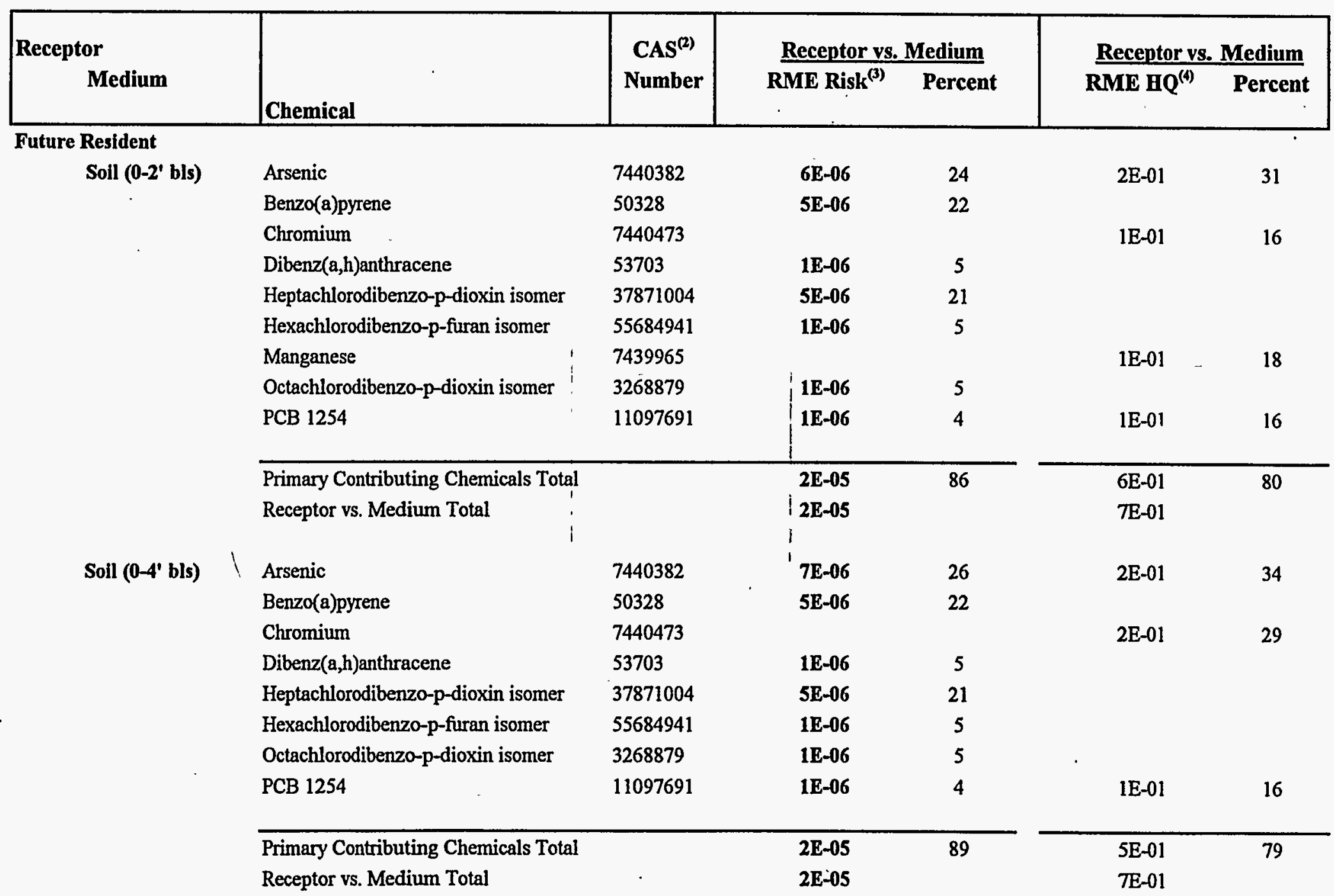


TABLE 3.39

CHEMICALS CONTRIBUTING SIGNIFICANTLY TO HUMAN HEALTH RISK (1)

SAVANNAH RIVER SITE

BURNING/RUBBLE PITS 231-F AND 231-1F

\begin{tabular}{|c|c|c|c|c|c|c|}
\hline $\begin{array}{l}\text { Receptor } \\
\text { Medium }\end{array}$ & Chemical & $\begin{array}{c}\text { CAS }^{(2)} \\
\text { Number }\end{array}$ & $\frac{\text { Receptor vs }}{\text { RME Risk }^{(3)}}$ & $\frac{\text { Ledium }}{\text { Percent }}$ & $\frac{\text { Receptor vs }}{\text { RME HQ }^{(4)}}$ & $\frac{\text { Medium }}{\text { Percent }}$ \\
\hline \multicolumn{7}{|l|}{ Future Resident (cont.) } \\
\hline \multirow[t]{3}{*}{ Surface Water } & \multicolumn{4}{|c|}{ NONE } & \multicolumn{2}{|l|}{ NONE } \\
\hline & Primary Contributing Chemicals Total & & NA & & NA & \\
\hline & Receptor vs. Medium Total & & $7 \mathrm{E}-07$ & & $2 \mathrm{E}-02$ & \\
\hline \multirow[t]{3}{*}{ Sediment } & \multicolumn{4}{|c|}{ NONE } & \multicolumn{2}{|l|}{ NONE } \\
\hline & Primary Contributing Chemicals otal & & $\mathrm{NA}$ & & NA & \\
\hline & Receptor vs. Medium Total & & 3E-06 & & $4 \mathrm{E}-03$ & \\
\hline \multirow[t]{9}{*}{ Groundwater } & Arsenic & 7440382 & 4E-05 & 58 & $5 \mathrm{E}-01$ & 18 \\
\hline & Cadmium & 7440439 & & & $6 \mathrm{E}-01$ & 21 \\
\hline & Carbon tetrachloride & 56235 & 1E-05 & 22 & $1 E+00$ & 33 \\
\hline & Dichloromethane & 75092 & 3E-06 & 4 & $4 \mathrm{E}-02$ & 1 \\
\hline & Tetrachloroethylene & 127184 & 8E-06 & 12 & $9 \mathrm{E}-02$ & 3 \\
\hline & Manganese & 7439965 & & & $6 \mathrm{E}-01$ & 20 \\
\hline & \multicolumn{2}{|l|}{ Primary Contributing Chemicals Total } & 6E-05 & 95 & $3 \mathrm{E}+00$ & 96 \\
\hline & \multicolumn{2}{|l|}{ Receptor vs. Medium Total } & 7E-05 & & \multicolumn{2}{|l|}{$3 \mathrm{E}+00$} \\
\hline & \multicolumn{2}{|l|}{ Receptor Total } & $1 \mathrm{E}-04$ & & $4 \mathrm{E}+00$ & \\
\hline
\end{tabular}

1. Chemical for which calculated cancer risk exceeded $10^{-6}$ or hazard quotient exceeded 0.1 .

2. Chemical Abstracts Service.

3. Carcinogenic Risk

4. "Receptor Total" combines "Receptor vs. Medium" totals across media for a given receptor, where applicable.

X.XE+XX: Italicized, bold print represents carcinogenic risk exceeding USEPA minimum target risk of 1 E-06. 
TABLE 3.40

\section{CHEMICALS CONTRIBUTING SIGNIFICANTLY TO HUMAN HEALTH RISK (1) \\ SAVANNAH RIVER SITE \\ RUBBLE PIT 231-2F}

\begin{tabular}{|c|c|c|c|c|}
\hline $\begin{array}{l}\text { Receptor } \\
\text { Medium }\end{array}$ & Chemical & $\begin{array}{c}\text { CAS }^{(2)} \\
\text { Number }\end{array}$ & $\underset{\text { RME Risk }^{(3)} \quad \text { Percent }}{\text { Receptor ys, Medium }}$ & $\begin{array}{c}\text { Recentor vs. Medium } \\
\text { RME HQ }^{(4)} \quad \text { Percent }\end{array}$ \\
\hline \multirow[t]{2}{*}{$\begin{array}{l}\text { Current Visitor } \\
\text { Soil 0-2' bls }\end{array}$} & & & NONE & NONE \\
\hline & $\begin{array}{l}\text { Primary Contributing Chemicals Total } \\
\text { Receptor vs. Medium Total }\end{array}$ & & $\begin{array}{c}\text { NA } \\
2 \mathrm{E}-07\end{array}$ & $\begin{array}{c}\text { NA } \\
\text { 3E-03 }\end{array}$ \\
\hline Surface Water & & & NONE & NONE \\
\hline & $\begin{array}{l}\text { Primary Contributing Chemicals Total } \\
\text { Receptor vs. Medium Total }\end{array}$ & & $\begin{array}{c}\text { NA } \\
1 \mathrm{E}-08\end{array}$ & $\begin{array}{c}\text { NA } \\
8 \mathrm{E}-04\end{array}$ \\
\hline Sediment & & & NONE & NONE \\
\hline & $\begin{array}{l}\text { Primary Contributing Chemicals Total } \\
\text { Receptor vs. Medium Total }\end{array}$ & & $\begin{array}{c}\text { NA } \\
\text { 3E-07 }\end{array}$ & $\begin{array}{c}\text { NA } \\
6 \mathrm{E}-04\end{array}$ \\
\hline$i$ & Receptor Total ${ }^{(3)}$ & & $5 \mathrm{E}-07$ & 4E-03 \\
\hline
\end{tabular}


TABLE 3.40

CHEMICALS CONTRIBUTING SIGNIFICANTLY TO HUMAN HEALTH RISK (1)

SAVANNAH RIVER SITE

RUBBLE PIT 231-2F

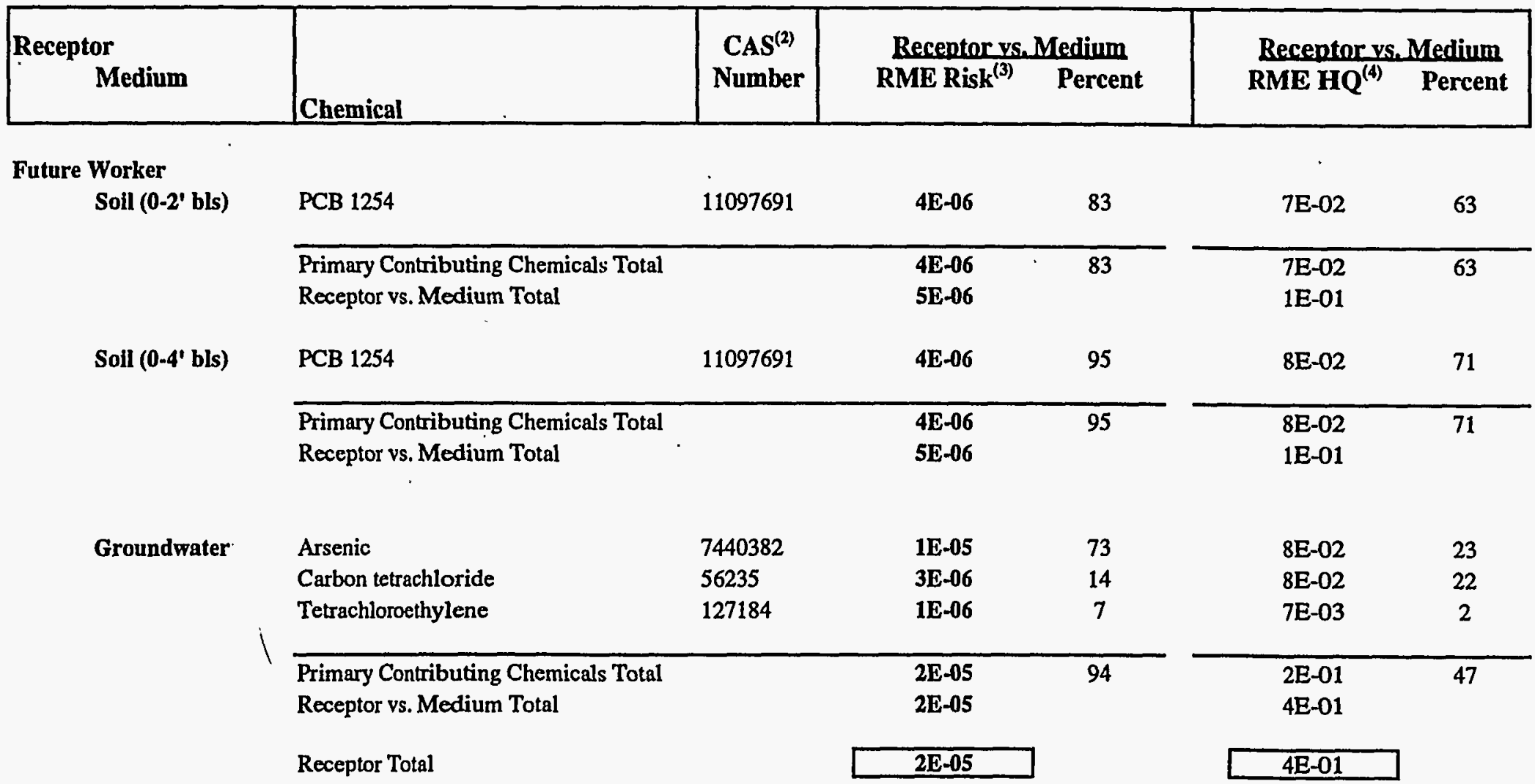


TABLE 3.40

CHEMICALS CONTRIBUTING SIGNIFICANTLY TO HUMAN HEALTH RISK (1)

SAVANNAH RIVER SITE

RUBBLE PIT 231-2F

\begin{tabular}{|c|c|c|c|c|c|}
\hline $\begin{array}{l}\text { Receptor } \\
\text { Medium }\end{array}$ & Chemical & $\begin{array}{c}\text { CAS }^{(2)} \\
\text { Number }\end{array}$ & $\begin{array}{l}\text { Receptor ys. Medium } \\
\text { RME Risk }^{(3)} \quad \text { Percent }\end{array}$ & $\begin{array}{l}\text { Recentor ys } \\
\text { RME HQ }^{(4)}\end{array}$ & $\frac{\text { Medium }}{\text { Percent }}$ \\
\hline \multicolumn{6}{|l|}{ Future Resident } \\
\hline \multirow[t]{4}{*}{ Soil $(0-2 '$ bls $)$} & Chromium & 7440473 & & 5E-01 & 22 \\
\hline & PCB 1254 & 11097691 & $2 \mathrm{E}-05$ & $2 \mathrm{E}+00$ & 88 \\
\hline & Primary Contributing Chemicals Total & & $2 \mathrm{E}-05$ & $2 \mathrm{E}+00$ & 110 \\
\hline & Receptor vs. Medium Total & & $2 \mathrm{E}-05$ & $2 \mathrm{E}+00$ & \\
\hline \multirow[t]{4}{*}{ Soil (0-4' bls) } & Chromium & 7440473 & & $1 \mathrm{E}-01$ & 7 \\
\hline & РCB 1254 & 11097691 & $2 \mathrm{E}-05$ & $2 \mathrm{E}+00$ & 88 \\
\hline & Primary Contributing Chemicals Total & & $2 \mathrm{E}-05$ & $2 \mathrm{E}+00$ & 95 \\
\hline & Receptor vs. Medium Total & & $2 \mathrm{E}-05$ & $2 \mathrm{E}+00$ & \\
\hline \multirow[t]{3}{*}{ Surface Water } & & & NONE & NONE & \\
\hline & Primary Contributing Chemicals Total & & NA & NA & \\
\hline & Receptor vs. Medium Total & & 7E-07 & $2 \mathrm{E}-02$ & \\
\hline \multirow[t]{3}{*}{ Sediment } & & & NONE & NONE & \\
\hline & Primary Contributing Chemicals Total & & NA & NA & \\
\hline & Receptor vs. Medium Total & & 3E-06 & 4E-03 & \\
\hline
\end{tabular}


BASELINE RISK ASSESSMENT RUBBLE PIT

TABLE 3.40

CHEMICALS CONTRIBUTING SIGNIFICANTLY TO HUMAN HEALTH RISK (1)

SAVANNAH RIVER SITE

RUBBLE PIT 231-2F

\begin{tabular}{|c|c|c|c|c|c|c|}
\hline $\begin{array}{r}\text { Receptor } \\
\text { Medium }\end{array}$ & Chemical & $\begin{array}{c}\text { CAS }^{(2)} \\
\text { Number }\end{array}$ & $\begin{array}{l}\text { Receptor ys } \\
\text { RME Risk }{ }^{(3)}\end{array}$ & Ledium & $\frac{\text { Receptor vs }}{\text { RME HQ }^{(4)}}$ & $\begin{array}{l}\text { Medium } \\
\text { Percent }\end{array}$ \\
\hline \multicolumn{7}{|l|}{ Future Resident (cont.) } \\
\hline \multirow[t]{9}{*}{ Groundwater } & Arsenic & 7440382 & 4E-05 & 58 & 5E-01 & 18 \\
\hline & Cadmium & 7440439 & & & $6 \mathrm{E}-01$ & 21 \\
\hline & Carbon tetrachloride & 56235 & 1E-05 & 22 & $1 \mathrm{E}+00$ & 33 \\
\hline & Dichloromethane & 75092 & 3E-06 & 4 & 4E-02 & 1 \\
\hline & Tetrachloroethylene & 127184 & 8E-06 & 12 & $9 \mathrm{E}-02$ & 3 \\
\hline & Manganese & 7439965 & & & $6 \mathrm{E}-01$ & 20 \\
\hline & Primary Contributing Chemicals Total & & 6E-05 & 95 & $3 \mathrm{E}+00$ & 96 \\
\hline & Receptor vs. Medium Total & & 7E-05 & & $3 E+00$ & \\
\hline & Receptor Total & & 1E-04 & & $5 E+00$ & \\
\hline
\end{tabular}

1. Chemical for which calculated cancer risk exceeded $10^{-6}$ or hazard quotient exceeded 0.1 .

2. Chemical Abstracts Service.

3. Carcinogenic Risk

4. "Receptor Total" combines "Receptor vs. Medium" totals across media for a given receptor, where applicable.

$X . X E+X X$ : Italicized, bold pritt represents carcinogenic risk exceeding USEPA minimum target risk of 1 E-06 
TABLE 3.41

Savannah River Site

Buming/Rubble Pits (231-F, 231-1F)

Radionuclide Carcinogenic Risk Summary by Receptor and Medium

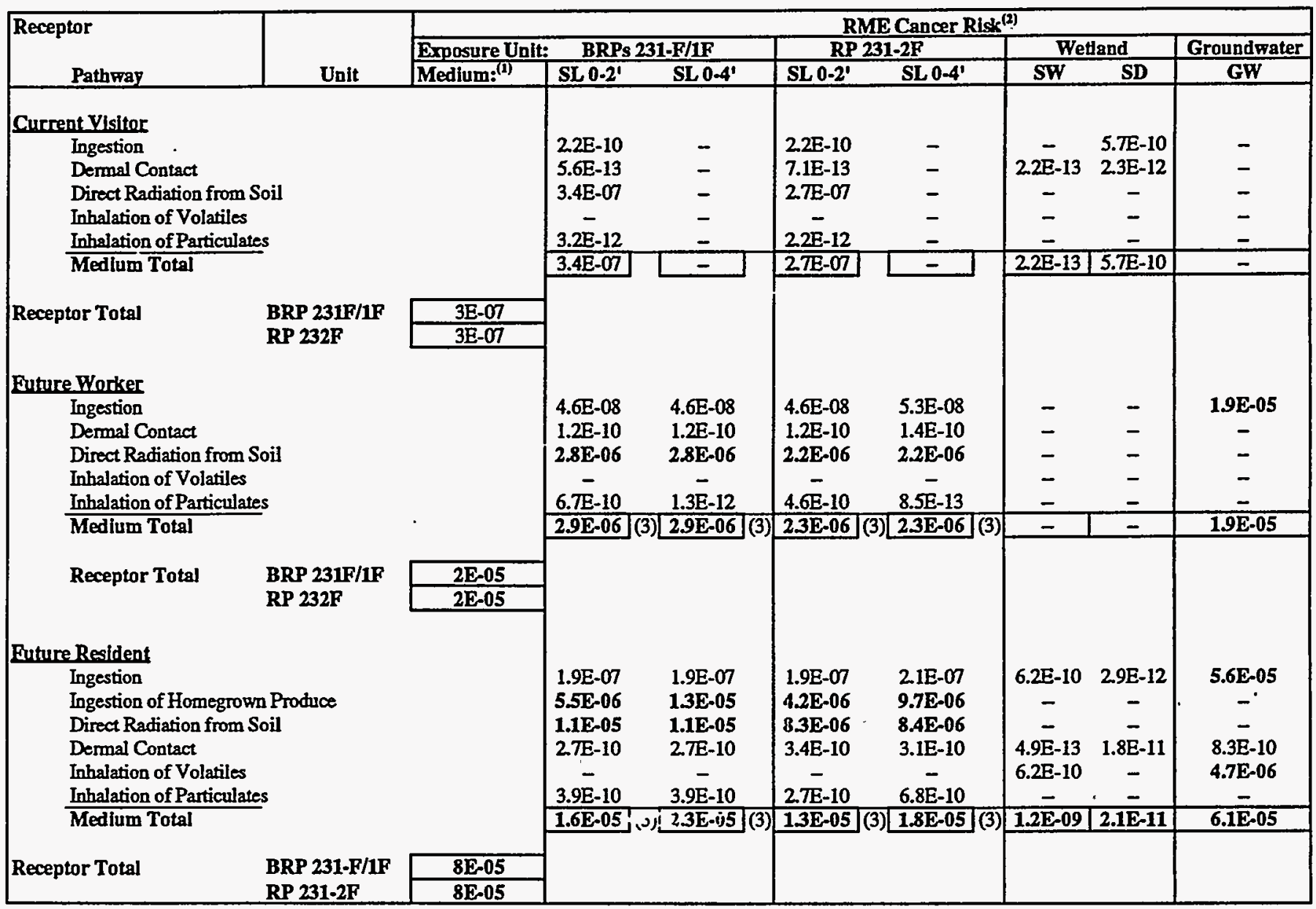

(1) SL- soil from 0-2 bis, and O-4' bls, SD - sediment; SW - surface water, GW - groondwater.

-- Indicates incomplete pathway for this receptor at this sire and medium.

NC - nox calalable doe to lact of toxiciny datu for COPC.

(2) RME - ressonble maximen exposte

(3) Risks and bazerds were derived for future receptors eypeed wo both the 0-2ft and 0-4ft intervals. Given that the risks

frezerds calcalated are, in most cases, equal, the total receptar rist/hazard reflects only the 0-4 finterval.

BOWD - Receptar risk equals or excods USEPA target riat nange of 1 E-06 to 1 E-04. 
TABLE 3.42

CHEMICALS WITHOUT TOXICITY VALUES

SAVANNAH RIVER SITES

BURNING/RUBBLE PITS - F AREA

\begin{tabular}{|l|}
\hline Constituent \\
\hline \\
Semivolatiles \\
Acenaphthylene \\
Benzo(g,h,i)perylene \\
Dibenzofuran \\
Diethylphthalate \\
2-Methylnaphthalene \\
Phenanthrene \\
Pesticides \\
delta-BHC \\
Endrin ketone \\
Metals \\
Lead \\
\hline
\end{tabular}


SECTION 4

ECOLOGICAL RISK ASSESSMENT 


\subsection{ECOLOGICAL RISK ASSESSMENT}

The purpose of the ecological risk assessment component of the BRA is to evaluate the likelihood that adverse ecological effects are occurring or may occur as a result of exposure to chemicals associated with the unit. The methodology used in this assessment was based on and complies with the intent of the 'Draft Ecological Risk Assessors' Guide for Evaluation of Waste Units on the Savannah River Site (WSRC, 1993), the Framework for Ecological Risk Assessment (EPA, 1992f), and the Risk Assessment Guidance for Superfund, Volume II, Environmental Evaluation Manual (EPA, 1989b).

The ecological assessment methodology consists of four major elements: characterization of the unit and the ecological components it may affect, exposure assessmeni, toxicity assessment, and risk characterization. The first element, unit characterization, is described in Subsections 4.1 through 4.3.

- Subsection 4.1, "Identification of Chemicals of Potential Concern," identifies chemicals of potential concern (COPCs) at the unit;

- Subsection 4.2, "Environmental Setting," describes the environmental setting, including the physical and ecological setting, the potentially complete exposure pathways (based on a conceptual site model), and any ecological effects observed at the unit; and

- Subsection 4.3, "Endpoint Selection," discusses the selection of endpoints related to the ecological values to be protected at the unit.

The second major element of the methodology is described in Subsection 4.4, "Exposure Assessment." The exposure assessment describes the spatial distribution of COPCs at the unit, the spatial and temporal distribution of ecological receptors, and the magnitude and patterns of exposure that may result from the overlap of these distributions. Daily intakes of COPCs through exposure to environmental media are quantified based on a range of potential exposures.

The assessment of ecological effects that potentially may result from exposures of receptors to COPCs at the unit is discussed in Subsection 4.5, "Effects Assessment." This section includes evaluation of the potential toxicity of each COPC in each medium, including the derivation of toxicity benchmark values and bioconcentration factors. 
Section 4.6, "Risk Characterization," integrates the results of the preceding elements of the assessment. It estimates risk with respect to the assessment endpoints, based on the predicted exposure to and toxicity of each COPC. The risk characterization also summarizes the ecological risk and interprets its ecological significance, discusses uncertainties in the assessment, and based on the weight of evidence, identifies those chemicals that should be considered chemicals of concern (COCs) for each medium and location at the unit.

\subsection{Identification of Chemicals of Potential Concern}

As described in Section 2.0, environmental media were sampled at the F-Area BRPs during investigations to characterize the nature and extent of contamination. COPCs were identified in each medium following qualification and evaluation of analytical data and comparison to unit background concentrations (for inorganics in soil, including radionuclides), and preliminary screening values. The analytes detected in soil samples are listed in Tables 4.1 through 4.4 , along with their frequency of detection and maximum detected concentration.

Data for BRPs 231-F/1F were combined because they are contiguous; data for the ephemeral wetland and RP 231-2F were evaluated independently. The data were evaluated in accordance with EPA data validation guidance contained in Risk Assessment Guidance for Superfund: Volume I - Human Health Evaluation Manual (EPA, 1989a). All data qualifiers were considered. Unit-specific background data were used to identify elevated concentrations of inorganic analytes and radionuclides related to the unit. Organic compounds were screened against background for comparison purposes only; no organic compounds were eliminated from further assessment as a result of screening against background.

After combining analytical data and eliminating those analytes not detected in any samples in a particular medium, the analytical data were evaluated on the basis of quality with respect to sample quantitation limits, laboratory qualifiers and codes, and blanks. Data were selected for use in the BRA based on EPA guidance (EPA, 1989a) and included data with no qualifiers, data with qualifiers that indicated uncertainties in concentrations but not in chemical identification, and data for chemical concentrations detected at levels significantly elevated above concentrations detected in associated sample blanks. Contaminant data that were rejected were those with an $\mathbf{R}$ (unreliable) qualifier and those with a $\mathrm{J} / \mathrm{V}$ result qualifier/analysis qualifier pair for groundwater data or a $U / V$ result qualifier/analysis qualifier pair for soil, surface water, or sediment data. 
Following the elimination of unreliable data, concentrations in soil were compared to appropriate background levels, as shown in Tables 4.1 through 4.4. Non-radioactive inorganic analytes and naturally occurring radionuclides in each medium were eliminated from the unit risk assessment if the maximum detected concentration was less than twice the mean background concentration (EPA, 1991d).

Those analytes not eliminated by the background screening were compared to preliminary screening values using the EPA Region IV methodology (EPA, 1995a). For soil screening, the maximum concentration of each analyte detected in the soil intervals used for current and future risk assessment was incorporated into a dietary intake equation and then compared to a risk-based dietary benchmark. The screening intake equation, based on EPA Region IV Guidance for Wildlife Screening Values (EPA, 1995), conservatively assumed that the entire diet of the receptor would consist of soil, all of which would contain the maximum detected concentration of the analyte. Receptors were not unit-specific, but instead, were the animals used in the analytespecific toxicity studies. However, since an oldfield mouse (Peromyscus polionotus) was selected as a unit-specific receptor for the later phases of the risk assessment, toxicity tests using species most closely related to a mouse were sought. The screening intake equation is:

$$
\begin{aligned}
& \mathrm{SI}=\left(\mathrm{SC}_{\max }\right)(\mathrm{f})(\mathrm{l} / \mathrm{w}) \\
& \text { where: } \\
& \text { SI = screening intake in milligrams per kilogram body weight per day }(\mathrm{mg} / \mathrm{kg} / \text { day) } \\
& \mathrm{SC}_{\max }=\text { maximum soil concentration in } \mathrm{mg} / \mathrm{kg} \\
& \text { f = feeding rate of test animal in } \mathrm{kg} / \mathrm{d} \text { dry weight ingested } \\
& \mathrm{w} \quad=\text { body weight of test animal in } \mathrm{kg}
\end{aligned}
$$

Maximum concentrations of the soil analytes are shown in Tables 4.1 through 4.4.

Toxicity benchmarks reflecting a dietary no observed adverse effect level (NOAEL) were sought for benchmarks in the soil screening. Where NOAEL tests could not be found, other tests with endpoints of lowest observed adverse effect level (LOAEL) or lethal dose for 50 percent of the test animals $\left(L D_{50}\right)$ were used, with appropriate uncertainty factors to equate the test to a NOAEL. Uncertainty factors were those published by EPA Region VIII (EPA, 1994c), as follows: 


\begin{tabular}{|c|c|c|c|}
\hline \multicolumn{4}{|l|}{$\begin{array}{l}\text { Category of Uncertainty } \\
\text { Study Duration Extrapolation }\end{array}$} \\
\hline \multicolumn{4}{|l|}{ Study Duration Extrapolation } \\
\hline Chronic studies, equilibrium attained & & 1 & \\
\hline Subchronic studies & & 5 & \\
\hline Acute studies & & 10 & \\
\hline Single dose & & 20 & \\
\hline Unknown & & 20 & \\
\hline Endpoint Extrapolation & Nonlethal & & Lethal \\
\hline No observed effects level . & 1 & & 1 \\
\hline No observed adverse effects level & 1 & & 1 \\
\hline Lowest observed effects level & 3 & & 10 \\
\hline Lowest observed adverse effects level & 5 & & 10 \\
\hline $\begin{array}{l}\text { Effect concentration to } 50 \% \text { of test } \\
\text { organisms }\end{array}$ & 10 & & 15 \\
\hline Unknown & 10 . & & 15 \\
\hline
\end{tabular}

1 The product of the appropriate uncertainty value from each uncertainty category becomes the . uncertainty factor applied to develop the chemical-specific screening benchmark.

Toxicity tests and test endpoints used to develop the preliminary screening values for soil contaminants are shown in Table 4.5. Feeding rates and body weights of the animals used in the toxicity tests are shown in Tables 4.6 through 4.12. Results of the soil screening are presented in Tables 4.6 through 4.12 .

For surface water in the ephemeral wetland, the maximum concentration of each detected analyte was compared to Region IV Freshwater Surface Water Screening Values for Hazardous Waste Sites (EPA, 1995a). These values generally represent federal chronic ambient water quality criteria (AWQC) values for protection of aquatic life. The AWQC for lead is based on hardness of the water; in the absence of unit-specific hardness data, a default hardness value of $50 \mathrm{mg} / \mathrm{L}$ as $\mathrm{CaCO}_{3}$ was used to determine the lead criterion. Chemicals detected in surface water, frequency of detection, and maximum detected concentration are shown in Table 4.14, along with the comparison to Region IV screening values. No background screening was done for surface water chemicals as no equivalent background sample location was available. 
For sediment in the ephemeral wetland, the maximum concentration of each detected analyte was compared to Region IV Sediment Screening Values for Hazardous Waste Sites (EPA, 1995a). These values are derived from effects-based data reported by the state of Florida, the National Oceanic and Atmospheric Administration, and Long et -al. (1995). Chemicals detected in sediment, frequency of detection, and maximum detected concentration are shown in Table 4.16, along with the comparison to Region IV screnning values (EPA, 1995a). No background screening was done for sediment chemicals as no equivalent background sample location was available.

The ecological COPCs identified as a result of the data eve'uation and screening are summarized by medium and location in Table 4.18. Identification as a COPC was considered an indication that a contaminant warranted further evaluation with regard to its potential to adversely affect ecological assessment endpoints.

\subsubsection{Exposure Point Concentrations}

An exposure point concentration is the concentration of a COPC in an environmental medium at the location where a receptor contacts the medium. Exposure point concentrations were calculated based on the reasonable maximum exposure (RME) concentration, a conservative concentration that is still within the range of possible exposures, for each complete pathway. Sampling data collected during characterization investigations at the unit were used to calculate the exposure point concentrations of COPCs identified in surface soil, surface water, and sediment.

Surface soil sampling data were used to estimate exposure point concentrations of COPCs at the F-Area BRPs for current land use conditions. Data for BRPs 231-F/1F and for RP 231-2F included soil samples from a depth of 0 to $0.6 \mathrm{~m}(0$ to $2 \mathrm{ft}$ ). Risk from soil exposure under future land use conditions was estimated based on exposure point concentrations in soil from 0 to $1.2 \mathrm{~m}$ ( 0 to $4 \mathrm{ft}$ ) deep, to allow for the possibility of future excavation of deeper soils to the surface as a result of human activities such as construction or farming. Concentrations of COPCs measured in standing water at the wetland area to the west of the BRPs were used to estimate exposure point concentrations in surface water (assuming a constant concentration over time) for both the current and future conditions. Sediment exposure point concentrations were estimated from measured concentrations of COPCs in sediment at the wetland area for both current and future conditions. 
Because of the uncertainty associated with any estimate of exposure point concentration, the 95 percent upper confidence limit (UCL95) on the arithmetic mean was used as the RME concentration (EPA, 1989a). In cases where the UCL95 exceeded the maximum detected concentration, the maximum detected concentration was used to estimate the RME concentration, in accordance with EPA guidance (EPA, 1989a). For COPCs with both positive and nondetected results, sample -results reported as below the quantitation limit (non-detects) were included at one-half the quantitation limit as a proxy concentration in the calculation of the UCL95 (EPA, 1989a). RME exposure point concentrations were calculated for each COPC in each medium of concern (soil, surface water, and sediment) and are shown in Tables 4.1 through 4.4 for soil, Tables 4.14 and 4.15 for surface water, and Tables 4.16 and 4.17 for sediment. RME concentrations were derived by the procedure outlined in the human health risk assessment (Section 3).

\subsection{Environmental Setting}

\subsubsection{Characterization of Exposure Setting}

This section describes the physical and ecological characteristics of the F-Area BRPs and RP and the surrounding study area. The overall study area comprises approximately 80 hectares (200 acres), bounded on the west by Upper Three Runs Creek, on the south by Road C, on the east by. F Area, and on the north by an unnamed tributary of Upper Three Runs Creek (Figure 4.1). An ecological survey of this area was conducted in January 1994 for the purpose of characterizing existing habitats and mapping vegetative communities for use in the BRA. Additional information regarding the exposure setting was presented in Section 3.2 of the Human Health Baseline Risk Assessment.

\subsubsection{Physical Setting}

The F-Area BRPs and RP are located in the north-central portion of SRS, which is located within the Upper Loam Hills Region of the Coastal Plain Province (Myers et al., 1986). The topography of the area consists of sandhills deeply incised by streams. The unit is located on a ridge that extends in a northwesterly direction toward Upper Three Runs Creek. The area is characterized by significant relief, with elevations ranging from $36.6 \mathrm{~m} \cdot(120 \mathrm{ft})$ above mean sea level (msl) along Upper Three Runs Creek to $91.4 \mathrm{~m}$ (300 ft) msl near RP 231-2F. Soils are generally deep, loose, well-drained sands that are highly erodible, high in permeability, low in water capacity, and low in organic matter (USDA, 1990). 
Upper Three Runs Creek is the principal surface water feature in the vicinity of the unit (Figure 4.1). It is a relatively large, swiftly-flowing stream with significant depth and discharge and is surrounded by riparian wetlands. It bounds the study area to the north and west and eventually receives all drainage from the area of the unit. From this reach, Upper Three Runs Creek flows southwestward approximately $13.7 \mathrm{~km}$ (8.5 miles) to the Savannah River.

A small, unnamed tributary of Upper Three Runs Creek flows northwestward along the northern border of the study area. This tributary originates from the F-Area NPDES outfall F-001 and appears to be perennial. It is a small, shallow, gently-flowing stream with a sandy substrate. An intermittent stream (not shown on Figure 4.1), which carries stormwater runoff from the eastern portion of the ridge on which the unit is located, empties into the unnamed tributary northeast of the unit. This intermittent stream is characterized by a rather steep gradient, and small, isolated pools were present in the stream channel at the time of the field survey. Flow in the unnamed tributary increases noticeably with distance downstream from the confluence with the intermittent drainage, possibly indicating groundwater discharge to the tributary.

Stormwater drainage to the east and south from the unit location on the ridge flows principally toward Upper Three Runs Creek through an eroded ravine adjacent to the cross-tie pile, or collects in a small [0.1 hectare ( 0.2 acre)], ephemeral, palustrine wetland approximately $76 \mathrm{~m}$ $(250 \mathrm{ft})$ west of BRPs $231-\mathrm{F} / 1 \mathrm{~F}$. The wetland likely was formed due to human activity that resulted in the blockage of natural surface drainage routes and the impoundment of runoff. It appears to be an ephemeral pond, inundated during extremely heavy rainy periods and desiccated during normal to dry conditions (Metcalf and Eddy, 1993).

Another aspect of the physical setting that has a significant influence on the environmental conditions of the area is climate. The climate of the SRS region is warm temperate, characterized by hot summers and mild winters. The average annual temperature is $17.2^{\circ} \mathrm{C}\left(63^{\circ} \mathrm{F}\right)$. In winter, the average temperature is $8.9^{\circ} \mathrm{C}\left(48^{\circ} \mathrm{F}\right)$ and the average daily minimum is $2.8^{\circ} \mathrm{C}\left(37^{\circ} \mathrm{F}\right)$. In summer, the average temperature is $26.7^{\circ} \mathrm{C}\left(80^{\circ} \mathrm{F}\right)$ and the average daily maximum is $32^{\circ} \mathrm{C}\left(90^{\circ} \mathrm{F}\right)$. Average annual precipitation is approximately $122 \mathrm{~cm}$ (48 in.), consisting predominantly of rainfall, with little or no snowfall. The average annual wind speed is highest, $3.6 \mathrm{~m} / \mathrm{sec}(8 \mathrm{miles}$ per hour), in spring (USDA, 1990). 


\subsubsection{Ecological Setting Plant Communities}

Based on field observations and interpretation of infrared aerial photographs, eight major vegetative community types were identified within the study area. As noted in a previous study that described the major plant community types of the Savannah River Site (Workman and McLeod, 1990), vegetative patterns were found to be dependent on topography, soil type, moisture, and disturbance activities. Scientific and common names of plant species used in this characterization are those described by Radford et al. (1968). The following plant community types were identified and are mapped in Figure 4.1:

Community Type No. 1: Consists predominantly of shortleaf (Pinus echinata), loblolly (Pinus taeda), and longleaf (Pinus palustris) pine, with a small component of sweetgum (Liquidambar styraciflua) and blackgum (Nyssa sylvatica) in the overstory. Dominant species in the sapling/ shrub stratum included sparkleberry (Vaccinium arboreum) and winged sumac (Rhus copallina). Broom sedge (Andropogon virginicus) and yellow jessamine (Gelsemium sempervirens) were the dominant herbaceous species present. The area of this community is approximately $9 \mathrm{ha}(21 \mathrm{ac})$.

Community Type No. 2: Open field with no overstory or sapling/shrub stratum present: Dominant herbaceous species included rabbit tobacco (Gnaphalium obtusifolia), dog fennel (Eupatorium compositafolium), bahia grass (Paspalum notatum), and broom sedge. In addition to the herbaceous species present, groundsel-tree (Baccharis glomeruliflora) and shortleaf pine regeneration were present in some areas. The area of this community is approximately $3 \mathrm{ha}(6 \mathrm{ac})$.

Community Type No. 3: Clearcut (approximately three growing seasons old) with no overstory present. Dominant species in the sapling/shrub stratum included longleaf and shortleaf pine, sparkleberry, and oak (Quercus spp.). Dominant herbaceous species included yellow jessamine, broom sedge, and reindeer moss (Cladina sp.). The area of this community is approximately 2 ha (4 ac).

Community Type No. 4: Hardwood overstory comprised predominantly of white oak (Quercus alba), southern red oak (Quercus falcata), black oak (Quercus marilandica), scarlet oak (Quercus coccinea), hickory (Carya spp.), and blackgum. Dominant species in the sapling/shrub stratum included dogwood (Cornus florida), American holly (Ilex opaca), American beech (Fagus grandifolia), and sparkleberry. In addition, rusty blackhaw (Viburnum prunifolium), wax 
myrtle (Myrica cerifera), and azalea (Rhododendron sp.) were observed along the slope, adjacent to Upper Three Runs. Creek. Herbaceous species were sparse within the community. Those that were observed included smilax (Smilax sp.), grape (Vitis rotundifolia), yellow jessamine, ebony spleenwort (Asplenium platyneuron), Christmas fern (Polystichum acrostichoides), false wintergreen (Chimaphila maculata), and switch cane (Arundinaria gigantea). The area of this community is approximately 27 ha $(67 \mathrm{ac})$.

Community Type No. 5: Dominant overstory species included loblolly pine and longleaf pine, sweetgum, and blackgum. An additional component of shortleaf pine and hickory was present along the slope above Upper Three Runs Creek. Within Community No. 5, the sapling/shrub stratum consisted of dogwood, deciduous holly (Ilex decidua), American holly, and sparkleberry. Dominant herbaceous species included yellow jessamine, blackberiy (Rubus sp.), bracken fern (Pteridium aquilinium), and trailing arbutus (Epigaea repens). The area of this community is approximately $23 \mathrm{ha}$ ( $57 \mathrm{ac})$.

Species composition within the large ravine and associated drain located downgradient from the cross-tie pile differed from the remainder of Community No. 5. Dominant overstory species present in the drain included red maple (Acer rubrum), sweetgum, blackgum, and sourwood (Oxydendrum arboreum). Azalea, dogwood, wax myrtle, and American holly were the major species in the sapling/shrub stratum. Vegetation in the herbaceous stratum for the most part was absent. Due to the resolution and scale of the aerial photograph, actual delineation of the community type was not possible.

Community Type No. 6: Predominantly hardwood overstory with a small component of loblolly pine. Dominant hardwood species included southern red oak, water oak (Quercus nigra), hickory, and blackgum. The sapling/shrub stratum was composed primarily of sparkleberry, American holly, black cherry (Prunus serotina), and hawthorne (Crataegus flava). Yellow jessamine, smilax, grape, and false wintergreen were the dominant herbaceous species present. The area of this community is approximately $8 \mathrm{ha}(21 \mathrm{ac})$.

Community Type No. 7: Loblolly pine was the dominant overstory species present. Wax myrtle and sparkleberry were the dominant mid-stratum species. Yellow jessamine and broomsedge were the major herbaceous species present within the community. The area of this community is approximately 7 ha (16 ac). 
Comn.unity Type No. 8: The small, isolated, ephemeral, palustrine wetland located $76.2 \mathrm{~m}$ (250 ft) west of BRPs $231-\mathrm{F} / 1 \mathrm{~F}$ was not formally delineated. However, the area was extensively investigated and wetland boundaries were located in an earlier study (Metcalf and Eddy, 1993). The 1993 study reported that the dominant species in the sapling/shrub stratum were river birch (Betula nigra) and black willow (Salix nigra). Dominant herbaceous species included Cyperus sp. and woolgrass (Scirpus cyprinus). Data obtained during the present study were similar to those collected during the earlier investigation. Seasonal or periodic inundation has resulted in the elimination of the overstory component within the wetland boundary. However, the dominant overstory species in the area immediately adjacent to the wetland was loblolly pine. The area of this community is approximately $0.4 \mathrm{ha}(0.9 \mathrm{ac})$.

The plants that compose the vegetative communities of the study area potentially could be affected by environmental contaminants. However, plants are generally less sensitive than animals, and no areas of distressed vegetation were observed. Accordingly, plants were not evaluated as ecological receptors, but they were considered as a potential pathway by which animals could be exposed.

\section{Animals}

Observations of animal species inhabiting the F-Area BRPs and RP and surrounding study area were made during the January 1994 ecological survey and in three previous surveys: Dixon et al. conducted an ecological characterization of the unit in September 1993; a preliminary ecological characterization of the unit was conducted in August 1993; and the Savannah River Forest Station (SRFS) of the U.S. Forest Service conducted a threatened, endangered, and sensitive (TES) species survey of the area in June 1993. During these reconnaissance activities, animal species were recorded based on actual sightings, trapping, and other evidence, such as tracks, scats, nests, and burrows. Animal species not recorded but likely to inhabit the study area were inferred based on their geographical ranges and habitat requirements.

\section{Birds}

Birds recorded in the area include a variety of passerine species that principally inhabit woodland habitats and edges, such as Carolina wren (Thryothorus ludovicianus), indigo bunting (Passerina cyanea), northern cardinal (Cardinalis cardinalis), wood thrush (Hylochichla mustelina), summer tanager (Piranga rubra), yellow-throated vireo (Vireo flavifrons), and white-eyed vireo (Vireo griseus). In addition to Carolina wren and northern cardinal, other species that are resident in the area throughout the year include Carolina chickadee (Parus carolinensis), tufted 
titmouse (Parus bicolor), rufous-sided towhee (Pipilo erythrophthalmus), and common crow (Corvus brachyrhynchos). Game bird species that are permanent residents in the area include wild turkey (Meleagris gallopavo), common bobwhite (Colinus virginianus), and mourning dove (Zenaida macroura). The species most commonly observed in the census by Dixon et al. (1993) were rock dove (Columba livia), mourning dove, ruby-throated hummingbird (Archilochus colubris), blue grosbeak (Guiraca caerulea), and Carolina wren. No evidence of wading birds or waterfowl was found at the small, ephemeral wetland or other surface water features of the study area, although the green heron (Butorides striatus) possibly could forage along the streams.

Raptors that may occur in the study area include the red-tailed hawk (Buteo jamaicensis), red-shouldered hawk (Buteo lineatus), American kestrel (Falco sparverius), barred owl (Strix varia), great horned owl (Bubo virginianus), and common screech owl (Otus asio). The possibility of these raptor species occurring in the vicinity of the unit was inferred based on their geographical ranges and typical habitats. Although these raptors potentially could inhabit the types of plant communities identified in the area of the unit, their presence at and utilization of the unit has not been confirmed.

\section{Mammals}

The number of mammal species recorded in the study area was less than the number of bird species, and most observations were indirect. Among the mammals recorded in the area, the largest native species is the white-tailed deer (Odocoileus virginianus). Numerous deer tracks were observed at the location of the F-Area BRPs and RP, and deer frequently obtain forage along woodland edges such as those surrounding the cleared areas of the unit. The largest mammal recorded in the study area is a non-native species, the feral swine (Sus scrofa). Swine are essentially herbivorous and preferentially inhabit bottomland habitats at SRS, such as along Upper Three Runs Creek. However, swine rootings have been observed in upland pine forests nearby and they have the potential to utilize the area of the unit. Other mammals recorded in the study area include raccoon (Procyon lotor), opossum (Didelphis virginiana), gray squirrel (Sciurus carolinensis), and eastern cottontail (Sylvilagus floridamus). Dixon et al. (1993) captured a large family group (two adults, three subadults, and four juveniles) of oldfield mice (Peromyscus polionotus) inhabiting the unit. Other species that likely occur in the area include gray fox (Urocyon cinereoargenteus), bobcat (Felis rufus), spotted skunk (Spilogale putorius), and various small rodents and insectivores (e.g., moles and shrews). The overall evaluation made by Dixon et al. (1993) was that the unit "represented extremely poor habitat for mammals." 


\section{Reptiles and Amphibians}

Other potential vertebrate receptors include reptiles and amphibians. Reptiles recorded on the unit include green anole (Anolis carolinensis) and five-lined skink (Eumeces fasciatus), and numerous other lizard and snake species may occur in the study area. Box turtles (Terrapene carolina) also inhabit the area.

Amphibians may occur in both terrestrial and aquatic habitats in the study area. Toads are terrestrial and may occur anywhere in the area. Southern toads (Bufo terrestris) were captured at the unit during daylight (Dixon et al., 1993). The most likely vertebrate inhabitants of the isolated, ephemeral wetland on the unit are breeding toads, frogs, and their eggs and tadpoles, which could be exposed to contaminated surface water and sediment during periods of inundation, which primarily occur during the breeding season in late winter and spring. Small mammals may utilize the wetland area during dry periods. Fish were not considered to be potential receptors because the intermittent nature of the inundation of the ephemeral wetland precludes their occurrence. In addition, fish were not observed in the unnamed tributary draining the unit, and any incremental exposure of fish in Upper Three Runs Creek to contaminants potentially emanating from the F-Area BRPs and RP was considered unlikely to be significant.

Potential invertebrate receptors are ubiquitous throughout the study area and include insects, arachnids, earthworms, and nematode worms. Depending on life cycle, diet, and behavior, invertebrates may be exposed to contaminants in soil, sediment, and surface water.

\section{Threatened, Endangered, and Sensitive Species (TES)}

A TES species survey was conducted at the F-Area BRPs and RP by the SRFS of the U.S. Forest Service in June and July 1993. SRFS files were examined to determine past or present occurrences of any TES species at or around this unit. Records reviewed included aerial photographs, vegetative stand data, soils information, and existing TES species records. This review was followed by a field survey for any TES plant or animal species determined to potentially occur at the unit, based on recorded observations or the presence of required habitat elements. The survey found no TES plant or animal species on the unit or within the study area. Those TES species that were not found but that potentially could occur in area habitats are listed in Table 4.20 with their Federal and State Heritage Trust Program status rankings (Jarvis, 1993).

For the most part, the study area was found to contain habitat with little potential for SRS TES plants. One exception was the upland hardwood area, which had high potential for Oconee azalea 
(Rhododendron flammeum); however, none was found. Likewise, no SRS TES animal species were found to occur in the survey area. The red-cockaded woodpecker (RCW) currently does not occur in the area of concern (the closest active colony is more than $12.9 \mathrm{~km}$ [8 miles] to the northeast) nor has it been documented in this area in the past (the closest historic nest was 5 miles to the west-southwest). Current vegetative conditions essentially rule out this area as potential RCW nesting habitat. The other SRS threatened and endangered animal species (bald eagle, wood stork, shortnose sturgeon, American alligator) do not occur in this area, nor would they be expected to occur based on habitat requirements. Six SRS Sensitive (Federal Category 2) animals potentially could occur at or adjacent to the unit based on habitat availability (see Table 4.20), but none was observed in the survey (Jarvis, 1993).

\subsubsection{Conceptual Site Model}

The four elements necessary for an exposure pathway to be complete were discussed in the exposure assessment section of the human health BRA (Section 3.2.2). If any of these elements is missing, the pathway is incomplete and is not considered further in the ecological risk assessment. The following section describes potential pathways by which non-radioactive chemicals and radionuclides could migrate from source locations to ecological receptors.

A conceptual site model (CSM) was developed to illustrate the potential pathways for exposure of ecological receptors to COPCs at the F-Area BRPs and RP (Figure 4.2). Complete exposure pathways are represented in the diagram by a circle: an open circle indicates qualitative evaluation of a pathway and a filled circle indicates quantitative evaluation. A pathway is complete when all of the necessary elements are present to permit potential exposure of a receptor to a source of contamination. Quantification of some potentially complete pathways may not be warranted because of minimal contribution to risk relative to other major pathways. The principal complete pathways that were evaluated quantitatively are represented in the diagram by filled circles in the receptor columns. Those pathways lacking at least one exposure pathway element are incomplete and are represented in the diagram by empty boxes.

The primary source of contaminants at the F-Area BRPs and RP was the wastes that were burned and buried in the former burning/rubble and rubble pits. The wastes disposed of at the unit are listed in the human health BRA. The primary release mechanisms are: (1) deposition of ash from waste-burning and inadvertent deposition of waste materials or airborne particulates outside the pits on surface soils during disposal activities and (2) infiltration/percolation of contaminants to deeper soils. 
Exposire to surface soil contaminants may occur directly through ingestion, dermal contact, and/or external radiation from radionuclides. Chemicals also may migrate further in the environment by a variety of pathways following secondary release from surface soil and deeper soil. The following pathways result from these secondary release mechanisms:

- Suspension and dispersal by the wind of particulate contaminants or contaminants adsorbed to surface soil particles.

- Direct volatilization of volatile organic compounds from surface soil to air.

- Uptake of soil contaminants by terrestrial organisms or uptake of contaminants in surface water and sediments by aquatic oryanisms.

- Transport of soil contaminants by stormwater runoff to surface water and sediments of the ephemeral wetland and streams.

- Leaching of chemicals from soil into groundwater, with subsequent discharge of groundwater into surface water and sediment of streams.

As shown in the CSM, there are five media through which ecological receptors could be exposed to unit-related contaminants: air (dust and vapor), soil, surface water, sediments, and organisms in the food chain. An exposure point is a location where a receptor potentially could come into contact with a contaminated medium. An exposure route is the means by which a receptor comes into contact with a contaminated medium at an exposure point. Exposure to both radionuclides and nonradioactive contaminants may occur through the routes of ingestion, inhalation, and dermal contact. Radiological exposures also may occur through direct, external radiation.

Probable exposure routes (i.e., potentially complete pathways) were identified for each medium based on the physical characteristics of the waste unit and the potential ecological receptors that may occur there (see the previous description of the ecological setting). Exposure routes were identified for both terrestrial and aquatic ecological receptors. Principal pathways, for which analytical data were available for quantitative evaluation,-include; for terrestrial receptors, ingestion of soil, surface water, and sediment, as well as exposure to direct radiation from soil and sediment, and ingestion of other animals and plants that have accumulated contaminants; for aquatic receptors, they include ingestion of surface water.

Terrestrial animals potentially could be directly exposed to soil contaminants through ingestion of, dermal contact with, and/or direct radiation from unit soils. For species such as deer, raccoon, 
opossum, rabbits, rodents, and birds, such exposures likely would be incidentally associated with foraging activities. Burrowing species, such as rabbits, rodents, moles, and shrews, would probably receive the greatest exposures among vertebrates. Invertebrates living on and within the soil also may experience significant exposures. Although ingestion and external radiation are the principal soil exposure routes, dermal contact also may be important, particularly for burrowing species. However, the limited dermal permeability database available for ecological receptors and surrogate species precluded quantitative evaluation of the dermal exposure pathway.

Both terrestrial and aquatic species potentially could be exposed to unit-related contaminants that may be present in surface water and/or sediments. The principal pathway for exposure to those media is ingestion. Dermal contact is a complete pathway but, for the reasons discussed above with regard to soil, it was addressed qualitatively. Terrestrial species may be exposed to chemicals in surface water and sediment while drinking or foraging. However, aquatic species may be exposed to a greater extent through ingestion, inhalation, and dermal absorption. Therefore, aquatic species were chosen for evaluation of toxicity from surface water exposure. Conversely, due to the intermittent inundation of the wetland and the lack of a significant benthic community, terrestrial species were considered to have a greater potential for significant exposure to sediment at the ephemeral wetland than aquatic species. Terrestrial organisms could be exposed to sediment during both wet and dry periods. Therefore, terrestrial species were chosen for evaluation of toxicity from sediment exposure.

The surface water and sediment samples collected from the unit were obtained from the small, ephemeral wetland located immediately. west of BRPs 231-F/1F. Accordingly, the only aquatic receptors for which risk could be quantitatively evaluated were those capable of inhabiting this small, isolated wetland that is inundated only intermittently. Such organisms include mainly amphibians and invertebrates.

Analytical data for other surface water features in the area of the unit were limited or not available; therefore, risk associated with exposure of terrestrial and aquatic receptors to potential contaminants in those surface water bodies could not be quantitatively evaluated. Nevertheless, streams draining the unit, such as Upper Three Runs Creek and its unnamed tributary to the north-northeast of the F-Area BRPs, potentially could provide complete exposure pathways for ecological receptors as a result of transport of contaminants in stormwater runoff and groundwater discharge to the streams. However, any runoff from the waste. unit that drains into 
the unnamed tributary would likely be significantly diluted by the stream flow, a major portion of which originates from the F-Area NPDES outfall F-001 located at the head of the tributary.

Similarly, any unit-related contaminants reaching Upper Three Runs Creek would be greatly diluted in such a large stream, which also drains the areas of other SRS waste units that may contribute contaminants. Significant attenuation of contaminant influence would be expected to occur over such distances.

Surface water and sediment data collected from the creek in a 1992 study (Shealy Environmental Services, 1992) support the conclusion that. Upper Three Runs Creek has not been measurably influenced by chemicals originating from the F-Area BRPs and RP. Upper Three Runs Creek was sampled immediately downstream of the unit as well as upstream of potential influence from the unit. Samples were analyzed for metals, herbicides, pesticides, volatiles, base neutral/acid extractables, dioxins, and radionuclides. No organics were detected at the downstream location, except for acetone in sediment. A limited number of metals were detected at this location, all of which were at low concentrations and several of which were at concentrations lower than those detected at the upstream location. The only radionuclides detected downstream were uranium-234 and technetium-99 in water and sediment and tritium in water. These could not be attributed specifically to the unit. Therefore, the potential for risk to ecological receptors from transport of unit-related chemicals to Upper Three Runs Creek and its tributary was considered to be insignificant and was not quantitatively evaluated.

Ecological receptors potentially could be exposed to unit-related contaminants via the air medium. Contaminants in air may be in the form of vapor from volatilized organic compounds, or in particulate form (as dusts or adsorbed to soil particles) suspended by wind. Ecological receptors could be exposed to contaminants in either form through inhalation. However, the lack of applicable inhalation toxicity data for ecological receptors or similar species precluded quantitative evaluation of potential risks.

Plants may be considered ecological receptors as well as a pathway or medium through which wildlife receptors can be exposed to contaminants. Terrestrial plants may absorb unit-related contaminants from soil through their roots. Aquatic and hydrophytic plants likewise may absorb chemicals directly from the water or from sediments through their roots. Contaminants absorbed by plants may be transferred to wildlife when the plants are ingested for food. This exposure pathway was addressed by use of chemical-specific soil-to-plant uptake factors, obtained from the 
scientific literature, in the animal receptor exposure calculations. No plants on or near the unit showed visible signs of stress during the field reconnaissance.

Under the future residential land use scenario for the unit, it is assumed that contaminated deep soils would be excavated during home construction and distributed on the ground surface. As under current conditions, ecological receptors potentially could be exposed to chemicals in soil through ingestion, dermal contact, and direct radiation from radionuclides. Other exposure pathways also were assumed to remain essentially the same as under current conditions, except that possible inhalation exposures are likely to be reduced by paving and vegetation (e.g., lawns), and the abundance and diversity of some ecological receptres on the unit are likely to be reduced due to the presence of human residences or industrial buildings and their associated parking areas.

The principal pathways for which the exposure of ecological receptors was quantitatively evaluated are represented by the filled circles in Figure 4.2 and are summarized in Table 4.21.

\subsubsection{Ecological Effects}

No adverse ecological effects were identified at ihe unit that could be directly attributed to chemical contaminants associated with previous waste disposal activities. The unit has been significantly impacted physically by clearing and other land disturbance activities. No areas of stressed vegetation were observed on the unit, but much of the area around the BRPs is sparsely vegetated. The reason for the sparseness of the vegetation was not apparent but is likely due to physical disturbance of the area.

As a result of the limited vegetation and lack of cover, these disturbed areas on the unit generally provide extremely poor habitat for mammals. Nevertheless, a large family group of oldfield mice was found living on the unit. Also, the diversity of bird species inhabiting the unit was found to be relatively high, perhaps due to the structural diversity of the unit with its surrounding wooded areas (Dixon et al., 1993).

\subsection{Endpoint Selection}

To assess whether significant adverse ecological effects have occurred or may occur at the F-Area $\mathrm{BRP} / \mathrm{RP}$ waste unit as a result of exposure of ecological receptors to COPCs, ecological endpoints were selected. An ecological endpoint is a characteristic of an ecological component that may be affected by exposure to a stressor, such as a chemical contaminant. Assessment endpoints represent environmental values to be protected. Measurement endpoints are 
measu able responses to a stressor that are related to the valued characteristics chosen as assessment endpoints (EPA, 1992f). Assessment endpoints generally refer to characteristics of populations and ecosystems, and it is usually impractical to measure changes in these characteristics as a part of an assessment (Suter, 1993). Consequently, measurement endpoints are selected that can be measured and extrapolated to predict effects on assessment endpoints (EPA, 1992f). This section describes the process used in selecting assessment endpoints for this unit.

Given the diversity of the biological world and the multiple values placed on it by society, there is no universally applicable list of assessment endpoints. Therefore, Suter (1993) has suggested five criteria that should be considered in selecting assessment endpoints suitable for a specific ecological risk assessment. Three of these are listed as considerations in selecting assessment endpoints in the Framework for Ecological Risk Assessment (EPA, 1992f).

1. Ecological relevance. The assessment endpoint should have biological/ecological significance to a higher level of the ecological hierarchy. For example, an increase in mortality or a decrease in fecundity of individuals is ecologically significant if it affects the size or productivity of the population. Likewise, a decrease in the size of a population is ecologically significant if it affects the number of species, the productivity, or some other property of the ecosystem (Suter, 1993).

2. Susceptibility to the contaminant(s). The assessment endpoint should be susceptible to exposure to the contaminant(s) and should be responsive to such exposure (Suter, 1993). That is, assessment endpoints should be chosen that are likely to be exposed to

- contaminants at the unit, either directly or through the food chain, and they should be sensitive enough that such exposure may elicit an adverse response.

3. Accessibility to prediction and/or measurement. The response of the assessment endpoint should be measurable, or it should be predictable from measurements of related responses, responses of other species (Suter, 1993), or responses of other levels of the hierarchy of ecological organization. That is, data relevant to the assessment endpoint should be available for use in predicting responses to unit-related chemicals.

4. Societal relevance. Ideally, the assessment endpoint should be understood and valued by the public and decision makers (Suter, 1993).

5. Definable in clear, operational terms. The operational definition of the assessment endpoint should include a subject (e.g., a native species, a population, or a community) 
and a characteristic of the subject (e.g., a reduction in population size or community diversity) (Suter, 1993).

The above criteria were the basis for evaluation of potential assessment endpoints to be used in assessment of ecological risk at the F-Area BRPs and RP. Potential receptor species likely to be exposed to unit-related chemicals were judged by these criteria as part of the process of selecting the most appropriate assessment endpoints for this unit. This evaluation is graphically presented in Table 4.22. The results of this analysis indicated that native mouse species inhabiting the area of the unit were the most appropriate assessment endpoint species for soil and sediment (when the ephemeral wetland is dry), and the relevant assessment endpoint was defined as a reduction in resident mouse populations. The maintenance of southern toad populations (representing amphibian and other semi-aquatic animals that may inhabit the small, ephemeral wetland area when inundated) was the assessment endpoint selected for evaluation of ecological risk associated with potential surface water contamination.

In addition, effects on individual species (e.g., native mice) may be used to identify potential effects on assessment endpoints at higher levels of ecological organization. Potential reduction in the abundance of individuals within species (i.e., population size) and potential reduction in the number of species making up the ecological community (i.e., biodiversity) at the unit were the assessment endpoints selected for evaluation of effects at the community level. The rationale for selecting these population- and community-level assessment endpoints is discussed below for each numbered criterion:

1. Ecological relevance. Native mouse species are subject to predation and to competition with other animals for food. An increase in mortality or decrease in fecundity of individual mice in the area as a result of exposure to toxicants will affect the size and productivity of mouse populations. In turn, decreases in mouse populations may alter the food supply of individual predators or competitors in the area, thereby affecting other members of the ecological community.

2. Susceptibility. Because unit-related chemicals are principally associated with discrete areas of soil contamination, to be susceptible to significant exposure a potential receptor species should be in intimate contact with soils for long periods of time, and/or should consume plants or animals which may have bioaccumulated unit-related chemicals. Native mouse species meet both of these requirements because they nest in burrows in the soil and feed on plants and insects within a small home range. In addition, a 
multitude of studies of closely-related laboratory rodents has demonstrated their sensitivity to a wide variety and large number of chemicals.

Other potential receptors considered for possible selection as assessment endpoint species, including white-tailed deer, feral swine, and predators such as raptors, were concluded to have very limited exposure potential because their home ranges are much larger than the size of the unit. For example, the contaminated area at the unit covers only around $0.6 \mathrm{ha}$, while the home range of a red-tailed hawk is approximately $100 \mathrm{ha}$ and that of a white-tailed deer is around 260 ha (see Table 4.23). In addition, the low productivity of the habitat at the unit is unlikely to provide sufficient forage or shelter to disproportionately attract these animals to that area.

3. Accessibility to prediction. Because the toxicological database is more extensive for rodents than for any other terrestrial group, native mice have a much greater database on which to draw for evaluation of potential toxicity effects from exposure to the various chemicals detected at the unit than any other native taxa. Because of the close phylogenetic relationship of native mice to commonly-studied laboratory mice and rats, toxicity data based on these laboratory species (measurement endpoints) are highly relevant, useful, and relatively low in uncertainty for predicting potential effects that may occur in populations of native species. Likely population effects can then be used to predict potential community effects.

4. Societal relevance. A TES species survey was conducted for the area of the unit, and no TES species were found. Other species commonly considered to be of special value, such as raptors and white-tailed deer, have home ranges much larger than the unit and are unlikely to utilize the area of the unit sufficiently to be significantly exposed. The societal relevance of native mouse populations at the unit resides principally in their role as primary consumers within the community, feeding on plants and animals and providing food for secondary consumers. Maintenance of the biological health of natural communities is generally considered of societal importance, and removal of mice from their role in the community could have adverse effects on the health of the community.

5. Definable. The assessment endpoints for this assessment were defined for operational purposes as: (1) a reduction in resident populations of native mouse species at the unit, in particular, populations of oldfield mice, which are known to inhabit the unit; and (2) a reduction in abundance of individuals and richness of species making up the ecological community at the unit. 
A large family group (nine individuals) of oldfield mice was found by Dixon et al. (1993) to inhabit the unit despite their description of the unit as representing "extremely poor habitat for mammals." These are the vertebrate receptors most likely to be maximally exposed to contaminants in soil or sediment (when the ephemeral wetland is dry) at the unit. They also represent a significant component of the food chain, feeding on seeds and berries and soil invertebrates and providing prey for predators. Therefore, the oldfield mouse was selected as the assessment endpoint species at this unit, and measurement endpoints were selected that could be extrapolated to predict effects on the assessment endpoints. Databases and available literature were searched for toxicity data for oldfield mice or other native rodent species. In the absence of unit-specific data, laboratory-derived data on mortality or reproductive effects were used as measurement endpoints, and in the absence of data on native species, data for closely related laboratory rodents such as laboratory mice (Mus musculus) and laboratory rats (Rattus norvegicus) were used.

The assessment endpoint selected for evaluation of ecological risk associated with potential surface water contamination at the wetland during periods of inundation was the maintenance of amphibian populations that may depend on the wetland for reproduction. Southern toads were trapped on the unit by Dixon et al. (1993), and these or other toad or frog species may reproduce in the ephemeral wetland. In the absence of available toxicity data for native amphibian species, laboratory-derived data for other amphibians were used as measurement endpoints. In the absence of amphibian data, data for fish or aquatic invertebrates were utilized.

Raptors were among the birds included in the evaluation of potential assessment endpoints (see Table 4.22) because there is the possibility that raptors may capture prey at the unit. However, the depauperate populations of mice and other prey species inhabiting the unit appear to be insufficient to support even an individual raptor, and the large home range areas typically utilized by raptors are indicative of their need to forage over extensive areas in order to satisfy their prey requirements. The small area of the unit would be unlikely to.fulfill these requirements even if it provided optimal habitat for prey species. Therefore, it is extremely unlikely that a raptor could feed on prey containing chemical residues from the unit to such an extent that residues in its own tissues would bioaccumulate to toxic levels. Even if it were assumed that this could occur in a raptor living at the unit, toxic effects on that individual would not be ecologically significant for the raptor population or for the health of the greater ecological community of the area. 
This concept is discussed by Suter et al. (1994) in Ecological Risk Assessment For Comprehensive Environmental Response, Compensation, and Liability Act Sites On Federal Facilities: An Introductory Guide For Facility Managers. The guide presents and discusses a generic conceptual model, developed for DOE's Oak Ridge Reservation, for a typical individual waste site or source operable unit (OU). Such a unit is typically covered by a mowed fescue lawn, which reduces the ecological value of the unit to most species other than soil invertebrates. Therefore, the ecological conceptual model focuses on the proximate receptors that can be associated with the OU (i.e., plants and soil invertebrates), though "conceptual models for OUs with natural vegetation might include more species (e.g., small mammals).".

The guide points out the importance of discriminating between receptors for an OU-level assessment and receptors for a larger-scale assessment: "While some wide-ranging species may periodically use a particular OU, these organisms are not dependent on that specific unit (i.e., most of their diet or daily activity comes from or occurs off the unit) so they should not be included in the OU-scale conceptual model. Because these mobile species may be exposed to multiple waste units, risks to such species must be represented in a larger-scale (watershed, facility, or reservation-wide) conceptual model." Furthermore, wide-ranging species at the apex of the food web, such as raptors, essentially will be exposed only to contaminants that significantly bioaccumulate/biomagnify. Potential ecological effects that may result from the toxicity of non-bioaccumulative contaminants would not be assessed if a raptor or other top predator were the assessment endpoint. Therefore, maintenance of the oldfield mouse population was considered to be the most appropriate assessment endpoint for assessing ecological risk at the F-Area BRPs and RP.

In the receptor species selection process shown in Table 4.22, raptors were given a rating of 1 under the criterion "susceptibility to contaminant" for the reasons discussed above. Because the potential for exposure to contaminants from this unit is much less for a raptor than for a mouse living in contact with the soil and feeding on the unit throughout its lifetime, tite raptor is much less susceptible to the toxic effects of the contaminants, particularly those that are nonbioaccumulative (including many radionuclides).

Raptors also were given a rating of 2 under the criterion "accessibility to prediction" because of the lack of toxicity data for raptur species. For the great majority of contaminants at the unit, there are no available toxicity data by which to evaluate the potential for toxic effects in raptors as a result of ingestion of a given intake of contaminant. Therefore, it is very. difficult to predict 
whether the levels of most contaminants detected in environmental media at the unit may result in toxicity to raptors exposed through the food chain. On the other hand, effects in mice are much more accessible to prediction because of the extensive toxicity database that is available for mice and rats.

Accordingly, maintenance of the oldfield mouse population was considered the most appropriate selection as the assessment endpoint for this unit.

\subsection{Exposure Assessment}

The objective of the exposure assessment is to assess potential exposure of ecological receptors to unit-related chemicals. The process includes description of the spatial distribution of COPCs at the unit, description of spatial and temporal distribution of ecological receptors, and quantification of exposure that may result from overlap of these distributions.

\subsubsection{Chemical Distribution}

The extent of measured chemical contamination at the unit is restricted to the areas sampled within and adjacent to BRPs 231-F/1F, RP 231-2F, and the small ephemeral wetland to the west of the BRPs (see Figure 2-1). Unit-related contamination of soils located beyond the immediate vicinity of the pits or of surface water and sediment located elsewhere than the ephemeral wetland have not been confirmed. The combined area of the contiguous BRPs $231-\mathrm{F} / 1 \mathrm{~F}$ is $0.4 \mathrm{ha}(1 \mathrm{ac})$, the area of RP 231-2F is $0.05 \mathrm{ha}(0.1 \mathrm{ac})$, and the area of the ephemeral wetland is $0.1 \mathrm{ha}(0.2$ ac). Thus, the total unit area in which COPCs were detected is approximately 0.6 ha $(1.3 \mathrm{ac})$.

The magnitude of the chemical exposures that may be experienced by ecological receptors is affected by the degree of their spatial and temporal associations with the unit.

\subsubsection{Receptor Distribution}

Approximately 80 ha $(200 \mathrm{ac})$ of the ecosystem surrounding the unit composed the study area in which ecological communities and receptors that potentially could be exposed to unit-related chemicals were characterized (see Section 4.2.1.2 and Figure 4.1). Of the plant communities identified, only the wetland community and edges of the loblolly/longleaf/shortleaf pine forest community overlap the areas of contamination detected at the unit. The former pit areas support a rather sparse plant community of low diversity and abundance. Consequently, these pit areas provide relatively poor habitat for animals, and the number of animal species and individuals for 
which the physically impacted areas of the unit provide a significant source of food, water, or cover appears to be very limited.

The structural diversity provided by the forest edges surrounding the open areas of the unit supports a relatively high diversity of birds, including passerines, doves, and bobwhite. The home ranges of these birds may overlap areas of contaminated media at the unit, qualifying them as potential receptors. However, the areas of the home ranges utilized by most birds are larger than the area of the unit, and the limited vegetation on the unit would tend to further reduce the amount of time spent there and the extent of exposure.

A variety of other factors also may affect the extent and significance of potential avian exposures. Receptor exposures are affected by the degree of both spatial and temporal association with the unit. For example, the mobility of birds may significantly affect their potential exposures to localized contaminants such as those at this unit. Many insectivorous songbirds, such as buntings, thrushes, tanagers, and vireos, inhabit the study area only during the summer breeding season. Other species that spend the winter in the study. area migrate northward for the summer. The majority of the recorded species are non-migratory and remain in the vicinity throughout the year. The latter species, particularly those with longer life spans such as raptors, have the greatest potential duration of exposure. Other factors affecting avian exposures include habitat preference, behavior, and diet. Diet is of particular importance in exposure of birds to bioaccumulative chemicals. Such chemicals also may tend to biomagnify in the food chain, with the result that predatory species at higher trophic levels, such as raptors, may receive their most significant exposures through their prey.

As discussed above in Section 4.3, the possibility of a population of raptors, or even an individual, utilizing the unit as a primary source of food is considered extremely remote. Although many raptors prefer to hunt in relatively open areas, there are other open areas in the vicinity, including grassy roadsides, that are probably more productive and better sources of prey than is this unit. The lack of major areas of open countryside in the vicinity, rather than being indicative of greater utilization of the unit by raptors, is an indication that the number of raptors utilizing the area is likely to be very limited due to the scarcity of habitat. The values shown in Table 4.23 of $0.6 \%$ for the red-tailed hawk and 7.5 percent for the American kestrel reflect the unit size relative to the raptor home range requirements and is considered to conservatively represent their potential utilization of the unit. 
For mammalian receptors, the magnitude of exposure to localized contaminants at the unit also is dependent on a variety of factors such as diet, behavior, and individual home ranges. Behaviors such as burrowing (mice, shrews) and rooting (swine) increase potential exposures to soil contaminants, and foraging in wetlands and streams (raccoon) increases potential exposures to sediment and surface water contaminants. Typically, larger mammals have large home ranges and would likely spend only a proportionately limited amount of their time foraging on the unit. For example, white-tailed deer have a home range of around 260 ha (640 ac), red fox typically range over an area of that size or larger, and opossum have a home range of 6-16 ha (15-40 ac) (Burt and Grossenheider, 1976). The combination of the large size of their ranges (see Table 4.23) and the limited sources of food provided by the habitat on the unit makes significant exposure of large mammals to unit contaminants very unlikely.

Small mammals, on the other hand, generally have small ranges, which could result in greater exposures if these ranges overlap or are encompassed by the contaminated area. For example, oldfield mice may have a home range of up to 6 ha $(15 \mathrm{ac}$ ), white-footed mice (Peromyscus leucopus) occupy a typical range of 0.2-0.6 ha (0.5-1.5 ac), and short-tailed shrews (Blarina brevicauda) have a home range of only around 0.2-0.4 ha (0.5-1 ac) (Burt and Grossenheider, 1976). The habitat on the unit could support these or other small mammal species, and oldfield mice have been trapped there. In addition, small mammals are much less mobile than birds, and are expected to have more extensive direct contact with environmental media such as soil and sediment. Therefore, small mammals such as the three species listed above are considered to have the greatest potential for direct exposure to contaminated media at the unit.

Trophic level and feeding behavior of potential receptors are significant factors in determining exposure to chemicals in the ecosystem. Predators (e.g., fox, raccoon, red-tailed hawk) are at the top of the food chain and thus may receive their greatest exposure to unit-related chemicals through ingestion of prey that have bioaccumulated contaminants. Small animals that utilize the unit as a significant part of their range are more likely than larger mammals or birds to be directly exposed to COPCs through ingestion of contaminated media, such as incidental ingestion of soil or sediment while burrowing or grooming, or drinking surface water. Small animals also can be exposed through the food chain, such as through ingestion of plants or invertebrates that have bioconcentrated chemicals from the soil. Table 4.23 lists typical home range areas of a number of potential receptor species and the percentage of each range that the unit would comprise if these overlapped completely. The data provide an indication of the relative likelihood of exposure of various potential receptors, based on spatial distribution only. 
Aquatic or semi-aquatic organisms are likely to be confined to the surface water of the wetland area during periods of inundation. For example, toads or frogs may reproduce in the wetland in late winter or early spring, and their offspring are obligately aquatic until they metamorphose into adults.

\subsubsection{Quantification of Exposure}

Evaluation of the degree to which contaminant distributions and receptor distributions (described in the previous two sections) coincide at the unit indicated that small mammals are the receptors likely to have the greatest potential exposures to COPCs in soil and sediment. As the small mammals likely to inhabit the unit in greatest abundance, mice represent an important component of the food chain, functioning as primary consumers as well as a food source for mammalian, avian, and reptilian predators. Therefore, as discussed in Section 4.3, toxicity effects of COPCs on mice were measurement endpoints for assessing ecological risk from soil and sediment contamination. Lethal effects on aquatic organisms, particularly amphibians, were endpoints for assessing risk from surface water contaminants at the ephemeral wetland.

In order to quantify exposures of terrestrial receptors to each COPC in soil or sediment, a daily intake of each chemical in each medium was calculated. Conversion of the environmental concentration of each COPC in soil or sediment to an estimated daily intake for a receptor at the unit was necessary prior to evaluation of potential toxicity effects. For terrestrial animal receptors, calculation of exposure intake rates relied upon determination of an organism's exposure to COPCs found in surface soil and sediment. Exposure rates for the oldfield mouse receptor were based upon ingestion of contaminants from these media and from consumption of other organisms. Given the scarcity of data available for wildlife dermal and/or inhalation exposure pathways, this risk assessment does not attempt to measure potential risk from these pathways.

The first step in measuring exposure rates for terrestrial wildlife was calculation of food ingestion rates for the oldfield mouse receptor. EPA's Wildlife Exposure Factors Handbook (EPA, 1993c) includes a variety of exposure information for a number of avian, herptile, and mammalian species. Data are directly available for body weights of various species. Similarly, information regarding food ingestion rates and dietary composition also are available for many species. For other species for which data are not provided, the document provides an allometric equation, from Nagy (1987), to estimate food intake based on body mass, as follows: 


$$
\mathrm{FI}=0.0687\left(\mathrm{BW}^{0.822}\right)
$$

where:

$$
\begin{aligned}
& \mathrm{FI}=\text { food intake rate }(\mathrm{kg} / \text { day }) \\
& \mathrm{BW}=\text { body weight }(\mathrm{kg})
\end{aligned}
$$

Average body weight for the oldfield mouse is $0.012 \mathrm{~kg}$ (Whittaker, 1966). Using this body weight in the above equation produces an estimated food intake rate of $0.0018 \mathrm{~kg} / \mathrm{day}$.

The Wildlife Exposure Factors Handbook also presents average values for intake of animal matter and plant matter for the deer mouse, as well as incidental soil ingestion. Soil ingestion has been measured at less than 2 percent of diet. As might be expected based on the opportunistic habits of mice, the proportion of animal to plant matter in the diet varies from around 65 percent animal : 35 percent plant to 25 percent animal : 75 percent plant depending on season and region of the country. In the south, where there is less restriction from cold and snow cover, reliance on plant material in the winter is not as pervasive. For this risk assessment, an approximate average of 50 percent animal : 50 percent plant was used, after subtracting the 2 percent for incidental soil ingestion. Based on similarity of diet between the oldfield mouse and the deer mouse, the ratios of animal to plant to soil in the diet was assumed to be the same for the two species. The dietary intakes calculated for this assessment, summarized in Table 4.24, are as follows:

$$
\begin{aligned}
& \text { total dietary intake }=0.0018 \mathrm{~kg} / \text { day } \\
& \text { plant matter intake }=\quad 0.000882 \mathrm{~kg} / \text { day } \\
& \text { animal matter intake }=0.000882 \mathrm{~kg} / \mathrm{day} \\
& \text { incidental soil intake }=0.000036 \mathrm{~kg} / \text { day }
\end{aligned}
$$

For the COPCs that exceeded the background and preliminary toxicity screening, a unit-specific exposure value was calculated, using a food chain uptake model consistent with EPA Region IV guidance (EPA, 1995a). This algorithm accounts for exposure via incidental ingestion of contaminated soil, ingestion of plants grown in contaminated soil, and ingestion of lower trophic level animals associated with contamination. The exposure equation for soil is as follows.

$\mathrm{ED}_{\text {soil }}=\left[\left(\mathrm{C}_{z} * \mathrm{SP} * \mathrm{I}_{\mathrm{p}}\right)+\left(\mathrm{Cs} * \mathrm{BAF} * \mathrm{I}_{2}\right)+\left(\mathrm{C}_{s} * \mathrm{I}_{8}\right)\right] * \mathrm{SFF} / \mathrm{BW}$ 
where:

EDsoil $=\quad$ soil exposure dose for terrestrial animal $(\mathrm{mg} / \mathrm{kg} /$ day $)$

$\mathrm{C}_{\mathrm{z}}=\mathrm{RME}$ concentration in soil $(\mathrm{mg} / \mathrm{kg})$

$\mathrm{SP}=$ soil-to-plant uptake factor (unitless)

$I_{p}=$ receptor-specific ingestion rate of plant material $(\mathrm{kg} /$ day $)=0.000882$ $\mathrm{kg} /$ day

$\mathrm{BAF}=$ chemical-specific bioaccumulation factor (unitless)

$\mathrm{I}_{\mathbf{z}}=$ receptor-specific ingestion rate of animal material $(\mathrm{kg} /$ day $)=0.000882$ $\mathrm{kg} /$ day

$\mathrm{I}_{\mathrm{s}}=$ receptor-specific ingestion rate of soil $(\mathrm{kg} /$ day $)=0.000036 \mathrm{~kg} /$ day

$\mathrm{SFF}=$ site foraging factor (unitless) $=0.73$ for $231-\mathrm{F} / 1 \mathrm{~F}, 0.09$ for $231-2 \mathrm{~F}$ (see explanation below)

$\mathrm{BW}=$ body weight $(\mathrm{kg})=0.012 \mathrm{~kg}$

For the terrestrial receptor's exposure to sediment, an algorithm similar to the exposure equation for soil was used, as follows.

\section{$\mathrm{ED}_{\text {sed }}=\left[\left(\mathrm{C}_{d} * \mathrm{SP} * \mathrm{I}_{\mathrm{p}}\right)+\left(\mathrm{C}_{\mathrm{d}} * \mathrm{BAF} * \mathrm{I}_{2}\right)+\left(\mathrm{C}_{\mathrm{d}} * \mathrm{I}_{s}\right)\right] * \mathrm{SFF} / \mathrm{BW}$}

where:

$\mathrm{ED}_{\text {sed }}=\quad$ sediment exposure dose for terrestrial animal $(\mathrm{mg} / \mathrm{kg} /$ day $)$

$\mathrm{C}_{\mathrm{d}}=\mathrm{RME}$ concentration in sediment $(\mathrm{mg} / \mathrm{kg})$

$\mathrm{SP}=$ soil-to-plant uptake factor (unitless)

$\mathrm{I}_{\mathrm{p}}=$ receptor-specific ingestion rate of plant material $(\mathrm{kg} /$ day $)=0.000882$ $\mathrm{kg} /$ day

$\mathrm{BAF}=$ chemical-specific bioaccumulation factor (unitless)

$I_{2}=$ receptor-specific ingestion rate of animal material $(\mathrm{kg} /$ day $)=0.000882$ $\mathrm{kg} /$ day

$I_{3}=$ receptor-specific ingestion rate of sediment $(\mathrm{kg} /$ day $)=0.000036 \mathrm{~kg} / \mathrm{day}$

$\mathrm{SFF}=$ site foraging factor (unitless) $=0.18$ (see explanation below)

BW $=$ body weight $(\mathrm{kg})=0.012 \mathrm{~kg}$ 
For the aquatic faunal receptor, the southern toad, intake rates were not calculated, as risk was characterized by comparing receptor toxicity concentrations in water with concentrations in surface water samples from the unit.

Because of the small area of their home ranges and their year-round residence, mice and other small mammals living on the unit and amphibian species using the ephemeral wetland potentially could use contaminated areas 100 percent of the time. However, the same mouse receptor would be exposed to both soil and sediment contaminants in the unit. The exposure dose calculations assumed the mouse would be exposed to each medium in proportion to the size of the nonwetland parts of the unit $(0.45 \mathrm{ha})$. compared to the size of the ephemeral wetland $(0.1 \mathrm{ha})$. Therefore, a site foraging factor (SFF) of $0.82(0.45$ ha $/ 0.55 \mathrm{ha})$ was used for soil and an exposure factor of $0.18(0.1 \mathrm{ha} / 0.55 \mathrm{ha})$ was used for sediment. The soil exposure is further broken down between BRPs $231-\mathrm{F} / 1 \mathrm{~F}$ and RP 231-2F, in proportion to their size. Therefore, the SFF for BRPs 231-F/1F is 0.73 ( $0.4 \mathrm{ha} / 0.55 \mathrm{ha})$ and for RP $231-2 \mathrm{~F}$ is $0.09(0.05 \mathrm{ha} / 0.55 \mathrm{ha})$.

Soil-to-plant uptake factors, bioaccumulation factors, and the calculation of daily intakes for soil and sediment are shown in Tables 4.25 through 4.29.

\subsection{Effects Assessment}

Effects assessment is the process by which potentially toxic effects on ecological receptors are estimated for each COPC. The assessment evaluates the relationship between levels of exposure and the levels and types of effects in representative species that may result from such exposures. The results of the toxicity assessment are used in Section 4.6 to identify ecological COCs and characterize ecological risk.

\subsubsection{Methodology}

This section describes the methodology used in assessing the potential for toxicity to ecological receptors posed by each COPC detected in surface soil, sediment, and surface water at the F-Area BRPs operable unit. This methodology is summarized in the lower half of Figure 3.1. Different assessment methodologies were followed for intake of contaminants and external exposure to radiation from radionuclides due to their essentially different mechanisms of toxicity. Toxicity data for all non-radioactive COPCs are shown in Table 4.30. 


\subsubsection{Non-radioactive Contaminants}

The methodology for assessing the potentially toxic effects of nonradioactive COPCs was based on the derivation of a toxicity reference value (TRV) for each COPC in each medium. The TRVs were derived to represent reasonable estimates of the chemical concentrations that, if exceeded in an environmental medium, may produce toxicity effects in ecological receptors exposed to that medium. Ideally, TRV values would be based on unit-specific toxicity data. However, in the absence of unit-specific data, toxicity data from the literature were used by establishing data selection criteria such that TRVs would be as relevant as possible to assessment endpoints at the unit. Furthermore, the conservativeness of the TRVs was reirforced by using the lowest availabie, appropriate toxicity values and modifying them by uncertainty factors when necessary. The derivation of TRVs is shown by medium in Table 4.30 .

For exposure of terrestrial receptors to soil and sediment at the unit, the preferred TRVs were derived from toxicity tests on native small mammal species. Terrestrial receptors were chosen for assessing sediment.toxicity because the isolated, shallow, and ephemeral character of the small wetland was considered to provide a greater exposure potential for terrestrial species than for aquatic species. The preferred toxicity test was the lowest appropriate chronic LOAEL (lowest-observed-adverse-effect level) for mortality or reproductive effects. When values were not available for these effects, LOAELs for other, less serious, toxic effects or NOAELs were used as available. Values based on chronic studies were preferred. Studies were considered to provide chronic toxicity data if conducted for a minimum duration of 1 year in mammals or 7 days in fish or invertebrates. Studies between 90 days and 1 year for mammals were considered subchronic. Studies shorter than 90 days were considered acute.

If LOAEL data were not available for a contaminant, the next preferred form of toxicity data for use in deriving a TRV was an $\mathrm{LD}_{50}$ (median lethal dose), or an $\mathrm{LC}_{50}$ (median lethal concentration) in diet.

The order of taxonomic preference when choosing appropriate toxicity values for terrestrial receptors was data for (1) small mammal species potentially present at the unit, or (2) proxy species such as commonly studied laboratory rodents. The lowest (most conservative) value from the preferred data, modified by uncertainty factors(s) as necessary, was used as the TRV for each COPC. 
Upon gathering relevant toxicity information, TRVs were calculated for each of the COPCs following EPA Region VIII (EPA, 1994c) guidance. The TRVs represent LOAELs with uncertainty factors incorporated for toxicity information derived from studies other than chronic lowest-effects studies and studies on species other than the receptors selected for this risk assessment. EPA Region VIII has identified major areas where receptors differ in response to contaminant introduction, as follows: type of study (i.e., chronic lowest-effects versus acute lethality); interspecies variation (such as laboratory mouse to oldfield mouse); variation -from genus (such as rat to oldfield mouse); or variation from family (such as cat to oldfield mouse). Each of these areas is then assigned an uncertainty factor from 1 to 20 based on the inherent variance. In addition, where toxicity information for a surrogate chemical was used, an additional uncertainty factor of 10 was applied. Uncertainty factors are listed below.

\begin{tabular}{|c|c|c|}
\hline CATEGORY OF UNCERTAINTY & \multicolumn{2}{|c|}{ UNCERTAINTY VALUE ${ }^{1}$} \\
\hline Use of surrogate chemical & \multicolumn{2}{|c|}{10} \\
\hline $\begin{array}{l}\text { Intertaxon Extrapolation } \\
\text { Same species } \\
\text { Same genus, different species } \\
\text { Same family, different genus } \\
\text { Same order, different family } \\
\text { Same class, different order }\end{array}$ & \multicolumn{2}{|c|}{$\begin{array}{c}1 \\
3 \\
5 \\
7 \\
10 \\
\end{array}$} \\
\hline $\begin{array}{l}\text { Study Duration Extrapolation } \\
\text { Chronic studies, equilibrium attained } \\
\text { Subchronic studies } \\
\text { Acute studies } \\
\text { Single dose } \\
\text { Unknown }\end{array}$ & \multicolumn{2}{|c|}{$\begin{array}{c}1 \\
5 \\
10 \\
20 \\
20\end{array}$} \\
\hline $\begin{array}{l}\text { Endpoint Extrapolation } \\
\text { No observed effects level } \\
\text { No observed adverse effects level } \\
\text { Lowest observed effects level } \\
\text { Lowest observed adverse effects level } \\
\text { Effect concentration to } 50 \% \text { of test organisms } \\
\text { Unknown }\end{array}$ & $\begin{array}{c}\text { Nonlethal } \\
1 \\
1 \\
1 \\
1 \\
10 \\
10 \\
\end{array}$ & $\begin{array}{c}\text { Lethal } \\
1 \\
1 \\
3 \\
3 \\
15 \\
15 \\
\end{array}$ \\
\hline
\end{tabular}

1 The product of the appropriate uncertainty value from each uncertainty category becomes the uncertainty factor applied to develop the chemical-specific TRV.

For aquatic receptors in surface water, aquatic toxicity data from the Aquatic Information Retrieval (AQUIRE) (1993) or Hazardous Substances Data Bank (HSBD, 1994) databases or 
other sources were used. The databases were searched for the lowest recorded $\mathrm{LC}_{50}$, or $\mathrm{EC}_{50}$ (effect concentration at which 50 percent of the test population exhibits an effect) for reproductive effects, in the following order of taxonomic preference: (1) amphibian, or invertebrate species potentially present at the unit; (2) proxy species commonly studied in the laboratory, such as fathead minnow (Pimephales promelas); or (3) other amphibian or invertebrate species most similar taxonomically and physiologically to native species. The lowest (most conservative) value from the preferred data, modified by uncertainty factors as necessary, was used as the TRV for each surface water COPC.

In evaluating the potential for a chemical to pose ecological risk, it is important to consider its propensity for bioaccumulation even though its concentration in an environmental medium may be below toxic levels. Therefore, all COPCs were evaluated with regard to their ecological persistence and tendency to bioaccumulate.

Bioaccumulation is the process of absorption and retention of a substance by an organism due to both uptake from water (or other surrounding media) and uptake from ingested residues in food, soil, and/or sediment. It is quantified by the calculation of a bioaccumulation factor (BAF). Bioconcentration is a component of bioaccumulation, accounting only for the process of uptake from the surrounding medium (usually water). It is quantified by the calculation of a bioconcentration factor (BCF). Both BAFs and BCFs are proportionality constants relating the concentration of a chemical in the tissues of an organism to the concentration in the surrounding environment (Amdur et al., 1991; EPA, 1989b).

Bioaccumulation and bioconcentration may be a significant component of exposure to COPCs for the BRP receptors. These processes were not evaluated for the aquatic receptor, as the risk evaluation was conducted by comparison of unit surface water COPC concentrations with ambient water concentrations used in toxicity tests. No food chain uptake was evaluated. Because inundation of the wetland is intermittent, the amphibians would not reside in the surface water permanently and long-term food uptake from the unit was not judged to be a significant route of exposure.

For the terrestrial receptor, bioaccumulation was evaluated by means of chemical-specific soil-toplant uptake factors and bioaccumulation factors. The soil-to-plant uptake factors were obtained from Baes et al. (1984) for metals and, for organic compounds, by using a regression equation for from Travis and Arms (1988). The latter is based on the chemical-specific octanol/water partition 
coefficient $\left(\log \mathrm{K}_{\text {ow }}\right.$ ). Bioaccumulation factors were obtained from the scientific literature. Factors reflecting accumulation of COPCs in earthworms were selected, based on the feeding habits of the oldfield mouse receptor. Values for $\log \mathrm{K}_{\text {ow }}$ are shown in Table 4.29 and soil-toplant uptake factors and bioaccumulation factors are shown in Tables 4.25 through 4.29.

\subsubsection{Radioactive Contaminants}

Radionuclides may produce toxic effects as a result of their chemical properties as well as their radioactive properties. It is the radioactive emissions that are considered to be responsible for most of the biologically deleterious effects that may be produced in exposed organisms as a result of intake of radionuclides (ATSDR, "1989j). In addition tc intake, however, ecological receptors also may be affected by radionuclides in the environment through direct exposure to external radiation. Therefore, both routes of exposure were considered in evaluating potential toxicity effects from radioactive contaminants at the unit.

The toxic effects induced in an organism by the chemical properties of an element or compound are characteristic of that specific substance. The adverse effects induced in an organism by radiation, on the other hand, are independent of the chemical toxicity of the radionuclide and are related to the amount of radiation absorbed by tissues and organs. While chemical properties affect the distribution and biological half-life of a radionuclide and influence the retention of the radionuclide within a target organ, the damage from a given type of radiation is independent of the source of that radiation (ATSDR, 1989k).

Radiation occurs as a result of the spontaneous disintegration of the nucleus of certain isotopes of certain elements, which results in the emission of one or more characteristic types of radiation. The types of radiation most commonly produced are alpha particles, beta particles, and gamma rays:

- Alpha particles are doubly-charged cations consisting of two protons and two neutrons. Due to their relatively large mass and charge, alpha particles tend to readily ionize nearby atoms, expending their energy in short distances. Alpha particles are not sufficiently energetic to penetrate the outer layer of the skin or other body coverings of most organisms and do not contribute significantly to exposure from external radiation. However, alpha particles present a significant hazard when taken into the body, where their energy is completely absorbed by small volumes of tissues. As a result, great local tissue damage can occur (EPA, 1989d; Wang et al., 1975). 
- Beta particles are electrons or positrons emitted at high velocities in radioactive decay. They have a smaller mass, higher velocity, and lower ionization potential than alpha particles. As a result, beta particles have more penetrating power and somewhat more range than alpha particles, but their energy from external exposure also is imparted primarily to the outer layers of the skin. Internally, beta particles have a much greater range than alpha particles in tissue. However, because they deposit much less energy to small volumes of tissue, they inflict much less damage than alpha particles (EPA, 1989d).

- Gamma rays are photons emitted from the nucleus of certain radionuclides when they decay. Because gamma rays are a form of electromagnetic radiation, they can readily penetrate matter and have an effective range that is much greater than that of alpha or beta particles of comparable energy. Consequently, gamma rays comprise the primary contribution to radiation dose from external exposures. The entire volume of the body of an organism can be irradiated from an external gamma source, and when taken internally, gamma-emitters can produce essentially whole-body radiation effects, regardless of the localization of the radionuclide within the body (EPA, 1989d; Wang et al., 1975).

The presence of radionuclides in environmental media can result in the two forms of potential radiation exposure discussed above: internal and external. The term, "exposure," when used with regard to radiation, refers to the physical interaction of radiation emitted by radionuclides with the cells and tissues of organisms. Internal exposures occur when radionuclides that have entered the body, such as through ingestion of contaminated soil, undergo radioactive decay, resulting in the deposition of energy to internal organs. External exposures occur when radiation enters the body directly from sources located outside the body, such as from radionuclides present in soil or dissolved in water.

In general, external exposures result from radionuclides that emit gamma radiation, which readily penetrates skin and other body coverings. Alpha and beta radiation from external sources are far less penetrating and deposit their energy primarily on the skin's outer layer. Consequently, their contribution to the total dose of external radiation absorbed by an organism is negligible compared to that deposited by gamma rays (ATSDR, 1989k). Therefore, in evaluating exposures of ecological receptors to external radiation from radionuclides in soil, sediment, and surface water at the F-Area BRPs operable unit, only the gamma-emitting radionuclides in each medium were included. 
Potential toxicity to ecological receptors from internal exposure to radionuclides was evaluated through the derivation of toxicity screening benchmarks. In this screening step, potential risk from external exposure to radionuclides was evaluated by calculating external gamma dose rates for both a large and a small wildlife species, represented by a deer and a mouse, respectively. These dose rates were compared to an external radiation toxicity benchmark of $0.1 \mathrm{rad} /$ day. This evaluation is shown by medium and location in Tables 4.7, 4.9, 4.11, 4.13, 4.15, and 4.17. The benchmark value was based on a study of the effects of ionizing radiation on aquatic biota that found no deleterious effects at dose rates below approximately $0.1 \mathrm{rad} /$ day (Templeton and Blaylock, 1990). The IAEA (1992) reports that irradiation at chronic dose rates of $1 \mathrm{mGray} / \mathrm{day}$ ( $0.1 \mathrm{rad} / \mathrm{day})$ or less does not appear likely to cause observable change in terrestrial animal populations. The $0.1 \mathrm{rad} /$ day value was used for screening purposes. The external dose was calculated by multiplying the maximum concentration of each gamma-emitting COPC by the appropriate external dose conversion factor (DCF) using the following equation (NRC, 1992):

Dose $(\mathrm{rem} / \mathrm{day})=\mathrm{CS} \times \mathrm{DCF} \times \mathrm{CFa} \times \mathrm{CFd}$

where

$$
\begin{array}{ll}
\mathrm{CS}= & \text { Radionuclide concentration in soil or sediment }(\mathrm{pCi} / \mathrm{g}) \\
\mathrm{DCF}= & \text { Dose conversion factor }(\mathrm{Sv} / \mathrm{d} \text { per } \mathrm{Bq} / \mathrm{m} 3) \\
\mathrm{Cfa}= & \text { Conversion factor for activity }(5.92 \times 104 \mathrm{~Bq} / \mathrm{m} 3=1 \mathrm{pCi} / \mathrm{g}) \\
\mathrm{Cfd}= & \text { Conversion factor for dose }(100 \mathrm{rem} / \mathrm{Sv})
\end{array}
$$

For conservatism and calculation convenience, the receptors were assumed to remain resident in the contaminated area. The external DCF values obtained from NRC (1992) were based on exposures at a height of one meter. Therefore, the calculated dose was used directly for deer. However, the dose rate from a large plane source of radiation declines as a function of height above the source. For example, the dose rate at $0.05 \mathrm{~m}$ ( $2 \mathrm{in})$ above a $20-\mathrm{m}(66-\mathrm{ft})$ diameter source would be 2 times the dose rate at $1 \mathrm{~m}$ (39 in).-Consequently, for small animals, represented by the rat, the calculated dose rates were multiplied by a factor of 2 to account for their greater proximity to the radiation source (soil or sediment).

The quantity obtained from the above equation is the dose equivalent $(\mathrm{H})$ of the radiation to which an organism may be exposed. Dose equivalent is equal to absorbed dose (D), measured in rads, multiplied by a quality factor to account for the relative biological effectiveness of a 
radiation type. Because the value of the quality factor for gamma rays is considered to be one, $H$ is essentially equal to $\mathrm{D}$, and the calculated dose equivalent in rems is equal to the absorbed dose in rads. Consequently, the calculated value could be directly compared to the benchmark value of $0.1 \mathrm{rad} /$ day. Estimated radiation doses from each gamma-emitting radionuclide in a medium were summed, and the total dose was compared to the screening benchmark dose. Dose rates from a given environmental medium that exceeded the screening benchmark dose were further evaluated due to the potential for deleterious radiation effects on ecological receptors exposed to that medium.

Risk to ecological receptors from external radiation sources at the unit was estimated based on chronic exposure to those media in which gamma-emitting radionuclides were detected (or gamma-emitting progeny were predicted). The potential for chronic exposure was considered significant only for those radionuclides with a half-life exceeding 14 days (Gillette, 1983). A radionuclide with a half-life equal to or less than 14 days would be significantly depleted within a fraction of the lifetime of most vertebrates and many invertebrates, and it would be of little concern for ecotoxicologic effects.

Risk due to external radiation from radionuclides in soil was estimated for current land use conditions based on detected radionuclides in surface soil from 0 to $0.6 \mathrm{~m}(0-2 \mathrm{ft})$ deep. Risk from soil exposure under future land use conditions was estimated based on detected radionuclides in soil from 0 to $1.2 \mathrm{~m}(0-4 \mathrm{ft})$ deep, to allow for the possibility of future excavation of deeper soils to the surface as a result of human activities such as new home construction. The radionuclides detected in wetland sediment were assumed to remain the same under current and future conditions. No gamma-emitting radionuclides were detected in ephemeral wetland surface water.

As a result of the preliminary toxicity screening step for radionuclides, only tritium remained as a COPC in soil and surface water, due to the lack of a means of calculating dose to the receptors. For sediment, potassium-40, strontium-90, technetium-99, and tritium were retained as COPCs, also due to lack of a means of calculating dose using this scréening method.

Following the screening evaluation for radionuclides, tritium and potassium-40 were qualitatively evaluated and the remaining COPCs in sediment were further evaluated by estimating intake dose to a receptor cotton mouse from sediment containing RME concentrations of strontium- 90 and technetium-99. For estimating radiological dose, it is assumed that the cotton mouse weighs $26 \mathrm{~g}$ and consumes $2.6 \mathrm{~g}$ of food and $10 \mathrm{~mL}$ of water daily. The soil/water ratio and plant/soil ratio 
were the same as those used for the human health evaluation. The concentration of a given radionuclide in the vegetation was calculated by multiplying the water concentration by the soil/water ratio and the plant/soil ratio.

The concentration in the mouse relative to the concentration in food and drinking water was modeled using a simple compartment model similar to the model used in human health evaluations. Intakes through food and drinking water were used in the calculations. The model assumes that a known fraction of the radionuclide entering the gastrointestinal system will be absorbed into the body. The absorbed fraction is distributed among several compartments in the body. For the nuclides of concern in this study, the bones and liver are identified as separate compartments and the rest of the body as another compartment. The distribution of the absorbed radionuclide is assumed to be instantaneous. Loss of the radionuclide from the compartment is calculated as one or the sum of more than one exponential releases. For the case of one exponential release component for each of three compartments, the balance of the radionuclide in the animal is described by the following:

$$
\begin{aligned}
\mathrm{DA}=\mathrm{f}_{\mathrm{i}} * \mathrm{I} * \mathrm{Dt} \quad-\quad & \mathrm{f}_{\mathrm{L}} * \mathrm{~A} *\left\{1-\exp \left[(-0.693 * \mathrm{Dt}) / \mathrm{t}_{\mathrm{L}}\right]\right\}- \\
& \mathrm{f}_{\mathrm{b}} * \mathrm{~A} *\left\{1-\exp \left[(-0.693 * \mathrm{Dt}) / \mathrm{t}_{\mathrm{b}}\right]\right\}- \\
& \mathrm{f}_{\mathrm{r}} * \mathrm{~A} *\left\{1-\exp \left[(-0.693 * \mathrm{Dt}) / \mathrm{t}_{\mathrm{r}}\right]\right\}
\end{aligned}
$$

where:
$\mathrm{A}=$ total radionuclide activity in an animal at any given time, $\mathrm{mCi}$;
$\mathrm{DA}=$ incremental change in total activity during time increment $\mathrm{Dt}, \mathrm{mCi}$;
$\mathrm{Dt}=$ time increment (one day in the calculations presented here);
$f_{i}=$ fraction of radionuclide entering body from gastrointestinal tract;
I $=$ the ingestion rate of the radionuclide in either water or food;
$\mathrm{f}_{\mathrm{L}}=$ fraction of radionuclide distributed to the liver;
$\mathrm{f}_{\mathrm{b}}=$ fraction of radionuclide distributed to the bones;
$\mathrm{f}_{\mathrm{r}}=$ fraction of radionuclide distributed to the rest of the body;
$t_{L}=$ biological half-life of the radionuclide in the liver;
$t_{b}=$ biological half-life of the radionuclide in the bones; and
$\mathfrak{t}_{\mathbf{r}}=$ biological half-life of the radionuclide in the rest of the body. 
Results of this evaluation are shown in Table 4.31. Doses for both strontium-90 and technetium99 were estimated at less than $0.1 \mathrm{rad} /$ day. The IAEA (1992) reports that irradiation at chronic dose rates of $1 \mathrm{mGy} /$ day $(0.1 \mathrm{rad} /$ day) or less does not appear likely to cause observable changes in terrestrial animal populations. Potassium- 40 was not evaluated quantitatively, but this radioisotope is typically not of concern because it occurs naturally in the environment. Although potassium- 40 has a long half-life ( $1.25 \mathrm{E}+08 \mathrm{yr}$ ), it is the most abundant radionuclide in foods and all tissues (Eisler, 1994). The potassium content of the bodies of vertebrates is under strict homeostatic control and is not influenced by variations in environmental levels. For this reason, the internal radiation dose from potassium- 40 is relatively constant (Eisenbud, 1987). However, tritium is a pure beta-emitter of very low energy that is not selectively concentrated in any organ and is metabolized as water. Accordingly, it is rapidly diluted and eliminated and has a relatively rapid turnover rate in the body (a biological half-life of around 10 days) (Eisenbud, 1987), which tends to further reduce the magnitude of the internal radiation dose it may administer. Because it is not a gamma-emitter, external radiation exposure from tritium in surface water was not evaluated.

\subsection{Risk Characterization}

The risk characterization integrates the results of the exposure assessment and the effects assessment in characterizing the potential for adverse effects on receptors that may result from exposure to unit-related chemicals. Risk characterization includes two main steps: risk estimation and risk description. Risk estimation (Section 4.6.1) uses the results of the exposure and effects assessments to calculate for each COPC an ecological quotient (EQ). The EQs are based on relevant measurement endpoints and are indicative of the potential of each chemical to pose ecological risk to receptors. Also in this step, uncertainty in the risk assessment is analyzed and discussed. Risk description (Section 4.6.2) summarizes the conclusions of the risk estimation and discusses confidence in the risk estimates based on the weight of evidence and the uncertainties involved in the assessment. Any chemicals in a given medium and location that were thereby identified as likely to pose significant risk to receptors were classified as ecological chemicals of concern (COCs).

\subsubsection{Risk Estimation}

Estimation of the potential for COPCs at the unit to pose significant risk to receptors was based on the magnitude of the EQ value calculated for each chemical, as well as other factors such as the bioaccumulation/biomagnification potential, mechanism of toxicity, physicochemical characteristics, environmental fate, and ecological relevance of each chemical. Tables 4.32 
through 4.37 present the calculation of EQs for COPCs in each medium and location at the unit. An EQ is a ratio of the estimated exposure dose (for terrestrial receptors) or exposure concentration (for aquatic receptors) of a chemical to the TRV. Generally, the greater this ratio, or quotient, the greater the likelihood of an effect. A quotient of 1 is considered the threshold level at which effects may occur. The TRVs on which the EQs were based were derived to be conservative and representative of chronic exposures, as described previously in Section 4.5.

The calculated EQs were used to assess the potential that toxicological effects will occur among the unit's receptors. The EQs were compared to EQ guidelines for assessing the risk posed from contaminants (Menzie et al., 1993). These guidelines suggest that EQs less than or equal to 1 present no probable risk, EQs from 1 up to but less than 10 present a small potential for environmental effects, EQs from 10 up to but less than 100 present a significant potential that effects could result from greater exposure, and EQs greater than 100 present the highest potential for expected effects. The likelihood that populations and, ultimately, the wildlife community at the unit could be significantly impacted by the toxicological effect(s) produced by a given COPC was a major factor in the subsequent determination (in Section 4.6.2) of whether that chemical should be classified as a chemical of concern (COC).

Ecological risk from non-radioactive chemicals and radionuclides was characterized for both current and potential future land use conditions at the unit (Sections 4.6.1.1 and 4.6.1.2, respectively). Under current conditions, ecological receptors are unlikely to be exposed to soils deeper than $0.6 \mathrm{~m}(2 \mathrm{ft})$. Therefore, soil data from the surface down to this depth was used in characterizing current risk at BRPs $231-\mathrm{F} / 1 \mathrm{~F}$ and at RP $231-2 \mathrm{~F}$. Under hypothetical future land uses involving home construction, deeper soils could be excavated and distributed on the surface, where ecological receptors might then be exposed. Therefore, soil data from the surface down to

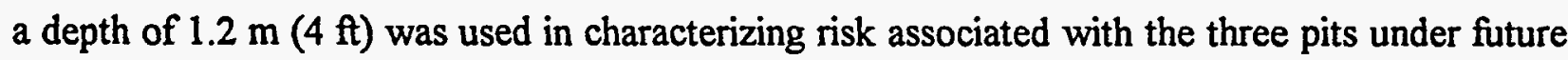
conditions. Current and future conditions were assumed to be the same for the ephemeral wetland area (surface water and sediment). Consequently, this area was discussed only under current land use.

Uncertainty in the assessment process is discussed in Section 4.6.1.3. Toxicity profiles for the COPCs are included in Appendix E. 


\subsubsection{Current Land Use \\ Soil}

BRPs 231-F/1F

For soil sampled to a depth of $0.6 \mathrm{~m}(2 \mathrm{ft})$ at BRPs $231-\mathrm{F} / 1 \mathrm{~F}$, EQs calculated for the nonradioactive COPCs exceeded a value of 1 for barium $(\mathrm{EQ}=6.3)$, chromium $(\mathrm{EQ}=1.1)$, and mercury $(\mathrm{EQ}=32)$ (Table 4.32). However, the magnitude of their exceedence of the TRV was small (less than one order of magnitude) for all but mercury. Dibenzofuran was carried through as a COPC because no toxicity data were available to calculate a TRV.

Barium intake estimated for maximal exposure of receptors at the unit marginally exceeded the TRV intake with an EQ of 6.3. However, the toxicity benchmark value was based on a study in which barium was administered to mice in drinking water. When ingested by animals, soluble barium compounds are absorbed to a limited extent, while insoluble forms, such as barium sulfate and barium carbonate, are only minimally absorbed. Thus, soluble forms are highly toxic relative to insoluble forms (ATSDR, 1990b), and barium that has not leached from surficial soils at the unit is likely to be in an insoluble form. Therefore, the study animals ingested soluble, more toxic forms of barium in water, while receptors at the unit would likely ingest insoluble, less toxic forms in soil. Based on these factors, barium in soil from this location was estimated to pose essentially no risk to assessment endpoints and is not considered to be a COC.

Chromium intake estimated for maximal exposure of receptors at the unit marginally exceeded the TRV intake with an EQ of 1.1. Chromium in its trivalent form (Cr III) is an essential trace nutrient in animals. $\mathrm{Cr} I I$ is the most common form of chromium found in nature. The known harmful effects of chromium in animals are primarily attributable to the hexavalent form (Cr VI) (Amdur et al., 1991), which is generally far more acutely toxic than the trivalent form. The form(s) composing the total chromium level measured at the unit is unknown, so a conservative toxicity benchmark from a subchronic study based on $\mathrm{Cr} V \mathrm{VI}$ was used. Thus, there is substantial uncertainty regarding the significance of chromium concentrations in soil from this area. The EQ for chromium, at 1.1; only marginally exceeded 1 , the level at which a small potential for adverse effects may be expected. Based on these factors in addition to the conservativeness of the exposure assumptions and the magnitude of the quotients, chromium was estimated to pose minimal risk to the assessment endpoints even if continuous use of the area is assumed. Therefore, chromium is not considered to be a COC. 
Mercury intake estimated for maximal exposure of receptors at the unit exceeded the TRV intake with an EQ of 32. Mercury and its compounds have no known biological function. Mercury toxicity in aquatic and terrestrial organisms is well documented. The kidney as well as the central nervous system is the probable critical organ for toxicity effects from mercury in mammals. Mercury also causes teratogenic, mutagenic, and carcinogenic effects. Organic forms of mercury are capable of biomagnifying, especially in aquatic systems. In terrestrial food chains, little is known of mercury bioaccumulation. Carnivores at the top of the food chain appear to have elevated mercury concentrations, indicating biomagnification. The form(s) of mercury composing the total mercury concentration at the unit is not known, so a conservative toxicity level from a chronic study was used for the TRV. . It is unlikely that all or most of the soil mercury is in the organic form, as organic mercury tends to be found more commonly in anaerobic sediments. Inorganic forms of mercury are generally less toxic than organic forms. Nothing is known of the bioavailability of the mercury in soil at the unit either. Absorption of inorganic mercury from oral exposure has been reported at 2 to 38 percent (ATSDR, 1994). With an EQ of 32, mercury in soil presents a possibility of adverse effects to the oldfield mouse receptor using BRPs 231-F/1F as part of its foraging area.

Dibenzofuran lacked data by which to evaluate its toxicity. Bioconcentration studies have shown that dibenzofuran can bioaccumulate significantly in aquatic organisms, but that depuration is rapid (HSDB, 1994). Therefore, long-term bioaccumulative effects and biomagnification are unlikely, and this compound was estimated to pose minimal ecological risk.

Evaluation of potential risk associated with radionuclides at BRPs $231-\mathrm{F} / 1 \mathrm{~F}$ was based on the results shown in Table 4.7. None of the doses calculated for radionuclides exceeded a value of $0.1 \mathrm{rad} / \mathrm{day}$. Toxicity data could not be found for tritium. However, tritium is a pure beta-emitter of very low energy that is not selectively concentrated in any organ and is metabolized as water. Accordingly, it is rapidly diluted and eliminated and has a relatively rapid turnover rate in the body (a biological half-life of around 10 days) (Eisenbud, 1987), which tends to further reduce the magnitude of the internal radiation dose it may administer. Therefore, tritium is not considered as a COC.

$R P$ 231-2F

At RP 231-2F (Table 4-34), of the EQs calculated for the COPCs detected in soil to a depth of $0.6 \mathrm{~m}(2 \mathrm{ft})$, only the $\mathrm{EQ}$ for lead $(\mathrm{EQ}=1.4)$ exceeded a value of 1 . 
Oral exposure to lead leads to increases in blood pressure in laboratory animals (lead-induced hypertension) as well as interference with heme synthesis. A LOAEL resulting in increased blood pressure in laboratory rats was used to generate the TRV for this assessment (ATSDR, 1991f). It is not known if such a physiological effect would have an effect on the populations of small mammals at the unit. In addition, this assessment assumed $100 \%$ availability of lead from the soil ingested by the receptor. As lead tends to be very strongly bound to organic matter in soil, such high bioavailability certainly is not the case at the unit. With an EQ only slightly exceeding 1 , lead was not judged to present a significant potential for adverse effects and is not considered a COC.

Evaluation of risk associated with radionuclides.at RP 231-2F was based on the results shown in Table 4.11. None of the doses calculated for radionuclides with available toxicity data exceeded a value of $0.1 \mathrm{rad} /$ day. Due to lack of toxicity data, tritium could not be evaluated. However, for reasons outlined in the discussion of current conditions at BRPs 231-F/1F, tritium is not judged to be a COC.

\section{Surface Water}

None of the EQs calculated for the non-radioactive COPCs in surface water at the ephemeral wetland exceeded a value of 1 (Table 4.36).

The only radionuclide detected in surface water at the ephemeral wetland was tritium, at a maximum concentration of $10,400 \mathrm{pCi} / \mathrm{L}$ (Table 4.15). An EQ could not be calculated for tritium due to the lack of available toxicity data. However, tritium is a pure beta-emitter of very low energy that is not selectively concentrated in any organ and is metabolized as water. Accordingly, it is rapidly diluted and eliminated and has a relatively rapid turnover rate in the body (a biological half-life of around 10 days) (Eisenbud, 1987); this tends to further reduce the magnitude of the internal radiation dose it may impart. Because it is not a gamma-emitter, external radiation exposure from tritium in surface water was not evaluated. Therefore, tritium in surface water at the ephemeral wetland is not a COC.

\section{Sediment}

With the exception of mercury ( $E Q=10)$, none of the $E Q s$ calculated for the non-radioactive COPCs in sediment at the ephemeral wetland exceeded a value of 1 (Table 4.37). For the reasons discussed above for soil, the mercury in sediment at the wetland was estimated to pose a possible risk to the oldfield mouse receptor that may use the ephemeral wetland as part of its foraging area. 
None of the doses calculated for those radionuclides in sediment for which toxicity data were available exceeded the benchmark screening value of $0.1 \mathrm{rad} /$ day (Table 4.17). A food chain uptake dose rate to the mouse receptor was calculated for strontium-90 (1.77 x $\left.10^{-5} \mathrm{rad} / \mathrm{day}\right)$, and technetium-99, $\left(3.51 \times 10^{-9} \mathrm{rad} /\right.$ day $)$, and found to be well below the threshold of $0.1 \mathrm{rad} /$ day (Table 4.31). Irradiation at chronic dose rates of $0.1 \mathrm{rad} /$ day or less dees not appear likely to cause observable changes in terrestrial animal populations. As previously discussed, while toxicity data could not be found for tritium or potassium-40, neither was determined to pose significant risk to ecological receptors.

\subsubsection{Future Land Use \\ Soil}

BRPs 231-F/1F

At BRPs 231-F/1F under hypothetical future conditions in which deep soils down to $1.2 \mathrm{~m}(4 \mathrm{ft}$ ) have been spread on the surface, the only COPCs with a calculated EQ exceeding a value of 1 were the metals barium ( $E Q=7.0)$, chromium $(E Q=1.9)$, and mercury $(E Q=21)$ (Table 4.33). As discussed above for soil at this location under current conditions, barium and chromium were estimated to pose essentially no risk to assessment endpoints. For the reasons discussed for soil under current conditions, mercury was estimated to pose a possible risk to the oldfield mouse receptor that may use the unit as part of its foraging area.

As with soil under current conditions, dibenzofuran was carried through as a COPC because toxicity data were not available to calculate a TRV. For reasons discussed under current soil conditions, dibenzofuran is not considered of concern.

Under hypothetical future land use conditions at BRPs 231-F/1F (Table 4.9), the radionuclides and activities to which ecological receptors may be exposed will likely be essentially unchanged from current conditions. All radionuclides were below the screening dose. Only tritium could not be evaluated, and for reasons described under current conditions, tritium was not determined to be of concern.

\section{RP 231-2F}

At RP 231-2F under the future land use scenario (Table 4.35), no EQs exceeded 1.

Under hypothetical future land use conditions at RP 231-2F (Table 4.13), radionuclides and activities to which ecological receptors may be exposed will likely be essentially unchanged from 
current conditions. All radionuclides were below the screening dose. Only tritium could not be evaluated, and for reasons described previously, tritium was not determined to be of concern.

\subsubsection{Uncertainty}

Uncertainty is inherent in each step of the ecological risk assessment process. Major factors contributing to uncertainty in this risk assessment are discussed qualitatively in the following sections.

\section{Chemicals of Potential Concern}

The sampling data may not represent the actual overall distribution of contamination at the unit, which could result in underestimation or overestimation of potential risk from identified chemicals. However, the use of RME concentrations provided conservative exposure estimates and it is, therefore, unlikely that the potential for deleterious levels of contaminants has been underestimated. The lack of analytical data for surface water or sediment in the tributary north of the unit and the drainage gully to the west of the unit potentially could have resulted in underestimation of the number of COPCs and their environmental concentrations. However, contaminants found in these areas likely would have been at lower concentrations than on the unit and would have been difficult to attribute to the unit.

\section{Exposure Assessment}

Uncertainty in the exposure assessment was minimized by conducting a unit-specific ecological characterization in addition to utilizing data from three previous field surveys and other available literature. Nevertheless, the potential receptor species listed as having been observed at the unit are inevitably a limited subset of the total list of species, that may utilize the unit to some extent as part of their home range during at least a portion of the year. However, the potential exposures of the species evaluated in this assessment are considered likely to be representative of the nature and magnitude of the exposures experienced by those species not discussed. In addition, it was not feasible to obtain actual field data quantifying the extent to which potential receptors actually utilize the unit for habitat. Therefore, a conservative assumption that all foraging is done in the unit was made. Given the small size of the unit $(0.55 \mathrm{ha})$, this is likely an overestimation. Typically, the foraging range of the oldfield mouse is about $2 \mathrm{ha}$ (Table 4.23).

Risk associated with intake of contaminants through the food chain was addressed by modeling food chain transfer of chemical residues through plants and earthworms. Beginning with bioconcentration directly from environmental media to plants or animals, followed by estimation 
of residue concentrations in animals at increasingly higher trophic levels due to bioaccumulation and biomagnification, the degree of uncertainty in the results of the analysis increases with the increasing distance of the receptor from the base of the food chain. Intakes from dermal contact with and inhalation of contaminants were not quantifiable for ecological receptors. However, this does not significantly increase the uncertainty of the estimated intakes, because for most receptors, intakes via these routes are likely to be minimal relative to intakes via ingestion.

Risk was estimated for both current land use and future land use conditions. Whereas estimates of the potential for risk to assessment endpoints under current conditions potentially could be underestimated because of data insufficiencies (although unlikely because of conservative assumptions), such estimates for hypothetical future conditions are more likely to overestimate the potential for adverse effects.

\section{Toxicity Assessment}

There is uncertainty associated with the TRVs calculated for this risk characterization because the toxicity benchmark data were not unit-specific. However, the TRVs used were conservative, being the lowest available, appropriate toxicity values, relevant to the assessment endpoints, and modified by uncertainty factors where necessary to increase the applicability of the data to the assessment. The EQs calculated from these conservative TRVs and RME exposure concentrations provide confidence that the risk assessment yielded reasonably conservative estimates of the potential risk of adverse ecological effects on the assessment endpoints.

In addition, most toxicity studies administer the chemical under study in a highly bioavailable form, and for the purpose of this assessment, each COPC in each environmental medium was assumed to be highly bioavailable. However, for most chemicals in most media, this is an overestimate (Dixon et al., 1993), which may result in an overestimation of the potential for ecological risk. Empirical information on bioavailability of the COPCs was not available. No leachability tests in soil or pore water partitioning analyses for sediment were conducted. It is possible that some of the contaminants, particularly the metals, may be bound to soil particles and not available for uptake by receptors. This would tend to overestimate risk. The soil-to-plant uptake equations and the bioaccumulation factors include a bioavailability factor; however, these data, taken from the scientific literature, are not specific to this unit and may under- or overestimate exposure. 
The potential for toxic effects to be produced in receptor organisms as a result of exposure to multiple chemicals in a single medium or in multiple media was not evaluated. Exposure to multiple chemicals may have synergistic, additive, or antagonistic effects which could not be predicted. Therefore, the potential for toxic effects in a receptor as a result of exposure to a given medium could be higher or lower than estimated, depending on such toxicological interactions. Exposure of a receptor to multiple contaminated media is likely to increase the risk of toxic effects.

\section{Risk Characterization}

The methodology, conservative assumptions, and toxicity benchmarks used in the risk estimation portion of the risk characterization are expected to overestimate, rather than underestimate, the potential for COPCs to pose risk to the ecological assessment endpoints. RME environmental concentrations were used, concentrations were assumed to remain constant over time, and the toxicity benchmarks used were the lowest reported LOAEL values for mortality or reproductive effects that were appropriate for extrapolation to effects on assessment endpoints. In many cases where LOAELs for these effects were not available, LOAELs for less serious effects or NOAELs were substituted. Therefore, the actual risks of deleterious effects are expected to be less than indicated by the values of the ecological quotients.

While bioaccumulation was determined not to be a significant fate process for the COPCs, it can be very significant for compounds such as organochlorine pesticides. However, as noted in Section 4.3, "Endpoint Selection," it is inappropriate to attempt to attribute possible bioaccumulation/biomagnification effects on wide-ranging, top-trophic-level predators such as raptors to soil contaminants at a small, relatively isolated, source unit. The uncertainties in such an evaluation are large and minimize confidence in any conclusions reached. However, potential effects of bioaccumulative contaminants in the food chain may be assessed based on native rodents because most species, including the oldfield mouse, feed on invertebrates (or even small vertebrates) in addition to vegetation. Because they may do so predominantly within the area of the unit, they may be particularly subject to exposure to bioaccumulative contaminants. Ecological risk due to bioaccumulation/biomagnification potential was assessed on that basis.

\subsubsection{Risk Description}

The risk description has two main elements: (1) the ecological risk summary, which summarizes the results of the risk estimation and uncertainty analysis and assesses confidence in the risk 
estimates based on the weight of evidence; and (2) the interpretation of ecological significance, which describes the magnitude of the identified risks to the assessment endpoint(s).

\subsubsection{Ecological Risk Summary}

The risk estimation step resulted in the identification of a subset of COPCs for each medium/location within the unit for both current and hypothetical future conditions. These subsets of COPCs include those chemicals estimated to have the potential to pose adverse effects to the assessment endpoints selected in Section 4.3. In this section, these COPCs are further evaluated based on the weight of evidence, and a determination will be made as to whether any have a high likelihood of being a significant risk to the receptor population analyzed for this risk assessment or the ecological community that encompasses the area of the unit.

A hierarchy of assessment endpoints was selected in order to assess both proximate and ultimate risks, that might be associated with unit-related chemicals. The proximate assessment endpoints were chosen to provide protection of the population levels of vertebrate species that utilize the area of the unit to a significant extent and that are important as indicators of potential effects on the health of the community. Oldfield mice represent terrestrial vertebrate populations at the unit, and southern toads represent amphibian and other semi-aquatic animal populations that may inhabit the small, ephemeral wetland area when it is inundated. Toxic effects that reduce these assessment endpoint populations or the populations they represent in the immediate vicinity of the unit are significant to the populations themselves; they are not necessarily significant to the ultimate, more important, assessment endpoint - the community of species that occupies the area surrounding and including the unit.

This ultimate assessment endpoint - maintenance of the health and diversity of the natural community in the area - is the most important ecological component to be protected with regard to this unit. Therefore, those COCs estimated to pose a potential for adverse effects to proximate assessment endpoints were subsequently evaluated with regard to the risk they may pose to the ultimate assessment endpoint.

As part of the weight of evidence evaluation, the environmental setting of the waste unit itself is an important consideration. The ecological setting of the unit, as described in Section 4.2, is not characterized by uniqueness or significance. There are no endangered, threatened, or special concern species in the vicinity that are likely to be dependent on or affected by the habitat at the unit. The species that inhabit the unit are not rare in the region and are not generally considered 
to be of special societal value. The area of the unit is small, and the habitat it provides appears to be relatively low in diversity and productivity.

The risk characterization step evaluated contaminants in soil, sediment, and surface water. Four of the COPCs identified in soil or sediment at the unit have an EQ greater than 1. These are listed below according to the location and medium in which they occurred.

\section{MEDIUM / location}

SOIL

BRPs 231-F/1F - current conditions.

BRPs 231-F/1F - future conditions

RP 231-2F - current conditions

RP 231-2F - future conditions

SEDIMENT

SURFACE WATER

\section{COPC}

barium $(\mathrm{EQ}=6.3)$, chromium $(\mathrm{EQ}=1.1)$,

mercury $(\mathrm{EQ}=32)$

barium $(E Q=7.0)$, chromium $(E Q=1.9)$, mercury $(\mathrm{EQ}=21)$

lead $(\mathrm{EQ}=1.4)$

none

mercury $(E Q=10)$

none

There is a low likelihood of risk to the proximate assessment endpoint - oldfield mouse populations at the unit - from the concentrations of barium, chromium, and lead based on the following weight of evidence.

- The EQs for barium, chromium, and lead are below 10 for each medium and soil depth interval. According to the guidelines originally proposed by Menzie et al. (1993), EQs from 1 up to but less than 10 present a small potential for environmental effects.

- Calculation of the TRVs and exposure rates for barium, chromium, and lead was a very conservative process.

- Barium is most likely present in an insoluble form in the soil, which is not very bioavailable for uptake by receptors. The TRV was derived from a toxicity test in which a soluble, and therefore much more bioavailable, form of barium was administered in drinking water. Therefore, use of this TRV is likely to overestimate risk. 
- While the chemistry of chromium would indicate that it is likely to be present in the soil at the unit in the trivalent (III) form, the TRV was based on a toxicity test for hexavalent (VI) chromium, a more toxic form. Therefore, use of this TRV is likely to overestimate risk.

- Lead is likely to be tightly bound to organic matter in the soil, and therefore not very bioavailable for uptake. The exposure assessment assumed $100 \%$ bioavailability from soil and will likely overestimate risk.

- The exposure assessment assumed that all the oldfield mouse's foraging is done on the unit. The unit is only $0.55 \mathrm{ha}$, and the usual foraging range for an oldfield mouse is approximately 2 ha. Therefore, the exposure calculation overestimates the mouse's foraging in the unit.

- Habitat quality of the unit is poor. While several individuals of the receptor species were found at the unit, it is unlikely that the unit provides important habitat that would support a significant portion (at least $20 \%$ ) of the oldfield mouse population in the local area.

Therefore, barium, chromium, and lead were not considered to be ecological COCs.

Only mercury in BRPs $231-\mathrm{F} / 1 \mathrm{~F}$ (current and future exposure scenarios) and in sediment equaled or exceeded an EQ of 10. The guidelines proposed by Menzie et al. (1993) suggest that EQs from 10 up to but less than 100 present a significant potential that effects could result from greater exposure. Mercury is considered to be a COC, but not likely to cause significant effects to the overall receptor population in the local area or to the ecological community based on the following weight of evidence.

- Calculation of the TRVs and exposure rates for mercury was a very conservative process.

- The form(s) of mercury composing the total mercury concentration at the unit is not known, so a conservative toxicity level from a chronic study was úsed for the TRV. It is unlikely that all or most of the soil mercury is in the organic form, as organic mercury tends to be found more commonly in anaerobic sediments. Inorganic forms of mercury are generally less toxic than organic forms. 
- Nothing is known of the bioavailability of the mercury in soil at the unit. Absorption of inorganic mercury from oral exposure has been reported at 2 to 38 percent (ATSDR, 1994). The exposure assessment assumed $100 \%$ bioavailability from soil.

- The exposure assessment assumed that all the oldfield mouse's foraging is done on the unit. The unit is only $0.55 \mathrm{ha}$, and the usual foraging range for an oldfield mouse is approximately 2 ha. Therefore, the exposure calculation overestimates the mouse's foraging in the unit.

- Habitat quality of the unit is poor. While several individuals of the receptor species were found at the unit, it is unlikely that the unit provides important habitat that would support a significant portion (at least $20 \%$ ) of the oldfield mouse population in the local area.

Therefore, while the EQ indicates a potential for significant effects to the oldfield mouse individuals using the unit as a foraging area as a result of mercury concentrations at BRPs 231$\mathrm{F} / 1 \mathrm{~F}$ and the wetland sediment, it is unlikely that these effects would cause a significant adverse effect (loss of $20 \%$ or more of the population) on the local oldfield mouse population or the ecological community. 


\section{FIGURES}


BASRLINE RISK ASSEŚSMENT

WWSRC-TR-94-108

F-AREA DURNING/RUDBLE PITS AND RUTEBLE PIT MARCH 1996

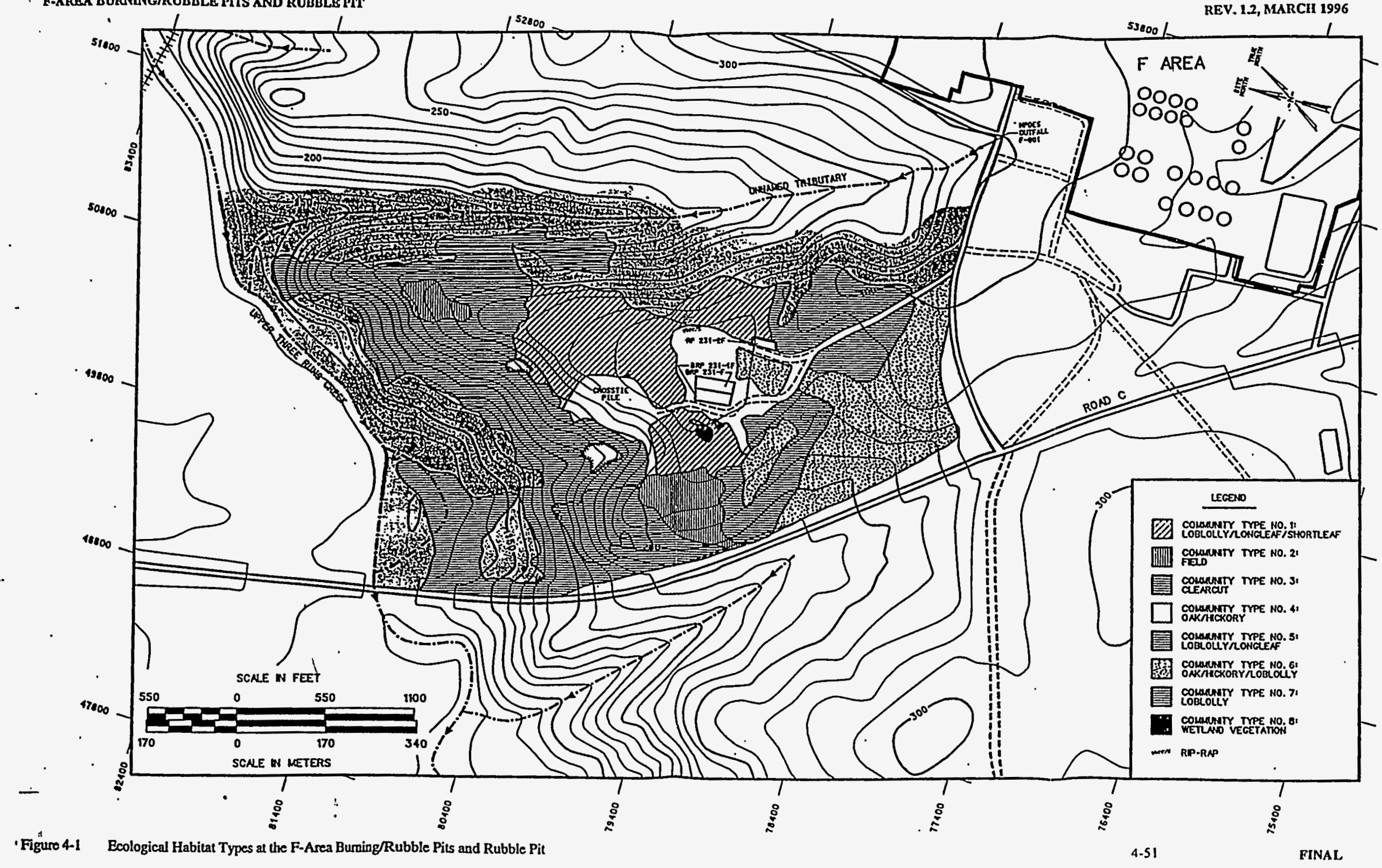


Figure 4.2. Ecologieal Concoptual Sito Model for the F-Aren Buming/Rubblo Pite and Rubblo Pit

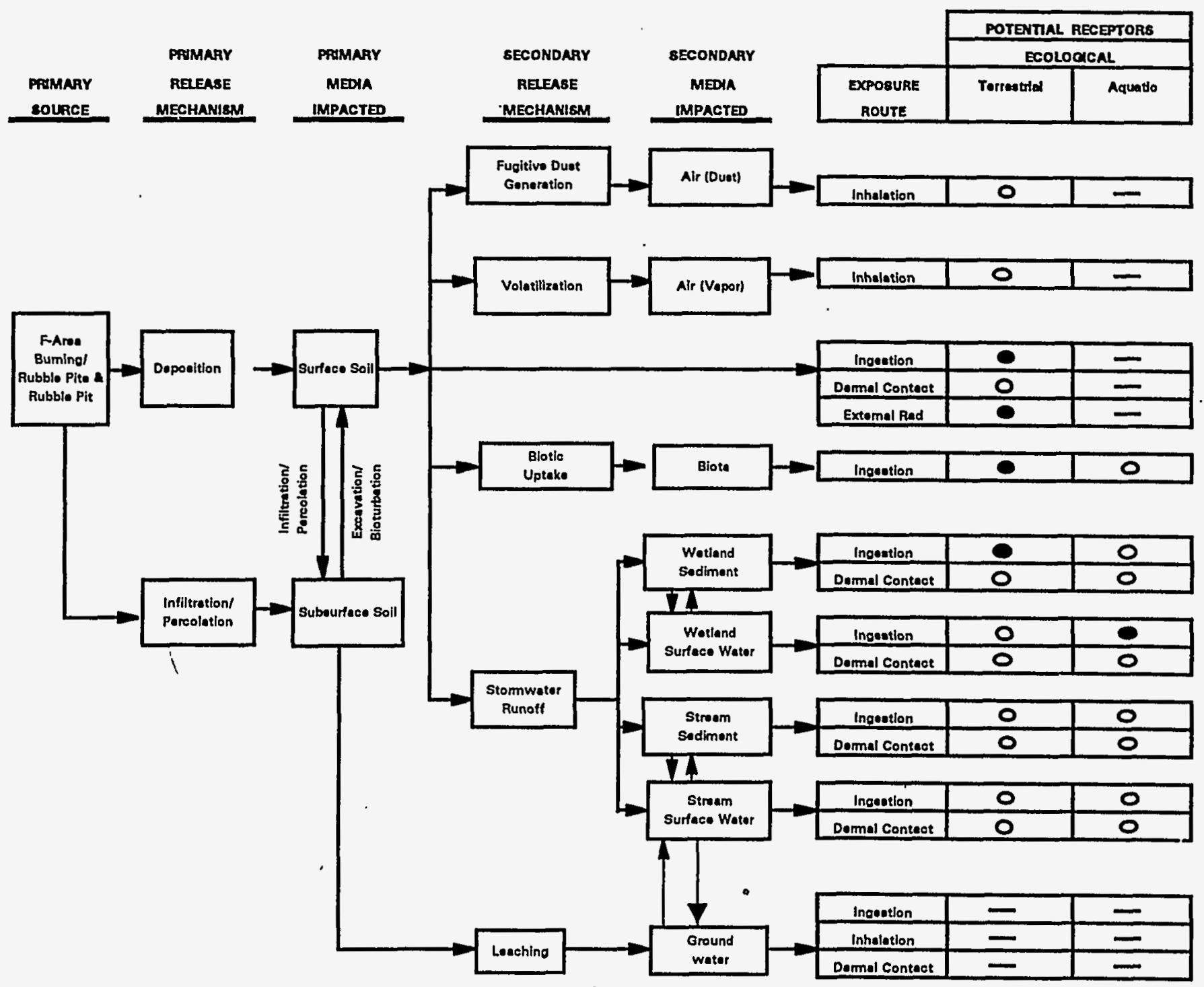

$\rightarrow$ = Pathwoys, both curront and hiotorical

- Principel Pothwayo for quentitetive ovaluation

- Pathways for qualitativo evaluation

- incomplosto pecthwayo 
Table 4.1

SOIL ANALYTES AND BACKGROUND SCREENING

BRP 231-F/1F; 0 - 0.6 M (0 - 2 FT)

CURRENT CONDITIONS

\begin{tabular}{|c|c|c|c|c|c|c|c|c|}
\hline Chemical & CASNo. & $\begin{array}{l}\text { Frequency of } \\
\text { Detection }\end{array}$ & Units & $\begin{array}{l}\text { Maximum } \\
\text { Detection }\end{array}$ & $\begin{array}{l}95 \% \text { UCL of Los- } \\
\text { Transformed Data }\end{array}$ & $\begin{array}{c}\text { Reasonable } \\
\text { Maximum } \\
\text { Expesure } \\
\text { Concentration }\end{array}$ & $\begin{array}{c}2 \text { times } \\
\text { Background } \\
\text { Concentration }\end{array}$ & $\begin{array}{l}\text { Marimum } \\
\text { Exceeds 2X } \\
\text { Background } \\
\text { Cencentration } \\
\left(\mathbf{Y N ) ^ { 2 }}\right.\end{array}$ \\
\hline
\end{tabular}

Non-Radionettve

Volatiles

1,1,2-Trichloroethano

1,2-Dichloroethene

Acetone

Chloroform

Dichloromethano (methylene chlorido)

Tetrachloroethyiens

i Tolueno

Xylenes

Semivolatiles

2-Methytraphthaleno

Acenaphthene

Acemaphthyieno

Anthraceno

Berood(a) anthrucene

Benzo(a)pyreno

Benzo(b) fluoranthene

Benzo(Bhi)perylene

Benzo(k)fluoremtheno

Benzoic acid

Bis(2-ethythexsyl)phthalato

Butylbenzyl phthalato

Chryseno

Dimbutyl phthalate

Di-n-octyl phthalate

Dibenz(a,h)amthracene

Parcon-Tampa: 223196

Sheet Tablef-1 of File TABSI $2 \times 1$ S

Pago 1 of 4

$\begin{array}{rcccc}79-00-5 & 2 & / & 16 & \text { MO/KO } \\ 107-06-2 & 2 & / & 16 & \text { MO/KO } \\ 67-64-1 & 14 & / & 16 & \text { MO/KO } \\ 67-66-3 & 1 & / & 16 & \text { MO/KO } \\ 75-09-2 & 14 & / & 16 & \text { MO/KO } \\ 127-18-4 & 1 & / & 16 & \text { MO/KO } \\ 108-88-3 & 10 & / & 16 & \text { MOKKO } \\ 1330-20-7 & 1 & / & 16 & \text { MO/KO }\end{array}$

$\begin{array}{rcccc}91-57-6 & 2 & / & 16 & \text { MO/KO } \\ 83-32-9 & 4 & / & 16 & \text { MO/KO } \\ 208-96-8 & 4 & / & 16 & \text { MO/KG } \\ 120-12-7 & 9 & / & 16 & \text { MO/KO } \\ 56-55-3 & 14 & / & 16 & \text { MG/KO } \\ 50-32-8 & 14 & / & 16 & \text { MO/KO } \\ 205-99-2 & 14 & / & 16 & \text { MO/KO } \\ 191-24-2 & 12 & / & 16 & \text { MO/KO } \\ 207-08-9 & 13 & / & 16 & \text { MO/KO } \\ 65-85-0 & 2 & / & 16 & \text { MO/KO } \\ 117-81-7 & 14 & / & 16 & \text { MO/KO } \\ 85-68-7 & 1 & / & 16 & \text { MO/KO } \\ 218-01-9 & 14 & / & 16 & \text { MO/KO } \\ 84-74-2 & 8 & / & 16 & \text { MO/KO } \\ 117-84-0 & 2 & / & 16 & \text { MO/KO } \\ 53-70-3 & 6 & / & 16 & \text { MO/KO }\end{array}$

$1.65 \mathrm{E}-02$
$2.16 \mathrm{E}-02$
$2.62 \mathrm{E}+01$
$1.01 \mathrm{E}-02$
$3.80 \mathrm{E}-02$
$2.87 \mathrm{E}-03$
$3.06 \mathrm{E}-02$
$1.34 \mathrm{E}-03$


$7.69 \mathrm{E}-02$
$3.16 \mathrm{E}-01$
$8.61 \mathrm{E}-02$
$6.74 \mathrm{E}-01$
$1.62 \mathrm{E}+00$
$1.33 \mathrm{E}+00$
$1.28 \mathrm{E}+00$
$9.52 \mathrm{E}-01$
$1.49 \mathrm{E}+00$
$4.86 \mathrm{E}-02$
$6.02 \mathrm{E}-01$
$2.08 \mathrm{E}-01$
$1.62 \mathrm{E}+00$
$2.42 \mathrm{E}-01$
$7.90 \mathrm{E}-02$
$4.73 \mathrm{E}-01$

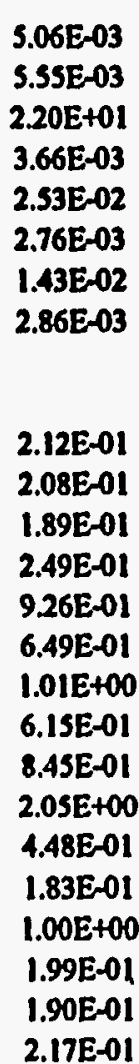

$5.06 \mathrm{E}-03$
$5.55 \mathrm{E}-03$
$2.20 \mathrm{E}+01$
$3.66 \mathrm{E}-03$
$2.53 \mathrm{E}-02$
$2.76 \mathrm{E}-03$
$1.43 \mathrm{E}-02$
$1.34 \mathrm{E}-03$


$7.69 \mathrm{E}-02$
$2.08 \mathrm{E}-01$
$8.61 \mathrm{E}-02$
$2.49 \mathrm{E}-01$
$9.26 \mathrm{E}-01$
$6.49 \mathrm{E}-01$
$1.01 \mathrm{E}+00$
$6.15 \mathrm{E}-01$
$8.45 \mathrm{E}-01$
$4.86 \mathrm{E}-02$
$4.48 \mathrm{E}-01$
$1.83 \mathrm{E}-01$
$1.00 \mathrm{E}+00$
$1.99 \mathrm{E}-01$
$7.90 \mathrm{E}-02$
$2.17 \mathrm{E}-01$

ND
ND
$1.06 \mathrm{E}-01$
ND
$1.40 \mathrm{E}-02$
ND
2.80E-02
ND

ND
ND
ND
ND
ND
ND
ND
ND
ND
ND
$1.56 E+00$
ND
ND
$1.26 E+00$
$1.70 E-01$
ND

$\mathbf{Y}(\mathbf{a})$

$\mathbf{Y}$

$\mathbf{Y}(\mathbf{a})$

$\mathbf{Y}(\mathbf{a})$

$\mathbf{Y}$

$\mathbf{Y}$
$\mathbf{Y}$
$\mathbf{Y}$
$\mathbf{Y}$
$\mathbf{Y}$
$\mathbf{Y}$
$\mathbf{Y}$
$\mathbf{Y}$
$\mathbf{Y}$
$\mathbf{Y}(\mathbf{a})$
$\mathbf{Y}$
$\mathbf{Y}$
$\mathbf{Y}(\mathbf{a})$
$\mathbf{Y}(\mathbf{a})$
$\mathbf{Y}$


SOIL ANALYTES AND BACKGROUND SCREENING

BRP 231-F/1F; 0 - $0.6 M(0-2$ FT)

CURRENT CONDITIONS

\begin{tabular}{|c|c|c|c|c|c|c|c|c|c|c|}
\hline Chemical & CASNo. & $\begin{array}{l}\text { Freq } \\
\text { De }\end{array}$ & & cy of & Units & $\begin{array}{l}\text { Maximum } \\
\text { Detection }\end{array}$ & $\begin{array}{l}95 \% \text { UCL of Log- } \\
\text { Transformed Data }\end{array}$ & $\begin{array}{c}\text { Rexsonable } \\
\text { Mnsimum } \\
\text { Exposure } \\
\text { Concentration' }\end{array}$ & $\begin{array}{c}2 \text { times } \\
\text { Brekground } \\
\text { Coricentration }\end{array}$ & $\begin{array}{l}\text { Masimum } \\
\text { Exceeds 2X } \\
\text { Background } \\
\text { Concentration } \\
\text { (YNN) }\end{array}$ \\
\hline Diberzofuran & $132-64-9$ & 1 & 1 & 16 & MG/KO & 2.22E-01 & $1.85 E-01$ & 1.85E-0! & ND & $\mathbf{Y}$ \\
\hline Fhoranthene & $206-44-0$ & 14 & I & 16 & $\mathrm{MO} / \mathrm{KO}$ & $3.91 E+00$ & $2.04 E+00$ & $2.04 E+00$ & 1.06E-01 & $\mathbf{Y}(a)$ \\
\hline Fluorene & $86-73-7$ & 3 & I & 16 & $\mathrm{MO} / \mathrm{KO}$ & 2.99E-01 & 2.17E-01 & 2.17E-01 & ND & $\mathbf{Y}$ \\
\hline Indeno $(1,2,3-\infty d)$ pyreno & $193-39-5$ & 12 & 1 & 16 & $\mathrm{MO} / \mathrm{KO}$ & 8.32E-01 & S.25E-01 & 5.25E-01 & ND & $\mathbf{Y}$ \\
\hline Naphthaleno & $91-20-3$ & 2 & 1 & 16 & MO/KG & $3.22 \mathrm{E}-01$ & 2.21E-01 & $2.21 E-01$ & ND & $\mathbf{Y}$ \\
\hline Phenenthreno & 85-01-8 & 12 & 1 & 16 & $\mathrm{MO} / \mathrm{KG}$ & $3.38 \mathrm{E}+00$ & $6.87 \mathrm{E}-01$ & $6.87 \mathrm{E}-01$ & ND & $\mathbf{Y}$ \\
\hline Pyrene & 129000 & 14 & 1 & 16 & MO/KO & $2.42 \mathrm{E}+00$ & $1.23 E+00$ & $1.23 E+\infty 0$ & ND & $\mathbf{Y}$ \\
\hline \multicolumn{11}{|l|}{ Dioxina/furans } \\
\hline Heptachlorodiberro-p-dioxin isomers & $37871-00-4$ & 10 & 1 & 16 & MOKKO & 8.30E-03 & $4.11 E-03$ & $4.11 \mathrm{E} 03$ & ND & $\mathbf{Y}$ \\
\hline Heptechlorodibenzo-p-furan isomers & $38998-75-3$ & 6 & 1 & 16 & $\mathrm{MG} / \mathrm{KO}$ & S.00E-04 & 2.58E-04 & $2.58 \mathrm{E}-04$ & ND & $\mathbf{Y}$ \\
\hline Hexachlorodiberzo-p-furan isomers & S5684-94-1 & 1 & 1 & 16 & $\mathrm{MG} / \mathrm{KO}$ & $1.00 \mathrm{E}-04$ & $1.40 \mathrm{E}-04$ & $1.00 \mathrm{E}-04$ & ND & $\mathbf{Y}$ \\
\hline Octechlorodiberro-p-dioxin isomers & $3268-87-9$ & 15 & 1 & 16 & $\mathrm{MO} / \mathrm{KO}$ & 1.10E-02 & 9.26E-03 & $9.26 \mathrm{E}-03$ & $1.00 \mathrm{E}-03$ & $\mathbf{Y}(\mathbf{a})^{\circ}$ \\
\hline Octachlorodibeneo-p-furan isomers & $39001-02-0$ & 6 & 1 & 16 & MO/KO & 8.00E-04 & $3.48 \mathrm{E}-04$ & $3.48 \mathrm{E}-04$ & ND & $\mathbf{Y}$ \\
\hline \multicolumn{11}{|l|}{ PCBa } \\
\hline PCB 1254 & $11097-69-1$ & 8 & 1 & 16 & MO/KG & $4.38 \mathrm{E}-01$ & $1.78 \mathrm{E}-01$ & $1.78 \mathrm{E}-01$ & ND & $\mathbf{Y}$ \\
\hline \multicolumn{11}{|l|}{ Pesticides } \\
\hline Aldrin & $309-00-2$ & 2 & 1 & 16 & $\mathrm{MO} / \mathrm{KO}$ & $4.80 E-03$ & 2.33E-03 & 2.33E-03 & ND & $\mathbf{Y}$ \\
\hline alpha-Chlondano & $5103-71-9$ & 4 & 1 & 16 & MOKKO & $7.43 E-03$ & $3.56 \mathrm{E}-03$ & $3.56 \mathrm{E}-03$ & ND & $\mathbf{Y}$ \\
\hline delta-Benzen hexachloride & $319-86-8$ & 1 & 1 & 16 & MO/KO & 1.84E-03 & , 1.89E-03 & $1.84 E-03$ & ND & $\mathbf{Y}$ \\
\hline Endosulfan 11 & $33213-65-9$ & 1 & 1 & 16 & $\mathbf{M G / K G}$ & 4.69E-03 & $3.88 E-03$ & $3.88 \mathrm{E}-03$ & ND & $\mathbf{Y}$ \\
\hline Endrin ketone & $53494-70-5$ & 1 & 1 & 16 & MO/KO & $3.20 \mathrm{E}-03$ & $5.28 E-03$ & $3.20 E-03$ & ND & $\mathbf{Y}$ \\
\hline gamma-Chlondens & $5566-34-7$ & 6 & 1 & 16 & MO/KO & $6.49 \mathrm{E}-03$ & $3.08 E-03$ & $3.08 \mathrm{E}-03$ & ND & $\mathbf{Y}$ \\
\hline P.P'-DDD & $72-54-8$ & 2 & 1 & 16 & $\mathrm{MO} / \mathrm{KG}$ & $1.39 \mathrm{E}-02$ & $5.05 E-03$ & 5.05E-03 & ND & $\mathbf{Y}$ \\
\hline p.p-DDE & $72-55-9$ & 2 & 1 & 16 & $\mathrm{MO} / \mathrm{KO}$ & 2.03E-02 & $5.62 \mathrm{E}-03$ & $5.62 \mathrm{E}-03$ & ND & $\mathbf{Y}$ \\
\hline P,P'-DDT & $50-29-3$ & 4 & 1 & 16 & $\mathrm{MO} / \mathrm{KO}$ & 4.31E-02 & 9.95E-03 & $9.95 \mathrm{E}-03$ & ND & $\mathbf{Y}$ \\
\hline
\end{tabular}


Table 4.1

SOIL ANALYTES AND BACKGROUND SCREENING

BRP 231-F/1F; 0 - 0.6 M (0-2 FT) CURRENT CONDITIONS

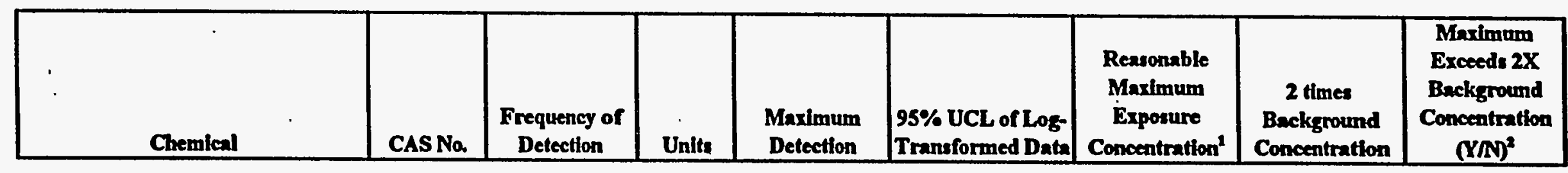

$\begin{array}{ll} & \text { Metals } \\ & \text { Arsenic } \\ & \text { Barium } \\ & \text { Cadmium } \\ & \text { Chromium } \\ & \text { Copper } \\ & \text { Lead } \\ & \text { Mengenese } \\ & \text { Mercury } \\ \text { if } & \text { Nickel } \\ \text { if } & \end{array}$

$\begin{array}{lllll}7440-38-2 & 16 & / & 16 & \text { MO/KO } \\ 7440-39-3 & 4 & / & 16 & \text { MO/KO } \\ 7440-43-9 & 4 & / & 16 & \text { MO/KO } \\ 7440-47-3 & 16 & / & 16 & \text { MG/KO } \\ 7440-50-8 & 15 & / & 16 & \text { MO/KO } \\ 7439-92-1 & 16 & / & 16 & \text { MO/KO } \\ 7439-96-5 & 16 & / & 16 & \text { MG/KO } \\ 7439-97-6 & 6 & / & 16 & \text { MO/KO } \\ 7440-02-0 & 11 & / & 16 & \text { MG/KO }\end{array}$

$7.80 E+00$
$3.30 E+01$
$1.40 E+00$
$9.75 E+01$
$6.25 E+01$
$5.62 E+01$
$1.43 E+03$
$8.31 E-01$
$3.26 E+01$

$4.82 E+00$
$1.88 E+01$
$6.14 E-01$
$4.42 E+01$
$5.40 E+01$
$2.48 E+01$
$4.78 E+02$
$5.48 E-01$
$2.04 E+01$

$4.82 E+00$
$1.88 E+01$
$6.14 E-01$
$4.42 E+01$
$5.40 E+01$
$2.48 E+01$
$4.78 E+02$
$5.48 E-01$
$2.04 E+01$

$2.20 \mathrm{E}+00$
ND
ND
$9.70 \mathrm{E}+00$
ND
$7.50 \mathrm{E}+00$
$1.02 \mathrm{E}+01$
ND
ND

$\mathbf{Y}$
$\mathbf{Y}$
$\mathbf{Y}$
$\mathbf{Y}$
$\mathbf{Y}$
$\mathbf{Y}$
$\mathbf{Y}$
$\mathbf{Y}$
$\mathbf{Y}$


Table 4.1

SOIL ANALYTES AND BACKGROUND SCREENING

BRP 231-F/1F; 0 - $0.6 \mathrm{M}(0-2$ FT)

CURRENT CONDITIONS

\begin{tabular}{|c|c|c|c|c|c|c|c|c|}
\hline Chemical & CAS No. & $\begin{array}{c}\text { Frequency of } \\
\text { Detection }\end{array}$ & Units & $\begin{array}{c}\text { Maximum } \\
\text { Detection }\end{array}$ & $\begin{array}{l}95 \% \text { UCL of Log- } \\
\text { Tranuformed Data }\end{array}$ & $\begin{array}{c}\text { Reasonable } \\
\text { Maximum } \\
\text { Exposure } \\
\text { Cencentration }\end{array}$ & $\begin{array}{c}2 \text { times } \\
\text { Background } \\
\text { Cencentration }\end{array}$ & $\begin{array}{c}\text { Maximum } \\
\text { Exceeds 2X } \\
\text { Backzround } \\
\text { Concentration } \\
\text { (YIN) }\end{array}$ \\
\hline
\end{tabular}

Ceaium-137
lodino-129
Plutonium-238
Plutonium-239
Potassium-40
Redium, total alpha-emitting
Strontium-90
Technetium-99
Tritium
Uranium-234
Uranium-235
Uranium-238

$\begin{array}{rccc}\text { CS-137 } & 15 & / & 16 \\ 1-129 & 2 & / & 16 \\ \text { PU-238 } & 1 & / & 16 \\ \text { PU-239 } & 6 & / & 16 \\ \text { K-40 } & 15 & / & 16 \\ \text { RA } & 4 & / & 16 \\ 10098-97-2 & 8 & / & 16 \\ 14133-76-7 & 4 & / & 16 \\ 1333-74-0 & 7 & / & 16 \\ 13966-29-5 & 16 & / & 16 \\ \text { U-235 } & 1 & / & 16 \\ 7440-61-1 & 16 & / & 16\end{array}$

\begin{tabular}{|c|c|c|}
\hline $\mathrm{pCi}_{\mathrm{B}}$ & $4.30 E+00$ & $1.77 \mathrm{E}+00$ \\
\hline $\mathrm{pCi} / \mathrm{B}$ & $2.70 \mathrm{E}-01$ & $1.32 \mathrm{E}+01$ \\
\hline $\mathrm{pCi} / \mathrm{B}$ & $2.16 \mathrm{E}+00$ & 4.94E-01 \\
\hline $\mathrm{pCi} / \mathrm{B}$ & $1.23 E+00$ & $5.27 E_{-01}$ \\
\hline $\mathrm{pCi} / \mathrm{B}$ & $4.80 E+00$ & 4.27E+00 \\
\hline $\mathrm{pCi} / \mathrm{g}$ & $2.30 \mathrm{E}+00$ & $2.49 \mathrm{E}+00$ \\
\hline $\mathrm{pCi} / \mathrm{g}$ & $1.64 \mathrm{E}+01$ & $2.96 \mathrm{E}+00$ \\
\hline $\mathrm{pCi} / \mathrm{g}$ & $9.20 \mathrm{E}-01$ & S.51E-01 \\
\hline $\mathrm{PCi} / \mathrm{B}$ & $8.02 E+00$ & $7.69 E+\infty 0$ \\
\hline $\mathrm{pCi} / \mathrm{g}$ & $3.58 \mathrm{E}+00$ & $1.80 \mathrm{E}+00$ \\
\hline $\mathrm{pCi} / \mathrm{g}$ & $1.10 \mathrm{E}-01$ & $1.04 \mathrm{E}-01$ \\
\hline $\mathrm{pCi} / \mathrm{g}$ & $3.28 \mathrm{E}+00$ & $1.79 E+00$ \\
\hline
\end{tabular}

$1.77 \mathrm{E}+00$
$2.70 \mathrm{E}-01$
$4.94 \mathrm{E}-01$
$5.27 \mathrm{E}-01$
$4.27 \mathrm{E}+00$
$2.30 \mathrm{E}+00$
$2.96 \mathrm{E}+00$
$5.51 \mathrm{E}-01$
$7.69 \mathrm{E}+00$
$1.80 \mathrm{E}+00$
$1.04 \mathrm{E}-01$
$1.79 \mathrm{E}+00$

ND
$N D$
$N D$
ND
$3.10 E+00$
$3.90 E+00$
$4.20 E+00$
ND
$1.24 E+01$
$1.30 E+00$
ND
$1.60 E+00$

$\mathbf{Y}$
$\mathbf{Y}$
$\mathbf{Y}$
$\mathbf{Y}$
$\mathbf{Y}$
$\mathbf{N}$
$\mathbf{Y}$
$\mathbf{Y}$
$\mathbf{Y}(\mathbf{a})$
$\mathbf{Y}$
$\mathbf{Y}$
$\mathbf{Y}$

(1) The leser of the $95 \%$ UCL of the trensformed data and the maximum was used as the reasonable maximum exposure concentration.

(2) If background data were not available, the chemical was retained.

(a) For organics and nonnaturally occurring radionuclides, the $2 \mathrm{X}$ background rule was used for comparative purposes only. Elimination of COPCs was only performed for naturally occurring analytes. 
Table 4.2

SOIL ANALYTES AND BACKGROUND SCREENING

BRP 231-F/IF; $0-1.2 \mathrm{M}(0-4$ FT)

FUTURE CONDITIONS

\begin{tabular}{|c|c|c|c|c|c|c|c|c|}
\hline Chemical & CASNa. & $\begin{array}{l}\text { Frequency of } \\
\text { Detection }\end{array}$ & Units & $\begin{array}{l}\text { Marimum } \\
\text { Detection }\end{array}$ & $\begin{array}{l}95 \% \text { UCL of Log- } \\
\text { Transformed Datal }\end{array}$ & $\begin{array}{l}\text { Reasonable } \\
\text { Maximum } \\
\text { Exposure } \\
\text { Cencentration' }\end{array}$ & $\begin{array}{c}2 \text { time: } \\
\text { Beckground } \\
\text { Cencentration }\end{array}$ & $\begin{array}{l}\text { Marimum } \\
\text { Excoeds 2X } \\
\text { Background } \\
\text { Concentration } \\
\text { (XNN }{ }^{2}\end{array}$ \\
\hline
\end{tabular}

Non-Radioactive

\section{Volatlies}

1,1,2-Trichloroethane

1,2-Dichloroethene

Acetone

Chloroform

Dichlorometheno (methylene chloride)

Ethylbenzene

ᄀ Tetrachloroethyleno

Toluene

Xylenes

Semtuolatiles

2-Methylnephthaleno

Aceraphthene

Aconaphthyleno

Anthracene

Benzo(a)anthraceno

Benzo(a)pyreas

Benzo(b)fluorenthene

Benzo(ghi)perylene

Benzo(k) fluorunthene

Benzoic acid

Bis(2-ethylhexyl)phthulate

Butyibenzeyl phthalato

Chrysens

Di-n-butyi phthalate

Din-octsil phthalato

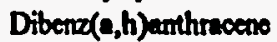

$\begin{array}{rccccc}79-00-5 & 2 & / & 24 & \text { MO/KG } & 1.65 E-02 \\ 107-06-2 & 2 & / & 24 & \text { MO/KG } & 2.16 E-02 \\ 67-64-1 & 20 & / & 24 & \text { MO/KO } & 2.62 E+01 \\ 67-66-3 & 1 & / & 24 & \text { MG/KO } & 1.01 E-02 \\ 75-09-2 & 20 & / & 24 & \text { MG/KO } & 3.80 E-02 \\ 100-41-4 & 1 & / & 24 & \text { MG/KG } & 1.29 E-03 \\ 127-18-4 & 1 & / & 24 & \text { MG/KO } & 2.87 E-03 \\ 108-88-3 & 16 & / & 24 & \text { MG/KO } & 3.06 E-02 \\ 1330-20-7 & 1 & / & 24 & \text { MO/KG } & 1.34 E-03\end{array}$

$91-57.62 / 16 \quad \mathrm{MG} / \mathrm{KO}$

83-32-9 $4 / 16$

208-96-8 4,16

$120.12-79 / 16$

$56.55-314 / 16$

$50-32-814 / 16$

205.99-2 $14 / 16$

191-24-2 12,16

207-08-9 $13 / 16$

$65-85-02 / 16$

$\begin{array}{cccc}117-81-7 & 14,16\end{array}$

85-68-7 $1 / 16$

$218-01-9 \quad 14,16$

84-74-2 $8 / 16$

$117-84-02 / 16$

$53-70-3$

16

$4.13 E-03$
$4.37 E-03$
$1.64 E+00$
$3.36 E-03$
$1.91 E-02$
$2.88 E-03$
$2.84 E-03$
$1.18 E-02$
$2.88 E-03$

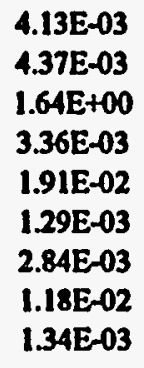

$2.12 E-01$

2.08E-01

$1.89 E-01$

2.49E-01

$9.26 E-01$

$6.49 E-01$

$1.01 E+00$

6.15E-01

8.45E-01

2.05E+00

4.48E-01

1.83E-01

$1.00 E+00$

1.99E-01

$1.90 E-01$

2.17E-01

7.69E- 02

2.08E-01

8.61E-02

2.49E-01

$9.26 \mathrm{E}-01$

$6.49 \mathrm{E}-01$

1.01E+00

6.15E-01

8.45E-01

4.86E-02

4.48E-01

1.83E-01

$1.00 \mathrm{E}+00$

$1.99 \mathrm{E}-01$

7.90E-Q2

2.17E-01

ND
ND
$1.06 \mathrm{E}-01$
ND
$1.40 \mathrm{E}-02$
ND
ND
$2.80 \mathrm{E}-02$
ND

$\mathbf{Y}$
$\mathbf{Y}$
$\mathbf{Y}(\mathbf{a})$
$\mathbf{Y}$
$\mathbf{Y}(\mathbf{a})$
$\mathbf{Y}$
$\mathbf{Y}$
$\mathbf{Y}(\mathbf{a})$
$\mathbf{Y}$

ND
ND
ND
ND
ND
ND
ND
ND
ND
ND
$1.56 E+00$
ND
ND
$1.26 E+00$
$1.70 E-01$
ND

ND
Parcon-Tampa: 2/23/96

Sheet Table $4-2$ of Filo TABS1 \&2 2 XIS

Page 1 of 4 
Table 4.2

SOIL ANALYTES AND BACKGROUND SCREENING

BRP 231-F/1F; 0 - $1.2 \mathrm{M}(0$ - 4 FT)

FUTURE CONDITIONS

\begin{tabular}{|c|c|c|c|c|c|c|c|c|c|}
\hline Chemleal & CAS No. & $\begin{array}{r}\text { Freq } \\
\text { De }\end{array}$ & $\begin{array}{l}\text { Lency of } \\
\text { ection }\end{array}$ & Units & $\begin{array}{l}\text { Maximum } \\
\text { Detection }\end{array}$ & $\begin{array}{l}95 \% \text { UCL of Log- } \\
\text { Transformed Data }\end{array}$ & $\begin{array}{c}\text { Reasonable } \\
\text { Maximum } \\
\text { Exposure } \\
\text { Concentration }\end{array}$ & $\begin{array}{c}2 \text { times } \\
\text { Background } \\
\text { Concentration }\end{array}$ & $\begin{array}{l}\text { Marrimum } \\
\text { Exceeds 2X } \\
\text { Background } \\
\text { Concentration } \\
\text { (XNN) }\end{array}$ \\
\hline Dibenzofuran & $132-64-9$ & 1 & 116 & MG/KG & $2.22 \mathrm{E}-01$ & 1.85E-01 & $1.85 \mathrm{E}-01$ & ND & $\mathrm{Y}$ \\
\hline Fluoranthene & $206-44-0$ & 14 & 16 & MO/KG & $3.91 E+00$ & $2.04 E+00$ & $2.04 E+00$ & $1.06 \mathrm{E}-01$ & $\mathbf{Y ( n )}$ \\
\hline Fluorene & $86-73-7$ & 3 & 16 & MO/KO & $2.99 \mathrm{E}-01$ & 2.17E-01 & 2.17E-0I & ND & $\dot{\mathbf{Y}}$ \\
\hline Indeno $(1,2,3-c d)$ pyrene & $193-39-5$ & 12 & 16 & $\mathrm{MO} / \mathrm{KO}$ & 8.32E-01 & $5.25 E-01$ & S.25E-01 & ND & $\mathbf{Y}$ \\
\hline Naphthalene & $91-20-3$ & 2 & 116 & $\mathrm{MG} / \mathrm{KO}$ & $3.22 \mathrm{E}-01$ & 2.21E-01 & 2.21E-01 & ND & $\mathbf{Y}$ \\
\hline Phenenthrene & $85-01-8$ & 12 & 16 & MO/KO & $3.38 \mathrm{E}+00$ & $6.87 \mathrm{E}-01$ & 6.87E-01 & ND & $\mathbf{Y}$ \\
\hline Pyrene & $129.00-0$ & 14 & 16 & $\mathrm{MO} / \mathrm{KO}$ & $2.42 \mathrm{E}+00$ & $1.23 E+00$ & $1.23 E+00$ & ND & $\mathbf{Y}$ \\
\hline \multicolumn{10}{|l|}{ Dioxins } \\
\hline Heptechlorodiberna-p-dioxin isomers & $37871-00-4$ & 10 & 16 & MG/KO & $8.30 \mathrm{E}-03$ & 4.11E-03 & 4.11E-03 & ND & $\mathbf{Y}$ \\
\hline Heptuchloradibereo-p-furan isomers & $38998-75-3$ & 6 & 116 & MO/KO & 5.00E-04 & 2.58E-04 & 2.58E-04 & ND & $\mathbf{Y}$ \\
\hline Hexachlorodibenzo-p-furan isomers & $55684-94-1$ & 1 & 116 & $\mathrm{MO} / \mathrm{KO}$ & $1.00 \mathrm{E}-04^{-}$ & $1.40 E-04$ & $1.00 \mathrm{E}-04$ & ND & $\mathbf{Y}$ \\
\hline Octachlorodibenzo-p-dioxin isomers & $3268-87-9$ & 15 & 16 & $\mathrm{MO} / \mathrm{KO}$ & $1.10 \mathrm{E}-02$ & 9.26E-03 & $9.26 \mathrm{E}-03$ & $1.00 \mathrm{E}-03$ & $Y(a)$ \\
\hline Octachlorodibenco-p-furan isomers & $39001-02-0$ & 6 & 16 & MG/KO & $8.00 \mathrm{E}-04$ & 3.48E-04 & $3.48 E-04$ & ND & $\mathbf{Y}$ \\
\hline \multicolumn{10}{|l|}{ PCBs } \\
\hline PCB 1254 & $11097-69-1$ & 8 & 16 & MO/KG & 4.38E-01 & $1.78 \mathrm{E}-01$ & $1.78 \mathrm{E}-01$ & ND & $\cdot \mathbf{Y}$ \\
\hline \multicolumn{10}{|l|}{ Pesticides } \\
\hline Aldrin & $309-00-2$ & 2 & 16 & MO/KO & $4.80 \mathrm{E}-03$ & 2.33E-03 & 2.33E-03 & ND & $\mathbf{Y}$ \\
\hline alpha-Chlordano & $5103-71-9$ & 4 & 16 & $\mathrm{MO} / \mathrm{KG}$ & $7.43 \mathrm{E}-03$ & $3.56 \mathrm{E}-03$ & $3.56 \mathrm{E}-03$ & ND & $\mathbf{Y}$ \\
\hline delia-Benzeno hoxachloride & $319-86-8$ & 1 & 16 & $\mathrm{MO} / \mathrm{KO}$ & $1.84 \mathrm{E}-03$ & $1.89 \mathrm{E}-03$ & $1.84 \mathrm{E}-03$ & ND & $\mathbf{Y}$ \\
\hline Endosulfan.ll & $33213-65-9$ & 1 & 16 & MG/KO & $4.69 \mathrm{E}-03$ & $3.88 \mathrm{E}-03$ & $3.88 E-03$ & ND & $\mathbf{Y}$ \\
\hline Endrin ketone & $53494-70-5$ & 1 & 16 & MG/KG & $3.20 \mathrm{E}-03$ & $5.28 E-03$ & $3.20 \mathrm{E}-03$ & ND & $\mathbf{Y}$ \\
\hline gamma-Chlordano & $5566-34-7$ & 6 & 16 & MG/KO & $6.49 \mathrm{E}-03$ & $3.08 E-03$ & $3.08 E-03$ & ND & $\mathbf{Y}$ \\
\hline P,P-DDD & $72-54-8$ & 2 & 16 & MO/KO & $1.39 \mathrm{E}-02$ & 5.05E-03 & 5.05E-03 & ṆD & $\mathbf{Y}$ \\
\hline P.P'-DDE & $72-55-9$ & 2 & 16 & $\mathrm{MO} / \mathrm{KO}$ & $2.03 \mathrm{E}-02$ & 5.62E-03 & $5.62 \mathrm{E}-03$ & ND & $\mathbf{Y}$ \\
\hline p,p-DDT & $50-29-3$ & 4 & 16 & MO/KO & 4.31E-02 & 9.95E-03 & 9.95E-03 & ND & $\mathbf{Y}$ \\
\hline
\end{tabular}

FINAL 
Table 4.2

SOIL ANALYTES AND BACKGROUND SCREENING

BRP 231-F/1F; 0 - $1.2 M(0-4$ FT)

FUTURE CONDITIONS

\begin{tabular}{|c|c|c|c|c|c|c|c|c|}
\hline Chemical & CAS No. & $\begin{array}{l}\text { Frequency of } \\
\text { Detection }\end{array}$ & Units & $\begin{array}{c}\text { Maximum } \\
\text { Detection }\end{array}$ & $\begin{array}{l}95 \% \text { UCL of Log- } \\
\text { Transformed Data }\end{array}$ & $\begin{array}{c}\text { Ressonable } \\
\text { Maximum } \\
\text { Exposure } \\
\text { Concentration }\end{array}$ & $\begin{array}{c}2 \text { times } \\
\text { Background } \\
\text { Concentration }\end{array}$ & $\begin{array}{l}\text { Marimum } \\
\text { Exceeds 2X } \\
\text { Background } \\
\text { Cencentration } \\
(\mathbf{Y} /)^{2}\end{array}$ \\
\hline
\end{tabular}

\section{Metals}

Arsenic

Barium

Cadmium

Chromium

Copper

lead

Manganeso

Mercury

Nickel

$\begin{array}{lclll}7440-38-2 & 24 & \text { / } & 24 & \text { MG/KO } \\ 7440-39-3 & 7 & / & 24 & \text { MG/KO } \\ 7440-43-9 & 6 & / & 24 & \text { MG/KO } \\ 7440-47-3 & 24 & / & 24 & \text { MG/KO } \\ 7440-50-8 & 22 & / & 24 & \text { MO/KO } \\ 7439-92-1 & 24 & / & 24 & \text { MO/KO } \\ 7439-96-5 & 24 & / & 24 & \text { MO/KO } \\ 7439-97-6 & 9 & / & 24 & \text { MO/KO } \\ 7440-02-0 & 18 & / & 24 & \text { MO/KO }\end{array}$

$7440-02-0,18,24$ MG/KG

$1.52 E+01$
$5.86 E+01$
$1.40 E+00$
$3.53 E+02$
$6.25 E+01$
$6.39 E+01$
$1.43 E+03$
$8.31 E-01$
$6.34 E+01$

$5.29 E+00$

2.07E+01

S.81E-01

7.65E+01

4.69E+01

$2.62 \mathrm{E}+01$

$3.61 \mathrm{E}+02$

3.58E-01

2.42E+01

$5.29 \mathrm{E}+00$.
$2.07 \mathrm{E}+01$
S.81E-01
$7.65 \mathrm{E}+01$
$4.69 \mathrm{E}+01$
$2.62 \mathrm{E}+01$
$3.61 \mathrm{E}+02$
$3.58 \mathrm{E}-01$
$2.42 \mathrm{E}+01$

$2.20 \mathrm{E}+00$
ND
ND
$9.70 \mathrm{E}+00$
ND
$7.50 \mathrm{E}+00$
$1.02 \mathrm{E}+01$
ND

ND 
Table 4.2

SOIL ANALYTES AND BACKGROUND SCREENING

BRP 231-F/1F; 0 - $1.2 M(0$ - 4 FT)

FUTURE CONDITIONS

\begin{tabular}{|c|c|c|c|c|c|c|c|c|}
\hline Chemical & CAS No. & $\begin{array}{l}\text { Frequency of } \\
\text { Detection }\end{array}$ & Units & $\begin{array}{l}\text { Maximum } \\
\text { Detection }\end{array}$ & $\begin{array}{l}95 \% \text { UCL of Log- } \\
\text { Transformed Data }\end{array}$ & $\begin{array}{c}\text { Reasonable } \\
\text { Marimum } \\
\text { Exposure } \\
\text { Concentrationt }\end{array}$ & $\begin{array}{c}2 \text { timex } \\
\text { Background } \\
\text { Concentration }\end{array}$ & $\begin{array}{l}\text { Maximum } \\
\text { Exceeds 2X } \\
\text { Background } \\
\text { Concentration } \\
\left(\mathrm{X} / \mathrm{N}^{2}\right.\end{array}$ \\
\hline
\end{tabular}

Resium-137
Jodine-129
Plutonium-238
Plutonium-239
Potnssium-40
Redium, total alphe-emitting
Strontium-90
Technetium-99
Tritium
Uranium-234
Urarium-235
Uranium-238

\begin{tabular}{|c|c|c|c|c|c|}
\hline CS-137 & 15 & 1 & 16 & $\mathrm{pCi} / \mathrm{g}$ & $4.30 \mathrm{E}+00$ \\
\hline |-129 & 2 & 1 & 16 & $\mathrm{pCi} / \mathrm{g}$ & $2.70 \mathrm{E}-01$ \\
\hline PU-238 & 1 & 1 & 16 & $\mathrm{pCi} / \mathrm{g}$ & $2.16 \mathrm{E}+00$ \\
\hline PU-239 & 6 & 1 & 16 & $\mathrm{pCi} / \mathrm{g}$ & $1.23 E+00$ \\
\hline$K-40$ & 15 & 1 & 16 & $\mathrm{pCi} / \mathrm{g}$ & $4.80 \mathrm{E}+00$ \\
\hline $\mathbf{R A}$ & 6 & I & 24 & $\mathrm{pCi}_{\mathbf{B}}$ & $2.30 \mathrm{E}+00$ \\
\hline $10098-97-2$ & 8 & 1 & 16 & $\mathrm{pCi} \mathrm{g}_{\mathrm{B}}$ & $1.64 E+01$ \\
\hline $14133-76-7$ & 4 & 1 & 16 & $\mathrm{pCi} / \mathrm{B}$ & $9.20 \mathrm{E}-01$ \\
\hline $1333-74-0$ & 14 & 1 & 24 & $\mathbf{p C i} /_{\mathbf{B}}$ & $8.25 \mathrm{E}+00$ \\
\hline $13966-29-5$ & 16 & 1 & 16 & $\mathrm{pCi}_{\mathrm{g}}$ & $3.58 \mathrm{E}+00$ \\
\hline U-235 & 1 & 1 & 16 & $\mathrm{pC} \mathbf{V}_{\mathbf{g}}$ & $1.10 E-01$ \\
\hline $7440-61-1$ & 16 & I & 16 & pCi/g & $3.28 \mathrm{E}+00$ \\
\hline
\end{tabular}

$\begin{array}{ll}1.77 E+00 & 1.77 \mathrm{E}+00 \\ 1.32 \mathrm{E}+01 & 2.70 \mathrm{E}-01 \\ 4.94 \mathrm{E}-01 & 4.94 \mathrm{E}-01 \\ 5.27 \mathrm{E}-01 & 5.27 \mathrm{E}-01 \\ 4.27 \mathrm{E}+00 & 4.27 \mathrm{E}+00 \\ 1.94 \mathrm{E}+00 & 1.94 \mathrm{E}+00 \\ 2.96 \mathrm{E}+00 & 2.96 \mathrm{E}+00 \\ 5.51 \mathrm{E}-01 & 5.51 \mathrm{E}-01 \\ 6.23 \mathrm{E}+\infty 0 & 6.23 \mathrm{E}+00 \\ 1.80 \mathrm{E}+00 & 1.80 \mathrm{E}+00 \\ 1.04 \mathrm{E}-01 & 1.04 \mathrm{E}-01 \\ 1.79 \mathrm{E}+00 & 1.79 \mathrm{E}+00\end{array}$

ND
ND
ND
ND
$3.10 E+00$
$3.90 E+00$
$4.20 \mathrm{E}+00$
ND
$1.24 \mathrm{E}+01$
$1.30 \mathrm{E}+00$
ND
$1.60 \mathrm{E}+00$

$\mathbf{Y}$
$\mathbf{Y}$
$\mathbf{Y}$
$\mathbf{Y}$
$\mathbf{Y}$
$\mathbf{N}$
$\mathbf{Y}(\boldsymbol{a})$
$\mathbf{Y}$
$\mathbf{Y}(\mathbf{a})$
$\mathbf{Y}$
$\mathbf{Y}$
$\mathbf{Y}$

(1) The lesser of the $95 \%$ UCL of the transformed data and the maximum was used as the reasonable maximum exposure concentration.

(2) If bestaground dets were for aveileble, the chemical was reteined.

(a) For orgenics and non-naturally occurring radiomuclides, the $2 X$ background rule was used for comparative purposes only. Elimination of COPCs was only performod for naturally occurring analytes. 
Table 4.3

SOIL ANALYTES AND BACKGROUND SCREENING

RP 231-2F; 0 - $0.6 \mathrm{M}$ (0 - 2 FT)

CURRENT CONDITIONS :

\begin{tabular}{|c|c|c|c|c|c|c|c|c|}
\hline Chemical & CASNo. & $\begin{array}{c}\text { Frequency of } \\
\text { Detection }\end{array}$ & Units & $\begin{array}{l}\text { Maximum } \\
\text { Detection }\end{array}$ & $\begin{array}{c}95 \% \text { UCL of Log- } \\
\text { Transformed } \\
\text { Data }\end{array}$ & $\begin{array}{c}\text { Reasonable } \\
\text { Maximum } \\
\text { Exposure } \\
\text { Concentration }\end{array}$ & $\begin{array}{c}2 \text { times } \\
\text { Background } \\
\text { Concentration }\end{array}$ & $\begin{array}{l}\text { Maximum. } \\
\text { Exceeds 2X } \\
\text { Background } \\
\text { Concentration } \\
\text { (YNN) }\end{array}$ \\
\hline
\end{tabular}

Non-Radloactive

\section{Volatiles}

1,1,2-Trichloroethano
1,2-Dichloroethane
Acetone
Chloroform
Dichloromethano (methylene chloride)
Toluene

$\begin{array}{rlllll}79-00-5 & 2 & / & 8 & \text { MO/KO } & 1.36 E-02 \\ 107-06-2 & 1 & / & 8 & \text { MO/KO } & 1.03 E-02 \\ 67-64-1 & 5 & / & 8 & \text { MO/KO } & 4.75 E-02 \\ 67-66-3 & 1 & / & 8 & \text { MG/KO } & 2.65 E-03 \\ 75-09-2 & 6 & / & 8 & \text { MO/KO } & 6.22 E-03 \\ 108-88-3 & 5 & / & 8 & \text { MOKKO } & 1.19 E-02\end{array}$

$\begin{array}{ccc}6.87 E-03 & 6.87 E-03 & \text { ND } \\ 5.50 E-03 & 5.50 E-03 & \text { ND } \\ 3.20 E-02 & 3.20 E-02 & 1.06 E-01 \\ 2.75 E-03 & 2.65 E-03 & \text { ND } \\ 5.32 E-03 & 5.32 E-03 & 1.40 E-02 \\ 1.87 E-02 & 1.19 E-02 & 2.80 E-02\end{array}$

$\mathbf{Y}$
$\mathbf{Y}$
$\mathbf{Y}(\mathbf{a})$
$\mathbf{Y}$
$\mathbf{Y}(\mathbf{a})$
$\mathbf{Y}(\mathbf{a})$

\section{Semholatiles}

Berzo(a)anthracene

Benzo(a)pyrene

Berro(b) lluoranthene

Benzor(k)fluorantheno

Bis(2-ethythexyi)phthalate

Chryeene

Di-n-butyt phthalate

Di-n-octyl phthalato

Fluorentheno

Phenanthreno

Pynemo

Dioxina/urans

Heptuchlorodiberno-p-dioxin isomers

Octuchlorodibenro-p-dioxin isomers

3268-87-9 6 / 8 MO/KO

6.90E-02

56-55-3 I / 8 MOKKO

50-32-8 $1 / 8$ MOKKO

6.0IE-02

205-99-2 / 8 MO/KO

8.14E-02

207-08-9 $1 / 8$ MO/KO

6.50E-02

$117-81.7 \quad 6 / 8$ MO/KO

2.77E-01

218-01-9 1 / 8 MOKKO

6.92E-02

$\begin{array}{lllll}117-84-0 & 1 & 1 & 8 & \mathrm{MO} / \mathrm{KO}\end{array}$

1.16E-01

206-44-0 I / 8 MG/KO

$1.66 \mathrm{E}-01$

$\begin{array}{llllll}85-01-8 & / & 8 & \text { MGKK } & 8.68 \mathrm{E}-02\end{array}$

1.14E-01

2.52E+00

2.77E+00

$6.90 \mathrm{E}-02$

$2.28 \mathrm{E}+00$

2.63E+00

7.61E-01

$2.52 \mathrm{E}+00$

$5.67 E+00$

$1.88 \mathrm{E}+00$

$1.67 \mathrm{E}+00$

2.18E+00

129-00-0 I / 8 MOKO

$1.90 E+00$

\subsection{E-02}

8.14E-02

6.50E-02

2.77E-01

$6.92 \mathrm{E}-02$

7.07E-02

$1.16 \mathrm{E}-01$

$1.66 \mathrm{E}-01$

8.68E-02

$1.14 E-01$

ND
ND
ND
ND

1.56E+00

ND

$1.26 \mathrm{E}+00$

1.70E-01

$1.06 \mathrm{E}-01$

ND

ND

$\mathbf{Y}$
$\mathbf{Y}$
$\mathbf{Y}$
$\mathbf{Y}$
$\mathbf{Y}(\boldsymbol{a})$
$\mathbf{Y}$
$\mathbf{Y}(\boldsymbol{a})$
$\mathbf{Y}(\boldsymbol{a})$
$\mathbf{Y}$
$\mathbf{Y}$
$\mathbf{Y}$

$6.00 \mathrm{E}-04$

6.36E-04

S.24E-04

6.00E-04

4.00E-04

ND

1.00E-03

$\mathbf{Y}$

PCBs

PCB 1254

11097-69-1 1 / 8 MO/KंO

2.87E+00

2.61E+01

PCB 1260

$\begin{array}{lllll}11096-82-5 & 1 & 8 & \text { MOKKO 9.01E-02 }\end{array}$

$3.48 E+00$

2.87E+00

9.01E-02

ND
ND

$\mathbf{Y}$
$\mathbf{Y}$

FINAL

Pancon-Tumpe: 2/20/96 
Table 4.3

SOIL ANALYTES AND BACKGROUND SCREENING .

RP 231-2F; 0 - $0.6 \mathrm{M}(0$ - 2 FT)

CURRENT CONDITIONS

\begin{tabular}{|c|c|c|c|c|c|c|c|}
\hline & & & & Maximum \\
Chemical & CAS No. & $\begin{array}{c}\text { Frequency of } \\
\text { Detection }\end{array}$ & Units & $\begin{array}{c}\text { Maximum } \\
\text { Detection }\end{array}$ & $\begin{array}{c}\text { 95\% UCL of Log- } \\
\text { Transformed } \\
\text { Data }\end{array}$ & $\begin{array}{c}\text { Maximumale } \\
\text { Exposure } \\
\text { Concentration' }\end{array}$ & $\begin{array}{c}2 \text { times } \\
\text { Backgraund } \\
\text { Background } \\
\text { Concentration } \\
\text { Concentration }\end{array}$ \\
\hline
\end{tabular}

\section{Metule}

Arseric

Chromium

Copper

Lead

Manganese

Mencury

Nickel

$\begin{array}{llllll}7440-38-2 & 1 & / & 7 & \mathrm{MO} / \mathrm{KO} & 1.00 \mathrm{E}+00 \\ 7440-47-3 & 7 & / & 7 & \mathrm{MO} / \mathrm{KO} & 1.82 \mathrm{E}+02 \\ 7440-50-8 & 4 & / & 7 & \mathrm{MO} / \mathrm{KO} & 2.43 \mathrm{E}+01 \\ 7439-92-1 & 7 & / & 7 & \mathrm{MO} / \mathrm{KO} & 1.00 \mathrm{E}+02 \\ 7439-96-5 & 7 & / & 7 & \mathrm{MO} / \mathrm{KO} & 2.03 \mathrm{E}+02 \\ 7439-97-6 & 1 & / & 7 & \mathrm{MO} / \mathrm{KO} & 8.65 \mathrm{E}-02 \\ 7440-02-0 & 1 & / & 7 & \mathrm{MO} / \mathrm{KO} & 7.71 \mathrm{E}+01\end{array}$

$7.39 \mathrm{E}+00$
$2.24 \mathrm{E}+02$
$6.19 \mathrm{E}+01$
$9.00 \mathrm{E}+01$
$1.72 \mathrm{E}+02$
$6.57 \mathrm{E}-02$
$1.26 \mathrm{E}+02$

$1.00 \mathrm{E}+00$

$1.82 \mathrm{E}+02$

2.43E+01

9.00E+01

$1.72 \mathrm{E}+02$

6.57E-02

7.71E+01

2.20E+00
$9.70 \mathrm{E}+00$
ND
$7.50 \mathrm{E}+00$
$1.02 \mathrm{E}+01$
ND
ND

N

$\mathbf{Y}$

$\mathbf{Y}$

$\mathbf{Y}$

$\mathbf{Y}$

$\mathbf{Y}$ 
Table 4.3

SOIL ANALYTES AND BACKGROUND SCREENING

RP 231-2F; 0 - $0.6 M(0-2$ FT)

CURRENT CONDITIONS

\begin{tabular}{|c|c|c|c|c|c|c|c|}
\hline & & & & Maximum \\
Chemical & CAS No. & $\begin{array}{c}\text { Frequency of } \\
\text { Detection }\end{array}$ & Units & $\begin{array}{c}\text { Maximum } \\
\text { Detection }\end{array}$ & $\begin{array}{c}\text { Reasonable } \\
\text { Transformed } \\
\text { Data }\end{array}$ & $\begin{array}{c}\text { Maximum } \\
\text { Exposure } \\
\text { Concentration' }\end{array}$ & $\begin{array}{c}2 \text { times } \\
\text { Background } \\
\text { Concentration }\end{array}$ \\
$\begin{array}{c}\text { Backgroumd } \\
\text { Concentration } \\
\text { (YNM) }\end{array}$ \\
\hline
\end{tabular}

Radlonuclides
Cesium-137
Plutonium-239
Polasitium-40
Radium, total alpha-emitting
Strontium-90
Technetium-99
Tritium
Uranium-234
Uranium-235
Uranium-238

\begin{tabular}{|c|c|c|c|c|c|c|c|}
\hline CS-137 & 8 & 1 & 8 & $\mathrm{pCi} / \mathrm{g}$ & $1.42 E+00$ & $1.49 E+00$ & $1.42 E+00$ \\
\hline PU-239 & 2 & 1 & 8 & $\mathrm{pCi} / \mathrm{g}$ & $6.60 \mathrm{E}-01$ & $1.44 E+00$ & $6.60 \mathrm{E}-01$ \\
\hline$K-40$ & 8 & 1 & 8 & $\mathrm{pCi} / \mathrm{g}$ & $3.78 E+00$ & $3.16 E+00$ & $3.16 \mathrm{E}+00$ \\
\hline $\mathbf{R A}$ & 5 & 1 & 8 & $\mathrm{pCi} / \mathrm{s}$ & $2.22 \mathrm{E}+00$ & $2.00 E+00$ & $2.00 \mathrm{E}+00$ \\
\hline $10098-97-2$ & 2 & 1 & 8 & $\mathrm{pCi} / \mathrm{g}$ & $6.00 \mathrm{E}-01$ & $1.24 E+00$ & $6.00 E-01$ \\
\hline $14133-76-7$ & 5 & I & 8 & $\mathrm{pCi} V_{B}$ & $4.68 E+00$ & $1.87 E+01$ & $4.68 \mathrm{E}+00$ \\
\hline $1333-74-0$ & 6 & I & 8 & $\mathrm{pCi} / \mathrm{g}$ & $6.20 \mathrm{E}+00$ & $8.02 E+00$ & $6.20 \mathrm{E}+00$ \\
\hline $13966-29-5$ & 8 & 1 & 8 & $\mathrm{pCi} / \mathrm{B}$ & $2.59 \mathrm{E}+00$ & $2.09 \mathrm{E}+00$ & $2.09 \mathrm{E}+00$ \\
\hline U-23s & 5 & 1 & 8 & $\mathrm{pCi} \mathrm{V}_{\mathrm{B}}$ & $1.40 \mathrm{E}-01$ & $1.65 \mathrm{E}-01$ & $1.40 \mathrm{E}-01$ \\
\hline $7440-61-1$ & 8 & 1 & 8 & $\mathrm{pCi} / \mathrm{g}$ & $2.00 \mathrm{E}+00$ & $1.81 E+00$ & $1.81 E+00$ \\
\hline
\end{tabular}

(1) The lesser of the $95 \%$ UCL of the transformed data and the maximum was used as the reasonablo maximum exposure concentration.

(2) If background data were not available, the chemical was retained.

(a) For organics and non-naturally occurring radionuclides, the $2 X$ background rulo was used for comparativo purposes only. Elimination of COPCs was only performed for naturally occurring analytes. 
Table 4.4

SOIL ANALYTES AND BACKGROUND SCREENING

RP 231-2F; 0 - $1.2 \mathrm{M}(0$ - 4 FT)

FUTURE CONDITIONS

\begin{tabular}{|c|c|c|c|c|c|c|c|c|}
\hline Chemical & CAS No. & $\begin{array}{l}\text { Frequency of } \\
\text { Detection }\end{array}$ & Units & $\begin{array}{l}\text { Maximum } \\
\text { Detection }\end{array}$ & $\begin{array}{c}95 \% \text { UCL of Log- } \\
\text { Transformed } \\
\text { Data }\end{array}$ & $\begin{array}{c}\text { Reasonable } \\
\text { Maximum } \\
\text { Exposure } \\
\text { Concentration }\end{array}$ & $\begin{array}{c}2 \text { times } \\
\text { Background } \\
\text { Concentration }\end{array}$ & $\begin{array}{l}\text { Maximum } \\
\text { Exceeds } 2 X \\
\text { Background } \\
\text { Concentration } \\
\text { (YN) }{ }^{2}\end{array}$ \\
\hline
\end{tabular}

Non-Radloactive

- Volatiles

1,1,2-Trichlorocthano

1,2-Dichloroethene

Acetono

Chloroform

Dichloromethme (methylene chloride)

Tolueno

$\begin{array}{rllll}79-00-5 & 2 & / & 12 & \text { MO/KO } \\ 107-06-2 & 1 & / & 12 & \text { MO/KO } \\ 67-64-1 & 7 & / & 12 & \text { MO/KO } \\ 67-66-3 & 1 & / & 12 & \text { MO/KO } \\ 75-09-2 & 8 & / & 12 & \text { MO/KO } \\ 108-88-3 & 7 & / & 12 & \text { MO/KO }\end{array}$

$1.36 \mathrm{E}-02$
$1.03 \mathrm{E}-02$
$4.75 \mathrm{E}-02$
$2.65 \mathrm{E}-03$
$6.22 \mathrm{E}-03$
$1.19 \mathrm{E}-02$

4.06E-03

$1.70 \mathrm{E}-02$

2.71E-03

4.05E-03

8.17E-03

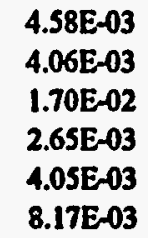

ND

ND

1.06E-01

ND

$1.40 \mathrm{E}-02$

$2.80 \mathrm{E}-02$

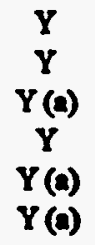

Semivolatiles

Berzo(a)enthracene

Benzo(a)pyreno

Benzo(b)fluoranthene

Benzo(k) fluorantheno

Bis(2-ethythexy) phthalate

Chryseno

Di-n-butyl phthalato

Di-n-octyl phthalate

Fluoranthene

Phenanthreno

Pyrene:

$\begin{array}{rlll}56-55-3 & 1 & 1 & 9 \\ 50-32-8 & 1 & 1 & 9 \\ 205-99-2 & 1 & 1 & 9 \\ 207-08-9 & 1 & 1 & 9 \\ 117-81-7 & 6 & 1 & 9 \\ 218-01-9 & 1 & 1 & 9 \\ 84-74-2 & 2 & 1 & 9 \\ 117-84-0 & 1 & 1 & 9 \\ 206-44-0 & 1 & 1 & 9 \\ 85-01-8 & 1 & 1 & \\ 129-00-0 & 1 & 1 & \end{array}$

MG/KO

MOKO

$6.90 \mathrm{E}-02$

6.01E-02

MO/KO

MO/KO

8.14E-02

6.50E-02

2.16E+00

2.33E+00

$1.97 \mathrm{E}+00$

2.23E+00

2.77E-01

MOIKO

6.92E-02

8.65E-01

2.16E+00

$4.23 E+00$

MG/KG

$7.07 \mathrm{E}-02$

1.16E-01

1.66E-01

MOKKG

$1.69 \mathrm{E}+00$

$1.52 \mathrm{E}+00$

$1.91 E+00$

8.68E-02

1.71E +00

6.90E-02

6.01E-02

8.14E-02

6.50E-02

2.77E-01

6.92E-02

7.07E-02

$1.16 \mathrm{E}-01$

$1.66 \mathrm{E}-01$

8.68E-02

MOIKO

1.14EOLI

1.14E-OI

$\begin{array}{cc}\text { ND } & Y \\ \text { ND } & Y \\ \text { ND } & Y \\ \text { ND } & Y \\ 1.56 E+00 & Y(a) \\ \text { ND } & Y \\ 1.26 E+00 & Y(a) \\ 1.70 E-01 & Y(a) \\ 1.06 E-01 & Y \\ \text { ND } & Y \\ \text { ND } & Y\end{array}$

\section{Dioxins/furans}

Heptachlorodibenzo-p-dioxin isomers

Octechlorodiberre-p-dioxin isomers

37871-00-4 $1, / 9 \mathrm{MO} / \mathrm{KO}$

$3268-87-9 \quad 6 \quad 1$

6.00E-04 4.75E-04

4.75E-04

4.00E-04

\section{ND}

1.00E-03

$\mathbf{Y}$

PCBs

PCB 1254

PCB 1260

11097-69-1 1,9

MOKKO

2.87E+00

1.17E+01

2.87E+00

11096-82-5 $1 / 9$

9.01E-02

$2.06 \mathrm{E}+00$

9.01E-02

ND

ND

$\mathbf{Y}$
$\mathbf{Y}$

FINAL

Sheet T: Iof File TABS384XXIS

Page 1 
Table 4.4

SOIL ANALYTES AND BACKGROUND SCREENING

RP 231-2F; 0 - $1.2 M(0-4$ FT)

FUTURE CONDITIONS

\begin{tabular}{|c|c|c|c|c|c|c|c|c|}
\hline Chemical & CASNo. & $\begin{array}{c}\text { Frequency of } \\
\text { Detection }\end{array}$ & Units & $\begin{array}{l}\text { Maximum } \\
\text { Detection }\end{array}$ & $\begin{array}{c}95 \% \text { UCL of } \log - \\
\text { Transformed } \\
\text { Date }\end{array}$ & $\begin{array}{c}\text { Reasonable } \\
\text { Maximum } \\
\text { Exposure } \\
\text { Concentration' }\end{array}$ & $\begin{array}{c}2 \text { times } \\
\text { Background } \\
\text { Concentration }\end{array}$ & $\begin{array}{l}\text { Maximum } \\
\text { Exceeds 2X } \\
\text { Background } \\
\text { Concentration } \\
(Y / N)^{2}\end{array}$ \\
\hline
\end{tabular}

Metals
Arsenic
Chromium
Copper
Lead
Manganese
Mercury
Nickel

$\begin{array}{cccccc}7440-38-2 & 1 & / & 11 & \text { MO/KO } & 1.00 E+00 \\ 7440-47-3 & 11 & / & 11 & \text { MG/KG } & 1.82 E+02 \\ 7440-50-8 & 6 & / & 11 & \text { MG/KG } & 2.43 E+01 \\ 7439-92-1 & 11 & / & 11 & \text { MG/KO } & 1.00 E+02 \\ 7439-96-5 & 11 & / & 11 & \text { MO/KO } & 2.03 E+02 \\ 7439-97-6 & 2 & / & 11 & \text { MO/KO } & 3.84 E-01 \\ 7440-02-0 & 1 & / & 11 & \text { MG/KO } & 7.71 E+01\end{array}$

$5.61 E+00$
$5.51 E+01$
$1.84 E+01$
$6.20 E+01$
$1.08 E+02$
$1.23 E-01$
$1.66 E+01$

$$
\begin{aligned}
& 1.00 E+00 \\
& 5.51 E+01 \\
& 1.84 E+01 \\
& 6.20 E+01 \\
& 1.08 E+02 \\
& 1.23 E-01 \\
& 1.66 E+01
\end{aligned}
$$

$2.20 \mathrm{E}+00$
$9.70 \mathrm{E}+00$
ND
$7.50 \mathrm{E}+00$
$1.02 \mathrm{E}+01$
ND
ND

$\mathbf{N}$
$\mathbf{Y}$
$\mathbf{Y}$
$\mathbf{Y}$
$\mathbf{Y}$
$\mathbf{Y}$
$\mathbf{Y}$

Radionuclides

Cesium-137
Plutonium-239
Potussium-10
Radium, toul alpha-emitting
Strontium-90
Technetium-99
Tritium
Uranium-234
Unuium-235
Uranium-238

$\begin{array}{rccc}\text { CS-137 } & 9 & / & 9 \\ \text { PU-239 } & 3 & / & 9 \\ \text { K-40 } & 9 & / & 9 \\ \text { RA } & 8 & / & 12 \\ 10098-97-2 & 3 & / & 9 \\ 14133-76-7 & 5 & / & 9 \\ 1333-74-0 & 10 & / & 12 \\ 13966-29-5 & 9 & / & 9 \\ \text { U-235 } & 6 & / & 9 \\ 7440-61-1 & 9 & / & 9\end{array}$

$\begin{array}{ll}\mathrm{pCi} /_{\mathrm{B}} & 1.42 \mathrm{E}+00 \\ \mathrm{pCi} /_{\mathrm{B}} & 3.80 \mathrm{E}+00 \\ \mathrm{pCi} /_{\mathrm{g}} & 3.78 \mathrm{E}+00 \\ \mathrm{pCi} /_{\mathrm{g}} & 2.22 \mathrm{E}+00 \\ \mathrm{pCi} \mathrm{g}_{\mathrm{g}} & 2.90 \mathrm{E}+00 \\ \mathrm{pCi} /_{\mathrm{g}} & 4.68 \mathrm{E}+00 \\ \mathrm{pCi} /_{\mathrm{g}} & 6.30 \mathrm{E}+00 \\ \mathrm{pCi} /_{\mathrm{g}} & 2.59 \mathrm{E}+00 \\ \mathrm{pCi} / \mathrm{g} & 1.60 \mathrm{E}-01 \\ \mathrm{pCi} /_{\mathrm{g}} & 2.00 \mathrm{E}+00\end{array}$

$1.60 \mathrm{E}+00$
$7.11 \mathrm{E}+00$
$3.26 \mathrm{E}+00$
$1.60 \mathrm{E}+00$
$2.42 \mathrm{E}+00$
$8.90 \mathrm{E}+00$
$5.89 \mathrm{E}+00$
$2.13 \mathrm{E}+00$
$1.71 \mathrm{E}-01$
$1.83 \mathrm{E}+00$

$1.42 E+00$

$3.80 \mathrm{E}+00$

$3.26 \mathrm{E}+00$

$1.60 \mathrm{E}+00$

2.42E+00

$4.68 E+00$

S.89E+00

2.13E+00

$1.60 \mathrm{E}-01$$$
\text { - }
$$

(1) The lesser of the $95 \%$ UCL of tho transformed data and the maximum was used as the reasonsblo maximum exposure concentration.

(2) If background data nere not available, the chemical was retained.

(a) For organics and non-naturally occurring radiomuclides, the $2 \mathrm{X}$ background rulo was used for comparative purposes only. Elimination of COPCs was onty porformod for naturally occurring analytes. 
TABLE 4.5

TOXICITY DATA FOR ECOLOGICAL SCREENING

BRP 231-F/1F and RP 231-2F - SOIL

\begin{tabular}{|c|c|c|c|c|c|c|c|c|c|}
\hline Analyte & $\begin{array}{c}\text { Test } \\
\text { Organism }\end{array}$ & Endpoint/Duration/ErTect & Source & $\begin{array}{l}\text { Erfect Dose } \\
(\mathrm{mg} / \mathrm{kg} / \mathrm{day})\end{array}$ & $\begin{array}{c}\text { Surrogate } \\
\text { Chemical } \\
\text { UF. }\end{array}$ & $\begin{array}{c}\text { Endpoint } \\
\text { UF }\end{array}$ & $\begin{array}{c}\text { Studiy } \\
\text { Durnation } \\
\text { UF }\end{array}$ & $\begin{array}{c}\text { Total } \\
\text { UF }\end{array}$ & $\begin{array}{l}\text { Toxicity } \\
\text { Sereening } \\
\text { Benchmark } \\
\text { (mp/ke/day) }\end{array}$ \\
\hline
\end{tabular}

\section{Volatiles}

\section{Acetone}

Chloroform

Dichloroethene, 1,2

Dichloromethane (methylene chloride)

Ethylbenzeno

Tetrachlorocthyleno

Tolueno

Trichlorocthane, 1,1,2-

Xylenes

Semivolatiles

Aceraphthene

Acenaphthylene

Anthraceno

Berzo(a)enthraceno

Benzo(a)pyreno

Benzo(b) fluoranthene

Benzo(ghi)perylene

Benzo(k)fluoranthene

Bemzoic ecid

Bis(2-ethylhexyl)phthalato

Butylbenzyl phithalato

Chrysene

Di-n-butyl phthalate

Di-noct f phthalate

Diberza, $(\mathrm{h})$ anthracene

Dibenzofuran

Fluoranthene

Fluorene

Indeno(1,2,3-cd)pyrene

Methyinaphthalene, 2-

Naphthalene

Phenanthreno

Pancons - Tom - 3/2196

Shest usble

: TABASXIS

\begin{abstract}
rat LOAEL, diet, 90-day, nephrotoxicity
rat LOAEL, gavage in oil, 78-week, decr. survival

rat LOAEL, gavage in oil, 78-woek, death

rat LOAEL, water, 90-day, hepatic nocrosis

rat LOAEL, diet, 182-day, hepatio and renal toxicity

mouse LOAEL, gavage in oil, 78-week, death

rat LOAEL, diet, 91-day, hepatic \& renal hypertrophy

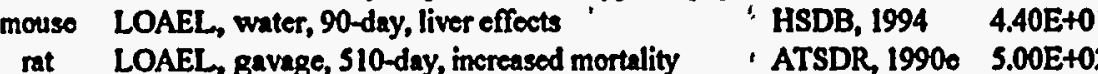

IRIS, $1994 \quad 5.00 E+02$

ATSDR, 1991j 9.00E+01

ATSDR, $19920 \quad 9.50 \mathrm{E}+0$

ATSDR, 1991d 1.20E+03

IRIS, $1994 \quad 2.91 \mathrm{E}+02$

ATSDR, 1989i 3.86E+02

rat LOAEL, gavage, 510-day, increased mortality ' ATSDR, 19906 5.00E+02
\end{abstract}

mouse LOAEL, oral (diet), 90-day, hepatotoxicity

mouse Usod berzo(a)pyrene as surrogate

rat LOAEL, injection (subcutencous), 232-day

rat NOAEL, diet, 4-day, gastro, hepatio, renal effocts

mouso LOAEL, diet, 8-day, fetal rosorption

mouse Used benzio(a)pyrene as surrogato

Imouse Used benzo(a)pyrene as surrogate

mouse Used benzo(a)pyrene as surrogato

rat NOAEL, diet, assume acute

mouse LOAEL, diet, 105-day, repro/fertility offects

rat LOAEL, diet, 6-month, kidney pathology

mouse Used benzo(a)pyrene as surrogato

rat LOAEL, diet, 52-week, death

mouse LDS0, oral

mouse Used benzo(a)pyrene as surrogate no data

rat NOAEL, scute, gavage

mouso LOAEL, diet, 91-day, decreased orythropoiesis

mouse Used benzo(a)pyrene as surrogate

rat LDSO, oral, acute

rat LDSO, gavago

mouse LDSO, oral (gavage)
IRIS, $1994 \quad 3.50 \mathrm{E}+02$

$1.20 \mathrm{E}+02$

HSDB, 1994 6.70E+01

ATSDR, $1993 \quad 1.50 \mathrm{E}+02$

ATSDR, $19890 \quad 1.20 \mathrm{E}+02$

$1.20 \mathrm{E}+02$

$1.20 \mathrm{E}+02$

$1.20 \mathrm{E}+02$

IRIS, $1994 \quad 8.00 \mathrm{E}+01$

ATSDR, 1989c 1.30E+02

IRIS, $1994 \quad 4.70 \mathrm{E}+02$

$1.20 \mathrm{E}+02$

ATSDR, 1989d 6.25E+02

HSDB, 1994 1.30E+04

$1.20 \mathrm{E}+02$

ATSDR, $1993 \quad 1.50 \mathrm{E}+02$

IRIS, 1994

2.50E+02

$1.20 \mathrm{E}+02$

RTECS, 199X 1.63E+03

RTECS, $1993 \quad 4.90 \mathrm{E}+02$

RTECS, $1993 \quad 7.00 E+02$

1
1
1
1
1
1
1
1
1

5
5
10
5
5
10
5
5
10

1

$25200 E+01$

$5 \quad 1.80 \mathrm{E}+01$

$4.80 \mathrm{E}+01$

$1.16 \mathrm{E}+01$

$3.86 \mathrm{E}+01$

$1.78 \mathrm{E}+01$

$1.76 \mathrm{E}+00$

$5.00 E+01$

$.40 E+01$

2.40E-01

2.68E+00

1.50E+01

$2.40 E+00$

2.40E-01

2.40E-01

$2.40 \mathrm{E}-01$

$8.00 \mathrm{E}+00$

$5.20 \mathrm{E}+00$

$1.88 \mathrm{E}+01$

2.40E-01

6.25E+01

8.67E+01

$2.40 \mathrm{E}-01$

no data

$1.50 \mathrm{E}+01$

$1.00 \mathrm{E}+01$

2.40E-01

$1.09 \mathrm{E}+01$

$3.27 \mathrm{E}+00$

4.67E+ 00 
TOXICITY DATA FOR ECOLOGICAL SCREENING BRP 231-F/1F and RP 231-2F - SOIL

\begin{tabular}{|c|c|c|c|c|c|c|c|c|c|}
\hline Analyto & $\begin{array}{c}\text { Test } \\
\text { Organism }\end{array}$ & Endpoint/Duration'ErTect & Source & $\begin{array}{l}\text { Erreet Dose } \\
(\mathrm{m} / \mathrm{l} / \mathrm{d} / \mathrm{day})\end{array}$ & $\begin{array}{c}\text { Surrogate } \\
\text { Chemical } \\
\text { UF }\end{array}$ & $\begin{array}{c}\text { Endpoint } \\
\text { UF }\end{array}$ & $\begin{array}{c}\text { Study } \\
\text { Duration } \\
\text { UF }\end{array}$ & $\begin{array}{c}\text { Total } \\
\text { UF }\end{array}$ & $\begin{array}{l}\text { Toxicity } \\
\text { Sereening } \\
\text { Benchmark } \\
\text { (mp/ke/day) }\end{array}$ \\
\hline Pyrene & rat & LOAEL, diet, 91-day, renal fibrosis & IRIS, 1994 & $1.25 E+02$ & 1 & 5 & 5 & 25 & $5.00 \mathrm{E}+00$ \\
\hline
\end{tabular}

\section{Diosina/furam}

Heptachlorodibenco-p-dioxin isomers Heptachlorodibenco-p-furan isomers Hexachlorodibenzo-p-funen isomers Octechlorodibenzo-p-dioxin isomer Octachlorodiberroo-p-furan isomers

PDBs

PCB 1254

PCB 1260

Pestlcides

Aldin

Benzeno hexachloride, delto

Chlordane, alpha

Chlordene, gamma

DDD, P,p'-

DDE, p,p'-

DDT, p.p'-

Endosulfan II

Endrin ketono

\section{Metals}

Arsenic .

Barium

Cedmium

Chromium

Copper

lead

Manganeso

Mercury

Nickel

\section{guinee pig LDSO, oral, acute}

mouse EDS0, subchronic, immunotoxicity

mouse EDSO, subchronic, teratogenicity

mouse NOAEL, oral, scuto

rat NOAEL, gavage in oil, acute, hepatic effects

rat LOAEL, diet, 104-woek, death

rat LOAEL, diet, 21-month, hepatocellular carcinoms

rat LOAEL, diet, 31-month, docreasod lifo spen

Lat LDSO, oral, acute

mouse LOAEL, diet, 80-week, docreased survival

mouse LOAEL, diet, 80-woek, decreased survival

mouse LOAEL, diet, 130-wook, lung/liver tumore

mouse LOAEL, diet, 78-week, liver carcinomas

rat LOAEL, diet, 70-week, decreased survival

mouse LOAEL, diet, 78-week, increased mortality

mouse substituted endrin value, LOAEL, diet, 80-wreek

mouse LOAEL, gavage, 19-days, reducod fertility

mouse NOAEL, drinking water, 2-year

mouso LOAEL, water, 12-month, hematological effocts

mouso LOAEL, diet, 7-week, decreased spermatogenesis

mouse LOAEL, drinking water, 850-day, decr survival

rat LOAEL, diet, 18-month, increased blood prossuro

LOAEL, diet, 103-week, death

mouso LOAEL, diet, 104-week, kidney effects

rat LOAEL, diet, 2-year, decr liver \& body weight

\section{HSDB, 1994}

Safo, 1990

Safo, 1990

HSDB, 1994

ATSDR, 1994

6.00E-0I

6.16E-03

S.T7E-03

4.00E+03

4.00E-02

ATSDR, 1991g 2.50E+00

ATSDR, $1991 \mathrm{~g}$ 5.00E+00

ATSDR, 19910 2.50E+00

RTECS, $1989 \quad 1.00 E+03$

ATSDR, 19920 3.90E+00

ATSDR $1992 \mathrm{C} \quad 3.90 \mathrm{E}+00$

ATSDR, 1992d 325E+01

ATSDR, 1992d 1.90E+0

ATSDR, $19920 \quad 1.30 \mathrm{E}+01$

ATSDR, 1990d 9.00E-0I

ATSDR, 1989h 5.30E-0

ATSDR, 1991. 5.50E+01

ATSDR, 1991k 9.50E-0I

ATSDR, 19916 5.70E+0!

ATSDR 19910 5.70E+01

ATSDR, 1989a 4.25E+01

ATSDR, $1992 \quad 2.80 E+00$

ATSDR, 1990c 9.30E+02

ATSDR, 1994 1.10E-0

ATSDR, 1991 S.00E+0

1
1
1
1
1

15
10
10
1
1

$4.00 \mathrm{E}-03$

1.23E-04

$1.15 \mathrm{E}-04$

$4.00 \mathrm{E}+02$

$4.00 \mathrm{E}-03$

Pursoms - Tumpa: 3 28/96

- 


\section{TABLE 4.6}

SOIL SCREENING AGAINST ECOLOGICAL TOXICITY SCREENING BENCHMARKS

BRP 231-R/1F; 0 - $0.6 \mathrm{M}(0-2 \mathrm{FT})$

CURRENT CONDITIONS

NON-RADIOACTIVE ANALYTES

\begin{tabular}{|c|c|c|c|c|c|c|}
\hline Analyte & $\begin{array}{c}\text { Marimum } \\
\text { Detected Conc. } \\
(\mathrm{mg} / \mathrm{kg})\end{array}$ & $\begin{array}{c}\text { Feeding Rate } \\
\text { (kg/day) }\end{array}$ & $\begin{array}{c}\text { Body Weight }{ }^{1} \\
\text { (kg) }\end{array}$ & $\mid \begin{array}{c}\text { Soil Sereening } \\
\text { Intake }^{2}(\mathrm{mg} / \mathrm{kg} / \mathrm{day})\end{array}$ & $\begin{array}{c}\text { Toxicity Screening } \\
\text { Benchmark } \\
\text { (mg/kg/day) }\end{array}$ & $\begin{array}{c}\text { Exceeds } \\
\text { Screening Value } \\
(\mathbf{Y} / \mathbf{N})\end{array}$ \\
\hline
\end{tabular}

\section{Volatiles}

\section{Acetone}

Chloroform

Dichloroethane, 1,2-

Dichloromethane

Tetrachloroethylene

Toluene

Trichloroethane, 1,1,2-

Xylene

$2.62 \mathrm{E}+01$
$1.01 \mathrm{E}-02$
$2.16 \mathrm{E}-02$
$3.80 \mathrm{E}-02$
$2.87 \mathrm{E}-03$
$3.06 \mathrm{E}-02$
$1.65 \mathrm{E}-02$
$1.34 \mathrm{E}-03$

$2.55 \mathrm{E}-02$
$2.55 \mathrm{E}-02$
$2.55 \mathrm{E}-02$
$2.55 \mathrm{E}-02$
$3.31 \mathrm{E}-03$
$2.55 \mathrm{E}-02$
$3.31 \mathrm{E}-03$
$2.55 \mathrm{E}-02$

3.00E-01

3.00E-01

3.00E-01 .

$3.00 \mathrm{E}-01$

2.50E-02

3.00E-01

2.50E-02

3.00E-01

$2.23 \mathrm{E}+00$
$8.60 \mathrm{E}-04$
$1.84 \mathrm{E}-03$
$3.23 \mathrm{E}-03$
$3.80 \mathrm{E}-04$
$2.60 \mathrm{E}-03$
$2.19 \mathrm{E}-03$
$1.14 \mathrm{E}-04$

$2.00 E+01$

$1.80 \mathrm{E}+01$

$9.50 \mathrm{E}+00$

$4.80 \mathrm{E}+01$

$3.86 \mathrm{E}+01$

$1.78 \mathrm{E}+01$

$1.76 \mathrm{E}+00$

$5.00 \mathrm{E}+01$

$\mathbf{N}$
$\mathbf{N}$
$\mathbf{N}$
$\mathbf{N}$
$\mathbf{N}$
$\mathbf{N}$
$\mathbf{N}$
$\mathbf{N}$

Semivolatiles

Acenaphthene

Acenaphthylene

$\begin{array}{ll}3.16 \mathrm{E}-01 & 3.31 \mathrm{E}-03 \\ 8.61 \mathrm{E}-02 & 3.31 \mathrm{E}-03 \\ 6.74 \mathrm{E}-01 & 2.55 \mathrm{E}-02 \\ 1.62 \mathrm{E}+00 & 2.55 \mathrm{E}-02 \\ 1.33 \mathrm{E}+00 & 3.31 \mathrm{E}-03 \\ 1.28 \mathrm{E}+00 & 3.31 \mathrm{E}-03 \\ 9.52 \mathrm{E}-01 & 3.31 \mathrm{E}-03 \\ 1.49 \mathrm{E}+00 & 3.31 \mathrm{E}-03 \\ 4.86 \mathrm{E}-02 & 2.55 \mathrm{E}-02 \\ 6.02 \mathrm{E}-01 & 3.31 \mathrm{E}-03 \\ 2.08 \mathrm{E}-01 & 2.55 \mathrm{E}-02 \\ 1.62 \mathrm{E}+00 & 3.31 \mathrm{E}-03 \\ 2.42 \mathrm{E}-01 & 2.55 \mathrm{E}-02\end{array}$

2.50E-02

2.50E-02

3.00E-01

3.00E-01

Benzo(a)anthracene

Benzo(a)pyrene

Benzo(b)fluoranthene

Benzo(ghi)perylene

Benzo(k)fluoranthene

Benzoic acid

Bis(2-ethylhexyl) phthalate

Butylbenzyl phthalate

Chrysene

Di-n-butyl phthalate

2.42E-01

2.50E-02

$2.50 \mathrm{E}-02$

2.50E-02

$2.50 \mathrm{E}-02$

$3.00 \mathrm{E}-01$

$2.50 \mathrm{E}-02$

$3.00 \mathrm{E}-01$

$2.50 \mathrm{E}-02$

$3.00 \mathrm{E}-01$

$4.19 \mathrm{E}-02$
$1.14 \mathrm{E}-02$
$5.74 \mathrm{E}-02$
$1.38 \mathrm{E}-01$
$1.76 \mathrm{E}-01$
$1.70 \mathrm{E}-01$
$1.26 \mathrm{E}-01$
$1.97 \mathrm{E}-01$
$4.14 \mathrm{E}-03$
$7.97 \mathrm{E}-02$
$1.77 \mathrm{E}-02$
$2.15 \mathrm{E}-01$
$2.06 \mathrm{E}-02$

$1.40 \mathrm{E}+01$

$5.20 \mathrm{E}-01$

$2.68 \mathrm{E}+00$

$1.50 \mathrm{E}+01$

$2.40 \mathrm{E}+00$

$2.40 \mathrm{E}-01$

$2.40 \mathrm{E}-01$

$2.40 \mathrm{E}-01$

$8.00 \mathrm{E}+00$

$5.20 \mathrm{E}+00$

$1.88 \mathrm{E}+01$

$5.00 \mathrm{E}+00$

$6.25 \mathrm{E}+01$

$\mathbf{N}$
$\mathbf{N}$
$\mathbf{N}$
$\mathbf{N}$
$\mathbf{N}$
$\mathbf{N}$
$\mathrm{N}$
$\mathbf{N}$
$\mathbf{N}$
$\mathbf{N}$
$\mathbf{N}$
$\mathbf{N}$

Parrons - Tempa: 3/8/96

Sheet Tabs 'file TAB6-7.XIS

Page 1 of 
TABLE 4.6

SOIL SCREENING AGAINST ECOLOGICAL TOXICITY SCREENING BENCHMARKS

BRP 231-F/1F; 0 - $0.6 \mathrm{M}(0$ - 2 FT)

CURRENT CONDITIONS

NON-RADIOACTIVE ANALYTES

\begin{tabular}{|c|c|c|c|c|c|c|}
\hline Analyte & $\begin{array}{c}\text { Maximum } \\
\text { Detected Conc. } \\
(\mathrm{mg} / \mathrm{kg})\end{array}$ & $\begin{array}{l}\text { Feeding Rate } \\
\text { (kg/day) }\end{array}$ & $\begin{array}{c}\text { Body Weight }{ }^{1} \\
\text { (kg) }\end{array}$ & $\begin{array}{c}\text { Soil Screening } \\
\text { Intake }{ }^{2}(\mathrm{mg} / \mathrm{kg} / \mathrm{day})\end{array}$ & $\begin{array}{c}\text { Toxicity Screening } \\
\text { Benchmark }{ }^{3} \\
\text { (mg/kg/day) }\end{array}$ & $\begin{array}{c}\text { Exceeds } \\
\text { Screening Value } \\
\text { (YIN) }\end{array}$ \\
\hline Di-n-octyl phthalate & $7.90 \mathrm{E}-02$ & $3.31 E-03$ & $2.50 \mathrm{E}-02$ & 1.05E-02 & $8.67 \mathrm{E}+01$ & $\mathbf{N}$ \\
\hline Dibenz(a,h)anthracene & 4.73E-01 & 3.31E-03 & $2.50 \mathrm{E}-02$ & $6.27 \mathrm{E}-02$ & $4.80 \mathrm{E}+00$ & $\mathbf{N}$ \\
\hline Dibenzofuran & $2.22 \mathrm{E}-01$ & no data & no data & no data & no data & $\mathbf{Y}$ \\
\hline Fluoranthene & $3.91 \mathrm{E}+00$ & 2.55E-02 & $3.00 \mathrm{E}-01$ & $3.33 \mathrm{E}-01$ & $1.50 \mathrm{E}+01$ & $\mathbf{N}$ \\
\hline Flourene & $2.99 E-01$ & 3.31E-03 & $2.50 \mathrm{E}-02$ & $3.96 \mathrm{E}-02$ & $1.00 E+01$ & $\mathbf{N}$ \\
\hline Indeno(1,2,3-cd)pyrẹne & $8.32 \mathrm{E}-01$ & 3.31E-03 & $2.50 \mathrm{E}-02$ & $1.10 \mathrm{E}-01$ & $0.00 \mathrm{E}+00$ & $\mathbf{Y}$ \\
\hline Methylnaphthylene, 2- & $7.69 \mathrm{E}-02$ & 2.55E-02 & $3.00 \mathrm{E}-01$ & $6.55 \mathrm{E}-03$ & $1.09 E+01$ & $\mathbf{N}$ \\
\hline Naphthalene & $3.22 \mathrm{E}-01$ & $2.55 \mathrm{E}-02$ & $3.00 \mathrm{E}-01$ & 2.74E-02 & 3.27E+00 & $\mathbf{N}$ \\
\hline Phenanthrene & $3.38 \mathrm{E}+00$ & 3.31E-03 & $2.50 \mathrm{E}-02$ & $4.48 \mathrm{E}-01$ & $4.67 \mathrm{E}+00$ & $\mathbf{N}$ \\
\hline Pyrene & $2.42 \mathrm{E}+00$ & 2.55E-02 & $3.00 \mathrm{E}-01$ & $2.06 \mathrm{E}-01$ & $5.00 \mathrm{E}+00$ & $\mathbf{N}$ \\
\hline \multicolumn{7}{|l|}{ Dioxins/furans } \\
\hline Heptachlorodibenzo-p-dioxin isomers & $8.30 \mathrm{E}-03$ & 2.55E-02 & $3.00 \mathrm{E}-01$ & $7.06 \mathrm{E}-04$ & $4.00 E-03$ & $\mathbf{N}$ \\
\hline Heptachlorodibenzo-p-furan isomers & $5.00 \mathrm{E}-04$ & $3.31 \mathrm{E}-03$ & $2.50 \mathrm{E}-02$ & $6.62 \mathrm{E}-05$ & $1.23 E-04$ & $\mathbf{N}$ \\
\hline Hexachlorodibenzo-p-furan isomers & $1.00 \mathrm{E}-04$ & 3.31E-03 & $2.50 \mathrm{E}-02$ & 1.32E-05 & 1.15E-04 & $\mathbf{N}$ \\
\hline Octachlorodibenzo-p-dioxin isomers & $1.10 \mathrm{E}-02$ & $3.31 \mathrm{E}-03$ & 2.50E-02 & $1.46 \mathrm{E}-03$ & $4.00 \mathrm{E}+02$ & $\mathbf{N}$ \\
\hline Octachlorodibenzo-p-furan isomers & 8.00E-04 & 2.55E-02 & $3.00 \mathrm{E}-01$ & $6.81 E-05$ & $4.00 \mathrm{E}-03$ & $\mathbf{N}$ \\
\hline PDBs & & & & & & - \\
\hline PCB 1254 & $4.38 \mathrm{E}-01$ & 2.55E-02 & $3.00 \mathrm{E}-01$ & 3.73E-02 & $2.50 \mathrm{E}-01$ & $\mathbf{N}$ \\
\hline \multicolumn{7}{|l|}{ Pesticides } \\
\hline Aldrin & $4.80 \mathrm{E}-03$ & 2.55E-02 & $3.00 \mathrm{E}-01$ & $4.09 \mathrm{E}-04$ & $5.00 \mathrm{E}-01$ & $\mathbf{N}$ \\
\hline Benzene hexachloride, delta- & $1.84 \mathrm{E}-03$ & $2.55 \mathrm{E}-02$ & $3.00 \mathrm{E}-01$ & $1.57 \mathrm{E}-04$ & $6.67 E+00$ & $\mathbf{N}$ \\
\hline Chlordane, alpha- & $7.43 \mathrm{E}-03$ & $3.31 \mathrm{E}-03$ & $2.50 \mathrm{E}-02$ & 9.84E-04 & $7.80 \mathrm{E}-01$ & $\mathbf{N}$ \\
\hline
\end{tabular}

Parrons - Tampa: 3/896 
TABLE 4.6

SOIL SCREENING AGAINST ECOLOGICAL TOXICITY SCREENING BENCHMARKS

BRP 231-F/1F; 0 - 0.6 M (0 - 2 FT)

CURRENT CONDITIONS

NON-RADIOACTIVE ANALYTES

\begin{tabular}{|c|c|c|c|c|c|c|c|}
\hline $\begin{array}{r}\text { Analyte } \\
\end{array}$ & : & $\begin{array}{c}\text { Maximum } \\
\text { Detected Conc. } \\
(\mathrm{mg} / \mathrm{kg})\end{array}$ & $\begin{array}{c}\text { Feeding Rate } \\
\text { (kg/day) }\end{array}$ & $\begin{array}{c}\text { Body Weight } \\
\text { (kg) }\end{array}$ & $\begin{array}{c}\text { Soil Screening } \\
\text { Intake }{ }^{2} \text { (mg/kg/day) }\end{array}$ & $\begin{array}{c}\text { Toxicity Screening } \\
\text { Benchmark }^{3} \\
\text { (mg/kg/day) }\end{array}$ & $\begin{array}{c}\text { Exceeds } \\
\text { Sereening Value } \\
(\mathbf{Y} / \mathbf{N}) \\
\end{array}$ \\
\hline Chlordane, gamma- & & $6.49 \mathrm{E}-03$ & $3.31 \mathrm{E}-03$ & $2.50 \mathrm{E}-02$ & $8.60 \mathrm{E}-04$ & $7.80 \mathrm{E}-01$ & $\mathbf{N}$ \\
\hline DDD, p,p'- & & $1.39 \mathrm{E}-02$ & $3.31 \mathrm{E}-03$ & $2.50 \mathrm{E}-02$ & $1.84 \mathrm{E}-03$ & $6.50 E+00$ & $\mathbf{N}$ \\
\hline DDE, p,p'- & & $2.03 \mathrm{E}-02$ & 3.31E-03 & 2.50E-02 & $2.69 \mathrm{E}-03$ & $3.80 \mathrm{E}+00$ & $\mathbf{N}$ \\
\hline DDT, p,p'- & & $4.31 \mathrm{E}-02$ & 2.55E-02 & $3.00 E-01$ & $3.67 \mathrm{E}-03$ & $2.60 \mathrm{E}+00$ & $\mathbf{N}$ \\
\hline Endosulfan II & & $4.69 \mathrm{E}-03$ & $3.31 E-03$ & $2.50 \mathrm{E}-02$ & $6.21 E-0.4$ & $9.00 \mathrm{E}-02$ & $\mathbf{N}$ \\
\hline Endrin ketone & & $3.20 \mathrm{E}-03$ & $3.31 \mathrm{E}-03$ & 2.50E-02 & $4.24 \mathrm{E}-0.4$ & $1.06 \mathrm{E}-02$ & $\mathbf{N}$ \\
\hline \multicolumn{8}{|l|}{ Metals } \\
\hline Arsenic & & $7.80 \mathrm{E}+00$ & 3.31E-03 & $2.50 \mathrm{E}-02$ & $1.03 \mathrm{E}+00$ & $2.20 \mathrm{E}+00$ & $\mathbf{N}$ \\
\hline Barium & & $3.30 E+01$ & 3.31E-03 & $2.50 \mathrm{E}-02$ & $4.37 \mathrm{E}+00$ & $9.50 \mathrm{E}-01$ & $\mathbf{Y}$ \\
\hline Cadmium & & $1.40 \mathrm{E}+00$ & $3.31 \mathrm{E}-03$ & $2.50 \mathrm{E}-02$ & $1.85 \mathrm{E}-01$ & $1.14 E+01$ & $\mathbf{N}$ \\
\hline Chromium & & $9.75 E+01$ & 3.31E-03 & $2.50 \mathrm{E}-02$ & $1.29 \mathrm{E}+01$ & $2.28 \mathrm{E}+00$ & $\mathbf{Y}$ \\
\hline Copper & 1 & $6.25 E+01$ & $3.31 \mathrm{E}-03$ & $2.50 \mathrm{E}-02$ & $8.28 \mathrm{E}+00$ & $8.50 E+00$ & $\mathbf{N}$ \\
\hline Lead & & $5.62 \mathrm{E}+01$ & $2.55 \mathrm{E}-02$ & $3.00 \mathrm{E}-01$ & $4.78 E+00$ & $5.60 \mathrm{E}-01$ & $\mathbf{Y}$ \\
\hline Manganese & & $1.43 E+03$ & $2.55 \mathrm{E}-02$ & $3.00 \mathrm{E}-01$ & $1.22 E+02$ & $9.30 E+01$ & $\mathbf{Y}$ \\
\hline Mercury & & 8.31E-01 & $3.31 \mathrm{E}-03$ & $2.50 \mathrm{E}-02$ & $1.10 \mathrm{E}-01$ & $2.20 E-02$ & $\mathbf{Y}$ \\
\hline Nickel & & $326 E+01$ & $255 \mathrm{E}-02$ & $3.00 \mathrm{E}-01$ & $2.77 \mathrm{E}+00$ & $100 \mathrm{E}+01$ & $\mathbf{N}$ \\
\hline
\end{tabular}

no data = no toxicological data could be found on this chemical.

1 Body weight and feeding rate are based on the species used in the toxicity study from which the toxicity screening benchmark for this chemical was derived (see Table 4-5).

2 Soil screening intake $=($ maximum concentration*feeding rate)/body weight

3 Toxicity screening benchmarks are calculated from toxicity values with appropriate EPA Region VIIl uncertainty factors to derive a NOAEL equivalent value (see Table 4-5). If a benchmark was not available the chemical was retained as a COPC. 
TABLE 4.7

SOIL SCREENINGG AGAINST ECOLOGICAL TOXICITY SCREENING BENCHMARKS

BRP 231-F/1F; 0 - $0.6 \mathrm{M}(0-2$ FT)

CURRENT CONDITIONS

RADIONUCLIDES

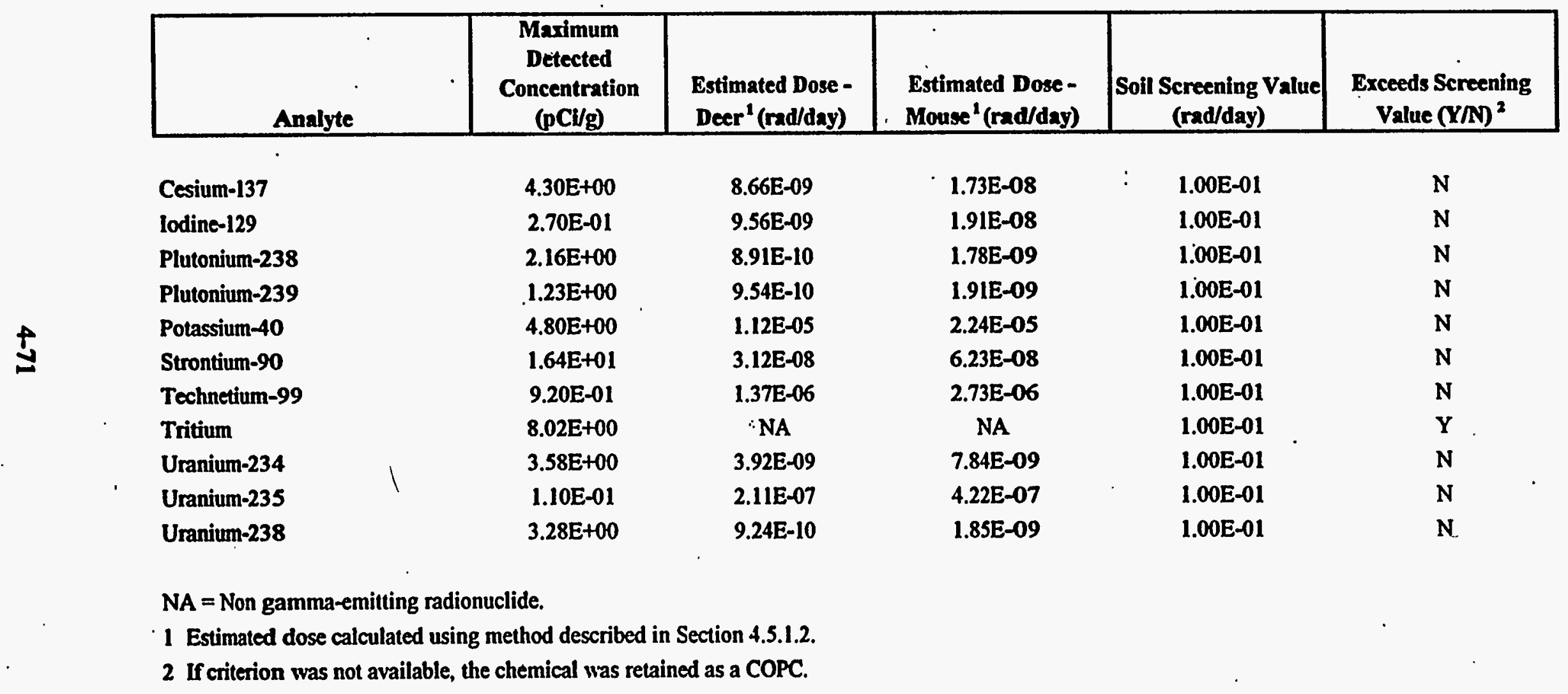


TABLE 4.8

SOIL SCREENING AGAINST ECOLOGICAL TOXICITY SCREENING BENCHMARKS

BRP 231-F/1F; 0 - 1.2 M (0 - 4 FT)

FUTURE CONDITIONS

NON-RADIOACTTVE ANALYTES

\begin{tabular}{|c|c|c|c|c|c|c|c|}
\hline Analyte & & $\begin{array}{c}\text { Marimum } \\
\text { Detected Conc. } \\
\text { (mg/kg) }\end{array}$ & $\begin{array}{c}\text { Feeding Rate } \\
\text { (kg/day) }\end{array}$ & $\begin{array}{c}\text { Body Weight }{ }^{1} \\
\text { (kg) }\end{array}$ & $\begin{array}{c}\text { Soil Screening } \\
\text { Intake }^{2}(\mathrm{~m} / / \mathrm{kg} / \text { day })\end{array}$ & $\begin{array}{c}\text { Toxicity Screening } \\
\text { Benchmark }^{3} \\
\text { (mg/kg/day) }^{-}\end{array}$ & $\begin{array}{c}\text { Exceeds } \\
\text { Screening Value } \\
\text { (Y/N }\end{array}$ \\
\hline \multicolumn{8}{|l|}{ Volatiles } \\
\hline Acetone & & $2.62 E+01$ & $2.55 \mathrm{E}-02$ & $3.00 \mathrm{E}-01$ & $2.23 E+00$ & $2.00 \mathrm{E}+01$ & $\mathbf{N}$ \\
\hline Chloroform & & $1.01 E-02$ & 2.55E-02 & $3.00 \mathrm{E}-01$ & $8.60 \mathrm{E}-04$ & $1.80 \mathrm{E}+01$ & $\mathbf{N}$ \\
\hline Dichloroethane, 1,2- & & $2.16 \mathrm{E}-02$ & $2.55 \mathrm{E}-02$ & $3.00 \mathrm{E}-01$ & $1.84 E-03$ & $9.50 \mathrm{E}+00$ & $\mathbf{N}$ \\
\hline Dichloromethane & & $3.80 \mathrm{E}-02$ & $2.55 \mathrm{E}-02$ & $3.00 \mathrm{E}-01$ & $3.23 E-03$ & $4.80 \mathrm{E}+01$ & $\mathbf{N}$ \\
\hline Ethylbenzene & & $1.29 \mathrm{E}-03$ & 2.55E-02 & $3.00 \mathrm{E}-01$ & $1.10 \mathrm{E}-04$ & $1.16 \mathrm{E}+01$ & $\mathbf{N}$ \\
\hline Tetrachloroethylene & & $2.87 \mathrm{E}-03$ & $3.31 E-03$ & $2.50 \mathrm{E}-02$ & $3.80 \mathrm{E}-04$ & $3.86 \mathrm{E}+01$ & $\mathbf{N}$ \\
\hline Toluene & & $3.06 \mathrm{E}-02$ & $2.55 \mathrm{E}-02$ & $3.00 \mathrm{E}-01$ & $2.60 \mathrm{E}-03$ & $1.78 \mathrm{E}+01$ & $\mathbf{N}$ \\
\hline Trichloroethane, 1,1,2- & & $1.65 \mathrm{E}-02$ & $3.31 \mathrm{E}-03$ & $2.50 \mathrm{E}-02$ & $2.19 \mathrm{E}-03$ & $1.76 \mathrm{E}+00$ & $\mathbf{N}$ \\
\hline Xylene & & $1.34 \mathrm{E}-03$ & 2.55E-02 & $3.00 \mathrm{E}-01$ & $1.14 \mathrm{E}-04$ & $5.00 \mathrm{E}+01$ & $\mathbf{N}$ \\
\hline \multicolumn{8}{|l|}{ Semivolatiles } \\
\hline Acenaphthene & & $3.16 \mathrm{E}-01$ & $3.31 E-03$ & $2.50 \mathrm{E}-02$ & $4.19 \mathrm{E}-02$ & $1.40 \mathrm{E}+01$ & $\mathbf{N}$ \\
\hline Acenaphthylene & 1 & $8.61 E-02$ & $3.31 E-03$ & $2.50 \mathrm{E}-02$ & $1.14 E-02$ & $5.20 \mathrm{E}-01$ & $\mathbf{N}$ \\
\hline Anthracene & & $6.74 E-01$ & 2.55E-02 & $3.00 \mathrm{E}-01$ & $5.74 \mathrm{E}-02$ & $2.68 \mathrm{E}+00$ & $\mathbf{N}$ \\
\hline Benzo(a)anthracene & & $1.62 \mathrm{E}+00$ & $2.55 \mathrm{E}-02$ & $3.00 \mathrm{E}-01$ & $1.38 E-01$ & $1.50 E+01$ & $\mathbf{N}$ \\
\hline Benzo(a)pyrene & & $1.33 E+00$ & $3.31 E-03$ & $2.50 \mathrm{E}-02$ & $1.76 \mathrm{E}-01$ & $2.40 \mathrm{E}+00$ & $\mathbf{N}$ \\
\hline Benzo(b)fluoranthene & & $1.28 \mathrm{E}+00$ & $3.31 E-03$ & $2.50 E-02$ & $1.70 E-01$ & $2.40 E-01$ & $\mathbf{N}$ \\
\hline Benzo(ghi)perylene & & $9.52 \mathrm{E}-01$ & $3.31 \mathrm{E}-03$ & $2.50 \mathrm{E}-02$ & $1.26 \mathrm{E}-01$ & $2.40 \mathrm{E}-01$ & $\mathbf{N}$ \\
\hline Benzo(k)fluoranthene & & $1.49 \mathrm{E}+00$ & $3.31 E-03$ & $2.50 \mathrm{E}-02$ & $1.97 E-01$ & $2.40 \mathrm{E}-01$ & $\mathbf{N}$ \\
\hline Benzoic acid & & $4.86 \mathrm{E}-02$ & $2.55 \mathrm{E}-02$ & $3.00 \mathrm{E}-01$ & 4.14E-03 & $8.00 \mathrm{E}+00$ & $\mathbf{N}$ \\
\hline Bis(2-ethylhexyl)phthalate & & $6.02 \mathrm{E}-01$ & $3.31 \mathrm{E}-03$ & $2.50 \mathrm{E}-02$ & $7.97 \mathrm{E}-02$ & $5.20 \mathrm{E}+00$ & $\mathbf{N}$ \\
\hline Butylbenzyl phthalate & & $2.08 \mathrm{E}-01$ & $2.55 \mathrm{E}-02$ & $3.00 \mathrm{E}-01$ & $1.77 \mathrm{E}-02$ & $1.88 E+01$ & $\mathbf{N}$ \\
\hline Chrysene & & $1.62 \mathrm{E}+00$ & $3.31 E-03$ & $2.50 E-02$ & $2.15 E-01$ & $5.00 E+00$ & $\mathbf{N}$ \\
\hline Di-n-butyl phthalate & & $2.42 \mathrm{E}-01$ & $2.55 \mathrm{E}-02$ & $3.00 \mathrm{E}-01$ & $2.06 \mathrm{E}-02$ & $6.25 \mathrm{E}+01$ & $\mathbf{N}$ \\
\hline
\end{tabular}

Pareons - Tampa: 3/8/96 
TABLE 4.8

SOIL SCREENING AGAINST ECOLOGICAL TOXICITY SCREENING BENCEMARKS

BRP 231-F/1F; 0 - 1.2 M (0-4 FT)

FUTURE CONDITIONS

NON-RADIOACTIVE ANALYTES

\begin{tabular}{|c|c|c|c|c|c|c|}
\hline Analyte & $\begin{array}{c}\text { Marimum } \\
\text { Detected Conc. } \\
(\mathrm{mg} / \mathrm{kg})\end{array}$ & $\begin{array}{c}\text { Feeding Rate }{ }^{2} \\
\text { (kg/day) }\end{array}$ & $\begin{array}{c}\text { Body Weight } \\
(\mathrm{kg})\end{array}$ & $\begin{array}{c}\text { Soil Screening } \\
\text { Intake }{ }^{2}(\mathrm{~m} / \mathrm{kg} / \mathrm{d} a \mathrm{y})\end{array}$ & $\begin{array}{c}\text { Toxicity Sereening } \\
\text { Benchmark } \\
\text { (mg/kg/day) }\end{array}$ & $\begin{array}{c}\text { Exceeds } \\
\text { Sereening Value } \\
\text { (XN) }\end{array}$ \\
\hline Di-n-octyl phthalate & $7.90 \mathrm{E}-02$ & $3.31 \mathrm{E}-03$ & $2.50 \mathrm{E}-02$ & $1.05 \mathrm{E}-02$ & $8.67 \mathrm{E}+01$ & $\mathbf{N}$ \\
\hline Dibenz(a,h)anthracene & $4.73 E-01$ & $3.31 E-03$ & $2.50 \mathrm{E}-02$ & $6.27 \mathrm{E}-02$ & $1.16 \mathrm{E}+00$ & $\mathbf{N}$ \\
\hline Dibenzofuran & $2.22 \mathrm{E}-01$ & no data & no data & no data & no data & $\mathbf{Y}$ \\
\hline Fluoranthene & $3.91 E+00$ & $2.55 \mathrm{E}-02$ & $3.00 \mathrm{E}-01$ & $3.33 \mathrm{E}-01$ & $1.50 \mathrm{E}+01$ & $\mathbf{N}$ \\
\hline Flourene & $2.99 \mathrm{E}-01$ & $3.31 \mathrm{E}-03$ & $2.50 \mathrm{E}-02$ & $3.96 \mathrm{E}-02$ & $1.00 \mathrm{E}+01$ & $\mathbf{N}$ \\
\hline Indeno(1,2,3-od)pyrene & $8.32 E-01$ & $3.31 \mathrm{E}-03$ & $2.50 \mathrm{E}-02$ & $1.10 \mathrm{E}-01$ & $0.00 \mathrm{E}+00$ & $\mathbf{Y}$ \\
\hline Methylnaphthylene, 2- & $7.69 \mathrm{E}-02$ & $2.55 \mathrm{E}-02$ & $3.00 \mathrm{E}-01$ & $6.54 \mathrm{E}-03$ & $1.09 \mathrm{E}+01$ & $\mathbf{N}$ \\
\hline Naphthalene & $3.22 \mathrm{E}-01$ & $2.55 \mathrm{E}-02$ & $3.00 \mathrm{E}-01$ & $2.74 E-02$ & $3.27 \mathrm{E}+00$ & $\mathbf{N}$ \\
\hline Phenanthrene & $3.38 \mathrm{E}+00$ & $3.31 \mathrm{E}-03$ & $2.50 \mathrm{E}-02$ & $4.48 E-01$ & $4.67 \mathrm{E}+00$ & $\mathbf{N}$ \\
\hline Pyrene & $2.42 E+00$ & 2.55E-02 & $3.00 \mathrm{E}-01$ & $2.06 \mathrm{E}-01$ & $5.00 \mathrm{E}+00$ & $\mathbf{N}$ \\
\hline \multicolumn{7}{|l|}{ Dioxins/furans } \\
\hline Heptachlorodibenzo-p-dioxin isomers & 8.30E-03 & 2.55E-02 & $3.00 \mathrm{E}-01$ & $7.06 \mathrm{E}-04$ & $4.00 \mathrm{E}-03$ & $\mathbf{N}$ \\
\hline Heptachlorodibenzo-p-furan isomers & $5.00 \mathrm{E}-04$ & $3.31 \mathrm{E}-03$ & $2.50 \mathrm{E}-02$ & $6.62 \mathrm{E}-05$ & $1.23 E-04$ & $\mathbf{N}$ \\
\hline Hexachlorodibenzo-p-furan isomers & $1.00 \mathrm{E}-04$ & $3.31 E-03$ & $2.50 \mathrm{E}-02$ & $1.32 E-05$ & $1.15 \mathrm{E}-04$ & $\mathbf{N}$ \\
\hline Octachlorodibenzo-p-dioxin isomers & $1.10 \mathrm{E}-02$ & $3.31 \mathrm{E}-03$ & $2.50 \mathrm{E}-02$ & $1.46 \mathrm{E}-03$ & $4.00 E+02$ & $\mathbf{N}$ \\
\hline Octachlorodibenzo-p-furan isomers & 8.00E-04 & 2.55E-02 & $3.00 \mathrm{E}-01$ & $6.80 \mathrm{E}-05$ & $4.00 \mathrm{E}-03$ & $\mathbf{N}$ \\
\hline \multicolumn{7}{|l|}{ PCBs } \\
\hline PCB 1254 & $4.38 \mathrm{E}-01$ & $2.55 \mathrm{E}-02$ & $3.00 \mathrm{E}-01$ & $3.73 \mathrm{E}-02$ & $2.50 E-01$ & $\mathbf{N}$ \\
\hline \multicolumn{7}{|l|}{ Pesticides } \\
\hline Aldrin & $4.80 \mathrm{E}-03$ & $2.55 \mathrm{E}-02$ & $3.00 \mathrm{E}-01$ & $4.09 \mathrm{E}-04$ & $5.00 \mathrm{E}-01$ & $\dot{N}$ \\
\hline Benzene hexachloride, delta- & $1.84 E-03$ & 2.55E-02 & $3.00 \mathrm{E}-01$ & $1.57 \mathrm{E}-04$ & 6.67Et00 & $\mathbf{N}$ \\
\hline Chlordane, alpha- & 7.43E-03 & $3.31 E-03$ & $2.50 E-02$ & 9.84E-04 & $\forall .80 \mathrm{E}-01$ & $\mathbf{N}$ \\
\hline
\end{tabular}

Parains - Tampa: 3/8/96

FINAL 
SOIL SCREENING AGAINST ECOLOGICAL TOXICITY SCREENING BENCHMARKS

BRP 231-F/1F; 0 - $1.2 \mathrm{M}(0$ - 4 FT)

FUTURE CONDITIONS

NON-RADIOACTIVE ANALYTES

\begin{tabular}{|c|c|c|c|c|c|c|}
\hline Analyte & $\begin{array}{l}\text { Maximum } \\
\text { Detected Conc. } \\
\text { (mg/kg) }\end{array}$ & $\begin{array}{l}\text { Feeding Rate } \\
\text { (kg/day) }\end{array}$ & $\begin{array}{c}\text { Body Weight }{ }^{1} \\
\text { (kg) }\end{array}$ & $\begin{array}{c}\text { Soil Screening } \\
\text { Intake }^{2} \text { (mg/kg/day) }\end{array}$ & $\begin{array}{c}\text { Toxicity Screening } \\
\text { Benchmark }{ }^{3} \\
\text { (mg/kg/day) }\end{array}$ & $\begin{array}{c}\text { Exceeds } \\
\text { Screening Value } \\
\text { (YIN) }\end{array}$ \\
\hline Chlordane, gamma- & $6.49 \mathrm{E}-03$ & $3.31 \mathrm{E}-03$ & $2.50 \mathrm{E}-02$ & $8.60 \mathrm{E}-04$ & $7.80 \mathrm{E}-01$ & $\mathbf{N}$ \\
\hline DDD, p,p'- & $1.39 \mathrm{E}-02$ & $3.31 E-03$ & $2.50 \mathrm{E}-02$ & $1.84 \mathrm{E}-03$ & $6.50 \mathrm{E}+00$ & $\mathbf{N}$ \\
\hline DDE, p,p'- & $2.03 E-02$ & 3.31E-03 & $2.50 \mathrm{E}-02$ & $2.69 \mathrm{E}-03$ & $3.80 E+00$ & $\mathbf{N}$ \\
\hline DDT, p,p'- & $4.31 E-02$ & 2.55E-02 & $3.00 \mathrm{E}-01$ & $3.67 \mathrm{E}-03$ & $2.60 \mathrm{E}+00$ & $\mathbf{N}$ \\
\hline Endosulfan II & $4.69 \mathrm{E}-03$ & $3.31 E-03$ & $2.50 \mathrm{E}-02$ & 6.21E-0.4 & $9.00 \mathrm{E}-02$ & $\mathbf{N}$ \\
\hline Endrin ketone & $3.20 \mathrm{E}-03$ & $3.31 E-03$ & $2.50 \mathrm{E}-02$ & $4.24 \mathrm{E}-04$ & $1.06 \mathrm{E}-02$ & $\mathbf{N}$ \\
\hline
\end{tabular}

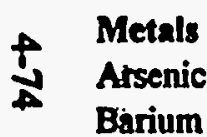

Cadmium

Chromium

Copper

Lead

Manganese

Mercury

Nickel

no'data = no toxicological data could be found on this chemical.

1 Body weight and feeding rate are based on the species used in the toxicity study from which the toxicity screening benchmark for this chemical was derived (see Table 4-5).

2 Soil screening intake $=$ (maximum concentration * feeding rate)/body weight

3 Toxicity screening benchmarks are calculated from toxicity values with appropriate EPA Region VIII uncertainty factors to derive a NOAEL equivalent value (see Table 4-5). If a benchmark was not available, the chemical was retained as a COPC.

$\begin{array}{lll}3.31 \mathrm{E}-03 & 2.50 \mathrm{E}-02 & 2.01 \mathrm{E}+00 \\ 3.31 \mathrm{E}-03 & 2.50 \mathrm{E}-02 & 7.76 \mathrm{E}+00 \\ 3.31 \mathrm{E}-03 & 2.50 \mathrm{E}-02 & 1.85 \mathrm{E}-01 \\ 3.31 \mathrm{E}-03 & 2.50 \mathrm{E}-02 & 4.68 \mathrm{E}+01 \\ 3.31 \mathrm{E}-03 & 2.50 \mathrm{E}-02 & 8.28 \mathrm{E}+00 \\ 2.55 \mathrm{E}-02 & 3.00 \mathrm{E}-01 & 5.44 \mathrm{E}+00 \\ 2.55 \mathrm{E}-02 & 3.00 \mathrm{E}-01 & 1.22 \mathrm{E}+02 \\ 3.31 \mathrm{E}-03 & 2.50 \mathrm{E}-02 & 1.10 \mathrm{E}-01 \\ 2.55 \mathrm{E}-02 & 3.00 \mathrm{E}-01 & 5.40 \mathrm{E}+00\end{array}$

$2.20 \mathrm{E}+00$
$9.50 \mathrm{E}-01$
$1.14 \mathrm{E}+01$
$2.28 \mathrm{E}+00$
$8.50 \mathrm{E}+00$
$5.60 \mathrm{E}-01$
$9.30 \mathrm{E}+01$
$2.20 \mathrm{E}-02$
$1.00 \mathrm{E}+01$

$\mathbf{N}$

$\mathbf{Y}$

$\mathbf{N}$

$\mathbf{Y}$

$\mathbf{N}$

$\mathbf{Y}$

$\mathbf{Y}$

$\mathbf{Y}$ 
TABLE 4.9

SOIL SCREENING AGAINST ECOLOGICAL TOXICITY SCREENING BENCHMARKS

BRP 231-F/1F; 0 - $1.2 \mathrm{M}(0$ - 4 FT)

FUTURE CONDITIONS

RADIONUCLIDES

\begin{tabular}{|c|c|c|c|c|c|c|}
\hline Analyte & & $\begin{array}{c}\text { Maximum Detected } \\
\text { Concentration } \\
(\mathbf{p C l} / \mathrm{g})\end{array}$ & $\begin{array}{c}\text { Estimated Dose - Deer } \\
1 \text { (rad/day) }\end{array}$ & $\begin{array}{l}\text { Estimated Dose - } \\
\text { Mouse (rad/day) }\end{array}$ & $\begin{array}{c}\text { Soil Screening Value } \\
\text { (rad/day) }\end{array}$ & $\begin{array}{c}\text { Exceeds Screening } \\
\text { Value }(\mathbf{Y} / \mathbb{N})^{2}\end{array}$ \\
\hline Cessium-137 & & $4.30 \mathrm{E}+00$ & $8.66 \mathrm{E}-09$ & $1.73 E-08$ & $1.00 \mathrm{E}-01$ & $\mathbf{N}$ \\
\hline lodine-129 & & $2.70 \mathrm{E}-01$ & $9.56 \mathrm{E}-09$ & $1.91 E-08$ & $1.00 \mathrm{E}-01$ & $\mathbf{N}$ \\
\hline Plutonium-238 & & $2.16 \mathrm{E}+00$ & 8.91E-10 & $1.78 \mathrm{E}-09$ & $1.00 \mathrm{E}-01$ & $\mathbf{N}$ \\
\hline Plutonium-239 & & $1.23 \mathrm{E}+00$ & $9.54 \mathrm{E}-10$ & $1.91 \mathrm{E}-09$ & $1.00 \mathrm{E}-01$ & $\mathbf{N}$ \\
\hline Potassium-40 & & $4.80 \mathrm{E}+00$ & $1.12 E-05$ & $2.24 \mathrm{E}-05$ & $1.00 \mathrm{E}-01$ & $\mathbf{N}$ \\
\hline Strontium-90 & & $1.64 \mathrm{E}+01$ & $3.12 E-08$ & $6.23 E-08$ & $1.00 \mathrm{E}-01$ & $\mathbf{N}$ \\
\hline Technetium-99 & & $9.20 \mathrm{E}-01$ & $1.37 \mathrm{E}-06$ & 2.73E-06 & $1.00 \mathrm{E}-01$ & $\mathbf{N}$ \\
\hline Tritium & & 8.25E+00 & NA & NA & $1.00 \mathrm{E}-01$ & $\mathbf{Y}$ \\
\hline Uranium-234 & & $3.58 \mathrm{E}+00$ & $3.92 \mathrm{E}-09$ & $7.84 \mathrm{E}-09$ & $1.00 \mathrm{E}-01$ & $\mathbf{N}$ \\
\hline Uranium-235 & 1 & $1.10 \mathrm{E}-01$ & 2.11E-07 & $4.22 \mathrm{E}-07$ & $1.00 \mathrm{E}-01$ & $\mathbf{N}$ \\
\hline Uranium-238 & & $3.28 \mathrm{E}+00$ & $9.24 \mathrm{E}-10$ & $1.85 \mathrm{E}-09$ & $1.00 \mathrm{E}-01$ & $\mathbf{N}$ \\
\hline
\end{tabular}


TABLE 4.10

SOIL SCREENING AGAINST ECOLOGICAL TOXICITY SCREENING BENCHMARKS

RP 231-2F; 0 - 0.6 M (0 - 2 FT)

CURRENT CONDITIONS

\section{NON-RADIOACTIVE ANALYTES}

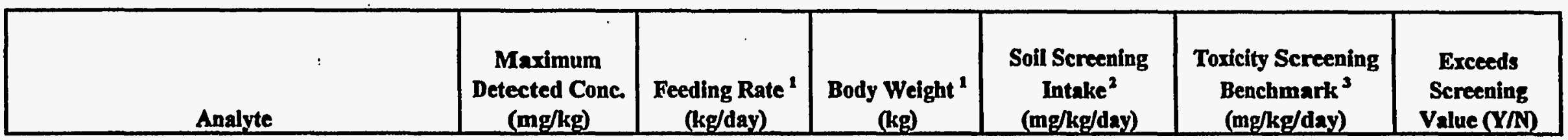

\section{Volatiles}

Acetone

Chloroform

Dichloroethane, 1,2-

Dichloromethane

Toluene

Trichloroethane, 1,1,2(kg/day)

$\begin{array}{ll}4.75 \mathrm{E}-02 & 2.55 \mathrm{E}-02 \\ 2.65 \mathrm{E}-03 & 2.55 \mathrm{E}-02 \\ 1.03 \mathrm{E}-02 & 2.55 \mathrm{E}-02 \\ 6.22 \mathrm{E}-03 & 2.55 \mathrm{E}-02 \\ 1.19 \mathrm{E}-02 & 2.55 \mathrm{E}-02 \\ 1.36 \mathrm{E}-02 & 3.31 \mathrm{E}-03\end{array}$

$3.00 \mathrm{E}-01$
$3.00 \mathrm{E}-01$
$3.00 \mathrm{E}-01$
$3.00 \mathrm{E}-01$
$3.00 \mathrm{E}-01$
$2.50 \mathrm{E}-02$

4.04E-03

2.26E-04

8.77E-04

5.29E-04

1.01E-03

$1.80 \mathrm{E}-03$

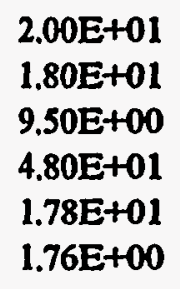

$N^{\prime}$

$N$

$\mathbf{N}$

$\mathbf{N}$

N

Semivolatiles

Benzo(a)anthracene

Benzo(a)pyrene

6.90E-02

\subsection{E-02}

6.01E-02

3.00E-01

2.50E-02

5.87E-03

7.96E-03

3.31E-03

8.14E-02

$2.50 \mathrm{E}-02$

6.05E-02

3.31E-03

Benzo(k) Mouranthene

2.77E-01

3.31E-03

6.92E-02

$3.31 \mathrm{E}-03$

7.07E-02

2.55E-02

Di-n-butyl phthalate

$1.61 \mathrm{E}-01$

3.31E-03

2.50E-02

2.50E-02

$1.08 \mathrm{E}-02$

8.01E-03

2.50E-02

3.67E-02

3.00E-01

9.17E-03

6.02E-03

$2.50 \mathrm{E}-02$

2.55E-02

$1.66 \mathrm{E}-01$

$3.00 \mathrm{E}-01$

2.13E-02

$3.31 \mathrm{E}-03$

Phenanthrene

8.68E-02

2.55E-02

2.50E-02

1.41E-02

1.15E-02

3.00E-01

9.70E-03

$1.50 \mathrm{E}+01$

2.40E+00

2.40E-01

2.40E-01

$5.20 \mathrm{E}+00$

$1.76 \mathrm{E}-01$

6.25E+01.

8.67E+01

$1.50 \mathrm{E}+01$

4.67E+00

$5.00 \mathrm{E}+00$

$\mathbf{N}$

\section{Dioxins/furans}

Heptochlorodibenzo-p-dioxin isomers

Octachlorodibenzo-p-dioxin isomers

$\begin{array}{lll}6.00 \mathrm{E}-04 & 2.55 \mathrm{E}-02 & 3.00 \mathrm{E}-01 \\ 4.00 \mathrm{E}-04 & 3.31 \mathrm{E}-03 & 2.50 \mathrm{E}-02\end{array}$

5.11E-05
5.30E-05

$4.00 \mathrm{E}-03$
$4.00 \mathrm{E}+02$

$\mathrm{N}$

$\mathbf{N}$

Parsons - Tp- 3/8/96

Steet Tab f file TAB10-11.XIS
Page 1 of. 
TABLE 4.10

SOIL SCREENING AGAINST ECOLOGICAL TOXICITY SCREENING BENCHMARKS

RP 231-2F; 0 - $0.6 \mathrm{M}(0$ - 2 FT)

CURRENT CONDITIONS

NON-RADIOACTIVE ANALYTES

\begin{tabular}{|c|c|c|c|c|c|c|}
\hline Analyte & $\begin{array}{c}\text { Maximum } \\
\text { Detected Conc. } \\
(\mathrm{mg} / \mathrm{kg})\end{array}$ & $\begin{array}{c}\text { Feeding Rate } \\
\text { (kg/day) }\end{array}$ & $\begin{array}{c}\text { Body Weight } \\
\text { (kg) }\end{array}$ & $\begin{array}{c}\text { Soil Screening } \\
\text { Intake } \\
\text { (mig/kg/day) }\end{array}$ & $\begin{array}{c}\text { Toxicity Screening } \\
\text { Benchmark } \\
\text { (mg/kg/day) }\end{array}$ & $\begin{array}{l}\text { Exceeds } \\
\text { Sereening } \\
\text { Value }(\mathbf{Y} / \mathbf{N})\end{array}$ \\
\hline
\end{tabular}

\section{PCBs}

PCB 1254

PCB 1260

$2.87 \mathrm{E}+00$

9.01E-02

2.55E-02

2.55E-02

3.00E-01

3.00E-01

2.44E-01

7.67E-03

$2.50 \mathrm{E}-01$

$1.00 \mathrm{E}+00$

$\mathbf{N}$

Metals

Chromium

$\begin{array}{lll}1.82 \mathrm{E}+02 & 3.31 \mathrm{E}-03 & 2.50 \mathrm{E}-02 \\ 2.43 \mathrm{E}+01 & 3.31 \mathrm{E}-03 & 2.50 \mathrm{E}-02 \\ 1.00 \mathrm{E}+02 & 2.55 \mathrm{E}-02 & 3.00 \mathrm{E}-01 \\ 2.03 \mathrm{E}+02 & 2.55 \mathrm{E}-02 & 3.00 \mathrm{E}-01 \\ 8.65 \mathrm{E}-01 & 3.31 \mathrm{E}-03 & 2.50 \mathrm{E}-02 \\ 7.71 \mathrm{E}+01 & 2.55 \mathrm{E}-02 & 3.00 \mathrm{E}-01\end{array}$

2.41E+01

$3.22 \mathrm{E}+00$

8.51E+00

Copper

Manganese

Mercury

Nickel

3.31E-03

1.73E+01

$1.15 \mathrm{E}-01$

$6.56 \mathrm{E}+00$

$2.28 \mathrm{E}+00$
$8.50 \mathrm{E}+00$
$5.60 \mathrm{E}-01$
$9.30 \mathrm{E}+01$
$2.20 \mathrm{E}-02$
$1.00 \mathrm{E}+01$

$\mathbf{Y}$

$\mathrm{N}$

$\mathbf{Y}$

$\mathbf{N}$

1 Body weight and feeding rate are based on the species used in the toxicity study from which the toxicity screening benchmark for this chemical was derived (see Table 4.5).

2 Soil screening intake $=$ (maximum concentration*feeding rate)/body weight

3 Toxicity screening benchmarks are calculated from toxicity values with appropriate EPA,

Region VIII uncertainty factors to derive a NOAEL equivalent value (see Table 4.5). If a benchmark was not available, the chemical was retained as a COPC. 
SOLL SCREENING AGAINST ECOLOGICAL TOXICITY SCREENING BENCHMARKS

RP 231-2F; 0 - 0.6 M (0 - 2 FT)

CURRENT CONDITIONS

RADIONUCLIDES

\begin{tabular}{|c|c|c|c|c|c|}
\hline Analyte & $\begin{array}{c}\text { Maximum Detected } \\
\text { Concentration } \\
(\mathrm{pC} / \mathrm{g})\end{array}$ & $\begin{array}{c}\text { Estimated Dose - Deer } \\
1 \text { (rad/day) } \\
\end{array}$ & $\begin{array}{l}\text { Estimated Dose - } \\
\text { Mouse' (rad/day) }\end{array}$ & $\begin{array}{c}\text { Soil Screening Value } \\
\text { (rad/day) }\end{array}$ & $\begin{array}{c}\text { Exceeds Screening } \\
\text { Value }(\mathbf{Y} / \mathbf{N})^{2} \\
\end{array}$ \\
\hline Cesium-137 & $1.42 E+00$ & 2.86E-09 & 5.72E-09 & $1.00 \mathrm{E}-01$ & $\mathbf{N}$ \\
\hline Plutonium-239 & $6.60 \mathrm{E}-01$ & 5.12E-10 & $1.02 \mathrm{E}-09$ & $1.00 \mathrm{E}-01$ & $\mathbf{N}$ \\
\hline Potassium-40 & $3.78 \mathrm{E}+00$ & 8.84E-06 & 1.77E-05 & $1.00 \mathrm{E}-01$ & $\mathbf{N}$ \\
\hline Strontium-90 & $6.00 \mathrm{E}-01$ & $1.14 \mathrm{E}-09$ & $2.28 \mathrm{E}-09$ & $1.00 \mathrm{E}-01$ & $\mathbf{N}$ \\
\hline Technetium-99 & $4.68 \mathrm{E}+00$ & 6.95E-06 & $1.39 \mathrm{E}-05$ & $1.00 \mathrm{E}-01$ & $\mathbf{N}$ \\
\hline Tritium & $6.20 \mathrm{E}+00$ & NA & NA & $1.00 \mathrm{E}-01$ & $\mathbf{Y}$ \\
\hline Uranium-234 & $2.59 E+00$ & 2.84E-09 & 5.67E-09 & $1.00 \mathrm{E}-01$ & $\mathbf{N}$ \\
\hline Uranium-235 & $1.40 E-01$ & 2.69E-07 & 5.37E-07 & $1.00 \mathrm{E}-01$ & $\mathbf{N}$ \\
\hline Uranium-238 & $2.00 \mathrm{E}+00$ & $5.64 \mathrm{E}-10$ & 1.13E-09 & $1.00 \mathrm{E}-01$ & $\mathbf{N}$ \\
\hline
\end{tabular}

NA = Non gamma-emitting radionuclide.

1 Estimated dose calculated using method described in Section 4.5.1.2.

2 If criterion was not available, the chemical was retained as a COPC. 
TABLE 4.12

SOIL SCREENING AGAINST ECOLOGICAL TOXICITY SCREENING BENCHMARKS

RP 231-2F; 0 - $1.2 \mathrm{M}(0-4 \mathrm{FT})$

FUTURE CONDITIONS

NON-RADIOACTIVE ANALYTES

\begin{tabular}{|c|c|c|c|c|c|c|}
\hline Analyte & $\begin{array}{c}\text { Maximum } \\
\text { Detected Conc. } \\
(\mathrm{mg} / \mathrm{kg})\end{array}$ & $\begin{array}{c}\text { Feeding Rate } \\
\text { (kg/day) }\end{array}$ & $\begin{array}{c}\text { Body Weight }{ }^{1} \\
\text { (kg) }\end{array}$ & $\begin{array}{c}\text { Soil Screening } \\
\text { Intake }{ }^{2} \\
\text { (mg/kg/day) }\end{array}$ & $\begin{array}{c}\text { Toxicity Sereening } \\
\text { Benchmark }{ }^{3} \\
\text { (mg/kg/day) }\end{array}$ & $\begin{array}{c}\text { Exceeds } \\
\text { Screening Value } \\
(\mathbf{Y} / \mathbf{N}) \\
\end{array}$ \\
\hline
\end{tabular}

\section{Volatiles}

Acetone

Chiloroform

Dichloroethane, 1,2-

Dichloromethane

Toluene

$\frac{f}{6}:$

Trichloroethane, 1,1,2-

Semivolatiles

Benzo(a)anthracene

Benzo(a)pyrene

Benzo(b)flouranthene

Benzo(k)flouranthene

Bis(2-ethylhexyl)phthalate

Chrysene

Di-n-butyl phthalate

Di-n-octyl phthalate

Fluoranthene

Phenanthrene

Pyrene

\section{Dioxins/furans}

Heptachlorodibenzo-p-dioxin isomers

Octachlorodibenzo-p-dioxin isomers

$4.75 \mathrm{E}-02$
$2.65 \mathrm{E}-03$
$1.03 \mathrm{E}-02$
$6.22 \mathrm{E}-03$
$1.19 \mathrm{E}-02$
$1.36 \mathrm{E}-02$

2.55E-02

2.55E-02

2.55E-02

2.55E-02

2.55E-02

3.31E-03

$6.90 \mathrm{E}-02$

6.01E-02

8.14E-02

$6.50 \mathrm{E}-02$

2.77E-01

$6.92 \mathrm{E}-02$

7.07E-02

1.16E-01

$1.66 \mathrm{E}-01$

8.68E-02

$1.14 \mathrm{E}-01$

2.55E-02

3.31E-03

3.31E-03

3.31E-03

3.31E-03

3.31E-03

2.55E-02

3.31E-03

2.55E-02

3.31E-03

2.55E-02

6.00E-04

4.00E-04

2.55E-02

3.31E-03

$3.00 \mathrm{E}-01$
$3.00 \mathrm{E}-01$
$3.00 \mathrm{E}-01$
$3.00 \mathrm{E}-01$
$3.00 \mathrm{E}-01$
$2.50 \mathrm{E}-02$

4.04E-03

2.26E-04

8.77E-04

5.29E-04

1.01E-03

$1.80 \mathrm{E}-03$

3.00E-01

2.50E-02

2.50E-02

2.50E-02

2.50E-02

2.50E-02

3.00E-01

2.50E-02

$3.00 \mathrm{E}-01$

2.50E-02

3.00E-01
$3.00 \mathrm{E}-01$

$2.50 \mathrm{E}-02$
$5.87 \mathrm{E}-03$
$7.96 \mathrm{E}-03$
$1.08 \mathrm{E}-02$
$8.61 \mathrm{E}-03$
$3.67 \mathrm{E}-02$
$9.17 \mathrm{E}-03$
$6.02 \mathrm{E}-03$
$1.54 \mathrm{E}-02$
$1.41 \mathrm{E}-02$
$1.15 \mathrm{E}-02$
$9.70 \mathrm{E}-03$

5.11E-05

5.30E-05

$\begin{array}{ll}2.00 \mathrm{E}+01 & \mathrm{~N} \\ 1.80 \mathrm{E}+01 & \mathrm{~N} \\ 9.50 \mathrm{E}+00 & \mathrm{~N} \\ 4.80 \mathrm{E}+01 & \mathrm{~N} \\ 1.78 \mathrm{E}+01 & \mathrm{~N} \\ 1.76 \mathrm{E}+00 & \mathrm{~N}\end{array}$

$1.50 \mathrm{E}+01$

2.40E+00

2.40E-01

2.40E-01

$5.20 \mathrm{E}+00$

1.76E-01

6.25E+01

8.67E+01

$1.50 \mathrm{E}+01$

4.67E+00

$5.00 E+00$

4.00E-03

$4.00 E+02$
$\mathbf{N}$
$\mathbf{N}$
$\mathbf{N}$
$\mathbf{N}$
$\mathbf{N}$
$\mathbf{N}$

$\mathbf{N}$

$\mathbf{N}$

N

$\mathbf{N}$

N

N

N

N

N

N

$\mathbf{N}$

N

N 
SOIL SCREENING AGAINST ECOLOGICAL TOXICITY SCREENING BENCHMARKS

RP 231-2F; 0 - $1.2 \mathrm{M}(0$ - 4 FT)

FUTURE CONDITIONS

NON-RADIOACTIVE ANALYTES

\begin{tabular}{|c|c|c|c|c|c|c|}
\hline Analyte & $\begin{array}{c}\text { Maximum } \\
\text { Detected Conc. } \\
(\mathrm{mg} / \mathrm{kg})\end{array}$ & $\begin{array}{c}\text { Feeding Rate } \\
\text { (kg/day) }\end{array}$ & $\begin{array}{c}\text { Body Weight }{ }^{2} \\
\text { (kg) }\end{array}$ & $\begin{array}{l}\text { Soil Screening } \\
\text { Intake } \\
\text { (mg/kg/day) }\end{array}$ & $\begin{array}{c}\text { Toxicity Screening } \\
\text { Benchmark. } \\
\text { (mg/kg/day) }\end{array}$ & $\begin{array}{c}\text { Excceds } \\
\text { Sereening Value } \\
\text { (Y/N) }\end{array}$ \\
\hline
\end{tabular}

\section{PCBs \\ PCB 1254 \\ PCB 1260}

\section{Metals}

Chromium

Copper

Lead

Manganese

Mercury

Nickel

$\begin{array}{ll}2.87 \mathrm{E}+00 & 2.55 \mathrm{E}-02 \\ 9.01 \mathrm{E}-02 & 2.55 \mathrm{E}-02 \\ & \\ 1.82 \mathrm{E}+02 & 3.31 \mathrm{E}-03 \\ 2.43 \mathrm{E}+01 & 3.31 \mathrm{E}-03 \\ 1.00 \mathrm{E}+02 & 2.55 \mathrm{E}-02 \\ 2.03 \mathrm{E}+02 & 2.55 \mathrm{E}-02 \\ 3.84 \mathrm{E}-01 & 3.31 \mathrm{E}-03 \\ 7.71 \mathrm{E}+01 & 2.55 \mathrm{E}-02\end{array}$

$\begin{array}{ll}3.00 \mathrm{E}-01 & 2.44 \mathrm{E}-01 \\ 3.00 \mathrm{E}-01 & 7.67 \mathrm{E}-03 \\ & \\ 2.50 \mathrm{E}-02 & 2.41 \mathrm{E}+01 \\ 2.50 \mathrm{E}-02 & 3.22 \mathrm{E}+00 \\ 3.00 \mathrm{E}-01 & 8.51 \mathrm{E}+00 \\ 3.00 \mathrm{E}-01 & 1.73 \mathrm{E}+01 \\ 2.50 \mathrm{E}-02 & 5.09 \mathrm{E}-02 \\ 3.00 \mathrm{E}-01 & 6.56 \mathrm{E}+00\end{array}$

1 Body weight and feeding rate are based on the species used in the toxicity study from which the toxicity screening benchmark for this chemical was derived (see Table 4.5).

2 Soil screening intake $=$ (maximum concentration*feeding rate)/body weight

${ }^{3}$ Toxicity screening benchmarks are calculated from toxicity values with appropriate EPA

Region VII uncertainty factors to derive a NOAEL equivalent value (see Table 4.5). If a benchmark was not available, the chemical was retained as a COPC. 
SOIL SCREENING AGAINST ECOLOGICAL TOXICITY SCREENING BENCHMARKS

RP 231-2F; 0 - $1.2 \mathrm{M}(0-4$ FT)

FUTURE CONDITIONS

RADIONUCLIDES

\begin{tabular}{|c|c|c|c|c|c|}
\hline Analyte & $\begin{array}{c}\text { Maximum Detected } \\
\text { Concentration } \\
(\mathrm{pCl} / \mathrm{g})\end{array}$ & $\begin{array}{l}\text { Estimated Dose - } \\
\text { Deer' (rad/day) } \\
\end{array}$ & $\begin{array}{l}\text { Estimated Dose - } \\
\text { Mouse (rad/day) }\end{array}$ & $\begin{array}{c}\text { Soil Screening Value } \\
\text { (rad/day) }\end{array}$ & $\begin{array}{c}\text { Exceeds Screening } \\
\text { Value }\left(\mathbf{X} / \mathbf{N}^{2}\right.\end{array}$ \\
\hline Cesium-137 & $1.42 E+00$ & $2.86 \mathrm{E}-09$ & $5.72 E-09$ & $1.00 \mathrm{E}-01$ & $\mathbf{N}$ \\
\hline Plutonium-239 & $3.80 E+00$ & 2.95E-09 & $5.89 \mathrm{E}-09$ & $1.00 E-01$ & $\mathbf{N}$ \\
\hline Potassium-40 & $3.78 \mathrm{E}+00$ & $8.84 E-06$ & $1.77 \mathrm{E}-05$ & $1.00 \mathrm{E}-01$ & $\mathbf{N}$ \\
\hline Strontium-90 & $2.90 E+00$ & 5.51E-09 & $1.10 \mathrm{E}-08$ & $1.00 \mathrm{E}-01$ & $\mathbf{N}$ \\
\hline Tochnethum-99 & $4.68 \mathrm{E}+00$ & 6.95E-06 & $1.39 \mathrm{E}-05$ & $1.00 \mathrm{E}-01$ & $\mathbf{N}$ \\
\hline Tritium & $6.30 \mathrm{E}+00$ & NA & NA & $1.00 \mathrm{E}-01$ & $\mathbf{Y}$ \\
\hline Uranium-234 & $2.59 E+00$ & 2.84E-09 & 5.67E-09 & $1.00 \mathrm{E}-01$ & $\mathbf{N}$ \\
\hline Uranium-235 & $1.60 \mathrm{E}-01$ & $3.07 \mathrm{E}-07$ & $6.14 \mathrm{E}-07$ & $1.00 \mathrm{E}-01$ & $\mathbf{N}$ \\
\hline Uranium-238 & $2.00 \mathrm{E}+00$ & $5.64 \mathrm{E}-10$ & 1.13E-09 & $1.00 \mathrm{E}-01$ & $\mathbf{N}$ \\
\hline
\end{tabular}

NA = Non gamma-emitting radionuclide.

1 Estimated dose calculated using method described in Section 4.5.1.2.

2 If criterion was not available, the chemical was retained as a COPC. 
SURFACE WATER SCREENING AGAINST ECOLOGICAL TOXICITY SCREENING BENCHMARKS

BRP 231-F/1F and RP 231-2F - WETLAND

CURRENT AND FUTURE CONDITIONS

NON-RADIOACTIVE ANALYTES

\begin{tabular}{|c|c|c|c|c|c|c|c|c|c|}
\hline Analyte & - & CAS Na. & $\begin{array}{c}\text { Frequency of } \\
\text { Detection }\end{array}$ & Units & $\begin{array}{c}\text { Maximum } \\
\text { Detected } \\
\text { Concentration }\end{array}$ & $\begin{array}{l}\text { 95\% UCL of } \\
\text { Log- } \\
\text { Transformed } \\
\text { Data }\end{array}$ & $\begin{array}{c}\text { Reasonable } \\
\text { Maximum } \\
\text { Exposure } \\
\text { Concentration }\end{array}$ & $\begin{array}{l}\text { EPA Region IV } \\
\text { Freshwater } \\
\text { Screening Value }\end{array}$ & \begin{tabular}{|c|} 
Maximum \\
Exceeds \\
Screening Value \\
$\left(\mathbf{Y} / \mathbf{N}^{2}\right.$
\end{tabular} \\
\hline
\end{tabular}

Non-radloactive

Volatiles

Acetone

Dichloromethane (methylene chloride)

$6 / 9$ ugR 3.05E-02

2.26E-02

$2.26 \mathrm{E}-02$

NA

$1.12 E-02$

$1.00 \mathrm{E}-02$

1.93E+03

$\mathbf{Y}$

Semivolatiles

Bis(2-ethylhexyl)phthalate

Di-n-butyl phthalate

$\begin{array}{rrrrrr}117-81-7 & 5 & / & 5 & \text { ug/L } & 3.45 E-01 \\ 84-74-2 & 4 & / & 5 & \text { ug/L } & 1.74 E-03 \\ & & & & & \\ 7439-92-1 & 4 & / & 5 & \text { ug/L } & 7.90 \mathrm{E}-03\end{array}$

$6.32 \mathrm{E}+$

4.97E-03

3.45E-01

$<3.00 \mathrm{E}-01$

$9.40 \mathrm{E}+00$

$\mathbf{Y}$

\section{Metals}

Lead

Manganese

7439-96-5 $5 / 5$ ug/ $2.13 E-01$

$2.10 \mathrm{E}-02 \quad 7.90 \mathrm{E}-03$

$1.32 E+00$ (a)

NA

$\mathbf{N}$

$\mathrm{NA}=$ not available.

(1) The lesser of the $95 \%$ UCL of the transformed data and the maximum was used as the reasonable maximum exposure concentration

(2) If criterion was unavailable, chemical was retained as a cliemical of potential concern. 
SURFACE WATER SCREENING AGAINST ECOLOGICAL TOXICITY SCREENING BENCHMARKS

BRP 231-F/1F and RP 231-2F - WETLAND

CURRENT AND FUTURE CONDITIONS

RADIONUCLIDES

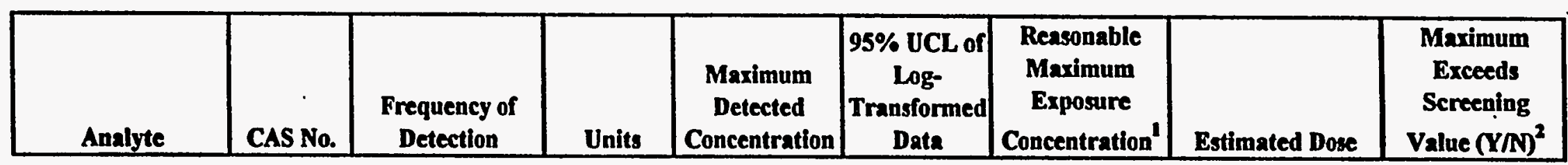

$\begin{array}{lllllllllllll}\text { Tritium } & 1333-74-0 & 3 & / & 3 & \mathrm{pCi} / \mathrm{L} & 1.04 \mathrm{E}+04 & - & 1.04 \mathrm{E}+04 & \mathrm{NA} & \mathrm{Y}\end{array}$

"-n : chemical for which $95 \%$ UCL is incalculable due to sample size below 5.

$\mathrm{NA}=$ non gamma-emitting radionuclide.

(1) The lesser of the $95 \%$ UCL of the transformed data and the maximum was used as the reasonable maximum exposure concentration

(2) If criterion was unavailable, chemical was retained as a chemical of potential concem.

$\stackrel{t}{\infty}$ 
TABLE 4.16

SEDIMENT SCREENING AGAINST TOXICITY SCREENING BENCHMARKS

BRP 231-F/1F and RP 231-2F - WETLAND

CURRENT AND FUTURE CONDITIONS

NON-RADIOACTIVE ANALYTES

\begin{tabular}{|c|c|c|c|c|c|c|c|c|}
\hline Analyte & CAS No. & $\begin{array}{c}\text { Frequency of } \\
\text { Detection }\end{array}$ & Units & $\begin{array}{c}\text { Maximum } \\
\text { Detected } \\
\text { Concentration }\end{array}$ & $\begin{array}{c}\text { 95\% UCL of } \\
\text { Log- } \\
\text { Transformed } \\
\text { Data }\end{array}$ & $\begin{array}{c}\text { Reasonable } \\
\text { Maximum } \\
\text { Exposure } \\
\text { Concentration' }\end{array}$ & \begin{tabular}{|l|} 
EPA Region IV \\
Sediment \\
Screening Value
\end{tabular} & $\begin{array}{c}\text { Maximum } \\
\text { Exceeds } \\
\text { Screening Value } \\
(\mathbf{Y} / \mathbf{N})^{2} \\
\end{array}$ \\
\hline
\end{tabular}

\section{Volatiles}

Acelone

Chloromethane (methyl chloride)

Dichloromethane (methylene chloride)

Toluene

67-64-1 5 / 5 mg/kg

74-87-3 2 / $5 \mathrm{mg} / \mathrm{kg}$

75-09-2 5 / 5 mg/kg

108-88-3 1 / $5 \mathrm{mg} / \mathrm{kg}$

$6.43 \mathrm{E}-01$

1.38E-02

4.19E-02

1.13E-02

$\begin{array}{rllll}83-32-9 & 1 & / & 5 & \mathrm{mg} / \mathrm{kg} \\ 208-96-8 & 1 & / & 5 & \mathrm{mg} / \mathrm{kg} \\ 120-12-7 & 1 & / & 5 & \mathrm{mg} / \mathrm{kg} \\ 56-55-3 & 1 & / & 5 & \mathrm{mg} / \mathrm{kg} \\ 50-32-8 & 1 & / & 5 & \mathrm{mg} / \mathrm{kg} \\ 205-99-2 & 1 & / & 5 & \mathrm{mg} / \mathrm{kg} \\ 191-24-2 & 1 & / & 5 & \mathrm{mg} / \mathrm{kg} \\ 207-08-9 & 1 & / & 5 & \mathrm{mg} / \mathrm{kg} \\ 117-81-7 & 4 & / & 5 & \mathrm{mg} / \mathrm{kg} \\ 218-01-9 & 1 & / & 5 & \mathrm{mg} / \mathrm{kg} \\ 84-74-2 & 2 & / & 5 & \mathrm{mg} / \mathrm{kg} \\ 53-70-3 & 1 & / & 5 & \mathrm{mg} / \mathrm{kg} \\ 132-64-9 & 1 & / & 5 & \mathrm{mg} / \mathrm{kg} \\ 206-44-0 & 1 & 1 & 5 & \mathrm{mg} / \mathrm{kg} \\ 86-73-7 & 1 & / & 5 & \mathrm{mg} / \mathrm{kg} \\ 193-39-5 & 1 & / & 5 & \mathrm{mg} / \mathrm{kg} \\ 85-01-8 & 1 & / & 5 & \mathrm{mg} / \mathrm{kg} \\ 129-00-0 & 1 & 1 & 5 & \mathrm{mg} / \mathrm{kg}\end{array}$

2.18E-01

7.75E-01

4.21E+00

4.31E+00

2.99E+00

4.34E+00

$1.74 \mathrm{E}+00$

$3.99 \mathrm{E}+00$

$1.68 \mathrm{E}+00$

5.33E+00

$5.96 \mathrm{E}-02$

5.62E-01

$1.33 \mathrm{E}-01$

$1.21 \mathrm{E}+01$

5.22E-01

$1.78 \mathrm{E}+00$

$5.36 \mathrm{E}+00$

7.83E+00

$1.60 E-01$
$1.30 E-02$
$5.07 E-02$
$1.09 E-02$

$3.90 E-01$
$7.49 E-01$
$2.79 E+01$
$3.01 E+01$
$1.04 E+01$
$3.08 E+01$
$2.84 E+00$
$2.36 E+01$
$1.56 E+01$
$6.11 E+01$
$3.35 E+00$
$5.34 E-01$
$5.09 E-01$
$1.42 E+03$
$5.01 E-01$
$2.98 E+00$
$6.23 E+01$
$2.43 E+02$

$1.60 \mathrm{E}-01$
$1.30 \mathrm{E}-02$
$4.19 \mathrm{E}-02$
$1.09 \mathrm{E}-02$

2.18E-01

7.49E-01

4.21E+00

4.31E+00

2.99E+00

4.34E+00

$1.74 \mathrm{E}+00$

3.99E+00

$1.68 \mathrm{E}+00$

5.33E+00

5.96E-02

5.3+E-01

1.33E-01

1.21E+01

5.01E-01

$1.78 \mathrm{E}+00$

$5.36 \mathrm{E}+00$

$7.83 \mathrm{E}+00$

$$
\begin{aligned}
& \text { NA } \\
& \text { NA } \\
& \text { NA } \\
& \text { NA }
\end{aligned}
$$

$\mathbf{Y}$
$\mathbf{Y}$
$\mathbf{Y}$
$\mathbf{Y}$

$\begin{array}{cc}6.71 E-03 & Y \\ 5.87 E-03 & Y \\ 4.69 E-02 & Y \\ 7.48 E-02 & Y \\ 8.88 E-02 & Y \\ \text { NA } & Y \\ \text { NA } & Y \\ \text { NA } & Y \\ 1.82 E-01 & Y \\ 1.08 E-01 & Y \\ \text { NA } & Y \\ 6.22 E-03 & Y \\ \text { NA } & Y \\ 1.13 E-01 & Y \\ 2.12 E-02 & Y \\ \text { NA } & Y \\ 8.67 E-02 & Y \\ 1.53 E-01 & Y\end{array}$

FINAL 
TABLE 4.16

SEDIMENT SCREENING AGAINST TOXICITY SCREENING BENCHMARKS

BRP 231-F/1F and RP 231-2F - WETLAND

CURRENT AND FUTURE CONDITIONS

NON-RADIOACTIVE ANALYTES

\begin{tabular}{|c|c|c|c|c|c|c|c|c|c|c|}
\hline $\begin{array}{l}\text { Analyte } \\
\end{array}$ & CAS No. & $\begin{array}{r}\text { Freq } \\
\text { De }\end{array}$ & uenc & on of & Units & $\begin{array}{c}\text { Marimum } \\
\text { Detected } \\
\text { Concentration }\end{array}$ & $\begin{array}{c}\text { 95\% UCL of } \\
\text { Log- } \\
\text { Transformed } \\
\text { Data } \\
\end{array}$ & \begin{tabular}{|c|} 
Reasonable \\
Maximum \\
Exposure \\
Concentration \\
\end{tabular} & \begin{tabular}{|c|} 
EPA Region IV \\
Sediment \\
Screening Value
\end{tabular} & $\begin{array}{c}\text { Maximum } \\
\text { Exceeds } \\
\text { Screening Value } \\
\left(\mathbf{Y} / \mathbf{N}^{2}\right.\end{array}$ \\
\hline $\begin{array}{l}\text { Dioxinsfiurans } \\
\text { Octachlorodibenzo-p-dioxin isomers }\end{array}$ & $268-87-9$ & 4 & I & 5 & mg/kg & $8.00 \mathrm{E}-01$ & $1.81 E+00$ & $8.00 \mathrm{E}-01$ & NA & $\mathbf{Y}$ \\
\hline $\begin{array}{l}\text { Metals } \\
\text { Arsenic } \\
\text { Chromium } \\
\text { Copper } \\
\text { Lead } \\
\text { Manganese } \\
\text { Mercury }\end{array}$ & $\begin{array}{l}440-38-2 \\
440-47-3 \\
440-50-8 \\
439-92-1 \\
439-96-5 \\
439-97-6\end{array}$ & $\begin{array}{l}2 \\
5 \\
4 \\
5 \\
5 \\
5\end{array}$ & $\begin{array}{l}1 \\
1 \\
1 \\
1 \\
1 \\
1\end{array}$ & $\begin{array}{l}5 \\
5 \\
5 \\
5 \\
5 \\
5\end{array}$ & $\begin{array}{l}\mathrm{mg} / \mathrm{kg} \\
\mathrm{mg} / \mathrm{kg} \\
\mathrm{mg} / \mathrm{kg} \\
\mathrm{mg} / \mathrm{kg} \\
\mathrm{mg} / \mathrm{kg} \\
\mathrm{mg} / \mathrm{kg}\end{array}$ & $\begin{array}{l}2.90 \mathrm{E}+00 \\
2.80 \mathrm{E}+01 \\
2.56 \mathrm{E}+01 \\
1.90 \mathrm{E}+01 \\
5.75 \mathrm{E}+01 \\
7.10 \mathrm{E}-01\end{array}$ & $\begin{array}{l}6.05 E+00 \\
1.44 E+02 \\
2.28 E+02 \\
1.86 E+01 \\
1.68 E+02 \\
8.01 E-01\end{array}$ & $\begin{array}{l}2.90 E+00 \\
2.80 E+01 \\
2.56 E+01 \\
1.86 E+01 \\
5.75 E+01 \\
7.10 E-01\end{array}$ & $\begin{array}{c}7.24 E+00 \\
5.23 E+01 \\
1.87 E+01 \\
3.02 E+01 \\
\text { NA } \\
1.30 E-01\end{array}$ & $\begin{array}{l}\mathbf{N} \\
\mathbf{N} \\
\mathbf{Y} \\
\mathbf{N} \\
\mathbf{Y} \\
\mathbf{Y}\end{array}$ \\
\hline
\end{tabular}


SEDIMENT SCREENING AGAINST TOXICITY SCREENING BENCHMARKS

BRP 231-F/1F and RP 231-2F - WETLAND

CURRENT AND FUTURE CONDITIONS

RADIONUCLIDES

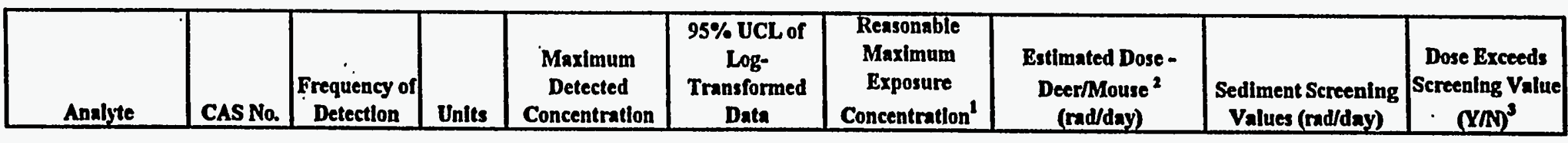

$\begin{array}{lrlllllll}\text { Cesium-137 } & \text { CS-137 } & 3 & 1 & 3 & \mathrm{pCi} / \mathrm{g} & 3.10 \mathrm{E}+00 & - & 3.10 \mathrm{E}+00 \\ \text { Plutonium-239 } & \mathrm{PU}-239 & 1 & 1 & 3 & \mathrm{pCi} / \mathrm{g} & 5.70 \mathrm{E}-01 & - & 5.70 \mathrm{E}-01 \\ \text { Potassium-40 } & \mathrm{K}-40 & 3 & 1 & 3 & \mathrm{pCi} / \mathrm{g} & 2.24 \mathrm{E}+00 & - & 2.24 \mathrm{E}+00 \\ \text { Strontium-90 } & 0098-97-2 & 3 & 1 & 3 & \mathrm{pCi} / \mathrm{g} & 1.70 \mathrm{E}+00 & - & 1.70 \mathrm{E}+00 \\ \text { Technetium-99 } & 4133-76-7 & 1 & 1 & 3 & \mathrm{pCi} / \mathrm{g} & 3.43 \mathrm{E}+00 & - & 3.43 \mathrm{E}+00 \\ \text { Tritium } & 1333-74-0 & 3 & 1 & 3 & \mathrm{pCi} / \mathrm{g} & 1.04 \mathrm{E}+01 & - & 1.04 \mathrm{E}+01 \\ \text { Uranium-234 } & \mathrm{U}-234 & 3 & 1 & 3 & \mathrm{pCi} / \mathrm{g} & 1.50 \mathrm{E}+00 & - & 1.50 \mathrm{E}+00 \\ \text { Uranium-238 } & 7440-61-1 & 3 & 1 & 3 & \mathrm{pCi} / \mathrm{g} & 1.60 \mathrm{E}+00 & - & 1.60 \mathrm{E}+00\end{array}$

$\begin{array}{cc}6.24 \mathrm{E}-09 / 1.25 \mathrm{E}-08 & 1.00 \mathrm{E}-01 \\ 4.42 \mathrm{E}-10 / 8.84 \mathrm{E}-10 & 1.00 \mathrm{E}-01 \\ \text { NA } & 1.00 \mathrm{E}-01 \\ \text { NA } & 1.00 \mathrm{E}-01 \\ \text { NA } & 1.00 \mathrm{E}-01 \\ \text { NA } & 1.00 \mathrm{E}-01 \\ 1.64 \mathrm{E}-09 / 3.29 \mathrm{E}-09 & 1.00 \mathrm{E}-01 \\ 4.51 \mathrm{E}-10 / 9.02 \mathrm{E}-10 & 1.00 \mathrm{E}-01\end{array}$

$\mathbf{N}$
$\mathbf{N}$
$\mathbf{Y}$
$\mathbf{Y}$
$\mathbf{Y}$
$\mathbf{N}$
$\mathbf{N}$

"n : denotes chemicals for which $95 \%$ UCL is incalculable due to sample size below 5 .

$N A=$ Comparison could not be made because no criteria were available for this chemical.

(1) The lesser of the $95 \%$ UCL of the transformed data and the maximum was used as the reasonable maximum exposure concentration.

(2) Estimated dose calculated using method described in Section 4.5.1.2.

(3) If criterion was not available, the chemical was retained. 
TABLE 4.18

ECOLOGICAL COPCS IN EACH MEDIUM

BRP 231-F/1F and RP 231-2F

NON-RADIOACTIVE ANALYTES

\begin{tabular}{|c|c|c|c|c|c|c|c|}
\hline COPC & $\begin{array}{l}\text { CAS } \\
\text { Na. }\end{array}$ & $\begin{array}{c}231-\mathrm{F} \& 231-1 \mathrm{~F} \\
0-0.6 \mathrm{~m}(0-2 \mathrm{ft}) \\
\text { RME Concentration } \\
\text { (me/kg) }\end{array}$ & $\begin{array}{c}231-\mathrm{F} \& 231-1 \mathrm{~F} \\
0-1.2 \mathrm{~m}(0-4 \mathrm{~m}) \\
\mathrm{RME} \text { Concentration } \\
\text { (mo/kg) }\end{array}$ & $\begin{array}{c}231-2 \mathrm{~F} \\
0-0.6 \mathrm{~m}(0-2 \mathrm{ft}) \\
\mathrm{RMB} \text { Concentration } \\
\text { (mg/kg) }\end{array}$ & \begin{tabular}{c|}
$231-2 \mathrm{~F}$ \\
$0-1.2 \mathrm{~m}(0-4 \mathrm{fr})$ \\
RME Concentration \\
(mg/kg)
\end{tabular} & $\begin{array}{c}\text { Surface Water } \\
\text { RME Concentration } \\
\text { (mg/) }\end{array}$ & $\begin{array}{c}\text { Sediment } \\
\text { RME Concentrution } \\
\text { (melkg) }\end{array}$ \\
\hline
\end{tabular}

Volatiles

Acetone

Chloromethane (methyl chloride)

Toluene

\section{Semivolatiles}

Acenaphthene

Acenaphthylene

Anthracene

Benzo(a)anthracene

Benzo(a)pyrene

Benzo(b)fluoranthene

Benzo(ghi)perylene

Benzo(k)fluoranthene

Bis(2-ethythexyl)phthalate

Chrysene

Di-n-butyl phthalate

Dibenz(a,h)anthracene

Dibenzofuran

Fluoranthene

Fluorene

Indeno(1,2,3-cd)pyrene

Phenanthrene

Pyrene

67-64-1

108-88-3

. 83-32-9

208-96-8

120-12-7

56-55-3

50-32-8

205-99-2

191-24-2

207-08-9

$117-81-7$

218-01-9

84-74-2

53-70-3

132-64-9

$206-44-0$

86-73-7

193-39-5

85-01-8

129-00-0

Dioxins/furans

Octachlorodibenzo-p-dioxin isomers

\subsection{E-02}

1.60E-01

$1.30 \mathrm{E}-02$

4.19E-02

$1.90 \mathrm{E}-02$

$1.85 \mathrm{E}-01$

1.85E-01

3.45E-01

2.18E-01

7.49E-01

$4.21 \mathrm{E}+00$

$4.31 \mathrm{E}+00$

$2.99 \mathrm{E}+00$

4.34E+00

$1.74 \mathrm{E}+00$

$3.99 \mathrm{E}+00$

$1.68 \mathrm{E}+00$

$5.33 \mathrm{E}+00$

$5.96 \mathrm{E}-02$

5.34E-01

1.33E-01

$1.21 \mathrm{E}+01$

5.01E-01

$1.78 \mathrm{E}+00$

$5.36 \mathrm{E}+00$

$7.83 \mathrm{E}+00$

8.00E-01 


\section{TABBLE 4.18}

ECOLOGICAL COPCs IN EACH MEDIUM

BRP 231-F/1F and RP 231-2F

NON-RADIOACTIVE ANALYTES

\begin{tabular}{|c|c|c|c|c|c|c|c|}
\hline COPC & $\begin{array}{l}\text { CAS } \\
\text { No. }\end{array}$ & $\begin{array}{c}231-\mathrm{F} \& 231-1 \mathrm{~F} \\
0-0.6 \mathrm{~m}(0-2 \mathrm{~m}) \\
\mathrm{RME} \text { Concentration } \\
(\mathrm{m} / \mathrm{kg})\end{array}$ & $\begin{array}{c}231-F \& 231-1 F \\
0-1.2 \mathrm{~m}(0-4 \mathrm{f}) \\
\mathrm{RME} \text { Concentration } \\
(\mathrm{mg} / \mathrm{kg})\end{array}$ & \begin{tabular}{|c|}
$231-2 F$ \\
$0-0.6$ m (0-2 ft) \\
RME Concentration \\
$(m g / k q)$
\end{tabular} & $\begin{array}{c}231-2 \mathrm{~F} \\
0-1.2 \mathrm{~m}(0-4 \mathrm{ft}) \\
\text { RME Concentration } \\
\text { (mg/kg) }\end{array}$ & $\begin{array}{c}\text { Surface Water } \\
\text { RME Concentration } \\
\text { (mg/L) }\end{array}$ & $\begin{array}{c}\text { Sediment } \\
\text { RME Concentration } \\
\text { (mg/kg) }\end{array}$ \\
\hline
\end{tabular}

\section{Metals}

Chromium

Copper

Lead

Manganese

Mercury

$\begin{array}{ll}440-39-3 & 1.88 E+01 \\ 440-47-3 & 4.42 E+01 \\ 440-50-8 & \\ 439-92-1 & 2.48 E+01 \\ 439-96-5 & 4.78 E+02 \\ 439-97-6 & 5.48 E-01\end{array}$

2.07E+01

7.65E+01

$1.82 \mathrm{E}+02$

5.51E+01

$2.62 \mathrm{E}+01$

$3.61 \mathrm{E}+02$

3.58E-01

$9.00 \mathrm{E}+01$

6.57E-02

$6.20 \mathrm{E}+01$

1.23E-01
$2.56 E+01$

2.13E-01

5.75E+01

7.10E-01 
TABLE 4.19

ECOLOGICAL COPC\& IN EACH MEDIUM

BRP 231-F/1F and RP 231-2F

RADIONUCLIDES

\begin{tabular}{|c|c|c|c|c|c|c|c|}
\hline COPC & $\begin{array}{l}\text { CAS } \\
\text { No. }\end{array}$ & $\begin{array}{c}231-\mathrm{F} \& 231-1 \mathrm{~F} \\
0-0.6 \mathrm{~m} \text { (0-2 ft) } \\
\text { RME Concentration } \\
(\mathrm{pCi} / \mathrm{g})\end{array}$ & $\begin{array}{c}\text { 231-F \& 231-1F } \\
0-1.2 \mathrm{~m}(0-1 \mathrm{ft}) \\
\text { RME Concentration } \\
\text { (pCi/g) }\end{array}$ & $\begin{array}{c}231-2 \mathrm{~F} \\
0-0.6 \mathrm{~m} \text { (0-2 ft) } \\
\text { RME Concentration } \\
\text { (nCi/g) }\end{array}$ & $\begin{array}{c}231-2 \mathrm{~F} \\
0-1.2 \mathrm{~m}(0-4 \mathrm{ft}) \\
\text { RME Concentration } \\
(\mathrm{pC} / \mathrm{g})\end{array}$ & $\begin{array}{c}\text { Surface Water } \\
\text { RME Concentration } \\
\text { (pCi/L) }\end{array}$ & $\begin{array}{c}\text { Sediment } \\
\text { RME Concentration } \\
(\mathrm{pCi} / \mathrm{g})\end{array}$ \\
\hline Potassium-40 & $K-40$ & & & & & & $2.24 E+C 0$ \\
\hline Strontium-90 & $10098-97-2$ & & & & & & $1.70 E+00$ \\
\hline Technetium-99 & $14133-76-7$ & & & & & & $3.43 \mathrm{E}+00$ \\
\hline Tritium & $1333-74-0$ & $7.69 \mathrm{E}+00$ & $6.23 E+00$ & $6.20 \mathrm{E}+00$ & $5.89 \mathrm{E}+00$ & $1.04 E+04$ & $1.04 E+01$ \\
\hline
\end{tabular}


THREATENED, ENDANGERED, AND SENSITTVE SPECIES THAT POTENTIALLY COULD OCCUR IN THE AREA OF THE F-AREA BRP

\begin{tabular}{|c|c|c|c|c|}
\hline Common Name & Scientific Name & $\begin{array}{l}\text { Federal } \\
\text { Status }\end{array}$ & $\begin{array}{l}\text { State Heritage } \\
\text { Program Status }\end{array}$ & Preferred Habitat \\
\hline $\begin{array}{l}\text { BIRDS } \\
\text { Loggorhead shrike }\end{array}$ & Lanius Iudovicianus & C2 & G4S4 & Large grass/shrub openings \\
\hline Bachman's sparrow & Aimophila aestivalis & $\mathbf{C 2}$ & G3 & $\begin{array}{l}\text { Open fire-maintained longleaf } \\
\text { pine grass-forb-shrub openings }\end{array}$ \\
\hline AMPEIIBLANS & & & . & \\
\hline Carolina gopher frog & Rana areolasa capito & $\mathbf{C 2}$ & sc & $\begin{array}{l}\text { Pine sandhills w/underground burrows, } \\
\text { reproduce in shallow wetlands }\end{array}$ \\
\hline REPTILES & & & & \\
\hline Florida pine snake & Pituophis melanoleucus mugitus & $\mathbf{C 2}$ & & Pine sandhill areas \\
\hline Northern pine snake & Pituophis melanoleucus melanoleucus & $\mathbf{C 2}$ & & Pine sandhill areas \\
\hline Southern hognose snake & Heterodon simus & $\mathbf{C 2}$ & & Pine sandhill areas \\
\hline $\begin{array}{l}\text { PLANTS } \\
\text { Oconeo azalea }\end{array}$ & Rhododendron flammeum & & SL & $\begin{array}{l}\text { Steep, north-facing bluffes with upland } \\
\text { hardwood }\end{array}$ \\
\hline Wild-indigo & Baptisia lanceolata & & $\mathbf{G 2 G 3}$ & Xeric sandhills \\
\hline Smooth purple coneflower & Echinacea laevigata & $\mathbf{E}$ & & Prairie remnants-calcareous soils \\
\hline Nestronia & Nestronia umbellula & $\mathbf{C 2}$ & $\mathbf{0 3 G 4}$ & Upland mixed pine-hardwood or bayheads \\
\hline Sandhill lily & Nolina georgiana & & G3G5 & Xeric sandhills \\
\hline American nailwort & Paromychia americana & & $\mathbf{0 3}$ & Xeric sandhills \\
\hline Milk pea(*) & Astragalus villosus & $\therefore$ & G2G3 & Sandhills \\
\hline Chapman's sedge(*) & Carex chapmanii & $\mathbf{C 2}$ & $\mathbf{G 2 G 3}$ & Sandhills \\
\hline Georgia plume(*) & Elliottia racemosa & & $\mathbf{G} 2 \mathbf{G 3}$ & Sandhill ridges \\
\hline Sarvis holly(*) & Ilex amelanchier & $:$ & G3G4 & Wooded streambanks, mostly in sandhills \\
\hline Relict trillium(*) & Trillium reliquum & $\mathbf{E}$ & $\mathbf{G 1}$ & Upland mesic hardwood \\
\hline
\end{tabular}

\section{Legend}

$\mathbf{E}=$ Endangered

C2 $=$ Category 2

$\mathrm{SC}=$ State concern-animal

RC $=$ Regional concern-plant

NCN $=$ No common name

TSA $=$ Threatened due to similarity of appearance

SL $=$ State concern-plant

G1, G2, G3, G4, G5 = Globally rare, listed in deceasing importance

S1, S2, S3, S4, S5 = Statewide rare, listed in decreasing importance

$(*)=$ species found in proximity to SRS that could occur at site 


\section{TABLE 4.21
ThATH}

PRINCIPAL EXPOSURE PATHWAYS SELECTED

FOR QUANTITATIVE EVALUATION

OF RISK TO ECOLOGICAL RECEPTORS

\begin{tabular}{|c|c|c|}
\hline \multirow[b]{2}{*}{ PATHWAY } & \multicolumn{2}{|c|}{ POTENTIAL RECEPTOR } \\
\hline & Terrestrial & Aquatic \\
\hline $\begin{array}{l}\text { Fugitive Dust } \\
\text { inhalation }\end{array}$ & & \\
\hline $\begin{array}{l}\text { Organic Vapor } \\
\text { inhalation }\end{array}$ & & \\
\hline $\begin{array}{l}\text { Surface Soil } \\
\text { ingestion } \\
\text { dermal contact } \\
\text { direct radiation }\end{array}$ & $\begin{array}{l}\mathbf{x} \\
\mathbf{x}\end{array}$ & \\
\hline $\begin{array}{l}\text { Plants } \\
\text { ingestion }\end{array}$ & $\mathbf{x}$ & \\
\hline $\begin{array}{l}\text { Animals } \\
\text { ingestion }\end{array}$ & $\mathbf{x}$ & \\
\hline $\begin{array}{l}\text { Surface Water } \\
\text { ingestion } \\
\text { dermal contact }\end{array}$ & & $\mathbf{X}$ \\
\hline $\begin{array}{l}\text { Sediment } \\
\text { ingestion } \\
\text { dermal contact } \\
\text { direct radiation }\end{array}$ & $\begin{array}{l}\mathbf{x} \\
\mathbf{x}\end{array}$ & \\
\hline
\end{tabular}

$\mathrm{X}=$ Denotes receptor for which completo pathway was quantitatively evaluated. 
TABLE 4.22

EVALUATION OF POTENTIAL ECOLOGICAL RECEPTORS AS ASSESSMENT ENDPOINTS BRP 231-F/1F and RP 231-2F

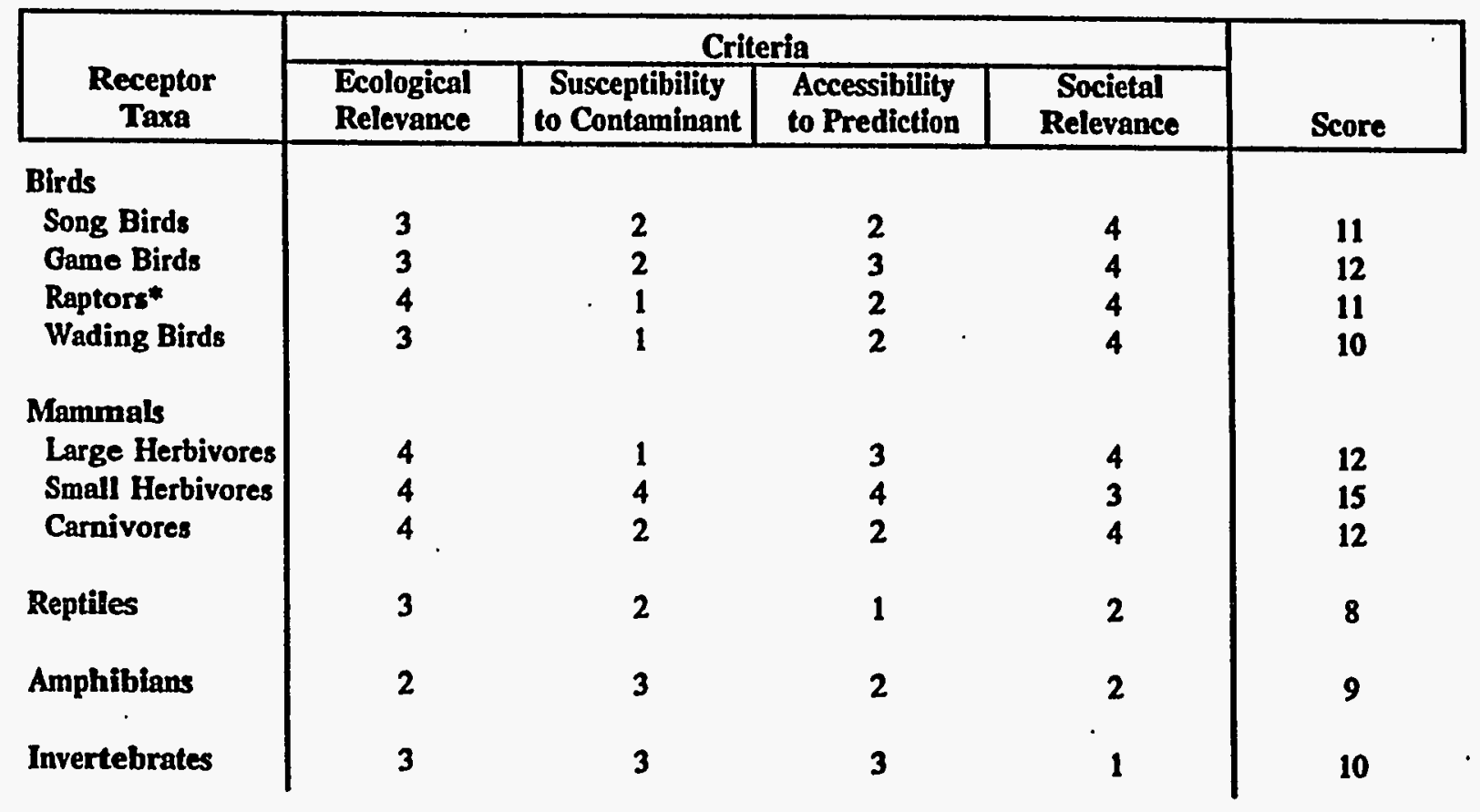

Ratings based on degrees of satisfaction of criterion by receptor:

$4=$ Excellent

$3=$ Good

2 = Fair

$1=$ Poor

Professional judgement was used in rating each receptor group with regard to how well it satisfied each criterion for selecting assessment endpoints. Section 4.3 describes the process of criteria evaluation as it pertains to endpoint selection including examples of how professional judgement was used in evaluation of potential receptor endpoints.

Ratings were totaled to derive a score for each receptor group. A suilablo representativo of the group with the highest score was selected as the assessment endpoint species. An equivalent operational definition could be stated for any of the potential receptors: therefore, they were not rated by this criterion.

* No direct evidence of utilization of the unit 


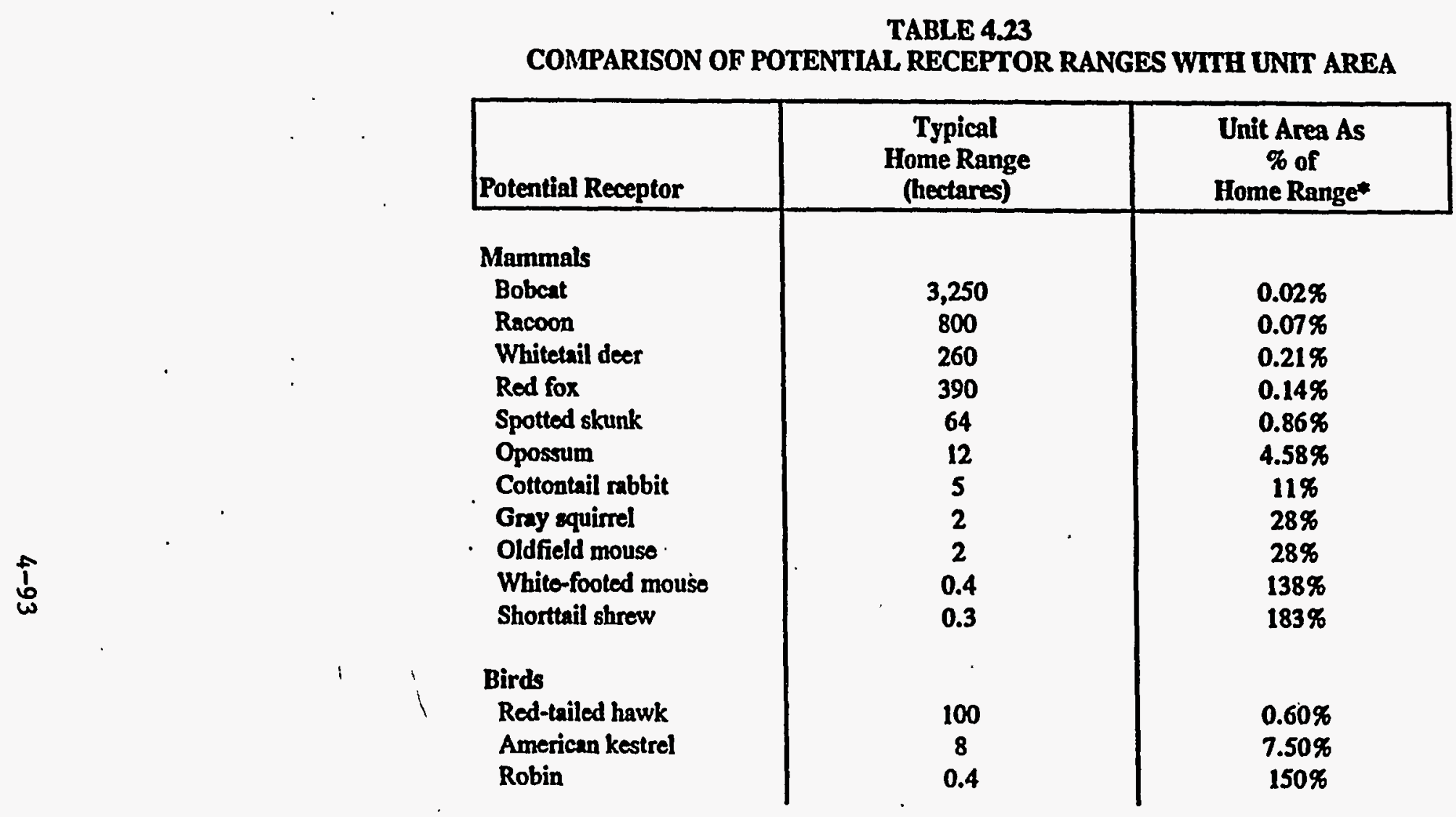

- Are of unit is approximately 0.55 hectares. Calculation assumes maximal overlap of unit and home ranges. 
TABLE 4.24

OLDFIELD MOUSE INTAKE RATES

\begin{tabular}{|c|c|c|c|c|c|c|c|}
\hline Body & \multirow{2}{*}{$\begin{array}{c}\text { Trophic } \\
\text { Wevel }\end{array}$} & \multicolumn{3}{|c|}{ Site Foraging Factor } & \multicolumn{2}{c|}{ Dietary Breakdown } \\
\cline { 3 - 7 } & BRP 231-F \& 231-1F & RP 231-2F & Wetland & Plant (kg/day) & Animal (kg/dav) & $\begin{array}{c}\text { Soil/Sediment } \\
(\mathrm{kg} / \mathrm{dav})\end{array}$ \\
\hline 0.012 & 3 & 0.73 & 0.09 & 0.18 & 0.000882 & 0.000882 & 0.000036
\end{tabular}

(1) Trophic level: organisms are assigned to trophic levels of 1 (producer), 2 (herbivore), 3 (1st order carnivore), and 4 (top carnivore) within the food web.

(2) Site foraging factor: adjustment factor (from 0 to l) based upon an organism's total time of exposure to site-based contaminants. Organisms associated solely with a contaminated site are assigned a factor of 1 , while organisms exhibiting mobility to areas outside the site are assigned a value less than 1 . In addition, aadjustment factors for subareas within the site can be used. In this assessment, the relative proportion of the size of each site (BRP 231-F \& 231-1F, RP 231-2F, and wetland) to the whole site is used as a SFF for the exposure to contaminants at that site. 
TABLE 4.25

CALCULATED SOIL RECEPTOR EXPOSURE BRP 231-F/1F; 0 - $0.6 M(0-2$ FT)

CURRENT CONDITIONS

\begin{tabular}{|c|c|c|c|c|c|}
\hline & CAS No. & $\begin{array}{c}\text { RME Concentration } \\
(\mathrm{m} / \mathrm{kg})\end{array}$ & $\mathrm{SP}^{1}$ & $\mathrm{BAF}^{2}$ & $\begin{array}{c}\text { Oldfield Mouse } \\
\text { Exposure } \\
\text { (mg/kg/day) }^{3}\end{array}$ \\
\hline Non-Radioactive
\end{tabular}

\section{Semivolatiles}

Dibenzofuran

\section{Metals}

Barium

Chromium

Lead

i

Manganese

Mercury
(1) SP: soil-10-plant uptake frictor.

(2) BAF: bionccumulation factor.

(3) Oldfiedd mouse exposure calculated as

$E D=\left[\left(C_{s} \cdot S P \bullet I^{\bullet}\right)+\left(C_{s} \bullet^{\bullet} B A F \cdot I_{a}\right)+\left(C_{3}{ }^{\bullet} I_{s}\right)\right] \bullet S F F / B W$

Where, ED - exposure dose

$\mathrm{Cs}=\mathrm{RME}$ conc in soil (mg/kg)

$S P=$ soiltro-plame upplake factor

Ip = plant-matter intake rate (kg/day, see Table 4.24)

BAf $=$ bioscrumulation factor (unitless)

la = animal-matter intake rate (kg/day, seec Table 4.24)

Is = incidental soil intake rate (kg/day, see Table 4.24)

SFF - site foraging frector (unitless, see Table 4.24)

$\mathrm{BW}=$ boty wreight (Kg, sce Table 4.24)

$\begin{array}{cccccc}1.85 \mathrm{E}-01 & 1.72 \mathrm{E}-01 & 5 & 1.00 \mathrm{E}+00 & 6 & 1.20 \mathrm{E}-02 \\ & & & & & \\ 1.88 \mathrm{E}+01 & 1.50 \mathrm{E}-01 & 4 & 1.00 \mathrm{E}+00 & 6 & 1.20 \mathrm{E}+00 \\ 4.42 \mathrm{E}+01 & 7.50 \mathrm{E}-03 & 4 & 1.00 \mathrm{E}+00 & 6 & 2.49 \mathrm{E}+00 \\ 2.48 \mathrm{E}+01 & 4.50 \mathrm{E}-02 & 4 & 2.69 \mathrm{E}-01 & 7 & 4.72 \mathrm{E}-01 \\ 4.78 \mathrm{E}+02 & 2.50 \mathrm{E}-01 & 4 & 1.00 \mathrm{E}+00 & 6 & 3.31 \mathrm{E}+01 \\ 5.48 \mathrm{E}-01 & 9.00 \mathrm{E}-01 & 4 & 2.30 \mathrm{E}+01 & 8 & 7.04 \mathrm{E}-01\end{array}$

(4) Source: Becs et al, 1984.

(5) Source: Travis and Ams, 1988.

(6) Defaut where no experimemtal data available, no evidence of bioconcentration.

(7) Source: Ma, 1983.

(8) Source: USEPA, 1994. 
TABLE 4.26

CALCULATED SOIL RECEPTOR EXPOSURE

BRP 231-F/1F; 0 - 1.2 M (0 - 4 FT)

FUTURE CONDITIONS

\begin{tabular}{|c|c|c|c|c|c|}
\hline Chemical & CAS No. & $\begin{array}{l}\text { RME Concentration } \\
(\mathrm{mg} / \mathrm{kg})\end{array}$ & $\mathbf{S P}^{1}$ & $\mathbf{B A} \mathbf{F}^{2}$ & $\begin{array}{l}\text { Oldfield Mouse } \\
\text { Exposure } \\
\text { (me/ke/day) }\end{array}$ \\
\hline
\end{tabular}

\section{Semivolatiles}

Dibenzofuran

$\begin{array}{ll}132-64-9 & 1.85 \mathrm{E}-01 \\ & \\ 440-39-3 & 2.07 \mathrm{E}+01 \\ 440-47-3 & 7.65 \mathrm{E}+01 \\ 439-92-1 & 2.62 \mathrm{E}+01 \\ 439-96-5 & 3.61 \mathrm{E}+02 \\ 439-97-6 & 3.58 \mathrm{E}-01\end{array}$

Metals
Barium
Chromium
Lead
Manganese
Mercury

(l) SP: soilto-plent uptake factor.

(2) BAF: biosocumulation factor.

(3) Oldfietd mouse exposure calculated es

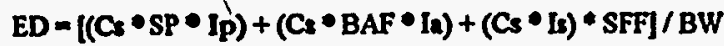

Where, ED = exposure dose

$\mathrm{Cs}=\mathrm{RME}$ conc in soil (mg/kg)

$$
\text { SP = soil-to-plaint uptake factor }
$$

Ip = plant-matter imake rate (kg/day, see Table 4.24)

BAf - biorocumulation factor (unilless)

la = animal-mater intake rate (kg/day, see Table 4.24)

Is = incidental soil intake rate (kg/day, see Table 4.24)

SFF $=$ site foraging factor (unitless, $2 e 0$ Table 4.24)

BW - body weight (Kg, see Table 4.24)

$\begin{array}{lllll}1.72 \mathrm{E}-01 & 3 & 1.00 \mathrm{E}+00 & 6 & 1.20 \mathrm{E}-02 \\ & & & & \\ 1.50 \mathrm{E}-01 & 4 & 1.00 \mathrm{E}+00 & 6 & 1.32 \mathrm{E}+00 \\ 7.50 \mathrm{E}-03 & 4 & 1.00 \mathrm{E}+00 & 6 & 4.30 \mathrm{E}+00 \\ 4.50 \mathrm{E}-02 & 4 & 1.72 \mathrm{E}-01 & 7 & 3.62 \mathrm{E}-01 \\ 2.50 \mathrm{E}-01 & 4 & 1.00 \mathrm{E}+00 & 6 & 2.50 \mathrm{E}+01 \\ 9.00 \mathrm{E}-01 & 4 & 2.30 \mathrm{E}+01 & 8 & 4.60 \mathrm{E}-01\end{array}$

(4) Source: Baces et al, 1984.

(3) Source: Travis and Arms, 1988.

(6) Defruit where no experimertal data available, no evidence of bioconcentration.

(7) Source: Ma, 1983.

(8) Source: USEPA, 1994. 


\section{CALCULATED SOIL RECEPTOR EXPOSURE \\ RP 231-2F; 0 - $0.6 \mathrm{M}(0$ - 2 FT) \\ CURRENT CONDITIONS}

\begin{tabular}{|c|c|c|c|c|c|}
\hline Chemical & CAS No. & $\begin{array}{c}\text { RME Concentration } \\
(\mathrm{mg} / \mathrm{kg})\end{array}$ & $\mathrm{SP}^{1}$ & BAF $^{2}$ & $\begin{array}{c}\text { Oldfield Mouse } \\
\text { Exposure } \\
(\mathrm{mg} / \mathrm{kg} / \mathrm{day})^{3}\end{array}$ \\
\hline
\end{tabular}

Non-Radioactive

\section{Metals}

Chromium

Lead

Mercury

$\begin{array}{ll}440-47-3 & 1.82 \mathrm{E}+02 \\ 439-92-1 & 9.00 \mathrm{E}+01 \\ 439-97-6 & 6.57 \mathrm{E}-02\end{array}$

(1) SP: soitho-plant uptake factor.

(2) BAF: biamocumulation fector.

(3) Oldfietd mouse exposure calculated as

$E D=[(C s \bullet S P \bullet I P)+(C s \bullet B A F * I R)+(C s * I s) * S F F] / B W$

Where, $E D=$ expoesure dose

$\mathrm{Cs}=\mathrm{RME}$ conc in soil (mg/kg)

$S P=$ soiltoiplant uptake factor

Ip = plemt-matter intake rate (kg/day, ste Table 4.24)

BAF - bionceumulation factor (unitless)

In = animal-matter intake rate (kg/day, see Table 4.24)

Is = incidemal soil intake rate (kg/day, see Table 4.24)

SFF = site foraging factor (unitless, see Table 4.24)

BW' = body weight (kg. see Table 4.24)
(4) Source: Bace ct al., 1984.

(5) Default where no experimental data available, no evidence of bioconcentration.

(6) Source: M2, 1983.

(7) Source: USEPA, 1994. 
TABLE 4.28

CALCULATED SOIL RECEPTOR EXPOSURE

RP 231-2F; 0 - 1.2.M (0 - 4 FT)

FUTURE CONDITIONS

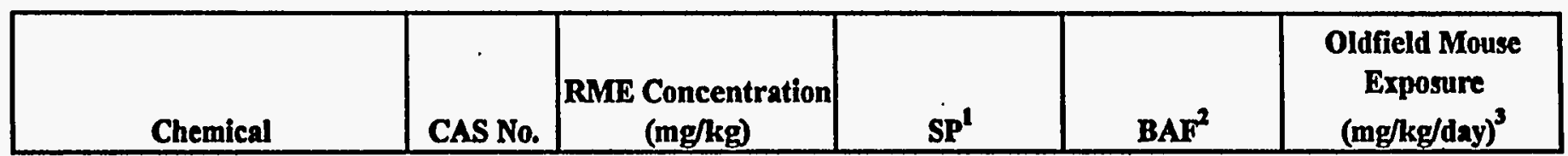

Non-Radioactive

$\begin{array}{lllllllll}\text { Metals } & & & & & \\ \text { Chromium } & 440-47-3 & 5.51 E+01 & 7.50 \mathrm{E}-03 & 4 & 1.00 \mathrm{E}+00 & 5 & 3.82 \mathrm{E}-01 \\ \text { Lead } & 439-92-1 & 6.20 \mathrm{E}+01 & 4.50 \mathrm{E}-02 & 4 & 1.12 \mathrm{E}+00 & 6 & 4.95 \mathrm{E}-01 \\ \text { Mercury } & 439-97-6 & 1.23 \mathrm{E}-01 & 9.00 \mathrm{E}-01 & 4 & 2.30 \mathrm{E}+01 & 7 & 1.95 \mathrm{E}-02\end{array}$

(1) SP: soittoplant uptake factor.

(2) BAF: biasceumulation factor.

(3) Oldfield mouse exposure calculated as

$E D=\left[\left(C:{ }^{\bullet} \mathrm{SP} \bullet \mathrm{Ip}\right)+(\mathrm{Cs} \cdot \mathrm{BAF} \bullet \mathrm{I})+\left(\mathrm{C} \cdot{ }^{\bullet} \mathrm{Is}\right) \cdot \mathrm{SFF}\right] / \mathrm{BW}$

Where, ED - exposure dose

$\mathrm{Cs}_{\mathrm{s}}=\mathrm{RME}$ conc in roil ( $\left.\mathrm{mg} / \mathrm{k}_{\mathrm{B}}\right)$

$S P=$ soil-to-plamt uptake factor

Ip = plamt-matter intake rate (kg/day, see Table 4.24)

BAF - bionocumulation factor (unitless)

Ia = animal-maitler intake rate (kg/day, see Table 4.24)

ls = incidemal soil intake rate (kg/day, see Table 4.24)

SFF = site foraging factor (unilless, see Table 4.24)

$\mathrm{BW}=$ body weight ( $\mathrm{kg}$, see Table 4.24)

(4) Source: Bues ct al, 1984.

(5) Defauth where no experimental data available,

no evidence of bioconcentration.

(6) Source: Ma, 1983

(7) Sourne: USEPA, 1994. 
TABLE 4.29

CALCULATED SEDIMENT RECEPTOR EXPOSURE BRP 231-F/1F and RP 231-2F - WETLAND CURRENT AND FUTURE CONDITIONS

\begin{tabular}{|c|c|c|c|c|c|c|}
\hline Chemical & CAS No. & $\begin{array}{c}\text { RME Concentration } \\
(\mathrm{m} / \mathrm{kg})\end{array}$ & $\log \mathrm{Kow}^{\mathrm{x}}$ & $\mathrm{SP}^{2}$ & BAF & $\begin{array}{c}\text { Oldfield Mouse } \\
\text { Exposure } \\
(\mathrm{mg} / \mathrm{kg} / \mathrm{day})^{3}\end{array}$ \\
\hline
\end{tabular}

Non-Radioactive

\section{Volatiles}

Acetone

Chloromethane (methyl chloride)

Dichloromethane (methylene chloride)

Toluene

Semivolatiles

Acenaphthene

Acenaphthylene

Anthracene

Benzo(a)anthracene

Benzo(a)pyrene

Benzo(b)fluoranthene

Benzo(ghi)perylene

Benzo(k) lluoranthene

Bis(2-ethylhexyl)phthalate

Chrysene

Di-n-butyl phthalate

Dibenz(a,h)anthracene

Dibenzofuran

Fluoranthene

Fluorene

Indeno $(1,2,3-\infty d)$ pyrene

Phenanthrene

Pyrene

$\begin{array}{rr}67-64-1 & 1.60 \mathrm{E}-01 \\ 74-8-73 & 1.30 \mathrm{E}-02 \\ 75-09-2 & 4.19 \mathrm{E}-02 \\ 108-88-3 & 1.90 \mathrm{E}-02 \\ & \\ & \\ 83-32-9 & 2.18 \mathrm{E}-01 \\ 208-96-8 & 7.49 \mathrm{E}-01 \\ 120-12-7 & 4.21 \mathrm{E}+00 \\ 56-55-3 & 4.31 \mathrm{E}+00 \\ 50-32-8 & 2.99 \mathrm{E}+00 \\ 205-99-2 & 4.34 \mathrm{E}+00 \\ 191-24-2 & 1.74 \mathrm{E}+00 \\ 207-08-9 & 3.99 \mathrm{E}+00 \\ 117-81-7 & 1.68 \mathrm{E}+00 \\ 218-01-9 & 5.33 \mathrm{E}+00 \\ 84-74-2 & 5.96 \mathrm{E}-02 \\ 53-70-3 & 5.34 \mathrm{E}-01 \\ 132-64-9 & 1.33 \mathrm{E}-01 \\ 206-44-0 & 1.21 \mathrm{E}+01 \\ 86-73-7 & 5.01 \mathrm{E}-01 \\ 193-39-5 & 1.78 \mathrm{E}+00 \\ 85-01-8 & 5.36 \mathrm{E}+00 \\ 129-00-0 & 7.83 \mathrm{E}+00\end{array}$

$\begin{array}{lrllll}-0.24 & 5.33 E+01 & 5 & 6.90 E-01 & 13 & 1.14 E-01 \\ 0.91 & 1.15 E+01 & 5 & 1.00 E+00 & 10 & 2.16 E-03 \\ 1.25 & 7.34 E+00 & 3 & 1.00 E+00 & 10 & 4.64 E-03 \\ 2.75 & 9.97 E-01 & 5 & 1.00 E+00 & 10 & 5.12 E-04\end{array}$

2.10E-01 S 3.42E-01 99 2.02E-01 S 3.42E-01 8,9

9.08E-02 S 5.11E-02 8

1.97E-02 \& 1.25E-01 8

$1.14 \mathrm{E}-02$ s $3.42 \mathrm{E}-01$ \&

$1.01 E-02$ s $3.19 \mathrm{E}-01$ \&

$5.19 E-03$ s $2.44 E-01$ \&

1.01E-02 s 2.53E-01 \&

2.34E-03 S 1.20E+01 11

$1.97 \mathrm{E}-02$ s $1.75 \mathrm{E}-01$ \&

8.38E.02 s 4.90E+03 is

$5.26 \mathrm{E}-03$ s $3.68 \mathrm{E}-018$

$1.72 E-01$ s $1.00 E+10$ 10

4.2SE-02 3 7.92E-02 \&

$1.43 \mathrm{E}-01$ s $3.42 \mathrm{E}-01 \quad 8,9$

$5.55 \mathrm{E}-03$ s $4.19 \mathrm{E}-01 \quad 8$

9.08E-02 s 1.22E-01 8

$\begin{array}{lll}4.31 E-02 & \text { s } 9.20 \mathrm{E}-02 \text { 8 }\end{array}$
1.71E-03

S.79E- 03

1.02E-02

$1.06 \mathrm{E}-02$

$1.56 \mathrm{E}-02$

2.13E-02

6.69E-03

1.61E-02

2.68E-01

$1.66 \mathrm{E}-02$

$3.86 \mathrm{E}+00$

2.92E-03

2.13E-03

$2.60 \mathrm{E}-02$

3.48E-03

$1.10 \mathrm{E}-02$

$1.80 \mathrm{E}-02$

1.82E-02 
CALCULATED SEDIMENT RECEPTOR EXPOSURE

BRP 231-F/1F and RP 231-2F - WETLAND

CURRENT AND FUTURE CONDITIONS

\begin{tabular}{|c|c|c|c|c|c|c|c|c|}
\hline $\begin{array}{r}\text { Chemical } \\
\end{array}$ & CAS No. & $\begin{array}{c}\text { RME Concentration } \\
(\mathrm{mg} / \mathrm{kg})\end{array}$ & $\log K_{0 w}{ }^{\prime}$ & $\mathbf{S P}^{2}$ & & BAF & & $\begin{array}{l}\text { Oldfield Mou } \\
\text { Exposure } \\
\text { (me/kg/day) }\end{array}$ \\
\hline \multicolumn{9}{|l|}{ Dloxing/furans } \\
\hline Octachlorodibenzo-p-dioxin isomers & $3268-87-9$ & $8.00 E-01$ & 6.53 & $6.51 E-03$ & 5,7 & $1.50 E+01$ & 11 & $1.59 \mathrm{E}-01$ \\
\hline \multicolumn{9}{|l|}{ Metals } \\
\hline Copper & $7440-50-8$ & $2.56 \mathrm{E}+01$ & NA & 4.00E-01 & 6 & $9.25 E-01$ & 12 & $4.63 \mathrm{E}-01$ \\
\hline Manganese & $7439-96-5$ & $5.75 E+01$ & NA & 2.50E-01 & 6 & $1.00 \mathrm{E}+00$ & 10 & $9.82 \mathrm{E}-01$ \\
\hline Mercury & $7439-97-6$ & $7.10 \mathrm{E}-01$ & NA & $9.00 \mathrm{E}-01$ & 6 & $2.30 \mathrm{E}+01$ & 11 & $2.25 \mathrm{E}-01$ \\
\hline
\end{tabular}

(1) Log Kow values obtained from Kerickhoff and Long. 1995.

(5) Source: Travis and Arms, 1988.

(6) Source: Baes er al., 1984.

(2) SP: soiltoplant uptake factor.

(3) BAF: biosccumulation fector.

(4) Otdifld mouse exposure calculated as

$\mathrm{ED}=[(\mathrm{Cd} \bullet \mathrm{SP} \bullet \mathrm{Ip})+(\mathrm{Cd} \bullet \mathrm{BAF} \bullet \mathrm{la})+(\mathrm{Cd} \bullet \mathrm{l}) \bullet \mathrm{SFF}] / \mathrm{BW}$ Where, ED - exposure doce

$\mathrm{Cd}$ - RME conc in sedimenk (mg/kg)

SP = soilto-plank uptuke factor

lp - plart-matter intake rate (kg/day, see Table 4.24)

BAF = biosccumulation factor (unitlexs)

Ia - animal-matter intake rate (kg/day, sec Table 4.24)

is - incidemal sediment intake rate (kg/dny, see Table 4.24)

SFF = site foraging factor (unitless, see Table 4.24)

BW = body weight (kg, see Table 4.24)

(7) Substitured value for 2,3,7,8-TCDD.

(8) Source: Marquenic et al., 1987 from Beyer, 1990.

(9) Subatituted value for berwo(a)pyrence.

(10) Default where no experimental data available, no evidence of bioconcentration.

(11) Source: USEPA, 1994.

(12) Source: Ma, 1983.

(13) Source HSDB, 1995 
TABLE 430

TOXICTTY REFERENCE VALUES FOR ECOLOGICAL COPC BRP 231-F/1F and RP 231-2F

\begin{tabular}{|c|c|c|c|c|c|c|c|c|c|c|}
\hline Analute & $\begin{array}{c}\text { Test } \\
\text { Orranism }\end{array}$ & Endpolnt/Duration/Erfect & Source & $\begin{array}{l}\text { Erreet Dose } \\
\text { (m/les/day) }\end{array}$ & $\begin{array}{c}\text { Surrogate } \\
\text { Chemical } \\
\text { UR }\end{array}$ & $\begin{array}{c}\text { Endpoint } \\
\text { UF }\end{array}$ & $\begin{array}{c}\text { Study } \\
\text { Durnition } \\
\text { UIF }\end{array}$ & $\begin{array}{c}\text { Inter } \\
\text { taxon } \\
\text { UR }\end{array}$ & $\begin{array}{c}\text { Total } \\
\text { UF }\end{array}$ & $\begin{array}{l}\text { Toxicty } \\
\text { Reference } \\
\text { Value } \\
\text { (melef/day) }\end{array}$ \\
\hline
\end{tabular}

\section{Volatiles}

Actione

Chloromethane (methyl chloride)

Dichloromethane (methylene chloride)

Toluene

Semivolitilea

Acensphthene

Acensphthinglene

Anthrecens:

\section{Benseo(a) anthracene}

Benzo(a)pyrene

Benzo(b) Aluoranthene

Beneo(thi)peryiene

Berwoo(k) Aluorenthere

Bie(2-ethylhexyl)phthalate

Bio(2-ethylhexyl)phthalate

Chysene

Dirsbutyl phthalate

Dibenze(a,h)enthracene

Diberzofurm

Fluoranthene

Fhuorene

Indeno(1,2,3-od)pyrene

Fhenenthrene

Pyrene

Dioxbu/furas:

Octachlorodibenzo-p-dioxin isomers

\begin{tabular}{|c|c|}
\hline $\begin{array}{l}\text { rat } \\
\text { clawed tond } \\
\text { rat } \\
\text { rat } \\
\text { rat }\end{array}$ & $\begin{array}{l}\text { LOAEL, diet, 90-day, nephrotoxicity } \\
\text { LCSO, acute, ambient water } \\
\text { LDSO, oral } \\
\text { LOAEL, water, 90-day, hepatic necroxis } \\
\text { LOAEL, diet, 91-day, hepatic \& renal hypertropl }\end{array}$ \\
\hline $\begin{array}{l}\text { mouse } \\
\text { mouse } \\
\text { rat } \\
\text { mot } \\
\text { mouse: } \\
\text { mouse } \\
\text { mouse } \\
\text { mouse } \\
\text { mouse } \\
\text { fish } \\
\text { mouse } \\
\text { rat } \\
\text { mouse } \\
\text { rat } \\
\text { mouse } \\
\text { mouse } \\
\text { mouse } \\
\text { rat }\end{array}$ & 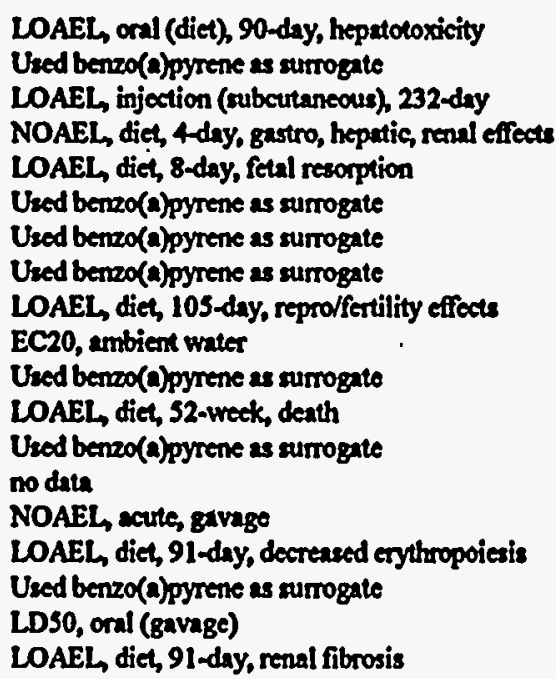 \\
\hline
\end{tabular}

$5.00 E+02$

HSDB, 1994

HSDB, 1994

ATSDR, 1991d

2.4E+0.4 ug/L

$1.80 \mathrm{E}+03$

$1.20 \mathrm{E}+03$

$4.46 \mathrm{E}+02$

IRIS, 1994

HSDB, 1994

ATSDR, 1993

ATSDR, 1989e

$3.50 \mathrm{E}+02$

$1.20 \mathrm{E}+02$

$6.70 \mathrm{E}+01$

$1.50 \mathrm{E}+02$

$1.20 \mathrm{E}+02$

$1.20 \mathrm{E}+02$

$120 \mathrm{E}+02$

$1.20 \mathrm{E}+02$

ATSDR, 1989c $\quad 1.30 \mathrm{E}+02$

Suter \& Mabry, 1994 5.4E+01 ug/L.

ATSDR, 1989d $\quad 6.25 \mathrm{E}+02$

$1.20 \mathrm{E}+02$

$1.20 \mathrm{E}+02$

ATSDR, $1993 \quad 1.50 \mathrm{E}+02$

IRIS, 1994

$2.50 \mathrm{E}+02$

$2.50 \mathrm{E}+02$
$1.20 \mathrm{E}+02$

RTECS, 1993

IRIS, 1994

.25E+02

HSDB, 1994

$4.00 \mathrm{E}+03$

\section{1}

11

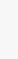

1

10

1
10
1
1
1
10
10
10
1
1
10
1
10
1
1
10
1
1

(1)

1
1
1
1
1
1
1
1
1
1
1
3
1
1
1
1
15
1

$\begin{array}{cccc}5 & 5 & 25 & 2.00 E+01 \\ 10 & 7 & 1050 & 2.3 E+01 \text { ugl } \\ 10 & 5 & 750 & 2.40 E+00 \\ 5 & 5 & 25 & 4.80 E+01 \\ 5 & 5 & 25 & 1.78 E+01\end{array}$

$\begin{array}{ccc}5 & 5 & 25 \\ 10 & 5 & 500 \\ 5 & 5 & 25 \\ 10 & 5 & 50 \\ 10 & 5 & 50 \\ 10 & 5 & 500 \\ 10 & 5 & 500 \\ 10 & 5 & 500 \\ 5 & 5 & 25 \\ 10 & 10 & 100 \\ 10 & 5 & 500 \\ 1 & 5 & 15 \\ 10 & 5 & 500 \\ 10 & 5 & 50 \\ 5 & 5 & 25 \\ 10 & 5 & 500 \\ 10 & 5 & 750 \\ 5 & 5 & 25\end{array}$

$\begin{array}{cc}25 & 1.40 \mathrm{E}+01 \\ 500 & 2.40 \mathrm{E}-01 \\ 25 & 2.68 \mathrm{E}+00 \\ 50 & 3.00 \mathrm{E}+00 \\ 50 & 2.40 \mathrm{E}+00 \\ 500 & 2.40 \mathrm{E}-01 \\ 500 & 2.40 \mathrm{E}-01 \\ 500 & 2.40 \mathrm{E}-01 \\ 25 & 5.20 \mathrm{E}+00 \\ 100 & 5.4 \mathrm{E}-01 \mathrm{ug} / \mathrm{l} \\ 500 & 2.40 \mathrm{E}-01 \\ 15 & 4.17 \mathrm{E}+01 \\ 500 & 2.40 \mathrm{E}-01 \\ & n 0 \mathrm{n}+1 \mathrm{k} \\ 50 & 3.00 \mathrm{E}+00 \\ 25 & 1.00 \mathrm{E}+01 \\ 500 & 2.40 \mathrm{E}-01 \\ 750 & 9.33 \mathrm{E}-01 \\ 25 & 5.00 \mathrm{E}+00\end{array}$

FINAL 
TABLE 4.30

TOXICITY REFERENCE VALUES FOR ECOLOGICAL COPC

BRP 231-F/1P and RP 231-2

\begin{tabular}{|c|c|c|c|c|c|c|c|c|c|c|}
\hline Analyte & $\begin{array}{c}\text { Test } \\
\text { Orranlom }\end{array}$ & Endpolnt/Duration/Errect & Source & $\begin{array}{l}\text { Enrect Dose } \\
\text { (me/kg/day) }\end{array}$ & $\begin{array}{c}\text { Surrogate } \\
\text { Cremical } \\
\text { UR }\end{array}$ & $\begin{array}{c}\text { Endpoint } \\
\text { UF }\end{array}$ & $\begin{array}{c}\text { Study } \\
\text { Duration } \\
\text { UF }\end{array}$ & \begin{tabular}{|} 
Inter- \\
taron \\
UR \\
\end{tabular} & $\begin{array}{c}\text { Total } \\
\text { UF }\end{array}$ & $\begin{array}{c}\text { Toxicty } \\
\text { Reference } \\
\text { Value } \\
\text { (melceldas) }\end{array}$ \\
\hline \multicolumn{11}{|l|}{ Metali } \\
\hline Burium & mouse & NOAEL, drinking water, 2-year & ATSDR, 1991k & $9.50 \mathrm{E}-01$ & 1 & 1 & 1 & $\mathbf{s}$ & 5 & $1.90 \mathrm{E}-01$ \\
\hline Chromium & mouse & LOAEL, diet, 7-weeck, decr ppermatogenesis & ATSDR, 1991c & $5.70 E+01$ & 1 & 1 & $\mathbf{s}$ & 5 & 25 & $2.28 E+00$ \\
\hline Copper & mouse & LOAEL, drinking water, 850-day, decr survival & ATSDR, 1989a & 4.25E+01 & 1 & 1 & 1 & $\mathbf{s}$ & $\mathbf{s}$ & $8.50 \mathrm{E}+00$ \\
\hline Lead & $\mathbf{r a t}$ & LOAEL, diet, 18 -month, increased blood presure & ATSDR, 1992 & $2.80 \mathrm{E}+00$ & 1 & 1 & 1 & $\mathbf{s}$ & 5 & $5.60 \mathrm{~B}-01$ \\
\hline Manganese & $\mathbf{r a t}$ & LOAEL, diet, 103-week, death & ATSDR, 19900 & $9.30 E+02$ & 1 & 3 & 1 & $\mathbf{s}$ & 15 & $6.20 \mathrm{E}+01$ \\
\hline Minganese & fish & EC20, 28-day earty life stage test & Suter \& Mabry, 199 & $1.77 \mathrm{E}+03$ ug/L & $\mathbf{I}$ & 10 & 5 & 20 & 1000 & $1.77 E+00$ ug $/$ \\
\hline Mereury & mouse & LOAEL, diet, 104-week, kidney effects & ATSDR, 1994 & $1.10 \mathrm{E}-01$ & 1 & 1 & 1 & 5 & $\mathbf{s}$ & 2.20E-02 \\
\hline
\end{tabular}


TABLE 4.31

EXPOSURE TO RADIATION FROM SEDIMENT

BRP 231-F/1F and RP 231-2F-

CURRENT AND FUTURE CONDITIONS

\begin{tabular}{|c|c|c|c|c|c|c|}
\hline Chemical & CAS No. & $\begin{array}{c}\text { RME } \\
\text { Concentration } \\
(\mathrm{pCi} / \mathrm{g})\end{array}$ & $\begin{array}{c}\text { Plant Radionuclide } \\
\text { Concentration } \\
(\mathrm{pCi} / \mathrm{g})\end{array}$ & $\begin{array}{c}\text { Animal } \\
\text { Radionuclide } \\
\text { Concentration } \\
(\mathrm{pCi} / \mathrm{g})\end{array}$ & $\begin{array}{c}\text { Dose Rate to } \\
\text { Receptor } \\
\text { (mGy/yr) }\end{array}$ & $\begin{array}{c}\text { Dose Rate to } \\
\text { Receptor } \\
\text { (rad/day) }\end{array}$ \\
\hline $\begin{array}{l}\text { Strontium-90 } \\
\text { Technetium-99 }\end{array}$ & $10098-97-2$ & $1.70 \mathrm{E}+00$ & $1.70 \mathrm{E}-01$ & $2.99 \mathrm{E}+00$ & $6.46 \mathrm{E}-02$ & $1.77 \mathrm{E}-05$ \\
\end{tabular}


CALCULATION OF SOIL ECOLOGICAL QUOTIENTS

RP 231-2F; 0 - 0.6 M (0 - 2 FT)

CURRENT CONDITIONS

\begin{tabular}{|c|c|c|c|c|}
\hline Chemical & CAS No. & $\begin{array}{c}\text { Oldfield Mouse } \\
\text { Exposure } \\
\text { (mg/kg/day) }^{1}\end{array}$ & $\begin{array}{c}\text { Toxicity Reference } \\
\text { Value (mg/kg/day) }\end{array}$ & $\begin{array}{c}\text { Ecological } \\
\text { Quotient }^{3}\end{array}$ \\
\hline
\end{tabular}

Non-Radioactive

\begin{tabular}{lllll} 
Metals & & & & \\
Chromium & $440-47-3$ & $1.26 \mathrm{E}+00$ & $2.28 \mathrm{E}+00$ & $5.5 \mathrm{E}-01$ \\
Lead & $439-92-1$ & $7.80 \mathrm{E}-01$ & $5.60 \mathrm{E}-01$ & $1.4 \mathrm{E}+00$ \\
\hline Mercury & $439-97-6$ & $1.04 \mathrm{E}-02$ & $2.20 \mathrm{E}-02$ & $4.7 \mathrm{E}-01$ \\
\hline
\end{tabular}

(1) Receptor exposure from Table 4.27.

(2) Toxicity reference value from Table 4.30.

(3) Ecological quotient calculated as $E Q=$ exposure rate / toxicity reference value

with $\mathrm{EQ}<1$, no effects expected

$1<\mathrm{EQ}=<10$, small potential for effects

$10<\mathrm{EQ}=<100$, potential for greater exposure to result in effects, and

$E Q>100$, highest potential for effects.

BOLD: represents receptor $E Q>=1$.

FINÁL 
TABLE 4.32

CALCULATION OF SOL ECOLOGICAL QUOTIENTS

BRP 231-F/1F; 0 - 0.6 M (0 - 2 FT) -

CURRENT CONDITIONS

\begin{tabular}{|c|c|c|c|c|}
\hline & CAS No. & $\begin{array}{c}\text { Oldfield Mouse } \\
\text { Exposure } \\
(\mathrm{mg} / \mathrm{kg} / \mathrm{day})^{1}\end{array}$ & $\begin{array}{c}\text { Toxicity Reference } \\
\text { Value (mg/kg/day) }\end{array}$ & $\begin{array}{c}\text { Ecological } \\
\text { Quotient }^{3}\end{array}$ \\
\hline
\end{tabular}

Non-Radioactive

\begin{tabular}{lllll|}
$\begin{array}{l}\text { Semivolatiles } \\
\text { Dibenzofuran }\end{array}$ & $132-64-9$ & $1.20 \mathrm{E}-02$ & no data & \\
Metals & & & & \\
Barium & $440-39-3$ & $1.20 \mathrm{E}+00$ & $1.90 \mathrm{E}-01$ & $\mathbf{6 . 3 \mathrm { E } + 0 0}$ \\
Chromium & $440-47-3$ & $2.49 \mathrm{E}+00$ & $2.28 \mathrm{E}+00$ & $1.1 \mathrm{E}+00$ \\
Lead & $439-92-1$ & $4.72 \mathrm{E}-01$ & $5.60 \mathrm{E}-01$ & $8.4 \mathrm{E}-01$ \\
Manganese & $439-96-5$ & $3.31 \mathrm{E}+01$ & $6.20 \mathrm{E}+01$ & $5.3 \mathrm{E}-01$ \\
Mercury & $439-97-6$ & $7.04 \mathrm{E}-01$ & $2.20 \mathrm{E}-02$ & $3.2 \mathrm{E}+01$ \\
\hline
\end{tabular}

(1) Receptor exposure from Table 4.25 .

(2) Toxicity reference value from Table 4.30

(3) Ecological quotient calculated as EQ = exposure rate / toxicity reference value with $E Q<1$, no effects expected $1<E Q=<10$, small potential for effects

$10<E Q=<100$, potential for greater exposure to result in effects, and $E Q>100$, highest potential for effects.

BOLD: represents receptor EQ $>=1$.

- : no EQ could be calculated, as no toxicity data could be found. 
TABLE 4.33

CALCULATION OF SOIL ECOLOGICAL QUOTITNTS

BRP 231-F/1F; 0 - 1.2 M (0 - 4 FT $)$

FUTURE CONDITIONS

\begin{tabular}{|c|c|c|c|c|}
\hline Chemical & CAS Na. & $\begin{array}{c}\text { Oldfield Mouse } \\
\text { Exposure } \\
(\mathrm{mg} / \mathrm{kg} / \mathrm{day})\end{array}$ & $\begin{array}{c}\text { Toxicity Reference } \\
\text { Value (mg/kg/day) }\end{array}$ & $\begin{array}{c}\text { Ecological } \\
\text { Quotient }^{3}\end{array}$ \\
\hline
\end{tabular}

Non-Radioactive

Semivolatiles

Dibenzofuran

$132-64-9 \quad 1.20 \mathrm{E}-02$

no data

\section{Metals}

Barium

Chromium

点

Lead

$440-47-3$

439-92-1

$439-96-5$

Manganese

$439-97-6$

$\begin{array}{ll}1.32 \mathrm{E}+00 & 1.90 \mathrm{E}-01 \\ 4.30 \mathrm{E}+00 & 2.28 \mathrm{E}+00 \\ 3.62 \mathrm{E}-01 & 5.60 \mathrm{E}-01 \\ 2.50 \mathrm{E}+01 & 6.20 \mathrm{E}+01 \\ 4.60 \mathrm{E}-01 & 2.20 \mathrm{E}-02\end{array}$

\begin{tabular}{|c|}
\hline $7.0 \mathrm{E}+00$ \\
\hline $1.9 \mathrm{E}+00$ \\
\hline $6.5 \mathrm{E}-01$ \\
$4.0 \mathrm{E}-01$ \\
\hline $2.1 \mathrm{E}+01$ \\
\hline
\end{tabular}

(1) Receptor exposure from Table 4.26.

(2) Toxicity reference value from Table 4.30.

(3) Ecological quotient calculated as $\mathrm{EQ}=$ exposure rate / toxicity reference value with $\mathrm{EQ}<1$, no effects expected

$1<E Q=<10$, small potential for effects

$10<E Q=<100$, potential for greater exposure to result in effects, and $E Q>100$, highest potential for effects.

BOLD: represents receptor $E Q>>1$.

- : no EQ could be calculated, as no toxicity data could be found. 
TABLE 4.34

CALCULATION OF SOIL ECOLOGICAL OUOTIENTS

RP 231-2F; 0 - 0.6 M (0 - 2 FT)

CURRENT CONDITIONS

\begin{tabular}{|c|c|c|c|c|}
\hline Chemical & CAS No. & $\begin{array}{c}\text { Oldfield Mouse } \\
\text { Exposure } \\
\text { (mg/kg/day) }\end{array}$ & $\begin{array}{c}\text { Toxicity Reference } \\
\text { Value (mg/kg/day) }\end{array}$ & $\begin{array}{c}\text { Ecological } \\
\text { Quotient }^{3}\end{array}$ \\
\hline
\end{tabular}

Non-Radioactive

\section{Metals}

Chromium

Lead

440-47-3

$\begin{array}{ll}1.26 \mathrm{E}+00 & 2.28 \mathrm{E}+00 \\ 7.80 \mathrm{E}-01 & 5.60 \mathrm{E}-01 \\ 1.04 \mathrm{E}-02 & 2.20 \mathrm{E}-02\end{array}$

$5.5 \mathrm{E}-01$

Mercury

(1) Receptor exposure from Table 4.27.

(2) Toxicity reference value from Table 4.30.

(3) Ecological quotient calculated as EQ = exposure rate / toxicity reference value with $E Q<1$, no effects expected

$1<E Q=<10$, small potential for effects

$10<E Q=100$, potential for greater exposure to result in effects, and

$E Q>100$, highest potential for effects.

BOLD: represents receptor $\mathrm{EQ}>=1$. 
TABLE 4.35

CALCULATION OF SOIL ECOLOGICAL QUOTIENTS

RP 231-2F; 0 - 1.2 M (0 - 4 FT) FUTURE CONDITIONS

\begin{tabular}{|c|c|c|c|c|}
\hline & CAS No. & $\begin{array}{c}\text { Oldfield Mouse } \\
\text { Exposure } \\
(\mathrm{mg} / \mathrm{kg} / \mathrm{day})^{1}\end{array}$ & $\begin{array}{c}\text { Toxicity Reference } \\
\text { Value (mg/kg/day) }\end{array}$ & $\begin{array}{c}\text { Ecological } \\
\text { Quotient }^{3}\end{array}$ \\
\hline
\end{tabular}

Non-Radioactive

\section{Metals}

Chromium

Lead

440-47-3

439-92-1

3.82E-01

4.95E-01

2.28E+00

1.7E-01

Mercury

$439-97-6$

1.95E-02

$5.60 \mathrm{E}-01$

$8.8 \mathrm{E}-01$

8.9E-01

(1) Receptor exposure from Table 4.28.

(2) Toxicity reference value from Table 4.30.

(3) Ecological quotient calculated as $E Q=$ exposure rate / toxicity reference value with $E Q<1$, no effects expected

$1<E Q=<10$, small potential for effects

$10<\mathrm{EQ}=<100$, potential for greater exposure to result in effects, and

$E Q>100$, highest potential for effects.

BOLD: represents receptor EQ >= 1 . 
TABLE 4.36

CALCULATION OF SURFACE WATER ECOLOGICAL QUOTIENTS

BRP 231-F/1F and RP 231-2F - WETLAND

CURRENT AND FUTURE CONDITIONS

\begin{tabular}{|c|c|c|c|c|}
\hline Chemical & CAS No. & $\begin{array}{c}\text { RME Concentration } \\
(n g / L)\end{array}$ & $\begin{array}{c}\text { Toxicity Reference } \\
\text { Value (ng/L) }\end{array}$ & $\begin{array}{c}\text { Ecological } \\
\text { Qwotient }^{3}\end{array}$ \\
\hline
\end{tabular}

Non-radioactive

\begin{tabular}{|c|c|c|c|c|}
\hline \multicolumn{5}{|l|}{ Volatiles } \\
\hline Acetone & $67-64-1$ & $2.26 \mathrm{E}-02$ & $2.30 E+01$ & 9.8E-04 \\
\hline \multicolumn{5}{|l|}{ Semivolatiles } \\
\hline Bis(2-ethylhexyl)phthalate & $117-81-7$ & $3.45 \mathrm{E}-01$ & $5.40 \mathrm{E}-01$ & $6.4 E-01$ \\
\hline \multicolumn{5}{|l|}{ Metals } \\
\hline Manganese & $7439-96-5$ & $2.13 \mathrm{E}-01$ & $1.77 \mathrm{E}+\infty 0$ & $1.2 \mathrm{E}-01$ \\
\hline
\end{tabular}

$\stackrel{t}{\stackrel{\circ}{\circ}}$

(1) RME concentration from Table 4.14.

(2) Toxicity reference value from Table 4.30.

(3) Ecological quotient calculated as $E Q=$ exposure rate / toxicity reference value with $E Q<1$, no effects expected

$1<\mathrm{EQ}=<10$, small potential for effects

$10<\mathrm{EQ}=<100$, potential for greater exposure to result in effects, and $E Q>100$, highest potential for effects.

BOLD: represents receptor EQ $>=1$. 
TABLE 4.37

CALCULATION OF SEDIMENT ECOLOGICAL QUOTIENTS

BRP 231-F/1F and RP 231-2F - WETLAND

CURRENT AND FUTURE CONDTTIONS

\begin{tabular}{|c|c|c|c|c|}
\hline & Chemical & $\begin{array}{c}\text { Oldfield Mouse } \\
\text { Exposure (mg/kg/day) }\end{array}$ & $\begin{array}{l}\text { Toxicity Reference } \\
\text { Value (mg/kg/day) }\end{array}$ & $\begin{array}{c}\text { Ecological } \\
\text { Quotient }\end{array}$ \\
\hline
\end{tabular}

Nom-Radioactive

Volatiles

Acetone

Dichloromethane (methylene chloride) 75-09-2

Toluene

Semivolatiles

Acenaphthene

Acenaphthylene

Anthracene

Benzo(a)anthracene

Benzo(a)pyrene

Benzo(b)fluoranthene

Benzo(ghi)perylene

Benzo(k)fluoranthene

Bis(2-ethylhexyl)phthalate

Chrysene

Di-n-butyl phthalate

Dibenz(a,h)anthracene

Dibenzofuran

Fluoranthene

Fluorene

Indeno(1,2,3-cd)pyrene

Phenanthrene

Pyrene
67-64-1

74-8-73

108-88-3

$1.14 \mathrm{E}-01$
$2.16 \mathrm{E}-03$
$4.64 \mathrm{E}-03$
$5.12 \mathrm{E}-04$

$1.71 \mathrm{E}-03$
$5.79 \mathrm{E}-03$
$1.02 \mathrm{E}-02$
$1.06 \mathrm{E}-02$
$1.56 \mathrm{E}-02$
$2.13 \mathrm{E}-02$
$6.69 \mathrm{E}-03$
$1.61 \mathrm{E}-02$
$2.68 \mathrm{E}-01$
$1.66 \mathrm{E}-02$
$3.86 \mathrm{E}+00$
$2.92 \mathrm{E}-03$
$2.13 \mathrm{E}-03$
$2.60 \mathrm{E}-02$
$3.48 \mathrm{E}-03$
$1.10 \mathrm{E}-02$
$1.80 \mathrm{E}-02$
$1.82 \mathrm{E}-02$

83-32-9

208-96-8

120-12-7

56-55-3

$50-32-8$

205-99-2

191-24-2

207-08-9

117-81-7

218-01-9

84-74-2

$53-70-3$

132-64-9

206-44-0

86-73-7

193-39-5

85-01-8

129-00-0
2.00E+01

2.40E+00

$4.80 E+01$

$1.78 E+01$

1.40E+01

2.40E-01

$2.68 \mathrm{E}+00$

$3.00 \mathrm{E}+00$

2.40E+00

$2.40 \mathrm{E}-01$

$2.40 \mathrm{E}-01$

2.40E-01

$5.20 \mathrm{E}+\infty 0$

$2.40 \mathrm{E}-01$

$4.17 \mathrm{E}+01$

$2.40 \mathrm{E}-01$

no data

$3.00 \mathrm{E}+00$

$1.00 \mathrm{E}+01$

$2.40 \mathrm{E}-01$

9.33E-01

$5.00 E+00$
5.7E-03

9.0E-04

9.7E-05

2.9E-05

$1.2 \mathrm{E}-04$
$2.4 \mathrm{E}-02$
$3.8 \mathrm{E}-03$
$3.5 \mathrm{E}-03$
$6.5 \mathrm{E}-03$
$8.9 \mathrm{E}-02$
$2.8 \mathrm{E}-02$
$6.7 \mathrm{E}-02$
$5.1 \mathrm{E}-02$
$6.9 \mathrm{E}-02$
$9.3 \mathrm{E}-02$
$1.2 \mathrm{E}-02$
$-\mathrm{E}-$
$8.7 \mathrm{E}-03$
$3.5 \mathrm{E}-04$
$4.6 \mathrm{E}-02$
$1.9 \mathrm{E}-02$
$3.6 \mathrm{E}-03$


TABLE 4.37

CALCULATION OF SEDIMENT ECOLOGICAL QUOTIENTS

BRP 231-F/1F and RP 231-2F - WETLAND

CURRENT AND FUTURE CONDITIONS

\begin{tabular}{|c|c|c|c|c|}
\hline & CAS Na & $\begin{array}{c}\text { Oldfield Mouse } \\
\text { Expoure (mg/kg/day) }\end{array}$ & $\begin{array}{l}\text { Toxicity Reference } \\
\text { Value (mg/kg/day) }\end{array}$ & $\begin{array}{c}\text { Ecological } \\
\text { Quotiear }\end{array}$ \\
\hline
\end{tabular}

\section{Dioxineffurans}

$\begin{array}{lllll}\text { Octachlorodibenzo-p-dioxin isomers } & 3268-87-9 & 1.59 \mathrm{E}-01 & 8.00 \mathrm{E}+01 & 2.0 \mathrm{E}-03\end{array}$

Metals

Copper

Manganese

Mercury

$\begin{array}{ll}7440-50-8 & 4.63 \mathrm{E}-01 \\ 7439-96-5 & 9.82 \mathrm{E}-01 \\ 7439-97-6 & 2.25 \mathrm{E}-01\end{array}$

$8.50 \mathrm{E}+00$

$6.20 \mathrm{E}+01$

2.20E-02

(1) Receptor exposure from Table 4.29.

(2) Toxicity reference value from table 4.30.

(3) Ecological quotient calculated as $\mathrm{EQ}=$ exposure rate / toxicity reference value with $E Q<1$, no effects expocted

$1<\mathrm{EQ}=<10$, small potential for effects

$10<\mathrm{EQ}=<100$, porential for greater exposure to result in effects, and

$\mathrm{EQ}>100$, highest potential for effects.

BOLD: represents receptor EQ $>=1$.

- : no EQ could be calculated, as no toxicity data could be found. 
TABLE 4.37

CALCULATION OF SEDIMENT ECOLOGICAL QUOTIENTS

- BRP 231-F/1F and RP 231-2F - WETLAND

CURRENT AND FUTURE CONDITIONS

\begin{tabular}{|c|c|c|c|c|}
\hline & CAS No. & $\begin{array}{c}\text { Oldfield Mouse } \\
\text { Exposure (mg/kg/day) }\end{array}$ & $\begin{array}{c}\text { Toxicity Reference } \\
\text { Value (mg/kg/day) }\end{array}$ & $\begin{array}{c}\text { Ecołogical } \\
\text { Quotient }\end{array}$ \\
\hline
\end{tabular}

\section{Dioxing/furans}

Octachlorodibenzo-p-dioxin isomers

3268-87-9

$1.59 \mathrm{E}-01$

8.00E+01

2.0E-03

\section{Metals}

Copper

Manganese

7440-50-8

7439-96-5

4.63E-01

9.82E-01

2.25E-01

\begin{tabular}{lr}
$8.50 \mathrm{E}+00$ & $5.4 \mathrm{E}-02$ \\
$6.20 \mathrm{E}+01$ & $\quad \begin{array}{r}1.6 \mathrm{E}-02 \\
2.20 \mathrm{E}-02\end{array}$ \\
\hline $1.0 \mathrm{E}+01$ \\
\hline
\end{tabular}

(1) Receptor exposure from Table 4.29.

(2) Toxicity reference value from table 4.30 .

(3) Ecological quotient calculated as $\mathrm{EQ}=$ exposure rate / toxicity reference value with $\mathrm{EQ}<1$, no effects expected

$1<E Q=<10$, small potential for effects

$10<E Q=<100$, potential for greater exposure to result in effects, and $E Q>100$, highest potential for effects.

\section{BOLD: represents receptor EQ $>=1$.}

- : no EQ could be calculated, as no toxicity data could be found. 
BASELINE RISK ASSESSMENT

F-AREA BURNING/RUBBLE PITS AND R RoBLE PIT
WSRC-TR-94-108

REV. 1.2, MARCH 1996

SECTION 5

SUMMARY

FINAL 


\subsection{SUMMARY .}

The F-Area BRPs Operable Unit consists of two burning/rubble pits and one rubble pit that were used prior to 1978 for the disposal of a variety of wastes. As a result of past disposal activities, contaminants may have been released from the pits via infiltration and percolation through deep soils to groundwater, surface water runoff, vegetative uptake, and volatile or particulate emissions from soil. To evaluate risks to human and ecological receptors, a BRA was performed. This section summarizes the results of the BRA.

Chemicals of potential concern (COPCs) were identified ...1lowing data evaluation and quality screening against unit-specific background concentrations and risk-based concentrations. COPCs were identified for both current and hypothetical future conditions, in which deeper soils may be excavated and spread on the surface. Current and future human and ecological receptors were identified and a conceptual site model was developed to determine potential routes of exposure.

\subsection{Human Health Risk Assessment}

Both current and hypothetical future receptors were quantitatively evaluated in the human health BRA. Current receptors include visitors to the units. Hypothetical future receptors include potential on-unit residents and workers.

Current visitors are expected to be exposed to surface soils ( 0 to $0.6 \mathrm{~m}=0$ to $2 \mathrm{ft}$ ) as well as media in the ephemeral wetland area (surface water and sediment). Tables 5.1 and 5.2 present the overall risks and hazards to the current on-unit visitor at BRP 231-F/1F and RP 231-2F, respectively.

Unrestricted future development of the site is assumed, per EPA guidance (EPA, 1989a). Consequently, risks were derived for both future residents and workers although, according to the Savannah River Site: Future Use Project Report (January 1996), "residential uses of SRS land should be prohibited." Additionally, the report recommends that the F-Area BRPs and RP be located within the future use "Industrial (Nuclear) Zone." Consequently, the only realistic future receptor are the workers. The potential risks and hazards to hypothetical future residents, therefore, were derived for comparative purposes. 
Both the residents and workers are assumed to be exposed to surface soils ( 0 to $0.6 \mathrm{~m}=0$ to $2 \mathrm{ft}$ ) and groundwater. Given that future use could result in excavation of the unit during development, these receptors were also assumed to be exposed to soils located in the 0 to $1.2 \mathrm{~m}$ $(0$ to $4 \mathrm{ft})$ depth. Risks and hazards were derived for both the 0 to $0.6 \mathrm{~m}(0$ to $2 \mathrm{ft}$ ) and 0 to $1.2 \mathrm{~m}$ ( 0 to $4 \mathrm{ft}$ ) exposure scenarios. Risks reported in summary results for the soils are the maximum of the 0 to $0.6 \mathrm{~m}(0$ to $2 \mathrm{ft}$ ) and 0 to $1.2 \mathrm{~m}(0$ to $4 \mathrm{ft}$ ) depths. Hypothetical future residents were also assumed to be exposed to homegrown produce as well as media located in the ephemeral wetland area (surface water and sediment).

The overall risks and hazards to the hypothetical future resident and workers at BRP 231-F/1F and RP 231-2F are presented in Tables 5.3, 5.4, 5.5 and 5.6.

Tables 5.7, 5.8, 5.9 and 5.10 present the risks and hazards associated with the following exposure units: soil at BRP 231-F/1F, soil at RP 231-2F, groundwater, and media located in the ephemeral wetland (surface water/sediment), respectively. Figures 5-1 through 5-8 visually present the risks and hazards associated with the exposure units at BRP 231-F/1F and RP 231-2F.

\subsubsection{Current Land Use}

\subsubsection{On-Unit Visitor Carcinogenic Risk}

On-unit visitors were assumed to be exposed to soil and surface water/sediment located in the ephemeral wetland area. At BRPs 231-F/1F, and RP 231-2F, total carcinogenic risk (radiologic and nonradiologic) for the current on-unit visitor were $6 \times 10^{-7}(231-\mathrm{F} / 1 \mathrm{~F})$ and $8 \times 10^{-7}(231-2 \mathrm{~F})$, neither of which exceeded the EPA target range of $10^{-6}$ to $10^{-4}$.

\subsubsection{On-Unit Visitor Noncarcinogenic Hazard}

Hazard indices for exposure of the on-unit visitor for all media and all pathways were 0.02 (231F/1F) and $0.004(231-2 F)$, indicating that adverse effects are not likely to occur in receptors exposed to soil or surface water/sediment. 


\subsubsection{Future Land Use}

\subsubsection{Resident Carcinogenic Risks}

The hypothetical future resident was assumed to be exposed to soil, groundwater, homegrown produce and surface water/sediment located in the ephemeral wetland area. At BRPs 231-F/1F, total carcinogenic risk (nonradiologic and radiologic) values for all media and all pathways for the future on-unit resident (adult and child) were $2 \times 10^{-4}$ for both the 0 to $0.6 \mathrm{~m}(0$ to $2 \mathrm{ft}$ ) and 0 to $1.2 \mathrm{~m}$ ( 0 to $4 \mathrm{ft}$ ) scenarios. At RP 231-2F, the total risk was $1 \times 10^{-4}$ for the 0 to $0.6 \mathrm{~m}(0$ to $2 \mathrm{ft})$ scenario and $2 \times 10^{-4}$ for the 0 to $1.2 \mathrm{~m}(0$ to $4 \mathrm{ft})$ scenario. The risks for both units exceed the EPA target risk range of $10^{-4}$ to $10^{-6}$. These increased risks primarily resulted from potential exposure to radionuclides (I-129, Ra-226, Ra-228, Sr-90, Tc-99, tritium) and nonradionuclides (carbon tetrachloride, dichloromethane, tetrachloroethylene, arsenic) in groundwater. Exposure to soil, homegrown produce and surface water/sediment did not result in increased risks to the future resident.

\subsubsection{Resident Noncarcinogenic Hazards}

Total noncarcinogenic hazard indices, summing all exposure media, were 3 for the future residents (adult and child) exposed to both 0 to $0.6 \mathrm{~m}$ ( 0 to $2 \mathrm{ft}$ ) and 0 to $1.2 \mathrm{~m}(0$ to $4 \mathrm{ft}$ ) soil intervals at BRPs $231-\mathrm{F} / 1 \mathrm{~F}$ and 5 for residents exposed to these intervals at RP 231-2F. Both indices exceeded the EPA target of 1 . These increased hazards resulted primarily from potential exposure to chemicals (carbon tetrachloride, arsenic, cadmium, manganese) in groundwater, and potential exposure to PCB-1254 in the soil at RP 231-2F. Exposure to homegrown produce and surface water/sediment did not result in increased hazards to the future resident.

\subsubsection{Commercial/Industrial Worker Carcinogenic Risks}

The hypothetical future worker was assumed to be exposed to soil and groundwater at the units. Total carcinogenic risk (radiologic and nonradiologic) values for the future on-unit commercial/industrial worker was $4 \times 10^{-5}$ for workers exposed to both the 0 to $0.6 \mathrm{~m}$ ( 0 to $2 \mathrm{ft}$ ) and 0 to $1.2 \mathrm{~m}$ ( 0 to $4 . \mathrm{ft}$ ) intervals at both BRPs $231-\mathrm{F} / 1 \mathrm{~F}$ and $\mathrm{RP} 231-2 \mathrm{~F}$. This risk falls within the EPA target risk range of $10^{-4}$ to $10^{-6}$. 


\subsubsection{Commercial/Industrial Worker Noncarcinogenic Hazards}

Noncarcinogenic indices for the future worker at BRPs 231-F/1F were 0.4 for both the 0 to $0.6 \mathrm{~m}$ $(0$ to $2 \mathrm{ft})$ and 0 to $1.2 \mathrm{~m}(0$ to $4 \mathrm{ft})$. intervals and 0.5 at RP $231-2 \mathrm{~F}$ for both the 0 to $0.6 \mathrm{~m}(0$ to 2 $\mathrm{ft}$ ) and 0 to $1.2 \mathrm{~m} \mathrm{(0} \mathrm{to} 4 \mathrm{ft})$ intervals. Neither of these hazard indices exceeded 1.0, indicating that adverse effects resulting from exposure to media at the units are not expected.

\subsubsection{Uncertainties}

The evaluation of chemical and radiological risks to human health and ecological receptors presented in this baseline risk assessment was, by necessity, based on a number of assumptions. In addition, many uncertainties are inherent in the risk assessment process. This section briefly discusses the uncertainties associated with the BRA and their potential impact on the results contained herein.

\subsubsection{Uncertainties Leading to Potential Over Estimation of Risk}

Several potential sources of contamination located adjacent to the F-Area BRPs have documented soil and groundwater contamination associated with them. Contaminants from these sources may be inadvertently attributed to the BRPs, thereby overestimating the risk contribution from the site.

Radiological uncertainties are tentative due to conservative modeling assumptions concerning leaching of iodine-129 and technetium-99 from deep soils to groundwater. Iodine-129 and technetium-99 are not currently detected, nor have they been historically detected, in the groundwater at the unit and it is unlikely that they will reach detectable levels from leaching in the future.

The summing of risk across pathways is inherently uncertain because:

a. Summing the risk across pathways combines risks as if they were additive.

b. Summing risk assumes that all concentrations of COPCs will be at their reasonable maximum concentrations for all pathways for all times ( i.e., there is no depletion of sources of contamination). Present day concentrations used in the BRA are not reduced 
to account for degradation, dilution, or dispersion on any media evaluated. However, the leaching to groundwater from deep soils did consider chemical and radioactive degradation mechanisms and dilution in the aquifer.

c. Scenario specific assumptions concerning exposure pathways, duration of exposure, and COPCs contribute to a conservative estimation of risk.

\subsubsection{Uncertainties Leading to Potential Under Estimation of Risk}

It should be noted, that for any subsurface characterization, short of complete excavation, there will always remain some level of uncertainty as to the completeness of the characterization. The sampling strategy at the F-BRP was developed utilizing professional judgment upon thorough review of process history and historical data, in conjunction with financial, logistic, administrative, regulatory, and schedule limitations.

In the COPC screening process used in this BRA, chemicals were eliminated following screening against background, risk-based concentrations, essential human nutrients and frequency of detection. Limited background data in soil and groundwater, and the lack of background data for surface water and sediment, resulted in a uncertainty in the background screening portion of the COPC process. Very few chemicals in soil and groundwater, however, were eliminated using the background screening process. The majority of the chemicals were eliminated following the screening against risk-based concentrations. Risk-based concentrations are dynamic and, therefore, uncertainty in these values cannot be eliminated. Eliminating contaminants in the COPC screening process can lead to lower estimates of potential health effects than inclusion of all analytes.

The major source of uncertainty contributing to the potential underestimation of risk to current and future receptors was due to the uncertainty inherent in any characterization effort. That is, since it is not economically justifiable to sample and analyze each gram of material in a unit, it is necessary to devise a sampling program which provides a reasonable certainty with respect to the nature and extent of contamination present in a unit. While the characterization work did not eliminate this uncertainty, it was performed at a reasonable level of uncertainty. The nature and extent of contaminants at the unit have been adequately characterized. 


\subsection{Ecological Risk Assessment}

The purpose of the ecological risk assessment component of the BRA was to evaluate the likelihood that adverse ecological effects are occurring or may occur as a result of exposure of ecological components to unit-related chemicals. The methodology used in this assessment was based on and complies with the intent of the Draft Ecological Risk Assessors' Guide for Evaluation of Waste Units on the Savannah River Site (WSRC, 1993), the Framework for Ecological Risk Assessment (EPA, 1992f), and the Risk Assessment Guidance for Superfund, Volume II, Environmental Evaluation Mamual (EPA, 1989b). Thз methodology consisted of four main elements: characterization of the unit, including the nature of the contamination that may exist and the ecological components that may be affected; exposure assessment, describing the magnitude and patterns of exposure; effects assessment, evaluating the potential toxicity of contaminants based on comparison of estimated intakes to toxicity reference by calculation of quotients, as well as an evaluation of other chemical characteristics; and risk characterization, integrating the results of the preceding elements in estimating risk, analyzing uncertainty, and interpreting the ecological significance of the assessment results.

Ecological COPCs were identified following data evaluation and quality screening, screening of inorganics against unit-specific background levels, and screening against toxicological benchmarks. COPCs were present in soil from the BRPs (231-F/1F) and RP (231-2F) and in sediment from the small ephemeral wetland area that receives runoff from the BRPs (231-F/1F) area. COPCs were identified in soil for both current and hypothetical future conditions, in which deeper soils may be excavated and spread on the surface.

Based on characterization of the environmental setting and identification of potential receptor organisms, a conceptual site model was developed to determine the complete exposure pathways through which receptors could be exposed to COPCs. Assessment endpoints were selected to represent ecological values to be protected with regard to exposure to unit-related chemicals, and measurement endpoints were selected that could be measured and related to the assessment endpoints.

The proximate assessment endpoints chosen were protection of small mammal populations at the unit, as represented by the native oldfield mouse population, and protection of populations of amphibians that may reproduce in the wetland and be exposed to surface water during the limited period of surface water availability. The ultimate assessment endpoint chosen was protection of 
the overall health and biodiversity of the community of species inhabiting the vicinity of the unit. Because these assessment endpoints could not be directly measured, measurement endpoints were chosen that could be extrapolated to the assessment endpoints. The measurement endpoints used were the best available, most relevant and appropriate toxicity data from studies on native species or surrogate laboratory species, or ARARs based on such data. The studies were preferentially chosen to represent chronic exposures and effects likely to impact population levels, such as increased mortality or decreased fecundity of individuals.

Food chain intakes of COPCs (terrestrial) or concentrations of COPCs (aquatic) were derived for the receptors, and quotients were calculated to represent the ratio of estimated intake to the intake (terrestrial) or concentration (aquatic) at which toxicity is likely to occur. The threshold for ecological effects is generally considered to be a quotient of 1 , and greater quotients indicate greater likelihood of effects. Those COPCs with quotients exceeding were further evaluated as part of the risk estimation in order to estimate the likelihood that they might pose risk to assessment endpoints.

The results of the risk estimation step of the risk characterization indicated that, although several metals in soil (barium, chromium, mercury, lead), and mercury in sediment, had quotients exceeding a value of 1 , only mercury actually posed more than minimal risk to assessment endpoints. All other COPCs for which toxicity data or ARARs were available had quotients less than 1 , indicating that they do not pose significant risk to assessment endpoints through exposure to $\mathrm{RME}$ concentrations in environmental media.

Only mercury in soil at BRPs $231-\mathrm{F} / 1 \mathrm{~F}$ (under both current and future scenarios) and sediment in the ephemeral wetland was identified in the risk estimation step of the risk characterization as having the potential to pose risk to assessment endpoints for the F-Area BRP Operable Unit. Actual community-level ecological effects are highly unlikely given the small area of the unit, its limited biological productivity and diversity, and its minimal utilization as a food source for predators. As a result, the likelihood of unit-related chemicals causing an ecologically significant decline in populations of native mice at the unit was estimated to be low; the likelihood of significant effects on amphibian populations due to surface water contaminants at ' the wetland was estimated to be negligible; and there was concluded to be no likelihood that significant impacts to the community of species in the vicinity of the unit will occur. 


\subsection{Remedial Goal Options}

Chemical-specific remedial goal options (RGOs) are concentration goals for individual chemicals for specific media and land use scenarios at CERCLA sites. General sources of chemical-specific RGOs include: (1) concentrations based on ARARs, and (2) concentrations based on risk values produced from the risk assessment.

\subsubsection{Human Health RGOs}

The COCs identified from the human health portion of the baseline risk assessment include several organics (carbon tetrachloride, dichloromethane, tetrachloroethylene), metals (arsenic, cadmium, manganese) and radionuclides (iodine-129, radium-226, radium-228, technetium-99, tritium) in groundwater and PCB-1254 in soil at RP 231-2F. These chemicals and radionuclides were evaluated to determine whether Remedial Goal Options (RGO) were appropriate. A comparison to Applicable and Relevant and Appropriate Requirements (ARARs) was completed to establish whether the risk drivers were present in concentrations that exceeded regulatory levels. Although a chemical or radionuclide may result in a calculated risk or hazard in the risk assessment, a comparison to ARARs is necessary to establish whether the concentrations exceed regulatory standards. Regulatory standards may be higher than risk-based standards because regulatory standards take into account the feasibility of achieving a risk-based goal. If a risk-based goal is unachievable, an achievable value is designated as the regulatory standard. RGOs were derived for those chemicals that exceeded the ARARs.

Given that only soil located at the RP 231-2F and area-wide groundwater exceeded EPA targets for carcinogenic and noncarcinogenic effects in the BRA, only these media will be evaluated to determine whether chemical-specific RGOs are appropriate.

\subsubsection{Groundwater}

For groundwater, ARARs included Federal and State of South Carolina maximum contaminant levels (MCLs). Neither iodine-129 nor technetium-99 were detected in groundwater. Their inclusion as risk drivers in the BRA resulted from the modeling of soil leaching to groundwater. Given that they have not and are not detected in groundwater, a comparison to ARARs and derivation of an RGO are not appropriate. 


\subsubsection{Derivation of RGO values}

\section{Hypothetical Future Industrial Worker}

Exposure to Surface Soil - Nonradiological

Total Risk $=$ Risk from Dermal Contact + Risk from Ingestion + Risk from Inhalation

Exposure to Groundwater - Nonradiological

Total Risk $=$ Risk from Ingestion

\section{Hypothetical Future Resident Adult and Child}

Exposure to Surface Soil - Nonradiological

Total Risk $=$ Risk from Dermal Contact + Risk from Ingestion + Risk from Inhalation

Exposure to Groundwater - Nonradiological

Total Risk $=$ Risk from Ingestion + Risk from Inhalation (groundwater VOCs only)

The results of the risk-based RGO calculations are provided in Table 5.12. RGOs are expressed as a range of concentrations in relation to the carcinogenic risk range of $1 \times 10^{-4}$ to $1 \times 10^{-6}$ and/or hazard indices of $0.1,1$, and 3 . The estimated RGOs for radionuclides may be lower than site-wide background concentrations. Table 5.13 provides a summary of the COCs and identifies the maximum concentration of each $\mathrm{COC}$ in a given media.

RGOs are estimated to provide remedial engineers an early indication of the COCs which are primary contributors to risk and specific media of concern. RGOs are not final remediation goals and should not be regarded as such.

\subsubsection{Ecological Risk-Based RGOs}

Ecological risk-based RGOs were not estimated for the F-Area BRPs Operable Unit since only mercury in soil at BRPs $231-\mathrm{F} / 1 \mathrm{~F}$ and the ephemeral wetland sediment was identified in the risk estimation step of the risk characterization as having the potential to pese risk to assessment 
endpoints for the unit. Actual community-level ecological effects are highly unlikely given the small area of the unit, its limited biological productivity and diversity, and its minimal utilization as a food source for predators. As a result, the likelihood of unit-related chemicals causing an ecologically significant decline in populations of native mice at the unit was estimated to be low; the likelihood of ecologically significant effects on amphibian populations due to surface water contaminants at the wetland was estimated to be negligible; and there was concluded to be essentially no likelihood that ecologically significant impacts to the community of species in the vicinity of the unit will occur.

\subsection{Conclusions}

Exposures to current unit visitors, hypothetical future workers and residents, and ecological receptors were evaluated in the BRA. Risk to the human and ecological receptors generally fell below or within the EPA target risk and hazard ranges. Chemicals of concern (COCs) were developed for chemical or radiological exposures that exceeded the EPA target ranges. Remedial Goal Options (RGOs) were derived for the specific exposure pathways that exceeded the EPA target ranges.

In the human health baseline risk assessment, only PCB-1254 in Rubble Pit 231-2F surface soil exceeded the EPA noncancer hazard index (HI) threshold of unity (1.0). PCB-1254 was detected in only 1 of 8 samples in the 0 to $0.6 \mathrm{~m}$ ( 0 to $2 \mathrm{ft}$ ) soil interval at RP 231-2F. The maximum detected value of PCB-1254 (2.87 mg/kg) was quantitatively evaluated for human health risk over the entire pit area, which likely overestimates the risk from PCBs at RP 231-2F. Given the low frequency of detection and the low level of the maximum detected concentration, it is unlikely that PCBs are a unit-wide contaminant at levels that would cause concern for human health protection, but, rather represent an isolated area (or "hot spot") of contamination.

According to the Savannah River Site Future Use Project Report (January, 1996), "residential uses of SRS land should be prohibited." Additionally, the report recommends that the F-Area BRPs and RP be located within the future use "Industrial (Nuclear) Zone." Consequently, the most reasonable future use of the unit is industrial and the concentrations of PCB-1254 detected in the soil do not pose an unacceptable hazard to future workers.

According to $A$ Guide on Remedial Actions at Superfund Sites with PCB Contamination (EPA, 1990a), soil action levels for PCBs in soil are $1 \mathrm{mg} / \mathrm{kg}$ for residential land use and $10-25 \mathrm{mg} / \mathrm{kg}$ for industrial land use. The $1 \mathrm{mg} / \mathrm{kg}$ action level for residential exposure. was exceeded and, 
therefore, an RGO was derived for PCB-1254. Given that the most likely future land use will be industrial, however, the action level of $10-25 \mathrm{mg} / \mathrm{kg}$ for the industrial worker is more appropriate. The maximum detected concentration of PCB-1254 does not exceed the 10-25 $\mathrm{mg} / \mathrm{kg}$ action level for industrial workers.

Groundwater contaminants that caused the hypothetical future residential exposure to equal the EPA cancer risk threshold of $1 \times 10^{-4}$ included carbon tetrachloride, dichloromethane, and tetrachloroethylene. While this is the risk result, the maximum detected level of tetrachloroethylene is in upgradient wells and the maximum detected levels of dichloromethane are in sidegradient wells, indicating an F-Area-wide volatile organic chemical contamination plume that makes it difficult to distinguish the contaminants detected in groundwater at the BRPs from other sources in the general area. Additionally, the volatile organic chemicals driving the risk will biodegrade within the next few years and do not, therefore, appear as risk drivers in the future groundwater levels derived from modeling. Also, since the area is located within the future use industrial (nuclear) zone and there is no unacceptable risk to future workers, it is unlikely that any receptors will ever be exposed to these levels of organic contaminants in the groundwater.

In the ecological risk assessment, only mercury in soil at BRPs $231-\mathrm{F} / 1 \mathrm{~F}$ (under both current and future scenarios) and sediment in the ephemeral wetland was identified in the risk estimation step of the risk characterization as having the potential to pose risk to assessment endpoints for the FArea BRP Operable Unit. Actual community-level ecological effects are highly unlikely given the small area of the unit, its limited biological productivity and diversity, and its minimal utilization as a food source for predators. As a result, the likelihood of unit-related chemicals causing an ecologically significant decline in populations of native mice at the unit was estimated to be low; the likelihood of significant effects on amphibian populations due to surface water contaminants at the wetland was estimated to be negligible; and there was concluded to be essentially no likelihood that significant impacts to the community of species in the vicinity of the unit will occur.

In conclusion, given that the F-Area Burning/Rubble Pits and Rubble Pit area is contained within the future use industrial (nuclear) zone at SRS, thereby eliminating the potential for future residential development and the resulting hypothetical residential exposures, there are no current nor realistic future risks to human health that exceed the EPA remedial action thresholds of $1 \mathrm{x}$ $10^{-4}$ risk and/or $1.0 \mathrm{HI}$. Additionally, the marginal utility of the ephemeral wetland as an 
ecological resource makes the potential ecological impact from mercury in sediments inconsequential. The results of this BRA leave no COCs requiring remedial action to protect actual human or sensitive ecological receptors. 


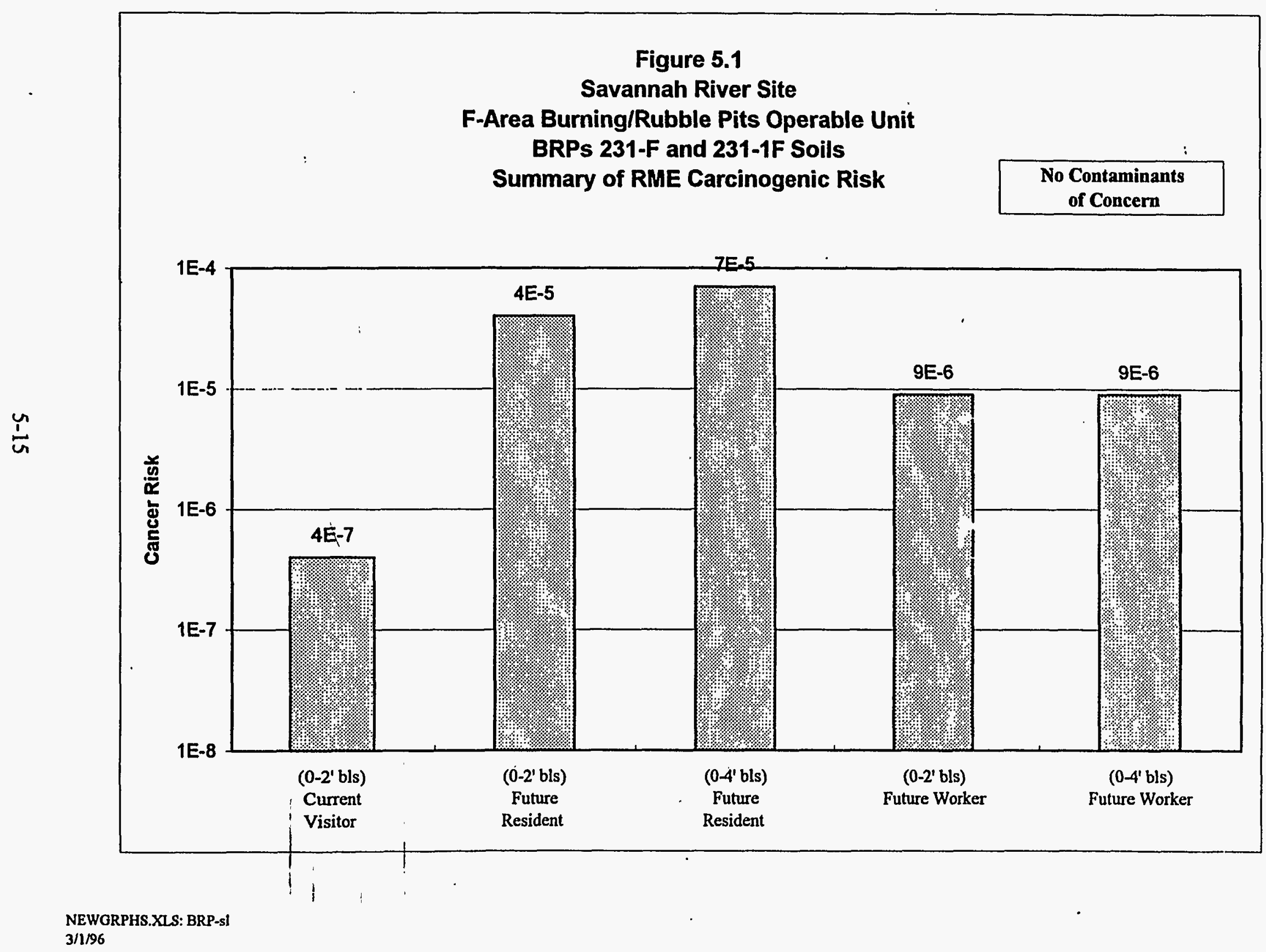




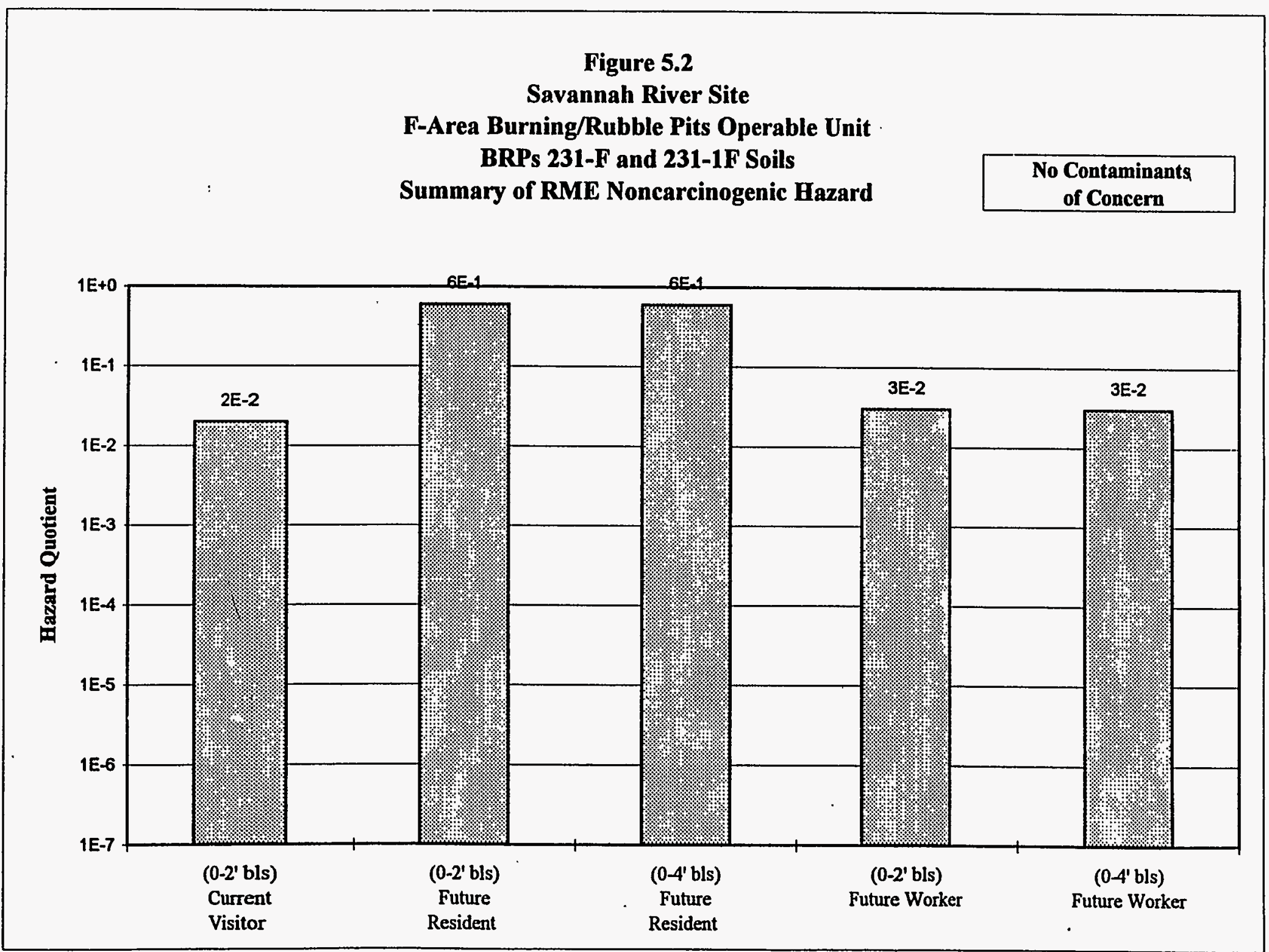

FINAL 


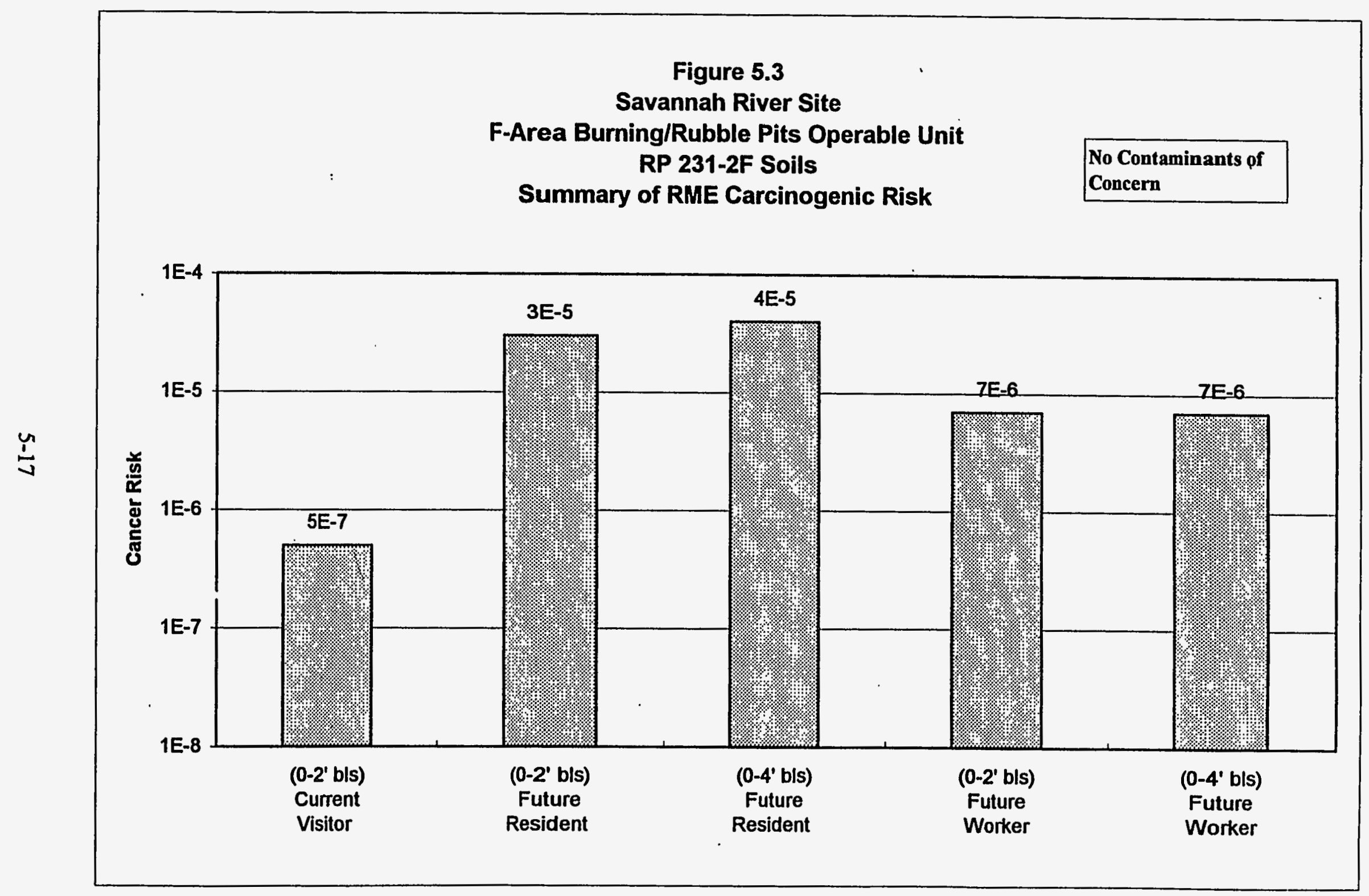

FINAL 


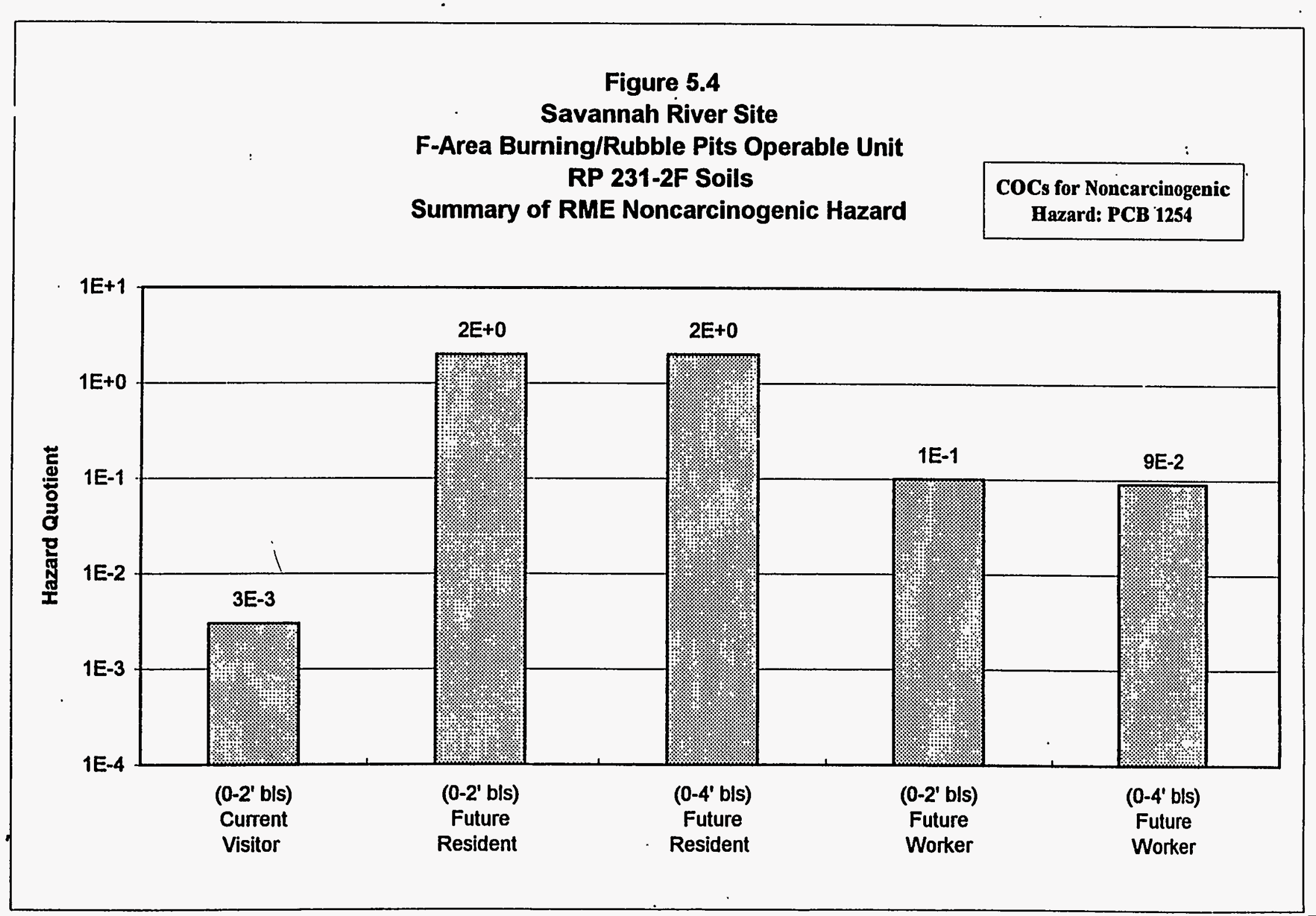

FINAL 


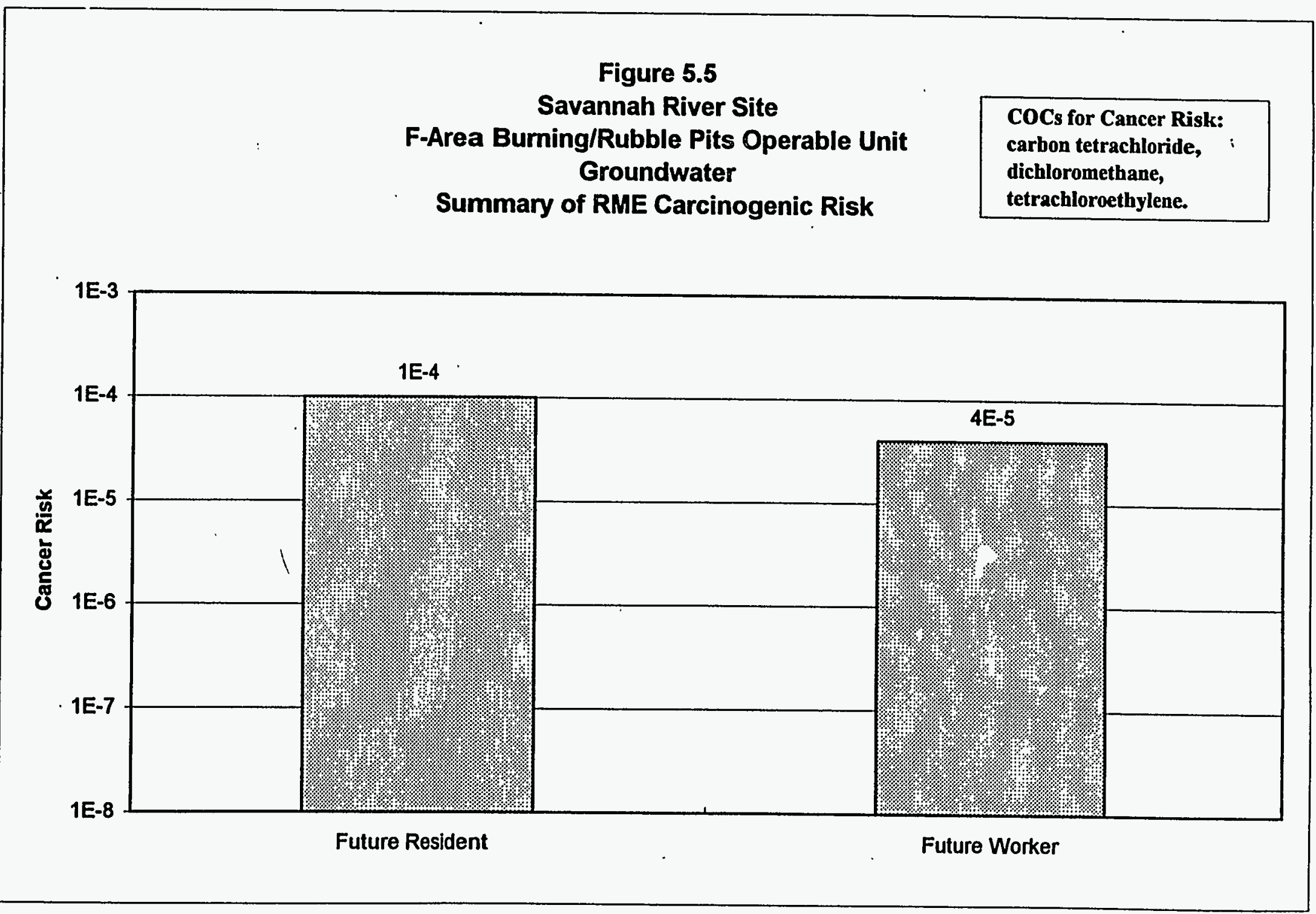




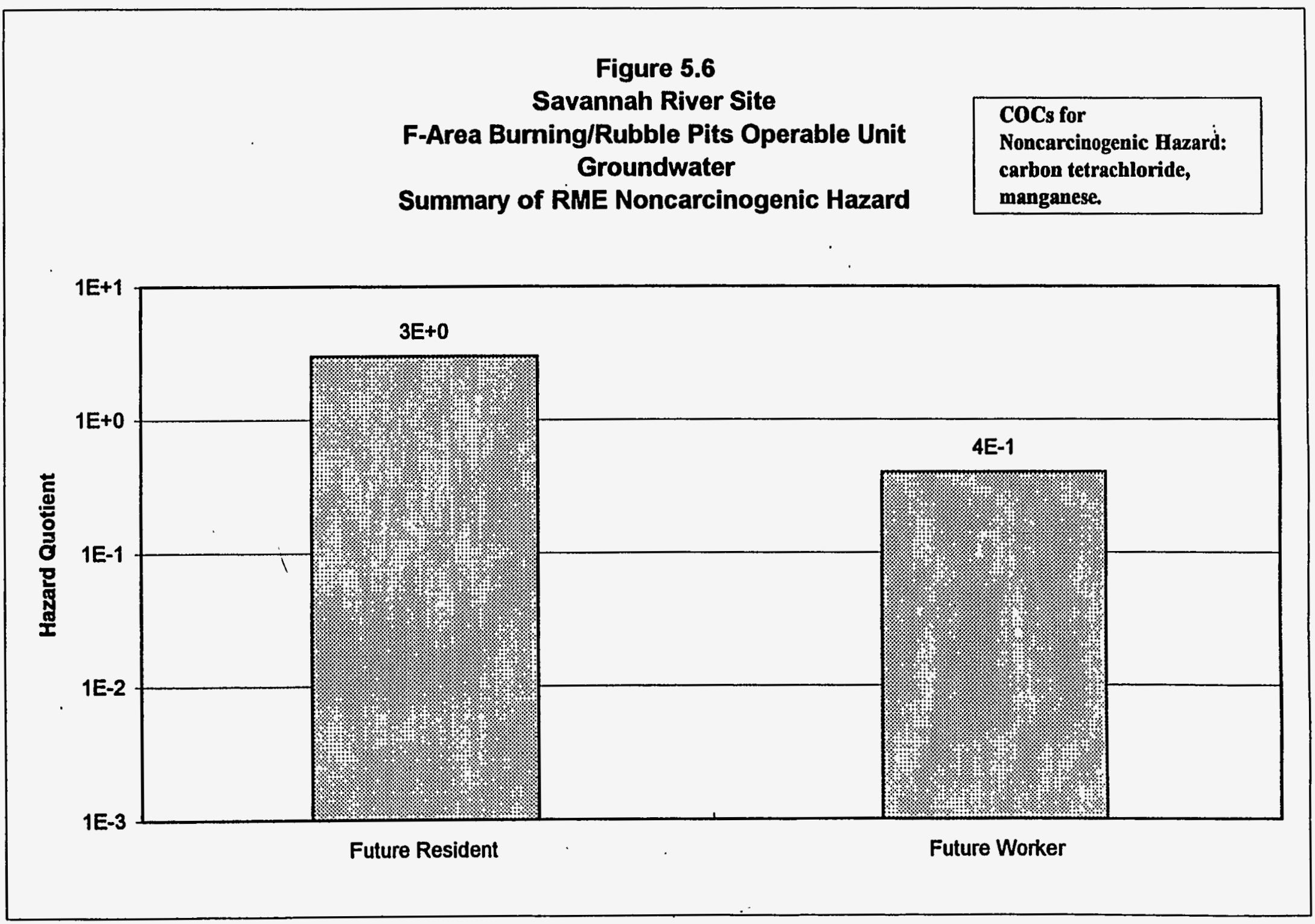




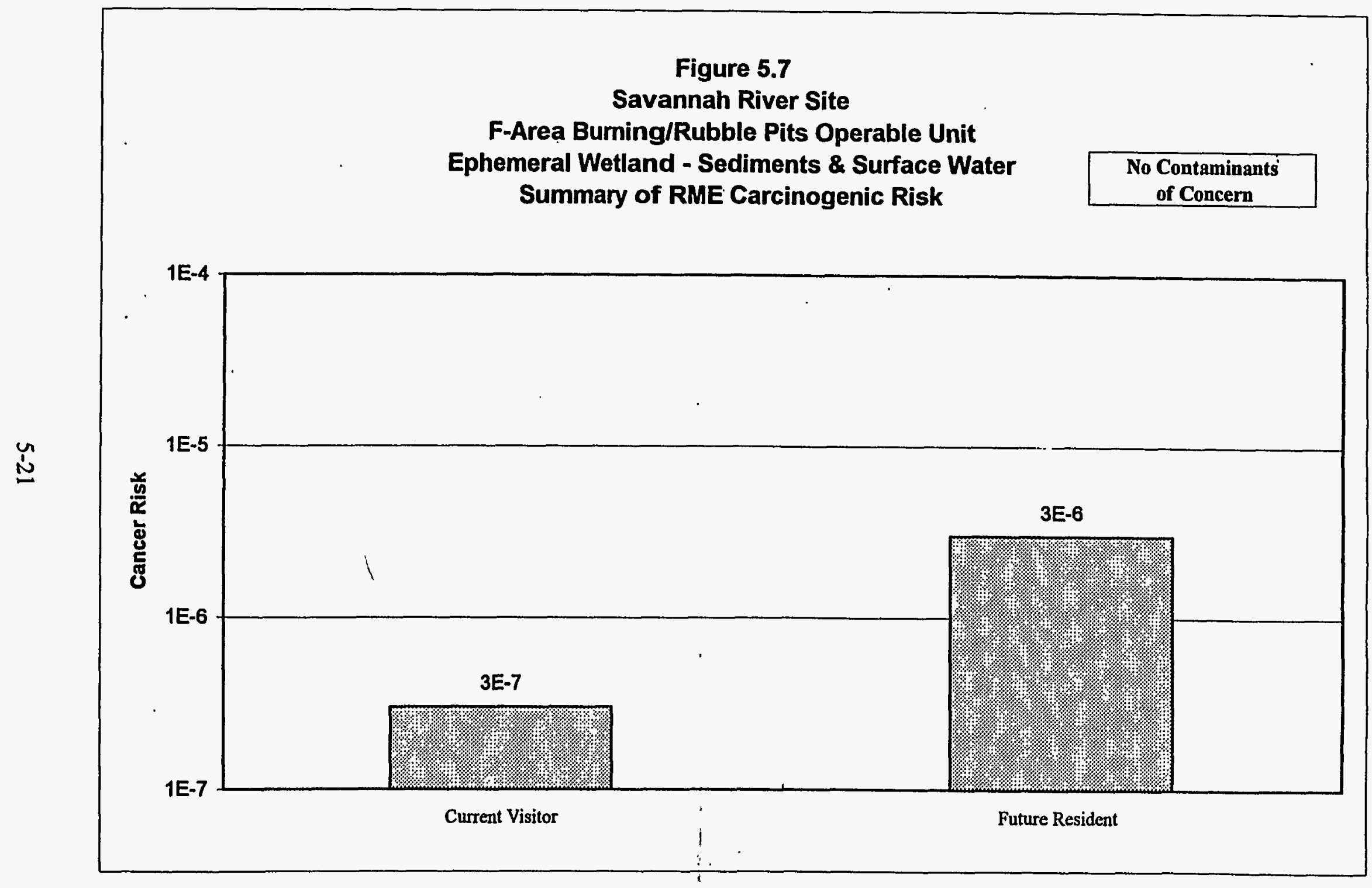




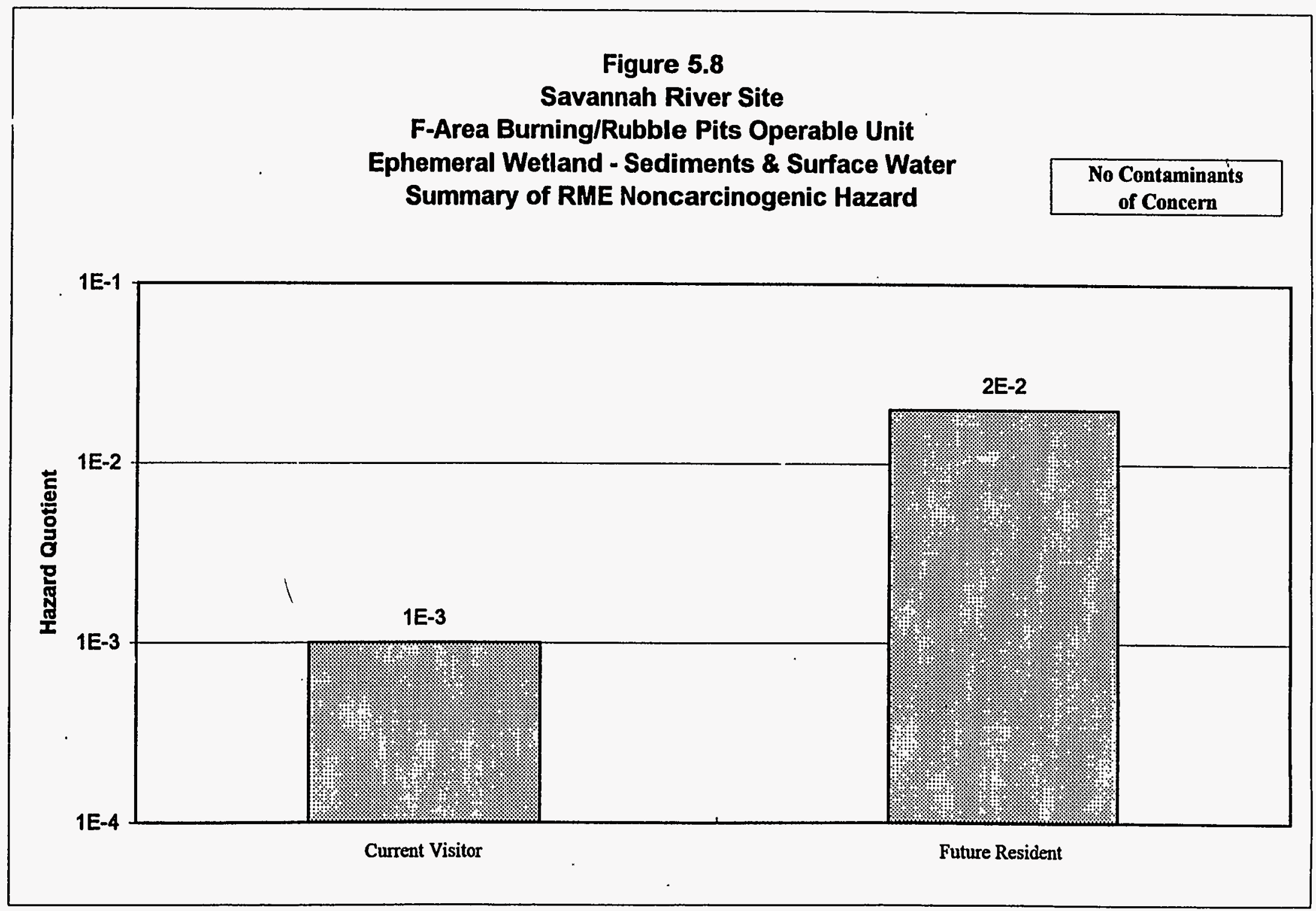

FINAL

NEW' 'HS.XLS: SW-Sd 
Table 5.1

Summary of Carcinogenic Risk and

Noncarcinogenic Hazards for Potential

On-Unit Visitor Exposure Pathways for

Burning Rubble Pits 231-F and 231-1F

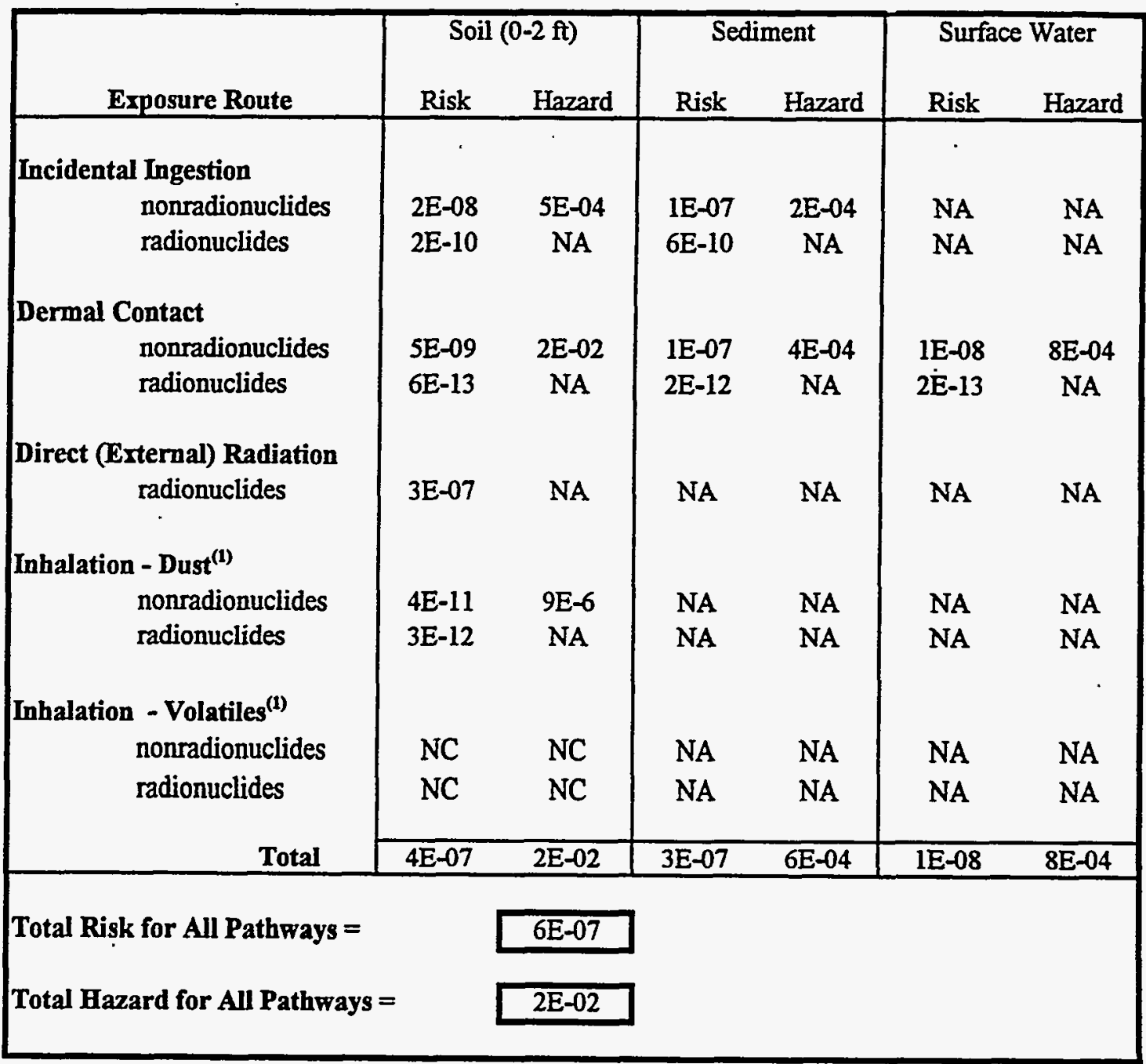

NA - Not Applicable

NC - Not Calculated due to a lack of data

(1) Values for inhalation of dust and volatiles in air are estimated from COPC concentrations in soil. 
Table 5.2

Summary of Carcinogenic Risk and

Noncarcinogenic Hazards for Potential

On-Unit Visitor Exposure Pathways for

Rubble Pit 231-2F

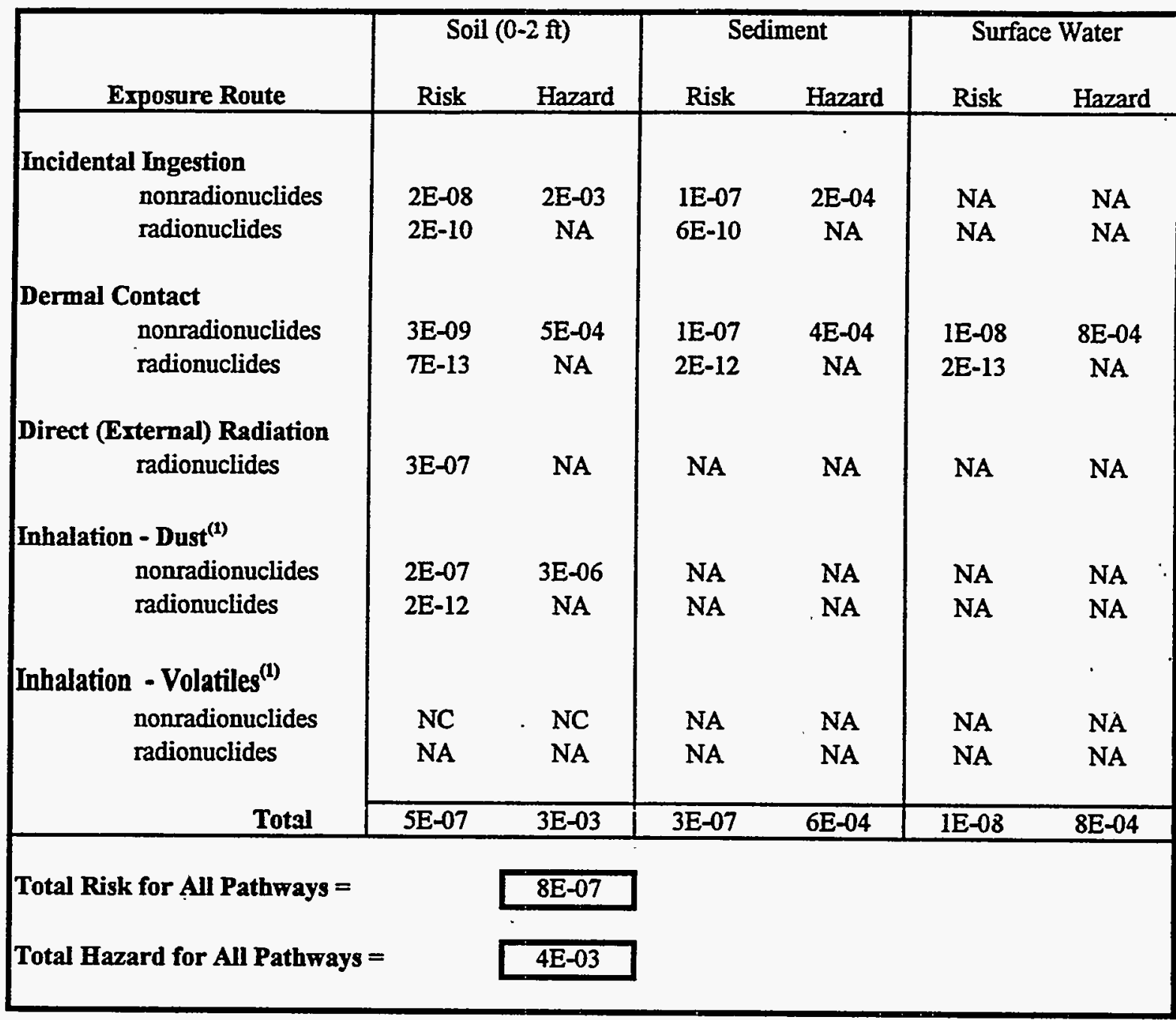

NA - Not Applicable

NC - Not Calculated due to a lack of data

(1) Values for inhalation of dust and volatiles in air are estimated from COPC concentrations in soil. 
Table 5.3

Summary of Carcinogenic Risk and

Noncarcinogenic Hazards for Potential Future

On-Unit Resident Exposure Pathrays for

Burning Rubble Pits 231-F and 231-1F

\begin{tabular}{|c|c|c|c|c|c|c|c|c|c|c|}
\hline \multirow[b]{2}{*}{ Exposure Route } & \multicolumn{2}{|c|}{ Soil $(0-2 \mathrm{ft})$} & \multicolumn{2}{|c|}{ Soil $(0-4 \mathrm{ft})$} & \multicolumn{2}{|c|}{ Groundwater } & \multicolumn{2}{|c|}{ Sediment } & \multicolumn{2}{|c|}{ Surface Water } \\
\hline & Risk & Hazand & Risk & Hazard & Risk & Hazard & Risk & Hazard & Risk & Hazard \\
\hline \multicolumn{11}{|l|}{ Incidental Ingestion } \\
\hline nonradionuclides & $2 \mathrm{E}-5$ & $6 \mathrm{E}-1$ & 2E-5 & $6 \mathrm{E}-1$ & $5 \mathrm{E}-5$ & $2 E+0$ & $1 E-6$ & $1 \mathrm{E}-3$ & $3 E-8$ & 1E-3 \\
\hline radionuclides & $2 \mathrm{E}-7$ & NA & 2.E-7 & NA & $6 \mathrm{E}-5$ & NA & $3 E-12$ & NA & $6 \mathrm{E}-10$ & NA \\
\hline \multicolumn{11}{|l|}{ Dermal Contact } \\
\hline nonradionuclides & $3 \mathrm{E}-6$ & $2 \mathrm{E}-2$ & $3 E-6$ & $3 E-2$ & $2 \mathrm{E}-7$ & $8 \mathrm{E}-2$ & $2 \mathrm{E}-6$ & 3E-3 & $7 \mathrm{E}-7$ & $2 \mathrm{E}-2$ \\
\hline radionuclides & $3 \mathrm{E}-10$ & NA & $3 \mathrm{E}-10$ & NA & $8 \mathrm{E}-10$ & NA & $2 \mathrm{E}-11$ & NA & SE-13 & NA \\
\hline \multicolumn{11}{|l|}{ Direct (Erternal) Radiation } \\
\hline radionuclides & IE-5 & NA & 1.E-5 & NA & NA & NA & NC & NA & NA & NA \\
\hline \multicolumn{11}{|l|}{ Inhalation - Dust( } \\
\hline nonradionuclides & 8E-8. & 2.E-2 & L.E-7 & 1.E-2 & NA & NA & NA & NA & NA & NA \\
\hline radionuclides & $4 \mathrm{E}-10$ & NA & 4.E-10 & NA & NA & NA & NA & NA & NA & NA \\
\hline \multicolumn{11}{|l|}{ Inhalation - Volatiles ${ }^{(1)}$} \\
\hline nonradionuclides & NC & NC & NC & NC & IE-5 & $6 \mathrm{E}-1$ & NA & NA & NA & NA \\
\hline radionuclides & NA & NA & NA & NA & $5 E-6$ & NA & NA & $\mathbf{N A}$ & NA & NA \\
\hline \multicolumn{11}{|l|}{ Ingestion - Leafy Produce ${ }^{(2)}$} \\
\hline nonradionuclides & NA & NA & NA & NA & NA & NA & NA & NA & NA & NA \\
\hline radionuclides & $5 E-7$ & NA & 6.E-6 & NA & NA & NA & NA & NA & NA & NA \\
\hline \multicolumn{11}{|l|}{ Ingestion -Tuberous Produce (2) } \\
\hline nonradionuclides & NA & NA & NA & NA & NA & NA & NA & NA & NA & NA \\
\hline radionuclides & $2 \mathrm{E}-6$ & NA & 4.E-6 & NA & NA & NA & NA & NA & NA & NA \\
\hline \multicolumn{11}{|l|}{ Ingestion - Fruit Produce $($ ) } \\
\hline nonradionuclides & NA & NA & NA & NA & NA & NA & NA & NA & NA & NA \\
\hline radionuclides & $4 \mathrm{E}-6$ & NA & 3.E-5 & NA & NA & NA & NA & NA & NA & NA \\
\hline Total & $4 \mathrm{E}-5$ & 6E-1 & $7 \mathrm{E}-5$ & $6 \mathrm{E}-1$ & $1 \mathrm{E}-4$ & $3 E+0$ & $3 \mathrm{E}-6$ & $4 \mathrm{E}-3$ & $7 \mathrm{E}-7$ & $2 \mathrm{E}-2$ \\
\hline Total Risk for All Pathways = & $0-2 \mathrm{ft}=$ & $2 \mathrm{E}-4$ & & & Total Ha & $\operatorname{ird}$ for $A$ & Pathwa: & & $0-2 \mathrm{ft}=$ & $3 \mathrm{E}+0$ \\
\hline & $0.4 \mathrm{ft}=$ & $2 \mathrm{E}-4$ & & & & & & & $0-4 \mathrm{ft}=$ & $3 E+0$ \\
\hline
\end{tabular}

NA - Not Applicable

NC - Not Calculated duo to a lack of data

(1) Values for inhalation of dust and volatiles in air are estimated from COPC concentrations in soil.

(2) Values for produce are estimated from COPC concentrations in soil. 
Table 5.4

Summary of Carcinogenic Risk and

Noncarcinogenic Hazards for Potential Future

On-Unit Resident Exposure Pathways for

Rubble Pit 231-2F

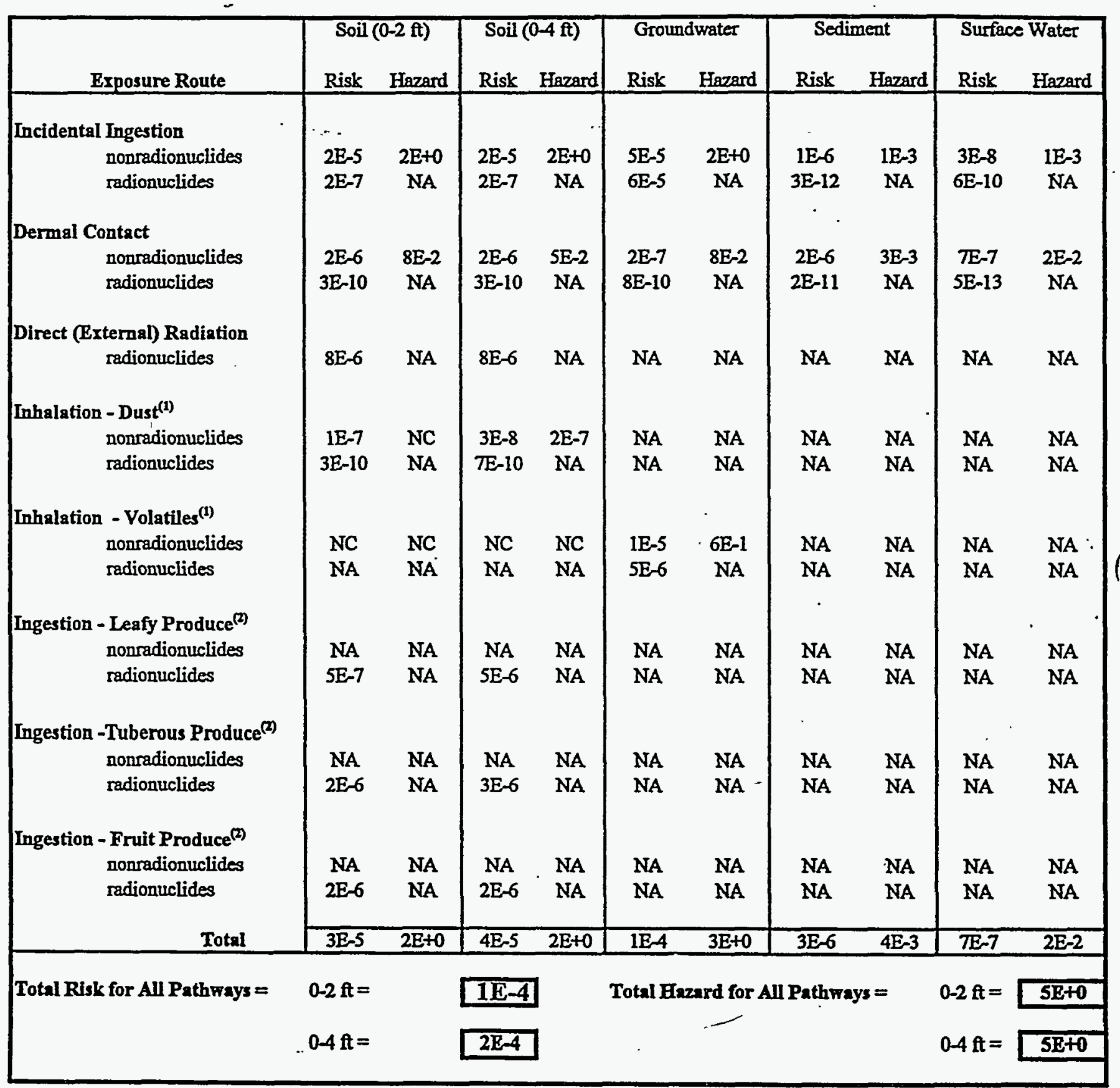

NA - Not Applicable

NC - Not Calculate i due to a lack of dats

(1) Values for inhalation of dust and volatiles in air are estimated from COPC concentrations in soil.

(2) Values for produce are estimated from COPC concentrations in soil. 
Table 5.5

Summary of Carcinogenic Risk and

Noncarcinogenic Hazards for Potential Future

On-Unit Commercial/tndustrial Worker Exposure Pathways for

Burning Rubble Pits 231-F and 231-1F

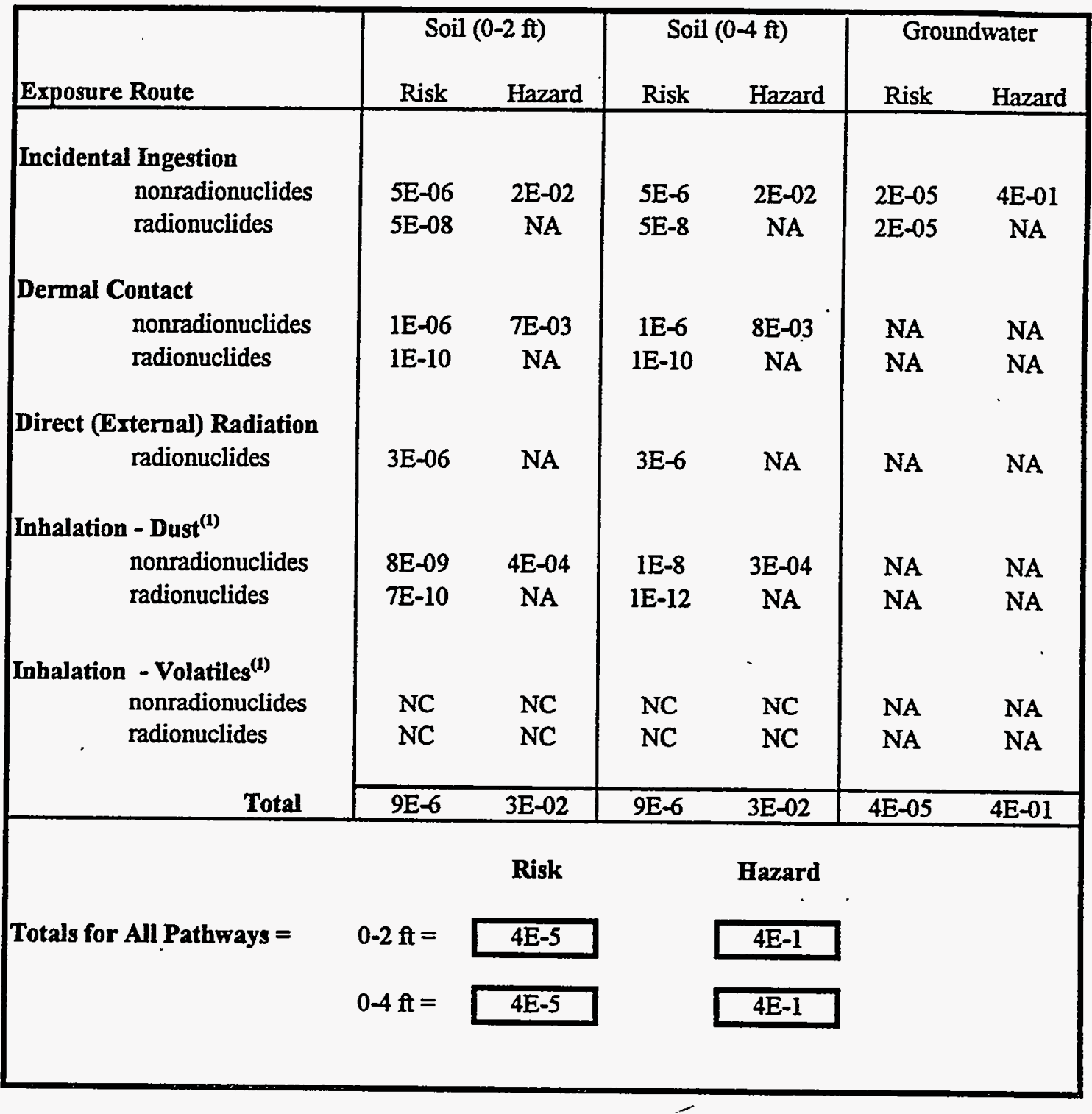

NA - Not Applicable

NC - Not Calculated due to a lack of data

(1) Values for inhalation of dust and volatiles in air are estimated from COPC concentrations in soil. 
Table 5.6

Summary of Carcinogenic Risk and

Noncarcinogenic Hazards for Potential Future

. On-Unit Commercial/Industrial Worker Exposure Pathways for

Rubble Pit 232-2F

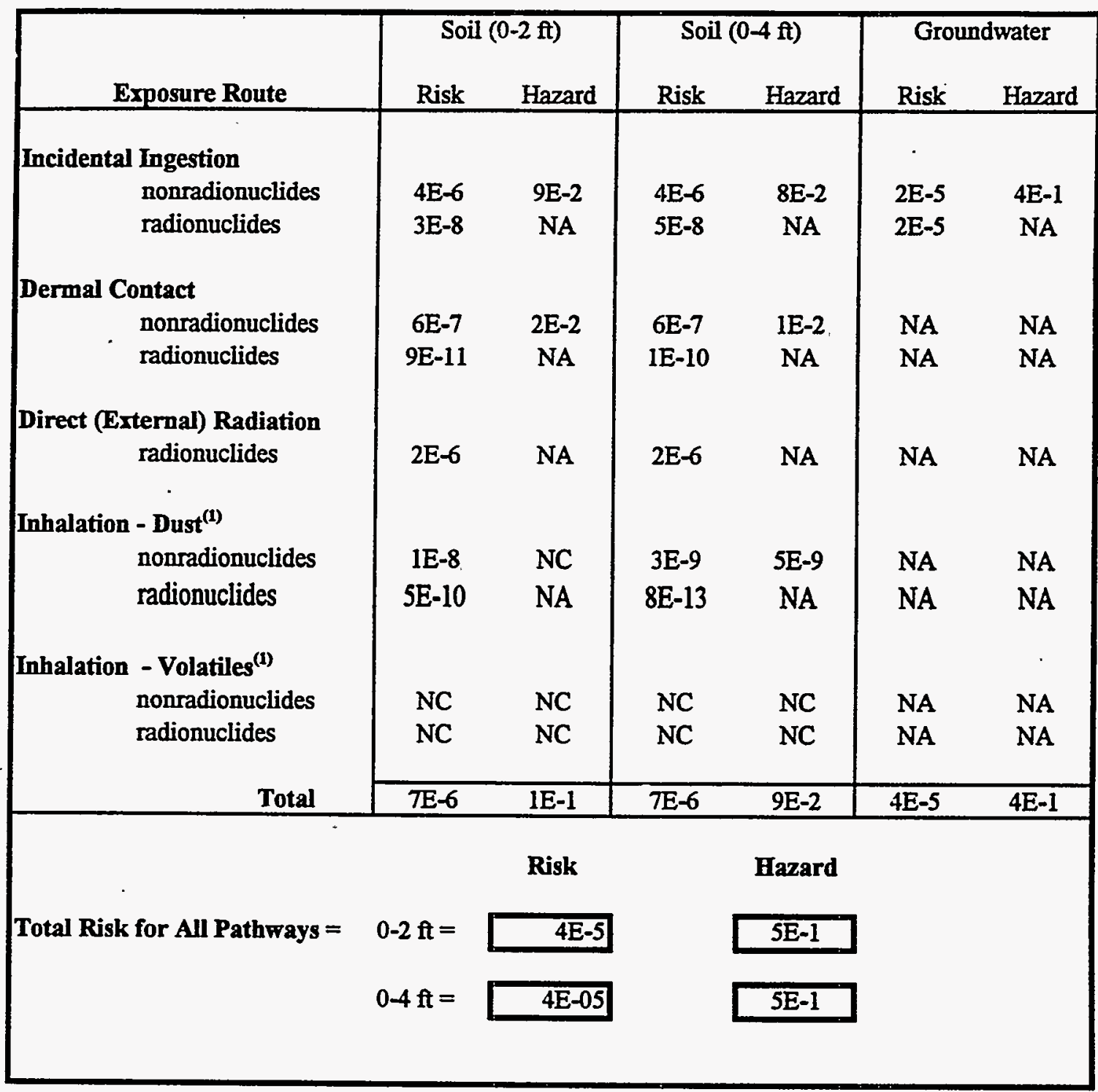

NA - Not Applicable

NC - Not Calculated due to a lack of data

(1) Values for inhalation of dust and volatiles in air are estimated from COPC concentrations in soil. 
Table 5.7

Summary of Carcinogenic Risk and

Noncarcinogenic Hazands for Soll

Current Visitors and Hypothetical Future Resldents and Workers

Burning Rubble Pits 231-F and 231-1 F

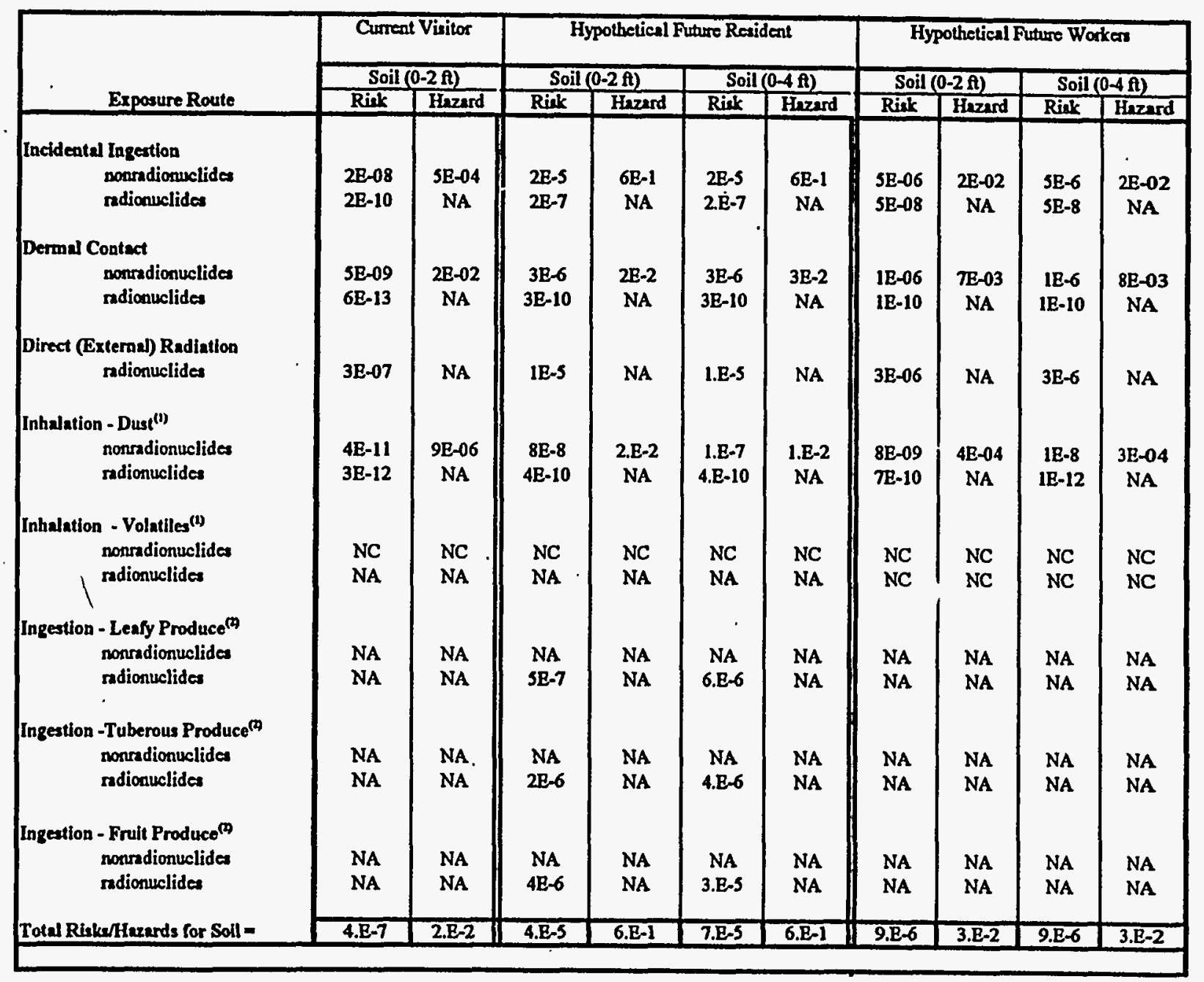

NA - Not Applicable

NC - Not Calculated due to a lack of dats

(1) Values for inhalation of duat and volatiles in air are estimated from COPC concentrations in soil.

FINAL,

(2) Values for produce are extimaled from COPC concentrations in soil. 
Table 5.8

Summary of Carcinogenic Risk and

Noncarcinogenic Hazards for Current and

Hypothetical Future Receptor Exposure Pathways to Soll for Rubble Pit 231-2F

\begin{tabular}{|c|c|c|c|c|c|c|c|c|c|c|}
\hline \multirow{3}{*}{ Exposure Route } & \multirow{2}{*}{\multicolumn{2}{|c|}{$\begin{array}{c}\text { Current On-Site Visitor } \\
\text { Soil (0-2 } \mathrm{A})\end{array}$}} & \multicolumn{4}{|c|}{ Hypothetical Future Resident } & \multicolumn{4}{|c|}{ Hypothetical Future Worker } \\
\hline & & & \multicolumn{2}{|c|}{ Soil $(0-2 \mathrm{~A})$} & \multicolumn{2}{|c|}{ Soil $(04 \mathrm{ft})$} & \multicolumn{2}{|c|}{ Soil $(0-2 \Omega)$} & \multicolumn{2}{|c|}{ Soil $(0-4 \mathrm{~A})$} \\
\hline & & & & & & & & & Risik & \\
\hline $\begin{array}{l}\text { Inctdental Ingertlon } \\
\text { nonradionuclides } \\
\text { radionuclides }\end{array}$ & $\begin{array}{l}2 \mathrm{E}-08 \\
2 \mathrm{E}-10\end{array}$ & $\begin{array}{l}2 \mathrm{R}-03 \\
\mathrm{NA}\end{array}$ & $\begin{array}{l}2 \mathrm{E}-5 \\
2 \mathrm{E}-7\end{array}$ & $\begin{array}{c}2 \mathrm{E}+0 \\
\mathrm{NA}\end{array}$ & $\begin{array}{l}2 \mathrm{2B}-\mathrm{S} \\
2 \mathrm{E}-7\end{array}$ & $\begin{array}{l}2 \mathrm{~B}+0 \\
\mathrm{NA}\end{array}$ & $\begin{array}{l}4 \mathrm{E}-6 \\
3 \mathrm{E}-8\end{array}$ & $\begin{array}{l}9 \mathrm{E}-2 \\
\mathrm{NA}\end{array}$ & $\begin{array}{l}4 \mathrm{E}-6 \\
5 \mathrm{E}-8\end{array}$ & $\begin{array}{l}8 \mathrm{8E}-2 \\
\mathrm{NA}\end{array}$ \\
\hline $\begin{array}{l}\text { Dermal Contact } \\
\text { nonradionuclides } \\
\text { radionuclides }\end{array}$ & $\begin{array}{l}3 \mathrm{3E}-09 \\
7 \mathrm{E}-13\end{array}$ & $\begin{array}{l}\text { SE-04 } \\
\text { NA }\end{array}$ & $\begin{array}{l}2 \mathrm{E}-6 \\
3 \mathrm{E}-10\end{array}$ & $\begin{array}{l}8 \mathrm{8E}-2 \\
\mathrm{NA}\end{array}$ & $\begin{array}{r}2 \mathrm{E}-6 \\
3 \mathrm{E}-10\end{array}$ & $\begin{array}{l}5 \mathrm{SB}-2 \\
\mathrm{NA}\end{array}$ & $\begin{array}{l}6 \mathrm{E}-7 \\
9 \mathrm{E}-11\end{array}$ & $\begin{array}{l}2 \mathrm{E}-2 \\
\mathrm{NA}\end{array}$ & $\begin{array}{c}6 \mathrm{E}-7 \\
1 \mathrm{E}-10\end{array}$ & $\begin{array}{l}\text { IE-2 } \\
\mathrm{NA}\end{array}$ \\
\hline $\begin{array}{c}\text { Direct (Extermal) Radiation } \\
\text { radionuclides }\end{array}$ & $3 \mathrm{E}-07$ & NA & $8 \mathrm{E}-6$ & NA & $8 \mathrm{E}-6$ & NA & 2B-6 & NA & $2 \mathrm{E}-6$ & NA \\
\hline $\begin{array}{c}\text { Inhalation - Dust(1) } \\
\text { nonradionuclides } \\
\text { redionuclides }\end{array}$ & $\begin{array}{l}2 \mathrm{E}-07 \\
2 \mathrm{E}-12\end{array}$ & $\begin{array}{l}\text { 3E-06 } \\
\text { NA }\end{array}$ & $\begin{array}{l}1 \mathrm{E}-7 \\
3 \mathrm{E}-10\end{array}$ & $\begin{array}{l}\mathrm{NC} \\
\mathrm{NA}\end{array}$ & $\begin{array}{l}\text { 3E-8 } \\
7 \mathrm{E}-10\end{array}$ & $\begin{array}{l}\text { 2E-7 } \\
\mathrm{NA}\end{array}$ & $\begin{array}{c}\text { 1E-8 } \\
\text { SE-10 }\end{array}$ & $\begin{array}{l}\mathrm{NC} \\
\mathrm{NA}\end{array}$ & $\begin{array}{l}3 \mathrm{E}-9 \\
8 \mathrm{E}-13\end{array}$ & $\begin{array}{l}5 \mathrm{E}-9 \\
\mathrm{NA}\end{array}$ \\
\hline $\begin{array}{c}\text { Inhatertion - Volatllese } \\
\text { nonrodionuclides } \\
\text { radionuclides }\end{array}$ & $\begin{array}{l}\mathrm{NC} \\
\mathrm{NA}\end{array}$ & $\begin{array}{l}\mathrm{NC} \\
\mathrm{NA}\end{array}$ & $\begin{array}{l}\mathrm{NC} \\
\mathrm{NA}\end{array}$ & $\begin{array}{l}\mathrm{NC} \\
\mathrm{NA}\end{array}$ & $\begin{array}{l}\mathrm{NC} \\
\mathrm{NA}\end{array}$ & $\begin{array}{l}\text { NC } \\
\text { NA }\end{array}$ & $\begin{array}{l}\mathrm{NC} \\
\mathrm{NC}\end{array}$ & $\begin{array}{l}\mathrm{NC} \\
\mathrm{NC}\end{array}$ & $\begin{array}{l}\mathrm{NC} \\
\mathrm{NC}\end{array}$ & $\begin{array}{l}\mathrm{NC} \\
\mathrm{NC}\end{array}$ \\
\hline $\begin{array}{l}\text { Ingestlon - Lealy Produce } e^{(a)} \\
\text { nonradionuclides } \\
\text { radionuclides }\end{array}$ & $\begin{array}{l}\text { NA } \\
\text { NA }\end{array}$ & $\begin{array}{l}\mathrm{NA} \\
\mathrm{NA}\end{array}$ & $\begin{array}{l}\text { NA } \\
\text { SE-7 }\end{array}$ & $\begin{array}{l}\text { NA } \\
\text { NA }\end{array}$ & $\begin{array}{c}\mathrm{NA} \\
\text { SE-6 }\end{array}$ & $\begin{array}{l}\mathrm{NA} \\
\mathrm{NA}\end{array}$ & $\begin{array}{l}\text { NA } \\
\text { NA }\end{array}$ & $\begin{array}{l}\text { NA } \\
\text { NA }\end{array}$ & $\begin{array}{l}\mathrm{NA} \\
\mathrm{NA}\end{array}$ & $\begin{array}{l}\text { NA } \\
\text { NA }\end{array}$ \\
\hline $\begin{array}{c}\text { Ingestion - Tuberous Produceo } \\
\text { nonradionuclides } \\
\text { radionuclides }\end{array}$ & $\begin{array}{l}\text { NA } \\
\text { NA }\end{array}$ & $\begin{array}{l}\mathrm{NA} \\
\mathrm{NA}\end{array}$ & $\begin{array}{c}\mathrm{NA} \\
2 \mathrm{~B}-6\end{array}$ & $\begin{array}{l}\text { NA } \\
\text { NA }\end{array}$ & $\underset{3 E-6}{N A}$ & $\begin{array}{l}\text { NA } \\
\text { NA }\end{array}$ & $\begin{array}{l}\mathrm{NA} \\
\mathrm{NA}\end{array}$ & $\begin{array}{l}\text { NA } \\
\text { NA }\end{array}$ & $\begin{array}{l}\text { NA } \\
\text { NA }\end{array}$ & $\begin{array}{l}\mathrm{NA} \\
\mathrm{NA}\end{array}$ \\
\hline $\begin{array}{l}\text { Ingetlon - Frutt Produce }{ }^{(\boldsymbol{)})} \\
\text { nonradionuclides } \\
\text { radionuclides }\end{array}$ & $\begin{array}{l}\text { NA } \\
\text { NA }\end{array}$ & $\begin{array}{l}\text { NA } \\
\text { NA }\end{array}$ & $\begin{array}{c}\mathrm{NA} \\
2 \mathrm{~B}-6\end{array}$ & $\begin{array}{l}\text { NA } \\
\text { NA }\end{array}$ & $\begin{array}{l}\mathrm{NA} \\
2 \mathrm{E}-6\end{array}$ & $\begin{array}{l}\text { NA } \\
\text { NA }\end{array}$ & $\begin{array}{l}\text { NA } \\
\text { NA }\end{array}$ & $\begin{array}{l}\text { NA } \\
\text { NA }\end{array}$ & $\begin{array}{l}\text { NA } \\
\text { NA }\end{array}$ & $\begin{array}{l}\mathrm{NA} \\
\mathrm{NA}\end{array}$ \\
\hline Total for All Soll Pathmye = & 5B-7 & 3B-3 & 3E-5 & $2 \mathrm{E}+0$ & $4 \mathrm{E}-5$ & $2 E+0$ & $7 \mathrm{E}-6$ & IE-1 & $\overline{7 \mathrm{E}-6}$ & 9E-2 \\
\hline
\end{tabular}

NA - Not Applicable

NC - Not Calculated due to a leck of data

(1) Values for inhalution of dust and volatiles in air are cotimated from COPC concentrations in soil.

(2) Values for protuce are extimuted from COPC concentrations in soil. 
Table 5.9

Summary of Carcinogenic Risk and

Noncarcinogenic Hazards for Potential Future

On-Unit Resident and Worker Exposure Pathways for Groundwater

F-Area

\begin{tabular}{|c|c|c|c|c|}
\hline \multirow[b]{3}{*}{ Exposure Route } & \multicolumn{2}{|c|}{ Future Resident } & \multicolumn{2}{|c|}{ Future Worker } \\
\hline & \multicolumn{2}{|c|}{ Groundwater } & \multicolumn{2}{|c|}{ Groundwater } \\
\hline & Risk & Hazard & Risk & Hazard \\
\hline \multicolumn{5}{|l|}{ Incidental Ingestion } \\
\hline nonradionuclides & $5 E-5$ & $2 E+0$ & $2 \mathrm{E}-05$ & $4 \mathrm{E}-01$ \\
\hline radionuclides & $6 \mathrm{E}-5$ & NA & $2 \mathrm{E}-05$ & NA \\
\hline \multicolumn{5}{|l|}{ Dermal Contact } \\
\hline nonradionuclides & $2 \mathrm{E}-7$ & $8 \mathrm{E}-2$ & NA & NA \\
\hline radionuclides & $8 \mathrm{E}-10$ & NA & NA & NA \\
\hline \multicolumn{5}{|l|}{ Inhalation - Volatiles } \\
\hline nonradionuclides & $1 E-5$ & $6 \mathrm{E}-1$ & NA & NA \\
\hline radionuclides & $5 E-6$ & NA & NA & NA \\
\hline Total for Groundwater Pathways = & $1 \mathrm{E}-4$ & $3 \mathrm{E}+0$ & $4 \mathrm{E}-5$ & $4 \mathrm{E}-1$ \\
\hline
\end{tabular}

NA - Not Applicable 
Table 5.10

Summary of Carcinogenic Risk and Noncarcinogenic Hazards for Current and Hypothetical Future Receptors

Ephemeral Wetland (Surface Water/Sediment)

$$
\text { F-Area }
$$

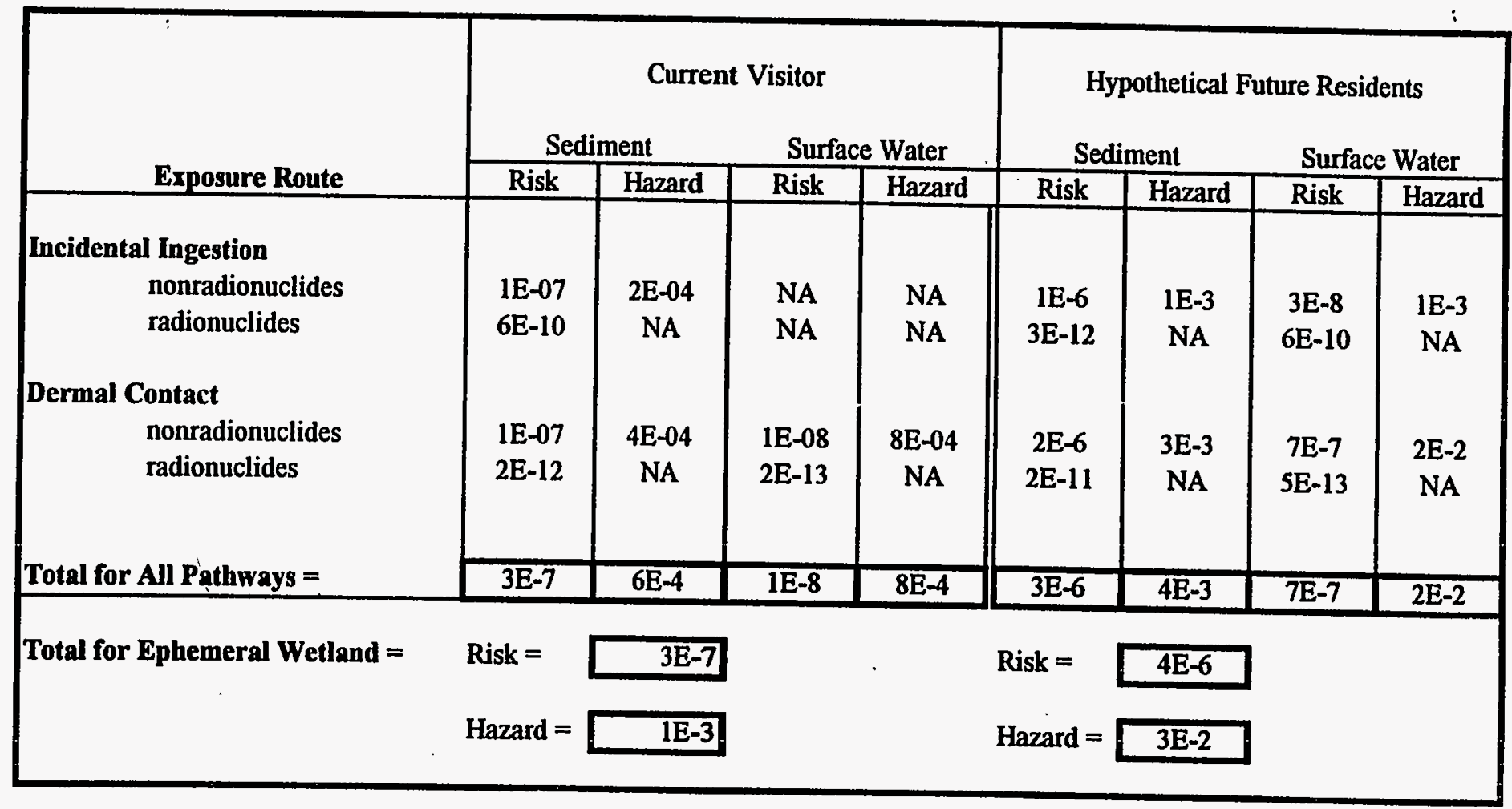

NA - Not Applicable 
TABLE 5.11

APPLICABLE OR RELEVANT AND APPROPRIATE

REQUIREMENTS FOR GROUND WATER

SAVANNAH RIVER SITE

BURNING/RUBBLE PIT - F AREA

\begin{tabular}{|c|c|c|c|c|}
\hline Analyte & $\begin{array}{c}\text { Maximum } \\
\text { Detected } \\
\text { Concentration } \\
\end{array}$ & $\begin{array}{c}\text { Federal } \\
\text { MCL (1) }\end{array}$ & $\begin{array}{l}\text { South Carolina } \\
\text { MCL (2) } \\
\end{array}$ & $\begin{array}{c}\text { Calculate: } \\
\text { RGO } \\
(\mathbf{Y} / \mathbf{N}) \\
\end{array}$ \\
\hline \multicolumn{5}{|l|}{ Volatiles (mo/L) } \\
\hline Carbon Tetrachloride & 0.00541 & 0.005 & 0.005 & $\mathbf{Y}$ \\
\hline Dichloromethane & 0.0302 & 0.005 & 0.005 & $\mathbf{Y}$ \\
\hline Tetrachloroethylene & 0.0328 & 0.005 & 0.005 & $\mathbf{Y}$ \\
\hline \multicolumn{5}{|l|}{ Metals (mo/L) } \\
\hline Arsenic & 0.0025 & 0.05 & 0.05 & $\mathbf{N}$ \\
\hline Cadmium & 0.002 & 0.005 & 0.005 & $\mathbf{N}$ \\
\hline Manganese & 0.453 & $0.05^{*}$ & $0.05^{*}$ & $\mathbf{Y}$ \\
\hline \multicolumn{5}{|l|}{ Radionuclides pCi/L } \\
\hline Radium-226 & 1.7 & 20 & 5 & $\mathbf{N}$ \\
\hline Radium-228 & 2.7 & 20 & 5 & $\mathbf{N}$ \\
\hline Tritium & 5400 & NA & 20,000 & $\mathbf{N}$ \\
\hline Radium, total alpha emitting & 35 & 15 & NA & ND \\
\hline
\end{tabular}

RGO - Remedial Goal Option

NA - Not Available; ND = Not Derived

* - Secondary MCL

** - Modeled Values

(1) USEPA, Drinking Water Regulations and Health Advisories, May 1995.

(2) South Carolina, Maximum Contaminant Levels for Organic and Inorganic Chemicals, 1996. 
Table 5.12

Savannah River Site

Burning/Rubble Pits (231-F/1F) and Rubble Pit (231-2F)

Remedial Goals for Contaminants of Concern by Receptor and Medium

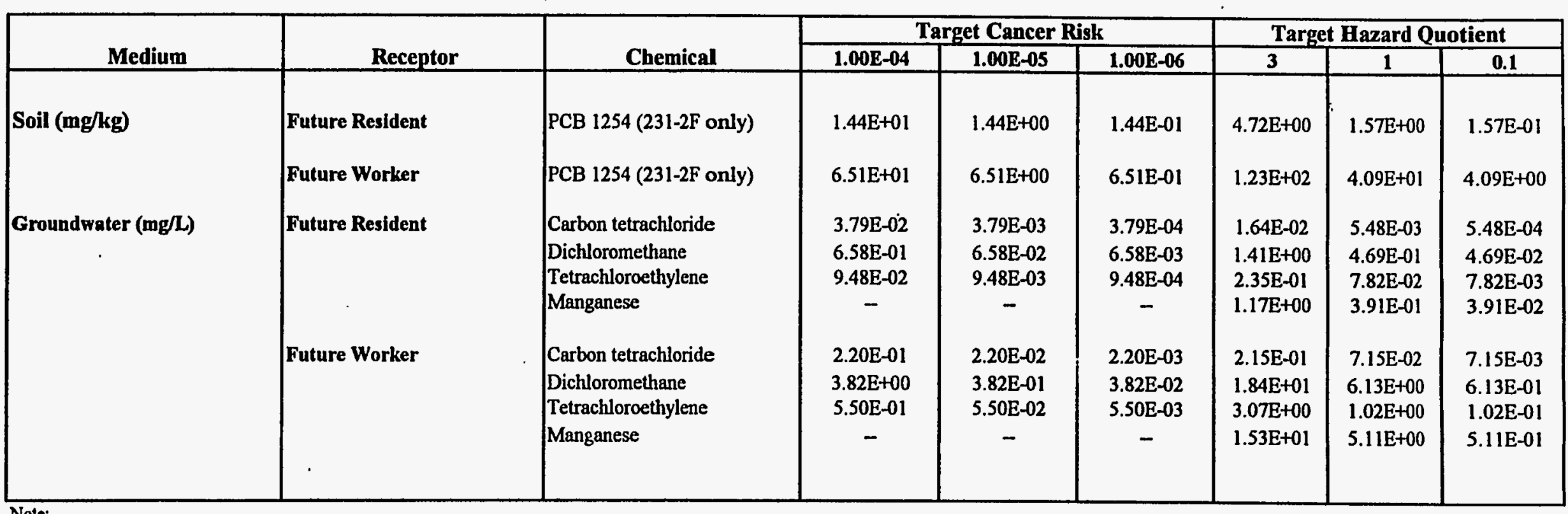

PCB 1254 is a contaminant of concern for 231-2F soil only.

Potential future resident/worker exposure to soil contaminants includes ingestion, dermal contact, and inhalation of particulates.

Potential future worker exposure to groundwater contaminants was considered by ingestion only;

Potential future resident exposure to groundwater contaminants includes ingestion, and inhalation/dermal exposure which was estimated as equivalent to oral exposure (USEPA Region IV, $11 / 95$.

FINAL 
Table 5-13

Summary of COCs and Maximum Detected Concentrations in Groundwater

\begin{tabular}{|l|c|c|}
\hline \multicolumn{1}{|c|}{ Contaminant } & Media & $\begin{array}{c}\text { Maximum } \\
\text { Concentration }\end{array}$ \\
\hline Carbon Tetrachloride & Groundwater & $0.0054 \mathrm{mg} / \mathrm{L}$ \\
\hline Dichloromethane & Groundwater & $0.030 \mathrm{mg} / \mathrm{L}$ \\
\hline Tetrachloroethylene & Groundwater & $0.033 \mathrm{mg} / \mathrm{L}$ \\
\hline & & \\
\hline Manganese & Groundwater & $0.453 \mathrm{mg} / \mathrm{L}$ \\
\hline & & \\
\hline
\end{tabular}

1. Radioactive COCs modeled in groundwater from soil leachability using conservative assumptions. Iodine-129 and technetium-99 are not and have not been detected in the groundwater. 
REFERENCES

FINAL 


\section{REFERENCES}

ATSDR (Agency for Toxic Substances and Disease Registry), 1988. Draft Toxicological Profile for Nickel. Prepared by Syracuse Research Corporation and Clement Associates, Inc., U.S. Department of Health and Human Services.

ATSDR (Agency for Toxic Substances and Disease Registry), 1989a. Draft Toxicological Profile for Copper. Prepared by Syracuse Research Corporation, U.S. Department of Health and Human Services.

ATSDR (Agency for Toxic Substances and Disease Registry), 1989c. Draft Toxicological Profile for Di(2-ethylhexyl)phthalate. Prepared by Life Systems, Inc., U.S. Department of Health and Human Services.

ATSDR (Agency for Toxic Substances and Disease Registry), 1989d. Draft Toxicological Profile for Di-n-butyl phthalate. Prepared by Life Systems, Inc. and Clement Associates, Inc., U.S. Department of Health and Human Services.

ATSDR (Agency for Toxic Substances and Disease Registry), 1989e. Draft Toxicological Profile for Polycyclic Aromatic Hydrocarbons. Prepared by Clement Associates, Inc., U.S. Department of Health and Human Services.

ATSDR (Agency for Toxic Substances and Disease Registry), 1989f. Draft Toxicological Profile for Chlorobenzene. Prepared by Life Systems, Inc. and Clement Associates, Inc., U.S. Department of Health and Human Services.

ATSDR (Agency for Toxic Substances and Disease Registry), 1989g. Draft Toxicological Profile for 1,2-Dichloroethylene. Prepared by Syracuse Research Corporation and Clement Associates, Inc., U.S. Department of Health and Human Services.

ATSDR (Agency for Toxic Substances and Disease Registry), 1989h. Draft Toxicological Profile for Endrin Ketone. Prepared by Life Systems, Inc. and Clement Associates, Inc., U.S. Department of Health and Human Services.

ATSDR (Agency for Toxic Substances and Disease Registry), 1989i. Draft Toxicological Profile for Silver. Prepared by Clement International Corporation. U.S. Department of Health and Human Services. 
ATSDR (Agency for Toxic Substances and Disease Registry), 1989j. Draft Toxicological Profile for Thorium. Prepared by Syracuse Research Corporation and Clement Associates, Inc., U.S. Department of Health and Human Services.

ATSDR (Agency for Toxic Substances and Disease Registry), 1989k. Draft Toxicological Profile for Uranium. Prepared by Syracuse Research Corporation and Clement Associates, Inc., U.S. Department of Health and Human Services.

ATSDR (Agency for Toxic Substances and Disease Registry), 1989l. Draft Toxicological Profile for Radium. Prepared by Life Systems, Inc. and Clement Associates, Inc., U.S. Department of Health and Human Services.

ATSDR (Agency for Toxic Substances and Disease Registry), 1989m. Draft Toxicological Profile for Plutonium. Prepared by Clement Associates, Inc., U.S. Department of Health and Human Services.

ATSDR (Agency for Toxic Substances and Disease Registry), 1989n. Draft Toxicological Profile for Heptachlor. Prepared by Dynamac Corporation, U.S. Department of Health and Human Services.

ATSDR (Agency for Toxic Substances and Disease Registry), 1990a. Draft Toxicological Profile for Aluminum. Prepared by Clement Associates, Inc. U.S. Department of Health and Human Services.

ATSDR (Agency for Toxic Substances and Disease Registry), 1990b. Draft Toxicological Profile for Barium. Prepared by Clement Associates, Inc., U.S. Department of Health and Human Services.

ATSDR (Agency for Toxic Substances and Disease Registry), 1990c. Draft Toxicological Profile for Manganese. Prepared by Life Systems, Inc. and Clement International Corporation. U.S. Department of Health and Human Services.

ATSDR (Agency for Toxic Substances and Disease Registry), 1990d. Draft Toxicological Profile for Endosulfan. Prepared by Clement Associates, Inc., U.S. Department of Health and Human Services. 
ATSDR (Agency for Toxic Substances and Disease Registry), 1990e. Draft Toxicological Profile for Vanadium. Prepared by Clement Associates, Inc., U.S. Department of Health and Human Services.

ATSDR (Agency for Toxic Substances and Disease Registry), 1991a. Draft Toxicological Profile for Arsenic. Prepared by Life Systems, Inc., U.S. Department of Health and Human Services.

ATSDR (Agency for Toxic Substances and Disease Registry), 1991b. Draft Toxicological Profile for Cadmium. Prepared by Life Systems, Inc., U.S. Department of Health and Human Services.

ATSDR (Agency for Toxic Substances and Disease Registry), 1991c. Draft Toxicological Profile for Chromium. Prepared by Syracuse Research Corporation and Clement Associates, Inc., U.S. Department of Health and Human Services.

ATSDR (Agency for Toxic Substances and Disease Registry), 1991d. Draft Toxicological Profile for Methylene Chloride. Prepared by Clement Associates, Inc., U.S. Department of Health and Human Services.

ATSDR (Agency for Toxic Substances and Disease Registry), 1991e. Draft Toxicological Profile for Aldrin. Prepared by Clement International Corporation, U.S. Department of Health and Human Services.

ATSDR (Agency for Toxic Substances and Disease Registry), 1991f. Draft Toxicological Profile for Lead. Prepared by Clement International Corporation. U.S. Department of Health and Human Services.

ATSDR (Agency for Toxic Substances and Disease Registry), 1991g. Draft Toxicological Profile for Polychlorinated Biphenyls. Prepared by Syracuse Research Corporation and Clement International Corporation. U.S. Department of Health and Human Services.

ATSDR (Agency for Toxic Substances and Disease Registry), 1991h. Draft Toxicological Profile for Tetrachloroethylene. Prepared by Clement International Corporation. U.S. Department of Health and Human Services. 
ATSDR (Agency for Toxic Substances and Disease Registry), 1991i. Draft Toxicological Profile for Heptachlor Epoxide. Prepared by Clement International Corporation. U.S. Department of Health and Human Services.

ATSDR (Agency for Toxic Substances and Disease Registry), 1991j. Draft Toxicological Profile for Chloroform. Prepared by Syracuse Research Corporation and Clement Associates, Inc., U.S. Department of Health and Human Services.

ATSDR (Agency for Toxic Substances and Disease Registry), 1992A. Draft Toxicological Profile for Pentachlorophenol. Prepared by Clement International Corporation. U.S. Department of Health and Human Services.

ATSDR (Agency for Toxic Substances and Disease Registry), 1992b. Draft Toxicological Profile for Carbon Tetrachloride. Prepared by Clement International Corporation. U.S. Department of Health and Human Services.

ATSDR (Agency for Toxic Substances and Disease Registry), 1992c. Draft Toxicological Profile for Alpha, Beta, Gamma Chlordane. Prepared by Clement International Corporation, U.S. Department of Health and Human Services.

ATSDR (Agency for Toxic Substances and Disease Registry), 1992d. Draft Toxicological Profile for $D D T, D D E$, and $D D D$. Prepared by Clement International Corporation, U.S. Department of Health and Human Services.

ATSDR (Agency for Toxic Substances and Disease Registry), 1992e. Draft Toxicological Profile for 1,2-Dichloroethane. Prepared by Syracuse Research Corporation and Clement International Corporation, U.S. Department of Health and Human Services.

ATSDR (Agency for Toxic Substances and Disease Registry), 1993. Draft Toxicological Profile for Polycyclic Aromatic Hydrocarbons. Prepared by Clement. International Corporation. U.S. Department of Health and Human Services.

ATSDR (Agency for Toxic Substances and Disease Registry), 1994a. Draft Toxicological Profile for Mercury. Prepared by Clement International Corporation. U.S. Department of Health and Human Services. 
ATSDR (Agency for Toxic Substances and Disease Registry), 1994b. Draft Toxicological Profile for Chlorodibenzofurans. Prepared by Clement International Corporation. U.S. Department of Health and Human Services.

Amdur, M. O., J. Doull, C. D. Klaassen (eds.), 1991. Casarett and Doull's Toxicology: The Basic Science of Poisons. Fourth Edition, Pergamon Press, New York.

AQUIRE (Aquatic Information Retrieval Database), 1993.

Baes, C.F., III, R.D. Sharp, A.L. Sjoreen and R.W. Shor, 1984 A Review and Analysis of Parameters for Assessing Transport of Environmentally Released Radionuclides through Agriculture. Oak Ridge National Laboratory, ORNL - 5786.

Beyer, W.N., 1990. Evaluating Soil Contamination. U.S. Fish and Wildlife Service, Biological Report No. 90(2).

BNA (Bureau of National Affairs), 1992. Project Summary, Guidance on Estimating Exposure to VOCs During Showering. "BNA, Environment Report, February 26, 1992, pp. E-13 E-14.

Burt, W.H. and R.P. Grossenheider, 1976. A Field Guide to the Mammals: North American North of Mexico. Houghton Mifflin, Boston, MA.

Bysshe, Sara E., 1982. Bioconcentration Factor in Aquatic Organisms. Chapter Five in Handbook of Chemical Property Estimation Methods - Environmental Behavior of Organic Compounds. W.J. Lyman; W. F. Reehl, and D. H. Rosenblatt, eds. McGrawHill Book Company.

Calder II, W. A. and E. J. Braun, 1983. Scaling of Osmotic Regulation in Mammals and Birds. American Journal of Physiology. 244: 601-606.

Dixon, K., T. LaPoint, R. Dickerson, K. Brooks, C. Bens, R. Mellott, S. Anderson, A. Edinger, L. Esman, M. Feken, and S. Wall, 1993. Characterization of Waste Sites at Savannah River Site - Draft. Project 09268 Report. The Institute of Wildlife and Environmental Toxicology at Clemson University, Pendleton, SC. 
DOE (U.S. Department of Energy), 1991. Draft Envrionmental Impact Statement for the Siting, Construction, and Operation of New: Production Reactor Capacity. DOE/EIS-0144D. Office of New Production Reactors.

DOE (U.S. Department of Energy), 1992. Permit Renewal Application for a Hazardous Waste Part B Post-Closure Care Permit: F-Area Hazardous Waste Management Facility, Volume IV, Section E, Rev. 2, December 3, 1992. Westinghouse Savannah River Company, Aiken, SC.

DOE (U.S. Department of Energy), 1994. Programmatic Spent Nuclear Fuel Management and Idaho National Engineering Laboratory Environmental Restoration and Waste Management Programs Environmental Impact Statement, Volume 1, Appendix C. Office of Environmental Restoration and Waste Management, DOE Idaho Operations Office.

DOE (U.S. Department of Energy), 1996. Savannah River Site: Future Use Project Report. Stakeholder Recommendations for SRS Land and Facilities. January, 1996. Cover letter: Fiori, Mario P., "SRS Future Use Project Report (Reference: Transmittal of Final Draft "Forging the Missing Link: A Resource Document for Identifying Future Use Options," Grumbly/Pearlmann letter, 1-12-94)", USDOE Letter EB-96-015, Savannah River Site, Aiken, SC 29808, January 29, 1996.

Eisenbud, M. 1987. Environmental Radioactivity from Natural, Industrial, and Military Sources. Academic Press, San Diego, CA.

Eisler, R., 1988. Lead Hazards to Fish, Wildlife, and Invertebrates: A Synoptic Review. U.S. Fish and Wildlife Service, Biological Report No. 85(1.14).

Eisler, R., 1994. Radiation Hazards to Fish, Wildlife, and Invertebrates: A Synoptic Review. U.S. Dept. Interior, National Biological Service, Report No. 29, Washington, DC.

EPA (U.S. Environmental Protection Agency), 1989a. Risk Asssessment Guidance for Superfund (RAGS) Volume I - Human Health Evaluation Manual (Part A). EPA/540/1-89/002, Office of Emergency and Remedial Response, USEPA, Washington, DC.

EPA (U.S. Environmental Protection Agency), 1989b. Risk Assessment Guidance for Superfund, Volume II: Environmental Evaluation Manual. EPA/540/1-89/001, USEPA, Washington, DC. 
EPA (U.S. Environmental Protection Agency), 1989c. Assessing Human Health Risks from Chemically Contaminated Fish and Shellfish: A Guidance Manual. Office of Marine and Estuarine Protection. EPA-503/8-89-002.

EPA (U.S. Environmental Protection Agency), 1989d. Risk Assessments Methodology; Environmental Impact Statement; NESHAPS for Radionuclides; Background Document Volume 1. EPA/520/1-89-005. Office of Relations Programs. Washington, DC.

EPA (U.S. Environmental Protection Agency), 1989e. Interim Procedures for Estimating Risks Associated With Exposures to Mixtures of Chlorinated Dibenzo-p-Dioxins and Dibenzofurans (CDDs and CDFs) and 1989 Update. EPA/625/3-89/016. Risk Assessment Forum, Washington, DC.

EPA (U.S. Environmental Protection Agency), 1990. Exposure Factors Handbook. EPA/600/880/043. Office of Health \& Environmental Assessment. USEPA, Washington, DC.

EPA (U.S. Environmental Protection Agency), 1990a. A Guide on Remedial Actions at Superfund Site with PCB.Contamination. Office of Emergency and Remedial Response. Directive 9355.4-01 FS. August 1990.

EPA (U.S. Environmental Protection Agency); 1991a. Risk Assessment Guidance for Superfund: Volume I - Human Health Evaluation Manual. Supplemental Guidance "Standard Default Exposure Factors" - Interim Final. OSWER Directive 9285.6-03, Office of Emergency \& Remedial Response Toxics Integration Branch, USEPA, Washington, DC.

EPA (U.S. Environmental Protection Agency), 1991b. Health Effects Assessment Summary Tables. FY1991, EPA 9200.6-303 (91-1), Office of Emergency and Remedial Response, USEPA, Washington, DC.

EPA (U.S. Environmental Protection Agency), 1991c. "Risk Assessment Guidance for Superfund: Volume I - Human Health Evaluation Manual (Part B, Development of Riskbased Preliminary Remediation Goals), Interim, ".. Office of Emergency and Remedial Response, Washington, DC, Pub.No. 9285.7-01B.

EPA (U.S. Environmental Protection Agency), 1991d. Supplemental Region IV Risk Assessment Guidance. U.S. Environmental Protection AGency - Region IV, Atlanta, GA. 
EPA (U.S. Environmental Protection Agency), 1991e. Role of the Baseline Risk Assessment in Superfund Remedy Selection Decisions. OSWER Directive 9355.0-30. Office of Solid Waste and Emergency Responses, Washington, D.C.

EPA (U.S. Environmental Protection Agency), 1992b. Guidance on Risk Characterization for Risk Managers and Risk Assessors. Memorandum from F.H. Habicht to Assistant and Regional Administrators, USEPA, Washington, DC.

EPA (U.S. Environmental Protection Agency), 1992c. Dermal Assessment: Principles and Applications, Interim Report, EPA 600/8-91-011B, Office of Research \& Development, USEPA, Washington, DC.

EPA (U.S. Environmental Protection Agency). 1992d. New Interim Region IV Guidance.

EPA (U.S. Environmental Protection Agency), 1992e. Chemical Specific Issue Papers, 3rd QuarterSuperfund Health Risk Technical Support Center, Environmental Criteria and Assessment Office, Cincinnati, $\mathrm{OH}$.

EPA (U.S. Environmental Protection Agency), 1992f. Framework for Ecological Risk Assessment. EPA/630/R-92/001. Risk Assessment Forum, Washington, D.C.

EPA (U.S. Environmental Protection Agency), 1993a. Health Effects Assessment Summary Table (HEAST). Washington, D.C.

EPA (U.S. Environmental Protection Agency), 1993b. Provisional Guidance for Quantitative Risk Assessment of Polycyclic Aromatic Hydrocarbons. Office of Research and Development. EPA-600/R-93-089.

EPA (U.S. Environmental Protection Agency), 1993c. Wildlife Exposure Factors Handbook. Office of Research and Development, EPA/600/R-93/187a \& b.

EPA (U.S. Environmental Protection Agency), 1994a. Soil Screening Guidance. Office of Solid Waste and Emergency Response. EPA/540/R-94/101 PB95-963529.

EPA (U.S. Environmental Protection Agency), 1994b. Integrated Exposure Uptake Biokinetic Model for Lead. Version .99d. PB93-963511. February, 1994. 
EPA (U.S. Environmental Protection Agency), 1994b Integrated Exposure Uptake Biokinetic Model for Lead. Version .99D. February, 1994.

EPA (U.S. Environmental Protection Agency), 1994b. Supplemental Guidance to RAGS: Region IV Bulletin, Development of Health Based Preliminary Remediation Goals, Remedial Goal Options, and Remediation Levels. EPA Region IV, Waste Management Division, Atlanta, GA.

EPA (U.S. Environmental Protection Agency), Region VIII, 1994c. Memorandum from Dr. Gerry Henningaen, EPA Region VIII toxicologist, to EPA Region VIII. Hazardous Waste Management Site Remedial Program Managers and Contractors. September 28.

EPA (U.S. Environmental Protection Agency), 1994d. Development of Ecological Exit Criteria for the Hazardous Waste Identification Project, Review Draft. Office of Solid Waste.

EPA (U.S. Environmental Protection Agency), 1995a Region IV, Supplemental Guidance to RAGS: Region 4 Bulletins. November 1995.

EPA (U.S. Environmental Protection Agency), 1995b. Health Effects Assessment Summary Tables (HEAST). Office of Research and Development and Office of Emergency and Remedial Response. FY-1995 Annual.

EPA (U.S. Environmental Protection Agency), 1996a. Integrated Risk Management System (IRIS), on-line January, 1996.

EPA (U.S. Environmental Protection Agency), 1996b. Technical Support Personal Communication between M. Cowles and R. Tudor. January 29, 1996.

Gilbert, R.O., 1987. "Statistical Methods For Environmental Pollution Monitoring." Van Nostrand Reinhold Co., Inc., New York, IJew York.

Gilbert, T.L., C. Yu,; Y.C. Yuan, A.J. Zielen, M.J. Jusho, and A. Wallo, III, 1989. "A Manual For Implementing Residual Radioactive Material Guidelines," DOE/CH/8901, Argonne National Laboratory, Argonne, II.

Gillett, James W., 1983. A Comprehensive Prebiological Screen For Ecotoxicological Effects. In Environmental Toxicology and Chemistry, Vo!. 2, pp. 463-476. 
Glasstone, Samuel and W.H. Jordan, 1980. Nuclear Power and Its Environmental Effects. American Nuclear Society, La Grange Park, Illinois.

HSDB (Hazardous Substances Data Bank), 1994. National Library of Medicine (NLM) on-line Toxicological Network (TOXNET). Bethesda, MD.

Howard, P.H., 1990. Handbook of Environmental Fate and Exposure Data for Organic Chemicals, Volume II Solvents, Lewis Publishers, Chelsea, MI.

IAEA (International Atomic Energy Agency), 1992. Effects of Ionizing Radiation on Plants and Animals at Levels Implied by Current Radiation Protection Standards. Technical Reports Series No. 332. Vienna.

.Jarvis, William L., 1993. Threatened, Endangered and Sensitive Species Listing, F-Area Burning/Rubble Pits. U. S. Department of Agriculture - Savannah River Forest Station, Savannah River Site, Aiken, SC.

Karickhoff, S.W. and J.M. Long, 1995. Internal Report on Summary of Measured, Calculated and Recommended Log Kow Values. U.S. Environmental Protection Agency Environmental Research Laboratory, Athens, GA.

Killough, G.G., and R. F. Eckerman, 1983. Internal Dosimetry. In: Radiological Assessment. Till, J.E. and Meyer, H.R. eds. pp. 7-1 to 7-98. United States Nuclear Regulatory Commission (NUREG/CR-3332, ORNL-5966), Washington, D.C.

Long, E.R., D.D. MacDonald, S.L. Smith and F.D. Calader, 1995. Incidence of Adverse Biological Effects within Ranges of Chemical Concentrations in Marine anad Estuarine Sediments. Environmental Manaagement, Vol 19(1), pp. 81-97.

Looney, B. B., C. A. Eddy, M. Ramdeen, J. Pickett, V. Rogers, M. T. Scott, and P. A. Shirley, 1990. Geochemical and Physical Properties of Soils and Shallow Sediments at the Savannah River Site (U). WSRC-RP-90-1031, Westinghouse Savannah River Company, Aiken, SC.

Looney, B.B., K.M. Jerome, and M.T. Scott, 1994. "Detailed Quality Assurance (QA)/Quality Control (QC) Documentation Supporting the Savannah River Site (SRS) Baseline Study of Upland Soils $(U)$. WSRC-RP-94-203, Westinghouse Savannah River Company, Aiken, SC. 
Ma, W., Th. Edelman, I van Beersum and Th. Jans, 1983. Uptake of Cadmium, Zinc, Lead, and Copper by Earthworms near a Zinc-smelting Coimplex: Influence of Soil pH and Organic Matter. Bull. Environ. Contam. Toxicol. Vol. 30, pp. 424-427.

Marquenie, J.M., J.W. Simmers and S.H. Kay, 1987. Preliminary Assessment of bioaccumulation of Metals and Organic Contaminaants at the Times Beach Confined Disposal Site, Buffalo, N.Y. Miscellaneous paper EL-87-6, U.S. Army Corps of Engineers, Waterways Experiment Station, Vicksburg, MS, 67 pp.

Menzie, C., J. Cura, J. Freshman and S. Svirsky, 1993. Evaluating Ecological Risks and Developing Remedial Objectives at Forested Wetland Systems in New England. pp. 89100 in Application of Ecological Risk Assessment to Hazardous Waste Site Remediation. Workshop Proceedings. USEPA Science Advisory Board.

Metcalf and Eddy, 1993. Preliminary Site Characterization - Final Report. Contract No. AA46327P; Task 41, December 1993. Prepared for Westinghouse Savannah River Company, Aiken, SC.

Myers, R. K., R. Zahner, and S. M. Jones, 1986. Forest Habitat Regions of South Carolina from Landsat Imagery. Forest Research Series No. 42. Department of Forestry, Clemson University, Clemson, SC.

Nagy, K. A. 1987. Field Metabolic Rate and Food Requirement Scaling in Mammals and Birds. Ecological Monographs. 57(2):111-128.

NAS (National Academy of Science), 1989. Recommended daily allowances Table.

National Research Council, 1989. Subcommittee on the Tenth Edition of the Recommended Daily Allowances, Food and Nutrition Committee, Commision on Life Sciences.

NLM (National Library of Medicine, 1992. Hazardous Substances Data Base (HSDB). Toxicological Netowrk, Bethesda, MD.

NRC (Nuclear Regulatory Commission), 1992. Residual Radioactive Contamination From Decommissioning, Technical Basis for Translating Contamination Levels to Anmual Total Effective Dose Equivalent. Final Report, NUREG/CR-5512, PNL-7994, Vol.1. 
Pendleton, R.C., C. W. Mays, R. D. Lloyd, and B. W. Church, 1965. A Trophic Level Effect on Cesium-137 Concentration. Health Physics, II: 1503-1510.

Radford, A. E., H. E. Ahles and C. R. Bell, 1968. Manual of the Vascular Flora of the Carolinas. University of North Carolina Press, Chapel Hill, NC.

Rand McNalley, 1992. Commercial Atlas and Markeing Guide. 123rd edition, Skokie, II.

Rogers, V., 1994. Soil Characterization Waste Site 231-F and IF, Memorandum to L. Smith, January 28, 1994. SRT-ESS-94-202, Westinghouse Savannah River Company, Aiken, SC.

RTECS (Registry of Toxic Effects of Chemical Substances), 1989. U.S. National Institute of Occupational Safety and Health (NIOSH) on-line database. Chemical Information System, Inc., Bethesda, MD.

RTECS (Registry of Toxic Effects of Chemical Substances), 1993. U.S. National Institute of Occupational Safety and Health (NIOSH) on-line database. Chemical Information System, Inc., Bethesda, MD.

Safe, Stephen. 1990. Polychlorinated Biphenyls (PCBs), Dibenzo-p-Dioxins (PCDDs), Dibenzofurans (PCDFs), and related compounds: Environmental and Mechanistic Considerations which support the Development of Toxic Factors (TEFs). In Critical Reviews in Toxicology, Roger O. McClellan, ed. Vol. 21, No. 1, pp. 51-88.

SAS Institute, Inc., 1989. "JMP User's Guide - Version 2 of JMP," SAS Institute, Cary, NC.

SCDHEC (South Carolina DHEC), 1995. Comments regarding F-Area Burning/Rubble Pits (231-F, 1F, and -2F) Baseline Risk Assessment (WSRC-TR94-108, Rev.1.1) received on Novemer 28, 1995.

Shealy Environmental Services, Inc., 1992. UTRC Water and Sediment Chemical/Radiological Analyses Conducted for the Environmental Restoration Department, In Support of Waste Site Investigations and Assessments. Report to Westinghouse Savannah River Company, Aiken, SC.

Suter, G.W., II, 1993. Ecological Risk Assessment. Lewis Publishers, Boca Raton, FL. 
Suter, G.W., II, R.N. Hull, and B.E. Sample, 1994. Ecological Risk Assessment For Comprehensive Environmental Response, Compensation, and Liability Act Sites on Federal Facilities: An Introductory Guide For Facility Managers. U.S. Department of Energy, DOE/HWP-152.

Suter, G.W., II, anad J.B. Mabrey, 1994. Toxicological Benchmarks for Screening Potnetial Contaminants of Concern for Effects on Aquatic Biota: 1994 Revision. Oak Ridge National Laboratory, ES/ER/TM-96/R1.

Templeton, W.L., and B.G. Blaylock, 1990. The Effects of Ionizing Radiation On Aquatic Organisms. Presented at the Biological Effects of Chernobyl Accident - USSR. National Council on Radiation Protection (NCRP) Report No. 109.

Travis, C.C. and A.D. Arms, 1988. Bioconcentration of Organics in Beef, Milk, and Vegetation. Environ. Sci. Technol. Vol. 22(3), pp. 271-274.

Turner, J.E., 1986. Atoms, Radiation, and Radiation Protection, Pergamon Press, New York, NY.

Urban, D. J. and N. J. Cook, 1986. Hazard Evaluation, Standard Evaluation Procedure, Ecological Risk Assessment. EPA-540/9-85-001, U. S. Environmental Protection Agency, Washington, DC.

USDA (U. S. Department of Agriculture), 1990. Soil Survey of Savannah River Plant Area, Parts of Aiken, Barnwell, and Allendale Counties, South Carolina: USDA Soil Conservation Service

Wang, C.H., D.L. Willis, and W.D. Loveland, 1975. Radiotracer Methodology in the Biological, Environmental, and Physical Sciences. Prentice-Hall, Inc. Englewood Cliffs, New Jersey.

Whittaker, J.O., Jr., 1966. Food of Mus musculus, Peromyscus maniculatus bairdi, and Permoyscus leucopus in Vigo County, Indiana. J. Mammal., Vol 47, pp. 473-486.

Workman, S. W. and K. W. McLeod, 1990. Vegetation of the Savannah River Site: Major Community Types. Publication SRO-NERP-19, Savannali River Ecology Laboratory, Aiken, SC. 
WSRC (Westinghouse Savannah River Company), 1991. RCRA/CERCLA Integration Document for the F-Area Seepage Basins Groundwater Unit (U). WSRC-RP-91-1019, Savannah River Site, Aiken, SC.

WSRC (Westinghouse Savannah River Company), 1992. Phase II, Revision 2, RCRA Facility Investigation/Remedial Investigation Plan for the F-Area Burning/Rubble Pits (231-F and 231-IF) and Rubble Pit (231-2F) (U). WSRC-RP-90-486, Rev. 2, Aiken, SC.

WSRC (Westinghouse Savannah River Company), 1993. Ecological Risk Assessors Guide for Evaluation of Waste Units on the Savannah River Site. WSRC-RP-92-232. Westinghouse Savannah River Company, Aiken, SC.

WSRC (Westinghouse Savannah River Company), 1994. F-Area Burning/Rubble Pits and Rubble Pit Data Summary Report. WSRC-TR-94-108, Rev. 0, Westinghouse Savannah River Company, Aiken, SC. 
BASELINE" RISK ASSESSMENT

F-AREA BURNING/RUBBLE PITS AND RUBBLE PIT
WSRC-TR-94-108

REV. 1.2, MARCH 1996

\section{APPENDIX A}

STATISTICAL METHODS AND SAMPLE CALCULATIONS - 


\section{THIS PAGE LEFT INTENTIONALLY BLANK}




\section{APPENDIX A STATISTICAL METHODS FOR SUMMARIZING DATA}

The statistical methods used to summarize the analytical sampling data and derive the exposure point concentration values for risk calculation are described in this appendix. Analytical data from soil, sediment, surface water, and groundwater media were used in this BRA. These data are

- summarized using statistical procedures to calculate the deterministic value that is used to represent the concentration of chemicals in each medium during exposure assessment, i.e., exposure point concentration . Concentrations in water media are expressed in units of $\mu \mathrm{g} / \mathrm{L}$ (ppb) and solid media (soil, sediment) in units of $\mathrm{mg} / \mathrm{kg}(\mathrm{ppm})$. Surrogate values set at one-half of the sample quantitation limit (SQL) are included for all non-detects for those chemicals with at least one detection.

For oral and dermal exposure pathways, the representative concentration of the COPCs in the relevant media is used reflect the level to which receptors are exposed. In addition, representative concentrations from the media are used as the basis for calculating the modeled concentrations in air and groundwater that are used for additional exposure calculation.

The parameters that are characterized in determining representative concentrations include means, variances, and confidence limits on the means. In addition, transformation by the natural logarithm (ln ( $x)$ ) is used in many cases to ensure that the distributional requirements for estimating these parameters are optimized. The methods for carrying out these steps are described below.

\section{DATA DISTRIBUTIONS}

In accordance with USEPA guidance (USEPA, 1992), lognormal distributions were assumed to be representative for analytes in soil. Analytes in sediment were treated similarly. Therefore, soil and sediment data were always transformed using the natural logarithm prior to the calcuation of summary statistics.

For groundwater and surface water, the decision to consider data lognormally distributed was based on the detection frequency since transformation of very small datasets or those containing a large proportion of censored values (i.e., non-detects) is inappropriate. The specific criteria will be discussed below in the section describing selection of the exposure point concentrations. 
BASELINE RISK ASSESSMENT

F-AREA BURNING/RUBBLE PITS AND RUBBLE PIT
WSRC-TR-94-108

REV. 1.2, MARCH 1996

\section{SUMMARY STATISTICS}

Means were calculated as the arithmetic mean of the transformed or untransformed values, as appropriate, including surrogate values.

$$
\begin{array}{rll}
\bar{x}=\frac{\sum_{i=1}^{n} x_{i}}{n} & \\
\overline{\mathrm{x}} & = & \\
x & = & \text { arithmetic mean of the data } \\
i & = & \text { index for sample numbers } \\
n & = & \text { number of samples }
\end{array}
$$

Sample standard deviations were calculated from the transformed and untransformed values, as appropriate, including surrogate values.

$$
s=\sqrt{\frac{\sum_{i=1}^{n}\left(x_{i}-\bar{x}\right)^{2}}{n-1}}
$$

$$
\begin{array}{lll}
s & = & \text { standard deviation (sample) } \\
x & = & \text { sample value } \\
i & = & \text { index for sample numbers } \\
n & = & \text { number of samples }
\end{array}
$$

In order to ensure that the extent of the variability found in the datasets is reflected in the summary statistics, the 95 percent upper confidence limits (95\% UCL) on the mean are also calculated. To calculate the $95 \%$ UCL of the arithmetic mean for lognormally distributed data, the data were first transformed using the natural logarithm function $(\ln (x))$. The arithmetic mean and standard deviation of the transformed data were be calculated, and the H-statistic determined or extrapolated (Gilbert, 1987). The $95 \%$ UCL is calculated as follows for transformed data:

$95 \% U C L=e^{\left(\bar{x}+0.5 s^{2}+s H / \sqrt{n-1}\right)}$ 
wherè:

$$
\begin{aligned}
& \text { 95\% UCL }=95 \% \text { upper confidence limit of mean, } \\
& \mathbf{e} \quad=\quad \text { constant (base of the natural log, equal to 2.718), } \\
& \overline{\mathrm{x}} \quad=\quad \text { arithmetic mean of the transformed data, } \\
& \mathrm{s} \quad=\quad \text { standard deviation of the transformed data, } \\
& \mathrm{H} \quad=\quad \text { H-statistic (from Table X in Gilbert, 1987, or extrapolated), } \\
& \mathrm{n} \quad=\quad \text { number of samples. }
\end{aligned}
$$

To calculate the $95 \%$ UCL for normally distributed data, the arithmetic mean and standard deviation of the untransformed data are calculated, and the one-tailed t-statistics are determined from Gilbert (1987). The 95\% UCL is then calculated for each chemical as follows for untransformed data:

$95 \% U C L=\bar{x}+t\left(\frac{s}{\sqrt{n}}\right)$ where:

$95 \% \mathrm{UCL}=95 \%$ upper confidence limit of the mean,

$$
\begin{array}{ll}
\overline{\mathrm{x}} & =\text { arithmetic mean of the untransformed data, } \\
\mathrm{s} & =\text { standard deviation of the untransformed data, } \\
\mathrm{t} & =\quad \text { one-tailed student-t statistic (from Gilbert, 1987), } \\
\mathrm{n} & =\text { number of samples. }
\end{array}
$$

The summary statistics calculated by these methods are used as the basis for determining the representative concentrations -- exposure point concentrations, used in risk calculations. 


\section{EXPOSURE PONT CONCENTRATIONS}

Exposure point concentrations are the concentrations of chemicals in a given medium to which a hypothetical receptor may be exposed at a specific location known as the "exposure point." Exposure point concentrations must be determined for each chemical from each medium. Exposure point concentrations can be based on analytical data obtained from on-site sampling, or they may be estimated through modeling.

The exposure point concentrations for exposure to soil, sediment, and surface water through oral and dermal pathways are based directly on the summarized analytical data for the respective media. The determination of these exposure point concentrations is discussed below.

The exposure point concentrations for the inhalation of volatiles and fugitive dust released from soil are modeled based on the representative concentration of chemicals in soil, and are discussed in a separate appendix. In addition, groundwater migration models have been used to develop exposure point concentrations for groundwater exposure pathways, and these are also discussed in a separate appendix.

In accordance with current USEPA recommendations, exposure assessment for this BRA is based on reasonable maximal exposure (RME) assumptions. The RME is defined as the highest . exposure that could reasonably be expected to occur for a given exposure pathway at a site, and is intended to account for both uncertainty in the contaminant concentration and variability in the exposure parameters (such as exposure frequency or averaging time).

For the RME scenario, exposure point concentrations for direct contact pathways (e.g., ingestion of soil) are set at the lower of the 95\% UCL of the mean (calculated as above), or the maximumdetected level from the appropriate medium. This approach ensures that a conservative estimate of the site concentrations is used in exposure calculation, but prevents the exposure point concentration from exceeding the maximum detected site concentration.

Since lognormal distributions were not assumed in all cases for surface water and groundwater, it was necessary to decide whether $95 \%$ UCLs based on transformed or untransformed data should be used. Summary statistics were calculated for both transformed and untransformed datasets, and the following frequency of detection criteria were used in the final determination of the exposure point concentration. 
For chemicals detected in at least 50 percent of samples, the log-transformed data were used as the bias caused by surrogate values (i.e., one-half of the SQL) exceeding detected values is expected to be minimized. For chemicals detected in between 25 percent to 50 percent of samples, a comparison was made between untransformed and transformed data, and the more conservative value (higher exposure concentration) was used. Finally, for chemicals detected in less than 25 percent of samples (or with less than 5 total samples), log-transformation was deemed inappropriate, and untransformed data were used for identifying the exposure point concentration. In addition, in all cases, the limitation that the exposure point concentration could not exceed the maximum detected value in the appropriate medium was retained. Thus, the following decision matrix illustrates the derivation of exposure point concentrations for surface water and groundwater.

\section{RME exposure-point concentration -- Surface water and groundwater}

1. For chemicals detected in $50 \%$ or more of the samples, the $95 \%$ UCL of the logtransformed data is compared to the maximum detected concentration and the smaller value is used as the exposure point concentration.

2. For chemicals detected in at least $25 \%$, but less than $50 \%$, of the samples, the $95 \%$ UCL from the untransformed data is compared to the $95 \% \mathrm{UCL}$ from the transformed data. The larger of these two values is then compared to the maximum detected concentration, and the smaller value of this second comparison is used as the exposure point concentration.

3. For chemicals detected in fewer than $25 \%$ of the samples, the maximum detected concentration is used as the exposure point concentration.

\section{SUMMARY}

The calculations and methods described in this appendix illustrate how site analytical data was summarized and used to determine the point estimates that are used in exposure estimation. The equations for deriving summary statistics are presented and the manner in which these are used to derive exposure point concentrations relevant for ingestion and dermal exposure to soil, sediment, surface water, and groundwater is described. See the additional appendices related to calculating exposure point concentrations for inhalation exposure and on the use of groundwater modelling in some cases for additional exposure estimation information. 
BASELINE RISK ASSESSMENT

F-AREA BURNING/RUBBLE PITS AND RUBBLE PIT
WSRC-TR-94-108

REV. 1.2, MARCH 1996

\section{CITATIONS}

USEPA, 1992. Supplemental Guidance to RAGS: Calculating the concentration term. OSWER 9285.7-081. May, 1992.

Gilbert R.O., 1987. Statisitical Methods for Environmental Pollution Monitoring. New York: Van Nostrand Reinhold. 


\section{APPENDIX B}




\section{THIS PAGE LEFT INTENTIONALLY BLANK}




\section{APPENDIX B}

\section{Leachability of Contaminants from Soil to Groundwater}

\subsection{INTRODUCTION}

As part of the RFI/BRA for the F-Area Burning/Rubble and Rubble Pits (FBRP), analysis of leachability to groundwater of soil contaminants is required. This leachability analysis provides estimated future groundwater concentrations in a hypothetical supply well at the edge of the Pits. The estimated groundwater concentrations are then used to estimate a risk or hazard quotient for chemicals of potential concern (COPCs) that may leach to groundwater in the future. The method and results of the soil leachability evaluation and risk analysis are provided below.

\subsection{SOIL LEACHABILITY APPROACH}

The soil leachability analysis consisted of several steps. These steps inciuded: 1) selection of the modeling approach, 2) review of reports to estimate physical setting parameters (hydrogeologic data, Pit descriptions, etc.), 3) review of literature to obtain chemical and biological properties of COPCs, 4) review and analysis of measured soil contaminant data to estimate reasonable maximum exposure (RME) soil concentrations, 5) screening of RME soil concentration data, 6) estimation of groundwater concentrations, 6) estimation of carcinogenic and noncarcinogenic risk, and 7) sensitivity analysis for contaminants that are estimated to pose a potentially significant risk. Each of these steps are discussed below.

\subsection{Modeling Approach}

The soil leachability analysis consisted of two distinct calculations. The first calculation estimated the leachability of contaminants from the source area. For the FBRP, the source of contaminants is considered four feet below ground surface to the bottom of the Pits, per SCDHEC guidance for the FBRP. The leachability analysis provides an estimate of the soil water contaminant concentration at the water table surface. This soil water concentration is used to calculate a groundwater concentration at the receptor by dividing by a groundwater dilution factor. The groundwater dilution factor accounts for mixing that occurs when the leached contaminants are transported into the "clean" groundwater.

\subsubsection{Soil Leachability Modeling}

The soil leachability estimate was performed using an analytical equation that is programmed into an Excel $\otimes$ spreadsheet. Steady one-dimensional flow in the vadose zone is assumed to represent average flow over the period of interest. Reversible, equilibrium adsorption of contaminants was incorporated through a distribution coefficient 
$\left(\mathrm{K}_{\mathrm{d}}\right)$. First-order decay of contaminants was incorporated by utilizing published half-lives. For radionuclides, the half-life is due to radioactive decay, and for organic contaminants the half-life is due to biological reaction. EPA (1994) recommends assuming an infinite source of contaminants for a simple site-specific soil screening model, so dispersion is not incorporated into the vadose zone estimate because it will not affect the maximum groundwater concentration or the time that it occurs. Volatilization of potentially volatile organic compounds is not incorporated into the estimate because the bulk of potential volatilization has already occurred at the FBRP and not incorporating volatilization is slightly more conservative.

The equation that describes the soil water concentration is given by:

$$
C_{w}(z=L, t=T \max )=C_{w}(z=0, t=0) \exp \left(-0.693^{*} T \max / t_{1 / 2}\right)
$$

where $\mathrm{Cw}$ is the soil water concentration

$\mathrm{L}$ is the distance between the bottom of the Pit and the water table surface

Tmax is the time that the maximum soil water concentration occurs at the water table surface

$t_{1 / 2}$ is the biological or radiological half-life

$z$ is the vertical axis with the origin at the bottom of the Pits

Tmax is estimated by dividing the distance between the bottom of the Pit and the top of the water table surface $(\mathrm{L})$ by the retarded soil water velocity. That is,

$$
\operatorname{Tmax}=\left(L^{*} R / V\right)
$$

where $V$ is the soil water velocity in the vadose zone, and $R$ is the retardation coefficient. $V$ is estimated from the recharge rate, effective porosity, and average percent saturation (Flach et al., 1996) as follows:

$$
\mathrm{V}=\mathrm{I} /\left(\theta_{\mathrm{e}} * \mathrm{~S}_{\mathrm{w}}\right)
$$

where $I$ is the average recharge rate, $\theta_{c}$ is the effective porosity, and $S_{w}$ is the average percent water saturation. The retardation coefficient is calculated as:

$$
\mathbf{R}=1+\left(\mathbf{K}_{\mathrm{d}} \rho_{\mathrm{b}} / \theta_{\mathrm{T}}\right)
$$

where $K_{d}$ is the distribution coefficient, $\theta_{T}$ is the total porosity, and $\rho_{b}$ is the bulk soil density. The soil concentration at the bottom of the Pit is calculated from the total soil concentration as follows (EPA, 1994, page 2-23): 


$$
C_{w}=\frac{C_{T}}{K D}
$$

where $\mathrm{C}_{T}$ is the reasonable maximum exposure concentration (RME) within the Pits. This formula corrects for the contaminants that are in the water phase of the soil sample and assumes a linear equilibrium between the soil and water. Any potential contamination in . the air phase of the sample has been ignored (i.e., we have consistently assumed no volatilization).

\subsubsection{Groundwater Dilution Factor}

A groundwater dilution factor is calculated as described by EPA (1994, pages 2-32,33). This calculation requires an estimate of the water table hydraulic conductivity; hydraulic gradient, water table "thickness", source length parallel to groundwater flow, recharge rate, and longitudinal and vertical dispersivity. These parameters were obtained from WSRC (1994) or from recommended values provided by EPA (1994). The soil water concentration at the surface of the water table is divided by the groundwater dilution factor to obtain a groundwater concentration. The groundwater concentration is converted to a risk for the future unit resident or worker via the groundwater exposure pathway.

The values of parameters used in the groundwater dilution factor are provided in the Table below.

Table B1. Parameters for Estimating the Groundwater Dilution Factor

\begin{tabular}{|c|c|c|}
\hline PARAMETER & PITS 231-F \& -1F & PIT 231-2F \\
\hline $\begin{array}{c}\text { Saturated Hydraulic } \\
\text { Conductivity (ft/day) }\end{array}$ & 10 & 10 \\
\hline Infiltration Rate & 17 inches per year & 17 inches per year \\
\hline Hydraulic Gradient & 0.036 & 0.036 \\
\hline $\begin{array}{c}\text { Saturated Thickness of } \\
\text { Water Table }\end{array}$ & 70 feet & 70 feet \\
\hline $\begin{array}{c}\text { Source Length Parallel to } \\
\text { Water Table }\end{array}$ & 300 feet & 164 feet \\
\hline $\begin{array}{c}\text { Calculated Mixing Zone }: \\
\text { Depth }\end{array}$ & 2 & 19 feet \\
\hline Calculated Dilution Factor & 11.8 & 11.7 \\
\hline
\end{tabular}


The dilution factor calculation is fairly simple and is premised on "basic" mixing principles. It is slightly conservative from an exposure viewpint because it does not account for dilution that would occur when groundwater is pumped at a supply well. That is, the mixing depth would probably be different from the screen location of a water supply well, which would result in greater dilution. Also, the zone of capture of a supply well can be wider than the width of the plume, which would also decrease the contaminant exposure concentration.

\subsubsection{Modeling Approach Summary}

The modeling approach consisted of series of conservative screening steps and estimates. First, the RME soil concentrations are compared to generic soil screening levels (EPA, 1994) with no dilution or attenuation. If the RME soil concentration is less than the generic screening level, the contaminant is eliminated from further consideration. For those contaminants that pass the generic screening, a conservative dilution attenuation estimate is made based on an approach that is consistent with the Simple Site-Specific Method of EPA's Soil Screening Guidance (EPA, 1994). That is, parameters that are fairly well known and easily obtained are used in addition to reference values. Risks and hazard quotients are calculated for contaminants after this simple, site-specific screening estimate. For those contaminants that potentially pose a risk after the simple site-specific screening, less conservative but still defensibly conservative parameters are used to estimate a final groundwater exposure concentration.

The following summarizes the major assumptions for the soil leachability analysis performed to support the risk assessment.

1. One-dimensional, steady flow with uniform average soil properties.

2. Reversible, equilibrium, linear soil-water distribution of contaminants.

3. Biological decay is aerobic and is described by a first-order rate constant.

4. No volatilization of contaminants.

5. Dilution factor (EPA, 1994) calculation method is reasonable.

6. Infinite source of contaminants within the Pits.

7. The $\mathrm{pH}$ of the soil water is approximately five units.

\subsection{Physical Setting and Hydrogeological Parameters}

The FBRP are located in the F-Area within the SRS. The nearest receiving steam is Upper Three Runs Creek. Burning/Rubble Pits 231-F and 231-1F are adjacent to each other and are therefore simulated as a single Pit. Rubble Pit 231-2F is located approximately 350 feet from the others Pits and is therefore modeled separately. The nature of the contamination at $231-2 \mathrm{~F}$ is also markedly different, because materials were reportedly not burned there. Pits $231-\mathrm{F}$ is approximately 275 feet by 63 feet, and 231-1F is approximately 325 feet by 89 feet, with the longer side being parallel to groundwater flow. Pit 231-2F has dimensions of approximately 164 feet by 33 feet with the longer side 
again being approximately parallel to groundwater flow. The depth of the Pits extends to approximately 12 feet below land surface (WSRC, 1995).

The water table is approximately 70 feet below land surface (WSRC, 1994). The first confining layer is sporadic so the water table aquifer thickness is assumed from the top of the Gordon Confining Unit (formerly "Green Clay") to the top of the water table.

Therefore, the water table saturated thickness was taken to be approximately 70 feet thick (WSRC, 1994). The hydraulic conductivity in the water table is estimated as 10 feet per day, with a horizontal gradient of approximately 0.036 (WSRC, 1995).

The recharge rate to the vadose zone is estimated as 17 inches per year (Flach et al., 1996). The effective porosity is estimated as 0.20 and the bulk soil density as $1.65 \mathrm{gm} / \mathrm{ml}$ (Looney et al., 1987), and the average percent water saturation is estimated as 0.50 (Flach et al., 1996).

These data are summarized in the Table below.

Table B2. Additional Hydrogeological and Physical Properties at the FBRP

\begin{tabular}{|l|l|}
\hline Property & Estimated Value \\
\hline Depth to Bottom of Pits & 12 feet \\
\hline $\begin{array}{l}\text { Approximate Areal Pit Dimensions (231-F } \\
\&-1 F)\end{array}$ & 150 feet by 300 feet (combined) \\
\hline Areal Pit Dimensions (231-2F) & 33 feet by 164 feet \\
\hline Depth to Water Table & 70 feet \\
\hline Total Porosity & 0.50 \\
\hline Effective Porosity & 0.20 \\
\hline Recharge Rate & 17 inches per year \\
\hline Water Saturation & 0.50 \\
\hline Soil Bulk Density & $1.65 \mathrm{gm} /($ cubic $\mathrm{cm})$ \\
\hline
\end{tabular}

\subsection{RME Concentrations and Generic Soil Screening Levels}

The soil concentration data from four feet below land surface to the bottom of the Pits were analyzed to support the soil leachability analysis. The summarized results of that analysis are provided in Tables B3 and B4. If a value was never detected, it was not considered as a COPC. The RME soil concentration is the lesser of the $95 \%$ upper confidence limit (UCL) of the log-transformed data and the maximum level detected.

The RME soil concentrations for the COPCs were compared against generic SSLs (EPA, 1994, pgs. 3-31 to 33) for the migration to ground water pathway levels with a dilution attenuation factor (DAF) of 1.0. If the RME soil concentration, as determined by the above process, is less than the generic SSL, the COPC is eliminated from further consideration, i.e., it is not included in the soil leachability analysis. If a generic SSL is not 
available, as is the case for all radionuclides, the COPC is retained in the soil leachability analysis. The results of this comparison process are also presented in Tables B3 and B4.

It is important to note that contaminants with data qualifiers (such as " $\mathrm{f}$ " qualifiers) are retained in the soil leachability analysis. However, COPCs that contribute significantly to the estimated risk and have a significant fraction of data qualifiers are caveated in the discussion of risk.

\subsection{Contaminant Specific Data}

For the contaminants that are retained in the soil leachability analysis, chemical specific information are required such as biological half-lives for the organic contaminants, radiological half-lives for the radioactive contaminants, and distribution coefficients for all contaminants. Metals were assumed not to undergo any non-adsorptive type reactions, which is reasonable and slightly conservative. The distribution coefficients are used to calculate retardation coefficients by the equation presented previousiy.

Distribution coefficients can vary widely depending upon soil chemistry (cation exchange capacity, organic carbon, etc.) and soil water chemistry ( $\mathrm{pH}$, total dissolved ions, etc.). The value selected for the distribution coefficient significantly affects the estimated groundwater concentrations and thus the risk assessment. Initial conservative, yet reasonable, estimates of distribution coefficients of the COPCs are provided in Table B5, along with the reference where the value is obtained. A few of the parameters are different than those utilized by WSRC (1995). For example, "contaminants" such as iron affect adsorption and are controlled by solubility at a $\mathrm{pH}$ of five in oxidizing environments, and thus a $\mathrm{Kd}$ for iron is not appropriate. This is also true for aluminum, magnesium, calcium, etc. Sensitivity analyses will be performed for contaminants that are estimated to create adverse risk because there is greatest uncertainty with the distribution coefficients.

The biological and radiological half-lives are taken from available references and are predominantly the same as WSRC (1995). The main exception is that WSRC (1995) used reported biological half-lives for organics in groundwater versus in soil. Because the soil leachability is in the unsaturated zone (soil), the less conservative but still defensibie biological half-lives are those published for soil. Aerobic biological half-lives in soil are typically two to twenty times greater than those in groundwater because oxygen is much more readily available in soil and is more "mobile." The radiological and biological halflives are provided in the tables that provide the results of the soil leachability analysis. Note that some compounds are given a biological half-life of $1 \mathrm{E}+99$ years which means that they are assumed to be non-biodegradable.

Selecting distribution coefficients for several of the metal COPCs is difficult. Those constituents are identified with asterisks in Table B5. For example, iron is a major component of soil and typically provides the adsorption sites for several other compounds. Also, several of the metal COPCs are commonly occurring elements in natural 
groundwater. This is supported by EPA (1994, pg 5-28) which input aluminum, calcium (and carbonate), iron, manganese, magnesium, and potassium into their predictive model for simulating distribution coefficients. Distribution coefficients are estimated for these COPCs based on observed groundwater and soil concentrations in the vicinity of FBRP at Pits 231-F and 231-1F.

\subsection{Estimated Groundwater Concentrations}

The estimated groundwater concentrations based on the simple site-specific screening approach are provided in Tables B6 through B8 for organics, radionuclides and metals for Pits 231-F and 231-1F, respectively and in Tables B9 through B11 for organics, radionuclides and metals for Pit 231-2F. The Tables include the distribution coefficient, calculated retardation coefficient, years from 1994 that the maximum concentration occurs, soil water concentration at the water table surface, and COPC concentration at a hypothetical supply well at the edge of the Pits. The time for the maximum concentration is referenced to 1994 because that was the year that the soil data were collected. The concentrations presented in Tables B6 through B 11 should be viewed as second tier screening concentrations, because very conservative assumptions were used to develop them. For contaminants that potentially pose a risk based on the estimates provided in Tables B5 through B11, more reasonable yet still conservative and defensible assumptions are made. This further refinement is discussed under the Risk and Hazard Analysis Section of this Appendix.

The only organic compounds that are estimated to appear in the groundwater (above $10^{-6}$ $\mu \mathrm{g} / \mathrm{L}$ ) in the next one hundred years are 1,2-dichloroethylene, dichloromethane, tetrachloroethylene, delta benzene hexachloride, and heptachlor epoxide. PCB-1254 is estimated to appear in groundwater substantially in the future, but this results because PCBs are strongly bound to soil and we did not allow for any biological decay of the PCB.

The only radionuclides estimated to appear in groundwater in the next 100 years or at appreciable activities are iodine-129, technetium-99, and tritium. The radionuclide activities were estimated as previously discussed, with the exception of tritium. Tritium in soil and groundwater at the Pits (and at the SRS) is in the form of tritium oxide (HTO). This form behaves essentially as water in the environment. As a result, the appropriate distribution coefficient for tritium is zero. However, dividing the total RME concentration by zero, would translate to an infinite tritium soil water concentration (or activity), which is aphysical. Tritium in soil is measured by removing the water phase and measuring the tritium activity in the water. The activity is then reported-for the soil sample. Therefore, for tritium the soil water concentration must be estimated by utilizing the average saturation and density of the soil as follows.

Soil water activity $=(\operatorname{RME} \text { Soil Activity, } \mathrm{pCi} / \mathrm{gm} \text { soil })^{*}($ bulk density soil, gm soil/ml soil $) /$ (total porosity, $\mathrm{ml}$ voids/ml soil)/(saturation, $\mathrm{ml}$ water/mi voids) 
This soil water activity is then used to calculate a groundwater activity as was done with the other radionuclides. That is, the tritium decays as it travels to the top of the water table surface and then it is further diluted when it mixes with the groundwater.

Because tritium (tritiated water) behaves essentially as water in the environment, it is an excellent tracer and therefore provides an excellent comparison for a vadose zone transport estimate. As shown in Table B7, the estimated maximum groundwater tritium activity is $4.45 \mathrm{pCi} / \mathrm{ml}$. The maximum observed activity of tritium in monitoring wells in the vicinity of the FBRP is $5.1 \mathrm{pCi} / \mathrm{ml}$ (Table 3-3). The predicted and observed activities . are in excellent agreement. This provides significant evidence that the unretarded soil water velocity and dilution factor estimates are reasonably accurate. Thus for this analysis, the greatest uncertainty for metals and radionuclides is most likely the distribution coefficient. For organic COPCs there is additional uncertainty for the biological half-lives.

The only hazardous metal that may appear in groundwater in the next one hundred years is cadmium. The abundantly naturally occurring metals (aluminum, calcium, magnesium, magnesium, iron, and potassium) are not estimated to appear because the distribution coefficient was chosen to estimate the observed groundwater concentrations. These constituents are most likely driven by solubility and a distribution coefficient is not appropriate. Presently observed groundwater concentrations may be the best indicator of their potential risk.

\subsection{Risk and Hazard Analysis}

The final estimated risks and hazards from the soil leachability to groundwater pathway are summarized in Table B12. (The equations that were used to estimate the risks and hazards are provided in Section 3.2.4.3 in the main body of this document.) Some of the concentrations used to estimate the risks and hazards are different from those presented in Tables B6 through B12; they are noted with asterisks in Table B12. Different estimated exposure concentrations occur because the concentrations presented in Tables B6 through B11 are based on fairly conservative assumptions. Therefore, for some constituents slightly less conservative but still defensible assumptions were used to refine the exposure estimate. The assumptions and sensitivity analysis for major COPCs are discussed below for each of the constituents identified in Table B12.

As reported in Table B12, none of the contaminants have cancer risks that exceed $10^{-4}$. Iodine-129, technetium-99, and tritium are the only contaminants estimated to have cancer risks above $10^{-6}$. Iodine-129 is estimated to produce the greatest cancer risk, but that risk is driven by J-qualified data. That is, iodine-129 was never detected above the detection limit in any of the soil samples. However, it was reported at below half the SQL for two measurements that were J-qualified. The tritium that produces the risk is most likely the result of tritium in rainfall. 
None of the COPCs are estimated to result in a hazard quotient greater than unity. Cadmium is estimated to result in the greatest hazard quotient of approximately 0.6, but it occurs approximately 83 years from 1994 . The total hazard index from the soil leachability to groundwater pathway is below one (Appendix D).

Below is a discussion of uncertainty for the COPCs that are presented in Table B12.

\section{1,2-Dichloroethylene}

The estimated 1,2-dichloroethylene (DCE) groundwater concentration is below typical detection limits. DCE is not presently detected in the groundwater in the vicinity of the FBRP. In an aerobic environment, DCE rapidly degrades to carbon dioxide, hydrogen and chloride ions. Dichloroethylene was only detected in one soil sample within the interval of four feet to bottom. As a resuit, the DCE exposure estimate from the soil leachability to groundwater estimate does not warrant sensitivity analysis because the risk is estimated to be negligible after applying fairly conservative assumptions.

\section{Dichloromethane}

The groundwater concentration used to calculate the risks and hazard associated with soil leachability is less than that reported in Table B.6. The RME soil concentration of 0.265 $\mathrm{mg} / \mathrm{Kg}$ used in Table B6 is based on all data including J qualified data. Dichloromethane was only detected 3 times out of 21 samples when J qualified data is excluded. Also, one J-qualified value of $12.4 \mathrm{mg} / \mathrm{Kg}$ substantially impacts the exposure estimate. Therefore; the $95 \%$ UCL for the RME soil concentration was recalculated by considering an average value in place of the maximum. In this manner, the $95 \%$ UCL for the RME soil concentration becomes 0.013 or a factor of 20.4 times less. Therefore, the exposure concentration presented in Table B12 for dichloromethane is 20.4 times less than that reported in Table B6. Thus the risk presented is for an average in the absence of the hot spot. Even if the "hot spot" value is considered, the risk and hazard for dichoromethane from soil leachability would be considerably less than $10^{-6}$ or one, respectively. Dichloromethane may currently appear in groundwater at the FBRP because it is reasonably mobile and may have leached from the Pits. Also, dichloromethane does not readily undergo aerobic biodegradation.

\section{Tetrachloroethylene}

The estimated tetrachloroethylene (PCE) exposure concentration from Table B6 was used to estimate the risk and hazard. Most PCE would have already leached from the Pits. Also, the highest detected PCE at the Pits is in an upgradient well. Therefore, the estimated exposure concentration is believed to be reasonable. 


\section{Heptachlor Epoxide}

The estimated carcinogenic risk from heptachlor epoxide is based on a single detected value within Pit 231-1F. It was not detected in the soil below the bottom of the Pit nor is it presently detected in groundwater in the vicinity of the Pits. However, based on the estimate that was made with available data, it should have already reached the groundwater because the travel time between the bottom of the Pits and the top of the water table is estimated as 7.4 years and the Pits were in "operation" until the late 1970's. Therefore, the distribution coefficient that applied is overly conservative.

Another important consideration is the dilution factor. Because heptachlor epoxide is detected at a single core, the size of the source area can be justifiably reduced. The dimensions of 231-1F are approximately 325 feet by 89 feet. There were four core samples collected within Pit 231-1F, so one can assume a source area of approximately 81 feet by 22 feet, which is approximately 0.04 acres. The dilution factor on the 95 th percentile according to EPA (1994, pg. 2-36) is approximately 330 (assuming a linear relation). The actual applied dilution factor was 11.8 , therefore the concentration exposure concentration can be reduced by a factor of approximately 30 from Table B6, as was done for Table B12.

Therefore, the estimated exposure concentration from Table B6 was too large because of the overly conservative distribution coefficient and the overly large estimated source area.

\section{Cadmium}

The input RME soil concentration $(1.11 \mathrm{mg} / \mathrm{Kg}$ ) is based on the maximum of 4 out of 21 detected values. One value of $22.2 \mathrm{mg} / \mathrm{Kg}$ substantially impacts the exposure estimate. Therefore, the $95 \%$ UCL for the RME soil concentration was recalculated by considering an average value in place of the maximum. In this manner, the $95 \%$ UCL for the RME soil concentration becomes 0.36 or a factor of 2.5 times less. Therefore, the exposure concentration presented in Table B12 for cadmium is 2.5 times less than that reported in Table B8. Thus the risk presented is for an average in the absence of the hot spot. Use of the average exposure concentration without the "hot spot" is defensible because the dilution factor would increase by an order of magnitude if the source was considered from a single core (see discussion on heptachlor epoxide).

The hazard coefficients in Table B12 are based on a distribution coefficient of $6.3 \mathrm{~L} / \mathrm{Kg}$ (Looney et al., 1987). If a value of $0.9 \mathrm{~L} / \mathrm{Kg}$ (EPA, 1994) is used, the exposure concentration would increase by a factor of 7.0 and the time for the maximum exposure decrease by a factor of 7.0 to approximately 25 years. However, even with the value of $6.3 \mathrm{~L} / \mathrm{Kg}$, the estimated exposure concentration is $4.83 \mathrm{ug} / \mathrm{L}$ which is twice the maximum detected already. Use of the greater distribution coefficient is justified because the cadmium only appears in the does not appear within the Pits deeper than 8 feet, which suggests a distribution coefficient greater than $6.3 \mathrm{~L} / \mathrm{Kg}$. 
Iodine-129

The estimated cancer risk for groundwater ingestion of I-129 by a resident is $2.62 \mathrm{e}-5$ based on an exposure concentration of $7.29 \mathrm{pCi} / \mathrm{ml}$. This value must be viewed with caution, because it is based on 2 detects out of 13 samples. The two detected values have $\mathrm{J}$ qualifiers which means they are estimates below the practical quantification limit (PQL) and that the estimates have extreme uncertainty. Iodine-129 is also currently undetected in the groundwater at the FBRP. Another factor to be considered is that the I-129 could be associated with atmospheric deposition of anthroprogenic sources, and it may not be associated with FBRP. The carcinogenic risk for I-129 could easily be $<<1 e-6$ and it may not be associated with activities at the unit.

The exposure concentration is based on a $\mathrm{Kd}$ of $25 \mathrm{~L} / \mathrm{Kg}$, which is for organic soils (Sheppard and Thibault, 1990). This value is 25 times higher than that recommended for clay and sand. However, employing the lower Kd would mean that the I-129 would have already leached from the FBRP because the travel time would be approximately four years. Also, the estimated groundwater activity would be about twenty-five times higher, which is not currently observed. The use of the $\mathrm{Kd}$ for organic soils is also justified by the practice of burning materials at the Pits which produced organic adsorption sites.

\section{Technetium-99}

The greatest estimated cancer risk for Tc-99 is $5.8 \mathrm{e}-6$ for ingestion of groundwater by a resident based on a groundwater activity of $236 \mathrm{pCi} / \mathrm{ml}$. The risk is based on 8 out of 13 detected values that do not have significant variability. Tc-99 is extremely mobile (low $\mathrm{Kd}$ ) is most forms in the environment; the estimated transport time to the water table is approximately 18 years (Table B6). The Pits were used for much longer than 18 years and it has been approximately 18 years since they have been in use. Despite this, Tc-99 is currently not observed in the groundwater at the FBRP. Therefore, the Tc-99 exposure concentration is believed to be fairly conservative, and at the estimated concentration, still not does not pose a risk near $1 \mathrm{e}-6$.

\section{Tritium}

The estimated carcinogenic risk for tritium is $4.54 \mathrm{e}-6$ (Table B12). The estimated activity of tritium in the groundwater is reasonably accurate. However, the tritium is most likely from rainfall depositing atmospheric tritium from the Separations Area and not associated with disposal practices at the Pits. Also, the estimated concentration is a factor of four below the drinking water standard for tritium.

\subsection{REFERENCES}


EPA, 1994. Technical Background Document for Soil Screening, Review Draft, November 1994, EPA540/R-94/106.

EPA, 1995. Interim Supplemental Guidance to RAGS: Region 4 Bulletins Human Health Risk Assessment; November 1995, Atlanta, GA.

Flach, G. P., L. L. Hamm, M. K. Harris, P. A. Thayer, J. S. Haselow and A. D. Smits, 1996, "Groundwater flow and tritium migration from the SRS Old Burial Ground to Fourmile Branch (U)", WSRC-TR-96-0037.

Howard, P.H., 1991. Handbook of Environmental Degradation Rates, Lewis Publishers, 1991.

Looney, B.B., M.W. Grant and C.M. King, 1987. Estimation of Geochemical Parameters for Assessing Subsurface Transport at the Savannah River Plant, DPST-85-903.

Montgomery, J.H. and L. K. Welkom, 1990. Groundwater Chemicals Desk Reference, Lewis Publishers, 1990.

U.S. DHEW, 1970. Radiological Health Handbook.

Sheppard, M.I. and D.H. Thibault, 1990. Default Soil Solid/Liquid Partition Coefficients, $\mathrm{K}_{d}$ s for Four Major Soil Types: A Compendium, Health Physics, 59(4), 471-482.

Superfund Public Health Manual, USEPA, EPA/540/1-86/060. October 1986.

WSRC, 1994. RCRA Facility Investigation Remedial Investigation Report for the F-Area Burning/Rubble Pits (231-F, 231-1F and 231-2F) (U), WSRC-RP-94-938, Revision 0, September, 1994.

WSRC, 1995. Baseline Risk Assessment for the F-Area Buming/Rubble Pits and Rubble Pit, WSRC-TR-94-108, Revision 1, April, 1995. 


\begin{tabular}{|c|c|c|c|c|c|c|c|c|c|c|c|c|c|c|c|c|c|c|c|c|c|c|c|}
\hline & & 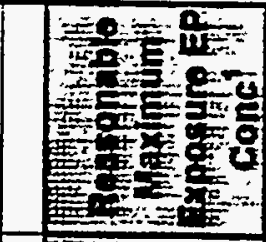 & 另 & 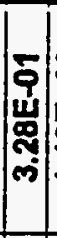 & : & 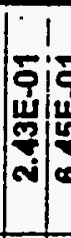 & & & & & & & & 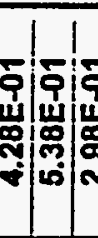 & & 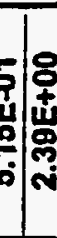 & 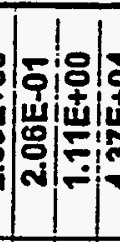 & : & & & & & \\
\hline ह̂: & & 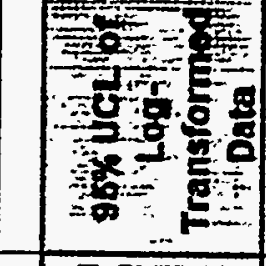 & 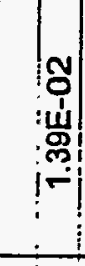 & 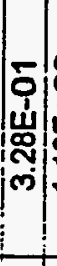 & $\begin{array}{l}8 \\
\\
+ \\
\\
\\
\\
\end{array}$ & & im & & & & & & & 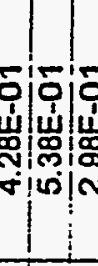 & 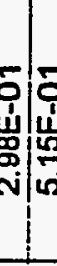 & 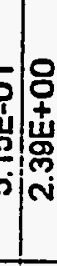 & 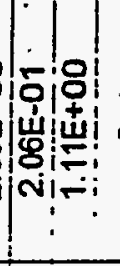 & & & & & $\left|\begin{array}{l}\dot{w} \\
0 \\
0 \\
-\end{array}\right|$ & \\
\hline 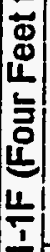 & & 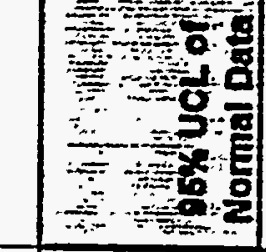 & 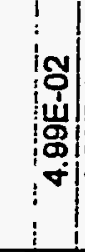 & 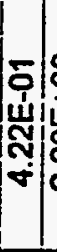 & $\begin{array}{l}8 \\
\\
+ \\
\\
\\
\text { ง. }\end{array}$ & & & & $\sigma^{\circ}$ & ov & $\mid$ & & & 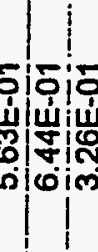 & & 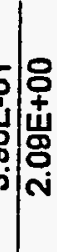 & 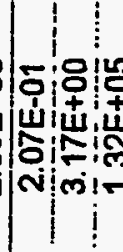 & 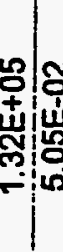 & 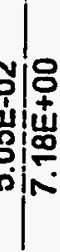 & & 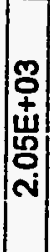 & ง̛ & \\
\hline 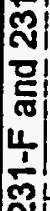 & & 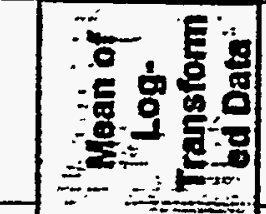 & 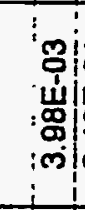 & & & & 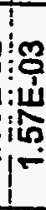 & & $\infty$ & & | & : & & 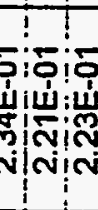 & & 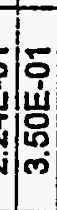 & & זiv & pin & & 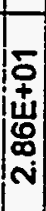 & 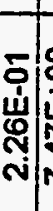 & |con \\
\hline 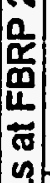 & $!$ & 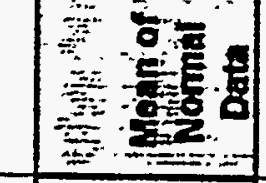 & 染 & & : & 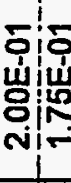 & i⿱口 & & $\mid$ & & 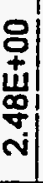 & & & 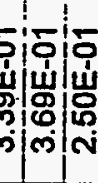 & & : & 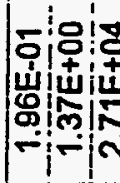 & & & & & & 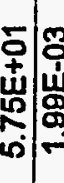 \\
\hline 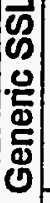 & $!$ & 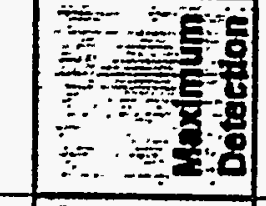 & $\mid$ & & 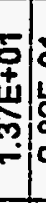 & 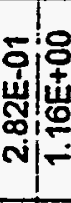 & & & & & $\begin{array}{c}8 \\
\vdots \\
\\
\\
⿱ 亠 䒑 \\
\infty\end{array}$ & & 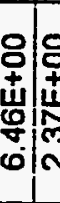 & 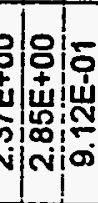 & 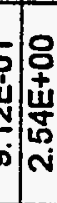 & 客 & 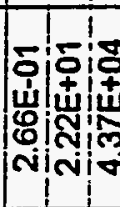 & 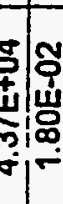 & : & 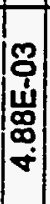 & 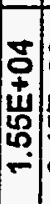 & 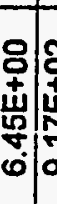 & 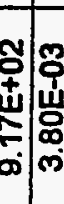 \\
\hline 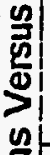 & ; & \begin{tabular}{ll|} 
& \\
$\therefore$ & $\therefore$ \\
& $\therefore$
\end{tabular} & $\begin{array}{l}0 \\
0 \\
0 \\
0\end{array}$ & & ê & 诰 & & & $<1$ & & $\begin{array}{l}0 \\
\frac{1}{3} \\
\frac{9}{2}\end{array}$ & 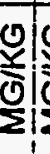 & 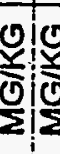 & 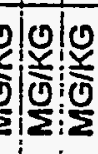 & $\frac{0}{3}$ & $\mid \frac{0}{0}$ & 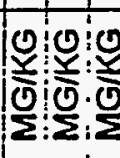 & : & : & $\mid \begin{array}{l}0 \\
\frac{y}{0} \\
\Sigma\end{array}$ & & 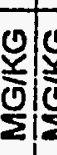 & : \\
\hline 흔 & & $\therefore$ 开要要 & : & 1 & & & & & & & & & & & & & minin & & & $\overline{v i}$ & & $F$ & $\Sigma$ \\
\hline & & & $=!$ & -is & -15 & 2.2 & $=$ & - & $=1$ & -1 & 5 & $=1-$ & -12 & $6-10$ & $2=$ & $1=1$ & I-í & 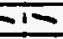 & 10 & & & & -12 \\
\hline & ! & $\therefore \quad \therefore=0$ & & & & & & & & & "20 & n & & & & in & roin & & & & & & $=N$ \\
\hline $\begin{array}{l}\overline{\bar{c}} \\
\text { co } \\
\text { uI }\end{array}$ & $\vdots$ & 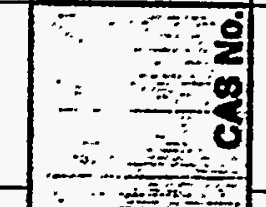 & 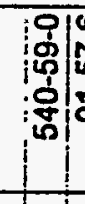 & का: & 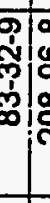 & 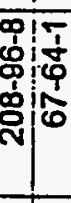 & & & & & 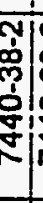 & 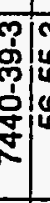 & & 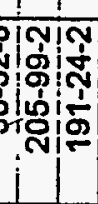 & 要 & $\frac{1}{7}$ & 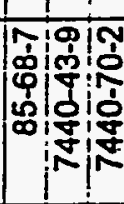 & & 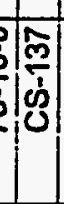 & 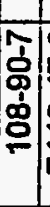 & $\begin{array}{l}\frac{p}{3} \\
\frac{y}{y} \\
o \\
\frac{y}{2}\end{array}$ & 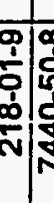 & 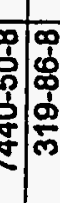 \\
\hline & & 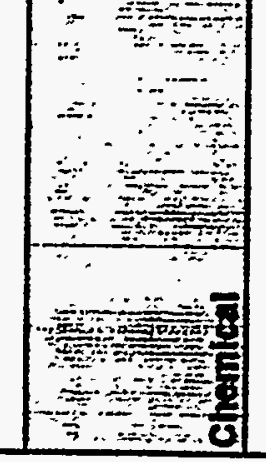 & 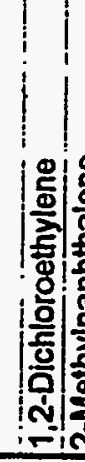 & 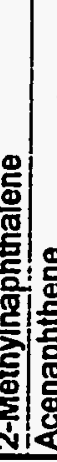 & & 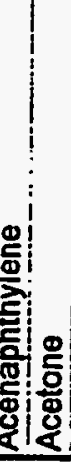 & & & & & & & 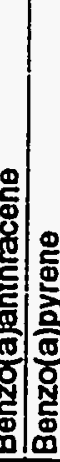 & 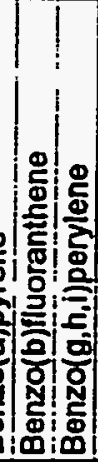 & & 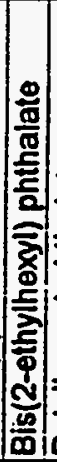 & 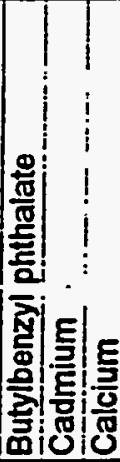 & 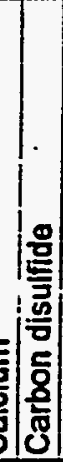 & $\mid$ & & & : & 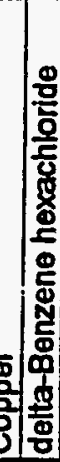 \\
\hline
\end{tabular}




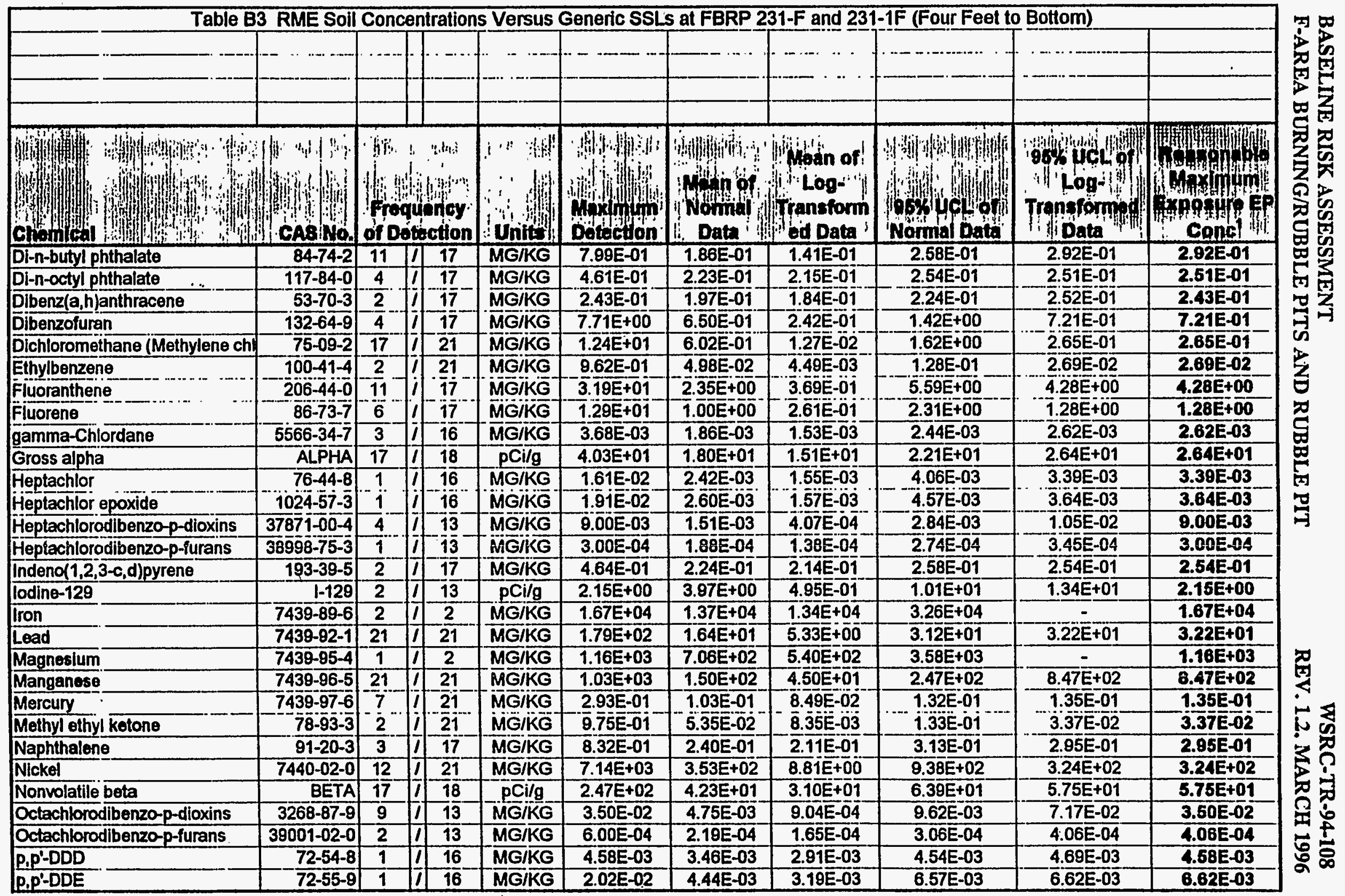




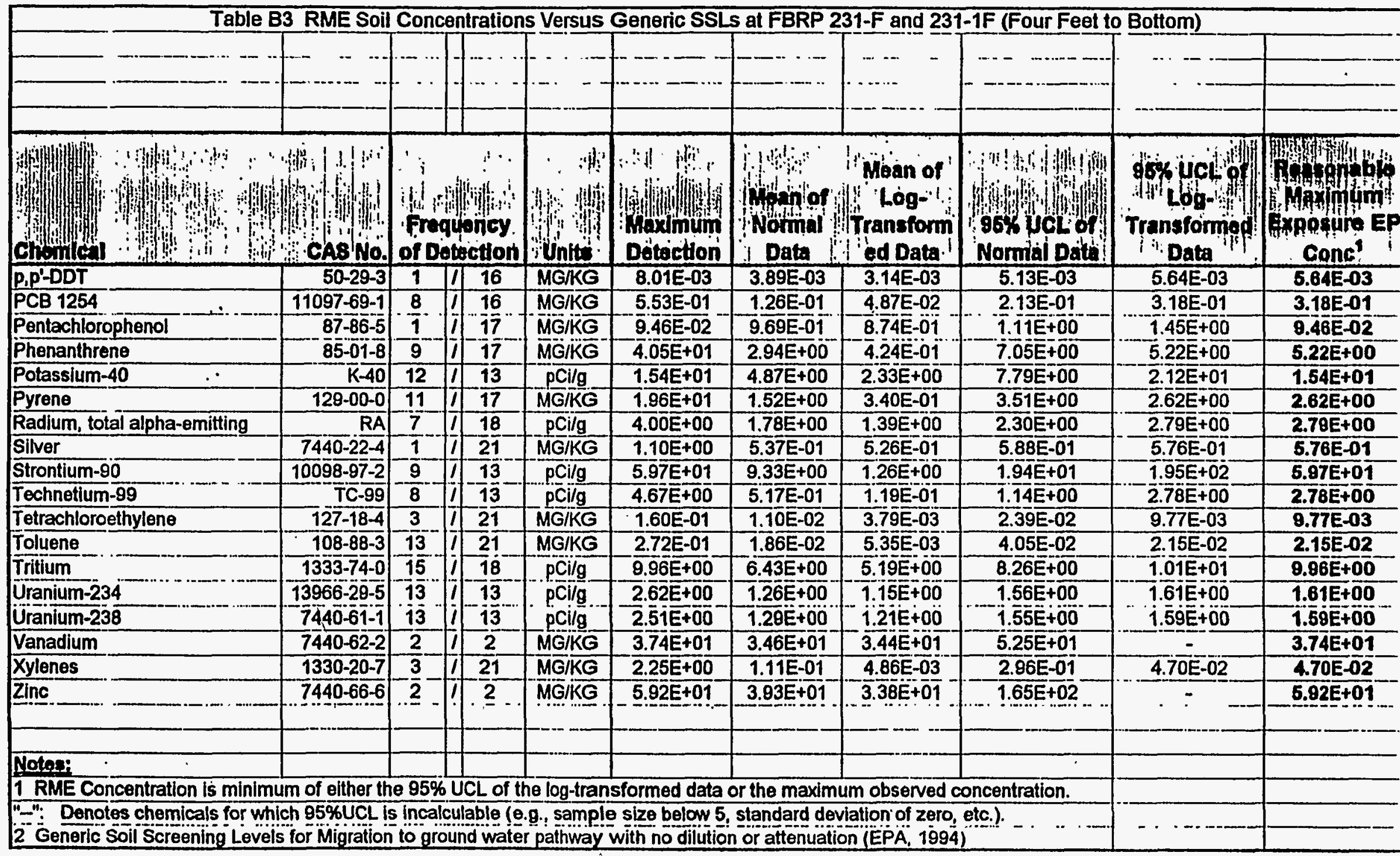




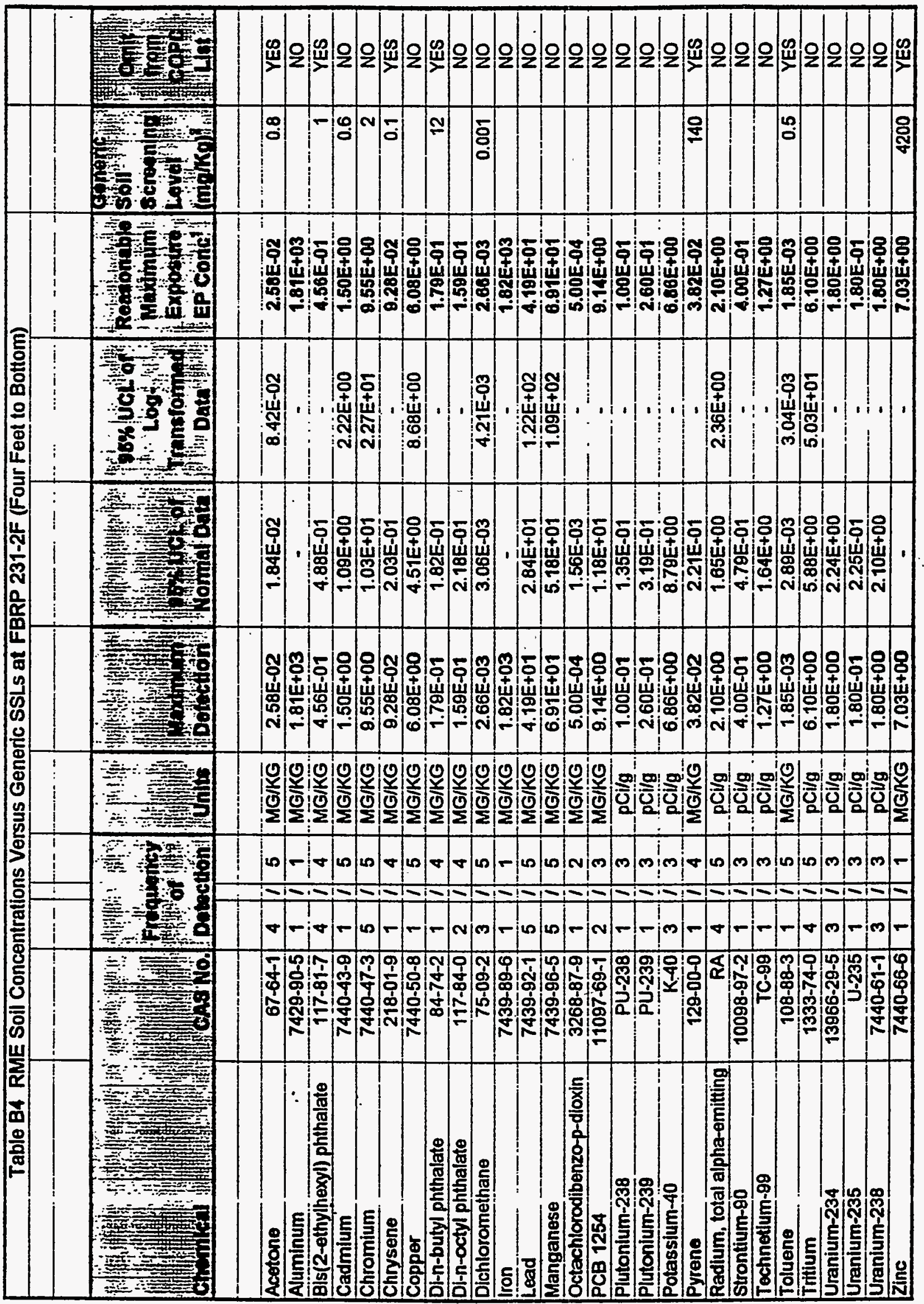




\section{Table B4 RME Soil Concentrations Versus Generic SSLS at FBRP 231-2F (Four Feet to Bottom)}

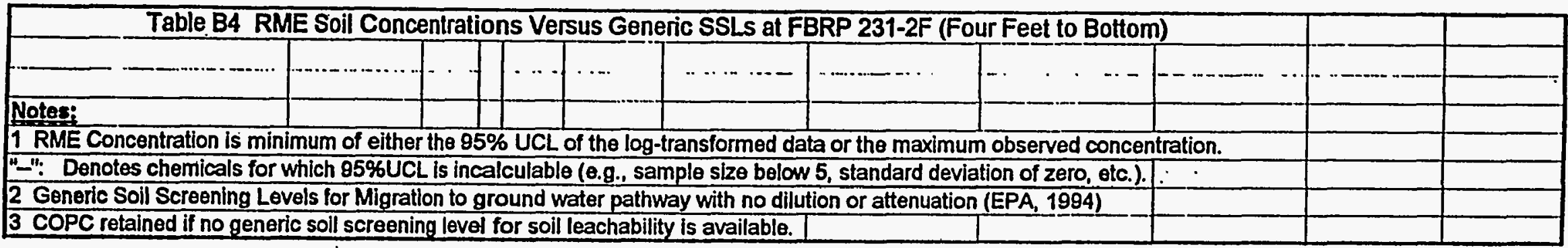


Table B.5 Distribution Coefficients for Chemicals of Potential Concern

\begin{tabular}{|c|c|c|c|c|c|c|}
\hline Organic C & OPC & $\mathrm{Kd}(\mathrm{L} / \mathrm{Kg})$ & Reference & Metal COPC & $\mathrm{Kd}(\mathrm{L} / \mathrm{Kg})$ & Reference \\
\hline 1,2-Dichlor & ethylene & 6.5E-02 & 1 & Aluminum ${ }^{*}$ & $1.2 \mathrm{E}+02$ & \\
\hline 2-Methyina & hthalene & $7.4 E+00$ & 3 & \begin{tabular}{|l|} 
Arsenic \\
\end{tabular} & $2.5 E+01$ & 4 \\
\hline Acenaphth & lene & $2.5 E+00$ & 1 & Barium & $5.3 E+02$ & 7 \\
\hline Aldrin & & $9.6 \mathrm{E}+01$ & 1 & Cadmium & $6.3 \mathrm{E}+00$ & 2 \\
\hline Benzo(a)ar & thracene & $1.4 E+03$ & 1 & Calcium ${ }^{\text {t* }}$ & $4.5 E+02$ & \\
\hline Benzo(a)py & rene & $5.5 E+03$ & 1 & Chromium & $4.0 E+01$ & 2 \\
\hline Benzo(b)fic & oranthene & $5.5 E+02$ & 1 & \begin{tabular}{|l|} 
Copper \\
\end{tabular} & $2.5 E+01$ & 4 \\
\hline Benzo(g,h, & perylene & $1.6 E+03$ & 1 & Iron & $5.0 \mathrm{E}+01$ & \\
\hline Benzo(k)fiu & oranthene & $5.5 E+02$ & 1 & \begin{tabular}{|l|l} 
Lead & \\
\end{tabular} & $1.0 E+02$ & 2 \\
\hline Bis(2-ethyll & exyl) phthalate & $1.0 E+02$ & 3 & Magnesium & $8.0 E+01$ & \\
\hline Chrysene & & $2.0 E+02$ & 1 & Manganese & $2.0 E+02$ & \\
\hline delta-Benz & ne hexachloride & $1.0 E+00$ & Estimated & \begin{tabular}{|l|l} 
Nickel & \\
\end{tabular} & $1.0 E+02$ & 2 \\
\hline Di-n-octyi p & hthalate & $1.9 E+01$ & 5 & Silver & $1.0 E+02$ & 2 \\
\hline Dibenzofur & & $8.1 E+00$ & 3 & Zinc & $1.6 E+01$ & 2 \\
\hline Dichlorome & thane & 1.0E-03 & 1 & & & \\
\hline Ethylbenze & & $1.1 \mathrm{E}+00$ & 1 & Radionuclide COPC & $\mathrm{Kd}(\mathrm{L} / \mathrm{g})$ & Reference \\
\hline gamma-Ch & ordane & $1.0 E+03$ & 3 & Cesium-137 & 5.0E-01 & 2 \\
\hline Heptachlor & & $1.2 E+01$ & 1 & lodine-129 & $2.5 E-02$ & 6 (Organic soil) \\
\hline Heptachlor & epoxide & 2.2E-01 & 1 & \begin{tabular}{|l|} 
Plutonium \\
\end{tabular} & $1.0 \mathrm{E}-01$ & 2 \\
\hline Heptachloro & ibenzo-p-dioxins & $3.3 E+02$ & $1^{*}$ & Potassium -40 & 4.0E-02 & 6 (sand/clay) \\
\hline Heptachloro & ibenzo-p-furans & $1.0 \mathrm{E}+02$ & $1^{*}$ & \begin{tabular}{|l|} 
Radium \\
\end{tabular} & 1.0E-01 & 2 \\
\hline Methyl ethy & ketone & 4.5E-03 & 1 & Strontium-90 & $7.9 E-03$ & 2 \\
\hline Octachloro & dibenzo-p-dioxins & $1.0 E+04$ & 5 & Technetium-99 & $1.0 \mathrm{E}-03$ & 6 (Organic soil) \\
\hline Octachloro & dibenzo-p-furans & $1.0 E+04$ & 5 & \begin{tabular}{|l|l|} 
Tritium & \\
\end{tabular} & 0 & \\
\hline$p, p^{\prime}-D D D$ & & $7.7 E+02$ & 1 & Uranium-234 & 4.0E-01 & 2 \\
\hline$p, p^{\prime}-D D E$ & & $4.4 E+03$ & 1 & Uranium-238 & 4.0E-01 & 2 \\
\hline$p, p^{\prime}-D D T$ & & $2.4 E+02$ & 1 & & & \\
\hline PCB 1254 & & $4.1 E+02$ & 3 & & & \\
\hline Pentachlor & aphenol & $5.3 E+01$ & 1 & & & \\
\hline Phenanthr & & $1.4 E+01$ & 1 & & & \\
\hline Tetrachlorc & ethylene & 3.8E-03 & 2 & & & \\
\hline Toluene & & $3.0 \mathrm{E}-01$ & 1 & & & \\
\hline Xylenes & & 1.0E-03 & 1 & & & \\
\hline & & & & & & \\
\hline Notes: & & & & & & \\
\hline Disributio & coefficient for 0 & ganics is the & product of $\mathrm{pl}$ & blished Koc and foc ( & raction orga & (c carbon_) \\
\hline The fracti & of of organic car & on is assum & d to be 0.00 & 1 (WSRC, 1995). & & \\
\hline Estimate & d based on $2,3,8$ & $8-T C D D(W$ & SRC, 1995) & & & \\
\hline * Predon & inant water spec & es and may & affect adsorp & ion. & & \\
\hline Referenc & & & & & & \\
\hline 1 Superfund & Public Heatth Manua & USEPA, EPAE & $40 / 1-86 / 060,0 c$ & ober 1986 & & \\
\hline 2 Estimation & of Geochmical Parar & ters for assess & ing subsurtace 7 & ransport at the Savanah Rive & & \\
\hline Plant, B. B. & Looney et al, E. I. Di & ont, DPST-85-s & 04, March $\overline{1987}$ & & & \\
\hline 3 Groundwa & er Chemicals DeskF & ference, John F & Montgomery a & d Linda K Welkom, Lewis $\mathrm{P}$ & ublishers, 1990 & \\
\hline 4. EPA, 199 & 4. Technical Backgrc & nd Document $f$ & r Soil Screening & Review Draft, November 19 & 94, EPA540/R- & 1106. \\
\hline 5 HSDB 195 & 4. Same reference $p$ & vided by WSR & (1995). & & & \\
\hline 6. Sheppar & M.l. and D.H. Thibe & 1t, 1990. Defau & Soil Solid/Liquil & Partition Coefficients, Kds & & \\
\hline Four Major & oil Types: A Comper & Ium, Heath $\mathrm{Ph}$ & sics, 59(4), 471 & & & \\
\hline 7. Battelle A & lemorial Institute, 198 & Chamiolnat & Bases for the I & ultimedia Environmental Pol & utant Asse & $\mathbf{m}$ \\
\hline (MEPAS): & ersion 1. Prepared & the U.S. Depa & tment of Energy & & & \\
\hline
\end{tabular}


Table B6. Estimated Organic Concentrations in Grour. Ater from Soil Leachability at Pits 231-F and 231-1F

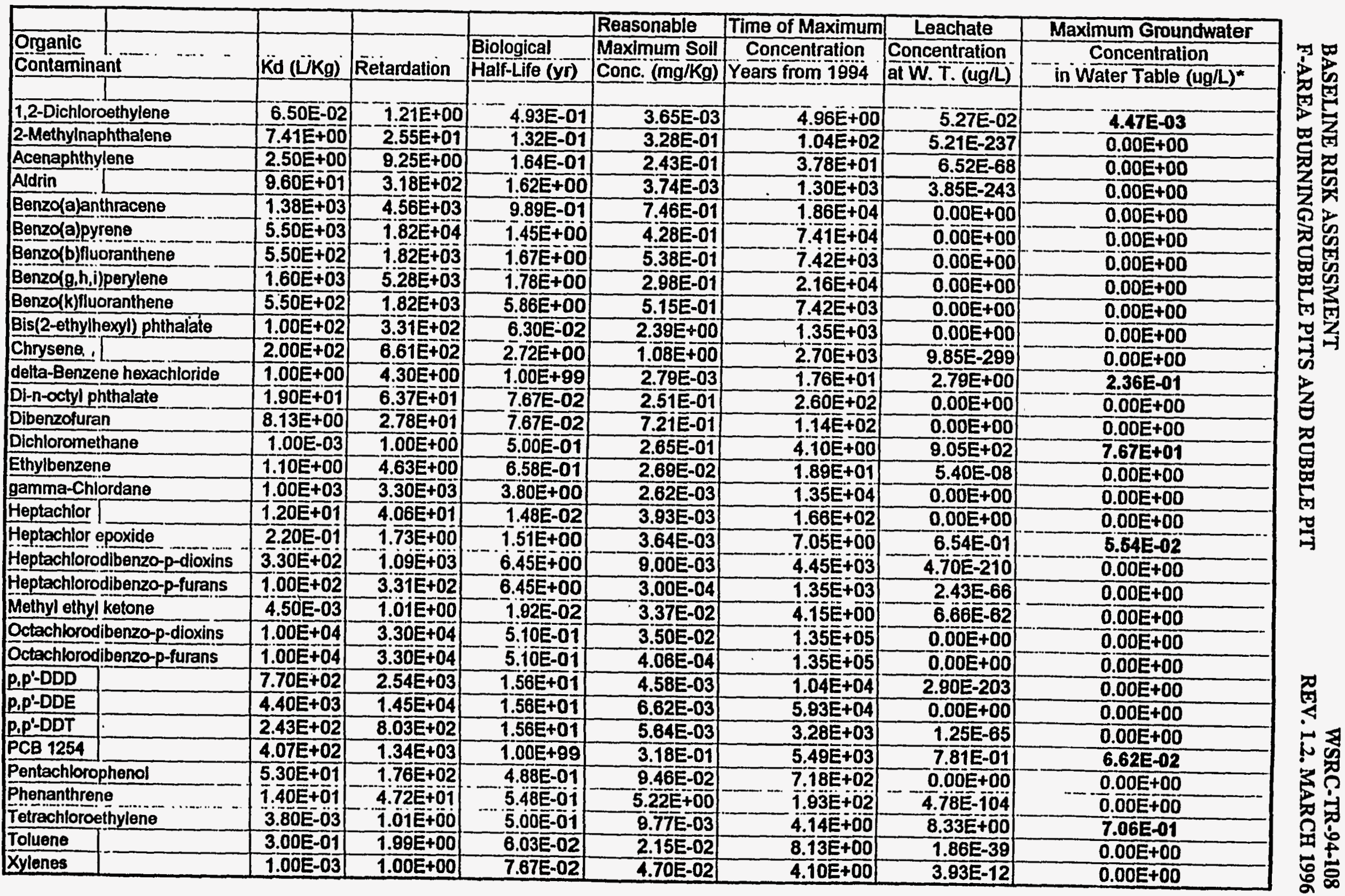


Table B.8 Estimated Metal Concentrations in Groundwater from Soll Leachability at Pits 231-F and 231-1F

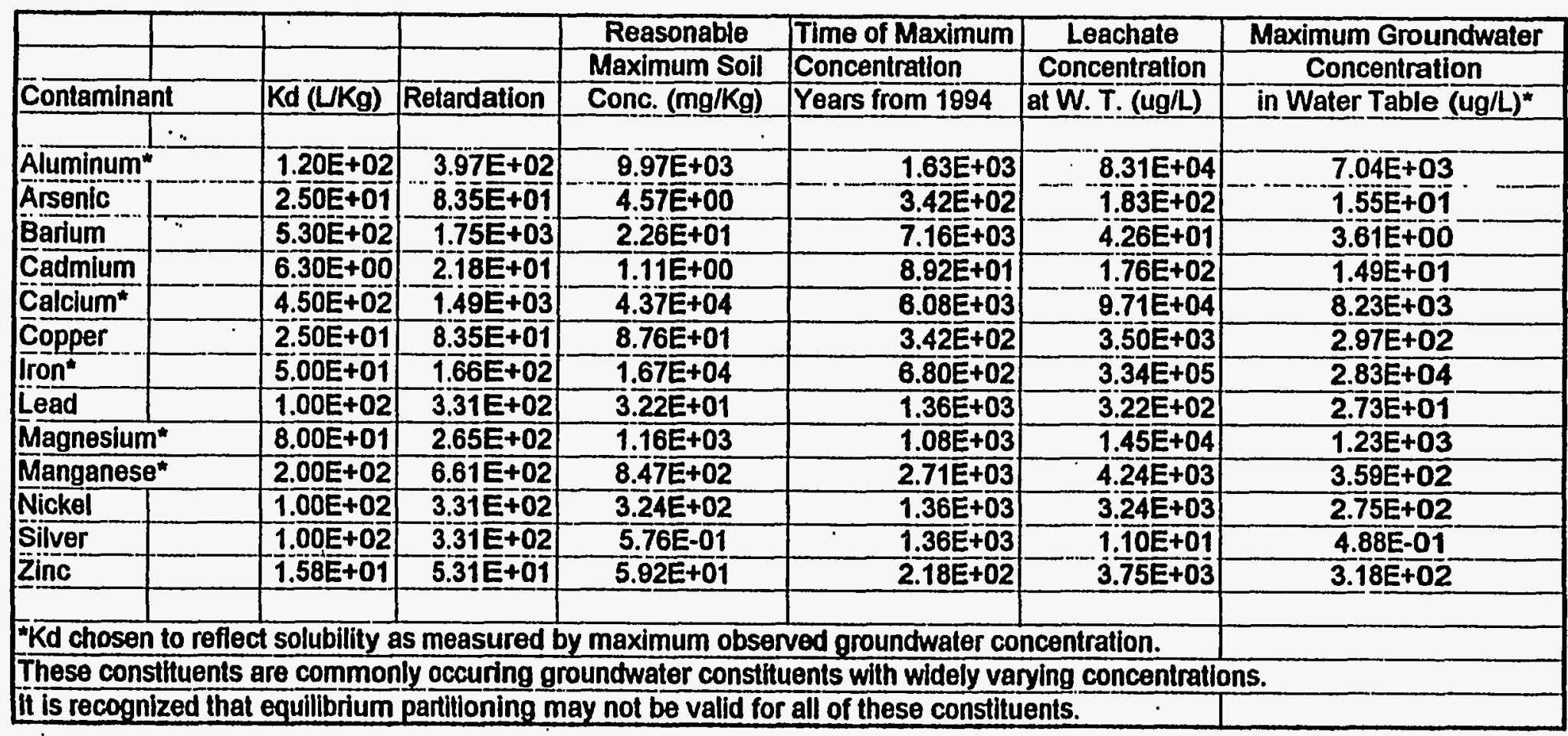


Table B9. Estimated Órganic Concentrations from Soil Leachability at Pit 231-2F

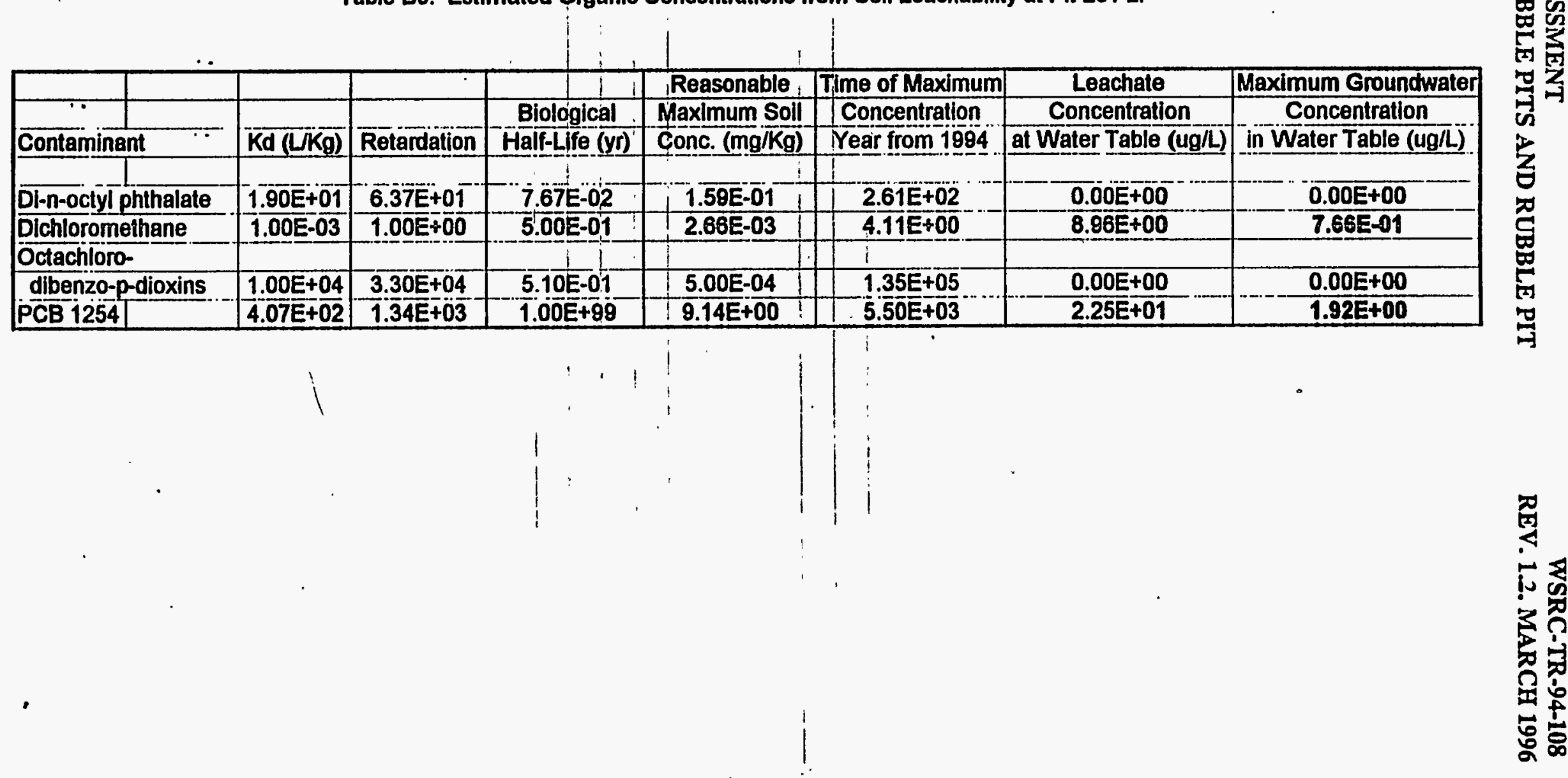


Table B10. Estimated Radionuclide Activities in Groundwater from Soil Leachability at Pit 231-2F

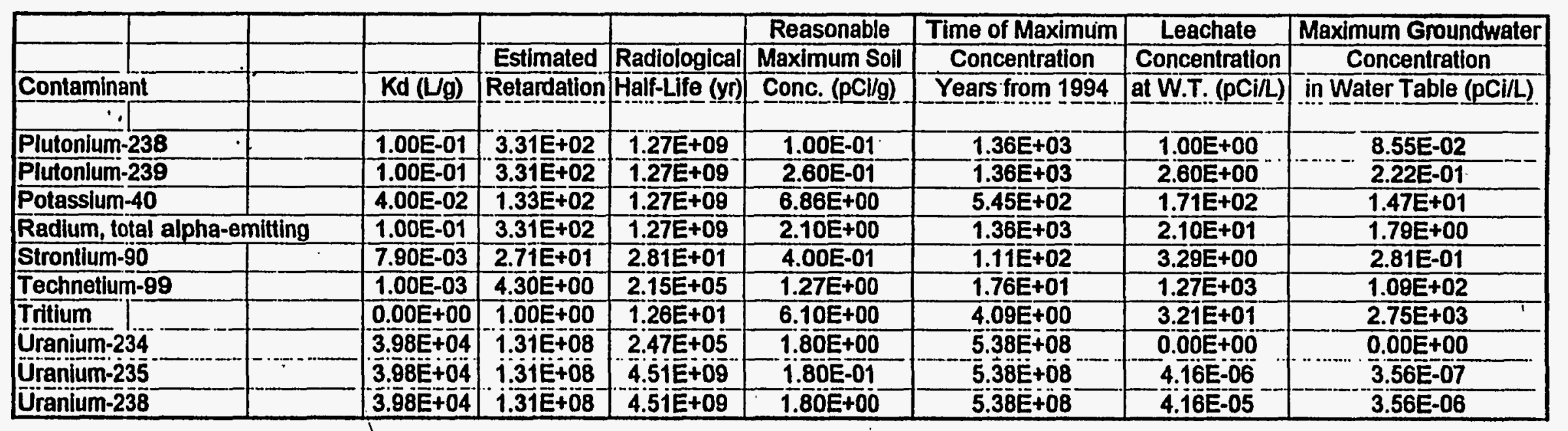


Table B11. Estimated Metals Concentrations in Groundwater from Soil Leachability at Pit 231-2F

\begin{tabular}{|c|c|c|c|c|c|c|c|c|}
\hline & & & & Reasonable & Time of Maximum & Leachate & Maximum Groundwater & \\
\hline \multirow{2}{*}{\multicolumn{2}{|c|}{ Contaminant }} & & & Maximum Soll & Concentration & Concentratlon & Concentration & \\
\hline & & $\mathrm{Kd}(\mathrm{LKg})$ & Retardation & Conc. (mg/Kg) & Years from 1994 & at $\mathrm{W}$. T. (ug/L) & in Water Table (ug/L) & \\
\hline \multicolumn{2}{|l|}{ Alluminum* } & $1.20 \mathrm{E}+02$ & 3.97E+02 & $181 \mathrm{E}+03$ & $1.63 E+03$ & $1.51 E+04$ & $1.29 E+03$ & \\
\hline \multicolumn{2}{|l|}{ Cadmium } & $\overline{6} . \overline{30 E+00}$ & $2.18 \bar{E}+01$ & $1.50 \mathrm{E}+00$ & $8.82 E+01$ & $2.38 \bar{E}+02$ & $2.04 \bar{E}+01$ & \\
\hline \multicolumn{2}{|l|}{ Chromium } & $3.98 E+01$ & $1.32 \bar{E}+02$ & $8.55 \mathrm{E}+00$ & $5.42 E+02$ & $2.40 \mathrm{E}+02$ & $2.05 E+0 ?$ & \\
\hline \multicolumn{2}{|l|}{ Copper } & $2.50 \mathrm{E}+01$ & $8.35 \bar{E}+01$ & $6.08 \bar{E}+00$ & $3.42 \mathrm{E}+02$ & $2.43 \overline{E+02}$ & $2.08 \bar{E}+0 i$ & \\
\hline \multicolumn{2}{|l|}{ Iron" } & $5.00 \bar{E}+01$ & $1.6 \overline{6} \bar{E}+02$ & $1.82 E+03$ & $6.80 \bar{E}+02$ & $3.64 E+04$ & $3.11 \mathrm{E}+03$ & \\
\hline \multirow{2}{*}{\multicolumn{2}{|c|}{$\begin{array}{l}\text { Lead } \\
\text { Manganese }\end{array}$}} & $1.00 E+02$ & $3.31 E+02$ & 4.19E+01 & 1.36E+03 & 4.19E+02 & $3.58 E+01$ & \\
\hline & & $2.00 E+02$ & $6.61 \overline{E+02}$ & $6.91 E+01$ & 2.71E+03 & $3.46 \bar{E}+02$ & $2.85 \bar{E}+01$ & \\
\hline \multicolumn{2}{|c|}{ K K chosen to reflect: } & & & & & & & \\
\hline \multicolumn{9}{|c|}{ 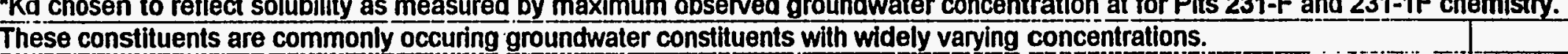 } \\
\hline \multicolumn{7}{|c|}{ It is recognized that equilibrium partitioning may not be valid for all of these constituents. } & & \\
\hline
\end{tabular}


Table B12. Summary of Carcinogenic and Noncarcinogenic Risk from Soil Leachability to Groundwater

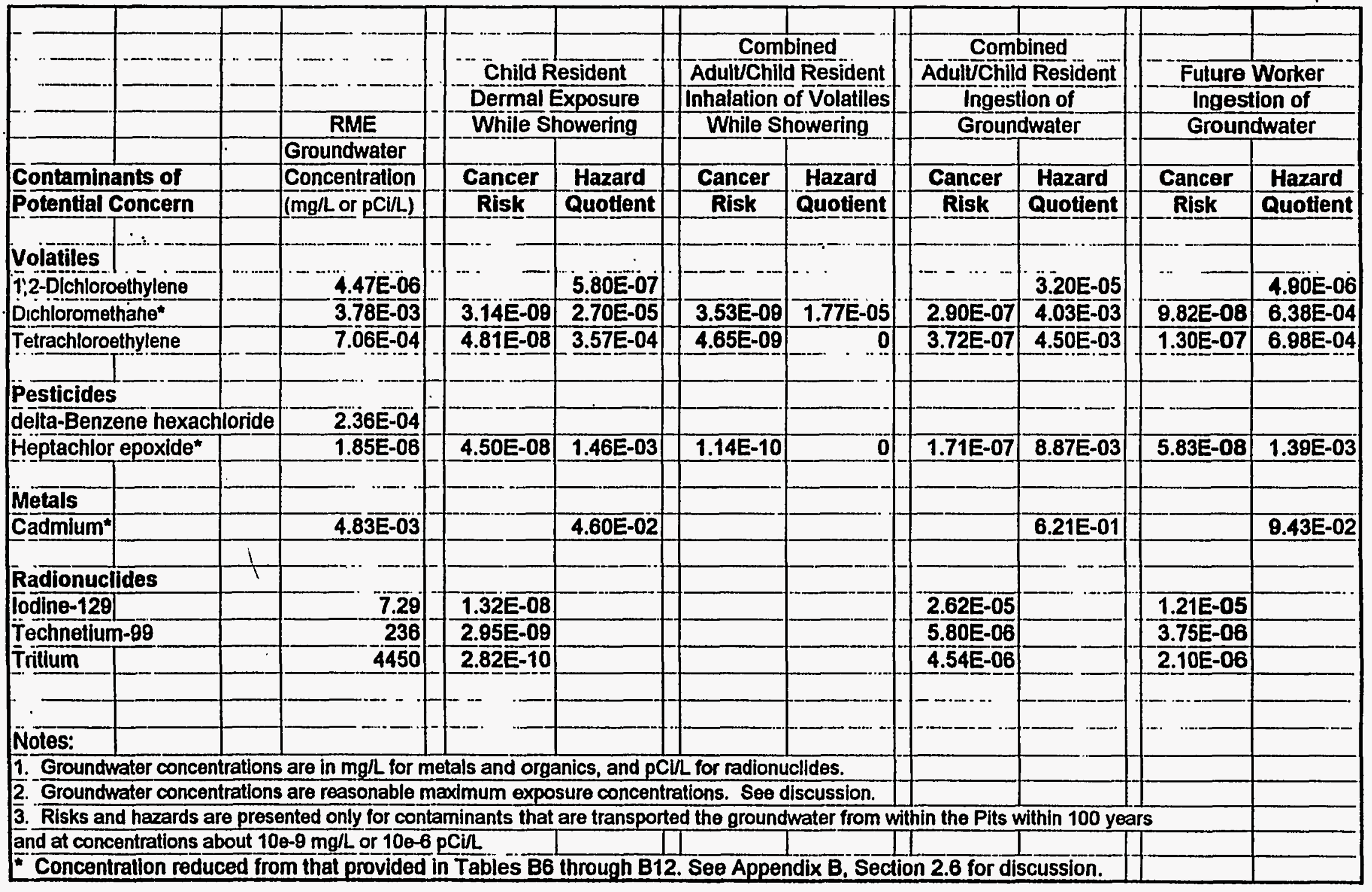




\section{APPENDIX C}

\section{AIR MODELING - VOLATILES AND PARTICULATES}




\section{THIS PAGE LEFT INTENTIONALLY BLANK}




\section{APPENDIX C \\ CALCULATIONS FOR INHALATION EXPOSURE}

The approach for estimating inhalation exposures to volatiles and particulates released from soil in this BRA was to convert chemical concentrations in soil to volatile and dust concentrations in air using simplified soil-to-air transmission relationships specified by USEPA $(1991,1994)$. These estimated air concentrations were then used in the exposure calculations for inhalation pathways.

\section{VOLATILIZATION FROM SOIL TO AIR}

For calculating air concentrations of volatilized chemicals, the soil concentration is multiplied by the soil-to-air Volatilization Factor (VF). The VF is calculated from factors describing the volatility of specific chemicals, the porosity of the soil, and the estimations of the air flow across the contaminated soil. While this is an very simplified method for modelling air concentration of volatile chemicals, it is consistent with USEPA recommendations and the degree of precision and uncertainty is adequate for the needs of a BRA. Some factors require chemical-specific inputs, and site-specific information can be substituted for some of the input variables, but there are default values provided by USEPA $(1991,1994)$.

The equations used for calculating the VF and the parameters that were used for the calculations in this BRA are presented below.

Volatilization Factor (VF), $\mathrm{m} 3 / \mathrm{kg}=\frac{L S \times V \times D H \times \sqrt{3.14(\text { alpha })(T)}}{A \times 2 \times D e i \times E \times K a s \times C F}$ 
BASELINE RISK ASSESSMENT

WSRC-TR-94-108

F-AREA BURNING/RUBBLE PITS AND RUBBLE PIT

REV. 1.2, MARCH 1996

\section{CONSTANTS:}

Length of side of contam'd. area (LS), $m=$

Wind speed in mixing zone $(\mathrm{V}), \mathrm{m} / \mathrm{sec}=$

3.6

Diffusion height (DH), $\mathrm{m}=$

1.66

Exposure interval $(T), \sec =$

788400000 . Based on 25 years

Area of contamination (A), $\mathrm{cm}^{2}=$

5000000

True soil porosity $(\mathrm{E})$, unitless =

0.35

Conversion factor (CF), $\mathrm{kg} / \mathrm{g}=$

0.001

INTERMEDIATE CALCULATIONS:

alpha, $\mathrm{cm}^{2} / \mathrm{sec}=$

$($ Dei $\times E) /(E+(P s)(1-E)) /$ Kas

Effective diffusivity (Dei), $\mathrm{cm}^{2} / \mathrm{sec}=$

$\left(\mathrm{Di} \times\left(\mathrm{E}^{0.33}\right)\right)$

Soil-air partition coefficient (Kas), g-soil/ $\mathrm{cm}^{3}$-air $((\mathrm{H} / \mathrm{Kd}) \times 41)$

$=$

Soil-water partition coefficient $(\mathrm{Kd}), \mathrm{cm}^{3} / \mathrm{kg}=\quad(\mathrm{Koc} \times \mathrm{OC})$

\section{ADDITIONAL VARIABLES IN INTERMEDIATE CALCULATIONS}

True soil density (Ps), $\mathrm{g} / \mathrm{cm}^{3}=$

Gas diffusivity (Di), $\mathrm{cm}^{2} / \mathrm{sec}=\quad$ chemical specific

Henry's Law constant $(\mathrm{H})$, atm- $\mathrm{m}^{3} / \mathrm{mol}=\quad$ chemical specific

Organic carbon partition coef. (Koc), $\mathrm{cm}^{3} / \mathrm{kg}=\quad$ chemical specific

Organic carbon content $(\mathrm{OC})$, unitless $=\quad 0.02$

\section{CONCENTRATIONS ON RESUSPENDED PARTICULATES}

Soil concentrations are multiplied by a different factor, the Particulate Emmissions Factor (PEF), to estimate the concentration of chemicals that will be available for inhalation on resuspended soil particles in the air. The PEF depends on physical features of the soil and site that affect the resuspension of soil particles. Again, this is a simplified resuspension model, but it is adqeuate for the needs of the BRA and conforms to USEPA recommendations. USEPA provides default assumptions that were used for most parameters in this BRA (USEPA, 1991, 1994). 
Particulate emission factor (PEF) $=\frac{L S \times V \times D H \times C F a \times C F b}{A \times R F \times(1-G) \times\left(\frac{U m}{U t}\right)^{3} \times F x}$.

Length of side of contaminated area (LS) =

Wind speed in mixing zone $(\mathrm{V})=$

Diffusion height $(\mathrm{DH})=$

Conversion factor $(\mathrm{CFa})=$

Conversion factor $(\mathrm{CFb})=$

Area of contamination $(A)=$

Respirable fraction $(\mathrm{RF})=$ site specific meters

3.6 meters/sec

1.66 meters

$3600 \mathrm{sec} / \mathrm{hr}$

$1000 \mathrm{~g} / \mathrm{kg}$

500 meters $^{2}$

$0.003 \mathrm{~g} /$ meters $^{2}-\mathrm{hr}$

3

0.9 unitless

3.6 meters/sec

Mean annual wind speed $(\mathrm{Um})=$

Equivalent threshold value of wind speed at $10 \mathrm{~m}(\mathrm{Ut})=$

Fraction dependent on $\mathrm{Um} / \mathrm{Ut}(\mathrm{Fx})=$
$5.04 \mathrm{~meters} / \mathrm{sec}$

1.27 unitless

\section{SUMMARY}

The methodologies for calculating air concentrations of volatiles and particulates from soil concentrations are presented in this appendix. The derivation of the representative values used for soil concentrations is described in Appendix A. The methods used in this BRA are adopted from simplified modeling equations developed by the USEPA. In addition, the USEPA default parameters were adopted in most cases for the inputs to the model equations.

\section{CITATIONS}

USEPA, 1991. Risk Assessment Guidance for Superfund: Volume 1 - Human Health Evaluation Manual (Part B, Development of Risk-Based Preliminary Remediation Goals). Office of Emergency and Remedial Response.

USEPA, 1994. Soil Screening Guidance. Office of Solid Waste and Emergency Response. EPA/540/R-94/101. 


\section{APPENDIX D}

CALCULATION TABLES FOR CARCINOGENIC RISK AND NONCARCINOGENIC HAZARDS FOR CURRENT AND FUTURE RECEPTORS 


\section{THIS PAGE LEFT INTENTIONALLY BLANK}




\section{APPENDIX D \\ TABLE OF CONTENTS \\ LIST OF TABLES}

Table No.

Title

Nonradioactive Chemicals

\begin{tabular}{|c|c|c|c|c|c|}
\hline D-1 & Summary of Exposure & ctors Use & for Receptors & & \\
\hline $\mathrm{D}-2$ & $\mathrm{BRP}(231-\mathrm{F}, 231-1 \mathrm{~F})$ & Current & Visitor & Ingestion of Soil & $0-2^{\prime}$ bls \\
\hline D-3 & $\mathrm{BRP}(231-\mathrm{F}, 231-1 \mathrm{~F})$ & Current & Visitor & Dermal Exposure to Soil & $0-2^{\prime}$ bls \\
\hline D-4 & $\mathrm{BRP}(231-\mathrm{F}, 231-1 \mathrm{~F})$ & Current & Visitor & Inhalation of Volatile Soil Contaminants & $0-2^{\prime}$ bls \\
\hline D-5 & $\mathrm{BRP}(231-\mathrm{F}, 231-1 \mathrm{~F})$ & Current & Visitor & Inhalation of Resuspended Soil Particulates & $0-2$ bls \\
\hline D-6 & $\mathrm{RP}(231-2 \mathrm{~F})$ & Current & Visitor & Ingestion of Soil & $0-2^{\prime}$ bls \\
\hline D.7 & $R P(231-2 F)$ & Current & Visitor & Dermal Exposure to Soil & $0-2^{\prime} \mathrm{bls}$ \\
\hline D-8 & $R P(231-2 F)$ & Current & Visitor & Inhalation of Volatile Soil Contaminants & $0-2^{\prime}$ bls \\
\hline D-9 & $R P(231-2 F)$ & Current & Visitor & Inhalation of Resuspended Soil Particulates & $0-2^{\prime}$ bls \\
\hline D-10 & $\mathrm{BRP}$ and $\mathrm{RP}$ & Current & Visitor & Dermal Exposure to Surface Water (Wetland) & \\
\hline D-11 & $B R P$ and $R P$ & Current & Visitor & Ingestion of Sediment (Wetland) & \\
\hline D-12 & $\mathrm{BRP}$ and RP & Current & Visitor & Dermal Exposure to Sediment (Wetland) & \\
\hline$\overline{\mathrm{D}-13}$ & $\overline{B R P}(231-F, 231-1 F)$ & Future & Worker & Ingestion of Soil & $0-2^{\prime}$ bls \\
\hline D-14 & $\operatorname{BRP}(231-\mathrm{F}, 231-1 \mathrm{~F})$ & Future & Worker & Dermal Exposure to Soil & $0-2^{\prime}$ bls \\
\hline D-15 & BRP (231-F, 231-1F) & Future & Worker & Inhalation of Volatile Soil Contaminants & $0-2$ bls \\
\hline D-16 & $\mathrm{BRP}(231-\mathrm{F}, 231-1 \mathrm{~F})$ & Future & Worker & Inhalation of Resuspended Soil Particulates & $0-2^{\prime}$ bls \\
\hline$\overline{\mathrm{D}-17}$ & $\overline{B R P}(231-F, 231-1 F)$ & Future & Worker & Ingestion of Soil & $0-4^{\prime}$ bls \\
\hline D-18 & $\mathrm{BRP}(231-\mathrm{F}, 231-1 \mathrm{~F})$ & Future & Worker & Dermal Exposure to Soil & $0-4^{\prime}$ bls \\
\hline D-19 & BRP (231-F, 231-1F) & Future & Worker & Inhalation of Volatile Soil Contaminants & $0-4^{\prime}$ bls \\
\hline D-20 & $\mathrm{BRP}(231-\mathrm{F}, 231-1 \mathrm{~F})$ & Future & Worker & Inhalation of Resuspended Soil Particulates & $0-4^{\prime}$ bls \\
\hline$\overline{D-21}$ & $\mathrm{RP}(231-2 \mathrm{~F})$ & Future & Worker & Ingestion of Soil & $0-2$, bls \\
\hline D-22 & $R P(231-2 F)$ & Future & Worker & Dermal Exposure to Soil & 0-2' bls \\
\hline D-23 & $R P(231-2 F)$ & Future & Worker & Inhalation of Volatile Soil Contaminants & $0-2$ bls \\
\hline D-24 & $R P(231-2 F)$ & Future & Worker & Inhalation of Resuspended Soil Particulates & $0-22^{\prime}$ bls \\
\hline D-25 & $\mathrm{RP}(231-2 \mathrm{~F})$ & Future & Worker & Ingestion of Soil & $0-4^{\prime}$ bls \\
\hline D-26 & $R P(231-2 F)$ & Future & Worker & Dermal Exposure to Soil & $0-4$ bls \\
\hline D-27 & $\operatorname{RP}(231-2 F)$ & Future & Worker & Inhalation of Volatile Soil Contaminants. & $0-4$ bls \\
\hline D-28 & $R P(231-2 F)$ & Future & Worker & Inhalation of Resuspended Soil Particulates & $0-4^{\prime}$ bls \\
\hline D-29 & BRP and RP & Future & Worker & Ingestion of Groundwater & \\
\hline D.30 & $\overline{B R P}(231-F, 231-1 F)$ & Future & Combined Adult/Child Resident & Ingestion of Soil & $0-2^{\prime} \mathrm{bls}$ \\
\hline D-31 & $\mathrm{BRP}(231-\mathrm{F}, 231-1 \mathrm{~F})$ & Future & Combined Adult/Child Resident & Dermal Exposure to Soil & $0-2^{\prime}$ bls \\
\hline D-32 & $\mathrm{BRP}(231-\mathrm{F}, 231-1 \mathrm{~F})$ & Future & Combined Adult/Child Resident & Inhalation of Volatile Soil Contaminants & $0-2$ ' bls \\
\hline D-33 & $\mathrm{BRP}(231-\mathrm{F}, 231-1 \mathrm{~F})$ & Future & Combined Adult/Child Resident & Inhalation of Resuspended Soil Particulates & $0-2^{\prime}$ bls \\
\hline $\mathrm{D}-34$ & $\mathrm{BRP}(231-\mathrm{F} ; 231-1 \mathrm{~F})$ & Future & Combined Adult/Child Resident & Ingestion of Soil & $0-4^{\prime}$ bls \\
\hline D-35 & $\mathrm{BRP}(231-\mathrm{F}, 231-1 \mathrm{~F})$ & Future & Combined Adult/Child Resident & Dermal Exposure to Soil & $0-4^{\prime}$ bls \\
\hline D-36 & $\mathrm{BRP}(231-F, 231-1 F)$ & Future & Combined Adult/Child Resident & Inhalation of Volatile Soil Contaminants & $0-4$ ' bls \\
\hline D.37 & BRP (231-F, 231-1F) & Future & Combined Adult/Child Resident & Inhalation of Resuspended Soil Particulates & $0-4^{\prime}$ bls \\
\hline D-38 & $R P(231-2 F)$ & Future & Combined Adult/Child Resident & Ingestion of Soil & $0-2^{\prime}$ bls \\
\hline D-39 & $R P(231-2 F)$ & Future & Combined Adult/Child Resident & Dermal Exposure to Soil & $0-2$ ' bls \\
\hline$D-40$ & $R P(231-2 F)$ & Future & Combined Adult/Child Resident & Inhalation of Volatile Soil Contaminants & $0-2$ bls \\
\hline D-41 & $R P(231-2 F)$ & Future & Combined Adult/Child Resident & Inhalation of Resuspended Soil Particulates & $0-2$ bls \\
\hline$\overline{\mathrm{D}-42}$ & $R P(231-2 F)$ & Future & Combined Adult/Child Resident & Ingestion of Soil & $0-4^{\prime}$ bls \\
\hline D-43 & $R P(231-2 F)$ & Future & Combined Adult/Child Resident & Dermal Exposure to Soil & $0-4$ ' bis \\
\hline D-44 & $R P(231-2 F)$ & Future & Combined Adult/Child Resident & Inhalation of Volatile Soil Contaminants & $0-4$ ' bls \\
\hline D-45 & $R P(231-2 F)$ & Future & Combined Adult/Child Resident & Inhalation of Resuspended Soil Particulates & $0-4^{\prime}$ bls \\
\hline$\overline{\mathrm{D}-46}$ & $\overline{B R P}$ and $\mathrm{RP}$ & Future & Combined Adult/Child Resident & \multirow{3}{*}{\multicolumn{2}{|c|}{$\begin{array}{l}\text { Ingestion of Groundwater } \\
\text { Dermal Exposure to Groundwater while Showering } \\
\text { Inhalation of Groundwater Volatiles while Showering }\end{array}$}} \\
\hline D-47 & $\mathrm{BRP}$ and $\mathrm{RP}$ & Future & Combined Adult/Child Resident & & \\
\hline D-48 & $\mathrm{BRP}$ and $\mathrm{RP}$ & Future & Combined Adult/Child Resident & & \\
\hline$\overline{D-49}$ & $\mathrm{BRP}$ and $\mathrm{RP}$ & Future & Child Resident & \multicolumn{2}{|c|}{ Ingestion of Surface Water (Wetland) } \\
\hline D-50 & $\mathrm{BRP}$ and $\mathrm{RP}$ & Future & Child Resident & \multicolumn{2}{|l|}{ Dermal Exposure to Surface Water (Wetland) } \\
\hline D-51 & BRP and RP & Future & Child Resident & \multicolumn{2}{|l|}{ Ingestion of Sediment (Wetland) } \\
\hline D-52 & BRP and RP & Future & Child Resident & \multicolumn{2}{|l|}{ Dermal Exposure to Sediment (Wetland) } \\
\hline
\end{tabular}




\section{APPENDIX D \\ TABLE OF CONTENTS \\ LIST OF TABLES}

Table No.

Title

Radionuclides

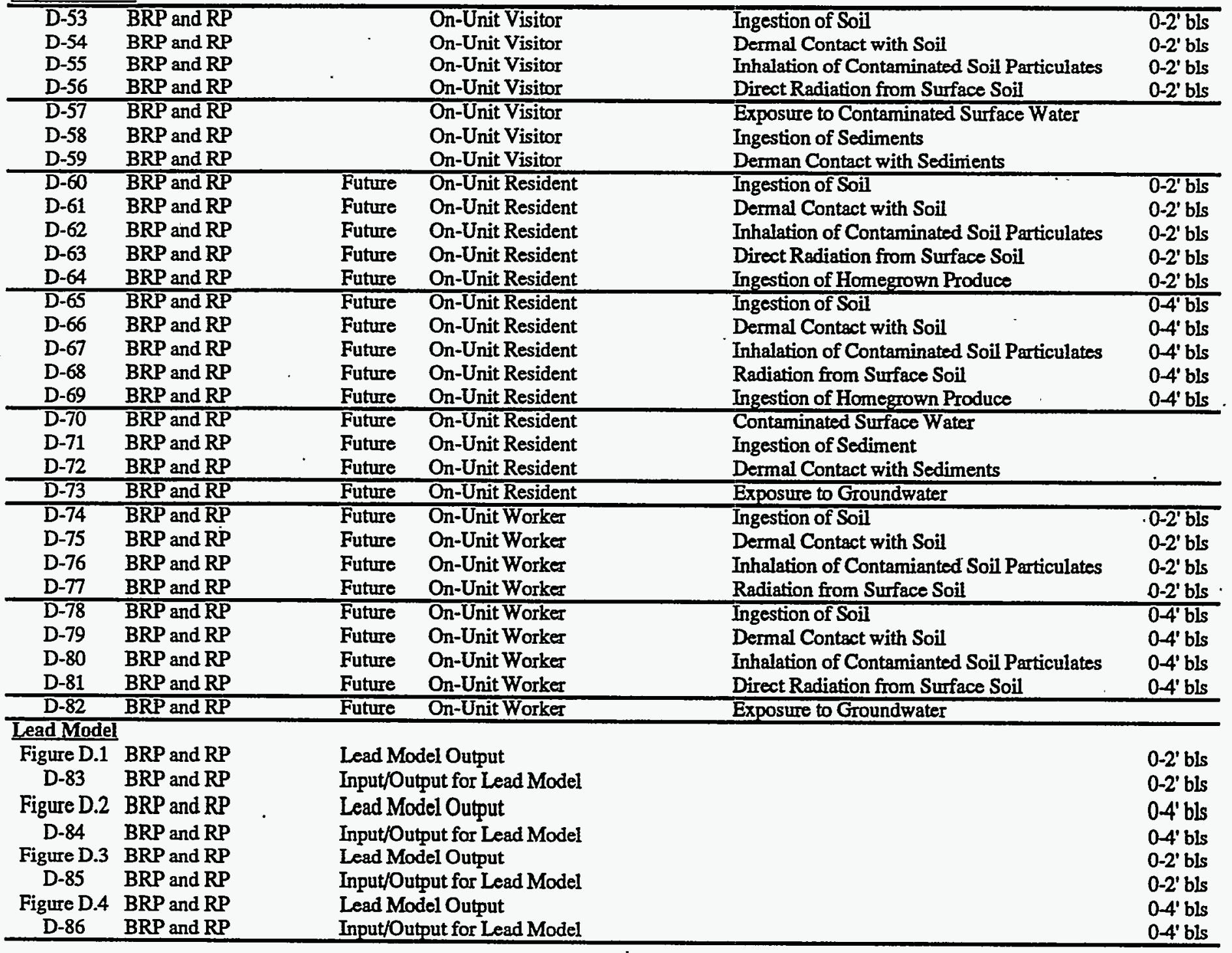




\section{NONRADIOACTIVE CHEMICALS}


THIS PAGE LEFT INTENTIONALLY BLANK 
Table D.1

Savannah River Site

Burning /Rubble Pits (321-F, 231-1F) and Rubble Pit (231-2F)

Summary of Human Health RME Exposure Factors

\begin{tabular}{|c|c|c|c|c|c|c|c|c|c|c|c|c|c|c|c|}
\hline \multirow{2}{*}{$\begin{array}{l}\text { Exposare } \\
\text { Perameter }\end{array}$} & \multirow[b]{2}{*}{ Units } & \multicolumn{3}{|c|}{$\begin{array}{c}\text { Current } \\
\text { On-Unut Viator }\end{array}$} & \multicolumn{3}{|c|}{$\begin{array}{c}\text { Future } \\
\text { Aduk Readdent }\end{array}$} & \multicolumn{5}{|c|}{$\begin{array}{c}\text { Future } \\
\text { Culli Restient }\end{array}$} & \multicolumn{2}{|c|}{$\begin{array}{l}\text { Future } \\
\text { Woriver }\end{array}$} & \multirow[b]{2}{*}{ REFERENCE } \\
\hline & & Soll & sw & Sed & Soll & $\mathbf{G W}$ & Vef. & Soll & GW & Vep. & sw & Sed & Soll & GW & \\
\hline Body Weight (BW) & kg & 70 & 70 & 70 & 70 & 70 & 70 & 15 & 15 & 15 & 45 & 45 & 70 & 70 & 2 \\
\hline Exposure Duration (ED) & yr & 5 & 5 & 5 & 24 & 24 & 24 & 6 & 6 & 6 & 10 & 10 & 25 & 25 & $\begin{array}{l}\text { Resident and } \\
\text { Wotker }=1,2 \\
\text { Visitor }=\text { Site-pecific }\end{array}$ \\
\hline $\begin{array}{l}\text { Exposure Frequency } \\
\text { (EF) }\end{array}$ & $d / y r$ & 6 & 6 & 6 & 350 & 350 & 350 & 350 & 350 & 350 & 50 & 39 & 250 & 250 & $\begin{array}{l}\text { Resident and } \\
\text { Worker = } 3 \\
\text { Viritor = Site-pecific }\end{array}$ \\
\hline Exposure Time (ET) & hr/d & 1.0 & 0.5 & NA & 1.5 & $0.20^{6}$ & NA & 18 & $0.20^{6}$ & NA & 2 & NA & 1.0 & 0.2 & $\begin{array}{l}\text { Resident = } 2 \\
\text { Viritor and Worker } \\
\text { = Site-specific }\end{array}$ \\
\hline $\begin{array}{l}\text { Adherence Factor of soil } \\
\text { to skin (AF) }\end{array}$ & $\mathrm{mg} / \mathrm{cm}^{2}$ & 0.2 & $\overline{\text { NA }}$ & 0.6 & 0.2 & NA & $\overline{\text { NA }}$ & 0.2 & NA & $\overline{N A}$ & $\overline{\text { NA }}$ & 0.6 & 0.2 & NA & 4 \\
\hline $\begin{array}{l}\text { Skin Surface Area } \\
\text { Available for Contuct } \\
\text { (SA) }\end{array}$ & $\mathrm{cm}^{2}$ & 3,200 & 5,000 & 5,000 & 5,000 & 20,000 & $\overline{\text { NA }}$ & 1,800 & 7,300 & $\mathbf{N A}$ & 3,100 & 3,100 & 3,200 & $\overline{\mathbf{N A}}$ & $\begin{array}{l}\text { Rexident - 2,4 } \\
\text { Vixitor and Worker } \\
=2\end{array}$ \\
\hline Ingestion Rate (IR) & d & 50 & NA & 50 & 100 & 2 & $\begin{array}{l}113^{\circ} \\
202^{r} \\
123^{8}\end{array}$ & 200 & 1 & $\begin{array}{l}42^{*} \\
75^{t} \\
45^{*}\end{array}$ & 0.01 & 20 & 30 & 1.0 & 2 \\
\hline Inhulation Rate (IR) & $\mathrm{m}^{3} / \mathrm{hr}$ & 2.5 & $\overline{N A}$ & NA & 0.83 & $0.83^{\circ}$ & NA & 1 & $0.6^{6}$ & $\overline{\text { NA }}$ & NA & NA & 2.5 & NA & $\begin{array}{l}\text { Adult, Visitor and } \\
\text { Worker }=2 \\
\text { Child }=3\end{array}$ \\
\hline $\begin{array}{l}\text { Fraction Ingerted from } \\
\text { Contuminated Source } \\
\text { (FI) }\end{array}$ & unitless & 1.0 & NA & 1.0 & 1.0 & NA & $\begin{array}{l}0.042^{c} \\
0.119^{r} \\
0.487^{r}\end{array}$ & 1.0 & NA & $\begin{array}{l}0.042^{6} \\
0.119^{f} \\
0.487^{\circ}\end{array}$ & NA & 1.0 & 1.0 & NA & 2.7 \\
\hline
\end{tabular}


Table D.1

Savannah River Site

Burning /Rubble Pits (321-F, 231-1F) and Rubble Pit (231-2F)

Summary of Human Health RME Exposure Factors

\begin{tabular}{|c|c|c|c|c|c|c|c|c|c|c|c|c|c|c|c|}
\hline \multirow{2}{*}{$\begin{array}{l}\text { Exposire } \\
\text { Parpecter } \\
\end{array}$} & \multirow[b]{2}{*}{ Units } & \multicolumn{3}{|c|}{$\begin{array}{c}\text { Curreal } \\
\text { On-Unit Viltor }\end{array}$} & \multicolumn{3}{|c|}{$\begin{array}{c}\text { Future } \\
\text { Adwit Reakent }\end{array}$} & \multicolumn{5}{|c|}{$\begin{array}{c}\text { Future } \\
\text { Crild Readean }\end{array}$} & \multicolumn{2}{|c|}{$\begin{array}{l}\text { Future } \\
\text { Worlicer }\end{array}$} & \multirow[b]{2}{*}{ REFERENCE } \\
\hline & & Soll & sw & Sed & Soll & $\mathbf{G W}$ & Vef. & Soll & $\mathbf{G W}$ & Vex. & sw & Sed & Soll & GW & \\
\hline $\begin{array}{l}\text { Abeorption Fector } \\
\text { (ABS) }\end{array}$ & unitless & $0.01 / 0.001$ & NA & $0.01 / 0.001$ & $0.01 / 0.001$ & NA & NA & $0.01 / 0.001$ & NA & NA & NA & $0.01 / 0.001$ & $0.01 / 0.001$ & NA & 6 \\
\hline $\begin{array}{l}\text { Dermal Permatibility } \\
\text { Condent (PC) }\end{array}$ & $\mathbf{c m} / \mathbf{h r}$ & NA & $\begin{array}{l}\text { sex } \\
\text { Table }\end{array}$ & NA & NA & $\begin{array}{l}\text { rece } \\
\text { Table }\end{array}$ & NA & NA & $\begin{array}{l}200 \\
\text { Table }\end{array}$ & NA & $\begin{array}{c}\text { rece } \\
\text { Table }\end{array}$ & NA & NA & $\begin{array}{l}200 \\
\text { Table }\end{array}$ & Chemical-epecific \\
\hline $\begin{array}{l}\text { Externil Gamma } \\
\text { Shielding Fector (SF) }\end{array}$ & unitless & 0.7 & NA & 0.7 & 0.7 & NA & $\mathbf{N A}$ & 0.7 & NA & NA & NA & 0.7 & 0.7 & NA & 5 \\
\hline
\end{tabular}

Note:

- 0-6 years old for soil and groundwater contact/4-12 years old for surfece water and sediment contact.

b For dermal contact while bathing.

c Inhalation of volatiles in groundwater while showering.

d Ingeation rates for soil and sediment are expressed in mg/d; aurfice water and groundwater are expressed in V/d.

c lenves (lettuce and toosed salad)

$f$ roots (white potutoes)

8 fruits (tomatoes)
(1) EPA, 1989a
(2) EPA, 1990a
(3) EPA, 1991:
(4) EPA, $1992 c$
(5) Gitbert a al., 1989
(6) EPA, 1992d
(7) EPA Region IV 
Table D.2

Savannah River Site

Burning/Rubble Pits (231-F, 231-1F)

Current Visitor

Ingestion of Soil (0-2' bls)

Carcinogenic and Noncarcinogenic Risk

\begin{tabular}{|c|c|c|}
\hline EXPOSURE ASSUMPTIONS: & & NTAKE FACTOR CALCULATIONS \\
\hline $\begin{array}{l}\text { Receptor } \\
\text { Exposure } \\
\text { Intake Rate (IR), mg/day } \\
\text { Fraction Ingested (FI), unitless } \\
\text { Exposure Frequency (EF), days/yr } \\
\text { Exposure Duration (ED), yrs } \\
\text { Body Weight (BW), kg } \\
\text { Avging Time, Carc (R) (ATC), days } \\
\text { Avging Time, Noncarc ((3) (ATN), days } \\
\text { Conversion Factor (CF), kg/mg }\end{array}$ & $\begin{array}{cc}\text { Current V/sitor } \\
\text { RME }^{\text {(1) }} \\
50 & \text { (4) } \\
1 & (4) \\
6 & (4) \\
5 & (4) \\
70 & (4) \\
25,550 & \\
1,825 & \\
1.00 \mathrm{E}-06 & \end{array}$ & $\begin{array}{l}\text { Carcinogenic Intake Factor }(\mathrm{CFF}), \mathrm{kg} / \mathrm{kg} \text {-day }= \\
(\mathrm{R} \bullet \mathrm{FI} * \mathrm{EF} * \mathrm{ED} \bullet \mathrm{CF}) /(\mathrm{BW} \bullet \mathrm{ATC}) \\
\mathrm{RMECF}=8.39 \mathrm{E}-10 \\
\text { Noncarcinogenic Intake Factor (NIF), kg/kg-day }= \\
(\mathrm{IR} * \mathrm{FI} \bullet \mathrm{EF} \bullet \mathrm{ED} \bullet \mathrm{CF}) /(\mathrm{BW} \bullet \mathrm{ATN}) \\
\mathrm{RME} \mathrm{NIF}=1.17 \mathrm{E}-08\end{array}$ \\
\hline 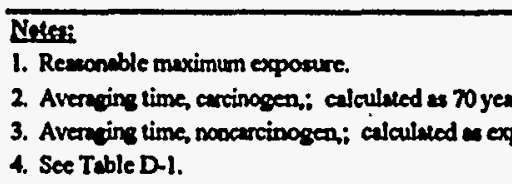 & 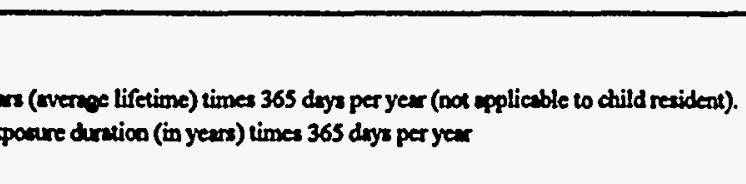 & . \\
\hline
\end{tabular}


Table D.2

Burning/Rubble Pits (231-F, 231-1F)

Current Visitor

Ingestion of Soil (0-2' bls)

Carcinogenic and Noncarcinogenic Risk

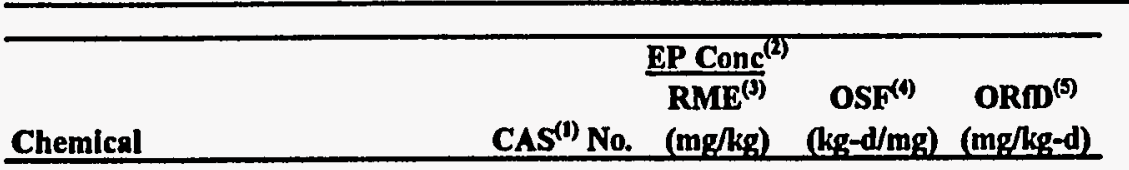

\begin{tabular}{|c|c|c|}
\hline Cancer & isk $^{(\text {()) }}$ & Hazard Quotient \\
\hline RME & $\begin{array}{l}\% \text { of } \\
\text { Total }\end{array}$ & $\begin{array}{ll}\text { RME of } & \text { Total } \\
\text { RME }\end{array}$ \\
\hline
\end{tabular}

\section{Semivolatiles}

2-Methyinaphthalene

Acenaphthylene

Benzo(a)anthracene

Benzo(a)pyrene

Benzo(b)fluoranthene

Benzo(g,h,i)perylene

Dibenz(a,h)anthracene

91-57-6 7.69E-02 -

208-96-8 8.61E-02 -

56-55-3 9.26E-01 7.30E-01

50-32-8 6.49E-01 7.30E+00 -

205-99-2 1.01E+00 7.30E-01 -

191-24-2 6.15E-01 -

53-70-3 2.17E-01 7.30E+00 -

Phenanthrene

85-01-8 6.87E-01 -

\section{Dioxins/furans}

Heptachlorodibenzo-p-dioxin isomer 37871-00-4 4.11E-03 1.50E+03 -

Heptachlorodibenzo-p-furan isomer 38998-75-3 2.58E-04 1.50E+03 -

Hexachlorodibenzo-p-furan isomer 55684-94-1 $1.00 \mathrm{E}-04 \quad 1.50 \mathrm{E}+04 \quad-$

Octachlorodibenzo-p-dioxin isomer $3268-87-9 \quad 9.26 \mathrm{E}-03 \quad 1.50 \mathrm{E}+02$ -

Octachlorodibenzo-p-furan isomer 39001-02-0 3.48E-04 1.50E+02 -

PCBs 1 .

PCB 1254

$11097-69-1 \quad 1.78 \mathrm{E}-01 \quad 7.70 \mathrm{E}+00 \quad 2.00 \mathrm{E}-05$

$\begin{array}{cccc}- & - & - & - \\ 5.7 \mathrm{E}-10 & 03 \% & \pm & - \\ 4.0 \mathrm{E}-09 & 18 \% & \pm & - \\ 6.2 \mathrm{E}-10 & 03 \% & - & - \\ \overline{-} & - & - & - \\ 1.3 \mathrm{E}-09 & 06 \% & - & - \\ - & - & - & -\end{array}$

Pesticides

delta-Beniene hexachloride

Endrin ketone

319-86-8 1.84E-03 $53494-70-5$ 3.20E-03 - 
Table D.2

Burning/Rubble Pits (231-F, 231-1F)

Current Visitor

Ingestion of Soil (0-2' bls)

Carcinogenic and Noncarcinogenic Risk

\begin{tabular}{|c|c|c|c|c|c|c|c|c|}
\hline Chemical & $\mathrm{CAS}^{(1)} \mathrm{No}$ & $\begin{array}{l}\text { EPConc( }{ }^{(2)} \\
\mathrm{RME}^{(3)} \\
(\mathrm{mg} / \mathrm{kg})\end{array}$ & $\underset{(\mathrm{kg}-\mathrm{d} / \mathrm{mg})}{\mathrm{OSF}(())}$ & $\begin{array}{c}\operatorname{ORfD}^{(s)} \\
(\mathrm{mg} / \mathrm{kg}-\mathrm{d})\end{array}$ & $\begin{array}{l}\text { Cancer F } \\
\text { RME }\end{array}$ & $\begin{array}{l}\text { isk } \\
\% \text { of } \\
\text { Total } \\
\end{array}$ & $\begin{array}{l}\text { Hazard Q2 } \\
\text { RME } \\
\end{array}$ & $\begin{array}{l}\text { otient" } \\
\% \text { of } \\
\text { Total } \\
\end{array}$ \\
\hline \multicolumn{9}{|l|}{ Metals } \\
\hline Arsenic & $7440-38-2$ & $4.82 \mathrm{E}+00$ & $1.50 \mathrm{E}+00$ & $3.00 \mathrm{E}-04$ & $6.1 \mathrm{E}-09$ & $28 \%$ & $1.9 \mathrm{E}-04$ & $36 \%$ \\
\hline Chromium & $7440-47-3$ & $4.42 \mathrm{E}+01$ & - & $5.00 \mathrm{E}-03$ & - & - & $1.0 \mathrm{E}-04$ & $20 \%$ \\
\hline Lead & $7439-92-1$ & $2.48 \mathrm{E}+01$ & - & - & - & - & - & - \\
\hline Manganese & $7439-96-5$ & $4.78 \mathrm{E}+02$ & - & $5.00 \mathrm{E}-02$ & - & - & 1.1E-04 & $21 \%$ \\
\hline \multirow[t]{2}{*}{ Mercury } & $7439-97-6$ & $5.48 \mathrm{E}-01$ & - & $3.00 \mathrm{E}-04$ & - & - & 2.1E-05 & $04 \%$ \\
\hline & & & PATHWAY SUMS: & WAY SUMS: & $\begin{array}{r}\text { Cencer } \\
\text { RMI } \\
2 \mathrm{E}-1 \\
\end{array}$ & 8 & \multicolumn{2}{|c|}{$\begin{array}{c}\text { Hazard Index } \\
\text { RME } \\
\text { SE-04 }\end{array}$} \\
\hline
\end{tabular}

Notes:

1. Chemical Abotracts Service.

2. Exposure point concentration

3. Ressonable muximum exposure.

4. Slope factor.

5. Reference doce.

6. Cancer Risk = (Chemical Concentration, mg/kg * Carcinogenic Ineake Factor, kg/kg-day * Slope Factor, kg-day/mg), where

RME carcinogenic intake $f$ 8.39E-10

7. Hazard Quotiend - (Chemical Concentration, mg/kg * Noncarcinogenic Intake Factor, kg/kg-day) / (Referenoc Dowe, mg/kg-day), where RME noncarcinozanic inta $1.17 E-08$ 
Table D.3

Savannah River Site

Burning/Rubble Pits (231-F, 231-1F)

Current Visitor

Dermal Exposure to Soil (0-2'bls)

Carcinogenic and Noncarcinogenic Risk

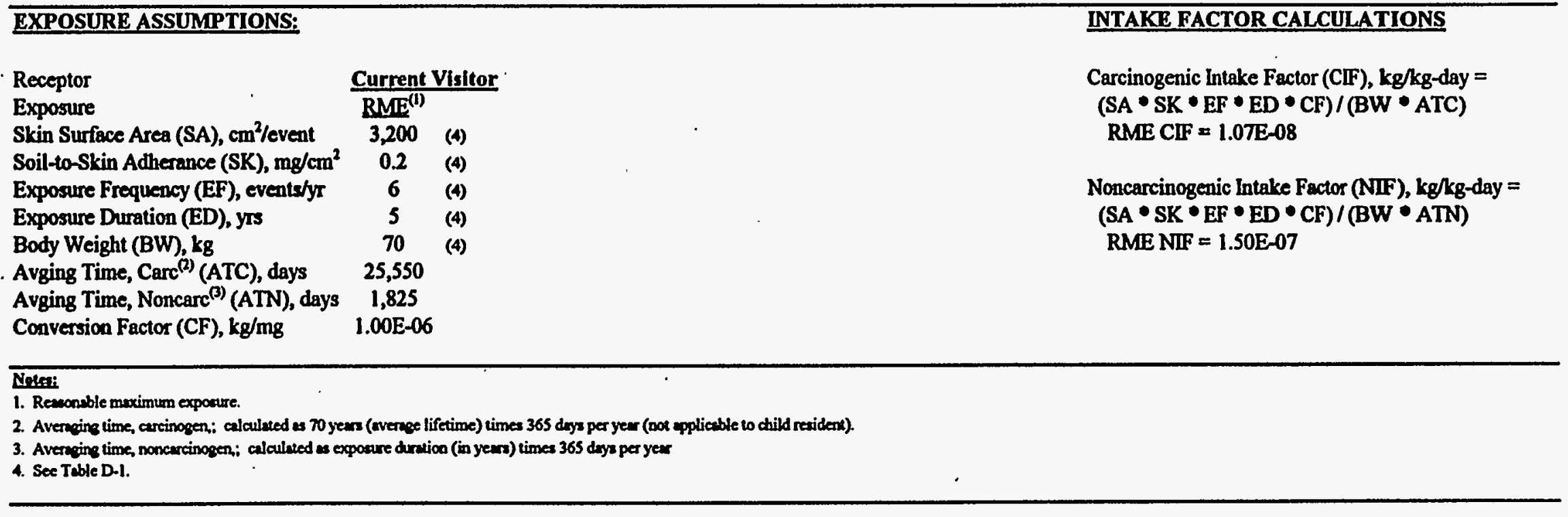


Table D.3

Savannah River Site

Burning/Rubble Pits (231-F, 231-1F)

Current Visitor

Dermal Exposure to Soil (0-2'bls)

Carcinogenic and Noncarcinogenic Risk

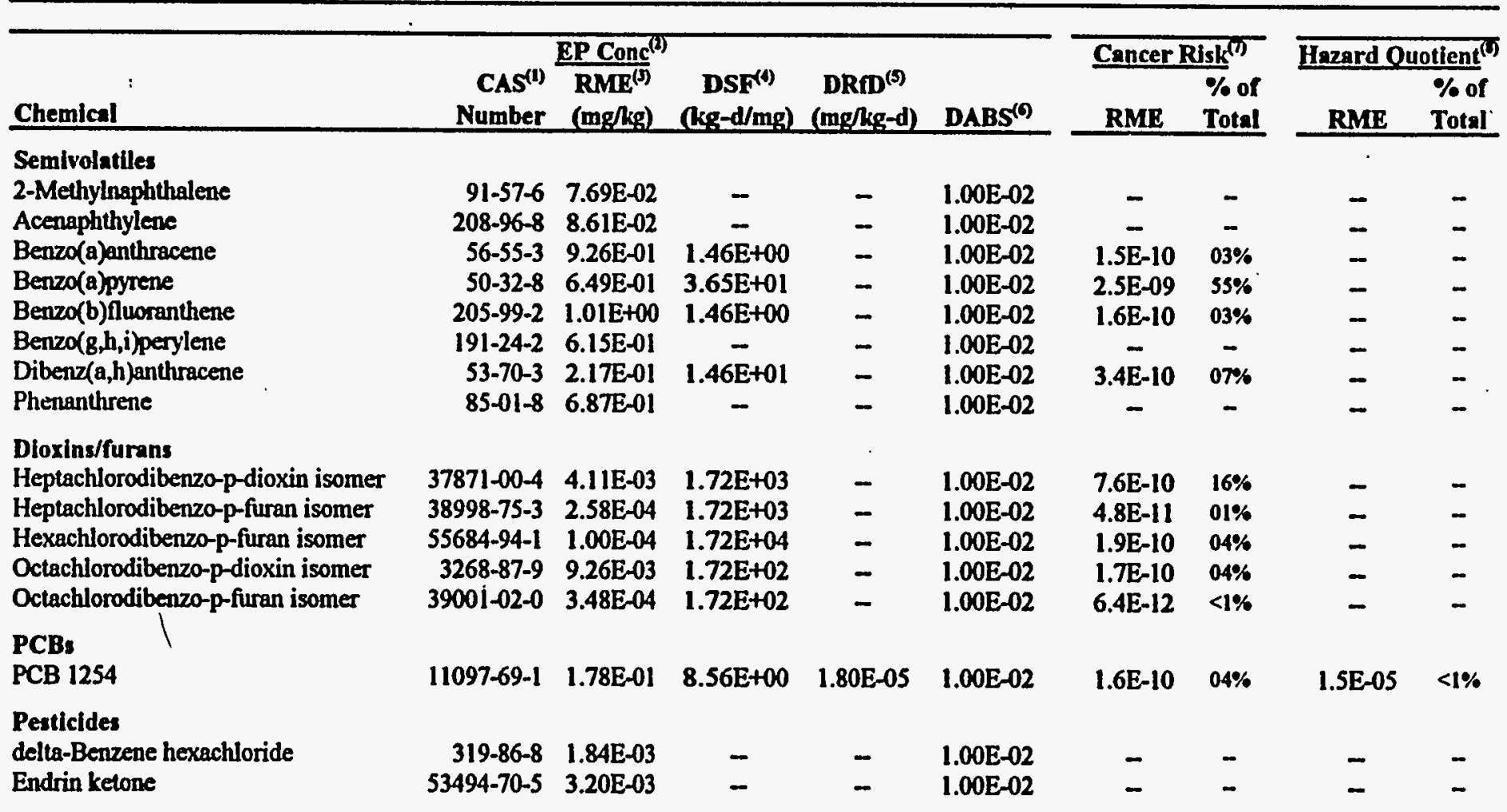


Table D.3

Savannah River Site

Burning/Rubble Pits (231-F, 231-1F)

Current Visitor

Dermal Exposure to Soil (0-2'bls)

Carcinogenic and Noncarcinogenic Risk

\begin{tabular}{|c|c|c|c|c|c|c|c|c|c|}
\hline Chemical & $\begin{array}{r}\text { CAS }^{(1)} \\
\text { Number }\end{array}$ & $\begin{array}{c}\text { EPConc }{ }^{(2)} \\
\operatorname{RME}^{(3)} \\
(\mathrm{m} / \mathrm{kg})\end{array}$ & $\begin{array}{c}\mathrm{DSF}(t) \\
(\mathrm{kg}-\mathrm{d} / \mathrm{mg})\end{array}$ & $\begin{array}{c}\mathrm{DRD}^{(\mathrm{s})} \\
\left(\mathrm{m} / \mathrm{kg}^{-\mathrm{d}}\right)\end{array}$ & DABS ${ }^{(0)}$ & Cancer & $\begin{array}{l}\text { Risk }^{(1)} \\
\% \text { of } \\
\text { Total } \\
\end{array}$ & $\begin{array}{l}\text { Hazard O } \\
\text { RME } \\
\end{array}$ & $\begin{array}{l}\frac{\text { atient }}{(1)}^{\% \text { of }} \\
\text { Total } \\
\end{array}$ \\
\hline & & & & ATHWA & SUMS: & $\begin{array}{r}\text { Cancer } \\
\text { RM } \\
\text { 5E- }\end{array}$ & $\begin{array}{l}\text { Risk } \\
9 \\
9\end{array}$ & $\begin{array}{r}\text { Hurnod } \\
\frac{\mathrm{RM}}{2 \mathrm{E}-}\end{array}$ & \\
\hline
\end{tabular}

Noten:

1. Chemical Abstract Service.

2. Exposure point concentration.

3. Resuonable maximum exposure.

4. Slope fuctor, dermal.

5. Reference douc, dermal.

6. Abworption factor.

7. Cancer Risk = (Chemical Concentration, mg/kg * Carcinogenic Intake Factor, kg/kg-day * Abeorption Factor, unitless * Slope Factor, kg-day/mg), where

RME carcinogenic intake f $1.07 E-08$

8. Hazard Quotient = (Chemical Concentration, mg/kg * Noncancinogenic Inxake Factor, kg/kg-day * Abeorption Factor, unillew)/(Reference Doce, mg/kg-day), where RME noncarcinogenic inta $1.50 \mathrm{E}-07$ 
Table D.4

Savannah River Site

Burning/Rubble Pits (231-F, 231-1F)

Current Visitor

Inhalation of Volatile Soil Contaminants (0-2'bls)

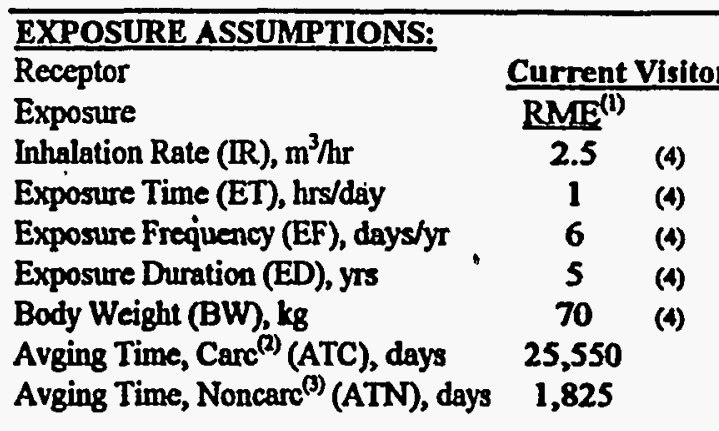

INTAKE FACTOR CALCULATIONS

Carcinogenic Intake Factor (CFF), kg/kg-day =

$(\mathrm{R} \bullet \mathrm{ET} * \mathrm{EF} \bullet \mathrm{ED} \bullet \mathrm{CF}) /(\mathrm{BW} * \mathrm{ATC})$

RME CIF $=4.19 \mathrm{E}-05$

Noncarcinogenic Intake Factor (NIF), kg/kg-day = $(\mathrm{R} \bullet \mathrm{ET} \bullet \mathrm{EF} \bullet \mathrm{ED} \bullet \mathrm{CF}) /(\mathrm{BW} \bullet \mathrm{ATN})$

RME NIF $=5.87 \mathrm{E}-04$

Nini:

1. Remonuble muximum expoutre

2. Avernaing time, carcinogen; calculated as 70 yeun (merage lifetime) times 365 deys per yeser (nox eppliceble to child rasident)

3. Averaging time, noncercinogen; calculated as exposture duretion (in yems) times 365 drye per year

4. See Teble D. 1 . 
Table D.4

Savannah River Site

Burning/Rubble Pits (231-F, 231-1F)

Current Visitor

Inhalation of Volatile Soil Contaminants (0-2'bls)

Calculation of Volatilization Factor (VF)

\begin{tabular}{|c|c|c|c|c|c|c|c|c|c|c|c|}
\hline Chemical & $\mathrm{CAS}^{(2)} \mathrm{No}$ & Class ${ }^{(3)}$ & MolWt. & $\mathrm{Koc}^{(\boldsymbol{(})}$ & $\mathbf{K} \mathbf{d}^{(0)}$. & $\mathbf{H}^{(())}$ & $\mathrm{Kas}^{(1)}$ & $\mathrm{Di}^{(())}$ & $\operatorname{Dei}^{(t)}$ & alpha(t) & $\begin{array}{l}\text { Volat. } \\
\text { Factor }{ }^{(0)} \\
\left(\mathrm{m}^{3} / \mathrm{kg}\right)\end{array}$ \\
\hline \multicolumn{12}{|l|}{ Semivolatlles } \\
\hline 2-Methylnaphthalene & $91-57-6$ & $\mathbf{S}$ & $1.42 \mathrm{E}+02$ & 8511.4 & $7.40 \mathrm{E}+00$ & 2.90E-04 & $1.61 \mathrm{E}-03$ & $6.43 E-02$ & $4.53 \mathrm{E}-02$ & $1.48 \mathrm{E}-05$ & $1.55 E+04$ \\
\hline Acenaphthylene & $208-96-8$ & $\mathbf{S}$ & $1.52 \mathrm{E}+02$ & 4786.3 & $2.50 \mathrm{E}+00$ & $2.80 \mathrm{E}-04$ & $4.59 \mathrm{E}-03$ & $6.22 \mathrm{E}-02$ & $4.38 \mathrm{E}-02$ & $4.08 \mathrm{E}-05$ & $9.34 E+03$ \\
\hline Phenanthrene & $85-01-8$ & $\mathbf{S}$ & $1.78 E+02$ & 38904.5 & $1.40 \mathrm{E}+01$ & $2.56 \mathrm{E}-05$ & $7.50 \mathrm{E}-05$ & $5.74 \mathrm{E}-02$ & $4.05 E-02$ & $6.17 \mathrm{E}-07$ & 7.61E+04 \\
\hline
\end{tabular}

Noter:

1. USEPA, 1991: RAGS: Vol 1 - Human Health Evaluation Manual (Part B, Development of Risk-Based Preliminary Remodiation Coals)

2. Chemical Abruract Service.

3. Chemical clas: $v$ - volutile; - semivolatile; $p$ - pexticide/pct; $x$ - dioxin; and $m$-metal.

4. Calculation of organic cortaminare volatilization factor:

CONSTANTS:

Length of side of contam'd. area (LS), $m=$ Wind speed in mixing zone $(V), \mathrm{m} / \mathrm{zec}$ Diffusion height (DH), $m=$ Area of contamination (Am), $\mathrm{m}^{2}=$ Aren in iquare centimeters (Acm), $\mathrm{cm}^{2}=$ true soil porocity $(E)$, unitless = True eoil dencity (P), $\mathrm{d}^{\mathrm{cm}^{3}}=$ Exposure inerval (Tyr), yns = Exponure interval (Tsec), sec = Organic carbon content (OC), unitless = Conversion factor (CF), $\mathrm{kg} / \mathrm{g}=$

NPUT VARIABLES:

Organic cerbon pertition coef. (Koc), $\mathrm{cm}^{3} / \mathrm{kg}_{\mathbf{8}}=$ Heary/s Law conatant $(H)$, atm- $\mathrm{m}^{3} / \mathrm{mol}=$ Gas diffusivity (Di), $\mathrm{cm}^{2} / \mathrm{sec}=$ INTERMEDIATE STEPS:

Soil-water partition coefficien (Kd), $\mathrm{cm}^{3} / \mathrm{kg}=$ Soil-air partition coefficient (Kes), g-6oil/cm $\mathrm{cm}^{3}-2 i r=$ Effoctive diffurivity (Dei), $\mathrm{cm}^{2} / \mathrm{sec}=$

OUTPUT: Ilpha (alphs), $\mathrm{cm}^{2} / \mathrm{sec}=$

Volatilization Factor (VF), $\mathrm{m}^{\mathbf{3}} / \mathbf{k g}=$

$\begin{array}{cl}65.00 & (5) \\ 3.60 & (5) \\ 1.66 & (5) \\ 4,200.00 & (5) \\ 4.20 E+07 & \left(\mathrm{Am} * 10,000 \mathrm{~cm}^{2} / \mathrm{m}^{2}\right) \\ 0.350 & (1) \\ 2.650 & (1) \\ 5.00 & (5) \\ 1.58 E+08 & (T y x * 31536000 \text { sec/yr) } \\ 0.020 & (1) \\ 0.001 & \end{array}$

chem-epec

chem-spec

chem-spec

chem-spec $(\mathrm{Koc} \times \mathrm{OC})$

chem-ipec $((H / K d) \times 41)$

chem-ppec (Di $\times\left(E^{\wedge} 0.33\right)$ )

chem-epec $(($ Dei $\times \mathrm{E})(\mathrm{E}+(\mathrm{PB})(1-\mathrm{E})) / \mathrm{K} \Omega)$

chem-spec $((L S \times V \times D H \times(3.14 \times 1$ phe $\times T) 0.5)(A \times 2 \times D e i \times E \times K a s \times C F))$ 
Table D.4

Savannah River Site

Burning/Rubble Pits (231-F, 231-1F)

Current Visitor

Inhalation of Volatile Soil Contaminants (0-2'bls)

Carcinogenic and Noncarcinogenic Risk

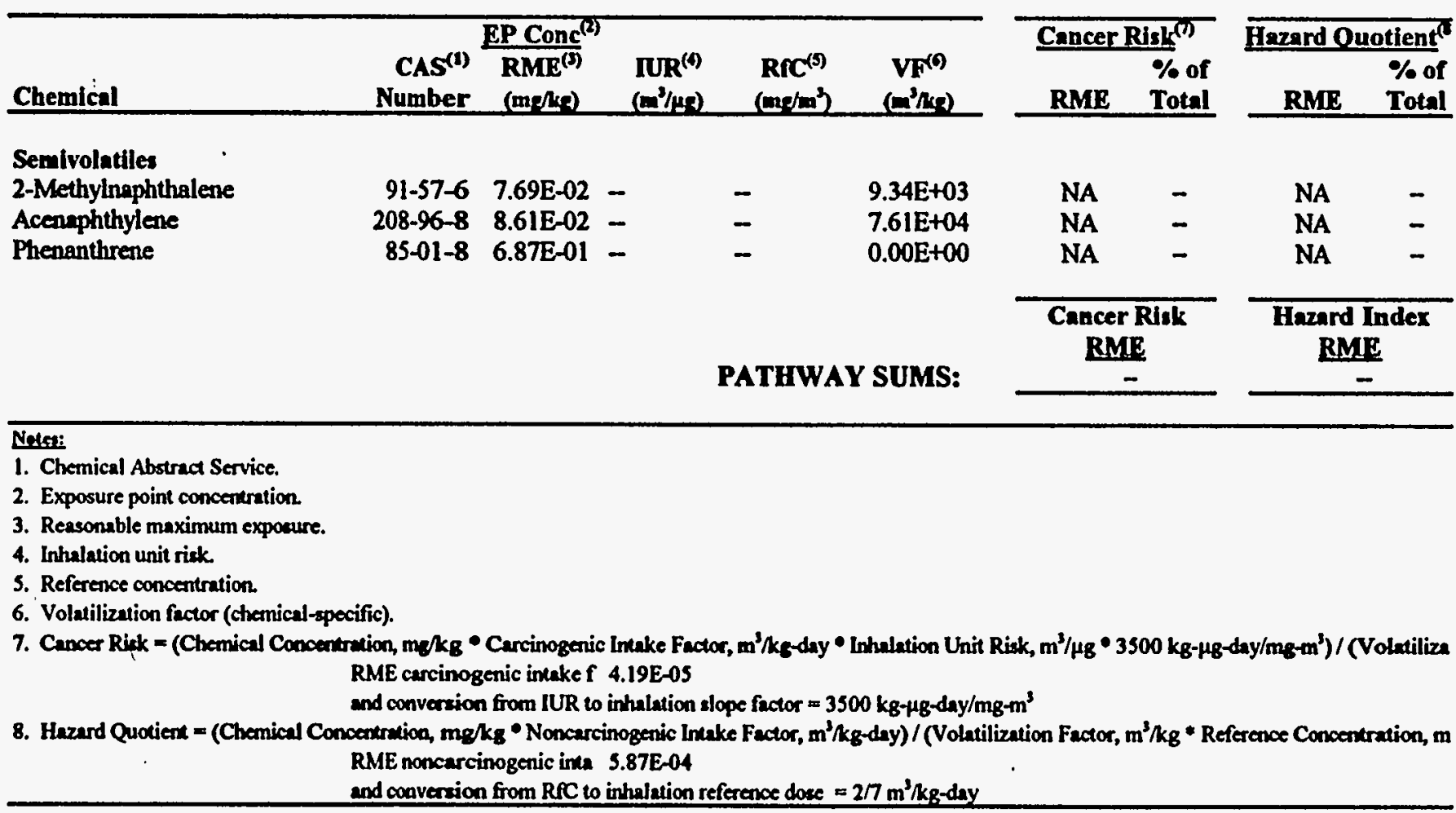


Table D.5

Savannah River Site

Burning/Rubble Pits (231-F, 231-1F)

Current Visitor

Inhalation of Resuspended Soil Particulate Contaminants (0-2'bls)

Carcinogenic and Noncarcinogenic Risk

\section{EXPOSURE ASSUMPTIONS:}

\section{Receptor}

Exposure

Inhalation Rate (IR), $\mathrm{m}^{3} / \mathrm{hr}$

Exposure Time (ET), hrs/day

Exposure Frequency (EF), days/yr

Exposure Duration (ED), yrs

Body Weight (BW), kg

Avging Time, Carc (2) (ATC), days

Avging Time, Noncarc ${ }^{(3)}$ (ATN), days

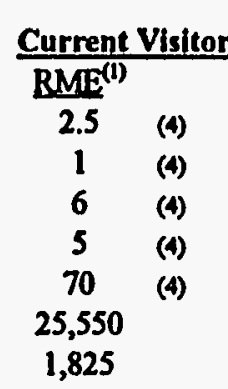

\section{INTAKE FACTOR CALCULATIONS}

Carcinogenic Intake Factor (CFF), $\mathrm{m}^{3} / \mathrm{kg}$-day = $(\mathbb{R} \bullet \mathrm{F} \bullet \mathrm{EF} \bullet \mathrm{ED} \bullet \mathrm{CF}) /(\mathrm{BW} \bullet \mathrm{ATC})$ $\mathrm{RME} C \mathrm{CF}=4.19 \mathrm{E}-05$

Noncarcinogenic Intake Factor (NIF), $\mathrm{m}^{3} / \mathrm{kg}$-day $=$ $(\mathbb{R} \bullet \mathrm{FI} * \mathrm{EF} * \mathrm{ED} \bullet \mathrm{CF}) /(\mathrm{BW} \bullet \mathrm{ATN})$

RME NIF $=5.87 \mathrm{E}-04$

\section{Netri:}

1. Ressonable maximum exposure.

2. Averaping time, carcinogen; calculbed as 70 years (wveruge lifetime) times 365 days per year (not uppliceble to child resident).

3. Averning time, noncarcinogen, calouleted as exposure deration (in years) times 365 deys par year

4. See Table D.1. 
Table D.5

Savannah River Site

Burning/Rubble Pits (231-F, 231-1F)

Current Visitor

Inhalation of Resuspended Soil Particulate Contaminants (0-2'bls)

Carcinogenic and Noncarcinogenic Risk

\begin{tabular}{llll}
\hline CALCULATION OF SOIL PARTICULATE RESUSPENSION FACTOR: & & \\
CONSTANTS: & & & \\
Length of side of contaminated area (LS) $=$ & 65.00 & meters & $(1)$ \\
Wind gpeed in mixing zone $(\mathrm{V})=$ & 3.60 & meters/sec & $(1)$ \\
Diffusion height $(\mathrm{DH})=$ & 1.66 & meters & $(1)$ \\
Area of contamination $(\mathrm{A})=$ & $4,200.00$ & meters & $(1)$ \\
Respirable fraction $(\mathrm{RF})=$ & 0.003 & $\mathrm{~g} /$ meters $^{2}$-hr & $(1)$ \\
Fraction of vegetative cover $(\mathrm{G})=$ & 0.90 & unitless & $(1)$ \\
Mean annual wind speed $(\mathrm{Um})=$ & 3.60 & meters/sec & $(1)$ \\
Equivalent threshold value of wind speed at $10 \mathrm{~m}(\mathrm{Ut})=$ & 5.04 & meters/sec & $(1)$ \\
Fraction dependent on $\mathrm{Um} / \mathrm{Ut}(\mathrm{Fx})=$ & 1.2700 & unitless & $(1)$ \\
Conversion factor $(\mathrm{CFa})=$ & $3,600.00$ & sec/hr & \\
Conversion factor $(\mathrm{CFb})=$ & $1,000.00$ & $\mathrm{~g} / \mathrm{kg}$ & \\
& & & \\
\hline
\end{tabular}

Nen:

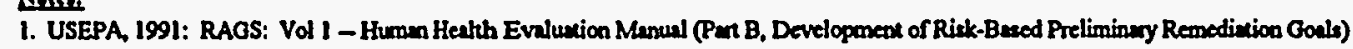

\section{INTERMEDIATE STEPS:}

Particulate emission factor (PEF) $=\quad 2.16 \mathrm{E}+09$ meters $^{3} \mathrm{~kg}$

$(\mathrm{LS} \times \mathrm{V} \times \mathrm{DH} \times \mathrm{CFa} \times \mathrm{CFb}) /\left(\mathrm{A} \times \mathrm{RF} \times(1-\mathrm{G}) \times\left((\mathrm{Um} / \mathrm{Ut})^{n} 3\right) \times(\mathrm{Fx})\right)$ 
Table D.5

Savannah River Site

Burning/Rubble Pits (231-F, 231-1F)

Current Visitor

Inhalation of Resuspended Soil Particulate Contaminants (0-2'bls)

Carcinogenic and Noncarcinogenic Risk

\begin{tabular}{|c|c|c|c|c|c|c|c|c|}
\hline \multirow[b]{2}{*}{ Chemical } & \multicolumn{3}{|c|}{ EP Conc $^{(t)}$} & \multirow[b]{2}{*}{$\begin{array}{l}\operatorname{RrC}^{(\theta)} \\
\left(m / m^{2}\right)\end{array}$} & \multicolumn{2}{|c|}{ CancerRisk ${ }^{(0)}$} & \multicolumn{2}{|c|}{ Hezard Quotient $^{(7}$} \\
\hline & $\begin{array}{r}\text { CAS }^{(1)} \\
\text { Number }\end{array}$ & $\begin{array}{l}\mathbf{R M E}^{(3)} \\
(m / \mathcal{L})\end{array}$ & $\begin{array}{l}\operatorname{TUR}^{(0)} \\
\left(\mathrm{m}^{3} / \mu q\right)\end{array}$ & & $\mathbf{R M E}$ & $\begin{array}{l}\% \text { of } \\
\text { Total } \\
\end{array}$ & $\mathbf{R M E}$ & $\begin{array}{l}\% \text { of } \\
\text { Total } \\
\end{array}$ \\
\hline \multicolumn{9}{|l|}{ Semivolatiles } \\
\hline 2-Methylnaphthalene & $91-57-6$ & $7.69 \mathrm{E}-02$ & - & - & - & - & - & - \\
\hline Acenaphthylene & $208-96-8$ & 8.61E-02 & - & - & - & - & - & - \\
\hline Benzo(a)anthracene & $56-55-3$ & 9.26E-01 & - & - & - & - & - & - \\
\hline Benzo(a)pyrene & $50-32-8$ & $6.49 E-01$ & - & - & - & - & - & - \\
\hline Benzo(b)fluoranthene & $205-99-2$ & $1.01 \mathrm{E}+00$ & - & - & - & - & - & - \\
\hline Benzo(B,h,i)perylene & $191-24-2$ & $6.15 E-01$ & - & - & - & - & - & - \\
\hline Dibenz(a,h)anthracene & $53-70-3$ & 2.17E-01 & - & - & - & - & - & - \\
\hline Phenanthrene & $85-01-8$ & $6.87 E-01$ & - & - & - & - & - & - \\
\hline \multicolumn{9}{|l|}{ Dloxins/furans } \\
\hline Heptachlorodibenzo-p-dioxin isomer & $37871-00-4$ & 4.11E-03 & $3.30 \mathrm{E}-01$ & - & $9.2 \mathrm{E}-14$ & $<1 \%$ & - & - \\
\hline Heptachlorodibenzo-p-furan isomer & 38998-75-3 & $2.58 \mathrm{E}-04$ & $3.30 \mathrm{E}-01$ & - & $5.8 \mathrm{E}-15$ & $<1 \%$ & - & - \\
\hline Hexachlorodibenzo-p-furan isomer & 55684-94-1 & $1.00 E-04$ & $3.30 E+00$ & - & $2.2 \mathrm{E}-14$ & $<1 \%$ & - & - \\
\hline Octachlorodibenzo-p-dioxin isomer & $3268-87-9$ & $9.26 \mathrm{E}-03$ & $3.30 \mathrm{E}-02$ & - & 2.1E-14 & $<1 \%$ & - & - \\
\hline Octechlorodibenzo-p-furan isomer & $39001-02-0$ & $3.48 \mathrm{E}-0.4$ & $3.30 \mathrm{E}-02$ & - & 7.8E-16 & $<1 \%$ & - & - \\
\hline \multicolumn{9}{|l|}{ PCBs } \\
\hline PCB 1254 & $11097-69-1$ & $1.78 \mathrm{E}-01$ & - & - & - & - & - & - \\
\hline \multicolumn{9}{|l|}{ Pesticides } \\
\hline delta-Benzene hexachloride & $319-86-8$ & $1.84 \mathrm{E}-03$ & - & - & - & - & - & - \\
\hline Endrin ketone & $53494-70-5$ & $3.20 \mathrm{E}-03$ & - & - & - & - & - & - \\
\hline
\end{tabular}


Table D.5

Savannah River Site

Burning/Rubble Pits (231-F, 231-1F)

Current Visitor

Inhalation of Resuspended Soil Particulate Contaminants (0-2'bls)

Carcinogenic and Noncarcinogenic Risk

\begin{tabular}{|c|c|c|c|c|c|c|c|c|}
\hline \multirow[b]{2}{*}{ Chemical: } & \multicolumn{3}{|c|}{ EP Conc ${ }^{(2)}$} & \multirow[b]{2}{*}{$\begin{array}{r}\operatorname{RfC}^{(5)} \\
\left(\mathrm{m} / \mathrm{m}^{2}\right)\end{array}$} & \multicolumn{2}{|c|}{ Cancer Risk ${ }^{(0)}$} & \multicolumn{2}{|c|}{ Hazard Ouotient } \\
\hline & $\begin{array}{r}\text { CAS }^{(1)} \\
\text { Number }\end{array}$ & $\begin{array}{l}\mathrm{RMB}^{(3)} \\
\left(\mathrm{m} / \mathrm{LS}^{\prime}\right)\end{array}$ & $\begin{array}{l}\mathrm{IUR}^{(0)} \\
\left(\mathrm{m}^{3} / \mu q\right)\end{array}$ & & $\mathbf{R M E}$ & $\begin{array}{l}\% \text { of } \\
\text { Total }\end{array}$ & $\mathbf{R M E}$ & $\begin{array}{l}\% \text { of } \\
\text { Total } \\
\end{array}$ \\
\hline \multicolumn{9}{|l|}{ Metals } \\
\hline Arsenic & $7440-38-2$ & $4.82 E+00$ & $4.30 \mathrm{E}-03$ & - & $1.4 E-12$ & $04 \%$ & 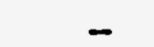 & - \\
\hline Chromium & $7440-47-3$ & $4.42 \mathrm{E}+01$ & $1.20 \mathrm{E}-02$ & - & $3.6 \mathrm{E}-11$ & $96 \%$ & - & - \\
\hline Lead & $7439-92-1$ & $2.48 E+01$ & - & - & - & - & - & - \\
\hline Manganese & $7439-96-5$ & $4.78 \mathrm{E}+02$ & - & 5.00E-05 & - & - & $9.1 \mathrm{E}-06$ & $100 \%$ \\
\hline \multirow[t]{2}{*}{ Mercury } & $7439-97-6$ & $5.48 \mathrm{E}-01$ & - & $3.00 \mathrm{E}-04$ & - & - & 1.7E-09 & $<1 \%$ \\
\hline & & - & PAT & HWAY SUMS: & \multicolumn{2}{|c|}{$\begin{array}{c}\text { Cancer RIsk } \\
\frac{\text { RME }}{4 \mathrm{E}-11}\end{array}$} & \multicolumn{2}{|c|}{$\begin{array}{c}\text { Hazard Index } \\
\text { RME } \\
9 \mathrm{E}-06\end{array}$} \\
\hline
\end{tabular}

Notes:

1. Chemical Abotract Service.

2. Exposure point concentration.

3. Reasonable maximum exposure.

4. Inhalation unit risk.

5. Referenoe concentrution.

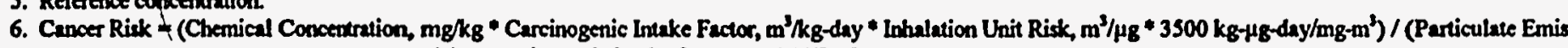
RME carcinogenic intake fuctor - 4.19E-0S

Particulate emission factor = $\quad 2.16 \mathrm{E}+09$

and conversion from IUR to inhalation slope factor $=3500 \mathrm{~kg}-\mu \mathrm{g}-\mathrm{day} / \mathrm{mg}^{3} \mathrm{~m}^{3}$

7. Hazard Quotient = (Chemical Concentration, $\mathrm{mg} / \mathrm{kg}$ * Noncarcinogenic Intake Factor, $\mathrm{m}^{3} / \mathrm{kg}$-day) $/$ (Particulate Emission Fector, $\mathrm{m}^{3} / \mathrm{kg}_{\mathrm{g}}$ * Reference Concentration,

RME noncarcinogenic intake factor 5.87E-04

Particulate emission fictor = $\quad 2.16 \mathrm{E}+09$

and conversion from RfC to inhalation reference dose $=2 / 7 \mathrm{~m}^{3} / \mathrm{kg}$-day 
Table D.6

Savannah River Site

Rubble Pit (231-2F)

Current Visitor

Ingestion of Soil (0-2' bls)

Carcinogenic and Noncarcinogenic Risk

\section{EXPOSURE ASSUMPTIONS:}

\section{Receptor}

Exposure

Intake Rate (IR), mg/day

Fraction Ingested (FI), unitless

Exposure Frequency (EF), days/yr

Exposure Duration (ED), yrs

Body Weight (BW), kg

Avging Time, $\operatorname{Carc}^{(2)}$ (ATC), days

Avging Time, Noncarc ${ }^{(3)}$ (ATN),

Conversion Factor (CF), kg/mg

\section{INTAKE FACTOR CALCULATIONS}

Carcinogenic Intake Factor (CIF), kg/kg-day = $(\mathrm{IR} \bullet \mathrm{FI} \bullet \mathrm{EF} \bullet \mathrm{ED} \bullet \mathrm{CF}) /(\mathrm{BW} \bullet \mathrm{ATC})$

RME CIF $=8.39 \mathrm{E}-10$

Noncarcinogenic Intake Factor (NIF), kg/kg-day = $(\mathrm{IR} \bullet \mathrm{FI} \bullet \mathrm{EF} \bullet \mathrm{ED} \bullet \mathrm{CF}) /(\mathrm{BW} \bullet \mathrm{ATN})$

RME NIF $=1.17 E-08$

\section{Noter:}

1. Ressonable maximum exposure.

2. Averaging time, carcinogen; calculated as 70 yean (nerage lifetime) times 365 days per year (nox applieable to child resident).

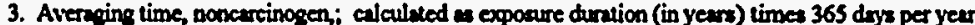

4. See Table D-1. 
Table D.6

Savannah River

Rubble Pit (231-2F)

Current Visitor

Ingestion of Soil (0-2' bls)

Carcinogenic and Noncarcinogenic Risk

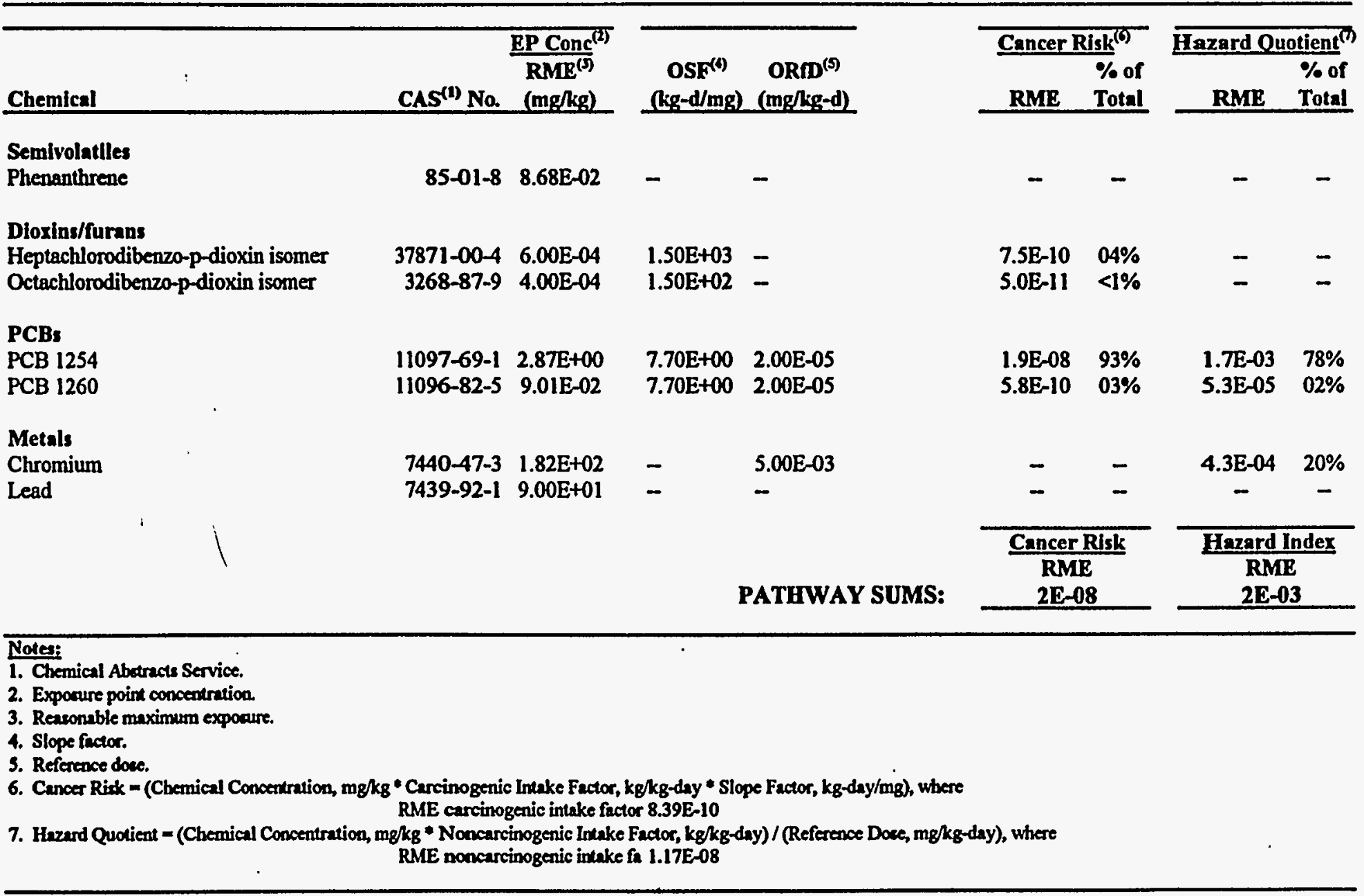


Table D.7

Savannah River Site

Rubble Pit (231-2F)

Current Visitor

Dermal Exposure to Soil

Carcinogenic and Noncarcinogenic Risk

EXPOSURE ASSUMPTIONS:

\section{INTAKE FACTOR CALCULATIONS}

\section{-Receptor}

Exposure

Skin Surface Area (SA), $\mathrm{cm}^{2} /$ event

Soil-to-Skin Adherance (SK), $\mathrm{mg} / \mathrm{cm}^{2}$

Exposure Frequency (EF), events/yr

Exposure Duration (ED), yrs

Body Weight (BW), kg

Avging Time, Carce (ATC), days

Avging Time, Noncarc ${ }^{(3)}$ (ATN), days

Conversion Factor (CF), kg/mg

Current Visitor

$\mathrm{RME}^{(1)}$

$3,200 \quad$ (4)

$0.2 \quad$ (4)

$6 \quad(4)$

$5 \quad(4)$

$70 \quad$ (4)

25,550

1,825

$1.00 \mathrm{E}-06$
Carcinogenic Intake Factor (CFF), kg/kg-day =

$(\mathrm{SA} \bullet \mathrm{SK} * \mathrm{EF} \bullet \mathrm{ED} \bullet \mathrm{CF}) /(\mathrm{BW} \bullet \mathrm{ATC})$

RME CFF $=1.07 \mathrm{E}-08$

Noncarcinogenic Intake Factor (NIF), $\mathrm{kg} / \mathrm{kg}$-day = $(\mathrm{SA} * \mathrm{SK} * \mathrm{EF} * \mathrm{ED} \bullet \mathrm{CF}) /(\mathrm{BW} \bullet \mathrm{ATN})$

RME NIF $=1.50 \mathrm{E}-07$

\section{Neferi:}

1. Remonuble muximum expocure

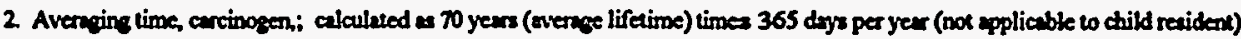

3. Averaing time, noncarcinogen; calculmed as exposure duretion (in yern) times 365 doy per yeer

4. See Table D-1. 
Table D.7

Savannah River

Rubble Pit (231-2F)

Current Visitor

Dermal Exposure to Soil

Carcinogenic and Noncarcinogenic Risk

\begin{tabular}{|c|c|c|c|c|c|c|c|c|c|}
\hline Chemical & \multicolumn{2}{|c|}{ 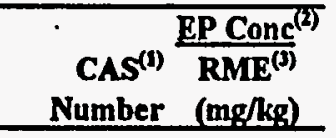 } & $\begin{array}{c}\mathrm{DSF}^{(1)} \\
(\mathrm{kg}-\mathrm{d} / \mathrm{mg})\end{array}$ & 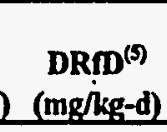 & DABS $^{(0)}$ & Cancer & $\begin{array}{l}\text { isk }^{(1)} \\
\% \text { of } \\
\text { Total }\end{array}$ & Hazard & $\begin{array}{l}\frac{\text { lotient }^{(\mathrm{(})}}{\% \text { of }} \\
\text { Total }\end{array}$ \\
\hline $\begin{array}{l}\text { Semivolatlles } \\
\text { Phenenthrene } \\
\text { Dloxins/furans }\end{array}$ & $85-01-8$ & 8.68E-02 & - & - & $1.00 \mathrm{E}-02$ & - & - & - & - \\
\hline $\begin{array}{l}\text { Heptachlorodibenzo-p-dioxin isomer } \\
\text { Octachlorodibenzo-p-dioxin isomer }\end{array}$ & $\begin{array}{r}37871-00-4 \\
3268-87-9\end{array}$ & $\begin{array}{l}6.00 \mathrm{E}-04 \\
4.00 \mathrm{E}-04\end{array}$ & $\begin{array}{l}1.72 E+03 \\
1.72 E+02\end{array}$ & - & $\begin{array}{l}1.00 \mathrm{E}-02 \\
1.00 \mathrm{E}-02\end{array}$ & $\begin{array}{l}1.1 \mathrm{E}-10 \\
7.4 \mathrm{E}-12\end{array}$ & $\begin{array}{l}04 \% \\
<1 \%\end{array}$ & - & - \\
\hline $\begin{array}{l}\text { PCBs } \\
\text { PCB } 1254 \\
\text { PCB } 1260\end{array}$ & $\begin{array}{l}11097-69-1 \\
11096-82-5\end{array}$ & $\begin{array}{l}2.87 \mathrm{E}+00 \\
9.01 \mathrm{E}-02\end{array}$ & $\begin{array}{l}8.56 \mathrm{E}+00 \\
8.56 \mathrm{E}+00\end{array}$ & $\begin{array}{l}1.80 \mathrm{E}-05 \\
1.80 \mathrm{E}-05\end{array}$ & $\begin{array}{l}1.00 \mathrm{E}-02 \\
1.00 \mathrm{E}-02\end{array}$ & $\begin{array}{l}2.6 \mathrm{E}-09 \\
8.3 \mathrm{E}-11\end{array}$ & $\begin{array}{l}93 \% \\
03 \%\end{array}$ & $\begin{array}{l}2.4 \mathrm{E}-04 \\
7.5 \mathrm{E}-06\end{array}$ & $\begin{array}{l}46 \% \\
01 \%\end{array}$ \\
\hline $\begin{array}{l}\text { Metals } \\
\text { Chromium } \\
\text { Lead }\end{array}$ & $\begin{array}{l}7440-47-3 \\
7439-92-1\end{array}$ & $\begin{array}{l}1.82 \mathrm{E}+02 \\
9.00 \mathrm{E}+01\end{array}$ & - & $\begin{array}{l}1.00 \mathrm{E}-04 \\
-\end{array}$ & $\begin{array}{l}1.00 \mathrm{E}-03 \\
1.00 \mathrm{E}-03\end{array}$ & - & - & $\begin{array}{c}\text { 2.7E-04 } \\
-\end{array}$ & $\begin{array}{c}53 \% \\
-\end{array}$ \\
\hline 1 & & & & PATHWAY & Y SUMS: & $\begin{array}{r}\text { Cancer } \\
\text { RM } \\
\text { 3E- }\end{array}$ & & $\begin{array}{r}\text { Hazar } \\
\frac{R}{5 E}\end{array}$ & $\begin{array}{l}\text { Index } \\
4 \\
\end{array}$ \\
\hline $\begin{array}{l}\text { Notes: } \\
\text { 1. Chemical Abatract Service. } \\
\text { 2. Expoeure point concentration. } \\
\text { 3. Rearonable maximum exposure. } \\
\text { 4. Slope factor, dermal. } \\
\text { 3. Reference doce, dermal. } \\
\text { 6. Abeorption factor. } \\
\text { 7. Cancer Risk = (Chemical Concentration }\end{array}$ & $\mathrm{mg} / \mathrm{kg} * \mathrm{Carci}$ & inogenic Intake & ctor, kg/kg-da & ay * Absorption & Fuctor, unit! & Slope Facto & $k g-d a$ & where & \\
\hline 8. Hezard Quotien = (Chemical Concentrs & $\begin{array}{l}\text { RME carcinog } \\
\text { ation, mg/kg * } \mathrm{N} \\
\text { RME noncarci }\end{array}$ & $\begin{array}{l}\text { genic intake fac } \\
\text { Noncarcinogeni } \\
\text { inogenic intake }\end{array}$ & $\begin{array}{l}1.07 \mathrm{E}-08 \\
\text { Intuke Factor, } \\
1.50 \mathrm{E}-07\end{array}$ & $k g / k g-d a y \cdot A b$ & berplion Fac & nitless)/( $R$ & erence D & $m g / k g-d a j$ & here \\
\hline
\end{tabular}


Table D.8

Savannah River

Rubble Pit (231-2F)

Current Visitor

Inhalation of Volatile Soil Contaminants (0-2'bls)

\section{EXPOSURE ASSUMPTIONS:}

Receptor

Exposure

Inhalation Rate (IR), $\mathrm{m}^{3} \mathrm{hr}$

Exposure Time (ET), hrs/day

Exposure Frequency (EF), days/yr

Exposure Duration (ED), yrs

Body Weight (BW), kg

Avging Time, Carc $^{(2)}$ (ATC), days

Avging Time, Noncarc ${ }^{(3)}$ (ATN), days

\begin{tabular}{cc}
\multicolumn{2}{c}{ Current Visito } \\
\hline RME $^{(1)}$ & \\
2.5 & (4) \\
1 & (4) \\
6 & $(4)$ \\
5 & $(4)$ \\
70 & $(4)$ \\
25,550 & \\
1,825 &
\end{tabular}

INTAKE FACTOR CALCULATIONS

Carcinogenic Intake Factor (CIF), $\mathrm{kg} / \mathrm{kg}$-day =

$(\mathrm{R} * \mathrm{ET} \bullet \mathrm{EF} \bullet \mathrm{ED} \bullet \mathrm{CF}) /(\mathrm{BW} \bullet \mathrm{ATC})$

$\mathrm{RME} C \mathrm{CF}=4.19 \mathrm{E}-05$

Noncarcinogenic Intake Factor (NIF), kg/kg-day = $(\mathrm{R} \bullet \mathrm{ET} \bullet \mathrm{EF} \bullet \mathrm{ED} \bullet \mathrm{CF}) /(\mathrm{BW} \bullet \mathrm{ATN})$

RME NIF $=5.87 E-04$

\section{Nesini:}

1. Remonsble maximum exposire.

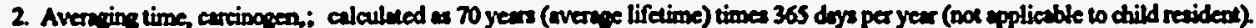

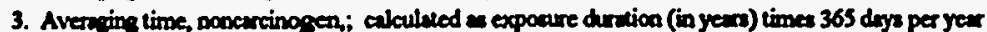

4. See Tuble D-1. 
Table D.8

Rubble Pit (231-2F)

Current Visitor

Inhalation of Volatile Soil Contaminants (0-2'bls)

Carcinogenic and Noacarcinogenic Risk

\begin{tabular}{|c|c|c|c|c|c|c|c|c|c|c|}
\hline Chemical & $\mathrm{CAS}^{(2)} \mathrm{Na}$ & Class $^{(3)}$ & $\mathrm{Kac}^{(\boldsymbol{l})}$ & $\mathbf{K d}^{(1)}$ & $\mathbf{H}^{(\boldsymbol{(})}$ & $\mathrm{Kas}^{(4)}$ & $\mathbf{D H}^{(0)}$ & $\mathrm{Dei}^{(1)}$ & $\operatorname{alpha}(1)$ & $\begin{array}{l}\text { FolaL } \\
\text { Factor } \\
\left(\mathrm{m}^{3} / \mathrm{kg}\right)\end{array}$ \\
\hline $\begin{array}{l}\text { Semivolatiles } \\
\text { Phenanthrene }\end{array}$ & $85-01-8$ & $\mathbf{S}$ & $1.78 \mathrm{E}+02$ & $3.56 \mathrm{E}+00$ & 2.56E-05 & 2.95E-04 & $5.74 \mathrm{E}-02$ & 4.05E-02 & $2.43 E-06$ & $5.66 \mathrm{E}+04$ \\
\hline
\end{tabular}

Notec:

1. USEPA, 1991: RAGS: Vol 1 - Humen Health Evaluation Manual (Part B, Developmeat of Risk-Bered Preliminary Remediation Goals)

2. Chemical Abatraca Service.

3. Chemical class: $v$-volatile; $s$ - remivolatile; $p$-pesticide/pab; $x$-dioxin; and $m$-menal.

4. Calculation of organic contaminarn volatilization factor:

CONSTANTS:

Length of side of contem'd, area (LS), $m=24.00$

Wind spoed in mixing zone ( $V$, $\mathrm{m} / \mathrm{woc}=3.60$

Diffusion heigtx (DH), $\mathrm{m}=0.79$

Area of contamination $(\mathrm{Am}), \mathrm{m}^{2}=\mathbf{5 0 0 . 0 0}$

Area in square centimeters $(\mathrm{Acm}), \mathrm{cm}^{2}=5.00 \mathrm{E}+06$

True soil porocity $(E)$, unitless $=0.350$

True soil density $(\mathrm{Px}), \mathrm{g} / \mathrm{cm}^{3}=2.650$

Exposure interval (Tyr), yrs $=5.00^{\circ}$

Exposure interval (Tsac), $200=1.58 \mathrm{E}+08$

Organic carbon content $(O C)$, unitleas $=0.020$

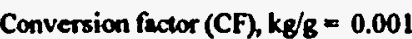

INPUT VARIABLES

Organic carton partition coef. (Koc), $\mathrm{cm}^{3} / \mathrm{kg}=\mathrm{chem}-\mathrm{spec}$

Henry's Law constank (H), atm-m $3 / \mathrm{mol}=$ chem-spec

Gas diffusivity (Di), $\mathrm{cm}^{2} / \mathrm{scc}=$ chem-spec

INTERMEDIATE STEPS:

Soil-water partition coefficient (Kd), $\mathrm{cm}^{3} / \mathrm{kg}$ a chem-spec Soil-air partition coefficiern (Kas), E-soil/ $\mathrm{cm}^{3}-\mathrm{air}=\mathrm{cthem-spec}$ Effoctive diffusivity (Dei), $\mathrm{cm}^{2} / 12 \mathrm{cos}$ chem-spoc alpha (alpha), $\mathrm{cm}^{2} / \mathrm{sec}=$ chem-spoc

OUTPUT:

Volatilization Factor $(V F), m^{3} / k_{B}=$ chem-spec

(1)

(1)

(Am*10,000 $\mathrm{cm}^{2} / \mathrm{m}^{2}$ )

(1)

(1)

(1)

(Tyr*31536000 200/yr)

(1)

$(\mathrm{Koc} \times \mathrm{OC})$

$((\mathrm{H} / \mathrm{K} d) \times 41)$

(Di $\left.\times\left(E^{\prime} 0.33\right)\right)$

$(($ Dei $\times E)(E+(P z)(1-E)) / K a s)$

$((L S \times V \times D H \times(3.14 \times 2 / p h a \times T) \sim .5)(A \times 2 \times D e i \times E \times K e s \times C F))$

Page 1 of 1 
Table D.8

Rubble Pit (231-2F)

Current Visitor

Inhalation of Volatile Soil Contaminants (0-2'bls)

Carcinogenic and Noncarcinogenic Risk

\begin{tabular}{|c|c|c|c|c|c|c|c|c|c|}
\hline \multirow[b]{2}{*}{ Chemical } & \multirow{2}{*}{\multicolumn{2}{|c|}{ 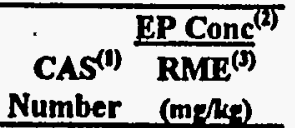 }} & \multirow{2}{*}{$\begin{array}{l}\text { IUR } \\
\left(\mathrm{m}^{3} / \mathrm{s}^{(\mathrm{s})}\right.\end{array}$} & \multirow{2}{*}{ 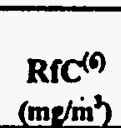 } & \multirow{2}{*}{$\begin{array}{c}V_{\left(m^{\prime}\right)} \\
\left(m^{\prime} / g\right)\end{array}$} & \multicolumn{2}{|c|}{ Cancer Risk ${ }^{(1)}$} & \multicolumn{2}{|c|}{ Fazard Quntient } \\
\hline & & & & & & RME & $\begin{array}{l}\text { \% of } \\
\text { Total }\end{array}$ & RME & $\begin{array}{l}\% \text { of } \\
\text { Total }\end{array}$ \\
\hline $\begin{array}{l}\text { Semivolatiles } \\
\text { Phenanthrese }\end{array}$ & $85-01-8$ & $2.90 \mathrm{E}+00$ & - & - & $5.66 \mathrm{E}+04$ & - & - & - & - \\
\hline & & & \multicolumn{3}{|c|}{ PATHWAY SUMS: } & $\begin{array}{r}\text { Cance } \\
\text { RN }\end{array}$ & & \multicolumn{2}{|c|}{$\begin{array}{c}\text { Hazard Index } \\
\text { RME } \\
\text { - }\end{array}$} \\
\hline
\end{tabular}

Netre:

1. Chemical Abrtruct Service.

2. Exposure point concentration.

3. Reaconable maximum expocure.

4. Central tendency (exposure).

5. Inhalation unit risk.

6. Reference concentration

7. Volatilization factor (chemical-specific).

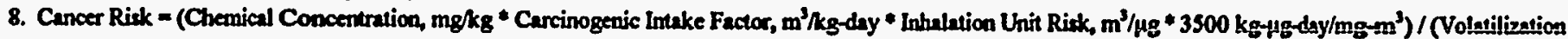
RME carcinogenic inuke fictor 4.19E-05

CT carcinogenic ineake frutor $=0.00 \mathrm{E}+00$

and conversion from IUR to inhalation slope fictor $-3300 \mathrm{~kg}-\mu \mathrm{g}-\mathrm{day} / \mathrm{mg}-\mathrm{m}^{3}$

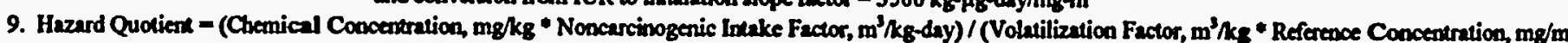
RME noncarcinogenic inake fin 5.87E-04

CT noncarcinogenic intake fact $0.00 E+00$

and conversion from RRC to intalation referenoe dose $=277 \mathrm{~m}^{3} / \mathrm{kg}$-day 
Table D.9

Savannah River

Rubble Pit (231-2F)

Current Visitor

Inhalation of Resuspended Soil Particulate Contaminants (0-2'bls)

Carcinogenic and Noncarcinogenic Risk

\section{EXPOSURE ASSUMPTIONS:}

Receptor

Exposure

Inhalation Rate (IR), $\mathrm{m}^{\mathbf{3}} / \mathrm{hr}$

Exposure Time (ET), hrs/day

Exposure Frequency (EF), days/yr

Exposure Duration (ED), yrs

Body Weight (BW), kg

Avging Time, Carc ${ }^{(2)}$ (ATC), days

Avging Time, Noncarc ${ }^{(3)}$ (ATN), days

\begin{tabular}{cc}
\multicolumn{3}{c}{ Current Visitor } \\
\hline BME $^{(1)}$ & \\
2.5 & $(4)$ \\
1 & $(4)$ \\
6 & $(4)$ \\
5 & $(4)$ \\
70 & $(4)$ \\
25,550 & \\
1,825 &
\end{tabular}

INTAKE FACTOR CALCULATIONS

Carcinogenic Intake Factor (CIF), kg/kg-day = $(\mathrm{IR} \bullet \mathrm{FI} \bullet \mathrm{EF} \bullet \mathrm{ED} \bullet \mathrm{CF}) /(\mathrm{BW} \bullet \mathrm{ATC})$

RME CIF $=4.19 \mathrm{E}-05$

Noncarcinogenic Intake Factor (NIF), kg/kg-day = $(\mathrm{IR} \bullet \mathrm{FI} \bullet \mathrm{EF} \bullet \mathrm{ED} \bullet \mathrm{CF}) /(\mathrm{BW} \bullet \mathrm{ATN})$

RME NIF $=5.87 E-04$

1. Reacondic maximum exposure.

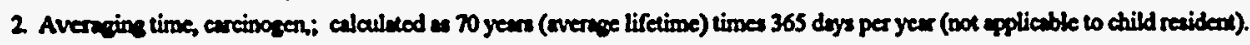

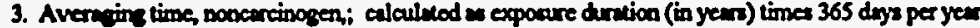

4. See Table D.1. 
Table D.9

Savannah River

Rubble Pit (231-2F)

Current Visitor

Inhalation of Resuspended Soil Particulate Contaminants (0-2'bls)

Carcinogenic and Noncarcinogenic Risk

\begin{tabular}{|c|c|c|c|c|}
\hline \multicolumn{5}{|c|}{ CALCULATION OF SOH PARTICULATE RESUSPENSION FACTOR: } \\
\hline Length of side of contaminated area (IS) $=$ & 24.00 & meters & (1) & Particulate emission factor (PEF) $=$ \\
\hline Wind speed in mixing zone $(V)=$ & 3.60 . & meters/sec & (1) & $(\mathrm{LS} \times \mathrm{V} \times \mathrm{DH} \times \mathrm{CFa} \times \mathrm{CFb}) /\left(\mathrm{A} \times \mathrm{RF} \times(1-\mathrm{G}) \times\left((\mathrm{Um} / \mathrm{Ut})^{\wedge} 3\right) \times(\mathrm{Fx})\right)$ \\
\hline Diffusion height $(\mathrm{DH})=$ & 0.79 & meters & (1) & \\
\hline Area of contamination $(A)=$ & 500.00 & meters $^{2}$ & (1) & \\
\hline Respirable fraction (RF) = & 0.003 & g/meters ${ }^{2}-h r$ & (1) & $\cdot$ \\
\hline Fraction of vegetative cover $(G)=$ & 0.90 & unitless & (1) & \\
\hline Mean annual wind speed $(\mathrm{Um})=$ & 3.60 & meters/sec & (1) & \\
\hline Equivalent threshold value of wind speed at $10 \mathrm{~m}(\mathrm{Ut})=$ & 5.04 & meters/sec & (1) & \\
\hline Fraction dependent on Um/Ut (Fx) $=$ & 1.2700 & unitless & (1) & \\
\hline Conversion factor $(\mathrm{CFa})=$ & $3,600.00$ & $\sec / \mathrm{hr}$ & & \\
\hline Conversion factor $(\mathrm{CFb})=$ & $1,000.00$ & g/kg & & \\
\hline
\end{tabular}

Nolen:

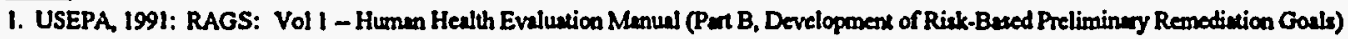


Table D.9

Savannah River

Rubble Pit (231-2F)

Current Visitor

Inhalation of Resuspended Soil Particulate Contaminants (0-2'bls)

Carcinogenic and Noncarcinogenic Risk

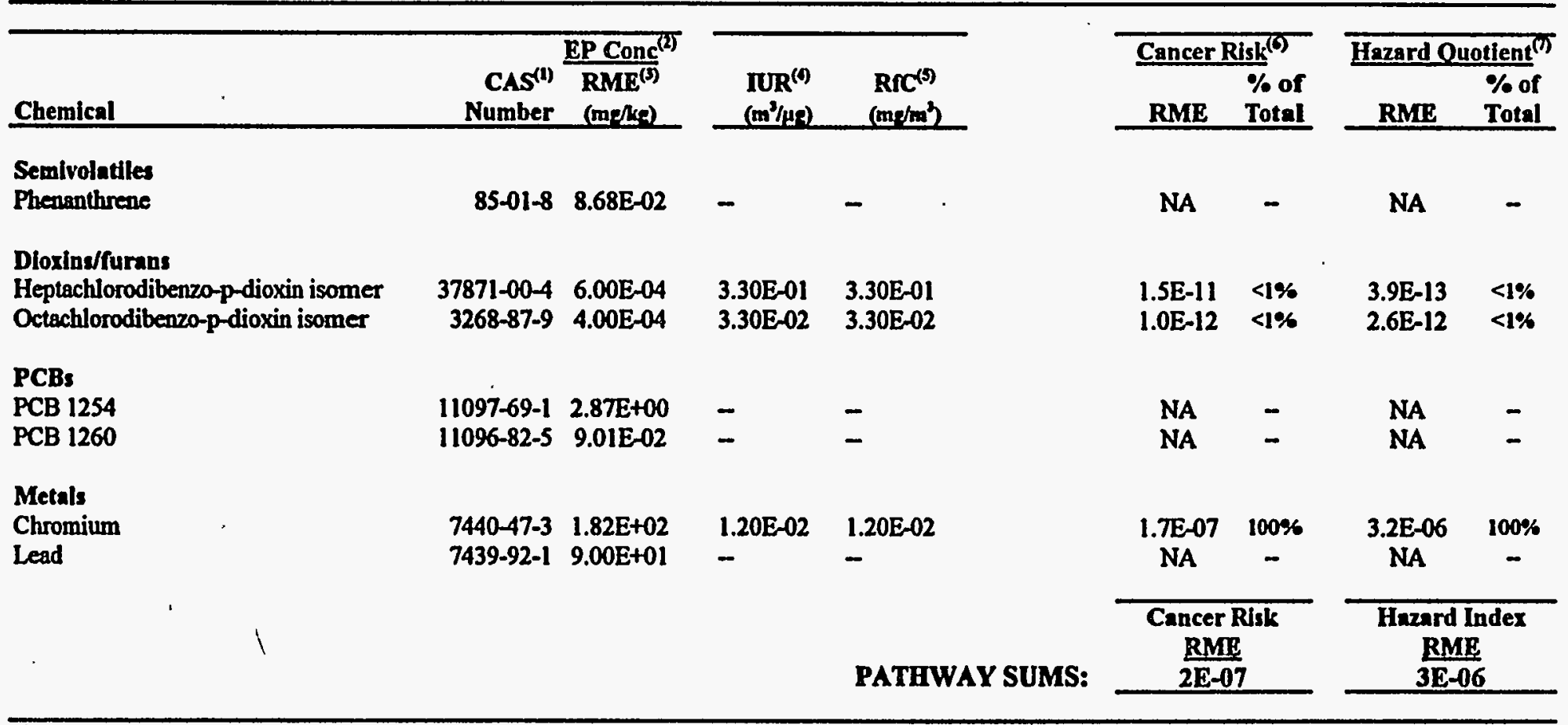

Noter:

1. Chemical Abatract Service.

2. Exposure point concentration

3. Reasoneble maximum expocure.

4. Inhalation unit risk.

5. Referenoc concentration

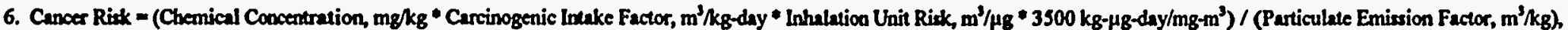
RME carcinogenic intake fuctor 6.99E-02

Particulate emission factor $=3.22 \mathrm{E}+09$

and convertion from IUR to inhalation slope factor $=3500 \mathrm{~kg}-\mu g-d a y / \mathrm{mg}^{3} \mathrm{~m}^{3}$

7. Hazard Quotient = (Chemical Concentration, $\mathrm{mg} / \mathrm{kg}_{\mathrm{g}}$ * Noncarcinogenic Intake Factor, $\mathrm{m}^{3} / \mathrm{kg}_{\mathrm{g}}$ day) $/\left(\right.$ Particulate Emirsion Factor, $\mathrm{m}^{3} / \mathrm{kg}_{\mathrm{g}}$ * Reference Concentration, $\mathrm{mg}^{3} \mathrm{~m}^{3}$ * $2 / 7 \mathrm{~m} / \mathrm{kg}$-day),

RME noncarcinogenic intake fa $1.96 \mathrm{E}-01$

Particulate cmivsion factor $=\quad 3.22 \mathrm{E}+09$

and convervion from RCC to inhalation reference doce $=2 / 7 \mathrm{~m}^{3} / \mathrm{kg}$-day 
Table D.10

Savannah River Site

Burning/Rubble Pits (231-F, 231-1F, 231-2F)

Current Visitor

Dermal Exposure to Surface Water (Wetland)

Carcinogenic and Noncarcinogenic Risk

\section{EXPOSURE ASSUMPTIONS:}

\section{Receptor}

Exposure

Skin Surface Area (SA), $\mathrm{cm}^{2} /$ event

Exposure Time (ET), hrs/day

Exposure Frequency (EF), events/yr

Exposure Duration (ED), yrs

Body Weight (BW), kg

Avging Time, Carc ${ }^{(2)}$ (ATC), days

Avging Time, Noncarc ${ }^{(3)}$ (ATN), days

Conversion Factor (CF), $\mathrm{L} / \mathrm{cm}^{3}$

\section{Current Visitor}

$\mathrm{BME}^{(\text {() }}$

5,000

$0.5 \quad$ (4)

6 (4)

5 (4)

70 (4)

25,550

1,825

$1.00 \mathrm{E}-03$

\section{INTAKE FACTOR CALCULATIONS}

Carcinogenic Intake Factor (CIF), L-hr/cm/kg-day = $(\mathrm{SA} \bullet \mathrm{ET} * \mathrm{EF} * \mathrm{ED} * \mathrm{CF}) /(\mathrm{BW} * \mathrm{ATC})$

RME CFF $=4.19 E-05$

Noncarcinogenic Intake Factor (NIF), L-hr/cm/kg-day $(\mathrm{SA} * \mathrm{ET} * \mathrm{EF} \otimes \mathrm{ED} * \mathrm{CF}) /(\mathrm{BW} * \mathrm{ATN})$

RME NIF $=5.87 \mathrm{E}-04$

\section{Notri:}

1. Reanonuble maximum exposure.

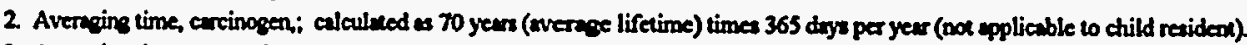

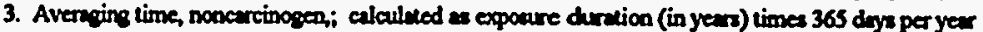

4. See Table D-1. 
Table D.10

Savannah River Site

Burning/Rubble Pits (231-F, 231-1F, 231-2F)

Current Visitor

Dermal Exposure to Surface Water (Wetland)

Carcinogenic and Noncarcinogenic Risk

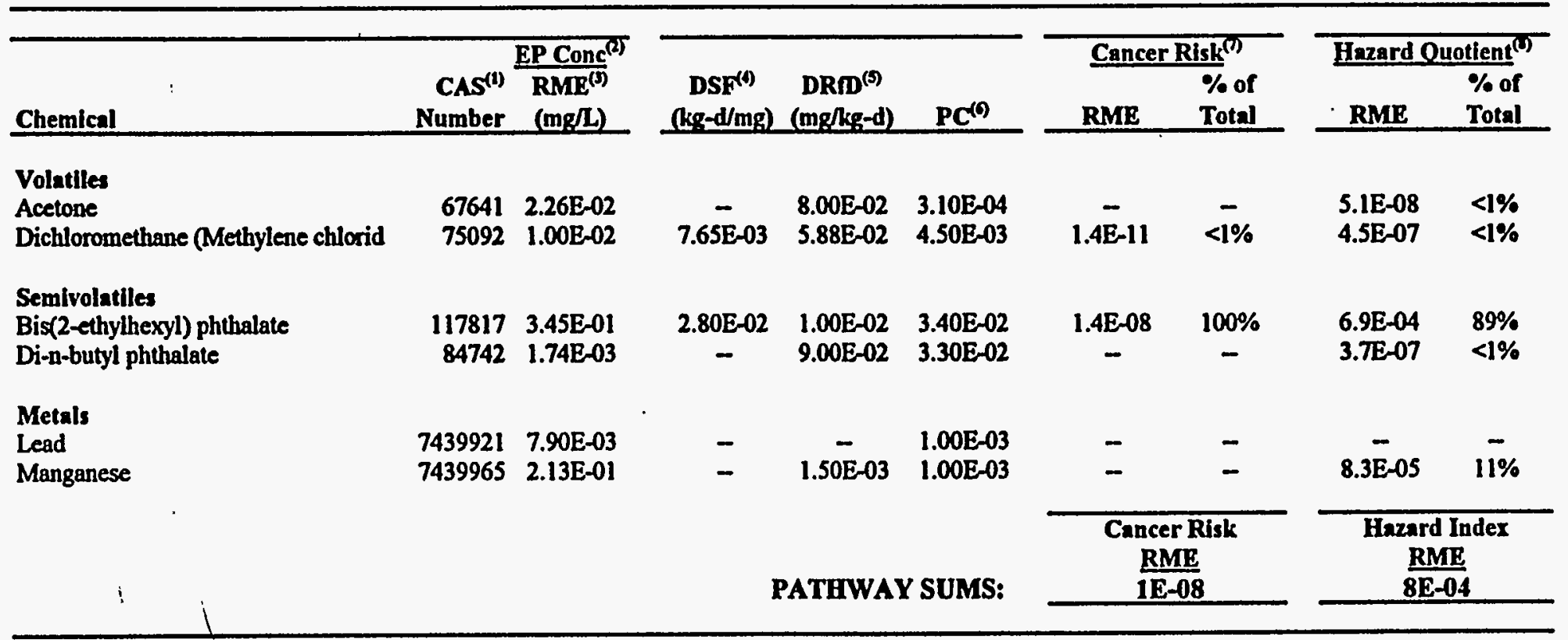

Notes:

1. Chemical Abatract Service.

2. Exposure point concentration.

3. Reasonable maximum expoutre.

4. Slope fector, dermal.

5. Reference dove, dermal.

6. Permeability content.

7. Cancer Riak = (Chemical Concentration, mgl * Carcinogenic Intake Fuctor, Lftr/cm/kg-day * Permeability Conatant, cm/hr * Slope Factor, kg-day/mg), whete RME carcinogenic intake factor 4.19E-05

8. Hazard Quotient = (Chemical Concentration, mg/L * Noncancinogenic Intake Factor, Lhr/cm/kg-day * Permeability Constant, cm/hr) /(Reference Dose, mg/kg-day), where

RME noncarcinogenic intake fo 5.87E-04 
Table D.11

Savannah River Site

Burning/Rubble Pits (231-F, 231-1F, 231-2F)

Current Visitor

Ingestion of Sediment (Wetland)

Carcinogenic and Noncarcinogenic Risk

EXPOSURE ASSUMPTIONS:

\section{Receptor}

Exposure

Intake Rate (IR), mg/day

Fraction Ingested (FI), unitless

Exposure Frequency (EF), days/yr

Exposure Duration (ED), yrs

Body Weight (BW), kg

Avging Time, Carc ${ }^{(2)}$ (ATC), days

Avging Time, Noncarc ${ }^{(3)}$ (ATN), days

Conversion Factor (CF), kg/mg

\section{INTAKE FACTOR CALCULATIONS}

Carcinogenic Intake Factor (CIF), kg/kg-day = $(\mathrm{R} * \mathrm{FI} \bullet \mathrm{EF} * \mathrm{ED} * \mathrm{CF}) /(\mathrm{BW} * \mathrm{ATC})$

RME CIF $=8.39 \mathrm{E}-10$

Noncarcinogenic Intake Factor (NIF), kg/kg-day = $(\mathrm{R} \bullet \mathrm{FI} \bullet \mathrm{EF} * \mathrm{ED} \bullet \mathrm{CF}) /(\mathrm{BW} \bullet \mathrm{ATN})$

RME NIF $=1.17 \mathrm{E}-08$

\section{Noter:}

1. Reasonuble maximum exposure.

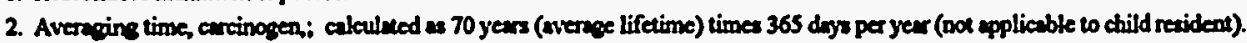

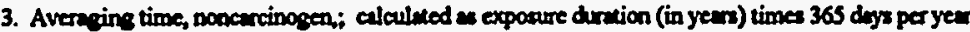

4. See Tuble D-1.

(4)

1 (4)

70 (4)

25,550

1,825

$1.00 \mathrm{E}-06$ 
Table D.11

Savannah River Site

Burning/Rubble Pits (231-F, 231-1F, 231-2F)

Current Visitor

Ingestion of Sediment (Wetland)

Carcinogenic and Noncarcinogenic Risk

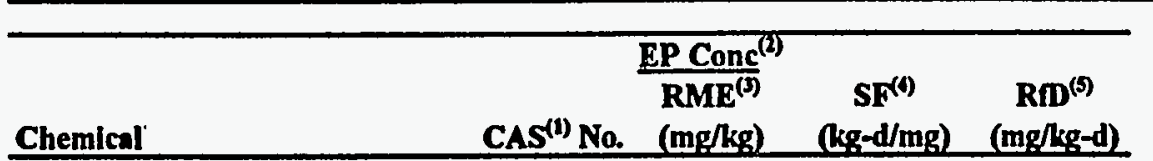

\begin{tabular}{|c|c|}
\hline Cancer Risk & Hazard Ouotient \\
\hline Total & $\begin{array}{l}\text { \% of } \\
\text { Total }\end{array}$ \\
\hline
\end{tabular}

\section{Volatiles}

Acetone

Chloromethene (Methyl ctloride)

Dictloromethene (Methylene chloride)

Toluene

$\begin{array}{rccc}67641 & 6.43 \mathrm{E}-02 & - & 1.00 \mathrm{E}-01 \\ 74873 & 1.30 \mathrm{E}-02 & 1.30 \mathrm{E}-02 & - \\ 75092 & 4.19 \mathrm{E}-02 & 7.50 \mathrm{E}-03 & 6.00 \mathrm{E}-02 \\ 108883 & 1.13 \mathrm{E}-02 & - & 2.00 \mathrm{E}-01\end{array}$.

\section{Semivolattles}

Acenaphthene

Acenuphthylene

Anthracene

Benzo(a) anthracene

Benzo(a)pyrene

Benzo(b) fluoranthene

Benzo(g,hi)perylene

Benzo(k)fluoranthene

Bis(2-ethylhexyl) phthalate

Chryeere

Di-nbutyl phthalate

Dibenz(a,h)anthracene

Dibenzofuran

Fluoranthene

Fluorene

Indeno(1,2,3-e,d)pyrene

Phenamuluence

Pyrene

\section{$833292.18 E-01$}

208968 7.75E-01

$120127 \quad 4.21 E+00$

$56533 \quad 4.31 E+00$

$50328 \quad 2.99 \mathrm{E}+00$

$205992 \quad 4.34 E+00$

$191242 \quad 1.74 \mathrm{E}+00$

$2070893.99 E+00$

$117817 \quad 1.68 \mathrm{E}+00$

$218019 \quad 5.33 \mathrm{E}+00$

$84742 \quad 5.96 \mathrm{E}-02$

$53703 \quad 5.62 \mathrm{E}-01$

$132649 \quad 1.33 \mathrm{E}_{-01}$

$206440 \quad 1.21 \mathrm{E}+01$

86737 S.22E-01

$193395 \quad 1.78 \mathrm{E}+00$

$850185.36 \mathrm{E}+00$

$129000 \quad 7.83 \mathrm{E}+00$

$\begin{array}{cc}- & 6.00 \mathrm{E}-02 \\ - & - \\ 7.30 \mathrm{E}-01 & 3.00 \mathrm{E}-01 \\ 7.30 \mathrm{E}+00 & - \\ 7.30 \mathrm{E}-01 & - \\ - & - \\ 7.30 \mathrm{E}-02 & - \\ 1.40 \mathrm{E}-02 & 2.00 \mathrm{E}-02 \\ 7.30 \mathrm{E}-03 & - \\ - & 1.00 \mathrm{E}-01 \\ 7.30 \mathrm{E}+00 & - \\ - & - \\ - & 4.00 \mathrm{E}-02 \\ - & 4.00 \mathrm{E}-02 \\ 7.30 \mathrm{E}-01 & - \\ - & - \\ - & 3.00 \mathrm{E}-02\end{array}$

Dloxins/furans

Oetactlorodibenzo-p-dioxin isomers

$3268879 \quad 8.00 \mathrm{E}-01$

$1.50 \mathrm{E}+02$

$\begin{array}{cc}1.4 \mathrm{E}-13 & <- \\ 2.6 \mathrm{E}-13 & <1 \% \\ - & -\end{array}$

$7.5 E-09<1 \%$

$8 . \overline{\mathrm{E}}-09<\overline{1 \%}$

$6.6 \mathrm{E}-10<1 \%$

$-\quad-\quad 4.3 \mathrm{E}-08<1 \%$

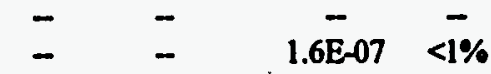

$2.6 \mathrm{E}-09 \quad 02 \%$

$1.8 \mathrm{E}-08 \quad 14 \%$

$2.7 \mathrm{E}-09 \quad 02 \% \quad-$

-

$2.0 \mathrm{E}-11<1 \% \quad 9.9 \mathrm{E}-07<\overline{1 \%}$

3.3E-11 $<1 \%<-$

$-\bar{E}-09 \quad$ 7.0E-09 $<1 \%$

- - $\quad$ - $\quad-$

- $\quad-\quad 3.6 \mathrm{E}-0602 \%$

$\overrightarrow{1 E}-09_{109}^{-} \quad 1.5 \mathrm{E}-07 \quad<1 \%$

- - - - -

- $\quad$ - 3.1E-06 $01 \%$

$1.0 \mathrm{E}-07 \quad 76 \% \quad-$ 
Table D.11

Savannah River Site

Burning/Rubble Pits (231-F, 231-1F, 231-2F)

Current Visitor

Ingestion of Sediment (Wetland)

Carcinogenic and Noncarcinogenic Risk

\begin{tabular}{|c|c|c|c|c|c|c|c|c|}
\hline & & $\frac{\mathrm{EP} \operatorname{Conc}^{(2)}}{\mathrm{RME}^{(3)}}$ & $\mathbf{S F}^{(0)}$ & $\mathbf{R} \mathbf{D}^{(\mathbf{f})}$ & Cancer $\mathbf{R}$ & $\frac{s_{k}^{(0)}}{\% \text { of }}$ & Hazard Que & $\frac{\operatorname{tient}^{\prime \prime}}{\% \text { of }}$ \\
\hline \multicolumn{9}{|l|}{ Metals } \\
\hline Arzenic & 7440382 & $2.90 \mathrm{E}+00$ & $1.50 E+00$ & $3.00 \mathrm{E}-04$ & $3.6 \mathrm{E}-09$ & $03 \%$ & $1.1 E-04$ & $48 \%$ \\
\hline Chromium & 7440473 & $2.80 \mathrm{E}+01$ & - & $5.00 \mathrm{E}-03$ & - & - & $6.6 \mathrm{E}-05$ & $28 \%$ \\
\hline Copper & 7440508 & $2.56 \mathrm{E}+01$ & - & $4.00 \mathrm{E}-02$ & - & - & $7.5 E-06$ & $03 \%$ \\
\hline Lead & 7439921 & $1.86 \mathrm{E}+01$ & - & - & - & - & - & - \\
\hline Manganese & 7439965 & 5.75E+01 & - & $5.00 \mathrm{E}-02$ & - & - & $1.4 E-05$ & $06 \%$ \\
\hline \multirow[t]{2}{*}{ Mercury } & 7439976 & $7.10 \mathrm{E}-01$ & - & $3.00 \mathrm{E}-04$ & - & - & $2.8 \mathrm{E}-05$ & $12 \%$ \\
\hline & & & & ATHWAY SUMS: & \multicolumn{2}{|c|}{$\begin{array}{c}\text { Cancer Risk } \\
\text { RMA } \\
1 \mathrm{E}-07\end{array}$} & \multicolumn{2}{|c|}{$\begin{array}{c}\text { Hazard Inder } \\
\mathbf{R M E} \\
2 \mathrm{E}-04\end{array}$} \\
\hline \multicolumn{9}{|c|}{ 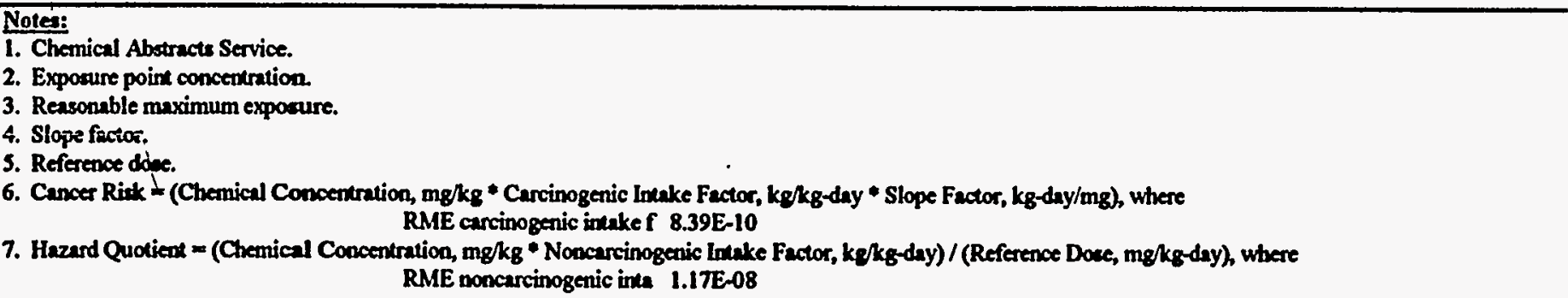 } \\
\hline
\end{tabular}


Table D.12

Savannah River

Burning/Rubble Pits (231-F, 231-1F, 231-21)

Current Visitor

Dermal Exposure to Sediment (Wetland)

Carcinogenic and Noncarcinogenic Risk

EXPOSURE ASSUMPTIONS:

\section{Receptor}

Exposure

Skin Surface Area (SA), $\mathrm{cm}^{2} /$ event

Soil-to-Skin Adherance (SK), $\mathrm{mg} / \mathrm{cm}^{2}$

Exposure Frequency (EF), events/yr

Exposure Duration (ED), yrs

Body Weight (BW), kg

Avging Time, $\operatorname{Carc}^{(2)}$ (ATC), days

Avging Time, Noncarc ${ }^{(3)}$ (ATN), days

Conversion Factor (CF), kg/mg

\section{INTAKE FACTOR CALCULATIONS}

Carcinogenic Intake Factor (CIF), kg/kg-day = $(\mathrm{SA} \bullet \mathrm{SK} \bullet \mathrm{EF} \bullet \mathrm{ED} \bullet \mathrm{CF}) /(\mathrm{BW} \bullet \mathrm{ATC})$ RME CIF $=5.03 E-08$

Noncarcinogenic Intake Factor (NIF), kg/kg-day = $(\mathrm{SA} \bullet \mathrm{SK} \bullet \mathrm{EF} \bullet \mathrm{ED} \bullet \mathrm{CF}) /(\mathrm{BW} * \mathrm{ATN})$

RME NIF $=7.05 E-07$

Netst:

1. Resconsble maximum exposure.

3. Averning time, carcinogen; calculued as 70 years (everaece lifetime) times 365 deys per year (nol upplieable to child residens).

3. Averaine time, noncercinogen; calculated ex exposure duration (in yema) times 365 drya per year

4. Sec Tuble D-1. 
Table D.12

Savannah River

Burning/Rubble Pits (231-F, 231-1F, 231-2F)

Current Visitor

Dermal Exposure to Sediment (Wetland)

Carcinogenic and Noncarcinogenic Risk

\begin{tabular}{|c|c|c|c|c|c|c|c|c|c|}
\hline \multirow[b]{2}{*}{ Chemical } & \multicolumn{3}{|c|}{ EPConc $^{(2)}$} & \multirow[b]{2}{*}{$\begin{array}{c}\text { DRm }^{(5)} \\
\left(\mathrm{m} / \mathrm{kg}^{-\mathrm{d}}\right)\end{array}$} & \multirow[b]{2}{*}{ DABS $^{(0)}$} & \multicolumn{2}{|c|}{ Cancer Risk (") } & \multicolumn{2}{|c|}{ Hazard Quatient $(")$} \\
\hline & $\begin{array}{r}\text { CAS }^{(1)} \\
\text { Number }\end{array}$ & $\begin{array}{l}\mathrm{RME}^{(3)} \\
(\mathrm{m} / / \mathrm{kg})\end{array}$ & $\begin{array}{c}\operatorname{DSF}^{(4)} \\
(\mathrm{kg}-\mathrm{d} / \mathrm{mg})\end{array}$ & & & RME & $\begin{array}{l}\% \text { of } \\
\text { Total } \\
\end{array}$ & $\mathbf{R M E}$ & $\begin{array}{l}\% \text { of } \\
\text { Total }\end{array}$ \\
\hline \multicolumn{10}{|l|}{ Voiatiles } \\
\hline Acetone & 67641 & $6.43 \mathrm{E}-02$ & - & 8.00E-02 & $1.00 \mathrm{E}-02$ & - & - & $5.7 \mathrm{E}-09$ & $<1 \%$ \\
\hline Chloromethane (Methyl chloride) & 74873 & $1.30 \mathrm{E}-02$ & $1.63 \mathrm{E}-02$ & - & $1.00 \mathrm{E}-02$ & $1.1 \mathrm{E}-13$ & $<1 \%$ & - & - \\
\hline Dichloromethane (Methylene chlorid & 75092 & 4.19E-02 & $7.65 \mathrm{E}-03$ & $5.88 \mathrm{E}-02$ & $1.00 \mathrm{E}-02$ & $1.6 \mathrm{E}-13$ & $<1 \%$ & $5.0 \mathrm{E}-09$ & $<1 \%$ \\
\hline Toluene & 108883 & $1.13 E-02$ & - & $1.60 \mathrm{E}-01$ & $1.00 \mathrm{E}-02$ & - & - & $5.0 \mathrm{E}-10$ & $<1 \%$ \\
\hline \multicolumn{10}{|l|}{ Semivolatiles } \\
\hline Acenaphthene & 83329 & $2.18 \mathrm{E}-01$ & - & $3.00 \mathrm{E}-02$ & $1.00 \mathrm{E}-02$ & - & - & 5.1E-08 & $<1 \%$ \\
\hline Acenaphthylene & 208968 & 7.75E-01 & - & - & $1.00 \mathrm{E}-02$ & - & - & - & - \\
\hline Anthracene & 120127 & $4.21 E+00$ & - & $1.50 \mathrm{E}-01$ & $1.00 \mathrm{E}-02$ & - & - & $2.0 \mathrm{E}-07$ & $<1 \%$ \\
\hline Benzo(a) anthracene & 56553 & $4.31 E+\infty 0$ & $1.46 \mathrm{E}+00$ & - & $1.00 \mathrm{E}-02$ & $3.2 \mathrm{E}-09$ & $02 \%$ & - & - \\
\hline Benzo(a)pyrene & 50328 & $2.99 E+\infty 0$ & $3.65 \mathrm{E}+01$ & - & $1.00 \mathrm{E}-02$ & $5.5 \mathrm{E}-08$ & $40 \%$ & - & - \\
\hline Benzo(b)fluoranthene & 205992 & $4.34 E+00$ & $1.46 \mathrm{E}+00$ & - & $1.00 \mathrm{E}-02$ & $3.2 \mathrm{E}-09$ & $02 \%$ & - & - \\
\hline Benzo $(g, h, i)$ perylene & 191242 & $1.74 E+00$ & - & - & $1.00 \mathrm{E}-02$ & - & - & - & - \\
\hline Benzo(k)fluoranthene & 207089 & $3.99 \mathrm{E}+00$ & $1.46 \mathrm{E}-01$ & - & $1.00 \mathrm{E}-02$ & $2.9 \mathrm{E}-10$ & $<1 \%$ & - & - \\
\hline Bis(2-ethylhexyl) phthalate & 117817 & $1.68 E+00$ & $2.80 \mathrm{E}-02$ & $1.00 \mathrm{E}-02$ & $1.00 \mathrm{E}-02$ & $2.4 \mathrm{E}-11$ & $<1 \%$ & $1.2 \mathrm{E}-06$ & $<1 \%$ \\
\hline Chrysene & 218019 & $5.33 E+00$ & $1.22 \mathrm{E}-02$ & - & $1.00 \mathrm{E}-02$ & $3.3 \mathrm{E}-11$ & $<1 \%$ & - & - \\
\hline Di-n-butyl phthalate & 84742 & $5.96 \mathrm{E}-02$ & - & $9.00 \mathrm{E}-02$ & $1.00 \mathrm{E}-02$ & - & - & 4.7E-09 & $<1 \%$ \\
\hline Dibenz( $\mathbf{n}, \mathrm{h})$ anthracene & 53703 & $5.62 \mathrm{E}-01$ & $1.46 \mathrm{E}+01$ & - & $1.00 \mathrm{E}-02$ & 4.1E-09 & $03 \%$ & - & - \\
\hline Dibenzofuran & 132649 & $1.33 E-01$ & - & - & $1.00 \mathrm{E}-02$ & - & - & - & - \\
\hline Fluoranthene & 206440 & $1.21 E+01$ & - & $2.00 \mathrm{E}-02$ & $1.00 \mathrm{E}-02$ & - & - & 4.3E-06 & $01 \%$ \\
\hline Fluorene & 86737 & $5.22 \mathrm{E}-01$ & $\cdot$ & $2.00 \mathrm{E}-02$ & $1.00 \mathrm{E}-02$ & - & - & $1.8 \mathrm{E}-07$ & $<1 \%$ \\
\hline Indeno $\{1,2,3-c, d\} p y r e n e$ & 193395 & $1.78 E+00$ & $1.46 \mathrm{E}+00$ & - & $1.00 \mathrm{E}-02$ & 1.3E-09 & $<1 \%$ & - & - \\
\hline Phenanthrene & 85018 & $5.36 \mathrm{E}+00$ & - & - & $1.00 \mathrm{E}-02$ & - & - & - & - \\
\hline Pyrene & 129000 & $7.83 E+00$ & - & $1.50 \mathrm{E}-02$ & $1.00 \mathrm{E}-02$ & - & - & 3.7E-06 & $<1 \%$ \\
\hline Dioxins/furans & & & & & & & & & \\
\hline Octachlorodibenzo-p-dioxin isomers & 3268879 & $8.00 \mathrm{E}-01$ & $1.72 E+02$ & - & $1.00 \mathrm{E}-02$ & $6.9 \mathrm{E}-08$ & $51 \%$ & - & - \\
\hline
\end{tabular}


Table D.12

Savannah River

Burning/Rubble Pits (231-F, 231-1F, 231-2F)

Current Visitor

Dermal Exposure to Sediment (Wetland)

Carcinogenic and Noncarcinogenic Risk

\begin{tabular}{|c|c|c|c|c|c|c|c|c|c|}
\hline \multirow[b]{2}{*}{ Chemical } & \multicolumn{3}{|c|}{ EP Conc ${ }^{(2)}$} & \multirow[b]{2}{*}{$\begin{array}{c}\operatorname{DRm}^{(0)} \\
(\mathrm{mg} / \mathrm{kg}-\mathrm{d})\end{array}$} & \multirow[b]{2}{*}{$\mathbf{D A B S}^{(0)}$} & \multicolumn{2}{|c|}{ Cancer Risk ${ }^{(\prime)}$} & \multicolumn{2}{|c|}{ Hazard Quotient ${ }^{(\prime)}$} \\
\hline & $\begin{array}{c}\text { CAS }^{(1)} \\
\text { Number } \\
\end{array}$ & $\begin{array}{l}\mathrm{RME}^{(3)} \\
(\mathrm{mg} / \mathrm{kg})\end{array}$ & $\begin{array}{c}\operatorname{DSF}^{(0)} \\
(\mathrm{lg}-\mathrm{d} / \mathrm{mg})\end{array}$ & & & RME & $\begin{array}{l}\% \text { of } \\
\text { Total } \\
\end{array}$ & $\mathbf{R M R}$ & $\begin{array}{l}\% \text { of } \\
\text { Total } \\
\end{array}$ \\
\hline \multicolumn{10}{|l|}{ Metals } \\
\hline Arsenic & 7440382 & $2.90 \mathrm{E}+00$ & $1.88 E+00$ & $2.40 \mathrm{E}-04$ & $1.00 \mathrm{E}-03$ & 2.7E-10 & $<1 \%$ & 8.5E-06 & $02 \%$ \\
\hline Chromium & 7440473 & $2.80 \mathrm{E}+01$ & - & $1.00 \mathrm{E}-0.4$ & $1.00 \mathrm{E}-03$ & - & - & $2.0 \mathrm{E}-04$ & $48 \%$ \\
\hline Copper & 7440508 & $2.56 \mathrm{E}+01$ & - & $2.40 \mathrm{E}-02$ & $1.00 \mathrm{E}-03$ & - & - & $7.5 \mathrm{E}-07$ & $<1 \%$ \\
\hline Lead & 7439921 & $1.86 \mathrm{E}+01$ & - & - & $1.00 \mathrm{E}-03$ & - & - & - & - \\
\hline Manganese & 7439965 & $5.75 \mathrm{E}+01$ & - & $1.50 \mathrm{E}-03$ & $1.00 \mathrm{E}-03$ & - & - & 2.7E-05 & $07 \%$ \\
\hline \multirow[t]{2}{*}{ Mercury } & 7439976 & $7.10 \mathrm{E}-01$ & - & $3.00 \mathrm{E}-06$ & $1.00 \mathrm{E}-03$ & - & - & $1.7 \mathrm{E}-04$ & $41 \%$ \\
\hline & & & & ATHWA & SUMS: & \multicolumn{2}{|c|}{$\begin{array}{c}\text { Cancer Risk } \\
\text { RME } \\
\text { 1E-07 }\end{array}$} & \multicolumn{2}{|c|}{$\begin{array}{c}\text { Hazard Index } \\
\text { RME } \\
\text { 4E-04 }\end{array}$} \\
\hline
\end{tabular}

Notes:

1. Chemical Abstruct Service.

2. Exposure point concentration

3. Reasonable maximum exposure

4. Slope factor, dermal.

5. Reference doje, dermal.

6. Absorption fuctor.

7. Canoer Rik = (Chemical Concentration, mg/kg * Carcinogenic Intake Fuctor, kg/kg-day * Abeorption Factor, unitless * Slope Factor, kg-day/mg), where RME carcinogenic intuke $f$ S.03E-08

8. Hazand Quotient = (Chemical Concentration, m\&/kg * Noncarcinogenic lntake Factor, ke/kg-day * Abeorption Factor, unilleas)/(Reference Dose, mg/kg-day), wher RME noncarcinogenic inta 7.05E-07 
Table D.13

Savannah River

Burning/Rubble Pits (231-F, 231-1F)

Future Worker

Ingestion of Soil (0-2'bls)

Carcinogenic and Noncarcinogenic Risk

\section{EXPOSURE ASSUMPTIONS;}

\section{Receptor \\ Exposure}

Intake Rate (IR), mg/day

Fraction Ingested (FI), unitless

Exposure Frequency (EF), days/yr

Exposure Duration (ED), yrs

Body Weight (BW), kg

Avging Time, Carc (2) (ATC), days

Avging Time, Noncarc ${ }^{(3)}$ (ATN), days

Conversion Factor (CF), kg/mg

\begin{tabular}{cc}
\multicolumn{2}{c}{ Euture Worker } \\
\hline RME $^{(1)}$ & \\
50 & $(4)$ \\
1 & $(4)$ \\
250 & $(4)$ \\
25 & $(4)$ \\
70 & $(4)$ \\
25,550 & \\
9,125 & \\
$1.00 \mathrm{E}-06$ &
\end{tabular}

INTAKE FACTOR CALCULATIONS

Carcinogenic Intake Factor (CIF), kg/kg-day = $(\mathrm{IR} \bullet \mathrm{FI} * \mathrm{EF} \bullet \mathrm{ED} \bullet \mathrm{CF}) /(\mathrm{BW} * \mathrm{ATC})$ RME CIF $=1.75 E-07$

Noncancinogenic Intake Factor (NIF), kg/kg-day = $(\mathrm{IR} * \mathrm{FT} * \mathrm{EF} * \mathrm{ED} * \mathrm{CF}) /(\mathrm{BW} * \mathrm{ATN})$

$\mathrm{RMENIF}=4.89 \mathrm{E}-07$

\section{Notsi:}

1. Resoneble maximum exposure.

2. Averaing time, carcinogen,; calculeted as 70 years (wernoce lifetime) times 365 deyn per year (not upplicuble to child resident).

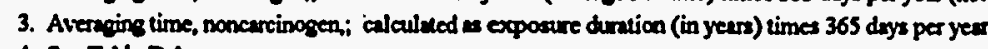

4. See Table D-1. 
Table D.13

Savannah River

Burning/Rubble Pits (231-F, 231-1F)

Future Worker

Ingestion of Soil (0-2'bls)

Carcinogenic and Noncarcinogenic Risk

\begin{tabular}{|c|c|c|c|c|c|c|c|c|c|}
\hline Chemical & $\mathrm{CAS}^{(1)} \mathrm{Na}$ & $\begin{array}{l}\mathbf{E P C o n c} c^{(2)} \\
\operatorname{RME}^{(3)} \\
(m g / k g)\end{array}$ & $\begin{array}{c}\text { OSF(t) } \\
(\mathrm{kg}-\mathrm{d} / \mathrm{mg})\end{array}$ & $\begin{array}{c}\mathrm{ORID}^{(-)} \\
(\mathrm{mg} / \mathrm{kg}-\mathrm{d})\end{array}$ & & \multicolumn{2}{|c|}{ Cancer Risk ${ }^{\left({ }^{()}\right.}$} & $\begin{array}{l}\text { Hazard } 0 \\
\text { RME }\end{array}$ & $\begin{array}{l}\text { tient }^{(1)} \\
\% \text { of } \\
\text { Total } \\
\end{array}$ \\
\hline \multicolumn{10}{|l|}{ Semivolatiles } \\
\hline 2-Methylnaphthalene & $91-57-6$ & $7.69 \mathrm{E}-02$ & - & - & & - & - & - & - \\
\hline Acenaphthylene & $208-96-8$ & 8.61E-02 & - & - & & - & - & - & - \\
\hline Benzo(a)anthracene & $56-55-3$ & $9.26 \mathrm{E}-01$ & $7.30 \mathrm{E}-01$ & - & & $1.2 \mathrm{E}-07$ & $03 \%$ & - & - \\
\hline Benzo(a)pyrene & $50-32-8$ & $6.49 \mathrm{E}-01$ & $7.30 \mathrm{E}+00$ & - & & 8.3E-07 & $18 \%$ & - & - \\
\hline Benzo(b)fluoranthene & $205-99-2$ & $1.01 E+00$ & $7.30 \mathrm{E}-01$ & - & & $1.3 \mathrm{E}-07$ & $03 \%$ & - & - \\
\hline Benzo( $(8, h, i)$ perylene & $191-24-2$ & 6.15E-01 & - & - & & - & - & - & - \\
\hline Dibenz $(a, h)$ anthracene & $53-70-3$ & 2.17E-01 & $7.30 \mathrm{E}+\infty 0$ & - & & $2.8 \mathrm{E}-07$ & $06 \%$ & - & - \\
\hline Phenanthrene & $85-01-8$ & $6.87 \mathrm{E}-01$ & - & - & & - & - & - & - \\
\hline \multicolumn{10}{|l|}{ Dioxins/furans } \\
\hline Heptachlorodibenzo-p-dioxin isomer & $37871-00-4$ & $4.11 \mathrm{E}-03$ & $1.50 E+03$ & - & & $1.1 \mathrm{E}-06$ & $24 \%$ & - & - \\
\hline Heptachlorodibenzo-p-furan isomer & $38998-75-3$ & 2.58E-04 & $1.50 E+03$ & - & & $6.8 E-08$ & $01 \%$ & - & - \\
\hline Hexachlorodibenzo-p-furan isomer & $55684-94-1$ & $1.00 \mathrm{E}-04$ & $1.50 \mathrm{E}+04$ & - & & $2.6 \mathrm{E}-07$ & $06 \%$ & - & - \\
\hline Octachlorodibenzo-p-dioxin isomer & $3268-87-9$ & $9.26 \mathrm{E}-03$ & $1.50 E+02$ & - & & $2.4 E-07$ & $05 \%$ & - & - \\
\hline Octachlorodibenzo-p-furan isomer & $39001-02-0$ & $3.48 \mathrm{E}-04$ & $1.50 \mathrm{E}+02$ & - & & 9.1E-09 & $<1 \%$ & - & - \\
\hline \multicolumn{10}{|l|}{ PCBs } \\
\hline PCB 1254 & $11097-69-1$ & $1.78 \mathrm{E}-01$ & $7.70 \mathrm{E}+00$ & $2.00 \mathrm{E}-05$ & & 2.4E-07 & $05 \%$ & $4.4 \mathrm{E}-03$ & $20 \%$ \\
\hline \multicolumn{10}{|l|}{ Pesticides } \\
\hline delta-Benzene hexachloride & $319-86-8$ & $1.84 \mathrm{E}-03$ & - & - & . & - & - & - & - \\
\hline Endrin ketone & $53494-70-5$ & $3.20 \mathrm{E}-03$ & - & - & & - & - & - & - \\
\hline \multicolumn{10}{|l|}{ Metals } \\
\hline Arsenic & $7440-38-2$ & $4.82 \mathrm{E}+00$ & $1.50 \mathrm{E}+00$ & $3.00 \mathrm{E}-04$ & & $1.3 \mathrm{E}-06$ & $28 \%$ & $7.9 \mathrm{E}-03$ & $36 \%$ \\
\hline Chromium & $7440-47-3$ & $4.42 \mathrm{E}+01$ & - & $5.00 \mathrm{E}-03$ & & - & - & 4.3E-03 & $20 \%$ \\
\hline Lead & $7439-92-1$ & $2.48 \mathrm{E}+01$ & - & - & & - & - & - & - \\
\hline Manganese & $7439-96-5$ & $4.78 \mathrm{E}+02$ & - & $5.00 \mathrm{E}-02$ & & - & - & 4.7E-03 & $21 \%$ \\
\hline \multirow[t]{2}{*}{ Mercury } & $7439-97-6$ & $5.48 \mathrm{E}-01:$ & - & $3.00 \mathrm{E}-04$ & & - & - & $8.9 E-04$ & $04 \%$ \\
\hline & & & • & ATHWAY & & \multicolumn{2}{|c|}{$\begin{array}{c}\text { Cancer Risk } \\
\text { RME } \\
\text { SE-06 }\end{array}$} & \multicolumn{2}{|c|}{$\begin{array}{c}\text { Hazard Index } \\
\text { RME } \\
2 \mathrm{E}-02 \\
\end{array}$} \\
\hline
\end{tabular}


Table D.13

Savannah River

Burning/Rubble Pits (231-F, 231-1F)

Future Worker

Ingestion of Soil (0-2'bls)

Carcinogenic and Noncarcinogenic Risk

\section{Notes:}

1. Chemical Abstracts Service.

2. Expocure point concentration.

3. Reasonable maximum expoure.

4. Slope factor.

5. Reference dose.

6. Cancer Risk = (Chemical Concentration, me/kg ${ }^{\bullet}$ Cercinogenic Imake Factor, kg/kg-day ${ }^{\circ}$ Slope Factor, kg-day/mg), where

7. RME cancinogenic intuke factor 1.75E-07

7. Hazard Quotient = (Chemical Concentration, mg/kg ' Noncarcinogenic Intake Factor, kg/kg-day) / (Reference Dose, mg/kg-day), where

RME noncancinogenic intuke fa $4.89 \mathrm{E}-07$ 
Table D.14

Savannah River

Burning/Rubble Pits (231-F, 231-1F)

Future Worker

Dermal Exposure to Soil (0-2'bls)

Carcinogenic and Noncarcinogenic Risk

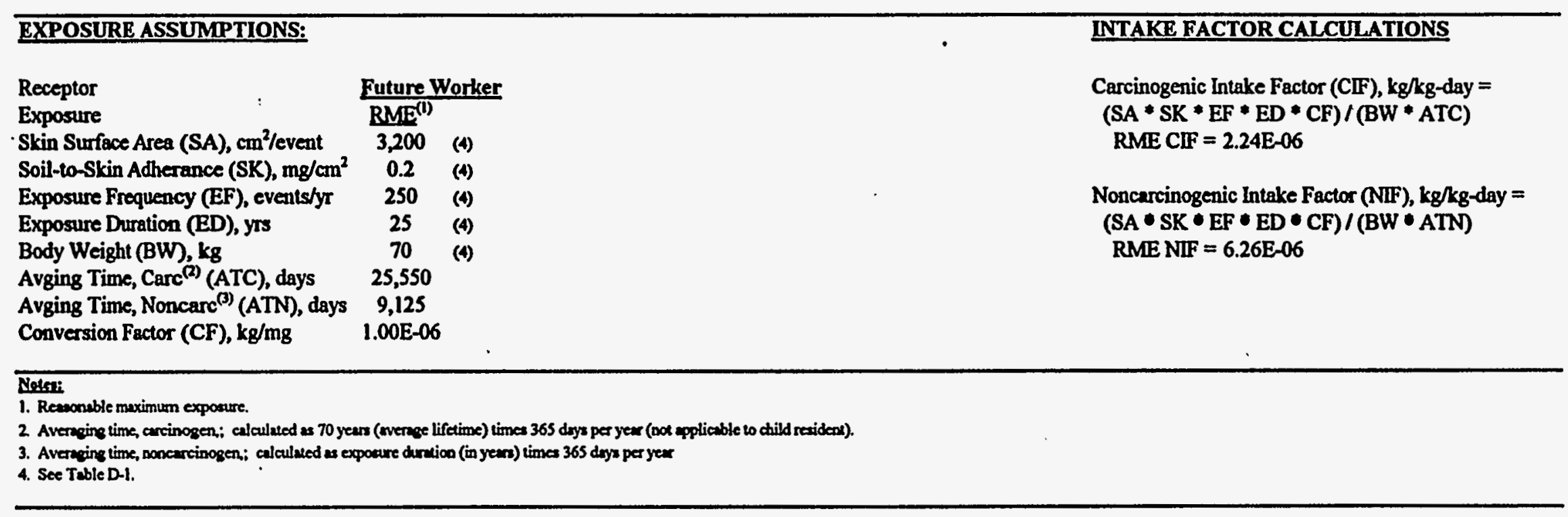


Table D.14

Savannah River

Burning/Rubble Pits (231-F, 231-1F)

Future Worker

Dermal Exposare to Soil (0-2'bls)

Carcinogenic and Noncarcinogenic Risk

\begin{tabular}{|c|c|c|c|c|c|c|c|c|c|}
\hline Chemical & \multicolumn{2}{|c|}{ EP Conc ${ }^{(2)}$} & $\begin{array}{c}\operatorname{DSF}^{(0)} \\
(\mathrm{kg}-\mathrm{d} / \mathrm{mg})\end{array}$ & $\begin{array}{c}\text { DRID }^{(s)} \\
(\mathrm{mq} / \mathrm{kg}-\mathrm{d})\end{array}$ & $\mathbf{D A B S}^{(0)}$ & \multicolumn{2}{|c|}{ Cancer Risk ${ }^{(7)}$} & \multicolumn{2}{|c|}{ Hazard Quotient } \\
\hline \multicolumn{10}{|l|}{ Semivolatiles } \\
\hline 2-Methylnaphthalene & $91-57-6$ & 7.69E-02 & - & - & $1.00 \mathrm{E}-02$ & - & - & - & - \\
\hline Acenaphthylene & $208-96-8$ & $8.61 \mathrm{E}-02$ & - & - & $1.00 \mathrm{E}-02$ & - & - & - & - \\
\hline Benzo(b)fluoranthene & $205-99-2$ & $1.01 \mathrm{E}+00$ & $1.46 E+00$ & - & $1.00 \mathrm{E}-02$ & 3.3E-08 & $03 \%$ & - & - \\
\hline Benzo $(B, h, i)$ perylene & $191-24-2$ & $6.15 E-01$ & - & - & $1.00 \mathrm{E}-02$ & - & - & - & - \\
\hline Dibenz(a,h)anthracene & $53-70-3$ & $2.17 \mathrm{E}-01$ & $1.46 \mathrm{E}+01$ & - & $1.00 \mathrm{E}-02$ & $7.1 \mathrm{E}-08$ & $07 \%$ & - & - \\
\hline Phenanthrene & $85-01-8$ & $6.87 \mathrm{E}-01$ & - & - & $1.00 \mathrm{E}-02$ & - & - & - & - \\
\hline \multicolumn{10}{|l|}{ Dioxinv/furans } \\
\hline Octachlorodibenzo-p-furan isomer & $39001-02-0$ & $3.48 \mathrm{E}-04$ & $1.72 \mathrm{E}+02$ & - & $1.00 \mathrm{E}-02$ & $1.3 \mathrm{E}-09$ & $<1 \%$ & - & - \\
\hline \multicolumn{10}{|l|}{ PCB: } \\
\hline PCB 1254 & $11097-69-1$ & $1.78 \mathrm{E}-01$ & $8.56 \mathrm{E}+00$ & $1.80 \mathrm{E}-05$ & $1.00 \mathrm{E}-02$ & $3.4 \mathrm{E}-08$ & $04 \%$ & $6.2 \mathrm{E}-04$ & $09 \%$ \\
\hline \multicolumn{10}{|l|}{ Pesticides } \\
\hline delta-Benzene hexachloride & $319-86-8$ & $1.84 E-03$ & - & - & $1.00 \mathrm{E}-02$ & - & - & - & - \\
\hline Endrin ketone & $53494-70-5$ & $3.20 \mathrm{E}-03$ & - & - & $1.00 \mathrm{E}-02$ & - & - & - & - \\
\hline
\end{tabular}


Table D.14

Savannah River

Burning/Rubble Pits (231-F, 231-1F)

Future Worker

Dermal Exposure to Soil (0-2'bls)

Carcinogenic and Noncarcinogenic Risk

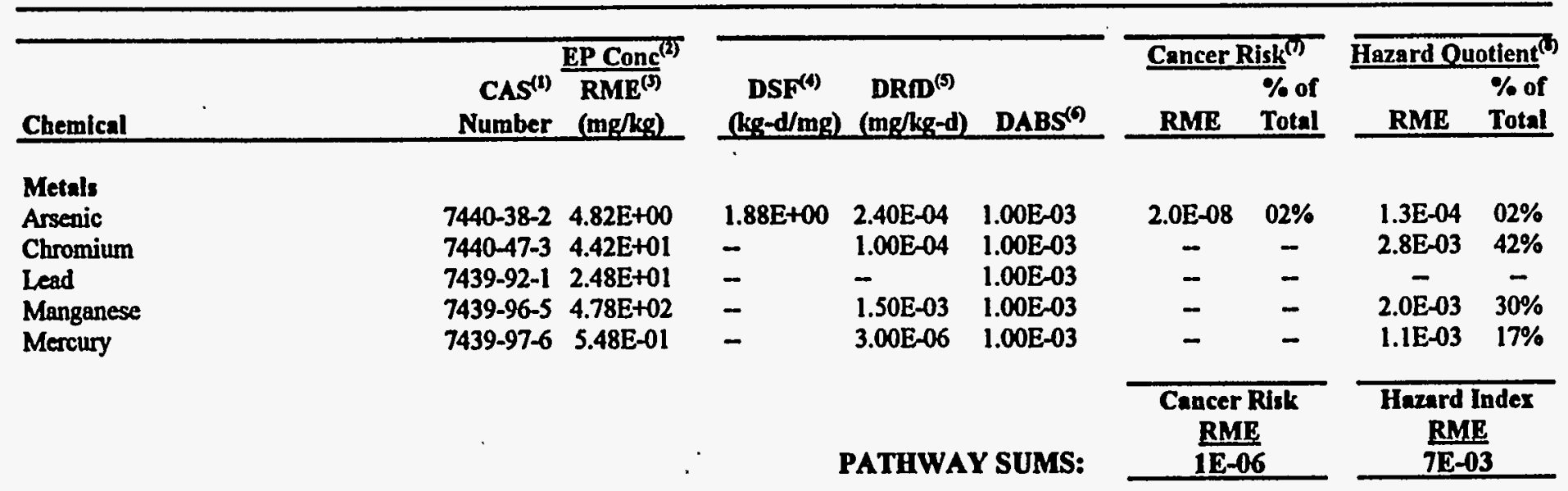

\section{Noten:}

1. Chemical Abstract Service.

2. Exposure point concentration.

3. Reasomable maximum exposure.

4. Slope fictor, dermal.

5. Reference dose, dermil.

6. Abrorption fuctor.

7. Cancer Risk = (Chemical Concentration, mg/kg * Carcinogenic Intake Factor, kg/kg-day * Abeorption Factor, unitleas * Slope Factor, kg-day/mg), where RME carcinogenic intake factor 2.24E-06

8. Hazard Quotient = (Chemical Concentration, mg/kg * Noncarcinogenic Intake Factor, kg/kg-day * Abrorption Factor, unitless)/(Reference Dose, mg/kg-day), where

RME noncarcinogenic intake fa 6.26E-06 
Table D.15

Savannah River

Burning/Rubble Pits (231-F, 231-1F)

Future Worker

Inhalation of Volatile Soil Contaminants (0-2'bls)

\section{EXPOSURE ASSUMPTIONS:}

Receptor

Exposure

Inhalation Rate (IR), $\mathrm{m}^{3} / \mathrm{hr}$

Exposure Time (ET), hrs/day

Exposure Frequency (EF), days/yr

Exposure Duration (ED), yrs

Body Weight (BW), kg

Avging Time, $\mathrm{Carc}^{(2)}$ (ATC), days

Avging Time, Noncarc ${ }^{(3)}$ (ATN), days

\begin{tabular}{|c|c|}
\hline \multirow{2}{*}{\multicolumn{2}{|c|}{ Future Worke }} \\
\hline $\mathrm{BME}^{(1)}$ & \\
\hline 2.5 & $(4)$ \\
\hline 1 & (4 \\
\hline 250 & (4) \\
\hline 25 & ( \\
\hline 70 & (4) \\
\hline 25,550 & $(4$ \\
\hline
\end{tabular}

\section{Nesi:}

1. Remonsble maximum oxposure.

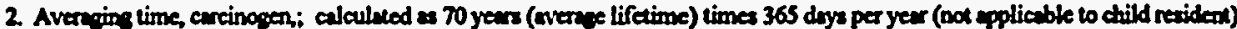

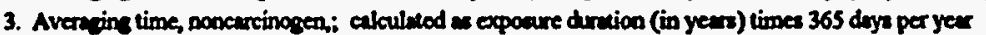

4. See Twble D-1.

INTAKR FACTOR CALCULATIONS

Carcinogenic Intake Factor (CIF), kg/kg-day =

$(\mathrm{IR} * \mathrm{ET} \bullet \mathrm{EF} \bullet \mathrm{ED} \bullet \mathrm{CF}) /(\mathrm{BW}$ * ATC)

RME CIF $=8.74 \mathrm{E}-03$

Noncarcinogenic Intake Factor (NIF), kg/kg-day = $(\mathbb{R} \bullet \mathrm{ET} \bullet \mathrm{EF} \bullet \mathrm{ED} \bullet \mathrm{CF}) /(\mathrm{BW} \bullet \mathrm{ATN})$

RME NIF $=2.45 \mathrm{E}-02$ 
Table D.15

Savannah River

Burning/Rubble Pits (231-F, 231-1F)

Future Worker

Inhalation of Volatile Soil Contaminants (0-2'bls)

Calculation of Volatilization Factor (VF) ${ }^{(1)}$

\begin{tabular}{|c|c|c|c|c|c|c|c|c|c|c|c|}
\hline Chemical & $\mathrm{CAS}^{(2)} \mathrm{Na}$ & Class $^{(3)}$ & MolWt & $\mathrm{Koc}^{(1)}$ & $\mathbf{K} \mathbf{d}^{(\mathbf{( )}}$ & $\mathbf{H}^{(0)}$ & $\mathrm{Kag}_{\mathbf{8}}^{(1)}$ & $\mathbf{D i}^{(4)}$ & $\operatorname{Del}^{(0)}$ & alpha(t) & $\begin{array}{l}\text { Volat. } \\
\text { Factor }{ }^{(4)} \\
\left(\mathrm{m}^{3} / \mathrm{kg}\right)\end{array}$ \\
\hline \multicolumn{12}{|l|}{ Semivolatiles } \\
\hline 2-Methylnaphthalene & $91-57-6$ & $\mathbf{S}$ & $1.42 \mathrm{E}+02$ & $0511.4^{\circ}$ & $1.70 \mathrm{E}+02$ & $2.90 \mathrm{E}-04$ & $6.98 \mathrm{E}-05$ & $6.43 \mathrm{E}-02$ & $4.53 \mathrm{E}-02$ & $6.43 \mathrm{E}-07$ & $1.67 \mathrm{E}+05$ \\
\hline Acenuphthylene & $208-96-8$ & $\mathbf{s}$ & $1.52 \mathrm{E}+02$ & 4786.3 & 9.57E+01 & $2.80 \mathrm{E}-0.4$ & $1.20 \mathrm{E}-04$ & $6.22 \mathrm{E}-02$ & $4.38 \mathrm{E}-02$ & $1.07 E-06$ & $1.29 E+05$ \\
\hline Phenenthrene & $85-01-8$ & $\mathbf{S}$ & $1.78 E+02$ & 38004.5 & $7.78 \mathrm{E}+02$ & $2.56 \mathrm{E}-05$ & $1.35 \mathrm{E}-06$ & $5.74 \mathrm{E}-02$ & 4.05E-02 & $1.11 \mathrm{E}-08$ & $1.27 \mathrm{E}+06$ \\
\hline
\end{tabular}

\section{Noter:}

1. USEPA, 1991: RAGS: Vol 1 - Human Heath Evalumion Mmunl (Pant B, Development of Riak-Bmed Preliminary Remodiation Caals)

2. Chemical Abutract Service.

3. Chemical class: $v$-volatile; s-semivolatile; $p$-pexticide/pcb; $x$ - dioxin; and $m$ - metal.

4. Calculation of organic contaminunt volatilization factor:

CONSTANTS:

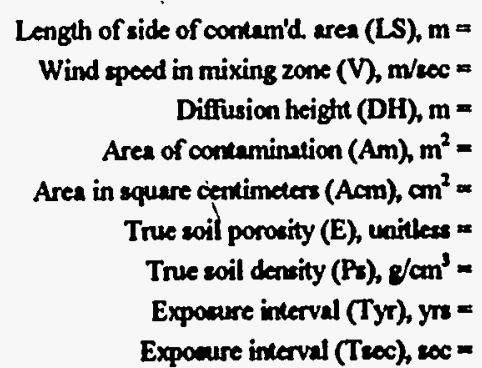

Length of side of contem'd. area (LS), $m=$

Wind speed in mixing zone $(V), m / 20 c=$ Diffusion height $(\mathrm{DH}), \mathrm{m}=$ Area of contamination $(\mathrm{Am}), \mathrm{m}^{2}=$ Area in square centimeters (Acm), $\mathrm{cm}^{2}=$ True soil porocity (E), unitless " True soil dencity (Pr), g/ $\mathrm{cm}^{3}$ Exposure interval (Tyr), yrs = Expomure interval (Troc), $200=$ Organic carbon comean (OC), unitless Conversion factor (CF), $\mathrm{ke} / \mathrm{g}=$

INPUT VARIABLES

Organic carbon partition coef. (Koc), $\mathrm{cm}^{3} / \mathrm{kg}=$ Henry's Law condant (H), atm-m $3 / \mathrm{mol}^{3}=$ Gas diffusivity (Di), $\mathrm{cm}^{2} / 10 \mathrm{c}=$

INTERMEDIATE STEPS

Soil-water partition coefficiext (Kd), $\mathrm{cm}^{3} / \mathrm{kg}=$ Soil-air partition coefficient (Kas), B-toil// $\mathrm{cm}^{3}$-air = Effective diffusivity (Dei), $\mathrm{cm}^{2} / \mathrm{sec}=$ alpha (alpha), $\mathrm{cm}^{2} / \mathrm{sec}=$

OUTPUT:

Volatilization Factor (VF), $\mathrm{m}^{3} / \mathrm{kg}=$

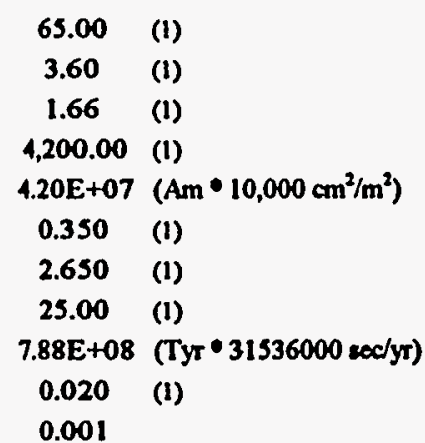


Table D.15

Savannah River

Burning/Rubble Pits (231-F, 231-1F)

Future Worker

Inhalation of Volatile Soil Contaminants (0-2'bls)

Carcinogenic and Noncarcinogenic Risk

\begin{tabular}{|c|c|c|c|c|c|c|c|c|c|}
\hline \multirow[b]{2}{*}{ Chemical } & \multirow{2}{*}{\multicolumn{2}{|c|}{ 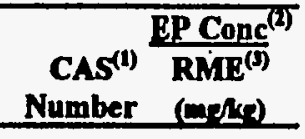 }} & \multirow[b]{2}{*}{$\begin{array}{l}\operatorname{IUR}^{(0)} \\
\left(\mathrm{m}^{3} / \mu \theta\right)\end{array}$} & \multirow[b]{2}{*}{ 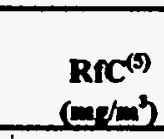 } & \multirow[b]{2}{*}{$\begin{array}{c}V F^{(n)} \\
\left(m^{2} / K^{\prime}\right)\end{array}$} & \multicolumn{2}{|c|}{ Cancer Risk ${ }^{(1)}$} & \multicolumn{2}{|c|}{ Hazard Quotient } \\
\hline & & & & & & RME & $\begin{array}{l}\% \text { of } \\
\text { Total } \\
\end{array}$ & RME & $\begin{array}{l}\% \text { of } \\
\text { Total } \\
\end{array}$ \\
\hline $\begin{array}{l}\text { Semivolatiles } \\
\text { 2-Methylnaphthalene } \\
\text { Acenaphthylene } \\
\text { Phenanthrene }\end{array}$ & $\begin{array}{r}91-57-6 \\
208-96-8 \\
85-01-8\end{array}$ & $\begin{array}{l}7.69 \mathrm{E}-02 \\
8.61 \mathrm{E}-02 \\
6.87 \mathrm{E}-01\end{array}$ & $\overline{-}$ & $\bar{z}$ & $\begin{array}{l}1.29 \mathrm{E}+05 \\
1.2 \mathrm{E}+06 \\
0.00 \mathrm{E}+00\end{array}$ & $\begin{array}{l}\text { NA } \\
\text { NA } \\
\text { NA }\end{array}$ & - & $\begin{array}{l}\text { NA } \\
\text { NA } \\
\text { NA }\end{array}$ & - \\
\hline & & & & PATHWA & SUMS: & $\begin{array}{r}\text { Cance: } \\
\mathbf{R M} \\
\end{array}$ & $\begin{array}{l}\text { Risk } \\
q^{2}\end{array}$ & $\begin{array}{r}\text { Hazard } \\
\mathbf{R N} \\
\end{array}$ & \\
\hline
\end{tabular}

Neati:

1. Chemical Abstract Service.

2. Expocure point concentration.

3. Reasonable maximum exposure.

4. Intulation unit risk.

5. Reference concentration.

6. Volatilization factor (ctremical-epecific).

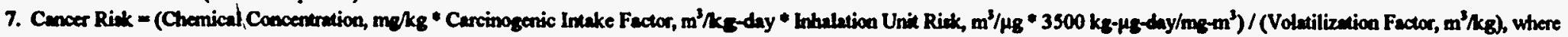
RME carcinogenic intake factor 8.74E-03

and conversion from IUR to inhalation slope factor $=3500 \mathrm{ks}-\mu \mathrm{g}-\mathrm{day} / \mathrm{mg}^{3}-\mathrm{m}^{3}$

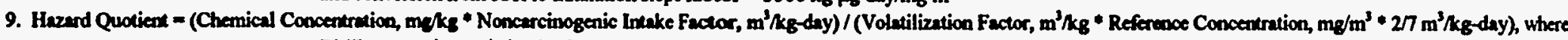
RME noncercinogenic ineake fa $2.45 E-02$

and conversion from RRC to inhalation reference doee $=277 \mathrm{~m}^{3} / \mathrm{kg}-$ day 
Table D.16

Savannah River

Burning/Rubble Pits (231-F, 231-1F)

Future Worker

Inhalation of Resuspended Soil Particulate Contaminants (0-2'bls)

Carcinogenic and Noncarcinogenic Risk

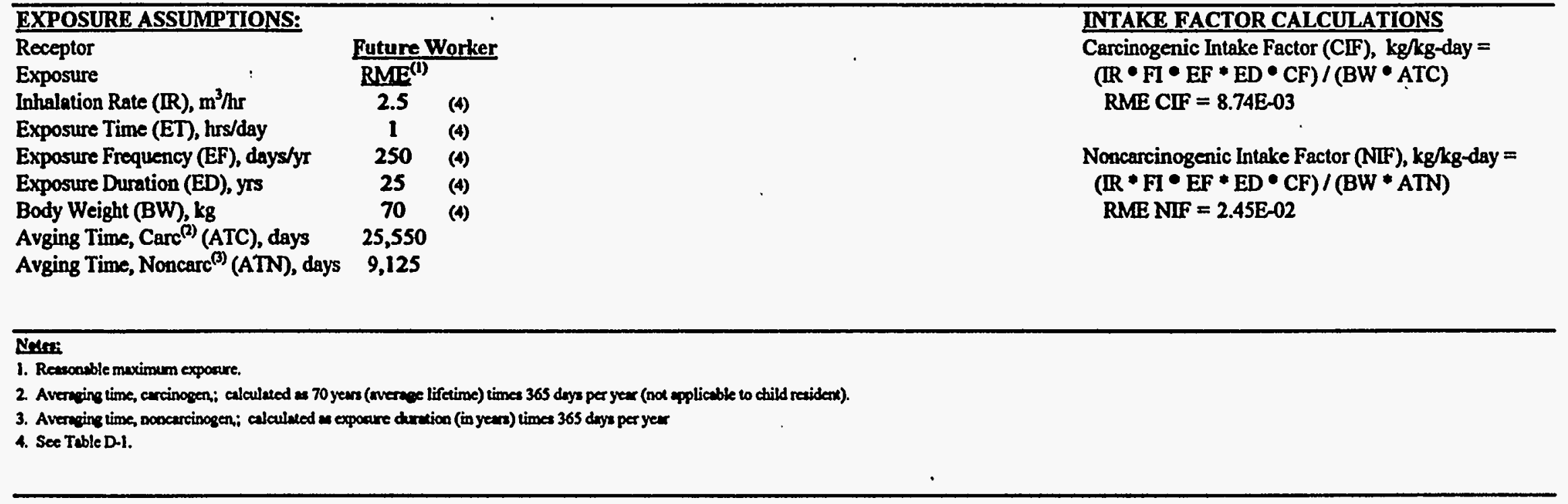


Table D.16

Savannah River

Burning/Rubble Pits (231-F, 231-1F)

Future Worker

Inhalation of Resuspended Soil Particulate Contaminants (0-2'bls)

Carcinogenic and Noncarcinogenic Risk

\begin{tabular}{|c|c|c|c|}
\hline $\begin{array}{l}\text { CALCULATION OF SOIL PARTICULATE RESUSPE } \\
\text { CONSTANTS: }\end{array}$ & CTOR: & & \\
\hline Length of side of contaminated area (LS) $=$ & 65.00 & meters & \\
\hline Wind spoed in mixing zone $(V)=$ & 3.60 & meters/sec & (1) \\
\hline Diffusion height $(\mathrm{DH})=$ & 1.66 & meters & \\
\hline Area of contamination $(A)=$ & $4,200.00$ & meters $^{2}$ & (1) \\
\hline Respirable fraction $(\mathrm{RF})=$ & 0.003 & $g /$ meters ${ }^{2}-\mathrm{hr}$ & \\
\hline Fraction of vegetative cover $(G)=$ & 0.90 & unitless - & (1) \\
\hline Mean ennual wind speed (Um) = & 3.60 & meters/sec & (1) \\
\hline Equivalent threshold value of wind speed at $10 \mathrm{~m}(\mathrm{Ut})=$ & 5.04 & meters/sec & (1) \\
\hline Fraction dependent on Um/Ut $(F x)=$ & 1.2700 & unitless & (1) \\
\hline Conversion factor $(\mathrm{CFa})=$ & $3,600.00$ & $\sec / \mathrm{hr}$ & \\
\hline Conversion factor $(\mathrm{CFb})=$ & $1,000.00$ & $g / k g$ & \\
\hline
\end{tabular}

Nels::

1. USEPA, 1991: RAGS: Vol 1 - Human Health Evalumion Manual (Pmt B, Development of Risk-Bmed Preliminary Remediation Goals)

\section{INTERMEDLATE STEPS:}

Particulate emission factor $(\mathrm{PEF})=2.18 \mathrm{E}+09$ meters $^{3} / \mathrm{kg}$ $(\mathrm{LS} \times \mathrm{V} \times \mathrm{DH} \times \mathrm{CFa} \times \mathrm{CFb}) /\left(\mathrm{A} \times \mathrm{RF} \times(1-\mathrm{G}) \times\left((\mathrm{Um} / \mathrm{Ut})^{-3}\right) \times(\mathrm{Fx})\right)$ 
Table D.16

Savannah River

Burning/Rubble Pits (231-F, 231-1F)

Future Worker

Inhalation of Resuspended Soil Particulate Contaminants (0-2'bls)

Carcinogenic and Noncarcinogenic Risk

\begin{tabular}{|c|c|c|c|c|c|c|c|c|}
\hline \multirow[b]{2}{*}{ Chemical } & \multicolumn{2}{|r|}{ EP Conctid) } & \multirow[b]{2}{*}{$\begin{array}{l}\mathrm{IUR}^{(4)} \\
\left(\mathrm{m}^{3} / \mu \rho\right)\end{array}$} & \multirow[b]{2}{*}{$\underset{\left(m / m^{3}\right)}{\operatorname{RrC}^{(S)}}$} & \multicolumn{2}{|c|}{ Cancer Risk ${ }^{(0)}$} & \multicolumn{2}{|c|}{$\overline{\text { Hazard Quotient }}^{(7)}$} \\
\hline & $\begin{array}{l}\text { CAS }^{(1)} \\
\text { Number }\end{array}$ & $\begin{array}{l}\mathbf{R M E}^{(3)} \\
(\mathrm{m} / \mathrm{LP})\end{array}$ & & & $\mathbf{R M E}$ & $\begin{array}{l}\% \text { of } \\
\text { Total }\end{array}$ & $\mathbf{R M E}$ & $\begin{array}{l}\% \text { of } \\
\text { Total }\end{array}$ \\
\hline \multicolumn{9}{|l|}{ Semivolatiles } \\
\hline 2-Methylnaphthalene & $91-57-6$ & $7.69 \mathrm{E}-02$ & - & - & NA & - & NA & - \\
\hline Acenaphthylene & $208-96-8$ & $8.61 \mathrm{E}-02$ & - & - & NA & - & NA & - \\
\hline Benzo(a)anthracene & $56-55-3$ & $9.26 \mathrm{E}-01$ & - & - & NA & - & NA & - \\
\hline Benzo(a)pyrene & $50-32-8$ & $6.49 \mathrm{E}-01$ & - & - & NA & - & NA & - \\
\hline Benzo(b) lluoranthene & $205-99-2$ & $1.01 \mathrm{E}+00$ & - & - & NA & - & NA & - \\
\hline Benzo( $(g, h, i)$ perylene & $191-24-2$ & $6.15 E-01$ & - & - & NA & - & NA & - \\
\hline Dibenz(a,h)anthracene & $53-70-3$ & $2.17 \mathrm{E}-01$ & - & - & NA & - & NA & - \\
\hline Phenanthrene & 85-01-8 & $6.87 \mathrm{E}-01$ & - & - & NA & - & NA & - \\
\hline \multicolumn{9}{|c|}{ Dloxins/furans } \\
\hline Heptachlorodibenzo-p-dioxin isomer & $37871-00-4$ & 4.11E-03 & $3.30 \mathrm{E}-01$ & - & $1.9 \mathrm{E}-11$ & $<1 \%$ & NA & - \\
\hline Heptachlorodibenzo-p-furan isomer & 38998-75-3 & $2.58 \mathrm{E}-04$ & $3.30 \mathrm{E}-01$ & - & $1.2 \mathrm{E}-12$ & $<1 \%$ & NA & - \\
\hline Hexachlorodibenzo-p-furan isomer & $55684-94-1$ & $1.00 \mathrm{E}-04$ & $3.30 \mathrm{E}+00$ & - & $4.6 \mathrm{E}-12$ & $<1 \%$ & NA & - \\
\hline Octachlorodibenzo-p-dioxin isomer & $3268-87-9$ & $9.26 \mathrm{E}-03$ & $3.30 \mathrm{E}-02$ & - & 4.3E-12 & $<1 \%$ & NA & - \\
\hline Octachlorodibenzo-p-furan isomer & $39001-02-0$ & $3.48 \mathrm{E}-04$ & 3.30E-02 & - & $1.6 \mathrm{E}-13$ & $<1 \%$ & NA & - \\
\hline \multicolumn{9}{|l|}{ PCBs } \\
\hline PCB 1254 & $11097-69-1$ & $1.78 \mathrm{E}-01$ & - & - & NA & - & NA & - \\
\hline \multicolumn{9}{|l|}{ Pesticides } \\
\hline delta-Benzene hexachloride & $319-86-8$ & $1.84 E-03$ & - & - & NA & - & NA & - \\
\hline Endrin ketone & $53494-70-5$ & $3.20 \mathrm{E}-03$ & - & - & NA & - & NA & - \\
\hline
\end{tabular}


Table D.16

Savannah River

Burning/Rubble Pits (231-F, 231-1F)

Future Worker

Inhalation of Resuspended Soil Particulate Contaminants (0-2'bls)

Carcinogenic and Noncarcinogenic Risk

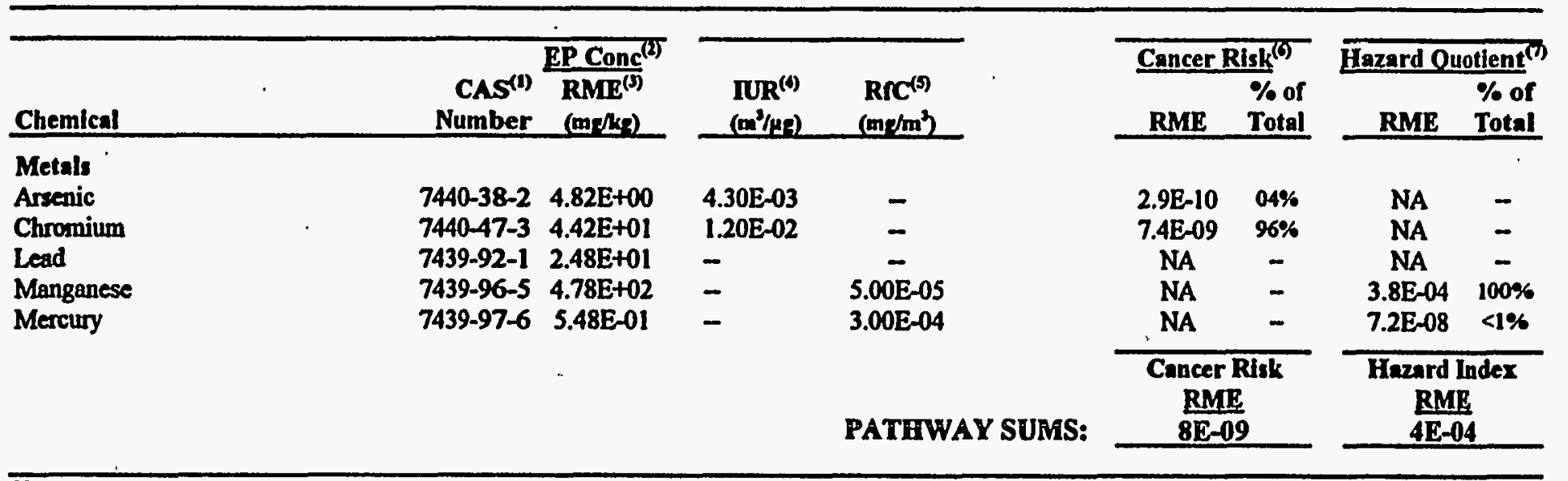

Note:

1. Chemical Abotract Service.

2. Expocure point concentration.

3. Reasonable maximum exposure.

4. Inhalation unit risk.

5. Referenoe concentration.

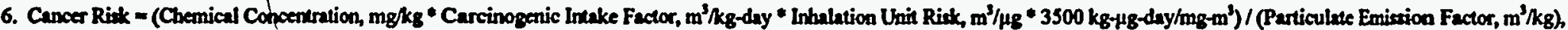

RME carcinogenic intake factor 8.74E-03

Particulate emission factor $=\quad 2.18 \mathrm{E}+09$

and convenzion from IUR to inhalation slope factor $=3500 \mathrm{~kg}-\mu g-\mathrm{day} / \mathrm{mg}^{3} \mathrm{~m}^{3}$

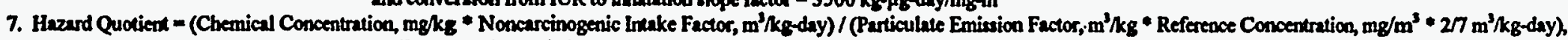

RME noncarcinogenic intake fi 2.45E-02

Particulate emivion factor $=2.18 \mathrm{E}+09$

and convervion from RfC to inhalation reference doue $=27 \mathrm{~m}^{3} / \mathrm{kg}$-day 
Table D.17

Savannah River

Burning/Rubble Pits (231-F, 231-1F)

Future Worker

Ingestion of Soil (0-4'bls)

Carcinogenic and Noncarcinogenic Risk

\section{EXPOSURE ASSUMPTIONS:}

\section{Receptor}

Exposure

Intake Rate (IR), mg/day

Fraction Ingested (FI), unitless

Exposure Frequency (EF), days/yr

Exposure Duration (ED), yrs

Body Weight (BW), kg

Avging Time, $\operatorname{Carc}^{(\mathfrak{l})}$ (ATC), days

Avging Time, Noncarc ${ }^{(3)}$ (ATN), days

Conversion Factor (CF), kg/mg

\section{Future Worker}

$\mathrm{RME}^{(1)}$

$50 \quad$ (4)

1 (4)

$250 \quad$ (4)

25 (4)

$70 \quad(4)$

25,550

9,125

1.00E-06

\section{INTAKE FACTOR CALCULATIONS}

Carcinogenic Intake Factor (CIF), kg/kg-day = $(\mathrm{IR} \bullet \mathrm{FI} \bullet \mathrm{EF} * \mathrm{ED} \bullet \mathrm{CF}) /(\mathrm{BW} * \mathrm{ATC})$ RME CIF $=1.75 E-07$

Noncarcinogenic Intake Factor (NIF), kg/kg-day = $(\mathrm{IR} \bullet \mathrm{FI} \bullet \mathrm{EF} \bullet \mathrm{ED} \bullet \mathrm{CF}) /(\mathrm{BW} * \mathrm{ATN})$

RME NIF $=4.89 \mathrm{E}-07$

\section{Noter:}

1. Ressonuble maximum exposure.

2. Averaing time, carcinogen; calculuted as 70 years (average lifetime) times 365 day par year (not applicable to child resident).

3. Averning time, noncurcinogen; calculsted es exposure duration (in years) times 365 days per year

4. See Table D-1. 
Table D.17

Savannah River

Burning/Rubble Pits (231-F, 231-1F)

Future Worker

Ingestion of Soil (0-4'bls)

Carcinogenic and Noncarcinogenic Risk

\begin{tabular}{|c|c|c|c|c|c|c|c|c|c|}
\hline Chemical & $\mathrm{CAS}^{(1)} \mathrm{Na}$ & $\begin{array}{c}\mathrm{EPConc} \mathrm{c}^{(2)} \\
\mathrm{RME}^{(3)} \\
(\mathrm{mg} / \mathrm{kg}) \\
\end{array}$ & $\begin{array}{c}\operatorname{OS} f^{(1)} \\
(k \rho-d / m q)\end{array}$ & $\begin{array}{c}\operatorname{ORD}^{(\sigma)} \\
(\mathrm{m} / \mathrm{kg} \mathrm{g}-\mathrm{d})\end{array}$ & & Cancer & $\begin{array}{l}\frac{1 s k^{())}}{\%} \text { of } \\
\text { Total } \\
\end{array}$ & Hazard 0 & $\begin{array}{l}\text { otient }^{(7)} \\
\% \text { of } \\
\text { Total }\end{array}$ \\
\hline \multicolumn{10}{|l|}{ Semivolatiles } \\
\hline 2-Methylnaphthalene & $91-57-6$ & $7.69 \mathrm{E}-02$ & - & - & & - & - & - & - \\
\hline Acenaphthylene & $208-96-8$ & $8.61 E-02$ & - & - & & - & - & - & 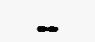 \\
\hline Benzo(a)anthracene & $56-55-3$ & $9.26 \mathrm{E}-01$ & $7.30 \mathrm{E}-01$ & - & & $1.2 E-07$ & $03 \%$ & - & - \\
\hline Benzo(a)pyrene & $50-32-8$ & $6.49 \mathrm{E}-01$ & $7.30 \mathrm{E}+00$ & - & & 8.3E-07 & $18 \%$ & - & - \\
\hline Benzo(b)Iluoranthene & $205-99-2$ & $1.01 E+00$ & $7.30 \mathrm{E}-01$ & - & & $1.3 \mathrm{E}-07$ & $03 \%$ & - & - \\
\hline Benzo(g,h,i)perylene & $191-24-2$ & $6.15 \mathrm{E}-01$ & - & - & & - & - & - & - \\
\hline $\operatorname{Dibenz}(\mathrm{a}, \mathrm{h})$ anthracene & $53-70-3$ & 2.17E-01 & $7.30 \mathrm{E}+00$ & - & & $2.8 \mathrm{E}-07$ & $06 \%$ & - & - \\
\hline Phenanthrene & $85-01-8$ & $6.87 \mathrm{E}-01$ & - & - & & - & - & - & - \\
\hline \multicolumn{10}{|l|}{ Dloxing/furans } \\
\hline Heptachlorodibenzo-p-dioxin isomer & $37871-00-4$ & $4.11 \mathrm{E}-03$ & $1.50 \mathrm{E}+03$ & - & & $1.1 \mathrm{E}-06$ & $23 \%$ & - & - \\
\hline Heptachlorodibenzo-p-furan isomer & $38998-75-3$ & $2.58 \mathrm{E}-04$ & $1.50 \mathrm{E}+03$ & - & & $6.8 \mathrm{E}-08$ & $01 \%$ & - & - \\
\hline Hexachlorodibenzo-p-furan isomer & $55684-94-1$ & $1.00 \mathrm{E}-04$ & $1.50 \mathrm{E}+04$ & - & & $2.6 \mathrm{E}-07$ & $06 \%$ & - & - \\
\hline Octachlorodibenzo-p-dioxin isomer & $3268-87-9$ & $9.26 \mathrm{E}-03$ & $1.50 \mathrm{E}+02$ & - & · & $2.4 \mathrm{E}-07$ & $05 \%$ & - & - \\
\hline Octachlorodibenzo-p-furan isomer & $39001-02-0$ & $3.48 \mathrm{E}-04$ & $1.505+02$ & - & & $9.1 E-09$ & $<1 \%$ & - & - \\
\hline \multicolumn{9}{|l|}{ PCBs } & $18 \%$ \\
\hline \multicolumn{10}{|l|}{ Peaticides } \\
\hline Endrin ketone & $53494-70-5$ & $3.20 \mathrm{E}-03$ & - & - & & - & - & - & - \\
\hline \multicolumn{10}{|l|}{ Metals } \\
\hline Arsenic & $7440-38-2$ & $5.29 E+00$ & $1.50 \mathrm{E}+00$ & $3.00 \mathrm{E}-04$ & & $1.4 \mathrm{E}-06$ & $30 \%$ & 8.6E-03 & $35 \%$ \\
\hline Chrọmium & $7440-47-3$ & $7.65 \mathrm{E}+01$ & - & $5.00 \mathrm{E}-03$ & & - & - & $7.5 \mathrm{E}-03$ & $30 \%$ \\
\hline Lead & $7439-92-1$ & $2.62 E+01$ & - & - & & - & - & - & - \\
\hline Manganese & $7439-96-5$ & $3.61 E+02$ & - & $5.00 \mathrm{E}-02$ & & - & - & $3.5 \mathrm{E}-03$ & $14 \%$ \\
\hline \multirow[t]{2}{*}{ Mercury } & $7439-97-6$ & $3.58 \mathrm{E}-01$ & - & $3.00 \mathrm{E}-04$ & & - & - & $5.8 \mathrm{E}-04$ & $02 \%$ \\
\hline & & & & PATHWAY & SUMS: & \multicolumn{2}{|c|}{$\frac{\text { Cancer Risk }}{\text { RMR }}$} & \multicolumn{2}{|c|}{$\begin{array}{c}\text { Hazard Index } \\
\mathrm{RME} \\
\mathbf{2 E - 0 2}\end{array}$} \\
\hline
\end{tabular}


Table D.17

Savannah River

Burning/Rubble Pits (231-F, 231-1F)

Future Worker

Ingestion of Soil $\left(0-4^{\prime} \mathrm{bls}\right)$

Carcinogenic and Noncarcinogenic Risk

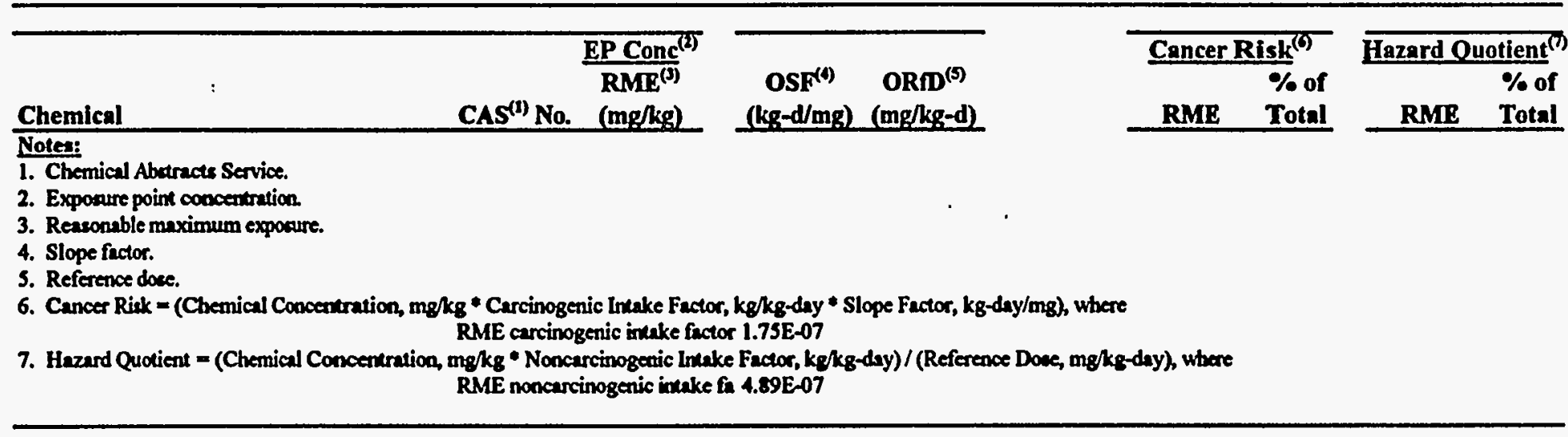


Table D.18

Savannah River Site

Burning/Rubble Pits (231-F, 231-1F)

Future Worker

Dermal Exposure to Soil (0-4'bls)

Carcinogenic and Noncarcinogenic Risk

\section{EXPOSURE ASSUMPTIONS:}

\section{Receptor}

Exposure

Skin Surface Area (SA), $\mathrm{cm}^{2} /$ event

Soil-to-Skin Adherance (SK), $\mathrm{mg} / \mathrm{cm}^{2}$

Exposure Frequency (EF), events/yr

Exposure Duration (ED), yrs

Body Weight (BW), kg

Avging Time, Carc ${ }^{(2)}$ (ATC), days

Avging Time, Noncarc ${ }^{(3)}$ (ATN), days

Conversion Factor (CF), kg/ms

\section{Future Worker}

$\mathrm{RME}^{(1)}$

3,200 (4)

0.2 (4)

250 (4)

25 (4)

70 (4)

25,550

$1.00 \mathrm{E}-06$

\section{INTAKE FACTOR CALCULATIONS}

Carcinogenic Intake Factor (CIF), $\mathrm{kg} / \mathrm{kg}$-day =

$(\mathrm{SA} * \mathrm{SK} * \mathrm{EF} * \mathrm{ED} * \mathrm{CF}) /(\mathrm{BW} * \mathrm{ATC})$

$\mathrm{RME} \mathrm{CFF}=2.24 \mathrm{E}-06$

Noncarcinogenic Intake Factor (NIF), kg/kg-day =

$(S A * S K * E F * E D * C F) /(B W * A T N)$

RME NIF $=6.26 \mathrm{E}-06$

\section{Ness:}

1. Resuonable maximum expoture.

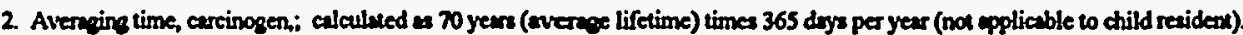

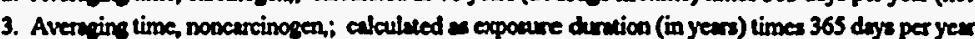

4. See Table D-1. 
Table D.18

Savannah River Site

Burning/Rubble Pits (231-F, 231-1F)

Future Worker

Dermal Exposure to Soil (0-4'bls)

Carcinogenic and Noncarcinogenic Risk

\begin{tabular}{|c|c|c|c|c|c|c|c|c|c|}
\hline \multirow[b]{2}{*}{ Chemical } & \multicolumn{2}{|c|}{ EPConc ${ }^{(2)}$} & \multirow[b]{2}{*}{$\begin{array}{c}\operatorname{DSF}^{(4)} \\
(\mathbf{k g}-\mathrm{d} / \mathrm{mg})\end{array}$} & \multirow[b]{2}{*}{ 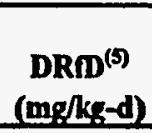 } & \multirow[b]{2}{*}{ DABS $^{(0)}$} & \multicolumn{2}{|c|}{ Cancer Risls") } & \multicolumn{2}{|c|}{ Hazard Quotient ${ }^{(1)}$} \\
\hline & $\begin{array}{r}\text { CAS }^{(1)} \\
\text { Number }\end{array}$ & $\begin{array}{l}\mathbf{R M E}^{(3)} \\
(\mathrm{m} / \mathbf{k g})\end{array}$ & & & & RME & $\begin{array}{l}\% \text { of } \\
\text { Total }\end{array}$ & RME & $\begin{array}{l}\% \text { of } \\
\text { Total }\end{array}$ \\
\hline \multicolumn{10}{|l|}{ Semivolatiles } \\
\hline 2-Methyinuphthalene & $91-57-6$ & 7.69E-02 & - & - & $1.00 \mathrm{E}-02$ & - & - & - & - \\
\hline Acenophthylene & $208-96-8$ & $8.61 \mathrm{E}-02$ & - & - & $1.00 \mathrm{E}-02$ & - & - & - & - \\
\hline Benzo(a)anthracene & $56-55-3$ & $9.26 \mathrm{E}-01$ & $1.46 \mathrm{E}+00$ & - & $1.00 \mathrm{E}-02$ & $3.0 \mathrm{E}-08$ & $03 \%$ & - & - \\
\hline Benzo(a)pyrene & $50-32-8$ & $6.49 \mathrm{E}-01$ & $3.65 E+01$ & - & $1.00 \mathrm{E}-02$ & $5.3 \mathrm{E}-07$ & $55 \%$ & - & - \\
\hline Benzo(b)fluoranthene & $205-99-2$ & $1.01 E+00$ & $1.46 \mathrm{E}+00$ & - & $1.00 \mathrm{E}-02$ & 3.3E-08 & $03 \%$ & - & - \\
\hline Benzo(g,h,i)perylene & $191-24-2$ & $6.15 E-01$ & $\rightarrow$ & - & $1.00 \mathrm{E}-02$ & - & - & - & - \\
\hline Dibenz $(a, h)$ anthracenc & $53-70-3$ & 2.17E-01 & $1.46 \mathrm{E}+01$ & - & $1.00 \mathrm{E}-02$ & $7.1 \mathrm{E}-08$ & $07 \%$ & - & - \\
\hline Phenanthrene & $85-01-8$ & $6.87 \mathrm{E}-01$ & - & - & $1.00 \mathrm{E}-02$ & - & - & - & - \\
\hline \multicolumn{10}{|l|}{ Dioxins/furans } \\
\hline Heptachlorodibenzo-p-dioxin isomer & $37871-00-4$ & 4.11E-03 & $1.72 \mathrm{E}+03$ & - & $1.00 \mathrm{E}-02$ & $1.6 \mathrm{E}-07$ & $16 \%$ & - & - \\
\hline Heptachloradibenzo-p-furan isomer & $38998-75-3$ & $2.58 \mathrm{E}-04$ & $1.72 E+03$ & - & $1.00 \mathrm{E}-02$ & $9.9 E-09$ & $01 \%$ & - & - \\
\hline Hexachlorodibenzo-p-furan isomer & $55684-94-1$ & $1.00 \mathrm{E}-04$ & $1.72 E+04$ & - & $1.00 \mathrm{E}-02$ & $3.9 E-08$ & $04 \%$ & - & - \\
\hline Octachlorodibenzo-p-dioxin isomer & $3268-87-9$ & $9.26 \mathrm{E}-03$ & $1.72 \mathrm{E}+02$ & - & $1.00 \mathrm{E}-02$ & $3.6 \mathrm{E}-08$ & $04 \%$ & - & - \\
\hline Octachlorodibenzo-p-furan isomer & $39001-02-0$ & $3.48 \mathrm{E}-04$ & $1.72 E+02$ & - & $1.00 \mathrm{E}-02$ & $1.3 \mathrm{E}-09$ & $<1 \%$ & - & - \\
\hline $\begin{array}{l}\text { PCBs } \\
\text { PCB } 1254\end{array}$ & $11097-69-1$ & $1.78 \mathrm{E}-01$ & $8.56 \mathrm{E}+00$ & $1.80 \mathrm{E}-05$ & $1.00 \mathrm{E}-02$ & $3.4 \mathrm{E}-08$ & $04 \%$ & $6.2 \mathrm{E}-04$ & $08 \%$ \\
\hline \multicolumn{10}{|l|}{ Pexticides } \\
\hline delta-Benzene hexachloride & $319-86-8$ & $1.84 \mathrm{E}-03$ & - & - & $1.00 \mathrm{E}-02$ & - & - & - & - \\
\hline Endrin ketone & $53494-70-5$ & $3.20 \mathrm{E}-03$ & - & - & $1.00 \mathrm{E}-02$ & - & - & - & - \\
\hline
\end{tabular}


Table D.18

Savannah River Site

Burning/Rubble Pits (231-F, 231-1F)

Future Worker

Dermal Exposure to Soil (0-4'bls)

Carcinogenic and Noncarcinogenic Risk

\begin{tabular}{|c|c|c|c|c|c|c|c|c|c|}
\hline \multirow[b]{2}{*}{ Chemical } & \multicolumn{2}{|r|}{ EPConc ${ }^{(2)}$} & \multirow[b]{2}{*}{$\begin{array}{c}\operatorname{DSF}^{(0)} \\
(\mathrm{kg}-\mathrm{d} / \mathrm{mg})\end{array}$} & \multirow[b]{2}{*}{ 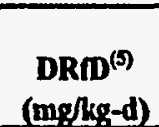 } & \multirow[b]{2}{*}{ DABS $^{(0)}$} & \multicolumn{2}{|c|}{ Cancer Risk (v) } & \multicolumn{2}{|c|}{ Hazard Ouotient $^{(3)}$} \\
\hline & $\begin{array}{l}\text { CAS }^{(1)} \\
\text { Number }\end{array}$ & $\begin{array}{l}\mathrm{RME}^{(3)} \\
(\mathrm{mq} / \mathrm{kg})\end{array}$ & & & & $\mathbf{R M E}$ & $\begin{array}{l}\% \text { of } \\
\text { Total }\end{array}$ & $\mathbf{R M E}$ & $\begin{array}{l}\% \text { of } \\
\text { Total }\end{array}$ \\
\hline \multicolumn{10}{|l|}{ Metals } \\
\hline Arsenic & $7440-38-2$ & $5.29 \mathrm{E}+00$ & $1.88 E+00$ & $2.40 \mathrm{E}-04$ & $1.00 \mathrm{E}-03$ & $2.2 E-08$ & $02 \%$ & $1.4 E-04$ & $02 \%$ \\
\hline Chromium & $7440-47-3$ & $7.65 E+01$ & - & $1.00 \mathrm{E}-04$ & $1.0,0 \mathrm{E}-03$ & - & - & $4.8 \mathrm{E}-03$ & $61 \%$ \\
\hline \multirow[t]{2}{*}{ Mercury } & 7439.97 .6 & $3.58 \mathrm{E}-01$ & - & $3.00 \mathrm{E}-06$ & $1.00 \mathrm{E}-03$ & - & - & $7.5 \mathrm{E}-04$ & $10 \%$ \\
\hline & · & & & PATHWA & SUMS: & \multicolumn{2}{|c|}{$\begin{array}{c}\text { Cancer Risk } \\
\text { RMR } \\
1 \mathrm{E}-06\end{array}$} & \multicolumn{2}{|c|}{$\begin{array}{c}\text { Harand Index } \\
\text { RME } \\
\text { 8E-03 }\end{array}$} \\
\hline
\end{tabular}

Note:

1. Chemical Abstrect Service.

2. Exposure point concentration.

3. Reasonable maximum exposure.

4. Slope factor, dermal.

5. Reference dose, dermal.

6. Abeorption factor.

7. Cenoer Risk = (Chemical Concentration, mg/k8 * Carcinogenic Intake Fector, kg/kg-day * Abeorption Factor, unitless * Slope Factor, kg-day/mg), where RME carcinogenic intake factor 2.24E-06

8. Hazand Quotient - (Chemical Concentrution, mg/kg * Noncarcinogenic Irntake Factor, kg/kg-day * Abeorption Factor, unitless) /(Reference Dose, mg/kg-day), where RME noncarcinogenic intake fa 6.26E-06 
Table D.19

Savannah River Site

Burning/Rubble Pits (231-F, 231-1F)

Future Worker

Inhalation of Volatile Soil Contaminants (0-4'bls)

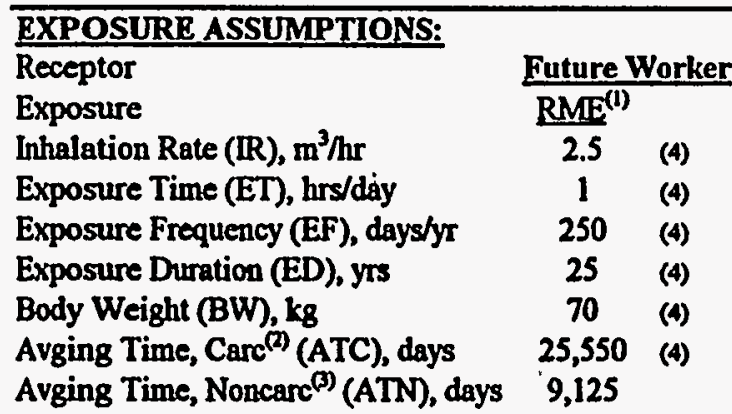

INTAKE FACTOR CALCULATIONS

Carcinogenic Intake Factor (CFF), kg/kg-day =

$(\mathrm{IR} \bullet \mathrm{ET} \bullet \mathrm{EF} \bullet \mathrm{ED} \bullet \mathrm{CF}) /(\mathrm{BW} \bullet \mathrm{ATC})$

$\mathrm{RME} C \mathrm{CIF}=8.74 \mathrm{E}-03$

Noncarcinogenic Intake Factor (NIF), kg/kg-day = $(\mathbb{R} \bullet \mathrm{ET} \bullet \mathrm{EF} \bullet \mathrm{ED} \bullet \mathrm{CF}) /(\mathrm{BW} \bullet \mathrm{ATN})$

RME NIF $=2.45 E-02$

Netri:

1. Remeconble muximum expoeure.

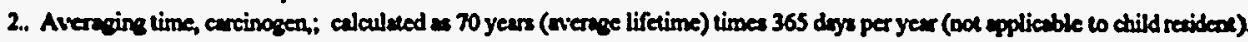

3. Averaging time, noncarcinogen; calculmod es exposure duration (in yemn) times 365 days par yesr

4. See Table D-1. 
Table D.19

Savannah River Site

Burning/Rubble Pits (231-F, 231-1F)

Future Worker

Inhalation of Volatile Soil Contaminants (0-4'bls)

Calculation of Volatilization Factor (VF) ${ }^{(1)}$

\begin{tabular}{|c|c|c|c|c|c|c|c|c|c|c|c|c|}
\hline Chemical & $\mathrm{CAS}^{(2)} \mathrm{Na}$ & Class $^{(3)}$ & MolWt. & $\log K a c$ & $\operatorname{Kac}^{(1)}$ & $\mathbf{K} \mathbf{d}^{(4)}$ & $\mathbf{H}^{(4)}$ & $\mathrm{Kas}^{(1)}$ & $D_{1}^{(())}$ & $\operatorname{Def}^{(1)}$ & alpha(t) & $\begin{array}{l}\text { Volat. } \\
\text { Factor }{ }^{(4)} \\
\left(\mathrm{m}^{3} / \mathrm{kg}\right)\end{array}$ \\
\hline \multicolumn{13}{|l|}{ Semivolatiles } \\
\hline 2-Methylnaphthalene & $91-57-6$ & $\mathbf{S}$ & $1.42 E+02$ & 3.93 & 8511.4 & $1.70 E+02$ & $2.90 \mathrm{E}-04$ & $6.98 \mathrm{E}-05$ & $6.43 E-02$ & 4.53E-02 & $6.43 \mathrm{E}-07$ & $1.67 \mathrm{E}+05$ \\
\hline Acenaphthylene & $208-96-8$ & $\mathbf{S}$ & $1.52 \mathrm{E}+02$ & 3.68 & 4786.3 & $9.57 \mathrm{E}+01$ & $2.80 E-04$ & $1.20 \mathrm{E}-04$ & $6.22 \mathrm{E}-02$ & $4.38 \mathrm{E}-02$ & 1.07E-06 & $1.29 E+05$ \\
\hline Phenanthrene & $85-01-8$ & $\mathbf{S}$ & $1.78 \mathrm{E}+02$ & 4.59 & 38904.5 & $7.78 \mathrm{E}+02$ & 2.56E-05 & $1.35 \mathrm{E}-06$ & $5.74 E-02$ & 4.05E-02 & $1.11 \mathrm{E}-08$ & $1.27 E+06$ \\
\hline
\end{tabular}

\section{Notet:}

1. USEPA, 1991: RAOS: Vol 1 - Human Heath Evaluation Manual (Pan B, Development of Risk-Based Preliminary Remediation Goals)

2. Chemical Abract Service.

3. Chemical class: $v$ - volatile; s-remivolatile; $p$-pesticide/pct; $x$-dioxin; and $m$-metal.

4. Calculation of organic contaminant volatilization factor:

CONSTANTS:

Length of side of contam'd. area (LS), $m=$

Wind speed in mixing zone (V), m/rec =

Diffusion heigth (DH), $m=$ Area of contamination (Am), $\mathrm{m}^{2}$. Area in square cemtimeters (Acm), $\mathrm{cm}^{2}=$ True edil porosity (E), unitless = True roil density $(\mathrm{Pz}), \mathrm{E} / \mathrm{cm}^{3}=$ Exposure interval (Tyt), yrs = Expocure interval (Trex), sec = Organic carbon content $(O C)$, unilless = Conversion factor (CF) kg/B =

INPUT VARIABLES:

Organic carbon partition coef. (Koc), $\mathrm{cm}^{3} / \mathrm{kg}=$ Hearys Law constanx (H), atm-m $3 / \mathrm{mol}=$ Ges diffusivity (Di), $\mathrm{cm}^{2} / \mathrm{sec}=$

INTERMEDLATE STEPS:

Soil-water partition coefficient (Kd), $\mathrm{cm}^{3} / \mathrm{kg}_{\mathrm{g}}$ " Soil-air partition coefficient (Kes), s-0il/cm'-air = Effective diffusivity (Dei), $\mathrm{cm}^{2} / 20 \mathrm{c}=$

OUTPUT:

$$
\text { alpha (Alpha), } \mathrm{cm}^{2} / \mathrm{sec}=
$$

Volatilization Factor (VF), $\mathrm{m}^{3} / \mathrm{kg}=$

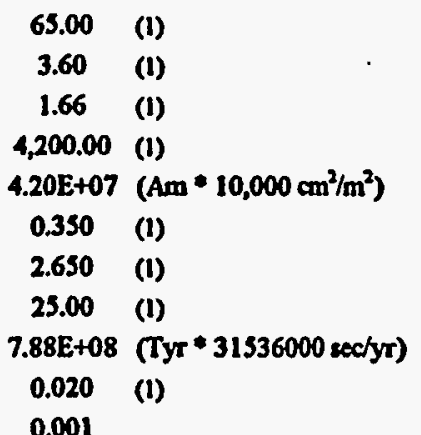


Table D.19

Savannah River Site

Burning/Rubble Pits (231-F, 231-1F)

Future Worker

Inhalation of Volatile Soil Contaminants ( $0-4$ 'bls)

Carcinogenic and Noncarcinogenic Risk

\begin{tabular}{|c|c|c|c|c|c|c|c|c|c|}
\hline Chemical & $\begin{array}{r}\text { CAS(1) } \\
\text { Number }\end{array}$ & $\begin{array}{c}\mathrm{EPConc} c^{(2)} \\
\mathrm{RME}^{(1)} \\
(\mathrm{O} / \mathrm{R})\end{array}$ & 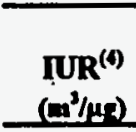 & $\begin{array}{l}\operatorname{RrC}^{(\theta)} \\
\text { (exples }\end{array}$ & $\begin{array}{l}V F^{(0)} \\
\left(m^{3} / k_{c}\right)\end{array}$ & $\begin{array}{l}\text { Cencer } \\
\text { RME }\end{array}$ & $\begin{array}{l}\text { isk } \\
\% \text { of } \\
\text { Total }\end{array}$ & $\begin{array}{l}\text { Hazard 0 } \\
\text { RME }\end{array}$ & $\begin{array}{l}\text { tient }^{(1)} \\
\% \text { of } \\
\text { Total }\end{array}$ \\
\hline $\begin{array}{l}\text { Semivolatiles } \\
\text { 2-Methylnaphthalene } \\
\text { Acenaphthylene } \\
\text { Phenanthrene }\end{array}$ & $\begin{array}{r}91-57-6 \\
208-96-8 \\
85-01-8\end{array}$ & $\begin{array}{l}7.69 \mathrm{E}-02 \\
8.61 \mathrm{E}-02 \\
6.87 \mathrm{E}-01\end{array}$ & - & $\begin{array}{l}- \\
-\end{array}$ & $\begin{array}{l}1.29 \mathrm{E}+05 \\
1.27 \mathrm{E}+06 \\
0.00 \mathrm{E}+00\end{array}$ & $\begin{array}{l}\text { NA } \\
\text { NA } \\
\text { NA }\end{array}$ & $\overline{-}$ & $\begin{array}{l}\text { NA } \\
\text { NA } \\
\text { NA }\end{array}$ & - \\
\hline & & & & PATHWA & Y SUMS: & $\begin{array}{r}\text { Cance } \\
\mathrm{RN} \\
\end{array}$ & Risk & $\begin{array}{r}\text { Hazard } \\
\text { RN } \\
\end{array}$ & ndex \\
\hline
\end{tabular}

1. Chemical Abstract Service.

2. Expocure point concentration.

3. Reasonable maximum expoeure.

4. Inhulation unit risk

5. Reference concentration

6. Volatilization factor (chemical-epecific)

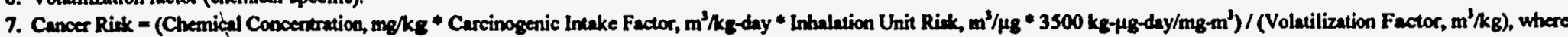
RME carcinonenic intake factor 8.74E-03

and conversion from IUR to inhalation slope factor $=3500 \mathrm{~kg}-\mu \mathrm{g}-$ day $/ \mathrm{mg}_{\mathrm{g}} \mathrm{m}^{3}$

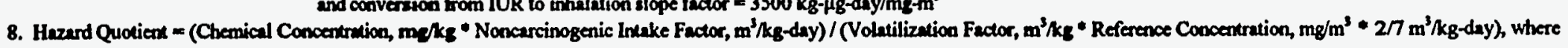
RME noncarcinogenic intake f 2.45E-02

and conversion from RfC to inhralation referenoe dose $=277 \mathrm{~m}^{3} / \mathrm{kg}-\mathrm{day}$ 
Table D.20

Savannah River Site

Burning/Rubble Pits (231-F, 231-1F)

Future Worker

Inhalation of Resuspended Soil Particulate Contaminants (0-4'bls)

Carcinogenic and Noncarcinogenic Risk

\section{EXPOSURE ASSUMPTIONS:}

Receptor

Exposure

Inhalation Rate (IR), $\mathbf{m}^{\mathbf{3}} / \mathrm{hr}$

Exposure Time (ET), hrs/day

Exposure Frequency (EF), days/yr

Exposure Duration (ED), yrs

Body Weight (BW), kg

Avging Time, $\mathrm{Carc}^{(2)}$ (ATC), days

Avging Time, Noncarc ${ }^{(3)}$ (ATN), days

\begin{tabular}{cc}
\multicolumn{3}{c}{ Future Worker } \\
\hline $\mathrm{BME}^{(1)}$ & \\
2.5 & (4) \\
1 & $(4)$ \\
250 & $(4)$ \\
25 & $(4)$ \\
70 & $(4)$ \\
25,550 & \\
9,125 &
\end{tabular}

\section{INTAKE FACTOR CALCULATIONS}

Carcinogenic Intake Factor (CIF), kg/kg-day = $(\mathrm{IR} * \mathrm{FI} \bullet \mathrm{EF} * \mathrm{ED} * \mathrm{CF}) /(\mathrm{BW} * \mathrm{ATC})$

$\mathrm{RME}$ CIF $=8.74 \mathrm{E}-03$

Noncarcinogenic Intake Factor (NIF), kg/kg-day = $(\mathrm{IR} \bullet \mathrm{FI} \bullet \mathrm{EF} \bullet \mathrm{ED} \bullet \mathrm{CF}) /(\mathrm{BW} \bullet \mathrm{ATN})$

RME NIF $=2.45 E-02$

\section{Noden:}

1. Remonsuble maximum expoure.

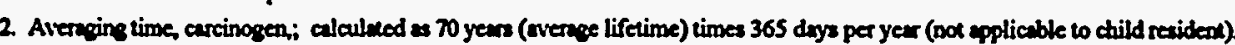

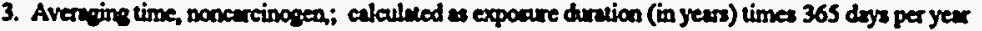

4. Sec Table D-1. 
Table D.20

Savannah River Site

Burning/Rubble Pits (231-F, 231-1F)

Future Worker

Inhalation of Resuspended Soil Particulate Contaminants (0-4'bls)

Carcinogenic and Noncarcinogenic Risk

\section{CALCULATION OF SOIL PARTICULATE RESUSPENSION FACTOR:}

\section{CONSTANTS:}

Length of side of contaminated area (LS) =

Wind speed in mixing zone $(V)=$

Diffusion height $(\mathrm{DH})=$

Area of contamination $(A)=$

Respirable fraction (RF) =

Fraction of vegetative cover $(G)=$

Mean annual wind speed (Um) =

Equivalent threshold value of wind speed at $10 \mathrm{~m}(\mathrm{Ut})=$

Fraction dependent on $\mathrm{Um} / \mathrm{Ut}(\mathrm{Fx})=$

Conversion factor (CFa) $=$

Conversion factor $(\mathrm{CFb})=$

$\begin{array}{lll}65.00 & \text { meters } & \text { (1) } \\ 3.60 & \text { meters/sec } & \text { (1) } \\ 1.66 & \text { meters } & \text { (1) } \\ 4,200.00 & \text { meters }^{2} & \text { (1) } \\ 0.003 & \text { g/meters }^{2}-\mathrm{hr} & \text { (1) } \\ 0.90 & \text { unitless } & \text { (1) } \\ 3.60 & \text { meters/sec } & (1) \\ 5.04 & \text { meters/sec } & (1) \\ 1.2700 & \text { unitless } & (1) \\ 3,600.00 & \text { sec/hr } & \\ 1,000.00 & \text { g/kg } & \end{array}$

Noter:

1. USEPA, 1991: RAGS: Vol I - Human Health Evaluation Manual (Pan B, Development of Risk-Based Preliminary Remodiation Coals)

\section{INTERMEDIATE STEPS:}

Particulate emission factor (PEF) $=2.18 \mathrm{E}+09$ meters $^{3} / \mathrm{kg}$ $(\mathrm{LS} \times \mathrm{V} \times \mathrm{DH} \times \mathrm{CFa} \times \mathrm{CFb}) /\left(\mathrm{A} \times \mathrm{RF} \times(1-G) \times\left((\mathrm{Um} / \mathrm{Ut})^{\wedge} 3\right) \times(\mathrm{Fx})\right)$ 
Table D.20

Savannah River Site

Burning/Rubble Pits (231-F, 231-1F)

Future Worker

Inhalation of Resuspended Soil Particulate Contaminants (0-4'bls)

Carcinogenic and Noncarcinogenic Risk

\begin{tabular}{|c|c|c|c|c|c|c|c|c|}
\hline \multirow[b]{2}{*}{ Chemical } & \multicolumn{2}{|r|}{ EP Conc ${ }^{(2)}$} & \multirow[b]{2}{*}{$\begin{array}{l}\mathrm{IUR}^{(0)} \\
\left(\mathrm{m}^{2} / \mu s\right)\end{array}$} & \multirow[b]{2}{*}{$\begin{array}{l}\operatorname{RrC}^{(s)} \\
\left(m>m^{2}\right)\end{array}$} & \multicolumn{2}{|c|}{ Cancer Risk ${ }^{(0)}$} & \multicolumn{2}{|c|}{ Hazard Quatlent } \\
\hline & $\begin{array}{r}\text { CAS }^{(1)} \\
\text { Number }\end{array}$ & $\begin{array}{l}\mathbf{R M E}^{(1)} \\
(\text { (mese })\end{array}$ & & & RME & $\begin{array}{l}\% \text { of } \\
\text { Total }\end{array}$ & RME & $\begin{array}{l}\% \text { of } \\
\text { Total }\end{array}$ \\
\hline \multicolumn{9}{|l|}{ Semivolatiles } \\
\hline 2-Methylnaphthalene & $91-57-6$ & $7.69 \mathrm{E}-02$ & - & - & NA & - & NA & - \\
\hline Acenaphthylene & 208-96-8 & 8.61E-02 & - & - & NA & - & NA & - \\
\hline Benzo(a)anthracene & $56-55-3$ & $9.26 \mathrm{E}-01$ & - & - & NA & - & NA & - \\
\hline Benzo(a)pyrene & $50-32-8$ & 6.49E-01 & - & - & NA & - & NA & - \\
\hline Benzo(b) fluoranthene & 205-99-2 & $1.01 E+00$ & - & - & NA & - & NA & - \\
\hline Benzo $(\mathrm{g}, \mathrm{h}, \mathrm{i})$ perylene & 191-24-2 & 6.15E-01 & - & - & NA & - & NA & - \\
\hline Dibenz $(\mathrm{a}, \mathrm{h})$ anthracene & $53-70-3$ & $2.17 \mathrm{E}-01$ & - & - & NA & - & NA & - \\
\hline Phenanthrene & $85-01-8$ & $6.87 \mathrm{E}-01$ & - & - & NA & - & NA & - \\
\hline \multicolumn{9}{|c|}{ Dioxinz/furans } \\
\hline Heptachlorodibenzo-p-dioxin isomer & $37871-00-4$ & 4.11E-03 & $3.30 \mathrm{E}-01$ & - & $1.9 \mathrm{E}-11$ & $<1 \%$ & NA & - \\
\hline Heptachlorodibenzo-p-furan isomer & 38998-75-3 & $2.58 \mathrm{E}-04$ & $3.30 \mathrm{E}-01$ & - & $1.2 \mathrm{E}-12$ & $<1 \%$ & NA & - \\
\hline Hexachlorodibenzo-p-furan isomer & $55684-94-1$ & $1.00 \mathrm{E}-04$ & $3.30 \mathrm{E}+00$ & - & $4.6 \mathrm{E}-12$ & $<1 \%$ & NA & - \\
\hline Octachlorodibenzo-p-dioxin isomer & $3268-87-9$ & $9.26 \mathrm{E}-03$ & 3.30E-02 & - & 4.3E-12 & $<196$ & NA & - \\
\hline Octachlorodibenzo-p-furan isomer & $39001-02-0$ & $3.48 \mathrm{E}-04$ & 3.30E-02 & - & $1.6 \mathrm{E}-13$ & $<1 \%$ & NA & - \\
\hline \multicolumn{9}{|l|}{ PCBs } \\
\hline PCB 1254 & $11097-69-1$ & $1.78 \mathrm{E}-01$ & - & - & NA & - & NA & - \\
\hline \\
\hline delta-Benzene hexachloride & $\begin{array}{r}319-86-8 \\
53109-70-5\end{array}$ & $1.84 \mathrm{E}-03$ & - & - & NA & - & NA & - \\
\hline Endrin ketone & & $3.20 \mathrm{E}-03$ & - & - & NA & - & NA & - \\
\hline
\end{tabular}


Table D.20

Savannah River Site

Burning/Rubble Pits (231-F, 231-1F)

Future Worker

Inhalation of Resuspended Soil Particulate Contaminants (0-4'bls)

Carcinogenic and Noncarcinogenic Risk

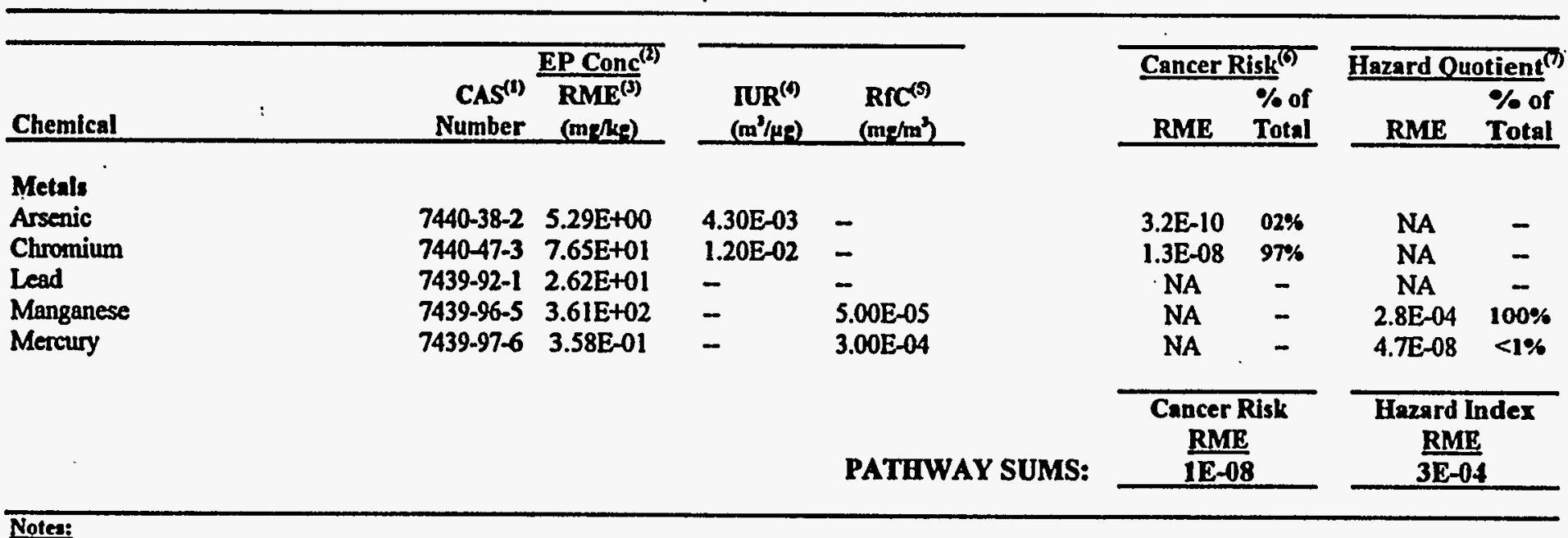

1. Chemical Abatract Service.

2. Expocure point concentration.

3. Reasonable maximum exposure

4. Inhalation unit risk.

5. Reference concentration.

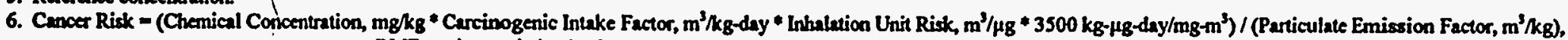
RME carcinosenic intake factor 8.74E- 03

Particulale emixsion factor = $2.18 \mathrm{E}+09$

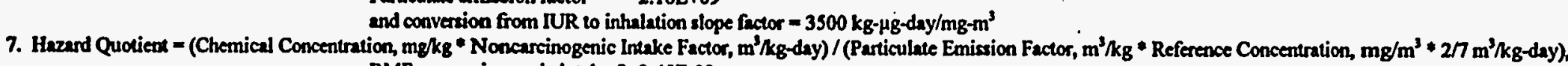
RME poncarcinogenic intake fa 2.45E-02

Particulate emiserion factor $=2.18 E+09$

and converion from RfC to inhalation reference doee $=2 / 7 \mathrm{~m}^{3} / \mathrm{kg}$-day 
Table D.21

Savannah River

Rubble Pit (231-2F)

Future Worker

Ingestion of Soil (0-2'b/s)

Carcinogenic and Noncarcinogenic Risk

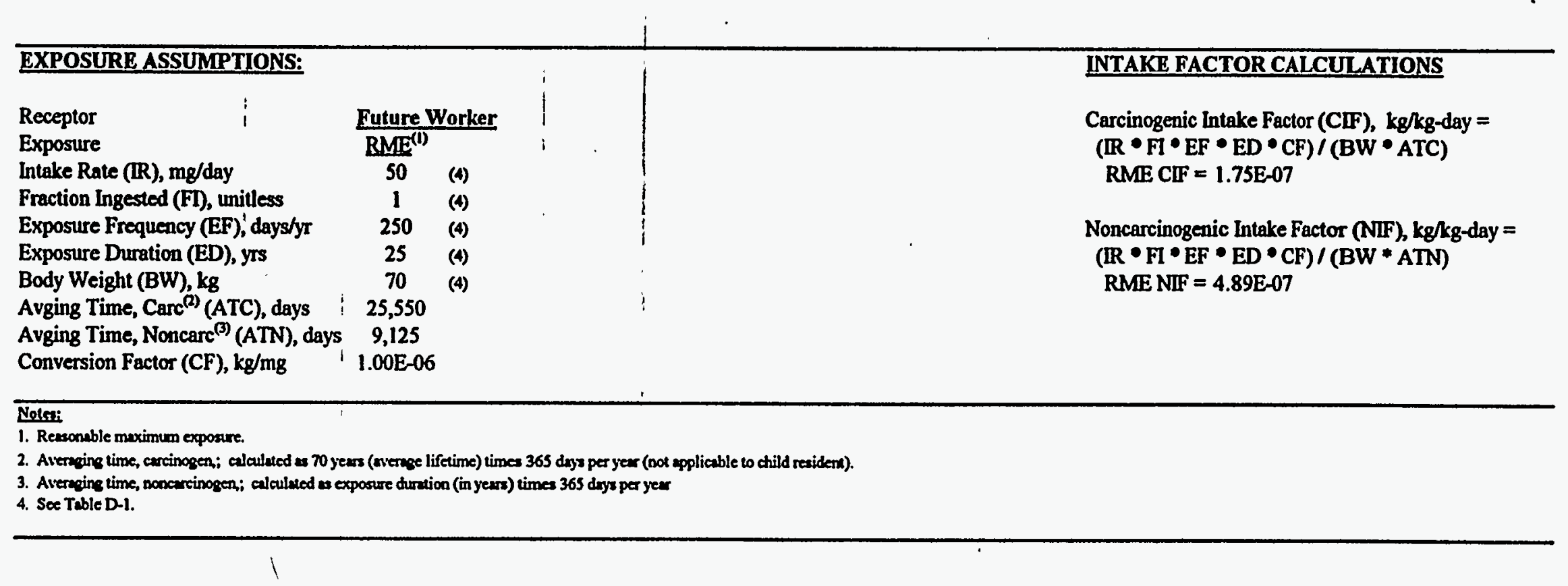


Table D.21

Savannah River

Rubble Pit (231-2F)

Future Worker

Ingestion of Soil (0-2'bls)

Carcinogenic and Noncarcinogenic Risk

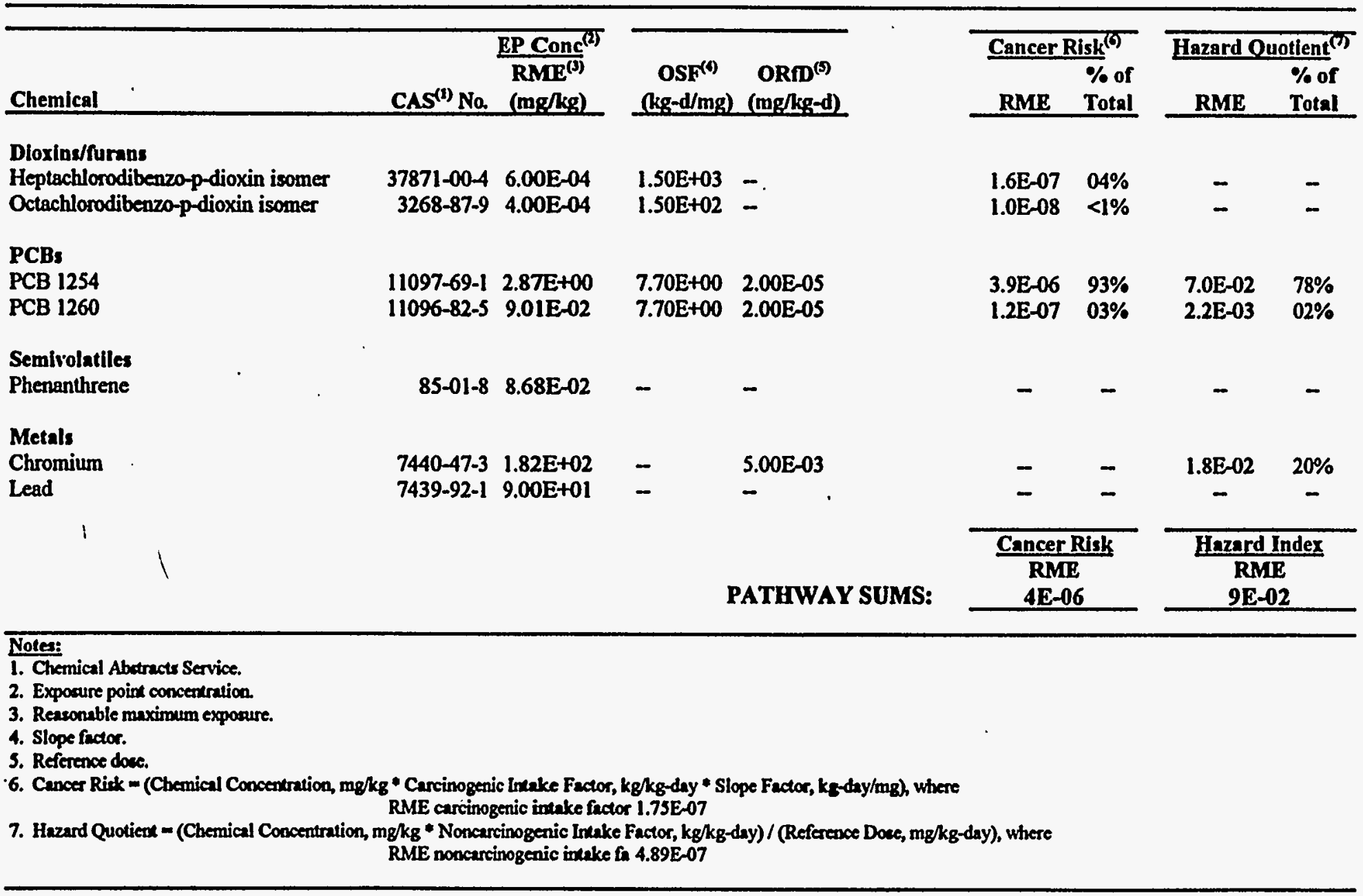


Table D.22

Savannah River Site

Rubble Pit (231-2F)

Future Worker

Dermal Exposure to Soil (0-2'bls)

Carcinogenic and Noncarcinogenic Risk

\section{EXPOSURE ASSUMPTIONS:}

Receptor

Exposure

Skin Surface Area (SA), $\mathrm{cm}^{2} /$ event

Soil-to-Skin Adherance (SK), $\mathrm{mg} / \mathrm{cm}^{2}$

Exposure Frequency (EF), eventstyr

Exposure Duration (ED), yrs

Body Weight (BW), kg

Avging Time, $\mathrm{Carc}^{(2)}$ (ATC), days

Avging Time, Noncarc ${ }^{(3)}$ (ATN), days

Conversion Factor (CF), kg/mg

Future Worker

$\mathrm{BME}^{(1)}$

3,200 (4)

0.2 (4)

250 (4)

25 (4)

$70 \quad$ (4)

25,550

9,125

1.00E-06

\section{INTAKE FACTOR CALCULATIONS}

Carcinogenic Intake Factor (CIF), kg/kg-day = $(\mathrm{SA} \bullet \mathrm{SK} \bullet \mathrm{EF} \bullet \mathrm{ED} \bullet \mathrm{CF}) /(\mathrm{BW} \bullet \mathrm{ATC})$

RME CIF $=2.24 \mathrm{E}-06$

Noncarcinogenic Intake Factor (NIF), kg/kg-day = $(\mathrm{SA} \bullet \mathrm{SK} * \mathrm{EF} \bullet \mathrm{ED} * \mathrm{CF}) /(\mathrm{BW} \bullet \mathrm{ATN})$

RME NIF $=6.26 \mathrm{E}-06$

Nolsen:

1. Ressonable maximum exposure.

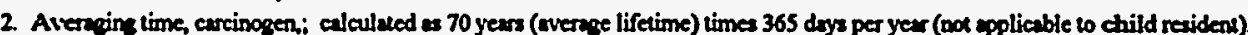

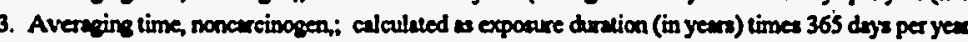

4.. See Trble D-1. 
Table D.22

Savannah River Site

Rubble Pit (231-2F)

Future Worker

Dermal Exposure to Soil (0-2'bls)

Carcinogenic and Noncarcinogenic Risk

\begin{tabular}{|c|c|c|c|c|c|c|c|c|c|}
\hline Chemical & $\begin{array}{r}\text { CAS(1) } \\
\text { Number }\end{array}$ & $\begin{array}{l}\operatorname{EPConc} c^{(2)} \\
\operatorname{RME}^{(3)} \\
(\mathrm{mg} / \mathrm{kg})\end{array}$ & $\begin{array}{c}\mathrm{DSF}^{(1)} \\
(\mathrm{kg}-\mathrm{d} / \mathrm{mg})\end{array}$ & $\begin{array}{c}\text { DRID }^{(\vartheta)} \\
\text { (mg/kg-d) }\end{array}$ & DABS $^{(0)}$ & Cancer I & $\begin{array}{l}\overline{i s k}^{(7)} \\
\% \text { of } \\
\text { Total }\end{array}$ & $\begin{array}{l}\text { Hazard Ou } \\
\text { RME }\end{array}$ & $\begin{array}{l}\text { tient }^{(3)} \\
\% \text { of } \\
\text { Total }\end{array}$ \\
\hline $\begin{array}{l}\text { Diorins/furans } \\
\text { Heptachlorodibenzo-p-dioxin isomer } \\
\text { Octachlorodibenzo-p-dioxin isomer }\end{array}$ & $\begin{array}{r}37871-00-4 \\
3268-87-9\end{array}$ & $\begin{array}{l}6.00 \mathrm{E}-04 \\
4.00 \mathrm{E}-04\end{array}$ & $\begin{array}{l}1.72 E+03 \\
1.72 E+02\end{array}$ & - & $\begin{array}{l}1.00 \mathrm{E}-02 \\
1.00 \mathrm{E}-02\end{array}$ & $\begin{array}{l}2.3 \mathrm{E}-08 \\
1.5 \mathrm{E}-09\end{array}$ & $\begin{array}{l}04 \% \\
<1 \%\end{array}$ & - & - \\
\hline $\begin{array}{l}\text { Semivolatiles } \\
\text { Phenanthrene }\end{array}$ & $85-01-8$ & $8.68 \mathrm{E}-02$ & - & - & $1.00 \mathrm{E}-02$ & - & - & - & - \\
\hline 1 & & & & PATHWAY & Y SUMS: & $\begin{array}{r}\text { Cancer } \\
\text { RM } \\
\text { 6E- }\end{array}$ & & $\begin{array}{r}\text { Hazurd } \\
\mathbf{R M} \\
\mathbf{E}-1\end{array}$ & ndex \\
\hline
\end{tabular}

Notea:

1. Chemical Abetract Service.

2. Exponure point concentration

3. Reasonable maximum exposure.

4. Slope fuctor, dermal.

5. Reference doue, dermal.

6. Abeorption fictor.

7. Cencer Risk = (Chemical Concentration, mg/kg * Carcinogenic Intake Factor, kg/kg-dny * Abeorption Factor, unitless * Slope Factor, kg-day/mg), where RME carcinogenic intake factor 2.24E-06

8. Hazard Quotient = (Chemical Concentration, mg/kg " Noncarcinogenic Intake Factor, kg/kg-day * Abcorption Factor, unitlens)/(Reference Doce, mg/kg-day), where RME noncercinogenic intake fa 6.26E-06 
Table D.23

Savannah River

Rubble Pit (231-2F)

Future Worker

Inhalation of Volatile Soil Contaminants (0-2'bls)

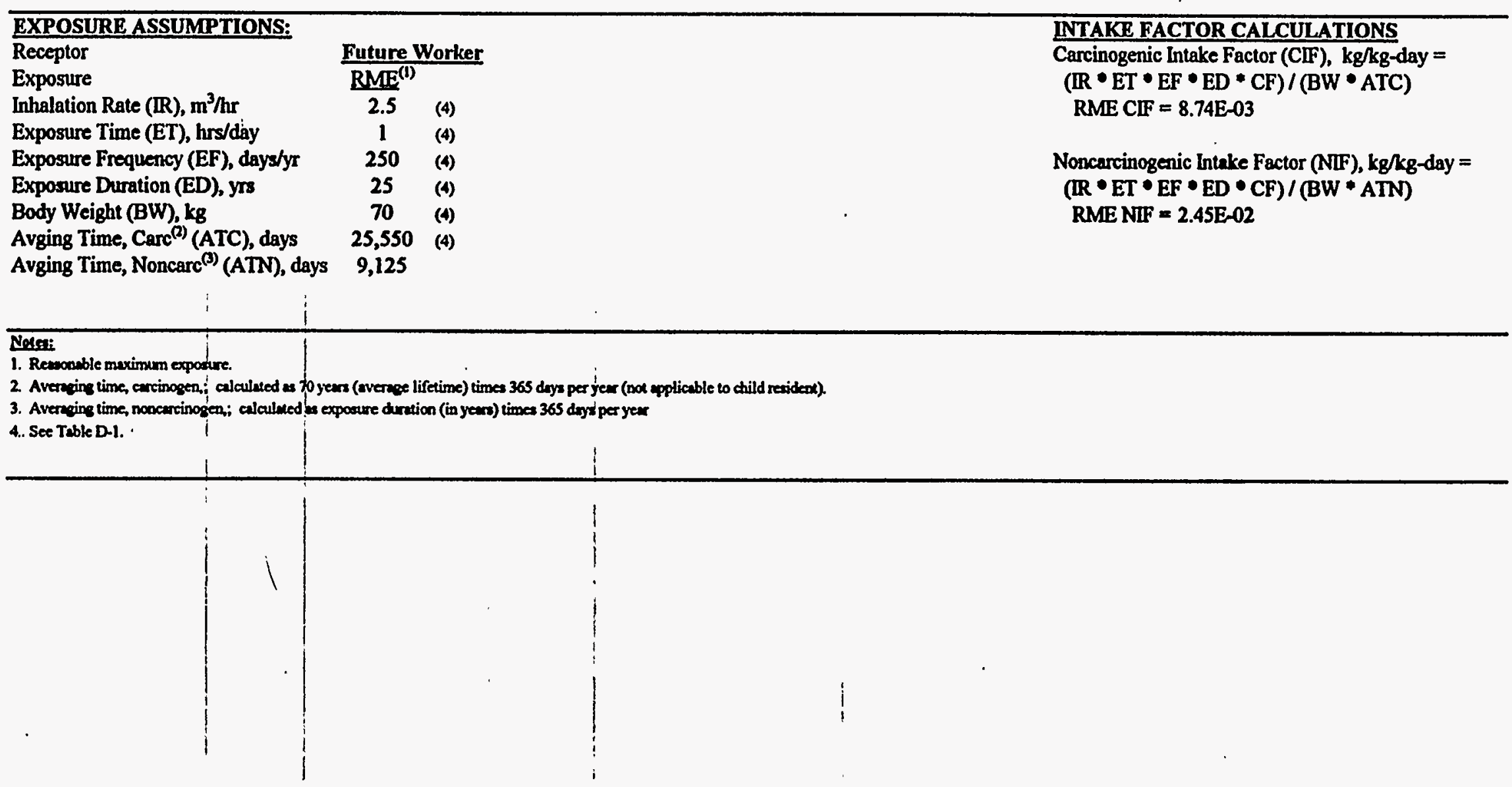


Table D.23

Savannah River

Rubble Pit (231-2F)

Future Worker

Inhalation of Volatile Soil Contaminants (0-2'bls)

Calculation of Volatilization Factor (VF)

\begin{tabular}{|c|c|c|c|c|c|c|c|c|c|c|c|c|}
\hline Chemical & : & $\mathrm{CAS}^{(2)}$ No. & Class ${ }^{(1)}$ & MolWt. & $\mathrm{Koc}^{(1)}$ & $\mathbf{K} \mathbf{d}^{(1)}$ & $H^{(t)}$ & $\mathrm{Kas}^{(1)}$ & $D^{(\theta)}$ & $\mathrm{De}^{(1)}$ & alpha(1) & $\begin{array}{l}\text { Volat. } \\
\text { Factor } \\
\left(\mathrm{m}^{3} / \mathrm{kg}^{(4)}\right)\end{array}$ \\
\hline $\begin{array}{l}\text { Semivolatiles } \\
\text { Phenanthrene }\end{array}$ & & $85-01-8$ & $\mathbf{s}$ & $1.78 E+02$ & 38904.5 & $7.78 \mathrm{E}+02$ & $2.56 \mathrm{E}-05$ & $1.35 \mathrm{E}-06$ & $5.74 \mathrm{E}-02$ & 4.05E-02 & $1.11 \mathrm{E}-08$ & $1.27 \mathrm{E}+06$ \\
\hline
\end{tabular}

\section{Noter:}

1. USEPA, 1991: RAGS: Vol 1 - Human Health Evaluation Menual (Part B, Development of Risk-Based Preliminary Remediation Gaals)

2. Chemical Abutract Service.

3. Chemical class: $v$-volatile; $s$ - semivolatile; $p$-pesticide/pcb; $x$ - dioxin; and m-metal.

4. Calculation of organic contaminant volutilization factor: CONSTANTS:

$$
\begin{aligned}
& \text { Length of side of contam'd area (LS), } m \text { = } \\
& \text { Wind speed in mixing zone }(V), \mathrm{m} / \mathrm{sec}= \\
& \text {. Diffusion heighk (DH), } m= \\
& \text { Area of contamination (Am), } \mathrm{m}^{2}= \\
& \text { Area in equare centimeters (Acm), } \mathrm{cm}^{2}= \\
& \text { True soil porosity (E), unitless = } \\
& \text { True soil density (Ps), } \mathrm{g} \mathrm{cm}^{3}= \\
& \text { Expocure imeerval (Tyr), yrs = } \\
& \text { Expocure interval (Trec), sec = }
\end{aligned}
$$

Organic cubon content $(O C)$, unitless =

Conversion factor (CF), $\mathrm{kg} / \mathrm{g}=$

INPUT VARIABLES:

Organic carbon partition coef. $(\mathrm{Koc}), \mathrm{cm}^{3} / \mathrm{k}_{\mathrm{B}}=$ Henry's Law constant $(\mathrm{H})$, atm- $\mathrm{m}^{3} / \mathrm{mol}=$ Gas diffusivity (Di), $\mathrm{cm}^{2} / 2 e c=$

INTERMEDIATE STEPS:

Soil-water partition coefficient (Kd), $\mathrm{cm}^{3} / \mathrm{kg}_{\mathrm{B}}=$ Soilair partition coefficient (Kes), \&-coil/ $\mathrm{cm}^{3}-2$ ir =

Effective diffusivity (Dei), $\mathrm{cm}^{2} / \mathrm{soc}=$ alpha (alpha), $\mathrm{cm}^{2} / 200=$

OUTPUT:

Volatilization Factor (VF), $\mathrm{m}^{3} / \mathrm{kg}_{\mathbf{B}}=$

\author{
$65.00 \quad(1)$ \\ $3.60 \quad(1)$ \\ $1.66 \quad(1)$ \\ $4,200.00$ (1) \\ 4.20E+07 (Am $\left.\cdot 10,000 \mathrm{~cm}^{2} / \mathrm{m}^{2}\right)$ \\ $0.350 \quad(1)$ \\ 2.650 (1) \\ $25.00 \quad(1)$ \\ $7.88 \mathrm{E}+08(\mathrm{Tyr} * 31536000 \mathrm{sec} / \mathrm{yr})$ \\ 0.020
}

0.001

chem-epec

chem-spec

chem-spec (Koc $\times \mathrm{OC})$

chem-spec $((\mathrm{H} / \mathrm{Kd}) \times 41)$

chem-4pec (Di $\times\left(E^{\prime} 0.33\right)$ )

chem-ppes $(($ Dei $\times E)(E+($ PB $)(1-E)) / K e s)$

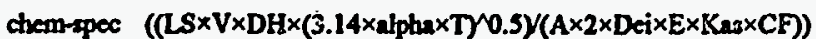


Table D.23

Savannah River

Rubble Pit (231-2F)

Future Worker

Inhalation of Volatile Soil Contaminants (0-2'bls)

Carcinogenic and Noncarcinogenic Risk

\begin{tabular}{|c|c|c|c|c|c|c|c|c|c|}
\hline Chemical & $:$ & 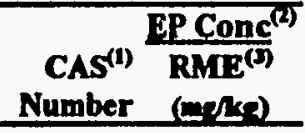 & 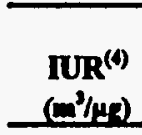 & $\begin{array}{l}\operatorname{RrC}^{(0)} \\
\text { (nertm) }\end{array}$ & $\begin{array}{c}V F^{(0)} \\
\left(m^{3} /(x)\right.\end{array}$ & $\begin{array}{l}\text { Cancer } \\
\text { RMR } \\
\end{array}$ & $\begin{array}{l}\text { inle } \\
\% \text { of } \\
\text { Total } \\
\end{array}$ & \multicolumn{2}{|c|}{ Hazard Ouotient ${ }^{(3)}$} \\
\hline \multirow[t]{2}{*}{$\begin{array}{l}\text { Semivolutiles } \\
\text { Phenmthrene }\end{array}$} & $85-01-8$ & 6.87E-01 & - & - & $1.27 E+06$ & NA & - & NA & - \\
\hline & & , & 1. & PATHWA & SUMS: & $\begin{array}{r}\text { Cance } \\
\text { RS } \\
\end{array}$ & Risk & $\begin{array}{r}\text { Hazand } \\
\mathbf{R y} \\
\end{array}$ & adex \\
\hline
\end{tabular}

Nexp:

1. Chemical Abotrea Service.

2. Exposure point concentration

3. Retconable maximum exposure

4. Inhalation unit riak

5. Reference concentration.

6. Volatilization factor (chemical-apecific).

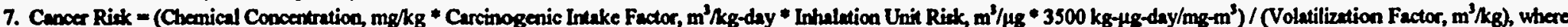
RME carcinogenic intake fector 8.74E-03

and conversion from IUR to inhalation slope fuctor $=3500 \mathrm{ks}-4 \mathrm{~g}-\mathrm{day} / \mathrm{mg}-\mathrm{m}$

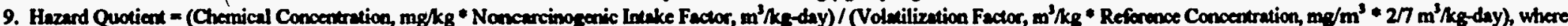
RME noncercinogenic intake fi 2.45E-02

and convertion from $\mathrm{RPC}$ Lo inhlalation reference dowe $=2 / 7 \mathrm{~m}^{3} / \mathrm{kg}$ - day 
Table D.24

Savannah River Site

Rubble Pit (231-2F)

Future Worker

Inhalation of Resuspended Soil Particulate Contaminants (0-2'bls)

Carcinogenic and Noncarcinogenic Risk

\begin{tabular}{|c|c|c|}
\hline \multicolumn{3}{|l|}{ EXPOSURE ASSUMPTIONS: } \\
\hline Receptor & Future & arke \\
\hline Exposure & $\mathrm{RME}^{(1)}$ & \\
\hline Inhalation Rate (IR), $\mathrm{m}^{3} \mathrm{hr}$ & 2.5 & (4) \\
\hline Exposure Time (ET), hrs/day & 1 & (4) \\
\hline Exposure Frequency (EF), days/yr & 250 & (4) \\
\hline Exposure Duration (ED), yrs & 25 & (4) \\
\hline Body Weight (BW), kg & 70 & (4) \\
\hline Avging Time, Carc (2) (ATC), days & 25,550 & \\
\hline Avging Time, Noncarc ${ }^{(3)}$ (ATN), days & 9,125 & \\
\hline
\end{tabular}

INTAKE FACTOR CALCULATIONS

Carcinogenic Intake Factor (CIF), $\mathrm{kg} / \mathrm{kg}$-day = $(\mathrm{R} * \mathrm{FI} \bullet \mathrm{EF} * \mathrm{ED} * \mathrm{CF}) /(\mathrm{BW} * \mathrm{ATC})$

RME CIF $=8.74 \mathrm{E}-03$

Noncarcinogenic Intake Factor (NIF), $k g / k g$-day = $\left(\mathbb{R}^{\bullet} \mathrm{FI} \bullet \mathrm{EF} \bullet \mathrm{ED} \bullet \mathrm{CF}\right) /(\mathrm{BW} \bullet \mathrm{ATN})$

RME NIF $=2.45 \mathrm{E}-02$

Noter:

1. Ressonable maximum exposume

2. Averaing time, carcinogen; calculued as 70 years (everwe lifetime) times 365 deys per year (not appliesble to child rexideno).

3. Averaing time, noncarcinogen; calculued as exposure duration (in years) times 365 doys per year

4. See Table D-1. 
Table D.24

Savannah River Site

Rubble Pit (231-2F)

Future Worker

Inhalation of Resuspended Soil Particulate Contaminants (0-2'bls)

Carcinogenic and Noncarcinogenic Risk

\section{CALCULATION OF SOIL PARTICULATE RESUSPENSION FACTOR:}

\section{CONSTANTS:}

Length of side of contaminated area (LS) =

Wind speed in mixing zone $(V)=$

Diffusion height $(\mathrm{DH})=$

Area of contamination $(A)=$

Respirable fraction (RF) =

Fraction of vegetative cover $(G)=$

Mean annual wind speed (Um) =

Equivalent threshold value of wind speed at $10 \mathrm{~m}(\mathrm{Ut})=$

Fraction dependent on Um/Ut (Fx) =

Conversion factor $(\mathrm{CFa})=$

Conversion factor $(\mathrm{CFb})=$

$\begin{array}{lll}24.00 & \text { meters } & \text { (1) } \\ 3.60 & \text { meters/sec } & \text { (1) } \\ 1.66 & \text { meters } & \text { (1) } \\ 500.00 & \text { meters } & \text { (1) } \\ 0.003 & \text { g/meters }{ }^{2} \text { hr } & \text { (1) } \\ 0.90 & \text { unitless } & \text { (1) } \\ 3.60 & \text { meters/sec } & \text { (1) } \\ 5.04 & \text { meters/sec } & \text { (1) } \\ 1.2700 & \text { unitless } & \text { (1) } \\ 3,600.00 & \text { sec/hr } & \\ 1,000.00 & \text { g/kg } & \end{array}$

Nefri:

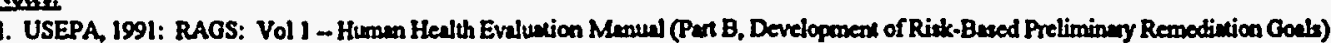

\section{INTERMEDIATE STEPS:}

Particulate emission factor (PEF) $=\quad 6.76 \mathrm{E}+09$ meters $^{3} / \mathrm{kg}$

$(L S \times V \times D H \times C F a \times C F b) /\left(A \times R F \times(1-G) \times\left((U m / U t)^{\wedge}\right) \times(F \times)\right)$ 
Table D.24

Savannah River Site

Rubble Pit (231-2F)

Future Worker

Inhalation of Resuspended Soil Particulate Contaminants (0-2'bls)

Carcinogenic and Noncarcinogenic Risk

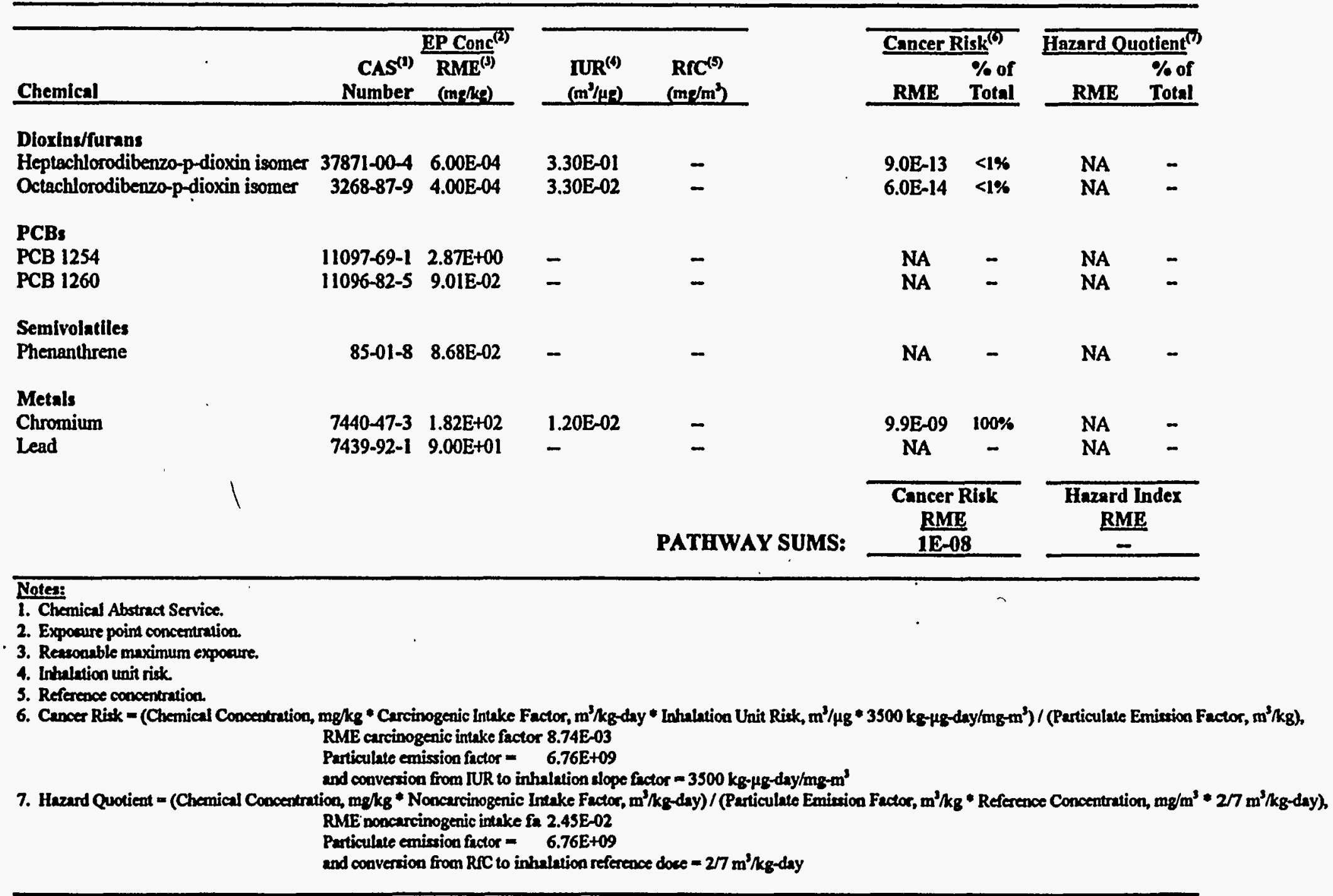


Table D.25

Savannah River Site

Rubble Pit (231-2F)

Future Worker

Ingestion of Soil (0-4'bls)

Carcinogenic and Noncarcinogenic Risk

EXPOSURE ASSUMPTIONS:

\section{INTAKE FACTOR CALCULATIONS}

\section{Receptor}

Exposure

Intake Rate (IR), mg/day

Fraction Ingested (FI), unitless

Exposure Frequency (EF), days/yr

Exposure Duration (ED), yrs

Body Weight (BW), kg

Avging Time, Carc ${ }^{(2)}$ (ATC), days

Avging Time, Noncarc ${ }^{(3)}$ (ATN), days

Conversion Factor (CF), kg/mg

\section{Future Worker}

$\mathrm{BME}^{(1)}$

50 (4)

$1 \quad(4)$

$250 \quad(4)$

$25 \quad$ (4)

70 (4)

25,550

9,125

$1.00 \mathrm{E}-06$
Carcinogenic Intake Factor (CIF), kg/kg-day = $(\mathbb{R} \bullet \mathrm{Fl} \bullet \mathrm{EF} \bullet \mathrm{ED} \bullet \mathrm{CF}) /(\mathrm{BW} \bullet \mathrm{ATC})$ RME CFF $=1.75 E-07$

Noncarcinogenic Intake Factor (NIF), kg/kg-day = $(\mathrm{R} \bullet \mathrm{FI} \bullet \mathrm{EF} \bullet \mathrm{ED} \bullet \mathrm{CF}) /(\mathrm{BW} * \mathrm{ATN})$

RME NIF $=4.89 \mathrm{E}-07$

Noleti:

1. Ressoneble maximum exposure.

2. Averaging time, carcinogen; calculated as 70 years (nverwoe lifetime) times 365 day per year (not eppliesble to child resident).

3. Averaing time, noncercinogen; calculued as exposure duration (in yems) times 365 days per year

4. See Table D-1. 
Table D.25

Savannah River Site

Rubble Pit (231-2F)

Future Worker

Ingestion of Soil (0-4'bls)

Carcinogenic and Noncarcinogenic Risk

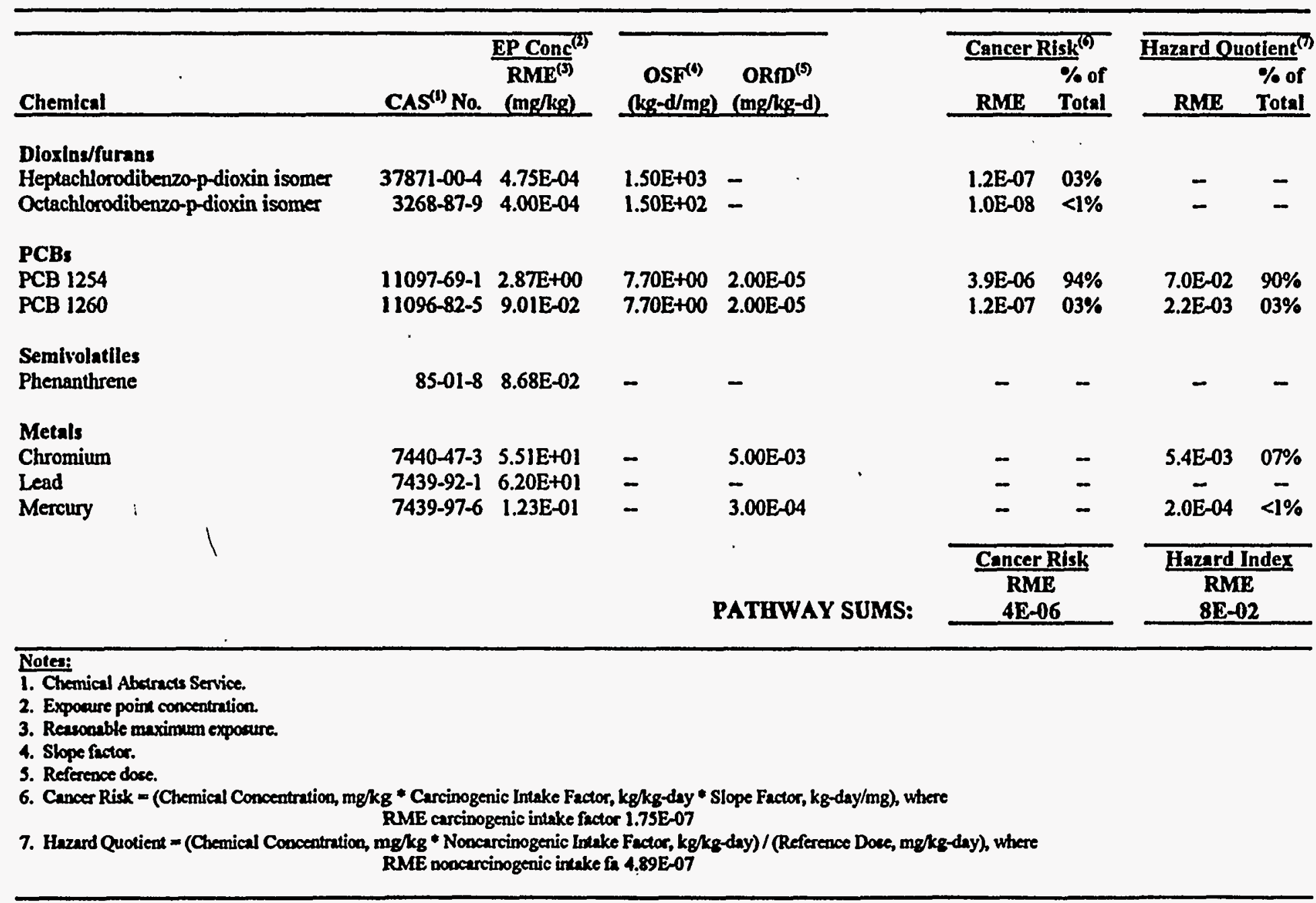


Table D.26

Savannah River Site

Rubble Pit (231-2F)

Future Worker

Dermal Exposure to Soil (0-4'bls)

Carcinogenic and Noncarcinogenic Risk

\section{EXPOSURE ASSUMPTIONS:}

\begin{tabular}{|c|c|c|}
\hline \multirow{2}{*}{$\begin{array}{l}\text { Receptor } \\
\text { Exposure }\end{array}$} & \multirow{2}{*}{\multicolumn{2}{|c|}{$\frac{\text { Future Worker }}{\text { RMF }^{(1)}}$}} \\
\hline & & \\
\hline Skin Surface Area (SA), $\mathrm{cm}^{2} /$ event & 3,200 & (4) \\
\hline Soil-to-Skin Adherance (SK), $\mathrm{mg} / \mathrm{cm}^{2}$ & 0.2 & (4) \\
\hline Exposure Frequency (EF), cvents/yr & 250 & (4) \\
\hline Exposure Duration (ED), yrs & 25 & (4) \\
\hline Body Weight (BW), kg & 70 & (4) \\
\hline Avging Time, $\operatorname{Carc}^{(2)}$ (ATC), days & 25,550 & \\
\hline Avging Time, Noncarc ${ }^{(3)}$ (ATN), days & 9,125 & \\
\hline Conversion Factor (CF), kg/mg & $1.00 \mathrm{E}-06$ & \\
\hline
\end{tabular}

\section{INTAKE FACTOR CALCULATIONS}

Carcinogenic Intake Factor (CIF), kg/kg-day = $(\mathrm{SA} * \mathrm{SK} * \mathrm{EF} * \mathrm{ED} * \mathrm{CF}) /(\mathrm{BW} * \mathrm{ATC})$ RME CIF $=2.24 \mathrm{E}-06$

Noncurcinogenic Intake Factor (NIF), kg/kg-day = $(\mathrm{SA} * \mathrm{SK} \bullet \mathrm{EF} \bullet \mathrm{ED} * \mathrm{CF}) /(\mathrm{BW} \bullet \mathrm{ATN})$

RME NIF $=6.26 \mathrm{E}-06$

Netse:

1. Reuromble maximum expoure.

2. Averaing time, carcinogen,; caleuleted as 70 years (nvernge lifetime) times 365 days per yeur (nox spplicable to child resident).

3. Avernaing time, noncircinogen; calculeled as exposure durmion (in yeurs) times 365 deys par year

4. See Table D-1. 
Table D.27

Savannah River Site

Rubble Pit (231-2F)

Future Worker

Inhalation of Volatile Soil Contaminants (0-4'bls)

\begin{tabular}{|c|c|c|}
\hline \multicolumn{3}{|l|}{ JMPTIONS: } \\
\hline & $\frac{\text { Future }}{\mathrm{RME}^{(1)}}$ & Yorke \\
\hline ) $\mathrm{m}^{3} / \mathrm{hr}$ & 2.5 & (4) \\
\hline D), hrs/day & 1 & (4) \\
\hline$y(E F)$, days/yr & 250 & (4) \\
\hline (ED), yrs & 25 & (4) \\
\hline . kg & 70 & (4) \\
\hline (2) (ATC), days & 25,550 & (4) \\
\hline
\end{tabular}

INTAKE FACTOR CALCULATIONS

Carcinogenic Intake Factor (CIF), kg/kg-day =

$(\mathrm{R} * \mathrm{ET} * \mathrm{EF} * \mathrm{ED} * \mathrm{CF}) /(\mathrm{BW} \bullet \mathrm{ATC})$

RME CIF $=8.74 \mathrm{E}-03$

Noncarcinogenic Intake Factor (NIF), kg/kg-day = $(\mathrm{R} \bullet \mathrm{ET} \bullet \mathrm{EF} \bullet \mathrm{ED} \bullet \mathrm{CF}) /(\mathrm{BW} \bullet \mathrm{ATN})$

RME NIF $=2.45 \mathrm{E}-02$

\section{exposurc.}

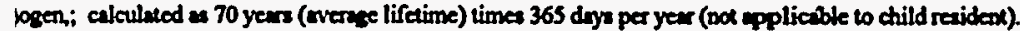

reinogen; celcultued as expoutre dration (in years) times 365 days per year 
Table D.28

Savannah River Site

Rubble Pit (231-2F)

Future Worker

Inhalation of Resuspended Soil Particulate Contaminants (0-4'bls)

Carcinogenic and Noncarcinogenic Risk

\begin{tabular}{|c|c|c|}
\hline \multicolumn{2}{|l|}{ EXPOSURE ASSUMPTIONS: } & INTAKE FACTOR CALCULATIONS \\
\hline Receptor & Future Worker & Carcinogenic Intake Factor (CIF), kg/kg-day = \\
\hline Exposure & $\mathrm{RME}^{(1)}$ & $(\mathbb{R} \bullet \mathrm{FI} \bullet \mathrm{EF} * \mathrm{ED} \bullet \mathrm{CF}) /(\mathrm{BW} \bullet \mathrm{ATC})$ \\
\hline Inhalation Rate (IR), $\mathrm{m}^{3} \mathrm{hr}$ & 2.5 & RME CIF $=8.74 \mathrm{E}-03$ \\
\hline Exposure Time (ET), hrs/day & 1 & \\
\hline Exposure Frequency (EF), days/yr & 250 & Noncarcinogenic Intake Factor (NIF), kg/kg-day = \\
\hline Exposure Duration (ED), yrs & 25 & $(\mathbb{R} \bullet \mathrm{FI} \bullet \mathrm{EF} \bullet \mathrm{ED} \bullet \mathrm{CF}) /(\mathrm{BW} \bullet \mathrm{ATN})$ \\
\hline Body Weight (BW), kg & 70 & $\mathrm{RME}$ NIF $=2.45 \mathrm{E}-02$ \\
\hline Avging Time, Carc $^{(2)}$ (ATC), days & 25,550 & \\
\hline Avging Time, Noncarc ${ }^{(3)}($ ATN), days & 9,125 & \\
\hline
\end{tabular}

\section{Neteri:}

I. Ressonable meximum exposure.

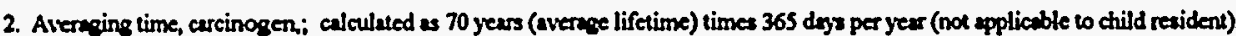

3. Averaging time, noncarcinogen; calculated as exposure duration (in years) times 365 days per year

4. See Table D-1. 
Table D.28

Savannah River Site

Rubble Pit (231-2F)

Future Worker

Inhalation of Resuspended Soil Particulate Contaminants (0-4'bls)

Carcinogenic and Noncarcinogenic Risk

\section{CALCULATION OF SOIL PARTICULATE RESUSPENSION FACTOR:}

\section{CONSTANTS:}

Length of side of contaminated area (LS) $=$

Wind speed in mixing zone $(\mathrm{V})=$

Diffusion height $(\mathrm{DH})=$

Area of contamination $(A)=$

Respirable fraction (RF) $=$

Fraction of vegetative cover $(G)=$

Mean annual wind speed $(\mathrm{Um})=$

Equivalent threshold value of wind speed at $10 \mathrm{~m}(\mathrm{Ut})=$

Fraction dependent on $\mathrm{Um} / \mathrm{Ut}(\mathrm{Fx})=$

Conversion factor $(\mathrm{CFa})=$

Conversion factor $(\mathrm{CFb})=$
24.00

3.60

1.66

500.00

0.003

0.90

3.60

5.04

1.2700

$3,600.00$

$1,000.00$

\begin{tabular}{|c|c|}
\hline meters & (1) \\
\hline meters/sec & (1) \\
\hline meters & (1) \\
\hline meters $^{2}$ & (1) \\
\hline$g /$ meters ${ }^{2}-\mathrm{hr}$ & (l) \\
\hline unitless & (1) \\
\hline meters/sec & (1) \\
\hline meters/sec & (1) \\
\hline unitless & (1) \\
\hline $\mathrm{sec} / \mathrm{hr}$ & \\
\hline g/kg & \\
\hline
\end{tabular}

\section{INTERMEDLATE STEPS:}

Particulate emission factor $(\mathrm{PEF})=6.76 \mathrm{E}+09$ meters ${ }^{3} / \mathrm{kg}$ $(\mathrm{LS} \times V \times \mathrm{DH} \times \mathrm{CFa} \times \mathrm{CFb}) /\left(\mathrm{A} \times \mathrm{RF} \times(1-G) \times\left((\mathrm{Um} / \mathrm{Ut})^{\wedge} 3\right) \times(\mathrm{Fx})\right)$

Notes:

1. USEPA, 1991: RAGS: Vol I - Human Heath Evaluation Manual (Part B. Development of Risk-Bated Preliminury Remediation Goals) 
Table D.28

Savannah River Site

Rubble Pit (231-2F)

Future Worker

Inhalation of Resuspended Soil Particulate Contamingnts (0-4'bls)

Carcinogenic and Noncarcinogenic Risk

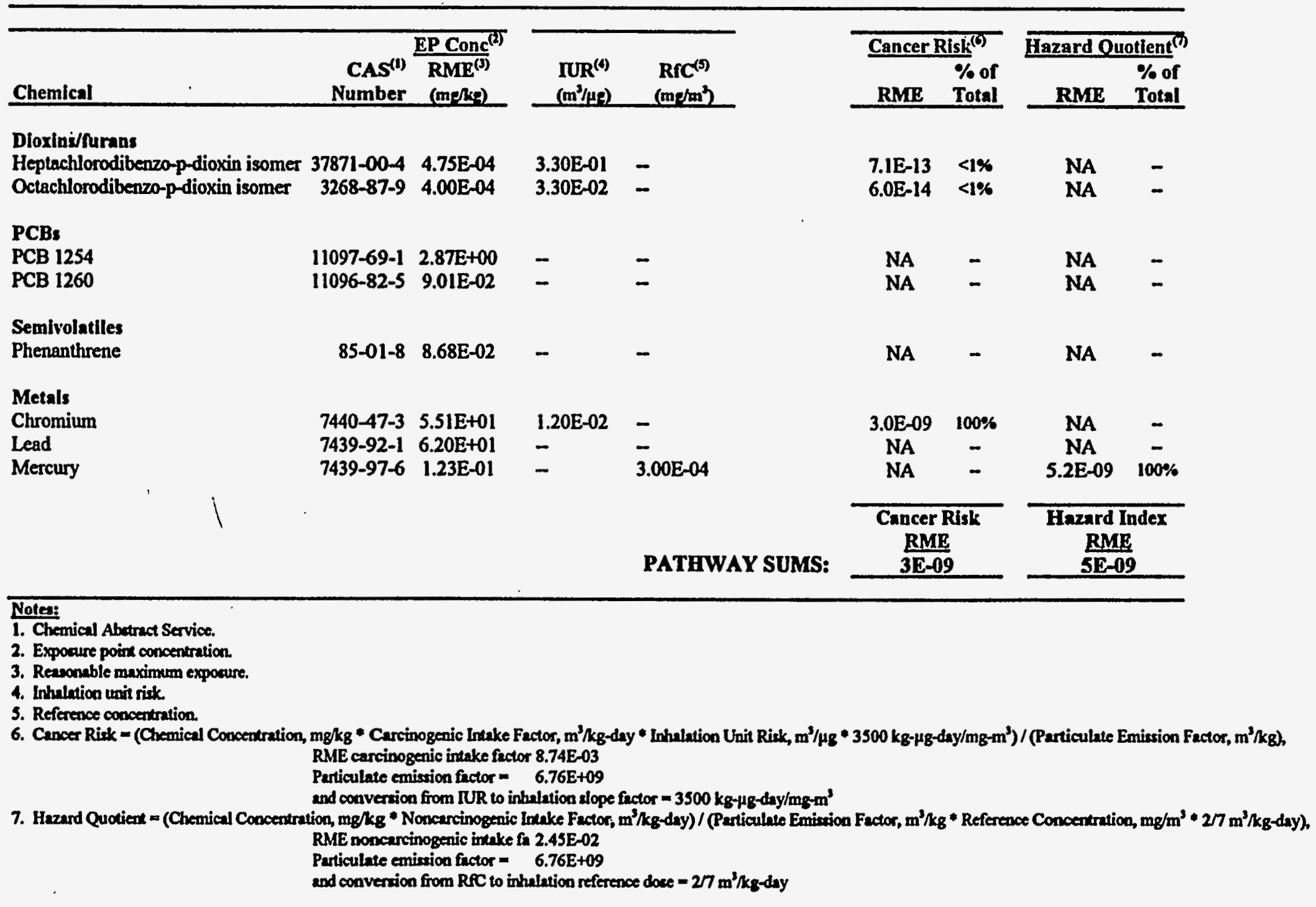


Table D.29

Savannah River

Burning/Rubble Pits (231-F, 231-1F, 231-2F)

Future Worker

Ingestion of Ground Water

Carcinogenic and Noncarcinogenic Risk

\begin{tabular}{lccc}
\hline EXPOSURE ASSUMPTIONS: & & \\
\hline Receptor & Future Worke \\
Exposure & RME $^{(1)}$ & \\
Intake Rate (IR), L/day & 1 & $(4)$ \\
Exposure Frequency (EF), days/yr & 250 & $(4)$ \\
Exposure Duration (ED), yrs & 25 & $(4)$ \\
Body Weight (BW), kg & 70 & $(4)$ \\
Avging Time, Car ${ }^{(2)}$ (ATC), days & 25,550 & \\
Avging Time, Noncarc $^{(3)}$ (ATN), days & 9,125 &
\end{tabular}

INTAKE FACTOR CALCULATIONS

Carcinogenic Intake Factor (CFF), L/kg-day =

$(\mathrm{IR} \bullet \mathrm{EF} \bullet \mathrm{ED}) /(\mathrm{BW} \bullet \mathrm{ATC})$

$\mathrm{RME}$ CIF $=3.49 \mathrm{E}-03$

Noncarcinogenic Intake Factor (NIF), L/kg-day =

$(\mathrm{IR} \bullet \mathrm{EF} \bullet \mathrm{ED}) /(\mathrm{BW} \bullet \mathrm{ATN})$

RME NIF $=9.78 E-03$

\section{Netri:}

1. Rewonsble muximum exposure.

2. Averaing time, carcinogen; calculued as 70 yems (wenase lifetime) times 365 dys per year (not upplicable to child resident).

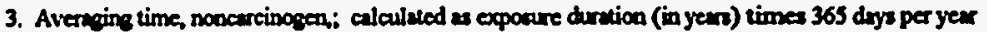

4. See Teble D-1. 
Table D.29

Savannah River

Burning/Rubble Pits (231-F, 231-1F, 231-2F)

Future Worker

Ingestion of Ground Water

Carcinogenic and Noncarcinogenic Risk

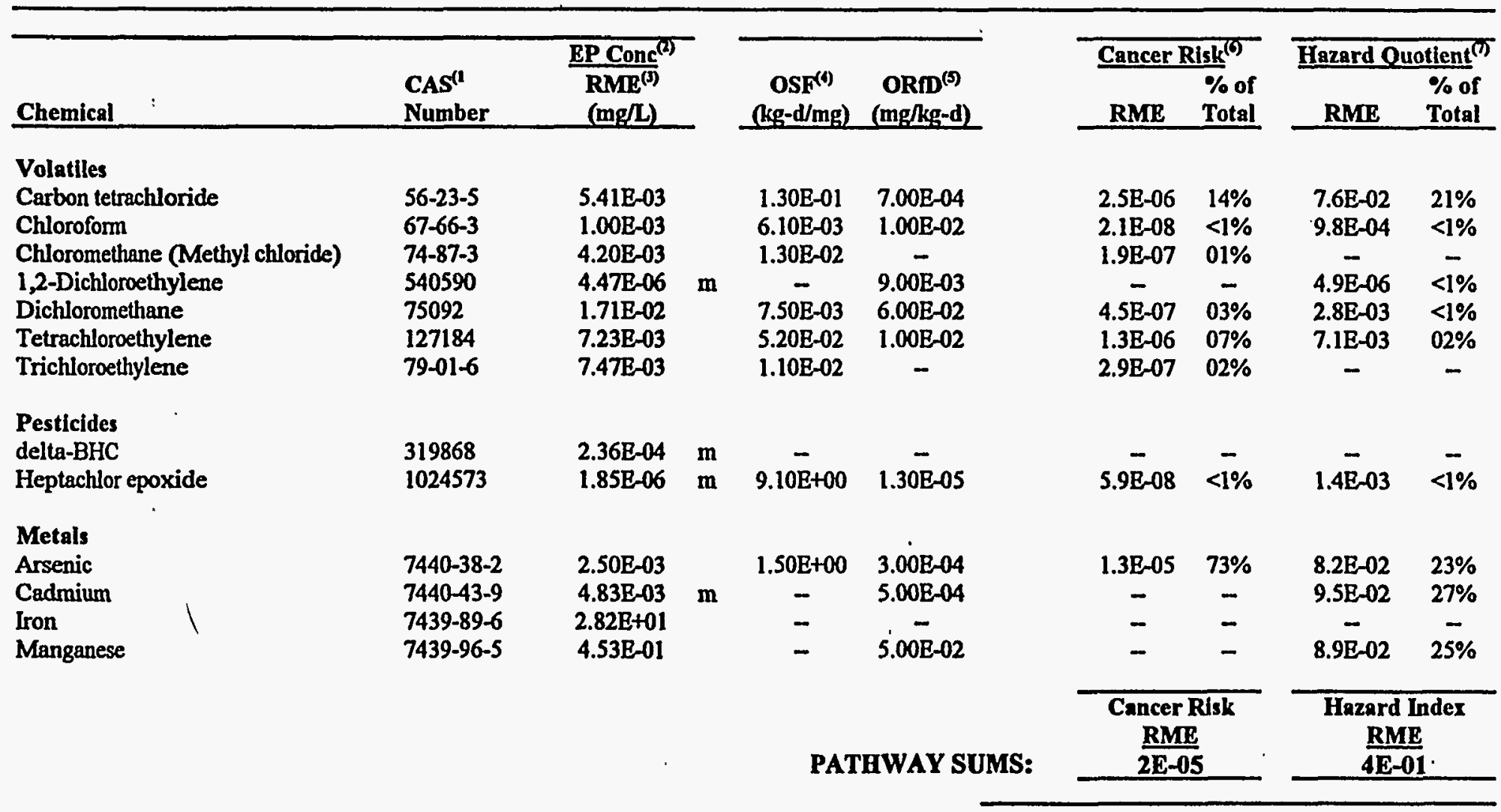

2. Exposure point concentration.

3. Reasonable maximum exposure; " $\mathrm{m}$ " indicates modeled concentration.

4. Slope factor, oral.

5. Reference dose, oral.

6. Cancer Risk = (Chemical Concentration, mg/L * Carcinogenic Intake Pactor, L/kg-day * Slope Factor, kg-day/mg), whace RME carcinogenic intake frctor $=3.49 \mathrm{E}-03$

7. Hazard Quotient = (Chemical Conocntration, mg/L * Noncarcinogenic Intake Factor, L/kg-day) / (Reference Doee, mg/kg-day), where

RME noncencinogenic intake factor $=9.78 \mathrm{E}-03$ 
Table D.30

Savannah River. Site

Burning/Rubble Pits (231-F, 231-1F)

Combined Adult/Child Resident (Future)

Ingestion of Soil (0-2'bls)

Carcinogenic and Noncarcinogenic Risk

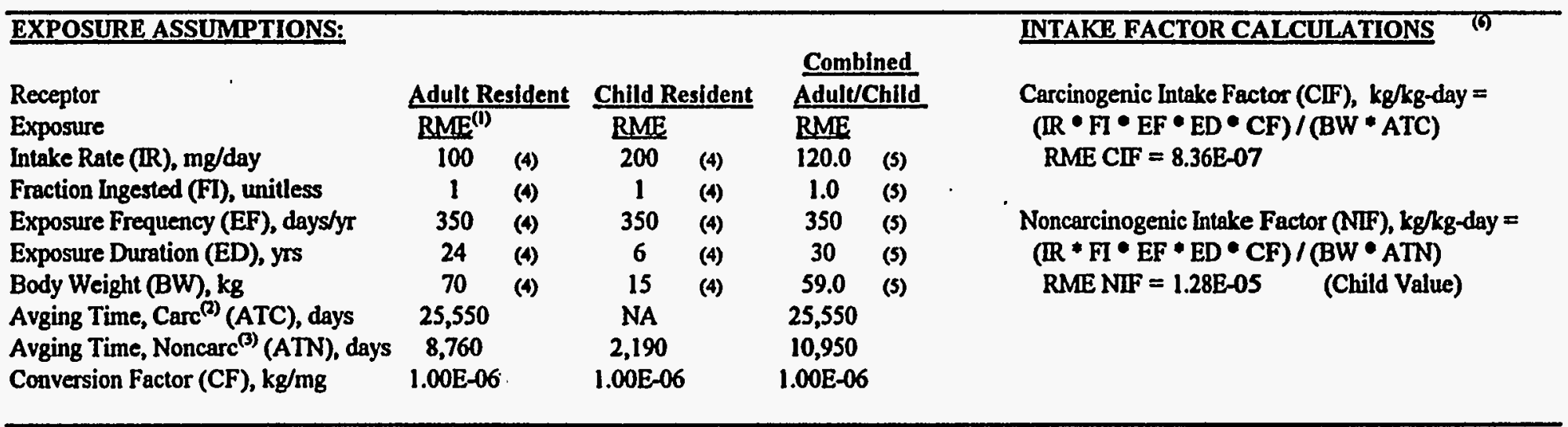

Noter:

1. Ressonable maximum exposure.

2. Averaing time, carcinogen; calculsted as 70 years (nverae lifetime) times 365 dayz per year (not upplicable to child rexiderx).

3. Averning time, noncercinogen; calculued as exposure duration (in years) times 365 days per year

4. See Teble D-1.

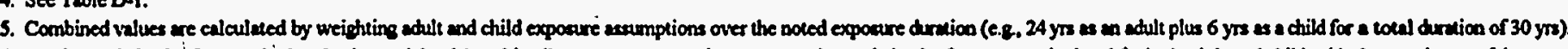

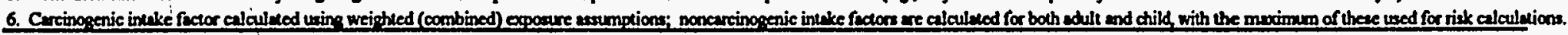


Table D.30

Savannah River Site

Burning/Rubble Pits (231-F, 231-1F)

Combined Adult/Child Resident (Future)

Ingestion of Soil (0-2'b/s)

Carcinogenic and Noncarcinogenic Risk

\begin{tabular}{|c|c|c|c|}
\hline Chemical & $\mathrm{CAS}^{(1)} \mathrm{Na}$ & $\begin{array}{c}\text { EP Conc } c^{(2)} \\
\text { RME }^{(j)} \\
(\mathrm{mg} / \mathrm{kg})\end{array}$ & 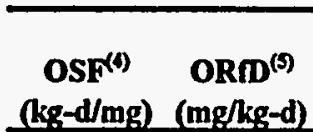 \\
\hline
\end{tabular}

\section{Semivolatiles}

\section{$91-57-6 \quad 7.69 \mathrm{E}-02$}

2-Methylnaphthalene

Acenaphthylene

Benzo(a)anthracene

Benzo(a)pyrene

Benzo(b)fluoranthene

Benzo(g,h,i)perylene

Dibenz(a,h)anthracene

208-96-8 8.61E-02

56-55-3 9.26E-01

$50-32-8 \quad 6.49 \mathrm{E}-01$

205-99-2 1.01E+00

191-24-2 6.15E-01

53-70-3 2.17E-01

Phenanthrene

85-01-8 $\quad 6.87 E-01$

$-$

$7.30 \mathrm{E}-01$

$7.30 \mathrm{E}+00$ -

7.30E-01 -

$-$

$7.30 \mathrm{E}+00$

\section{Dloxins/furans}

Heptachlorodibenzo-p-dioxin isomer 37871-00-4 4.11E-03

Heptachlorodibenzo-p-furan isomer 38998-75-3 2.58E-04

Hexachlorodibenzo-p-furan isomer 55684-94-1 1.00E-04

Octachlorodibenzo-p-dioxin isomer $\quad 3268-87-9$ 9.26E-03

Octachlorodibenzo-p-furan isomer

39001-02-0 3.48E-04

PCBs

PCB 1254

11097-69-1 1.78E-01

319-86-8 1.84E-03

$53494-70-5 \quad 3.20 \mathrm{E}-03$

\section{Pesticides}

delta-Benzene hexachloride

Endrin ketone
7.70E+00 2.00E-05

$1.50 \mathrm{E}+03-$

$1.50 \mathrm{E}+03-$

$1.50 \mathrm{E}+04-$

$1.50 \mathrm{E}+02$

$1.50 \mathrm{E}+02$

\begin{tabular}{|c|c|}
\hline Cancer Risk ${ }^{(\mathrm{S})}$ & Hazard Quotient $^{(7)}$ \\
\hline $\begin{array}{cc}\% \text { of } \\
\text { RME } \quad \text { Total } \\
\end{array}$ & $\begin{array}{cc} & \% \text { of } \\
\text { RME } & \text { Total } \\
\end{array}$ \\
\hline
\end{tabular}

$\begin{array}{cccc}- & - & - & - \\ 5.6 \mathrm{E}-07 & 03 \% & - & - \\ 4.0 \mathrm{E}-06 & 18 \% & - & - \\ 6.1 \mathrm{E}-07 & 03 \% & - & - \\ - & - & - & - \\ 1.3 \mathrm{E}-06 & 06 \% & - & - \\ - & - & - & -\end{array}$

5.2E-06 24\%

3.2E-07 $01 \%$

$1.3 \mathrm{E}-06 \quad 06 \%$

$1.2 \mathrm{E}-06 \quad 05 \%$

$4.4 \mathrm{E}-08<1 \%$

$1.1 \mathrm{E}-06 \quad 05 \%$

I.1E-01 20\% 
Table D.30

Savannah River Site

Burning/Rubble Pits (231-F, 231-1F)

Combined Adult/Child Resident (Future)

Ingestion of Soil (0-2'bls)

Carcinogenic and Noncarcinogenic Risk

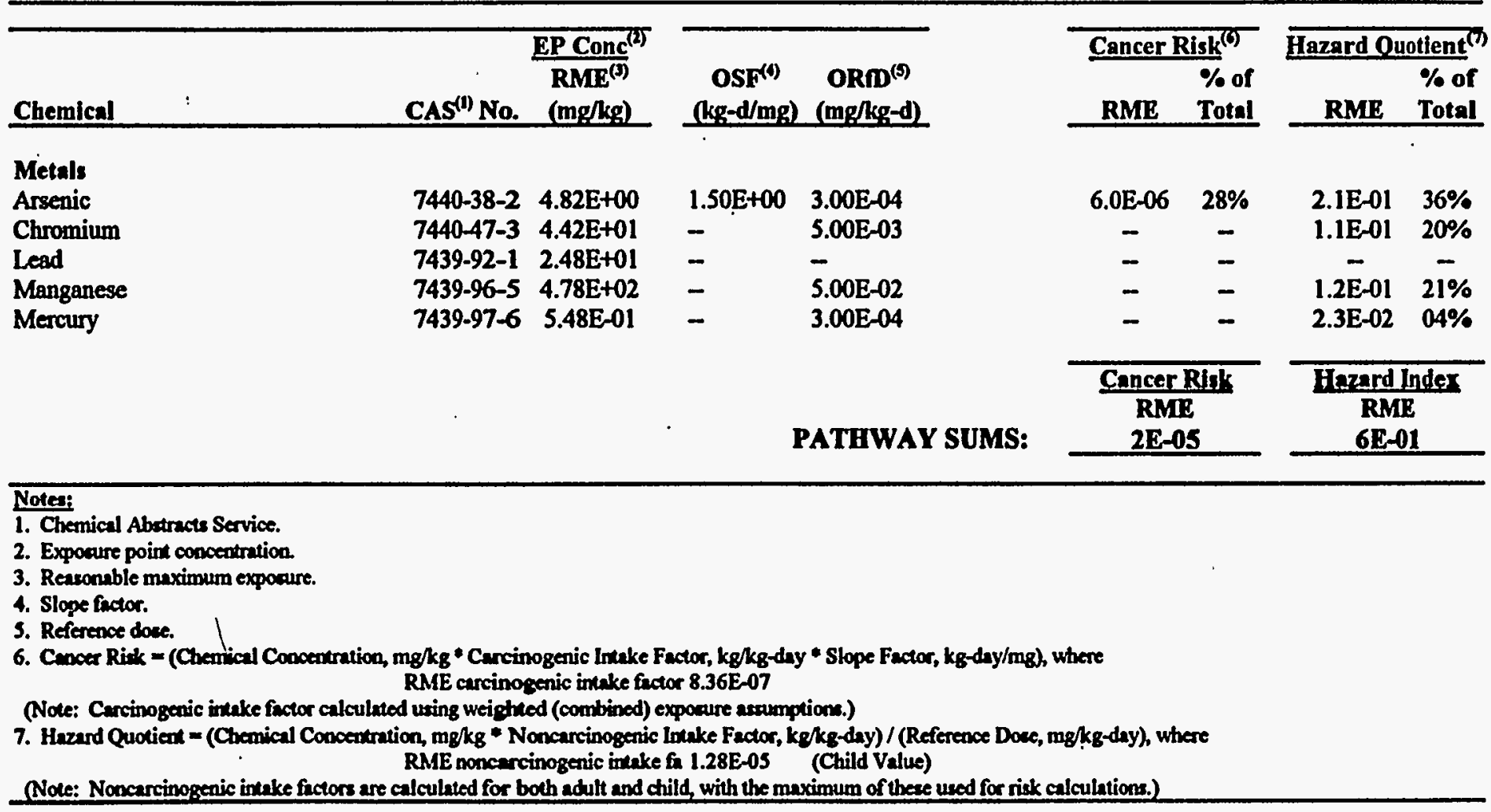


Table D.31

Savannah River Site

Burning/Rubble Pits (231-F, 231-1F)

Combined Adult / Child Resident (Future)

Dermal Exposure to Soil (0-2'bls)

Carcinogenic and Noncarcinogenic Risk

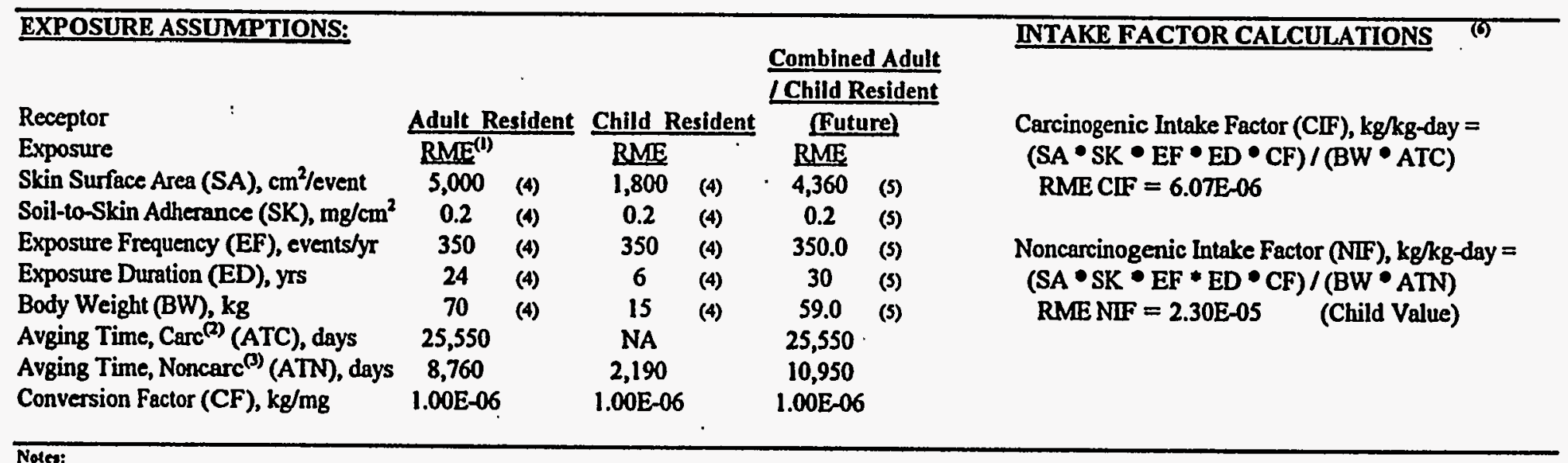

1. Ressonsble maximum exposure.

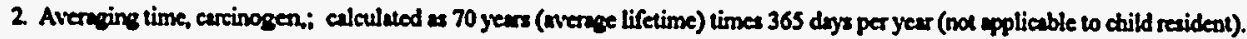

3. Averaging time, nọncarciniogen; celloulesed es expocure duration (in years) times 365 dens per yeer

4. See TrbleD.1.

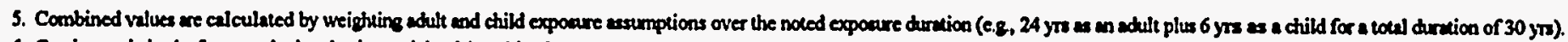

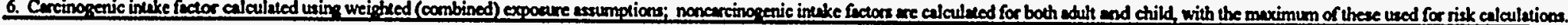


Table D.31

Savannah River Site

Burning/Rubble Pits (231-F, 231-1F)

Combined Adult / Child Resident (Future)

Dermal Exposure to Soil (0-2'bls)

Carcinogenic and Noncarcinogenic Risk

\begin{tabular}{|c|c|c|c|c|c|c|c|c|c|}
\hline \multirow[b]{2}{*}{ Chemical } & \multirow{2}{*}{$\begin{array}{r}\text { CAS"1) } \\
\text { Number }\end{array}$} & \multirow{2}{*}{ 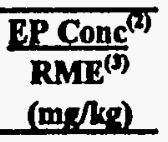 } & \multirow[b]{2}{*}{$\begin{array}{c}\operatorname{DSF}^{(4)} \\
(\text { (se-d/mg) }\end{array}$} & \multirow[b]{2}{*}{$\begin{array}{c}\operatorname{DRD}^{(\theta)} \\
\left(\mathrm{m} / \mathbf{L g}^{-\mathrm{d}}\right)\end{array}$} & \multirow[b]{2}{*}{ DABS(9) } & \multicolumn{2}{|c|}{ Cancer Risk ${ }^{(7)}$} & \multicolumn{2}{|c|}{ Hazard Ouotient } \\
\hline & & & & & & RME & $\begin{array}{l}\% \text { of } \\
\text { Total }\end{array}$ & RME & $\begin{array}{l}\% \text { of } \\
\text { Total }\end{array}$ \\
\hline \multicolumn{10}{|l|}{ Semlvolatiles } \\
\hline 2-Methylnaphithalene & $91-57-6$ & $7.69 \mathrm{E}-02$ & - & - & $1.00 \mathrm{E}-02$ & - & - & - & - \\
\hline Acenaphthylene & $208-96-8$ & 8.61E-02 & - & - & $1.00 \mathrm{E}-02$ & - & - & - & - \\
\hline Benzo(a)anthracene & $56-55-3$ & $9.26 \mathrm{E}-01$ & $1.46 \mathrm{E}+00$ & - & $1.00 \mathrm{E}-02$ & $8.2 \mathrm{E}-08$ & $03 \%$ & - & - \\
\hline Benzo(a)pyrene & $50-32-8$ & $6.49 \mathrm{E}-01$ & $3.65 E+01$ & - & $1.00 \mathrm{E}-02$ & $1.4 \mathrm{E}-06$ & $55 \%$ & - & - \\
\hline Benzo(b)fluoranthene & $205-99-2$ & $1.01 E+\infty 0$ & $1.46 \mathrm{E}+00$ & - & $1.00 \mathrm{E}-02$ & 8.9E-08 & $03 \%$ & - & - \\
\hline Benzo(g,h,i)perylene & $191-24-2$ & 6.15E-01 & - & - & $1.00 \mathrm{E}-02$ & - & - & - & - \\
\hline Dibenz $(a, h)$ anthracene & $53-70-3$ & 2.17E-01 & $1.46 \mathrm{E}+01$ & - & $1.00 \mathrm{E}-02$ & $1.9 \mathrm{E}-07$ & $07 \%$ & - & - \\
\hline Phenanthrene & $85-01-8$ & 6.87E-01 & - & - & $1.00 \mathrm{E}-02$ & - & - & - & - \\
\hline \multicolumn{10}{|l|}{ Dloxinz/furans } \\
\hline Heptachlorodibenzo-p-dioxin isomer & $37871-00-4$ & $4.11 \mathrm{E}-03$ & $1.72 E+03$ & - & $1.00 \mathrm{E}-02$ & 4.3E-07 & $16 \%$ & - & - \\
\hline Heplachlorodibenzo-p-furan isomer & 38998-75-3 & $2.58 \mathrm{E}-04$ & $1.72 E+03$ & - & $1.00 \mathrm{E}-02$ & $2.7 \mathrm{E}-08$ & $01 \%$ & - & - \\
\hline Hexachlorodibenzo-p-furan isomer & $55684-94-1$ & $1.00 \mathrm{E}-04$ & $1.72 \mathrm{E}+04$ & - & $1.00 \mathrm{E}-02$ & $1.0 \mathrm{E}-07$ & $04 \%$ & - & - \\
\hline Octachlorodibenzo-p-dioxin isomer & $3268-87-9$ & $9.26 \mathrm{E}-03$ & $1.72 E+02$ & - & $1.00 \mathrm{E}-02$ & $9.7 E-08$ & $04 \%$ & - & - \\
\hline Octechlorodibenzo-p-furan isomer & $39001-02-0$ & $3.48 E-04$ & $1.72 E+02$ & - & $1.00 \mathrm{E}-02$ & $3.6 \mathrm{E}-09$ & $<1 \%$ & - & - \\
\hline \multicolumn{10}{|l|}{ PCBs } \\
\hline PCB 1254 & $11097-69-1$ & $1.78 \mathrm{E}-01$ & $8.56 \mathrm{E}+00$ & $1.80 \mathrm{E}-05$ & $1.00 \mathrm{E}-02$ & $9.3 \mathrm{E}-08$ & $04 \%$ & 2.3E-03 & $09 \%$ \\
\hline \multicolumn{10}{|l|}{ Pesticide: } \\
\hline $\begin{array}{l}\text { delta-Benzene hexachloride } \\
\text { Endrin ketone }\end{array}$ & $\begin{array}{r}319-86-8 \\
52104-70-5\end{array}$ & $\begin{array}{l}1.84 \mathrm{E}-03 \\
320 \mathrm{E}-03\end{array}$ & - & - & $1.00 \mathrm{E}-02$ & - & - & - & - \\
\hline Endrin ketone & $53494-70-5$ & $3.20 \mathrm{E}-03$ & - & - & $1.00 \mathrm{E}-02$ & - & - & - & - \\
\hline
\end{tabular}


Table D.31

Savannah River Site

Burning/Rubble Pits (231-F, 231-1F)

Combined Adult / Child Resident (Future)

Dermal Exposure to Soil (0-2'bls)

Carcinogenic and Noncarcinogenic Risk

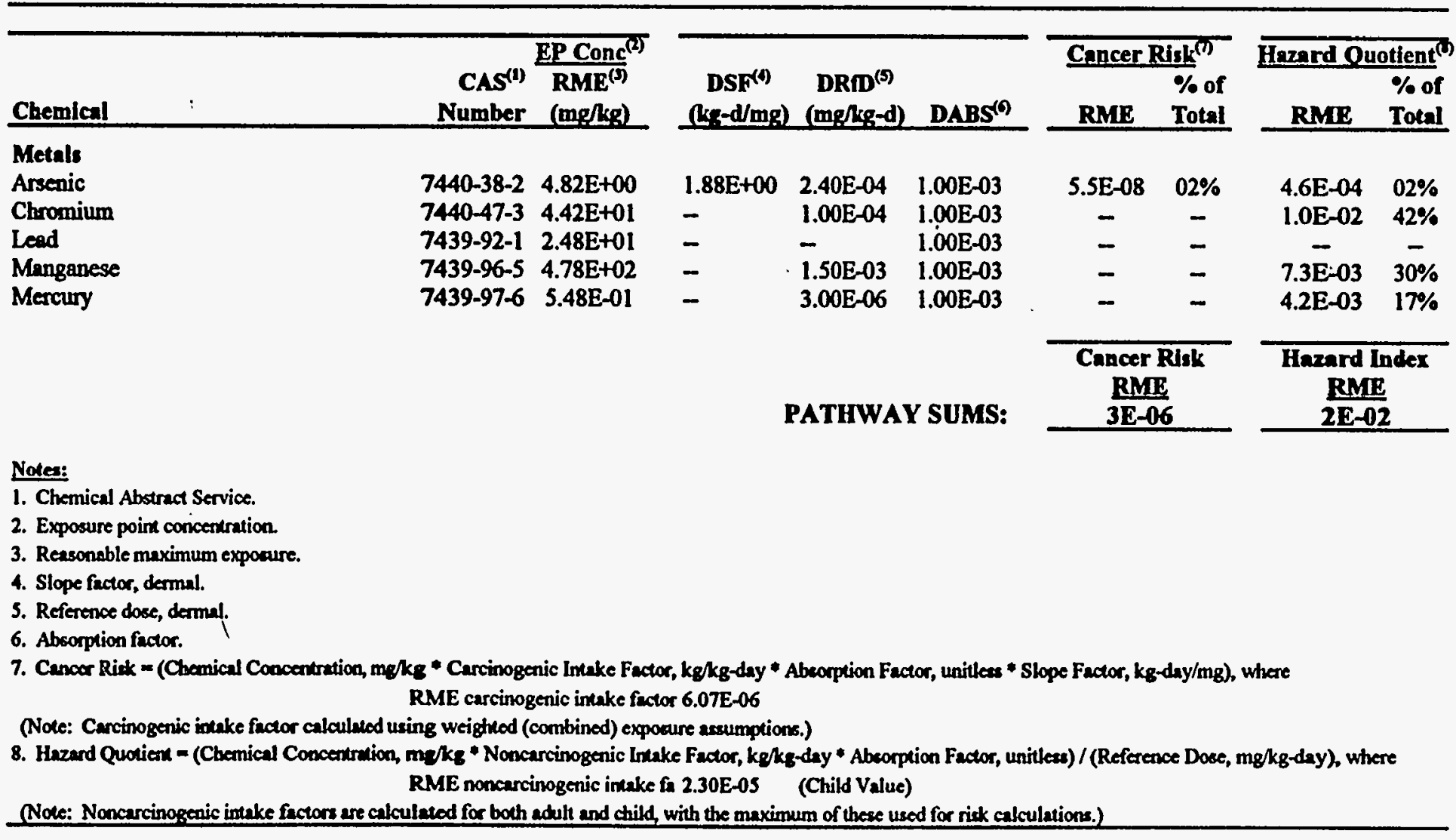


Table D.32

Savannah River Site

Burning/Rubble Pits (231-F, 231-1F)

Combined Adult / Child Resident (Future)

Inhalation of Volatile Soil Contaminants (0-2'bls)

\section{EXPOSURE ASSUMPTIONS:}

\section{Receptor}

Exposure

Inhalation Rate (IR), $\mathrm{m}^{\mathbf{3}} / \mathrm{hr}$

Exposure Time (ET), hrs/day

Exposure Frequency (EF), days/yr

Exposure Duration (ED), yrs

Body Weight (BW), kg

Avging Time, $\operatorname{Carc}^{(2)}$ (ATC), days

\begin{tabular}{|c|c|c|c|c|c|}
\hline & & & & \multicolumn{2}{|c|}{$\frac{\text { Combined Adult }}{\text { Child Resident }}$} \\
\hline \multicolumn{2}{|c|}{ Adult Resident } & \multicolumn{2}{|c|}{ Child Resident } & \multicolumn{2}{|c|}{ (Future) } \\
\hline $\mathrm{RME}^{(1)}$ & & RME & & $\mathrm{RME}$ & \\
\hline 0.83 & (4) & 1 & (4) & 0.9 & (5) \\
\hline 15 & (4) & 18 & (4) & 16 & (5) \\
\hline 350 & (4) & 350 & (4) & 350 & (5) \\
\hline 24 & (4) & 6 & (4) & 30 & (5) \\
\hline 70 & (4) & 15 & (4) & 59.0 & (5) \\
\hline 25,550 & & NA & & 25,550 & \\
\hline 8,760 & & 2,190 & & 10,950 & \\
\hline
\end{tabular}

INTAKE FACTOR CALCULATIONS

Carcinogenic Intake Factor (CIF), kg/kg-day = $(\mathrm{IR} * \mathrm{ET} \bullet \mathrm{EF} \bullet \mathrm{ED} \bullet \mathrm{CF}) /(\mathrm{BW} * \mathrm{ATC})$

RME CIF $=9.39 \mathrm{E}-02$

Noncarcinogenic Intake Factor (NIF), kg/kg-day = $(\mathrm{IR} \bullet \mathrm{ET} \bullet \mathrm{EF} \bullet \mathrm{ED} \bullet \mathrm{CF}) /(\mathrm{BW} * \mathrm{ATN})$

RME NIF $=1.15 E+00 \quad$ (Child Value)

Avging Time, Noncarc ${ }^{(3)}$ (ATN), days $\quad \mathbf{8 , 7 6 0}$

2,190

10,950

\section{Netra:}

1. Remonsble maximum exponume.

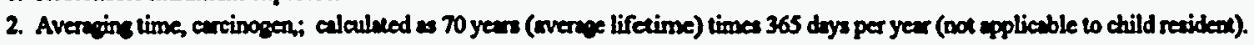

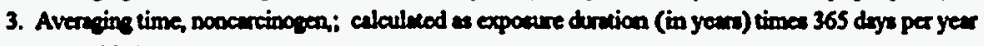

4. See Table D-1.

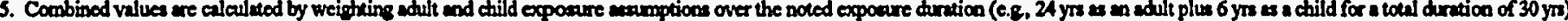

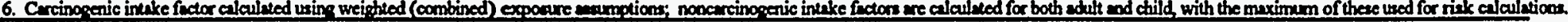


Table D.32

Savannah River Site

Burning/Rubble Pits (231-F, 231-1F)

Combined Adult / Child Resident (Future)

Inhalation of Volatile Soil Contaminants (0-2'bls)

Calculation of Volatilization Factor (VF) ${ }^{(1)}$

\begin{tabular}{|c|c|c|c|c|c|c|c|c|c|c|c|}
\hline Chemical & $\mathrm{CAS}^{(2)} \mathrm{Na}$ & $\mathrm{Class}^{(3)}$ & MolWt & $\operatorname{Koc}^{(1)}$ & $\mathbf{K} \mathbf{d}^{(1)}$ & $\mathbf{H}^{(\boldsymbol{(})}$ & $\mathrm{Kag}_{\mathbf{n}}^{(1)}$ & $\mathrm{Di}^{(4)}$ & $\operatorname{Dei}^{(\ell)}$ & alpha ${ }^{(1)}$ & $\begin{array}{c}\text { Volat. Factor } \\
\left(\mathrm{m}^{3} / \mathrm{kg}\right)\end{array}$ \\
\hline Semivolatiles & & & & & & & & & & & \\
\hline 2-Methylnaphthalene & $91-57-6$ & $\mathbf{S}$ & $1.42 E+02$ & 8511.4 & $1.70 E+02$ & $2.90 \mathrm{E}-04$ & 6.98E-05 & 6.43E-02 & 4.53E-02 & 6.43̣E-07 & $1.82 \mathrm{E}+05$ \\
\hline Acenaphthylene & $208-96-8$ & $\mathbf{S}$ & $1.52 E+02$ & 4786.3 & $9.57 E+01$ & $2.80 \mathrm{E}-04$ & $1.20 \mathrm{E}-04$ & $6.22 \mathrm{E}-02$ & $4.38 \mathrm{E}-02$ & $1.07 \mathrm{E}-06$ & $1.42 E+05$ \\
\hline Phenenthrene & $85-01-8$ & $\mathbf{S}$ & $1.78 E+02$ & 38004.5 & $7.78 \mathrm{E}+02$ & $2.56 \mathrm{E}-05$ & $1.35 \mathrm{E}-06$ & $5.74 \mathrm{E}-02$ & $4.05 E-02$ & $1.11 \mathrm{E}-08$ & $1.39 \mathrm{E}+06$ \\
\hline
\end{tabular}

1. USEPA, 1991: RAGS: Vol 1 - Human Heath Evaluation Mapual (Part B, Development of Risk-Basod Preliminary Remodiation Goals)

2. Chemical Abetract Service.

3. Chemical clas: $v$ - volatile; 8 - semivolatile; $p$ - pexticide/pct; $x$ - dioxin; and $m$ - metal.

4. Calculation of organic contaminart volatilization factor:

cONSTANTS:

INPUT VARLABLES:

Length of side of contarid. area (LS), $m=$ Wind speed in mixing zone $(V), m / s o c=$ - Diffusion heigtx (DH), $m=$ Area of contemination (Am), $\mathrm{m}^{2}=$ Area in squime centimeters $(\mathrm{Acm}), \mathrm{cm}^{2}=$ True soil porosity $(E)$, unitless = True eoil denity (Pr), $\varepsilon^{\prime \mathrm{cm}^{3}}=$ Expouture interval (Tyr), yrs = Expomure interval (Troc), sec = Organic carbon content (OC), unitless = Conversion fector (CF), $\mathrm{kg} / \mathrm{l}=$

Organic cartion partition coef. (Koc), $\mathrm{cm}^{3} / \mathrm{kg}=$ Herry's Law conatan (H), atm-m ${ }^{3} / \mathrm{mol}^{\mathrm{m}}=$ Gas diffusivity (Di), $\mathrm{cm}^{2} / \mathrm{sec}=$ INTERMEDLATE STEPS

Soil-water partition coefficient $(\mathrm{Kd}), \mathrm{cm}^{3} / \mathrm{kg}=$

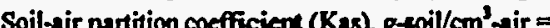
Effective diffusivity (Dei), $\mathrm{cm}^{2} / \mathrm{sec}=$ alpha (Alpha), $\mathrm{cm}^{2} / 20 \mathrm{c}=$

OUTPUT:

Volatilization Factor (VF), m $\mathrm{m}^{3} / \mathrm{kg}=$

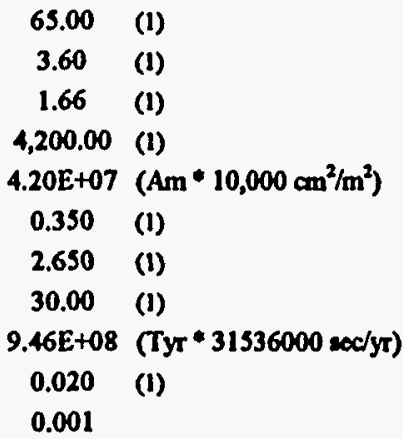

chem-epec

chem-spec

chem-apec

chem-spec (Koc $\times$ OC)

chem-spec $((\mathrm{H} / \mathrm{Kd}) \times 41)$

chem-spec (Di $\times\left(E^{\wedge}\right.$ 0.33))

chem-epec $(($ Dei $\times E)(E+($ Ps) $)(1-E)) / K a s)$

chem-apec $((\mathrm{LS} \times \dot{V} \times \mathrm{DH} \times(3.14 \times$ alphax $\times \mathrm{T}) \mathbf{0 . 5})(\mathrm{A} \times 2 \times \mathrm{Dei} \times \mathrm{E} \times \mathrm{Kas} \times \mathrm{CF}))$ 
Table D.32

Savannah River Site

Burning/Rubble Pits (231-F, 231-1F)

Combined Adult / Child Resident (Future)

Inhalation of Volatile Soil Contaminants (0-2'bls)

Carcinogenic and Noncarcinogenic Risk

\begin{tabular}{|c|c|c|c|c|c|c|c|c|c|}
\hline \multirow[b]{2}{*}{ Chemical } & \multicolumn{2}{|c|}{ EP Conc ${ }^{(2)}$} & \multirow[b]{2}{*}{$\begin{array}{l}\operatorname{TUR}^{(4)} \\
\left(\boldsymbol{m}^{2} / \mathbf{x}^{\prime}\right)\end{array}$} & \multirow[b]{2}{*}{$\begin{array}{l}\operatorname{RrC}^{(\theta)} \\
\left(n / \ln ^{2}\right)\end{array}$} & \multirow[b]{2}{*}{$\begin{array}{c}V F^{(0)} \\
\left(\square^{3} /(x)\right.\end{array}$} & \multicolumn{2}{|c|}{ Concer Risk } & \multicolumn{2}{|c|}{ Hozard Quotient ${ }^{()}$} \\
\hline & $\begin{array}{r}\text { CAS }^{(1)} \\
\text { Number }\end{array}$ & $\begin{array}{l}\mathbf{R M E}^{(\lambda)} \\
(m y / 2)\end{array}$ & & & & $\mathbf{R M P}$ & $\begin{array}{l}\% \text { of } \\
\text { Total }\end{array}$ & RME & $\begin{array}{l}\% \text { of } \\
\text { Total } \\
\end{array}$ \\
\hline \multicolumn{10}{|l|}{ Semivolatiles } \\
\hline 2-Methylnuphthalene & $91-57-6$ & 7.69E-02 & - & - & $1.82 E+05$ & NA & - & NA & - \\
\hline Acenaphthylene & $208-96-8$ & 8.61E-02 & - & - & $1.42 \mathrm{E}+05$ & NA & - & NA & - \\
\hline \multirow[t]{3}{*}{ Phenanthrene } & $85-01-8$ & $6.87 \mathrm{E}-01$ & - & - & $1.39 E+06$ & NA & - & NA & - \\
\hline & & & & & & \multicolumn{2}{|c|}{$\begin{array}{c}\text { Cancer Rlsk } \\
\text { RMI }\end{array}$} & \multicolumn{2}{|c|}{$\begin{array}{c}\text { Harand Index } \\
\text { RME }\end{array}$} \\
\hline & & & & \multicolumn{2}{|c|}{ PATHWAY SUMS: } & \multicolumn{2}{|c|}{-} & \multicolumn{2}{|c|}{-} \\
\hline
\end{tabular}

Nols:

1. Chemical Abstract Service.

2. Expocure point concentration.

3. Reasonable maximum expocure.

4. Inhalation unit risk.

3. Referenoe concentration.

6. Voletilization factor (chemical-apecific).

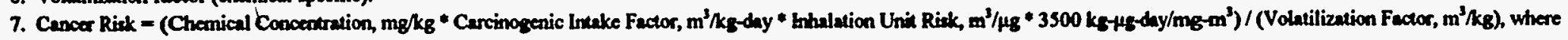
RME carcinogenic intake factor $9.39 \mathrm{E}-02$

and conversion fram IUR to inhalution slope factor $=3500 \mathrm{~kg}-14 \mathrm{~g}-\mathrm{day} / \mathrm{ms}-\mathrm{m}^{3}$

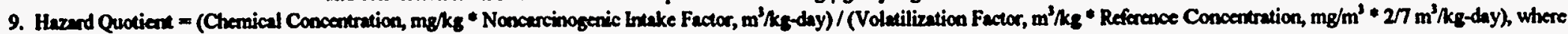
RME noncarcinogenic intake fo 1.15E+00 and convervion from RFC to inhalation reference dose $=2 \pi \mathrm{m}^{3} / \mathrm{kg}$-day 
Table D.33

Savannah River Site

Burning/Rubble Pits (231-F, 231-1F)

Combined Adult / Child Resident (Future)

Inhalation of Soil Particulates (0-2'bls)

Carcinogenic and Noncarcinogenic Risk

EXPOSURE ASSUMPTIONS:

Receptor

Exposure

Inhalation Rate (IR), $\mathrm{m}^{3} / \mathrm{hr}$

Exposure Time (ET), hrs/day

Exposure Frequency (EF), days/yr

Exposure Duration (ED), yrs

Body Weight (BW), kg

Avging Time, $\mathrm{Carc}^{(\Omega)}$ (ATC), days

Avging Time, Noncarc ${ }^{(3)}$ (ATN), days

\begin{tabular}{cc} 
& \\
Adult Resident \\
\hline RME $^{(1)}$ \\
0.83 & $(4)$ \\
15 & $(4)$ \\
350 & $(4)$ \\
24 & $(4)$ \\
70 & $(4)$ \\
25,550 & $(4)$ \\
8,760 &
\end{tabular}

\begin{tabular}{|c|c|c|c|}
\hline & & \multicolumn{2}{|c|}{$\begin{array}{l}\text { Combined Adult } \\
\text { Lhild Resident }\end{array}$} \\
\hline \multicolumn{2}{|c|}{ Child Resident } & \multicolumn{2}{|c|}{ (Future) } \\
\hline $\mathbf{R M E}$ & & $\mathrm{RME}$ & \\
\hline 1.0 & (4) & 0.9 & (5) \\
\hline 18 & (4) & 16 & (5) \\
\hline 350 & (4) & 350 & (5) \\
\hline 6 & (4) & 30 & (5) \\
\hline 15 & (4) & 59.0 & (5) \\
\hline NA & (4) & 25,550 & \\
\hline 2,190 & & 10,950 & \\
\hline
\end{tabular}

INTAKE FACTOR CALCULATIONS

Carcinogenic Intake Factor (CIF), kg/kg-day = $(\mathrm{IR} \bullet \mathrm{FI} \bullet \mathrm{EF} \bullet \mathrm{ED} \bullet \mathrm{CF}) /(\mathrm{BW} \bullet \mathrm{ATC})$ RME CIF $=9.39 \mathrm{E}-02$

Noncarcinogenic Intake Factor (NIF), $\mathbf{k g} / \mathbf{k g}$-day = $(\mathrm{IR} \bullet \mathrm{FI} \bullet \mathrm{EF} \bullet \mathrm{ED} \bullet \mathrm{CF}) /(\mathrm{BW} \bullet \mathrm{ATN})$

RME NIF $=1.15 E+00 \quad$ (Child Value)

Noleti:

1. Ressonuble maximum exposure.

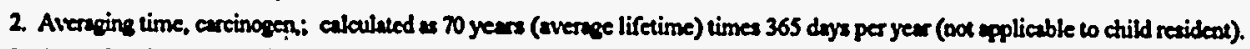

3. Averaging time, noncurcinopen; calculuted as exposure duration (in yems) times 365 deys per year

4. See Table D.1.

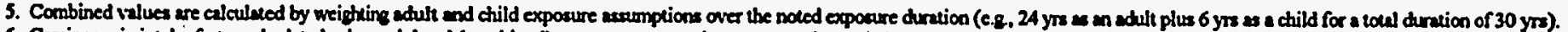

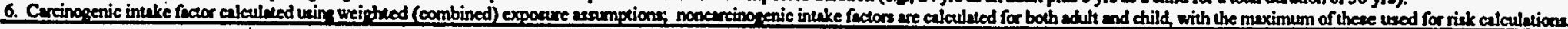


Table D.33

Savannah River Site

Burning/Rubble Pits (231-F, 231-1F)

Combined Adult / Child Resident (Future)

Inhalation of Soil Particulates (0-2'bls)

Carcinogenic and Noncarcinogenic Risk

\begin{tabular}{|c|c|c|c|c|}
\hline \multicolumn{4}{|c|}{ CALCULATION OF SOIL PARTICULATE RESUSPENSION FACTOR: } & \multirow{2}{*}{$\begin{array}{l}\text { INTERMEDIATE STEPS: } \\
\text { Particulate emission factor }(\mathrm{PEF})=\quad 2.18 \mathrm{E}+09 \mathrm{~meters}^{3} / \mathrm{kg}\end{array}$} \\
\hline Length of side of contaminated area (LS) $=$ & 65.00 & meters & (1) & \\
\hline Wind speed in mixing zone $(V)=$ & 3.60 & meters/sec & (1) & $(\mathrm{LS} \times \mathrm{V} \times \mathrm{DH} \times \mathrm{CFa} \times \mathrm{CFb}) /\left(\mathrm{A} \times \mathrm{RF} \times(1-\mathrm{G}) \times\left((\mathrm{Um} / \mathrm{Ut})^{\wedge} 3\right) \times(\mathrm{Fx})\right)$ \\
\hline Diffusion height $(\mathrm{DH})=$ & 1.66 & meters & (1) & \\
\hline Area of contamination $(A)=$ & $4,200.00$ & meters $^{2}$ & (1) & \\
\hline Respirable fraction (RF) = & 0.003 & g/meters ${ }^{2}-h r$ & (1) & ' \\
\hline Fraction of vegetative cover $(\mathbf{G})=$ & 0.90 & unitless & (1) & \\
\hline Mean annual wind speed $(\mathrm{Um})=$ & 3.60 & meters/sec & (1) & \\
\hline Equivalent threshold value of wind speed at $10 \mathrm{~m}(\mathrm{Ut})=$ & 5.04 & meters/sec & (1) & \\
\hline Fraction dependent on Um/Ut (Fx) $=$ & 1.2700 & unitless & (1) & \\
\hline Conversion factor $(\mathrm{CFa})=$ & $3,600.00$ & $\sec / \mathrm{hr}$ & & \\
\hline Conversion factor $(\mathrm{CFb})=$ & $1,000.00$ & g/kg & & \\
\hline
\end{tabular}

Noter:

1. USEPA, 1991: RAGS: Vol I - Humm Health Evaluation Munual (Pm B, Development of Risk-Bued Preliminary Remediation Goals) 
Table D.33

Savannah River Site

Burning/Rubble Pits (231-F, 231-1F)

Combined Adult / Child Resident (Future)

Inhalation of Soil Particulates (0-2'bls)

Carcinogenic and Noncarcinogenic Risk

\begin{tabular}{|c|c|c|c|}
\hline Chemical & : & $\begin{array}{r}\text { CAS }^{(1)} \\
\text { Number }\end{array}$ & $\frac{\operatorname{EPConc} c^{(2)}}{\operatorname{RME}_{(\mathrm{m})}^{(3)}}$ \\
\hline
\end{tabular}

\begin{tabular}{rr}
$\operatorname{IUR}^{(t)}$ & $\operatorname{RfC}^{(5)}$ \\
$\left(\mathrm{m}^{3} / \mu \mathrm{g}\right)$ & $\left(\mathrm{mg} / \mathrm{m}^{3}\right)$ \\
\hline
\end{tabular}

\begin{tabular}{|c|c|c|}
\hline Cancer & $\operatorname{isk}^{(6)}$ & Hazard Quotient $^{(7)}$ \\
\hline RME & $\begin{array}{l}\% \text { of } \\
\text { Total }\end{array}$ & RME Total $\begin{array}{c}\% \text { of } \\
\text { TME }\end{array}$ \\
\hline
\end{tabular}

Semivolatiles

2-Methylnaphthalene

Acenaphthylene

Benzo(a)anthracene

Benzo(a)pyrene

Benzo(b)iluoranthene

Benzo(g,h,i)perylene

Dibenz( $a, h)$ anthracene

Phenanthrene

$\begin{array}{rl}91-57-6 & 7.69 \mathrm{E}-02 \\ 208-96-8 & 8.61 \mathrm{E}-02 \\ 56-55-3 & 9.26 \mathrm{E}-01 \\ 50-32-8 & 6.49 \mathrm{E}-01 \\ 205-99-2 & 1.01 \mathrm{E}+00 \\ 191-24-2 & 6.15 \mathrm{E}-01 \\ 53-70-3 & 2.17 \mathrm{E}-01 \\ 85-01-8 & 6.87 \mathrm{E}-01\end{array}$

-
-
-
-
-

\section{Dioxins/furans}

Heptachlorodibenzo-p-dioxin isomer

Heptachlorodibenzo-p-furan isomer

Hexachlorodibenzo-p-furan isomer

Octachlorodibenzo-p-dioxin isomer

Octachlorodibenzo-p-furan isomer

PCBs

PCB 1254

$\begin{array}{rlll}37871-00-4 & 4.11 \mathrm{E}-03 & 3.30 \mathrm{E}-01 & - \\ 38998-75-3 & 2.58 \mathrm{E}-04 & 3.30 \mathrm{E}-01 & - \\ 55684-94-1 & 1.00 \mathrm{E}-04 & 3.30 \mathrm{E}+00 & - \\ 3268-87-9 & 9.26 \mathrm{E}-03 & 3.30 \mathrm{E}-02 & - \\ 39001-02-0 & 3.48 \mathrm{E}-04 & 3.30 \mathrm{E}-02 & -\end{array}$

11097-69-1 1.78E-01 -

\section{Pesticides}

delta-Benzene hexachloride

Endrin ketone

319-86-8 1.84E-03

$53494-70-5 \quad 3.20 \mathrm{E}-03$


Table D.33

Savannah River Site

Burning/Rubble Pits (231-F, 231-1F)

Combined Adult / Child Resident (Future)

Inhalation of Soil Particulates (0-2'bls)

Carcinogenic and Noncarcinogenic Risk

\begin{tabular}{|c|c|c|c|c|c|c|c|c|}
\hline Chemical & $\begin{array}{c}\text { CAS }^{(1)} \\
\text { Number }\end{array}$ & $\begin{array}{l}\text { EP Conc } \text { c }^{(2)} \\
\mathrm{RMEE}^{(3)} \\
(\mathrm{mIg} / \mathrm{kg})\end{array}$ & $\begin{array}{l}\mathrm{IUR}^{(4)} \\
\left(\mathrm{m}^{3} / \mu \mathrm{g}\right)\end{array}$ & $\begin{array}{l}\operatorname{RfC}^{(\theta)} \\
\left(\mathrm{m}^{\prime} / \mathrm{m}^{3}\right)\end{array}$ & $\begin{array}{l}\text { Cancer } \\
\text { RME }\end{array}$ & $\begin{array}{l}\text { isk } \\
\% \text { of } \\
\text { Total }\end{array}$ & $\begin{array}{r}\text { Hazard Q } \\
\text { RME }\end{array}$ & $\begin{array}{l}\text { otient } \\
\% \text { of } \\
\text { Total }\end{array}$ \\
\hline \multicolumn{9}{|l|}{ Metals } \\
\hline Arsenic & $7440-38-2$ & $4.82 E+00$ & $4.30 \mathrm{E}-03$ & - & 3.1E-09 & $04 \%$ & NA & - \\
\hline Chromium & $7440-47-3$ & 4.42E+01 & $1.20 \mathrm{E}-02$ & - & 8.0E-08 & $96 \%$ & NA & - \\
\hline Lead & $7439-92-1$ & $2.48 \mathrm{E}+01$ & - & - & NA & - & NA & - \\
\hline Manganese & $7439-96-5$ & $4.78 \mathrm{E}+02$ & - & $5.00 \mathrm{E}-05$ & NA & - & $1.8 \mathrm{E}-02$ & $100 \%$ \\
\hline Mercury & $7439-97-6$ & $5.48 \mathrm{E}-01$ & - & $3.00 \mathrm{E}-04$ & NA & - & $3.4 \mathrm{E}-06$ & $<1 \%$ \\
\hline - & . & & & & Cance & Visk & Hazard & inder \\
\hline & & & & PATHWAY SUMS: & $8 \mathrm{E}-$ & & $2 \mathrm{E}$ & \\
\hline
\end{tabular}

Noten:

1. Chemical Abstract Service.

2. Exposure point concentration

3. Reasonable maximum exposure.

4. Inhalation unit risk

5. Reference concentration.

6. Cancer Risk = (Chemical Concentration, mg/kg * Carcinogenic Intake Factor, $\mathrm{m}^{3} / \mathrm{kg}_{\mathrm{g}}$ day *Inhalation Unit Risk, $\left.\mathrm{m}^{3} / \mu \mathrm{g} * 3500 \mathrm{~kg}-\mu \mathrm{g}-\mathrm{day} / \mathrm{mg}_{\mathrm{g}} \mathrm{m}^{3}\right) /\left(\mathrm{Particulate}\right.$ Emission Factor, $\left.\mathrm{m}^{3} / \mathrm{kg}\right)$ RME carcinogenic intake factor $=9.39 \mathrm{E}-02$

Particulate emixion factor $=\quad 2.18 \mathrm{E}+09$

and converrion from IUR to inhulation slope factor $=3500 \mathrm{~kg}-\mu \mathrm{g}-\mathrm{day} / \mathrm{mg}-\mathrm{m}^{3}$

(Note: Curcinogenic intake factor calculated using weigtled (combined) exponure asmumptions.)

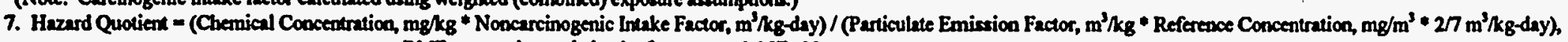
RME noncarcinogenic intake factor $=1.15 \mathrm{E}+00$

Particulate conistion factor = 2.18E+09

and conversion from RPC to inhalation reference doce $=2 / 7 \mathrm{~m}^{3} / \mathrm{kg}$-day

(Note: Noncarcinogenic intake factors are calculated for both achut and child with the maximum of these used for risk calculalions.) 
Table D.34

Savannah River Site

Burning/Rubble Pits (231-F, 231-1F)

Combined Adult/Child Resident (Future)

Ingestion of Soil (0-4'bls)

Carcinogenic and Noncarcinogenic Risk

\begin{tabular}{|c|c|c|c|c|c|c|c|}
\hline \multirow[t]{2}{*}{ EXPOSURE ASSUMPTIONS: } & & & & & & & \multirow[t]{2}{*}{ INTAKE FACTOR CALCULATIONS } \\
\hline & & & & & \multicolumn{2}{|c|}{ Combined } & \\
\hline Receptor & \multicolumn{2}{|c|}{ Adult Resident } & \multicolumn{2}{|c|}{ Child Resident } & \multicolumn{2}{|c|}{ Adult/Child } & \multirow{2}{*}{$\begin{array}{l}\text { Carcinogenic Intake Factor }(\mathrm{CIF}), \mathrm{kg} / \mathrm{kg} \text {-day }= \\
(\mathrm{IR} \bullet \mathrm{FI} \bullet \mathrm{EF} \bullet \mathrm{ED} \bullet \mathrm{CF}) /(\mathrm{BW} \bullet \mathrm{ATC})\end{array}$} \\
\hline Exposure & BME(1) & & RME & & RME & & \\
\hline Intake Rate (IR), mg/day & 100 & (4) & 200 & (4) & $\overline{120.0}$ & (5) & $\mathrm{RME} C \mathrm{CF}=8.36 \mathrm{E}-07$ \\
\hline Fraction Ingested (FI), unitless & 1 & (4) & 1 & (4) & 1.0 & (5) & \\
\hline Exposure Frequency (EF), days/yr & 350 & (4) & 350 & (4) & 350 & (5) & Noncarcinogenic Intake Factor (NIF), kg/kg-day = \\
\hline Exposure Duration (ED), yrs & 24 & (4) & 6 & (4) & 30 & (5) & $(\mathrm{IR} \bullet \mathrm{FI} \bullet \mathrm{EF} \bullet \mathrm{ED} \bullet \mathrm{CF}) /(\mathrm{BW} \bullet \mathrm{ATN})$ \\
\hline Body Weight (BW), kg & 70 & (4) & 15 & (4) & 59.0 & (5) & RME NIF $=1.28 \mathrm{E}-05 \quad$ (Child Value) \\
\hline Avging Time, Carc ${ }^{()}$(ATC), days & 25,550 & & NA & & 25,550 & & \\
\hline Avging Time, Noncarc ${ }^{(3)}$ (ATN), days & 8,760 & & 2,190 & & 10,950 & & \\
\hline Conversion Factor (CF), kg/mg & 1.00E-O & & $1.00 \mathrm{E}-06$ & & $1.00 \mathrm{E}-\mathrm{OC}$ & & \\
\hline
\end{tabular}

1. Remoneble maximum exposume.

2. Averaping time, carcinogen; calculued a 70 years (averne lifetime) times 365 deys per year (not applicable to child resident).

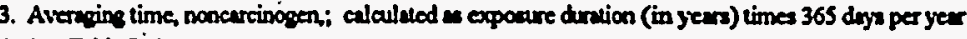

4. See Tuble D-1.

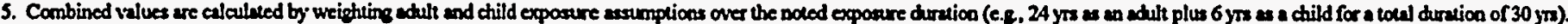

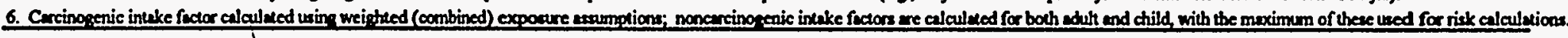


Table D.34

Savannah River Site

Burning/Rubble Pits (231-F, 231-1F)

Combined Adult/Child Resident (Future)

Ingestion of Soil (0-4'bls)

Carcinogenic and Noncarcinogenic Risk

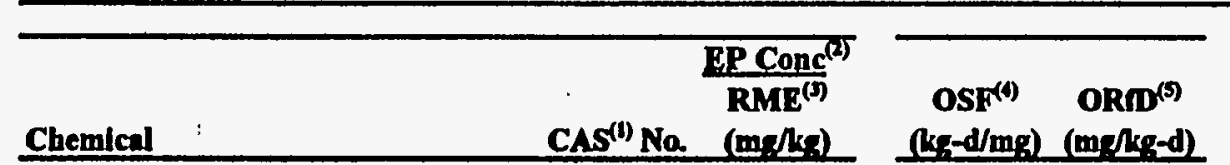

Semivelatiles

2-Methylnuphthalene

Acenaphthylene

Benzo(a) anthrecene

Benzo(a)pyrenc

Benzo(b)(luoranthene

Benzo(g,h,i)perylene

Dibenz(a,h)anthracene

Phenanthrene

$91-57-6 \quad 7.69 \mathrm{E}-02$

91-57-6 7.69E-02

$\begin{array}{cc}208-96-8 & 8.61 \mathrm{E}-02 \\ 56-55-3 & 9.26 \mathrm{E}-01\end{array}$

60-32-8 6.49E-01

205-99-2 1.01E+ +0

191-24-2 6.15E-01

53-70-3 2.17E-01

85-01-8 6.87E-01

$-$

-

$7.30 \mathrm{E}-01$

7.30E+00 -

$7.30 \mathrm{E}-01$

$-$

$7.30 \mathrm{E}+00-$

Dioxias/furans

Heptachlorodibenzo-p-dioxin isomer

Heptachlorodibenzo-p-furan isomer

$37871-00-4 \quad 4.11 E-03$

38998-75-3 2.58E-04

Hexachlorodibenzo-p-furan isomer

Octachlorodibenzo-p-dioxin isomer

55684-94-1 1.00E-04

3268-87-9 9.26E-03

Octachlorodibenzo-p-furan isomer

39001-02-0 3.48E-04

$1.50 \mathrm{E}+03-$

$1.50 \mathrm{E}+03$

$1.50 \mathrm{E}+04-$

$1.50 \mathrm{E}+02$

PCBs

PCB 1254

11097-69-1 1.78E-01

$7.70 \mathrm{E}+00 \mathrm{0} \quad 2.00 \mathrm{E}-05$

319-86-8 1.84E-03

delta-Benzene hexachloride

Endrin ketone

53494-70-5 3.20E-03

\begin{tabular}{|c|c|}
\hline Cancer Bisk ${ }^{(1)}$ & Hezard Quotient ${ }^{(7)}$ \\
\hline $\begin{array}{c}\text { \% of } \\
\text { RME Total } \\
\end{array}$ & $\begin{array}{l}\% \text { of } \\
\text { Total }\end{array}$ \\
\hline
\end{tabular}

$\begin{array}{cccc}- & - & - & - \\ - & - & - & - \\ 5.6 \mathrm{E}-07 & 03 \% & - & - \\ 4.0 \mathrm{E}-06 & 18 \% & - & - \\ 6.1 \mathrm{E}-07 & 03 \% & - & - \\ - & - & - & - \\ 1.3 \mathrm{E}-06 & 06 \% & - & - \\ - & - & - & -\end{array}$

$\begin{array}{llll}5.2 \mathrm{E}-06 & 23 \% & - & - \\ 3.2 \mathrm{E}-07 & 01 \% & - & - \\ 1.3 \mathrm{E}-06 & 06 \% & - & - \\ 1.2 \mathrm{E}-06 & 05 \% & - & - \\ 4.4 \mathrm{E}-08 & <1 \% & - & -\end{array}$

$1.1 \mathrm{E}-06 \quad 05 \% \quad 1.1 \mathrm{E}-01 \quad 18 \%$

$\begin{array}{llll}- & - & -\end{array}$ 
Table D.34

Savannah River Site

Burning/Rubble Pits (231-F, 231-1F)

Combined Adult/Child Resident (Future)

Ingestion of Soil (0-4'bls)

Carcinogenic and Noncarcinogenic Risk

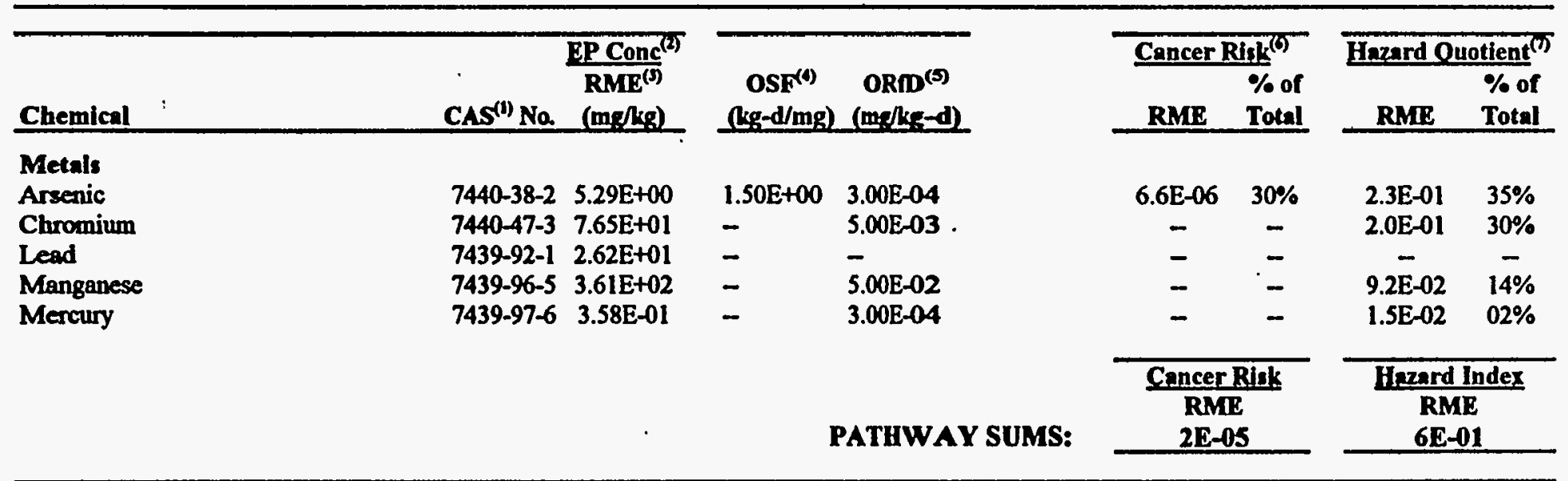

\section{Noter:}

1. Chemical Abstracts Service.

2. Exposure point concentration.

3. Reasonable maximum exposure.

4. Slope factor.

5. Reference doue.

6. Canor Risk - (Chemical Concentration, mg/kg * Carcinogenic Intake Factor, kg/kg-day * Slope Factor, kg-day/mg), where RME carcinogenic intake factor 8.36E-07

(Note: Cancinogenic intake factor calculated using weightod (combined) expoeure astumptions.)

7. Hazand Quotien = (Chemical Concentration, mg/kg * Noncarcinopenic Intake Factor, kg/kg-day)/(Reference Dowe, mg/kg-day), where

RME noncarcinogenic intake fa 1.28E-05 (Child Value)

(Note: Noncarcinogenic intake factors are calculated for both aduth and child, with the maximum of these used for risk calculations.) 
Table D.35

Savannah River Site

Burning/Rubble Pits (231-F, 231-1F)

Combined Adult / Child Resident (Future)

Dermal Exposure to Soil (0-4'bls)

Carcinogenic and Noncarcinogenic Risk

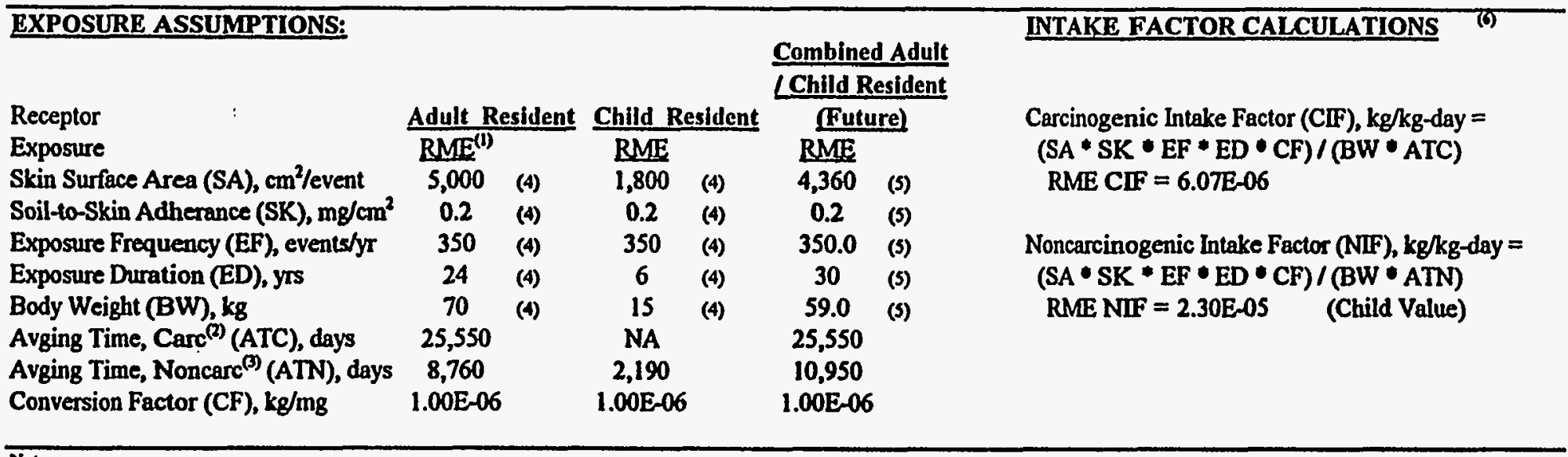

\section{Noter:}

1. Remomble muximum expoure.

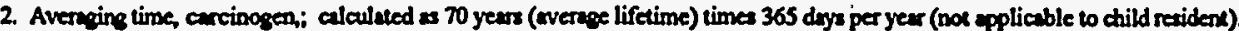

3. Avenging time, noscarcisogen; calculated as expocure duration (in yean) times 365 dense per year

4. See Table D-1.

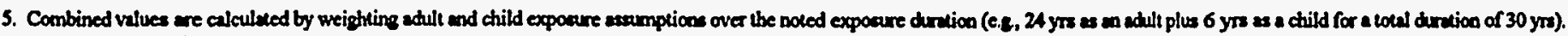

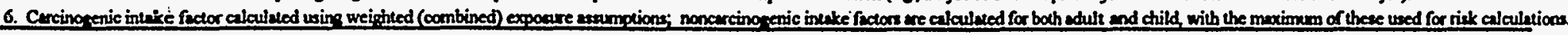


Table D.35

Savannah River Site

Burning/Rubble Pits (231-F, 231-1F)

Combined Adult / Child Resident (Future)

Dermal Exposure to Soil (0-4'bls)

Carcinogenic and Noncarcinogenic Risk

\begin{tabular}{|c|c|c|c|c|c|c|c|c|c|}
\hline \multirow[b]{2}{*}{ Chemical } & \multicolumn{2}{|c|}{ EPConct } & \multirow[b]{2}{*}{$\begin{array}{c}\operatorname{DSF}^{(0)} \\
(\mathrm{kg}-\mathrm{d} / \mathrm{mg})\end{array}$} & \multirow[b]{2}{*}{$\begin{array}{c}\operatorname{DRm}^{(N)} \\
\left(m / 2 / g^{-d}\right)\end{array}$} & \multirow[b]{2}{*}{ DABS ${ }^{(0)}$} & \multicolumn{2}{|c|}{ Cancer Risk ${ }^{(3)}$} & \multicolumn{2}{|c|}{ Harard Quotient ${ }^{(0)}$} \\
\hline & $\begin{array}{r}\text { CAS }^{(1)} \\
\text { Number }\end{array}$ & $\begin{array}{l}\mathbf{R M E}^{(3)} \\
(\mathbf{m q R} \mathbf{R}) \\
\end{array}$ & & & & $\mathbf{R M E}$ & $\begin{array}{l}\% \text { of } \\
\text { Total }\end{array}$ & $\mathbf{R M E}$ & $\begin{array}{l}\% \text { of } \\
\text { Total } \\
\end{array}$ \\
\hline \multicolumn{10}{|l|}{ Semivolatiles } \\
\hline 2-Methylnaphthalene & $91-57-6$ & $7.69 \mathrm{E}-02$ & - & - & $1.00 \mathrm{E}-02$ & - & - & - & - \\
\hline Acenaphthylene & $208-96-8$ & $8.61 E-02$ & - & - & $1.00 \mathrm{E}-02$ & - & - & - & - \\
\hline Benzo(a)anthracene & $56-55-3$ & $9.26 \mathrm{E}-01$ & $1.46 \mathrm{E}+00$ & - & $1.00 \mathrm{E}-02$ & 8.2E-08 & $03 \%$ & - & - \\
\hline Benzo(a)pyrene & $50-32-8$ & $6.49 \mathrm{E}-01$ & $3.65 \mathrm{E}+01^{\circ}$ & - & $1.00 \mathrm{E}-02$ & $1.4 \mathrm{E}-06$ & $55 \%$ & - & - \\
\hline Benzo(b)fluoranthene & $205-99-2$ & $1.01 E+00$ & $1.46 \mathrm{E}+00$ & - & $1.00 \mathrm{E}-02$ & 8.9E-08 & $03 \%$ & - & - \\
\hline Benzo(g,h,i)perylene & $191-24-2$ & $6.15 E-01$ & - & - & $1.00 E-02$ & - & - & - & - \\
\hline Dibenz $(a, h)$ anthracene & $53-70-3$ & 2.17E-01 & $1.46 \mathrm{E}+01$ & - & $1.00 \mathrm{E}-02$ & $1.9 \mathrm{E}-07$ & $07 \%$ & - & - \\
\hline Phenanthrene & $85-01-8$ & $6.87 \mathrm{E}-01$ & - & - & $1.00 \mathrm{E}-02$ & - & - & - & - \\
\hline \multicolumn{10}{|l|}{ Dioxins/furans } \\
\hline Heptachlorodibenzo-p-dioxin isomer & $37871-00-4$ & 4.11E-03 & $1.72 E+03$ & - & $1.00 \mathrm{E}-02$ & 4.3E-07 & $16 \%$ & - & - \\
\hline Heptachlorodibenzo-p-furan isomer & $38998-75-3$ & $2.58 \mathrm{E}-04$ & $1.72 E+03$ & - & $1.00 \mathrm{E}-02$ & 2.7E-08 & $01 \%$ & - & - \\
\hline Hexachlorodibenzo-p-furan isomer & $55684-94-1$ & $1.00 \mathrm{E}-04$ & $1.72 \mathrm{E}+04$ & - & $1.00 \mathrm{E}-02$ & $1.0 \mathrm{E}-07$ & $04 \%$ & - & - \\
\hline Octachlorodibenzo-p-dioxin isomer & $3268-87-9$ & $9.26 \mathrm{E}-03$ & $1.72 E+02$ & - & $1.00 \mathrm{E}-02$ & 9.7E-08 & $04 \%$ & - & - \\
\hline Octachlorodibenzo-p-furan isomer & $39001-02-0$ & $3.48 \mathrm{E}-04$ & $1.72 E+02$ & - & $1.00 \mathrm{E}-02$ & $3.6 \mathrm{E}-09$ & $<1 \%$ & - & - \\
\hline $\begin{array}{l}\text { PCB: } \\
\text { PCB } 1254\end{array}$ & $11097-69-1$ & $1.78 \mathrm{E}-01$ & $8.56 \mathrm{E}+00$ & $1.80 \mathrm{E}-05$ & $1.00 \mathrm{E}-02$ & 9.3E-08 & $04 \%$ & 2.3E-03 & $08 \%$ \\
\hline Pesticides & & & & & & & & & \\
\hline delta-Benzene hexachloride & $319-86-8$ & $1.84 \mathrm{E}-03$ & - & - & $1.00 \mathrm{E}-02$ & - & - & - & - \\
\hline Endrin ketone & $53494-70-5$ & $3.20 \mathrm{E}-03$ & - & - & $1.00 \mathrm{E}-02$ & - & - & - & - \\
\hline
\end{tabular}


Table D.35

Savannah River Site

Burning/Rubble Pits (231-F, 231-1F)

- Combined Adult / Child Resident (Future)

Dermal Exposure to Soil (0-4'bls)

Carcinogenic and Noncarcinogenic Risk

\begin{tabular}{|c|c|c|c|c|c|c|c|c|c|}
\hline \multirow[b]{2}{*}{ Chemical } & \multirow{2}{*}{$\begin{array}{r}\text { CAS(1) } \\
\text { Number }\end{array}$} & \multirow{2}{*}{ 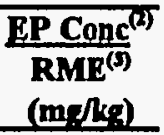 } & \multirow[b]{2}{*}{$\begin{array}{c}\mathrm{DSF}()^{(0)} \\
(\mathrm{kq}-\mathrm{d} / \mathrm{m} \mathrm{g})\end{array}$} & \multirow[b]{2}{*}{$\begin{array}{c}\operatorname{DRID}^{(5)} \\
(\mathbf{m} q / k g-d)\end{array}$} & \multirow[b]{2}{*}{ DABS $^{(0)}$} & \multicolumn{2}{|c|}{ Cancer Risks") } & \multicolumn{2}{|c|}{ Hazard Ouotient $^{(\mathrm{t})}$} \\
\hline & & & & & & RME & $\begin{array}{l}\% \\
\text { Total }\end{array}$ & RME & $\begin{array}{l}\text { \% of } \\
\text { Total }\end{array}$ \\
\hline \multicolumn{10}{|l|}{ Metals } \\
\hline Arsenic & $7440-38-2$ & $5.29 E+00$ & $1.88 E+\infty 0$ & $2.40 \mathrm{E}-04$ & $1.00 \mathrm{E}-03$ & $6.0 \mathrm{E}-08$ & $02 \%$ & 5.1E-04 & $02 \%$ \\
\hline Chromium & $7440-47-3$ & $7.65 \mathrm{E}+01$ & - & $1.00 \mathrm{E}-04$ & $1.00 \mathrm{E}-03$ & - & - & $1.8 \mathrm{E}-02$ & $61 \%$ \\
\hline Lead & $7439-92-1$ & $2.62 E+01$ & - & - & $1.00 \mathrm{E}-03$ & - & - & - & - \\
\hline Manganese & $7439-96-5$ & $3.61 \mathrm{E}+02$ & - & $1.50 \mathrm{E}-03$ & $1.00 \mathrm{E}-03$ & - & - & $5.5 \mathrm{E}-03$ & $19 \%$ \\
\hline \multirow[t]{2}{*}{ Mercury } & $7439-97-6$ & $3.58 \mathrm{E}-01$ & - & $3.00 \mathrm{E}-06$ & $1.00 \mathrm{E}-03$ & - & - & 2.7E-03 & $10 \%$ \\
\hline & & & & PATHWAY & / SUMS: & \multicolumn{2}{|c|}{$\begin{array}{c}\text { Cancer Risk } \\
\text { RMER } \\
\text { 3E-06 }\end{array}$} & \multicolumn{2}{|c|}{$\begin{array}{l}\text { Hazard Index } \\
\text { RME } \\
\text { 3E-02 }\end{array}$} \\
\hline
\end{tabular}

Noles:

1. Chemical Abetract Service.

2. Exposure point concentration

3. Reaconable maximum expocure.

4. Slope factor, dermal.

3. Referenoe dose, dermal.

6. Abeorption factor.

7. Cenoer Risk = (Chemical Concentration, me/kg * Carcinogenic Intuke Factor, kg/kg-day * Abeorption Factor, unitless * Slope Factor, kg-day/mg), uhtere RME carcinogenic inrake factor 6.07E-06

(Nole: Cancinogenic inkake factor calculated using weigtied (combinod) exponure assumptions.)

8. Hazand Quotient = (Chemical Concentration, mg/kg * Noncarcinogenic Intake Factor, kg/kg-day * Abworption Factor, unitless) / (Reference Doce, mg/kg-day), where RME noncarcinogenic intake fa 2.30E-05 (Child Value)

(Nose: Noncercinogenic inetke fectors ene calculesed for both edult and child, with the maximum of these used for risk calculations.) 
Table D.18

Savannah River Site

Burning/Rubble Pits (231-F, 231-1F)

Future Worker

Dermal Exposure to Soil (0-4'bls)

Carcinogenic and Noncarcinogenic Risk

\begin{tabular}{|c|c|c|c|c|c|c|c|c|c|}
\hline \multirow[b]{2}{*}{ Chemical } & \multicolumn{2}{|c|}{ EP Conc ${ }^{(2)}$} & \multirow[b]{2}{*}{$\begin{array}{c}\operatorname{DSF}^{(1)} \\
(\mathrm{kg}-\mathrm{d} / \mathrm{m} g)\end{array}$} & \multirow[b]{2}{*}{$\begin{array}{c}\text { DRm } \\
(\mathrm{mg} / \mathrm{kg}-\mathrm{d})\end{array}$} & \multirow[b]{2}{*}{ DABS $^{(9)}$} & \multicolumn{2}{|c|}{ Cancer Risk ${ }^{(7)}$} & \multicolumn{2}{|c|}{ Hazard Ountient ${ }^{(3)}$} \\
\hline & $\begin{array}{c}\text { CAS }^{(1)} \\
\text { Number }\end{array}$ & $\begin{array}{l}\mathrm{RME}^{(3)} \\
\text { (mg/kg) }\end{array}$ & & & & $\mathbf{R M E}$ & $\begin{array}{l}\% \text { of } \\
\text { Total }\end{array}$ & $\mathbf{R M E}$ & $\begin{array}{l}\% \text { of } \\
\text { Total }\end{array}$ \\
\hline \multicolumn{10}{|l|}{ Semivolatiles } \\
\hline 2-Methylnaphthalene & $91-57-6$ & $7.69 E-02$ & - & - & $1.00 \mathrm{E}-02$ & - & - & - & - \\
\hline Acensphthylene & $208-96-8$ & $8.61 E-02$ & - & - & $1.00 \mathrm{E}-02$ & - & - & - & - \\
\hline Benzo(a) anthracence & $56-55-3$ & $9.26 \mathrm{E}-01$ & $1.46 \mathrm{E}+00$ & - & $1.00 \mathrm{E}-02$ & $3.0 \mathrm{E}-08$ & $03 \%$ & - & - \\
\hline Benzo(a)pyrene & $50-32-8$ & $6.49 \mathrm{E}-01$ & $3.65 \mathrm{E}+01$ & - & $1.00 \mathrm{E}-02$ & 5.3E-07 & $55 \%$ & - & - \\
\hline Benzo(b)fluoranthene & $205-99-2$ & $1.01 E+00$ & $1.46 \mathrm{E}+00$ & - & $1.00 \mathrm{E}-02$ & $3.3 \mathrm{E}-08$ & $03 \%$ & - & - \\
\hline Benzo( $(B, h, i)$ perylene & $191-24-2$ & $6.15 \mathrm{E}-01$ & - & - & $1.00 \mathrm{E}-02$ & - & - & - & - \\
\hline Dibenz $(a, h)$ anthracene & $53-70-3$ & $2.17 E-01$ & $1.46 \mathrm{E}+01$ & - & $1.00 \mathrm{E}-02$ & $7.1 E-08$ & $07 \%$ & - & - \\
\hline Phenanthrene & $85-01-8$ & $6.87 E-01$ & - & - & $1.00 \mathrm{E}-02$ & - & - & - & - \\
\hline \multicolumn{10}{|l|}{ Dioxins/furans } \\
\hline Heptachlorodibenzo-p-dioxin isomer & $37871-00-4$ & $4.11 \mathrm{E}-03$ & $1.72 \mathrm{E}+03$ & - & $1.00 \mathrm{E}-02$ & $1.6 \mathrm{E}-07$ & $16 \%$ & - & - \\
\hline Heptachlorodibenzo-p-furan isomer & $38998-75-3$ & $2.58 \mathrm{E}-04$ & $1.72 E+03$ & - & $1.00 \mathrm{E}-02$ & $9.9 \mathrm{E}-09$ & $01 \%$ & - & - \\
\hline Hexachlorodibenzo-p-furan isomer & $55684-94-1$ & $1.00 \mathrm{E}-04$ & $1.72 \mathrm{E}+04$ & - & $1.00 \mathrm{E}-02$ & $3.9 \mathrm{E}-08$ & $04 \%$ & - & - \\
\hline Octachlorodibenzo-p-dioxin isomer & $3268-87-9$ & $9.26 \mathrm{E}-03$ & $1.72 E+02$ & - & $1.00 E-02$ & $3.6 \mathrm{E}-08$ & $04 \%$ & - & - \\
\hline Octachlorodibenzo-p-furan isomer & $39001-02-0$ & $3.48 \mathrm{E}-0.4$ & $1.72 \mathrm{E}+02$ & - & $1.00 \mathrm{E}-02$ & $1.3 \mathrm{E}-09$ & $<1 \%$ & - & - \\
\hline $\begin{array}{l}\text { PCBs } \\
\text { PCB } 1254\end{array}$ & $11097-69-1$ & $1.78 \mathrm{E}-01$ & $8.56 \mathrm{E}+\infty 0$ & $1.80 \mathrm{E}-05$ & $1.00 \mathrm{E}-02$ & $3.4 \mathrm{E}-08$ & $04 \%$ & 6.2E-04 & $08 \%$ \\
\hline \multicolumn{10}{|l|}{ Pesticides } \\
\hline delta-Benzene hexachloride & $319-86-8$ & $1.84 \mathrm{E}-03$ & - & - & $1.00 E-02$ & - & - & - & - \\
\hline Endrin ketone & $53494-70-5$ & $3.20 \mathrm{E}-03$ & - & - & $1.00 \mathrm{E}-02$ & - & - & - & - \\
\hline
\end{tabular}




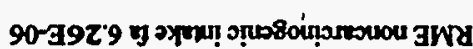

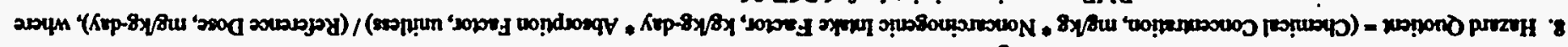

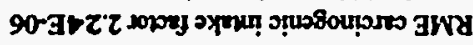

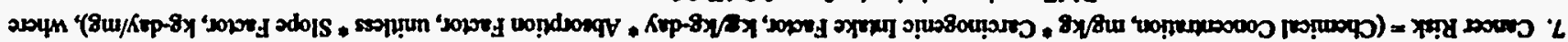

1 roporj uondrosqy is

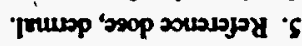

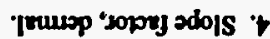

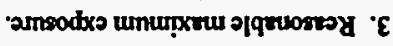

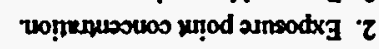

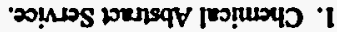

:TON

\begin{tabular}{|c|c|c|c|}
\hline \multicolumn{2}{|c|}{ 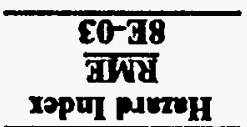 } & \multicolumn{2}{|c|}{ 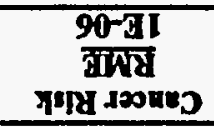 } \\
\hline$\% 01$ & to-GSL & - & - \\
\hline$\% 6 \mathrm{I}$ & EO-AS'1 & - & - \\
\hline- & - & - & - \\
\hline$\% 19$ & c0-98: & - & - \\
\hline$\% 20$ & to-3b 1 & \%zo & $80 \mathrm{ar} 2$ \\
\hline $\begin{array}{l}510 \Omega \\
j 0 \%\end{array}$ & âd & $\begin{array}{l}1010 \mathrm{I} \\
10 \%\end{array}$ & âd \\
\hline \multicolumn{2}{|c|}{ 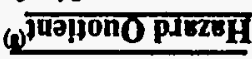 } & \multicolumn{2}{|c|}{ (1) } \\
\hline
\end{tabular}

\section{:SWIS XVMHLVd}

\begin{tabular}{|c|c|c|c|c|}
\hline 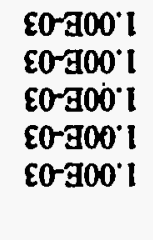 & $\begin{array}{l}\text { 90-G00' } \\
\text { EO-G05'I } \\
\text { t0-300'I } \\
\text { t0-30t }\end{array}$ & & 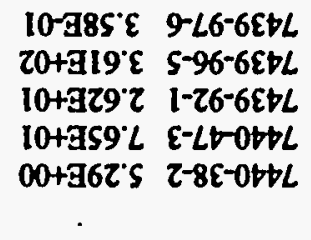 & 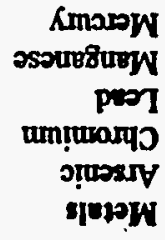 \\
\hline oSAva & 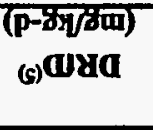 & $\begin{array}{l}\text { (8m/p-in) } \\
\text { (1) } 150\end{array}$ & 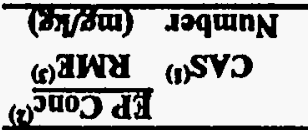 & 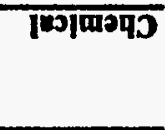 \\
\hline
\end{tabular}

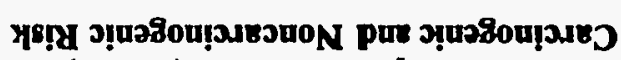

$(819,0-0)$ jos of amsodxg fruusa

dospoM aunjng

(HI-IEZ 'H-IEZ) s7!d गүqqm/8u!ung

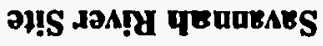

$81^{\circ}$ व गपष्L 
Table D.19

Savannah River Site

Burning/Rubble Pits (231-F, 231-1F)

Future Worker

Inhalation of Volatile Soil Contaminants (0-4'bls)

\begin{tabular}{|c|c|c|}
\hline \multicolumn{3}{|l|}{ EXPOSURE ASSUMPTIONS: } \\
\hline Receptor & Future & Vorke \\
\hline Exposure & $\mathrm{RME}^{(1)}$ & \\
\hline Inhalation Rate (IR), $\mathrm{m}^{3} / \mathrm{hr}$ & 2.5 & (4) \\
\hline Exposure Time (ET), hrs/dày & 1 & (4) \\
\hline Exposure Frequency (EF), days/yr & 250 & (4) \\
\hline Exposure Duration (ED), yrs & 25 & (4) \\
\hline Body Weight (BW), kg & 70 & (4) \\
\hline Avging Time, Carc ${ }^{(2)}$ (ATC), days & 25,550 & (4) \\
\hline Avging Time, Noncarc ${ }^{(B)}$ (ATN), days & 9,125 & \\
\hline
\end{tabular}

INTAKR FACTOR CALCULATIONS

Carcinogenic Intake Factor (CFF), kg/kg-day =

$(\mathrm{R} \bullet \mathrm{ET} \bullet \mathrm{EF} * \mathrm{ED} * \mathrm{CF}) /(\mathrm{BW} \bullet \mathrm{ATC})$

$\mathrm{RME} C \mathrm{CIF}=8.74 \mathrm{E}-03$

Noncarcinogenic Intake Factor (NIF), kg/kg-day = $(\mathrm{R} \bullet \mathrm{ET} \bullet \mathrm{EF} \bullet \mathrm{ED} \bullet \mathrm{CF}) /(\mathrm{BW} \bullet \mathrm{ATN})$

RME NIF $=2.45 E-02$

\author{
Noter: \\ 1. Ressonable maximum exposure. \\ 2. Averaging time, curcinogen; calculated as 70 years (average lifetime) times 365 days per year (not upplicable to child residenx).

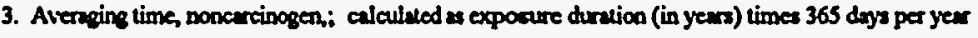

4. See Table D-1. 
Table D.19

Savannah River Site

Burning/Rubble Pits (231-F, 231-1F)

Future Worker

Inhalation of Volatile Soil Contaminants (0-4'bls)

Calculation of Volatilization Factor (VF)

\begin{tabular}{|c|c|c|c|c|c|c|c|c|c|c|c|c|}
\hline Chemical & $\operatorname{CAS}^{(2)} \mathrm{Na}$ & Class(3) & MolWt. & $\log K a c$ & $\operatorname{Koc}^{(t)}$ & $\mathbf{K} \mathbf{d}^{(0)}$ & $H^{(4)}$ & $\mathrm{Kas}^{(1)}$ & $\mathbf{D i}^{(0)}$ & $\operatorname{Def}^{(t)}$ & alpha $a^{(4)}$ & $\begin{array}{l}\text { Volat } \\
\text { Factor } \\
\left(\mathrm{m}^{3} / \mathrm{kg}\right)\end{array}$ \\
\hline \multicolumn{13}{|l|}{ Semivolatlles } \\
\hline 2-Methylnaphthalene & $91-57.6$ & $\mathbf{S}$ & $1.42 \mathrm{E}+02$ & 3.83 & 8511.4 & $1.70 E+02$ & $2.90 \mathrm{E}-04$ & $6.98 \mathrm{E}-05$ & $6.43 \mathrm{E}-02$ & $4.53 \mathrm{E}-02$ & 6.43E-07 & $1.67 \mathrm{E}+05$ \\
\hline Acenaphthylene & $208-96-8$ & $\mathbf{S}$ & $1.52 \mathrm{E}+02$ & 3.68 & 4786.3 & $9.57 E+01$ & $2.80 \mathrm{E}-04$ & $1.20 \mathrm{E}-04$ & $6.22 \mathrm{E}-02$ & $4.38 \mathrm{E}-02$ & $1.07 \mathrm{E}-06$ & $1.29 \mathrm{E}+05$ \\
\hline Phenanthrene & $85-01-8$ & $\mathbf{S}$ & $1.78 \mathrm{E}+02$ & 4.59 & 38904.5 & $7.78 \mathrm{E}+02$ & $2.56 \mathrm{E}-05$ & $1.35 \mathrm{E}-06$ & $5.74 \mathrm{E}-02$ & 4.05E-02 & $1.11 \mathrm{E}-08$ & $1.27 \mathrm{E}+06$ \\
\hline
\end{tabular}

Noter:

1. USEPA, 1991: RAGS: Vol 1 - Humen Heath Evaluation Manual (Part B, Development of Risk-Beced Preliminary Remediation Gaals)

2. Chemical Abutract Service.

3. Chemical class: v-volatile; s-semivolatile; $p$-pesticide/pcb; $x$-dioxin; and $m$-metal.

4. Calculation of organic contaminant volatilization factor:

CONSTANTS:

Length of side of contan'd. area (LS), $m=$

Wind speed in mixing zone $(\mathrm{V}) \mathrm{m} / \mathrm{sec}=$ Diffusion height (DH), $m=$ Area of contamination (Am) $\mathrm{m}^{2}=$ Area in square centimeters $(A \mathrm{~cm}), \mathrm{cm}^{2}=$ True soil porosity (E), unitless = True soil density (P3), g/cm' = Expocure interval (Tyr), yis = Expoure interval (Trec), sec = Organic carbon content (OC), unilless = INPUT VARIABLES: Converrion factor (CF), $\mathrm{kg} / \mathrm{g}=$

Organic curbon partition coef. (Koc), $\mathrm{cm}^{3} / \mathrm{kg}=$ Henry's Law constant $(\mathrm{H})$, atm-m $3 / \mathrm{mol}=$ Gas diffusivity (Di), $\mathrm{cm}^{2} / \mathrm{sec}=$

INTERMEDLATE STEPS:

Soil-water partition coefficient (Kd), $\mathrm{cm}^{3} / \mathrm{kg}=$ Soil-air partition coefficient (Kas), g-0il/ $\mathrm{cm}^{3}$-eir = Effective diffurivity (Dei), $\mathrm{cm}^{2} / \mathrm{sec}=$ alpha (alpha), $\mathrm{cm}^{2} / \mathrm{sec}=$

OUTPUT:

Volatilization Factor (VF), $\mathrm{m}^{3} / \mathrm{kg}=$

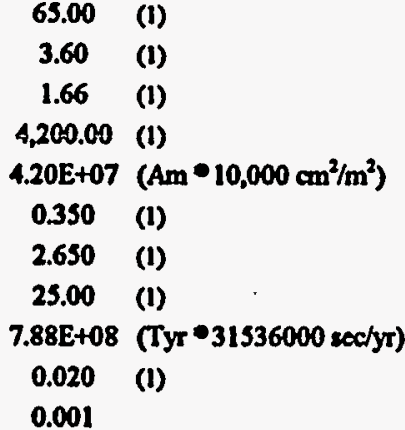

chem-epec

chem-pec

chem-epec

chem-spec (Koc $\times$ OC)

deim-epec $((\mathrm{H} / \mathrm{Kd}) \times 41)$

chem-ipec (Di $\times$ (EM.33))

chem-pec (Dei $\times$ E) $\left.\left(E+\left(P_{3}\right)(1-E)\right) / K 2 s\right)$

chemepec $((L S \times V \times D H \times(3.14 \times a j p h a \times T) 0.5)(A \times 2 \times D e i \times E \times K a s \times C F))$ 
Table D.19

Savannah River Site

Burning/Rubble Pits (231-F, 231-1F)

Future Worker

Inhalation of Volatile Soil Contaminants ( $0-4$ 'bls)

Carcinogenic and Noncarcinogenic Risk

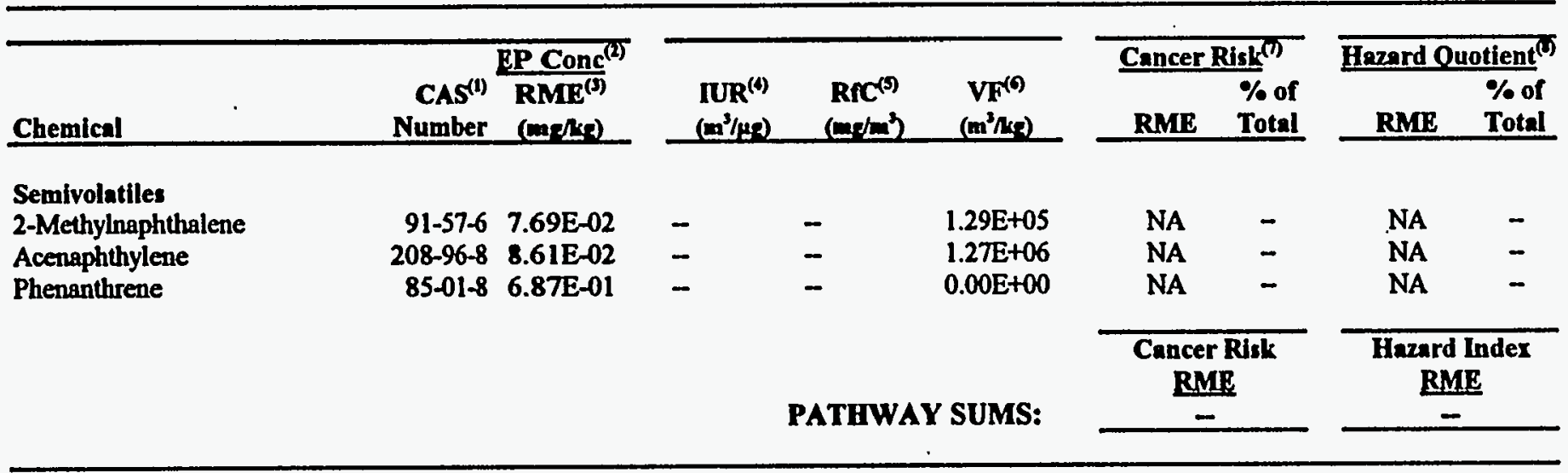

Notsi:

1. Chemical Abstract Service.

2. Exposure poim concentration.

3. Reasonable maximum exposure.

4. Inhalation unit risk

5. Reference concentration.

6. Volatilization factor (chemical-specific)

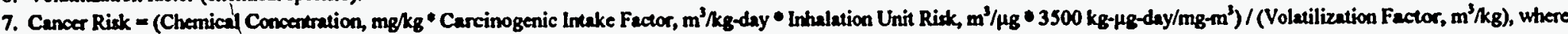
RME carcinogenic intake factor 8.74E-03

and converion from IUR to inhalation slope factor $=3500 \mathrm{~kg}-\mu \mathrm{g}-$ day $/ \mathrm{mg}^{3} \mathrm{~m}^{3}$

8. Hezard Quotient = (Chemical Concentration, mg/kg Noncarcinogenic Intake Factor, m $\mathrm{m}^{3} / \mathrm{kg}_{\mathrm{g}}$-day) $/\left(\right.$ Volatilization Factor, $\mathrm{m}^{3} / \mathrm{kg}_{\mathrm{g}} \bullet \mathrm{Reference}$ Concentration, $\mathrm{mg} / \mathrm{m}^{3} * 2 / 7 \mathrm{~m}^{3} / \mathrm{kg}_{\mathrm{g}}$-day), where RME noncarcinogenic intake fa $2.45 \mathrm{E}-02$

and conversion from RSC to inhalation reference dose $=2 / 7 \mathrm{~m}^{3} / \mathrm{kg}$-day 
Table D.20

Savannah River Site

Burning/Rubble Pits (231-F, 231-1F)

Future Worker

Inhalation of Resuspended Soil Particulate Contaminants (0-4'bls)

Carcinogenic and Noncarcinogenic Risk

\section{EXPOSURE ASSUMPTIONS:}

Receptor

Exposure

Inhalation Rate (R), $\mathrm{m}^{3} \mathrm{hr}$

Exposure Time (ET), hrs/day

Exposure Frequency (EF), days/yr

Exposure Duration (ED), yrs

Body Weight (BW), kg

Avging Time, $\operatorname{Carc}^{(2)}$ (ATC), days

Avging Time, Noncarc ${ }^{(\mathfrak{)})}$ (ATN), days

\section{Future Worker}

$\mathrm{BME}^{(1)}$

$2.5 \quad$ (4)

1 (4)

$250 \quad$ (4)

25 (4)

(4)

25,550

\section{INTAKE FACTOR CALCULATIONS}

Carcinogenic Intake Factor (CF), kg/kg-day = $(\mathbb{R} \bullet \mathrm{FI} \bullet \mathrm{EF} \bullet \mathrm{ED} \bullet \mathrm{CF}) /(\mathrm{BW} \bullet \mathrm{ATC})$

$\mathrm{RME} C \mathrm{CF}=8.74 \mathrm{E}-03$

Noncarcinogenic Intake Factor (NIF), kg/kg-day = $(\mathrm{R} * \mathrm{FI} * \mathrm{EF} \bullet \mathrm{ED} * \mathrm{CF}) /(\mathrm{BW} \bullet \mathrm{ATN})$

RME NIF $=2.45 E_{-02}$

\section{Noten}

1. Reconsteble meximum exposure.

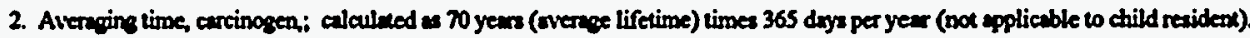

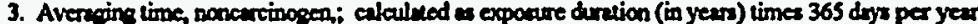

4. See Teble D.1. 
Table D.20

Savannah River Site

Burning/Rubble Pits (231-F, 231-1F)

Future Worker

Inhalation of Resuspended Soil Particulate Contaminants (0-4'bls)

Carcinogenic and Noncarcinogenic Risk

\begin{tabular}{|c|c|c|c|c|}
\hline \multicolumn{4}{|c|}{ CALCULATION OF SOL PARTICULATE RESUSPENSION FACTOR: } & \multirow{2}{*}{$\begin{array}{l}\text { INTERMEDIATE STEPS: } \\
\text { Particulate emission factor }(P E F)=\quad 2.18 E+09 \text { meters }{ }^{3} / \mathrm{kg}\end{array}$} \\
\hline Length of side of contaminated area (LS) $=$ & 65.00 & meters & (1) & \\
\hline Wind speed in mixing zone $(V)=$ & 3.60 & meters/sec & (1) & $(\mathrm{LS} \times \mathrm{V} \times \mathrm{DH} \times \mathrm{CFa} \times \mathrm{CFb}) /\left(\mathrm{A} \times \mathrm{RF} \times(1-\mathrm{G}) \times\left((\mathrm{Um} / \mathrm{Ut})^{\wedge} 3\right) \times(\mathrm{Fx})\right)$ \\
\hline Diffusion height $(\mathrm{DH})=$ & 1.66 & meters & (1) & \\
\hline Area of contamination $(A)=$ & $4,200.00$ & meters $^{2}$ & (1) & \\
\hline Respirable fraction (RF) $=$ & 0.003 & g/meters ${ }^{2}-h r$ & (1) & $\cdot$ \\
\hline Fraction of vegetative cover $(G)=$ & 0.90 & unitless & (1) & \\
\hline Mean annual wind speed $(\mathrm{Um})=$ & 3.60 & meters/sec & (1) & \\
\hline Equivalent threshold value of wind speed at $10 \mathrm{~m}(\mathrm{Ut})=$ & 5.04 & meters/sec & (1) & \\
\hline Fraction dependent on Um/Ut $(F x)=$ & 1.2700 & unitless & (1) & \\
\hline Conversion factor $(\mathrm{CFa})=$ & $3,600.00$ & $\sec / \mathrm{hr}$ & & \\
\hline Conversion factor $(\mathrm{CFb})=$ & $1,000.00$ & g/kg & & \\
\hline
\end{tabular}

Nolen:

1. USEPA, 199I: RAGS: Vol I - Humen Health Evaluation Menual (Pan B, Development of Risk-Based Preliminary Remediation Gials) 
Table D.20

Savannah River Site

Burning/Rubble Pits (231-F, 231-1F)

Future Worker

Inhalation of Resuspended Soil Particulate Contaminants (0-4'bls)

Carcinogenic and Noncarcinogenic Risk

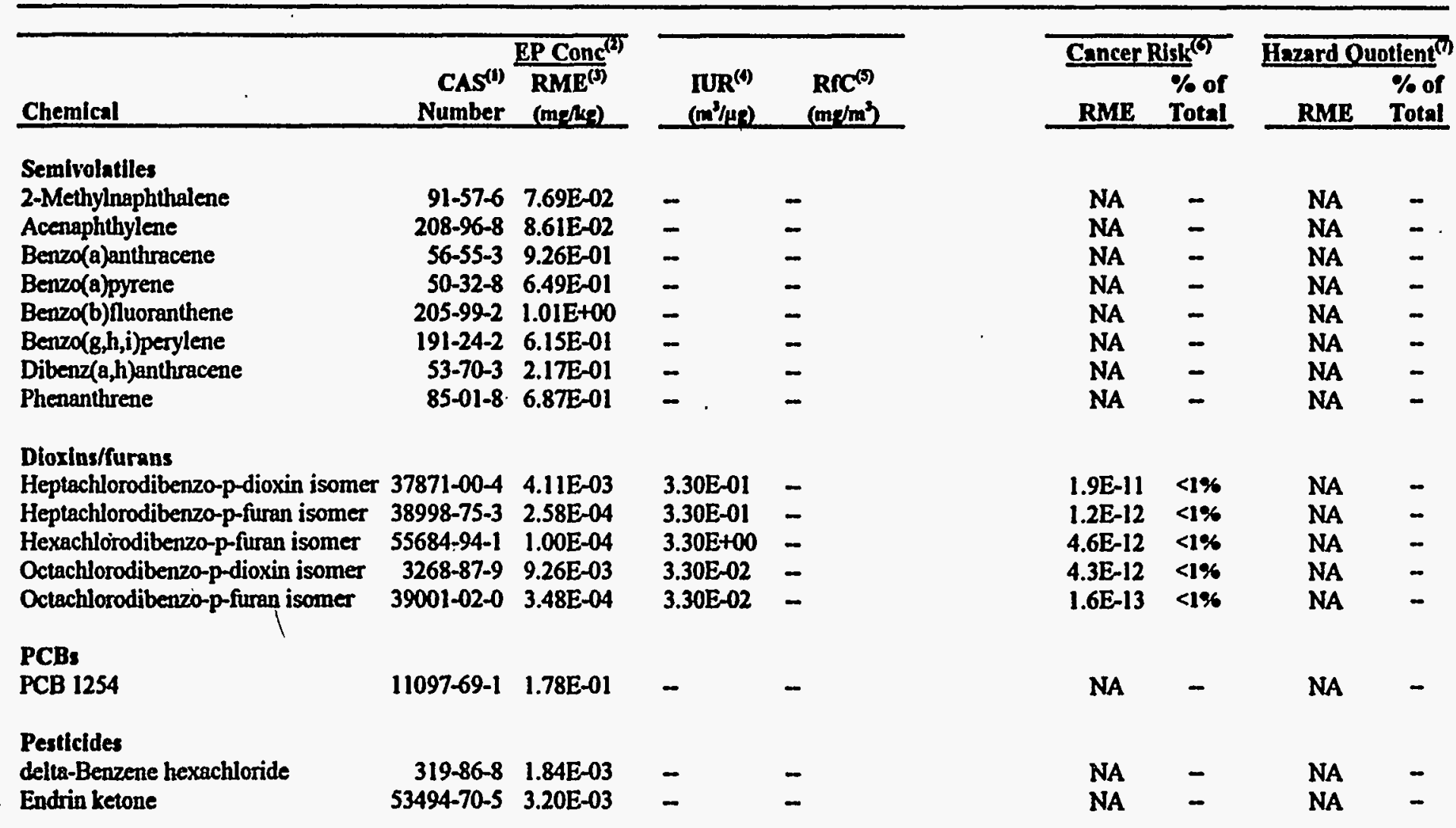


Table D.20

Savannah River Site

Burning/Rubble Pits (231-F, 231-1F)

Future Worker

Inhalation of Resuspended Soil Particulate Contaminants (0-4'bls)

Carcinogenic and Noncarcinogenic Risk

\begin{tabular}{|c|c|c|c|c|c|c|c|}
\hline Chemical & $\begin{aligned} & \operatorname{EPConc}(1) \\
& \operatorname{CAS}^{(1)} \operatorname{RME}^{(3)} \\
& \text { Number }(\mathrm{m} / \mu \mathrm{kg}) \\
&\end{aligned}$ & $\begin{array}{l}\mathrm{TR}^{(0)} \\
\left(\mathrm{m}^{3} / \mu\right)\end{array}$ & $\begin{array}{r}\operatorname{RfC}^{(5)} \\
\left(m / m^{3}\right) \\
\end{array}$ & $\begin{array}{l}\text { Cancer } \\
\text { RME }\end{array}$ & $\begin{array}{l}\text { isk } \\
\% \text { of } \\
\text { Total } \\
\end{array}$ & $\begin{array}{r}\text { Hazard Qu } \\
\text { RME } \\
\end{array}$ & $\begin{array}{l}\text { otient }^{(\%)} \\
\% \text { of } \\
\text { Total } \\
\end{array}$ \\
\hline $\begin{array}{l}\text { Metals } \\
\text { Arsenic } \\
\text { Chromium } \\
\text { Lead } \\
\text { Manganese } \\
\text { Mercury }\end{array}$ & $\begin{array}{ll}7440-38-2 & 5.29 E+00 \\
7440-47-3 & 7.65 E+01 \\
7439-92-1 & 2.62 E+01 \\
7439-96-5 & 3.61 E+02 \\
7439-97-6 & 3.58 E-01\end{array}$ & $\begin{array}{l}4.30 \mathrm{E}-03 \\
1.20 \mathrm{E}-02 \\
- \\
- \\
-\end{array}$ & $\begin{array}{l}- \\
- \\
\overline{5.00 E-05} \\
3.00 \mathrm{E}-04\end{array}$ & $\begin{array}{c}3.2 \mathrm{E}-10 \\
1.3 \mathrm{E}-08 \\
\text { NA } \\
\text { NA } \\
\text { NA }\end{array}$ & $\begin{array}{l}02 \% \\
97 \% \\
- \\
-\end{array}$ & $\begin{array}{c}\text { NA } \\
\text { NA } \\
\text { NA } \\
2.8 \mathrm{E}-04 \\
4.7 \mathrm{E}-08\end{array}$ & $\begin{array}{l}- \\
- \\
100 \% \\
<1 \%\end{array}$ \\
\hline & & & PATHWAY SUMS: & $\begin{array}{r}\text { Cancer } \\
\frac{\mathrm{RM}}{1 \mathrm{E}-} \\
\end{array}$ & Risk & $\begin{array}{r}\text { Hezard } \\
\qquad \begin{array}{r}\text { RM } \\
\mathbf{E}-1\end{array} \\
\end{array}$ & $\begin{array}{l}\text { Index } \\
\\
\end{array}$ \\
\hline $\begin{array}{l}\text { Noter: } \\
\text { 1. Chemical } \\
\text { 2. Exposure } \\
\text { 3. Reasonabi } \\
\text { 4. Inhalation } \\
\text { 5. Reference } \\
\text { 6. Cancer Ris } \\
\text { 7. Hazard Qu }\end{array}$ & $\begin{array}{l}\text { mg/kg * Carcinogenic Intak } \\
\text { RME carcinogenic intake fa } \\
\text { Particulate emission factor } \\
\text { and conversion from IUR to } \\
\text { tion, mg/kg * Noncarcinogen } \\
\text { RME noncarcinogenic intak } \\
\text { Particulate cmission factor } \\
\text { and conversion from RPC to }\end{array}$ & $\begin{array}{l}\text { actor, } \mathrm{m}^{3} / \mathrm{kB}_{\mathrm{B}} \\
8.74 \mathrm{E}-03 \\
2.18 \mathrm{E}+09 \\
\text { halation slope } \\
\text { Intake Factor } \\
2.45 \mathrm{E}-02 \\
2.18 \mathrm{E}+09 \\
\text { calation refer }\end{array}$ & $\begin{array}{l}\text { * Inhalation Unit Risk, } \mathrm{m}^{3} / \mu_{8} \\
\mathrm{n}^{3} / \mathrm{kg} \text {-day) } /(\text { Particulate Emissi } \\
\text { Le dose }=2 / 7 \mathrm{~m}^{3} / \mathrm{kg}_{\mathrm{B}} \text {-day }\end{array}$ & $\begin{array}{l}3500 \mathrm{~kg}-\mu \mathrm{g} \\
\text { Factor, } \mathrm{m}^{3} / 1\end{array}$ & Refer & / (Particulate) & $\mathrm{m}, \mathrm{mg} / \mathrm{m}^{3}$ \\
\hline
\end{tabular}


Table D.21

Savannah River

Rubble Pit (231-2F)

Future Worker

Ingestion of Soil (0-2'bls)

Carcinogenic and Noncarcinogenic Risk

\section{EXPOSURE ASSUMPTIONS:}

Receptor

Exposure

Intake Rate (IR), mg/day

Fraction Ingested (FI), unitless

Exposure Frequency (EF), days/yr

Exposure Duration (ED), yrs

Body Weight (BW), kg

Avging Time, $\mathrm{Carc}^{(2)}$ (ATC), days

Avging Time, Noncarc ${ }^{(3)}$ (ATN), days

Conversion Factor (CF), kg/mg

\section{INTAKE FACTOR CALCULATIONS}

Carcinogenic Intake Factor (CIF), $\mathrm{kg} / \mathrm{kg}_{\mathrm{g}}$-day = $(\mathbb{R} \bullet \mathrm{FI} \bullet \mathrm{EF} \bullet \mathrm{ED} \bullet \mathrm{CF}) /(\mathrm{BW} \bullet \mathrm{ATC})$ RME CIF $=1.75 \mathrm{E}-07$

Noncarcinogenic Intake Factor (NIF), kg/kg-day = $(\mathrm{IR} \bullet \mathrm{F} \bullet \mathrm{EF} \bullet \mathrm{ED} \bullet \mathrm{CF}) /(\mathrm{BW} * \mathrm{ATN})$

RME NIF $=4.89 \mathrm{E}-07$

\section{Nolse:}

1. Rearonable maximum exposure.

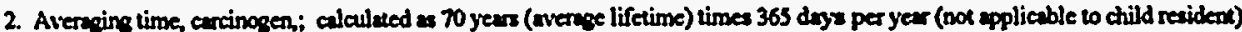

3. Avereging time, noscircinogen; calculted es exposure diration (in years) times 365 deys per year

4. See Trble D-1. 
Table D.21

Savannah River

Rubble Pit (231-2F)

Future Worker

Ingestion of Soil (0-2'bls)

Carcinogenic and Noncarcinogenic Risk

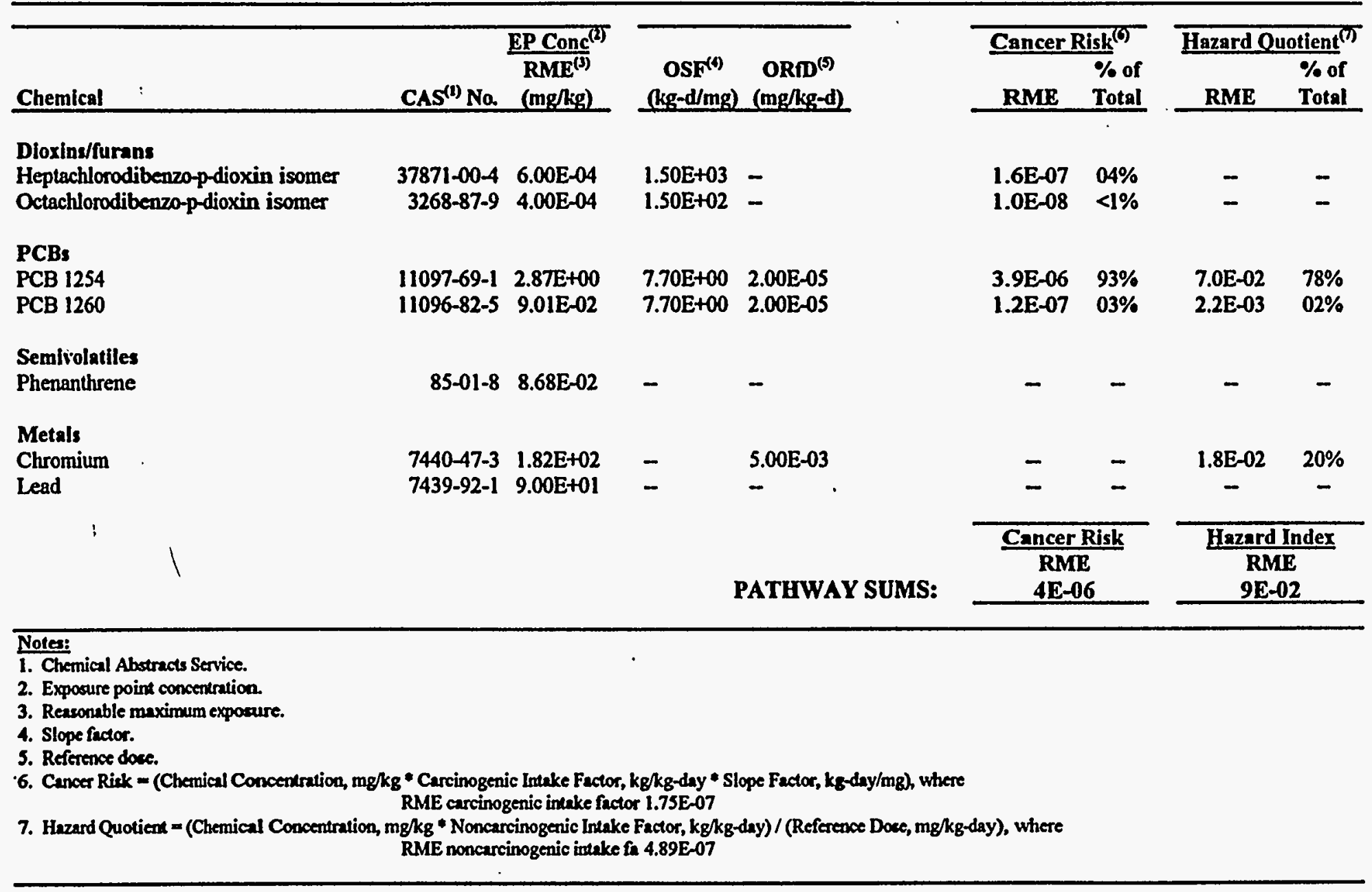


Table D.22

Savannah River Site

Rubble Pit (231-2F)

Future Worker

Dermal Exposure to Soil (0-2'bls)

Carcinogenic and Noncarcinogenic Risk

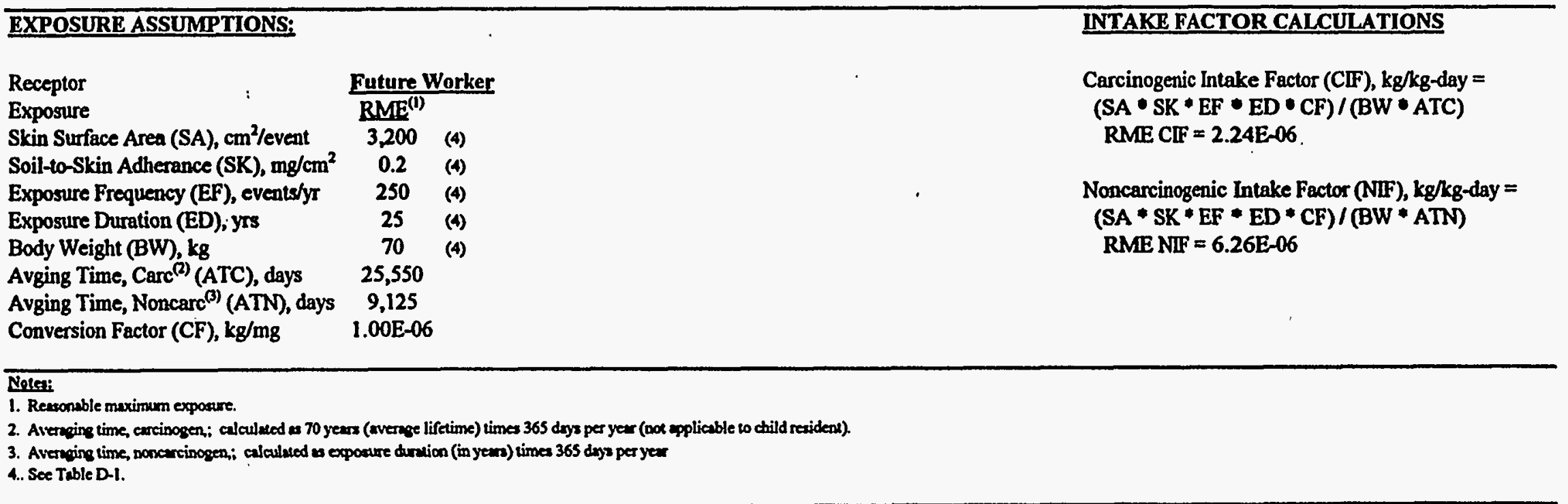


Table D.22

Savannah River Site

Rubble Pit (231-2F)

Future Worker

Dermal Exposure to Soil (0-2'bls)

Carcinogenic and Noncarcinogenic Risk

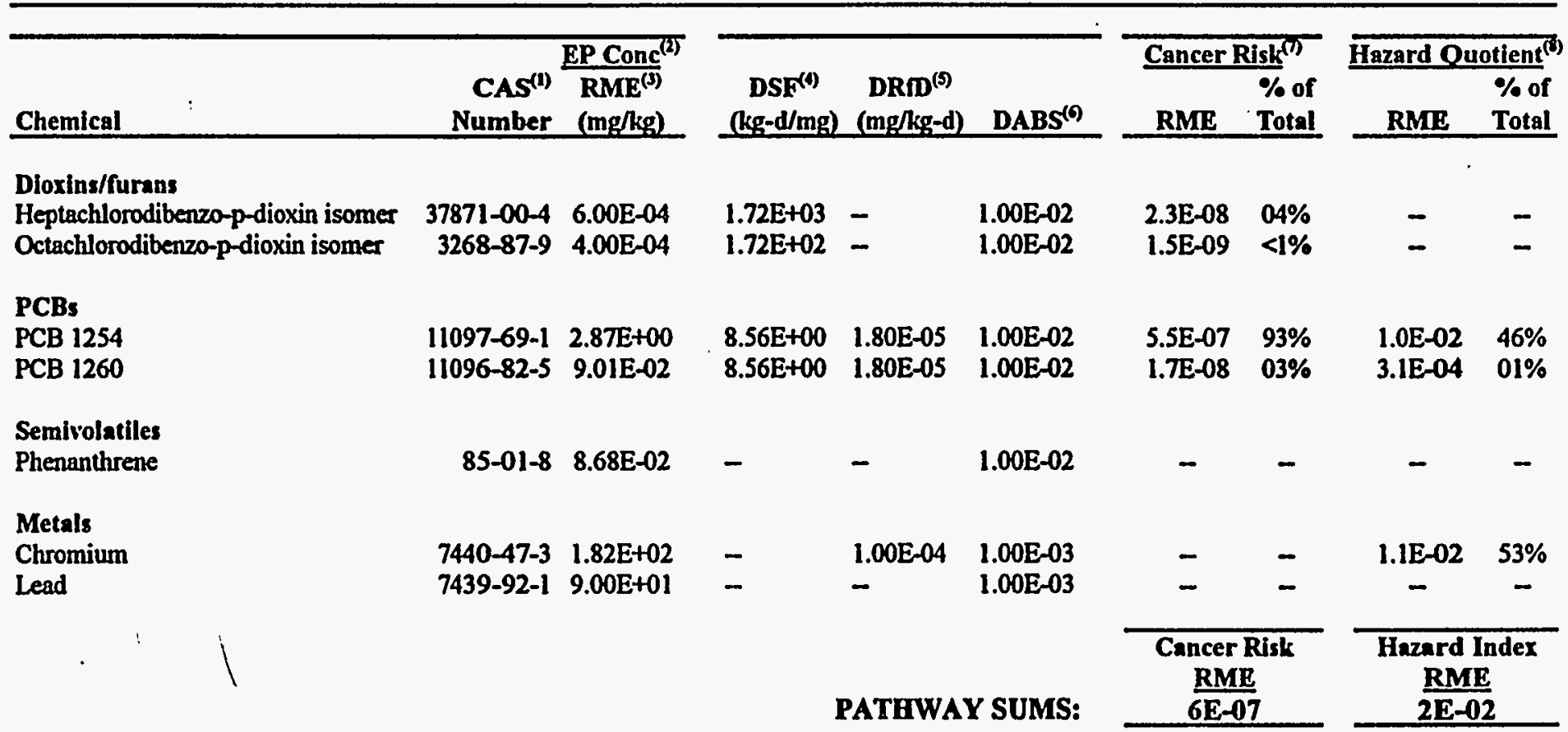

Notes:

1. Chemical Abstract Service.

2. Exposure point concentration.

3. Reasonable maximum exposure.

4. Slope factor, dermal.

5. Reference doce, dermal

6. Absorption factor.

7. Cancer Risk = (Chemical Concentration, mg/kg * Carcinogenic Intake Factor, k\&/kg-day • Absorption Factor, unitless * Slope Factor, kg-day/mg), where RME carcinogenic intake factor 2.24E-06

8. Hazard Quotient = (Chemical Concentration, mg/kg • Noncarcinogenic Intake Factor, kg/kg-day ${ }^{\bullet}$ Abrorption Factor, unitless)/(Reference Dose, mg/kg-day), where RME noncarcinogenic intake fa $6.26 \mathrm{E}-06$ 
Table D.23

Savannah River

Rubble Pit (231-2F)

Future Worker

Inhalation of Volatile Soil Contaminants (0-2'bls)

\begin{tabular}{|c|c|c|c|}
\hline \multicolumn{2}{|l|}{ EXPOSURE ASSUMPTIONS: } & INTAKE FACTOR CALCULATIONS & . \\
\hline Receptor & Future Worker & Cancinogenic Intake Factor (CIF), kg/kg-day $=$ & \\
\hline Exposure & $\mathrm{RME}^{(1)}$ & $(\mathrm{IR} \bullet \mathrm{ET} \bullet \mathrm{EF} * \mathrm{ED} \bullet \mathrm{CF}) /(\mathrm{BW} \bullet \mathrm{ATC})$ & \\
\hline Inhalation Rate (IR), $\mathrm{m}^{\mathbf{3}} / \mathrm{hr}$ & $2.5 \quad$ (4) & $\mathrm{RME}$ CIF $=8.74 \mathrm{E}-03$ & \\
\hline Exposure Time (ET), hrs/day & $1 \quad(4)$ & & \\
\hline Exposure Frequency (EF), days/yr & $250 \quad$ (4) & Noncarcinogenic Intake Factor (NIF), kg/kg-day = & \\
\hline Exposure Duration (ED), yrs & 25 (4) & $(\mathrm{IR} \bullet \mathrm{ET} \bullet \mathrm{EF} \bullet \mathrm{ED} \bullet \mathrm{CF}) /(\mathrm{BW} * \mathrm{ATN})$ & \\
\hline Body Weight (BW), kg & $70 \quad(4)$ & RME NIF $\approx 2.45 E-02$ & \\
\hline Avging Time, $\operatorname{Carc}^{(2)}$ (ATC), days & 25,550 (4) & & \\
\hline Avging Time, Noncarc ${ }^{(3)}$ (ATN), days & 9,125 & & \\
\hline \multicolumn{4}{|c|}{$\begin{array}{l}\text { Nodst: } \\
\text { 1. Reasonsble maximum exposure. }\end{array}$} \\
\hline \multicolumn{4}{|c|}{ 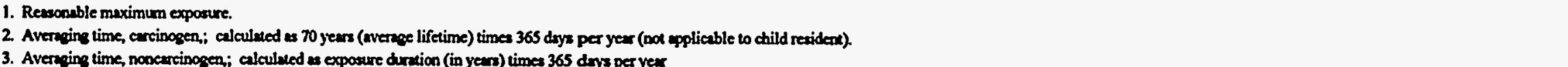 } \\
\hline \multicolumn{2}{|c|}{ 4.. See Table D-1. } & & \\
\hline
\end{tabular}


Table D.23

Savannah River

Rubble Pit (231-2F)

Future Worker

Inhalation of Volatile Soil Contaminants (0-2'bls)

Calculation of Volatilization Factor (VF) ${ }^{(\mathbf{1})}$

\begin{tabular}{|c|c|c|c|c|c|c|c|c|c|c|c|}
\hline Chemical & $\mathrm{CAS}^{(2)}$ No. & $\mathrm{Class}^{(3)}$ & MolWt & $\mathrm{Koc}^{(1)}$ & $\mathbf{K} \mathbf{d}^{(0)}$ & $\mathbf{H}^{(0)}$ & $\mathrm{Kas}^{(1)}$ & $\mathrm{Di}^{(4)}$ & $\mathrm{Dei}^{(0)}$ & alpha $a^{(4)}$ & $\begin{array}{l}\text { Volat } \\
\text { Factor }{ }^{(0)} \\
\left(\mathrm{m}^{3} / \mathrm{kg}\right)\end{array}$ \\
\hline $\begin{array}{l}\text { Semivolatiles } \\
\text { Phenanthrene }\end{array}$ & $85-01-8$ & $\mathbf{s}$ & $1.78 E+02$ & 38904.5 & $7.78 \mathrm{E}+02$ & $2.56 \mathrm{E}-05$ & $1.35 \mathrm{E}-06$ & $5.74 \mathrm{E}-02$ & $4.05 E-02$ & $1.11 \mathrm{E}-08$ & $1.27 \mathrm{E}+06$ \\
\hline
\end{tabular}

\section{Notes:}

1. USEPA, 1991: RAGS: Vol 1 - Human Health Evaluation Manual (Pant B, Development of Risk-Bused Preliminery Remediation Goals)

2. Chemical Abetract Service.

3. Chemical class: $v$ - volatile; s-semivolatile; $p$ - pesticide/peb; $x$ - dioxin; and $m$ - metal.

4. Calculation of onganic contaminant volatilization factor: CONSTANTS:

INPUT VARIABLES

$$
\begin{array}{r}
\text { Length of side of contum'd. area (LS), } \mathrm{m}= \\
\text { Wind speed in mixing zone (V), } \mathrm{m} / \mathrm{sec}= \\
\text { = Diffusion height (DH), } \mathrm{m}= \\
\text { Area of contamination (Am), } \mathrm{m}^{2}= \\
\text { = } \\
\text { Area in equare centimeters (Acm), } \mathrm{cm}^{2}= \\
\text { True coil porosity (E), unitless = } \\
\text { True soil density (Ps), } \mathrm{g} / \mathrm{cm}^{3}= \\
\text { Exposure interval (Tyr), yrs = } \\
\text { Exposure interval (Trec), sec = } \\
\text { Organic carbon content (OC), unitless = }
\end{array}
$$
Conversion factor (CF), $\mathrm{kg} / \mathrm{g}=$

Organic carbon partition coef. (Koc), $\mathrm{cm}^{3} / \mathrm{kg}=$ Henry's Law conatant (H), atm-m ${ }^{3} / \mathrm{mol}$ = Gas diffusivity (Di), $\mathrm{cm}^{2} / \mathrm{sec}=$

\section{INTERMEDIATE STEPS:}

Soil-water partition coefficient $(\mathrm{Kd}), \mathrm{cm}^{3} / \mathrm{kg}=$ Soil-air pertition coefficient (Kas), 8-soil//cm'-2ir = Effective diffusivity (Dei), $\mathrm{cm}^{2} / \mathrm{coc}=$ alphe (alpha), $\mathrm{cm}^{2} / 20 \mathrm{c}=$

OUTPUT:

Volatilization Factor (VF), $\mathrm{m}^{3} / \mathrm{kg}_{\mathrm{g}}=$

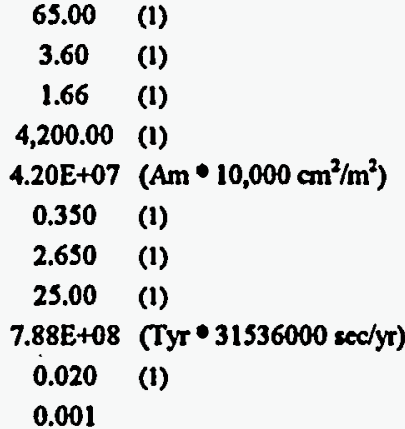

chem-spec $((L S \times V \times D H \times(3.14 \times a / p h a \times T) 0.5)(A \times 2 \times D e i \times E \times K a s \times C F))$ 
Table D.23

Savannah River

Rubble Pit (231-21)

Future Worker

Inhalation of Volatile Soil Contaminants (0-2'bls)

Carcinogenic and Noncarcinogenic Risk

\begin{tabular}{|c|c|c|c|c|c|c|c|c|c|}
\hline Chemical & \multicolumn{2}{|c|}{ 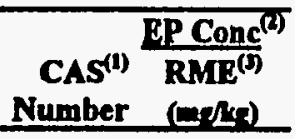 } & $\begin{array}{l}\operatorname{IUR}^{(0)} \\
\left(\boldsymbol{E}^{3} / \mathbf{y}^{\prime}\right)\end{array}$ & 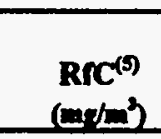 & $\begin{array}{l}V F^{(\theta)} \\
\left(\omega^{3} / n(s)\right.\end{array}$ & $\begin{array}{l}\text { Cancer } \\
\text { RME }\end{array}$ & $\begin{array}{l}\text { inkring } \\
\% \text { of } \\
\text { Total }\end{array}$ & $\begin{array}{l}\text { Hazrd 01 } \\
\text { RME } \\
\end{array}$ & $\begin{array}{l}\text { 2tient } \\
\% \text { of } \\
\text { Total }\end{array}$ \\
\hline $\begin{array}{l}\text { Semivolatiles } \\
\text { Phenunthrene }\end{array}$ & $85-01-8$ & $6.87 \mathrm{E}-01$ & \multirow{2}{*}{\multicolumn{3}{|c|}{ PATHWAY SUMS: }} & NA & - & NA & - \\
\hline & & & & & & \multicolumn{2}{|c|}{$\begin{array}{c}\text { Cancer Risk } \\
\text { RME } \\
-\end{array}$} & \multicolumn{2}{|c|}{$\begin{array}{c}\text { Hazard Index } \\
\text { RMI }\end{array}$} \\
\hline
\end{tabular}

Nesti:

1. Chemical Abetract Service.

2. Expoaure point concentration.

3. Reasomable maximum exposure.

4. Inhalation unit risk.

5. Reference concentration.

6. Volatilization factor (chemical-epecific).

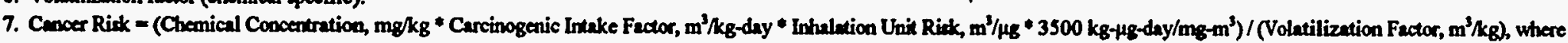
RME carcinogenic intake factor 8.74E-03

and conversion from IUR to inhalation alope factor $=3300 \mathrm{~kg}+48-\mathrm{day} / \mathrm{m}_{\mathrm{g}}-\mathrm{m}^{3}$

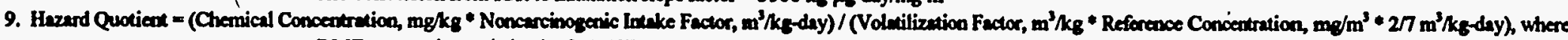
RME noncarcinogenic intake fin 2.45E-02

and conversion from RPC to inhalation reference doee $=2 / 7 \mathrm{~m}^{3} / \mathrm{kg}$ - day 
Table D.24

Savannah River Site

Rubble Pit (231-2F)

Future Worker

Inhalation of Resuspended Soil Particulate Contaminants (0-2'bls)

Carcinogenic and Noncarcinogenic Risk

\begin{tabular}{|c|c|c|}
\hline \multicolumn{3}{|l|}{ EXPOSURE ASSUMPTIONS: } \\
\hline Receptor & Future & Vorker \\
\hline Exposure & $\mathrm{RME}^{(1)}$ & \\
\hline Inhalation Rate $(\mathbb{R}), \mathrm{m}^{3} / \mathrm{hr}$ & 2.5 & (4) \\
\hline Exposure Time (ET), hrs/day & 1 & (4) \\
\hline Exposure Frequency (EF), days/yr & 250 & (4) \\
\hline Exposure Duration (ED), yrs & 25 & (4) \\
\hline Body Weight (BW), kg & 70 & (4) \\
\hline Avging Time, Carc (2) (ATC), days & 25,550 & \\
\hline Avging Time, Noncarc ${ }^{(3)}$ (ATN), days & 9,125 & \\
\hline
\end{tabular}

INTAKE FACTOR CALCULATIONS

Carcinogenic Intake Factor (CFF), $\mathrm{kg} / \mathrm{kg}$-day $=$

$(\mathrm{R} \bullet \mathrm{FI} \bullet \mathrm{EF} \bullet \mathrm{ED} \bullet \mathrm{CF}) /(\mathrm{BW} \bullet \mathrm{ATC})$

RME CFF $=8.74 \mathrm{E}-03$

Noncarcinogenic Intake Factor (NIF), $\mathrm{kg} / \mathrm{kg}$-day = $(\mathbb{R} * \mathrm{FI} * \mathrm{EF} * \mathrm{ED} * \mathrm{CF}) /(\mathrm{BW} * \mathrm{ATN})$

RME NIF $=2.45 E-02$

\section{Noter:}

1. Reasoruble maximum exposure.

2. Averaging time, carcinogen; calculated as 70 years (werage lifetime) times 365 day ser year (not applicable to child resident).

3. Averaging time, noncarcinogen; calculated es exposure duration (in years) times 365 days per year

4. See Table D-1. 
Table D.24

Savannah River Site

Rubble Pit (231-2F)

Future Worker

Inhalation of Resuspended Soil Particulate Contaminants (0-2'bls)

Carcinogenic and Noncarcinogenic Risk

\begin{tabular}{llll}
\hline CALCULATION OF SOIL PARTICULATE RESUSPENSION FACTOR: & & \\
CONSTANTS: & & & \\
Length of side of contaminated area $(\mathrm{LS})=$ & 24.00 & meters & (1) \\
Wind speed in mixing zone $(\mathrm{V})=$ & 3.60 & meters/sec & $(1)$ \\
Diffusion height $(\mathrm{DH})=$ & 1.66 & meters & $(1)$ \\
Area of contamination $(\mathrm{A})=$ & 500.00 & meters & $(1)$ \\
Respirable fraction $(\mathrm{RF})=$ & 0.003 & $\mathrm{~g} / \mathrm{meters}^{2}-\mathrm{hr}$ & $(1)$ \\
Fraction of vegetative cover $(\mathrm{G})=$ & 0.90 & unitless & $(1)$ \\
Mean annual wind speed $(\mathrm{Um})=$ & 3.60 & meters/sec & $($ (1) \\
Equivalent threshold value of wind speed at $10 \mathrm{~m}(\mathrm{Ut})=$ & 5.04 & meters/sec & $(1)$ \\
Fraction dependent on Um/Ut $(\mathrm{Fx})=$ & 1.2700 & unitless & $(1)$ \\
Conversion factor $(\mathrm{CFa})=$ & $3,600.00$ & sec/hr & \\
Conversion factor $(\mathrm{CFb})=$ & $1,000.00$ & $\mathrm{~g} / \mathrm{kg}$ &
\end{tabular}

Notsi:

1. USEPA, 1991: RAGS: Vol 1 - Humm Health Evaluation Menual (Part B, Development of Risk-Besed Preliminwy Remodielion Coals)

\section{INTERMEDIATE STEPS:}

Particulate emission fector (PEF) $=6.76 \mathrm{E}+09$ meters ${ }^{3} / \mathrm{kg}$ $(\mathrm{LS} \times \mathrm{V} \times \mathrm{DH} \times \mathrm{CFa} \times \mathrm{CFb}) /\left(\mathrm{A} \times \mathrm{RF} \times(1-\mathrm{G}) \times\left((\mathrm{Um} / \mathrm{Ut})^{\wedge} 3\right) \times(\mathrm{Fx})\right)$ 
Table D.24

Savannah River Site

Rubble Pit (231-2F)

Future Worker

Inhalation of Resuspended Soil Particulate Contaminants (0-2'bls)

Carcinogenic and Noncarcinogenic Risk

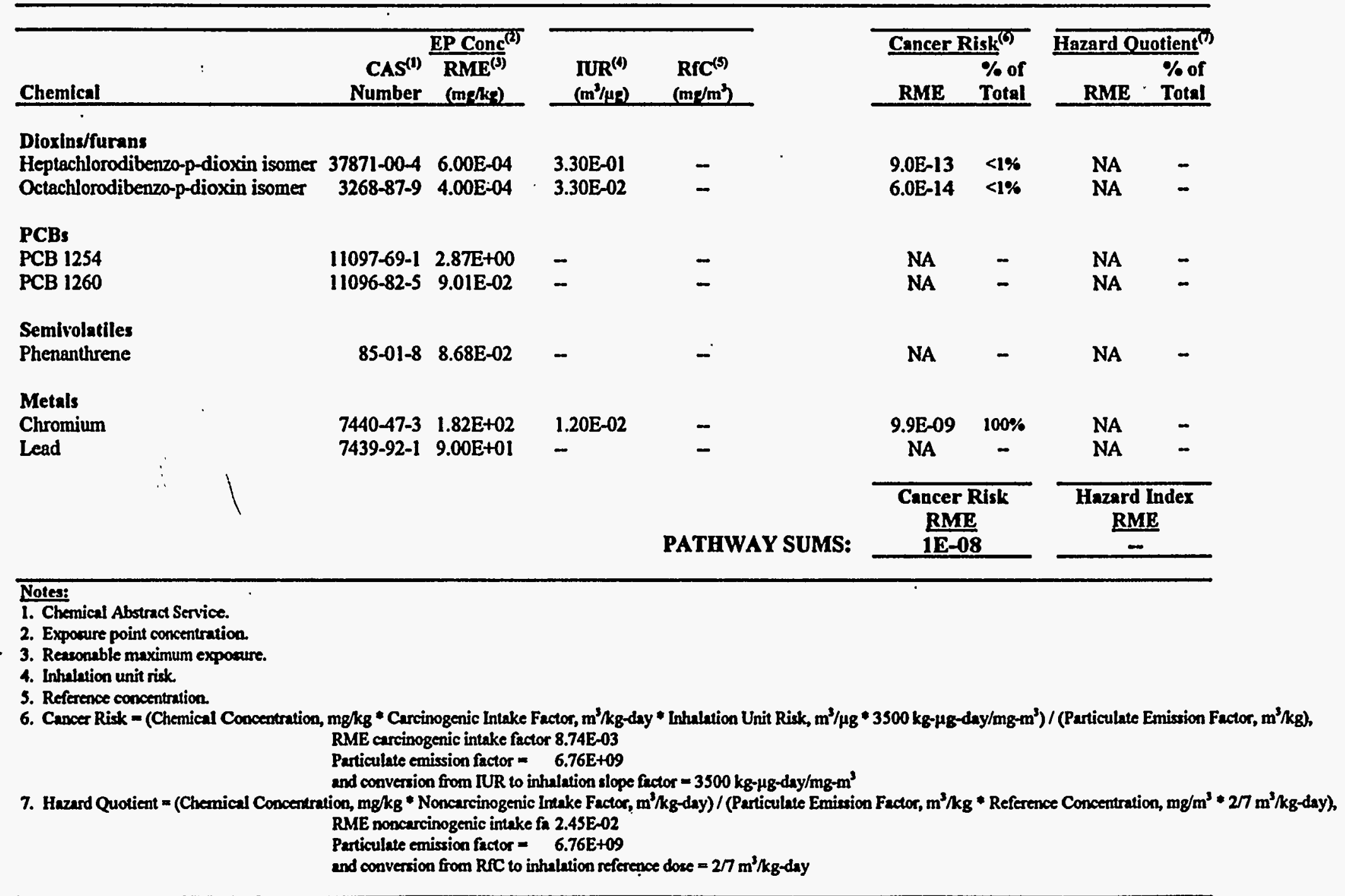


Table D.25

Savannah River Site

Rubble Pit (231-2F)

Future Worker

Ingestion of Soil (0-4'bls)

Carcinogenic and Noncarcinogenic Risk

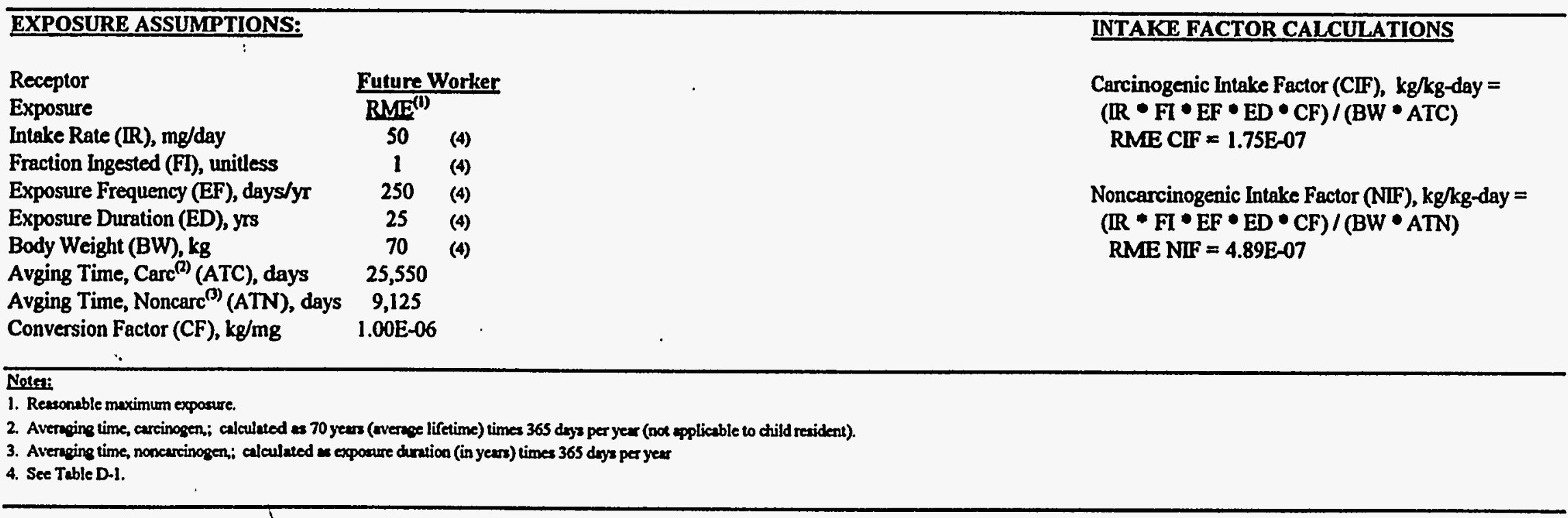


Table D.25

Savannah River Site

Rubble Pit (231-2F)

Future Worker

Ingestion of Soil ( $0-4$ 'bls)

Carcinogenic and Noncarcinogenic Risk

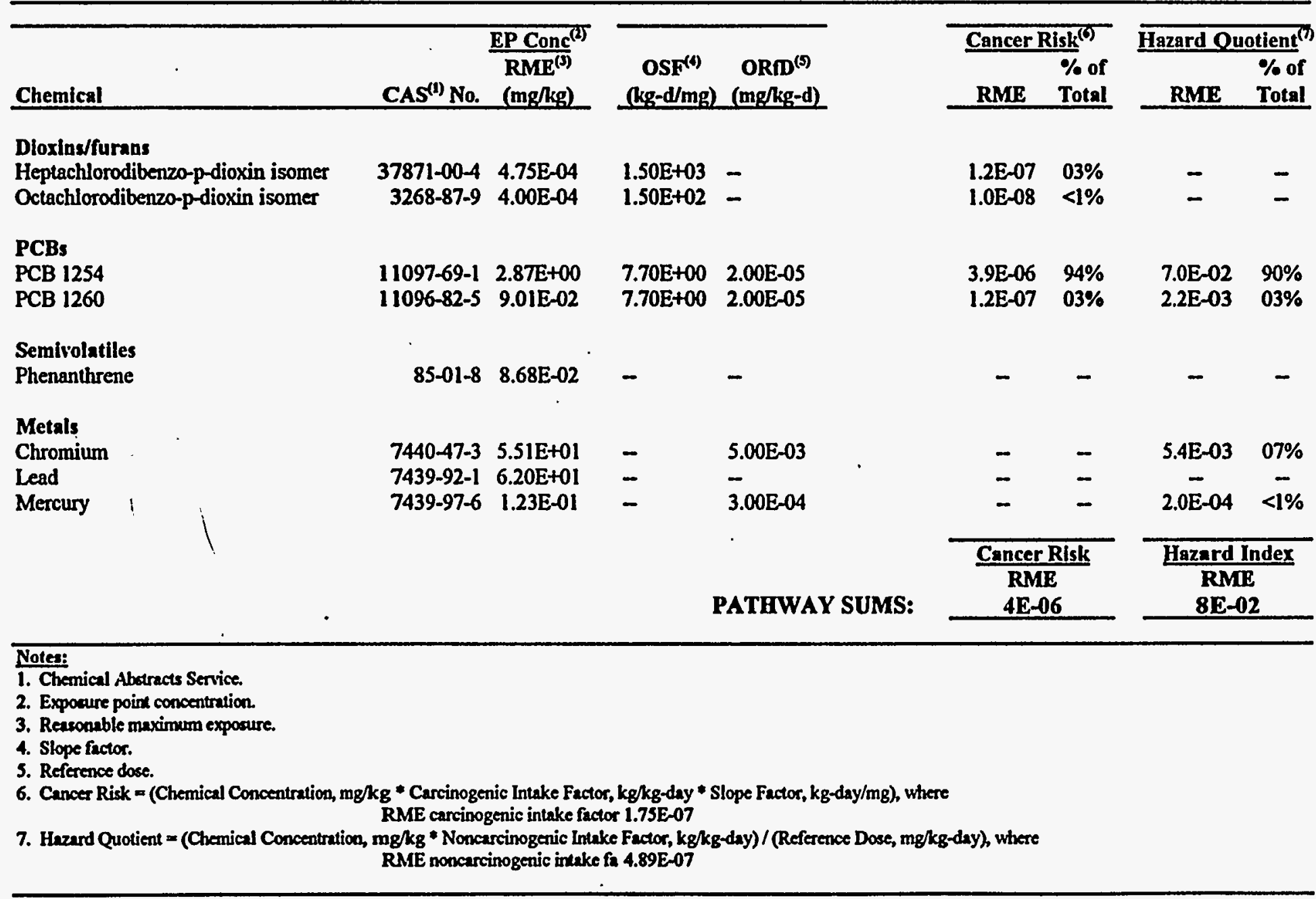


Table D.26

Savannah River Site

Rubble Pit (231-2F)

Future Worker

Dermal Exposure to Soil (0-4'bls)

Carcinogenic and Noncarcinogenic Risk

\section{EXPOSURE ASSUMPTIONS:}

\section{Receptor}

Skin Surface Area (SA), $\mathrm{cm}^{2} /$ event $\quad 3,200$

Future Worker

$\mathrm{RMF}^{(1)}$

Soil-to-Skin Adherance (SK), $\mathrm{mg} / \mathrm{cm}^{2} \quad 0.2 \quad$ (4)

Exposure Frequency (EF), events/yr 250 (4)

Exposure Duration (ED), yrs 25 (4)

Body Weight (BW), kg 70 (4)

Avging Time, Carc (2) (ATC), days $\quad 25,550$

Avging Time, Noncarc ${ }^{(3)}$ (ATN), days 9,125

Conversion Factor (CF), kg/mg $\quad 1.00 \mathrm{E}-06$

\section{Noter:}

1. Resconable maximum exposure.

2. Averaging time, carcinogen; calculuted as 70 years (vienge lifetime) times 365 dys per year (noc upplicable to child resident).

3. Ateraging time, noncarcinogen; calculeced as exposure duration (in yeans) times 365 days per year

4. See Table D-1.

\section{INTAKE FACTOR CALCULATIONS}

Carcinogenic Intake Factor (CIF), $\mathrm{kg} / \mathrm{kg}$-day =

$(\mathrm{SA} \bullet \mathrm{SK} \bullet \mathrm{EF} \bullet \mathrm{ED} \bullet \mathrm{CF}) /(\mathrm{BW} \bullet \mathrm{ATC})$

$\mathrm{RME} \mathrm{CIF}=2.24 \mathrm{E}-06$

Noncarcinogenic Intake Factor (NIF), $\mathbf{k g} / \mathbf{k g}$-day = $(\mathrm{SA} * \mathrm{SK} * \mathrm{EF} \bullet \mathrm{ED} * \mathrm{CF}) /(\mathrm{BW} * \mathrm{ATN})$

RME NIF $=6.26 \mathrm{E}-06$ 
Table D.26

Savannah River Site

Rubble Pit (231-2F)

Future Worker

Dermal Exposure to Soil (0-4'bls)

Carcinogenic and Noncarcinogenic Risk

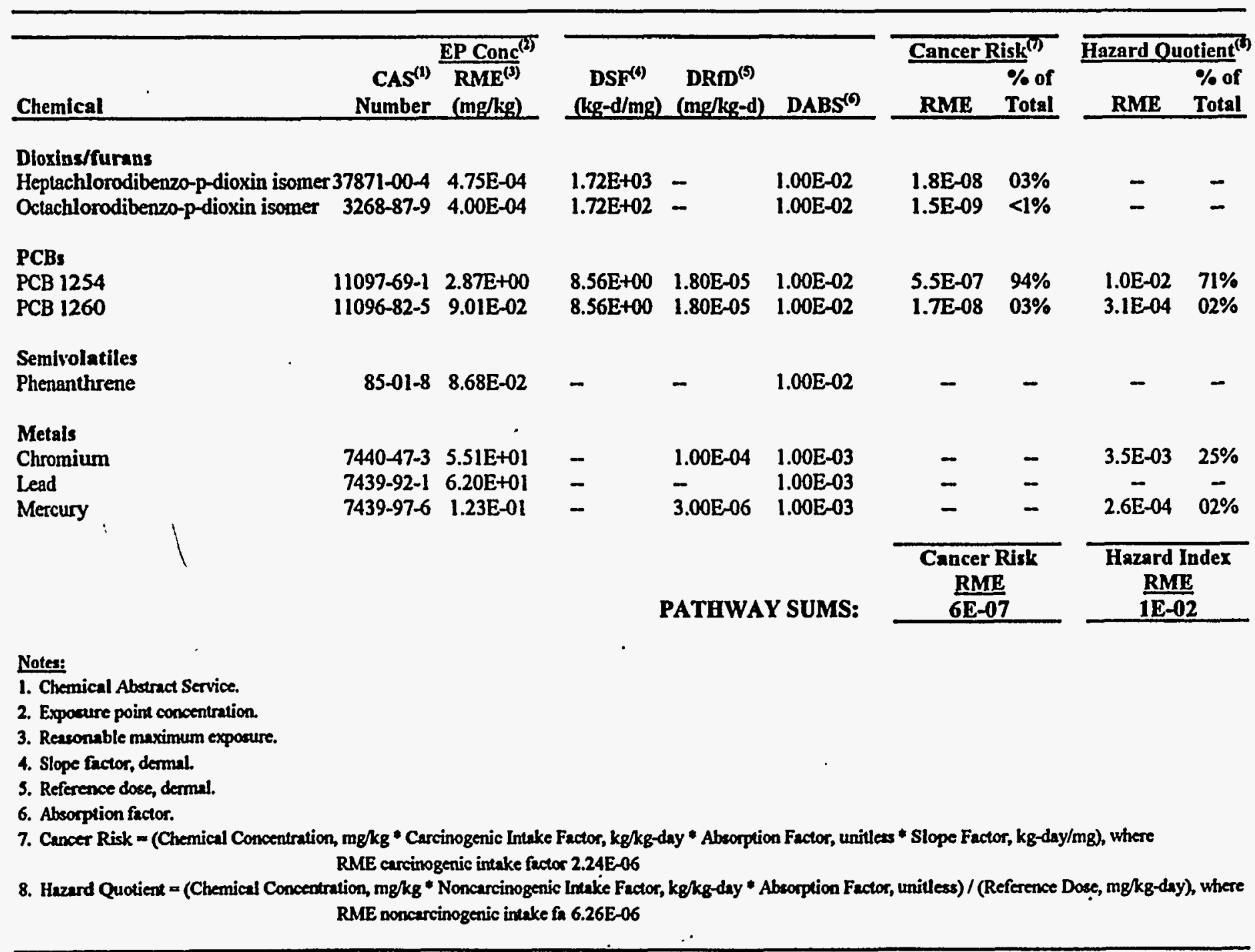


Table D.27

Savannah River Site

Rubble Pit (231-2F)

Future Worker

Inhalation of Volatile Soil Contaminants (0-4'bls)

\begin{tabular}{|c|c|c|}
\hline \multirow{2}{*}{\multicolumn{2}{|c|}{ EXPOSURE ASSUMPTIONS: }} & INTAKE FACTOR CALCULATIONS \\
\hline & & Carcinogenic Intake Factor (CIF), $\mathrm{kg} / \mathrm{kg}$-day $=$ \\
\hline Exposure & $\mathrm{RME}^{(\mathrm{I})}$ & $(\mathrm{IR} \bullet \mathrm{ET} \bullet \mathrm{EF} \bullet \mathrm{ED} \bullet \mathrm{CF}) /(\mathrm{BW} * \mathrm{ATC})$ \\
\hline Inhalation Rate (IR), $\mathbf{m}^{\mathbf{3}} / \mathbf{h r}$ & $2.5 \quad$ (4) & $\mathrm{RME} C \mathrm{CIF}=8.74 \mathrm{E}-03$ \\
\hline Exposure Time (ET), hrs/day & $1 \quad(4)$ & \\
\hline Exposure Frequency (EF), days/yr & $250 \quad(4)$ & Noncarcinogenic Intake Factor (NIF), kg/kg-day = \\
\hline Exposure Duration (ED), yrs & 25 & $(\mathrm{IR} * \mathrm{ET} \bullet \mathrm{EF} \bullet \mathrm{ED} \bullet \mathrm{CF}) /(\mathrm{BW} \bullet \mathrm{ATN})$ \\
\hline Body Weight (BW), kg & 70 (4) & $\mathrm{RME} \mathrm{NIF}=2.45 \mathrm{E}-02$ \\
\hline Avging Time, $\operatorname{Carc}^{(2)}$ (ATC), days & 25,550 (4) & \\
\hline Avging Time, Noncarc ${ }^{(3)}$ (ATN), days & 9,125 & \\
\hline \multicolumn{3}{|l|}{ Nodsti } \\
\hline \multicolumn{3}{|c|}{ 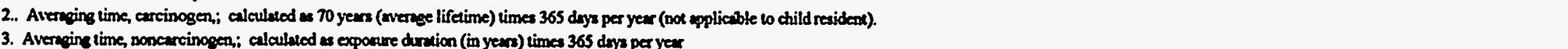 } \\
\hline \multicolumn{3}{|c|}{ 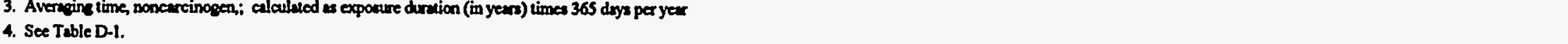 } \\
\hline
\end{tabular}


Table D.27

Savannah River Site

Rubble Pit (231-2F)

Future Worker

Inhalation of Volatile Soil Contaminants (0-4'bls)

Calculation of Volatilization Factor (VF)

\begin{tabular}{|c|c|c|c|c|c|c|c|c|c|c|c|c|}
\hline Chemical & $\mathrm{CAS}^{(2)} \mathrm{Na}$ & Class $^{(3)}$ & MolWt. & $\log \mathrm{Koc}$ & $\mathrm{Koc}^{(1)}$ & $K d^{(0)}$ & $\mathbf{H}^{(0)}$ & $\mathbf{K}_{\mathbf{g s}}(0)$ & Di $^{(4)}$ & $\mathrm{De}^{\left({ }^{(4)}\right.}$ & alpha(4) & $\begin{array}{l}\text { Volat. } \\
\text { Factor }{ }^{(s)} \\
\left(\mathrm{m}^{3} / \mathrm{kg}\right)\end{array}$ \\
\hline Phenanthrene & $85-01-8$ & $\mathbf{S}$ & $1.78 E+02$ & 4.59 & 38904.5 & $7.78 \mathrm{E}+02$ & $2.56 \mathrm{E}-05$ & $1.35 \mathrm{E}-06$ & $5.74 \mathrm{E}-02$ & $4.05 \mathrm{E}-02$ & $1.11 \mathrm{E}-08$ & $1.27 \mathrm{E}+06$ \\
\hline
\end{tabular}

Notes:

1. USEPA, 1991: RAGS: Vol 1 - Human Heath Evaluation Masueal (Part B, Development of Risk-Based Preliminary Remediation Goals)

2. Chemical Abatract Service.

3. Chemical class: $v$-volatile; 8 -semivolutile; $p$-pesicide/pcb; $x$-dioxin; and $m$-metal.

4. Calculation of organic contaminant volatilization factor:

CONSTANTS:

Length of side of contam'd. area (LS), $m=$

Wind speed in mixing zone (V), $m / s e c=$

Diffusion height (DH), $m=$ Area of conteminution (Am), $\mathrm{m}^{2}=$ Area in square centimeters (Acm), $\mathrm{cm}^{2}=$

True soil porosity $(E)$, unitless =

Trie woil density (Ps), $\mathrm{g} / \mathrm{cm}^{3}=$

Expoutre interval (Tyr), yrs =

Exposure interval (Trec), $200=$

Organic carbon contern (OC), unitless =

Conversion factor (CF), $\mathrm{kg} / \mathrm{g}=$

INPUT VARIABLES:

Organic cartion partition coef. (Koc), $\mathrm{cm}^{3} / \mathrm{kg}=$

Herry's Law constam $(H)$, atm-m $3 / m o l=$

Gas diffusiviny (Di), $\mathrm{cm}^{2} / \mathrm{sec}=$

INTERMEDIATE STEPS:

Soil-water partition coefficient $(\mathrm{Kd}), \mathrm{cm}^{3} / \mathrm{kg}=$ Soil-eir partition coefficient (Kas), B-soil/cm'-2ir = Effective diffusivity (Dei), $\mathrm{cm}^{2} / \mathrm{sec}=$ alpha (alpha), $\mathrm{cm}^{2} / \mathrm{sec}=$

oUTPUT:

Volatilization Factor (VF), $\mathrm{m}^{3} / \mathrm{kg}=$

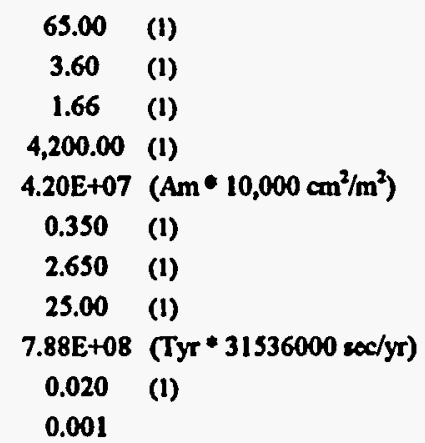

chem-spoc

chem-spec

chem-epec

chem-spec $(\mathrm{Koc} \times \mathrm{OC})$

chem-spec $((\mathrm{H} / \mathrm{Kd}) \times 41)$

chem-spec (Di $\left.\times\left(E^{\wedge} 0.33\right)\right)$

chem-spec $((D e i \times E) /(E+(P s)(1-E)) / K a s)$

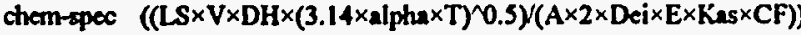


Table D.27

Savannah River Site

Rubble Pit (231-2F)

Future Worker

Inhalation of Volatile Soil Contaminants (0-4'bls)

Carcinogenic and Noncarcinogenic Risk

\begin{tabular}{|c|c|c|c|c|c|c|c|c|c|}
\hline \multirow[b]{2}{*}{ Chemical } & \multirow{2}{*}{\multicolumn{2}{|c|}{ 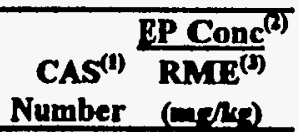 }} & \multirow[b]{2}{*}{ 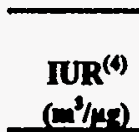 } & \multirow[b]{2}{*}{ 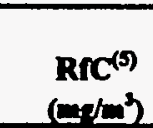 } & \multirow[b]{2}{*}{ 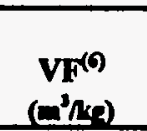 } & \multicolumn{2}{|c|}{ Cancer Risk" } & \multicolumn{2}{|c|}{ Hazard Ouotient ${ }^{(1)}$} \\
\hline & & & & & & RME & $\begin{array}{l}\% \text { of } \\
\text { Total }\end{array}$ & $\mathbf{R M E}$ & $\begin{array}{l}\% \text { of } \\
\text { Total }\end{array}$ \\
\hline \multirow[t]{2}{*}{ Phenanthrene } & $85-01-8$ & 6.87E-01 & - & - & $1.27 \mathrm{E}+06$ & NA & - & NA & - \\
\hline & & & & \multicolumn{2}{|c|}{ PATHWAY SUMS: } & \multicolumn{2}{|c|}{$\begin{array}{c}\text { Cancer Risk } \\
\text { RMIE } \\
-\end{array}$} & \multicolumn{2}{|c|}{$\begin{array}{c}\text { Hezard Index } \\
\text { RME } \\
-\end{array}$} \\
\hline
\end{tabular}

1. Chemical Abetract Service.

2. Exposure point concentration.

3. Reasonable maximum expocure.

4. Inhalation unit risk.

5. Reference concentration.

6. Volutilization fuctor (chemical-specific)

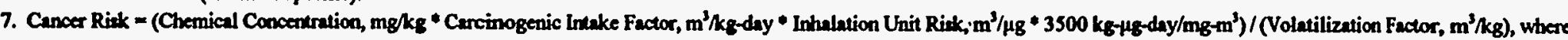
RME carcinogenic intake factor 8.74E-03

and conversion from IUR to inhalation slope factor $=3500 \mathrm{~kg}-\mu \mathrm{g}-\mathrm{day} / \mathrm{mg}^{-\mathrm{m}^{3}}$

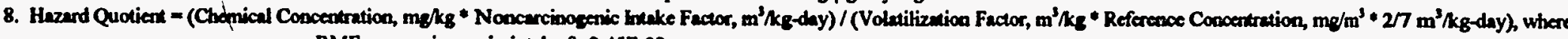
RME noncarcinogenic intake fa 2.45E-02 and conversion from RfC to inhalation referenoe doce $=2 / 7 \mathrm{~m}^{3} / \mathrm{kg}$-day 
Table D.28

Savannah River Site

Rubble Pit (231-2F)

Future Worker

Inhalation of Resuspended Soil Particulate Contaminants (0-4'bls)

Carcinogenic and Noncarcinogenic Risk

\begin{tabular}{|c|c|c|}
\hline \multicolumn{3}{|l|}{ EXPOSURE ASSUMPTIONS: } \\
\hline Receptor & Future & orke \\
\hline Exposure & $\mathrm{RMF}^{(1)}$ & \\
\hline Inhalation Rate ( $(\mathbb{R}), \mathrm{m}^{3} / \mathrm{hr}$ & 2.5 & (4) \\
\hline Exposure Time (ET), hrs/day & 1 & (4) \\
\hline Exposure Frequency (EF), days/yr & 250 & (4) \\
\hline Exposure Duration (ED), yrs & 25 & (4) \\
\hline Body Weight (BW), kg & 70 & (4) \\
\hline Avging Time, $\operatorname{Carc}^{(2)}$ (ATC), days & 25,550 & \\
\hline Avging Time, Noncarc ${ }^{(3)}($ ATN), days & 9,125 & \\
\hline
\end{tabular}

INTAKE FACTOR CALCULATIONS

Carcinogenic Intake Factor (CIF), $\mathrm{kg} / \mathrm{kg}$-day =

$(\mathrm{R} \bullet \mathrm{FI} \bullet \mathrm{EF} \bullet \mathrm{ED} \bullet \mathrm{CF}) /(\mathrm{BW} \bullet \mathrm{ATC})$

RME CIF $=8.74 \mathrm{E}-03$

Noncarcinogenic Intake Factor (NIF), $\mathrm{kg} / \mathrm{kg}$-day = $(\mathrm{R} \bullet \mathrm{FI} \bullet \mathrm{EF} \bullet \mathrm{ED} \bullet \mathrm{CF}) /(\mathrm{BW} \bullet \mathrm{ATN})$

RME NIF $=2.45 \mathrm{E}-02$

\section{Netsr:}

1. Reasonable maximum exposure.

2. Averaing time, carcinogen; calculated as 70 years (avernge lifetime) times 365 days per year (not applicable to child resident).

3. Averaging time, noncercinogen; calculated as exposture duration (in years) times 365 deys per year

4. See Table D-1. 
Table D.28

Savannah River Site

Rubble Pit (231-2E)

Future Worker

Inhalation of Resuspended Soil Particulate Contaminants (0-4'bls)

Carcinogenic and Noncarcinogenic Risk

\begin{tabular}{llll}
\hline CALCULATION OF SOIL PARTICULATE RESUSPENSION FACTOR: & & \\
CONSTANTS: & 24.00 & meters & $(1)$ \\
Length of side of contaminated area $(\mathrm{LS})=$ & 3.60 & meters/sec & $(1)$ \\
Wind speed in mixing zone $(\mathrm{V})=$ & 1.66 & meters & $(1)$ \\
Diffusion height $(\mathrm{DH})=$ & 500.00 & meters $^{2}$ & $(1)$ \\
Area of contamination $(\mathrm{A})=$ & 0.003 & $\mathrm{~g} /$ meters $^{2}-\mathrm{hr}$ & $(1)$ \\
Respirable fraction $(\mathrm{RF})=$ & 0.90 & unitless & $(1)$ \\
Fraction of vegetative cover $(\mathrm{G})=$ & 3.60 & meters/sec & $(1)$ \\
Mean annual wind speed $(\mathrm{Um})=$ & 5.04 & meters/sec & $(1)$ \\
Equivalent threshold value of wind speed at $10 \mathrm{~m}(\mathrm{Ut})=$ & 1.2700 & unitless & $(1)$ \\
Fraction dependent on Um/Ut $(\mathrm{Fx})=$ & $3,600.00$ & sec/hr & \\
Conversion factor $(\mathrm{CFa})=$ & $1,000.00$ & $\mathrm{~g} / \mathrm{kg}$ & \\
Conversion factor $(\mathrm{CFb})=$ & & &
\end{tabular}

Nolet:

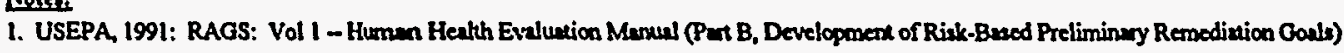

\section{INTERMEDLATE STEPS: \\ Particulate emission factor (PEF) $=6.76 \mathrm{E}+09$ meters $^{3} / \mathrm{kg}$ $(\mathrm{LS} \times \mathrm{V} \times \mathrm{DH} \times \mathrm{CFa} \times \mathrm{CFb}) /\left(\mathrm{A} \times \mathrm{RF} \times(1-G) \times\left((\mathrm{Um} / \mathrm{Ut})^{-1} 3\right) \times(\mathrm{Fx})\right)$}


Table D.28

Savannah River Site

Rubble Pit (231-2F)

Future Worker

Inhalation of Resuspended Soil Particulate Contaminants (0-4'bls)

Carcinogenic and Noncarcinogenic Risk

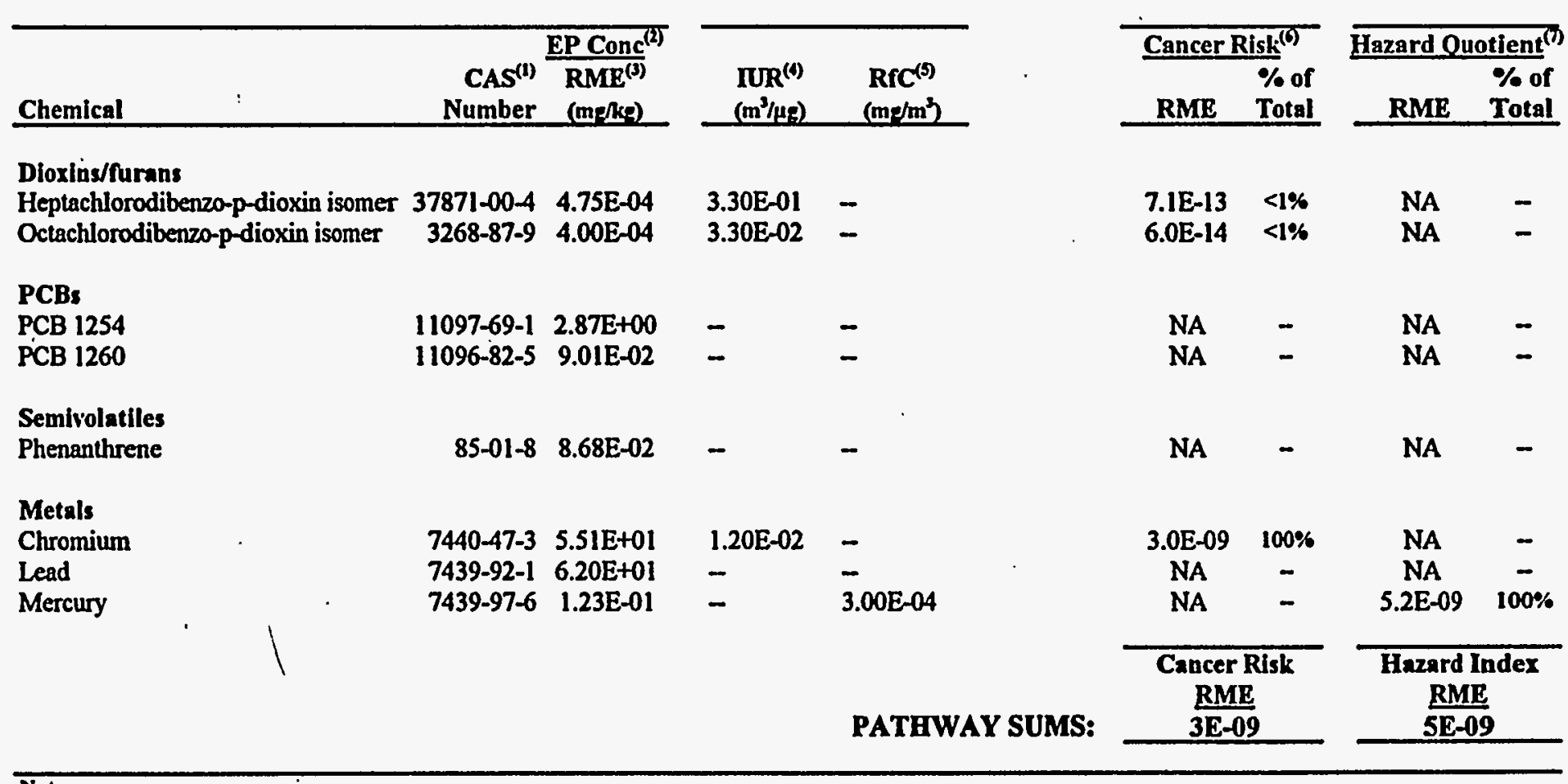

Notes:

1. Chemical Abctract Service.

2. Exposure point concentration.

3. Rearonable maximum exporure.

4. Inhalation unit risk.

5. Reference concentration.

6. Cancer Risk = (Chemical Concentration, mg/kg * Carcinogenic Intake Factor, $\mathrm{m}^{3} / \mathrm{kg}_{\mathrm{g}}$-day * Inhalation Unit Risk, $\left.\mathrm{m}^{3} / \mu \mathrm{g} * 3500 \mathrm{~kg}-\mu \mathrm{g}-\mathrm{day} / \mathrm{mg}_{\mathrm{g}} \mathrm{m}^{3}\right) /\left(\mathrm{Particulate}\right.$ Emission Factor, $\left.\mathrm{m}^{3} / \mathrm{kg}\right)$, RME carcinogenic intake factor $8.74 \mathrm{E}-03$

Particulate emission fuctor = $6.76 \mathrm{E}+09$

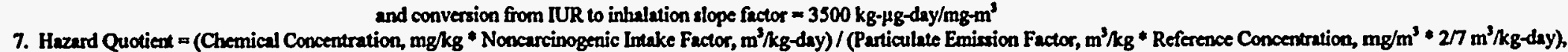

RME noscarcinogenic intuke fa 2.45E-02

Particulate emistion factor $=6.76 \mathrm{E}+09$

and conversion from RfC to inhalation referenoe doce $=2 / 7 \mathrm{~m}^{3} / \mathrm{kg}_{\mathrm{g}}$-day 
Table D.29

Savannah River

Burning/Rubble Pits (231-F, 231-1F, 231-2F)

Future Worker

Ingestion of Ground Water

Carcinogenic and Noncarcinogenic Risk

\section{EXPOSURE ASSUMPTIONS:}

Receptor

Exposure

Intake Rate (IR), L/day

Exposure Frequency (EF), days/yr

Exposure Duration (ED), yrs

Body Weight (BW), kg

Avging Time, $\mathrm{Car}^{(2)}$ (ATC), days

Avging Time, Noncarc ${ }^{(3)}$ (ATN), days

\section{Future Worker}

$\mathrm{RME}^{(1)}$

$1 \quad(4)$

$250 \quad$ (4)

$25 \quad$ (4)

$70 \quad(4)$

25,550
INTAKE FACTOR CALCULATIONS

Carcinogenic Intake Factor (CIF), L/kg-day =

$(\mathrm{IR} \bullet \mathrm{EF} \bullet \mathrm{ED}) /(\mathrm{BW} * \mathrm{ATC})$

RME CIF $=3.49 \mathrm{E}-03$

Noncarcinogenic Intake Factor (NIF), L/kg-day =

$(\mathbb{R} \bullet \mathrm{EF} \bullet \mathrm{ED}) /(\mathrm{BW} * \mathrm{ATN})$

RME NIF $=9.78 E-03$

\section{Nistri:}

1. Resonable maximum exporure.

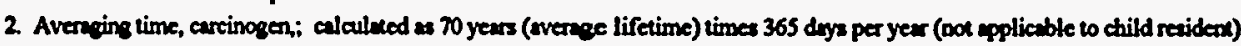

3. Averaing time, noncercinogen: calculled as expoeure durmion (in year) timea 365 days per year

4. Sec Table D-1. 
Table D.29

Savannah River

Burning/Rubble Pits (231-F, 231-1F, 231-2F)

Future Worker

Ingestion of Ground Water

Carcinogenic and Noncarcinogenic Risk

\begin{tabular}{|c|c|c|c|c|c|c|c|c|c|}
\hline \multirow[b]{2}{*}{ Chemical } & \multicolumn{3}{|c|}{ EP Conc ${ }^{(2)}$} & \multirow[b]{2}{*}{$\begin{array}{c}\text { OSfF(t) } \\
\text { (kg-d/mg) }\end{array}$} & \multirow[b]{2}{*}{$\begin{array}{c}\text { ORID } \\
(\mathrm{mg} / \mathrm{kg}-\mathrm{d})\end{array}$} & \multicolumn{2}{|c|}{ Cancer Risk ${ }^{(0)}$} & \multicolumn{2}{|c|}{ Hazard Quotient ${ }^{(\prime)}$} \\
\hline & $\begin{array}{l}\text { CAS }^{\mathbf{( I}} \\
\text { Number } \\
\end{array}$ & $\begin{array}{l}\mathbf{R M E}^{(3)} \\
(\mathrm{mg} / \mathbf{L})\end{array}$ & & & & $\mathbf{R M E}$ & $\begin{array}{l}\% \text { of } \\
\text { Total } \\
\end{array}$ & RME & $\begin{array}{l}\% \text { of } \\
\text { Total } \\
\end{array}$ \\
\hline \multicolumn{10}{|l|}{ Volatiles } \\
\hline Carbon tetrachloride & $56-23-5$ & $5.41 \mathrm{E}-03$ & & $1.30 \mathrm{E}-01$ & $7.00 \mathrm{E}-04$ & 2.5E-06 & $14 \%$ & $7.6 \mathrm{E}-02$ & $21 \%$ \\
\hline Chloroform & $67-66-3$ & $1.00 \mathrm{E}-03$ & & $6.10 \mathrm{E}-03$ & $1.00 \mathrm{E}-02$ & $2.1 \mathrm{E}-08$ & $<1 \%$ & $9.8 \mathrm{E}-04$ & $<1 \%$ \\
\hline Chloromethane (Methyl chloride) & $74-87-3$ & $4.20 \mathrm{E}-03$ & & $1.30 \mathrm{E}-02$ & - & $1.9 \mathrm{E}-07$ & $01 \%$ & - & - \\
\hline 1,2-Dichloroethylene & 540590 & $4.47 \mathrm{E}-06$ & $\mathbf{m}$ & - & $9.00 \mathrm{E}-03$ & - & - & $4.9 \mathrm{E}-06$ & $<1 \%$ \\
\hline Dichloromethane & 75092 & $1.71 \mathrm{E}-02$ & & $7.50 \mathrm{E}-03$ & $6.00 \mathrm{E}-02$ & 4.5E-07 & $03 \%$ & $2.8 \mathrm{E}-03$ & $<1 \%$ \\
\hline Tetrachloroethylene & 127184 & $7.23 \mathrm{E}-03$ & & $5.20 \mathrm{E}-02$ & $1.00 \mathrm{E}-02$ & $1.3 \mathrm{E}-06$ & $07 \%$ & $7.1 \mathrm{E}-03$ & $02 \%$ \\
\hline Trichloroethylene & $79-01-6$ & $7.47 \mathrm{E}-03$ & & $1.10 \mathrm{E}-02$ & - & $2.9 \mathrm{E}-07$ & $02 \%$ & - & - \\
\hline \multirow{2}{*}{$\begin{array}{l}\text { Pesticides } \\
\text { delta-BHC } \\
\text { Heptachlor epoxide }\end{array}$} & 319868 & $236 \mathrm{E}-04$ & $\mathrm{~m}$ & - & - & _ & _ & - & 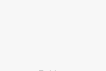 \\
\hline & 1024573 & $1.85 \mathrm{E}-06$ & $\mathrm{~m}$ & $9.10 \bar{E}+00$ & $1.30 \mathrm{E}-05$ & $5.9 \overline{\mathrm{E}}-08$ & $<\overline{1 \%}$ & $1.4 \overline{\mathrm{E}}-03$ & $<\overline{1 \%}$ \\
\hline \multirow{6}{*}{$\begin{array}{l}\text { Metals } \\
\text { Arsenic } \\
\text { Cadmium } \\
\text { Iron } \\
\text { Manganese }\end{array}$} & & & & & & & & & \\
\hline & $7440-38-2$ & $2.50 \mathrm{E}-03$ & & $1.50 \mathrm{E}+00$ & $3.00 \mathrm{E}-04$ & $1.3 \mathrm{E}-05$ & $73 \%$ & 8.2E-02 & $23 \%$ \\
\hline & $7440-43-9$ & 4.83E-03 & $\mathbf{m}$ & - & $5.00 \mathrm{E}-04$ & - & - & $9.5 \mathrm{E}-02$ & $27 \%$ \\
\hline & $7439-89-6$ & $2.82 E+01$ & & - & - & - & - & - & - \\
\hline & $7439-96-5$ & $4.53 \mathrm{E}-01$ & & - & $5.00 \mathrm{E}-02$ & - & - & $8.9 \mathrm{E}-02$ & $25 \%$ \\
\hline & & & & PAT & HWAY SUMS: & \multicolumn{2}{|c|}{$\begin{array}{c}\text { Cancer Risk } \\
\frac{\text { RME }}{2 \mathrm{E}-05}\end{array}$} & \multicolumn{2}{|c|}{$\begin{array}{l}\text { Hazard Index } \\
\frac{\text { RME }}{4 \mathrm{E}-01}\end{array}$} \\
\hline
\end{tabular}

2. Exposure point concentration.

3. Reasonable maximum exposure; " $\mathrm{m}$ " indicales modeled concentration.

4. Slope factor, oral.

5. Reference dose, oral.

6. Cancer Risk = (Chemical Concentration, mg/L • Carcinogenic Intake Factor, L/kg-day * Slope Factor, kg-day/mg), where

RME carcinogenic intake factor $=\quad 3.49 \mathrm{E}-03$

7. Hazard Quotient = (Chemical Concentration, $\mathrm{mg} / \mathrm{L} \bullet$ Noncarcinogenic Intake Factor, L/kg-day) /(Reference Dose, mg/kg-day), where

RME noncurcinogenic intake fuctor $=9.78 \mathrm{E}-03$ 
Table D.30

Savannah River Site

Burning/Rubble Pits (231-F, 231-1F)

Combined Adult/Child Resident (Future)

Ingestion of Soil (0-2'bls)

Carcinogenic and Noncarcinogenic Risk

\begin{tabular}{|c|c|c|c|c|c|c|}
\hline \multirow[t]{2}{*}{ EXPOSURE ASSUMPTIONS: } & & & & & & \multirow[t]{2}{*}{ INTAKE FACTOR CALCULATIONS } \\
\hline & \multicolumn{5}{|r|}{ Combined } & \\
\hline Receptor & \multicolumn{2}{|c|}{ Adult Resident } & \multicolumn{2}{|c|}{ Child Resident } & Adult/Child & \multirow{2}{*}{$\begin{array}{l}\text { Carcinogenic Intake Factor }(\mathrm{CIF}), \mathrm{kg} / \mathrm{kg}-\text { day }= \\
(\mathrm{IR} \bullet \mathrm{FI} \bullet \mathrm{EF} \bullet \mathrm{ED} * \mathrm{CF}) /(\mathrm{BW} \bullet \mathrm{ATC})\end{array}$} \\
\hline Exposure & \multicolumn{2}{|c|}{$\mathrm{RME}^{(1)}$} & \multicolumn{2}{|c|}{$\mathrm{RME}$} & RME & \\
\hline Intake Rate (IR), mg/day & 100 & (4) & 200 & (4) & 120.0 & $\mathrm{RME} \mathrm{CIF}=8.36 \mathrm{E}-07$ \\
\hline Fraction Ingested (FI), unitless & 1 & (4) & 1 & (4) & 1.0 & \\
\hline Exposure Frequency (EF), days/yr & 350 & (4) & 350 & (4) & 350 & Noncarcinogenic Intake Factor (NIF), kg/kg-day = \\
\hline Exposure Duration (ED), yrs & 24 & (4) & 6 & (4) & 30 & $(\mathrm{R} \bullet \mathrm{FI} \bullet \mathrm{EF} \bullet \mathrm{ED} \bullet \mathrm{CF}) /(\mathrm{BW} * \mathrm{ATN})$ \\
\hline Body Weight (BW), kg & 70 & (4) & 15 & (4) & 59.0 & RME NIF $=1.28 \mathrm{E}-05 \quad$ (Child Value) \\
\hline Avging Time, $\operatorname{Carc}^{(2)}$ (ATC), days & 25,550 & & NA & & 25,550 & \\
\hline Avging Time, Noncarc ${ }^{(3)}$ (ATN), days & 8,760 & & 2,190 & & 10,950 & \\
\hline Conversion Factor (CF), kg/mg & $1.00 \mathrm{E}-06$ & & $1.00 \mathrm{E}-06$ & & $1.00 \mathrm{E}-06$ & \\
\hline
\end{tabular}

\section{Nolex:}

1. Ressonable maximum exposure.

2. Averaing time, carcinogen; calculuted as 70 yeans (nvernge lifetime) times 365 days per year (not upplicable to child resident).

3. Averaing time, noncercisiogen; calculted as exposure duration (in years) times 365 day per year

4. See Table D-1.

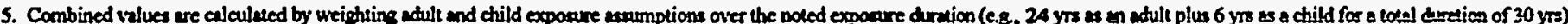

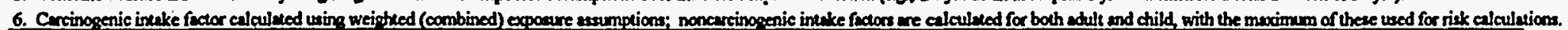


Table D.30

Savannah River Site

Burning/Rubble Pits (231-F, 231-1F)

Combined Adult/Child Resident (Future)

Ingestion of Soil (0-2'bls)

Carcinogenic and Noncarcinogenic Risk

\begin{tabular}{|c|c|c|c|c|}
\hline Chemical & CAS $^{(\mathbf{1})}$ No. & $\begin{array}{l}\frac{\text { EP Conc }}{(2)} \\
\mathrm{RME}^{(3)} \\
(\mathrm{mg} / \mathrm{kg})\end{array}$ & $\begin{array}{c}\operatorname{OSF}^{(1)} \\
(\mathrm{kg}-\mathrm{d} / \mathrm{mg})\end{array}$ & $\begin{array}{c}\text { ORID }^{(s)} \\
(\mathrm{mg} / \mathrm{kg}-\mathrm{d})\end{array}$ \\
\hline
\end{tabular}

\begin{tabular}{|c|c|}
\hline Cancer Risk $^{(6)}$ & Hazard Quatient $^{(7)}$ \\
\hline $\begin{array}{l}\text { \% } \% \text { of } \\
\text { RME } \quad \text { Total }\end{array}$ & $\begin{array}{cc} & \% \text { of } \\
\text { RME } & \text { Total }\end{array}$ \\
\hline
\end{tabular}

\section{Semivolatiles}

2-Methylnaphthalene

Acenaphthylene

Benzo(a)anthracene

Benzo(a)pyrene

(kg-d/mg) (mg/kg-d)

Benzo(b) lluoranthene

Benzo(g,h,i)perylene

Dibenz(a,h)anthracene

Phenanthrene

$\begin{array}{rlll}91-57-6 & 7.69 \mathrm{E}-02 & - & - \\ 208-96-8 & 8.61 \mathrm{E}-02 & - & - \\ 56-55-3 & 9.26 \mathrm{E}-01 & 7.30 \mathrm{E}-01 & - \\ 50-32-8 & 6.49 \mathrm{E}-01 & 7.30 \mathrm{E}+00 & - \\ 205-99-2 & 1.01 \mathrm{E}+00 & 7.30 \mathrm{E}-01 & - \\ 191-24-2 & 6.15 \mathrm{E}-01 & - & - \\ 53-70-3 & 2.17 \mathrm{E}-01 & 7.30 \mathrm{E}+00 & - \\ 85-01-8 & 6.87 \mathrm{E}-01 & - & -\end{array}$

Dioxins/furans

Heptachlorodibenzo-p-dioxin isomer 37871-00-4 4.11E-03 1.50E+03 -

Heptachlorodibenzo-p-furan isomer 38998-75-3 2.58E-04 1.50E+03 -

Hexachlorodibenzo-p-furan isomer 55684-94-1 1.00E-04 1.50E+04 -

Octachlorodibenzo-p-dioxin isomer $\quad 3268-87-9 \quad 9.26 \mathrm{E}-03 \quad 1.50 \mathrm{E}+02 \quad-$

Octachlorodibenzo-p-furan isomer $\quad 39001-02-0 \quad 3.48 \mathrm{E}-04 \quad 1.50 \mathrm{E}+02$ -

PCBs

PCB 1254

$11097-69-1 \quad 1.78 \mathrm{E}-01 \quad 7.70 \mathrm{E}+00 \quad 2.00 \mathrm{E}-05$

RME Total

\section{Pesticides}

delta-Benzene hexachloride

Endrin ketone

319-86-8 1.84E-03

$53494-70-5 \quad 3.20 \mathrm{E}-03$

$\begin{array}{cccc}- & - & - & - \\ - & - & - & - \\ 5.6 \mathrm{E}-07 & 03 \% & - & - \\ 4.0 \mathrm{E}-06 & 18 \% & - & - \\ 6.1 \mathrm{E}-07 & 03 \% & - & - \\ - & - & - & - \\ 1.3 \mathrm{E}-06 & 06 \% & - & - \\ - & - & - & -\end{array}$

$5.2 \mathrm{E}-06 \quad 24 \%$

$3.2 \mathrm{E}-07 \quad 01 \%$

$1.3 \mathrm{E}-06 \quad 06 \%$

$1.2 \mathrm{E}-06 \quad 05 \%$

$4.4 \mathrm{E}-08<1 \%$

$1.1 \mathrm{E}-06 \quad 05 \% \quad 1.1 \mathrm{E}-01 \quad 20 \%$

$-$

$-$

$-$

$-$ 
Table D.30

Savannah River Site

Burning/Rubble Pits (231-F, 231-1F)

Combined Adult/Child Resident (Future)

Ingestion of Soil (0-2'bls)

Carcinogenic and Noncarcinogenic Risk

\begin{tabular}{|c|c|c|c|c|c|c|c|c|}
\hline \multirow[b]{2}{*}{ Chemical } & \multirow{2}{*}{\multicolumn{2}{|c|}{$\begin{array}{ll} & \frac{\text { EP Conc }^{(2)}}{\mathrm{RME}^{(1)}} \\
\text { CAS }^{(1)} \text { No. } & (\mathrm{mg} / \mathrm{kg}) \\
\end{array}$}} & \multirow[b]{2}{*}{$\begin{array}{c}\operatorname{OSF}^{(4)} \\
(\mathrm{kg}-\mathrm{d} / \mathrm{mg})\end{array}$} & \multirow[b]{2}{*}{$\begin{array}{c}\text { ORID }^{(\theta)} \\
(\mathbf{m g / k g - d )}\end{array}$} & \multicolumn{2}{|c|}{ Cancer Risk $^{(0)}$} & \multicolumn{2}{|c|}{ Hazard Quotient } \\
\hline & & & & & RME & $\begin{array}{l}\% \text { of } \\
\text { Total }\end{array}$ & RME & $\begin{array}{l}\% \text { of } \\
\text { Total }\end{array}$ \\
\hline \multicolumn{9}{|l|}{ Metals } \\
\hline Arsenic & $7440-38-2$ & $4.82 E+00$ & $1.50 \mathrm{E}+00$ & 3.00E-04 & $6.0 \mathrm{E}-06$ & $28 \%$ & 2.1E-01 & $36 \%$ \\
\hline Chromium & $7440-47-3$ & $4.42 E+01$ & - & $5.00 \mathrm{E}-03$ & - & - & $1.1 \mathrm{E}-01$ & $20 \%$ \\
\hline Lead & $7439-92-1$ & $2.48 E+01$ & - & - & - & - & - & - \\
\hline Manganese & $7439-96-5$ & $4.78 E+02$ & - & $5.00 \mathrm{E}-02$ & - & - & $1.2 \mathrm{E}-01$ & $21 \%$ \\
\hline \multirow[t]{2}{*}{ Mercury } & $7439-97-6$ & $5.48 \mathrm{E}-01$ & - & $3.00 \mathrm{E}-04$ & - & - & 2.3E-02 & $04 \%$ \\
\hline & & & & PATHWAY SUMS: & \multicolumn{2}{|c|}{$\begin{array}{c}\text { Cancer Rink } \\
\text { RME } \\
2 \mathrm{E}-05\end{array}$} & \multicolumn{2}{|c|}{$\begin{array}{c}\text { Hozard Index } \\
\text { RME } \\
\text { 6E-01 }\end{array}$} \\
\hline
\end{tabular}

1. Chemical Abetracts Service.

2. Exposure point concentration.

3. Reasonable maximum exposure.

4. Slope facior.

5. Reference dose.

6. Cancer Risk = (Chemical Concentration, mg/kg * Cancinogenic Intake Fector, kg/kg-day * Slope Fuctor, kg-day/mg), where RME carcinogenic intake factor 8.36E-07

(Note: Carcinogenic intuke factor calculated using weighted (combined) exposure assumptions.)

7. Hazard Quotient $=$ (Chemical Concentration, mg/kg * Noncarcinogenic Intake Factor, kg/kg-day) /(Reference Doee, mg/kg-day), where RME noncarcinogenic intake fa 1.28E-05 (Child Value)

(Note: Noncancinogenic intake factors are calculated for both adult and child, with the maximum of these used for risk calculations.) 
Table D.31

Savannah River Site

Burning/Rubble Pits (231-F, 231-1F)

Combined Adult / Child Resident (Future)

Dermal Exposure to Soil (0-2'bls)

Carcinogenic and Noncarcinogenic Risk

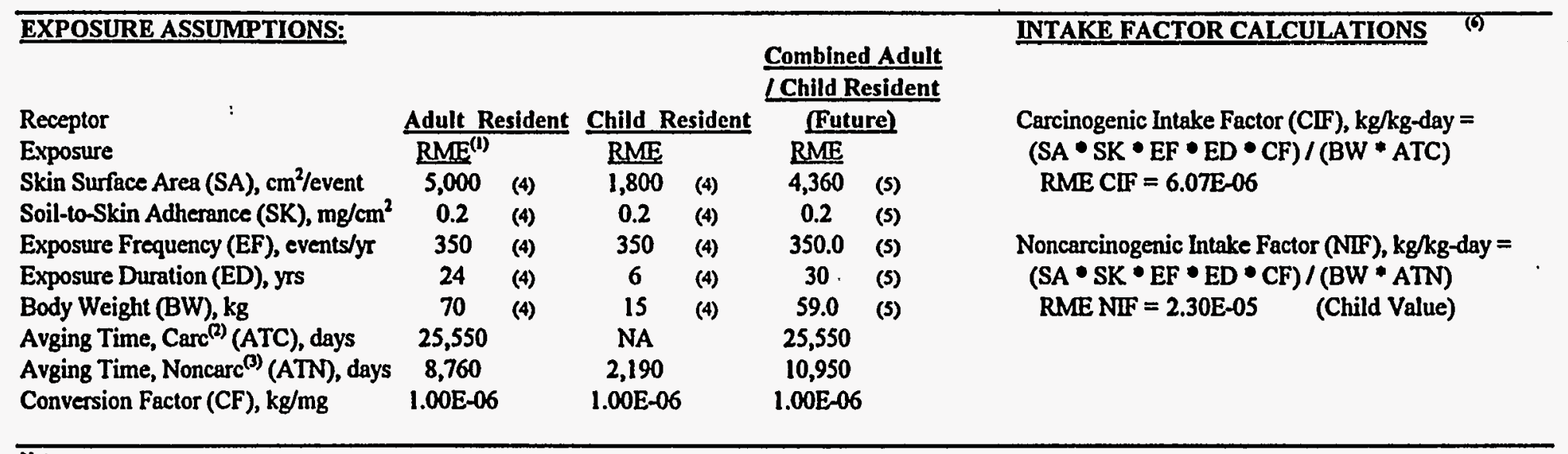

Noteri:

1. Resuonable maximum exposure.

2. Averesing time, cercinogen; calculated as 70 yean (averane lifetime) times 365 days per year (nok spplicable to child resident).

3. Averaging time, noncurciniogen; calculued as exposure duration (in years) times 365 dayx per year

4. See Teble D-1.

5. Combined values are calculated by weighting adult and child exposure assumptions over the noted exposure duration (e.g., $24 \mathrm{yga}$ a an adult plus $6 \mathrm{yms}$ as a child for a toul duration of $30 \mathrm{yrs}$ ).

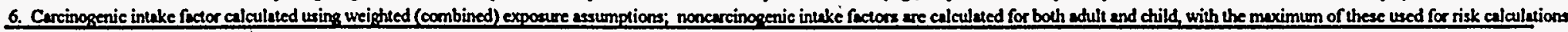


Table D.31

Savannah River Site

Burning/Rubble Pits (231-F, 231-1F)

- Combined Adult / Child Resident (Future)

Dermal Exposure to Soil (0-2'bls)

Carcinogenic and Noncarcinogenic Risk

\begin{tabular}{|c|c|c|c|c|c|c|c|c|c|}
\hline \multirow[b]{2}{*}{ Chemical } & \multicolumn{2}{|c|}{$\operatorname{EPConc}{ }^{(2)}$} & \multirow[b]{2}{*}{$\begin{array}{c}\operatorname{DSF}^{(0)} \\
\mathrm{Lg}(\mathrm{d}-\mathrm{d} / \mathrm{mg})\end{array}$} & \multirow[b]{2}{*}{$\begin{array}{c}\operatorname{DRm}^{(\sigma)} \\
\left(\mathrm{m} / \mathrm{Lg}_{\mathrm{g}-\mathrm{d})}\right.\end{array}$} & \multirow[b]{2}{*}{ DABS } & \multicolumn{2}{|c|}{ Cancer Risk ${ }^{(\prime)}$} & \multicolumn{2}{|c|}{ Hazard Ouotient ${ }^{(3)}$} \\
\hline & $\begin{array}{r}\text { CAS }^{(1)} \\
\text { Number }\end{array}$ & $\begin{array}{l}\mathbf{R M E}^{(3)} \\
(\mathbf{m} / \mathbb{L}(q)\end{array}$ & & & & RME & $\begin{array}{l}\% \text { of } \\
\text { Total }\end{array}$ & RME & $\begin{array}{l}\% \text { of } \\
\text { Total }\end{array}$ \\
\hline \multicolumn{10}{|l|}{ Semivolatiles } \\
\hline 2-Methylnaphthalene & $91-57-6$ & $7.69 E-02$ & - & - & $1.00 \mathrm{E}-02$ & - & - & - & - \\
\hline Acenaphthylene & $208-96-8$ & $8.61 E-02$ & - & - & $1.00 \mathrm{E}-02$ & - & - & - & - \\
\hline Benzo(a)anthracene & $56-55-3$ & $9.26 \mathrm{E}-01$ & $1.46 \mathrm{E}+00$ & - & $1.00 \mathrm{E}-02$ & 8.2E-08 & $03 \%$ & - & - \\
\hline Benzo(a)pyrenc & $50-32-8$ & $6.49 \mathrm{E}-01$ & $3.65 E+01$ & - & $1.00 \mathrm{E}-02$ & $1.4 \mathrm{E}-06$ & $55 \%$ & - & - \\
\hline Benzo(b)fluoranthene & $205-99-2$ & $1.01 E+00$ & $1.46 E+00$ & - & $1.00 \mathrm{E}-02$ & $8.9 \mathrm{E}-08$ & $03 \%$ & - & - \\
\hline Benzo(g,h,i)perylene & $191-24-2$ & $6.15 E-01$ & - & - & $1.00 \mathrm{E}-02$ & - & - & - & - \\
\hline Dibenz $(a, h)$ anthracene & $53-70-3$ & 2.17E-01 & $1.46 \mathrm{E}+01$ & - & $1.00 E-02$ & $1.9 E-07$ & $07 \%$ & - & - \\
\hline Phenanthrene & $85-01-8$ & $6.87 \mathrm{E}-01$ & - & - & $1.00 \mathrm{E}-02$ & - & - & - & - \\
\hline \multicolumn{10}{|l|}{ Dloxins/furans } \\
\hline Heptachlorodibenzo-p-dioxin isomer & $37871-00-4$ & $4.11 \mathrm{E}-03$ & $1.72 E+03$ & - & $1.00 \mathrm{E}-02$ & 4.3E-07 & $16 \%$ & - & - \\
\hline Heptechlorodibenzo-p-furan isomer & 38998-75-3. & 2.58E-04 & $1.72 \mathrm{E}+03$ & - & $1.00 \mathrm{E}-02$ & 2.7E-08 & $01 \%$ & - & - \\
\hline Hexachlorodibenzo-p-furan isomer & $55684-94-1$ & 1.00E-04 & $1.72 E+04$ & - & $1.00 \mathrm{E}-02$ & $1.0 \mathrm{E}-07$ & $04 \%$ & - & - \\
\hline Octachlorodibenzo-p-dioxin isomer & $3268-87-9$ & $9.26 \mathrm{E}-03$ & $1.72 \mathrm{E}+02$ & - & $1.00 \mathrm{E}-02$ & 9.7E-08 & $04 \%$ & - & - \\
\hline Octachlorodibenzo-p-furan isomer & $39001-02-0$ & $3.48 E-04$ & $1.72 E+02$ & - & $1.00 \mathrm{E}-02$ & $3.6 \mathrm{E}-09$ & $<1 \%$ & - & - \\
\hline \multicolumn{10}{|l|}{ PCBs } \\
\hline PCB 1254 & $11097-69-1$ & $1.78 \mathrm{E}-01$ & $8.56 \mathrm{E}+00$ & $1.80 \mathrm{E}-05$ & $1.00 \mathrm{E}-02$ & 9.3E-08 & $04 \%$ & 2.3E-03 & $09 \%$ \\
\hline \multicolumn{10}{|l|}{ Pesticides } \\
\hline delta-Benzene hexachloride & $319-86-8$ & $1.84 \mathrm{E}-03$ & - & - & $1.00 \mathrm{E}-02$ & - & - & - & - \\
\hline Endrin ketone & $53494-70-5$ & $3.20 \mathrm{E}-03$ & - & - & $1.00 \mathrm{E}-02$ & - & - & - & - \\
\hline
\end{tabular}


Table D.31

Savannah River Site

Burning/Rubble Pits (231-F, 231-1F)

- Combined Adult / Child Resident (Future)

Dermal Exposure to Soil (0-2'bls)

Carcinogenic and Noncarcinogenic Risk

\begin{tabular}{|c|c|c|c|c|c|c|c|c|c|}
\hline \multirow[b]{2}{*}{ Chemical } & \multicolumn{2}{|c|}{ EPConc $c^{(k)}$} & \multirow[b]{2}{*}{$\begin{array}{c}\mathrm{DSF}\left({ }^{())}\right. \\
(\mathrm{kg}-\mathbf{d} / \mathbf{m g})\end{array}$} & \multirow[b]{2}{*}{$\begin{array}{c}\operatorname{DRID}^{(\mathfrak{)})} \\
\text { (mg/kg-d) }\end{array}$} & \multirow[b]{2}{*}{ DABS $^{(0)}$} & \multicolumn{2}{|c|}{ Cancer Risk ${ }^{(7)}$} & \multicolumn{2}{|c|}{ Hazard Ouotient ${ }^{(k)}$} \\
\hline & $\begin{array}{r}\text { CAS }^{(1)} \\
\text { Number }\end{array}$ & $\begin{array}{l}\mathrm{RME}^{(3)} \\
(\mathrm{mg} / \mathrm{kg}) \\
\end{array}$ & & & & RME & $\begin{array}{l}\% \text { of } \\
\text { Total }\end{array}$ & RME & $\begin{array}{l}\% \text { of } \\
\text { Total }\end{array}$ \\
\hline \multicolumn{10}{|l|}{ Metals } \\
\hline Arsenic & $7440-38-2$ & $4.82 \mathrm{E}+00$ & $1.88 E+00$ & $2.40 \mathrm{E}-04$ & $1.00 \mathrm{E}-03$ & $5.5 E-08$ & $02 \%$ & 4.6E-04 & $02 \%$ \\
\hline Chromium & $7440-47-3$ & $4.42 E+01$ & - & $1.00 \mathrm{E}-04$ & $1.00 \mathrm{E}-03$ & - & - & $1.0 \mathrm{E}-02$ & $42 \%$ \\
\hline Lead & $7439-92-1$ & $2.48 \mathrm{E}+01$ & - & - & $1.00 \mathrm{E}-03$ & - & - & - & - \\
\hline Manganese & $7439-96-5$ & $4.78 \mathrm{E}+02$ & - & $1.50 \mathrm{E}-03$ & $1.00 \mathrm{E}-03$ & - & - & 7.3E-03 & $30 \%$ \\
\hline \multirow[t]{2}{*}{ Mercury } & $7439-97-6$ & $5.48 \mathrm{E}-01$ & - & $3.00 \mathrm{E}-06$ & $1.00 \mathrm{E}-03$ & - & - & $4.2 \mathrm{E}-03$ & $17 \%$ \\
\hline & & & & PATHWAY & SUMS: & \multicolumn{2}{|c|}{$\begin{array}{c}\text { Cancer Risk } \\
\frac{\text { RME }}{3 E-06} \\
\end{array}$} & \multicolumn{2}{|c|}{$\begin{array}{l}\text { Hazard Index } \\
\text { RME } \\
2 \mathrm{E}-02\end{array}$} \\
\hline
\end{tabular}

Noter:

1. Chemical Abstract Service.

2. Exposure poim concentration.

3. Reasonable maximum exposure.

4. Slope factor, dermal.

5. Reference dose, dermal,

6. Absorption factor.

7. Cancer Risk = (Chemical Concentration, mg/kg * Carcinogenic Intake Factor, kg/kg-day * Abcorption Factor, unitless * Slope Factor, kg-day/mg), where RME carcinogenic intake factor 6.07E-06

(Note: Cancinogenic intake factor calculated using weighted (combined) exposure sssumptions.)

8. Hazurd Quotient = (Chemical Concentration, mg/kg * Noncarcinogenic Intake Factor, kg/kg-day * Abeorption Factor, unitless)/(Reference Dose, mg/kg-day), where RME noncarcinogenic intake fa 2.30E-05 (Child Value)

(Note: Noncarcinogenic intake factors are calculated for both adult and child, with the maximum of these used for risk calculations.) 
Table D.32

Savanngh River Site

Burning/Rubble Pits (231-F, 231-1F)

Combined Adult / Child Resident (Future)

Inhalation of Volatile Soil Contaminants (0-2'bls)

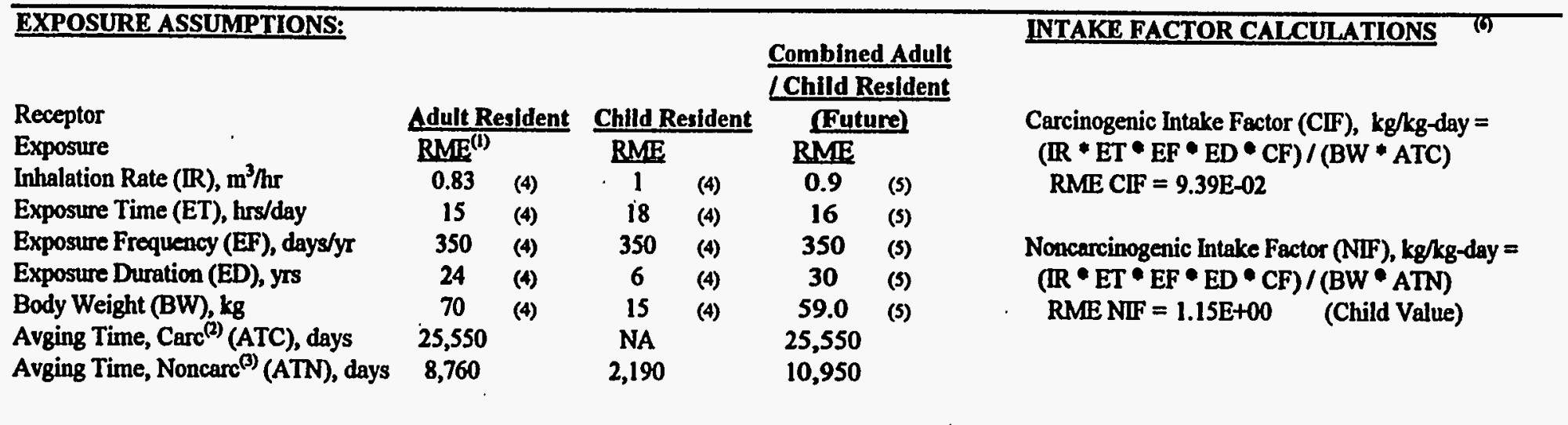

Notri:

1. Resconsble maximum exposure.

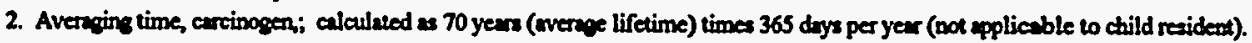

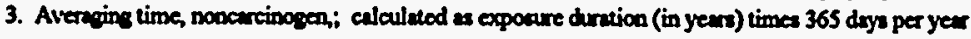

4. See Table D-1.

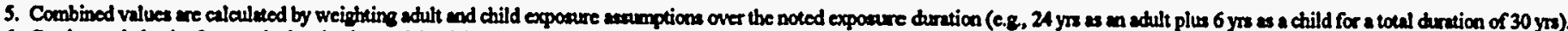

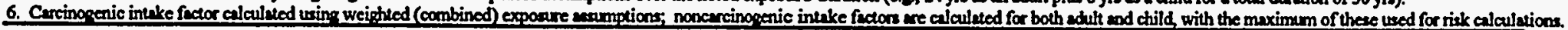


Table D.32

Savannah River Site

Burning/Rubble Pits (231-F, 231-1F)

Combined Adult / Child Resident (Future)

Inhalation of Volatile Soil Contaminants (0-2'bls)

Calculation of Volatilization Factor (VF)

\begin{tabular}{|c|c|c|c|c|c|c|c|c|c|c|c|}
\hline Chemical & $\mathrm{CAS}^{(2)} \mathrm{No}$ & Class $^{(3)}$ & MolWt & $\mathrm{Koc}^{(0)}$ & $\mathbf{K} \mathbf{d}^{(1)}$ & $\mathbf{H}^{(\ell)}$ & $\mathrm{Kas}^{(1)}$ & $\mathbf{D i}^{(4)}$ & $\mathbf{D e i}^{(t)}$ & alpha $\left({ }^{(1)}\right.$ & $\begin{array}{c}\text { Volat. Factor }{ }^{(4)} \\
\left(m^{3} / \mathrm{kg}\right)\end{array}$ \\
\hline \multicolumn{12}{|l|}{ Semivolatiles } \\
\hline 2-Methylnaphthalene & $91-57-6$ & $\mathbf{S}$ & $1.42 E+02$ & 8511.4 & $1.70 \mathrm{E}+02$ & $2.90 \mathrm{E}-04$ & $6.98 \mathrm{E}-05$ & $6.43 \mathrm{E}-02$ & $4.53 \mathrm{E}-02$ & $6.43 E-07$ & $1.82 E+05$ \\
\hline Acenaphthylene & $208-96-8$ & $\mathbf{S}$ & $1.52 E+02$ & 4786.3 & $9.57 \mathrm{E}+01$ & $2.80 \mathrm{E}-04$ & $1.20 \mathrm{E}-04$ & $6.22 \mathrm{E}-02$ & $4.38 \mathrm{E}-02$ & $1.07 \mathrm{E}-06$ & $1.42 E+05$ \\
\hline Phenanthrene & $85-01-8$ & $\mathbf{S}$ & $1.78 E+02$ & 38904.5 & $7.78 \mathrm{E}+02$ & $2.56 \mathrm{E}-05$ & $1.35 \mathrm{E}-06$ & $5.74 \mathrm{E}-02$ & 4.05E-02 & $1.11 E-08$ & $1.39 E+06$ \\
\hline
\end{tabular}

\section{Notea:}

1. USEPA, 1991: RAGS: Vol 1 - Human Health Evaluation Manual (Part B, Development of Risk-Based Preliminary Remediation Goals)

2. Chemical Abstract Service.

3. Chemical cless: v-volatile; s-semivolatile; $p$ - pesticide/peb; $x$ - dioxin; and m-metal.

4. Calculation of organic contaminarx volatilization factor:

CONSTANTS:

$$
\begin{aligned}
& \text { Length of side of contarid. area (LS), } m= \\
& \text { Wind speod in mixing zone }(V, m / s e c= \\
& \text { Diffusion height (DH), } m= \\
& \text { Area of contamination (Am), } \mathrm{m}^{2}= \\
& \text { Area in square centimeters }(A \mathrm{~cm}), \mathrm{cm}^{2}= \\
& \text { True soil porosity }(E) \text {, unitless = } \\
& \text { True toil density }\left(\mathrm{P}_{\mathrm{x}}\right), \mathrm{g} / \mathrm{cm}^{\mathrm{3}}= \\
& \text { Exposure interval (Tyr), yrs = } \\
& \text { Exponure interval }(\mathrm{Trec}), \mathrm{rec}= \\
& \text { Organic carbon content (OC), unitless = } \\
& \text { Convervion factor (CF), } \mathrm{kg} / \mathrm{g}=
\end{aligned}
$$

INPUT VARIABLES:

Organic cartion partition coef. (Koc), $\mathrm{cm}^{3} / \mathrm{kg}=$ Henry's Law condanx $(H), 2 t m-m^{3} / \mathrm{mol}^{2}=$ Gas diffusivity (Di), $\mathrm{cm}^{2} / \mathrm{sec}=$

INTERMEDIATE STEPS:

Soil-water partition coefficient (Kd), $\mathrm{cm}^{3} / \mathrm{kg}=$ Soil-air partition coefficient (Kas), $8-60 i l / \mathrm{cm}^{3}-2 i r=$

$$
\text { Effective diffurivity (Dei), } \mathrm{cm}^{2} / \mathrm{sec}=
$$$$
\text { Ilpha (alphu), } \mathrm{cm}^{2} / \mathrm{sec}=
$$

OUTPUT:

Volatilization Fuctor (VF), $\mathrm{m}^{3} / \mathrm{kg}=$

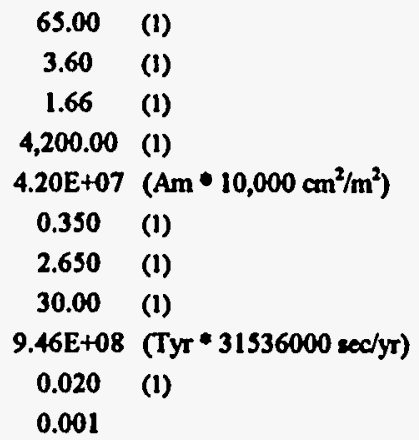


Table D.32

Savannah River Site

Burning/Rubble Pits (231-F, 231-1F)

Combined Adult / Child Resident (Future)

Inhalation of Volatile Soil Contaminants (0-2'bls)

Carcinogenic and Noncarcinogenic Risk

\begin{tabular}{|c|c|c|c|c|c|c|c|c|c|}
\hline \multirow[b]{2}{*}{ Chemical } & \multirow{2}{*}{\multicolumn{2}{|c|}{ 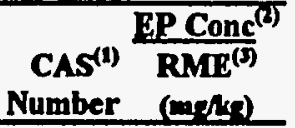 }} & \multirow[b]{2}{*}{$\begin{array}{l}\operatorname{IUR}^{(0)} \\
\left(m^{3} / 2 g\right)\end{array}$} & \multirow[b]{2}{*}{$\begin{array}{l}\operatorname{RrC}^{(9)} \\
\left(m / m^{3}\right)\end{array}$} & \multirow[b]{2}{*}{$\begin{array}{c}V F^{(0)} \\
\left(m^{3} / R()\right)\end{array}$} & \multicolumn{2}{|c|}{ Cancer Risk } & \multicolumn{2}{|c|}{ Hazard Ouotient ${ }^{(1)}$} \\
\hline & & & & & & RME & $\begin{array}{l}\% \text { of } \\
\text { Total }\end{array}$ & RME & $\begin{array}{l}\text { \% of } \\
\text { Total }\end{array}$ \\
\hline \multirow{5}{*}{$\begin{array}{l}\text { Semivolatiles } \\
\text { 2-Methylnaphthalene } \\
\text { Acenaphthylene } \\
\text { Phenanthrene }\end{array}$} & & & & & & & & & \\
\hline & $91-57-6$ & $7.69 \mathrm{E}-02$ & - & - & $1.82 E+05$ & NA & - & NA & - \\
\hline & $208-96-8$ & 8.61E-02 & - & - & $1.42 E+05$ & NA & - & NA & - \\
\hline & $85-01-8$ & 6.87E- -01 & - & - & $1.39 E+06$ & NA & - & NA & - \\
\hline & & & & \multicolumn{2}{|c|}{ PATHWAY SUMS: } & \multicolumn{2}{|c|}{$\begin{array}{c}\text { Cancer Risk } \\
\text { RME } \\
-\end{array}$} & \multicolumn{2}{|c|}{$\begin{array}{l}\text { Hazard Index } \\
\text { BME }\end{array}$} \\
\hline
\end{tabular}

1. Chemical Abstract Service.

2. Exposure point concentration

3. Reasonable maximum expocure.

4. Inhulation unit risk.

5. Reference concentration.

6. Volutilization factor (chemical-pecific).

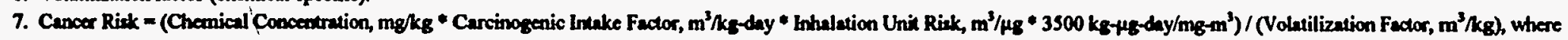
RME carcinogenic ineake fuctor 9.39E-02

and conversion from IUR wo inhulation stope fector $=3500 \mathrm{~kg}-\mu \mathrm{s}-\mathrm{day} / \mathrm{ms}^{-\mathrm{m}}$

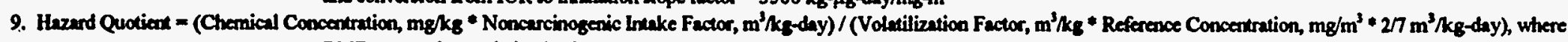
RME noncarcinogenic intake fa $1.15 E+00$ and conversion from RPC to inhhalation reference does $=2 / 7 \mathrm{~m}^{3} / \mathrm{kg}^{-}$-day 
Table D.33

Savannah River Site

Burning/Rubble Pits (231-F, 231-1F)

Combined Adult / Child Resident (Future)

Inhalation of Soil Particulates (0-2'bls)

Carcinogenic and Noncarcinogenic Risk

\begin{tabular}{|c|c|c|c|c|c|c|c|}
\hline EXPOSURE ASSUMPTIONS: & & & & & \multicolumn{2}{|c|}{ Combined Adult } & INTAKE FACTOR CALCULATIONS (11) \\
\hline Receptor & Adult R & sident & Child $\mathrm{E}$ & sident & Gut & & Carcinogenic Intake Factor (CIF), $\mathrm{kg} / \mathrm{kg}$-day = \\
\hline Exposure & $\mathbf{R M E}^{(1)}$ & & RME & & RME & & $(\mathrm{IR} \bullet \mathrm{FI} \bullet \mathrm{EF} \bullet \mathrm{ED} \bullet \mathrm{CF}) /(\mathrm{BW} \bullet \mathrm{ATC})$ \\
\hline Inhalation Rate (IR), $\mathrm{m}^{3} / \mathrm{hr}$ & 0.83 & (4) & 1.0 & (4) & 0.9 & (5) & RME CIF $=9.39 E-02$ \\
\hline Exposure Time (ET), hrs/day & 15 & (4) & 18 & (4) & 16 & (5) & \\
\hline Exposure Frequency (EF), days/yr & 350 & (4) & 350 & (4) & 350 & (s) & Noncarcinogenic Intake Factor (NIF), kg/kg-day = \\
\hline Exposure Duration (ED), yrs & 24 & (4) & 6 & (4) & 30 & (5) & $(\mathrm{IR} \bullet \mathrm{F} * \mathrm{EF} \bullet \mathrm{ED} * \mathrm{CF}) /(\mathrm{BW} * \mathrm{ATN})$ \\
\hline Body Weight (BW), kg & 70 & (4) & 15 & (4) & 59.0 & (5) & RME NIF $=1.15 E+00 \quad$ (Child Value) \\
\hline Avging Time, $\operatorname{Carc}^{(2)}$ (ATC), days & 25,550 & (4) & NA & (4) & 25,550 & & \\
\hline Avging Time, Noncarc ${ }^{(\mathfrak{)})}($ AIN), days & 8,760 & & 2,190 & & 10,950 & & \\
\hline
\end{tabular}

Nols:

1. Ressonuble maximum exponure.

2. Aleraging time, carcinosen; calculated as 70 years (average lifetime) times 365 days per year (bot upplicable wo child resident).

3. Averaping time, noncarcinogen; calculued us exporure duration (in years) times 365 dayz per yeur

4. See Table D-1.

5. Combined values are calculated by weigtting adult and child exposure assumptions over the noved expocure duration (c.g. 24 yrs as an adult plus 6 srs as a child for a loul duration of 30 yrs).

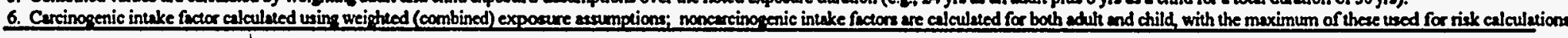
1 
Table D.33

Savannah River Site

Burning/Rubble Pits (231-F, 231-1F)

Combined Adult / Child Resident (Future)

Inhalation of Soil Particulates (0-2'bls)

Carcinogenic and Noncarcinogenic Risk

\begin{tabular}{|c|c|c|c|c|}
\hline \multicolumn{4}{|c|}{$\begin{array}{l}\text { CALCULATION OF SOIL PARTICULATE RESUSPENSION FACTOR: } \\
\text { CONSTANTS: }\end{array}$} & \multirow{2}{*}{$\begin{array}{l}\text { INTERMEDIATE STEPS: } \\
\text { Particulate emission factor }(\mathrm{PEF})=\quad 2.18 \mathrm{E}+09 \mathrm{~meters}^{3} / \mathrm{kg}\end{array}$} \\
\hline Length of side of contaminated area (LS) $=$ & 65.00 & meters & (1) & \\
\hline Wind speed in mixing zone $(V)=$ & 3.60 & meters/sec & (1) & $(\mathrm{LS} \times \mathrm{V} \times \mathrm{DH} \times \mathrm{CFa} \times \mathrm{CFb}) /\left(\mathrm{A} \times \mathrm{RF} \times(1-\mathrm{G}) \times\left((\mathrm{Um} / \mathrm{Ut})^{\wedge} 3\right) \times(\mathrm{Fx})\right)$ \\
\hline Diffusion height $(\mathrm{DH})=$ & 1.66 & meters & (i) & \\
\hline Area of contamination $(A)=$ & $4,200.00$ & meters ${ }^{2}$ & (1) & \\
\hline Respirable fraction $(R F)=$ & 0.003 & g/meters ${ }^{2}-h r$ & (1) & . \\
\hline Fraction of vegetative cover $(\mathbf{G})=$ & 0.90 & unitless & (1) & \\
\hline Mean annual wind speed $(\mathrm{Um})=$ & 3.60 & meters/sec & (1) & \\
\hline Equivalent threshold value of wind speed at $10 \mathrm{~m}(\mathrm{Ut})=$ & 5.04 & meters/sec & (1) & \\
\hline Fraction dependent on Um/Ut $(F x)=$ & 1.2700 & unitless & (1) & \\
\hline Conversion factor $(\mathrm{CFa})=$ & $3,600.00$ & sec/hr & & \\
\hline Conversion factor $(\mathrm{CFb})=$ & $1,000.00$ & g/kg & & \\
\hline
\end{tabular}

Noter:

1. USEPA, 1991: RAGS: Vol I - Humm Health Evaluation Manual (Purt B. Development of Risk-Based Preliminmy Remodixtion Coals) 
Table D.33

Savannah River Site

Burning/Rubble Pits (231-F, 231-1F)

Combined Adult / Child Resident (Future)

Inhalation of Soil Particulates (0-2'bls)

Carcinogenic and Noncarcinogenic Risk

\begin{tabular}{|c|c|c|c|c|c|c|c|c|}
\hline Chemical & $\begin{array}{r}\text { CAS }^{(1)} \\
\text { Number }\end{array}$ & $\begin{array}{c}\frac{\text { EP Conc }}{(2)} \\
\mathrm{RME}^{(3)} \\
(\mathrm{mg} / \mathrm{kg})\end{array}$ & $\begin{array}{l}\mathrm{IUR}^{(4)} \\
\left(\mathrm{m}^{3} / \mu g\right)\end{array}$ & $\begin{array}{r}\operatorname{RfC}^{(5)} \\
\left(\mathbf{m} g / \mathbf{m}^{3}\right)\end{array}$ & Cancer & $\begin{array}{l}\mathrm{isk}^{(6)} \\
\% \text { of } \\
\text { Total } \\
\end{array}$ & Hazard Q & $\begin{array}{l}\text { otient }^{(7)} \\
\% \text { of } \\
\text { Total } \\
\end{array}$ \\
\hline \multicolumn{9}{|l|}{ Semivolatiles } \\
\hline 2-Methylnaphthalene & $91-57-6$ & $7.69 \mathrm{E}-02$ & - & - & NA & - & NA & - \\
\hline Acenaphthylene & $208-96-8$ & 8.61E-02 & - & - & NA & - & NA & - \\
\hline Benzo(a)anthracene & $56-55-3$ & $9.26 \mathrm{E}-01$ & - & - & NA & - & NA & - \\
\hline Benzo(a)pyrene & $50-32-8$ & $6.49 \mathrm{E}-01$ & - & - & NA & - & NA & - \\
\hline Benzo(b)fluoranthene & $205-99-2$ & $1.01 \mathrm{E}+00$ & - & - & NA & - & NA & - \\
\hline Benzo(g,h,i)perylene & $191-24-2$ & 6.15E-01 & - & - & NA & - & NA & - \\
\hline Dibenz(a,h)anthracene & $53-70-3$ & 2.17E-01 & - & - & NA & - & NA & - \\
\hline Phenanthrene & $85-01-8$ & $6.87 E-01$ & - & - & NA & - & NA & - \\
\hline \multicolumn{9}{|l|}{ Dloxins/furans } \\
\hline Heptachlorodibenzo-p-dioxin isomer & $37871-00-4$ & $4.11 \mathrm{E}-03$ & $3.30 \mathrm{E}-01$ & - & 2.0E-10 & $<1 \%$ & NA & - \\
\hline Heptachlorodibenzo-p-furan isomer & $38998-75-3$ & $2.58 \mathrm{E}-04$ & $3.30 \mathrm{E}-01$ & - & $1.3 \mathrm{E}-11$ & $<1 \%$ & NA & - \\
\hline Hexachlorodibenzo-p-furan isomer & $55684-94-1$ & $1.00 \mathrm{E}-04$ & $3.30 \mathrm{E}+00$ & - & 5.0E-11 & $<1 \%$ & NA & - \\
\hline Octachlorodibenzo-p-dioxin isomer & $3268-87-9$ & $9.26 \mathrm{E}-03$ & $3.30 \mathrm{E}-02$ & - & $4.6 \mathrm{E}-11$ & $<1 \%$ & NA & - \\
\hline Octachlorodibenzo-p-furan isomer & $39001-02-0$ & $3.48 \mathrm{E}-04$ & $3.30 \mathrm{E}-02$ & - & $1.7 \mathrm{E}-12$ & $<1 \%$ & NA & - \\
\hline \multicolumn{9}{|l|}{ PCBs } \\
\hline PCB 1254 & $11097-69-1$ & $1.78 \mathrm{E}-01$ & - & - & NA & - & NA & - \\
\hline \multicolumn{9}{|l|}{ Pesticides } \\
\hline $\begin{array}{l}\text { delta-Benzene hexachloride } \\
\text { Endrin ketone }\end{array}$ & $319-86-8$ & $1.84 \mathrm{E}-03$ & - & - & NA & - & NA & - \\
\hline Endrin ketone & $53494-70-5$ & $3.20 E-03$ & - & - & NA & - & NA & - \\
\hline
\end{tabular}


Table D.33

Savannah River Site

Burning/Rubble Pits (231-F, 231-1F)

Combined Adult / Child Resident (Future)

Inhalation of Soil Particulates (0-2'bls)

Carcinogenic and Noncarcinogenic Risk

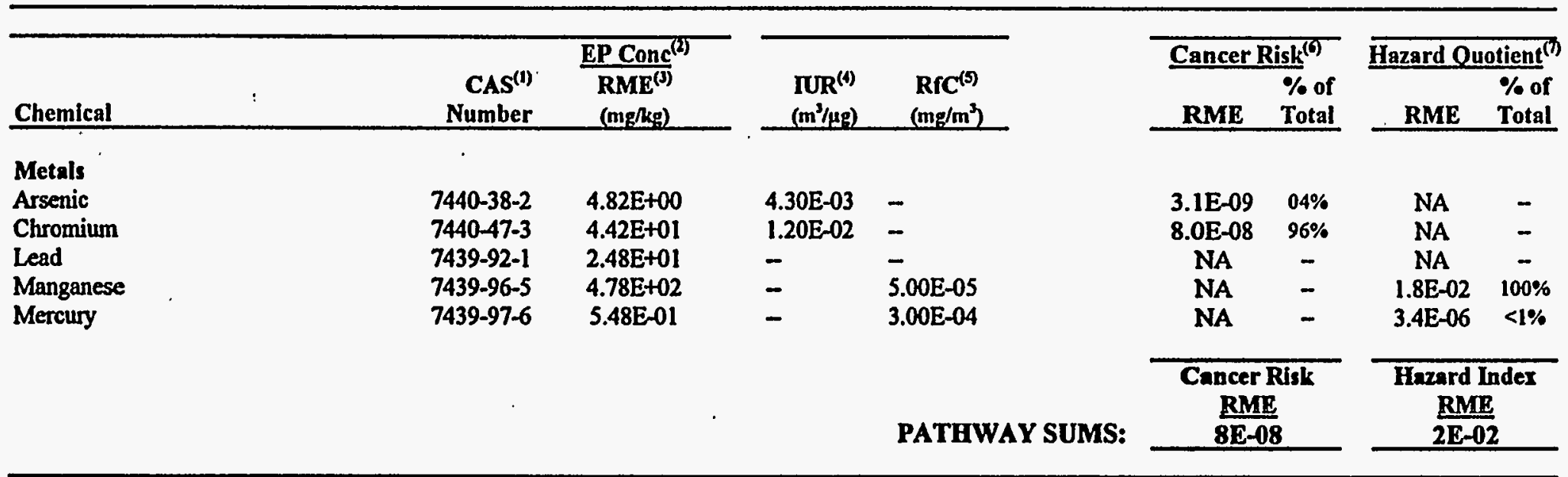

1. Chemical Abstract Service.

2. Exposure point concentration

3. Ressonable maximum exposure.

4. Inhulation unit risk

5. Referenoe concentration.

6. Cancer Risk = (Chemical Concentration, mg/kg * Carcinogenic Intake Factor, $\mathrm{m}^{3} / \mathrm{kg}$-day * Inhalation Unit Risk, $\mathrm{m}^{3} / \mu \mathrm{g}$ * $\left.3500 \mathrm{~kg}-\mu \mathrm{g}-\mathrm{day} / \mathrm{mg}_{\mathrm{g}}-\mathrm{m}^{2}\right) /\left(\mathrm{Particulale}\right.$ Emission Factor, $\left.\mathrm{m}^{3} / \mathrm{kg}\right)$

RME carcinogenic intake factor $=9.39 \mathrm{E}-02$

Particulate emission factor $=\quad 2.18 \mathrm{E}+09$

and conversion from IUR to inhalation slope factor $=3500 \mathrm{~kg}-\mu \mathrm{g}-$-day $/ \mathrm{mg}-\mathrm{m}^{3}$

(Note: Carcinogenic intake factor calculated using weighted (combined) exposure assumptions.)

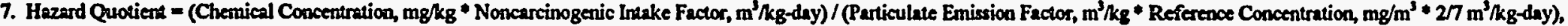

RME noncarcinogenic intuke factor $=1.15 E+00$

Particulate cmission fector $=\quad 2.18 \mathrm{E}+09$

and conversion from RfC to inhalation reference doce $=2 / 7 \mathrm{~m}^{3} / \mathrm{kg}$-day

(Note: Noncarcinogenic intuke factors are calculated for both aduh and child, with the maximum of these used for risk calculations.) 
Table D.34

Savannah River Site

Burning/Rubble Pits (231-F, 231-1F)

Combined Adult/Child Resident (Future)

Ingestion of Soil (0-4'bls)

Carcinogenic and Noncarcinogenic Risk

\begin{tabular}{|c|c|c|c|c|c|c|c|}
\hline \multirow[t]{2}{*}{ EXPOSURE ASSUMPTIONS: } & & \multirow{2}{*}{\multicolumn{2}{|c|}{ Combined }} & \multirow[t]{2}{*}{ INTAKE FACTOR CALCULATIONS (G) } \\
\hline & & & & & & & \\
\hline Receptor & \multicolumn{2}{|c|}{ Adult Resident } & \multicolumn{2}{|c|}{ Child Resident } & \multicolumn{2}{|c|}{ Adult/Child } & \multirow{3}{*}{$\begin{array}{l}\text { Carcinogenic Intake Factor }(\mathrm{CIF}), \mathrm{kg} / \mathrm{kg}-\text { day }= \\
(\mathrm{IR} \bullet \mathrm{FI} \bullet \mathrm{EF} \bullet \mathrm{ED} \bullet \mathrm{CF}) /(\mathrm{BW} * \mathrm{ATC}) \\
\mathrm{RMFCIF}=8.36 \mathrm{E}-07\end{array}$} \\
\hline Exposure & $\mathrm{RME}^{(1)}$ & & RME & & RME & & \\
\hline Intake Rate (IR), mg/day & 100 & (4) & 200 & (4) & 120.0 & (5) & \\
\hline Fraction Ingested (FI), unitless & 1 & (4) & 1 & (4) & 1.0 & (5) & \\
\hline Exposure Frequency (EF), days/yr & .350 & (4) & 350 & (4) & 350 & (5) & Noncarcinogenic Intake Factor (NIF), kg/kg-day = \\
\hline Exposure Duration (ED), yrs & 24 & (4) & 6 & (4) & 30 & (5) & $(\mathrm{IR} \bullet \mathrm{FT} \bullet \mathrm{EF} * \mathrm{ED} \bullet \mathrm{CF}) /(\mathrm{BW} \bullet \mathrm{ATN})$ \\
\hline Body Weight (BW), kg & 70 & (4) & 15 & (4) & 59.0 & (5) & RME NIF $=1.28 \mathrm{E}-05 \quad$ (Child Value) \\
\hline Avging Time, Carc ${ }^{(2)}$ (ATC), days & 25,550 & & NA & & 25,550 & & \\
\hline Avging Time, Noncarc ${ }^{(3)}$ (ATN), days & 8,760 & & 2,190 & & 10,950 & & \\
\hline Conversion Factor (CF), kg/mg & $1.00 \mathrm{E}-06$ & & $1.00 \mathrm{E}-06$ & & $1.00 \mathrm{E}-06$ & & \\
\hline
\end{tabular}

1. Restonsble maximum exposure.

2. Avernging time, carcinogen,; calculued a 70 years (average lifetime) times 365 days per year (not applicable to child resident).

3. Averaing time, noncercinogen; calculued as expoure duration (in yems) times 365 days per year

4. See Table D-1.

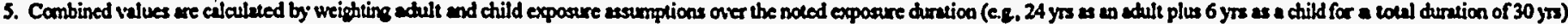

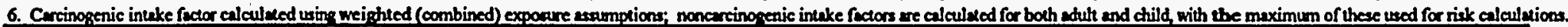


Table D.34

Savannah River Site

Burning/Rubble Pits (231-F, 231-1F)

- Combined Adult/Child Resident (Future)

Ingestion of Soil (0-4'bls)

Carcinogenic and Noncarcinogenic Risk

\begin{tabular}{|c|c|c|c|c|c|c|c|c|}
\hline Chemical & $\mathrm{CAS}^{(1)} \mathrm{No}$ & $\begin{array}{l}\text { EPConc }{ }^{(2)} \\
\text { RME }^{(1)} \\
\text { (mesks) }\end{array}$ & $\begin{array}{c}\operatorname{OSF}^{(1)} \\
(\mathrm{kg}-\mathrm{d} / \mathrm{mg})\end{array}$ & 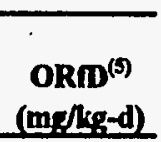 & $\begin{array}{l}\text { Cancer F } \\
\text { RME } \\
\end{array}$ & $\begin{array}{l}\text { ilik } \\
\% \text { of } \\
\text { Total }\end{array}$ & $\begin{array}{l}\text { Hezard } 0 \\
. \quad \text { RME } \\
\end{array}$ & $\begin{array}{l}\text { otient }^{(1)} \\
\% \text { of } \\
\text { Total }\end{array}$ \\
\hline Semivolatiles & & & & & & & & \\
\hline 2-Methylnuphthalene & $91-57-6$ & $7.69 \mathrm{E}-02$ & - & - & - & - & - & - \\
\hline Acenaphthylene & $208-96-8$ & $8.61 E-02$ & - & - & - & - & - & - \\
\hline Benzo $(a)$ anthracene & $56-55-3$ & $9.26 \mathrm{E}-01$ & $7.30 \mathrm{E}-01$ & - & $5.6 \mathrm{E}-07$ & $03 \%$ & - & - \\
\hline Benzo(a)pyrene & $50-32-8$ & $6.49 \mathrm{E}-01$ & $7.30 \mathrm{E}+00$ & - & 4.0E-06 & $18 \%$ & - & - \\
\hline Benzo(b) fluoranthene & 205-99-2 & $1.01 E+\infty$ & $7.30 \mathrm{E}-01$ & - & $6.1 \mathrm{E}-07$ & $03 \%$ & - & - \\
\hline Benzo(g,h,i)perylene & $191-24-2$ & 6.15E-01 & - & - & - & - & - & - \\
\hline Dibenz(a,h)anthracene & $53-70-3$ & 2.17E-01 & $7.30 \mathrm{E}+00$ & - & $1.3 \mathrm{E}-06$ & $06 \%$ & - & - \\
\hline Phenanthrene & $85-01-8$ & $6.87 \mathrm{E}-01$ & - & - & - & - & - & - \\
\hline Dioxins/furans & & & & & & & & \\
\hline Heptachlorodibenzo-p-dioxin isomer & $37871-00-4$ & $4.11 \mathrm{E}-03$ & $1.50 \mathrm{E}+03$ & - & $5.2 \mathrm{E}-06$ & $23 \%$ & - & - \\
\hline Heptachlorodibenzo-p-furan isomer & 38998-75-3 & $2.58 \mathrm{E}-04$ & $1.50 \mathrm{E}+03$ & - & $3.2 \mathrm{E}-07$ & $01 \%$ & - & - \\
\hline Hexachlorodibenzo-p-furan isomer & $55684-94-1$ & $1.00 \mathrm{E}-04$ & $1.50 \mathrm{E}+04$ & - & $1.3 \mathrm{E}-06$ & $06 \%$ & - & - \\
\hline Octachlorodibenzo-p-dioxin isomer & $3268-87-9$ & $9.26 \mathrm{E}-03$ & $1.50 \mathrm{E}+02$ & - & $1.2 \mathrm{E}-06$ & $05 \%$ & - & - \\
\hline Octachlọrodibenzo-p-furan isomer & $39001-02-0$ & $3.48 \mathrm{E}-04$ & $1.50 \mathrm{E}+02$ & - & 4.4E-08 & $<1 \%$ & - & - \\
\hline $\begin{array}{l}\text { PCBs } \\
\text { PCB 1254 }\end{array}$ & $11097-69-1$ & $1.78 \mathrm{E}-01$ & $7.70 E+00$ & $2.00 \mathrm{E}-05$ & $1.1 \mathrm{E}-06$ & $05 \%$ & $1.1 \mathrm{E}-01$ & $18 \%$ \\
\hline Pesticides & & & & & & & & \\
\hline delta-Benzene hexpchloride & $319-86-8$ & $1.84 \mathrm{E}-03$ & - & - & - & - & _- & _- \\
\hline Endrin ketone & $53494-70-5$ & $3.20 \mathrm{E}-03$ & - & - & - & - & - & - \\
\hline
\end{tabular}


Table D.34

Savannah River Site

Burning/Rubble Pits (231-F, 231-1F)

Combined Adult/Child Resident (Future)

Ingestion of Soil (0-4'bls)

Carcinogenic and Noncarcinogenic Risk

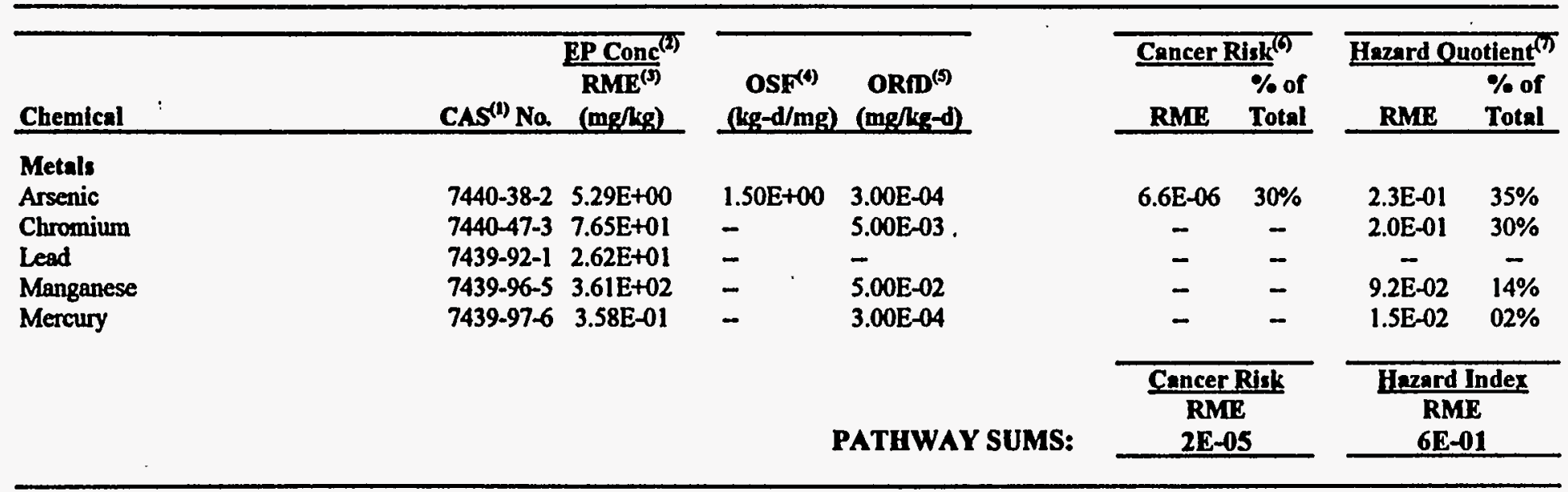

Notes:

1. Chemical Abstreats Service.

2. Exposure point concentration.

3. Reasonable maximum exposure.

4. Slope factor.

5. Reference doce.

6. Cancer Risk = (Chemical Concentration, mg/kg * Carcinogenic Intake Factor, kg/kg-day • Slope Factor, kg-day/mg), uhete RME carcinogenic intake factor 8.36E-07

(Note: Carcinogenic intake factor calculated using weighted (combined) exposure ascumptions.)

7. Hazard Quotient = (Chemical Concentration, mg/kg • Noncarcinogenic Intake Factor, kg/kg-day) / (Reference Dose, mg/kg-day), where

$$
\text { RME noncarcinogenic intake fa } 1.28 E-05 \text { (Child Value) }
$$

(Nole: Noncarcinogenic intake factors are calculated for both adult and child, with the maximum of these ured for risk calculations.) 
Table D.35

Savannah River Site

Burning/Rubble Pits (231-F, 231-1F)

- Combined Adult / Child Resident (Future)

Dermal Exposure to Soil (0-4'bls)

Carcinogenic and Noncarcinogenic Risk

\begin{tabular}{|c|c|c|c|c|c|c|c|}
\hline \multirow[t]{3}{*}{ EXPOSURE ASSUMPTIONS: } & & & & & \multirow{2}{*}{\multicolumn{2}{|c|}{ Combined Adult }} & \multirow[t]{3}{*}{ INTAKE FACTOR CALCULATIONS (ด) } \\
\hline & & & & & & & \\
\hline & & & & & \multicolumn{2}{|c|}{ LChild Resident } & \\
\hline Receptor & \multicolumn{2}{|c|}{ Adult Resident } & \multicolumn{2}{|c|}{ Child Resident } & \multicolumn{2}{|c|}{ (Future) } & \multirow{3}{*}{$\begin{array}{l}\text { Carcinogenic Intake Factor }(\mathrm{CIF}), \mathrm{kg} / \mathrm{kg}-\text { day }= \\
(\mathrm{SA} \bullet \mathrm{SK} * \mathrm{EF} * \mathrm{ED} \bullet \mathrm{CF}) /(\mathrm{BW} * \mathrm{ATC}) \\
\mathrm{RME} \mathrm{CFF}=6.07 \mathrm{E}-06\end{array}$} \\
\hline Exposure & \multicolumn{2}{|c|}{$\mathrm{RME}^{(1)}$} & \multicolumn{2}{|c|}{$\mathrm{RME}$} & \multicolumn{2}{|c|}{ RME } & \\
\hline Skin Surface Area (SA), $\mathrm{cm}^{2} /$ event & 5,000 & (4) & 1,800 & (4) & 4,360 & (5) & \\
\hline Soil-to-Skin Adherance (SK), $\mathrm{mg} / \mathrm{cm}^{2}$ & 0.2 & (4) & 0.2 & (4) & 0.2 & (5) & \\
\hline Exposure Frequency (EF), events/yr & 350 & (4) & 350 & (4) & 350.0 & (5) & Noncarcinogenic Intake Factor (NIF), kg/kg-day = \\
\hline Exposure Duration (ED), yrs & 24 & (4) & 6 & (4) & 30 & (5) & $(\mathrm{SA} \bullet \mathrm{SK} \bullet \mathrm{EF} \bullet \mathrm{ED} \bullet \mathrm{CF}) /(\mathrm{BW} \bullet \mathrm{ATN})$ \\
\hline Body Weight (BW), kg & 70 & (4) & 15 & (4) & 59.0 & (5) & RME NIF $=2.30 \mathrm{E}-05 \quad$ (Child Value) \\
\hline Avging Time, $\operatorname{Carc}^{(2)}$ (ATC), days & 25,550 & & NA & & 25,550 & & \\
\hline Avging Time, Noncarc ${ }^{(3)}$ (ATN), days & 8,760 & & 2,190 & & 10,950 & & \\
\hline Conversion Factor (CF), $\mathrm{kg} / \mathrm{mg}$ & $1.00 \mathrm{E}-06$ & & $1.00 \mathrm{E}-06$ & & $1.00 \mathrm{E}-06$ & & \\
\hline
\end{tabular}

Noter:

1. Resuonuble maximum exposure.

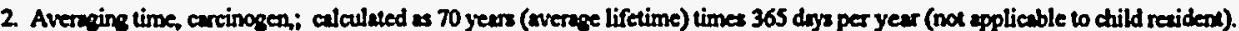

3. Averwing time, noncercisiogen; cilculated as exposure durution (in years) times 365 days per year

4. See Table D.1.

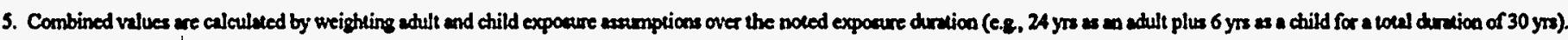

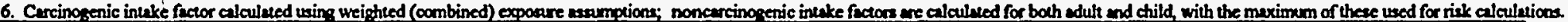


Table D.35

Savannah River Site

Burning/Rubble Pits (231-F, 231-1F)

- Combined Adult / Child Resident (Future)

Dermal Exposure to Soil (0-4'bls)

Carcinogenic and Noncarcinogenic Risk

\begin{tabular}{|c|c|c|c|c|c|c|c|c|c|}
\hline Chemical & $\begin{array}{r}\text { CAS }^{(1)} \\
\text { Number }\end{array}$ & $\begin{array}{l}\text { EP Conc }{ }^{(l)} \\
\operatorname{RME}^{(\lambda)} \\
(\mathrm{mg} / \mathrm{kg})\end{array}$ & $\begin{array}{c}\mathrm{DSF}^{(1)} \\
(\mathrm{kg}-\mathrm{d} / \mathrm{mg})\end{array}$ & $\begin{array}{c}\text { DRID }^{(9)} \\
(m / k g-d)\end{array}$ & DABS ${ }^{(0)}$ & Cancer I & $\begin{array}{l}\frac{1 \mathbf{l}^{(7)}}{\%} \\
\% \text { of } \\
\text { Total } \\
\end{array}$ & $\begin{array}{r}\text { Hazand OU } \\
\text { RME } \\
\end{array}$ & $\begin{array}{l}\text { otlent }^{(0)} \\
\% \text { of } \\
\text { Total } \\
\end{array}$ \\
\hline \multicolumn{10}{|l|}{ Semivolatikes } \\
\hline 2-Methylnaphthalene & $91-57-6$ & $7.69 \mathrm{E}-02$ & - & - & $1.00 \mathrm{E}-02$ & - & - & - & - \\
\hline Acenuphthylene & $208-96-8$ & 8.61E-02 & - & - & $1.00 \mathrm{E}-02$ & - & - & - & - \\
\hline Benzo(a)anthracene & $56-55-3$ & $9.26 \mathrm{E}-01$ & $1.46 \mathrm{E}+00$ & - & $1.00 E-02$ & 8.2E -08 & $03 \%$ & - & - \\
\hline Benzo(a)pyrene & $50-32-8$ & $6.49 \mathrm{E}-01$ & $3.65 \mathrm{E}+01$ & - & $1.00 \mathrm{E}-02$ & $1.4 \mathrm{E}-06$ & $55 \%$ & - & - \\
\hline Benzo(b)flworanthene & $205-99-2$ & $1.01 \mathrm{E}+00$ & $1.46 \mathrm{E}+00$ & - & $1.00 \mathrm{E}-02$ & 8.9E -08 & $03 \%$ & - & - \\
\hline Benzo(g,h,i)perylene & $191-24-2$ & $6.15 E-01$ & - & - & $1.00 \mathrm{E}-02$ & - & - & - & - \\
\hline Dibenz(a,h)anthracene & $53-70-3$ & $2.17 \mathrm{E}-01$ & $1.46 \mathrm{E}+01$ & - & $1.00 \mathrm{E}-02$ & $1.9 \mathrm{E}-07$ & $07 \%$ & - & - \\
\hline Phenanthrene & $85-01-8$ & $6.87 \mathrm{E}-01$ & - & - & $1.00 \mathrm{E}-02$ & - & - & - & - \\
\hline \multicolumn{10}{|l|}{ Dloxins/furans } \\
\hline Heptachlorodibenzo-p-dioxin isomer & $37871-00-4$ & 4.11E-03 & $1.72 E+03$ & - & $1.00 E-02$ & 4.3E-07 & $16 \%$ & - & - \\
\hline Heptachlorodibenzo-p-furan isomer & $38998-7.5-3$ & 2.58E-04 & $1.72 E+03$ & - & $1.00 E-02$ & 2.7E-08 & $01 \%$ & - & - \\
\hline Hexachlorodibenzo-p-furan isomer & $55684-94-1$ & $1.00 \mathrm{E}-04$ & $1.72 E+04$ & - & $1.00 \mathrm{E}-02$ & $1.0 \mathrm{E}-07$ & $04 \%$ & - & - \\
\hline Octachlorodibenzo-p-dioxin isomer & $3268-87-9$ & $9.26 \mathrm{E}-03$ & $1.72 \mathrm{E}+02$ & - & $1.00 \mathrm{E}-02$ & 9.7E-08 & $04 \%$ & - & - \\
\hline Octachlorodibenzo-p-furan isomer & $39001-02-0$ & $3.48 \mathrm{E}-04$ & $1.72 E+02$ & - & $1.00 E-02$ & $3.6 \mathrm{E}-09$ & $<1 \%$ & - & - \\
\hline \multicolumn{10}{|l|}{ PCBa } \\
\hline PCB 1254 & $11097-69-1$ & $1.78 \mathrm{E}-01$ & $8.56 E+00$ & $1.80 \mathrm{E}-05$ & $1.00 \mathrm{E}-02$ & $9.3 \mathrm{E}-08$ & $04 \%$ & $2.3 E-03$ & $08 \%$ \\
\hline \multicolumn{10}{|l|}{ Pesticides } \\
\hline delta-Benzene hexachloride & $319-86-8$ & $1.84 \mathrm{E}-03$ & - & - & $1.00 \mathrm{E}-02$ & - & - & - & - \\
\hline Endrin ketone & $53494-70-5$ & $3.20 \mathrm{E}-03$ & - & - & $1.00 \mathrm{E}-02$ & - & - & - & - \\
\hline
\end{tabular}


Table D.35

Savannah River Site

Burning/Rubble Pits (231-F, 231-1F)

Combined Adult / Child Resident (Future)

Dermal Exposure to Soil (0-4'bls)

Carcinogenic and Noncarcinogenic Risk

\begin{tabular}{|c|c|c|c|c|c|c|c|c|c|}
\hline Chemical & $\begin{array}{r}\text { CAS } \\
\text { Number }\end{array}$ & $\begin{array}{l}\frac{\mathrm{EPConc}}{(2)} \\
\mathrm{RME}^{(3)} \\
(\mathrm{m} / \mathrm{kg})\end{array}$ & $\begin{array}{c}\operatorname{DSF}^{(t)} \\
(\operatorname{Leg}-\mathrm{d} / \mathrm{m} \mathrm{x})\end{array}$ & $\begin{array}{c}\operatorname{DRID}^{(f)} \\
(\mathrm{mg} / \mathbf{k g}-\mathrm{d})\end{array}$ & DABS $^{(0)}$ & Cancer & $\begin{array}{l}\frac{13 k^{(y)}}{\%} \text { of } \\
\text { Total } \\
\end{array}$ & Hezard 0 & $\begin{array}{l}\text { otient }^{(0)} \\
\% \text { of } \\
\text { Total } \\
\end{array}$ \\
\hline \multicolumn{10}{|l|}{ Metals } \\
\hline Arsenic & $7440-38-2$ & $5.29 \mathrm{E}+00$ & $1.88 E+00$ & $2.40 \mathrm{E}-04$ & $1.00 \mathrm{E}-03$ & $6.0 \mathrm{E}-08$ & $02 \%$ & 5.1E 04 & $02 \%$ \\
\hline Chromium & $7440-47-3$ & $7.65 E+01$ & - & $1.00 \mathrm{E}-04$ & $1.00 \mathrm{E}-03$ & - & - & $1.8 \mathrm{E}-02$ & $61 \%$ \\
\hline Lead & $7439-92-1$ & $2.62 \mathrm{E}+01$ & - & - & $1,00 \mathrm{E}-03$ & - & - & - & - \\
\hline Manganese & $7439-96-5$ & $3.61 E+02$ & - & $1.50 \mathrm{E}-03$ & $1.00 \mathrm{E}-03$ & - & - & $5.5 \mathrm{E}-03$ & $19 \%$ \\
\hline \multirow[t]{2}{*}{ Mercury } & $7439-97-6$ & $3.58 \mathrm{E}-01$ & - & $3.00 \mathrm{E}-06$ & $1.00 \mathrm{E}-03$ & - & - & $2.7 \mathrm{E}-03$ & $10 \%$ \\
\hline & & & & PATHWAI & Y SUMS: & \multicolumn{2}{|c|}{$\begin{array}{c}\text { Cancer Risk } \\
\text { RME }\end{array}$} & \multicolumn{2}{|c|}{$\begin{array}{c}\text { Harard Inder } \\
\text { RME } \\
3 \mathrm{E}-02\end{array}$} \\
\hline
\end{tabular}

Noten:

1. Chemical Abstract Service.

2. Expoutre point concentration.

3. Reasonable maximum exposure.

4. Slope factor, dermal.

5. Referenoe doee, dermal.;

6. Abrooption factor.

7. Canocr Risk = (Chemical Concentration, mgkg * Carcinogenic Intake Factor, kg/kg-day * Abeorption Factor, unilless * Slope Fuctor, kg-day/mg), where RME cancinogenic intake fector 6.07E-06

(Note: Carcinogenic intake factor calculated using weighted (combined) exposure assumptions.)

8. Hezand Quotient = (Chemical Concentration, mg/kg * Noncancinogenic Intake Fuctor, kg/kg-day * Absosption Factor, unitless) /(Reference Dose, mg/kg-day), where

RME noncarcinogenic intake fa 2.30E-05 (Child Value)

Note: Noncarcinogenic intake factors are calculated for both adult and child, with the meximum of these used for risk calculations.) 
Table D.36

Savannah River Site

Burning/Rubble Pits (231-F, 231-1F)

Combined Adult / Child Resident (Future)

Inhalation of Volatile Soil Contaminants $\left(0-\mathbf{A}^{\prime} \mathrm{bls}\right)$

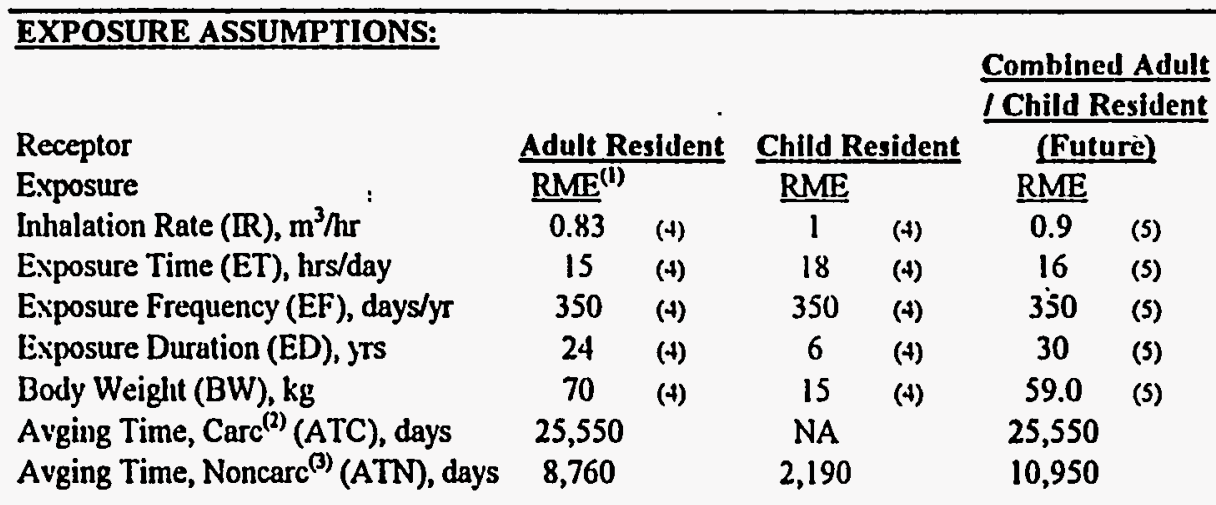

INTAKE FACTOR CALCULATIONS

Curcinogenic Intake Factor (CIF), $\mathrm{kg} / \mathrm{kg}$-day = $(\mathrm{R} \bullet \mathrm{ET} * \mathrm{EF} \bullet \mathrm{ED} \bullet \mathrm{CF}) /(\mathrm{BW} * \mathrm{ATC})$

RME CIF $=9.39 \mathrm{E}-02$

Noncarcinogenic Intake Factor (NIF), kg/kg-day = $(\mathrm{IR} \bullet \mathrm{ET} \bullet \mathrm{EF} \bullet \mathrm{ED} * \mathrm{CF}) /(\mathrm{BW} * \mathrm{ATN})$

RMI: NIF $=1.15 \mathrm{E}+00 \quad$ (Child Value)

\section{Noteri:}

I. Reasonable maximum exposure.

2. Averaging time, carcinogen; calculated as 70 years (average lifetime) times 365 dass per year (not applicable to child resident).

3. Averaging time, noncarcinogen; calculated as exposure duration (in years) times 365 days per year

4. See Table D-1.

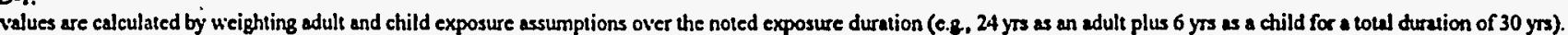

6. Carcinogenic intake factor calculated using weighted (combined) exposure assumptions; noncarcinogenic intake factors are calculated for both adult and child, with the maximum of these used for risk calculations. 
Table D.36

Savannah River Site

Burning/Rubble Pits (231-F, 231-1F)

Combined Adult / Child Resident (Future)

Inhalation of Volatile Soil Contaminants (0-f'bis)

Calculation of Volatilization Factor (VF)

\begin{tabular}{|c|c|c|c|c|c|c|c|c|c|c|c|}
\hline Chemical & $\mathrm{CAS}^{(2)}$ No. & Classs $^{(J)}$ & MolWt & $\mathrm{Koc}^{(1)}$ & $\mathbf{K} \mathbf{d}^{(4)}$ & $\mathrm{H}^{(4)}$ & $K_{i 1 s}^{(1)}$ & $\mathrm{Di}^{(1)}$ & $D \mathrm{ei}^{(t)}$ & alpha $a^{(t)}$ & $\begin{array}{l}\text { Volat. } \\
\text { Factor }^{(4)} \\
\left(\mathrm{m}^{3} / \mathrm{kg}\right)\end{array}$ \\
\hline \multicolumn{12}{|l|}{ Semivolatiles } \\
\hline 2-Methy'lnaphthalene & $91-57-6$ & S & $1.42 E+02$ & 8511.4 & $1.70 E+02$ & $2.90 \mathrm{E}-04$ & $6.98 E-05$ & $6.43 E-02$ & 4.53E-02 & $6.43 \mathrm{E}-07$ & $1.82 E+05$ \\
\hline Accnaphthyllene & $208-96-8$ & $\mathbf{S}$ & $1.52 E+02$ & 4786.3 & $9.57 \mathrm{E}+01$ & $2.80 \mathrm{E}-04$ & $1.20 \mathrm{E}-04$ & $6.22 \mathrm{E}-02$ & $4.38 \mathrm{E}-02$ & $1.07 \mathrm{E}-06$ & $1.42 E+05$ \\
\hline Phenanthrene & $85-01-8$ & $\mathbf{S}$ & $1.78 E+02$ & 38904.5 & $7.78 \mathrm{E}+02$ & $2.56 \mathrm{E}-05$ & $1.35 \mathrm{E}-06$ & $5.74 \mathrm{E}-02$ & $4.05 \mathrm{E}-02$ & $1.11 \mathrm{E}-08$ & $1.39 E+06$ \\
\hline
\end{tabular}

\section{Notes:}

1. USEPA, 1991: RAGS: Vol 1 - Human Health Evaluation Manual (Pan B, Development of Risk-Based Preliminary Remediation Goals)

2. Chemical Abstract Service.

3. Chemical class: $v$ - volatile; $s-$ semivolatile; $p$-pesticideipcb; $x$ - dioxin; and $m$-metal.

4. Calculation of organic contaminant volatilization factor:

CONSTANTS:

Length of side of contam'd. area (LS), $m=$ Wind speed in mixing zone $(\mathrm{V}), \mathrm{m} / \mathrm{sec}=$

Diffusion height (DH), $m=$ Area of contamination (Am), $\mathrm{m}^{2}=$ Area in square ceptimeters $(\mathrm{Acm}), \mathrm{cm}^{2}=$ True soil porosity (E), unitless = True soil density $\left(\mathrm{Ps}_{\mathrm{s}}\right) \mathrm{g}^{\prime} \mathrm{cm}^{3}=$ Exposure interval (Tyr), irs $=$ Exposure interval (Tsec), sec = Organic carbon content (OC), unitless = INPUT VARIABLES:

Organic carton partition coef. (Koc), $\mathrm{cm}^{3} \mathrm{~kg}=$ Heno's Laiv constant $(\mathrm{H}), \mathrm{atm}-\mathrm{m}^{3} / \mathrm{mol}=$ Gas diffusivity (Di), $\mathrm{cm}^{2} / \mathrm{sec}=$

INTERMEDIATE STEPS

Soil-water partition coefficient $(K d), \mathrm{cm}^{3} / \mathrm{kg}=$ Soil-air partition coefficient (Kas), g-soil/ $\mathrm{cm}^{3}$-air = Effective diffusisity (Dei), $\mathrm{cm}^{2} / \mathrm{sec}=$ alpha (alpha), $\mathrm{cm}^{2} / \mathrm{sec}=$

OUTPUT:

Volatilization Factor (VF), $\mathrm{m}^{3} / \mathrm{kg}=$

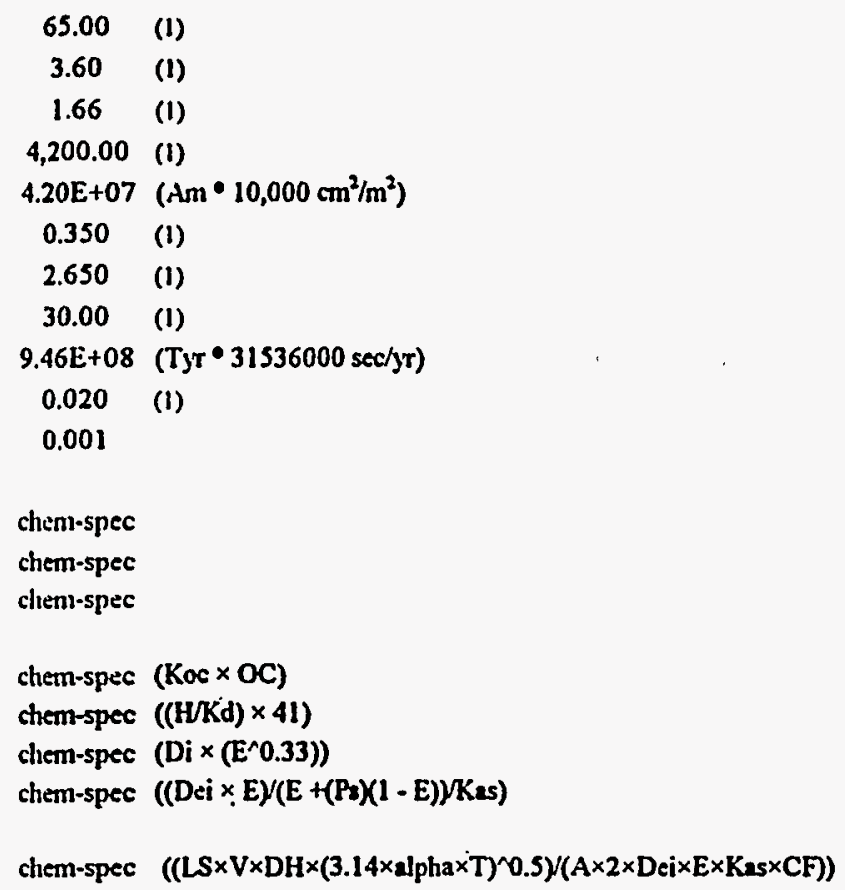


Table D.36

Savannah River Site

Burning/Rubble Pits (231-F, 231-1F)

Combined Adult / Child Resident (Future)

Inhalation of Volatile Soil Contaminants (0-4'bls)

Carcinogenic and Noncarcinogenic Risk

\begin{tabular}{|c|c|c|c|c|c|c|c|c|c|}
\hline \multirow[b]{2}{*}{ Chemical } & \multicolumn{2}{|c|}{$\underline{E P C o n c}^{(2)}$} & \multirow[b]{2}{*}{$\begin{array}{l}\operatorname{IUR}^{(4)} \\
\left(\mathrm{m}^{3} / \mu g\right) \\
\end{array}$} & \multirow[b]{2}{*}{$\begin{array}{r}\operatorname{RfC}^{(5)} \\
\left(\mathrm{m}^{\prime} / \mathbf{m}^{2}\right)\end{array}$} & \multirow[b]{2}{*}{$\begin{array}{l}V F^{(9)} \\
\left(m^{3} / k_{g}\right)\end{array}$} & \multicolumn{2}{|c|}{ Cancer Risk $^{(7)}$} & \multicolumn{2}{|c|}{ Hazard Quotient } \\
\hline & $\begin{array}{r}\text { CAS }^{(\mathbf{l})} \\
\text { Number }\end{array}$ & $\begin{array}{c}\mathrm{RME}^{(3)} \\
(m g / \mathrm{kg}) \\
\end{array}$ & & & & RME & $\begin{array}{l}\% \text { of } \\
\text { Total } \\
\end{array}$ & RME & $\begin{array}{l}\% \text { of } \\
\text { Total } \\
\end{array}$ \\
\hline \multicolumn{10}{|l|}{ Semivolutiles } \\
\hline 2-Methylnaphthalene & $91-57-6$ & $7.69 \mathrm{E}-(12$ & - & - & $1.42 \mathrm{E}+05$ & NA & - & NA & - \\
\hline Acenaphthylene & $208-96-8$ & $8.61 \mathrm{E}-02$ & - & - & $1.39 \mathrm{E}+06$ & NA & - & NA & - \\
\hline \multirow[t]{3}{*}{ Phenantlirene } & $85-01-8$ & $6.87 \mathrm{E}-01$ & - & - & $0.00 \mathrm{E}+00$ & NA & - & NA & - \\
\hline & & & & & & \multicolumn{2}{|c|}{$\begin{array}{c}\text { Cancer Risk } \\
\text { RME }\end{array}$} & \multicolumn{2}{|c|}{$\begin{array}{c}\text { Hazard Index } \\
\text { RME }\end{array}$} \\
\hline & & & & \multicolumn{2}{|c|}{ PATHWAY SUMS: } & \multicolumn{2}{|c|}{-} & \multicolumn{2}{|c|}{-} \\
\hline
\end{tabular}

1. Chemical Abstract Service.

2. Exposure point concentration.

3. Keasonable maximum exposure.

4. Inhalation unit risk.

5. Reference concentration.

6. Vulatilization factor (chemical-specific)

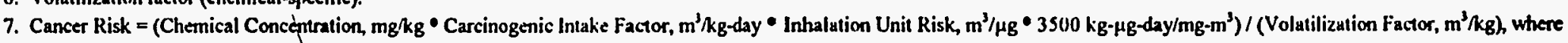
RMIE carcinogenic intake factor $9.39 \mathrm{E}-02$ and conversion from IUIR to inhalation slope factor $=3500 \mathrm{~kg}-\mu \mathrm{g}-\mathrm{day} / \mathrm{mg}^{-\mathrm{m}^{3}}$

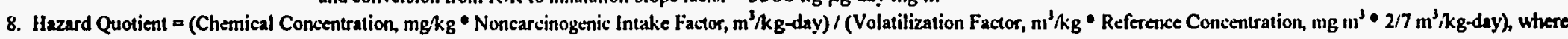
RME nontarcinogenic intake fa $1.15 \mathrm{E}+00$

and conversion from $R F C$ to iublalation reference dose $=2.7 \mathrm{~m}^{3} / \mathrm{k} \cdot \mathrm{g}$-dav 
Table D.37

Savannah River

Burning/Rublle Pits (231-F, 231-1F)

Combined Adult / Child Resident (Future)

Inlaalation of Soil Particulates ( $0-4$ 'bls)

Carcinogenic and Noncarcinogenic Risk

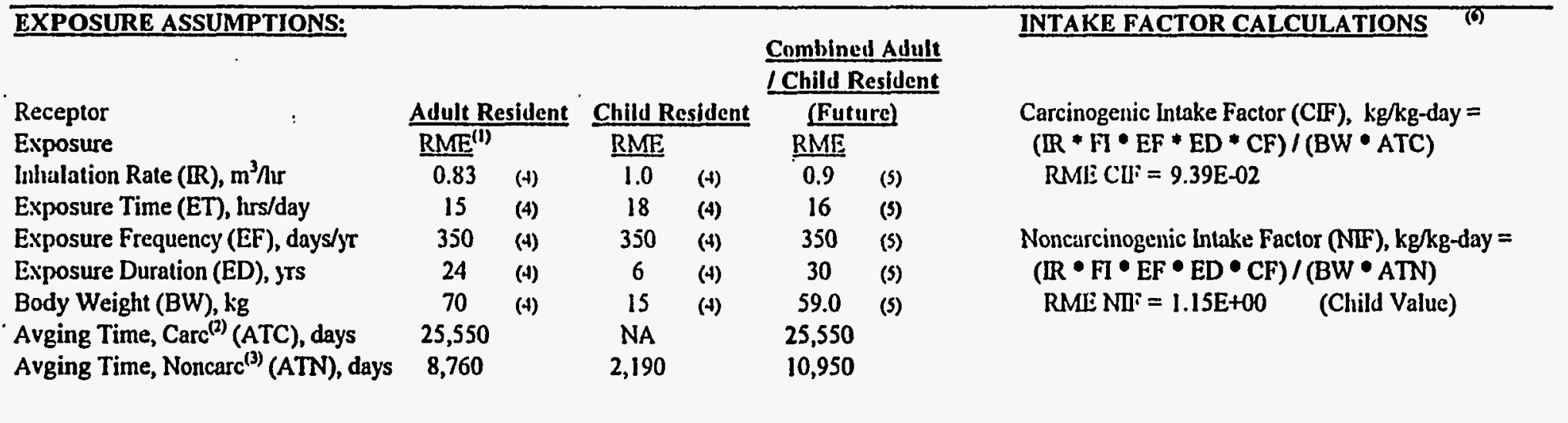

Noler:

1. Reasonable maximum exposure.

2. Averaging time, carcinogen;: calculated as 70 years (average lifetime) times 365 dass per year (not applicable to child resident).

3. Averaging time, noncarcinogen; calculated es exposure duration (in years) times 365 days per yeer

4. See Table D-1.

5. Combined values are calculated by weighting adult and child exposure assumptions over the noted exposure duration (e.g. 24 jrs as an adult plus 6 jts as a ctild for a toul duration of 30 yrs).

6. Carcinogenic intake factor calculaled wing, wieiphted (combined) exposure assumptions; noncercinogenic intuke factors ere calculated for both adult and child, with the maximum of these used for risk calculations 
Table D.37

Savannah River

Burning/Rubble Pits (231-F, 231-1F)

Combined Adult / Child Resident (Future)

Inhalation of Soil Particulates (0-4'bls)

Carcinogenic and Noncarcinogenic Risk

\begin{tabular}{llll}
\hline CALCULATION OF SOIL PARTICULATE RESUSPENSION FACTOR: & & \\
CONSTANTS: & & & \\
Length of side of contaminated area $(\mathrm{LS})=$ & 65.00 & meters & $(1)$ \\
Wind speed in mixing zone $(\mathrm{V})=$ & 3.60 & meters/sec & $(1)$ \\
Diffusion heiglt $(\mathrm{DH})=$ & 1.66 & meters & $(1)$ \\
Area of contumination $(\mathrm{A})=$ & $4,200.00$ & meters $^{2}$ & $(1)$ \\
Respirable fraction $(\mathrm{RF})=$ & 0.003 & $\mathrm{~g} / \mathrm{meters}^{2}$-hr & $(1)$ \\
Fraction of vegetative cover $(\mathrm{G})=$ & 0.90 & unitless & $(1)$ \\
Mean annual wind speed $(\mathrm{Um})=$ & 3.60 & meters/sec & $(1)$ \\
Equivalent tlireshold value of wind speed at $10 \mathrm{~m}(\mathrm{Ut})=$ & 5.04 & meters/sec & $(1)$ \\
Fraction dependent on Um/Ut $(\mathrm{Fx})=$ & 1.2700 & unitless & $(1)$ \\
Conversion factor $(\mathrm{CFa})=$ & $3,600.00$ & $\mathrm{sec} / \mathrm{hr}$ & \\
Conversion factor $(\mathrm{CFb})=$ & $1,000.00$ & $\mathrm{~g} / \mathrm{kg}$ &
\end{tabular}

\section{Noter;}

1. USEPA, 1991: RAGS: Vol 1 - Human Health Evaluation Mrenual (Pan B. Development of Risk-Based Preliminary Remediation Goals)

\section{INTERMEDIATE STEPS:}

Particulate emission factor $(\mathrm{PEF})=2.18 \mathrm{E}+09 \mathrm{~meters}^{3} / \mathrm{kg}$ $(\mathrm{L}, \mathrm{S} \times \mathrm{V} \times 1) \mathrm{I} \times \mathrm{CFa} \times \mathrm{CFb}) /\left(\mathrm{A} \times \mathrm{RF} \times(1-\mathrm{G}) \times\left((\mathrm{Um} / \mathrm{Ut})^{\wedge} 3\right) \times(\mathrm{Fx})\right)$ 
Table D.37

Savannah River

Burning/Rubble Pits (231-F, 231-1F)

Combined Adult / Child Resident (Future)

Inhalation of Soil Particulates (0-f'bls)

Carcinogenic and Noncarcinogenic Risk

\begin{tabular}{lll}
\hline & $\operatorname{CAS}^{(1)}$ & $\frac{\operatorname{EPConc}^{(2)}}{\operatorname{RME}^{(3)}}$ \\
Chemical & Number & $(1 m g / k g)$ \\
\hline
\end{tabular}

\begin{tabular}{rr}
$I U R^{(4)}$ & $\operatorname{RfC}^{(5)}$ \\
$\left(1 \mathrm{II}^{3} / \mu g\right)$ & $\left(\mathrm{nIg} / \mathrm{m}^{3}\right)$ \\
\hline
\end{tabular}

\begin{tabular}{|c|c|}
\hline Cancer Rish ${ }^{(6)}$ & Inzard Quotient $^{(t)}$ \\
\hline 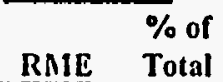 & $\begin{array}{ll} & \% \text { of } \\
\text { RME } & \text { Total }\end{array}$ \\
\hline
\end{tabular}

Semivolatiles

2-Methylnaphthalene

$91-57-6$

208-96-8

7.69E-02

8.61E-02

$56-55-3$

$9.26 \mathrm{E}-01$

Benzo(a)anthracen

Benzo(a)pyrene

Benzo(b)nuoranthene

50-32-8

$6.49 \mathrm{E}-01$

205-99-2

$1.01 \mathrm{E}+00$

191-24-2 6.15E-01

53-70-3 2.17E-0I

Dibenz(a,h)anthracene

$85-01-8$

$6.87 \mathrm{E}-01$

$\begin{array}{ll}- & - \\ - & - \\ - & - \\ - & - \\ - & - \\ - & -\end{array}$

NA

\section{Dioxins/furans}

Heptachlorodibenzo-p-dioxin isomer

Heptachlorodibenzo-p-furan isomer

Hexaclilorodibenzo-p-furan isomer

Oclachlorodibenzo-p-dioxin isomer

Octaclilorodibenzo-p-furan isomer

PCBs

PCB 1254

37871-00-4

38998-75-3

4.11E-03

3.30E-01

55684-94-1

2.58E-04

3.30E-01

3268-87-9

$1.00 \mathrm{E}-04$

$3.30 \mathrm{E}+00$

39001-02-0

9.26E-03

$3.30 \mathrm{E}-02$

3.30E-02 -

1 1 097-69-1

1.78E-01

319-86-8

delta-Benzene hexachloride

Endrin ketone

53494-70-5

1.84E-03

3.20E-03

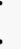

$-$

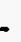

$\begin{array}{llll}\text { NA } & - & \text { NA } & - \\ \text { NA } & - & \text { NA } & - \\ \text { NA } & - & \text { NA } & - \\ \text { NA } & - & \text { NA } & - \\ \text { NA } & - & \text { NA } & - \\ \text { NA } & - & \text { NA } & - \\ \text { NA } & - & \text { NA } & - \\ \text { NA } & - & \text { NA } & -\end{array}$

$2.0 \mathrm{E}-10<1 \%$

1.3E-11 $<1 \%$

$5.0 \mathrm{E}-11<1 \%$

$4.6 \mathrm{E}-11<1 \%$

$1.7 \mathrm{E}-12<1 \%$

NA -

NA

NA

NA

NA

NA - NA

NA - $\quad$ NA

NA - $\quad$ NA 
Table D.37

Savannah River

Burning/Rubble Pits (231-F, 231-1F)

Combined Adult / Child Resident (Future)

Inhalation of Soil Particulates (0-4'bls)

Carcinogenic and Noncarcinogenic Risk

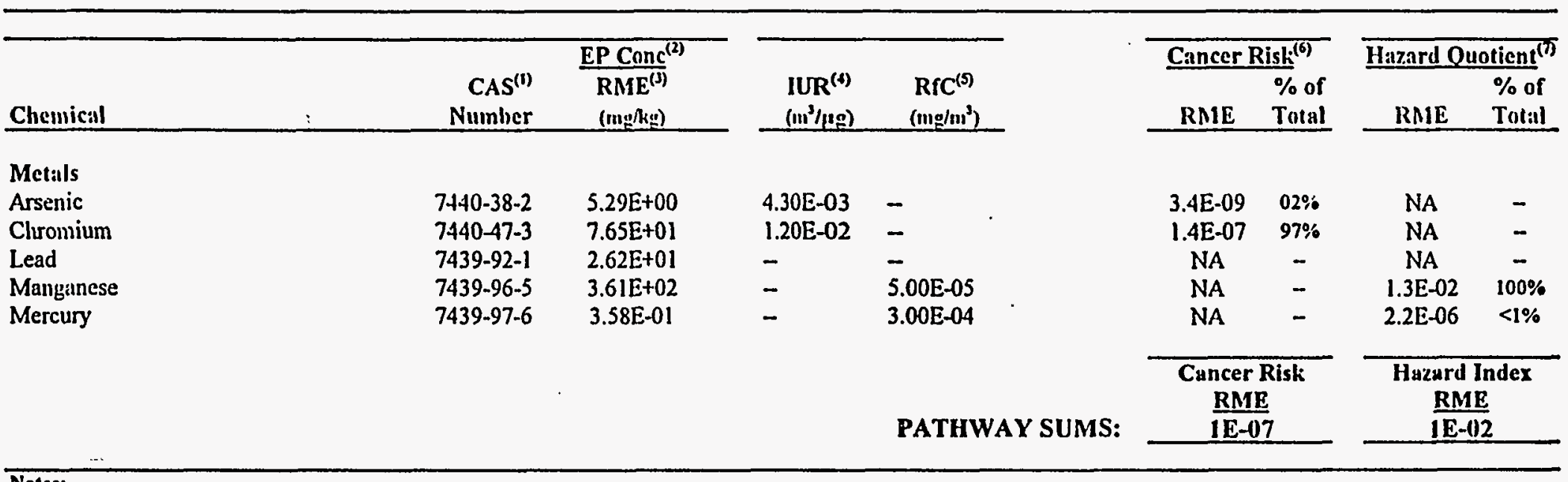

1. Chemical Abstract Service.

2. Esposure point concentration.

3. Reasonable maximum exposure.

4. Inlaalation unit risk.

5. Reference concentration.

6. Cancer Risk = (Chemical Concentration, $\mathrm{mg} / \mathrm{kg}$ * Carcinogenic Intake Factor, $\mathrm{m}^{3} / \mathrm{kg}$-day * Inhalation Unit Risk, $\mathrm{m}^{3} / \mu \mathrm{g} * 3500 \mathrm{~kg}-\mu \mathrm{g}$-day/mg-m $) /\left(\right.$ Particulate Emission Factor, $\left.\mathrm{m}^{3} / \mathrm{kg}\right)$

RME carcinogenic intake factor $\approx \quad 9.39 \mathrm{E}-02$

Particulate emission factor $=\quad 2.18 \mathrm{E}+09$

and conversion from IUR to inhalation slope factor $=3500 \mathrm{~kg}-\mu \mathrm{g}-\mathrm{day} / \mathrm{mg}_{\mathrm{g}}-\mathrm{m}^{3}$

(Note: Carcinogenic intake factor calculated using weighted (combined) exposure assumptions.)

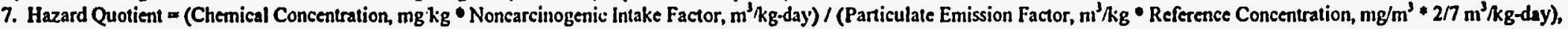

RAIE noncarcinogenic intake factor $=1.15 E+00$

Particulate enission factor $=\quad 2.18 \mathrm{E}+09$

and conversion from $R R C$ to inhalation reference dose $=217 \mathrm{~m}^{3} \mathrm{~kg}$-day

(Note: Noncarcinogenic intake factors are calculated for hoth adult and child. with the maximum of these used for risk calculations.) 
Table D.38

Savannah River Site

Rubble Pit (231-2F)

Combined Adult/Child Resident (Future)

Ingestion of Soil (0-2'bls)

Carcinogenic and Noncarcinogenic Risk

\begin{tabular}{|c|c|c|c|c|c|c|c|}
\hline \multirow[t]{2}{*}{ EXPOSURE ASSUMPTIONS: } & & & & & & & \multirow[t]{2}{*}{ INTAKE FACTOR CALCULATIONS .") } \\
\hline & & & & & \multicolumn{2}{|c|}{ Combined } & \\
\hline Receptor & \multicolumn{2}{|c|}{ Adult Resident } & \multicolumn{2}{|c|}{ Child Resident } & \multicolumn{2}{|c|}{ Adult/Child } & \multirow{2}{*}{$\begin{array}{l}\text { Carcinogenic Intake Factor }(\mathrm{CIF}), \mathrm{kg} / \mathrm{kg} \text {-day }= \\
(\mathbb{R} * \mathrm{H} \cdot \mathrm{EF} * \mathrm{ED} * \mathrm{CF}) /(\mathrm{BW} * \mathrm{ATC})\end{array}$} \\
\hline Exposure & \multicolumn{2}{|c|}{$\mathrm{RME}^{(1)}$} & RME & & RME & & \\
\hline Intake Rate (IR), mg/day & 100 & (t) & 200 & (4) & $\overline{120.0}$ & (5) & $\mathrm{RMI}: \mathrm{CIF}=8.36 \mathrm{E}-07$ \\
\hline Fraction Ingested (FI), unitless & 1 & (4) & 1 & (4) & 1.0 & (5) & \\
\hline Exposure Frequency (EF), days/yr & 350 & (4) & 350 & (4) & 350 & (5) & Noncarcinogenic Intake Factor (NIF), $\mathrm{kg} / \mathrm{kg}$-day = \\
\hline Exposure Duration (ED), yrs & 24 & (4) & 6 & (4) & 30 & (5) & $(\mathrm{IR} \bullet \mathrm{FI} \bullet \mathrm{EF} \bullet \mathrm{ED} \bullet \mathrm{CF}) /(\mathrm{BW} \bullet \mathrm{ATN})$ \\
\hline Body Weight (BW), kg & 70 & (4) & 15 & (4) & 59.0 & (5) & RMI: NIP $=1.28 \mathrm{E}-05 \quad$ (Child Value) \\
\hline Avging Time, Carc $^{(2)}$ (ATC), days & 25,550 & & NA & & 25,550 & & \\
\hline Avging Time, Noncarc ${ }^{(3)}$ (ATN), days & 8,760 & & 2,190 & & 10,950 & & \\
\hline Conversion Factor (CF), $\mathrm{kg} / \mathrm{mg}$ & $1.00 \mathrm{E}-1) 6$ & & $1.0(0 \mid ⿰-06$ & & $1 .(4) \mathrm{E}-106$ & & \\
\hline
\end{tabular}

Noter:

1. Reasonable maximum exposure.

2. Averaging tume, carcinogen,; calculuted as 70 years (average lifetıme) times 365 days per year (not applicable to child resident)

3. Averaging time, noncarcinogen; calculated es exposure duration (in years) tumes 365 days per year

5. See Table D-1.
5. Combined values are calculated by weighting adult and child exposure assumptions over the noted exposure duration (e. 8.24 grs as an adult plus 6 yrs as a child for a tousi duration of 30 yrs).

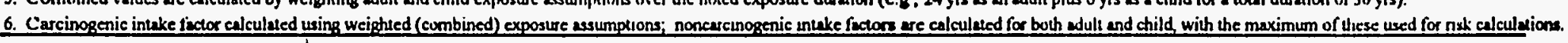


Table D.38

Savannah River Site

Rubble Pit (231-2F)

Combined Adult/Child Resident (Future)

Ingestion of Soil (0-2'bls)

Carcinogenic and Noncarcinogenic Risk

\begin{tabular}{|c|c|c|c|c|c|c|c|c|}
\hline Chemical & CAS $^{(1)}$ No. & $\begin{array}{l}\text { EP Conc }{ }^{(1)} \\
\operatorname{RME}^{(j)} \\
(\mathrm{mg} / \mathrm{kg} g) \\
\end{array}$ & $\begin{array}{c}\operatorname{OSF}^{(t)} \\
(\mathrm{kg}-\mathrm{d} / \mathrm{mg})\end{array}$ & $\begin{array}{c}\text { ORID }^{(9)} \\
(\mathrm{mg} / \mathrm{kg}-\mathrm{d}) \\
\end{array}$ & \multicolumn{2}{|c|}{\begin{tabular}{c}
\multicolumn{2}{c}{ Cinncer Risk } \\
\\
RME of \\
RME Total \\
\end{tabular}} & \multicolumn{2}{|c|}{$\begin{array}{r}\text { Hazard Quollent } \\
\begin{array}{r}\% \text { of } \\
\text { RME Total }\end{array} \\
\end{array}$} \\
\hline $\begin{array}{l}\text { PCBs } \\
\text { PCB } 1254 \\
\text { PCB } 1260\end{array}$ & $\begin{array}{l}11097-69-1 \\
11096-82-5\end{array}$ & $\begin{array}{c}2.87 \mathrm{E}+00 \\
9.01 \mathrm{E}-02\end{array}$ & $\begin{array}{l}7.70 \mathrm{E}+00 \\
7.70 \mathrm{E}+00\end{array}$ & $\begin{array}{l}2.00 \mathrm{E}-05 \\
2.00 \mathrm{E}-05\end{array}$ & $\begin{array}{l}1.8 \mathrm{E}-05 \\
5.8 \mathrm{E}-07\end{array}$ & $\begin{array}{l}93 \% \\
03 \%\end{array}$ & $\begin{array}{l}1.8 \mathrm{E}+00 \\
5.8 \mathrm{E}-02\end{array}$ & $\begin{array}{l}78 \% \\
02 \%\end{array}$ \\
\hline 1 & & & & PATHWAY SUMS: & $\begin{array}{r}\text { Cancer } \\
\mathbf{R M} \\
2 \mathrm{E}- \\
\end{array}$ & & $\begin{array}{r}\text { Hazard } \\
\mathbf{R M} \\
2 \mathbf{E}+ \\
\end{array}$ & $\frac{\text { index }}{20}$ \\
\hline $\begin{array}{l}\text { Notes: } \\
\text { 1. Chemical Abstracts Service. } \\
\text { 2. Exposure point concentration. } \\
\text { 3. Reasonable maximum exposure. } \\
\text { 4. Slope factor. } \\
\text { 5. Refertnce dose. } \\
\text { 6. Cancer Risk = (Chemical Concentration, } \\
\text { (Note: Carcinogenic intake factor calculat } \\
\text { 7. Hazard Quotient } \approx \text { (Chemical Concentra } \\
\text { (Note: Noncarcinogenic intake factors are }\end{array}$ & $\begin{array}{l}\text { mg/kg * Carcir } \\
\text { RME carcino } \\
\text { ed using weight } \\
\text { tion, migkg }{ }^{\circ} \\
\text { RMIE noncarc } \\
\text { calculated for b }\end{array}$ & $\begin{array}{l}\text { ogenic Intake } \\
\text { genic intake fac } \\
\text { ed (combined) } \\
\text { oncarcinogenic } \\
\text { inogenic intake }\end{array}$ & $\begin{array}{l}\text { tor, kg/kg-das } \\
8.36 \mathrm{E}-07 \\
\text { tasure assump } \\
\text { take Factor, } \mathrm{k} \\
\text { 1.28E-05 } \\
\text { d, with the ma }\end{array}$ & $\begin{array}{l}\text { " Slope Factor, kg-day/mg) } \\
\text { tions.) } \\
\text { (g/kg-day) / (Reference Dose, } \\
\text { (Child Value) } \\
\text { aximum of these used for risk }\end{array}$ & re & & & \\
\hline
\end{tabular}


Table D.39

Savannah River Site

Rubble Pit (231-2F)

Combined Adult / Child Resident (Future)

Dermal Exposure to Soil (0-2'bls)

Carcinogenic and Noncarcinogenic Risls

\begin{tabular}{|c|c|c|c|c|c|c|}
\hline \multirow[t]{3}{*}{ EXPOSURE ASSUMPTIONS: } & & & & & \multirow[b]{2}{*}{ Combined Adult } & \multirow[t]{3}{*}{ INTAKE FACTOR CALCULATIONS (\%) } \\
\hline & & & & & & \\
\hline & & & & & IChild Resident & \\
\hline Receptor & \multicolumn{2}{|c|}{ Adult Resident } & \multicolumn{2}{|c|}{ Child Resident } & (Fiture) & Carcinogenic Intake Factor (CFF), kg/kg-day = \\
\hline Exposure & $\mathrm{RME}^{(1)}$ & & RME & & RME & $(\mathrm{SA} \bullet \mathrm{SK} * \mathrm{EF} \bullet \mathrm{ED} \bullet \mathrm{CF}) /(\mathrm{BW} \bullet \mathrm{ATC})$ \\
\hline Skin Surface Area $(\mathrm{SA}), \mathrm{cm}^{2} / \mathrm{event}$ & 5,000 & (4) & 1,800 & (4) & 4,360 & RME: CIF = 6.07E-06 \\
\hline Soil-to-Skin Adherance (SK), $\mathrm{mg} / \mathrm{cm}^{2}$ & 0.2 & (4) & 0.2 & (4) & 0.2 & \\
\hline Exposure Frequency (EF), events/yr & 350 & (4) & 350 & (4) & 350.0 & Noncurcinogenic Intake Factor (NIF), kg/kg-day = \\
\hline Exposure Duration (ED), jts & 24 & (4) & 6 & (4) & 30 & $(\mathrm{SA} \bullet \mathrm{SK} \bullet \mathrm{EF} \bullet \mathrm{ED} \bullet \mathrm{CF}) /(\mathrm{BW} \bullet \mathrm{ATN})$ \\
\hline Body Weight (BW), kg & 70 & (4) & 15 & (4) & 59.0 & RME NIF $=2.30 \mathrm{E}-05 \quad$ (Child Value) \\
\hline Avging Time, $\operatorname{Carc}^{(2)}($ ATC), days & 25,550 & & NA & & 25,550 & \\
\hline Avging Time, Noncarc ${ }^{(3)}$ (ATN), days & 8,760 & & 2,190 & & 10,950 & \\
\hline Conversion Factor (CF), kg/mg & $1.00 \mathrm{E}-06$ & & $1.00 \mathrm{E}-0 \mathrm{C}$ & & $1.00 \mathrm{E}-06$ & \\
\hline
\end{tabular}

1. Ressonable meximum exposure.

2. Averaing time, carcinogen; calculated as 70 years (average lifetime) times 365 days per year (not applicable to child resident).

3. Averaging time, noncarcinogen,; calculated es exposure duration (in years) times 365 day per year

4. See Table D-1.

5. Combined values are calculated by weighting adult and child exposure assumplions over the noted exposure duratton (e a. 24 ses as an adult plus 6 yrs as a child for a total duration of 30 yra).

6. Carcinogenic intake factor.caleujated using Weighted (combined) exposure sssumptions; noncarcinogenic intule factors are calculated for both aduls and child, with the maximum of these used for risk caleulations. 
Table D.39

Savannah River Site

Rubble Pit (231-2F)

Combined Adult / Child Resident (Future)

Dermal Exposure to Soil (0-2'bls)

Carcinogenic and Noncarcinogenic Risk

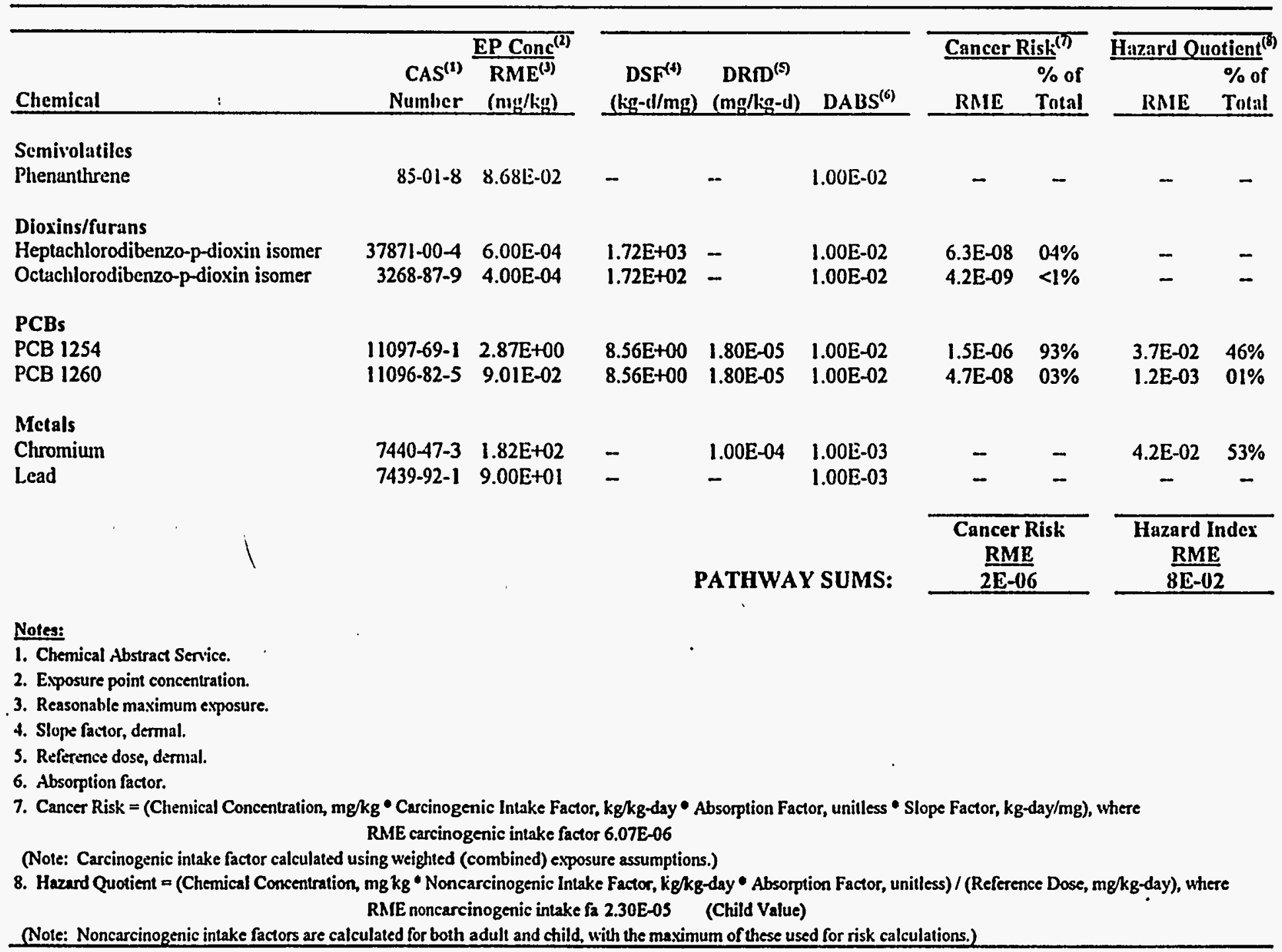


Table D.40

Savannah River Site

Rubble Pit (231-2F)

Combined Adult / Child Resident (Future)

Inhalation of Volatile Soil Contuminants (0-2'bls)

Carcinogenic and Noncarcinogenic Risk

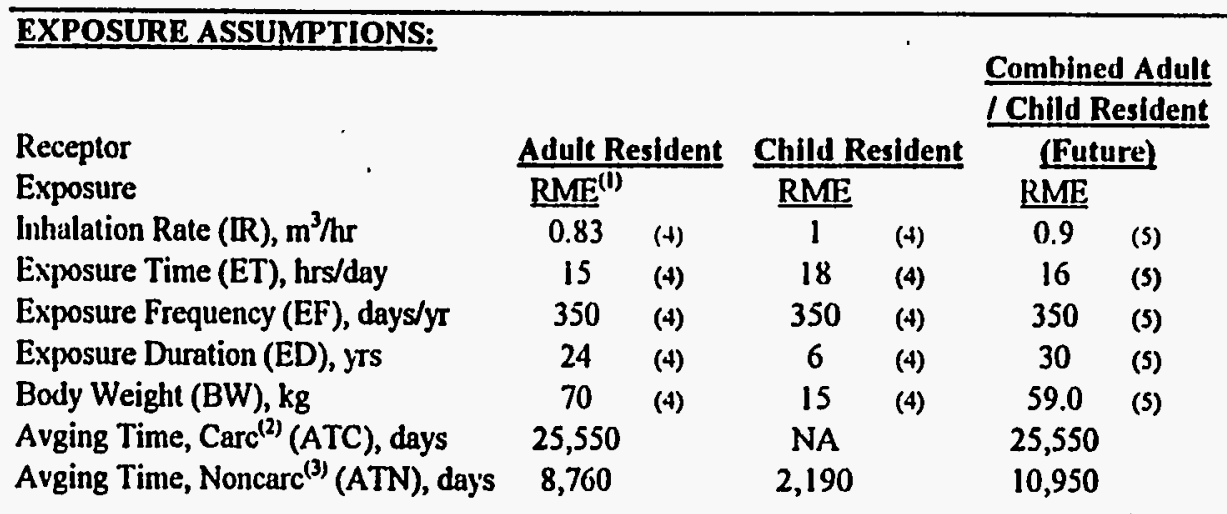

INTAKE FACTOR CALCULATIONS

Carcinogenic Intake Factor (CIF), kg/kg-day =

$(\mathrm{IR} \bullet \mathrm{ET} \bullet \mathrm{EF} \bullet \mathrm{ED} \bullet \mathrm{CF}) /(\mathrm{BW} \bullet \mathrm{ATC})$

RMI: CIF $=9.39 \mathrm{E}-02$

Noncarcinogenic Intake Factor (NIF), kg/kg-day =

$(\mathrm{IR} * \mathrm{ET} * \mathrm{EF} * \mathrm{ED} * \mathrm{CF}) /(\mathrm{BW} * \mathrm{ATN})$

RME NIF $=1.15 E+00 \quad$ (Child Value)

\section{Noten:}

1. Reasonable maximum exposure.

2. Averaging time, carcinogen; calculaled as 70 years (average lifetime) times 365 days per year (not applicable to child resident).

3. Averaging time, noncarcinogen; calculated es exposure duration (in years) times 365 days per year

4. See Table D-1.

5. Combined values are calculated by weighting edult and child exposure assumptions over the noted exposure duration (c.q. 24 yrs as an adult plus 6 yrs as a child for a total duration of 30 yrs).

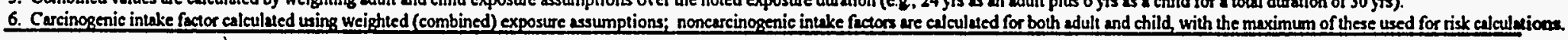


Table D.40

Savannah River Site

Rubble Pit (231-2F)

Combined Adult / Child Resident (Future)

Inhalation of Volatile Soil Contaminants (0-2'bls)

Calculation of Volatilization Factor (VF) ${ }^{(1)}$

\begin{tabular}{|c|c|c|c|c|c|c|c|c|c|c|c|}
\hline Chiemical & $\mathrm{CAS}^{(2)}$ No. & Class $^{(3)}$ & MolWt & $\mathrm{Koc}^{(1)}$ & $\mathrm{Kd}^{(0)}$ & $H^{(4)}$ & $\mathrm{Kas}^{(4)}$ & $D_{i}^{(4)}$ & Dei( ${ }^{(4)}$ & alpha $a^{(4)}$ & $\begin{array}{l}\text { Volat. } \\
\left.\text { Factor }{ }^{(0)}\right) \\
\left(\mathrm{m}^{3} / \mathrm{kg}\right)\end{array}$ \\
\hline Semivolatiles & 000 & 0 & & & & 256500 & 250 11 & $57150 ?$ & $\triangle 05 E_{\Omega} ?$ & S UE_ 08 & $303 \mathrm{~F}+\mathrm{O}$ \\
\hline
\end{tabular}

\section{Notes:}

1. USEPA, 1991: RAGS: Vol 1 - Human Health Evaluation Manual (Part B, Development of Risk-Based Preliminary Remediation Goals)

2. Chemical Abru act Service.

3. Chemical class: $v$-volatile; $s$-semivolatile; $p$-pesticide'pcb; $x$-dioxin; and $m$--metal.

4. Calculation of organic contaminant volatilization factor: CONSTANTS:

$$
\begin{array}{r}
\text { Length of side of contanid. area (LS), } \mathrm{m}= \\
\text { Wind speed in mixing zone (V), } \mathrm{m} / \mathrm{sec}= \\
\text { Diflusion height (DII), } \mathrm{m}= \\
\text { Area of contamination (Am), } \mathrm{m}^{2}= \\
\text { Area in square centimeters (Acm), } \mathrm{cm}^{2}= \\
\text { True soil porosity (E), unilless = } \\
\text { True soil density (Ps). g/cm }= \\
\text { Exposure interval (TyT), yts = } \\
\text { Exposure interval (Tsec), sec = }
\end{array}
$$
Organic carbon content $(O C)$, unitless = Conversion factor (CF), $\mathrm{kg}^{\prime} \mathrm{g}=$

INIUT VARIAIBLES:

Organic carbon partition coef. (Koc), $\mathrm{cm}^{3} \cdot \mathrm{hg}=$ Henry's Law constant $(\mathrm{H})$, atm $-\mathrm{m}^{3} / \mathrm{mol}=$ Gas diffusivity (Di), $\mathrm{cm}^{2} / \mathrm{sec}=$

\section{INTERMEDIATE STEPS:}

Soil-water partition coefficient (Kd), $\mathrm{cm}^{3} / \mathrm{kg}=$ Soil-air partition coefficient (Kas), g-soil//cm'-air = Effective diffusivity (Dej), $\mathrm{cm}^{2} / \mathrm{sec}=$ alpha (alpha), $\mathrm{cm}^{2} / \mathrm{sec}=$ OUTPUT:

Volatilization Factor (VF), $\mathrm{m}^{3} / \mathrm{kg}=$

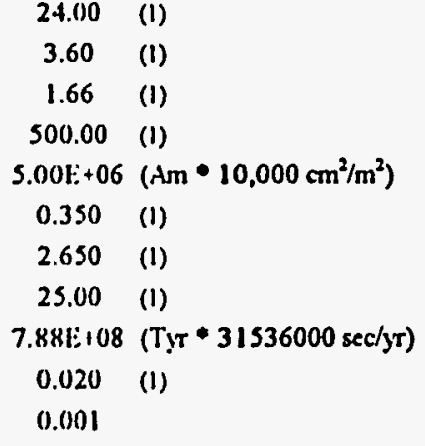


Table D.40

Savannah River Site

Rubble Pit (231-2F)

Combined Adult / Child Resident (Future)

Inhalation of Volatile Soil Contaminants (0-2'bls)

Carcinogenic and Noncarcinogenic Risk

\begin{tabular}{|c|c|c|c|c|c|c|c|c|c|c|}
\hline \multirow[b]{2}{*}{ Chemical } & \multirow[b]{2}{*}{$:$} & \multicolumn{2}{|c|}{ EP Conc $^{(2)}$} & \multirow[b]{2}{*}{$\begin{array}{l}1 \mathrm{IUR}^{(4)} \\
\left(1 \mathrm{II}^{3} / \mu \mathrm{g}\right)\end{array}$} & \multirow[b]{2}{*}{$\begin{array}{r}\operatorname{RfC}^{(5)} \\
\left(m g / m^{3}\right)\end{array}$} & \multirow[b]{2}{*}{$\begin{array}{l}V F^{(0)} \\
\left(m^{3} / k g\right)\end{array}$} & \multicolumn{2}{|c|}{ Cancer Riskib } & \multicolumn{2}{|c|}{ Hazard Quotient ${ }^{(8)}$} \\
\hline & & $\begin{array}{r}\text { CAS }^{(1)} \\
\text { Number } \\
\end{array}$ & $\begin{array}{l}\mathrm{RME}^{(3)} \\
(\mathrm{mg} / \mathrm{kg})\end{array}$ & & & & RME & $\begin{array}{l}\% \text { of } \\
\text { Total } \\
\end{array}$ & RME & $\begin{array}{l}\% \text { of } \\
\text { Total } \\
\end{array}$ \\
\hline \multirow[t]{2}{*}{$\begin{array}{l}\text { Semivolatiles } \\
\text { Phenanthrene }\end{array}$} & & $85-01-8$ & $6.87 \mathrm{E}-01$ & -- & - & $3.93 E+06$ & -- & - & - & - \\
\hline & & & & & PATHWA & SUMS: & $\begin{array}{r}\text { Cance } \\
\mathrm{RN} \\
\end{array}$ & Risk & $\begin{array}{r}\text { Hazar } \\
\mathbf{R} \\
\end{array}$ & index \\
\hline
\end{tabular}

Notes:

1. Chemical Abstract Service.

2. Exposure point concentration.

3. Reasonable maximum exposure.

4. Inhalation unit risk

5. Reference concentration.

6. Volatilization factor (chemical-specific).

7. Cancer Risk = (Chemical Concentration, $\mathrm{mg} / \mathrm{kg}$ * Carcinogenic Intake Factor, $\mathrm{m}^{3} / \mathrm{kg}$-day * Inhalation Unit Risk, $\mathrm{m}^{3} / \mu \mathrm{g} * 3500 \mathrm{~kg}-\mu \mathrm{g}$-day $\left./ \mathrm{mg}_{\mathrm{g}} \mathrm{m}^{3}\right) /\left(\right.$ Volatilization Factor, $\left.\mathrm{m}^{3} / \mathrm{kg}\right)$, where RME carcinugenic intake factor $9.39 \mathrm{E}-02$

and conversion from IUR to inhlalation slope factor $+3500 \mathrm{~kg}-\mu \mathrm{g} \cdot \mathrm{day} / \mathrm{mg}^{-}-\mathrm{m}^{3}$

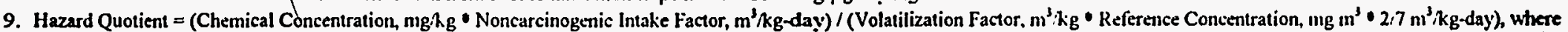
RMIE noncarcinogenic intake fa $1.15 \mathrm{E}+00$ and conversion from $R I C$ to inhalitition reference dose $=27 \mathrm{~m}^{3} / \mathrm{kg}$-dav 
Table D.41

Savannah River Site

Rubble Pit (231-2F)

Combined Adult / Child Resident (Future)

Inhalation of Soil Particulates (0-2'bls)

Carcinogenic and Noncarcinogenic Risk

\section{EXPOSURE ASSUMPTIONS:}

\section{Receptor}

\section{Exposure}

Inlalation Rute (IR), $\mathrm{m}^{\mathbf{3}} \mathrm{hr}$

Exposure Time (ET), hrs/day

Exposure Frequency (EF), days/yr

Exposure Duration (ED), yrs

Body Weight (BW), kg

Avging Time, $\operatorname{Carc}^{(2)}$ (ATC), days

Avging Time, Noncarc ${ }^{(3)}$ (ATN), days

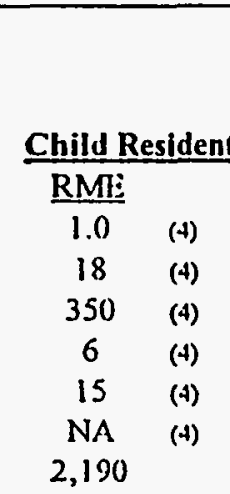

\begin{tabular}{|c|c|}
\hline \multicolumn{2}{|c|}{ Combined Adult } \\
\hline \multicolumn{2}{|c|}{ I Child Resident } \\
\hline \multicolumn{2}{|c|}{ (Future) } \\
\hline \multicolumn{2}{|c|}{$\mathrm{KME}$} \\
\hline 0.9 & (5) \\
\hline 16 & (5) \\
\hline 350 & (5) \\
\hline 30 & (S) \\
\hline 59.0 & (5) \\
\hline \multicolumn{2}{|l|}{25,550} \\
\hline 10,950 & \\
\hline
\end{tabular}

INTAKE FACTOR CALCULATIONS

Carcinogenic Intuke Factor (CIF), kg/kg-day = $(\mathrm{IR} * \mathrm{FI} * \mathrm{EF} \bullet \mathrm{ED}) /(\mathrm{BW} * \mathrm{ATC})$

RMI: (IF $=9.39 \mathrm{E}-() 2$

Noncarcinogenic Intuke Factor (NFF), $\mathrm{kg} / \mathrm{kg}$-day = $(\mathrm{IR} * \mathrm{FI} \bullet \mathrm{EF} * \mathrm{ED}) /(\mathrm{BW} \bullet \mathrm{ATN})$ RMI: NII" $=1.15 E+00 \quad$ (Child Value)

Niles:

1. Reasonabic maximum exposure.

2 Averaging time, carcinogen; calculated as 70 years (average lifetime) unres 365 days per year (not applicable to child resident)

3. Averaging time, noncarcinogen,; calculated as exposure duration (in years) times 365 days per year

4. See Table D-1.

5. Combined values are calculated by weighting adull and chld exposure assumptions over the noted exposure duration (eo 24 ym as an adule plus 6 grs as a child for a total duration of 30 yrs).

6. (arcinogenic intuke factor calculated using weighted (combined) exposure assumptrons; moncescinogenic intake factors ure calculated for both adult and child, with the maximum of tiese used for risk calculations. 
Table D.41

Savannah River Site

Rubble Pit (231-2F)

Combined Adult / Child Resident (Future)

Inhalation of Soil Particulates ((1)-2'b/s)

Carcinogenic and Noncarcinogenic Risk

\section{CALCULATION OF SOIL PARTICULATE RESUSPENSION FACTOR:}

\section{CONSTANTS:}

Length of side of contaminated area (LS) $=$

Wind speed in mixing zone $(\mathrm{V})=$

Dillusion heiglt (DII) $=$

Area of contumination $(A)=$

Respirable fraction $(\mathrm{RF})=$

Fraction of vigetative cover $(G)=$

Mean annual wind speed $(\mathrm{Um})=$

Equivalent threshold value of wind speed at $10 \mathrm{~m}(\mathrm{Ut})=$

Fraction dependent on $\mathrm{Um} / \mathrm{Ut}(\mathrm{Fx})=$

Conversion fuctor $(\mathrm{CF} a)=$

Conversion läctor $(\mathrm{Cl} b)=$

$\begin{array}{lll}24.00 & \text { meters } & (1) \\ 3.60 & \text { meters/sec } & (1) \\ 1.66 & \text { melers } & (1) \\ 500.00 & \text { meters }^{2} & (1) \\ 0.003 & \text { g/meters }^{2} \text {-hr } & (1) \\ 0.90 & \text { unitless } & (1) \\ 3.60 & \text { meters/sec } & (1) \\ 5.04 & \text { meters/sec } & (1) \\ 1.2700 & \text { unilless } & (1) \\ 3,600.00 & \text { sec/lur } & \\ 1,000.00 & \mathrm{~g} / \mathrm{kg} & \end{array}$

\section{INTERMEDIA TE STEPS:}

Particulate emission factor $(\mathrm{PEF})=6.76 \mathrm{E}+09$ meters $^{3} / \mathrm{kg}$ $($ I.S $\times V \times D H \times C l a \times C F b) /\left(A \times R F \times(I-G) \times\left((U m / U t)^{\wedge} 3\right) \times(F x)\right)$ 
Table D.41

Savannah River Site

Rubble Pit (231-2F)

Combined Adult / Child Resident (Future)

Inhalation of Soil Particulates (0-2'bls)

Carcinogenic and Noncarcinogenic Risk

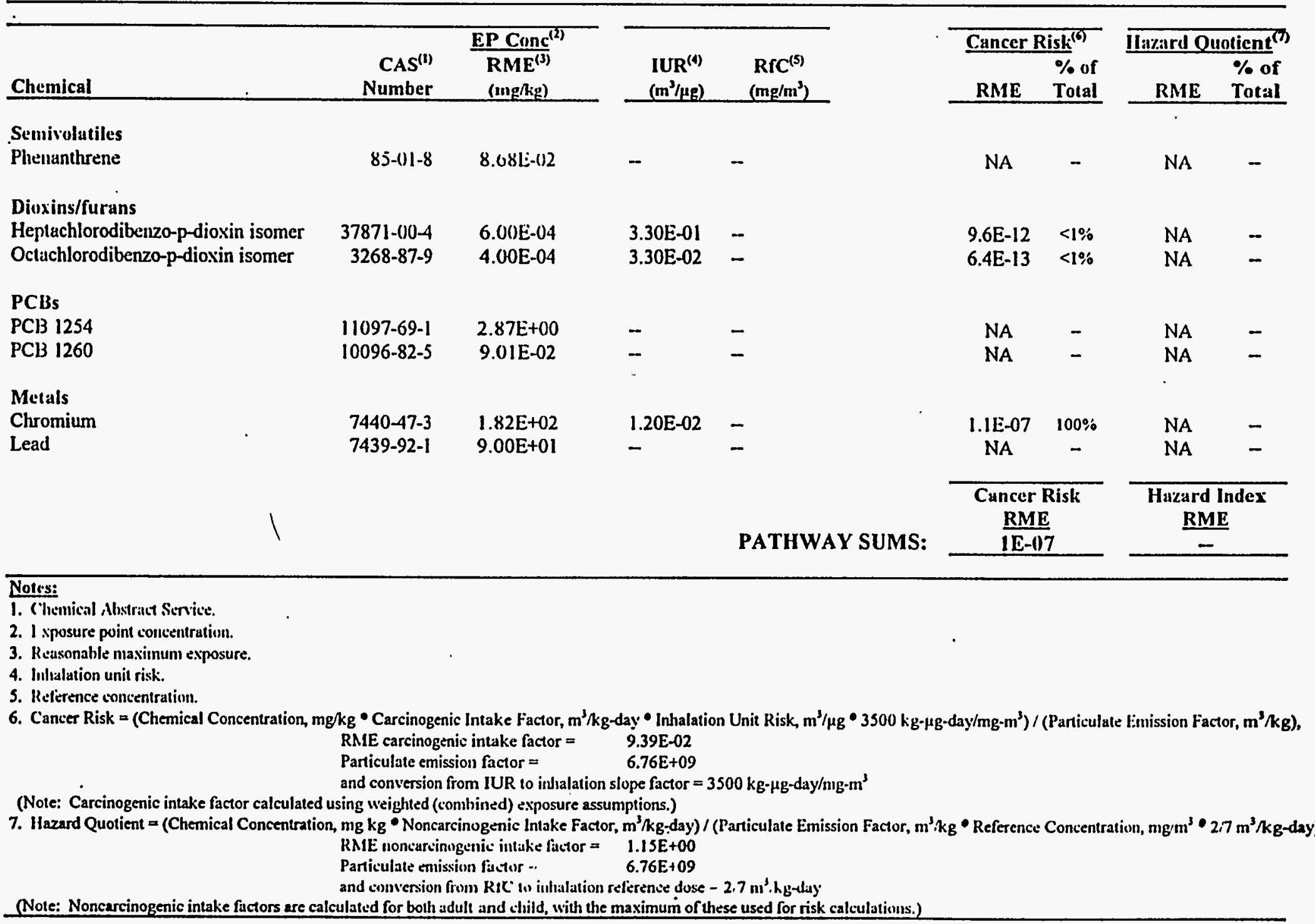

F-RES-SLPRI':riskcalc 
Table D.42

Savannah River Site

Rubble Pit (231-2F)

Combined Adult/Child Resident (Future)

Ingestion of Soil ((1)-t'lls)

Carcinogenic and Noncarcinogenic Risk

\begin{tabular}{|c|c|c|c|c|c|c|c|}
\hline \multicolumn{7}{|l|}{ EXPOSURE ASSUMPTIONS: } & \multirow[t]{2}{*}{ INTAKE FACTOR CALCULATIONS (*) } \\
\hline & & & & & \multicolumn{2}{|c|}{ Combined } & \\
\hline Receptor & \multicolumn{2}{|c|}{ Adult Resident } & \multicolumn{2}{|c|}{ Child Resident } & \multicolumn{2}{|c|}{ Aduit/Child } & \multirow{2}{*}{$\begin{array}{l}\text { Carcinogenic Intuke Fuctor }(\mathrm{ClF}), \text { kg/kg-day }= \\
(\mathrm{IR} * \mathrm{FI} * \mathrm{EF} \bullet \mathrm{ED} \bullet \mathrm{CF}) /(\mathrm{BW} \bullet \mathrm{ATC})\end{array}$} \\
\hline Exposure & \multicolumn{2}{|c|}{$\mathrm{RME}^{(1)}$} & RME & & $\mathrm{RME}$ & & \\
\hline Intake Rate (IR), mg/day & 100 & $(4)$ & 200 & (4) & 120.0 & (5) & RME CIF $=8.36 \mathrm{E}-(1) 7$ \\
\hline Fraction Ingested (FI), unitless & 1 & (4) & 1 & (4) & 1.0 & (5) & \\
\hline Exposure Frequency (EF), days/yr & 350 & (4) & 350 & (4) & 350 & (5) & Noncurcinogenic Intuke Factor (NIF), kg/kg-day = \\
\hline Exposure Duration (ED), jTs & 24 & (4) & 6 & (4) & 30 & (5) & $(\mathrm{IR} \bullet \mathrm{FI} \bullet \mathrm{EF} \bullet \mathrm{ED} * \mathrm{CF}) /(\mathrm{BW} * \mathrm{ATN})$ \\
\hline Body Weight (BW), kg & 70 & (4) & 15 & (4) & 59.0 & (s) & RMI: NIF $=1.28 \mathrm{E}-05 \quad$ (Child Value) \\
\hline Avging Time, $\operatorname{Carc}^{(2)}$ (ATC), days & 25,550 & & NA & & 25,550 & & \\
\hline Avging Time, Noncarc ${ }^{(3)}$ (ATN), days & 8,760 & & 2,190 & & 10,950 & & \\
\hline Conversion Factor (CF), $\mathrm{kg} / \mathrm{mg}$ & $1.00 \mathrm{E}-(16$ & & $1.0(1) \equiv-06$ & & 1.00E:-06 & & \\
\hline
\end{tabular}

\section{Sintes:}

1. Reasonable maxımum exposure.

2. Averaging time, carcinogen; calculated as 70 years (average lifecime) times 365 dajss per year (not applicable to child resident)

3. Averaging time, lloncarcinogeni: calculated es exposure duration (in years) times 365 dayy per year

4. See Table D-1.

5. Combined values are calculated by weighting adult and child exposure assumptions over the noted exposure duration (e g. 24 yrs as an adult plus 6 yrs as a child for a total duration of 30 yrs).

6. Carcinogenic intalie factor calculated using weighted (combined) exposure assumptions; noncurcinogenic intake factors are calculated for both adult and child. uith the maximum of tiese used for risk calculations. 
Table D.42

Savannah River Site

Rubble Pit (231-2F)

Combined Adult/Child Resident (Future)

Ingestion of Soil (0-t'hls)

Carcinogenic and Noncarcinogenic Risk

\begin{tabular}{|c|c|c|c|c|c|c|c|c|}
\hline Chemical & CAS ${ }^{(1)}$ No. & $\begin{array}{l}\frac{\mathrm{EP} \operatorname{Conc}^{(2)}}{\mathrm{RME}^{(3)}} \\
(\mathrm{mg} / \mathrm{kg}) \\
\end{array}$ & \multicolumn{2}{|c|}{$\begin{array}{cc}\text { OSF }^{(1)} & \text { ORfi }^{(S)} \\
(\mathrm{kg}-\mathrm{d} / \mathrm{mg}) & (\mathrm{mg} / \mathrm{kg}-\mathrm{d}) \\
\end{array}$} & \multicolumn{2}{|c|}{\begin{tabular}{ll}
\multicolumn{2}{l}{ Cancer Risk } \\
& $\%$ of \\
RME $\quad$ Total \\
\end{tabular}} & \multicolumn{2}{|c|}{ 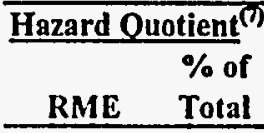 } \\
\hline $\begin{array}{l}\text { Semivolatiles } \\
\text { Phenanthrene }\end{array}$ & $85-01-8$ & $8.68 \mathrm{E}-(1) 2$ & - & - & - & - & - & - \\
\hline $\begin{array}{l}\text { Dioxins/furans } \\
\text { Heptachlorodibenzo-p-dioxin isomer } \\
\text { Octacllorodibenzo-p-dioxin isomer }\end{array}$ & $\begin{array}{r}37871-00-4 \\
3268-87-9\end{array}$ & $\begin{array}{l}4.75 \mathrm{E}-04 \\
4.00 \mathrm{E}-04\end{array}$ & $\begin{array}{l}1.50 \mathrm{E}+03 \\
1.50 \mathrm{E}+02\end{array}$ & - & $\begin{array}{l}6.0 \mathrm{E}-07 \\
5.0 \mathrm{E}-08\end{array}$ & $\begin{array}{l}03 \% \\
<1 \%\end{array}$ & $\overline{-}$ & - \\
\hline $\begin{array}{l}\text { PCBs } \\
\text { PCB } 1254 \\
\text { PCB } 1260\end{array}$ & $\begin{array}{l}11097-69-1 \\
10096-82-5\end{array}$ & $\begin{array}{l}2.87 \mathrm{E}+00 \\
9.01 \mathrm{E}-02\end{array}$ & $\begin{array}{l}7.70 \mathrm{E}+00 \\
7.70 \mathrm{E}+00\end{array}$ & $\begin{array}{l}2.00 \mathrm{E}-0.05 \\
2.00 \mathrm{E}-05\end{array}$ & $\begin{array}{l}1.8 \mathrm{E}-05 \\
5.8 \mathrm{E}-07\end{array}$ & $\begin{array}{l}94 \% \\
03 \%\end{array}$ & $\begin{array}{l}1.8 \mathrm{E} E+00 \\
5.8 \mathrm{E}-02\end{array}$ & $\begin{array}{l}90 \% \\
03 \%\end{array}$ \\
\hline $\begin{array}{l}\text { Metals } \\
\text { Chromium } \\
\text { l.ead } \\
\text { Mercury }\end{array}$ & $\begin{array}{l}7440-47-3 \\
7439-92-1 \\
7439-97-6\end{array}$ & $\begin{array}{l}5.51 \mathrm{E}+01 \\
6.20 \mathrm{E}+01 \\
1.23 \mathrm{E}-01\end{array}$ & $\begin{array}{l}- \\
-\end{array}$ & $\begin{array}{l}5.00 \mathrm{E}-03 \\
\overline{3.00 \mathrm{E}-04}\end{array}$ & $\begin{array}{l}- \\
- \\
-\end{array}$ & $\begin{array}{l}- \\
- \\
-\end{array}$ & $\begin{array}{c}1.4 \mathrm{E}-01 \\
- \\
5.2 \mathrm{E}-03\end{array}$ & $\begin{array}{l}07 \% \\
-\overline{1 \%}\end{array}$ \\
\hline 1 & & & & PATHWAY SUMS: & $\begin{array}{r}\text { Cancer } \\
\mathrm{RM} \\
2 \mathrm{E}-\end{array}$ & & $\begin{array}{r}\text { Hazard } \\
\mathbf{R N} \\
2 \mathbf{E}+ \\
\end{array}$ & ndex \\
\hline $\begin{array}{l}\text { Notes: } \\
\text { 1. Chemical Abstracts Service. } \\
\text { 2. Exposure point concentration. } \\
\text { 3. Reasomahle maximum expisure. } \\
\text { 4. Slope fastor. } \\
\text { 5. Referente dose. } \\
\text { 6. Cancer Risk = (Chemical Concentration, } \\
\text { (Note: Carcinogenic intake factor calculate } \\
\text { 7. Hazard Quotient = (Chemical Concentrat } \\
\text { (Note: Noncarcinogenic intake factors are }\end{array}$ & 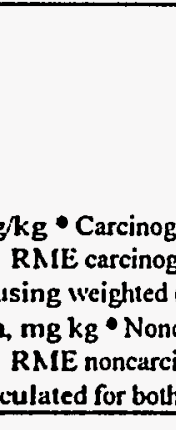 & $\begin{array}{l}\text { genic Intake Fac } \\
\text { genic intake fac } \\
\text { (combined) exp } \\
\text { carcinogenic In } \\
\text { inogenic intake } \\
\text { hodult and chil }\end{array}$ & $\begin{array}{l} \\
\text { sure assumptio } \\
\text { ke Factor, } \mathrm{kg} / \mathrm{kg} \text {-day } \\
1.28 \mathrm{E}-05 \\
\text { with the maxin }\end{array}$ & $\begin{array}{l}\text { - Slope Factor, kg-day/nig), } \\
\text { ons.) } \\
\text { kg-day') / (Reference Dose, } \\
\text { (Child Value) } \\
\text { imum of these used for risk c }\end{array}$ & -day), wher & & & \\
\hline
\end{tabular}


Table D.43

Savannah River Site

Rubble Pit (231-2F)

Combined Adult / Child Resident (Future)

Dermal Exposure to Soil (0-4'bls)

Carcinogenic and Noncarcinogenic Risk

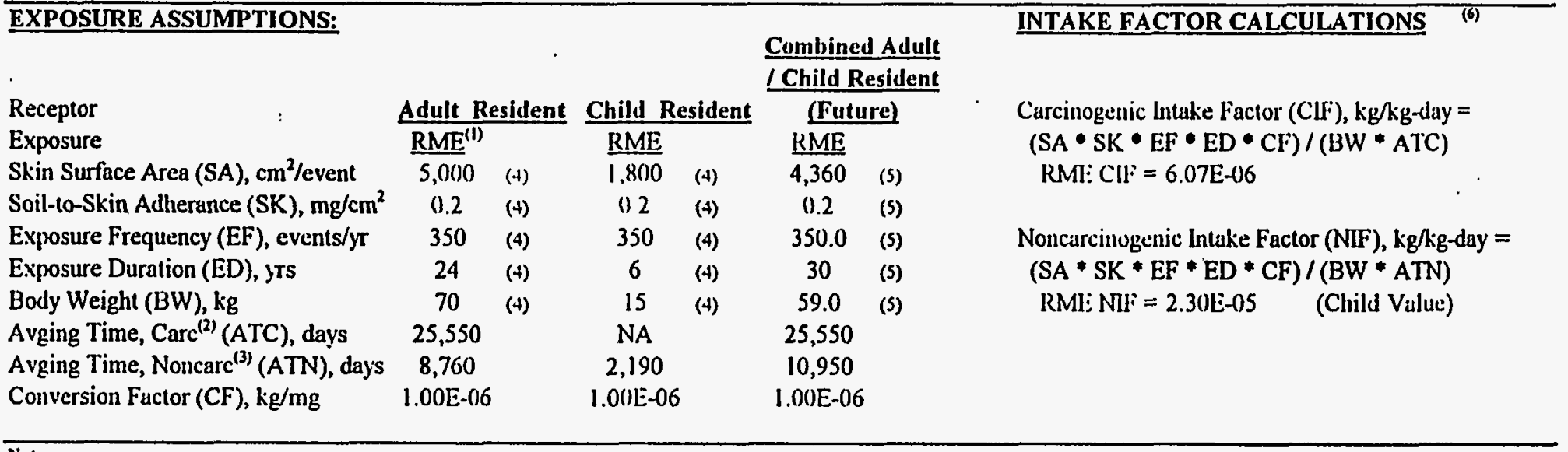

Nater:

1. Reasonable maximum exposure.

2. Aleraging time, carcinogen; calculated as 70 years (average lifetime) times 365 days per year (not applicable to child resident).

3. Averaging time, nonearcinogen; calculated as exposure duration (in years) times 365 days per year

4. Sec Table D-1.

5. Combined values are calculated by weighting adult and child exposure assumptions over the noted exposure duration (e 8.24 yrs as an adult plus 6 yrs as a child for a total duration of 30 yrs).

6. Carcinogenic intake factor calculated using weighted (combined) exposure assumptions; noncarcinogenic intake factors we colculated for both adult and child, with the maximum of these used for risk calculations. 
Table D.43

Savannah River Site

Rubble Pit (231-2F)

Combined Adult / Child Resident (Future)

Dermal Exposure to Soil (0-4'bls)

Carcinogenic and Noncarcinogenic Risk

\begin{tabular}{|c|c|c|c|c|c|c|c|c|c|}
\hline \multirow[b]{2}{*}{ Chemical } & \multicolumn{2}{|c|}{ EP Conc ${ }^{(2)}$} & \multirow[b]{2}{*}{$\begin{array}{c}\mathrm{DSF}^{(4)} \\
(\mathrm{kg}-\mathrm{d} / \mathrm{mg})\end{array}$} & \multirow[b]{2}{*}{$\begin{array}{c}\text { DRID }^{(5)} \\
\text { (mg/kg-d) } \\
\end{array}$} & \multirow[b]{2}{*}{$\mathrm{DABS}^{(6)}$} & \multicolumn{2}{|c|}{ Cancer Risk $^{(n)}$} & \multicolumn{2}{|c|}{ Hazard Quotient $^{(8)}$} \\
\hline & $\begin{array}{r}\text { CAS }^{(!)} \\
\text {Number }\end{array}$ & $\begin{array}{l}\mathrm{RME}^{(3)} \\
(\mathrm{mg} / \mathrm{kg})\end{array}$ & & & & RME & $\begin{array}{l}\% \text { of } \\
\text { Total } \\
\end{array}$ & RME & $\begin{array}{l}\% \text { of } \\
\text { Total } \\
\end{array}$ \\
\hline \multicolumn{10}{|l|}{ Semivolatiles } \\
\hline Phenanthrene & $85-01-8$ & $8.68 \mathrm{E}-02$ & - & - & $1.00 \mathrm{E}-02$ & - & - & - & - \\
\hline \multicolumn{10}{|l|}{ Dioxins/furans } \\
\hline Heptachlorodibenzo-p-dioxin isomer & $37871-00-4$ & $4.75 \mathrm{E}-0.4$ & $1.72 E+03$ & - & $1.00 \mathrm{E}-02$ & $5.0 \mathrm{E}-08$ & $03 \%$ & - & - \\
\hline Octachlorodibenzo-p-dioxin isomer & $3268-87-9$ & $4.00 \mathrm{E}-04$ & $1.72 \mathrm{E}+02$ & - & $1.00 \mathrm{E}-02$ & 4.2E-09 & $<1 \%$ & - & - \\
\hline \multicolumn{10}{|l|}{ PCBs } \\
\hline PCB 1254 & $\mid 1097-69-1$ & $2.87 \mathrm{E}+00$ & $8.56 \mathrm{E}+00$ & $1.80 \mathrm{E}-05$ & $1.00 \mathrm{E}-02$ & $1.5 \mathrm{E}-06$ & $94 \%$ & $3.7 \mathrm{E}-02$ & $71 \%$ \\
\hline PCB 1260 & $11096-82-5$ & $9.01 \mathrm{E}-02$ & $8.56 E+00$ & $1.80 \mathrm{E}-05$ & $1.00 \mathrm{E}-02$ & 4.7E-08 & $03 \%$ & $1.2 \mathrm{E}-03$ & $02 \%$ \\
\hline \multicolumn{10}{|l|}{ Metals } \\
\hline Cluromium & $7440-47-3$ & $5.51 E+01$ & - & $1.00 \mathrm{E}-04$ & $1.00 \mathrm{E}-03$ & - & - & $1.3 \mathrm{~L}-02$ & $25 \%$ \\
\hline Lead & $7439-92-1$ & $6.20 \mathrm{E}+01$ & - & - & $1.00 \mathrm{E}-03$ & - & - & - & - \\
\hline \multirow[t]{2}{*}{ Mercury } & $7439-97-6$ & $1.23 \mathrm{E}-01$ & - & $3.00 \mathrm{E}-06$ & $1.00 \mathrm{E}-03$ & - & - & $9.4 E-04$ & $02 \%$ \\
\hline & & & & PATHWA & Y SUMS: & \multicolumn{2}{|c|}{$\begin{array}{c}\text { Cancer Risk } \\
\text { RME }\end{array}$} & \multicolumn{2}{|c|}{$\begin{array}{l}\text { Hazard Index } \\
\frac{\mathrm{RME}}{5 \mathrm{E}-112} \\
\end{array}$} \\
\hline \multicolumn{10}{|l|}{ Noules: } \\
\hline \multicolumn{10}{|l|}{ 1. Chenvical Ahstract Service. } \\
\hline \multicolumn{10}{|l|}{ 2. Exposure point concentration. } \\
\hline \multicolumn{10}{|l|}{ 3. Reasonahle naximum exposure. } \\
\hline \multicolumn{10}{|l|}{ 4. Slope factor, derinal. } \\
\hline \multicolumn{10}{|l|}{ 5. Reference dose, dermal. } \\
\hline \multicolumn{10}{|c|}{ 6. Absorption factor. } \\
\hline \\
\hline \multicolumn{10}{|c|}{$\begin{array}{l}\text { 7. Cancer Risk = (Chemical Concentration, } \mathrm{mg} / \mathrm{kg} \bullet \text { Carcinogenic Intake Factór, } \mathrm{kg} / \mathrm{kg} \text {-day * Absorption Factor, unitless * Slope Factor, } \mathrm{kg} \text {-day/mg), where } \\
\text { RMIE carcinogenic intake factor } 6.07 E-06\end{array}$} \\
\hline \multirow{2}{*}{\multicolumn{10}{|c|}{$\begin{array}{l}\text { (Note: Carcinogenic intake factor calculated using weighted (comhined) exposure assumptions.) } \\
\text { 8. Hazard Quotiemt }=\text { (Chemical Concentration, mg'kg * Noncarcinogenic Intake Factor, kg/kg-day * Absorption Factor, un }\end{array}$}} \\
\hline & & & & & & & & & \\
\hline & RME noncarci & cinogenic intak & 2.30E-05 & (Child Value) & & & & & \\
\hline
\end{tabular}


Table D.44

Savannah River Site

Rubble Pit (231-2F)

Combined Adult / Child Resident (Future)

Inhalation of Volatile Soil Contaminants (0-4'bls)

Carcinogenic and Noncarcinogenic Risk

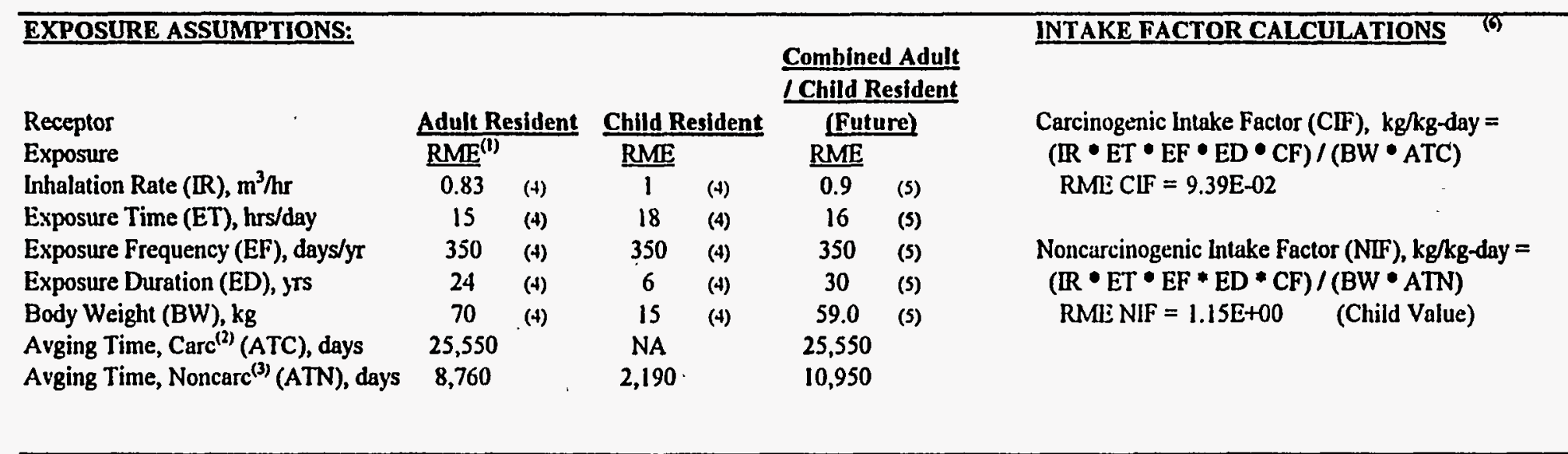

Ressonable maximum exposure

2. Averaging time, carcinogen; calculated as 70 years (average lifetime) times 365 days per year (not epplicable to child resident)

3. Averning time, noncarcinogen. calculuted es exposure duration (in years) times 365 days per year

4. See Table D.1.

5. Combined values are calculated by weighting edult and child exposure assumptions over the noted exposure duration (e g, 24 yrs as an adult plus 6 jrs as a child for a total duration of 30 yra).

6 Carcinogenic intake factor calculuted using wejghted (combined) exposure assumptions; noncarcinogenic inuke factors ure calculated for boch ad ult and child, with the maximum of these used for risk calcalyions. 
Table D.44

Savannah River Site

Rubble Pit (231-2F)

Combined Adult / Child Resident (Future)

Inhalation of Volatile Soil Contaminants (0-4'bls)

Calculation of Volatilization Factor (VF) ${ }^{(1)}$

\begin{tabular}{|c|c|c|c|c|c|c|c|c|c|c|c|}
\hline Chemical & $\mathrm{CAS}^{(2)}$ No. & Class $^{(3)}$ & MolWt & $K_{0(}^{(4)}$ & $\mathrm{Kd}^{(4)}$ & $\mathbf{H}^{(4)}$ & $\mathrm{Kgs}^{(1)}$ & $\mathrm{Di}^{(4)}$ & $\operatorname{Dei}^{(4)}$ & wlpha(4) & $\begin{array}{l}\text { Volat } \\
\text { Factor }^{(d)} \\
\left(\mathrm{m}^{3} / \mathrm{kg}\right)\end{array}$ \\
\hline
\end{tabular}

Notes:

1. USEPA, 1991: RAGS: Vol 1 - Human Health Evaluation Manual (Part B. Development of Risk-Based Preliminary Remediation Goals)

2. Chemical Abstract Service.

3. Chemical class: $v$-volatile; $s$-semivolatile; $p$-pesticide/pct); $x$-dioxin; and $m-$ metal.

4. Calculation of organic contaminant volatilization factor:

CONSTANTS:

Length of side of contam'd. area (LS), $m=$ Wind speed in mixing zone (V), $\mathrm{m} / \mathrm{sec}=$

Diffusion height (DH), $m=$. Area of contamination $(\mathrm{Am}), \mathrm{m}^{2}=$ Area in square centimeters $(\mathrm{Acm}), \mathrm{cm}^{2}=$ True soil porosily. (li), unitless = True soil densily (P's), $\mathrm{g}^{\prime} \mathrm{cm}^{3}$ Exposure interval (TyT), yTs = Exposure interial (Tsec), sec $=$ Organic carbon content (OC), unilless = Conversion factor (CF). $\mathrm{kg}^{\prime} \mathrm{g}=$

INPUT VARIAIBI.ES:

Organic turtion partition coef. (hice), $\mathrm{cm}^{\mathrm{l}} \cdot \mathrm{hg}=$ Henry's Law constant $(\mathrm{H}), \mathrm{atm}-\mathrm{m}^{3} / \mathrm{mol}=$ Gas diffusivity (Di), $\mathrm{cm}^{2} / \mathrm{sec}^{\mathrm{C}}=$ INTERMEDIATE STEPS:

Soil-water partition coeflicient (Kd), $\mathrm{cm}^{3}, \mathrm{~kg}=$ Soil-air partition coefficient (Kas), g-soil/ $\mathrm{cm}^{3}$-air a Effective diffusivity (Dei), $\mathrm{cm}^{2} / \mathrm{sec}=$ alpha (alpha), $\mathrm{cm}^{2} / \mathrm{sec}=$

OUTPUT:

Volatilization Factor (VF), $\mathrm{m}^{3} / \mathrm{kg}=$

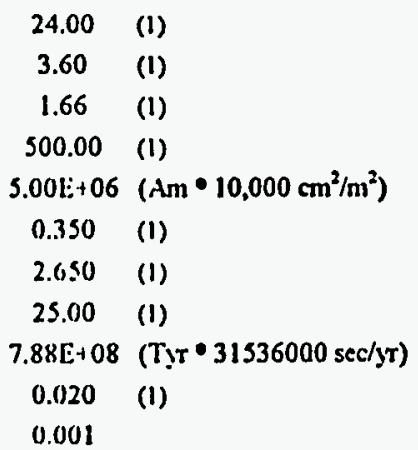

Page 1 of 2 
Table D.44

Savannah River Site

Rubble Pit (231-2F)

Combined Adult / Child Resident (Future)

Inhalation of Volatile Soil Contaminants ( $0-4$ 'bls)

Carcinogenic and Noncarcinogenic Risk

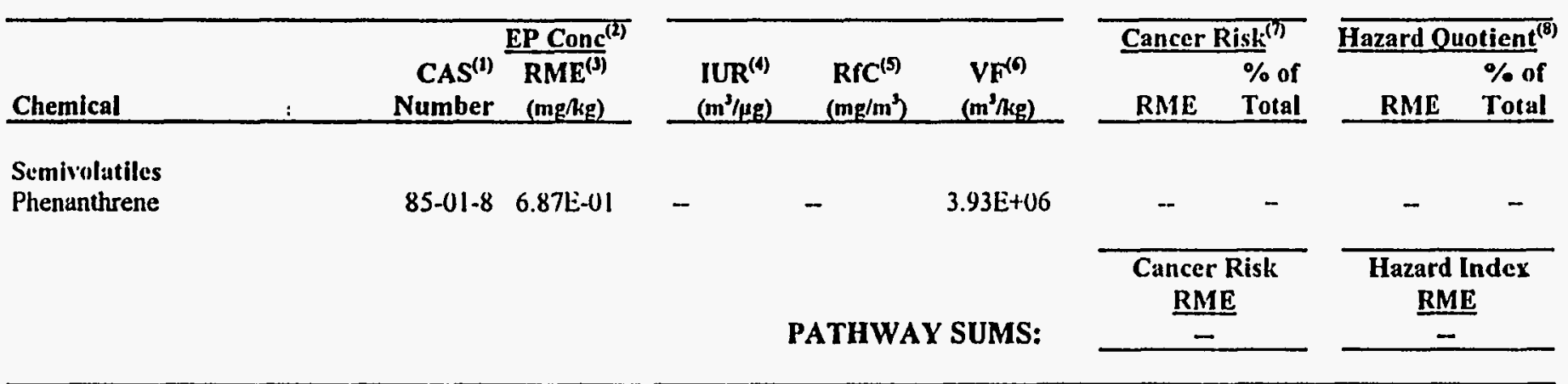

Noles:

1. Chemical Abstract Service.

2. Exposure point concentration.

3. Reasonable maximum exposure.

4. Inhalation unit risk.

5. Reference concentration

6. Volatilization factor (chemical-specific).

7. Cancer Risk $=\left(\right.$ Chemical Concentration, $\mathrm{mg} / \mathrm{kg} \bullet$ Carcinogenic Intake Factor, $\mathrm{m}^{3} / \mathrm{kg}$-day $\bullet$ Inhalation Unit Risk, $\left.\mathrm{m}^{3} / \mathrm{\mu g} \bullet 3500 \mathrm{~kg}-\mu \mathrm{g}-\mathrm{day} / \mathrm{mg}^{\circ} \mathrm{m}^{3}\right) /\left(\right.$ Volatilization Factor, $\left.\mathrm{m}^{3} / \mathrm{kg}\right)$, where RAIE carcinogenic intake factor $9.39 \mathrm{E}-02$

and conversion from IUR to inlualation slope factor $=3500 \mathrm{~kg}-\mu \mathrm{g}-$ day $/ \mathrm{mg}^{-1 \mathrm{~m}^{3}}$

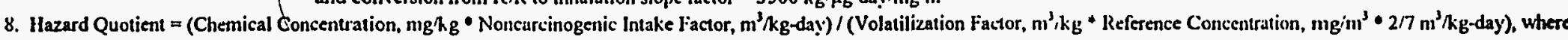
RME noncarcinogenic intake fa $1.15 \mathrm{E}+00$

and conversion from $R R$ to inhallation reference dose $=2.7 \mathrm{~m} / \mathrm{kg}$-day 
Table D.45

Savannah River Site

Rubble Pit (231-2F)

Combined Adult / Child Resident (Future)

Inhalation of Soil Particulates (0-4'bls)

Carcinogenic and Noncarcinogenic Risk

\begin{tabular}{|c|c|c|c|c|c|c|c|}
\hline \multirow[t]{2}{*}{ EXPOSURE ASSUMPTIONS: } & & & & & & \multirow[t]{2}{*}{ INTAKE FACTOR CALCULATIONS } \\
\hline & & & & & \multicolumn{2}{|c|}{$\frac{\text { Combined Adult }}{\text { / Child Resident }}$} & \\
\hline Receptor & Adult $R$ & sident & Child R & sident & (Fut & & Carcinogenic Intake Factor (CIF), $\mathrm{kg} / \mathrm{kg}$-day $=$ \\
\hline Exposure & $\underline{\mathrm{RME}}^{(1)}$ & & RME & & $\mathrm{RME}$ & & $(\mathrm{IR} * \mathrm{Fl} \bullet \mathrm{EF} \bullet \mathrm{ED} \bullet \mathrm{CF}) /(\mathrm{BW} \bullet \mathrm{ATC})$ \\
\hline Inlalation Rate (IR), $\mathrm{m}^{3} / \mathrm{hr}$ & 0.83 & (4) & 1.0 & (4) & 0.9 & (\$) & RME CII $=9.39 E-112$ \\
\hline lixposure Time (ET), hrs/day & 15 & (4) & 18 & (4) & 16 & (s) & \\
\hline lixposure Frequency (EF), days/yr & 350 & (4) & 350 & (4) & 350 & (5) & Noncatcinogenic Intuke Factor (NIF), kg/kg-day = \\
\hline Exposure Duration (ED), yts & 24 & (4) & 6 & (4) & 30 & (5) & $(\mathrm{R} \bullet \mathrm{FI} * \mathrm{EF} * \mathrm{ED} * \mathrm{CF}) /(\mathrm{BW} \bullet \mathrm{ATN})$ \\
\hline Body Weight (BW), kg & 70 & (4) & 15 & (4) & 59.0 & (5) & RMI NIF $=1.15 E+00 \quad$ (Child Value) \\
\hline Avging Time, $\operatorname{Carc}^{(2)}$ (ATC), days & 25,550 & & NA & & 25,550 & & \\
\hline Avging Time, Noncarc ${ }^{(3)}$ (ATN), days & 8,760 & & 2,190 & & 10,950 & & \\
\hline
\end{tabular}

Notes:

1. Reasonable maximum exposure.

2. Averaging time, cercinogen,; calculated as 70 years (average lifeume) unies 365 days per year (not applicable to child resident)

3. Averaging time, nonearcinogen: calculated as exposure duration (in years) times 365 dasy per year

4. Sec Table D-1.

5. Combined values are calculated by weighting adult and child exposure assumptions over the noted exposure duration (e.g, 24 yss as an adult plus 6 yrs as a child for a total duration of 30 yrs)

6. Carcinogenic intake factor calculated using wejehted (combined) exposure assumptions; noncarcinogenic intuke factors ere calcultied for both adult ind child with the maximum of these used for rist: calculations. 
Table D.45

Savannah River Site

Rubble Pit (231-2F)

Combined Adult / Child Resident (Future)

Inhalation of Soil Particulates (0-4'bls)

Carcinogenic and Noncarcinogenic Risk

\section{CALCULATION OF SOIL PARTICULATE RESUSPENSION FACTOR:}

\section{CONSTANTS:}

Length of side of contaminated ureu $(L S)=$

24.00

Wind speed in mixing zone $(\mathrm{V})=$

Diffusion height $(\mathrm{DH})=$

Area of contuminution $(A)=$

Respirable fraction $(R F)=$

Fraction of vegetative cover $(\mathbf{G})=$

Mean annual wind speed $(\mathrm{Um})=$

Equivalent threshold value of wind speed at $10 \mathrm{~m}(\mathrm{Ut})=$

Fraction dependent on Um/Ut (Fx)

Conversion lactor $(\mathrm{CF} a)=$

Conversion factor $(\mathrm{CFb})=$

$\begin{array}{lll}24.00 & \text { meters } & (1) \\ 3.60 & \text { meters/sec } & (1) \\ 1.66 & \text { meters } & (1) \\ 500.00 & \text { meters }^{2} & (1) \\ 0.003 & \text { g/meters }^{2}-\mathrm{hr} & (1) \\ 0.90 & \text { unitless } & (1) \\ 3.60 & \text { meters/sec } & (1) \\ 5.04 & \text { meters/sec } & (1) \\ 1.2700 & \text { unitless } & (1) \\ 3,600.00 & \mathrm{sec} / \mathrm{lr} & \\ 1,000.00 & \mathrm{~g} / \mathrm{kg} & \end{array}$

\section{INTERMEDIATE STEPS:}

Particulate emission factor $(\mathrm{PEF})=6.76 \mathrm{E}+09$ meters $^{3} / \mathrm{kg}$ $(\mathrm{LS} \times \mathrm{V} \times \mathrm{DH} \times \mathrm{CFa} \times \mathrm{CFb}) /\left(\mathrm{A} \times \mathrm{RF} \times(1-\mathrm{G}) \times\left((\mathrm{Um} / \mathrm{Ut})^{\wedge}\right) \times(\mathrm{Fx})\right)$

Noles:

1. USEPA, 1991: RAGS: Vol I .. Human Health Evaluation Mlanual (Part B, Development of Rish-Based Preliminary Remediation Goals) 
Table D.45

Savannah River Site

Rubble Pit (231-2F)

Combined Adult / Child Resident (Future)

Inhalation of Soil Particulates ((0-4'bls)

Carcinogenic and Noncarcinogenic Risk

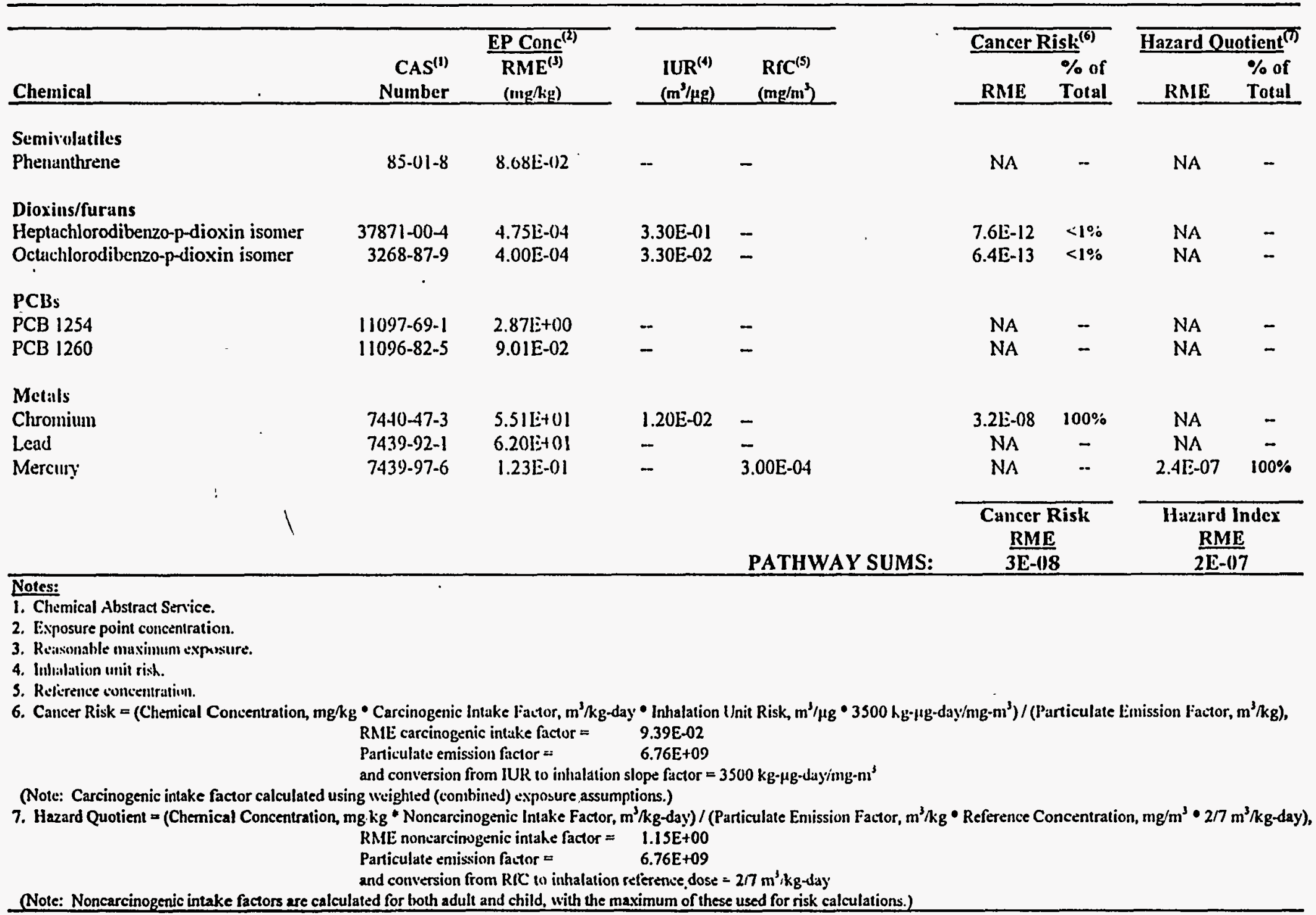


Table D.46

Savannah River Site

Burning/Rubble Pits (231-F, 231-1F, 231-2F)

Combined Adult / Child Resident

Ingestion of Groundwater

Carcinogenic and Noncarcinogenic Risk

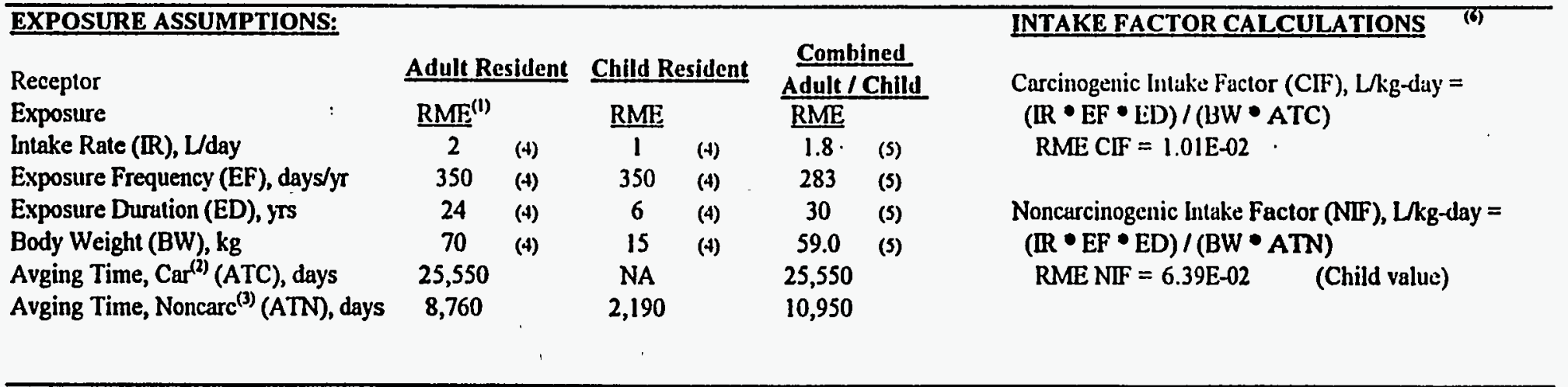

\section{Noter:}

1. Reasonable maximum exposure.

2. Averaging time, carcinogen; calculated as 70 years (average lifetime) times 365 day ser year (nol applicable to child resident).

3. Averaging time, noncurcinogen, calculated as exposure durntion (in years) times 365 days per yeer

4. See Table D-1.

5. Combined values are calculated by weighting adult and child exposure assumptions over the noted exposure duration (c.e. 24 yrs as an adult plus 6 yrs as a child for a total duration of 30 yrs).

6. Carcinogenic intake factor for residents calculated using weighted (combined) exposure assumptions; noncercinogenic intake factors are calculated for both adult and child with the maximum of these used for risk calculations. 
Table D.46

Savannah River Site

Burning/Rubble Pits (231-F, 231-1F, 231-2F)

Combined Adult / Child Resident

Ingestion of Groundwater

Carcinogenic and Noncarcinogenic Risk

\begin{tabular}{|c|c|c|c|c|c|c|c|c|c|}
\hline \multirow{2}{*}{ Chemical } & \multicolumn{3}{|c|}{$\underline{E P C o n c}^{(2)}$} & \multirow[b]{2}{*}{$\begin{array}{c}\mathrm{OSF}^{(4)} \\
(\mathrm{kg}-\mathrm{d} / \mathrm{mg})\end{array}$} & \multirow[b]{2}{*}{$\begin{array}{c}\text { ORID }^{(5)} \\
\text { (mg/kg-d) }\end{array}$} & \multicolumn{2}{|c|}{ Cancer Risk ${ }^{(0)}$} & \multicolumn{2}{|c|}{ Hazard Quotient $^{(7)}$} \\
\hline & $\begin{array}{r}\text { CAS }^{(1)} \\
\text { Number }\end{array}$ & $\begin{array}{l}\mathrm{RME}^{(3)} \\
(\mathrm{mg} / \mathrm{L}) \\
\end{array}$ & & & & RME & $\begin{array}{l}\% \text { of } \\
\text { Total } \\
\end{array}$ & RME & $\begin{array}{l}\% \text { of } \\
\text { Total } \\
\end{array}$ \\
\hline \multicolumn{10}{|l|}{ Volatiles } \\
\hline Carbon tetrachloride & $56-23-5$ & $5.41 \mathrm{E}-03$ & & 1.30E-01 & $7.00 \mathrm{E}-04$ & $7.1 \mathrm{E}-06$ & $14 \%$ & $4.9 \mathrm{E}-01$ & $21 \%$ \\
\hline Chloroform & $67-66-3$ & $1.00 \mathrm{E}-03$ & & $6.10 \mathrm{E}-03$ & $1.00 \mathrm{E}-02$ & $6.2 \mathrm{E}-08$ & $<1 \%$ & $6.4 \mathrm{E}-03$ & $<1 \%$ \\
\hline Cliloromethane (Methyl cliloride) & $74-87-3$ & $4.2015-03$ & & 1.30I:-(12 & -- & $5.5 E-07$ & $01 \%$ & - & -- \\
\hline 1,2-Dichloroethylene & 540590 & 4.471:-06 & $\mathbf{m}$ & - & $9.00 \mathrm{E}-(1) 3$ & - & -- & $3.2 \mathrm{E}-05$ & $<1 \%$ \\
\hline Dichloromethane & 75092 & 1.71E-02 & & $7.50 \mathrm{E}-03$ & $6.00 \mathrm{E}-02$ & $1.3 \mathrm{E}-06$ & $06 \%$ & $1.8 \mathrm{E}-02$ & $<1 \%$ \\
\hline Tetrachloroethylene & 127184 & 7.23E-03 & & 5.20E-02 & $1.00 \mathrm{E}-02$ & $3.8 \mathrm{E}-06$ & $07 \%$ & $4.6 \mathrm{I}-02$ & $02 \%$ \\
\hline Trichloroethylene & $79-01-6$ & 7.47L-03 & & 1.10E-02 & - & 8.3E-07 & $02 \%$ & -- & - \\
\hline \multicolumn{10}{|l|}{ Pesticides } \\
\hline delta-BHC & 319868 & $2.36 \mathrm{E}-04$ & m & - & - & - & - & - & - \\
\hline Heptachlor epoxide & 1024573 & $1.85 \mathrm{E}-06$ & m & $9.10 \mathrm{E}+00$ & $1.30 \mathrm{E}-05$ & $1.7 \mathrm{E}-07$ & $<1 \%$ & 9.1E-03 & $<1 \%$ \\
\hline \multicolumn{10}{|l|}{ Metals } \\
\hline Arsenic & $7440-38-2$ & $2.50 \mathrm{E}-03$ & & $1.50 \mathrm{E}+00$ & $3.00 \mathrm{E}-04$ & $3.8 \mathrm{E}-05$ & $73 \%$ & $5.3 E-01$ & $23 \%$ \\
\hline Cadmium & $7440-43-9$ & $4.83 \mathrm{E}-03$ & m & - & $5.00 \mathrm{E}-04$ & - & - & $6.2 \mathrm{E}-01$ & $27 \%$ \\
\hline Iron & $7439-89-6$ & $2.82 \mathrm{E}+01$ & & - & - & - & -- & - & - \\
\hline Manganese & $7439-96-5$ & $4.53 \mathrm{E}-01$ & & - & $5.00 \mathrm{E}-02$ & - & - & $5.8 \mathrm{E}-(1) 1$ & $25 \%$ \\
\hline & & & & & PATHWAY SUMS: & \multicolumn{2}{|c|}{$\begin{array}{c}\text { Cancer Risk } \\
\text { RME }\end{array}$} & \multicolumn{2}{|c|}{$\begin{array}{c}\text { Hazard Index } \\
\text { RME } \\
\text { 2E+100 }\end{array}$} \\
\hline
\end{tabular}

Notes:

1. Chemical Abstract Service.

2. Exposure point concentration.

3. Reasonable maximum exposure; " $\mathrm{m}$ " indicales modeled concentration

4. Slope factor, oral.

5. Reference dose, oral.

6. Cancer Risk $=$ (Chemical Concentration, $\mathrm{mg} / \mathrm{L}$ * Carcinogenic Intake Factor, L/kg-day ${ }^{\bullet}$ Slope Factor, $\mathrm{kg}$-day/mg), where RME carcinogenic intake factor $=$, $1.01 \mathrm{E}-02$

Carcinogenic intake factor for residents calculated using weighted (combined) exposure assumptions.

7. Hazand Quotient $=($ Chemical Concentration, $\mathrm{mg} / \mathrm{L}$ - Noncarcinogenic Intake Factor, $\mathrm{L} / \mathrm{kg}$-day) $/($ Reference Dose, $\mathrm{ng} / \mathrm{kg}$-day), where RME noncarcinogenic intake factor $=6.39 \mathrm{E}-02$ (Child value)

Noncarcinogenic intake factors are calculated for both adult and child, with the maximum of these used for risk calculations. 
Table D.47

Savannah River Site

Burning/Rubble Pits (231-F, 231-1F, 231-2F)

Combined Adult / Child Resident

Dermal Exposure to Ground Water while Showering

Carcinogenic and Noncarcinogenic Risk

\begin{tabular}{|c|c|c|c|c|c|c|c|}
\hline \multirow[t]{2}{*}{ EXPOSURE ASSUMPTIONS: } & & & & & \multirow{2}{*}{\multicolumn{2}{|c|}{ Combined }} & \multirow{2}{*}{ INTAKE FACTOR CALCULATIONS (क) } \\
\hline & \multirow{2}{*}{\multicolumn{2}{|c|}{ Adult Resident }} & & & & & \\
\hline Receptor & & & \multicolumn{2}{|c|}{ Child Resident } & \multicolumn{2}{|c|}{ Adult / Child } & \multirow{3}{*}{$\begin{array}{l}\text { Carcinogenic Intake Factor }(\mathrm{CIF}), \mathrm{L}-\mathrm{hr} / \mathrm{cm} / \mathrm{kg} \text {-day }= \\
(\mathrm{SA} \bullet \mathrm{ET} \bullet \mathrm{EF} \bullet \mathrm{ED} \bullet \mathrm{CF}) /(\mathrm{BW} \bullet \mathrm{ATC}) \\
\mathrm{RME} \mathrm{CWF}=2.43 \mathrm{E}-02\end{array}$} \\
\hline Exposure $\quad$ : & $\mathrm{RME}^{(1)}$ & & \multicolumn{2}{|c|}{ RME } & \multicolumn{2}{|c|}{$\mathrm{RME}$} & \\
\hline Skin Surface Area (SA), $\mathrm{cm}^{2} / \mathrm{event}$ & $\overline{20,000}$ & (4) & $\overline{7,300}$ & (4) & 17,460 & (5) & \\
\hline Exposure Time (ET), hrs/day & 0.2 & (4) & 0.2 & (4) & 0.20 & (5) & \\
\hline Exposure Frequency (EF), events/yr & 350 & (4) & 350 & (4) & 350 & (5) & Noncarcinogenic Intuke Factor (NIF), L-hr $/ \mathrm{cm} / \mathrm{kg}$-day = \\
\hline Exposure Duration (ED), yts & 24 & (4) & 6 & (4) & 30 & (5) & $(\mathrm{SA} \bullet \mathrm{ET} \bullet \mathrm{EF} \bullet \mathrm{ED} \bullet \mathrm{CF}) /(\mathrm{BW} * \mathrm{ATN})$ \\
\hline Body Weight (BW), kg & 70 & (4) & 15 & (4) & 59.0 & (s) & RME NIF $=9.33 \mathrm{E}-02 \quad$ (Child Value) \\
\hline Avging Time, $\operatorname{Carc}^{(2)}(\mathrm{ATC})$, days & 25,550 & & NA & & 25,550 & & \\
\hline Avging Time, Noncarc ${ }^{(3)}$ (ATN), days & 8,760 & & 2,190 & & 10,950 & & \\
\hline Conversion Factor (CF), $\mathrm{L} / \mathrm{cm}^{3}$ & $1.00 E-03$ & & $1.00 E-03$ & & $1.00 \mathrm{E}-03$ & & \\
\hline
\end{tabular}

\section{Noter:}

1. Reasonable muximum exposure.

2. Averaing time, cercinogen,; calculated as 70 years (average lifetime) times 365 days per year (not applicable to child resident).

3. Averaging time, noncarcinogen; calculated as exposure duration (in years) times 365 dayz per year

4. See Trable D-1.

5. Combined values are calculated by weighting adult and child exposure assumptions over the noted exposure duration (e.g. $24 \mathrm{yrs}$ as an adult plus $6 \mathrm{yrs}$ as a child for a total duration of $30 \mathrm{yrs}$ ).

6. Carcinogenic intake factor calculated using weighted (combined) exposure assumptions; noncarcinogenic intake fretons are calculated for both adult and child uith the maximum of these used for risk calculations. 
Table D.47

Savannah River Site

Burning/Rubble Pits (231-F, 231-1F, 231-2F)

Child Resident

Dermal Exposure to Ground Water while Showering (Non-Volatiles)

Carcinogenic and Noncarcinogenic Risk

\begin{tabular}{|c|c|c|c|c|c|c|c|c|c|c|c|}
\hline \multirow[b]{2}{*}{ Chemical } & \multirow[b]{2}{*}{ : } & \multicolumn{3}{|c|}{ EP Conc ${ }^{(2)}$} & \multirow[b]{2}{*}{$\begin{array}{c}\mathrm{DSF}^{(4)} \\
(\mathrm{kg}-\mathrm{d} / \mathrm{mg})\end{array}$} & \multirow[b]{2}{*}{$\begin{array}{c}\text { DRD }^{(5)} \\
\text { (mg/kg-d) }\end{array}$} & \multirow[b]{2}{*}{$P C^{(6)}$} & \multicolumn{2}{|c|}{ Cancer Risk $^{(3)}$} & \multicolumn{2}{|c|}{ Hazard Quotient $^{(8)}$} \\
\hline & & $\begin{array}{r}\text { CAS }^{(1)} \\
\text { Number }\end{array}$ & $\begin{array}{c}\mathrm{RME}^{(3)} \\
(\mathrm{mg} / \mathrm{l})\end{array}$ & & & & & IRME & $\begin{array}{l}\% \text { of } \\
\text { Total }\end{array}$ & RME & $\begin{array}{l}\% \text { of } \\
\text { Total }\end{array}$ \\
\hline \multicolumn{12}{|l|}{ Pesticides } \\
\hline delta-BHC & & 319868 & $2.36 \mathrm{E}-04$ & $\mathrm{~m}$ & - & - & $2.80 \mathrm{E}-02$ & - & - & -- & - \\
\hline Heptachlor epoxide & & 1024573 & 1.85E-06 & $\mathbf{m}$ & $1.82 \mathrm{E}+01$ & $6.50 \mathrm{E}-06$ & $5.50 \mathrm{E}-02$ & 4.5E-08 & $28 \%$ & $1.5 \mathrm{E}-03$ & $02 \%$ \\
\hline \multicolumn{12}{|l|}{ Metals } \\
\hline Arsenic & & $7440-38-2$ & $2.50 \mathrm{E}-03$ & & $1.88 E+00$ & $2.40 \mathrm{E}-04$ & 1.00E-03 & $1.1 \mathrm{E}-07$ & $72 \%$ & 9.71:-04 & $01 \%$ \\
\hline Cadmium & & $7440-43-9$ & 4.83E-03 & m & - & $1.00 \mathrm{E}-05$ & $1.00 E-03$ & - & -- & 4.5E-02 & $60 \%$ \\
\hline Iron & & $7439-89-6$ & $2.82 \mathrm{E}+01$ & & - & - & $1.00 \mathrm{E}-03$ & - & - & -- & - \\
\hline Manganese & & $7439-96-5$ & $4.53 \mathrm{E}-01$ & & - & $1.50 \mathrm{E}-03$ & 1.0OE-()3 & - & -- & $2.81 \mathrm{i}-112$ & $37 \%$ \\
\hline & . & & & & & PATHW & AY SUMS: & $\begin{array}{r}\text { Cancer } \\
\text { RM } \\
2 \mathrm{E}-1\end{array}$ & & $\begin{array}{r}\text { Hazard } \\
\frac{\mathrm{RN}}{8 \mathrm{E}}\end{array}$ & $\begin{array}{l}\text { ndex } \\
2 \\
2\end{array}$ \\
\hline
\end{tabular}

Notes:

2. Exposure point concentration.

3. Reasonable maximum exposure; " $m$ " indicates modeled concentration.

4. Slope factor, dermal.

5. Reference dose, dermal.

6. Permeability constant.

7. Cancer Risk $=$ (Chemical Concentration, $\mathrm{mg} /$ * Carcinogenic Intake Factor, Lhr/cm/kg-day $\bullet$ Permeability Constant, $\mathrm{cm} / \mathrm{hr} *$ Slope Factor, $\mathrm{kg}$-day/mg), where RME carcinogenic intake factor $=\quad 2.43 \mathrm{E}-02$

. (Note: Carcinogenic intake factor calculated using weighted (combined) exposure assuniptions.)

8. Hazard Quotient $=$ (Chemical Concentration, $\mathrm{mg} / \mathrm{L} \bullet$ Noncarcinogenic Intake Factor, $\mathrm{L} / \mathrm{hr} / \mathrm{cm} / \mathrm{kg}$-day $\bullet$ lecrmeability Constant, cm/hr) $/(\mathrm{Reference} \mathrm{Dose,} \mathrm{nIg/kg-day),} \mathrm{where}$ RME noncarcinogenic intake factor $=9.33 \mathrm{E}-02$ (Child Value)

(Note: Noncarcinogenic intake factors are calculated for both adult and child, with the maximum of these used for risk calculations.)

Carcinogenic and noncarcinogenic risks for dennal expsosure to volutile groundwater contaminants included in showering exposure (see Table D.46). 
Table D.48

Savannah River Site

Burning/Rubble Pits (231-F, 231-1F, 231-2F)

Combined Adult / Child Resident

Showering Exposure to Ground Water Volatiles (Inhalation and Dermal) ${ }^{(1)}$

Carcinogenic and Noncarcinogenic Risk

\begin{tabular}{|c|c|c|c|c|c|c|}
\hline \multicolumn{6}{|l|}{ EXPOSURE ASSUMPTIONS: } & INTAKE FACTOR CALCULATIONS (7) \\
\hline & & & & & Combined & \\
\hline Receptor & Aduit R & sident & Child $\mathrm{R}$ & sident & Adult / Child & Carcinogenic Intake Factor (CIF), L/kg-day = \\
\hline Exposure & $\underline{\mathrm{RME}}^{(2)}$ & & $\underline{\mathrm{RME}}$ & & RME & $(\mathbb{R} * \mathrm{EF} * \mathrm{ED}) /(\mathrm{BW} \bullet \mathrm{ATC})$ \\
\hline Intake Rate (IR), Uday & 2 & (4) & 1 & (5) & 1.8 & RME CIF $=1.01 \mathrm{E}-02$ \\
\hline Exposure Frequency (EF), days/yr & 350 & (4) & 350 & (5) & 283 & \\
\hline Exposure Duration (ED), yrs & 24 & (4) & 6 & (5) & 30 & Noncarcinogenic Intake Factor (NIF), L/kg-day = \\
\hline Body Weight (BW), kg & 70 & (4) & 15 & (5) & 59.0 & $(\mathrm{IR} \bullet \mathrm{EF} \bullet \mathrm{ED}) /(\mathrm{BW} \bullet \mathrm{ATN})$ \\
\hline Avging Time, $\mathrm{Car}^{(3)}$ (ATC), days & 25,550 & & NA & & 25,550 & RME NIF $=6.39 \mathrm{E}-02 \quad$ (Child valuc) \\
\hline Avging Time, Noncarc ${ }^{(4)}$ (ATN), days & 8,760 & & 2,190 & & 10,950 & \\
\hline
\end{tabular}

Notcri:

. Demal and inhulution exposure to groundwater volutes utile showering was considered equinalent to infestion of ground water (USEPA Region IV. 1995).

2. Reasonable maximum exposure.

3. Averaging time, carcinogen; calculated as 70 years (average lifetime) times 365 days per year (not applicable to child resident).

4. Averaging time, noncarcinogen,i calculated as exposure duration (in years) times 365 dass per year

5. See Table D-1.

6. Combined values are calculated by weighting adult end child exposure assumptions over the noted exposure duration (c 8,24 yrs as an adult plus 6 yrs as a child for a torel duration of 30 yrs).

7. Carcinogenic intale factor for residents calculated using weighted (combined) exposure assumptions; noncarcinogenic intuke factors are calculated for both adult and child, with the maximum of these used for riak calculations. 
Table D.48

Savannah River Site

Burning/Rubble Pits (231-F, 231-1F, 231-2F)

Combined Adult / Child Resident

Showering Exposure to Ground Water Volatiles (Inhalation and Dermal) ${ }^{(1)}$

Carcinogenic and Noncarcinogenic Risk

\begin{tabular}{|c|c|c|c|c|c|c|c|c|c|}
\hline Chemical & $\begin{array}{r}\text { CAS }^{(2)} \\
\text { Number } \\
\end{array}$ & $\begin{array}{l}\text { EP Conc } \\
\text { RME }^{(3)} \\
(\mathrm{mg} / \mathrm{L}) \\
\end{array}$ & & $\begin{array}{c}\mathrm{OSF}^{(5)} \\
(\mathrm{kg}-\mathrm{d} / \mathrm{mg})\end{array}$ & $\begin{array}{c}\mathrm{ORI}^{(\theta)} \\
\left(\mathrm{mg} / \mathrm{Kg}^{-\mathrm{d}}\right)\end{array}$ & $\begin{array}{l}\text { Cancer } \\
\text { RME }\end{array}$ & $\begin{array}{l}\text { isk" } \\
\% \text { of } \\
\text { Total }\end{array}$ & Hazard O & $\begin{array}{l}\text { otient }^{(1)} \\
\% \text { of } \\
\text { Total } \\
\end{array}$ \\
\hline \multicolumn{10}{|l|}{ Volatiles } \\
\hline Carbon tetrachloride & $56-23-5$ & $5.41 \mathrm{E}-03$ & . & $1.30 \mathrm{E}-01$ & $7.00 \mathrm{E}-04$ & 7.1E-06 & $52 \%$ & $4.9 \mathrm{E}-01$ & $87 \%$ \\
\hline Chloroform & $67-66-3$ & $1.00 \mathrm{E}-03$ & & $6.10 \mathrm{E}-03$ & $1.00 \mathrm{E}-02$ & $6.2 \mathrm{E}-08$ & $<1 \%$ & $6.4 \mathrm{E}-03$ & $01 \%$ \\
\hline Chloromethane (Methyl chloride) & $74-87-3$ & $4.20 \mathrm{E}-03$ & & $1.30 \mathrm{E}-02$ & - & 5.5E-07 & $04 \%$ & - & - \\
\hline 1,2-Dichloroethylene & 540590 & $4.47 \mathrm{E}-06$ & $\mathbf{m}$ & - & $9.00 \mathrm{E}-03$ & - & - & $3.2 \mathrm{E}-05$ & $<1 \%$ \\
\hline Dichloromethane & 75092 & $1.71 \mathrm{E}-02$ & & $7.50 \mathrm{E}-03$ & $6.00 \mathrm{~B}-02$ & $1.3 \mathrm{E}-06$ & $06 \%$ & $1.8 \mathrm{E}-02$ & $03 \%$ \\
\hline Tetrachloroethylene & 127184 & $7.23 \mathrm{E}-03$ & & $5.20 \mathrm{E}-02$ & $1.00 \mathrm{E}-02$ & $3.8 \mathrm{E}-06$ & $28 \%$ & $4.6 \mathrm{E}-02$ & $08 \%$ \\
\hline \multirow[t]{2}{*}{ Trichloroethylene } & $79-01-6$ & $7.47 \mathrm{E}-03$ & & $1.10 \mathrm{E}-02$ & - & 8.3E-07 & $06 \%$ & - & - \\
\hline & & & . & & PATHWAY SUMS: & \multicolumn{2}{|c|}{$\begin{array}{c}\text { Cancer Risk } \\
\frac{\text { RME }}{1 \mathrm{E}-05}\end{array}$} & \multicolumn{2}{|c|}{$\begin{array}{l}\text { Hazard Index } \\
\frac{\mathrm{RME}}{6 \mathrm{E}-01}\end{array}$} \\
\hline
\end{tabular}

1. Dermal and inhulation exposure to groundwater volatiles while showering was considered equivalent to ingeation of ground water (USEPA Region IV, 1995).

2. Chemical Abetract Service.

3. Exposure poirt concentration.

4. Reasonable maximum expoaure; " $m$ " indicates modeled concentration.

5. Slope factor, aral.

6. Reference douc, oral.

7. Cancer Risk - (Chemical Concentration, mg/L * Carcinogenic Intake Fuctor, LkB-day * Slope Fector, kg-day/mg), whero RME carcinogenic intake factor $=\quad$ 1.012-02

Carcinogenic intake factor for residents calculatod using weighted (combinod) exposure sesumption.

8. Hazard Quotient = (Chemical Concentration, mg/L * Noncarcinogenic Intake Factor, L/kg-day) /(Referenoc Dose, mg/kg-day), where

RME noncarcinogenic intake factor $=6.39 \mathrm{E} .02$ (Child value)

Noncarcinogenic intake factors are calculated for both adult and child, with the maximum of these used for riak calculation. 
Table D.49

Savannah River Site

Burning/Rubble Pits (231-F, 231-1F, 231-2F)

Child Resident

Ingestion of Surface Water (Wetland)

Carcinogenic and Noncarcinogenic Risk

\section{EXPOSURE ASSUMPTIONS}

Receptor

Exposure

Intake Rate (IR), L/hr

Exposure Time (ET), hrs/day

Exposure Frequency (EF), days/yr

Exposure Duration (ED), yrs

Body Weight (BW), kg

Avging Time, $\operatorname{Carc}^{(2)}$ (ATC), days

Avging Time, Noncarc ${ }^{(3)}($ ATN $)$, days

\begin{tabular}{cc}
\multicolumn{3}{c}{ Child Resident } \\
$\underline{\mathrm{RME}}^{(1)}$ & \\
0.01 & $(4)$ \\
2 & $(4)$ \\
39 & $(4)$ \\
10 & $(4)$ \\
45 & $(4)$ \\
25,550 & \\
3,650 &
\end{tabular}

\section{INTAKE FACTOR CALCULATIONS}

Carcinogenic Intake Factor (CIF), L/kg-day = $(\mathrm{IR} \bullet \mathrm{ET} \bullet \mathrm{EF} \bullet \mathrm{ED} * \mathrm{CF}) /(\mathrm{BW} \bullet \mathrm{ATC})$

$\mathrm{RME} \mathrm{CIF}=6.78 \mathrm{E}-06$

Noncarcinogenic Intake Factor (NIF), L/kg-day = $(\mathrm{IR} * \mathrm{ET} * \mathrm{EF} \bullet \mathrm{ED} * \mathrm{CF}) /(\mathrm{BW} * \mathrm{ATN})$

$\mathrm{RMENIF}=4.75 \mathrm{E}-05$

Notes:

1. Reasonable maximum exposure.

2. Averaging time, carcinogen; calculated as 70 years (average lifetime) times 365 days per year (not applicable to child resident).

3. Averaging time, noncarcinogen; calculated as exposure duration (in years) times 365 dsys per year

4. See Table D-1. 
Table D.49

Savannah River Site

Burning/Rubble Pits (231-F, 231-1F, 231-2F)

Child Resident

Ingestion of Surface Water (Wetland)

Carcinogenic and Noncarcinogenic Risk

\begin{tabular}{|c|c|c|c|c|c|c|c|c|c|}
\hline Chemical & CAS $^{(1)}$ No. & $\begin{array}{c}\text { EP Conc }{ }^{(2)} \\
\operatorname{RME}^{(3)} \\
(\mathrm{mg} / \mathrm{L})\end{array}$ & $\begin{array}{c}\operatorname{OSF}^{(1)} \\
(\mathrm{kg}-\mathrm{d} / \mathrm{mg})\end{array}$ & $\begin{array}{c}\operatorname{ORfD}^{(g)} \\
(\mathbf{m g} / \mathbf{k g}-\mathbf{d})\end{array}$ & & Cancer & $\begin{array}{l}\overline{\text { isk }}{ }^{(0)} \\
\% \text { of } \\
\text { Total }\end{array}$ & Fazard 0 & $\begin{array}{l}\text { otient }^{(7)} \\
\% \text { of } \\
\text { Total }\end{array}$ \\
\hline \multicolumn{10}{|l|}{ Volatiles } \\
\hline Acetone & 67641 & $2.26 \mathrm{E}-02$ & - & $1.00 \mathrm{E}-01$ & & - & - & $1.1 \mathrm{E}-05$ & $01 \%$ \\
\hline Dichloromethane (Methylene chlorid & 75092 & $1.00 \mathrm{E}-02$ & $7.50 \mathrm{E}-03$ & $6.00 \mathrm{E}-02$ & & $5.1 \mathrm{E}-10$ & $02 \%$ & $7.9 \mathrm{E}-06$ & $<1 \%$ \\
\hline \multicolumn{10}{|l|}{ Semivolatlles } \\
\hline Bis(2-ethylhexyl) phthalate & 117817 & $3.45 \mathrm{E}-01$ & $1.40 \mathrm{E}-02$ & $2.00 \mathrm{E}-02$ & & 3.3E-08 & $98 \%$ & 8.2E-04 & $79 \%$ \\
\hline Di-n-butyl phthalate & 84742 & $1.74 \mathrm{E}-03$ & - & $1.00 \mathrm{E}-01$ & & - & - & 8.3E-07 & $<1 \%$ \\
\hline Metals & & & & & $i$ & & & & \\
\hline Lead & 7439921 & $7.90 \mathrm{E}-03$ & - & - & & - & - & _- & - \\
\hline Manganese & 7439965 & 2.13E-01 & - & $5.00 \mathrm{E}-02$ & $!$ & - & - & $2.0 \mathrm{E}-04$ & $19 \%$ \\
\hline 1 & & & \multicolumn{3}{|c|}{ PATHWAY SUMS: } & \multicolumn{2}{|c|}{$\begin{array}{c}\text { Cancer Risk } \\
\text { RME } \\
\text { 3E-08 }\end{array}$} & \multicolumn{2}{|c|}{$\begin{array}{c}\text { Hazard Index } \\
\text { RME } \\
\text { 1E-03 }\end{array}$} \\
\hline
\end{tabular}

Notes:

1. Chemical Abstracts Service.

2. Exposure point concentration.

3. Reasonable maximum exposure.

4. Slope factor.

5. Ŕeference dose.

6. Cancer Risk = (Chemical Concentration, mg/L * Carcinogenic Intake Factor, L/kg-day * Slope Factor, kg-dny/mg), where

RME carcinogenic intake facto $6.78 \mathrm{E}-06$

7. Hazard Quotient = (Chemical Coscentration, mg/L * Noncarcinogenic Intake Factor, L/kg-day)/ (Reference Doue, mg/kg-day), where

RME noncarcinogenic intake fa 4.75E-05 
Table D.50

Savannah River Site

Burning/Rubble Pits (231-F, 231-1F, 231-2F)

Child Resident

Dermal Exposure to Surface Water (Wetland)

Carcinogenic and Noncarcinogenic Risk

\begin{tabular}{|c|c|c|}
\hline EXPOSURE ASSUMPTTONS: & & INTAKE FACTOR CALCULATIONS \\
\hline Receptor & Child Resident & Carcinogenic Intake Factor (CIF), L-hr/cm/kg-day = \\
\hline Exposure & $\underline{\mathrm{RME}^{(1)}}$ & $(\mathrm{SA} * \mathrm{ET} * \mathrm{EF} * \mathrm{ED} * \mathrm{CF}) /(\mathrm{BW} * \mathrm{ATC})$ \\
\hline Skin Surface Area (SA), $\mathrm{cm}^{2} /$ event & 3,100 & $\mathrm{RME} \mathrm{CIF}=2.10 \mathrm{E}-03$ \\
\hline Exposure Time (ET), hrs/day & 2 & \\
\hline Exposure Frequency (EF), days/yr & 39 & Noncarcinogenic Intake Factor (NIF), L-hr/cm/kg-day \\
\hline Exposure Duration (ED), yrs & 10 (4) & $(\mathrm{SA} \bullet \mathrm{ET} * \mathrm{EF} * \mathrm{ED} * \mathrm{CF}) /(\mathrm{BW} * \mathrm{ATN})$ \\
\hline Body Weight (BW), kg & $45 \quad(4)$ & RME NIF = 1.47E-02 \\
\hline Avging Time, Carc $^{(2)}$ (ATC), days & 25,550 & \\
\hline Avging Time, Noncarc ${ }^{(3)}$ (ATN), days & 3,650 & \\
\hline Conversion Factor (CF), $\mathrm{L} / \mathrm{cm}^{3}$ & $1.00 \mathrm{E}-03$ & \\
\hline \multicolumn{3}{|l|}{ Noten: } \\
\hline \multicolumn{3}{|l|}{ 1. Reasonable maximum exposure. } \\
\hline \multicolumn{3}{|c|}{ 2. Averaging time, carcinogen; caleulated as 70 years (average lifetime) times 365 days per year (not applicable to child resident). } \\
\hline \multicolumn{3}{|c|}{ 3. Averaging time, noncarcinogen, calculated as exposure duration (in years) times 365 days per year } \\
\hline 4. See Table D-1. & & \\
\hline
\end{tabular}


Table D.50

Savannah River Site

Burning/Rubble Pits (231-F, 231-1F, 231-2F)

Child Resident

Dermal Exposure to Surface Water (Wetland)

Carcinogenic and Noncarcinogenic Risk

\begin{tabular}{|c|c|c|c|c|c|c|c|c|c|}
\hline \multirow[b]{2}{*}{ Chemical } & \multicolumn{2}{|c|}{ EP Conc ${ }^{(2)}$} & \multirow[b]{2}{*}{$\begin{array}{c}\mathrm{DSF}^{(4)} \\
\text { (kg-d/mg) }\end{array}$} & \multirow[b]{2}{*}{$\begin{array}{c}\operatorname{DRm}^{(\rho)} \\
\text { (mg/kg-d) }\end{array}$} & \multirow[b]{2}{*}{$P \mathbf{C}^{(0)}$} & \multicolumn{2}{|c|}{ Cancer Risk $^{(n)}$} & \multicolumn{2}{|c|}{ Hazard Ouotient $^{(0)}$} \\
\hline & $\begin{array}{r}\text { CAS }^{(\mathbf{1})} \\
\text { Number } \\
\end{array}$ & $\begin{array}{l}\mathbf{R M B}^{(3)} \\
(\mathrm{mg} / \mathrm{L}) \\
\end{array}$ & & & & $\mathbf{R M E}$ & $\begin{array}{l}\% \text { of } \\
\text { Total } \\
\end{array}$ & RME & $\begin{array}{l}\% \text { of } \\
\text { Total } \\
\end{array}$ \\
\hline \multicolumn{10}{|l|}{ Volatiles } \\
\hline Acetone & 67641 & $2.26 \mathrm{E}-02$ & - & 8.00E-02 & $3.10 \mathrm{E}-04$ & - & - & $1.3 \mathrm{E}-06$ & $<1 \%$ \\
\hline Dichloromethane (Methylene chlorid & 75092 & $1.00 \mathrm{E}-02$ & $7.65 \mathrm{E}-03$ & $5.88 \mathrm{E}-02$ & $4.50 \mathrm{E}-03$ & 7.2E-10 & $<1 \%$ & 1.1E-05 & $<1 \%$ \\
\hline \multicolumn{10}{|l|}{ Semivolatiles } \\
\hline Bis(2-ethylhexyl) phthalate & 117817 & 3.45E-01 & $2.80 \mathrm{E}-02$ & $1.00 \mathrm{E}-02$ & $3.40 \mathrm{E}-02$ & $6.9 \mathrm{E}-07$ & $100 \%$ & 1.7E-02 & $89 \%$ \\
\hline Di-n-butyl phthalate & 84742 & $1.74 \mathrm{E}-03$ & - & $9.00 \mathrm{E}-02$ & $3.30 \mathrm{E}-02$ & - & - & $9.4 \mathrm{E}-06$ & $<1 \%$ \\
\hline \multicolumn{10}{|l|}{ Metals } \\
\hline Lead & 7439921 & $7.90 \mathrm{E}-03$ & - & - & $1.00 \mathrm{E}-03$ & - & - & - & - \\
\hline Manganese & 7439965 & $2.13 \mathrm{E}-01$ & - & $1.50 \mathrm{E}-03$ & $1.00 \mathrm{E}-03$ & - & - & $2.1 \mathrm{E}-03$ & $11 \%$ \\
\hline 1 & & & & ATHWAY & SUMS: & $\begin{array}{c}\text { Cancer Risk } \\
\text { RMR }\end{array}$ & $\begin{array}{l}\text { Risk } \\
7 \\
\end{array}$ & \multicolumn{2}{|c|}{$\begin{array}{l}\text { Hezard Index } \\
\frac{\text { RME }}{2 \mathrm{E}-02}\end{array}$} \\
\hline
\end{tabular}

Noten:

. Chemical Abstrect Service.

2. Exposure point concentration.

3. Reasonable maximum exposure.

4. Slope factor, dermal.

5. Refience dose, dermal.

6. Permeability constant

7. Cancer Risk = (Chemical Concentration, mg/L * Carcinogenic Intake Factor, L-hr/cm/kg-day * Permeability Constant, cm/hr * Slope Factor, kg-day/mg), where RME carcinogenic intake facto $2.10 \mathrm{E}-03$

8. Hazard Quotient = (Chemical Concentration, $\mathrm{mg} / \mathrm{L}$ * Noncarcinogenic Intake Factior, L-hr/cm/kg-day * Permeability Constant, cm/hr)/(Reference Dose, mg/kg-day), where RME noncarcinogenic intake fa $1.47 \mathrm{E}-02$ 
Table D.51

Savannah River Site

Burning/Rubble Pits (231-F, 231-1F, 231-2F)

Child Resident

Ingestion of Sediment (Wetland)

Carcinogenic and Noncarcinogenic Risk

\section{EXPOSURE ASSUMPTIONS:}

\section{Receptor}

Exposure

Intake Rate (IR), mg/day

Fraction Ingested (FI), unitless

Exposure Frequency (EF), days/yr

Exposure Duration (ED), yrs

Body Weight (BW), kg

Avging Time, $\mathrm{Carc}^{(2)}$ (ATC), days

Avging Time, Noncarc ${ }^{(3)}$ (ATN), days

Conversion Factor (CF), kg/mg

\section{INTAKE FACTOR CALCULATIONS}

Carcinogenic Intake Factor (CIF), kg/kg-day = $(\mathrm{R} * \mathrm{FI} \bullet \mathrm{EF} \bullet \mathrm{ED} \bullet \mathrm{CF}) /(\mathrm{BW} * \mathrm{ATC})$ $\mathrm{RME} C \mathrm{CF}=6.78 \mathrm{E}-09$

Noncarcinogenic Intake Factor (NIF), $\mathrm{kg} / \mathrm{kg}$-day = $(\mathbb{R} * \mathrm{FI} * \mathrm{EF} * \mathrm{ED} * \mathrm{CF}) /(\mathrm{BW} * \mathrm{ATN})$ RME NIF $=4.75 \mathrm{E}-08$

Notes:

1. Reasonable maximum exposure.

2. Averaging time, carcinogen; calculated as 70 years (average lifetime) times 365 days per year (not applicable to child resident).

3. Averaging time, noncarcinopen;' culculated es exposure duration (in years) times 365 days per year

4. See Teble D.1. 
Table D.51

Savannah River Site

Burning/Rubble Pits (231-F, 231-1F, 231-2F)

Child Resident

Ingestion of Sediment (Wetland)

Carcinogenic and Noncarcinogenic Risk

\begin{tabular}{|c|c|c|c|c|c|c|c|c|}
\hline \multirow[b]{2}{*}{ Chemical } & \multirow{2}{*}{ CAS $^{(1)}$ No. } & \multirow{2}{*}{$\begin{array}{l}\frac{\mathrm{EPConc}}{\left({ }^{(2)}\right.} \\
\mathrm{RME}^{(3)} \\
(\mathrm{mg} / \mathrm{kg})\end{array}$} & \multirow[b]{2}{*}{$\begin{array}{c}\text { OSF } \\
(\mathrm{kg}-\mathrm{d} / \mathrm{mg}) \\
\end{array}$} & \multirow[b]{2}{*}{$\begin{array}{c}\operatorname{ORID}^{(())} \\
(\mathrm{mg} / \mathrm{kg} \text {-d })\end{array}$} & \multicolumn{2}{|c|}{ Cancer Risk } & \multicolumn{2}{|c|}{ Bazard Quotient ${ }^{(7)}$} \\
\hline & & & & & RME & $\begin{array}{l}\% \text { of } \\
\text { Total }\end{array}$ & RME & $\begin{array}{l}\% \text { of } \\
\text { Total } \\
\end{array}$ \\
\hline \multicolumn{9}{|l|}{ Volatiles } \\
\hline Acetone & 67641 & $6.43 \mathrm{E}-02$ & - & $1.00 \mathrm{E}-01$ & - & - & $3.1 \mathrm{E}-08$ & $<1 \%$ \\
\hline Chloromethane (Methyl chloride) & 74873 & $1.30 \mathrm{E}-02$ & $1.30 \mathrm{E}-02$ & - & $1.1 \mathrm{E}-12$ & $<1 \%$ & - & - \\
\hline Dichloromethane (Methylene chlorid & 75092 & 4.19E-02 & $7.50 \mathrm{E}-03$ & $6.00 \mathrm{E}-02$ & $2.1 \mathrm{E}-12$ & $<1 \%$ & $3.3 \mathrm{E}-08$ & $<1 \%$ \\
\hline Toluene & 108883 & $1.13 \mathrm{E}-02$ & - & $2.00 \mathrm{E}-01$ & - & - & 2.TE-09 & $<1 \%$ \\
\hline \multicolumn{9}{|l|}{ Semivolatiles } \\
\hline Acenaphthene & 83329 & $2.18 \mathrm{E}-01$ & - & $6.00 \mathrm{E}-02$ & - & - & $1.7 \mathrm{E}-07$ & $<1 \%$ \\
\hline Acenaphthylene & 208968 & 7.75E-01 & - & - & - & - & - & - \\
\hline Anthracene & 120127 & $4.21 \mathrm{E}+00$ & - & $3.00 \mathrm{E}-01$ & - & - & $6.7 \mathrm{E}-07$ & $<1 \%$ \\
\hline Benzo(a)anthracene & 56553 & $4.31 \mathrm{E}+00$ & 7.30E-01 & - & $2.1 \mathrm{E}-08$ & $02 \%$ & - & - \\
\hline Benzo(a)pyrene & 50328 & $2.99 \mathrm{E}+00$ & $7.30 \mathrm{E}+00$ & - & $1.5 \mathrm{E}-07$ & $14 \%$ & - & - \\
\hline Benzo(b)fluoranthene & 205992 & $4.34 \mathrm{E}+00$ & 7.30E-01 & - & $2.1 \mathrm{E}-08$ & $02 \%$ & - & - \\
\hline Benzo(g,h,i)perylène & 191242 & $1.74 \mathrm{E}+00$ & - & - & - & - & - & - \\
\hline Benzo(k)fluoranthene & 207089 & $3.99 \mathrm{E}+00$ & 7.30E-02 & - & $2.0 \mathrm{E}-09$ & $<1 \%$ & - & - \\
\hline Bis(2-ethylhexyl) phthalate & 117817 & $1.68 \mathrm{E}+00$ & $1.40 \mathrm{E}-02$ & $2.00 \mathrm{E}-02$ & $1.6 \mathrm{E}-10$ & $<1 \%$ & $4.0 \mathrm{E}-06$ & $<1 \%$ \\
\hline Chrysene & 218019 & $5.33 \mathrm{E}+00$ & $7.30 \mathrm{E}-03$ & - & $2.6 \mathrm{E}-10$ & $<1 \%$ & - & - \\
\hline Di-n-butyl phthalate & 84742 & $5.96 \mathrm{E}-02$ & - & $1.00 \mathrm{E}-01$ & - & - & $2.8 \mathrm{E}-08$ & $<1 \%$ \\
\hline Dibenz(a,h)anthracene & 53703 & $5.62 \mathrm{E}-01$ & $7.30 \mathrm{E}+00$ & - & 2.8E-08 & $03 \%$ & - & - \\
\hline Dibenzofuran & 132649 & $1.33 \mathrm{E}-01$ & - & - & - & - & - & - \\
\hline Fluoranthene & 206440 & $1.21 \mathrm{E}+01$ & - & $4.00 \mathrm{E}-02$ & - & - & $1.4 \mathrm{E}-05$ & $02 \%$ \\
\hline Fluorene & 86737 & $5.22 \mathrm{E}-01$ & - & $4.00 \mathrm{E}-02$ & - & - & $6.2 \mathrm{E}-07$ & $<1 \%$ \\
\hline Inden $0(1,2,3-c, d)$ pyrene & 193395 & $1.78 \mathrm{E}+00$ & $7.30 \mathrm{E}-01$ & - & $8.8 \mathrm{E}-09$ & $<1 \%$ & - & - \\
\hline Phenanthrene & 85018 & $5.36 \mathrm{E}+00$ & - & - & - & - & - & - \\
\hline Pyrene & 129000 & $7.83 \mathrm{E}+00$ & - & $3.00 \mathrm{E}-02$ & - & - & $1.2 \mathrm{E}-05$ & $01 \%$ \\
\hline \multicolumn{9}{|l|}{ Diorins } \\
\hline Octachlorodibenzo-p-dioxin isomers & 3268879 & 8.00E-01 & $1.50 \mathrm{E}+02$ & - & 8.1E-07 & $76 \%$ & - & - \\
\hline
\end{tabular}


Table D.51

Savannah River Site

Burning/Rubble Pits (231-F, 231-1F, 231-2F)

Child Resident

Ingestion of Sediment (Wetland)

Carcinogenic and Noncarcinogenic Risk

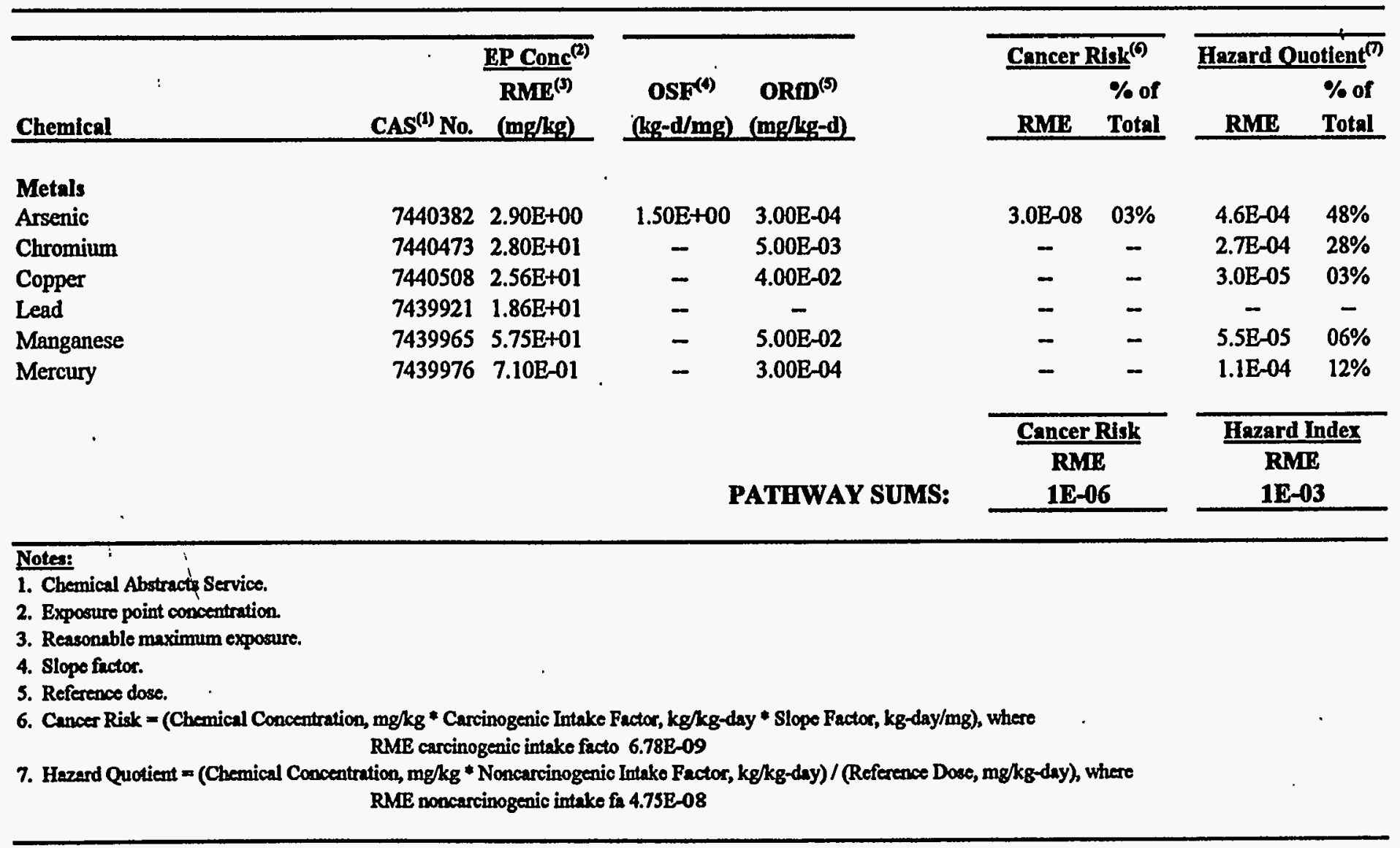


Table D.52

Savannah River Site

Burning/Rubble Pits (231-F, 231-1F, 231-2F)

Child Resident

Dermal Exposure to Sediment (Wetland)

Carcinogenic and Noncarcinogenic Risk

\section{EXPOSURE ASSUMPTIONS:}

\section{Receptor}

Exposure

Skin Surface Area (SA), $\mathrm{cm}^{2} /$ event

Soil-to-Skin Adherance (SK), $\mathrm{mg} / \mathrm{cm}^{2}$

Exposure Frequency (EF), events/yr

Exposure Duration (ED), yrs

Body Weight (BW), kg

Avging Time, $\mathrm{Canc}^{(3)}$ (ATC), days

Avging Time, Noncarc(4) (ATM), days

Conversion Factor (CF), kg/mg

\section{Child Resident}

$\mathrm{RME}^{(1)}$

3,100 (4)

$0.6 \quad$ (4)

$39 \quad$ (4)

10 (4)

$45 \quad$ (4)

25,550

3,650

$1.00 \mathrm{E}-06$

\section{INTAKE FACTOR CALCULATIONS}

Carcinogenic Intake Factor (CIF), $\mathrm{kg} / \mathrm{kg}$-day =

$(\mathrm{SA} \bullet \mathrm{SK} * \mathrm{EF} \bullet \mathrm{ED} * \mathrm{CF}) /(\mathrm{BW} * \mathrm{ATC})$

$\mathrm{RME}$ CIF $=6.31 \mathrm{E}-07$

Noncarcinogenic Intake Factor (NIF), kg/kg-day =

$(\mathrm{SA} * \mathrm{SK} * \mathrm{EF} * \mathrm{ED} * \mathrm{CF}) /(\mathrm{BW} * \mathrm{ATN})$

$\mathrm{RME}$ NIF $=4.42 \mathrm{E}-06$

\section{Notes:}

1. Reasonable maximum exposure.

2. Averaging time, carcinogen; calculsted as 70 years (average lifetime) times 365 days per year (not applicable to child resident).

3. Averaging time, noncarcinogen; calculated as exposure duration (in years) times 365 days per year

4. See Trable D-1. 
Table D.52

Savannah River Site

Burning/Rubble Pits (231-F, 231-1F, 231-2F)

Child Resident

Dermal Exposure to Sediment (Wetland)

Carcinogenic and Noncarcinogenic Risk

\begin{tabular}{|c|c|c|c|c|c|c|c|c|c|}
\hline \multirow[b]{2}{*}{ Chemical } & \multicolumn{2}{|r|}{ EPConc ${ }^{(x)}$} & \multirow[b]{2}{*}{$\begin{array}{c}\text { DSF(t) } \\
(\mathrm{kg}-\mathrm{d} / \mathrm{mg})\end{array}$} & \multirow[b]{2}{*}{ 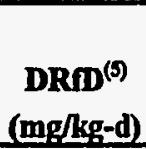 } & \multirow[b]{2}{*}{ DABS $^{(0)}$} & \multicolumn{2}{|c|}{ Cancer Risk } & \multicolumn{2}{|c|}{ Hazard Quotient ${ }^{(0)}$} \\
\hline & $\begin{array}{r}\text { CAS }^{(1)} \\
\text { Number }\end{array}$ & $\begin{array}{l}\mathbf{R M E}^{(3)} \\
(\mathrm{mg} / \mathrm{Kg}) \\
\end{array}$ & & & & RME & $\begin{array}{l}\% \text { of } \\
\text { Total }\end{array}$ & RME & $\begin{array}{l}\% \text { of } \\
\text { Total }\end{array}$ \\
\hline \multicolumn{10}{|l|}{ Volatiles } \\
\hline Acetone & 67641 & $6.43 \mathrm{E}-02$ & - & $8.00 \mathrm{E}-02$ & $1.00 \mathrm{E}-02$ & - & - & 3.5E-08 & $<1 \%$ \\
\hline Chloromethane (Methyl chloride) & 74873 & $1.30 \mathrm{E}-02$ & $1.63 \mathrm{E}-02$ & - & $1.00 \mathrm{E}-02$ & $1.3 \mathrm{E}-12$ & $<1 \%$ & - & - \\
\hline Dichloromethane (Methylene chlorid & 75092 & $4.19 \mathrm{E}-02$ & $7.65 \mathrm{E}-03$ & $5.88 \mathrm{E}-02$ & $1.00 \mathrm{E}-02$ & $2.0 \mathrm{E}-12$ & $<1 \%$ & $3.1 \mathrm{E}-08$ & $<1 \%$ \\
\hline Toluene & 108883 & $1.13 \mathrm{E}-02$ & - & $1.60 \mathrm{E}-01$ & $1.00 \mathrm{E}-02$ & - & - & $3.1 \mathrm{E}-09$ & $<1 \%$ \\
\hline \multicolumn{10}{|l|}{ Semivolatiles } \\
\hline Acenaphthene & 83329 & $2.18 \mathrm{E}-01$ & - & $3.00 \mathrm{E}-02$ & $1.00 \mathrm{E}-02$ & - & - & 3.2E-07 & $<1 \%$ \\
\hline Acenaphthylene & 208968 & 7.75E-01 & - & - & $1.00 \mathrm{E}-02$ & - & - & - & - \\
\hline Anthracene & 120127 & $4.21 E+00$ & - & $1.50 \mathrm{E}-01$ & $1.00 \mathrm{E}-02$ & - & _- & $1.2 \mathrm{E}-06$ & $<1 \%$ \\
\hline Benzo(a)anthracene & 56553 & $4.31 E+00$ & $1.46 \mathrm{E}+00$ & - & $1.00 \mathrm{E}-02$ & $4.0 \mathrm{E}-08$ & $02 \%$ & - & - \\
\hline Benzo(a)pyrene & 50328 & $2.99 \mathrm{E}+00$ & $3.65 \mathrm{E}+01$ & - & $1.00 \mathrm{E}-02$ & $6.9 \mathrm{E}-07$ & $40 \%$ & - & - \\
\hline Benzo(b)fluoranthene & 205992 & $4.34 \mathrm{E}+00$ & $1.46 \mathrm{E}+00$ & - & $1.00 \mathrm{E}-02$ & 4.0E-08 & $02 \%$ & - & - \\
\hline Benzo(g,h,i)perylehe & 191242 & $1.74 \mathrm{E}+00$ & - & - & $1.00 \mathrm{E}-02$ & - & - & - & - \\
\hline Benzo(k)fluoranthene & 207089 & $3.99 \mathrm{E}+00$ & $1.46 \mathrm{E}-01$ & - & $1.00 \mathrm{E}-02$ & 3.7E-09 & $<1 \%$ & - & - \\
\hline Bis(2-ethylhexyl) phthalate & 117817 & $1.68 \mathrm{E}+00$ & $2.80 \mathrm{E}-02$ & $1.00 \mathrm{E}-02$ & $1.00 \mathrm{E}-02$ & $3.0 \mathrm{E}-10$ & $<1 \%$ & 7.4E-06 & $<1 \%$ \\
\hline Chrysene & 218019 & $5.33 E+00$ & $1.22 \mathrm{E}-02$ & - & $1.00 \mathrm{E}-02$ & 4.1E-10 & $<1 \%$ & - & - \\
\hline Di-n-butyl phthalate & 84742 & $5.96 \mathrm{E}-02$ & - & $9.00 \mathrm{E}-02$ & $1.00 \mathrm{E}-02$ & - & - & $2.9 \mathrm{E}-08$ & $<1 \%$ \\
\hline Dibenz(a,h)anthracene & 53703 & $5.62 \mathrm{E}-01$ & $1.46 \mathrm{E}+01$ & - & $1.00 \mathrm{E}-02$ & $5.2 \mathrm{E}-08$ & $03 \%$ & - & - \\
\hline Dibenzofuran & 132649 & $1.33 \mathrm{E}-01$ & - & - & $1.00 \mathrm{E}-02$ & - & - & - & - \\
\hline Fluoranthene & 206440 & $1.21 \mathrm{E}+01$ & - & $2.00 \mathrm{E}-02$ & $1.00 \mathrm{E}-02$ & - & - & 2.7E-05 & $01 \%$ \\
\hline Fluorene & 86737 & $5.22 \mathrm{E}-01$ & - & $2.00 \mathrm{E}-02$ & $1.00 \mathrm{E}-02$ & - & - & $1.2 \mathrm{E}-06$ & $<1 \%$ \\
\hline Indeno $(1,2,3-c, d)$ pyrene & 193395 & $1.78 \mathrm{E}+00$ & $1.46 \mathrm{E}+00$ & - & $1.00 \mathrm{E}-02$ & $1.6 \mathrm{E}-08$ & $<1 \%$ & - & - \\
\hline Phenanthrene & 85018 & $5.36 \mathrm{E}+00$ & - & - & $1.00 \mathrm{E}-02$ & - & - & - & - \\
\hline Pyrene & 129000 & $7.83 \mathrm{E}+00$ & - & $1.50 \mathrm{E}-02$ & $1.00 \mathrm{E}-02$ & - & - & 2.3E-05 & $<1 \%$ \\
\hline \multicolumn{10}{|l|}{ Dioxing } \\
\hline Octachlorodibenzo-p-dioxin isomers & 3268879 & $8.00 \mathrm{E}-01$ & $1.72 \mathrm{E}+02$ & - & $1.00 \mathrm{E}-02$ & 8.TE-07 & $51 \%$ & - & - \\
\hline
\end{tabular}


Table D.52

Savannah River Site

Burning/Rubble Pits (231-F, 231-1F, 231-2F)

Child Resident

Dermal Exposure to Sediment (Wetland)

Carcinogenic and Noncarcinogenic Risk

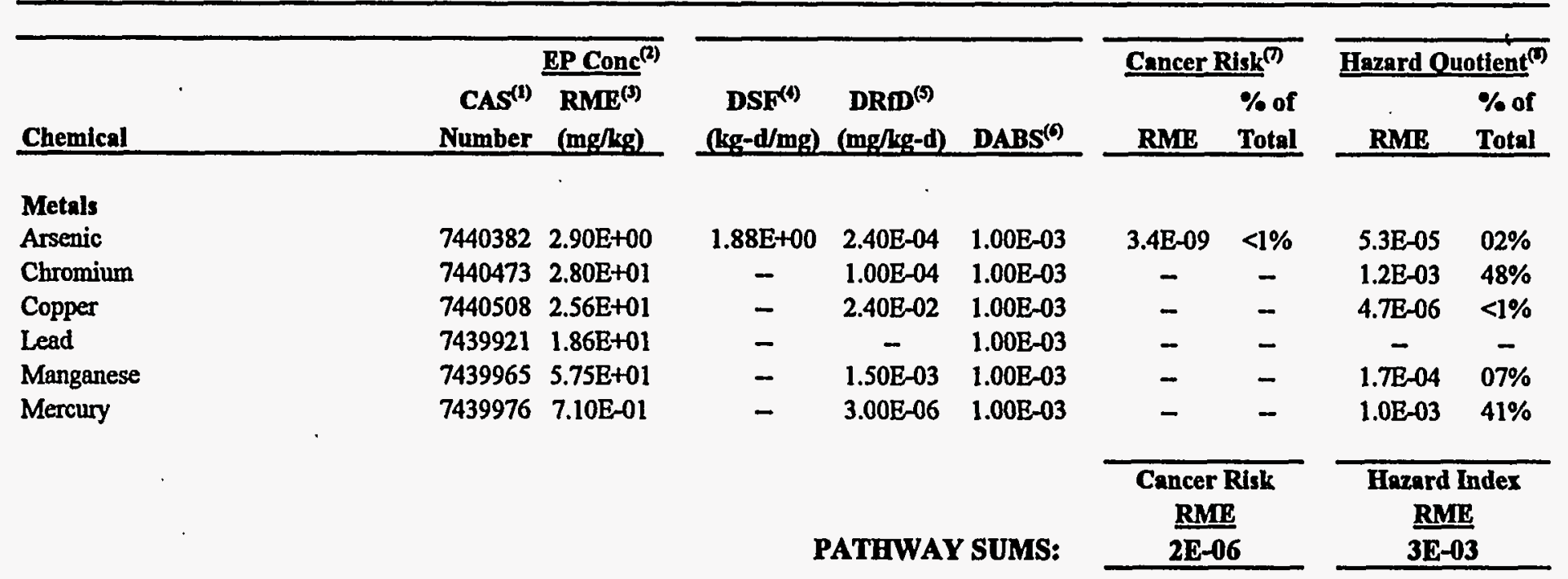

Notes:

1. Chemical Abstract Service.

2. Exposure point concentration.

3. Reasonable maximum exposure.

4. Slope factor, dermal.

5. Reference dose, dermal

6. Absorption factor.

7. Cancer Risk = (Chemical Concentration, mg/kg * Cancinogenic Intake Factor, kg/kg-day * Absorption Factor, unilless * Slope Factor, kg-day/mg), where RME carcinogenic intake facto 6.31E-07

8. Hazard Quotient = (Chemical Concentration, mg/kg * Noncarcinogenic Intake Factor, kg/kg-day * Absorption Fuctor, unitless)/(Reference Dose, mg/kg-day), where RME noncarcinogenic intake fa 4.42E-06 


\section{RADIONUCLIDES}




\section{THIS PAGE LEFT INTENTIONALLY BLANK}




\section{Appendix D.53 \\ F-Area Burning/Rubble Pits 231-F/1F and Rubble Pit 2F \\ On-Unit Visitor \\ Radionuclide Cancer Risk Based on Ingestion of Soil}

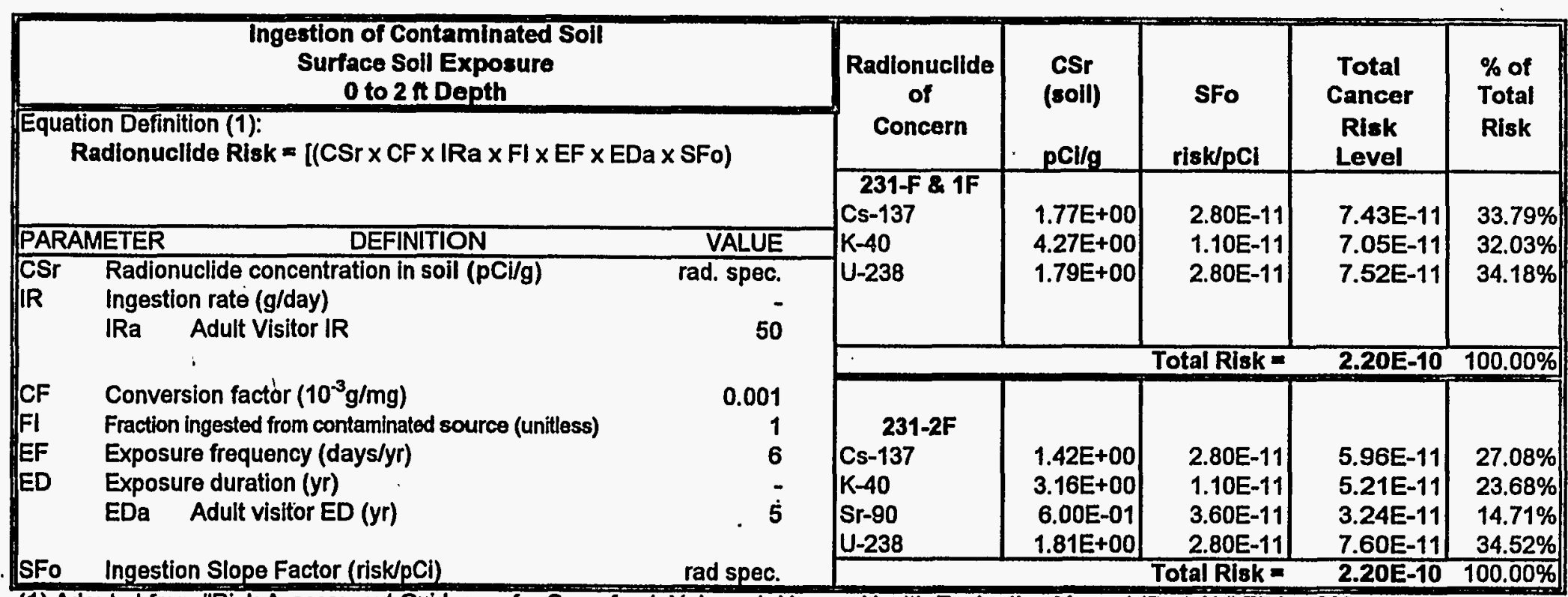

(1) Adapted from "Risk Assessment Guidance for Superfund, Volume I, Human Health Evaluation Manual (Part A)," EPA, 1989. 
Appendix D.64

F-Area Buming/Rubble Pits 231-F/1F and Rubble Pit 2F

On-Unit Visitor

Radionuclide Cancer Risk Based on Dermal Contact with Soil

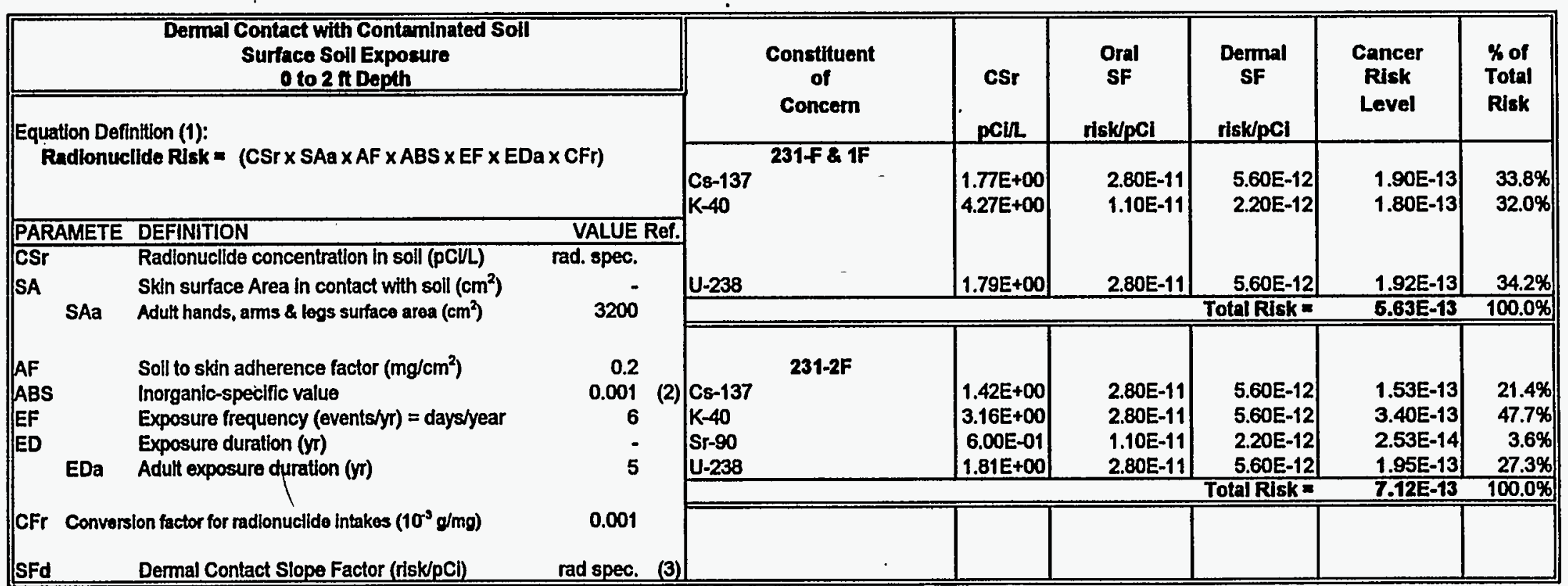

(1) Adapted from "Rlsk Assessment Guidance for Superfund, Volume I, Human Health Evaluation Manual (Part A)," EPA, 1989.

(2) ABS for organics $=0.01$; ABS for inorganics $=0.001$.

(3) Dermal Contact Slope Factors were derived from the ingestion SF by the following formula: SFd = SFo $\times 0.2$ 
Appendix D.55

F-Area Burning/Rubble Pits 231-F/1F and Rubble Pit 2F

On-Unit Visitor

Radionuclide Cancer Risk Based on Inhalation of Contaminated Soll Particulates

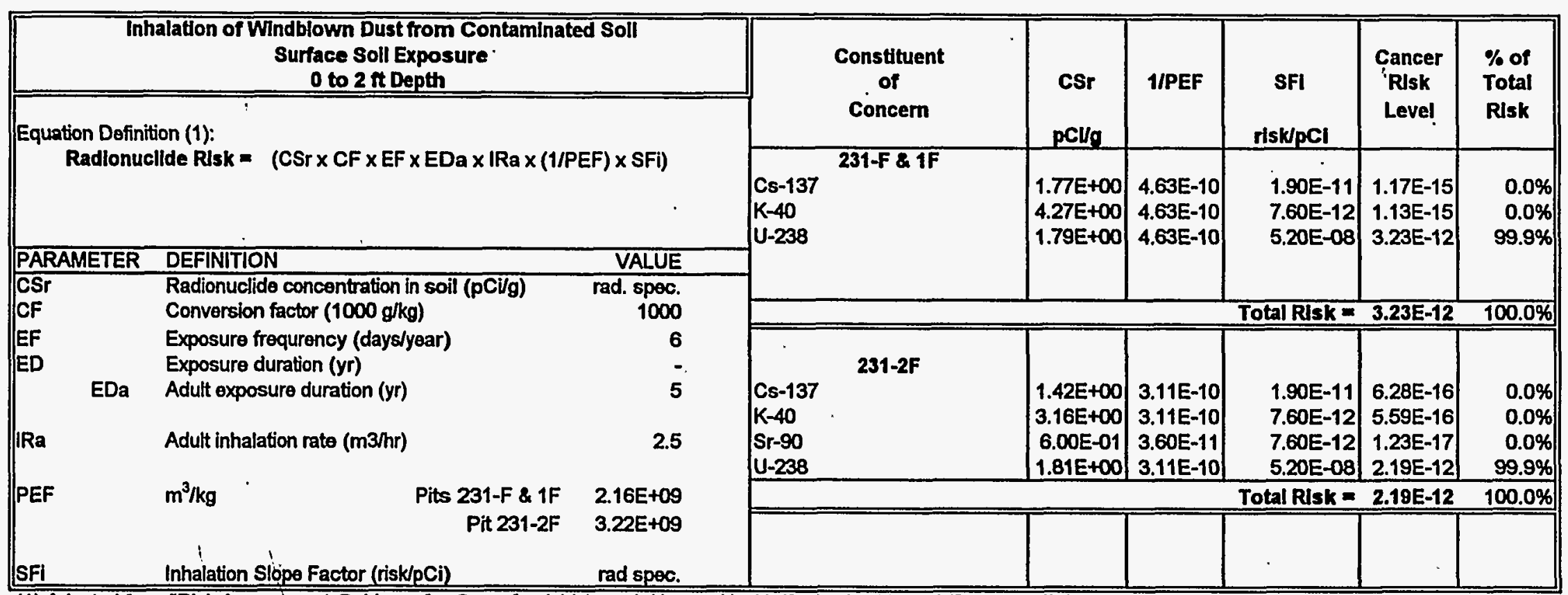

(1) Adapted from "Risk Assessment Guidance for Superfund, Volume I, Human Health Evaluation Manual (Part A), EPA, 1989. 
Appendix D.56

F-Area Burning/Rubble Pits 231-F/1F and Rubble Pit 2F

On-Unit Visitor

Radionucllde Cancer Risk Based on Direct Radiation from Surface Soll

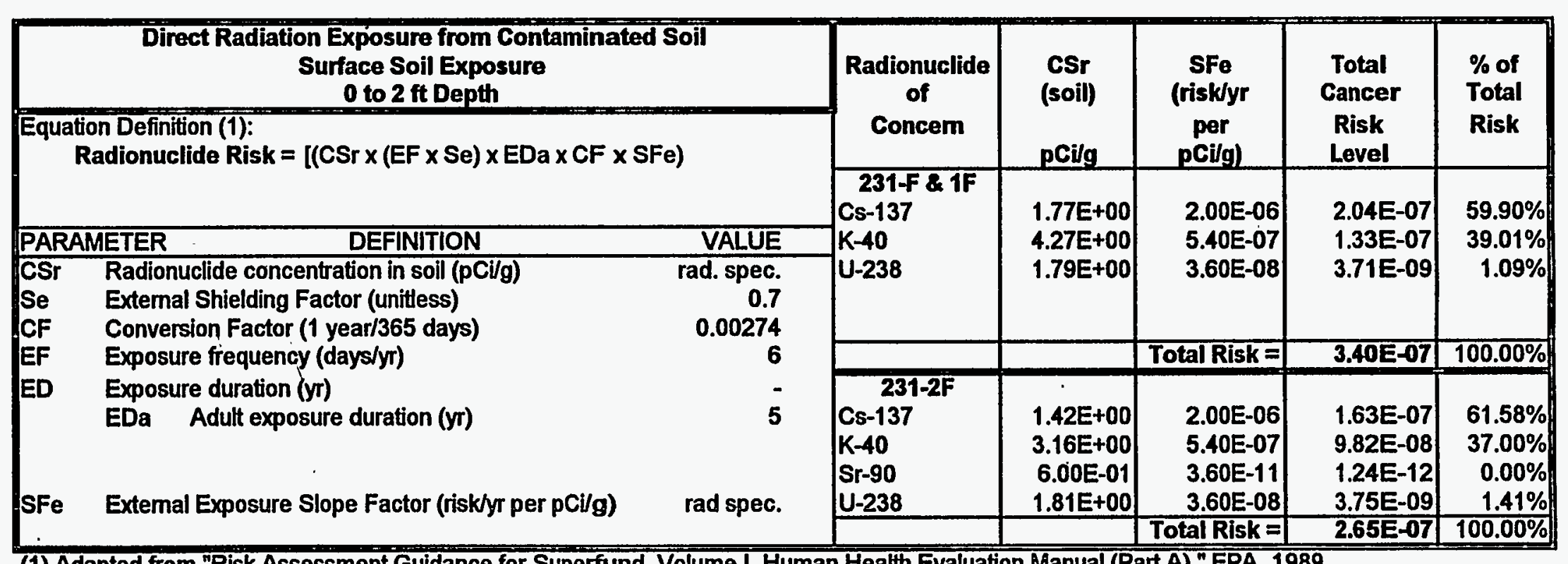

(1) Adapted from "Risk Assessment Guidance for Superfund, Volume I, Human Health Evaluation Manual (Part A)," EPA, 1989. 
Appendix D.57

F-Area Burning/Rubble Pits 231-F/1F and Rubble Pit 2F

On-Unit Visitor

Radionuclide Cancer Risk from Exposure to Contaminated Surface Water

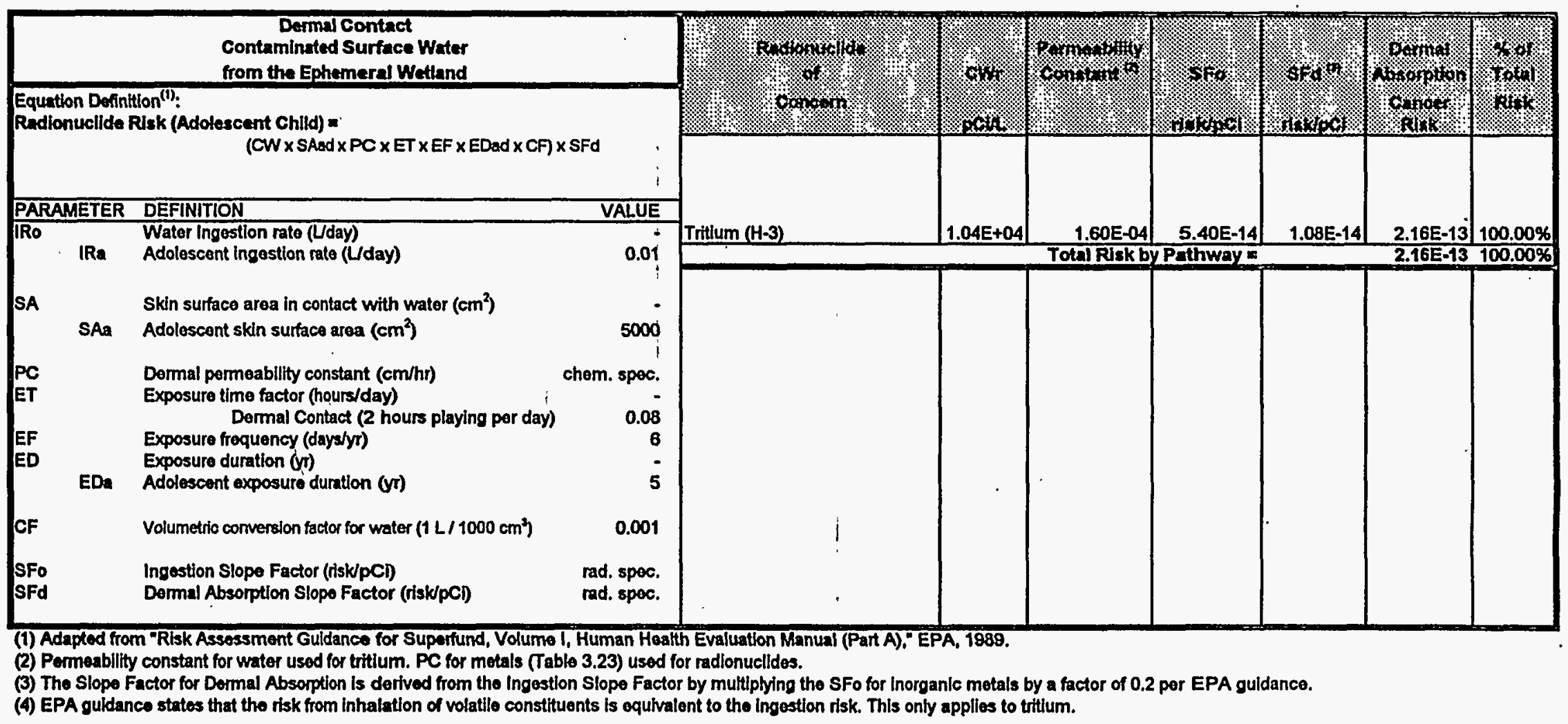


Appendix D.58

F-Area Burning/Rubble Pits 23:1-F/1F and Rubble PIt 2F

On-Unit Visitor

Radionuclide Cancer Risk Based on Ingestion of Sediments

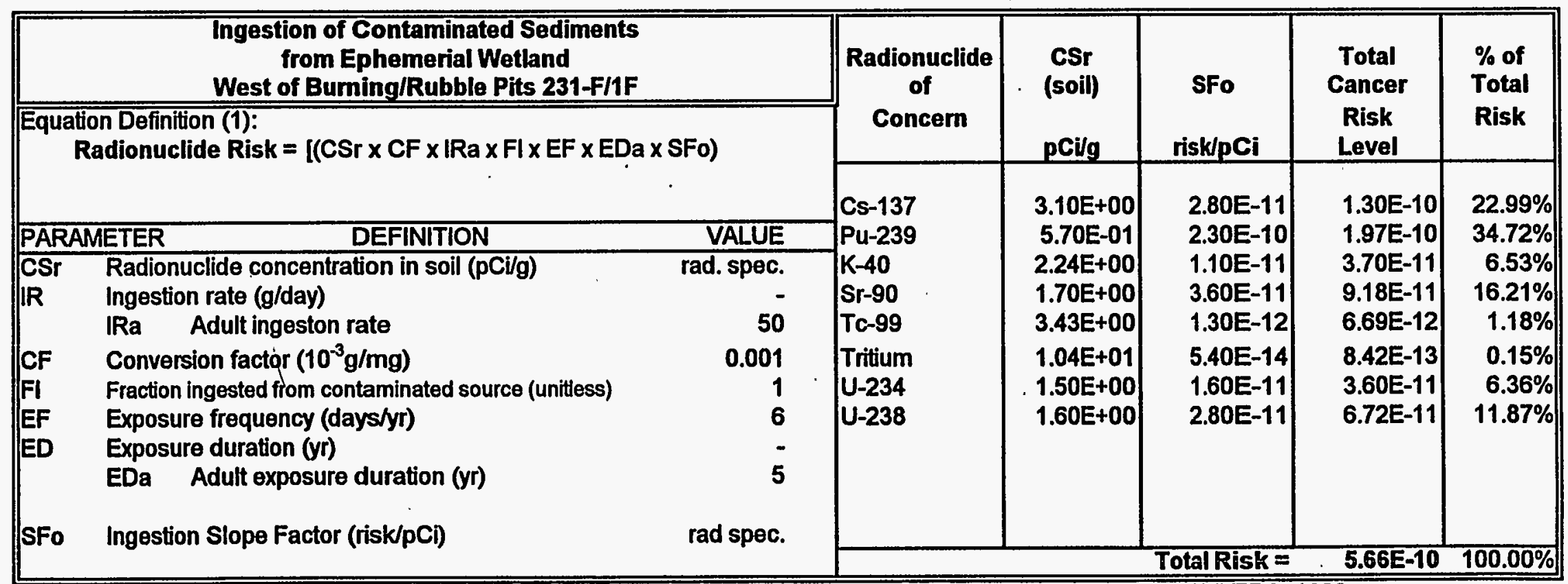

(1) Adapted from "Risk Assessment Guidance for Superfund, Volume I, Human Health Evaluation Manual (Part A)," EPA, 1989. 
Appendix D.59

F-Area Burning/Rubble Pits 231-F/1F and Rubble Pit 2F

On-Unit Visitor

Radionuclide Cancer Risk Based on Dermal Contact with Sediments

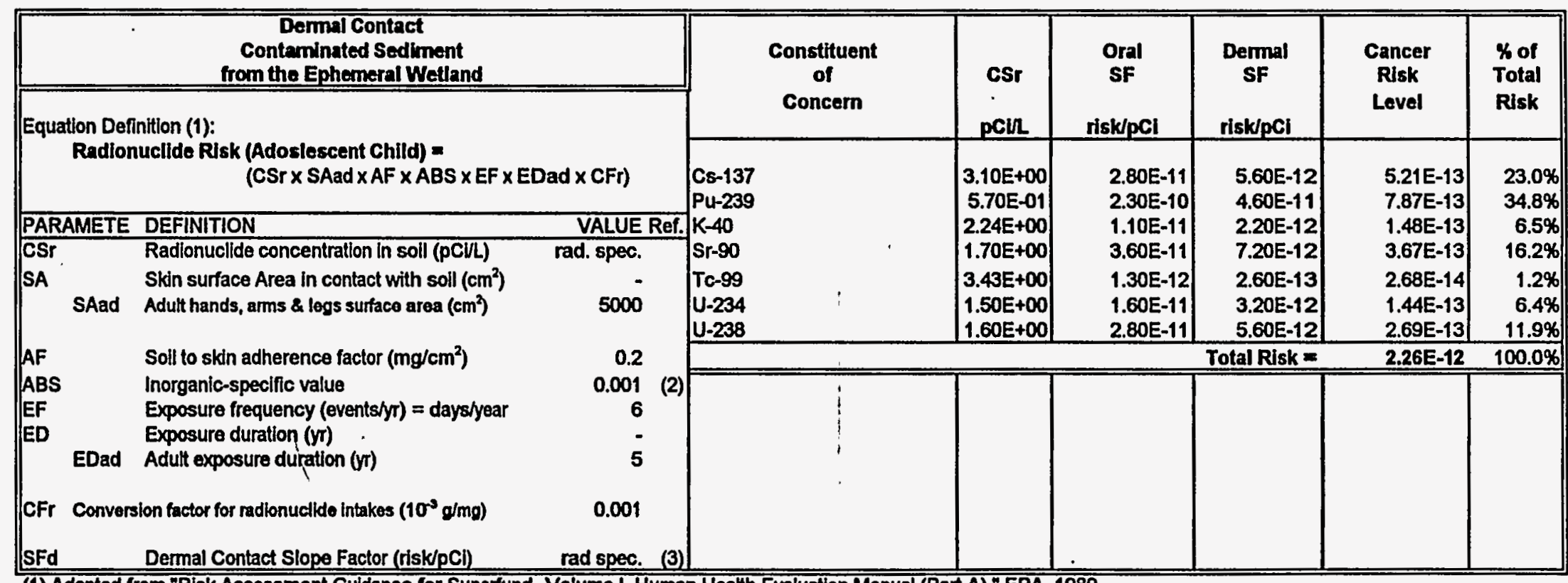

(1) Adapted from "Risk Assessment Guldance for Superfund, Volume 1, Human Health Evaluation Manual (Part A)," EPA, 1989.

(2) ABS for organics $=0.01$; ABS for inorganics $=0.001$.

(3) Dermal Contact Slope Factors were derived from the ingestion SF by the following formula: SFd = SFo $\times 0.2$ 


\section{Appendix D.60}

\section{F-Area Burning/Rubble Pits 231-F/1F and Rubble Pit 2F Future On-Unit Resident Scenario Radionuclide Cancer Risk Based on Ingestion of Soil}

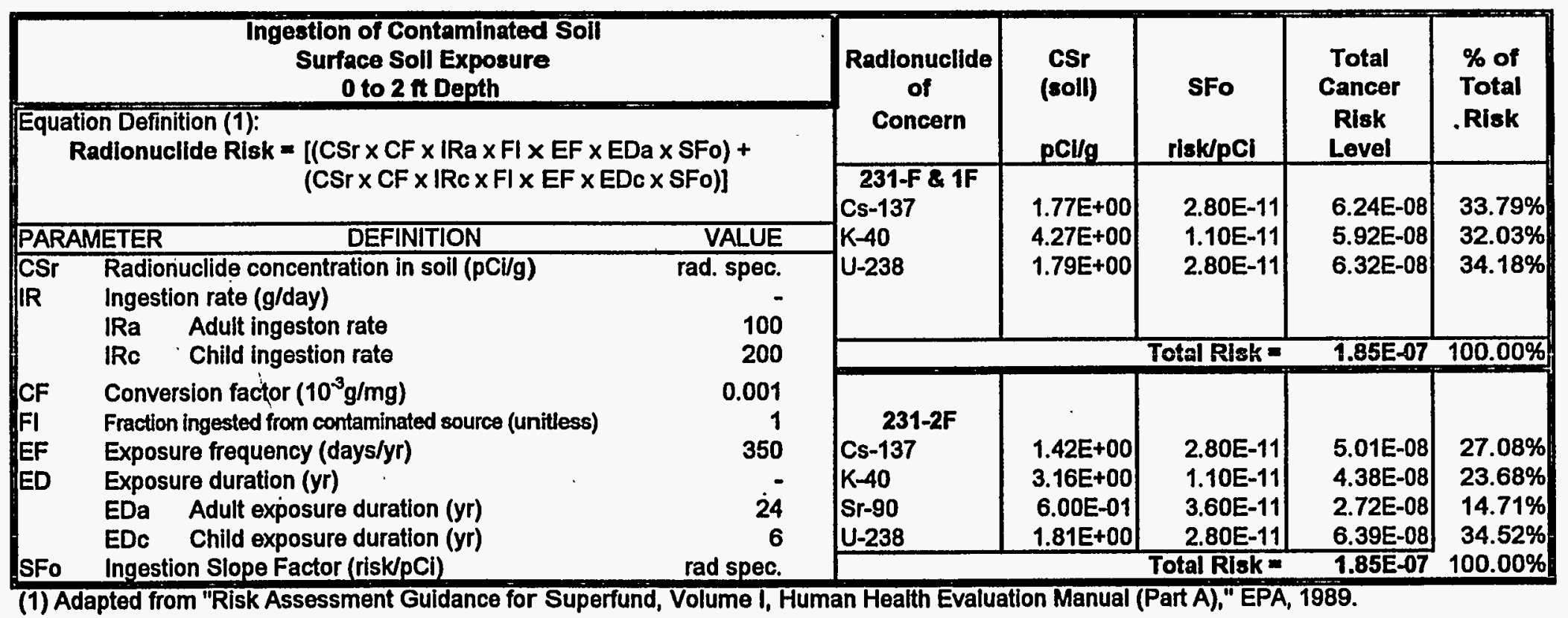

(1) Adapted from "Risk Assessment Guidance for Superfund, Volume I, Human Health Evaluation Manual (Part A)," EPA, 1989. 
Appendix 0.61

F-Area Buming/Rubble Pits 231-F/1F and Rubble Pit 2F

Future On-Unit Resident Scenario

Radionuclide Cancer Risk Based on Dermal Contact with Soil

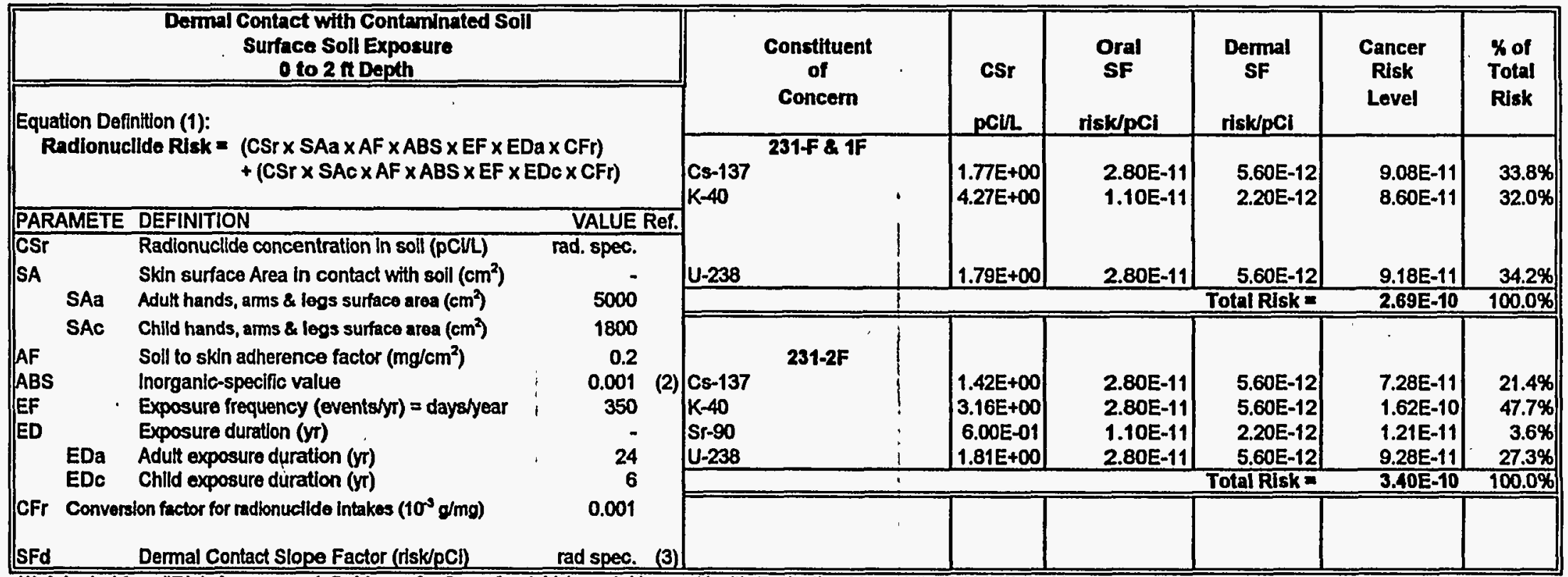

(1) Adapled from "Risk Assessment Guldance for Superfund, Volume I, Human Health Evaluation Manual (Part A)," EPA, 1989.

(2) ABS for organics $=0.01 ;$ ABS for inorganics $=0.001$.

(3) Dermal Contact Slope Factors were derived from the Ingestion SF by the following formula: SFd $=S F 0 \times 0.2$ 
Appendix D.62

F-Area Burning/Rubble Pits 231-F/1F and Rubble Pit 2F

Future On-Unit Resident Scenario

Radionuclide Cancer Risk Based on Inhalation of Contaminated Soll Particulates

\begin{tabular}{|c|c|c|c|c|c|c|c|c|}
\hline \multicolumn{3}{|c|}{\begin{tabular}{|c|} 
Inhalation of Windblown Dust from Contaminated Soll \\
Surface Soll Exposure \\
0 to $2 \mathrm{ft}$ Depth \\
\end{tabular}} & \multirow{2}{*}{$\begin{array}{l}\text { Constituent } \\
\text { of } \\
\text { Concern }\end{array}$} & \multirow{2}{*}{$\begin{array}{l}\text { Csr } \\
\text { pcug }\end{array}$} & \multirow[t]{2}{*}{ 1/PEF } & \multirow{2}{*}{ SFis/pci } & \multirow{2}{*}{$\begin{array}{l}\text { Cancer } \\
\text { Rlsk } \\
\text { Level }\end{array}$} & \multirow{2}{*}{$\begin{array}{l}\% \text { of } \\
\text { Total } \\
\text { Rlsk }\end{array}$} \\
\hline \multirow{2}{*}{\multicolumn{3}{|c|}{$\begin{array}{l}\text { Equation Definition (1): } \\
\begin{aligned} \text { Radlonucllde RIsk = } & (\operatorname{CSr} \times \text { CF } \times \text { EF } \times \text { EDa } \times \text { IRa } \times(1 / P E F) \times S F i) \\
& +(\operatorname{CSr} \times \text { CF } \times \text { EF } \times \text { EDc } \times \text { IRc } \times(1 / P E F) \times S F i)\end{aligned}\end{array}$}} & & & & & & \\
\hline & & & \multirow{3}{*}{$\begin{array}{l}\mathrm{C}-137 \\
\mathrm{~K}-40 \\
\mathrm{U}-238\end{array}$} & \multirow{3}{*}{$\begin{array}{l}1.77 E+\infty 0 \\
4.27 E+00 \\
1.79 E+\infty 0\end{array}$} & \multirow{3}{*}{$\begin{array}{l}4.63 E-10 \\
4.63 E-10 \\
4.63 E-10\end{array}$} & \multirow{2}{*}{$\begin{array}{l}1.90 \mathrm{E}-11 \\
7.60 \mathrm{E}-12 \\
5.20 \mathrm{E}-08\end{array}$} & \multirow{2}{*}{$\begin{array}{l}1.41 \mathrm{E}-13 \\
1.36 \mathrm{E}-13 \\
3.91 \mathrm{E}-10\end{array}$} & \multirow{2}{*}{$\begin{array}{r}0.0 \% \\
0.0 \% \\
99.9 \%\end{array}$} \\
\hline \multirow{2}{*}{\begin{tabular}{|l} 
PARAMETER \\
CSr \\
CF
\end{tabular}} & DEFINITION & \multirow{2}{*}{$\begin{array}{r}\text { VALUE } \\
\text { rad. spec. } \\
1000\end{array}$} & & & & & & \\
\hline & $\begin{array}{l}\text { Radionuclide concentration in soil (pCi/g) } \\
\text { Conversion factor }(1000 \mathrm{~g} / \mathrm{kg})\end{array}$ & & & & & \multicolumn{2}{|c|}{ Total RIsk $=3.91 \mathrm{E}-10$} & $100.0 \%$ \\
\hline $\begin{array}{ll} & \text { EDa } \\
\text { IRa } & \text { EDc } \\
\text { IRc }\end{array}$ & $\begin{array}{l}\text { Exposure frequrency (days/year) } \\
\text { Exposure duration (yr) } \\
\text { Adult exposure duration (yr) } \\
\text { Child exposure duration }(\mathrm{yr}) \\
\text { Adult inhalation rate }(\mathrm{m} 3 / \mathrm{hr}) \\
\text { Child Inhalation rate }(\mathrm{m} 3 / \mathrm{hr})\end{array}$ & $\begin{array}{r}350 \\
- \\
24 \\
6 \\
0.83 \\
1\end{array}$ & $\begin{array}{l}\text { Cs-137 } \\
K-40 \\
\text { Sr-90 } \\
U-238 \\
\end{array}$ & $\begin{array}{r}1.42 E+\infty 0 \\
3.16 E+00 \\
6.00 E-01 \\
1.81 E+00\end{array}$ & $\begin{array}{l}3.11 E-10 \\
3.11 E-10 \\
3.60 E-11 \\
3.11 E-10 \\
\end{array}$ & $\begin{array}{r}\text { 1.90E-11 } \\
7.60 \mathrm{E}-12 \\
7.60 \mathrm{E}-12 \\
5.20 \mathrm{E}-08 \\
\text { Total RIsk }=\end{array}$ & $\begin{array}{l}7.60 E-14 \\
6.77 E-14 \\
1.49 E-15 \\
2.65 E-10 \\
2.65 E-10 \\
\end{array}$ & $\begin{array}{r}0.0 \% \\
0.0 \% \\
0.0 \% \\
99.9 \% \\
100.0 \% \\
\end{array}$ \\
\hline PEF & $\begin{array}{l}\mathrm{m}^{3} / \mathrm{kg} \\
\text { Pits } 231-\mathrm{F} \& 1 \mathrm{~F} \\
\text { Pit 231-2F }\end{array}$ & $\begin{array}{l}2.16 E+09 \\
3.22 E+09 \\
\text { rad spec. }\end{array}$ & & & & & & \\
\hline
\end{tabular}

(1) Adapted from "Risk Assessment Guidance for Superfund, Volume I, Human Health Evaluation Manual (Part A)," EPA, 1989. 


\section{Appendix D.63 \\ F-Area Burning/Rubble Pits 231-F/1F and Rubble Pit 2F Future On-Unit Resident Scenario Radionuclide Cancer Risk Based on Direct Radiation from Surface Soll}

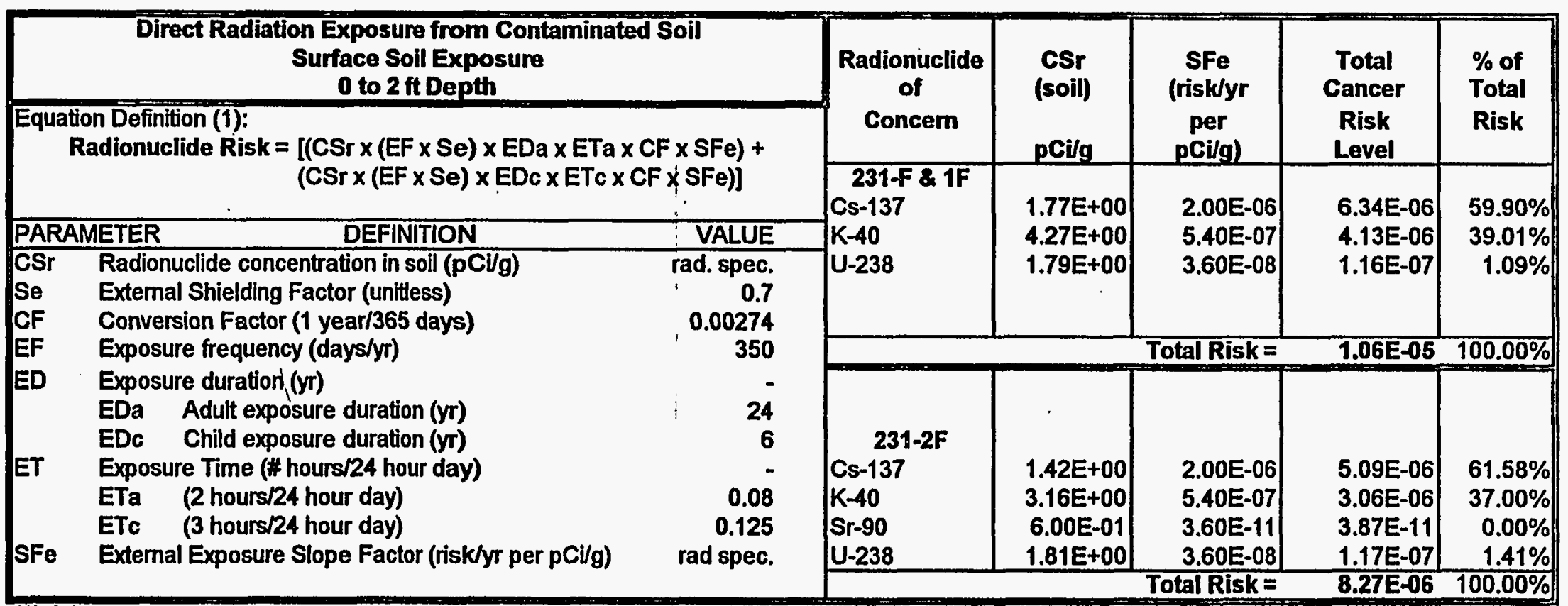

(1) Adapted from "Risk Assessment Guidance for Superfund, Volume I, Human Health Evaluation Manual (Part A)," EPA, 1989. 
Appendix D.64

F-Area Burning/Rubble PIts 231-F/1F and Rubble Pit 2F

Future On-Unit Resident Scenario

Radionuclide Cancer Risk Based on Ingestion of Homegrown Produce

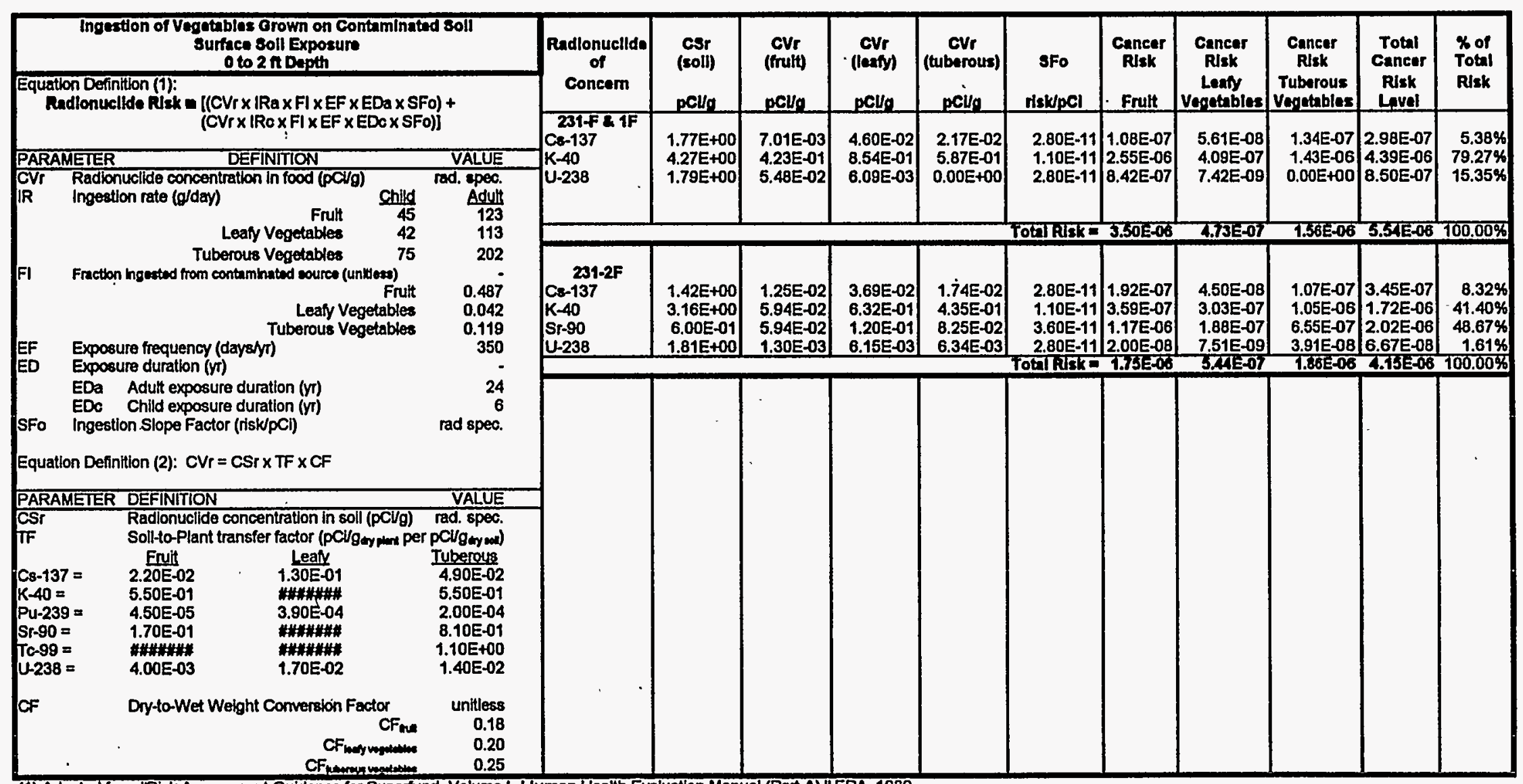

(1) Adapted from "Risk Assessment Guidance for Superfund, Volume I, Human Health Evaluation ManLal (Pait A)," EPA, 1889. 
Appendix D.65

F-Area Burning/Rubble Pits 231-F/1F and Rubble Pit 2F

Future On-Unit Resident Scenario

Radionuclide Cancer Risk Based on Ingestion of Soil

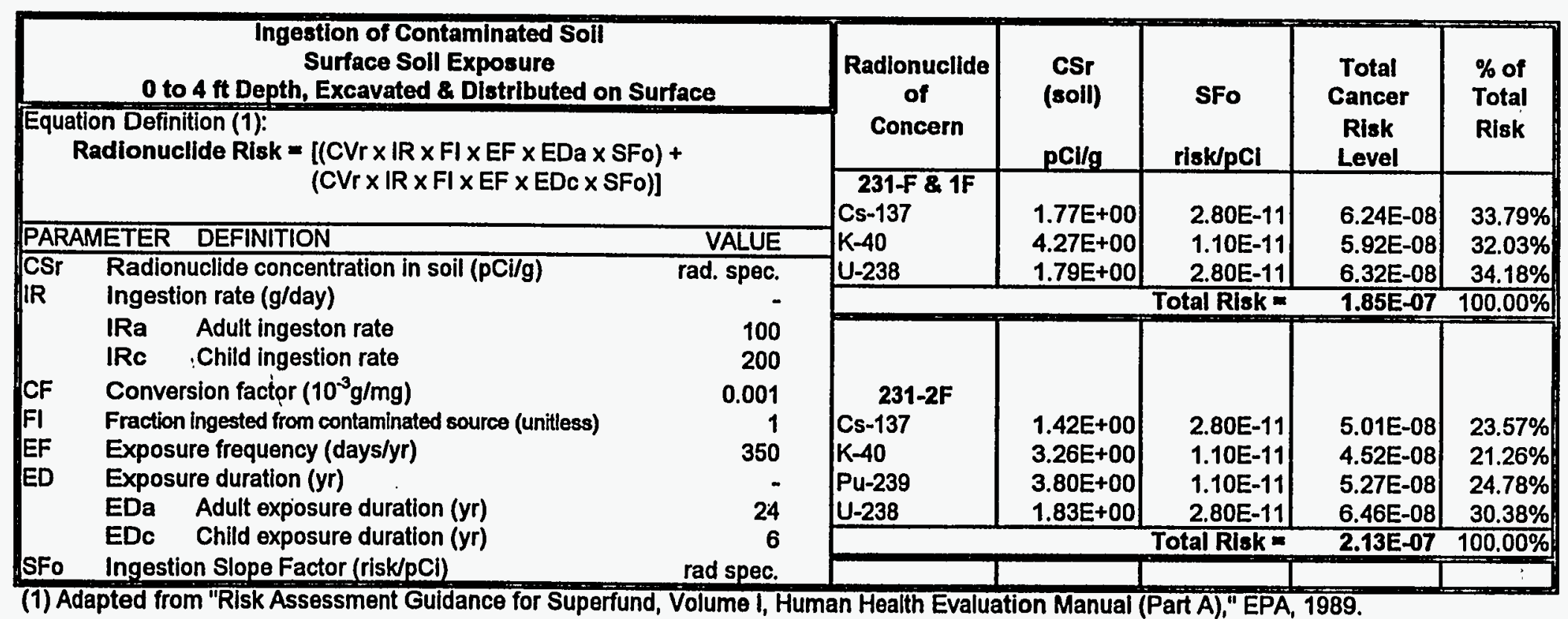


Appendix D.66

F-Area Buming/Rubble Pits 231-F/1F and Rubble Pit 2F

Future On-Unit Resident Scenario

Radionuclide Cancer Risk Based on Dermal Contact with Soil

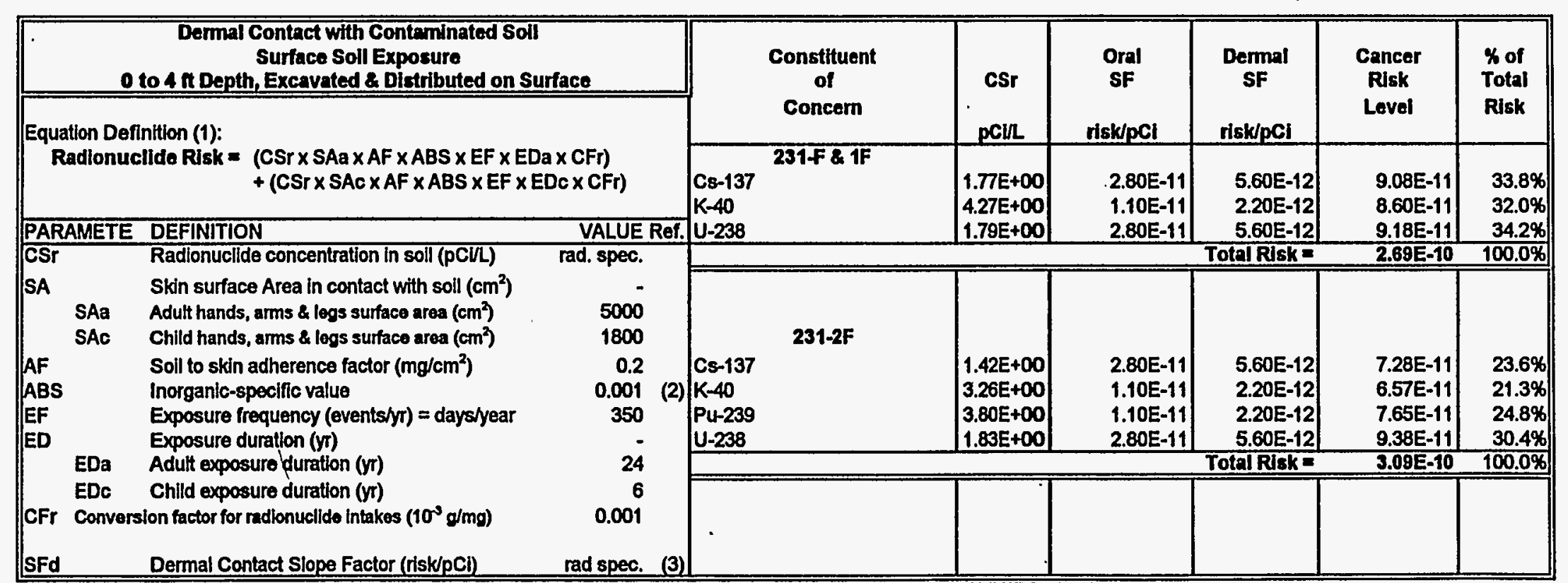

(1) Adapted from "Risk Assessment Guldance for Superfund, Volume I, Human Health Evaluation Manual (Part A)," EPA, 1989.

(2) ABS for organics $=0.01 ;$ ABS for inorganics $=0.001$.

(3) Dermal Contact Slope Factors were derived from the Ingestion SF by the following formula: SFd = SFo $\times 0.2$ 
Appendix D.67

F-Area Burning/Rubble Pits 231-F/1F and Rubble Pit 2F

Future On-Unit Resident Scenario

Radionuclide Cancer Risk Based on Inhalation of Contaminated Soil Particulates

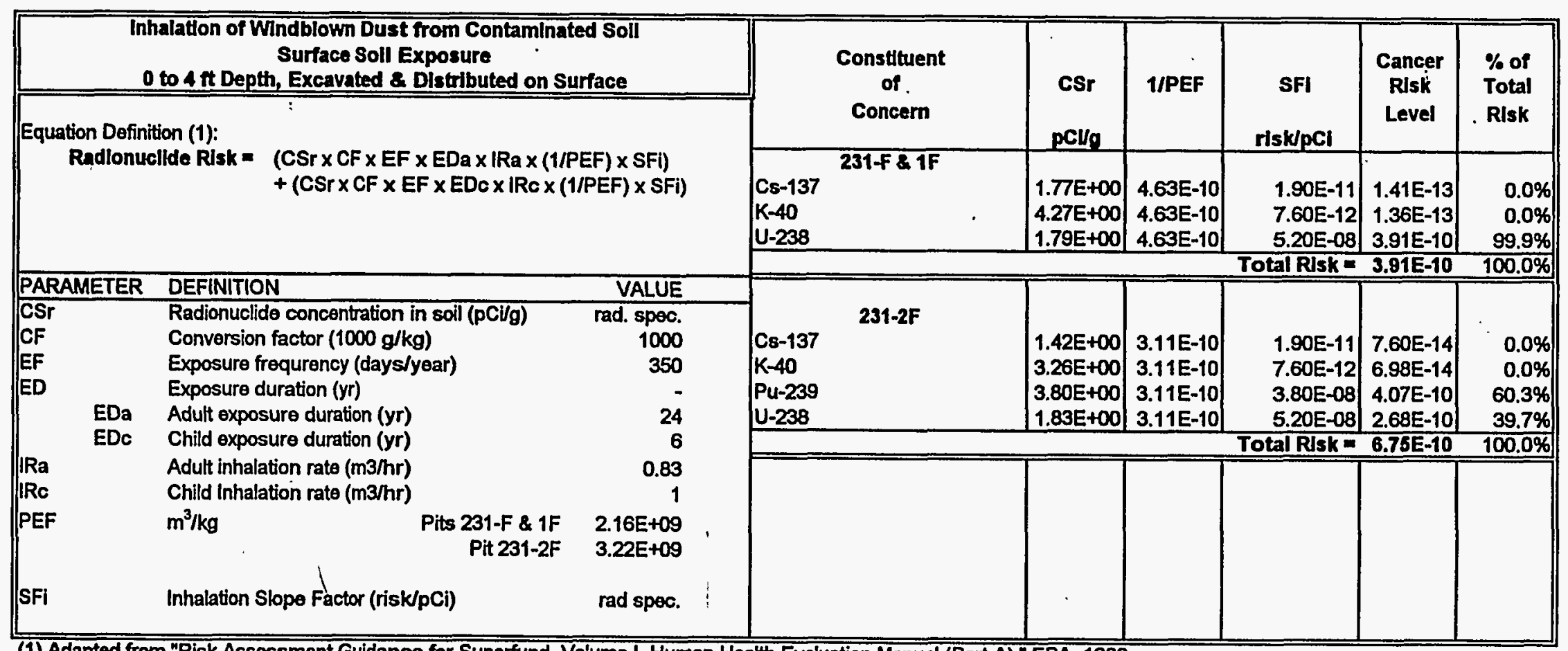

(1) Adapted from "Risk Assessment Guidance for Superfund, Volume 1, Human Health Evaluation Manual (Part A)," EPA, 1989. 


\section{Appendix D.68 \\ F-Area Burning/Rubble Pits 231-F/1F and Rubble PIt 2F \\ Future On-Unit Resident Scenario \\ Radionuclide Cancer Risk Based on Direct Radiation from Surface Soil}

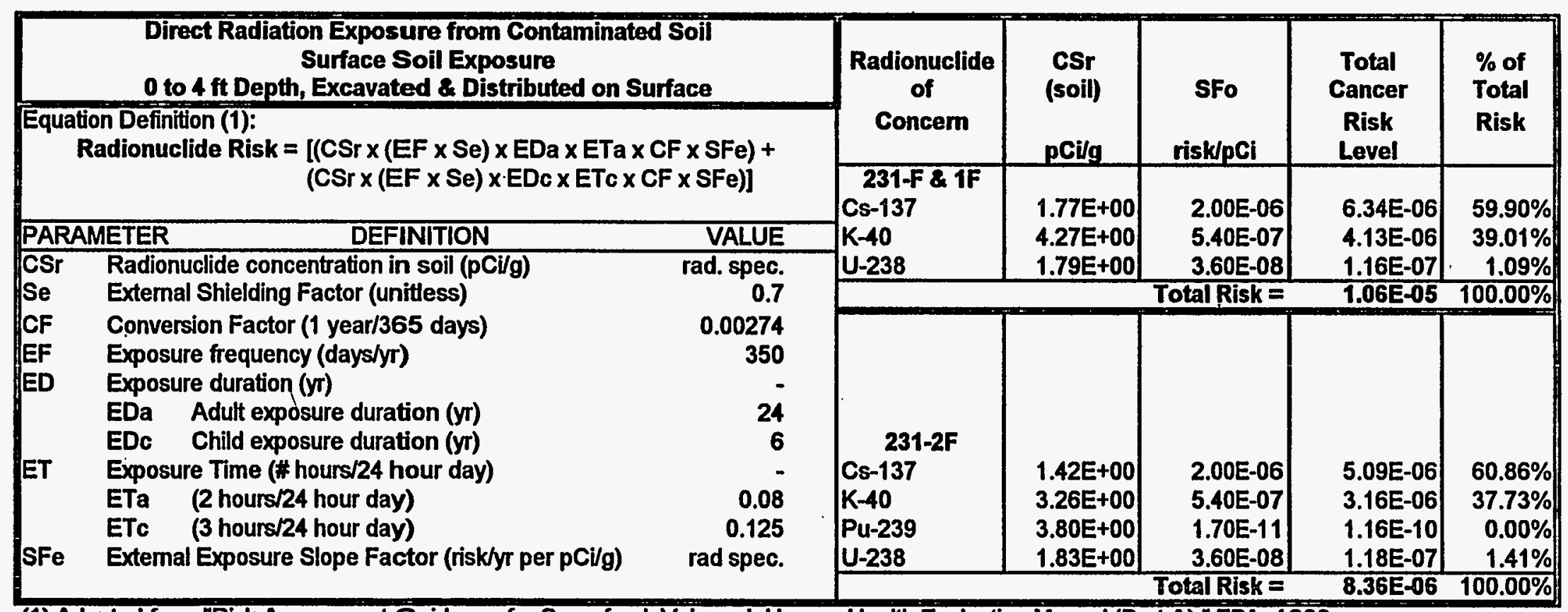

(1) Adapted from "Risk Assessment Guidance for Superfund, Volume I, Human Health Evaluation Mianual (Part A)," EPA, 1989. 
Appendix D.69

F-Area Buming/Rubble Pits 231-F/1F and Rubble Pit 2F Future On-Unit Resident Scenario

Radionuclide Cancer Risk Based on Ingestion of Homegrown Produce

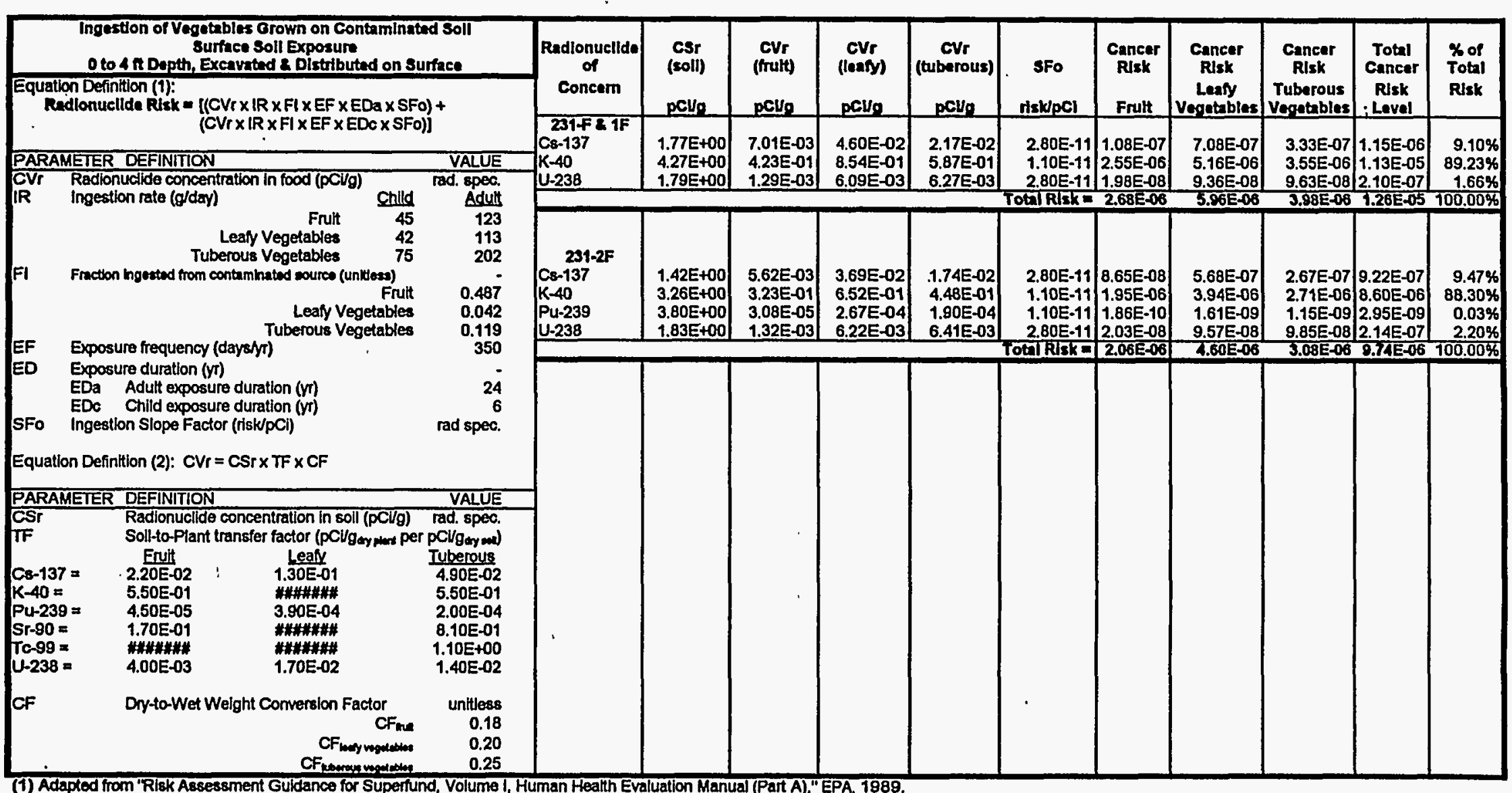




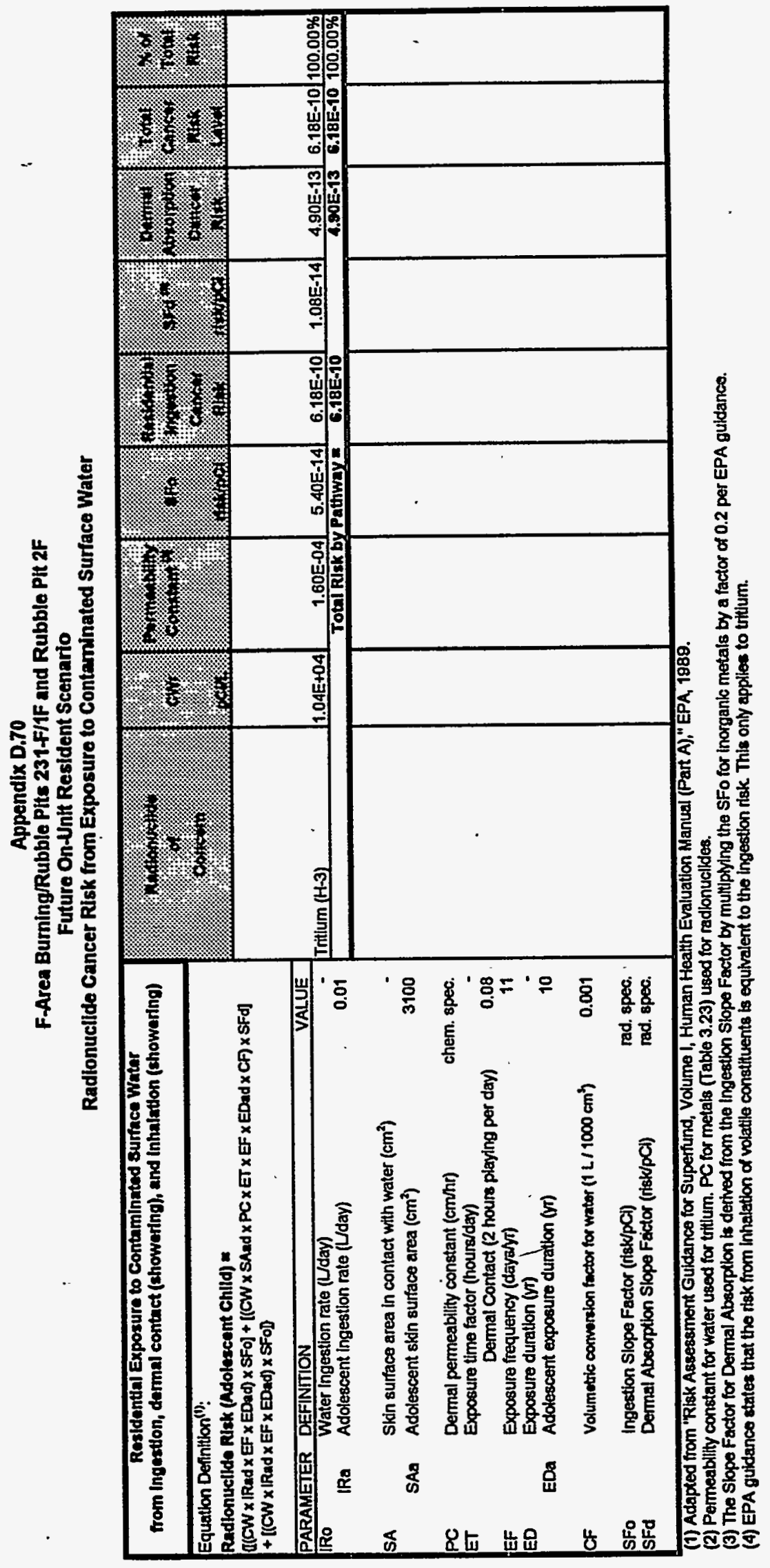

$\underset{m}{=}$ 


\section{Appendix D.71 \\ F-Area Burning/Rubble Pits 231-F/1F and Rubble Pit 2F \\ Future On-Unit Resident Scenario \\ Radionuclide Cancer Risk Based on Ingestion of Sediment}

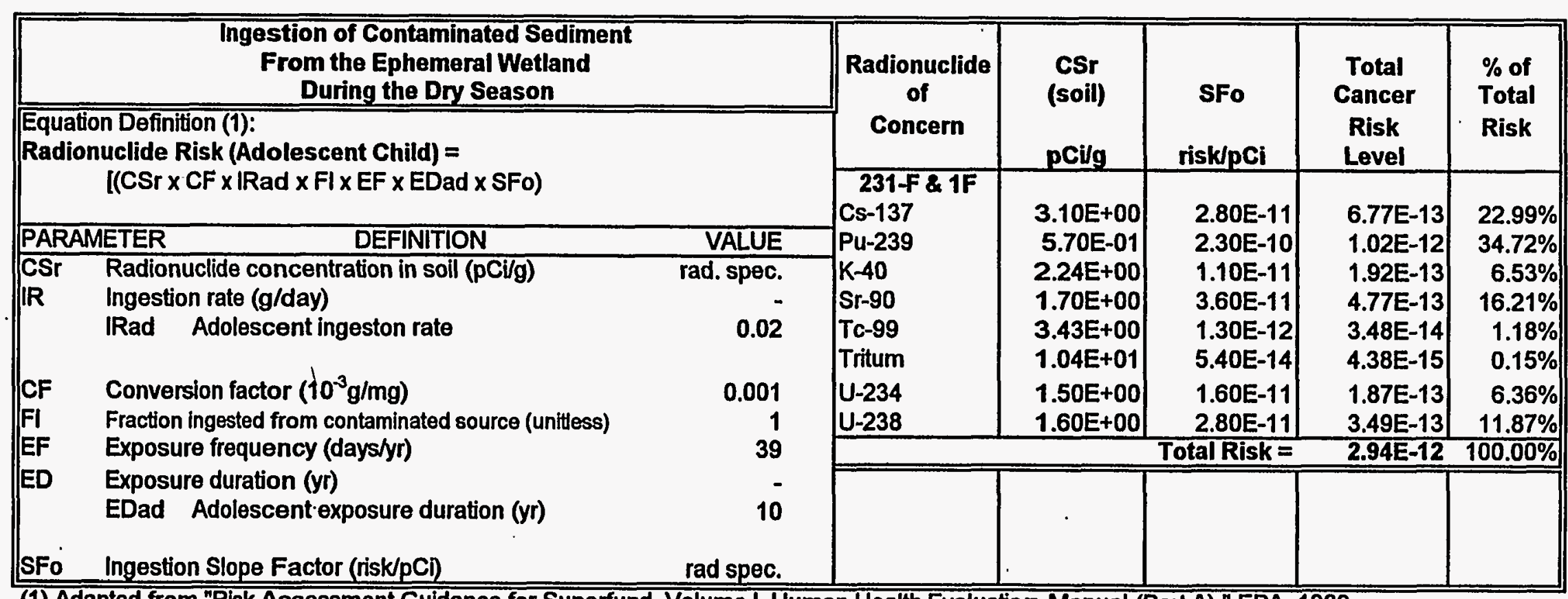

(1) Adapted from "Risk Assessment Guidance for Superfund, Volume I, Human Health Evaluation Manual (Part A), "EPA, 1989. 
Appendix D.72

F-Area Buming/Rubble Pits 231-F/1F and Rubble Pit 2F

Future On-Unit Resident Scenario

: Radionuclide Cancer Risk Based on Dermal Contact with Sediments

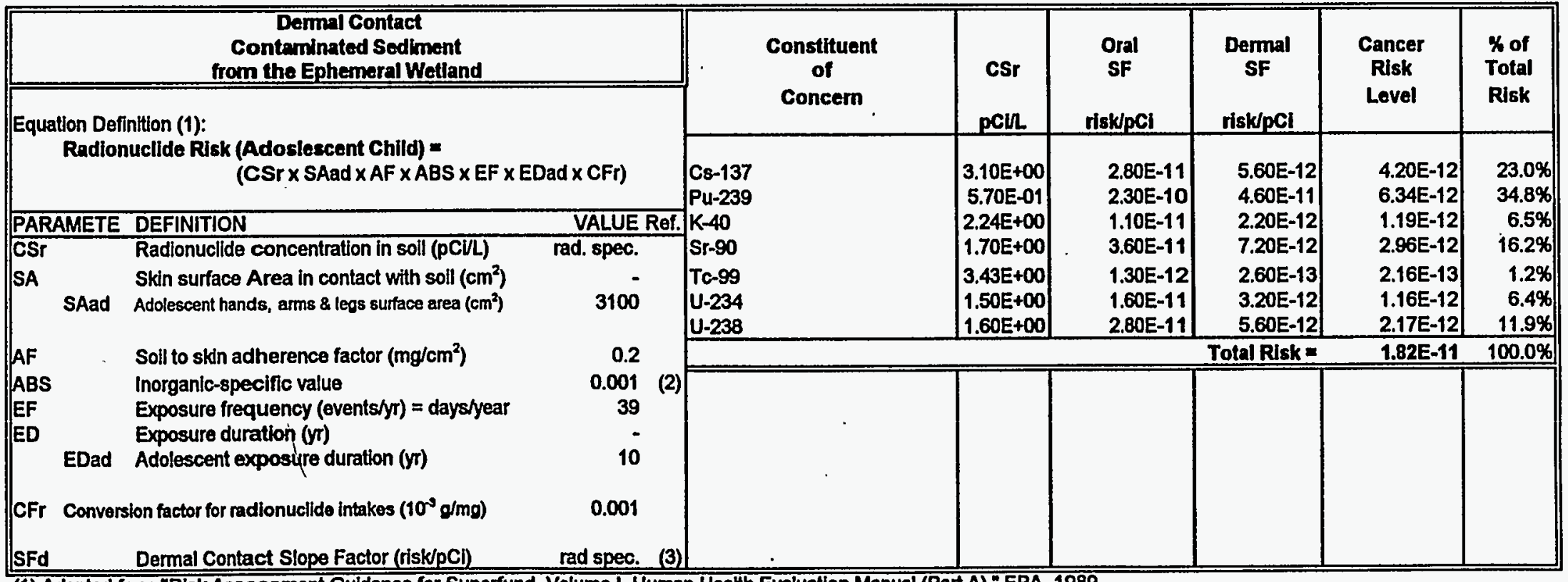

(1) Adapted from "Risk Assessment Guldance for Superfund, Volume I, Human Health Evaluation Manual (Part A)," EPA, 1989.

(2) $A B S$ for organlcs $=0.01 ; A B S$ for inorganics $=0.001$.

(3) Dermal Contact Slope Factors were derived from the ingestion SF by the following formula: SFd = SFo $\times 0.2$ 
Appendix 0.73

F-Aren Buming/Rubble Pits 231 FHF and Rubble Ptt 2F

Future OntUnk Resldent scennito

Radionucilde Cancer Rlsk Based on Exposure to Groundwater

i

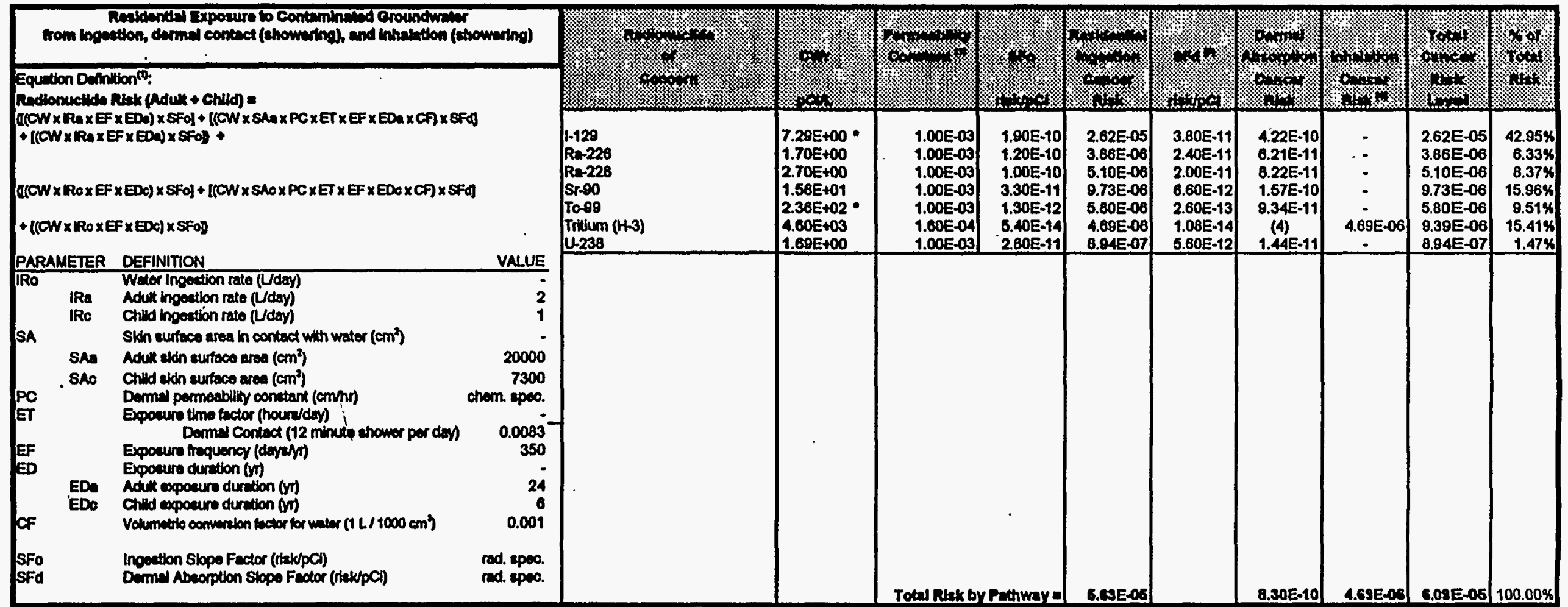

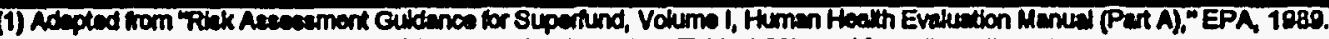

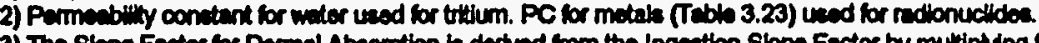

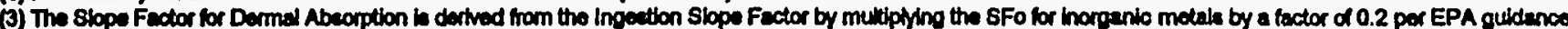

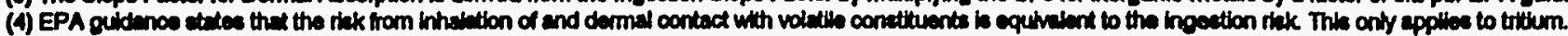

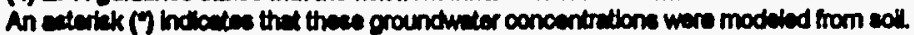




\section{Appendix 0.74}

\section{F-Area Buming/Rubble Pits 231-F/1F and Rubble Pit 2F}

Future On-Unit Worker Scenario

Radionuclide Cancer Risk Based on Ingestion of Soil

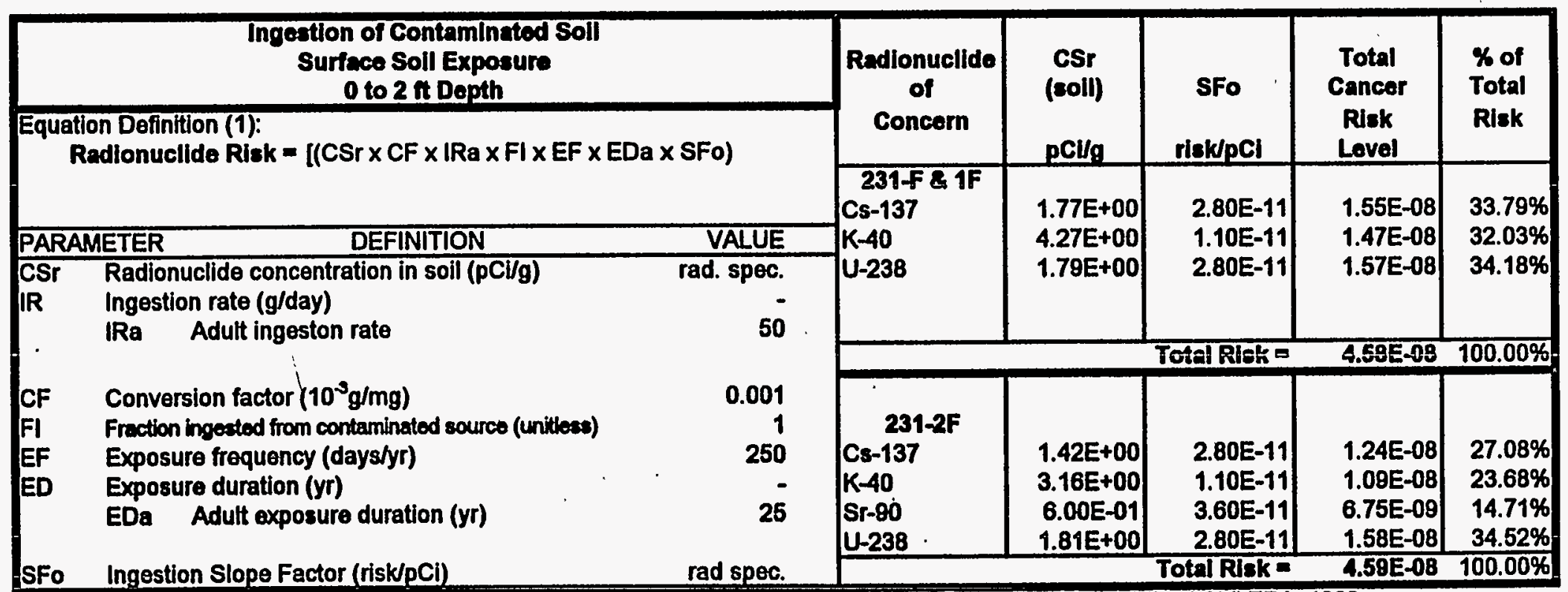

(1) Adaptẹd from "Risk Assessment Guidance for Superfund, Volume I, Human Health Evaluation Manual (Part A)," EPA, 1989. 
Appendix $\mathbf{D . 7 5}$

F-Area Buming/Rubble Pits 231-F/1F and Rubble Pit 2F

Future On-Unit Worker Scenario

Radionuclide Cancer Risk Based on Dermal Contact with Soil

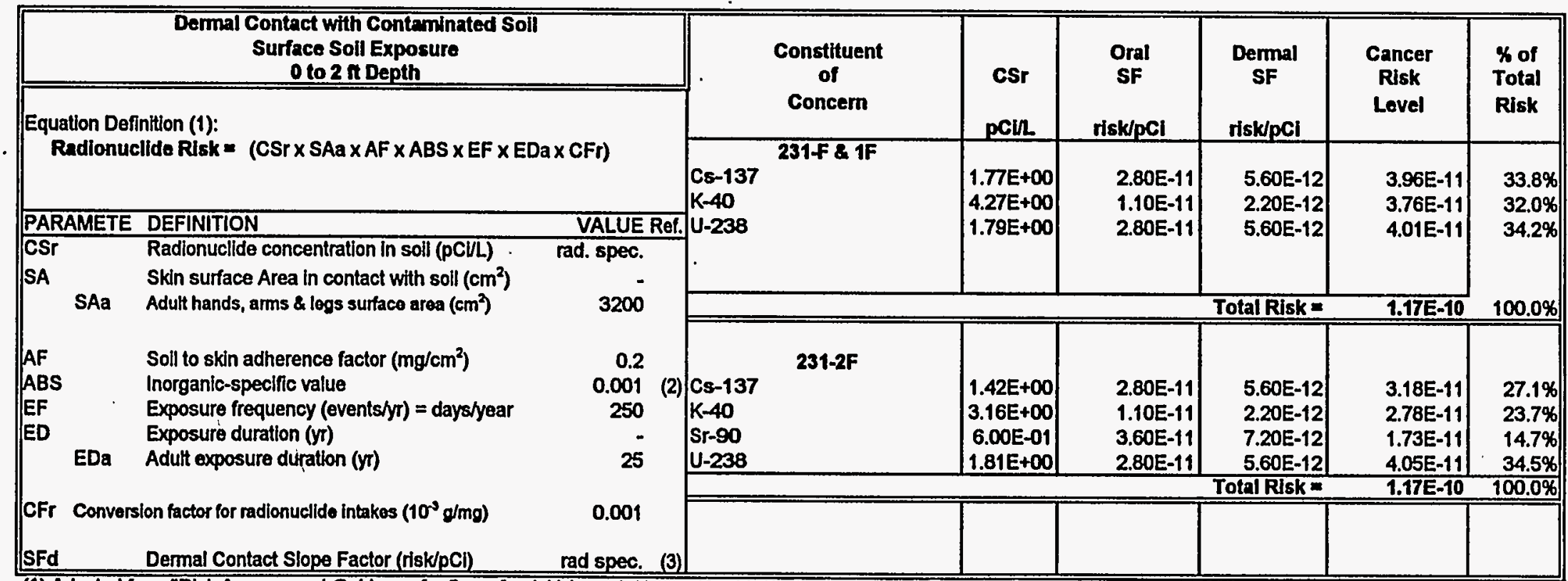

(1) Adapted from "Rlsk Assessment Guldance for Superfund, Volume I, Human Health Evaluation Manual (Part A)," EPA, 1989.

(2) ABS for organics $=0.01$; ABS for inorganics $=0.001$.

(3) Dermal Contact Slope Factors were derived from the Ingestion SF by the following formula: SFd $=$ SFo $\times 0.2$ 
Appendix D.76

F-Area Burning/Rubble Pits 231-F/1F and Rubble Pit 2F

Future On-Unit Worker Scenario

Radionuclide Cancer Risk Based on Inhalation of Contaminated Soll Particulates

\begin{tabular}{|c|c|c|c|c|c|c|c|c|}
\hline & $\begin{array}{c}\text { alation of Windblown Dust from Contaminat } \\
\text { Surface Soll Exposure } \\
\text { O to } 2 \text { ft Depth }\end{array}$ & Soll & $\begin{array}{c}\text { Constituent } \\
\text { of }\end{array}$ & & 1/PEF & & $\begin{array}{l}\text { Cancer } \\
\text { RI'sk }\end{array}$ & $\begin{array}{l}\% \text { of } \\
\text { Total }\end{array}$ \\
\hline Equation Defini & on (1): & & & pcisg & & riskJpcl & & \\
\hline Radloníc & Ide RIsk $=(C S r \times C F \times E F \times E D a \times I R a \times(1 / /$ & F) $\times$ SFi) & 231-F\& IF & & & & & \\
\hline PARAMETER & DEFINITION & VALUE & & & & & & \\
\hline CSr & Radionuclide concentration in soil $(\mathrm{pCi} / \mathrm{g})$ & rad. spec. & & & & & & \\
\hline CF & Conversion factor (1000 g/kg) & 1000 & & & & Total RIsk = & $6.74 E-10$ & $100.0 \%$ \\
\hline $\begin{array}{l}\text { EF } \\
\text { ED }\end{array}$ & $\begin{array}{l}\text { Exposure frequrency (days/year) } \\
\text { Exposure duration (yr) }\end{array}$ & 250 & 231-2F & & & & & \\
\hline IRa & $\begin{array}{l}\text { Adult exposure duration }(\mathrm{yr}) \\
\text { Adult inhalation rate }(\mathrm{m} 3 / \mathrm{hr})\end{array}$ & $\begin{array}{r}25 \\
2.5\end{array}$ & $\begin{array}{l}\mathrm{Cs}-137 \\
\mathrm{~K}-40 \\
\mathrm{Sr}-90 \\
\mathrm{U}-238 \\
\end{array}$ & $\begin{array}{r}1.42 E+00 \\
3.16 E+00 \\
6.00 E-01 \\
1.81 E+00 \\
\end{array}$ & $\begin{array}{l}3.11 E-10 \\
3.11 E-10 \\
3.11 E-10 \\
3.11 E-10 \\
\end{array}$ & $\begin{array}{l}1.90 \mathrm{E}-11 \\
7.60 \mathrm{E}-12 \\
3.60 \mathrm{E}-11 \\
5.20 \mathrm{E}-08\end{array}$ & $\begin{array}{l}1.31 \mathrm{E}-13 \\
1.17 \mathrm{E}-13 \\
1.05 \mathrm{E}-13 \\
4.57 \mathrm{E}-10 \\
\end{array}$ & $\begin{array}{r}0.0 \% \\
0.0 \% \\
0.0 \% \\
99.9 \% \\
\end{array}$ \\
\hline PEF & Pits 231-F \& 1F & $2.16 E+09$ & & & & Total Risk = & 4.57E-10 & $100.0 \%$ \\
\hline SFi & Inhalation SlopolFactor (risk/pCi) & $\begin{array}{l}3.22 \mathrm{E}+09 \\
\mathrm{rad} \text { spoc. }\end{array}$ & & & & & & \\
\hline
\end{tabular}

(1) Adapted from "Risk Assessment Guidance for Superfund, Volume I, Human Health Evaluation Manual (Part A)," EPA, 1889. 
Appendix D.77

F-Area Burning/Rubble Pits 231-F/1F and Rubble Pit 2F

Future On-Unit Worker Scenario

Radionuclide Cancer Risk Based on Direct Radiation from Surface Soil

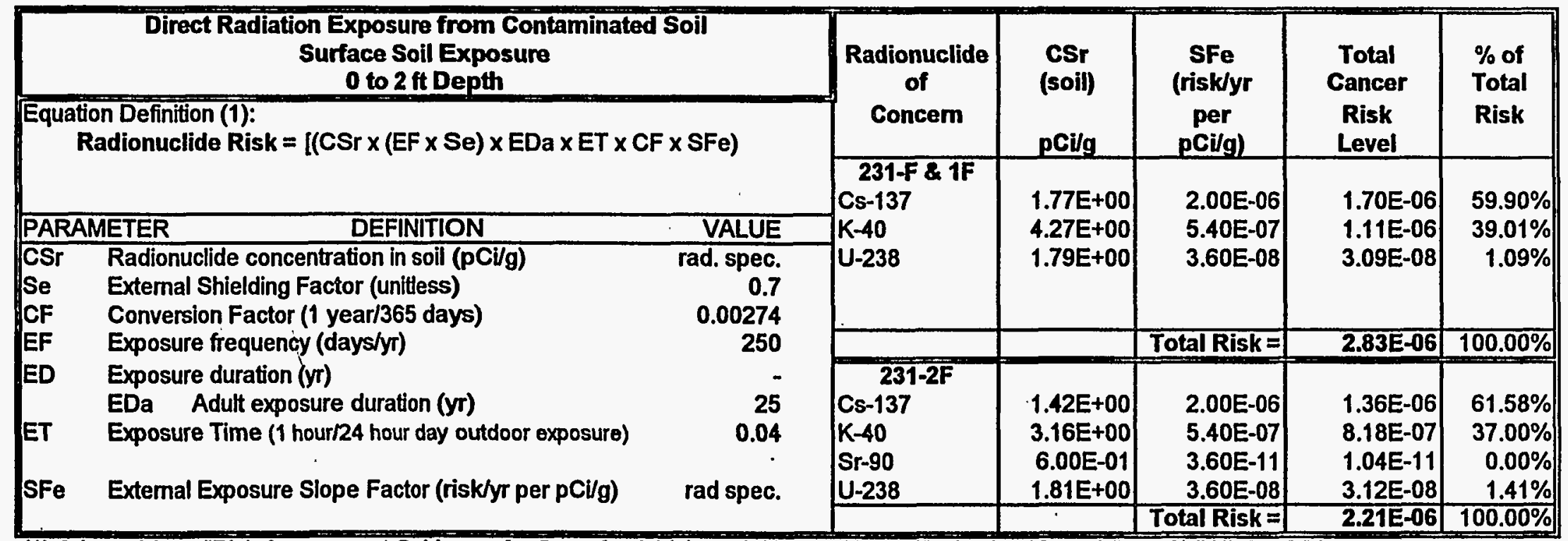

(1) Adapted from "Risk Assessment Guidance for Superfund, Volume I, Human Health Evaluation Manual (Part A)," EPA, 1989. 
Appendix $\mathrm{D.78}$

F-Area Burning/Rubble Pits 231-F/1F and Rubble Pit 2F

Future On-Unit Worker Scenario

Radionuclide Cancer Risk Based on Ingestion of Soil

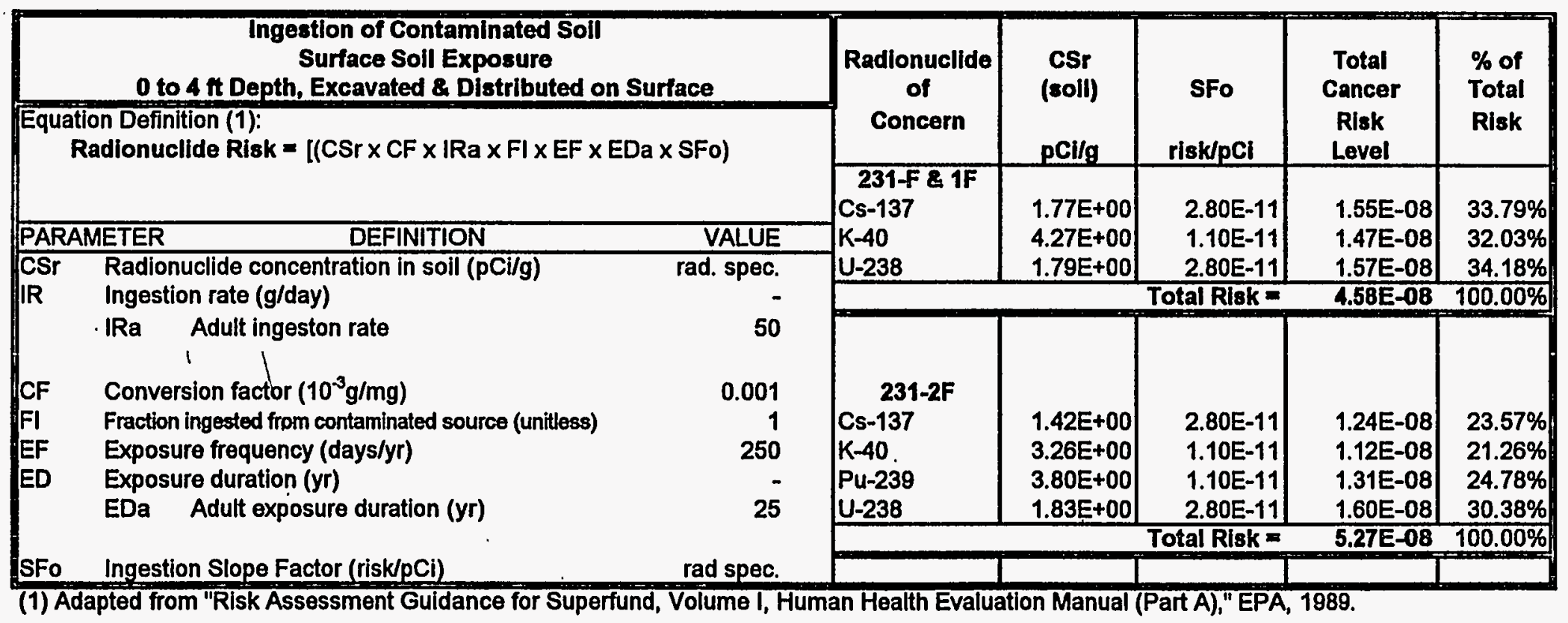


Appendix D.79

F-Area Buming/Rubble Pits 231-F/1F and Rubble Pit 2F

Future On-Unit Worker Scenario

Radionuclide Cancer Risk Based on Dermal Contact with Soil

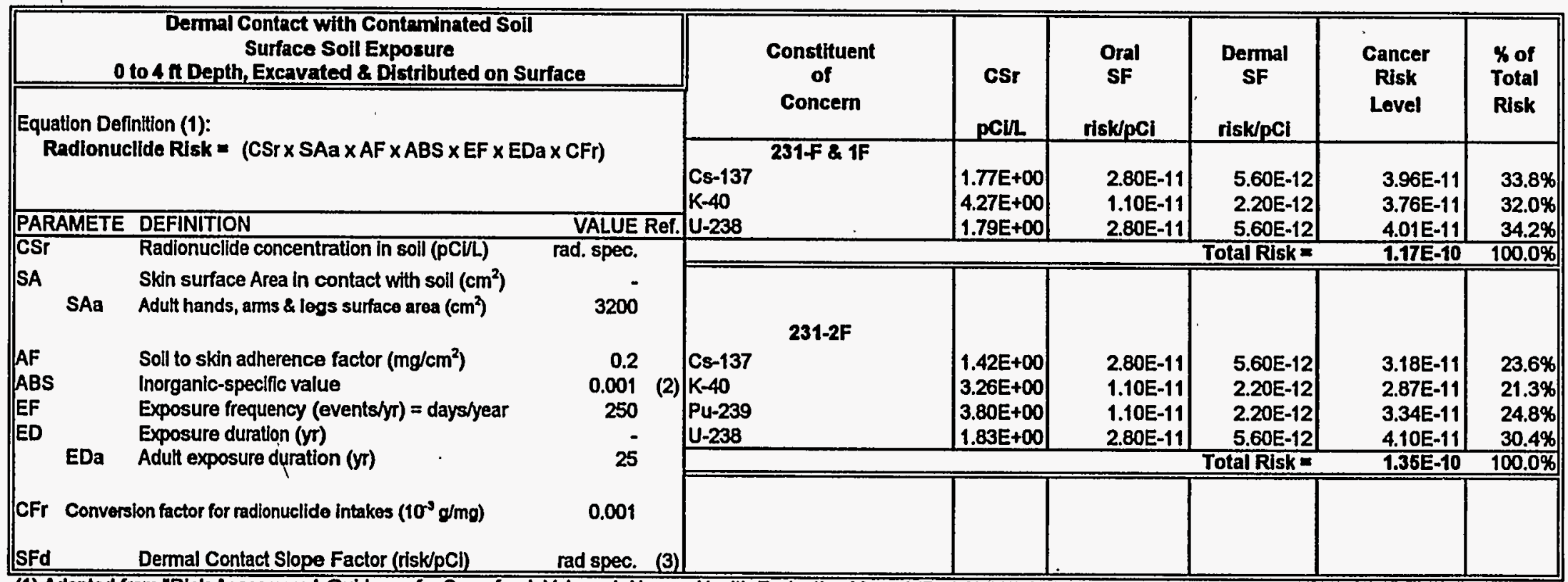

(1) Adapted from "RIsk Assessment Guidance for Superfund, Volume I, Human Health Evaluation Manual (Part A)," EPA, 1989.

(2) $\mathrm{ABS}$ for organics $=0.01 ; \mathrm{ABS}$ for inorganics $=0.001$.

(3) Dermal Contact Slope Factors were derived from the Ingestion SF by the following formula: SFd $=S F 0 \times 0.2$ 


\section{Appendix D.80 \\ F-Area Burning/Rubble Pits 231-F/1F and Rubble Pit 2F \\ Future On-Unit Worker Scenario}

Radionuclide Cancer Risk Based on Inhalation of Contaminated Soil Particulates

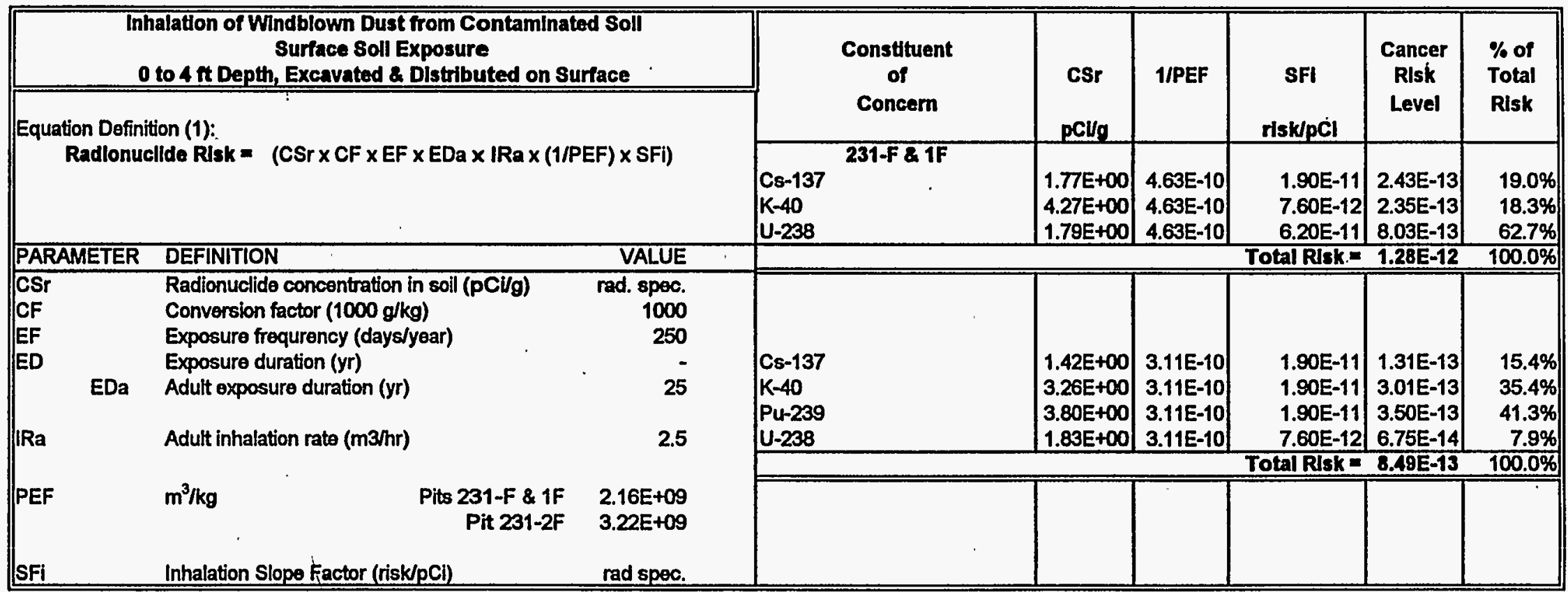

(1) Adapted from "Risk Assessment Guidance for Superfund, Volume I, Human Health Evaluation Manual (Part A)," EPA, 1989. 
Appendix D.81

F-Area Buming/Rubble Plts 231-F/1F and Rubble Pit 2F

Future On-Unit Worker Scenario

Radionuclide Cancer Risk Based on Direct Radlation from Surface Soll

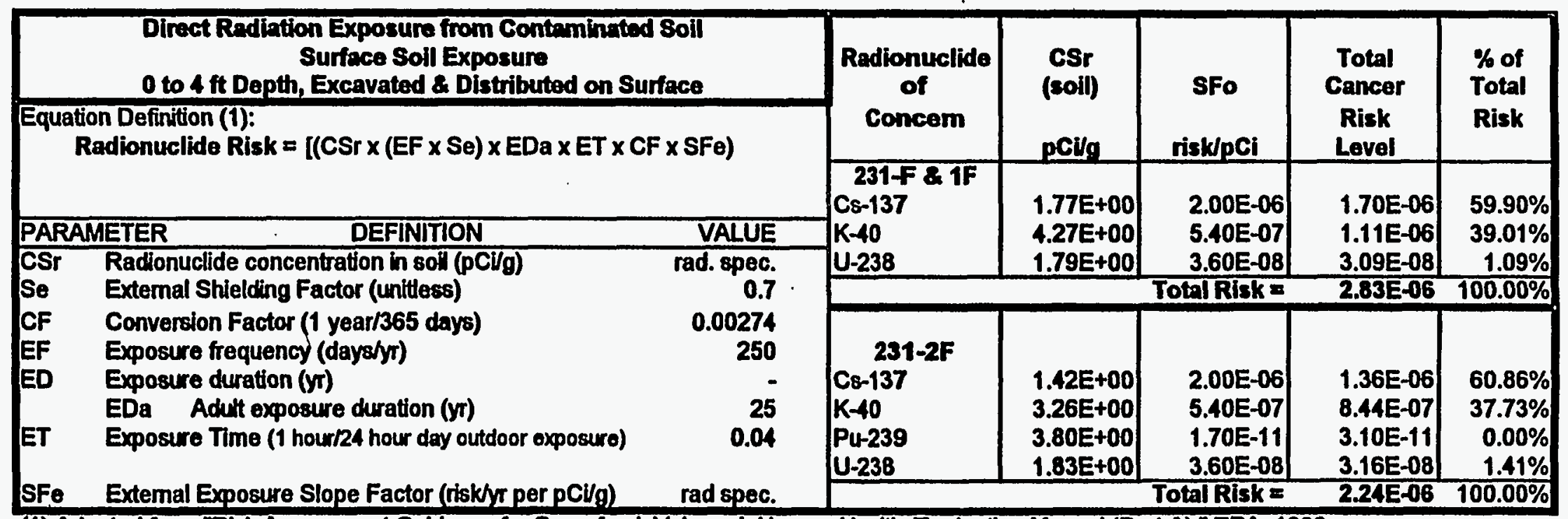

(1) Adapted from "Rlisk Assessment Guidance for Superfund, Volume I, Human Health Evaluation Manual (Part A)," EPA, 1989. 
F-Area Buming/Rubble Pits 231-F/1F and Rubble Plt 2F

Future On-Unit Worker Scenario

Radionuclide Cancer Rlsk Based on Exposure to Groundwater

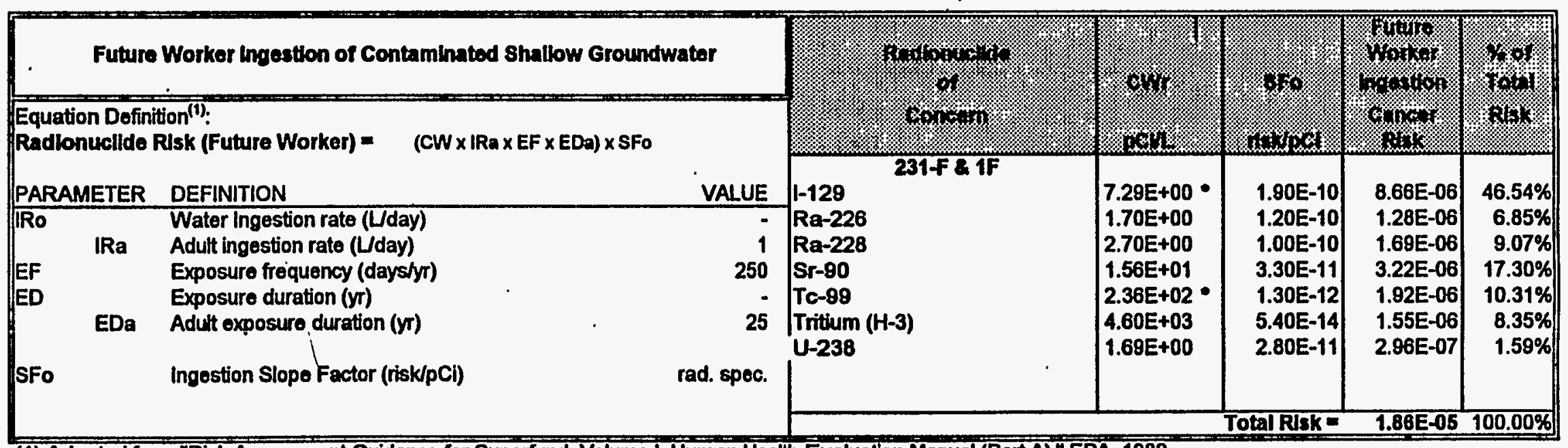

(1) Adepted from "Risk Assessment Guidance for Superfund, Volumo I, Human Health Evaluation Manual (Part A), EPA, 1989. 
BASELINE RISK ASSESSMENT

F-AREA BURNING/RUBBLE PITS AND RUBBLE PIT
WSRC-TR-94-108

REV.12, MARCH 1996

III. LEAD MODEL 
THIS PAGE LEFT INTENTIONALLY BLANK 


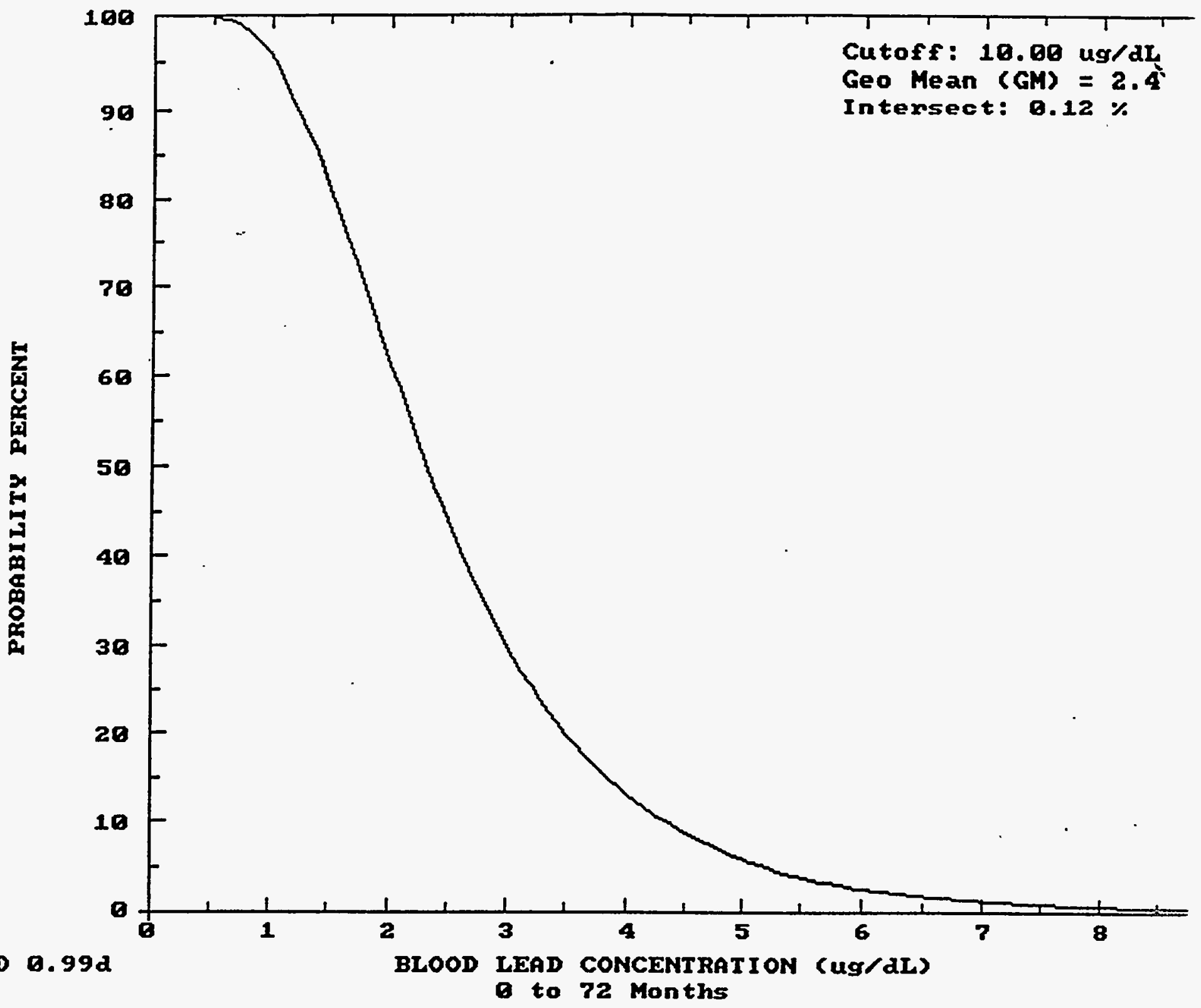

Figure D.1

Lead Model Output

Savannah River Site

Burning/Rubble Pits 231-F/1F

0-2 ft Interval 
Table D.83

LEAD MOOEL VerSion $0.99 d$

AIR CONCENTRATION: 0.100 ug Pb/m3 DEFAULT Indoor AIR Pb Conc: 30.0 percent of outdoor.

Other AIR Parameters:

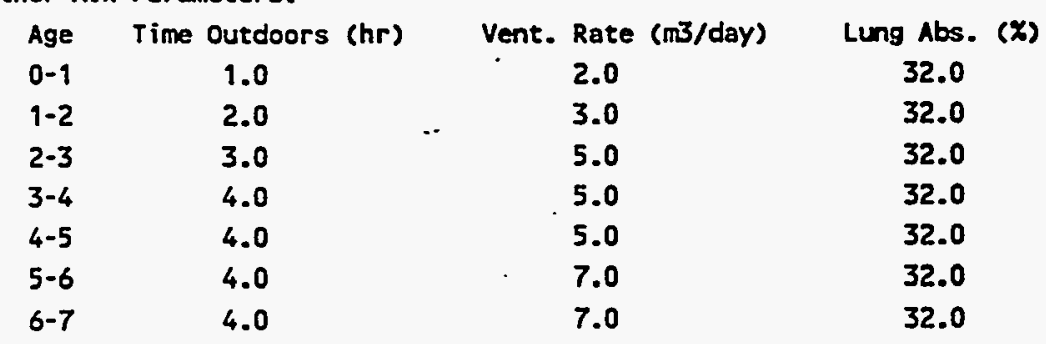

DIET: DEFAULT

DRINKING WATER CONC: 11.40 ug Pb/L

HATER Consumption: DEFAULT

SOIL \& DUST:

Soil: constant conc.

Dust: constant cone.

$\begin{array}{ccc}\text { Age } & \text { Soil (ug Pb/g) } & \text { House Dust (ug Pb/g) } \\ 0-1 & 24.8 & 24.8 \\ 1-2 & 24.8 & 24.8 \\ 2-3 & 24.8 & 24.8 \\ 3-4 & 24.8 & 24.8 \\ 4-5 & 24.8 & 24.8 \\ 5-6 & 24.8 & 24.8 \\ 6-7 & 24.8 & 24.8\end{array}$

Additional Dust Sources: Hone DEFAULT

PAINT Intake: 0.00 ug Pb/day DEFAULT

MATERHAL CONTRIBUTIOH: Infant Model

Maternal Blood Conc: 2.50 ug Pb/dL.

CALCULATED BLOOO Pb and Pb UPTAKES:

\begin{tabular}{cccc} 
YEAR & $\begin{array}{c}\text { Blood Level } \\
\text { (ug/dL) }\end{array}$ & $\begin{array}{c}\text { Total Uptake } \\
\text { (ug/day) }\end{array}$ & $\begin{array}{c}\text { Soil+oust Uptake } \\
\text { (Ug/day) }\end{array}$ \\
\hline 0.5-1: & 2.4 & -3.34 & 0.60 \\
1-2: & 2.7 & 6.41 & 0.95 \\
2-3: & 2.6 & 6.92 & 0.95 \\
3-4: & 2.4 & 6.91 & 0.96 \\
4-5: & 2.3 & 6.71 & 0.72 \\
5-6: & 2.1 & 7.00 & 0.65 \\
6-7: & 2.1 & 7.35 & 0.61
\end{tabular}

\begin{tabular}{|c|c|c|c|c|}
\hline YEAR & $\begin{array}{c}\text { Diet Uptake } \\
\text { (ug/day) }\end{array}$ & $\begin{array}{l}\text { Water Uptake } \\
\text { (ug/day) }\end{array}$ & $\begin{array}{l}\text { Paint Uptake } \\
\text { (ug/day) }\end{array}$ & $\begin{array}{c}\text { Air Uptake } \\
\text { (ug/day) }\end{array}$ \\
\hline 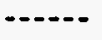 & - n-non & - & - & -..- \\
\hline $0.5-1:$ & 2.63 & 1.09 & 0.00 & 0.02 \\
\hline 1-2: & 2.73 & 2.70 & 0.00 & 0.03 \\
\hline 2-3: & 3.08 & 2.82 & 0.00 & 0.06 \\
\hline
\end{tabular}




$\begin{array}{lllll}\text { 3-4: } & 2.99 & 2.89 & 0.00 & 0.07 \\ \text { 4-5: } & 2.90 & 3.02 & 0.00 & 0.07 \\ \text { 5-6: } & 3.07 & 3.20 & 0.00 & 0.09 \\ \text { 6-7: } & 3.39 & 3.26 & 0.00 & 0.09\end{array}$

Input/Output for Lead Model Savannah River Site

Burning/Rubble Pits 231-F/1F 0-2 ft Interval 


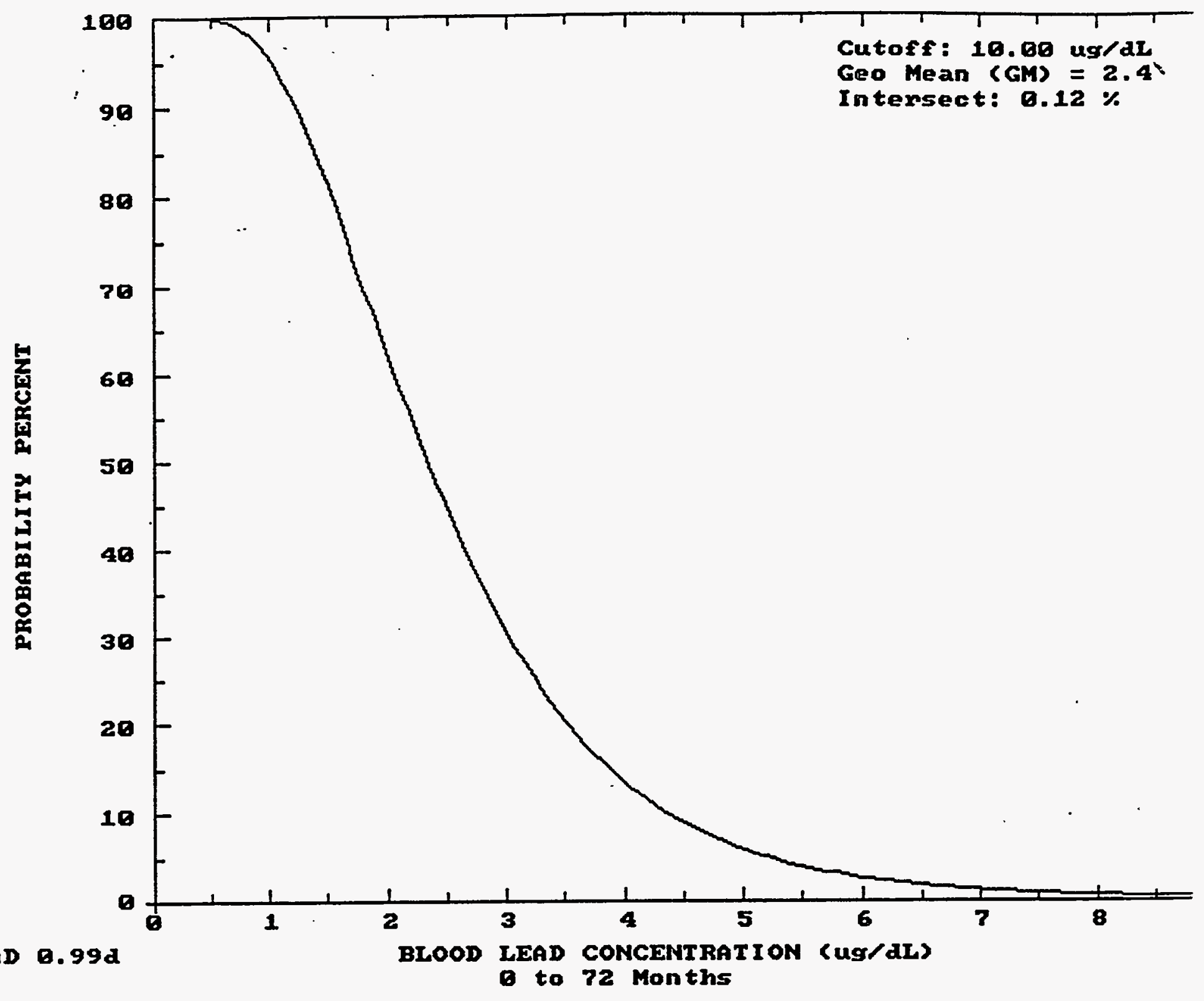

Figure D.2

Lead Model Output

Savannah River Site

Burning/Rubble Pits 231-F/1F

0-4 ft Interval - 
Table D.84

LEAD MOOEL Version $0.99 \mathrm{~d}$

IR COHCEHTRATION: 0.100 ug Pb/m3 DEFAULT Indoor AIR Pb conc: 30.0 percent of outdoor. Other AIR Paraneters:

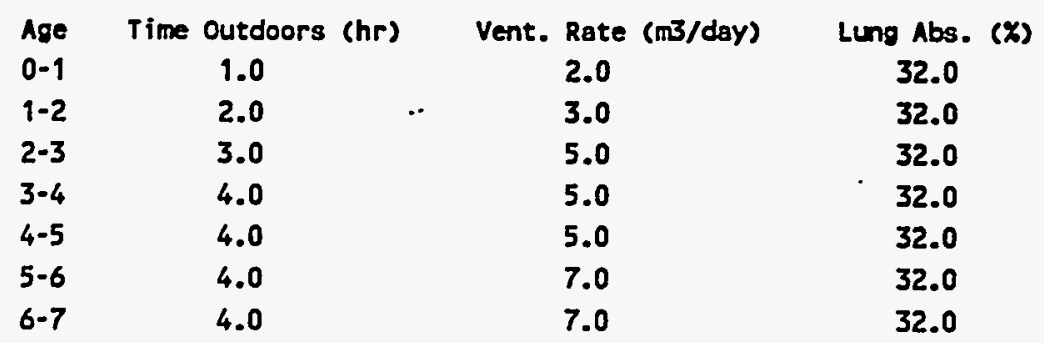

DIET: DEFAULT

DRIKKING HATER CONC: 11.40 ug Pb/L

HATER Consumption: DEFAULT

SOIL \& DUST:

Soil: constant conc.

Dust: constant conc.

$\begin{array}{ccc}\text { Age } & \text { Soil (ug } \mathrm{Pb} / \mathrm{g} \text { ) } & \text { House Dust (ug } \mathrm{Pb} / \mathrm{g} \text { ) } \\ 0-1 & 26.2 & 26.2 \\ 1-2 & 26.2 & 26.2 \\ 2-3 & 26.2 & 26.2 \\ 3-4 & 26.2 & 26.2 \\ 4-5 & 26.2 & 26.2 \\ 5-6 & 26.2 & 26.2 \\ 6-7 & 26.2 & 26.2\end{array}$

Additional Dust Sources: Hone DEFAULT

PAINT Intake: 0.00 ug Pb/day DEFAULT

MATERHAL CONTRIBUTION: Infant Model

Maternal Blood Conc: 2.50 ug Pb/dL

CALCULATED BLOOO Pb and Pb UPTAKES:

\begin{tabular}{|c|c|c|c|}
\hline YEAR & $\begin{array}{l}\text { Blood Level } \\
\text { (ug/dL) }\end{array}$ & $\begin{array}{l}\text { Total Uptake } \\
\text { (ug/day) }\end{array}$ & $\begin{array}{c}\text { Soil+Dust Uptake } \\
\text { (ug/day) }\end{array}$ \\
\hline 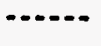 & - n & 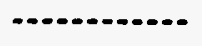 & $\ldots$ \\
\hline $0.5-1:$ & 2.4 & 4.37 & 0.64 \\
\hline 1-2: & 2.7 & .6 .46 & 1.00 \\
\hline 2-3: & 2.6 & 6.97 & 1.01 \\
\hline 3-4: & 2.5 & 6.96 & 1.02 \\
\hline 4-5: & 2.3 & 6.74 & 0.76 \\
\hline $5-6:$ & 2.2 & 7.04 & 0.68 \\
\hline 6-7: & 2.1 & 7.38 & 0.65 \\
\hline
\end{tabular}

\begin{tabular}{|c|c|c|c|c|}
\hline 'EAR & $\begin{array}{l}\text { Diet Uptake } \\
\text { (ug/day) }\end{array}$ & $\begin{array}{l}\text { Water Uptake } \\
\text { (ug/day) }\end{array}$ & $\begin{array}{l}\text { Paint Uptake } \\
\text { (ug/day) }\end{array}$ & $\begin{array}{l}\text { Air Uptake } \\
\text { (ug/day) }\end{array}$ \\
\hline$\ldots$ & 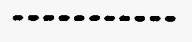 & - & - & - \\
\hline $0.5-1:$ & 2.63 & 1.09 & 0.00 & 0.02 \\
\hline 1-2: & 2.73 & 2.69 & 0.00 & 0.03 \\
\hline 2-3: & 3.08 & 2.82 & 0.00 & 0.06 \\
\hline
\end{tabular}




$\begin{array}{lllll}3-4: & 2.99 & 2.89 & 0.00 & 0.07 \\ 4-5: & 2.90 & 3.02 & 0.00 & 0.07 \\ 5-6: & 3.07 & 3.20 & 0.00 & 0.09 \\ 6-7: & 3.39 & 3.26 & 0.00 & 0.09\end{array}$

- Table D.84 (Continued) Input/Output for Lead Model Savannah River Site Burning/Rubble Pits 231-F/1F 0-4 ft Interval 


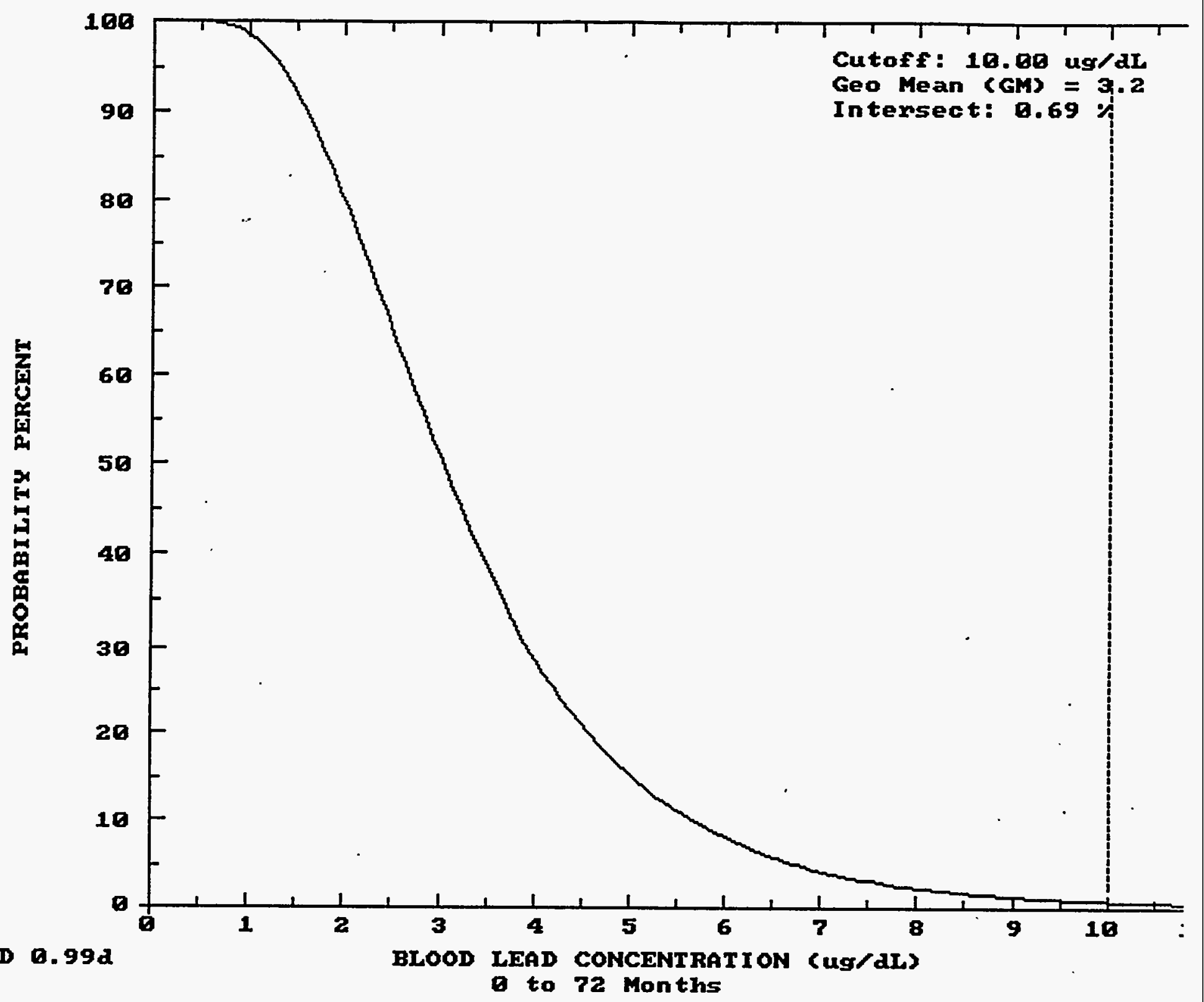

Figure D.3

Lead Model Output

Savannah River site

Rubble Pit 231-2F

0-2 ft Interval 


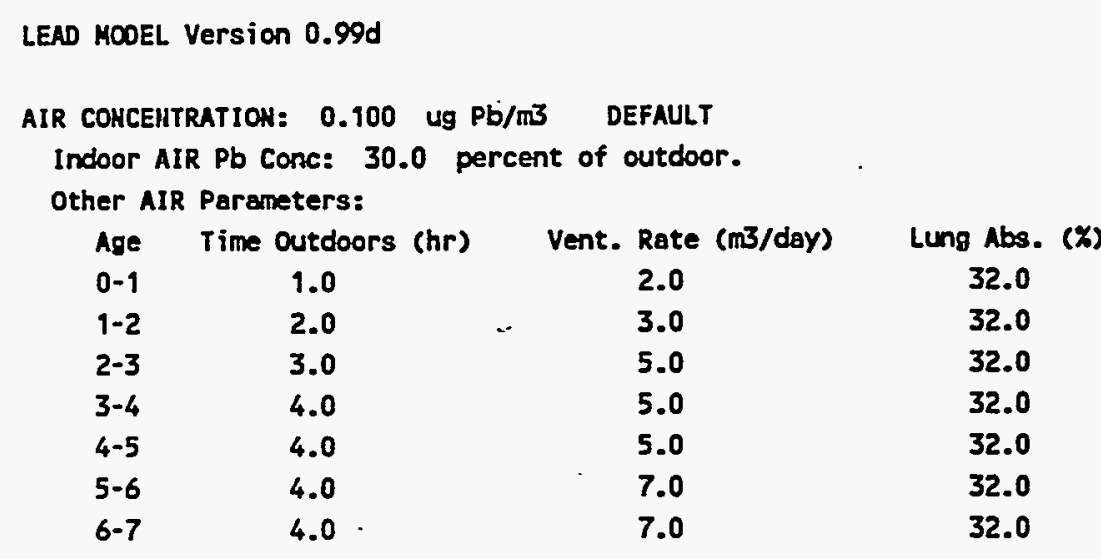

DIET: DEFAULT

DRINKIHG HATER CONC: $11.40 \mathrm{ug} \mathrm{Pb} / \mathrm{L}$

WATER Consumption: DEFAULT

SOIL \& DUST:

Soil: constant conc.

Dust: constant conc.

$\begin{array}{ccc}\text { Age } & \text { Soil (ug } \mathrm{Pb} / \mathrm{g} \text { ) } & \text { House Dust (ug } \mathrm{Pb} / \mathrm{g} \text { ) } \\ 0-1 & 90.0 & 90.0 \\ 1-2 & 90.0 & 90.0 \\ 2-3 & 90.0 & 90.0 \\ 3-4 & 90.0 & 90.0 \\ 4-5 & 90.0 & 90.0 \\ 5-6 & 90.0 & 90.0 \\ 6-7 & 90.0 & 90.0\end{array}$

Additional Dust Sources: None OEFAULT

PAInT Intake: 0.00 ug Pb/day DEFAULT

MATERHAL COHTRIBUTIOH: Infant Model

Maternal Blood Conc: 2.50 ug Pb/dL

CALCULATED BLOOO Pb and Pb UPTAKES:

\begin{tabular}{|c|c|c|c|c|}
\hline YEAR & $\begin{array}{l}\text { Blood Level } \\
\text { (ug/dl) }\end{array}$ & $\begin{array}{l}\text { Total Uptake } \\
\text { (ug/day) }\end{array}$ & $\begin{array}{c}\text { Soil+Dust Uptake } \\
\text { (ug/day) }\end{array}$ & \\
\hline$\cdots$ & - - n-n & - & 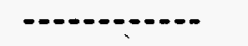 & \\
\hline $0.5-1:$ & 3.2 & 5.82 & 2.15 & \\
\hline 1-2: & 3.6 & 8.73 & 3.38 & \\
\hline $2-3:$ & 3.4 & 9.26 & 3.40 & \\
\hline 3-4: & 3.3 & 9.29 & 3.44 & \\
\hline 4-5: & 2.9 & 8.51 & 2.58 & \\
\hline 5-6: & 2.7 & 8.64 & 2.33 & \\
\hline 6-7: & 2.5 & 8.90 & 2.21 & \\
\hline YEAR & $\begin{array}{l}\text { Diet Uptake } \\
\text { (ug/day) }\end{array}$ & $\begin{array}{l}\text { Water Uptake } \\
\text { (ug/day) }\end{array}$ & $\begin{array}{l}\text { Paint Uptake } \\
\text { (ug/day) }\end{array}$ & $\begin{array}{l}\text { Air Uptake } \\
\text { (ug/day) }\end{array}$ \\
\hline$\ldots$ & -............. & - & 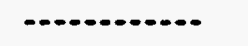 & .............. \\
\hline $0.5-1=$ & 2.59 & 1.07 & 0.00 & 0.02 \\
\hline 1-2: & 2.68 & 2.64 & 0.00 & 0.03 \\
\hline 2-3: & 3.03 & 2.77 & 0.00 & 0.06 \\
\hline
\end{tabular}

Input/Output for Lead Model Savannah River Site Rubble Pit 231-2F 0-2 ft Interval 


$\begin{array}{rrrrr}3-4: & 2.94 & 2.85 & 0.00 & 0.07 \\ 4-5: & 2.87 & 2.99 & 0.00 & 0.07 \\ 5-6: & 3.04 & 3.17 & 0.00 & 0.09 \\ -6-7: & 3.37 & 3.23 & 0.00 & 0.09\end{array}$

Table D.85 (Continued)

Input/Output for Lead Model Savannah River Site Rubble Pit 231-2F 0-2 ft Interval 


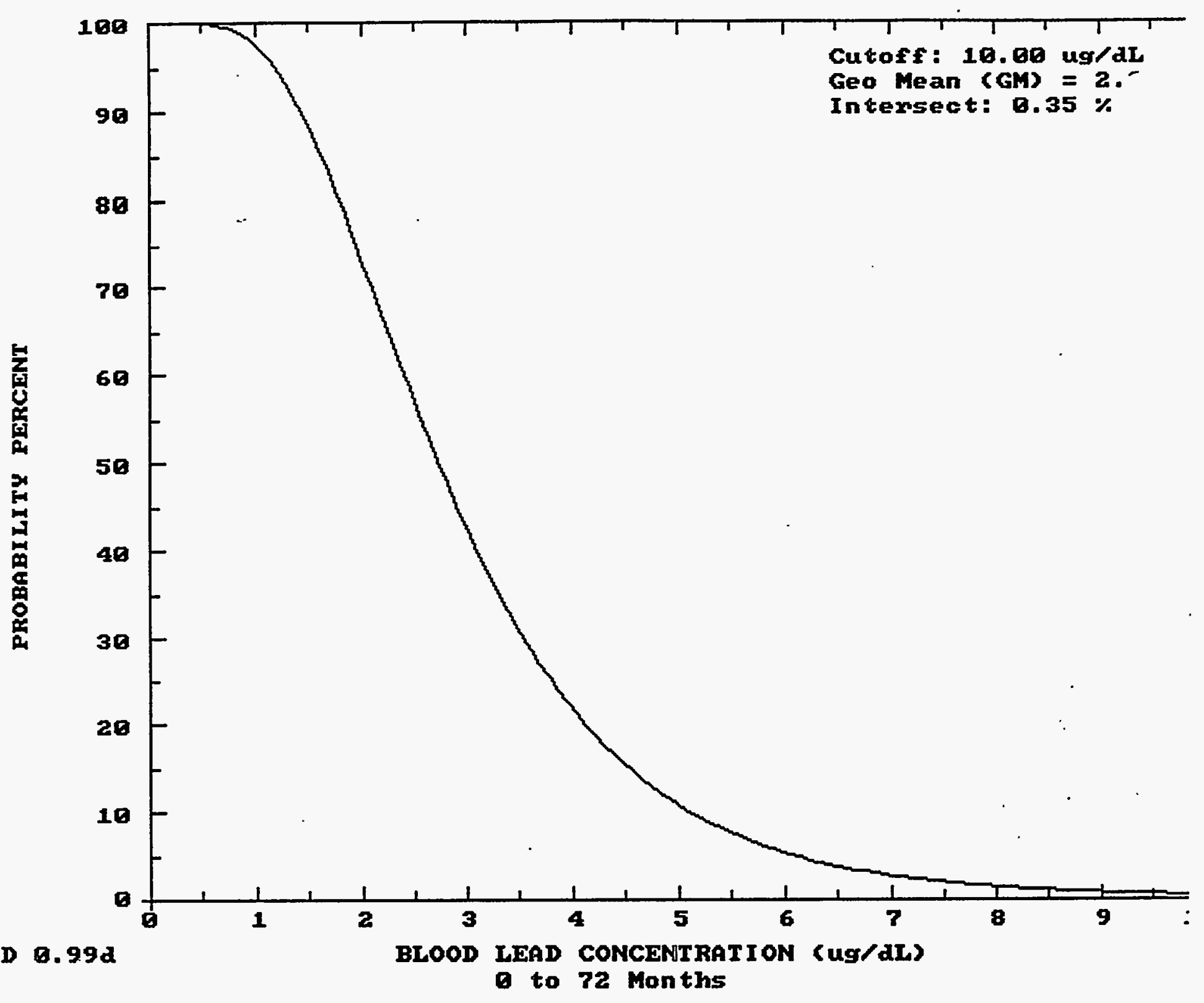

Figure D.4

Lead Model Output

Savannah River Site.

Rubble Pit 231-2F

0-4 ft Interval 
LEAD MCOEL Version $0.99 d$

- 'R COHCEntratioh: $0.100 \mathrm{ug} \mathrm{Pb} / \mathrm{m} 3$ Default Indoor AIR Pb Conc: 30.0 percent of outdoor. Other AIR Parameters:

$\begin{array}{cccc}\text { Age } & \text { Time outdoors (hr) } & \text { Vent. Rate (m3/day) } & \text { Lung Abs. (z) } \\ 0-1 & 1.0 & 2.0 & 32.0 \\ 1-2 & 2.0 & 3.0 & 32.0 \\ 2-3 & 3.0 & 5.0 & 32.0 \\ 3-4 & 4.0 & 5.0 & 32.0 \\ 4-5 & 4.0 & 5.0 & 32.0 \\ 5-6 & 4.0 & 7.0 & 32.0 \\ 6-7 & 4.0 & 7.0 & 32.0\end{array}$

DIET: DEFAULT

DRINKIHG HATER CONC: $11.40 \mathrm{ug} \mathrm{Pb} / \mathrm{L}$ WATER Consumption: DEFAULT

SOIL \& DUST:

$\begin{array}{lcc}\text { Soil: } & \text { constant conc. } \\ \text { Dust: constant conc. } & \\ & & \\ \text { Age } & \text { Soil (ug Pb/g) } & \text { House Dust (ug Pb/g) } \\ 0-1 & 62.0 & 62.0 \\ 9-2 & 62.0 & 62.0 \\ 2-3 & 62.0 & 62.0 \\ 3-4 & 62.0 & 62.0 \\ 4-5 & 62.0 & 62.0 \\ 5-6 & 62.0 & 62.0 \\ 6.7 & 62.0 & 62.0\end{array}$

Additional Dust Sources: Hone DEFAULT

PAIHT Intake: 0.00 ug Pb/day DEFAULT

MATERHAL COHTRIBUTION: Infant Model

Maternal Blood Conc: 2.50 ug Pb/dL

CALCULATED BLOOO Pb and Pb UPTAKES:

\begin{tabular}{|c|c|c|c|c|}
\hline YEAR & $\begin{array}{l}\text { Blood Level } \\
\text { (ug/dL) }\end{array}$ & $\begin{array}{l}\text { Total Uptake } \\
\text { (ug/day) }\end{array}$ & $\begin{array}{c}\text { Soil+Dust Uptake } \\
\text { (ug/day) }\end{array}$ & \\
\hline ....... & - & 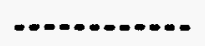 & ............... & \\
\hline $0.5-1:$ & 2.8 & 5.19 & 1.49 & \\
\hline 1-2: & 3.2 & 7.75 & 2.35 & \\
\hline 2-3: & 3.1 & 8.27 & 2.36 & \\
\hline $3-4:$ & 2.9 & 8.28 & 2.38 & \\
\hline 4-5: & 2.6 & 7.74 & 1.78 & \\
\hline 5-6: & 2.4 & 7.94 & 1.61 & \\
\hline 6-7: & 2.3 & 8.24 & 1.52 & \\
\hline EAR & $\begin{array}{c}\text { Diet Uptake } \\
\text { (ug/day) }\end{array}$ & $\begin{array}{c}\text { Water Uptake } \\
\text { (ug/day) }\end{array}$ & $\begin{array}{l}\text { Paint Uptake } \\
\text { (ug/day) }\end{array}$ & $\begin{array}{c}\text { Air Uptake } \\
\text { (ug/day) }\end{array}$ \\
\hline -.. & - & - & 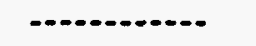 & $\ldots$ \\
\hline $0.5-1=$ & 2.61 & 1.07 & 0.00 & 0.02 \\
\hline 1-2: & 2.70 & 2.66 & 0.00 & 0.03 \\
\hline $2-3:$ & 3.05 & 2.79 & 0.00 & 0.06 \\
\hline
\end{tabular}

Table $\mathrm{D} .86$

Input/Output for Lead Model Savannah River Site Rubble Pit 231-2F 0-4 ft Interval 


$\begin{array}{lllll}\text { 3-4: } & 2.96 & 2.87 & 0.00 & 0.07 \\ \text { 4-5: } & 2.88 & 3.01 & 0.00 & 0.07 \\ \text { 5-6: } & 3.05 & 3.18 & 0.00 & 0.09 \\ \text { 6-7: } & 3.38 & 3.24 & 0.00 & 0.09\end{array}$

Table D.86 (Continued)

Input/Output for Lead Mode1 Savannah River Site Rubble Pit 231-2F $0-4 \mathrm{ft}$ Interval 


\section{THIS PAGE LEFT INTENTIONALLY BLANK}




\section{TABLE OF CONTENTS}

I. Toxicity Profiles for Nonradiological Chemicals of Potential Concern

Acetone

Aldrin

Aluminum

Arsenic

Barium

Benzoic Acid

Benzene Hexachloride

Bis(2-Ethylhexyl) Phthaiate

Butylbenzyl Phthalate

Cadmium

Calcium

Carbon Disulfide

Carbon Tetrachloride

Chlordane

Chloroform

Chloromethane

Chromium

Copper

Dichlorodiphenylethanes

1,2 Dichloroethane

Di-n-butyl Phthalate; Di-n-octyl Phthalate

Endosulfan

Endrin

Ethylbenzene

Heptachlor; Heptachlor Epoxide

Heptachlorodibenzo-p-dioxins isomers; octachlorodibenzo-p-doxin isomers;

Iron octachlorodibenzo-p-furan isomers; dibenzofuran

Lead Manganese

Magnesium

Methylene Chloride

Methyl Ethyl Ketone

Mercury

Naphthalene; 2-Methyl Naphthalene

Nickel

Pentachlorophenol

Polychlorinated Biphenyls-Aroclor 1254, Aroclor 1260

Polycyclic Aromatic Hydrocarbons

Silver

Tetrachloroethylene

Toluene

Trichloroethanes

Trichloroethylene

Trichlorofluoromethane

Vanadium

Xylenes 
Zinc

II. Toxicity Profiles for Radionuclides
Cesium
Plutonium
Potassium
Radium
Strontium
Technicium
Tritium
Uranium 
I. TOXICITY PROFILES FOR NONRADIOLOGICAL CHEMICALS OF POTENTIAL CONCERN

F.3 
THIS PAGE LEFT INTENTIONALLY BLANK 
ACETONE

CAS No:

67-64-1 .

Synonym(s) Dimethyl Ketone, 2-Propanone

Acetone is a naturally occurring compound produced in the body during fat oxidation and released in expired air (Conkle et al. 1975). Acetone is also naturally emitted into the environment from plants and trees (HSDB 1992), volcanic eruptions, and forest fires (Graedel 1978; Isidorov et al. 1985). Anthropogenic sources of acetone in the environment include automobile exhaust, tobacco smoke, and chemical manufacturing (Graedel 1978). The primary human uses of acetone are as a solvent and as an intermediate in chemical production (Nelson and Webb 1978). Additionally, acetone is a component of a number of consumer products such as waxes and polishes, detergents, nail polish remover, and some paint removers (Knoeppel and Schauenburg 1989; Tichenor and Mason 1988; Hahn and Werschulz 1986).

Acetone is a colorless liquid having a mildly pungent, aromatic odor that is detectable in air at a concentration of $3 \mathrm{ppm}$. Acetone is completely miscible in water and is soluble in benzene and ethanol (ATSDR 1992). It has a molecular weight of 58.08, a boiling point of $56.2^{\circ} \mathrm{C}$, and a melting point of $-95.35^{\circ} \mathrm{C}$ (Riddick et al. 1986).

\section{FATE AND TRANSPORT}

Acetone readily vaporizes and enters the atmosphere where it has a relatively long half-life of approximately 22 days (Meyrahn et al. 1986). Photolysis and reactions with hydroxyl radicals represent the major pathways for acetone degradation in air (Meyrahn et al. 1986).

Precipitation may transport acetone from air to surface waters and soil (Grosjean and Wright 1983). Additionally, acetone may enter surface waters as waste from chemical manufacturing and energy-related industries (Gordon and Gordon 1981; Mohr and King 1985). Groundwater contamination with acetone may result from leaching from municipal and industrial landfills (Brown and Donnelly 1988). Biodegradation and evaporation are the two primary mechanisms for removal of acetone from water (Thomas 1982; Urano and Kato 1986).

Acetone does not readily adsorb to sediments and therefore may be removed from soil through leaching and volatilization. Biodegradation may also be a significant pathway of removal of acetone from soil (ATSDR 1992).

\section{HUMAN HEALTH EFFECTS}

Exposure to acetone may occur through inhalation, ingestion, or dermal exposure. Typically the concentration of acetone in air and water is low and represents minimal exposure risks to the general public. Occupational exposure may occur in certain industries. Diabetics and persons with restricted dietary intakes are more susceptible to the effects of acetone exposure, primarily because these persons have higher than average background circulating concentrations of acetone. Additionally, acetone may potentiate the deleterious effects of 
exposure to other chemicals including carbon tetrachloride, chloroform, ethanol, and other ketones. Of particular interest, especially to industrial workers, is the potentiation of the hepatotoxic effects of acetominophen (Tylenol-like products) by acetone under certain conditions (ATSDR 1992).

Acute exposure to atmospheric acetone causes respiratory and eye irritation, and G.I. irritation, including hemorrhage and vomiting of blood. Central nervous system effects range from headache (for which one might take acetominophen), dizziness, and confusion to full CNS depression and coma, depending on the dose and length of exposure. There is no evidence to suggest that acetone is carcinogenic or mutagenic and the EPA has classified acetone in Group D (IRIS 1992).

\section{ECOLOGICAL EFFECTS}

Acetone does not bioaccumulate in aquatic organisms, however the possibility of bioaccumulation and magnification within terrestrial food chains has not been previously addressed. Acetone is readily degraded in water and soil by microorganisms, provided that the concentration of acetone is below the level which is toxic to microbes.

\section{US ENVIRONMENTAL PROTECTION AGENCY HUMAN HEALTH CRITERIA}

Oral Slope Factor

Inhalation Slope Factor

Chronic Oral RfD

Chronic Inhalation RfD
NA

NA

$1 \mathrm{E}-01 \mathrm{mg} / \mathrm{kg} /$ day

NA

\section{DRINKING WATER CRITERIA AND STANDARDS}

U.S. Environmental Protection Agency

Maximum Concentration Limit

Maximum Concentration Limit Goal

NA

NA

\section{AMBIENT WATER QUALITY CRITERIA}

U.S. Environmental Protection Agency Ambient Water Quality Criteria

$\begin{array}{ll}\text { Human Health-Water and Fish Ingestion } & \text { NA } \\ \text { Human Health-Fish Ingestion Only } & \text { NA } \\ \text { Aquatic Organisms-FW Acute } & \text { NA } \\ \text { Aquatic Organisms-FW Chronic } & \text { NA } \\ \text { Aquatic Organisms-Marine Acute } & \text { NA } \\ \text { Aquatic Organisms-Marine Chronic } & \text { NA }\end{array}$


South Carolina Department of Health and Environmental Control

Human Health Criteria

Fish and Aquatic Life-Continuous

Fish and Aquatic Life-Maximum
NA

NA

NA

$\mathrm{NA}=$ NOT AVAILABLE

\section{REFERENCES}

Agency for Toxic Substances ans Disease Registry. 1992. Prepared by Clement International Corp., contract no. 205-88-0608.

Brown, K.W., Donnelly, K.D. 1988. An estimation of the risk associated with the organic constituents of hazardous and municipal waste landfill leachates. Hazard. Waste Hazard. - Mater. 5:1-30.

Conkle, J.P., Camp, B.J., Welch, B.E. 1975. Trace composition of human respiratory gas. Arch. Environ. Health 30:290-295.

Gordon, A.W., Gordon, M. 1981. Analysis of volatile organic compounds in a textile finishing plant effluent. Trans Kentucky Academy of Science. 42:149-157.

Graedel, T.E. 1978. Chemical compounds in the atmosphere. New York. Academic Press $7,182$.

Grosjean, D., Wright, B. 1983. Carbonyls in urban fog, ice fog, cloudwater and rainwater. Atmos. Environ. 17:2093-2096.

Hahn, W.J., Werschulz, P.P. 1986. Evaluation of alternatives to toxic organic paint strippers. Washington, D.C. U.S. Environmental Protection Agency. NTIS. PB219-177/A.S.

HDSB. 1992. Hazardous substance data bank. NLM. National Toxicological Information Program. Bethesda, MD. Feb 1992.

Isidorov, V.A., Zenkevich, I.G., Ioffe ,B.V. 1985. Volatile organic compounds in the atmosphere of forests. Atmos. Environ. 19:1-18.

Knoeppel, H., Shauenberg, H. 1989. Screening of household products for the emission of volatile organic compounds. Environ. Int. 15:413-418.

Meyrhan, H., Pauly, J., Schneider, W. et al. 1986. Quantum yields for the photodissociation of acetone in air and an estimate for the life time of acetone in the lower troposphere. Atmos. Chem. 4:277-291. 
Mohr, D.H., King, C.J. 1985. Identification of polar organic compounds in coal-gasification condensate water by gas chromatography-mass spectrometry analysis of high pressure liquid chromatography functions. Environ. Sci. Tech. 19:929-935.

Nelson, D.L., Webb, B.P. 1978. Acetone. In: Grayson,M (ed.) Kirk-Othmer Encyclopedia of Chemical Technology, vol 1, New York: John Wiley and Sons 179-191.

Riddick, J.A., Bunger, W.B., Sakano, T.K. 1986. Techniques of chemistry, vol II. Organic solvents, physical properties, and methods of purification. 4th ed. New York:John Wiley and Sons, Inc. 337-338.

Thomas, R.G. 1982. Volatilization from water. In:Handbook of Chemical Property Estimation Methods. New York:McGraw-Hill. 15-21.

Tichenor, B.A., Mason ,M.A. 1988. Organic emissions from consumer products and building materials to the indoor environment. Japanese Air Pollution Control Association 38:264-268.

Urano, K., Kato, Z. 1986. A method to classify biodegradabilities of organic compounds. J. Haz. Mater. 13:135-145. 
ALDRIN

CAS No:

309-00-2

Synonym(s)

HHDN

Aldrin was first synthesized in the U.S. in 1948 and was used as an insecticide on crops such as corn and cotton from the 1950's until the early 1970's. Use of aldrin was approved for killing termites in 1972 and continued until 1987, when the manufacturer voluntarily cancelled its registration for use (ATSDR 1991). Although aldrin and a similar compound dieldrin are no longer used or manufactured in the U.S. they are still present in the environment from previous uses.

Aldrin is a white crystalline powder with a molecular weight of 364.93 . It has a density of 1.7 , a melting point of $104^{\circ} \mathrm{C}$, and a boiling point of $145^{\circ} \mathrm{C}$. Aldrin readily dissolves in most organic solvents and is somewhat soluble in water (HDSB 1990).

\section{FATE AND TRANSPORT}

Aldrin release to the atmosphere can occur as a result of volatilization from contaminated surface waters, primarily, although a small amount of soil-bound aldrin may evaporate from soils. Its presence in soils is primarily the result of improper disposal or treatment of soils for termite control and/or crop treatment. Aldrin may enter surface waters as runoff from contaminated soils. This is the major transport mechanism of aldrin in soils as it has very little leaching or volatilization potential. In biologically active soils, aldrin is rapidly converted to dieldrin by epoxidation (Gannon and Bigger 1958). Aldrin is estimated to have a half-life of 53 days in soil, where most of the aldrin is converted to dieldrin. Dieldrin is strongly bound to soils and is very resistant to further biodegradation, however in the presence of sunlight, dieldrin may be slowly converted to photodieldrin. The estimated halflife of dieldrin in soil is 868 days, however it loses most of its biological activity within 3 years (Jury et al. 1987).

\section{HUMAN HEALTH EFFECTS}

Human exposure to aldrin an/or dieldrin may occur through inhalation of indoor air of homes previously treated for termites or through consumption of food grown in aldrin contaminated soil. Aldrin and dieldrin were once widely used and are persistent in the environment, therefore there is a small risk of ingestion exposure from commercially obtained food products such as fish, milk, and certain other foods high in fat. Overall, however the risk of exposure to aldrin is greatest near hazardous waste sites where these products may be inhaled, ingested, or absorbed through the skin (ATSDR 1991).

Exposure to low levels of aldrin by factory workers caused symptoms such as headaches, dizziness, hyperirritability, nausea, vomiting, anorexia, muscle twitching, and myoclonic jerking (Jager 1970). Acute exposure to high concentrations of aldrin caused more severe central nervous system excitation which, in some cases, culminated in convulsions and death. 
Aldrin is metabolized to dieldrin by cytochrome P-450 enzymes in the liver (Wong and Tenure 1965), and also to some degree in the lung and skin (Graham et al. 1987). Dieldrin is further metabolized by the liver and excreted in the feces or stored as dieldrin in fat deposits (Hutson 1976). Studies have indicated that the ratio of the amount of dieldrin eliminated and the amount retained in the body are related to the amount of body fat of the individual. Dieldrin is quickly partitioned from the blood into fat stores because of its highly lipophilic nature (Hunter and Robinson 1967). Those individuals with a large amount of total body fat retain a greater percentage of dieldrin compared to the amount metabolized and excreted. The opposite is true for subjects with very small amounts of total body fat (Hunter and Robinson 1967). The same study concluded, however, that there was little danger of shifting toxic amounts of dieldrin from fat stores into the circulation during weight loss or times of excess stress. Persons unusually susceptible to aldrin and dieldrin include the very young, who do not have completely developed hepatofunction, and the unborn, who may be exposed by placental transport (ATSDR 1991).

The EPA has classified aldrin and dieldrin as probable human carcinogens, category B2, based on data obtained through animal experimentation (ATSDR 1991). Aldrin and dieldrin are thought to act as tumor promoters by interfering with cell-cell inhibition, which allows cellular hyperplasia and eventually neoplasia to occur (Klaunig and Ruch 1987).

\section{ECOLOGICAL EFFECTS}

A large percentage of aldrin is converted to dieldrin in the environment, where dieldrin is quite persistent. Plants grown in aldrin contaminated soil in an experimental ecosystem readily accumulated and concentrated both aldrin and dieldrin (Cole et al. 1976). Aquatic organisms have also been shown experimentally to bioaccumulate aldrin and dieldrin (Metcalf et al. 1973). Therefore, both aquatic and terrestrial food chains exhibit biomagnification of aldrin, and its metabolite dieldrin. Because of the persistent nature of aldrin and dieldrin in the environment, the most likely route of exposure for the general population is through consumption of contaminated food stuffs (ATSDR 1991).

US ENVIRONMENTAL PROTECTION AGENCY HUMAN HEALTH CRITERIA

Oral Slope Factor

Inhalation Slope Factor

Chronic Oral RfD

Chronic Inhalation RfD
$1.7 \mathrm{E}+1 \mathrm{~kg} / \mathrm{mg} /$ day $^{-1}$

$1.7 \mathrm{E}+1 \mathrm{~kg} / \mathrm{mg} /$ day $^{-1}$

$1.7 \mathrm{E}+1 \mathrm{mg} / \mathrm{kg} /$ day

NA

\section{DRINKING WATER CRITERIA AND STANDARDS}
U.S. Environmental Protection Agency
Maximum Concentration Limit Maximum Concentration Limit Goal
NA
NA 


\section{AMBIENT WATER QUALITY CRITERIA}

U.S. Environmental Protection Agency Ambient Water Quality Criteria

$\begin{array}{ll}\text { Human Health-Water and Fish Ingestion } & 0.074 \mathrm{ng} / \mathrm{L} \\ \text { Human Health-Fish Ingestion Only } & 0.079 \mathrm{ng} / \mathrm{L} \\ \text { Aquatic Organisms-FW Acute } & 3 \mu \mathrm{g} / \mathrm{L} \\ \text { Aquatic Organisms-FW Chronic } & \mathrm{NA} \\ \text { Aquatic Organisms-Marine Acute } & 13 \mu \mathrm{g} / \mathrm{L} \\ \text { Aquatic Organisms-Marine Chronic } & \mathrm{NA}\end{array}$

South Carolina Department of Health and Environmental Control

Human Health Criteria

Fish and Aquatic Life-Continuous

Fish and Aquatic Life-Maximum

$0.0136 \mu \mathrm{g} / \mathrm{L}$

NA

$4 \mu \mathrm{g} / \mathrm{L}$

$\mathrm{NA}=$ Not Available

\section{REFERENCES}

Agency for Toxic Substance and Disease Registry (ATSDR). 1991. Draft Toxicological Profile for Aldrin. Prepared by Dynamic Corp. U.S. Department of Health and Human Services.

Cole, L.K., Metcalf, R.L., Sanborn,J.R. 1976. Environmental fate of insecticides in terrestrial model ecosystems. Int. J. Environ. Studies 10:7-14.

Gannon, N., Bigger,J.H. 1958. The conversion of aldrin and heptachlor to their epoxides in soil. J. Econ. Entomol. 51:1-7.

Graham, MJ., Williams, F.M., Rettie, A.E., et al.1987. Aldrin metabolism in the skin: Invitro studies In: Shroot,B., Schaefer,H. eds. 7th Symposium on Advances in Skin Pharmacology, Skin Pharmacokinetics, Nice, France. September 26-28, 1986. New York. S.Karger, 252-255.

HSDB. 1990. Hazardous substance Data Bank. Aldrin. NLM. National Toxicological Information Program.

Hutson, D.H. 1976. Comparative metabolism of dieldrin in the rat (CFE) and in two strains of mouse (CF1 and LACG). Food Cosmet. Toxicol. 14:557-591.

Hunter, C.G., Robinson, J. 1968. Aldrin, dieldrin, and man. Food Cosmt. Toxicol. 6:253-260. 
Jager, KW. 1970. Aldrin, dieldrin, endrin, and telodrin: An epidemiological and toxicological study of long-term occupational exposure. New York: Elsevier.

Jury, W.A., Focht, D.D., Farmer, W.J. 1987. Evaluation of pesticide groundwater pollution potential from standard indices of soil-chemical adsorption and biodegradation. J. Environ. Qual. 16:422-428.

Metcalf, R.L., Kapoor, I.P., Lu, C.K., et al. 1973. Model ecosystem studies of environmental fate of six organochlorine pesticides. Environ. Health Perspect. 4:35-44.

Wong, D.T., Terriere, L.C. 1965. Epoxidation of aldrin, isodrin, and heptachlor by rat liver microsomes. Biochem. Pharmacol. 14:375-377. 


\section{ALUMINUM \\ CAS No.: 7429-90-5 \\ Synonym(s) None}

Aluminum is the third most abundant element (American Conference of Governmental Industrial Hygienists 1986) comprising about 8.8 percent of the earth's crust by weight (Merck Index 1983). Aluminum does not occur freely in nature, but is widely distributed combined with oxygen, fluorine, silicon, and other constituents. It is a silvery-white, malleable, ductile, light-weight metal. It is soluble in alkalies and in hydrochloric and sulfuric acid, and insoluble in concentrated nitric acid, hot acetic acid, and water (Weast 1979). It has a molecular weight of $26.98 \mathrm{~g} / \mathrm{mole}$, melts at $660^{\circ} \mathrm{C}$, boils at $2,450^{\circ} \mathrm{C}$ (at 1 atmosphere), has a vapor pressure of $1 \mathrm{~mm} \mathrm{Hg}$ at $1284^{\circ} \mathrm{C}$, and has a density of 2.70 at $20^{\circ} \mathrm{C} / 4^{\circ} \mathrm{C}$.

\section{FATE AND TRANSPORT}

Aluminum is abundant in soils although concentrations vary with solubility, which is a function of $\mathrm{pH}$ (National Research Council 1981). It is amphoteric, being more soluble in acidic and basic solutions than in more neutral solutions (U.S. Environmental Protection Agency 1988). Some acid soils contain sufficient aluminum in soluble form to kill some types of plants (Farm Chemicals Handbook 1984). Soil concentrations of aluminum have been recorded up to 150 to 600 grams per kilogram (Friberg et al. 1979). Human activities tend to increase aluminum content in surface waters (Friberg et al. 1979) and acid rain can cause aluminum to leach out of soil at a higher than normal rate, thus increasing the aluminum uptake by plants and animals. Aluminum concentrations in urban air. are reported as high as $10 \mu \mathrm{g}$ per cubic meter. In nonurban areas, values as low as $0.5 \mu \mathrm{g}$ per cubic meter have been reported (Friberg et al. 1979). Aluminum tends to bioaccumulate only slightly in aquatic organisms. Cleveland et al. (1986) reported BCFs of 50 to 230 among young brook trout.

\section{HUMAN HEALTH EFFECTS}

Humans are exposed to aluminum by ingestion of foods and beverages and by inhalation from the atmosphere. Human ingestion of aluminum is typically 30 to $50 \mathrm{mg}$ per day (Bjorksten 1982), with a daily absorption rate 0.1 to $2 \mathrm{mg}$ (Rosas 1978). Although not essential for human health, normal total body content of aluminum in humans is around 30 milligrams. Soluble forms of aluminum chloride, aluminum fluoride, and aluminum sulfate are potentially toxic, and may play a role in several disease conditions. Insoluble forms of aluminum do not seem to have a measurable acute response (Rumack and Spoerke 1989). Chronic inhalation of fine metallic aluminum dust, such as may occur in the manufacture of aluminum abrasives, explosives, and fireworks, has been shown to cause a distinctive type of pulmonary fibrosis with emphysema, chronic interstitial pneumonia, dyspnea, cough, and pneumothorax (Clayton and Clayton 1981). In addition, the inhalation of this aluminum dust has caused progressive encephalopathy, in which the brain shows evidence of neurofibrillary degeneration (Venugopal and Luckey 1978). Gastrointestinal problems develop when aluminum hydroxide is chronically administered orally, causing severe 
constipation by inhibiting muscle contraction of the intestines (Hamilton and Hardy 1974). It can also cause flatulence, inflammation, and colitis. Aluminum salts may cause a dialysis encephalopathy syndrome among kidney patients, resulting in progressive fatal brain disease characterized by impaired speech, dementia, and seizures (Rumack and Spoerke 1989). Increasing evidence indicates that the brains of Alzheimer's victims contain a much higher than normal level of aluminum (Rumack and Spoerke 1989). Repeated contact of the skin with soluble aluminum salts results in "acid irritation". Particles of aluminum deposited in the eye may cause necrosis of the cornea. Aluminum has not been shown to be carcinogenic among humans.

Focal pulmonary fibroses were observed among rats following intratracheal injection of $100 \mathrm{mg}$ of aluminum per animal (Clayton and Clayton 1981). Respiratory infections were observed in rats and rabbits where aluminum dust was inhaled or injected intratracheally (Browning, 1969). Accompanying the fibrosis was hyalinosis, emphysema, and hemorrhagic pneumonia. Fibrotic changes were also evident in the walls of the blood vessels and in the kidneys, while fibrous thickening of interstitial tissue in the spleen, liver, and meninges occurred.

\section{ECOLOGICAL EFFECTS}

Although oral toxic doses of aluminum are not well established for humans, oral mammalian $\mathrm{LD}_{s 0}$ 's range from 1 to 4 grams per $\mathrm{kg}$ body weight. Among aquatic plants, diatom growth is inhibited at aluminum concentrations of $810 \mu \mathrm{g} / \mathrm{L}$, concentrations greater than $45.7 \mathrm{mg} / \mathrm{L}$ reduced frond production in duckweeds, and an $\mathrm{EC}_{50}$ of $2.5 \mathrm{mg} / \mathrm{L}$ was reported for root development in Eurasian watermilfoil (Stanley 1974 and U.S. Environmental Protection Agency 1988). An aluminum concentration of $5.2 \mathrm{mg} / \mathrm{L}$ inhibited growth of young rainbow trout in water at a pH of 7.0 to 9.0 (Freeman 1973).

\section{U.S. ENVIRONMENTAL PROTECTION AGENCY HUMAN HEALTH CRITERIA}

Oral Slope Factor

- Inhalation Slope Factor

Chronic Oral RfD

Chronic Inhalation RfC

\author{
NA \\ NA \\ Aluminum $1 \mathrm{E}-01(\mathrm{mg} / \mathrm{kg} /$ day $)$ \\ Aluminum oxide NA \\ NA
}

\section{DRINKING WATER CRITERIA AND STANDARDS}

U.S. Environmental Protection Agency:

Maximum Concentration Limit

NA

Maximum Concentration Limit

NA 


\section{AMBIENT WATER QUALITY CRITERIA}

U.S. Environmental Protection Agency Ambient Water Quality Criteria

Human Health - Water \& Fish Ingestion NA

Human Health - Fish Ingestion Only NA

Aquatic Organisms - FW Acute NA

Aquatic Organisms - FW Chronic NA

Aquatic Organisms - Marine Acute NA

Aquatic Organisms - Marine Chronic NA

South Carolina Department of Health and Environmental Control

Fish and Aquatic Life - Continuous NA

Fish and Aquatic Life - Maximum $\quad \cdot \quad$ NA

$\mathrm{NA}=$ Not Available

\section{REFERENCES}

American Conference of Governmental Industrial Hygienists. 1986. Documentation of the Threshold Limit Values and Biological Exposure Indices, 5th ed. Cincinnati, OH.

Bjorksten, J.A. 1982. Comp. Therapy. 8:7 3-6.

Browning, E. 1969. Toxicity of Industrial Metals, 2nd Ed. Appleton-Century-Crofts, New York, NY.

Clayton, G.D. and F.E. Clayton, (eds). 1981. Patty's Industrial Hygiene and Toxicology, ois. 2A, 2B, 2C, Toxicology, 3rd ed. John Wiley and Sons, New York, NY.

Cleveland, L.; E.E. Little, S.J. Hamilton, R.D. Buckler, and J.B. Hunn. 1986. Interactive Toxicity of Aluminum and Acidity to Early Life Stages of Brook Trout. Trans. Amish. Soc. 115:610-620.

Farm Chemicals Handbook. 1984. Meister Publishing Co., Willoughby, OH.

Freeman, R.A. 1973. Recovery of Rainbow Trout from Aluminum Poisoning. Trans. Am. Fish. Soc. 102:152-154.

Friberg, L., G.R. Nordberg, and V.B. Vouk. 1979. Handbook on the Toxicology of Metals. Elsevier North Holland, New York, NY.

Hamilton, A. and H.L. Hardy. 1974. Industrial Toxicology, 3rd Ed. Publishing Sciences Group, Inc., Acton, MA. 
Merck Index. 1983. 10th Ed. Merck and Co. Inc., Rahway, NJ.

National Research Council (NRC). 1981. Drinking Water and Health, Vol 4. National Academy Press, Washington, D.C.

Rosas, V.V., et al. 1978. Arch. Intern. Med. 138: 1375.

Rumack, B.H. and D.G. Spoerke. 1989. Poisindex (R) Information Systems, CCIS CDROM, Vol. 62. Micromedex Inc., Denver, CO.

Stanley, R.A. 1974. Toxicity of Heavy Metals and Salts to Eurasian Watermilfoil (Myriophyllum spicatum L.). Arch. Environ. Contam Toxicol. 2:331-341.

U.S. Environmental Protection Agency. 1988. Ambient Water Quality Criteria for Aluminum, Doc. No.EPA 440/5-86-008. Washington,, DC.

Venugopal, B. and T.D. Luckey. 1978. Metal Toxicity in Mammals, Vol. 2. Plenum Press, New York, NY.

Weast, R.C. (ed.). 1979. Handbook of Chemistry and Physics, 60th Ed. CRC Press Inc., Boca Raton, FL. 
ARSENIC

CAS No: $\quad 7440-38-2$

Synonym(s) None

Arsenic exists in low concentrations in all environmental media as a natural component of the earth's crust. Typical arsenic concentrations are less than ten parts per billion in water and less than 40 parts per million in soil (U.S. Department of Health and Human Services 1991). It is a silver-gray, brittle, crystalline substance (International Labour Office 1983) that is soluble in nitric acid but insoluble in water (Weast 1987-1988) and in caustic and non-oxidizing acids. It has a molecular weight of $74.92 \mathrm{~g} / \mathrm{mole}$, sublimes at $613^{\circ} \mathrm{C}$, melts at $817^{\circ} \mathrm{C}$ (at 28 atmospheres), has a vapor pressure of $1 \mathrm{~mm} \mathrm{Hg}$ at $372^{\circ} \mathrm{C}$, and has a density of 5.73 at $14^{\circ} \mathrm{C}$ (U.S. Department of Health and Human Services 1991). Arsenic will vaporize when heated to $100^{\circ} \mathrm{C}$, with rapid vaporization at $450^{\circ} \mathrm{C}$ (Merck 1983).

\section{FATE AND TRANSPORT}

Arsenic is found primarily in soil or rock and is transported mainly adsorbed onto particulate material. In water, soluble forms of arsenic can be carried long distances, but may be adsorbed onto sediments or soils due to chemical or biological reactions that change arsenic speciation (U.S. Department of Health and Human Services 1991). In soil, arsenic is subject to a number of transformation processes, such as oxidation-reduction reactions, ligand exchange, biotransformation, precipitation, and adsorption. It is rarely encountered as a free element in natural waters (U.S. Environmental Protection Agency 1980), where it is also subject to processes such as oxidation-reduction reactions, ligand exchange, biotransformation, precipitation, and adsorption (U.S. Department of Health and Human Services 1991). Arsenic accumulates to varying degrees in some organisms such as algae and shellfish, but does not biomagnify in the food chain. Some arsenic is absorbed via inhalation and oral routes, but dermal absorption is considered to be minor (U. S. Department of Health and Human Services 1991).

\section{HUMAN HEALTH EFFECTS}

Inorganic arsenic compounds are generally more toxic than organic forms. For inorganic arsenic, human oral exposures of approximately 1 to $3 \mathrm{mg}$ per $\mathrm{kg}$ body weight appear to be the minimum lethal dose, based upon clinical reports (U.S. Department of Health and Human Services 1991). Populations at special risk from arsenic exposure include persons with existing diabetes, cardiovascular diseases, allergic or other skin diseases, neurologic or hepatic disease, or renal lesions. Children may be at special risk for the effects of inorganic arsenic. Exposure to high levels of arsenic results in a number of systemic effects on the respiratory system, heart and vascular system, gastrointestinal system, blood, liver, kidney, skin, eye, and nervous system. Specific effects reported include decreased production of red and white blood cells, abnormal heart and nerve function, damage to blood vessels, kidneys and liver, and possible mutagenic activity. The burden of evidence shows inorganic arsenic to be a carcinogen, resulting in an increased risk of lung and dermal cancer by inhalation and oral exposure, respectively. Organic arsenic has not been demonstrated to be 
carcinogenic to humans. The U.S. Environmental Protection Agency has classified arsenic as Class A, a known human carcinogen.

\section{ECOLOGICAL EFFECTS}

Arsenic toxicity to microorganisms produces a decline in growth and metabolic rates. The more tolerant species can withstand arsenic levels up to $1,000 \mathrm{ppm}$, whereas the most sensitive organisms are affected by levels less than 375 ppm (National Research Council Canada 1978). An $\mathrm{LC}_{50}$ of $10 \mathrm{mg} / \mathrm{l}$ is reported for black sea mussels (Pereladov and Erofeeva 1983). In rats an inhalation $L C_{50}$ of $2,100 \mathrm{ppm}$ is reported by Stevens et al. (1979). Among mice, dermal application of $2.5 \mathrm{mg}$ organic arsenic per $\mathrm{kg}$ body weight was reported to be the lowest observed adverse effect level for localized hyperplasia for 18 weeks of exposure (Boutwell 1963).

\section{U.S. ENVIRONMENTAL PROTECTION AGENCY HUMAN HEALTH CRITERIA}

Oral Slope Factor

Inhalation Slope Factor

Chronic Oral RfD

Chronic Inhalation RfD
$1.5 \mathrm{~kg} / \mathrm{mg} / \mathrm{day}^{-1}$

$5.0 \mathrm{E}+01 \mathrm{~kg} / \mathrm{mg} / \mathrm{day}^{-1}$

$3.0 \mathrm{E}-04 \mathrm{mg} / \mathrm{kg} /$ day

NA

\section{DRINKING WATER CRITERIA AND STANDARDS}

U.S. Environmental Protection Agency

Maximum Concentration Limit

Maximum Concentration Limit Goal

$0.05 \mathrm{mg} / \mathrm{L}$

NA

\section{AMBIENT WATER QUALITY CRITERIA}

U.S. Environmental Protection Agency Ambient Water Quality Criteria (as Arsenic III)

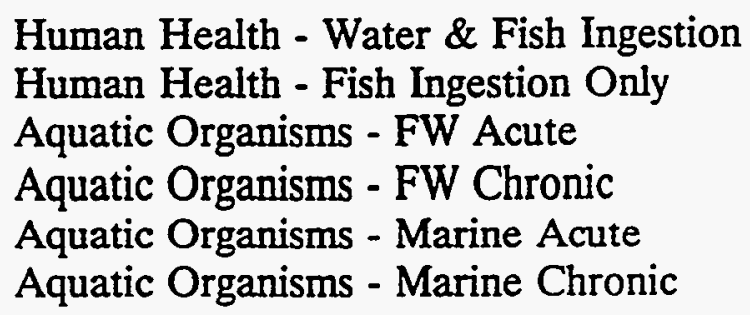

2.2E-06 mg/L

$1.7 \mathrm{E}-05 \mathrm{mg} / \mathrm{L}$

$3.6 \mathrm{E}-01 \mathrm{mg} / \mathrm{L}$

$1.9 \mathrm{E}-01 \mathrm{mg} / \mathrm{L}$

$6.9 \mathrm{E}-02 \mathrm{mg} / \mathrm{L}$

Aquatic Organisms - Marine Chronic

$3.6 \mathrm{E}-02 \mathrm{mg} / \mathrm{L}$

South Carolina Department of Health and Environmental Control (as Arsenic III)

Human Health Criteria

Fish and Aquatic Life - Continuous

Fish and Aquatic Life - Maximum
$1.4 \mu \mathrm{g} / \mathrm{L}$

$360 \mu \mathrm{g} / \mathrm{L}$

$190 \mu \mathrm{g} / \mathrm{L}$

NA $=$ Not Available 


\section{REFERENCES}

Agency for Toxic Substancees and Disease Registry. 1991. Toxicological Profile for Arsenic. Prepared by Clement International Corp., contract no. 205-88-0608.

Boutwell, R.K. 1963. A Carcinogenicity Evaluation of Potassium Arsenite and Arsenilic Acid. Agric. Food Chem. 11:381-385.

International Labour Office. 1983. Encyclopedia of Occupational Health and Safety. McGraw-Hill Book Co., New York, NY.

Merck. 1983. The Merck Index, 10th Ed. Merck Company, Inc., Rahway, NJ.

National Research Council of Canada. 1978. Effects of Arsenic in the Canadian Environment, Doc. No. NRCC 15391. National Research Council of Canada.

Pereladov, M.V. and M.P. Erofeeva. 1983. Ekol. Aspekty Khim Radioakt. Zagryaz. Vodn. redy. 61-4.

Stevens, J.T., L.C. DiPasquale, and J.D. Farmer. 1979. The acute inhalation toxicology of the clinical grade organoarsenical herbicides, cacodylic acid and disodium methanearsonic acid; a route comparison. Bull. Environ. Contam. Toxicol. 21:304-311.

U.S. Department of Health and Human Services. 1991. Toxicological Profile for Arsenic, Draft Update. Agency for Toxic Substances and Disease Registry, Atlanta, GA.

U.S. Environmental Protection Agency. 1980. Ambient Water Quality Criteria Document: Arsenic, EPA-440/5-80-021. U.S. Environmental Protection Agency, Washington, DC.

Weast, R.C. (ed.). 1987-1988. Handbook of Chemistry and Physics, 68th Ed., Vol. B. CRC Press Inc., Boca Raton, FL. 
THIS PAGE LEFT INTENTIONALLY BLANYK 


\section{BARIUM}

CAS No.:

7440-39-3

Synonym (s)

None

Barium occurs in nature primarily as barite $\left(\mathrm{BaSO}_{4}\right)$ and witherite $\left(\mathrm{BaCO}_{3}\right)$. Barite is a common mineral found in medium and low temperature hydrothermal veins associated with lead, silver, and antimony sulfides and in replacement veins, cavernous limestone formations, and dolomites. Witherite occurs in low temperature hydrothermal veins and is associated with barite and galena (Mottana 1978). Barium combines readily with oxygen, nitrogen, hydrogen, ammonia, water, halogens, and sulfides. The mineral forms of barium are relatively insoluble in water, have high melting and boiling points, and have very low vapor pressures (U.S. Environmental Protection Agency 1985). Barium acetate, nitrate, chloride, and hydroxide are very soluble in water. Solubility of barium compounds increases as $\mathrm{pH}$ decreases. Barium has no detectable odor or taste. Barium is soluble in alcohol and insoluble in benzene (Weast 1988-1989). It is somewhat malleable and has a distinctive property of absorbing gases (Clayton and Clayton 1981-1982). Barium has a molecular weight of 137.33 grams per mole, it melts at $725^{\circ} \mathrm{C}$ (at one atmosphere), boils at $1,640^{\circ} \mathrm{C}$ (at one atmosphere), has a vapor pressure of $10 \mathrm{~mm} \mathrm{Hg}$ at $1,049^{\circ} \mathrm{C}$ and density of 3.51 at $20^{\circ} \mathrm{C}$.

\section{FATE AND TRANSPORT}

Barium metal does not occur freely in nature (Kirk-Othmer Encyclopedia of Chemical Technology 1978-1984). Seawater contains about $0.03 \mathrm{ppm}$ barium (Venugopal and Luckey 1978). Background levels in natural soils range from 100 to $3,000 \mathrm{ppm}$ barium (Brown et al. 1983). The average concentration of barium in air was reported to be $5 \mathrm{ng} / \mathrm{m}^{3} \cdot(0$ to $1500 \mathrm{ng} / \mathrm{m}^{3}$ ) in 18 United States cities (Friberg et al. 1986). Only sketchy data are available describing environmental concentrations of barium in food, plants, fish, mammals, or milk. No data are available describing the environmental fate, environmental transformations, or transport of the compound.

\section{HUMAN HEALTH EFFECTS}

Humans are exposed to barium by ingestion or inhalation of dust or fume and by skin or eye contact. The average daily intake for the human adult is about 1.3 milligrams ( 0.65 to 1.7 milligrams) of total barium (Venugopal and Luckey 1978). About 90 percent of the total body barium occurs in the skeleton (Friberg et al., International Technical Information Institute, 1982). Insoluble forms of barium are not toxic by inhalation or ingestion because only minimal amounts are absorbed. Soluble barium compounds are highly toxic in humans by either route of exposure. The most important effect of acute barium exposure is a strong, prolonged stimulant action on muscle. Smooth, cardiac, and skeletal muscles are all affected, and a transient increase in blood pressure due to vasoconstriction can occur. Effects on the hematopoietic system and cerebral cortex have also been reported in humans. Accidental ingestion of soluble barium salts has resulted in gastroenteritis, muscle paralysis, and ventricular fibrillation and extra systoles. A benign pneumoconiosis, called baritosis, can result from inhaling barium dusts (Sittig 1985). There are no reports of carcinogenicity 
associated with exposure to barium or its compounds. Barium has not been evaluated by the U.S. Environmental Protection Agency for evidence of human carcinogenicity.

\section{ECOLOGICAL EFFECTS}

No information on the ecotoxicity of barium is available.

\section{U.S. ENVIRONMENTAL PROTECTION AGENCY HUMAN HEALTH CRITERIA}

Oral Slope Factor

Inhalation Slope Factor

Chronic Oral RfD

Chronic Inhalation RfD
NA

NA

$7 \mathrm{E}-02 \mathrm{mg} / \mathrm{kg} / \mathrm{day}$

$5 \mathrm{E}-4 \mathrm{mg} / \mathrm{m}^{3}$

\section{DRINKING WATER CRITERIA AND STANDARDS}

U.S. Environmental Protection Agency

Maximum Concentration Limit

Maximum Concentration Limit Goal

$2.0 \mathrm{mg} / \mathrm{L}$

$2.0 \mathrm{mg} / \mathrm{L}$

\section{AMBIENT WATER QUALITY CRITERIA}

U.S. Environmental Protection Agency Ambient Water Quality Criteria

Human Health - Water \& Fish Ingestion

Human Health - Fish Ingestion Only

Aquatic Organisms - FW Acute

Aquatic Organisms - FW Chronic

Aquatic Organisms - Marine Acute

Aquatic Organisms - Marine Chronic
$1.0 \mathrm{mg} / \mathrm{L}$

NA

NA

NA

NA

NA

South Carolina Department of Health and Environmental Control

Human Health Criteria

Fish and Aquatic Life - Continuous

Fish and Aquatic Life - Maximum
NA

NA

NA

$\mathrm{NA}=$ Not Available

\section{REFERENCES}

Brown, K.W., G.B. Evans, and B.D. Frentrup (eds.). 1983. Hazardous Waste Land Treatment. Butterworth Publishers, Boston, MA. 
Clayton, G.D. and F.E. Clayton (eds.). 1981-1982. Patty's Industrial Hygiene and Toxicology, 3rd Ed., Vols. 2A, 2B, 2C. John Wiley and Sons, New York, NY.

Friberg, L., G.F. Nordberg, E. Kessler, and V.B. Vouk (eds.). 1986. Handbook of Toxicology of Metals, 2nd Ed., Vols. I and II. Elsevier Science Publishers B.V., Amsterdam, The Netherlands.

International Technical Information Institute 1982. Toxic and Hazardous Industrial Chemicals Safety Manual. The International Technical Information Institute, Tokyo, Japan.

Kirk-Othmer Encyclopedia of Chemical Technology, 3rd Ed. 1978-1984.. John Wiley and Sons, New York, NY.

Mottana, A., R. Crespi, and G. Liborio. 1978. Rocks and Minerals. Simon and Schuster, New York, NY.

Sittig, M. 1985. Handbook of Toxic and Hazardous Chemicals. Noyes Publications, Park Ridge, NJ.

U.S. Environmental Protection Agency. 1985. Drinking Water Criteria Document for Barium (Draft), Doc. No. TR-540-60F. U.S. Environmental Protection Agency, Washington, DC.

Venugopal, B. and T.D. Luckey. 1978. Metal Toxicity in Mammals 2. Plenum Press, New York, NY.

Weast, R.C. (ed.). 1988-1989. Handbook of Chemistry and Physics, 69th Ed. CRC Press Inc., Boca Raton, FL 
THIS PAGE LEFT INTENTIONALLY BLANK 
BENZOIC ACID

CAS No.: $\quad$ 65-85-0

Synonym(s) none

In nature benzoic acid occurs in berries, prunes, cloves, anise seed, and the scent gland of beavers (HSDB 1993). It is widely used as a food preservative due to its antifungal/ antibacterial properties. Those same properties make benzoic acid useful also as a therapeutic agent. Industrial demand for benzoic acid includes its use in the manufacture of phenol benzoate plasticizers, benzoyl chloride, and cosmetics. Releases into the environment can result from industrial wastewater releases, fuel exhaust, refuse combustion, and tobacco smoke (HSDB 1994).

Benzoic acid is a white or tan solid that is nearly tasteless and odorless. It has a molecular weight of 122.13 , a melting point of $122.4^{\circ} \mathrm{C}$, and a boiling point of $249.2^{\circ} \mathrm{C}$. It has a density of 1.2659 at $15^{\circ} \mathrm{C}$ and a pka of 4.2 , indicating that it is generally in a dissociated state in nature (HSDB 1994).

\section{FATE AND TRANSPORT}

In soil, benzoic acid is biodegraded with a half-life of less than 1 week. In water, benzoic acid is degraded under both aerobic and anaerobic conditions with a half-life ranging from 8-3.6 days. Volatilization and adsorbance to sediments. would be minimal in soil as well as water (HSDB 1994).

When present in the atmosphere, benzoic acid is in the form of aerosols and therefore is subject to transport by precipitation and gravitational settling. Photolysis may be an important degradation pathway for removal of benzoic acid from air and soils such as sand. The half-life of benzoic acid in air is estimated to be 2 days (HSDB 1994).

\section{HUMAN HEALTH EFFECTS}

The most prominent route of entry into the body is through ingestion of food which has had benzoic acid added as a food preservative. In the GI tract, benzoic acid is rapidly ionized to benzoate, which is quickly absorbed. Benzoate is conjugated with glycine to form hippuric acid by the liver. Hippuric acid is then excreted, primarily in the urine. (Flower et al. 1980).

A daily intake of 4-6 grams of benzoic acid has not been associated with toxic symptoms, other than minor GI irritation. However, certain hypersensitive individuals, such as asthmatics, show a positive reaction to benzoic acid. In one case history, a chemical worker exhibited allergic reactions upon exposure to benzoic acid and suffered severe anaphylactic shock following ingestion of benzoic acid. (Flowers et al. 1980).

Ingestion of large doses of benzoic acid produces toxic symptoms similar to the systemic effects of salicylates (aspirin and related compounds). These effects range from headache, dizziness, tinnitus, hyperventilation, intoxication, and vertigo to more severe symptoms such as acid/base disturbances, convulsions, and coma. (Flower et al. 1980): 
ECOLOGICAL EFFECTS

Benzoic acid is degraded in the environment and readily metabolized by biota therefore it is not expected to bioaccumulate in the food chain. Researchers reported little potential for bioaccumulation in algae, fish, or mosquito larvae, but a greater potential does exist for bioaccumulation in snail and daphnia (HSDB 1994).

US ENVIRONMENTAL PROTECTION AGENCY HUMAN HEALTH CRITERIA

Oral Slope Factor

Inhalation Slope Factor

Chronic Oral RfD

Chronic Inhalation RfD
NA

NA

$4 \mathrm{mg} / \mathrm{kg} /$ day

NA

DRINKING WATER CRITERIA AND STANDARDS

U.S. Environmental Protection Agency

Maximum Concentration Limit

Maximum Concentration Limit Goal

NA

NA

\section{AMBIENT WATER QUALITY CRITERIA}

U.S. Environmental Protection Agency Ambient Water Quality Criteria

Human Health-Water and Fish Ingestion

NA

Human Health-Fish Ingestion Only

Aquatic Organisms-FW Acute

NA

Aquatic Organisms-FW Chronic

NA

Aquatic Organisms-Marine Acute

NA

Aquatic Organisms-Marine Chronic

NA

NA

South Carolina Department of Health and Environmental Control

Human Health Criteria

Fish and Aquatic Life-Continuous

Fish and Aquatic Life-Maximum
NA

NA

NA

$\mathrm{NA}=$ Not Available 


\section{REFERENCES}

Flower, R.J., Moncada, S., Vane, J.R. 1980. Analgesic-antipyretics and anti-inflammatory agents; Drugs employed in the treatment of gout. In: Goodman and Gilman's The Pharmacological Basis of Therapeutics., Gilman,AG, Goodman,LS., Gilman,A. eds. New York. MacMillan Publishing.

HSDB. 1994. Hazardous Substance Data Bank. NLM. On-line computer data base. September 2, 1994. 
THIS PAGE LEFT INTENTIONALLY BLANK 


\section{BENZENE HEXACHLORIDE}
CAS No.:
58-89-9
Synonym(s)
Hexachlorocyclohexane, lindane

Benzene hexachloride is a mixture of 8 isomers that has applications as an insecticide agriculturally on fruit, nut, and vegetable crops, and residentially as a fumigant for the control of roaches and flies. Only the gamma isomer (known as lindane) has insecticidal properties, even though it comprises only a small percentage of total benzene hexachloride (Klaasen 1980; Harte 1991).

Purified benzene hexachloride is a colorless crystalline solid with only slight solubility in water. It is soluble in chloroform, alcohol, acetone, ether and benzene. The molecular weight of benzine hexachloride is 290.85 , and it has a melting point of $112.5^{\circ} \mathrm{C}$ (Windholz Merkcitator 1983).

\section{FATE AND TRANSPORT}

Most uses of benzene hexachloride have been withdrawn by the EPA. Prior to its withdrawal, benzine hexachloride was used in dusts, sprays, and aerosols in large quantities, making airborne benzine hexachloride a potential exposure route (Harte 1991).

Benzine hexachloride is resistant to degradation in soils and water, and these two routes represent the primary potential exposure pathways to the general public since its use has been restricted.

\section{HUMAN HEALTH EFFECTS}

Lindane, or the gamma isomer, is the most toxic of the 8 isomers that make up benzine hexachloride. The toxic effects of benzine hexachloride exposure are similar to, but more pronounced than, DDT exposure. Tremors, ataxia, convulsions, and prostration result from benzine hexachloride poisoning. The onset of symptoms is rapid and is first characterized. by headache, weakness, diarrhea and dizziness. Increased heart rate and decreased blood pressure are signs of benzine hexachloride poisoning (Klaasen 1980, Mott and Snyder 1987, Harte 1991). Chronic exposure to benzine hexachloride produces aplastic anemia, renal and hepatic damage, reproductive dysfunction, and increased incidence of fetal death (Harte 1991, Loge 1965, West 1967).

\section{ECOLOGICAL EFFECTS}

Benzine hexachloride can enter the terrestrial food chain through uptake by plants from contaminated soils. Residues have been noted on corn, a crop currently restricted from benzine hexachloride use. Additionally, human consumption of poultry and dairy products are potential exposure routes. Aquatic food species, such as marine vertebrates and invertebrates, have limited tissue bioaccumulation (Harte 1991, N.A.S. 1987). 


\section{U.S. ENVIRONMENTAL PROTECTION AGENCY HUMAN HEALTH CRITERIA}

Oral Slope Factor

Inhalation Slope Factor

Chronic Oral RfD

Chronic Inhalation RfD
$1.30 \mathrm{~kg} / \mathrm{mg} / \mathrm{day}^{-1}$

NA

$3 \mathrm{E}-04 \mathrm{mg} / \mathrm{kg} /$ day

NA

\section{DRINKING WATER CRITERIA AND STANDARDS}

U.S. Environmental Protection Agency

Maximum Concentration Limit

$2 \mathrm{E}-04 \mathrm{mg} / \mathrm{L}$

Maximum Concentration Limit Goal

2E-04 mg/L

\section{AMBIENT WATER QUALITY CRITERIA}

U.S. Environmental Protection Agency Ambient Water Quality Criteria

Human Health - Water and Fish Ingestion

Human Health - Fish Ingestion Only

Aquatic Organisms - FW Acute

Aquatic Organisms - FW Chronic

Aquatic Organisms - Marine Acute

Aquatic Organisms - Marine Chronic
$9.2 \mathrm{ng} / \mathrm{L}$

$31 \mathrm{ng} / \mathrm{L}$

$2.0 \mu \mathrm{g} / \mathrm{L}$

$0.06 \mu \mathrm{g} / \mathrm{L}$

$0.16 \mu \mathrm{g} / \mathrm{L}$

NA

South Carolina Department of Health and Environmental Control Water Quality Criteria

Human Health Criteria

Fish and Aquatic Life - Continuous

Fish and Aquatic Life - Maximum
$0.625 \mu \mathrm{g} / \mathrm{L}$

NA

NA

$\mathrm{NA}=$ Not Available

\section{REFERENCES}

Harte, J., Holdren, C., Schneider, R., Shirley, C. 1991. Toxics A to Z. A Guide to everyday pollution hazards. Los Angeles, CA. Univ. California Press.

Mott, L., Snyder, K. 1987. Pesticide Alert. San Francisco: Sierra Club Books.

NAS. 1987. National Acad. Sci. 1987., Regulating pesticides in food. Washington, DC. National Acad. Press. 
Klaasen, C.D. 1980. Nonmetallic environmental toxicants: Air pollutants, solvents, and vapors, and pesticides: in Goodman \& Gilman's The Pharm. Basis of Therap. 6th ed. Gilman, A.G., Goodman, L.S., Gilman, A. (eds.) NY. MacMillan Pub. Co., Inc.

Loge, J.P. 1965. Aplastic anemia following exposure to benzene hexachloride (Lindane) JAMA 193:110-114.

West, I. 1967. Lindane \& hematologic reactions. Arch. Environ. Health 15:97-101. 
THIS PAGE LEFT INTENTIONALLY BLANK 
BIS (2-ETHYLHEXYL) PHTHALATE

CAS No(s):: $\quad$ 117-81-7

Synonym(s) DEHP, BEHP

Bis (2-ethylhexyl) phthalate is produced commercially for use as a plasticizer in polyvinyl chloride (PVC) and vinyl copolymer resins (EPA 1981). Other reported uses include bis (2ethylhexyl) phthalate as an inert component of pesticides, a solvent for erasable ink, as a component of dielectric fluids, as a replacement for PCBs, and others (EPA 1981, HSDB 1987).

Bis (2-ethylhexyl) phthalate is a colorless liquid having a molecular weight of 390.54. It has a melting point of $50^{\circ} \mathrm{C}$ and a boiling point of $385^{\circ} \mathrm{C}$. It has minimal solubility in water, but is miscible with oil, hexane, and organic solvents (ATSDR 1988).

\section{FATE AND TRANSPORT}

Bis (2-ethylhexyl) phthalate may be volatilized into the atmosphere during its manufacture and from commercial plastics use. Bis (2-ethylhexyl) phthalate adsorbs to particulate matter, therefore wet deposition would assist in transport of bis (2-ethylhexyl) phthalate from air to other media (ATSDR 1988). The most likely mechanism for degradation of bis (2ethylhexyl) phthalate in the atmosphere is through its reaction with hydroxyl radicals.

Bis (2-ethylhexyl) phthalate does not readily volatilize from surface waters to air. Its removal from water is principally through adsorption to suspended particulate matter and sediments and uptake and transformation by biotic organisms (ATSDR 1988).

In soils, bis (2-ethylhexyl) phthalate binds particles, particularly organic matter. There is little mobility of bis (2-ethylhexyl) phthalate in soils and biodegradation in soils occurs faster in aerobic versus anaerobic conditions.

\section{HUMAN HEALTH EFFECTS}

There is little data in the literature concerning the human health hazards of bis (2ethylhexyl) phthalate exposure. Morbidity and mortality studies were unable to correlate long-term bis (2-ethylhexyl) phthalate exposure with increased incidence of deaths and/or illness (Theiss et al. 1978).

Animal studies indicate bis (2-ethylhexyl) phthalate is hepatotoxic, producing chemical and morphological changes (Carpenter et al. 1953; Mitchell et al. 1985). Bis (2ethylhexyl) phthalate is a reproductive toxicant in rats and mice, causing testicular damage (Gangolli 1982; Reel et al. 1982). Bis (2-ethylhexyl) phthalate has also been associated with increased occurrence of hepatic carcinoma in a dose dependent manner (NTP 1982, Kluwe et al. 1982a). 


\section{ECOLOGICAL EFFECTS}

Bis (2-ethylhexyl) phthalate is highly lipid soluble and tends to partition into the lipid compartments of animals and plants. However, with the exception of a few aquatic crustacea and midge larvae, most organisms metabolize bis (2-ethylhexyl) phthalate at a rate sufficient to offset the tendency for bioconcentration of bis (2-ethylhexyl) phthalate.

\section{U.S. ENVIRONMENTAL PROTECTION AGENCY HUMAN HEALTH CRITERIA}

Oral Slope Factor

Inhalation Slope Factor

Chronic Oral RfD

Chronic Inhalation RfD
$1,4 \mathrm{E}-2 \mathrm{~kg} / \mathrm{mg} /$ day $^{-1}$

NA

$2 \mathrm{E}-2 \mathrm{mg} / \mathrm{kg} /$ day

NA

\section{DRINKING WATER CRITERIA AND STANDARDS}

U.S. Environmental Protection Agency

Maximum Concentration Limit

Maximum Concentration Limit Goal

$0.006 \mathrm{mg} / \mathrm{L}$

$0.0 \mathrm{mg} / \mathrm{L}$

\section{AMBIENT WATER QUALITY CRITERIA}

U.S. Environmental Protection Agency Ambient Water Quality Criteria

Human Health - Water and Fish Ingestion NA

Human Health - Fish Ingestion Only NA

Aquatic Organisms - FW Acute NA

Aquatic Organisms - FW Chronic NA

Aquatic Organisms - Marine Acute NA

Aquatic Organisms - Marine Chronic NA

South Carolina Department of Health and Environmental Control Water Quality Criteria

Human Health Criteria

Fish and Aquatic Life - Continuous

Fish and Aquatic Life - Maximum
$59.2 \mu \mathrm{g} / \mathrm{L}$

NA

NA

$\mathrm{NA}=$ Not Available

\section{REFERENCES}

Agency for Toxic Substances and Disease Registry (ATSDR). 1988. Draft Toxicological Profile for Bis (2-ethylhexyl) phthalate. Prepared by Clement International Corp., contract no. 205-88-0608. 
Carpenter, C.P., Weil, C.S., Smyth, H.F. 1953. Chronic oral toxicity of bis (2ethylhexyl) phthalate for rats, guinea pigs, and dogs. Am. Med. Assoc. Arch. Ind. Hyg. 8:219-226.

EPA. 1981. An exposure and risk assessment for phthalate esters. Final draft report. Office of Water Regulations and Standards, Washington, DC.

Gangolli, S.D. 1982. Testicular effects of phthalate esters. Environ. Health Perspect. 45:7784.

HSDB (Haz. Subs. Data Bank). 1987. Record for Bis (2-ethylhexyl) phthalate Computer Printout NLM April 9.

Kluwe, W.M., Hademan, J.R., Douglas, J.F., Huff, J.E. 1982. The carcinogenicity of dietary bis (2-ethylhexyl) phthalate in Fischer 344 rats and B6C3F(1) mice. J. Toxicol Environ. Health 10:797-815.

Mitchell, F.E., Price, S.C., Hinton, R.H., Grasso, P., Bridges, J.W. 1985. Time and does response study of the effects on rats of the plasticizer bis (2-ethylhexyl) phthalate. Toxicol Appl. Pharmacol. 81:371-392.

NTP (National Tox. Prog.). 1982. Carcinogenesis Bioassay of Bis (2-ethylhexyl) phthalate (CAS No. 117-81-7) in F344 rats and B63F1 mice (feed study) National Tox. Prog., Research Triangle Park, NC. NTP-80-37. NIH pub. 82-1772.

Reel, J.R., Lawton, A.D., Lamb, J.C. 1982. Diethylhexyl phthalate (bis (2ethylhexyl) phthalate): Reproduction and fertility assessment in CD-1 mice when administered in the feed. Report ISS RTI-31U-2344, RTI-72, NTP-84-079. Order PB84-181734 p. 221.

Theiss, A.M., Korte, A., Fleig, H. 1978. Mortality Study in workers exposed to di(2ethylhexyl)phthalate (OOP). In: Possibilities and Limits of Biological Monitoring. Problems of Occupational Medicine in Small Industries. Colloquium in Occ. Med. Frankfurt/M., AWGentner, pp 155-164 (Cited in IARC 1981). 


\section{THIS PAGE LEFT INTENTIONALLY BLANK}


BUTYLBENZYL PHTHALATE

CAS No.: $\quad 111-44-4$

Synonym(s) Dichloroethyl ether, Bis(2-chloroethyl)ether

Butylbenzyl phthalate, more commonly known as bis(2-chloroethyl)ether or BCEE, is a member of the group of compounds known as chloroalkyl ethers. These solvents are used in the production of ethylcellulose, gum, lacquer, paint, soap, tar, and insecticides.

Butylbenzyl phthalate has a molecular weight of 143.01 , a melting point of $-25.5^{\circ} \mathrm{C}$, and a boiling point of $176-178^{\circ} \mathrm{C}$. It is a colorless liquid which is practically insoluble in water but is soluble in many organic solvents (Sittig 1980). It has a nauseating odor resembling ethylene dichloride (HSDB).

\section{FATE AND TRANSPORT}

Releases of butylbenzyl phthalate to the environment may occur as wastewater from chemical plants which manufacture rubber, insecticides, or glycol products. Laboratory workers, chemical plant workers, and polymer manufacturers are most likely to be exposed to high concentrations of butylbenzyl phthalate vapors. Furthermore, in areas of labs or factories where HCL and formaldehyde are both used, the vapors may combine to form butylbenzyl phthalate in the air (Sittig 1980).

Monitoring studies of air, water, and soil have determined that butylbenzyl phthalate impacts all of these media. In air, butylbenzyl phthalate undergoes reactions with hydroxyl radicals, with a half-life of approximately 13.44 hours. It is not expected to undergo photolysis in the troposphere, because it does not absorb light at the wavelengths present in the troposphere (HSDB 1994).

Butylbenzyl phthalate contamination in surface waters may be subject to evaporation, with the rate of vaporization faster in streams and rivers ( half-life $=3.5-4.5$ days) than in lakes (half-life $=180.5$ days). Acclimated microorganisms can biodegrade butylbenzyl phthalate, however photolysis and hydrolysis are not thought to be significant degradation pathways (HSDB 1994).

In soils, butylbenzyl phthalate is mobile; very extensive leaching through the soil into groundwater is expected. Hydrolysis may degrade butylbenzyl phthalate in soils, and a limited amount of information on biodegradation in soils suggest that acclimated microbes may be able to transform butylbenzyl phthalate (HSDB 1994).

\section{HUMAN HEALTH EFFECTS}

Exposure to and absorption of butylbenzyl phthalate occur through inhalation, ingestion, or dermal contact. Vapor exposure is strongly irritating to skin, eyes, and mucous membranes. Exposure of humans to $500 \mathrm{ppm}$ butylbenzyl phthalate caused extreme irritation to the eyes and nose. This same dosage precipitated coughing, nausea, and vomiting in subjects. At the lower dose of $100 \mathrm{ppm}$ subjects suffered some irritation. No effects were observed at 35 
ppm. Repeated exposures to low concentrations, as may occur in industrial settings, causes mild bronchitis (NIOSH 1981). Rats and guinea pigs exposed to high concentrations of butylbenzyl phthalate for short periods of time suffered from unsteadiness, slowed and shallow respiration, unconsciousness, and death . The respiratory tract was the primary site of injury, and post mortem examination revealed emphysema and edema, whereas the trachea and bronchi were congested (Patty 1981).

The US EPA has given butylbenzyl phthalate a group B2 rating, or a ranking as a probable carcinogen in humans (EPA 1988). Mice given subcutaneous injections of butylbenzyl phthalate developed sarcomas at the injection site. Mice fed butylbenzyl phthalate by gavage developed hepatomas and lymphomas. Conversely, when induction of pulmonary tumors by butylbenzyl phthalate was studied, it was found that there was no correlation between oral ingestion of butylbenzyl phthalate and pulmonary tumor induction (NRC 1980).

\section{ECOLOGICAL EFFECTS}

Butylbenzyl phthalate is toxic to plants in the growing stage, however grasses are the most resistant to its deleterious effects (HSDB 1994). Conversely, when fat head minnows were exposed to high concentrations of butylbenzyl phthalate, no toxic effects were noted (EPA 1980). Barrows et al. (1980) determined bioconcentration factors experimentally in bluegill fish. According to their calculated factor, bioconcentration and biomagnification should not occur.

\section{US ENVIRONMENTAL PROTECTION AGENCY HUMAN HEALTH CRITERIA}

Oral Slope Factor

Inhalation Slope Factor

Chronic Oral RfD

Chronic Inhalation RfC
NA

NA

$2 \mathrm{E}-1 \mathrm{mg} / \mathrm{kg} /$ day

NA

\section{DRINKING WATER CRITERIA AND STANDARDS}

U.S. Environmental Protection Agency

Maximum Concentration Limit

Maximum Concentration Limit Goal

$0.1 \mathrm{mg} / \mathrm{L}$

0

\section{AMBIENT WATER QUALITY CRITERIA}

U.S. Environmental Protection Agency Ambient Water Quality Criteria

Human Health-Water and Fish Ingestion

NA

Human Health =Fish Ingestion Only NA

Aquatic Organisms-FW Acute NA

Aquatic Organisms-FW Chronic NA 
Aquatic Organisms-Marine Acute

NA

Aquatic Organisms-Marine Chronic

NA

South Carolina Department of Health and Environmental Control Water Quality Criteria

Human Health Criteria

Fish and Aquatic Life-Continuous

Fish and Aquatic Life-Maximum
$5202 \mu \mathrm{g} / \mathrm{L}$

NA

NA

$\mathrm{NA}=$ Not Available

\section{REFERENCES}

Barrows, M.E., et al. 1980. in: Dynamics, Exposure and Hazard Assessment of Toxic Chemicals. Ann Arbor, MI. Ann Arbor Science.

EPA. 1986. Methodology for evaluating potential carcinogenicity in support of reportable quantity adjustments pursuant to Cercla section 102 (final) p.38. EPA/600/8-89/053.

EPA. 1980. Ambient Water Quality Document. Chloroalkyl Ethers. EPA 440/5-90-030. p.B-1.

HSDB. 1994. Hazardous Substance Data Bank. NLM. On-Line Computer program. September 13, 1994.

NIOSH. 1981. OSHA Occupational Health Guide to Chemical Hazards. 1981. As cited in HSDB 1994.

NRC. 1980. Drinking water and health. Vol 3:89. As cited in HSDB 1994.

Patty. 1981. Industrial Hygiene and Toxicology. 3rd ed. Vol 2:1676. As cited in HSDB . 1984.

Sittig, M. 1980. Priority Toxic Pollutants: Health impacts and allowable limits. Environmental Health Review No 1. Park Ridge, NJ. Noyes Data Corp. 
THIS PAGE LEFT INTENTIONALLY BLANK

1 


\section{CADMIUM}

CAS No:7440-43-9

Synonym(s)none

Cadmium is a naturally occurring element which occurs in nature in association with other metals such as zinc and lead, and is usually not found in its pure form. It is often combined with other elements such as oxygen (cadmium oxide), chlorine (cadmium chloride), or sulfur (cadmium sulfide). Cadmium compounds are solids which are soluble in water to varying degrees ( $\mathrm{Cd}<$ cadmium carbonate $<$ cadmium sulfide $<$ cadmium oxide $<$ cadmium chloride). Pure cadmium is a white, odorless, tasteless solid with a molecular weight of 112.40 , a melting point of $320.9^{\circ} \mathrm{C}$, a boiling point of $767^{\circ} \mathrm{C}$, and a density of 8.642 (Sax and Lewis 1987).

\section{FATE AND TRANSPORT}

Extraction of zinc, lead, or copper also releases cadmium to the environment. Cadmium use was infrequent prior to the 20th century, however recognition of its resistance to corrosion increased its demand and it is now used in the manufacture of metal alloys, in nickel cadmium batteries, in pigments, metal coatings, and plastics.

Cadmium and cadmium compounds do not readily vaporize but may be suspended as particles in air. Cadmium emissions to the atmosphere result from combustion of fossil fuels, industrial emissions, or erosion of soils (Elinder 1985; Keitz 1980). Cadmium particles may be small enough to reach the gas-exchange surfaces of the lungs or large enough to be excluded from these areas, depending on the source. Small particles may be transported through the air over large distances, whereas larger particles may be deposited on the earth's surface due to gravitational settling (ATSDR 1991).

Cadmium may exist in water as the hydrated ion $(\mathrm{Cd}(+2) 6 \mathrm{H} 2 \mathrm{O})$ or may be complexed with organic material. It tends to be more mobile in water than heavier metals (Callahan et al. 1979), however sorption to mineral surfaces and humic materials are important transport pathways for removal of cadmium from water. Sediment bacteria are also important for transporting cadmium from the water column into the sediment (Burke and Pfister 1988). The partitioning of cadmium between water and aquatic sediments is related to the abovementioned factors, but is also affected by pH and salinity (Callahan et al. 1979).

\section{HUMAN HEALTH EFFECTS}

Occupational exposure to cadmium at metal processing plants and smeltering operations are the major risks of exposure to airborne cadmium. However, for the general public the primary source of exposure is through ingestion of contaminated food.

Acute poisoning from inhaled cadmium produces upper respiratory irritation, chest pains, nausea, dizziness, and diarrhea. Permanent damage to the lung tissue may result in emphysema and peribronchial and perivascular fibrosis (Benton et al. 1966). In severe cases, pulmonary edema can lead to death (Klaasen 1980). Chronic inhalation of cadmium can 
decrease the ventilatory capacity with a corresponding increase in residual lung volume (Klaasen 1980). These are the hallmarks of emphysema.

The primary toxic effects from acute ingestion of cadmium are nausea, vomiting, excess salivation, diarrhea, and abdominal cramps. Chronic ingestion exposure to cadmium produces renal dysfunction, hypertension, and osteomalacia. In a classic case of known etiology, Japanese women suffered from a disease known as itai-itai (ouch-ouch). Women who ingested rice contaminated by an upstream effluent from a lead-zinc-cadmium mine complained of rheumatic and myalgic pains. Osteomalacia was the most prominent feature of itai-itai and it is now believed that the toxic effects of cadmium were due to interference with renal regulation of calcium/phosphate balance (Klaasen 1980).

\section{ECOLOGICAL EFFECTS}

Aquatic and terrestrial organisms bioaccumulate cadmium (Callahan et al. 1979). Bioaccumulation in fish is dependent on the $\mathrm{pH}$ and organic content of the water, which are the major determinants of water/sediment partitioning. Because cadmium accumulates in - kidney and liver rather than muscle, and because intestinal absorption of cadmium is low, one would expect a low amount of biomagnification of cadmium in the food chain (ATSDR 1991). However, cadmium is known to be taken up and bioaccumulated by food crops grown in contaminated soil (Munshower 1977).

\section{US ENVIRONMENTAL PROTECTION AGENCY HUMAN HEALTH CRITERIA}

Oral Slope Factor

Inhalation Slope Factor

Chronic Oral RfD

Chronic Inhalation RfD
NA

$6.3 \mathrm{~kg} / \mathrm{mg} /$ day $^{-1}$

$5 \mathrm{E}-4 \mathrm{mg} / \mathrm{kg} /$ day

NA

\section{DRINKING WATER CRITERIA AND STANDARDS}
U.S. Environmental Protection Agency
Maximum Concentration Limit
Maximum Concentration Limit Goal
$0.005 \mathrm{mg} / 1$
$0.005 \mathrm{mg} / 1$

\section{AMBIENT WATER QUALITY CRITERIA}

U.S. Environmental Protection Agency Ambient Water Quality Criteria

$\begin{array}{ll}\text { Human Health-Water and Fish Ingestion } & \text { NA } \\ \text { Human Health-Fish Ingestion Only } & \text { NA } \\ \text { Aquatic Organisms-FW Acute } & 3.9 \mu \mathrm{g} / \mathrm{L} \\ \text { Aquatic Organisms-FW Chronic } & 1.1 \mu \mathrm{g} / \mathrm{L} \\ \text { Aquatic Organisms-Marine Acute } & 43 \mu \mathrm{g} / \mathrm{L} \\ \text { Aquatic Organisms-Marine Chronic } & 9.3 \mu \mathrm{g} / \mathrm{L}\end{array}$


South Carolina Department of Health and Environmental Control

Human Health Criteria

Fish and Aquatic Life-Continuous

Fish and Aquatic Life-Maximum
NA

0.66

1.79

$\mathrm{NA}=$ Not Available

\section{REFERENCES}

Agency for Toxic Substances and Disease Registry (ATSDR). 1991. Draft Toxicological Profile for Cadmium. Prepared by Life Systems, Inc., and Clement International Assoc. U.S.Department of Health and Human Services.

Beton, D.C., Andrew, G.S., Davies ,H.J., Howells, L., Smith, G.F. 1966. Acute cadmium fume poisoning, five cases with 1 death from renal necrosis. Br. J. Ind. Med. 23:292301.

Callahan, M.A.,'Slimak, M.W., Gable, M.W., et al. 1979. Water- related fate of 129 priority pollutants. Washington,D.C. U.S. Environmental Protection Agency. Office of Water Planning and Standards. EPA-440/4-79-129a.

Elinder, C.G., Edling ,C., Lindberg, E. et al. 1985. Assessment of renal function in workers previously exposed to cadmium:follow-up and dose response analyses. Am.J.Ind.Med. 8:553-564.

Keitz, E.L. 1980. Atmospheric cycles of cadmium and lead: Emissions, transport, transformation, and removal. McLean,V.A.: The Mitre Corporation.

Klaasen, C.D. 1980. Heavy metals, and heavy metal antagonists. In: Goodman and Gilman's The Pharmacological Basis of Therapeutics. Gilman, A.G., Goodman, L.S., Gilman, A. eds. New York. MacMillan Publishing. 1632-1633.

Munshower, F.F. 1977. Cadmium accumulation in plants and animals of polluted and nonpolluted grasslands. J. Environ. Qual. 6:411-413.

Sax, N.I., Lewis, R.J. 1987. Hawley's condensed chemical dictionary. 11th edition. New York. Van Nostrand-Reinhold Co. 664-672. 
THIS PAGE LEFT INTENTIONALLY BLANK 


\section{CALCIUM}

CAS No.: $\quad 7440-70-2$

Synonym(s) Calcitat

Calcium is the third most abundant metal and the fifth most abundant element. It occurs in nature in the form of compounds, never alone. Some of the compounds of calcium include calcium carbonate (limestone and marble), calcium sulfate (gypsum), calcium fluoride (fluorspar), and calcium phosphate (apatite). Metallic calcium is used as a hardener for lead bearings, in the electronics industry, and in the production of uranium an thorium (Parmeggiani 1983). The principal commercial source of calcium is limestone.

Calcium is an essential and abundant element of the human body, important in a variety of processes. Notably, calcium is important for nerve conduction, contraction of all types of muscle (smooth, cardiac, and skeletal), and in cellular membrane functions. It is also an important component of bones and teeth.

Calcium is a sliver-white, soft metal with a molecular weight of 40.08 . It melts at $842^{\circ} \mathrm{C}$ and boils at $1484^{\circ} \mathrm{C}$. It is soluble in water and ethanol (Weast 1985). Calcium fluoride is a transparent crystalline substance with a molecular weight of 78.1, a melting point of $1360^{\circ}$ $\mathrm{C}$, and a boiling point of $2500^{\circ} \mathrm{C}$. Metallic calcium can be extracted from calcium fluoride. Calcium fluoride is also used to make pottery and etch glass (Parmeggiani 1983). Calcium nitrate has a molecular weight of 236.2 and is highly soluble in water. This compound is used as fertilizer and in the explosive and pyrotechnics industry.

\section{FATE AND TRANSPORT}

Calcium compounds may be released into the atmosphere as dusts. Certain calcium compounds are highly reactive and may cause explosions and fires when tiny particles are released to the air. The less flammable forms of aerosols may bind particulate matter in the atmosphere and all are subject to wet and dry deposition. Some air-borne calcium compounds may be oxidized (Bowen 1979).

Calcium compounds in surface waters may be in solution and in temperate zones dissolved calcium and its compounds represent one of the major species present in rivers. They are typically transported in solution by water in flowing freshwater systems, however some calcium, particularly calcium carbonate, will be removed from lake water by sedimentation. In these cases where sedimentation occurs, aquatic sediments can act as sinks, or reservoirs, sequestering much of the calcium from the water column,-but releasing it slowly over a long period of time. Calcium which reaches the ocean's surface shows some degree of depletion through microbiotic assimilation (Bowen 1979).

\section{HUMAN HEALTH EFFECTS}

Metallic calcium reacts with moisture in the air, and owing to its alkaline properties, produces eye and skin irritation and burns. Fires and/or explosions may release toxic fumes into the atmosphere. Calcium chloride is also an irritant to skin and mucous membranes. 
It can produce erythema, facial skin peeling, burning in nasal cavities, nose bleed, and perforated septum. Calcium fluoride exerts harmful chronic effects on bones and teeth. These effects are due primarily to fluoride rather than calcium. Calcium fluoride also has been associated with silicosis of the lung. Fluorspar (calcium fluoride) contains only a small amount of silica, however calcium intensifies the fibrogenic effects of silica (Parmeggiani 1983).

\section{ECOLOGICAL EFFECTS}

Microorganisms degrade (and utilize) certain forms of calcium (Pargeggiani 1983; Bowen 1979). All organisms require calcium for physiologic functions therefore all have mechanisms for uptake of calcium from abiotic surroundings or through ingestion of food. The bioconcentration potential of calcium has not been well studied.

\section{US ENVIRONMENTAL PROTECTION AGENCY HUMAN HEALTH CRITERIA}

$\begin{array}{ll}\text { Oral Slope Factor } & \text { NA } \\ \text { Inhalation Slope Factor } & \text { NA } \\ \text { Chronic Oral RfD } & \text { NA } \\ \text { Chronic Inhalation RfC } & \text { NA }\end{array}$

\section{DRINKING WATER CRITERIA AND STANDARDS}

U.S. Environmental Protection Agency

Maximum Concentration Limit

NA

Maximum Concentration Limit Goal

NA

\section{AMBIENT WATER QUALITY CRITERIA}

U.S. Environmental Protection Agency Ambient Water Quality Criteria

Human Health-Water and Fish Ingestion NA

Human Health=Fish Ingestion Only NA

Aquatic Organisms-FW Acute NA

Aquatic Organisms-FW Chronic . NA

Aquatic Organisms-Marine Acute NA

Aquatic Organisms-Marine Chronic $\longrightarrow \mathrm{NA}$

South Carolina Department of Health and Environmental Control

Human Health Criteria NA

Fish and Aquatic Life-Continuous NA

Fish and Aquatic Life-Maximum NA

NA $=$ Not Available 


\section{REFERENCES}

Bowen. 1979. Environmental chemistry of the elements. New York, Academic Press.

Parmeggiani, L.(ed). 1983. Encyclopedia of occupational Health and Safety. Vol. 2. Geneva. International Labor Office.

Weast, R.C. 1985. CRC Handbook of chemistry and physics. 66 th edition. Boca Raton, FL., CRC Press. 


\section{THIS PAGE LEFT INTENTIONALLY BLANK}


CARBON DISULFIDE

CAS No.:

Synonym(s)
75-15-0

Carbon bisulfate, Carbon sulfide, Dithiocarbonic anhydride, sulfocarbonic anhydride, sulphuret of carbon

Carbon disulfide has been in industrial use since it was manufactured commercially in the 1980's (Timmerman 1978). Industrially, carbon disulfide is used as a solvent of fats, rubbers, sulfur, and other elements (Sine 1989), as a reagent in the production of carbon tetrachloride (Timmerman 1978), as an insecticide (Sine 1989), and in the manufacture of rayon and cellophane (Timmerman 1978). Carbon disulfide is produced in small amounts naturally in marshes and volcanic eruptions.

Carbon disulfide has a molecular weight of 76.14 and is a clear or yellowish liquid. Carbon disulfide melts at $-110.8^{\circ} \mathrm{C}$, boils at $46.5^{\circ} \mathrm{C}$, and has a density of 1.2632 at $20^{\circ} \mathrm{C}$. In its pure form, carbon disulfide has a sweet odor, however commercial grades have a foul odor similar to that of rotting radishes (Windholz 1983). Carbon disulfide has low solubility in water, but is miscible in organic solvents. It is flammable and has an autoignition temperature of $100^{\circ} \mathrm{C}$.

\section{FATE AND TRANSPORT}

Industrial releases of carbon disulfide to the environment occur mainly to air. Due to the low water solubility and high vapor pressure of carbon disulfide, releases to surface waters are quickly volatilized to the atmosphere. Carbon disulfide is mobile in soil, where it may also evaporate or may leach into ground water. Adsorption to soils is very limited (ATSDR).

One pathway for removal of carbon disulfide from the atmosphere is through reactions with hydroxyl radicals. Based on this reaction, the calculated half-life in the troposphere is estimated at approximately .2 years. Additionally, photo excited carbon disulfide reacts with oxygen to yield carbonyl sulfide (Wine et al. 1980). Based on this reaction, the calculated half-life for carbon disulfide in the troposphere is estimated at 0.2 years. Additionally, photo excited carbon disulfide reacts with oxygen to yield carbonyl sulfide (Wine et al. 1981). Considering the above reactions, the lifetime of carbon disulfide in the atmosphere was estimated to be 12 days (Khalil and Rasmussen 1984).

\section{HUMAN HEALTH EFFECTS}

Inhalation exposure results in rapid absorption of carbon disulfide. The effects from short term exposure include vascular changes in a number of organ systems, changes in brain chemistry, and sensory and motor nerve conduction abnormalities. Rats exposed to $777 \mathrm{ppm}$ carbon disulfide for 12 hours had swollen brain mitochondria and elevated central ATP levels (Tarkowski et al. 1980).

Epidemiological studies have demonstrated that long-term exposure to carbon disulfide produces significant cardiovascular and neurological alterations. Coronary heart disease was a consistent finding among chronically exposed individuals (Tiller et al. 1968; Tolonen et al. 
1979), as was a higher incidence of angina (Hernberg et al. 1971; Tolonen et al. 1979) and hypertension (Lieben et al. 1974; Tolonen et al. 1979). Gastrointestinal symptoms of stomach distress and impaired appetite have also been noted in chronically exposed individuals (Vigliani 1954; Rubin and Arieff 1945). Retinopathies have been another consistent pathologic change observed in epidemiological studies (Sugimoto et al. 1976; Szymankova 1968). Neurological alterations occur centrally as well as peripherally in individuals occupationally exposed to carbon disulfide. Workers exposed to higher concentrations of carbon disulfide had significantly delayed motor nerve conduction when compared with controls and individuals exposed to lower concentrations (Johnson et al.1983). Workers with high exposure levels also presented with reduced mental performance and behavioral changes (Foa et al. 1976; Cassitto et al. 1978). Typically, exposed individuals had decreased intelligence scores, less memory recall, poorer performance and attention span, and increased fatigue, anxiety, introversion, and depression.

In studies of human carcinogenicity, exposure to carbon disulfide showed a statistical correlation to increased incidence of lymphatic leukemia (Checkoway et al. 1984; Wilcoskey et al. 1984). However, interpretation of those data was confounded by the fact that participants in those studies were also exposed to a number of other solvents.

Individuals with a genetic predisposition for atherosclerosis may represent a special risk category because of the injury to vascular epithelium and changes in lipid metabolism caused by carbon disulfide. Alcoholics, vitamin-B deficient individuals, and those with neuropsychic disorders may also be unusually susceptible to the effects of carbon disulfide (ATSDR 1991).

\section{ECOLOGICAL EFFECTS}

There are no experimental data that suggest bioaccumulation or biomagnification of carbon disulfide in the food chain, The estimated values for solubility and the octanol/water partition coefficient suggest that bioaccumulation does not occur (EPA 1986).

\section{US.ENVIRONMENTAL PROTECTION AGENCY HUMAN HEALTH CRITERIA}

Oral Slope Factor

Inhalation Slope Factor

Chronic Oral RfD

Chronic Inhalation RfC
NA

NA

$1 \mathrm{E}-1 \mathrm{mg} / \mathrm{kg} /$ day

$2.9 \mathrm{E}-3 \mathrm{mg} / \mathrm{m}^{3}$

\section{DRINKING WATER CRITERIA AND STANDARDS}

U.S. Environmental Protection Agency

Maximum Concentration Limit

NA

Maximum Concentration Limit Goal

NA 


\section{AMBIENT WATER QUALITY CRITERIA}

U.S. Environmental Protection Agency Ambient Water Quality Criteria

$\begin{array}{ll}\text { Human Health-Water and Fish Ingestion } & \text { NA } \\ \text { Human Health-Fish Ingestion Only } & \text { NA } \\ \text { Aquatic Organisms-FW Acute } & \text { NA } \\ \text { Aquatic Organisms-FW Chronic } & \text { NA } \\ \text { Aquatic Organisms-Marine Acute } & \text { NA } \\ \text { Aquatic Organisms-Marine Chronic } & \text { NA }\end{array}$

South Carolina Department of Health and Environmental Control

Human Health Criteria

Fish and Aquatic Life-Continuous

Fish and Aquatic Life-Maximum
NA

NA

NA

$\mathrm{NA}=$ Not Available

\section{REFERENCES}

Agency for Toxic Substances and Disease Registry. 1990. Toxicological Profile for Carbon Disulfide. Prepared by Clement International, Corp., contract no. 205-88-0608.

Cassitto, M.G., Bertazzi,P.A., Camerina, D. et al. 1978. Subjective and objective behavioral alterations in carbon disulfide workers. Medicina del Lavoro 69:144-150.

Checkoway, H., Wilcosky, T., Wolf, P.et. al. 1984. An evaluation of the associations of leukemia and rubber industry solvent exposures. Am. J. Ind.Med. 5:239-249.

EPA. 1986. Health and Environmental Effects Profile for carbon disulfide. Cincinnati,OH: U.S. Environmental Protection Agency. Environmental Criteria and Assessment Office. EPA/600/X-86/155, 129.

Foa,V., Cassito, M.G., Forzi ,M. et al. 1976. Mental performance and personality disorders among workers exposed to carbon disulfide: Comparison between two different rayon plants. Adverse Effects of Environmental Chemicals and Psychotropic Drugs, Neurophysiological, and Behavioral Tests. Vol. 2, 173-182.

Johnson, B.L., Boyd, J., Burg, J.R. et al. 1983. Effects on the peripheral nervous system of workers exposed to carbon disulfide. Neurotoxicology 4:53-65.

Lieben ,J., Menduke, H.,Flegel, E.E., et al. 1974. Cardiovascular effects of carbon disulfide exposure. J. Occup. Med. 16:449-453. 
Rubin, H.H., Arieff, A.J. 1945. Carbon disulfide and hydrogen sulfide clinical study of chronic low-grade exposures. J. Ind. Hyg. Toxicol. 27:123-129.

Sugimoto, K., Goto, S., Hotta ,R. 1976. An epidemiological study on retinopathy due to carbon disulfide: Carbon disulfide exposure level and development of retinopathy. Int. Arch. Occup. Environ. Health 37:1-8.

Szymankova, G. 1968. Observations on the effects of cd on vision in workers engaged in the manufacture of synthetic fibers. Klinika Oczna (as cited in ATSDR 1990).

Vigliani, E.C. 1954. Cd poisoning in viscose rayon factories. Br. J. Ind. Med II:235-244.

Wilcosky,T.C., Checkoway ,H., Marshal, E.G., et al. 1984. Cancer mortality and solvent exposures in the rubber industry. Am. Ind. Hyg. Assoc. J. 45:809-811. 


\title{
CARBON TETRACHLORIDE
}

\author{
CAS No: $\quad 56-23-5$ \\ Synonym(s) Carbona, carbon chloride, carbon tetrachloride
}

The uses of carbon tetrachloride has been restricted to industrial production of the refrigerants, chlorofluorocarbons (CFCs). In the past however, carbon tetrachloride was a component of many household cleaning products used to remove spots from clothing, carpeting, and upholstery and as an aerosol propellant. It was used as an anaesthetic for a short time, as an industrial degreaser, a fumigant for grains, and as a component in fire extinguishers (HDSB 1992). Carbon tetrachloride is a colorless liquid with a sweet aromatic odor. It has a molecular weight of 153.8 , a melting point of $-23^{\circ} \mathrm{C}$, and a boiling point of $765^{\circ} \mathrm{C}$. It is soluble in water and miscible in organic solvents (Weast 1985).

\section{FATE AND TRANSPORT}

The majority of carbon tetrachloride released to the environment is to the atmosphere, and partitioning from soil and water releases to air also contributes to the atmospheric concentration of carbon tetrachloride (EPA 1991). Carbon tetrachloride is very persistent in air; it is resistant to troposphere photolysis and to interaction with hydroxyl radicals. The overall persistence, or lifetime, of carbon tetrachloride in air has been estimated at 30-100 years (Molina and Rowland 1974; Singh et al. 1979). Carbon tetrachloride that reaches the troposphere may be subject to photolysis and that which remains in the stratosphere may be transported to earth in rain water. The by-products of photolysis in the troposphere, chlorine atoms and chlorine compounds, act as catalysts for reactions that degrade ozone.

Carbon tetrachloride releases to water represent only a small fraction of the total emissions (Rams et al 1979), and evaporation is the primary transport mechanism for removal of carbon tetrachloride from water (Dilling 1977). Carbon tetrachloride dissolves in water and as such does not photodegrade or oxidize in detectable quantities (Howard et al. 1991). Biodegradation appears to be the most important degradation pathway in water.

Carbon tetrachloride rapidly evaporates from soil, and the remaining contents are fairly mobile (ATSDR 1992; Mackay et al. 1983). Degradation pathways of carbon tetrachloride in soil have not been elucidated (ATSDR 1992).

\section{HUMAN HEALTH EFFECTS}

Deaths from acute inhalation of carbon tetrachloride occurred more frequently when its use was more widespread (Norwood et al. 1950; Umiker and Pearce 1953). Pulmonary edema was a consistent finding at autopsy, but was a delayed effect which may have been secondary to the renal damage caused by exposure (Umiker and Pearce 1953). Liver injury, and renal nephritis and nephrosis are typical effects of acute carbon tetrachloride inhalation. Headache, weakness, lethargy, and stupor are CNS symptoms of exposure, and their severity is dose dependent (Cohen 1957). Similar effects have been noted following ingestion of carbon tetrachloride. Carbon tetrachloride is carcinogenic in experimental animals, causing a preponderance of hepatic tumors (Della Porta et al. 1961). 
Alcohol (ethanol) is contraindicated in persons who are exposed to carbon tetrachloride, due to its potentiation of carbon tetrachloride toxicity. Other alcohols, such as isopropyl, and ketones also enhance carbon tetrachloride toxicity (Folland et al. 1976). Because of the interactions between ketones and carbon tetrachloride, diabetics may be unusually susceptible to carbon tetrachloride poisoning. Phenobarbital, DDT and PCB stimulate the hepatic microsomal enzymes and increase toxicity of carbon tetrachloride. These and other agents which enhance the degradation of carbon tetrachloride to its more toxic metabolites increases the hepatotoxicity of carbon tetrachloride.

\section{ECOLOGICAL EFFECTS}

Estimates of carbon tetrachloride bioconcentration potentials in aquatic organisms are low (HDSB 1992), even though this substance is highly lipophilic. Most organisms metabolize and excrete carbon tetrachloride rapidly, thereby effectively reducing the amount of carbon tetrachloride available for storage in fat. The extent or potential of bioaccumulation in plants is not known.

\section{US ENVIRONMENTAL PROTECTION AGENCY HUMAN HEALTH CRITERIA}

Oral Slope Factor

Inhalation Slope Factor

Chronic Oral RfD

Chronic Inhalation RfD
$1.3 \mathrm{E}-1 \mathrm{~kg} / \mathrm{mg} /$ day $^{-1}$

$5.3 \mathrm{E}-2 \mathrm{~kg} / \mathrm{mg} /$ day $^{-1}$

$7 \mathrm{E}-4 \mathrm{mg} / \mathrm{kg} /$ day

NA

\section{DRINKING WATER CRITERIA AND STANDARDS}

U.S. Environmental Protection Agency

Maximum Concentration Limit

Maximum Concentration Limit Goal

$0.005 \mathrm{mg} / \mathrm{L}$

$0.0 \mathrm{mg} / \mathrm{L}$

\section{AMBIENT WATER QUALITY CRITERIA}

U.S. Environmental Protection Agency Ambient Water Quality Criteria

Human Health-Water and Fish Ingestion

Human Health-Fish Ingestion Only

Aquatic Organisms-FW Acute

Aquatic Organisms-FW Chronic

Aquatic Organisms-Marine Acute

Aquatic Organisms-Marine Chronic
$0.4 \mu \mathrm{g} / \mathrm{L}$

$6.94 \mu \mathrm{g} / \mathrm{L}$

$35,200 \mu \mathrm{g} / \mathrm{L}$

NA

$50,000 \mu \mathrm{g} / \mathrm{L}$

NA 
South Carolina Department of Health and Environmental Control

Human Health Criteria

Fish and Aquatic Life-Continuous

Fish and Aquatic Life-Maximum
$5 \mu \mathrm{g} / \mathrm{L}$

NA

NA

$\mathrm{NA}=$ Not Available

\section{REFERENCES}

Agency for Toxic Substances and Disease Registry. 1992. Draft toxicological profile for carbon tetrachloriderachloride. Prepared by Clement International Corp.US Department of Health and Human Services.

Dilling, W.L. 1977. Interphase transfer processes. II. Evaporation of chloromethanes, ethanes, propanes, and polypropylenes from dilute aqueous solutions. Comparison with theoretical predictions. Environ. Sci. Technol. 11:405-409.

EPA. 1991. US Environmental Protection Agency. Toxics in the community. National and local perspectives. Washington, D.C: Office of Toxic Substances. EPA 560/4-91-014.

HDSB. 1982. Hazardous Substance Data Bank. NLM. National Toxicology Program. Bethesda Md, April 14, 1992.

Howard, P.H., Boethling ,R.S., Jarvis ,W.F., et al. 1991. Handbook of environmental degradation rates. Chelsea, MI. Lewis Publishers, Inc.

Mackay ,D.M., Freyberg ,D.L., Goltz, M.N., et al. 1983. A field experiment of ground water transport of halogenated organic solutes (Extended Abstract). American Chemical Soc. 196th National Meeting of Division of Environmental Chemists.

Molina, M.J., Rowland ,F.S. 1974. Predicted present stratospheric abundances of chlorine species from photodissociation of carbon tetrachloriderachloride. Geophys. Res. Lett. $1: 309-312$

Norwood, W.D., Fuqua, P.A. ,Scudder ,B.C. 1950. Carbon tetrachloride poisoning. Arch.Ind.Hyg. Occup. Med. 1:90-100.

Rams, J.M., Pilgrim ,M., Rauth, S. et al. 1979. Level II materials balance: Carbon tetrachloride. (Draft Report) Washington D.C. US Environmental Protection Agency, Office of Pesticides and Toxic Substances. 
Singh, H.B., Salas, L.J., Shigeishi ,H., et al. 1979. Atmospheric distributions, sources, and sinks of selected halocarbons, hydrocarbons, SF6, and N20. Draft final report, prepared by SRI International for the US Environmental Protection Agency,Office of Research and Development. Research Triangle Park, NC.

Weast, R.C. 1985. CRC handbook of chemistry and physics. 66th edition. Boca Raton, FL CRC Press. 


\section{CHLORDANE}

CAS No.: $\quad$ 57-74-9 .

Synonym(s) 1,2,4,5,6,7,8,8-Octochloro-3a,4,7,7a-tetrahydro-4,7-methanoindan

Chlordane is a member of the chlorinated cyclodiene pesticide group and was once used agriculturally as an insecticide for a variety of crops and residentially to control termites. It was removed from use on food crops in 1978, non-food crops in 1983, and its use in the treatment of subterranean termites was finally canceled in 1988. Although chlordane is no longer.approved for use in the U.S., it is still manufactured here for export (ATSDR 1992).

Chlordane is a clear to amber-colored viscous liquid with a molecular weight of 409.76 . It has a melting point ranging between $104^{\circ} \mathrm{C}$ and $107^{\circ} \mathrm{C}$, depending on the isomeric form, and a boiling point of $175^{\circ} \mathrm{C}$. It has a density of $1.59-1.63 \mathrm{~g} / \mathrm{cm}$ and emits a mildly pungent odor. Chlordane has low water solubility but is miscible with hydrocarbon solvents (ATSDR 1992).

\section{FATE AND TRANSPORT}

Because of the nature of its past use, chlordane was intentionally applied directly onto the soil, where it has been found to persist up to 20 years or more (ATSDR 1992). Volatilization and stormwater runoff account for most of its presence in the air and surface waters, respectively, however some discharges to air may occur from current production at manufacturing facilities. Volatilization accounts for most of chlordane transport from surface water, as little evidence of biodegradation exists (ATSDR 1992). In the atmosphere, vapors of chlordane are subject to degradation by photolysis and oxidation (Oheme 1991).

In soil, chlordane adsorbs to organic matter and generally persists longer in rich, humus soils compared to sandy, non-organic soils (Weise and Basson 1966). The moisture content of the soil is also an important determinate in the fate of chlordane in soil. Volatilization, the major transport pathway for removal of chlordane from soils, occurs at a faster rate in moist soils, where chlordane does not adhere as tightly to organic particulates as it does in dry soils (Glotfelty and Schomburg 1989).

\section{HUMAN HEALTH EFFECTS}

Chlordane is highly lipophilic and as such is readily absorbed by the body through inhalation, ingestion, and dermal exposure. Most case studies of the effects of acute exposure to chlordane have resulted from accidental ingestion (ATSDR 1992). Gastrointestinal symptoms, ranging from upset stomach,-nausea, abdominal cramps, and diarrhea are the first symptoms of chlordane ingestion (EPA 1980). Neurologic effects such as headache, dizziness, irritability, excitability, confusion, incoordination, muscle tremors, seizures, convulsions, and coma also have been observed following acute ingestion of chlordane (EPA 1980).

Historically, inhalation exposure to chlordane typically occurred from occupational exposure or in homes treated with chlordane to control for termites. In epidemiological studies, patients reported chest pains, dyspnea, and shortness of breath (EPA 1980).The incidence 
of bronchitis and sinusitis was correlated to the concentration of chlordane pesticide in indoor air and the frequency of migraine headaches among individuals living in termite treated homes was proportional to the concentration of chlordane in the air (Menconi et al. 1988). Jaundice was frequently associated with continuous inhalation of chlordane in the indoor air (EPA 1980). and elevated serum concentrations of hepatic fats and enzymes have been measured in individuals who applied the pesticide occupationally (Ogata and Izushi 1991). Inhalation exposure also produces neurotoxic symptoms similar to those produced by acute chlordane ingestion.

There is little evidence that chlordane is carcinogenic in humans. One study reported that chronic exposure to chlordane in termite-treated homes was associated with an increased incidence of skin neoplasms (Menconi 1988). Unfortunately, the concommittant presence of aldrin and heptachlor in the indoor air of termite-treated homes limits interpretation of these data. Other studies have addressed the incidence of bladder cancer, non-Hodgkins lymphoma, and leukemia and found little or no statistical significance between chronically exposed individuals and controls (Epstein and Ozonoff 1987; Woods and Polissar 1989; Wang and MacMahon 1979).

\section{ECOLOGICAL EFFECTS}

Chlordane is bioconcentrated by aquatic organisms in both the marine and freshwater environments. One study exanined three trophic levels in the marine food chain of the Arctic and found biomagnification at each increasing trophic level (Muir et al. 1988).

Bioaccumulation of chlordane in terrestrial organisms also has been reported. Singh and others (1990) observed that small amounts of chlordane shifted from contaminated soil into plants, with the degree of uptake dependent on species and stage in the life cycle.

\section{US ENVIRONMENTAL PROTECTION AGENCY HUMAN HEALTH CRITERIA}

Oral Slope Factor

Inhalation Slope Factor

Chronic Oral RfD

Chronic Inhalation RfD
$1.3 \mathrm{~kg} / \mathrm{mg} / \mathrm{day}^{-1}$

$1.3 \mathrm{~kg} / \mathrm{mg} /$ day $^{-1}$

$6 \mathrm{E}-5 \mathrm{mg} / \mathrm{kg} /$ day

NA

\section{DRINKING WATER CRITERIA AND STANDARDS}

U.S. Environmental Protection Agency

Maximum Concentration Limit Maximum Concentration Limit Goal
$0.002 \mathrm{mg} / \mathrm{L}$

$0.0 \mathrm{mg} / \mathrm{L}$ 


\section{AMBIENT WATER QUALITY CRITERIA}

U.S. Environmental Protection Agency Ambient Water Quality Criteria

$\begin{array}{ll}\text { Human Health-Water and Fish Ingestion } & 0.46 \mathrm{ng} / \mathrm{L} \\ \text { Human Health-Fish Ingestion Only } & 0.48 \mathrm{ng} / \mathrm{L} \\ \text { Aquatic Organisms-FW Acute } & 2.4 \mu \mathrm{g} / \mathrm{L} \\ \text { Aquatic Organisms-FW Chronic } & 0.0043 \mu \mathrm{g} / \mathrm{L} \\ \text { Aquatic Organisms-Marine Acute } & 0.004 \mu \mathrm{g} / \mathrm{L} \\ \text { Aquatic Organisms-Marine Chronic } & 0.07 \mu \mathrm{g} / \mathrm{L}\end{array}$

South Carolina Department of Health and Environmental Control

Human Health Criteria

Fish and Aquatic Life-Continuous

Fish and Aquatic Life-Maximum
$0.00588 \mu \mathrm{g} / \mathrm{L}$

$0.0043 \mu \mathrm{g} / \mathrm{L}$

$2.4 \mu \mathrm{g} / \mathrm{L}$

NA $=$ Not Available

\section{REFERENCES}

Agency for Toxic Substance and Disease Registry (ATSDR). October 1992. Draft Toxicological Profile for Chlordane. Prepared by Syracuse Research Corp. and Clement International Corp. US Public Health Service.

EPA. 1980. Summary of Reported Pesticide Incidents Involving Chlordane. Pesticide Incident Monitoring System Report No. 360. Office of Pesticide Programs, Washington,D.C.

Epstein, S.S., Ozanoff, D. 1987. Leukemias and blood dyscrasias following exposure to chlordane and heptachlor. Teratogen Carcinogen Mutagen 7:527-540.

Glotfelty, D.E., Schomburg, C.L. 1989. Volatilization of pesticides from soil. In: Reactions and movement of organic chemicals in soils. Soil Science society of America and American Society of Agronomy. SSSA Special Publication. 22:181-200.

Menconi, S., Clark, J.M., Langenberg ,P. et al. 1988. A preliminary study of potential human health effects in private residences following chlordane applecations for termite control. Arch. Environ. Health 43:349-352.

Muir, D.C.G., Norstrum, R.J., Simon ,M. 1988. Organochlorine contaminants in arctic marine food chains:Accumulation of specific polychlorinated biphenyls and chlordane-related compounds. Environ. Sci. Tech. 22:1071-1079.

Oeheme, M. 1991. Dispersion and transport pathways of toxic persistent organochlorines to the Arctic levels and consequences. Sci.Tot.Environ. 106:43-53. 
Ogata, M., Izushi, F. 1991. Effects of chlordane on parameters of liver and muscle toxicity in man and experimental animals. Toxicol. Lett. 56:327-337.

Singh, G., Higginson ,F.R., Fenton, I.G., et al. 1990. Translocation of aged cyclodiene insecticide residues from soil into forage crops under optimal glasshouse conditions. J. Environ. Sci. Health [B]25:295-308.

Wang, H.H., MacMahon ,B. 1979. Mortality of pesticide applicators. J. Occup. Med. 21:741744.

Wiese , I.H., Basson, N.C.J. 1966. The degradation of some persistent chlorinated hydrocarbon insecticieds applied to different soil types. S. Afr. J. Agr. Sci. 9:945-969.

Woods, J.S., Polissar, L. 1989. Non-Hodgkin's lymphoma among phenoxy herbicide-exposed farm workers in Washington State. Chemosphere 18:401-406. 


\section{CHLOROFORM}

CAS No.:

67-66-3

Major Synonym(s) Methenyl Chloride, Methane Trichloride, Methyl Trichloride, Trichloromethane

Chloroform, or trichloromethane, is both a naturally occurring and man-made compound. It is used primarily in the production of fluorocarbon-22, however past uses included its application as a dry cleaning spot remover, as an extraction solvent, in fire extinguishers, as an anesthetic, and in the manufacture of dyes and pesticides.

Chloroform is a colorless liquid with a pleasant, ethereal odor. It has a molecular weight of 119.38 , a melting point of $-63.2^{\circ} \mathrm{C}$, and a boiling point of $61.3^{\circ} \mathrm{C}$. Chloroform is highly soluble in water and is miscible with principal organic solvents (Deshon 1979).

\section{FATE AND TRANSPORT}

Release of chloroform to the atmosphere may occur during the manufacture and handling of product and from its use in the production of other chemicals (EA 1985a): Volatilization from contaminated or chlorinated water sources and contaminated soil are major sources of chloroform vapors in the air. Interaction of chloroform with photochemically produced hydroxyl radicals degrades airborne chloroform, which may otherwise travel vast distances. Large amounts of chloroform may be transported from air to soil and water through wet deposition (Kawamura and Kaplan 1983).

There is evidence that some biodegradation of chloroform may occur in soil and water, however, this does not appear to be a primary mechanism for removal from sediment (Bouwer et al. 1981b) or water (Bouwer et al. 1981a). Most chloroform is removed from these media through volatilization.

\section{HUMAN HEALTH EFFECTS}

Acute exposure (including its use as an anesthetic) of humans to chloroform ha produced the following effects (U.S. EPA 1985; Ellenhorn and Barceloux 1988; ACGIH 1986; Torkelson and Rowe 1981): 1) Central nervous system depression that produces symptoms ranging from nausea, lightheadedness, headache and malaise to stupor, narcosis and coma, 2) heart effects such as ventricular arrhythmias, extrasystoles, bradycardia and cardiac arrest, and 3) liver toxicity characterized by centrilobular, and in severe cases, mid-zonal and massive necrosis. Symptoms include progressive weakness, vomiting, delirium, jaundice, abnormal liver and kidney function, coma, and possible death.

Bomski et al. (1967) found high incidences of hepatomegaly, toxic hepatitis, and splenomegaly in workers exposed to 2-205 ppm chloroform for one to four years. A 33-year-old male who habitually inhaled chloroform for 12 years had psychiatric and neurological symptoms of depression, appetite loss, hallucinations, ataxia and dysarthria (HSDB 1989). Phoon et al. (1983) reported two separate outbreaks of jaundice in workers exposed to chloroform in 
a household appliance manufacturing plant. The duration of exposure to chloroform prior to the onset of jaundice averaged one to four months.

Challen et al. (1958) reported that workers exposed to $\approx 77-237 \mathrm{ppm}$ chloroform complained of tiredness, irritability, depression, headache, gastrointestinal distress and frequent and scalding urination. There was no evidence of liver damage.

Acute inhalation exposure in laboratory animals results in narcosis, liver damage and respiratory tract irritation (NIOSH 1974; U.S. EPA 1985).

In a subchronic study, rats, guinea pigs and rabbits exposed to chloroform vapor at concentrations of up to $85 \mathrm{ppm}$ for seven hours/day, five days/week for six months developed increased liver and kidney weights, cloudy swelling in the kidneys and granular degeneration of the liver (Torkelson et al. 1976).

An increase in renal epithelial tumors was observed in mice chronically exposed by gavage to chloroform for 78 weeks followed by an observation period of 14-15 weeks (NCI 1976). This study was the basis for the development (by route-to-route extrapolation) of an inhalation slope factor of $0.081 \mathrm{mg} / \mathrm{kg} /$ day $^{-1}$ (U.S. EPA 1991).

Gosselin et al. (1984) have assigned a toxicity rating of 3 to chloroform, which means that it is moderately toxic to humans following a single oral dose. Chloroform has caused gonadal atrophy in male rats when administered by gavage (Palmer et al. 1979) and increased resorption rates and significant decreases in viable litter size and fetal body weights when administered to rats during gestation (Thompson et al. 1974).

Short-term gavage studies in mice (Munson et al. 1982) and rats (Palmer et al. 1979) demonstrated adverse effects on the liver at exposure levels of up to $410 \mathrm{mg} / \mathrm{kg} /$ day, including increased liver enzymes, increased liver weight and necrosis of hepatocytes. Chronic gavage studies in mice and rats showed kidney lesions (Roe et al. 1979) and necrosis of the hepatic parenchyma (NCI 1976).

Beagle dogs were administered chloroform in a toothpaste base for seven years at dose levels of 15 or $30 \mathrm{mg}$ chloroform $/ \mathrm{kg} /$ day for six days/week (Heywood et al. 1979). Treatment-related fatty cysts were observed in livers of some dogs in both treatment groups. A dose-related increase in SGPT levels was noted. A chronic oral RfD for chloroform of $1.0 \mathrm{E}-02$ has been established by the U.S. EPA (1991) based on the fatty cysts observed in orally exposed beagles.

NCI (1976) administered chloroform by gavage to rats for five days/week for 78 weeks followed by an observation period of 33 weeks. A statistically significant increase in renal epithelial tumors was observed in male rats. Jorgenson et al. (1985) found a statistically 
significant increase in the incidence of renal tumors in mice and rats administered chloroform in drinking water for 104 weeks.

The U.S. EPA (1991) considers chloroform to be a Class B2 carcinogen (probably carcinogenic to humans). The oral slope factor is $6.1 \mathrm{E}-03 \mathrm{mg} / \mathrm{kg} / \mathrm{day}^{-1}$.

Application of liquid chloroform to human skin results in a burning pain within 3-5 minutes with an associated erythema, followed by hyperemia, and finally vesication (NIOSH 1975).

\section{ECOLOGICAL EFFECTS}

Chloroform enters the environment from a variety of sources, as discussed previously. The general population may be exposed to chloroform via ingestion, inhalation of contaminated air, and to a lesser extent through dermal contact. Although chloroform is persistent in the environment, with degradation occurring almost exclusively in air, it does not tend to bioaccumulate in biota.

\section{U.S. ENVIRONMENTAL PROTECTION AGENCY HUMAN HEALTH CRITERIA}

Oral Slope Factor

Inhalation Slope Factor

Chronic Oral RfD

Chronic Inhalation RfD
$6.1 \mathrm{E}-3 \mathrm{~kg} / \mathrm{mg} / \mathrm{day}^{-1}$

$8.1 \mathrm{E}-2 \mathrm{~kg} / \mathrm{mg} /$ day $^{-1}$ $1.0 \mathrm{E}-2 \mathrm{mg} / \mathrm{kg} /$ day

NA

\section{DRINKING WATER CRITERIA AND STANDARDS}

U.S. Environmental Protection Agency

Maximum Concentration Limit

Maximum Concentration Limit Goal

$0.1 \mathrm{mg} / \mathrm{L}$

0

\section{AMBIENT WATER QUALITY CRITERIA}

U.S. Environmental Protection Agency Ambient Water Quality Criteria
Human Health - Water and Fish Ingestion
$0.2 \mu \mathrm{g} / \mathrm{L}$
Human Health - Fish Ingestion Only
$15.7 \mu \mathrm{g} / \mathrm{L}$
Aquatic Organisms - FW Acute
$2.9 \mathrm{mg} / \mathrm{L}$
Aquatic Organisms - FW Chronic
$1.2 \mathrm{mg} / \mathrm{L}$
Aquatic Organisms - Marine Acute
NA
Aquatic Organisms - Marine Chronic
NA

South Carolina Department of Health and Environmental Control Water Quality Criteria

Human Health Criteria

Fish and Aquatic Life - Continuous
$100 \mu \mathrm{g} / \mathrm{L}$

NA 
Fish and Aquatic Life - Maximum

NA

NA $=$ Not Available

\section{REFERENCES}

ACGIH (American Conference of Governmental and Industrial Hygienists). 1986. Documentation of the TLV and Biological Exposure Indices, 5th ed. ACGIH Inc., Cincinnati, $\mathrm{OH}$.

Agency for Toxic Substances and Disease Registry. 1991. Toxicological Profile for Chloroform. Prepared by Clement International Corp., contract no. 205-88-0608.

Bomski, H., A. Sobolweska and A. Strakowski. 1967. Toxic damage of the liver by chloroform in chemical industry workers. Arch. Gewerbepathol. Gewerbehy 24:127134.

Bouwer, E.J., Rittman, B., McCarty, P.L. 1981a. Anaerobic degradation of halogenated 1and 2-carbon organic compounds. Environmental Science and Technology 15:596599.

Bouwer, E.J., McCarty, P.L., Lance, J.C. 1981b. Trace organic behavior in soil columns during rapid infiltration of secondary wastewater. Water Res 15:151-159.

Challen, P., D. Hickish and J. Bedford. 1958. Chronic chloroform intoxication. Br. J. Ind. Med. 15:243-249.

Deshon, H.D. 1979. Carbon tetrachloride. In: Grayson, M., Eckroth, D., Ed. KirkOthmer Encyclopedia of Chemical Technology, 3rd ed. Vol 5. New York: John Wiley and Sons, 693-703.

Ellenhorn, M. and D. Barceloux. 1988. Medical Toxicology: Diagnosis and Treatment of Human Poisoning. Elsevier Publishing Co., New York, N.Y.

EPA. 1985a. U.S. Environmental Protection Agency: Health assessment document for chloroform. Washington, DC: Office of Health and Environmental Assessment. EPA/600/8-84-004F. NTIS PB86-105004/XAB.

Gosselin, R., R. Smith and H. Hodge. 1984. Clinical Toxicology of Commercial Products, 5th Ed. Williams and Wilkins, Baltimore, M.D.

Heywood, R., R.J. Sortwell, P.R.B. Noel et al. 1979. Safety evaluation of toothpaste containing chloroform. III. Long-term study in beagle dogs. J. Environ. Pathol. Toxicol. 2:835-851. 
HSDB (Hazardous Substances Data Bank). 1989. The National Library of Medicine (on-line via TOXNET).

Jorgenson, T., E. Meierhenry and C. Rushbrook. 1985. Carcinogenicity of chloroform in drinking water to male Osborne-Mendel rats and female B6C3F1 mice. Fund. Appl. Toxicol. 5:760-769.

Kawamura, K., Kaplan, I.R. 1983. Organic compounds in the rainwater of Los Angeles. Environ Sci Technol 17:497-501.

Munson, A., L. Sain and V. Sanders. 1982. Toxicology of organic drinking water contaminants: Trichloromethane, bromodichloromethane, dibromochloromethane and tribromoethane. Environ. Health Perspect. 46:117-126.

NCI (National Cancer Institute). 1976. Report on carcinogenesis bioassay of chloroform. NTIS PB76-264018.

NIOSH (National Institute for Occupational Safety and Health). 1974. Criteria for a recommended standard - occupational exposure to chloroform. Rockville, MD: National Institute for Occupational Safety and Health.

NIOSH. 1975. Criteria for a recommended standard - occupational exposure to chloroform. U.S. Department of Health, Education and Welfare. DHEW Publ. (NIOSH) 75-114.

Palmer, A. A. Street, F. Roe, A. Worden and N. Van Abbe. 1979. Safety evaluation of toothpaste containing chloroform. II. Long-term studies in rats. J. Environ. Pathol. Toxicol. 2:821-833.

Phoon, W., k. Goh, L. Lee, K. Tan and S. Kwok. 1983. Toxic jaundice from occupational exposure to chloroform. Med. J. Malaysia 30:31-34.

Roe, F., F. Palmer, A. Worden and N. Van Abbe. 1979. Safety evaluation of toothpaste containing chloroform. I. Long-term studies in mice. J. Environ. Toxicol. 2:799-819.

Thompson, D., S. Warnet and V. Robinson. 1974. Teratology studies on orally administered chloroform in the rat and rabbit. Toxicol. Appl. Pharmacol. 29:348-357.

Torkelson, T., F. Oyen and V. Rowe. 1976. The toxicity of chloroform as determined by single and repeated exposure of laboratory animals. am. Ind. Hyg. Assoc. J. 37:697704.

Torkelson, T. and V. Rowe. 1981. Halogenated aliphatic hydrocarbons containing chlorine, bromine and iodine. In: Clayton, G.D. and F.E. Clayton, Eds. Patty's Industrial Hygiene and Toxicology, 3rd ed. John Wiley and Sons, New York, N.Y. 
U.S. EPA (U.S. Environmental Protection Agency). 1985. Health assessment document for chloroform. Final report. Office of Health and Environmental Assessment. EPA600/8-84-004F. NTIS Document PB86-105004.

U.S. EPA (U.S. Environmental Protection Agency). 1991. Integrated Risk Information System (IRIS). Online. Office of Health and Environmental Assessment, Environmental Criteria and Assessment Office, Washington, D.C. 


\section{CHLOROMETHANE}

CAS No.: 74-87-3 .

Synonym (s) Methyl Chloride, Monochloromethane

Chloromethane is a natural component of the oceans and is produced biologically by some plants and fungi. Chloromethane was previously used in refrigerators as a refrigerant, but its current usage is mainly as a reactant in the production of other compounds. Chloromethane may be released naturally to the atmosphere from burning organic matter, or anthropogenically as a constituent of municipal and industrial solid waste, as well as from water treatment facilities (ATSDR 1989).

Chloromethane is a colorless gas with only a faint, nonirritating odor. It has a melting point of $-97.7^{\circ} \mathrm{C}$ and a boiling point of $-23.73^{\circ} \mathrm{C}$. The density of chloroethane is 1.74 at $0^{\circ} \mathrm{C}, 1 \mathrm{~atm}$. It is somewhat soluble in water and to a lesser degree in organic solvents (Ahlstrom and Steele 1979).

\section{FATE AND TRANSPORT}

Most releases of chloromethane are to the atmosphere since it is a gas at ambient temperature and pressure. Chloromethane may be transported from air by precipitation, due to its water solubility. The most prominent pathway for degradation of chloromethane in air is through reaction with hydroxyl radicals (Dilling 1982; Lovelock 1975). The half-life for chloromethane degradation by this process has been estimated to be approximately 1.5 years (Fabian 1986; Khalil and Rasmussen 1981; Crutzen and Gidel 1983). Others have presented data which suggests that photochemical degradation of chloromethane may occur at altitudes above $30 \mathrm{~km}$ (Crutzen et al. 1978).

Chloromethane release to water may occur from industrial discharges and waste treatment facility effluent. Groundwater contamination with chloromethane has been associated with municipal and hazardous waste landfills (Gould et al 1983; Brown and Donnelly 1988). Water-borne chloromethane will volatilize in surface waters with an estimated half-life of 25 hours in pond water and 18 days in lake water (ATSDR 1989). Hydrolysis of chloromethane to methane and hydrochloric acid represents an additional degradation pathway in surface waters, however the half-life (approx. 2 years) is sufficiently long that volatilization would account for the removal of most if not all of the contaminant. Hydrolysis may be more significant in the degradation of chloromethane in ground water (Heppolette and Robertson 1959; Hartmans et al. 1986; Janssen et al. 1985). In addition to the above two processes, a number of bacteria are capable of breaking down chloromethane in water under experimental conditions (Stirling and Dalton 1979), however the significance of this finding to environmental situations is unclear (ATSDR 1989).

Chloromethane contamination in soils usually occurs as a result of landfill activities: Volatilization of this product is probably the most important transportation and clearance pathway of chloromethane from the surface soils, whereas leaching to groundwater is expected to occur from the lower levels of soil. 


\section{HUMAN HEALTH EFFECTS}

The most common route of exposure to chloromethane is through inhalation of contaminated air. Historically, exposure of the general population occurred when chloromethane vapors leaked from home refrigerators and industrial cooling systems. Current exposure of the general public to chloromethane vapors is typically minimal, but ever-present. The greatest risk of exposure to elevated concentrations of airborne chloromethane is through occupational exposure in the production of chloromethane (ATSDR 1989).

Acute poisoning by chloromethane produces deleterious effects on the cardiovascular system, the gastrointestinal tract, and most notably the nervous system (ATSDR 1989). Case reports have indicated that chloromethane produce tachycardia (increased heart rate) decreased blood pressure, and heart conduction abnormalities (Spevak et al. 1976). Nausea and vomiting have been observed among exposed individuals (Baird 1954; Spevak et al. 1976), however it is unclear whether this is a direct action on the gastrointestinal tract or an indirect result of the neurotoxic effects of chloromethane. Neurologic signs and symptoms in humans range from staggering, blurred vision, coma, and death, depending on the dose and length of exposure. With the exception of severe neurological toxicity, most of the effects of acute exposure to high concentrations of chloromethane are reversible once the individual is removed from the exposure source.

Chronic exposure to chloromethane has been shown to produce clinical jaundice in humans (Mackie 1961) and hepatic necrosis in mice (Landry et al. 1985). Blurred vision, fatigue, vertigo, nausea, vomiting and tremor have been noted in individuals exposed to high levels of chloromethane for extended periods of time (ATSDR 1989).

Chloromethane exposure in humans has not been shown to statistically increase the incidence of cancer in an exposed population (Holmes et al. 1986). In several studies, however, chronic chloromethane produced renal cell proliferation and/or kidney tumors in male mice (Chellman et al. 1986).

Because of its central nervous system depressant effects, chloromethane is though to potentiate the effects of other centrally acting depressants such as diazepam and alcohol (Puts-Anderson et al. 1981).

\section{ECOLOGICAL EFFECTS}

Little information is available on the bioaccumulation of chloromethane. Based upon the octanol/water partition coefficient of chloromethane, little accumulation is expected in aquatic organisms. Additionally, the rapid elimination of chloromethane in vertebrates is suggestive that this compound will not bioaccumulate in these species (ATSDR 1989). 


\section{US ENVIRONMENTAL PROTECTION AGENCY HUMAN HEALTH CRITERIA}

Oral Slope Factor

Inhalation Slope Factor

Chronic Oral RfD

Chronic Inhalation RfD
$1.3 \mathrm{E}-2 \mathrm{~kg} / \mathrm{mg} / \mathrm{day}^{-1}$

$6.3 \mathrm{E}-3 \mathrm{~kg} / \mathrm{mg} /$ day $^{-1}$

NA

NA

\section{DRINKING WATER CRITERIA AND STANDARDS}

U.S. Environmental Protection Agency

Maximum Concentration Limit

NA

Maximum Concentration Limit Goal

NA

\section{AMBIENT WATER QUALITY CRITERIA}

U.S. Environmental Protection Agency Ambient Water Quality Criteria

Human Health-Water and Fish Ingestion NA Human Health-Fish Ingestion Only NA

Aquatic Organisms-FW Acute . NA

Aquatic Organisms-FW Chronic NA

Aquatic Organisms-Marine Acute NA

Aquatic Organisms-Marine Chronic NA

South Carolina Department of Health and Environmental Control

Human Health Criteria

Fish and Aquatic Life-Continuous

Fish and Aquatic Life-Maximum
$4708 \mu \mathrm{g} / \mathrm{L}$

NA

NA

NA $=$ Not Available

\section{REFERENCES}

Agency for Toxic Substances and Disease Registry. 1989. Toxicological Profile for Chloromethane. Prepared by Clement International Corp., contract no. 205-88-0608.

Ahlstrom, R.C., Steele, J.M. 1979. Chlorocarbons - hydrocarbons (Ch3Cl). In: Kirk-Othmer encyclopedia of chemical technology, 3rd edition. M.Grayson, D. Eckroth eds. New York. John Wiley and Sons, Inc. 5:677-685.

Baird, TT. 1954. Methylene chloride poisoning. Br. Med. J. 2:1353. 
Brown, K.W., Donnelly, K.C. 1988. An estimation of the risk associated with the organic constituents of hazardous and municipal waste landfill leachates. Haz. Waste Mater. 5:1-30.

Chellman, G.J., Morgan, K.T., Bus, J.S. et al. 1986. Inhibition of the acute toxicity of methyl chloride in male B6C3F1 mice by glutathione depletion. Toxicol. Appl. Pharmacol. 86:93-104.

Crutzen, P.J., Gidel, L.T. 1983. A two-dimensional photochemical model of the atoms. 2:The tropospheric budgets of the anthropogenic chlorocarbons, carbon monoxide, methane, chloromethane and the effect of various nitrogen oxide sources on the tropospheric ozone. J. Geophys. Res. 88:6641-6661.

Crutzen, P.J., Isaksen, I.S.A., McAfee, J.R. 1978. The impact of the chloroform industry on the ozone layer. J. Geophys. Res:88345-363.

Dilling W.L. 1982. Atmospheric environment, Chapt.5. In: Conway, R.A., Environmental Risk Analysis for Chemicals New York: Van Nostrand Reinhold Co., 154-197.

Fabian, P. 1986. Halogenated hydrocarbons in the atmosphere. In: O. Hutzinger, ed. The handbook of environmental chemistry, Vol 4, Part A., Berlin: Springer Verlag, 23-51.

Gould, J.P., Ramsey ,R.E., Giabbi, M., et al. 1983. Formation of volatile haloorganic compounds in the chlorination of municipal landfill leachates. In: Water Chlorination Environmental Impact Health Effects. 4:525- 539.

Hartmans, S. Schmuckle, A., Cook, A.M., et al. 1986. Methyl chloride:Naturally occurring toxicant and C-1 growth substrate. J. Gen. Microbiol. 132:1139-1142.

Heppolette,R.L., Robertson,R.E. 1959. The neutral hydrolysis of methyl halides. Proc. Royal Soc. London, Ser.A. 252:273-285.

Holmes, T.M., Buffler ,P.A., Holquin, A.H., et al. 1986. A mortality study of employees at a synthetic rubber manufacturing plant. Am. J. Ind. Med. 9:355-362.

Jannsen, D.B., Scheper, A., Kijkhuizen, L., et al. 1985. Degradation of halogenated aliphatic compounds by Xanthobacter autotrophicus. GJ10. Appl: Environ. Microbiol. 49:673-677.

Khalil, M.A.K., Rasmussen, R.A. 1981. Atmospheric methyl chloride (CH3Cl). Chemosphere 10:1019-1023.

Landry, T.D., Quast, J.F., Gushow, T.S. 1985. Neurotoxicity of methyl chloride in continuously versus intermittently exposed female C57BL/6 mice. Fund. Appl. Toxicol. 5:87-98. 
Lovelock, J.E. 1975. Natural halocarbons in the air and in the sea. Nature 256:193-194.

Mackie, I.J. 1961. Methyl chloride intoxication. Med. J. Australia 1:203-205.

Putz-Anderson, V., Setzer, J.V., Croxton, J.S., et al. 1981. Methyl chloride and diazepam effects on performance. Scand. J. Work Environ. Health 7:8-13.

Spevak, L., Nadj, V., Felle, D. 1976. Methyl chloride poisoning in four members of a family. Br. J. Ind. Med. 2:1353

Stirling, D.I., Dalton, H. 1979. The fortuitous oxidation and cometabolism of various carbon compounds by whole-cell suspensions of Methylococcus capsulatus (Bath), FEMS Microbiol. Lett. 5:315-318. 
THIS PAGE LEFT INTENTIONALLY BLANK 


\section{CHROMIUM}

$\begin{array}{ll}\text { CAS No.: } & 7440-47-3 \\ \text { Major Synonym(s) } & \text { Chromium III, Chromium VI }\end{array}$

Chromium is a naturally occurring element found in rocks, plants and animals, soil, and volcanic dust and gasses as Chromium (III). Other forms, such as Chromium (O) and Chromium (VI) are produced commercially. Chromium compounds of sodium potassium and aluminum are. also produced industrially, using the forms Chromium (III) and Chromium (VI) principally. These products are used for chrome plating, in the manufacture of dyes, leather (as a tanning agent), wood preservatives, in toner for copying machines, and in textiles.

Chromium is a steel-gray metal that can be highly polished. It has a molecular weight of 51.996. It melts at $1857^{\circ} \mathrm{C}$ and boils at $2672^{\circ} \mathrm{C}$ (Weast 1988).

\section{FATE AND TRANSPORT}

Chromium release to the atmosphere occurs primarily from industry and fuel combustion. Natural releases result form continental dust and volcanic dust and gases (Fisbein 1981; Towill et al. 1978).

The predominant form of atmospheric chromium is particulate, and transport and deposition are determined primarily by particle size and density (Cary 1982). Chromium is reduced by other elements and compounds present in air, however, removal of chromium from the atmosphere is accomplished through wet and dry deposition of the materials to soil and surface water (Schroeder et al. 1987).

Excessive chromium in surface and ground waters are generated by waste water form industry and deposition of airborne chromium. In water, oxidation and reduction reactions occur, generally at slow rates.

Disposal of commercial chromium-containing products, industrial disposal, agricultural wastes, animal wastes, and atmospheric deposition account for the presence of unnaturally high levels of chromium in soil. Most soil-bound chromium is in an insoluble form and not mobile, however, rainwater and anaerobic biodegradation of plant materials tend to increase mobility, particularly in acidic conditions (Stackhouse and Benson 1989). Mobile chromium may be transported to surface water, ground water, or the atmosphere.

\section{HUMAN HEALTH EFFECTS}

The primary damage from inhalation of hexavalent Chromium (Cr VI) compounds is to the respiratory tract as a consequence of their acidic and oxidative nature. Subchronic exposure in the workplace can lead to accumulation of $\mathrm{Cr} \mathrm{VI}$ compounds in the lining of the nose, with resultant perforation of the nasal septum (Royle 1975). Rhinitis and pharyngitis are frequently reported (Mancuso 1951). Kidney damage may result from absorption of soluble chromates through the lungs (Rinehart and Gad 1986). 
In laboratory animals exposure to $\mathrm{Cr}$ VI by inhalation, either acutely or chronically, has produced bronchitis and pneumonia, chronic irritation of the bronchi and lung parenchyma, and dystrophic changes in the liver and kidneys. Trivalent compounds ( $\mathrm{Cr}$ III) are far less toxic (Tandon 1982; NAS 1974; Blokin and Trop 1977).

Certain water-soluble $\mathrm{Cr}$ VI compounds are recognized as human respiratory carcinogens from inhalation exposure. This association has been confirmed by epidemiological investigations of the chromate industry (Taylow 1966; Alderson et al. 1981; Mancuso 1975) as well as in recent animal studies where animals were dosed intratracheally with $\mathrm{Cr}$ VI (Rinehart and Gad 1986). The U.S. EPA has classified hexavalent chromium as a Group A human carcinogen.

$\mathrm{Cr}$ VI compounds are generally far more acutely toxic than $\mathrm{Cr}$ III compounds. In man the lethal dose of various hexavalent compounds by ingestion ranges from 1.5-16 g/person. Toxic effects include severe inflammation of the digestive tract, and hepatic and renal tubular necrosis (IARC 1980). The oral $\mathrm{LD}_{50}$ for $\mathrm{Cr}$ VI (sodium chromate) in rats was 19.8 $\mathrm{mg} / \mathrm{kg}$ (NIOSH 1983). In acute and subchronic oral animal experiments, chromates (Cr VI) in general have been shown to primarily damage the convoluted proximal kidney tubules (Tandon 1982). Long-term ingestion of low levels of either Cr III or Cr VI apparently produces no ill effects in laboratory animals (MacKenzie et al. 1958; Anwar et al. 1961). The U.S. EPA has classified hexavalent chromium as a toxic substance from the oral route of exposure.

Contact with Cr VI compounds irritates and corrodes the skin, leading to painless, slowhealing lesions known as "chrome burns" (Pederson 1981). Dermal contact with Cr VI compounds (not noted for $\mathrm{Cr}$ III compounds) may also cause allergic dermatitis or sensitization.

\section{ECOLOGICAL EFFECTS}

Chromium concentration in plants grown in chromium contaminated soil have been shown to reach levels above background, however, most of the chromium remained in the plants' root structures (Cary 1982). Chromium is not expected to bioaccumulate in aquatic organisms, based on the bioconcentration factors (BCF) for Chromium (III) and Chromium (VI) in oyster, fish, mussel, and clam (EPA 1980, 1984a; Fisbein 1981).

\section{U.S. ENVIRONMENTAL PROTECTION AGENCY HUMAN HEALTH CRITERIA}

Oral Slope Factor

Inhalation Slope Factor

Chronic Oral RfD

Chronic Inhalation RfD
NA

$4.1 \mathrm{E}-1 \mathrm{~kg} / \mathrm{mg} /$ day $^{-1}$

$5 \mathrm{E}-3 \mathrm{mg} / \mathrm{kg} /$ day

NA 


\section{DRINKING WATER CRITERIA AND STANDARDS}

U.S. Environmental Protection Agency
Maximum Concentration Limit
$0.1 \mathrm{mg} / \mathrm{L}$ (Total)
Maximum Concentration Limit Goal
$0.1 \mathrm{mg} / \mathrm{L}$ (Total)

\section{AMBIENT WATER QUALITY CRITERIA}

U.S. Environmental Protection Agency Ambient Water Quality Criteria

Human Health-Water \& Fish Ingestion

Human Health - Fish Ingestion Only

Aquatic Organisms - FW Acute

Aquatic Organisms - FW Chronic

Aquatic Organisms - Marine Acute

Aquatic Organisms - Marine Chronic
$50 \mathrm{mg} / \mathrm{L}(\mathrm{VI})$

NA

$1700 \mathrm{mg} / \mathrm{L}$ (III); $16 \mathrm{mg} / \mathrm{L}$ (VI)

$210 \mathrm{mg} / \mathrm{L}$ (III); $11 \mathrm{mg} / \mathrm{L}$ (VI)

$10,300 \mathrm{mg} / \mathrm{L}$ (III); $1100 \mathrm{mg} / \mathrm{L}$ (VI)

$50 \mathrm{mg} / \mathrm{L}(\mathrm{VI})$

South Carolina Department Health and Environmental Control Water Quality Criteria

Human Health Criteria

Fish and Aquatic Life - Continuous

Fish and Aquatic Life - Maximum
$673,077 \mathrm{mg} / \mathrm{L}$ (III); $50 \mathrm{mg} / \mathrm{L}$ (VI)

$117.32 \mathrm{mg} / \mathrm{L}$ (III); $11 \mathrm{mg} / \mathrm{L}$ (VI)

$984.32 \mathrm{mg} / \mathrm{L}$ (III); $16 \mathrm{mg} / \mathrm{L}$ (VI)

$\mathrm{NA}=$ Not Available

\section{REFERENCES}

Alderson, M.R., N.S. Rattan, and L. Bidstrup. 1981. Health of workmen in the chromateproducing industry in Britain. Br. J. Ind. Med. 38:117-124.

Anwar, R.A., R.F. Langham, C.A. Hoppert, B.V. Alfredson, and R.U. Byerrum. 1961. Chronic toxicity studies: III. Chronic toxicity of cadmium and chromium in dogs. Arch. Environ. Health 3:456-460.

Blokin, V.S. and F.S. Trop. 1977. In: Dubinin, N.P., ed. Genetic Effects of the Pollution of the Environment, pp. 173-176. (Cited in Tandon, 1982).

Cary, E.E. 1982. - Chromium in air, soil and natural. In: Langard, S., ed. Topics in environmental health 5: Biological and environmental aspects of chromium. New York, NY: Elsevier Science Publishes, 49-64.

EPA. 1980. Ambient water quality criteria for chromium. Washington, DC: Office of Water Regulations and Standards, Criteria and Standards Division, U.S. Environmetnal Protection Agency. EPA-440/5-80-035. 
EPA. 1984a. Health assessment document for chromium. Research Triangle Park, NC: Environmental Assessment and Criteria Office, U.S. Environmental Protection Agency. EPA 600/8-83-014F.

Fishbein, L. 1981. Sources, transport and alterations of metal compounds: An overview. I. Arsenic, beryllium, cadmium, chromium and nickel. Environ Health Perspect 4043-64.

IARC (International Agency for Research on Cancer). 1980. IARC Monographs on the Evaluation of the Carcinogenic Risk of Chemicals to Humans: Some metals and metallic compounds. WHO, IARC, Lyon, France. Vol. 23, p. 205-323.

MacKenzie, R.D., R.U. Byerrum, C.F. Decker, C.A. Hoppert and R.F. Langham. 1958. Chronic toxicity studies. II. Hexavalent and trivalent chromium administered in drinking water to rats. AMA Arch. Ind. Health. 18:232-234.

Mancuso, T.F. 1951. Occupational cancer and other health hazards in a chromate plant: A medical appraisal. II. Clinical and toxicologic aspects. Ind. Med. Surg. 20:393-407.

Mancuso, T.F. 1975. Consideration of chromium as an industrial carcinogen. In: Hutchinson, T.C., ed. Proceedings of the International Conference on Heavy Metals in the Environment. Toronto, Canada: Toronto Institute for Environmental Studies, pp 343-356.

NAS (National Academy of Sciences). 1974. Medical and biological effects of environmental pollutants: Chromium. Washington, D.C. 155 pp.

NIOSH (National Institute of Occupational Safety and Health). 1983. Registry of toxic effects of chemical substances (RTECS). Vol. 2: p. 72.

Pederson, N.B. 1982. The effects of chromium on the skin In: Langard, ed. Biological and . Environmental Aspects of Chromium. Elsevier Biomedical Press, 249-275.

Rinehart, W.E. and S.C. Gad. 1986. Current concepts in occupational health: Metals chromium. Am. Ind. Hyg. Assoc. J. 47(11):696-699.

Royle, H. 1975. Toxicity of chromic acid in the chromium-plating industry (1). Environ. Res. 10:39-53.

Schroeder, W.H., Dobson, M., Kane, D.M., et al. 1987. Toxic trace elements associated with airborne particulate matter: A review. J Air Pollut Control Assoc 37:12671285.

Stackhouse, R.A., Benson, M.W.H. 1989. The effect of humic acid on the toxicity and vioavailability of trivalent chromium. Ecotoxicol environ Safety 17:105-111. 
Tandon, S.K. 1982. Organo toxicity of chromium in animals. In: Langard, S., Ed. Biological and Environmental Aspects of Chromium. Elsevier Biomedical Press B.V., Amsterdam.

Taylor, F.H. 1966. The relationship of mortality and duration of employment as reflected by a cohort of chromate workers. Am. J. Pub. Health 56:218-229.

Towill, L.E., Shriner, C.R., Drury, J.S. 1978. Reviews of the environmental effects of pollutants: III. Chromium. Report to Health Effects Research Laboratory, Office of Research and Development, U.S. Environmental Protection Agency, Cincinnati, $\mathrm{OH}$ by Information Center Complex/Information Division, Oak Ridge National Laboratory, Oak Ridge, TN. EPA-600/1-78-023.

Weast, R.C. editor. 1988. Handbook of Chemistry and Physics. 69th edition. Boca Raton, FL. CRC Press. 
THIS PAGE LEFT INTENTIONALLY BLANK 


\section{COPPER}

CAS No: $\quad 7440-50-8$

Synonym(s) none

Copper is an important metal that occurs naturally in rock, soil, water, sediment, air, and in plants and animals where it is an essential element. In its pure form, copper is used as the primary metal or an alloy in the manufacture of wire, sheet metal, pipes, etc. Copper is also combined with other elements to form copper compounds. Copper sulfate, the most abundant copper compound, is used as a fungicide on citrus, peanuts, potatoes, and other vegetable crops. Copper sulfate and other copper compounds are also used as algicides, for electroplating, in petroleum refining, and in the production of copper arsenate wood preservatives, azo dye, and the manufacture of other copper compounds (ATSDR 1989).

In its pure form, Copper exists as a reddish solid with a molecular weight of 63.546, a melting point of $1083.4^{\circ} \mathrm{C}$, and a boiling point of $2567^{\circ} \mathrm{C}$. It is insoluble in water and has a density of 8.92. Copper sulfate, a blue-white solid, has a molecular weight of 159.60 , a density of 3.603 , and begins to decompose at temperatures above $200^{\circ} \mathrm{C}$. Copper sulfate is relatively insoluble in water but soluble in ethanol and methanol (Weast 1980).

\section{FATE AND TRANSPORT.}

The amount of copper released to the air is only a small fraction of the total copper release to the environment (Perwack et al. 1980). The primary source of atmospheric copper is windblown dust, however other natural sources such as volcanoes, decaying vegetation, forest fires, and sea spray contribute (Davies and Bennet 1985). Human uses of copper in the production of wood, oil and gasoline consumption, and the manufacture of phosphate fertilizer are also sources of copper emissions.

Atmospheric copper is in the form of particulate matter or is adsorbed to particulate matter. Particle size is a major determinant of its fate, which may be deposition on the earths surfaces by precipitation and gravitational settling (Chan et al. 1986).

Copper binds tightly to soils. Leaching may occur in sites where acid rain is significant (Strain et al. 1984), however in most cases copper remains in the top layers of the soil. Of course the physical and organic characteristics of the soil, as well as the form of copper present affect its mobility (ATSDR 1989).

Unstable forms of copper present in water tend to complex with organic and inorganic ligands like ammonia and chloride ions, and humic substances. Insoluble, stable forms of copper bind particulate matter and settle onto aquatic sediments. Copper contamination in drinking water is generally derived from copper pipes and brass faucets that have held tap water overnight (ATSDR 1989). 


\section{HUMAN HEALTH EFFECTS}

Inhalation of copper acutely can produce respiratory irritation, anorexia, nausea, and diarrhea (Suciu et al. 1981). Metal fume fever, a syndrome characterized by 24-48 hours of chills, fever, aching muscles, dry mouth and throat, and headache has been reported by workers acutely exposed to high concentrations of copper fumes or dust (Armstrong et al. 1983). Acute oral exposure to copper results in GI distress (Chuttani et al. 1965), hepatotoxicity (Chuttani et al. 1965; Walsh et al. 1977), and renal tubular necrosis (Walsh et al. 1977). Suicidal death resulted from shock and hepatic and/or renal complications in patients who ingested copper sulfate (ATSDR 1989).

Chronic ingestion of copper has been associated with the development of hypertension in rats (Liu and Medeiros 1988), depressed skeletal growth (Rana and Kumar 1980), hepatic necrosis and renal tubular necrosis (Chuttani et al. 1965).

\section{ECOLOGICAL EFFECTS}

In all studies examined, copper was not found to bioaccumulate or bioconcentrate in the food chain. Bioconcentration factor estimates for fish yielded low potentials, however bottom dwelling aquatic species such as oysters have quite high potentials for bioconcentration. Regardless, there is no evidence of bioaccumulation, even in bottom dwelling species (Perwack et al. 1980).

Bioaccumulation was examined in several trophic levels of terrestrial vertebrates and no evidence of bioconcentration in the food chain was found (Hernandez et al. 1985). Likewise, examination of terrestrial invertebrates at the lower trophic levels indicated that organismal concentration of copper was poorly correlated with soil concentration (Beyer and Cromartie 1987).

\section{US ENVIRONMENTAL PROTECTION AGENCY HUMAN HEALTH CRITERIA}

Oral Slope Factor

Inhalation Slope Factor

Chronic Oral RfD

Chronic Inhalation RfD
NA

NA

$3.7 \mathrm{E}-2 \mathrm{mg} / \mathrm{kg} /$ day

NA

\section{DRINKING WATER CRITERIA AND STANDARDS}

U.S. Environmental Protection Agency

Maximum Concentration Limit

NA

Maximum Concentration Limit Goal

$1.3 \mathrm{mg} / \mathrm{l}$ 


\section{AMBIENT WATER QUALITY CRITERIA}

U.S. Environmental Protection Agency Ambient Water Quality Criteria

$\begin{array}{ll}\text { Human Health-Water and Fish Ingestion } & 170 \mathrm{mg} / \mathrm{L} \\ \text { Human Health-Fish Ingestion Only } & 3,433 \mathrm{mg} / \mathrm{L} \\ \text { Aquatic Organisms-FW Acute } & 18 \mu \mathrm{g} / \mathrm{L} \\ \text { Aquatic Organisms-FW Chronic } & 12 \mu \mathrm{g} / \mathrm{L} \\ \text { Aquatic Organisms-Marine Acute } & 2.9 \mu \mathrm{g} / \mathrm{L} \\ \text { Aquatic Organisms-Marine Chronic } & \mathrm{NA}\end{array}$

South Carolina Department of Health and Environmental Control

Human Health Criteria

Fish and Aquatic Life-Continuous

Fish and Aquatic Life-Maximum
NA

$6.5 \mu \mathrm{g} / \mathrm{L}$

$9.2 \mu \mathrm{g} / \mathrm{L}$

$\mathrm{NA}=$ Not Available

\section{REFERENCES}

Agency for Toxic Substances and Disease Registry (ATSDR). 1989. Draft Toxicological Profile for Copper. Prepared by Syracuse Research Corp. U.S. Department of Health and Human Services.

Armstrong, C.W., Moore ,L.W., Hackler, R.L., et al. 1983. An outbreak of metal fume fever: Diagnostic use of urinary copper and zinc determinations. J. Occup. Med. 25:886-888.

Beyer, W.N., Cromartie ,EJ. 1987. A survey of $\mathrm{Pb}, \mathrm{Cu}, \mathrm{Zn}, \mathrm{Cd}, \mathrm{Cr}, \mathrm{As}$, and $\mathrm{Se}$ in earthworms and soil from diverse sites. Environ. Monit. Assess. 8:27-36.

Chan, W.H., Tank ,AJ.S., Cheng ,D.H.S., et al. 1986. Concentration and deposition of trace metals in Ontario. 1982. Water Air Soil Pollut. 29:373-389.

Chuttani, H.K., Gupta, P.S., Gulati, S., et al. 1965. Acute copper sulfate poisoning. Am. J. Med. 39:849-854.

Davies, D.J.A., Bennet ,B.G. 1985. Exposure of man to environmental copper:An exposure commitment assessment. Sci.Total Environ. 46:215-227.

Hernandez, L.M., Gonzalez, J., Rico, C. et al. 1985. Presence and biomagnification of organochlorine pollutants and heavy metals in mammals of Donana-National Park (Spain) 1982-3. J. Environ. Sci. Health B20:633-650. 
Liu, C-C.F., Nadeiros ,D.M. 1986. Excess dietary copper increases systolic blood pressure in rats. Biol. Trace Element Res. 9:15-24.

Perwack, J.,Bysshe, S., Goyer, M., et al.1980. Exposure and risk assessment for copper. Cincinatti, OH : U.S. Environmental Protection Agency. EPA 440/4-81-015. NTS PB85-211985.

Rana, S.V.S., Kumar, A. 1980. Biological, hematological, and histological observations in copper-poisoned rats. Ind. Health 18:9-17.

Strain, W.H., Hershey, C.O., McInnes, S. et al.1984. Hazards to groundwater from acid rain. Trace Subst. Environ. health 18:178-184.

Suciu, H., Prodan ,L., Lazar, V. et al. 1981. Research on copper poisoning. Med. Lav. 72:190-197.

Walsh, F.M., Crosson, F.J., Bayley, J. et al. 1977. Acute copper intoxication. Am. J. Dis. Child 131:149-151.

Weast, R.C. 1980. CRC Handbook of chemistry and physics. 61st ed. Boca Raton, FL. CRC Press. 
DICHLORODIPHENYLETHANES (DDT, DDE, DDD)

CAS Nos.: $\quad 50-29-3,72-55-9,72-54-8$

Major Synonym(s) Dichlorodiphenyltrichloroethane (DDT), dihydrochloride (DDE)

DDT was first synthesized in 1874, however, its insecticidal properties were not discovered until 1939. From that time until its use was banned by the EPA in 1972, DDT was one of the most widely used insecticides in the United States. DDT was very effective in the control of insect vectors of malaria and typhus. It was extensively used on cotton, peanuts, and soybean crops, and at one time was registered for use on 334 agricultural commodities (ATSDR-1993).

Structurally, DDT, DDE, and DDD are very similarly, differing only in the number of Chlorine and hydrogen atoms. DDT has a molecular weight of 354.49, a melting point of $108-109^{\circ} \mathrm{C}$, and a boiling point of $260^{\circ} \mathrm{C}$. DDE has a molecular weight of 318.03 and a melting point of $88.4-90^{\circ} \mathrm{C}$. DDD has a molecular weight of 320.05 , a melting point of $109-$ $110^{\circ} \mathrm{C}$, and a boiling point of $193^{\circ} \mathrm{C}$. All are colorless crystals or white powder, insoluble in water, and soluble in organic solvents and/or lipids (HSDB 1988; Weast and Astlle 1981; Verschueren 1983).

\section{FATE AND TRANSPORT}

Prior to its ban in the United States, DDT was released to the atmosphere during application as an insecticide. Because it is currently used in other countries, DDT may still enter the atmosphere during pesticide application and may be transported to the U.S. by wind currents (Rapaport et al. 1985). Volatilization from soil and water also may contribute to atmospheric contamination by DDT (ATSDR 1003; Fishbein 1973). DDE and DDD occur in air and other environmental media as products of DDT degradation. DDT and its metabolites in are subject to decomposition by photo-oxidation and reaction with hydroxyl radicals (WHO 1979). Precipitation is thought to remove DDT from the air at a faster rate than other pathways (Woodwell et al. 1971) and leads to deposition in surface waters and sediments.

DDT in the water quickly adsorb to particulate matter, causing sedimentation. It may be transformed through photodegradation and biodegradation (Coulston 1985; Johnsen 1976) or DDT may be transported back to the atmosphere by evaporation (EPA 1986a).

DDT is persistent in soils where it binds to soil particles (EPA 1986). It may slowly volatilize to the atmosphere with an estimated half life of -100 days (Sleicher and Hopcraft 1984). DDT in soils is also subject to water runoff, photo-oxidation, and biodegradation in aerobic and anaerobic conditions (Fishbein 1973). Nonetheless, DDT may take up to 15 years to degrade in soil.

\section{HUMAN HEALTH EFFECTS}

Human volunteers experienced moderate irritation of the nose, throat and eyes when exposed to aerosols of DDT at levels which left a white deposit on the nasal hair of the 
volunteers (Neal et al. 1944). No other information was located in the available literature concerning the effects of inhalation exposure to DDT, DDE or DDD in humans or animals. The U.S. EPA (1992a) has derived an inhalation slope factor for DDT of $3.4 \mathrm{E}-01 \mathrm{mg} / \mathrm{kg}$ day $^{-1}$, based on data from oral exposure studies. No inhalation slope factors have been established for DDD or DDE.

Individuals are typically exposed to a mixture of DDT, DDE or DDD rather than the separate compounds, as DDE and DDD are contaminants as well as degradation and metabolic products of DDT. The following signs and symptoms have been reported in humans following accidental or intentional ingestion of DDT (up to $22 \mathrm{mg} / \mathrm{kg}$ ): disturbance of sensitivity of the lower part of the face, uncertain gait, malaise, cold, moist skin, confusion, tremors, malaise, headache, severe vomiting, and fatigue (Velbinger 1947a; 1947b; Francone et al. 1952; Garrett 1947; Hayes 1982).

The liver and the nervous system appear to be the primary organs affected in experimental animals following acute, subchronic, or chronic oral exposure to DDT. A dose-related increase in severity of hepatic cell hypertrophy, histopathologic alterations and microsomal enzyme activity were observed in rats following three to six months of exposure to DDT at doses ranging from 0.25 to $20 \mathrm{mg} / \mathrm{kg}$ /day (Hart and Fouts 1965; Laug et al. 1950; Ortega et al. 1956). Jonsson et al. (1981) reported spotty cellular necrosis with moderate hepatocyte regeneration in rats fed $3.75 \mathrm{mg} \mathrm{DDT} / \mathrm{kg}$ body weight/day continuously in the diet for 36 weeks. Necrosis, centrilogular hypertrophy, and hyperplasia have been noted in rats exposed to 10 to $40 \mathrm{mg}$ DDT/ $\mathrm{kg} /$ day for 24-27 months (Deichmann et al. 1967; Fitzhugh and Nelson 1947). Liver damage occurred in dogs exposed to $80 \mathrm{mg} \mathrm{DDT} / \mathrm{kg} / \mathrm{day}$ for 39-40 months (Lehman 1965).

Chronic exposure of experimental animals to DDT is associated with tremors and general hyperexcitability; the tremors persist for several weeks following cessation of exposure (NCI 1978).

The dichlorodiphenylethanes have been classified as probable human carcinogens (classification B2) by the U.S. EPA (1992a). Human epidemiological data are unavailable for DDD and DDE. The data for DDT are inadequate; evidence for the human carcinogenicity of this compound is based on three studies relating tissue levels of DDT to cancer incidence (Casarett et al. 1968;.Dacre and Jennings 1970; Wasserman et al. 1976). There is sufficient evidence, from over 25 studies in experimental animals, that DDD, DDE, and DDT are carcinogenic in laboratory animals. Long-term feeding studies have demonstrated that these compounds cause benign and malignant tumors in the liver and lungs of rats and mice (Walker et al. 1973; Terracini et al. 1973; Shabad et al. 1973; Cabral et al. 1982; Tomatis et al. 1974; Rossi et al. 1983). DDD and DDE have also reportedly induced thyroid tumors in rats (NCI 1978). Based on the evidence of these studies, the U.S. EPA (1992a) has derived an oral slope factor for both DDE and DDT of $3.4 \mathrm{E}-01 \mathrm{mg} / \mathrm{kg}$ day $^{-1}$; the oral slope factor for DDD is $2.4 \mathrm{E}-01 \mathrm{mg} / \mathrm{kg}$-day ${ }^{-1}$ (U.S. EPA 1992a).

\section{ECOLOGICAL EFFECTS}


Because of its persistence in the environment, coupled with its high lipophilicity, DDT, and its metabolites bioconcentrate readily within the food chain. DDT may be transported directly from contaminated soil into plants. Additionally, DDT enters the food chain through aquatic organisms. It has been found to bioaccumulate in plankton, mollusks, insects, other invertebrates, and fish (ATSDR 1993). Humans, as the top consumer, also ingest large amounts of DDT, which is stored in lipids.

\section{U.S. ENVIRONMENTAL PROTECTION AGENCY HUMAN HEALTH CRITERIA}

$\begin{array}{ll}\text { Oral Slope Factor } & 3.4 \mathrm{E}-1(\mathrm{DDT} / \mathrm{DDE}) \mathrm{kg} / \mathrm{mg} / \mathrm{day}^{-1} \\ & 2.4 \mathrm{E}-1(\mathrm{DDD}) \mathrm{kg} / \mathrm{mg} / \mathrm{day}^{-1} \\ \text { Inhalation Slope Factor } & 3.4 \mathrm{E}-1 \mathrm{~kg} / \mathrm{mg} / \mathrm{day}{ }^{-1} \\ \text { Chronic Oral RfD } & 5.0 \mathrm{E}-4 \mathrm{mg} / \mathrm{kg} / \mathrm{day} \\ \text { Chronic Inhalation RfD } & \mathrm{NA}\end{array}$

DRINKING WATER CRITERIA AND STANDARDS

U.S. Environmental Protection Agency

Maximum Concentration Limit

NA

Maximum Concentration Limit Goal NA

\section{AMBIENT WATER QUALITY CRITERIA}

U.S. Environmental Protection Agency Ambient Water Quality Criteria
Human Health - Water and Fish Ingestion
$0.059 \mathrm{ng} / \mathrm{L}$
Human Health - Fish Ingestion Only
Aquatic Organisms - FW Acute
$0.059 \mathrm{ng} / \mathrm{L}$
Aquatic Organisms - FW Chronic
$1.1 \mu \mathrm{g} / \mathrm{IL}$
Aquatic Organisms - Marine Acute
$0.001 \mu \mathrm{g} / \mathrm{L}$
Aquatic Organisms - Marine Chronic
$0.13 \mu \mathrm{g} / \mathrm{L}$
$0.001 \mu \mathrm{g} / \mathrm{L}$

South Carolina Department of Health and Environmental Control Water Quality Criteria

Human Health Criteria

Fish and Aquatic Life - Continuous

Fish and Aquatic Life - Maximum
$0.0059 \mu \mathrm{g} / \mathrm{L}$

$0.001 \mu \mathrm{g} / \mathrm{L}$

$1.1 \mu \mathrm{g} / \mathrm{L}$

$\mathrm{NA}=$ Not Available

\section{REFERENCES}

Agency for Toxic Substances and Disease Registry. 1992. Toxicological Profile for DDT, DDE, and DDD. Prepared by Clement International Corp., contract no. 205-88-0608. 
Ben-Dyke, R., Sanderson, D. and D. Noakes. 1970. Acute toxicity data for pesticides (1970). World Rev. Pest. Cont. 9:119-127.

Cabral, J.R.P., R.K. Hall, L. Rossi, S.A. Bronczyk and P. Shubik. 1982. Effects of long-term intake of DDT on rats. Tumorigenesis 68:11-17.

Cameron, G. and F. Burgess. 1945. The toxicity of 2,2-bis(p-chlorphenyl) 1,1,1trichloroethane (DDT). Br. Med. J. 1:865-871.

Casarett, L.J., G.C. Fryer, W.L. Yauger, Jr. and H. Klemmer. 1968. Organochlorine pesticide residues in human tissue. Hawaii. Arch. Environ. Health 17: 306-311.

Coulston, F. 1985. Reconsideration of the dilemma of DDT for the establishment of an acceptable daily intake. Regul Toxicol Pharmacol 5:332-383.

Dacre, J.C. and R.W. Jennings. 1970. Organochlorine insecticides in normal and carcinogenic human lung tissues. Toxicol. Appl. Pharmacol. 17:277.

Deichmann, W., M. Keplinger, F. Sala et al. 1967. Synergism among oral carcinogens. Toxicol. Appl. Pharmacol. 11:88-103.

EPA. 1986a. Environmental Protection Agency Superfund Public Health Evaluation . Manual. Office of Emergency and Remedial Response. EPA 540/1-86-060. October 1986.

Fishbein, L. 1973. Mutagens and potential mutagens in the biosphere I. DDT and its meteabolities, polychlorinated biphenyls, chlorodioxins, polycyclic aromatic hydrocarbons, haloethers. Sci Total Environ 4:305-340.

Fitzhugh, O. an dA. Nelson. 1947. The chronic oral toxicity of DDT (2,2-bis(p-chlorophenyl1,1,1-trichloroethane). J. Pharmacol. Exp. Ther. 89:18-30.

Francone, M., F. Mariani and Y. Demare. 1952. [Clinical signs of poisoning by DDT.] Rev. Assoc. Med. Argentina 66:56-59 (Spanish).

Gaines, T. 1969. Acute toxicity of pesticides. Tox. Appl. Pharmacol. 14:515-534.

Garrett, R. 1947. Toxicity of DDT for man. J. Med. Assoc. Alabama 17:74-78.

Gellert, R. and W. Heinrichs. 1975. Effects of DDT homologs administered to female rats during the perinatal period. Biol. Neonate 26:282-290.

Hart, M., R. Adamson and S. Fabro. 1971. Prematurity and intrauterine growth retardation induced by DDT in the rabbit (1)(2)(3). Arch. Int. Pharmacodyn. 192:286-290. 
Hart, M. and J. Fouts. 1965. Further studies on the stimulation of hepatic microsomal drug metabolizing enzymes by DDT and its analogs. Naunyn-Schmiedebergs Arch. Exp. Pathol.' Pharmakol. 249:486-500.

Hart, M., J. Whang-Peng, S. Sieber et al. 1972. Distribution and effects of DDT in the pregnant rabbit. Xenobiotica 2:567-574.

Hayes, W., Ed. 1982. Chlorinated hydrocarbon insecticides. In: Pesticides Studies in Man. Baltimore: Williams and Wilkins. pp. 186-195.

Herr, D. and H. Tilson. 1987. Modulation of p,p'-DDT-induced tremor by catechloaminergic agents. Toxicol. Appl. Pharmacol. 91:149-158.

HSDB. 1988. Hazardous Substances Data Bank. National Library of Medicine, National Toxicology Information Program, Bethesda, MD. November 11, 1988.

Johnsen, R. 1976. DDT metabolism in microbial systems. Residue Rev. 61:1-28.

Jonsson, H., E. Walker, W. Greene et al. 1981. Effects of prolonged exposure to dietary DDT and PCB on rat liver morphology. Arch. Environ. Contam. Toxicol. 10:171-183.

Laug, E.P., A.A. Nelson, O.G. Fitzhugh and F.M. Kunze. 1950. Liver cell alteration an DDT storage in the fat of the rat induced by dietary levels of $1-50 \mathrm{ppm}$ DDT. J. Pharmacol. Exp. Therap. 98:268-273.

Lehman, A. 1965. Summaries of Pesticide Toxicity, Topeka, Kansa, Association of Food and Drug Officials of the United States. (Cited in WHO, 1979).

Matin, M., F. Jaffery and R. Dissiqui. 1981. A possible neurochemical basis of the central stimulatory effects of pp'DDT. J. Neurochem 36:1000-1005.

NCI (National Cancer Institute). 1978. Bioassay of DDT, TDE and p,p'-DDE for possible carcinogenicity. NCI Report No. 131. DHEW Publ. No. (NIH) 78-1386.

Neal, P., W. Von Oettingen, W. Smith et al. 1944. Toxicity and potential dangers of aerosols, mists, and dusting powders containing DDT. Pub. Health Rep. 117:1-32.

Ortega, P. 1956. DDT in the diet of the rat. Pub. Health-Monogr. 43:1-27.

Rapaport, R., Urban, N., Chapel, P., et al. 1985. "New" DDT inputs to North America: Atmospheric deposition. Chemosphere 14:1167-1174.

Rossi, L., O. Barbieri, M. Sanguineti, J.R.P. Cabral, P. Bruzzi and L. Santi. 1983. Carcinogenicity study with technical-grade DDT and DDE in hamsters. Cancer Res. 43:776-781. 
Shabad, L.M., T.S. Kolesnichenko and T.V. Nikonova. 1973.f Transplacental and combined long-term effect of DDT in five generations of A-strain mice. Int. J. Cancer 11:688693.

Sleicher, C., Hopcraft, J. 1984. Persistence of pesticides in surface soil and relation to sublimation. Environ Sci Technol 18:514-518.

Terracini, B., M.C. Testa, J.R. Cabral and N. Day. 1973. The effects of long-term feeding of DDT to BALB/c mice. Int. J. Cancer 11:747-764.

Tomatis, L., V. Turusov, R.T. Charles and M. Boicchi. 1974. Effect of long-term exposure to 1,1-dichloro-2,2-bis()p-chlorophenyl)ethylene, to 1,1-dichloro-2,2-bis(pchlorophenyl) ethane, and the two chemicals combined on CF-1 mice. J. Natl. Cancer Inst. 52:883-891.

Velbinger, H. 1947a. [On the question of DDT toxicity of man.] Dtsch. Gesundheitwes. 2:355-238. (German).

Velbinger, H. 1947b. [Contribution to the toxicology of "ddt"-agent, dichlorodiphenyltrichloromethylmethane.] Pharmazie. 2:268-274. (German).

Verschueren, K ed. 1983. Handbook of environmental data on organic chemicals. 2nd ed. New York, NY: Van Nostrand Reinhold Company, 433-445.

Walker, A.I.T., E. Thorpe and D.E. Stevenson. 1973. The toxicology of dieldrin (HEOD). I. Long-term oral toxicity studies in mice. Food Cosmet. Toxicol. 11:415-432.

Wasserman, M., D.P. Nogueira, L. Tomatis et al. 1976. Organochlorine compounds in neoplastic and adjacent apparently normal breast tissue. Bull. Environ. Contam Toxicol. 15:478-484.

Weast, R., Astle, M. 1981. Handbook of chemistry and physics. Boca Raton, FL: CRC Press, Inc.

Woodwell, G., Craig, P., Johnson, H. 1971. DDT in the biosphere: Where does it go? Science 174:1101-1107.

WHO (World Health Organization). 1979. DDT and its derivatives. Environ. Health Criteria 9. WHO, Geneva. 
1,2-DICHLOROETHANE

CAS No: $\quad 107-06-2$

Synonym(s) Ethylene Dichloride, Sym-Dichloroethane, Ethylene Chloride, Glycol Dichloride, Beta-Dichloroethane, EDC

There are no natural sources of 1,2-dichloroethane. It is used primarily as a cleaning solvent, but also as a chemical intermediary, a diluent for pesticides, a grain fumigant, and in paint coatings and adhesives (Johns 1976). One source of chloroethanes in the environment may be from ethylene dichloride tars, by-products of vinyl chloride synthesis (Jensen et al. 1975). 1,2-Dichloroethane is a colorless liquid with a molecular weight of 99 $\mathrm{g} / \mathrm{mole}$ that melts at $-35.4^{\circ} \mathrm{C}$, boils at $83.5^{\circ} \mathrm{C}$, has a vapor pressure of $61 \mathrm{~mm} \mathrm{Hg}$ at $20^{\circ} \mathrm{C}$, has a specific gravity of 1.25 at $20^{\circ} / 4^{\circ} \mathrm{C}$, and has an aqueous solubility of $8,690 \mathrm{mg} / \mathrm{l}$ at $20^{\circ} \mathrm{C}$ (Verschueren 1983).

\section{FATE AND TRANSPORT}

1,2-Dichloroethane has a high vapor pressure and will volatilize rapidly when released into water or soil. Little absorption onto soil is expected and it will leach rapidly into the ground water (Swann et al. 1983). Biodegradation in soil or ground water will not be significant, nor will hydrolysis (Bouwer 1981). Some biodegradation will occur in surface water, although the primary removal system is by volatilization. One half of the 1,2-dichloroethane in water with an average depth of $6.5 \mathrm{~cm}$ and containing $1 \mathrm{ppm}$ was removed within 28 minutes at a temperature of $25^{\circ} \mathrm{C}$ (Verschueren 1983). 1,2-Dichloroethane is not expected to significantly bioconcentrate in aquatic organisms (Howard 1976). When released into the atmosphere, 1,2-dichloroethane will degrade with a half-life of a little over a month by reaction with hydroxyl radicals which are formed photochemically in the atmosphere. One would expect the chemical to be transported long distances and be washed out in rain.

\section{HUMAN HEALTH EFFECTS}

1,2-Dichloroethane is absorbed through inhalation, ingestion, and dermal contact. Repeated contact with 1,2-dichloroethane can produce a dry, scaly, fissured dermatitis. Liquid and vapor may also cause eye damage, including corneal opacity. Inhalation of high concentrations may cause nausea, vomiting, mental confusion, dizziness, and pulmonary edema. Chronic exposure has been associated with liver and kidney damage (Sittig 1985). Acute exposure can lead to death from respiratory and circulatory failure, caused by widespread damage and bleeding in most internal organs. Two human studies are reported in Verschueren (1983). One study with humans reported severe toxic effects with exposure for 60 minutes to $500 \mathrm{ppm}$, with symptoms of toxicity at $100 \mathrm{ppm}$. Another study reported no effect levels of $200 \mathrm{ppm}$ for 7 hours, $1,000 \mathrm{ppm}$ for 60 minutes, and 3,000 ppm for 6 minutes. Repeated long-term exposures to this compound have resulted in neurological changes, liver and kidney impairment, and death (National Institute of Occupational Safety and Health 1976). 1,2-Dichloroethane has been shown to be carcinogenic in both rats and mice (U.S. Department of Health and Human Services 1982). It has been classified in group B2, probable human carcinogen, under the U.S. Environmental Protection Agency weight-of-evidence classification. 


\section{ECOLOGICAL EFFECTS}

Toxicity threshold (cell multiplication inhibition test) for bacteria of $135 \mathrm{mg} / \mathrm{L}$, and 105 to $710 \mathrm{mg} / \mathrm{L}$ for algae have been reported (Verschueren 1983). Rainbow trout exposed to 5 ppm 1,2-dichloroethane for 24 hours showed no signs of toxic effects. A 7-day $\mathrm{LC}_{50}$ of 106 ppm has been reported for guppies. In mammals, an $\mathrm{LD}_{50}$ for $0.68 \mathrm{~g} / \mathrm{kg}$ is reported for rats, and inhalation studies with exposure as high as $150 \mathrm{ppm}$ did not produce any toxic affects.

\section{U.S. ENVIRONMENTAL PROTECTION AGENCY HUMAN HEALTH CRITERIA}

Oral Slope.Factor

Inhalation Slope Factor

Chronic Oral RfD

Chronic Inhalation RfD
9.1E-02 kg/mg/day ${ }^{-1}$

9:1E-02 kg/mg/ day $^{-1}$

NA

NA

\section{DRINKING WATER CRITERIA AND STANDARDS}

U.S. Environmental Protection Agency

Maximum Concentration Limit

Maximum Concentration Limit Goal

$0.005 \mathrm{mg} / \mathrm{L}$

Zero

\section{AMBIENT WATER QUALITY CRITERIA}

U.S. Environmental Protection Agency Ambient Water Quality Criteria
Human Health - Water \& Fish Ingestion
9.4E-03 $\mathrm{mg} / \mathrm{L}$
Human Health - Fish Ingestion Only
$2.4 \mathrm{E} \mathrm{mg} / \mathrm{L}$
Aquatic Organisms - FW Acute
Aquatic Organisms - FW Chronic
$1.2 \mathrm{E}+02 \mathrm{mg} / \mathrm{L}$
Aquatic Organisms - Marine Acute
Aquatic Organisms - Marine Chronic
$2.0 \mathrm{E}+01 \mathrm{mg} / \mathrm{L}$
$1.1 \mathrm{E}+02 \mathrm{mg} / \mathrm{L}$
NA

South Carolina Department of Health and Environmental Control

Human Health Criteria

Fish and Aquatic Life - Continuous

$5 \mu \mathrm{g} / \mathrm{l}$

Fish and Aquatic Life - Maximum

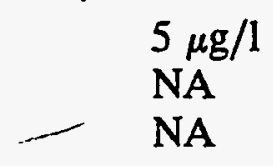

NA $=$ Not Available

\section{REFERENCES}

Agency for Toxic Substances and Disease Registry. 1992. Toxicological Profile for 1,2-

Dichloroethane. Prepared by Clement International Corp., contract no. 205-88-0608. 
Bouwer, E.J. et al. 1981. Environ. Sci. Technol. 15: 569.

Howard, P.H. et al. 1976. Investigations of Selected Environmental Contaminants: Nitroaromatics. U.S. Environmental Protection Agency 560/2-76-010 Research Triangle Park, NC.

Jensen, S., et al. 1975. Proc R Soc Lond B 189: 333-346.

Johns, R. 1976. Air Pollution Assessment. 1,2-Dichloroethane. MTR-7164 The Mitre Corp, McLean VA 34 pp.

Sittig, M. 1985. Handbook of Toxic and Hazardous Chemicals. Noyes Publications, Park Ridge, NJ.

Strafford, N., C.R.N. Strouts, W.V. Stubbings, no date. The Determination of Toxic Substances in Air. Heffer, Cambridge.

Swann, R.L., et al. 1983. Res Rev 85: 17-28.

U.S. Department of Health and Human Services. 1982. Third Annual Report on Carcinogens, Research Triangle Park, NC.

Verschueren, K. 1983. Handbook of Environmental Data on Organic Chemicals, 2nd Ed. Van Nostrand Reinhold, New York, NY. 
THIS PAGE LEFT INTENTIONALLY BLANK 


\section{DI-N-BUTYL Phthalate; DI-N-OCTYL Phthalate \\ CAS No: $\quad$ 84-74-2; \\ Synonym(s) - Butylphthalate; Dibutylphthalate}

For the purposes of this profile, Di-n-butyl phthalate will serve as a surrogate for di-n-octyl phthalate. di-n-butyl phthalate is commercially produced for use as a plasticizer for polyvinyl chloride and epoxy resins, as a concrete additive, a solvent for perfumes, as well as other industrial uses.

Di-n-butyl phthalate is an oily liquid at ambient conditions with a molecular weight of 278.35. It is odorless, colorless, melts at $-35^{\circ} \mathrm{C}$, and boils at $340^{\circ} \mathrm{C}$. Di-n-butyl phthalate is slightly more dense than water, with a density of 1.047. Di-n-butyl phthalate is insoluble in water, but soluble in alcohol, ether, and benzene (ATSDR 1989).

\section{FATE AND TRANSPORT}

Di-n-butyl phthalate use is widespread and as a result it is present in low doses in air, water, and soil. Di-n-butyl phthalate is released into the atmosphere from large, thin polymer surfaces and through disintegration of plastics at landfills. It occurs in air in both its vapor form and as particulate matter, and can be transported to water and soil by gravitational settling and wet deposition (precipitation) (Atlas and Giam 1981). The degradation half-life in air is predicted to be relatively short (approx. $18 \mathrm{hrs)} \mathrm{based} \mathrm{on} \mathrm{rates} \mathrm{of} \mathrm{reactions} \mathrm{with}$ hydroxyl radicals, however this compound may adsorb to particulates in the air, lengthening its half-life (ATSDR 1989).

Di-n-butyl phthalate is relatively insoluble in water, where it tends to form chemical complexes with humic materials which may settle out on bottom sediments (Callahan et al. 1979). Di-n-butyl phthalate degradation in water is primarily from microbial action, and to a lesser degree from photolysis. Evaporation is the major transport mechanism which aids the clearance of di-n-butyl phthalate from water (Wolfe et al. 1980).

In soils, a significant amount of adsortion occurs. The presence of certain organic solvents at hazardous waste sites may cause di-n-butyl phthalate to be released from soil particles, thereby making it a component of leachate which contaminates the groundwater. The most important degradation pathway for di-n-butyl phthalate in soils and aquatic sediments is through microbial action (Inman et al. 1984; Walker 1984).

\section{HUMAN HEALTH EFFECTS}

There is a dearth of information regarding the toxic effects of exposure to di-n-butyl phthalate from any absorption route. As a result, information on the toxicity of di-n-butyl phthalate has been extrapolated from animal studies.

The primary effect of inhalation exposure on rats to di-n-butyl phthalate over a 6 month period was a decrease in body weight. Acute oral ingestion increased microsomal enzyme levels in rat liver (Walseth and Nilsen 1986), whereas long term ingestion inhibited 
mitochondrial respiration (Murakami et al. 1986). The most striking toxicological findings have been the the ability of di-n-butyl phthalate to produce reproductive dysfunction. Seminiferous tubular atrophy was observed in male rats along with decreased sperm count and spermatogenesis (Cater et al. 1977). Oral doses of di-n-butyl phthalate given to pregnant mice and rats decreased the number of live births and reduced postnatal survival of those pups which were born live (Killinger et al. 1988). There is a limited amount of evidence which suggests that di-n-butyl phthalate causes teratogenic effects, primarily neural tube defects (Shiota and Nishimura 1982).

\section{ECOLOGICAL EFFECTS}

Di-n-butyl phthalate is bioconcentrated by a number of aquatic invertebrates (Sanders et al. 1973) and fish (Wofford et al. 1980). These organisms have been found to concentrate di-n-butyl phthalate at levels many fold above the ambient water concentration (Wofford et al. 1980). Food crops have also been observed to take up di-n-butyl phthalate from soil (Shea et al. 1982).

\section{US ENVIRONMENTAL PROTECTION AGENCY.HUMAN HEALTH CRITERIA}

Oral Slope Factor

Inhalation Slope Factor

Chronic Oral RfD

Chronic Inhalation RfD
NA

NA

1E-01(di-n-butyl)/

2E-02 (di-n-octyl)

NA

\section{DRINKING WATER CRITERIA AND STANDARDS}

U.S. Environmental Protection Agency

Maximum Concentration Limit

NA

Maximum Concentration Limit Goal

NA

\section{AMBIENT WATER QUALITY CRITERIA}

U.S. Environmental Protection Agency Ambient Water Quality Criteria

Human Health-Water and Fish Ingestion NA Human Health-Fish Ingestion Only _ • NA Aquatic Organisms-FW Acute NA Aquatic Organisms-FW Chronic . NA Aquatic Organisms-Marine Acute NA Aquatic Organisms-Marine Chronic NA 
South Carolina Department of Health and Environmental Control Water Quality Criteria

Human Health Criteria

Fish and Aquatic Life-Continuous

Fish and Aquatic Life-Maximum
NA

NA

NA

NA $=$ Data Not Available

\section{REFERENCES}

Agency for Toxic Substances and Disease Registry (ATSDR). October 1989. Draft Toxicological Profile for Di-n-butyl Phthalate. Prepared by Clement International Corp., contract no. 205-88-0608.

Atlas, E., Giam, C.S. 1981. Global transport of organic pollutants: Ambient concentrations in the remote marine atmosphere. Science 211:163-165.

Callahan, M.A., Slimak, M.W., Gabe, N.W., et al. 1979. Water-related environmental fate of 129 priority pollutants: Vol I: Introduction and technical background, metals and inorganics, pesticides and PCB's. Washington, D.C. U.S. Environmental Protection Agency, Office of Water Planning and Standards. EPA- 440/4-7-029a. NTIS No. PB 80-204373.

Cater, B.R., Cook, M.W., Gangolli, S.D., et al. 1977. . Studies on di-n-butyl phthalate-induced testicular atrophy in the rat: Effect on zinc metabolism. Toxicol. Appl. Pharmacol. 41:609-618.

Inman, J.C., Strachan, S.D., Sommers, L.E., et al. 1984. The decomposition of phthalate esters in soil. J. Environ. Sci. Health B19:245-257.

Killinger, J.M., Basaran, A.H., Persing, R.L., et al. 1988. Maximum perinatal dose feed study of di-n-butyl phthalate (CAS No. 84-74-2) in Fischer 344 rats. Report to the National Toxicological Program, Research Triangle Park, NC. by Batelle, Columbus. (as cited in ATSDR 1989).

Murakami, K., Nishiyama, K., Higuti, T. 1986. Mitochondrial effect of orally administered dibutyl phthalate in rats. Nippon Eiseigaku Zashi (Jpn. J. Hyg.) 41:769-774. (as cited in ATSDR 1989).

Sanders, H.O., Mayer, F.L., Jr., Walsh, D.F. 1973. Toxicity, residue dynamics, and reproduction effects of phthalate esters in aquatic invertebrates. Environ. Res. 6:84-90.

Shea, P.J., Weber, J.B., Overcash, M.R. 1982. Uptake and phototoxicity of di-n-butyl phthalate in corn (Zea mays). Bull. Environ. Contam. Toxicol. 29:153-158. 
Shiota, K., Nishimura, H. 1982. Teratogenicity of di (2-ethylhexyl) phthalate (DHEP) and di-n-butyl phthalate (DBP) in mice. Environ. Health Perspect. 45:65-70.

Walker, W.W. 1984. Development of a fate/toxicity screening test. Gulf Breeze, Fl. EPA-600/4-84-074. NTIS No. PB84-246370.

Walseth, F., Nilsen, O.G. 1986. Phthalate esters: Effects of orally administered di-n-butyl phthalate on cytochrome P-450 mediated metabolism in rat liver and lung. Acta Pharmacol Toxicol. 59:263-269.

Wolfe, N.L., Paris, D.F., Steen, W.C., et al. 1980. Correlation of microbial degradation rates with chemical structure. Environ. Sci. Technol. 14:1143-1146. 
ENDOSULFAN

CAS No:

115-29-7

Synonym(s)

6,7,8,9,10,10-hexachloro-1,5,5a,6,9,9a-hexa-

hydro-6,9-methano-2,4,3-benzo-dioxathiepin

Endosulfan, an insecticide, is a mixture of 2 different isomeric forms of the same compound (alpha-endosulfan and beta-endosulfan). Endosulfan is widely utilized throughout the U.S. on a large number of food and nonfood crops. Its popularity is based on its ability to control over 100 different insect pests (ATSDR 1990).

Endosulfan is a tan to brown solid at ambient conditions. This compound has a molecular weight of 406.95 and a melting point of $106^{\circ} \mathrm{C}$. It is soluble in organic solvents such as acetone, chloroform, xylene, and benzene (ATSDR 1990).

\section{FATE AND TRANSPORT}

Endosulfan is released directly to the air during its use as an insecticide on fruit trees and to the soil during its application to ground crops. Surface water contamination with endosulfan can occur through runoff from treated croplands or from manufacturing facility effluent (ATSDR 1990).

Airborne endosulfan may be in the form of aerosols from direct spraying activities or as vapors volatilized from surface waters (NRCC 1975; ATSDR 1990). Endosulfan does not decompose readily in the atmosphere (NRCC 1975), but may be transported over long distances and then be deposited in untreated areas by precipitation or gravitational settling (ATSDR 1990).

Deposition of airborne particles on soil surfaces represents only one of several mechanisms by which soil contamination occurs. The primary routes of soil contamination are crop spraying and intentional dumping of unused product. Once in the soil, endosulfan becomes strongly attached to sediment particles (Bowman et al. 1965) and shows little movement in experimental soil beds (Hodapp and Winterlin 1989; Stewart and Cairns 1974). A wide variety of soil microorganisms, including bacteria and fungi, are able to degrade endosulfan to endosulfan sulfate (Martens 1976; El Beit et al. 1981), a persistent metabolite in soils (Coleman and Dolinger 1982).

When endosulfan reaches surace water through runoff, effluent release, or deposition from air, it may volatilize at a very slow rate (Callahan et al-1989). Water-borne endosulfan attaches to particulate matter, undergoes biodegradation, or is subject to hydrolysis (ATSDR 1990).

\section{HUMAN HEALTH EFFECTS}

Acute oral ingestion of endosulfan can result in digestive (gagging, vomiting, diarrhea), respiratory (hypoventilation, pulmonary edema, and pneumonia), cardiovascular (tachycardia, hypertension, and cardiogenic shock), and neurological (agitàtion, hyperactivity, 
loss of consciousness) dysfunction in humans. Victims who have died following acute oral ingestion of endosulfan presented at autopsy with edema of the brain and lungs, hemorrhage of the renal medulla, and acute emphysema (Terziev et al. 1974). No data on acute exposure through inhalation or dermal absorption were obtained, however the potential for similar anomalies exists.

Chronic ingestion of endosulfan by experimental animals has not produced the striking neurotoxic effects seen with acute poisoning. In rats chronically administered endosulfan by gavage, central stimulation was evident for the first several days, but began to subside by day 5 (Dikshith et al. 1984). In human epidemiological studies, the central nervous system was also the primary target tissue, but was more pronounced and long-lasting than in rats. Neurotoxic effects ranged from EKG (conduction) abnormalities, convulsions, irreversible cognitive and emotional deterioration, memory loss, and impairment of visual motor coordination (Ely et al. 1967; Tiberin et al. 1970).

When gravid female animals ingest endosulfan it produces fetal toxicity at levels which are sub-clinical in the mother (ATSDR 1990). Genotoxic studies following oral administration of endosulfan in rats is questionable, however endosulfan does appear to enhance chromosomal aberrations and gene mutations in mice and Drosophila (Valaquez et al. 1984; Usha Rami and Reddy 1986.

\section{ECOLOGICAL EFFECTS}

Endosulfan does not bioaccumulate in terrestrial or aquatic ecosystems (NRCC 1975). In aquatic systems, endosulfan levels were elevated in fish tissues, however these levels decreased following removal to uncontaminated water or naturally with time. The tissue levels of endosulfan in fish eating birds and crocodiles followed a similar pattern as that seen in fish (HSDB 1989) and did not bioaccumulate. In plants, endosulfan is rapidly converted to endosulfan sulfate, however this metabolite has been shown to be toxic in animal studies (ATSDR 1990).

\section{US ENVIRONMENTAL PROTECTION AGENCY HUMAN HEALTH CRITERIA}

Oral Slope Factor

Inhalation Slope Factor

Chronic Oral RfD

Chronic Inhalation RfD
NA

NA

$6 \mathrm{E}-3 \mathrm{mg} / \mathrm{kg} /$ day

NA

\section{DRINKING WATER CRITERIA AND STANDARDS}

U.S. Environmental Protection Agency

Maximum Concentration Limit Maximum Concentration Limit Goal 


\section{AMBIENT WATER QUALITY CRITERIA}

U.S. Environmental Protection Agency Ambient Water Quality Criteria

Human Health-Water and Fish Ingestion $74 \mu \mathrm{g} / \mathrm{L}$

Human Health-Fish Ingestion Only

Aquatic Organisms-FW Acute

Aquatic Organisms-FW Chronic

Aquatic Organisms-Marine Acute

Aquatic Organisms-Marine Chronic

$159 \mu \mathrm{g} / \mathrm{L}$

$0.22 \mu \mathrm{g} / \mathrm{L}$

$0.056 \mu \mathrm{g} / \mathrm{L}$

$0.034 \mu \mathrm{g} / \mathrm{L}$

$0.0087 \mu \mathrm{g} / \mathrm{L}$

South Carolina Department of Health and Environmental Control Water Quality Criteria

Human Health Criteria

Fish and Aquatic Life-Continuous

Fish and Aquatic Life-Maximum
$1.99 \mu \mathrm{g} / \mathrm{L}$

$0.056 \mu \mathrm{g} / \mathrm{L}$

$0.22 \mu \mathrm{g} / \mathrm{L}$

$\mathrm{NA}=$ Not Available

\section{REFERENCES}

Agency for Toxic Substances and Disease Registry (ATSR). 1990. Draft Toxicological Profile for Endosulfan II. Prepared by Clement International Corp., contract no. 205-8-0608..

Bowman, M.C., Schecter, M.S., Carter, R.L. 1965. Behavior of chlorinated insecticides in a broad spectrum of soil types. J. Agric. Food Chem. 13:360-365.

Callahan, M.A., Slimak, M.W., Gabel, N.W., et al. 1979. Water-related environmental fate of 129 priority pollutants. Vol I. Introduction and technical background, metals and inorganics, pesticides and PCB's. Washington, D.C: U.S. Environmental Protection Agency, Office of Water Planning and Standards. EPA-440/4-79-029a, 27.1-27.16.

Coleman, P.F., Dolinger, P.M. 1982. Endosulfan monograph number 4: Environmental health evaluations of California restricted pesticides. Prepared by Peter M. Dolinger Assoc., Menlo Park, CA.

Diksith, T.S.S., Razaida, R.B., Srivastava, M.K., et al. 1984. Response of rats to repeated oral administration of endosulfan. Ind. Health 22:295-304.

El Beit, I.O.D., Wheelock, J.V., Cotton, D.E. 1981. Factors involved in the dynamics of pesticides in soils: The effect of pesticide concentration on leachability and adsorption. Int. J. Environ. Stud. 16:181-187. 
Ely, T.S., Macfarlane, J.W., Galen, W.P., et al. 1967. Convulsions in thiodan workers. J. Occup. Med. 9:35-37.

Hodapp, P.M., Winterlin, W. 1989. Pesticide degradation in model soil evaporation beds. Bull. Environ. Contam. Toxicol. 43:36-44.

HSDB. 1989. Hazardous Substance Data Bank. National Library of Medicine. Bethesda, MD.

Martens, R. 1976. Degradation of [8,9-carbon-14] endosulfan by soil microorganisms. Appl. Environ. Microbiol. 31:853-858.

NRCC. 1975. Endosulfan: Its effects on environmental quality. Ottawa, Ontario: National Research Council of Canada, Environmental Secretariat, Publication No. NRC 14098.

Stewart, D.K.R., Cairns, K.G. 1974. Endosulfan persistence in soil and uptake by potato tubers. J. Food Chem. 22:984-986.

Terziev, G. Dimitrova, N., Rusev, F. 1974. Forensic medical and forensic chemical study of acute lethal poisons with thiodan. Folia Med. 16:325-329.

Tïberin, P., Kristal, N., Israeli, R. 1970. EEG findings in poisoning by endosulfan (C9H603C165) [Abstract]. Electroencephalogy Clin. Neurophysiol. 28:642

Usha Rani, M.V., Reddy, P.P. 1986. Cytogenetic effects of aldrin and endosulfan in mice. IRCS J. Med. Sci. 14:1125-1126.

Valaquez, A., Creus, A., Xamena, N., et al. 1984. Mutagenicity of the insecticide endosulfan in Drosophila melanogaster. Mutat. Res. 136:115-118. 
ENDRIN

CAS No.: $\quad 72-20-8$

Synonym(s) endo-dimethanonapthalene

Endrin was produced commercially in the US for use as an insecticide, rodenticide and avicide to control cutworms, voles, grasshoppers, and other pests on cotton, sugarcane, tobacco, and grain crops (EPA 1979). The manufacturer voluntarily withdrew its registration with the Office of Pesticide Products in 1986 and all uses were withdrawn (ATSDR 1989).

Endrin, a chlorinated cyclodiene having a molecular weight of 380.90 is a stereoisomer of dieldrin. Endrin is a white crystalline solid at ambient conditions with little or no odor. Its density is 1.7 at $20^{\circ} \mathrm{C}$, its melting point is $235^{\circ} \mathrm{C}$ and boiling point data indicate it decomposes at $245^{\circ} \mathrm{C}$. It is relatively insoluble in water and moderately soluble inorganic solvents (ATSDR 1989).

\section{FATE AND TRANSPORT}

Endrin binds tightly to soil and has little mobility in this medium, therefore endrin has little potential to leach into groundwater (Sharom et al. 1980). Endrin has a low vapor pressure, yet one study demonstrated that $20-30 \%$ volatilization occurs from soil (Nash 1983). Laboratory experiments indicate that a large number of soil microorganisms are able to biodegrade endrin under aerobic conditions (Patil et al. 1970). Endrin was biodegraded most rapidly in organic-rich soils and most slowly in sandy soils (Gowda and Sethunathan 1976).

Endrin is persistent in surface waters (Sharom et al. 1980) but algae and other microorganisms biodegraded endrin in pond water. 12-ketoendrin was the metabolite produced by algal degradation (Patil et al. 1972).

Limited date indicate that some degree of photooxidation of endrin occurs in air (Burton and Pollard 1974).

\section{HUMAN HEALTH EFFECTS}

The most prominent route of exposure to endrin in the past bas been through ingestion of contaminated grain products. This route of exposure poses little risk today because use of this product in the US has been discontinued since 1986.

Acute ingestion of endrin has produced a large number of fatalities, particularly in children. Little information is available regarding deaths following inhalation exposure, however an increased incidence of respiratory disorders has been observed among workers exposed occupationally (Ditraglia et al. 1981). The primary target of endrin, however, is the central nervous system, regardless of the route of exposure. Convulsions, tonic-clonic seizures, sudden collapse, and death can result (Coble et al. 1967). Dermal exposure was the most common exposure route in occupational settings (Wolfe et al. 1963). 


\section{ECOLOGICAL EFFECTS}

Endrin is readily biodegraded by soil microbes in aerobic conditions, but is persistent in surface waters. Fish from around the country had endrin concentrations as high as $0.4 \mathrm{ppm}$ in 1977, but the amount concentrated by fish was declining by 1981 . The extent of biomagnification within the food chain is not known.

\section{US ENVIRONMENTAL PROTECTION AGENCY HUMAN HEALTH CRITERIA}

Oral Slope Factor

Inhalation Slope Factor

Chronic Oral RfD

Chronic Inhalation RfD
NA

NA

$6 \mathrm{E}-3 \mathrm{mg} / \mathrm{kg} /$ day

NA

\section{DRINKING WATER CRITERIA AND STANDARDS}

U.S. Environmental Protection Agency

Maximum Concentration Limit

Maximum Concentration Limit Goal

$0.002 \mathrm{ug} / \mathrm{L}$

$0.002 \mathrm{ug} / \mathrm{L}$

\section{AMBIENT WATER QUALITY CRITERIA}

U.S. Environmental Protection Agency Ambient Water Quality Criteria

Human Health-Water and Fish Ingestion

Human Health-Fish Ingestion Only

Aquatic Organisms-FW Acute

Aquatic Organisms-FW Chronic

Aquatic Organisms-Marine Acute

Aquatic Organisms-Marine Chronic $\operatorname{lug} / \mathrm{L}$

NA

$0.18 \mathrm{ug} / \mathrm{L}$

$0.0023 u g / L$

$0.037 \mathrm{ug} / \mathrm{L}$

$0.0023 \mathrm{ug} / \mathrm{L}$

South Carolina Department of Health and Environmental Control

Human Health Criteria

Fish and Aquatic Life-Continuous

Fish and Aquatic Life-Maximum
$0.2 \mathrm{ug} / \mathrm{L}$

$0.18 \mathrm{ug} / \mathrm{L}$

$0.0023 \mathrm{ug} / \mathrm{L}$

$\mathrm{NA}=$ Not Available

\section{REFERENCES}

ATSDR.1989. Agency for Toxic Substances and Disease Registry. Draft toxicologic profile for Endrin. Prepared by Life Systems and Clement Associates, Inc. US Department of Health and Human Services. 
Burton, W.B., Pollard, G.E. 1974. Rate of photochemical isomerization of endrin in sunlight. Bull. Environ. Contam. Toxicol. 12:113-116.

Coble, Y., Hildebrandt ,P., Davis, J. et al. 1967. Acute endrin poisoning. JAMA 202:489493.

Ditraglia, D., Brown, D.P., Namekata, T. et al. 1981. Mortality study of workers employed at organochlorine pesticide manufacturing plants. Scand. J.Work Environ. Health 7:140-146.

EPA. 1979. US Environmental Protection Agency: Part II. Federal Register 44:43632-43657.

Gowda, T.K., Sethunathan, N. 1976. Persistence of endrin in Indian rice soils under flooded conditions. J. Agric. Food Chem. 24:750-753.

Nash, R.G. 1983. Comparative volatilization and dissipation rates of several pesticides from soil. J. Agric. Food Chem. 31:210-217.

Patil , L.C., Matsumura, F., Boush ,G.M. 1970. Degradation of endrin, aldrin, and DDT by soil microorganisms. Appl. Microbiol. 19:879-881.

Patil, K.C., Matsumura, F., Boush, G.M. 1972. Metabolic transformation of DDT, dieldrin, aldrin, and endrin by marine microorganisms. Environ. Sci. Technol. 6:629-632.

Schmitt, C.J., Zajicek, J.L., Ribick, M.A. 1985. National pesticide monitoring program: Residues of organochlorine chemicals in freshwater fish. 1980-1981. Arch Environ. Contam. Toxicol. 14:225-260.

Sharom ,M.S., Miles, J.R., Harris, C.R., et al. 1980. Behavior of 12 insecticides in soil and aqueous suspensions of soil and sediment. Water Res. 14:1095-1100.

Wolfe, H.R., Durham, W.F., Armstrong ,J.F. 1963. Health hazards of the pesticides endrin and dieldrin:Hazards in some agricultural uses in the Pacific Northwest. Arch. Environ. Health 6:458-464. 
THIS PAGE LEFT INTENTIONALLY BLANK 


\section{ETHYLBENZENE}

CAS No.: $\quad 100-41-4$.

Synonym(s) Ethylbenzol, Phenlyethane

Ethylbenzene is an aromatic hydrocarbon produced anthropogenically for use in styrene production, as a solvent, as a component of asphalt and naptha, and in the manufacture of other compounds such as cellulose acetate, propylene oxide, and alpha-methyl benzyl alcohol. Ethylbenzene is a natural component of fossil fuels (ATSDR 1989).

Ethylbenzene is a colorless liquid with an odor similar to gasoline. It has a molecular weight of 106.6 , a melting point of $-95^{\circ} \mathrm{C}$, and a boiling point of $136.25^{\circ} \mathrm{C}$. Ethylbenzene is less dense than water, with a density of .866 . It is slightly soluble in water, miscible with many organic solvents, and soluble in alcohol and ether (Windholz 1983; Weast 1988).

\section{FATE AND TRANSPORT}

Ethylbenzene has been detected in all environmental media, however it is most prevalent in the atmoshpere. Releases into the air occur during manufacturing, fuel combustion, solvent use, as well as pesticide and other consumer product use (Fishbein 1985; NAS 1980). Reactions with atmospheric hydroxyl radicals appears to be an important pathway of ethylbenzene degradation (Atkinson et al. 1987). Reactions of gaseous ethylbenzene with ozone and toluene-like compounds has been observed (Atkinson and Carter 1984), and at least one product of these reactions, peroxyacetyl nitrate (PAN) is a toxic component of smog (Yanagihara et al. 1977).

Surface water contamination with ethylbenzene can occur through industrial discharges and gasoline spills, whereas groundwater discharges arise from leaking underground storage tanks, landfill leachate, and improper waste disposal (Snider and Manning 1982; Barker 1987; Eiceman et al. 1986). Biodegradation, photooxidation, and volatilization are the processes responbsible for removal of ethylbenzene from surface waters (ATSDR 1989).

Ethylbenzene releases to soil may result from fuel spills, disposal of solvents and certain household products such as paint, cleaners and degreasers, varnishes, pesticides, and leaking underground storage tanks (ATSDR 1989). Ethylbenzene does not readily adhere to soil, and therefore will either volatilize or migrate through soil until it reaches the groundwater. Rao et al (1985) reported that the solvent properties of ethylbenzene may release other organic compounds which would normally be tightly adsorbed to soil and thus increase their mobility. Biodegradation of ethylbenzene is significant in aerobic sediments, however migration of ethylbenzene to deeper, anaerobic soils is so rapid that the amount of aerobic transformation is limited (ATSDR 1989).

\section{HUMAN HEALTH EFFECTS}

Inhalation is the most likely route of exposure to ethylbenzene. In humans, acute exposure to airborne ethylbenzene causes irritation of the eyes and throat. Profuse lacrimation, dizziness, vertigo, and chest constriction also have been reported by exposed individuals 
Rao,, P.S.C., Hornsley, A.G., Kilcreae, D.F., et al. 1985. Sorption and transport of hydrophobic organic chemicals in aqueous and mixed solvent systems:Model development and preliminary evaluation. J. Environ. Qual. 14:376-382.

Snider, E.H., Manning, F.S., 1982. A survey of pollutant emission levels in wastewaters and residuals from the petroleum refining industry. Environ Int. 7:237-258.

Staples, C.A., Werner, A.F., Hoogheem, T.J. 1985. Assessment of priority pollutant concentrations in the United States using STORET database. Environ. Toxicol. Chem. 4:131-142.

Thienes, C., Haley, T.J., 1972. Clinical toxicology. 5th ed. Philadelphia,PA: Lea and Febiger, 126.

Weast ,R.C., ed. 1988. CRC Handbook of Chemistry and Physics. 69th ed. Boca Raton, FL: CRC Press, Inc., C-269.

Windholz, M. ed. 1983. The Merck Index. 10th ed. Rahway, NJ: Merck and Co., Inc. 546-: 547.

Yanagihara, S., Shimada ,I., Shinoyama, E.,et al. 1977. Photochemical reactivities with hydrocarbons. Proc,. Int. Clean Air Congr. 4th:427-477.

Yant, W.P. , Shrenk, H.H., Waite, C.P., et al. 1930. Acute response of guinea pigs to vapors of some new commercial organic coumpounds. II. Ethylbenzene. Public Health Report. 45:1241-1250. 
HEPTACHLOR/HEPTACHLOR EPOXIDE

CAS No.: $\quad 76-44-8,1024-57-3$

Major Synonym(s), none

Heptachlor is a cyclodiene type chlorinated hydrocarbon pesticide. It was isolated as an active constituent of chlordane in 1946. Commercial production began in 1953 by Velsicol Chemical Corporation: Its peak use occurred in the mid-1970s. Heptachlor is now restricted to one use, underground termite control. Heptachlor epoxide is the major biological metabolite of heptachlor. The chemical name for heptachlor is $1,4,5,6,7,8,8$,heptachloro-3a,4,7,7a-tetrahydro-4,7-methano-1H-indene.

Heptachlor is only slightly soluble in water, $0.056 \mathrm{ppm}$ at $25^{\circ} \mathrm{C}$. Its vapor pressure is $3 \times 10-4$ $\mathrm{mm} \mathrm{Hg}$ at $25^{\circ} \mathrm{C}$ making it moderately volatile (Khan 1980).

\section{FATE AND TRANSPORT}

Heptachlor is degraded, biologically, to heptachlor epoxide, which is resistant to further degradation and hence, more environmentally persistent than heptachlor. Past usage as an insecticide account for the presence of heptachlor and heptachlor epoxide in soil many years later, highlighting the persistence of these compounds. Heptachlor and heptachlor epoxide adsorb strongly to soil and while leaching is unlikely, some evaporation of heptachlor may occur (McLean et al. 1988, Chapman 1989). Laboratory tests indicate that certain microorganisms can slowly biodegrade heptachlor to heptachlor epoxide and other toxic and non-toxic metabolites (Miles et al. 1971).

Heptachlor and heptachlor epoxide adsorb strongly to sediment and particulate organic matter in water. Heptachlor may also evaporate slowly from water or be degraded by hydrolysis to heptachlor epoxide and 1-hydroxychlordane, in a ration of 2:3. A half-life of heptachlor epoxide in water was calculated to be four years (Eichelberger and Lichtenberg 1971). 1-hydroxy chlordane is further metabolized in aquatic systems (Ly et al. 1975).

Heptachlor and heptachlor epoxide can be released from soil to air in homes treated for termites and from soil and water contaminated by industrial discharges or past usage. Airborne heptachlor/heptachlor epoxide is subject to degradation by photolysis (Graham et al. 1973).

\section{HUMAN HEALTH EFFECTS}

No studies were found that specifically describe acute poisoning of heptachlor. It would be expected, however, based on the similarity of chemical structure between this compound and chlordane and the fact that their subchronic toxicities are identical, that the acute toxicities of these two compounds would be similar.

There have been several case reports of acute chlordane poisoning. Lehman (1949a,b) described a case of acute fatal poisoning. The onset of symptoms may occur within an hour of injection; death within four days. Irritability is the earliest symptom. This irritability 
progresses into convulsions alternating with periods of depression. Death occurs following deep central nervous system depression usually with terminal seizure.

Derbes et al. (1955) described two human fatalities from chlordane poisoning. Both cases exhibited marked CNS effects, confusion, agitation and convulsions within minutes. The first individually was exposed dermally (from a spill of a concentrated solution of chlordane on her dress); the second from ingestion.

Only one case study was found in which human exposure to heptachlor occurred over a relatively short period of time (Garrettson et-al. 1984-5). In this case, a 30-year-old woman was exposed to chlordane in her home for a four week period of time. The early symptoms of poisoning included circumoral numbness, anorexia, nausea and fatigue. After a one month delay, myoclonic jerks occurred. After six months, malaise and anorexia became the dominant symptoms.

Infante et al. (1978) reported six cases of blood dyscrasias and childhood tumors in association with long-term chlordane and heptachlor exposure. This article reviewed 31 other cases of chronic exposure and reported aplastic anemia, leukopenia and various anemias as consistent occurrences.

\section{ECOLOGICAL EFFECTS}

Heptachlor and heptachlor epoxide are strongly lipophilic and persistent in the environment and heptachlor epoxide, in particular, is bioconcentrated in the food chain. Crops grown in heptachlor contaminated soils absorbed the insecticide in amounts proportional to the soil concentration, up to a point where crop concentrations reached a plateau. As insecticide concentration in the soil declined, so too did the concentration accumulated by the crops, demonstrating a dose/response relationship (Lichtenstein et al. 1970). Beef cattle, as well as dairy cattle, which grazed on contaminated pastures concentrated heptachlor epoxide in muscle tissue and milk, respectively (Corrigan and Seneviratna 1989; Gannon and Decker 1960).

Aquatic organisms, including fish, clams, and shrimp, significantly bioaccumulated heptachlor and heptachlor epoxide in tissues sampled (Chan and Perkins 1989; Elder and Mattraw 1984; DeVault 1985). Estimated bioconcentration factors for heptachlor and heptachlor epoxide in mussels, oysters, and clam fat suggest that heptachlor bioconcentrates more readily than heptachlor epoxide in these species (Hartley and Johnston 1983). The.opposite may be true for terrestrial organisms that rapidly metabolize heptachlor to heptachlor epoxide (ATSDR.1991).

\section{U.S. ENVIRONMENTAL PROTECTION AGENCY HUMAN HEALTH CRITERIA}

Oral Slope Factor

Inhalation Slope Factor
$4.5 \mathrm{~kg} / \mathrm{mg} /$ day $^{-1}$ (heptachlor)

$9.1 \mathrm{~kg} / \mathrm{mg} /$ day $^{-1}$ (heptachlor epoxide)

$4.5 \mathrm{~kg} / \mathrm{mg} /$ day $^{-1}$ (heptachlor)

$9.1 \mathrm{~kg} / \mathrm{mg} /$ day $^{-1}$ (heptachlor epoxide) 
Chronic Oral RfD

Chronic Inhalation RfD
$5 \mathrm{E}-04 \mathrm{mg} / \mathrm{kg} /$ day (heptachlor)

$1.3 \mathrm{E}-05 \mathrm{mg} / \mathrm{kg} /$ day (heptachlor epoxide)

ND (heptachlor)

ND (heptachlor epoxide)

\section{DRINKING WATER CRITERIA AND STANDARDS}

U.S. Environmental Protection Agency
Maximum Concentration Limit
$0.0004 \mathrm{mg} / \mathrm{L}$ (heptachlor)
Maximum Concentration Limit Goal
$0.0002 \mathrm{mg} / \mathrm{L}$ (heptachlor epoxide)
$0.0 \mathrm{mg} / \mathrm{L}$ (heptachlor)
$0.0 \mathrm{mg} / \mathrm{L}$ (heptachlor epoxide)

\section{AMBIENT WATER QUALITY CRITERIA}

U.S. Environmental Protection Agency Ambient Water Quality Criteria (as measured for heptachlor)
Human Health - Water and Fish Ingestion
$0.28 \mathrm{ng} / \mathrm{L}$
Human Health - Fish Ingestion Only
$0.29 \mathrm{ng} / \mathrm{L}$
Aquatic Organisms - FW Acute
$0.2 \mu \mathrm{g} / \mathrm{L}$
Aquatic Organisms - FW Chronic
$0.0038 \mu \mathrm{g} / \mathrm{L}$
Aquatic Organisms - Marine Acute
$0.053 \mu \mathrm{g} / \mathrm{L}$
Aquatic Organisms - Marine Chronic
$0.0036 \mu \mathrm{g} / \mathrm{L}$

South Carolina Department of Health and Environmental Control Water Quality Criteria

Human Health Criteria

Fish and Aquatic Life - Continuous

Fish and Aquatic Life - Maximum
$0.00214 \mu \mathrm{g} / \mathrm{L}$

$0.0038 \mu \mathrm{g} / \mathrm{L}$

$0.52 \mu \mathrm{g} / \mathrm{L}$

NA $=$ Not Available

\section{REFERENCES}

Agency for Toxic Substances and Disease Registry. 1991. Toxicological Profile for Heptachlor/Heptachlor Epoxide. Prepared by Clement Internatnional Corp., contract no. 205-88-0608.

Chan, CH, Perkins, LH. 1989. Monitoring of trace organic contaminants in atmospheric precipitation. J Great Lakesk Res 15(3):465-475.

Chapman, PM. 1989. Current approaches to developing sediment quality criteria. Environ Toxicol Chem 8:589-600. 
Corrigan, PJ, Seneviratna, P. 1989. Pesticide residues in Australian meat. Vet Rec 125(8):180-181.

Derbes, V.J., Dent, J.H., Forrest, W.W. and Johnson, M.F. (1955). Fatal chlordane poisoning. J. Am. Med. Assoc. 158:1367-1369.

DeVault, DS. 1985. Contaminants in fish from Great Lakes harbors and tributary mouths. Arach Environ Contam Toxicol 14:587-594.

Eichelberger, JW, Lichtenberg, JJ. 1971. Persistence of pesticides in river water. Environ Sci Technol 5:541-544.

Elder, JF, Mattraw, HC, Jr. 1984. Accumulation of trace elements, pesticides, and polychlorinated biphenyls in sediments and the clam corbicula manilensis of the Apalachicola River, Florida. Arch Environ Contam Toxicol 13:453-469.

Gannon, N, and Decker, GC. 1960. The excretion of dieldrin, DDT, and heptachlor epoxide in milk of dairy cows fed on pastures treated with dieldrin, DDT, and heptachlor. J Econ Entomol 53(3):411-415.

Garrettson, L.K, Guzelian, P.S. and Blanke, R.V. (1984-5). Subacute chlordane poisoning. Clin. Toxicol. 22:565-571.

Graham, RE, Burson, KR, Hammer, CF, et al. 1973. Photochemical decomposition of heptachlor epoxide. J Agric Food Chem 21:824-834.

Hartley, DM, Johnston, JB. 1983. Use of the fresh water clam Corbicula manilensis as a monitor for organochlorine pesticides. Bull Environ Contam Toxicol 31:33-40.

Infante, P.F., Epstein, S.S., and Newton, W.A., Jr. (1978). Blood dyscrasias and childhood tumors and exposure to chlordane and heptachlor. Scane. J. Work Env. Hith. 4;137150.

Lehman, AJ. 1951. Chemicals in foods: A report to the Association of Food and Drug Officials on current developments. Part II. Pesticides. US Q Bull 15:122-133.

Lehman, A.J. (1965). Summaries of pesticide activity. p. 24, Assoc. of Food and Drug Officials of the U.S.

Lichtenstein, EP, Schultz, KR, Fuhremann, TW, et al. 1970. Degradation of aldrin and heptachlor in field soils during a ten-year period translocation into crops. J Agric Food Chem 18:100-106.

Lu, PY, Metcalf, RL, Hirwe, AS, et al. 1975. Evaluation of environmental distribution and fate of hexachlorocyclopentadiene, chlordene, heptachlor, and heptachlor epoxide in a laboratory model ecosystem. J Agric Food Chem 23(5):967-973. 
McLean, JE, Sims, RC, Doucette, WJ, et al. 1988. Evaluation of mobility of pesticides in soil using U.S. EPA methodology. J Environ Eng 114(3):689-703.

Miles, JRW, Tu, CM, Harris, CR. 1971. Degradation of heptachlor epoxide and heptachlor by a mixed culture of soil microorganisms. J Econ Entomol 64:839-841. 
THIS PAGE LEFT INTENTIONALLY BLANK 
HEPTACHLORDIBENZO-P-DIOXIN ISOMERS, OCTACHLORO-DIBENZO-P-DIOXIN ISOMERS, OCTACHLORODIBENZO-P-FURAN ISOMERS, DIBENZOFURAN

CAS No.: $\quad$ 1746-01-6 (TCDD Surrogate)

Major Synonym(s) Dioxin

For the purpose of this report, TCDD will be used as a surrogate compound for those chemicals listed above which exhibit dioxin (TCDD)-like effects both biologically and toxicologically. Collectively, these agents are known as dioxin.

Dioxin is produced as a by-product (and contaminant) of hexachlorophene, a germicide previously used to bathe babies, Agent Orange, an herbicide used extensively in the Vietnam War, and pentachlorophenol, a treatment for wood.

Chemically, dioxins are chlorinated phenolic compounds which are water insoluble. They are very persistent in the environment, as they are not readily biodegraded by most organisms.

\section{FATE AND TRANSPORT}

Although dioxin has an extremely low vapor pressure, it has been shown to volatilize and to occur in the atmosphere in both the gaseous and aerosol states (Podoll et al. 1986). Airborne dioxin has been shown to be subject to long-distance transportation prior to its removal from the atmosphere (ATSDR 1987). Dioxins react with hydroxyl radicals in the atmosphere with an estimated half-life of 8.3 days (Podoll et al. 1986). Although insoluble in water, dioxins may be removed from air through wet as well as dry deposition (NLM 1994).

Dioxin contaminants in water adsorb to sediments or suspended particulate matter which may slow down the rates of photolysis and evaporation, which occur at the water's surface. A half-life of 21 hours (in summer) to 118 hours (in winter) has been estimated for photolysis of dioxin at the water surface (ATSDR 1987). A volatilization half-life of 46 days has been estimated for dioxin, however, when adsorbance and sedimentation are accounted for, the total half-life is increased to 50 years, indicating that aquatic sediments represent a significant sink for dioxins (ATSDR 1987).

Soil-bound dioxin shows little vertical mobility and most is contained in the upper $8 \mathrm{~cm}$ (Di Domenico et al. 1980). Dioxin is very stable to metabolism and biodegradation is considered rare (IARC 1977; Isensee and Jones 1975)- Photolysis, however, may be significant, but confined to the upper $1 \mathrm{~cm}$.

\section{HUMAN HEALTH EFFECTS}

Human exposure to 2,3,7,8-TCDD, whether acute or chronic, results in chloracne, altered liver function, hematological problems and altered blood chemistry, porphyrin cutanea tarda, hyperpigmentation and hirsutism from all routes of exposure (Taylor 1979; Walker and Martin 1979; Crow 1981). Acute exposure can also cause nausea and vomiting, headache 
and eye irritation, and skin and respiratory tract irritation. Death usually occurs due to congestive heart failure (Reggiani 1982).

Occupational exposure to dioxin vapor has produced neurological problems of various kinds, including peripheral polyneuropathy, sensory impairments, weakness and impotence (Holmstedt 1980; Reggiani 1982; Kimbrough et al. 1984).

Several authors have reported cases of soft-tissue sarcoma and non-Hodgkins lymphoma in workers exposed to herbicides contaminated with 2,3,7,8-TCDD (Hardell 1977, 1981; Zack and Suskind 1980; Cook 1981; Moses and Selikoff 1981; Sarma and Jacobs 1981). In an extensive analysis of these and other studies, the U.S. EPA (1985) concluded that the studies suggested phenoxyacetic acid herbicides, chlorophenols, or their impurities are carcinogenic in humans.

\section{ECOLOGICAL EFFECTS}

Dioxin bioconcentrates in aquatic organisms (ATSDR 1987). Its persistence in the environment, as well as its resistance to metabolism indicate that dioxin is likely to biomagnify within the food chain.

\section{U.S. ENVIRONMENTAL PROTECTION AGENCY HUMAN HEALTH CRITERIA}

Oral Slope Factor

Inhalation Slope Factor

Chronic Oral RfD

Chronic Inhalation RfD
$1.5 \mathrm{E}+5 \mathrm{~kg} / \mathrm{mg} /$ day $^{-1}$

$1.5 \mathrm{E}+5 \mathrm{~kg} / \mathrm{mg} /$ day $^{-1}$

$1 \mathrm{E}-9 \mathrm{mg} / \mathrm{kg} /$ day (from heptachlorodibenzodioxin isomers) NA

\section{DRINKING WATER CRITERIA AND STANDARDS}

U.S. Environmental Protection Agency

Maximum Concentration Limit

Maximum Concentration Limit Goal
$3 \mathrm{E}-08 \mathrm{mg} / \mathrm{L}$

$0.0 \mathrm{mg} / \mathrm{L}$

\section{AMBIENT WATER QUALITY CRITERIA}

U.S. Environmental Protection Agency Ambient Water Quality Criteria

Human Health - Water and Fish Ingestion NA

Human Health - Fish Ingestion Only

Aquatic Organisms - FW Acute

Aquatic Organisms - FW Chronic

Aquatic Organisms - Marine Acute

Aquatic Organisms - Marine Chronic
NA

$0.01 \mu \mathrm{g} / \mathrm{L}$

$0.0056 \mu \mathrm{g} / \mathrm{L}$

NA

$\mathrm{NA}$ 
South Carolina Department of Health and Environmental Control Water Quality Criteria Human Health Criteria Fish and Aquatic Life - Continuous $1.2 \mathrm{ppq}$

Fish and Aquatic Life - Maximum

NA

NA

$\mathrm{NA}=$ Not Available

\section{REFERENCES}

Agency for Toxic Substances and Disease Registry. 1987. Toxicology Profile for 2,3,7,8TCDD. Prepared by Clement International Corp., contract no. 205-88-0608.

Cook, R.R. 1981. Dioxin, chloracne and soft-tissue sarcoma. Lancet 1:618-619.

Crow, K.D. 1981. Chloracne and its potential clinical implications. Clin. Exp. Dermatol. 6:243-257.

Di Domenico, A. et al. 1980. Ecotoxicol. Envir. Safety 4(3): 327-338. As cited in NLM 1994.

Hardell, L. 1977. Malignant mesenchymal tumors and exposure to phenoxyacids: A clinical observation. La Kartianingen 74:2753-2754.

Hardell, L. 1981. Relation of soft-tissue sarcoma, malignant lymphoma and colon cancer to phenoxyacids, chlorophenols and other agents. Scand. J. Work Environ. Health 7:119-130.

Holmstedt, B. 1980. Prolegomena to Seveso Ecclesiastes. I. Arch. Toxicol. 4:211-230.

IARC monographs. 1972-Present. v15:60, as cited in NLM 1994.

Isensee, A.R., Jones, G.E. 1975. Environ: Sci. Technol. 9:668-72, as cited in NLM 1994.

Kimbrough, R.D., H. Falk, P. Stehr and G. Fries. 1984. Health implications of 2, 3, 7, 8 tetrachlorodibenzo-p-dioxin (TCDD) contamination of residential soil. J. Tox. Env. Hlth. 14:47-93.

Moses, M. and I.J. Selikoff. 1981. Soft-tissue sarcomas, phenoxy herbicides and chlorinated phenols. Lancet 19:1370.

NLM. 1994. HDSB.

Podoll, R.T. et al. 1986. Environ. Sci. Technol. 20:490-492, as cited in NLM 1994. 
Reggiani, G. 1982. Toxicology of TCDD and related compounds: observation in man. In: Chlorinated Dioxins and Related Compounds. Impact on the environment. (Hutzinger, Frei, Merian and Pocchiari, Eds.) Pergamon Press, NY, pp. 463-493.

Sarma, P.R. and J. Jacobs. 1981. Thoracic soft tissue sarcoma in Vietnam veterans exposed to agent orange. Lancet 306: 1109.

Taylor, J.S. (1979) Environmental chloracne. Update and overview. Ann. NY Acad. Sci. 320: $295-307$.

U.S. EPA (1985) Develonment of statistical distributions on ranges of standard factors used in exposure assessments. Office of Health and Environmental Assessment, Washington DC. OHEA-E-161.

Walker, A.E. and J.V. Martin (1979) Lipid profiles in dioxin-exposed workers. Lancet 1:446-447.

Zack, J.A. and R.R. Suskind (1980) The mortality experience of workers exposed to tetrachlorodibenzo dioxin in a trichlorophenol process accident. J. Occup. Med. 22:11-14. 


\section{IRON}

CAS No.: 7439-89-6

Synonym(s) none

Iron is the 4th most abundant element in the earth's crust. Chemically unstable metallic iron is converted to the ferrous or ferric state. The ferric form of iron is insoluble in water and is found in rock and soils. Iron is used in the production of steel; oxides and carbonates are the primary ores from which the metal is extracted (Parmeggiani 1983).

Iron has a molecular weight of 55.85 , a melting point of $1,535^{\circ} \mathrm{C}$, and a boiling point of $2,750^{\circ} \mathrm{C}$. It is insoluble in water but will dissolve in acids. It is a tough, malleable, silver magnetic metal.

\section{FATE AND TRANSPORT}

Iron is not subject to significant environmental release by natural weathering processes. However, air pollution may occur as releases of aerosols during the processing of iron. Smaller particles suspended in the air will be washed out by rain, whereas larger particles fall to the earth by gravity (Bowen 1979).

Contamination of surface waters may result from run off due to the industrial practice of using water to quench coke and slag and from scrubbing of particulates. Iron tends to sink in water and aquatic sediments act as an effective sink in both fresh and estuarine systems (Moore and Moore 1976; Bowen 1979).

\section{HUMAN HEALTH EFFECTS}

Inhalation of silica or iron oxide dusts during mining and preparing iron ores may result in siderosis and pneumoconiosis (Parmeggiani 1983). Siderosis results when the bodie's iron storage capacities have been exceeded and excess iron is deposited in vital organs. The liver and pancreas are primary target organs and damage to these systems produces cirrhosis of the liver and diabetes, respectively. A small amount of iron may be deposited in myocardium, producing abnormal rhythms of the heart. Pneumoconiosis is a lung disease, most commonly observed among coal miners. This disease progresses from diffuse fibroids in lung tissue to progressive massive fibrosis, affecting the air exchange tissues of the lungs. This disease is reversible prior to entering the progressive stage. Iron workers also have an increased incidence of lung cancer and hepatic cell carcinoma (Jorgensen 1973; Morgan 1978).

Ingested iron is toxic but rarely fatal in adults. Young children are most susceptible to iron poisoning. Acute symptoms include abdominal pain, diarrhea, and vomiting. Life threatening effects are manifested as cyanosis, lassitude, drowsiness, acidosis and dehydration and may culminate in cardiovascular collapse and shock (Finch 1980). 


\section{ECOLOGICAL EFFECTS}

The availability of iron in nature is limited. It can be solubilized by acids or converted to organic compounds by microorganisms. Plants take up iron from soils and store it within their structures. Bacteria are also able to take up iron from soil and have adaptive mechanisms which make them efficient at this process. Iron is passed up the foodchain to higher vertebrates in the form of plant and animal food stuffs.

\section{US ENVIRONMENTAL PROTECTION AGENCY HUMAN HEALTH CRITERIA}

Oral Slope Factor

Inhalation Slope Factor

Chronic Oral RfD

Chronic Inhalation RfD
NA

NA

$5 E-1 \mathrm{mg} / \mathrm{kg} /$ day

NA

\section{DRINKING WATER CRITERIA AND STANDARDS}

U.S. Environmental Protection Agency

Maximum Concentration Limit

Maximum Concentration Limit Goal

NA

NA

\section{AMBIENT WATER QUALITY CRITERIA}

U.S. Environmental Protection Agency Ambient Water Quality Criteria

Human Health-Water and Fish Ingestion

Human Health-Fish Ingestion Only

Aquatic Organisms-FW Acute

Aquatic Organisms-FW Chronic

Aquatic Organisms-Marine Acute

Aquatic Organisms-Marine Chronic
$0.3 \mathrm{mg} / \mathrm{L}$

NA

$1,000 \mu \mathrm{g} / \mathrm{L}$

NA

NA

NA

South Carolina Department of Health and Environmental Control

Human Health Criteria

Fish and Aquatic Life-Continuous

Fish and Aquatic Life-Maximum
NA

NA

NA

NA $=$ Not Available 


\section{REFERENCES}

Bowen. 1979. Environmental chemistry of the elements. New York. Academic Press.

Finch, C.A. 1980. Drugs effective in iron deficiency and other hypochromic anemias in: Goodman and Gilman's The pharmacological basis of therapeutics. Gilman, A.G.,Goodman L.S., Gilman, A. (eds). Sixth edition. New York, MacMillan Publishing Co.

Jorgensen ,H.S. 1973. A study of mortality from lung cancer among miners in Kinuna 19501970. In : Work-Environment-Health. Helsinki. CIS 74-818.

Morgan, W.K.C. 1978. Magnetite pneumoconiosis. J.Occup. Med. 20(11):762-763.

Moore , J.W., Moore, E.H. 1976. Environmental Chemistry. New York, NY. Academic Press.

Parmeggiani, L. (ed). 1983. Encyclopedia of occupational Health and Safety. Vol 2. Geneva. International Labor Office. 
THIS PAGE LEFT INTENTIONALLY BLANK 
LEAD

CAS No.: 7439-92-1

Synonym(s) None

Lead is an important commercial silvery gray metal that is lustrous when freshly cut and is very soft and malleable, thus it is easily melted, cast, rolled, and extruded. It is insoluble in water (Weast 1987-1988) but is soluble in nitric acid and in hot concentrated sulfuric acid (Merck Index 1983). Lead has a molecular weight of $207.20 \mathrm{~g} / \mathrm{mole}$, melts at $327^{\circ} \mathrm{C}$ (at one atmosphere), boils at $1,740^{\circ} \mathrm{C}$ (at one atmosphere), and has a vapor pressure of $1.77 \mathrm{~mm} \mathrm{Hg}$ at $1,000^{\circ} \mathrm{C}$ and a density of 11.34 at $20^{\circ} \mathrm{C}$.

\section{FATE AND TRANSPORT}

In water, lead is effectively removed from the water column to the sediment by adsorption to organic matter and clay minerals, precipitation as an insoluble salt, and reaction with hydrous iron and manganese oxide. The amount of lead that can remain in solution in water is a function of the $\mathrm{pH}$ and the dissolved salt content. Much of the lead carried by river water is in the form of suspended solids. Atmospheric lead is generally be in the form of dust or adsorbed to particulate matter and subject to gravitational settling. If released or deposited on soil, lead will be retained in the upper 2 to $5 \mathrm{~cm}$, especially soils with 75 percent organic matter and a $\mathrm{pH}$ of greater than five. While corrosion may be expected in soil, an inert coating of insoluble salt usually forms and limits further corrosion. Lead will convert to more insoluble forms such as $\mathrm{PbSO}_{4}, \mathrm{~Pb}_{3}\left(\mathrm{PO}_{4}\right)_{2}, \mathrm{PbS}$, and $\mathrm{PbO}$. It also forms complexes with organic matter and clay minerals that limit its mobility. In lake sediments, microorganisms can directly methylate certain inorganic lead compounds. Biomethylation of lead by benthic microorganisms can lead to its remobilization into the aqueous environmental compartment (Frances 1985; and Stephenson and Lester 1987). Evidence suggests that lead uptake in fish is localized in the mucous on the epidermis, dermis, and scales, so that it poses no human health danger due to its lack of availability in edible portions of fish tissue (Schulz-Baldes et al. 1983; Biddinger and Glass 1984). Lead does not appear to bioconcentrate significantly in fish, but does in some shellfish, such as mussels. Log bioconcentration factors of 1.38 to 1.65 have been reported for freshwater fish; 2.70 to 3.23 for freshwater invertebrates; and 1.24 to 3.40 for various saltwater bivalves, mollusks, diatoms, and phytoplankton (Schulz-Baldes et al. 1983; and Biddinger and Glass 1984).

\section{HUMAN HEALTH EFFECTS}

The toxic effects of lead on the central nervous system are-eonsidered to be the most serious systemic effect in terms of human health. In children, cognitive or motor neurologic effects are of main concern, while peripheral neuropathy and nephropathy (effects on the kidney) are of concern in adults. Blood levels typically range from 30 to $50 \mu \mathrm{g}$ lead per deciliter (Goyer 1986). Blood levels of 60 to $70 \mu \mathrm{g}$ lead per deciliter, and greater than $80 \mu \mathrm{g}$ per deciliter, are associated with encephalopathy in children and adults, respectively. In addition to central nervous system dysfunction, high blood levels of lead are associated with hematologic and renal effects and shortened life-span of erythrocytes and impaired heme synthesis. Renal effects include reversible renal tubular dysfunction in children following 
acute exposure to lead, and incurable interstitial nephropathy. Studies on the carcinogenicity of lead indicate that lead can induce cancer in kidneys of rodents fed high doses, but there is little evidence to indicate that lead is a human carcinogen. Nevertheless, the U.S. Environmental Protection Agency has classed lead as B2, a probable human carcinogen.

\section{ECOLOGICAL EFFECTS}

High lead levels ( 0.5 to $16 \mathrm{mg} / \mathrm{kg}$ body weight) in animal diets cause abortions and poisoning (International Association Research on Cancer 1980). Symptoms of poisoning in waterfowl that ingest lead pellets include anorexia, weight loss, weakness, lethargy, diarrhea, coma, and death (Booth and McDonald 1982). The acute oral lethal dose of lead in various species is: calves, 50 to $600 \mathrm{mg} / \mathrm{kg}$ as lead or lead salts; cattle, 600 to $800 \mathrm{mg} / \mathrm{kg}$ from lead salts; sheep and goats, 600 to $800 \mathrm{mg} / \mathrm{kg}$ from lead salts, and fowl, 160 to $600 \mathrm{mg} / \mathrm{kg}$ from lead salts. The NOEL for sheep is $0.1 \mathrm{mg} / \mathrm{kg} /$ day for 60 weeks, biochemical aberrations occur when intake is 0.3 to $3 \mathrm{mg} / \mathrm{kg} /$ day. $\mathrm{An} \mathrm{LC}_{50}$ was established for Japanese quail at greater than $5,000 \mathrm{ppm}$.

\section{U.S. ENVIRONMENTAL PROTECTION AGENCY HUMAN HEALTH CRITERIA}

Oral Slope Factor

Inhalation Slope Factor

Chronic Oral RfD

Chronic Inhalation RfD
NA

NA

NA

$1.5\left(\mu \mathrm{g} / \mathrm{m}^{3}\right)$

\section{DRINKING WATER CRITERIA AND STANDARDS}

U.S. Environmental Protection Agency

Maximum Concentration Limit Maximum Concentration Limit Goal

$0.015 \mathrm{mg} / \mathrm{L}$ (action level) 0

\section{AMBIENT WATER QUALITY CRITERIA}

U.S. Environmental Protection Agency Ambient Water Quality Criteria

Human Health - Water \& Fish Ingestion

Human Health - Fish Ingestion Only

Aquatic Organisms - FW Acute

Aquatic Organisms - FW Chronic

Aquatic Organisms - Marine Acute

Aquatic Organisms - Marine Chronic
$5.00 \mathrm{E}-02 \mathrm{mg} / \mathrm{L}$

- NA

$8.30 \mathrm{E}-02 \mathrm{mg} / \mathrm{L} \mathrm{CaCO}_{3}$

$3.20 \mathrm{E}-03 \mathrm{mg} / \mathrm{L} \mathrm{CaCO}_{3}$

$1.4 \mathrm{E}-01 \mathrm{mg} / \mathrm{L}$

$5.6 \mathrm{E}-03 \mathrm{mg} / \mathrm{L}$ 
South Carolina Department of Health and Environmental Control

Human Health Criteria

Fish and Aquatic Life - Continuous

Fish and Aquatic Life - Maximum
$50 \mu \mathrm{g} / \mathrm{L}$

$34 \mu \mathrm{g} / \mathrm{L}$

$1.3 \mu \mathrm{g} / \mathrm{L}$

$\mathrm{NA}=$ Not Available

\section{REFERENCES}

Agency for Toxic Substances and Disease Registry. 1991. Toxicological Profile for Lead. Prepared by Clement International Corp., contract no. 205-88-0608.

Biddinger, G.R. and S.P. Glass. 1984. Res. Rev. 91: 103-45.

Booth, N.H. and L.E. McDonald (eds). 1982. Veterinary Pharmacology and Therapeutics. 5th Ed, Iowa State University Press, Ames, IA.

Frances, AJ. 1985. Anaerobic Microbial Dissolution of Toxic Metals in Subsurface Environments, BLN-36571. Brookhaven National Lab, Upton, NY.

Goyer, R.A. 1986. Toxic Effects of Metals, as cited in Casarett and Doull's Toxicology, The Basic Science of Poisons, 3rd Ed. MacMillan Publishing Co., New York, NY.

International Agency for Research on Cancer. 1980. Monographs on the Evaluation of the Carcinogenic Risk of Chemicals to Man. Geneva, World Health Organization, IARC Monogr. Eval. Carcinog. Risk Chem.

Merck Index, 10th Ed. 1983. Merck and Co., Rahway, NJ.

Schulz-Baldes, M. et al. 1983. Marine Biology 75: 307-18.

Stephenson, T. and J.N. Lester. 1987. Sci. Tot. Environ. 63: 199-214.

Weast, R.C. 1987-1988. Handbook of Chemistry and Physics, 68th Ed. CRC Press Inc., Boca Raton, FL. 
THIS PAGE LEFT INTENTIONALLY BLANK 


\section{MANGANESE}

CAS No: $\quad 7439-96-5$

Synonym(s): None

Manganese is a widely-distributed metal constituting 0.085 percent of the earth's crust. It is found in the minerals manganosite and braunite, and in minute quantities in water, plants, and animals (Merck Index 1983). Nodules on the floors of the Pacific Ocean and Lake Michigan contain manganese (Clayton and Clayton 1981-1982; Worthing and Walker 1987). Manganese may be gray-pink, steel-gray, whitish-gray, or silvery white in color, depending upon its form or refining process (Weast 1988-1989; Merck Index 1983; Browning 1969). It has a molecular weight of $54.938 \mathrm{~g} / \mathrm{mole}$, melts at $1,244+/-3^{\circ} \mathrm{C}$, boils at $1,962^{\circ} \mathrm{C}$, and has a density of 7.47 for the alpha form. It is readily dissolved in dilute mineral acids and in sodium or potassium bicarbonate (Worthing and Walker 1987; Sittig 1985).

\section{FATE AND TRANSPORT}

Manganese enters the atmosphere primarily through anthropogenic sources. Vegetation may concentrate manganese under some conditions. Oats and barley grown in acid sulfate soils have unusually high concentrations of cobalt, nickel, and manganese (Palko and Yli-Hala 1988) due to higher concentrations of extractable cobalt, nickel, and manganese in the acidic soils. The World Health Organization (1981) reported availability of soil manganese to vegetation is influenced by the activity of soil microorganisms that can alter $\mathrm{pH}$ and oxidation/reduction potentials of manganese compounds. Heavy fertilization of acidic soils without liming increases manganese solubility and availability. Addition of organic material to soils can increase manganese uptake by vegetation under certain $\mathrm{pH}$ and aeration conditions. However, the absorption capacity of plants varies from species to species with as much as a 60-fold difference in absorption capacity between species. Honda et al. (1987), studied manganese accumulation in Japanese serows, which are long-lived and free-ranging ruminants related to cattle. The body burden of manganese in serow fetuses was low compared to mothers (less than one percent of the adult females). High manganese concentrations in the intestinal organs of two adult serows was thought to be specific to the species because serows have not developed an excretory system for manganese via bile. The serow's uptake of manganese correlated with the concentration found in plants utilized as a source of food.

\section{HUMAN HEALTH EFFECTS}

Human exposure to manganese is typically via inhalation-although ingestion through diet, drinking water, and inhaled particles cleared from the respiratory tract also occurs (U.S. Environmental Protection Agency 1984). Manganese is considered essential to human health. No cases of manganese toxicity have been reported from excessive human intake in foods or beverages (National Research Council 1980). The World Health Organization (1981) reported sixteen cases of manganese poisoning, including three fatalities, in a small Japanese community where dry-cell batteries were buried near water supply wells in which manganese concentrations varied from $8 \mathrm{mg} / \mathrm{L}$ to $14 \mathrm{mg} / \mathrm{L}$. Individuals affected exhibited psychological and neurological disorders typical of industrial manganese poisoning. 
Susceptibility to inhaled manganese varies widely among individuals. The very young, individuals with iron deficiency, and workers exposed to manganese at or near the recommended threshold limit value are at greatest risk for adverse effects due to manganese exposure (U.S. Environmental Protection Agency 1984). High levels of manganese may increase anemia by interfering with iron absorption (National Research Council 1980). Inhalation of manganese and its compounds in aerosols or fine dusts may produce "metal fume fever" in exposed workers following acute exposure (Venugopal and Luckey 1978; Browning 1969). Chronic manganese poisoning usually affects the central nervous system, resulting in symptoms that include apathy, anorexia, weakness in the legs, uncontrollable laughter, and a stolid, mask-like appearance of the face (Clayton and Clayton 1981-1982). Chronic manganese poisoning is not usually fatal, although permanent disability may result if an individual does not receive treatment. No quantitative estimates of human carcinogenic risks from oral or inhalation exposure to manganese are available because existing studies are not deemed adequate for an assessment of carcinogenicity (U.S. Environmental Protection Agency 1984, 1990).

Repetitive oral administration of manganese in moderate doses for prolonged periods of time does not cause evident injury to various animals (Clayton and Clayton 1981-1982). Growth was stimulated in animals where manganese doses ranged up to 100 parts per million. Manganese concentrations were measured in liver tissue of fetuses from rats, fed a diet containing manganese in concentrations of 4 to $1,004 \mathrm{mg} / \mathrm{kg}$ dry weight, during weaning and pregnancy. Offspring whose dams ate diets with 154 to $1,004 \mathrm{mg} / \mathrm{kg}$ of manganese contained manganese in their livers (Friberg et al. 1986). The maximum biliary excretion of bilirubin is markedly diminished in manganese-loaded rats (Casarett and Doull 1975). Acute pneumonitis can be produced by intratracheal administration of manganese dust or solutions of its compounds (Browning 1969). Histological changes were observed in the central nervous system of a monkey that was exposed to manganese in the form of an aerosol (Browning 1969), while behavioral changes were observed in other monkeys upon exposure to manganese aerosols (Clayton and Clayton 1981-1982).

\section{U.S. ENVIRONMENTAL PROTECTION AGENCY HUMAN HEALTH CRITERIA}

Oral Slope Factor

Inhalation Slope Factor

Chronic Oral RfD

Chronic Inhalation RfD
NA

NA

$5 \mathrm{E}-02 \mathrm{mg} / \mathrm{kg}$.day

$4 \mathrm{E}-3 \mathrm{mg} / \mathrm{m}^{3}$

\section{DRINKING WATER CRITERIA AND STANDARDS -}

\section{U.S. Environmental Protection Agency}

Maximum Concentration Limit

NA

Maximum Concentration Limit Goal

NA 


\section{AMBIENT WATER QUALITY CRITERIA}

U.S. Environmental Protection Agency Ambient Water Quality Criteria

$\begin{array}{ll}\text { Human Health - Water \& Fish Ingestion } & 50 \mu \mathrm{g} / \mathrm{L} \\ \text { Human Health - Fish Ingestion Only } & 100 \mu \mathrm{g} / \mathrm{L} \\ \text { Aquatic Organisms - FW Acute } & \text { NA } \\ \text { Aquatic Organisms - FW Chronic } & \text { NA } \\ \text { Aquatic Organisms - Marine Acute } & \text { NA } \\ \text { Aquatic Organisms - Marine Chronic } & \text { NA }\end{array}$

South Carolina Department of Health and Environmental Control

Human Health Criteria

Fish and Aquatic Life - Continuous

NA

Fish and Aquatic Life - Maximum

NA

NA

$\mathrm{NA}=$ Not Available

\section{REFERENCES}

Agency for Toxic Substances and Disease Registry. 1990. Toxicological Profile for Manganese. Prepared by Clement International Corp., contract no. 205-88-0608.

Browning, E. 1969. Toxicity of Industrial Metals, 2nd Ed. Appleton-Century Crofts, New York, NY.

Casarett, L.J. and J. Doull. 1975. Toxicology: The Basic Science of Poisons. MacMillan Publishing Company, New York, NY.

Clayton, G. D. and F.E. Clayton (eds.). 1981-1982. Patty's Industrial Hygiene and Toxicology, Vol. 2A, 2B, 2C, 3rd Ed. John Wiley Sons, New York, NY.

Friberg, L., G.F. Nordberg, E. Kessler, and V.B. Vouk (ed.). 1986. Handbook of Toxicology of Metals, Vol. I and II, 2nd Ed. Elsevier Science Publishers B.V., Amsterdam, Netherlands.

Honda, K. et al. 1987. Arch. Environ. Contam. Toxicol-16: 551-561.

Merck Index. 1983. 10th Ed. Merck Company, Inc. Rahway, NJ.

National Research Council. 1980. Drinking Water and Health, Vol., 3. National Academy Press, Washington, DC.

Palko, J. and M. Yli-Hala. 1988. Acta. Agric. Scand. 38(2): 153-158. 
Sittig, M. 1985. Handbook of Toxic and Hazardous Chemicals and Carcinogens, 2nd Ed. Noyes Data Corporation, Park Ridge, NJ.

U.S. Environmental Protection Agency, 1984. Health Assessment Document: Manganese. Doc No. EPA-600/8-83-013F. U.S. EPA, Washington, DC.

U.S. Environmental Protection Agency. 1990. Health Evaluation Assessment Summary Tables, Third Quarter, Fiscal Year 1990. Washington, DC.

Venugopal, B. and T.D. Luckey. 1978. Metal Toxicity in Mammals, Vol.2. Plenum Press, New York, NY.

Weast, R.C. (ed.). 1988-1989. Handbook of Chemistry and Physics, 69th Ed. CRC Press, Inc., Boca Raton, FL.

World Health Organization. 1981. Environmental Health Criteria: Manganese. World Health Organization, Geneva, Switzerland.

Worthing, C.R. and S.B. Walker (eds.). 1987. The Pesticide Manual - A World Compendium, 8th ed. The British Crop Protection Council, Thornton Heath, U.K. 
MAGNESIUM

CAS No:

7439-95-4

Synonym(s)

None

Magnesium is ubiquitous in the earth's crust and is the second most abundant cation of human intracellular fluids. Magnesium does not occur in nature in the pure metallic form, rather it exists in combination with other elements such as dolomite $\left(\mathrm{CaCo}_{3}-\mathrm{MgCO}_{3}\right)$, magnesite $\left(\mathrm{MgCO}_{3}\right)$, brucite $\left(\mathrm{Mg}(\mathrm{OH})_{2}\right)$, and others. Most magnesium used industrially is obtained by electrolysis of magnesium chloride from brines, wells, and sea water. Magnesium is a light but strong metal used in alloys for components of military equipment, aircraft, ships, and handtools, where the above mentioned properties are desirable (Parmegianni 1983).

Magnesium has a molecular weight of 24.305 , a melting point of $648.8^{\circ} \mathrm{C}$, and a boiling point of $1090^{\circ} \mathrm{C}$. Its specific gravity is 1.738 . Magnesium is a silvery-white, very light, but tough metal. Fine particles of magnesium produced during grinding or polishing are flammable in air and represent a significant occupational hazard (Parmegianni 1983; Weast 1985).

\section{FATE AND TRANSPORT}

Anthropogenic emissions of magnesium occur as dust or fumes released into the atmosphere during grinding or finishing the metal. The dust may ignite in air. Particles which are less flammable will be removed from air by rainfall or by gravitational settling (Bowen 1979).

Magnesium does not appear to have a great affinity for suspended particulate matter in surface waters. That which is dissolved in water tends to be transported by currents, however settling may occur slowly in static waters (Bowen 1979).

\section{HUMAN HEALTH EFFECTS}

Magnesium toxicity is low, however the dust is irritating to eyes and mucous membranes. The fumes from magnesium oxide can cause metal -fume fever. Additionally, epidemiological studies have revealed an association between magnesium absorption and gastroduodenal ulcers. Small slivers of magnesium that penetrate skin and become embedded cause deep destructive lesions, or tumors, of the skin (Parmegianni 1983).

\section{ECOLOGICAL EFFECTS}

Magnesium is an essential element necessary for the production of chlorophyll in green plants and for oxidative phosphorylation, and thus energy transformations, in the mitochondria of living organisms (Parmegianni 1983) Hence, all organisms will take up magnesium in some form. The topics of bioaccumulation and biomagnification of magnesium in the food chain have not been addressed with any great emphasis, probably because of the low toxicity of magnesium and biota's essential need for this element. 


\section{US ENVIRONMENTAL PROTECTION AGENCY HUMAN HEALTH CRITERIA}

Oral Slope Factor

Inhalation Slope Factor

Chronic Oral RfD

Chronic Inhalation RfC
NA

NA

NA

NA

\section{DRINKING WATER CRITERIA AND STANDARDS}

U.S. Environmental Protection Agency

Maximum Concentration Limit

NA

Maximum Concentration Limit Goal

NA

\section{AMBIENT WATER QUALITY CRITERIA}

U.S. Environmental Protection Agency Ambient Water Quality Criteria

$\begin{array}{ll}\text { Human Health-Water and Fish Ingestion } & \text { NA } \\ \text { Human Health=Fish Ingestion Only } & \text { NA } \\ \text { Aquatic Organisms-FW Acute } & \text { NA } \\ \text { Aquatic Organisms-FW Chronic } & \text { NA } \\ \text { Aquatic Organisms-Marine Acute } & \text { NA } \\ \text { Aquatic Organisms-Marine Chronic } & \text { NA }\end{array}$

South Carolina Department of Health and Environmental Control

Human Health Criteria NA

Fish and Aquatic Life-Continuous NA

Fish and Aquatic Life-Maximum NA

$\mathrm{NA}=$ Not Available

\section{REFERENCES}

Bowen, 1979. Environmental chemistry of the elements. New York. Academic Press.

Parmeggiani, L. (ed). 1983. Encyclopedia of occupational Health and Safety. Vol. 2. Geneva. International Labor Office.

Weast, R.C. 1985. CRC Handbook of chemistry and physics. 66 th edition. Boca Raton, FL. CRC Press. 


\section{METHYLENE CHLORIDE}
CAS No.:
75-09-2
Major Synonym(s) Dichloromethane, methylene dichloride

Methylene chloride is produced anthropogenically from methane gas or wood alcohol. Its primary uses are as a solvent in paint strippers and removers and as a propellant in aerosols, however, it also functions as a metal cleaning solvent and a process solvent in the production of drugs and film coatings (NTP 1989). Methylene chloride is also used as a solvent in the extraction of caffeine from coffee, as a fumigant for harvested grains and strawberries, and as a degreening agent for citrus fruit (NTP 1989).

Methylene chloride is a colorless liquid with a molecular weight of 84.93 . It melts at $-95.1^{\circ} \mathrm{C}$ and boils at $40^{\circ} \mathrm{C}$. Its odor is sweet. The density of methylene chloride is 1.3266 and it is somewhat soluble in water and goes easily into solution in alcohol and ether (ATSDR 1991).

\section{FATE AND TRANSPORT}

Methylene chloride is highly volatile. The U.S. Environmental Protection Agency estimates that $85 \%$ of all methylene chloride produced in the United States is lost to the environment, primarily to air (EPA 1985).

Methylene chloride is released to the atmosphere during its production and handling yet the overwhelming majority of releases occur from industrial and consumer uses (EPA 1985). Degradation of atmospheric methylene chloride is slow and occurs primarily through interaction of this compound with hydroxyl radicals. Because of the long half-life of methylene chloride in air, its vapors may become widely dispersed (ATSDR 1991; Altshuller 1980).

A small percentage of methylene chloride releases are to water (EPA 1982) and generally results from industrial and municipal effluents or leachate (EPA 1983; Brown and Donnelly 1988). Degradation of methylene chloride in water may result slowly from hydrolysis or more rapidly through biodegradation in aerobic conditions (Dilling et al. 1975; Tagbel et al. 1981).

Methylene chloride disposal to landfills, primarily as consumer products waste, is the predominant source of soil contamination (TRI88 1990). Methylene chloride does not adsorb to soils or sediments readily and is therefore thought to be mobile in soils (Dilling et al. 1975; Bahnick and Dioucette 1988). Evaporation, leaching into groundwater, and biodegradation may all be important mechanisms for the removal of methylene chloride from soil (ATSDR 1991; EPA 1984; Sawhney 1989).

\section{HUMAN HEALTH EFFECTS}

Methylene chloride is a central nervous system depressant and is narcotic at high concentrations. Symptoms of toxicity include faintness, giddiness, fatigue, headache, nausea and respiratory distress (Hughes 1954; Lee 1981). Unconsciousness, narcosis and death may occur (Winek et al. 1981). After 1.5-3 hours of exposure to $200 \mathrm{ppm}$, neurobehavioral 
changes (vigilance disturbance and impaired combined tracking monitoring performance) were observed in human volunteers (Putz et al. 1976). Four hours' exposure to $750 \mathrm{ppm}$ resulted in decreased psychomotor performance (Winneke 1974). One to two-hour exposures to methylene chloride vapor in concentrations of 500-1000 ppm resulted in sustained elevation of carboxyhemoglobin levels (Stewart et al. 1972).

The effects of chronic exposure to methylene chloride were studies in workers exposed for several years to 75-100 ppm of a 9:1 mixture of methylene chloride-methanol in air. Neurological symptoms were significantly elevated in the exposed group when compared to controls, but the numbers of effective symptoms, such as irritability, tiredness and stomachaches were not significantly increased (Cherry et al. 1981). No effects on clinical chemistry, hematology or electrocardiogram values were noted on workers exposed to a TWA of 33 ppm methylene chloride (DiVincenzo and Kaplan 1981).

Occupational mortality studies conducted to date have not supported a carcinogenic effect of methylene chloride by the inhalation route of exposure (Friedlander et al. 1978; Hearne and Friedlander 1981; Hearne et al. 1987; Hearne et al. 1990). Exposure may have been inadequate in most persons for identification of excess cancer rates (OSHA 1986; IARC 1986).

No information could be found in the available literature on the oral toxicity of methylene chloride in humans. Decreased cytochrome P-450 in liver microsomes of rats resulted from a single oral dose of $1 \mathrm{~g} / \mathrm{kg}$ (Moody et al. 1981). Rats receiving oral doses of up to $5 \mathrm{~g} / \mathrm{kg}$ developed gastrointestinal lesions, pancreatitis and peritonitis, but no lesions were seen upon microscopic examination of liver and other tissues (Laham 1978).

Male and female Fischer rats were administered methylene chloride at dose of 1-250 $\mathrm{mg} / \mathrm{kg} /$ day in drinking water for two years (Serota et al. 1986a). No compound-related effects were observed on physical exam or gross necropsy. In groups given $50 \mathrm{mg} / \mathrm{kg} / \mathrm{day}$ and above, altered hepatic cell morphology was noted at 18 and 24 months. At the two highest dose levels there was also an increased incidence of fatty liver changes in both sexes at 18 and 24 months. The no-effect level for chronic toxicological effects was $5 \mathrm{mg} / \mathrm{kg} / \mathrm{day}$. A chronic oral RfD of $6.0 \mathrm{E}-02$ has been calculated for methylene chloride based on the hepatoxicity observed in the above drinking water study (U.S. EPA 1991a).

Methylene chloride has been classified as a probable human carcinogen (B2) by the oral route of exposure based on the development of excess numbers of hepatocellular adenomas and carcinomas in mice treated through drinking water (Serota et al. 1986b). An oral slope factor of $7.5 \mathrm{E}-03$ has been established using data from this study and the inhalation study conducted by NTP (1986).

Liquid methylene chloride is a severe skin irritant in man (Winek et al. 1981). No other information was located on the dermal toxicity of methylene chloride to animals or man. 


\section{ECOLOGICAL EFFECTS}

It is not presently known if methylene chloride is bioconcentrated in aquatic or terrestrial organisms. The U.S. EPA (1980a, 1984) estimated the bioconcentration factor (concentration in organism/concentration in ambient media) of methylene chloride and found this calculation to be low, suggesting that it does not bioconcentrate in biologic systems.

\section{U.S. ENVIRONMENTAL PROTECTION AGENCY HUMAN HEALTH CRITERIA}

Oral Slope Factor

Inhalation Slope Factor

Chronic Oral RfD

Chronic Inhalation RfD
$7.5 \mathrm{E}-3 \mathrm{~kg} / \mathrm{mg} /$ day $^{-1}$

$1.6 \mathrm{E}-3 \mathrm{~kg} / \mathrm{mg} /$ day $^{-1}$

$6 \mathrm{E}-2 \mathrm{mg} / \mathrm{kg} /$ day

$\mathrm{E}+00 \mathrm{mg} / \mathrm{m}^{3}$

\section{DRINKING WATER CRITERIA AND STANDARDS}

U.S. Environmental Protection Agency

Maximum Concentration Limit

Maximum Concentration Limit Goal

$0.005 \mathrm{mg} / \mathrm{L}$

$0 \mathrm{mg} / \mathrm{L}$

\section{AMBIENT WATER QUALITY CRITERIA}

U.S. Environmental Protection Agency Ambient Water Quality Criteria

Human Health - Water and Fish IngestioNA

Human Health - Fish Ingestion Only

NA

Aquatic Organisms - FW Acute

NA

Aquatic Organisms - FW Chronic

NA

Aquatic Organisms - Marine Acute

NA

Aquatic Organisms - Marine Chronic

NA

South Carolina Department of Health and Environmental Control Water Quality Criteria

Human Health Criteria

Fish and Aquatic Life - Continuous

Fish and Aquatic Life - Maximum
$1.6 \mathrm{E}+04 \mu \mathrm{g} / \mathrm{L}$

NA

NA

NA $=$ Not Available

\section{REFERENCES}

Agency for Toxic Substances and Disease Registry. 1991. Toxicological Profile for Methylene Chloride. Prepared by Life Systems, Inc., under subcontract to Clement International Corp., contract no. 205-88-0608. 
Bahnick, D.A., Doucette, W.J. 1988. Use of molecular connectivity indices to estimate soil sorption coefficients for organic chemicals. Chemosphere 17:1703-1715.

Berger, M. and G.G. Fodor. 1968. Central nervous system disorders under the influence of air mixtures containing dichloromethane. Zentralbl. Bakteril. 215:517 (Cited in HSE 1985).

Brown, K.W., Donnelly, K.C. 1988. An estimation of the risk associated with the organic constituents of hazardous and municipal waste landfill leachates. Hazardous Waste and Hazardous Materials 5:1-30.

Burek, J.D., K.D. Nitschke, T.J. Bell, L.W. Rampy and M.J. McKenna. 1984. Methylene chloride: A two-year inhalation toxicity and oncogenicity study in rats and hamsters. Fund. Appl. Toxicol. 4:30-47.

Cherry, N., H. Venables, H.A. Waldron and G.G. Wells. 1981. Some observations on workers exposed to methylene chloride. Br. J. Ind. Med. 38:351-355.

Dilling, W.L., Tefertiller, N.B., Kallos, G.J. 1975. Evaporation rates of methylene chloride, chloroform, 1,1,1-trichloroethane, trichloroethylene, tetrachloroethylene, and other chlorinated compounds in dilute aqueous solutions. Environ Sci Technol 9:833-838.

DiVincenzo, G.D. and C.J. Kaplan. 1981. Uptake, metabolism and elimination of methylene chloride vapour by humans. Toxicol. Appl. Pharmacol. 59:130-140.

EPA. 1982a. Chlorinated organic solvents: trichloroethene, tetrachloroethene, 1,1,1-trichloroethane, dichloromethane, and tetrachloromethane. In: Kayser, R., Sterling, D., Viviani, D., eds. Intermedia priority pollutant guidance documents. Washington, DC: U.S. Environmental Protection Agency, Office of Pesticides and Toxic Substances.

EPA. 1983b. Treatability manual. Vol. 1. Treatability data. Washington, DC: U.S. Environmental Protection Agency, Office of Research and Development. EPA$600 / 2-82-001 \mathrm{~A}$.

EPA. 1984. Health effects assessment for methylene chloride. Cincinnati, OH: U.S. Environmental Protection Agency, Environmental Criteria and Assessment Office. EPA/540/1-86/028. NTIS no. PB86 134392/AS. -

EPA. 1985a. Addendum to the health assessment document for dichloromethane (methylene chloride). Updated carcinogen assessment of dichloromethane (methylene chloride). Final report. Washington, DC: U.S. Environmental Protection Agency, Office of Research and Development. EPA 600/8-82-0044FF.

Friedlander, B.R., T. Hearne and S. Hall. 1978. Epidemiologic investigation of employees exposed to methylene chloride. Morality analysis. J. Occup. Med. 20:657-666. 
Hearne, T. and B.R. Friedlander. 1981. Follow-up of methylene chloride study. J. Occup. Med. 23:660.

Hearne, F.T., F. Grose, J.W. Pifer, B.R. Friedlander and R.L. Raleigh. 1987. Methylene chloride mortality study: Dose response characterization and animal model comparison. J. Occup. med. 29:217-228.

Hearne, F.T., J.W. Pifer and F. Grose. 1990. Absence of adverse mortality effects in workers exposed to methylene chloride: An update. J. Occup. Med. 32(3):324-340.

HSE (Health and Safety Executive). 1985. Toxicity Review 12-Dichloromethane (Methylene Chloride). Her Majesty's Stationery Office, London.

Hughes, J.P. 1943. Hazardous exposure to some so-called safe solvents. J.A.M.A. 156:234237.

IARC (International Agency for Research on Cancer). 1979. Monographs on the evaluation of the carcinogenic risk of chemicals to humans. vol. 20. Some halogenated hydrocarbons. IARC, Lyon. pp. 43-85.

IARC. 1986. IARC monograph on the evaluation of the carcinogenic risk of chemicals to humans. Some halogenated hydrocarbons and pesticide exposures. Vol. 41. World Health Organization, International Agency for Research on Cancer, Lyon, France, 43-85.

Laham, S. 1978. Toxicological studies on dichloromethane, a solvent simulating carbon monoxide poisoning. Toxicol. Eur. Res. 1:63-73. (Cited in HSE 1985).

Lee, B. 1981. Multisystem disorder after exposure to paint stripper (Nitromers). Br. Med. J. 282:1321.

Moody, D.E., J.L. James, G.A. Clawson and E.A. Smuckler. 1981. Correlations among the changes in hepatic microsomal components after intoxication with alkyl halides and other hepatotoxins. Mol. Pharamcol. 20:685-693.

Nitschke, K.D., J.D. Burek, T.J. Bell, L.W. Rampy and M.J. McKenna. 1988. Methylene chloride: A 2-year inhalation toxicity and oncogenicity study in rats. Fund. Appl. Toxicol. (In press) (Cited in U.S. EPA 1991b).

NTP (National Toxicology Program). 1986. Toxicology and carcinogenesis studies of dichloromethane (methylene chloride) (CAS No. 75-09-2) in F344/N rats and $\mathrm{B}_{3} \mathrm{C}_{3} \mathrm{~F}_{1}$ mice (inhalation studies). Technical Report No. 306. U.S. DHEW, Research Triangle Park, N.C.

NTP. 1989. National Toxicology Program. Fifth annual report on carcinogens: Summary 1989. Report to the National Institute of Environmental Health Sciences, Research 
Triangle Park, NC, by Technical Resources, Inc., Rockville, MD, 110-113. NTP 89239.

OSHA (Occupational Safety and Health Administration). 1986. Occupational safety of methylene chloride. Fed. Reg. 51(226):42257-42266.

Putz, V.R., B.L. Johnson and J.V. Setzer. 1976. A comparative study of the effects of carbon monoxide and methylene chloride on human performance. J. Environ. Pathol. Toxicol. 2:97-112.

Sawhney, B.L., 1989. Movement of organic chemicals through landfill and hazardous waste disposal sites. In: Reactions and movement of organic chemicals in soils. Madison, WI: Soil Science society of America and American and American Society of Agronomy, 447-474.

Serota, D.G., A.K. Thakur, B.M. Ulland, J.C. Kirschman, N.M. Brown, R.H. Coots and K. Morgareidge. 1986a. A two-year drinking water study of dichloromethane in rodents. I. Rats. Food Chem. Toxicol. 24:951-958.

Serota, D.G., A.K. Thakur, B.M. Ulland, J.C. Kirschman, N.M. Brown, R.H. Coots and K. Morgareidge. 1986b. A two-year drinking water study of dichloromethane in rodents. I. Mice. Food Chem. Toxicol. 24:959-963.

Stewart, R.D., T.N. Fisher, M.J. Hoske, J.E. Peterson, E.D. Beretta and H.C. Dodd. 1972. Experimental human exposure to methylene chloride. Arch. Environ. Health 25:342348.

TRI88. 1990. Toxic chemical release inventory. National Library of Medicine, National Toxicology Information Program, Bethesda, MD.

U.S. EPA (U.S. Environmental Protection Agency). 1991a. Integrated Risk Information System (IRIS). Online. Office of Health and Environmental Assessment, Environmental Criteria and Assessment Office, Washington, D.C.

U.S. EPA (U.S. Environmental Protection Agency). 1991b. Health Effects Assessment Summary Tables (HEAST). Office of Research and Development, Office of Emergency and Remedial Response, Washington, D.C.

Winek, C.L., W.D. Collom and F. Esposito. 1981. Accidental methylene chloride fatality. Forensic Sci. Int. 18:165-168.

Winneke, G. 1974. Behavioral effects of methylene chloride and carbon monoxide as assessed by sensory and psychomotor performance. In: Xintaras, C., B. Johnson and I. deGroot, Eds. Behavioral Toxicology. U.S. Government Printing Office, Washington, D.C. pp. 130-144. 
METHYL ETHYL KETONE

CAS No.: $\quad 78-93-3$

Major Synonym(s) 2-Butanone, 3-Butanone, Methyl Acetone

Methyl ethyl ketone (MEK) is a natural product of certain plants such as ferns, cypress trees, firs, junipers, and cedar trees (Isidorov et al. 1985). It is produced commercially for its outstanding solvent and quick drying properties, and its applications include its use as a solvent for adhesives, coatings, printing inks, paint removers, and as a metal degreaser (Papa and Sherman 1981; Sak and Lewis 1987).

MEK is a colorless liquid having a molecular weight of 72.1. It has a sweet odor and rapidly vaporizes with a vapor pressure of $77.5 \mathrm{mmHg}$ at $20^{\circ} \mathrm{C}$ and a vapor density of 2.4 . MEK has a boiling point of $79.6^{\circ} \mathrm{C}$ and a melting point of $-86.6^{\circ} \mathrm{C}$.

\section{FATE AND TRANSPORT}

MEK can be released into the atmosphere by natural sources, however, emissions primarily occur from anthropogenic activities. Automobile emissions, photochemical degradation of hydrocarbons, production, and handling of product, and curing of adhesives and coatings comprise some of the sources of atmospheric emissions of MEK. MEK is found predominantly in the vapor phase in air, therefore, little deposition of dry particulate matter occurs (ATSDR 1990). Wet deposition, conversely, may contribute significantly to the removal of MEK from air, especially since MEK is highly water soluble (Tewari et al. 1982). Hydroxyl radicals interact with MEK and act as a degradation pathway for removal of product from air (Cox et al. 1980).

MEK is highly mobile in soil (Swann et al. 1983) and readily leaches into groundwater (Halvorsen and Ohneck 1985; Francis et al. 1980). Evaporation will potentiate the clearance of MEK from soil (ATSDR 1990), however, hydrolysis, photolysis, or chemical degradation are not anticipated to be removal pathways. Biodegradation may occur, but is yet to be documented (ATSDR 1990).

MEK enters the waterways in industrial effluents, as atmospheric fallout in rainwater, and as leachate from contaminated soils (Dunovant et al. 1986; Grosjean and Wright 1983; Francis et al. 1980). Microbes degrade waterborne MEK to propionate in aerobic conditions (Phillips and Perry 1974), or to methane in anaerobic conditions (Chou et al. 1979).

\section{HUMAN HEALTH EFFECTS}

Kresavage et al. (1982) state that "methyl ethyl ketone has a low order of toxicity following both acute and chronic exposures". Acute exposure of men to MEK caused irritation to the eyes, nose, and throat (Patty et al. 1935; Nelson et al. 1943). In other studies, acute MEK exposure produced headache, irritation, nausea, facial dermatitis, and numbness of the fingers and legs (Krasavage et al. 1982, NIOSH 1978). MEK has not been shown to produce neurotoxic effects in humans or animals, regardless of whether exposure was acute or chronic. 


\section{ECOLOGICAL EFFECTS}

Methyl ethyl ketone has been identified as a natural volatile compound in a number of food types. It has been detected in raw chicken breast, certain nuts, Gruyere cheese, nectarines, dried beans, split peas, and lentils (Dumont and Adda 1978; Grey and Shrimpton 1967; Kinlin et al. 1972; Takeoka et al. 1988; Lovegren et al. 1979). In spite of its widespread occurrence in the environment, methyl ethyl ketone is not anticipated to bioconcentrate in aquatic organisms nor to biomagnify within the food chain (ATSDR 1990).

\section{U.S. ENVIRONMENTAL PROTECTION AGENCY HUMAN HEALTH CRITERIA}

Oral Slope Factor

Inhalation Slope Factor

Chronic Oral RfD

Chronic Inhalation RfD
NA

NA $6 \mathrm{E}-01 \mathrm{mg} / \mathrm{kg} /$ day

$1 \mathrm{E}+01 \mathrm{mg} / \mathrm{m}^{3}$

\section{DRINKING WATER CRITERIA AND STANDARDS}

U.S. Environmental Protection Agency

Maximum Concentration Limit

NA

Maximum Concentration Limit Goal

NA

\section{AMBIENT WATER QUALITY CRITERIA}

U.S. Environmental Protection Agency Ambient Water Quality Criteria

$\begin{array}{ll}\text { Human Health - Water and Fish Ingestion } & \text { NA } \\ \text { Human Health - Fish Ingestion Only } & \text { NA } \\ \text { Aquatic Organisms - FW Acute } & \text { NA } \\ \text { Aquatic Organisms - FW Chronic } & \text { NA } \\ \text { Aquatic Organisms - Marine Acute } & \text { NA } \\ \text { Aquatic Organisms - Marine Chronic } & \text { NA }\end{array}$

South Carolina Department of Health and Environmental Control Water Quality Criteria

Human Health Criteria

Fish and Aquatic Life - Continuous

Fish and Aquatic Life - Maximum
NA

NA

NA

$\mathrm{NA}=$ Not Available

\section{REFERENCES}

Agency for Toxic Substances and Disease Registry. 1990. Toxicological Profile for 2Butanone. Prepared by Clement International Corp., contract no. 205-88-0608. 
Chou, WL, Speece, RE, Siddiqi, RH. 1979. Acclimation and degradation of petrochemical wastewater components by methane fermentation. Biotechnol Bioeng Symp 8:391-414.

Cox, RA, Derwent, RG, Williams, MR. 1980. Atmospheric photooxidation reactions. Rates, reactivity, and mechanism fo reaction of organic compounds with hydroxyl radicals. Environ Sci Technol 14:57-61.

Dumont, JP, Adda, J. 1978. Occurrence of sesquiterpenes in mountain cheese volatiles. J Agric Food Chem 26:364-367.

Dunovant, VS, Clark, CS, Que Hee SS, et al. 1986. Volatile organics in the wastewater and airspaces of three wastewater plants. J Wwater Pollut Contr Fed 58:8860895.

Francis, AJ, Iden, CR, Nine, BJ, et al. 1980. Characterization of organics in leachates from low-level radioactive waste disposal sites. Nuclear Tech 50:158-163.

Grey, TC, Shrimpton, DH. 1967. Volatile components of raw breast muscle. Br Poultry Sci 8:23-33.

Grosjean, D, Wright, B. 1983. Carbonyls in urban fog, ice fog, cloudwater and rainwater. Atmos Environ 17:2093-2096.

Halvorsen, F, Ohneck, R. 1985. MEK groundwater decontamination. In: Hazardous wastes and environmental emergencies. Silver Springs, MD: Hazardous Materials Control Research Institute, 193-195.

Kinlin, TE, Muralidhara, R, Pittet, AO. 1972. Volatile components in roasted filberts. J Agric Food Chem 20:1021-1028.

Kresavage, W., O'Donoghue, J., Divincenzo, G. 1982. Ketones. Patty's Industrial Hygiene and Toxicology (G. D. Clayton and F. E. Clayton, eds.) Vol. 2C, ppp. 4709-4800. Wiley, New York.

Lovegren, NV, Fisher, GS, Legendre, MG, et al. 1979. Volatile constituents of dried legumes. J Agric Food Chem 27:851-853.

Nelson, K. Egre, J. Ross, N. Woodman, L. Silverman. 1943. Sensory response to industrial solvent vapors. J. Industrial Hyg. Toxic. 25'282-2.

NIOSH. 1978. Criteria for a Recommended Standard...Occupational Exposure to Ketones. National Institute for Occupational Safety and Health. U.S. Dept. of Health, Education, and Welfare. Pub. No. 78-173.

Patty, F., Shrenk, and Yant, W. 1935. Butanone. U.S. Public Health Rep. 50.1217. 
Phillips, WE, Jr., Perry, JJ. 1974. Metabolism of butane and 2-butanone by Mycobacterium vaccae. J Bacteriol 120:987-989.

Swann, RL, Laskowski, DA, McCall, PJ, et al. 1983. A rapid method for the estimation of the environmental parameters octanol/water partition coefficient, soil sorption constant, water to air ratio and water solubility. Residue Rev 85:17-28.

Takeoka, GR, Flath, RA, Guntert, M, et al. 1988. Nectarine volatiles: Vacuum steam distillation versus headspace sampling. J Agric Food Chem 36:553-560.

Tewari, YB, Miller, MM, Wasik, SP, et al. 1982. Aqueous solubility and octanol/water partition coefficient of organic compounds at $25^{\circ} \mathrm{C}$. J Chem Eng Data 27:451-454. 


\section{MERCURY}

CAS No.:
Major Synonyms

Mercury $(\mathrm{Hg})$ is a liquid at ambient temperatures and is a good electrical conductor. These and other properties such as its ability to form alloys with other metals and its uniform volume expansion render mercury an important metal industrially and commercially. Aside from its well-known use in thermometers, barometers, and pressure-sensing devices, mercury is also used in hearing aids, cameras, toys, portable radios, dental fillings, and many other everyday consumer products. It was once used in fungicides and marine paint, although most of this applications have been cancelled (ATSDR 1993).

Mercury may occur in its metallic form and as inorganic salts or organic mercury. Metallic mercury has a molecular weight of 200.59 and is a silver-white, heavy liquid. It melts at $38.87^{\circ} \mathrm{C}$ and boils at $356.72^{\circ} \mathrm{C}$. Inorganic salts and organic forms of $\mathrm{Hg}$ are solids at ambient temperature. All are relatively soluble in water.

\section{FATE AND TRANSPORT}

Mercury occurs naturally in the earth's crust and may be released into the environment by wind and water. Overall, the majority of total $\mathrm{Hg}$ in the environment is due to release from natural processes, however, $\mathrm{Hg}$ may be concentrated at particular sites due to human activities. Inorganic $\mathrm{Hg}$ is the most prevalent form of $\mathrm{Hg}$ found in soil, water, and air. Organic mercury is typically produced from transformation of inorganic mercury, but was used in the past in fungicides and paints.

Mercury volatilizes from soil and water and is subject to transportation and deposition, due to its long residence time in air (EPA 1984b). Wet deposition, dry deposition, and binding to particulate matter help to remove mercury from air (EPA 1984b; Lindovist 1991b)

Transport and fate of mercury in soils and water is determined by the particular form of mercury. The gaseous form of metal mercury is often dissolved in water, but may evaporate along with other volatile forms (Vandel et al. 1991). Solid mercury compounds adhere to particulates in the water column and are transported down into the sediments (Hurley et al. 1991). Transformation of mercury occurs in aquatic sediments as well as within the water column, with bacterial biodegradation as the most important pathway.

Organic mercury, the product of bacterial action, can be-photolytically degraded in surface waters (Callahan et al. 1979).

Mercury released to soils demonstrates little mobility, as it is tightly sorbed to particulate matter, especially in organic soils (Hurley et al. 1991(). Some $\mathrm{Hg}$ may be transported by surface runoff or evaporation (Meili 1991; EPA 1984b). Soil = bound mercury is also subject to biodegradation, like that which occurs in aquatic systems (Andersson 1979). 


\section{HUMAN HEALTH EFFECTS}

Acute toxicity can result from exposure to mercury vapor at concentrations greater than 2 $\mathrm{mg} / \mathrm{M} 3$. Signs include febrile illness with cough and dyspnea, chest pains, heotysis, impairment of pulmonary function, diffuse pulmonary infiltrates, and evidence of interstitial pneumonitis (McFarmland and Reigel, 1978; Milne et al., 1970). Prolonged central nervous system (CNS) effects have also been reported following severe acute exposure to mercury vapor (McFarland and Reigel, 1978).

Subchronic or chronic low dose exposure of mercury vapor causes primarily effects on the central nervous system. Early signs are nonspecific and include neurasthenia (weakness, fatigue)( and three or more of the following clinical findings: tremor, enlargement of the thyroid with increased uptake of radioiodine in the thyroid, labile pulse, tachycardia, urticaria, hematologic changes, or increased excretion of mercury in urine (Goyer, 1986). More severe exposures produce the classic mercurialism; the three characteristic signs being erethism (characterized by irritability and increased excitability, excessive shyness, insomnia and indecision), gingivitis and trembling of the body (NIOSH, 1973).

The kidney has a remarkable capacity to concentrate mercury and is a target organ when inhalation exposure to metallic mercury occurs. Case studies of acute inhalation exposure in humans note renal insufficiency, proteinuria, hematuria, and degeneration of the convoluted tubules (Campbell, 1984.; Jaffe et al., 1983; Snodgrass et al., 1981). The first symptoms seen following chronic low dose inhalation exposure are mild proteinuria and biochemical alterations, both of which are reversible. More severe damage becomes evident as glomerular dysfunction, acute nephrotic syndrome and edema occur (Agner and Jans, 1978).

Maternal inhalation results in uptake of mercury vapor by the fetus and might be expected to be hazardous to the fetus (Clarkson et al., 1972).

Several animal studies have shown interference with the estrous cycle and increased prenatal and postnatal mortality after subchronic exposure of rats to $2.5 \mathrm{mg} / \mathrm{M}^{3}$ of mercury vapor (Barlow and Sullivan, 1982).

Ingestion of mercuric mercury can cause corrosive damage to the entire gastrointestinal tract. Symptoms are abdominal pain, vomiting and bloody diarrhea. Acute oral exposure also damages the proximal renal tubules. The lethal dose for adult humans is 1-4 $\mathrm{gm} /$ person $\left(\mathrm{HgCl}_{2}\right)$ (Berlin, 1986; Goyer, 1986).

Skin absorption can be an important route of exposure for mercuric compounds (Berlin, 1986). The only studies located regarding health effects in humans or animals following dermal exposure to inorganic mercury are those reports describing neurological effects after contact with mercury. Nephrotic syndrome has been observed following skin applications of mercury ointment (Batigelli, 1988). acrodynia is a classical syndrome seen in children repeatedly exposed dermally to mercury-containing compounds (inorganic mercury). One aspect of the syndrome is neurotoxicity characterized by fine postural or action tremor of 
face or extremities, sudden jerky movements, a lack of muscle tone, impaired reflexes, seizures, light sensitivity; deafness, insomnia, and irritability (DeBone et al., 1986). Allergic hypersensitivity has been occasionally associated with elemental mercury used in dental amalgam (Duxbury et al., 1982).

\section{ECOLOGICAL EFFECTS}

Aquatic organisms assimilate and accumulate mercury, particularly methyl mercury, which is soluble and mobile. Aquatic plants bioaccumulate mercury in proportion to the water concentration of mercury (Ribewyre et al. 1991). Fish, on the other hand, bioconcentrate mercury at levels far exceeding that of the water concentration (Callahan et al. 1979). likewise, aquatic macrophytes bioconcentrate mercury (Ribeyre), however, fish consumption is thought to be the most probable source of human exposure.

\section{U.S. ENVIRONMENTAL PROTECTION AGENCY HUMAN HEALTH CRITERIA}

Oral Slope Factor

Inhalation Slope Factor

Chronic Oral RfD

Chronic Inhalation RfD
N/A
N/A
$3 \mathrm{E}-4$
$8.6 \mathrm{E}-5$

\section{DRINKING WATER CRITERIA AND STANDARDS}

U.S. Environmental Protection Agency

$\begin{array}{ll}\text { Maximum Concentration Limit } & .002 \mathrm{mg} / \mathrm{l} \\ \text { Maximum Concentration Limit Goal } & .002 \mathrm{mg} / \mathrm{l}\end{array}$

South Carolina Department of Health and Environmental Control
Drinking Water Standard
N/A

\section{AMBIENT WATER QUALITY CRITERIA}

U.S. Environmental Protection Agency Ambient Water Quality Criteria

Human Health - Water and Fish Ingestion $144 \mathrm{ng}$

Human Health - Fish Ingestion Only $\quad 146 \mathrm{ng}$

Aquatic Organisms - FW Acute

Aquatic Organisms - FW Chronic

Aquatic Organisms - Marine Acute

Aquatic Organisms - Marine Chronic

$2.4 \mu \mathrm{g} / \mathrm{l}$

$.012 \mu \mathrm{g} / \mathrm{l}$

$2.1 \mu \mathrm{g} / 1$

$.025 \mu \mathrm{g} / 1$ 
South Carolina Department of Health and Environmental Control

Human Health Criteria

Fish and Aquatic Life - Continuous

Fish and Aquatic Life - Maximum
$.153 \mu \mathrm{g} / \mathrm{l}$.

$.012 \mu \mathrm{g} / \mathrm{l}$

$2.4 \mu \mathrm{g} / \mathrm{l}$

\section{REFERENCES}

U.S. EPA (U.S. Environmental Protection Agency). 1986a. Air Quality Criteria for Lead. Volumes I-IV. EPA 600/83/0286 CF. 
NAPHTHALENE; 2-METHYL NAPHTHALENE

CAS Nos.: $\quad$ 91-20-3; 91-57-6

Synonym(s) Tar camphor, albocarbon; Beta-methyl-naphthalene, naphthalene

Naphthalene and 2-methyl naphthalene are produced from distillation and fractionation of petroleum and coal tar (Sax and Lewis 1987; Coons et al. 1982). Naphthalene is used in the synthesis of carbonate insecticides, as a tanning agent, a moth repellant, and in the production of dyes and resins (EPA 1988d).

Naphthalene is a ringed structure with a molecular weight of 128.19. It is a white solid having the distinctive odor of mothballs, a boiling point of $218^{\circ} \mathrm{C}$, and a melting point of $80.5^{\circ} \mathrm{C}$. 2-methyl naphthalene is structurally similar to naphthalene, with the addition of a $\mathrm{CH}_{3}$ group in the 2 position. It is solid at ambient conditions, has a molecular weight of 142.20 , a melting point of $34.6^{\circ} \mathrm{C}$, and a boiling point of $241^{\circ} \mathrm{C}$. 2-methyl naphthalene is used in the production of certain insecticides (Weast et al. 1985).

\section{FATE AND TRANSPORT}

Most releases of naphthalene are to the air and result from the combustion of fossil fuels and the use of mothballs (Coons et al. 1982). Smaller amounts are released atmospherically during coal tar production and distillation and from aeration of public water treatment product (Coons et al. 1982). Naphthalene reacts with hydroxyl radicals in the atmosphere and degrades rapidly, with a half-life of 3-8 hours (Atkinson et al. 1984).

A small fraction of naphthalene releases occur to water, primarily from the coal tar industry (Coons et al. 1982). Degradation of naphthalene in water by microbial action occurs at a rate proportional to the concentration of naphthalene (Herbes 1981).

Very minor emissions of naphthalene occur to soil and those that do occur are from coal tar production, naphthalene production, sludge disposal from water treatment facilities, and use of pesticides (Coons et al. 1982). Biodegradation of naphthalene occurs in aerated soils with a half-life estimated at 8 days (Kappeler and Wuhrmann 1978).

\section{HUMAN HEALTH EFFECTS}

Acute inhalation exposure to naphthalene produces headaches, nausea, vomiting, and gastrointestinal pain. Longer exposure times also result in hemolytic anemia in neonates and renal disease (Linick 1983; Valaes et al. 1963), as-well as malaise, confusion, and anemia (Linick 1983).

Ingestion of naphthalene is associated with gastrointestinal distress (nausea, vomiting, diarrhea and pain), renal tubular necrosis and renal failure, hepatic insufficiency, respiratory collapse, and CNS dysfunction (convulsions, coma) (ATSDR 1989).

Most exposures to naphthalene have occurred in homes where mothballs were extensively used, and through accidental (through contaminated food) or intentional (suicidal) ingestion. 


\section{ECOLOGICAL EFFECTS}

Naphthalene is not expected to bioconcentrate in the environment, because most organisms readily metabolize it. There is data, however, that moderate amounts of naphthalene may be bioconcentrated in fish and aquatic invertibrates (Bysshe 1982). Other studies indicate that these organisms quickly eliminate naphthalene when placed in contaminant-free water (Eastmond et al. 1984; Callahan et al. 1979.)

\section{U.S. ENVIRONMENTAL PROTECTION AGENCY HUMAN HEALTH CRITERIA}

Oral Slope Factor

Inhalation Slope Factor

Chronic Oral RfD

Chronic Inhalation RfD
NA

NA

$4 \mathrm{E}-2 \mathrm{mg} / \mathrm{kg} /$ day

NA

\section{DRINKING WATER CRITERIA AND STANDARDS}

U.S. Environmental Protection Agency

Maximum Concentration Limit

Maximum Concentration Limit Goal

NA

NA

\section{AMBIENT WATER QUALITY CRITERIA}

U.S. Environmental Protection Agency Ambient Water Quality Criteria

$\begin{array}{ll}\text { Human Health - Water and Fish Ingestion } & \text { NA } \\ \text { Human Health - Fish Ingestion Only } & \text { NA } \\ \text { Aquatic Organisms - FW Acute } & 2300 \mu \mathrm{g} / \mathrm{L} \\ \text { Aquatic Organisms - FW Chronic } & 620 \mu \mathrm{g} / \mathrm{L} \\ \text { Aquatic Organisms - Marine Acute } & 2350 \mu \mathrm{g} / \mathrm{L} \\ \text { Aquatic Organisms - Marine Chronic } & \text { NA }\end{array}$

South Carolina Department of Health and Environmental Control Water Quality Criteria

Human Health Criteria

Fish and Aquatic Life - Continuous

Fish and Aquatic Life - Maximum
NA

NA

NA

$\mathrm{NA}=$ Not Available

\section{REFERENCES}

Agency for Toxic Substances and Disease Registry. 1989. Toxicological Profile for Naphthalene. Prepared by Clement International Corp., contract no. 205-88-0608. 
Atkinson, R., Aschmann, S.M., Pitts, JN, Jr. 1984. Kinetics of the reactions of naphthalene and biphenyl with hydroxyl radicals and with oxygen at $294 \pm 1 \mathrm{~K}$, Environ. Sci. Tech. 18: 1014-1022.

Bysshe, S.E. 1982. Bioconcentration factor in aquatic organisms. In: Lyman, W.J., ed. Handbook of chemical property estimation methods. New York, NY: McGray Hill, 5-1 - 5-30.

Callahan, M.A., Slimak, M.W., Gabel, N.W., et al. 1979. Water-relatead environmental fate of 129 priority pollutants: Volume I: Introduction and technical background, metals and inorganics, pesticide and PCBs. Washington, DC: U.S. Environmental Protection Agency, Office of Water Planning and Standards. EPA-440/4-79-029a. NTIS No. PB80-204373.

Coons, S., Burne, M., Goyer, M., et al. 1982. An exposure and risk assessment for benzo[a]pyrene and other polycyclic hydrocarbons: Vol. II. Naphthalene Final Draft Report. Washington, DC: US EPA, Office water regulations and standards.

Eastmond, D.A., Booth, G.M., Lee, M.L. 1984. Toxicity, accumulation, and elimination of polycyclic aromatic sulfur heterocycles in Daphnia magna Arch. Environ. Contam. 13:105-111.

EPA. 1988. US Environmental Protection Agency Federal Regulations 53:9138-9141.

Herbes. 1981. Rates of microbial transformation of polycyclic aromatic hydrocarbons in pristine and petroleum-contaminated sediments. Applied Environmental Microbiology 35:306-316.

Kappeler, T., Wuhrmann, K 1978. Microbial degradation of the water-soluble fraction of gas oil-I. Water Res. 12:327-333.

Linick, M. 1983. Illness associated with exposure to naphthalene in mothballs - Indiana. MMWR 32:34-35.

Sax, N.I. and Lewis, R.J., Sr. 1987. Hawley's Condensed Chemical Dictionary, NY, NY. Van Nostrand Reinhold Co. 775, 806.

Valaes, T., Doxiadis, S.A., Fessas, P. 1963. Acute hemolysis due to naphthalene inhalation. Journal of Pediatrics 63:904-915.

Weast, R.C., Astle, MJ., Beyer, W.H., eds. 1985. CRC handbook of chemistry and physics: Boca Raton, FL: CRC Press, Inc. 
THIS PAGE LEFT INTENTIONALLY BLANK 
NICKEL

CAS No.: 7440-02-0

Major Synonym(s) NP-2, Raney Alloy, NI-4303T

Nickel is a hard metal often alloyed with iron, copper, chromium, or zinc for use in making coins, jewelry, industrial valves, and stainless steel. Nickel compounds composed of nickel and other elements such as chlorine, sulfur, and oxygen, are used for coloring ceramics, nickel plating, battery production, and as catalysts of chemical reactions.

Nickel is a silvery white, odorless metal having a molecular weight of 58.69 , a melting point of $1453^{\circ} \mathrm{C}$, and a boiling point of $2732^{\circ} \mathrm{C}$ (Weast 1988).

\section{FATE AND TRANSPORT}

Nickel occurs naturally in the earth's crust and may be released to the environment from natural sources such as volcanos, forest fires, wind blown soil, and from human usage. The general population is exposed to nickel present in air, water, and food in low concentrations.

Nickel releases from anthropogenic sources are often in the form of airborne particulate matter. Large particles may be deposited to land and water through gravitational settling, whereas smaller particles will be deposited by precipitation (Schroeder et al. 1987)

Nickel adheres tightly to soil particles, especially those containing iron or manganese. Nickel is more tightly adsorbed to the sediment in alkaline pHs and may become detached and mobile in acidic environments.

\section{HUMAN HEALTH EFFECTS}

Inhalation exposures to nickel compounds in occupational settings have been associated with asthma (Tolot et al. 1956; Block and Young 1981) and pneumoconiosis (Zislin et al. 1969; Jones and Warner 1972). Irritation of the nasal membranes and epithelial dysplasia of the nasal mucosa have also be reported in workers exposed to nickel dust (Suschenkjo and Rafikova 1972; Torjussen et al. 1979).

In two studies assessing chronic nickel toxicity by inhalation in animals, severe effects, including death and pathological changes in the respiratory system were observed (Hueper 1958; Wehner et al. 1975).

A number of studies provide evidence that nickel refinery workers have increased risk of nasal and lung cancers from nickel vapors, dusts and aerosols (Sunderman 1977). Rats and guinea pigs, when exposed to nickel in a variety of forms, by inhalation, developed a significantly higher number of benign and malignant lung tumors (squamous cell carcinomas, fibrosarcomas and adenocarcinomas) when compared to control subjects (Hueper 1958; Sunderman et al. 1957, 1959; Ottolenghi et al. 1974). 
Nickel carbonate vapor has been shown to induce teratogenesis in rats (Sunderman et al. 1959). The U.S. EPA has classified nickel as a Group A carcinogen from the inhalation route of exposure.

Laboratory rats exposed to nickel for life experienced only minor effects (a mild decrease in mean body weight) (Schroeder et al. 1964; 1974). Subchronic dietary exposure of rats to nickel produced signs of hematological damage (decreased hematocrit and hemoglobin concentration) and a reduction in weight gain (Whanger 1973; Clary 1975). Other investigators also reported degenerative changes in the liver, kidney, and testes (Waltschewa et al. 1972). Nickel chloride, in doses of $1.2-20 \mathrm{mg} / \mathrm{kg}$, was found to cause increased resorption sites and malformations in fetuses of treated pregnant females (Storeng and Jonsen 1981). The U.S. EPA classified nickel as a toxic substance from the oral route of exposure.

Dermatitis and other dermatological effects are the most frequent results of dermal exposure to nickel (NAS 1975). No systemic effects have been reported due to dermal exposure.

\section{U.S. ENVIRONMENTAL PROTECTION AGENCY HUMAN HEALTH CRITERIA}

Oral Slope Factor

Inhalation Slope Factor

Chronic Oral RfD

Chronic Inhalation RfD
NA

$8.4 \mathrm{E}-01 \mathrm{~kg} / \mathrm{mg} /$ day $^{-1}$

$2 \mathrm{E}-02 \mathrm{mg} / \mathrm{kg} /$ day

$6 \mathrm{E}-04 \mathrm{mg} / \mathrm{m}^{3}$

\section{DRINKING WATER CRITERIA AND STANDARDS}

\section{U.S. Environmental Protection Agency}
Maximum Concentration Limit
$0.1 \mathrm{mg} / \mathrm{L}$
Maximum Concentration Limit Goal
$0.1 \mathrm{mg} / \mathrm{L}$

\section{AMBIENT WATER QUALITY CRITERIA}

U.S. Environmental Protection Agency Ambient Water Quality Criteria

Human Health - Water and Fish Ingestion

$13.4 \mu \mathrm{g} / \mathrm{L}$

Human Health - Fish Ingestion Only

$100 \mu \mathrm{g} / \mathrm{L}$

Aquatic Organisms - FW Acute

Aquatic Organisms - FW Chronic

$1800 \mu \mathrm{g} / \mathrm{L}$

Aquatic Organisms - Marine Acute

$96 \mu \mathrm{g} / \mathrm{L}$

Aquatic Organisms - Marine Chronic

$140 \mu \mathrm{g} / \mathrm{L}$

$7.1 \mu \mathrm{g} / \mathrm{L}$ 
South Carolina Department of Health and Environmental Control Water Quality Criteria

Human Health Criteria .

Fish and Aquatic Life - Continuous

Fish and Aquatic Life - Maximum
$4584 \mu \mathrm{g} / \mathrm{L}$

$87.71 \mu \mathrm{g} / \mathrm{L}$

$789 \mu \mathrm{g} / \mathrm{L}$

NA $=$ Not Available

\section{REFERENCES}

Agency for Toxic Substances and Disease Registry. 1990. Toxicological Profile for Nickel. Prepared by Clement International Corp., contract no. 205-88-0608.

Block, G.T. and M. Young. 1982. Asthma induced by nickel. J. Am. Med. Assoc. 247:16001602 .

Clary, J.J. 1975. Nickel chloride-induced metabolic changes in the rat and guinea pig. Toxicol Appl Pharmacol 31:55-65.

Hueper., W.C. 1958. Experimental studies in metal carcinogenesis. IX. Pulmonary lesions in guinea pigs and rats exposed to prolonged inhalation of powdered metallic nickel. arch. Pathol. 65:600-607.

NAS. 1975. Nickel. National Academy of Sciences. Washington, DC: National Academy of Sciences, 4, 5, 17.

Ottolenghi, A.D., J.K. Haseman, W.W. Payne, H.L. Falk and H.N. MacFarland. 1974. Inhalation studies of nickel sulfide in pulmonary carcinogenesis of rats. J. natl. Cancer Inst. 54-1.165-1172.

Schroeder, H.A., J.J. Balassa and W. H. Vinton. 1964. Chromium, lead, cadmium, nickel and titanium in mice: Effect on mortality, tumors, and tissue levels. J. Nutr. 104:239243.

Schroeder, H.A., M. Mitchener and A. P. Nason. 1974. Life-term effects of nickel in rats: Survival, tumors, interactions with trace elements and tissue levels. J. Nutr. 104: 239243.

Schroeder WH, Dobson M, Kane DM. 1987. Toxic trace elements associated with airborne particulate matter: A review. Air Pollut Control Assoc 11:1267-1287.

Storeng, R. and J. Jonsen. 1981. Nickel toxicity in early embryogenesis in mice. Toxicology 20:45-51. 
Sunderman, F.W. Jr. 1977. The metabolism and toxicology of nickel. In: Brow, S.S., Ed. Clinical Chemistry and Chemical Toxicology of Metals. Elsevier, Amsterdam. pp. 231-259.

Sunderman, F.S., J.F. Kinkcaid, A.J. Donnelly and B. West. 1957. Nickel poisoning. IV. Chronic exposure of rats to nickel carbonyl: A report after one year of observation. Arch. Ind. Health. 16:4800-485.

Suschenkjo, A.V. and K.S. Rafinkova. 1972. [Problems of occupational health in hydrometallurgy of copper, nickel and cobalt based on sulfide ores]. Gig. Tr. Prof. Zabol. 16:42-45.

Tolot, F., P. Brodeur and G. Neulat. 1956. [Troubles pulmonaires asthmatiformes chez des ouviers a l'inhalation de chrome, nickel at aniline]. Arch. Mal. Prof. Med. Trav. Secur. Soc. 18:288-293

Torjussen, W., L.A. Solberg and A.C. Hogetveit. 1979. Histopathological changes of the nasal mucosa in active and retired nickel workers. Br. J. Cancer 40(4); 568-580.

Waltschewa, V.W. et al. 1972. [Testicular changes due to long-term administration of nickel sulfate in rats]. Exp. Pathol. 6:116.

Weast, R.C. editor. 1988. Handbook of Chemistry and Physics. 69th edition. Boca Raton, FL. CRC Press.

Wehner, A.P., R.H. Busch, R.J. Olson and D.K. Craig. 1975. Chronic inhalation of nickel oxide and cigarette smoke by hamsters. Am. Ind. Hyg. Assoc. J. 36:801-8910.

Whanger, P.D. 1973. Effects of dietary nickel on enzyme activities and mineral content of rats. Toxicol. Appl. Pharmacol. 25:323-331.

Zislin, D.M., S.M. Ganjuskina, E.S. Dubilina and N.V. Tjusnjakova. 1969. The residual volume of the lungs in complex assessment of the functional status of the respiratory system in initial and suspected pneumoconiosis. Gig. Tr. Prof. Zabol. 13:26-29. 
PENTACHLOROPHENOL

CAS No.: $\quad 87-86-5$

Synonym(s) PCP, Penchlorol, Penta

Pentachlorophenol is a pesticide which gained wide usage before its large number of applications were restricted. Historically, pentachlorophenol was used extensively as a wood treatment and as a slimicide in industrial cooling towers. At one time it was also registered as a termiticide, fungicide, herbicide, molluscicide, and algicide, and as a disinfectant (Cirelli 1978). Its use is currently limited to treatment of wooden utility poles and for formulations of fungicides and insecticides (ATSDR 1992).

Pentachlorophenol is a colorless or white crystalline solid in its pure form, but appears as dark grey or brown pellets in its crude form. It has a molecular weight of 266.35 , a melting point of $19^{\circ} \mathrm{C}$, and a boiling point of $309-310^{\circ} \mathrm{C}$. When pentachlorophenol reaches its boiling point it decomposes: It is very soluble in alcohol, ether, and other organic solvents (HSDB 1992).

\section{FATE AND TRANSPORT}

Pentachlorophenol is ubiquitous in the environment and occurs in virtually every media (Hazdat 1992). Pentachlorophenol volatilizes from treated wood, during production and handling, during pesticide application, and historically, evaporation from cooling towers occurred. Transport of pentachlorophenol to other media can be attributed to wet deposition. Degradation of pentachlorophenol in air is most likely the result of photolysis (Gab et al. 1975).

In the past, pentachlorophenol was released onto the soil through herbicide spraying and from wood treatment evaporation ponds. Current contamination of soil may occur as leachate from treated wood poles, as spills during production and handling, from atmospheric deposition, and from hazardous waste sites. Vertical transport through soils occurs in neutral or alkaline environments, whereas pentachlorophenol is adsorbed to soils in acidic conditions (Kuwatsuka and Igarashi 1975). Pentachlorophenol is rapidly metabolized by soil microbes, and this is the major degradation pathway in soils (Kaufman 1978). The degradation rate increases with increasing organic content an/or increasing moisture content (Young and Carroll 1951).

Surface water contamination with pentachlorophenol occurs through direct release or is deposited from other media and non-point sources (EPA 1979). Pentachlorophenol is structurally stable from hydrolysis and oxidation in surface waters (Callahan et al. 1979) and evaporative losses are minimal (Pignatello et al. 1983). Conversely, pentachlorophenol photolysis occurs rapidly at neutral $\mathrm{pH}$, with an estimated half life of 3.5 hours at $\mathrm{pH} 7.3$ or 100 hours at pH 3.3 (Wong and Cosby 1981). Biodegradation is also an important mechanism for decomposition of pentachlorophenol and occurs more rapidly in aerobic conditions than in anaerobic environments (Pignatello et al. 1983). 


\section{HUMAN HEALTH EFFECTS}

Acute inhalation of pentachlorophenol vapors has caused a number of deaths in humans and experimental animals. Tachycardia, jaundice, and hemolytic anemia have been observed following acute inhalation exposure (Hassan et al. 1985). Other effects of chronic inhalation of pentachlorophenol include respiratory inflammation and bronchitis (Baader and Bauer 1951), enlarged liver (Gordon 1956), and decreased glomerular filtration rate and tubular function (Begley et al. 1977).

Tachycardia, vascular damage, and heart failure have been associated with oral pentachlorophenol ingestion in humans and experimental animals (Haley 1977; Deichmann et al. 1942). Hepatic and renal function are compromised following ingestion of pentachlorophenol. Changes in hepatic enzymes and glycogen storage levels occurred in pentachlorophenol treated animals, as did hepatic necrosis (Johnson et al. 1973; Kimbrough and Linder 1978). The ratio of kidney weight to body weight was increased in pentachlorophenol treated rats (Johnson et al.1973). Although no pathological changes were observed under histological examination, renal function appears to be compromised by chronic pentachlorophenol exposure. For example, Greichus and coworkers (1979) reported blood urea nitrogen (BUN) levels were elevated in pigs after 15 days of 10 or $15 \mathrm{mg} / \mathrm{kg}$ oral ingestion of pentachlorophenol. Immunologic suppression and neurological effects such as hyperthermia have also accompanied chronic pentachlorophenol ingestion.

Pentachlorophenol is fetotoxic and embryotoxic, but not mutagenic (Larsen 1976; Courtney et al. 1976). Dwarfism, exencephaly, micropthalmia, and missing tails were common malformations in rats exposed to pentachlorophenol gestationally (Larsen 1976; Larsen et al. 1975). The EPA has classified pentachlorophenol as a group B2 substance (probable human carcinogen) based on animal studies (IRIS 1992).

\section{ECOLOGICAL EFFECTS}

Fish and freshwater mussel were observed to accumulate pentachlorophenol in contaminated water but quickly purged the tissue of pentachlorophenol after placement in uncontaminated water (Pruitt et al. 1977; Makela et al. 1991). Fish, algae, and aquatic invertebrates all have a high calculated bioconcentration potential (EPA 1986), however this has not been substantiated experimentally. All evidence to date indicates that neither aquatic nor terrestrial species bioconcentrate pentachlorophenol to any significant degree. Likewise, biomagnification in the food chain has not been demonstrated (ATSDR 1991).

\section{US ENVIRONMENTAL PROTECTION AGENCY HUMAN HEALTH CRITERIA}

Oral Slope Factor

Inhalation Slope Factor

Chronic Oral RfD

Chronic Inhalation RfD
$1.2 \mathrm{E}-1 \mathrm{mg} / \mathrm{kg} /$ day $^{-1}$

NA

$3 E-2 \mathrm{mg} / \mathrm{kg} /$ day

NA 


\section{DRINKING WATER CRITERIA AND STANDARDS}

U.S. Environmental Protection Agency

Maximum Concentration Limit Maximum Concentration Limit Goal

$0.001 \mathrm{mg} / \mathrm{L}$

$0.0 \mathrm{mg} / \mathrm{L}$

\section{AMBIENT WATER QUALITY CRITERIA}

U.S. Environmental Protection Agency Ambient Water Quality Criteria

Human Health-Water and Fish Ingestion

NA

Human Health-Fish Ingestion Only

NA

Aquatic Organisms-FW Acute

$55 \mu \mathrm{g} / \mathrm{l}$

Aquatic Organisms-FW Chronic

$32 \mu \mathrm{g} / \mathrm{l}$

Aquatic Organisms-Marine Acute

$53 \mu \mathrm{g} / \mathrm{l}$

Aquatic Organisms-Marine Chronic

$34 \mu \mathrm{g} / \mathrm{l}$

South Carolina Department of Health and Environmental Control

Human Health Criteria

Fish and Aquatic Life-Continuous

Fish and Aquatic Life-Maximum
$1010 \mu \mathrm{g} / \mathrm{l}$

$2.1 \mu \mathrm{g} / \mathrm{l}$

$3.32 \mu \mathrm{g} / \mathrm{l}$

NA $=$ Not Available

\section{REFERENCES}

Agency for Toxic Substance and Disease Registry (ATSDR). 1992. Draft Toxicological Profile for Pentachlorophenol. Produced by Clement International Assoc. U.S. Department of Health and Human Services.

Baader, E.W., Bauer, H.J. 1951. Industrial intoxication due to pentachlorophenol. Ind. Med.Surg. 20:286-290.

Begley,J.Reichert, A.W., Siesmen, A.W. et al. 1977. Association between renal function tests and pentachlorophenol exposure. Clin.Toxicol. 11:97-106.

Cirelli, D.P. 1978. Patterns of pentachlorophenol usage in America-an overview. In: Rao, K.R. ed. Pentachlorophenol, Chemistry, Pharmacology, and Environmental Toxicology. New York, Plenum Press, 13-18.

Courtney, K.D., Copeland ,M.F. ,Robbins, A. 1976. The effects of pentachloronitrobenzene, hexachlorobenzene, and related compounds on fetal development. Toxicol. Appl. Pharmacol. 35:239-256. 
Deichmann, W., Machle, W., Kitzmiller, K.V. ,et al. 1942. Acute and chronic effects of pentachlorophenol and sodium pentachlorophenate upon experimental animals. J. Pharmacol. Exp. Therap. 76:104-117.

EPA 1979. Timber Products. U.S.Environmental Protection Agency. Federal Register 45:62810-62846.

EPA. 1986. Ambient water quality criteria for pentachlorophenol. Washington,D.C: U.S. Environmental Protection Agency, Office of Water Regulations and Standards, Criteria and Standards Division. EPA 440/5-86-009.

Gab, S., Nitz, H., Korte, F. 1983. Photomineralization of certain aromatic xenobiotica. Chemosphere 4:251-257.

Gordon, D. 1956. How dangerous is pentachlorophenol? Med.J.Australia 43:485-488.

Greichus, Y.A., Libal, G.W., Johnson, D.D. 1979. Diagnosis and physiologic effects of pentachlorophenol on young pigs: Part I. Effects of purified pentachlorophenol. Bull.Environ.Contam.Toxicol. 23:418-422.

Haley, T.J. 1977. Human poisoning with pentachlorophenol and its treatment. Entotoxicol. Environ .Saf. 1:343-347.

Hassan, A.B., Hiseligmann, H., Bassan, H.M. 1985. Intravascular hemolysis induced pentachlorophenol. Br. Med. J. J291:21-22.

HAZDAT. 1992. Agency for Toxic Substances and Disease Registry (ATSDR). Atlanta, GA.

HSDB. 1992. Hazardous Substance Data Bank. National Library of Medicine, National Toxicology Information Program, Bethesda, MD.

IRIS. 1992. Integrated risk Information Systems. u.S.Environmental Protection Agency, Office of Health and Environmental Assessment, Environmental Criteria and Assessment Office, Cincinnati,OH.

Johnson , R.L., Gehring, P.J., Kociba, R.J., et al. 1973. Chlorinated dibenzodioxins and pentachlorophenol. Environ.Health Perspect. 5:171-175.

Kaufman, D.D. 1978. Degradation of pentachlorophenol in soil, and by soil organisms. In: Rao, K.R., ed. Pentachlorophenol: Chemistry, Pharmacology, and Environmental Toxicology. New York. Plenum Press 27-39.

Kimbrough, R.D., Linder, R.E. 1978. The effect of technical and purified pentachlorophenol on the rat liver. Toxicol. Appl. Pharmacol. 46:151-162. 
Kuwatsuka, S., Igarashi ,M. 1975. Degradation of Pentachlorophenol in soil: II. The relationship between the degradation. Soil Sci. Plant Nutr. 21:405-414.

Larsen, R.V. 1976. The placental transfer and teratology of pentachlorophenol in rats. Diss. Abstr. Intr. B37:1184-

Larsen, R.V., Born, G.S., Kessler, W.V., et al. 1975. Placental transfer and teratology of pentachlorophenol in rats. Environ.Lett. 10:121-128.

Pignatello, JJ., Martinson, M.M., Steiert, J.G. et al: 1983. Biodegradation and photolysis of pentachlorophenol in artificial freshwater streams. Appl. Environ. Microbiol. 46:1024- 1031.

Pruitt, G.W., Grantham, B.J., Pierce, R.H. 1977. Accumulation and elimination of pentachlorophenol by the bluegill, Lepomis macrochirus. Transactions of the American Fisheries Society. 106:462-465.Makela,T.P.,

Petanan, T., Kukkonen, J. et al. 1991. Accumulation and depuration of chlorinated phenolics in the freshwater mussel (Anodonta anatina L.). Ecotoxicol. Environ. Safety 22:153-163.

Scow, K., Goyer, M., Payne, E. et al. 1980. Exposure and risk assessment for pentachlorophenol. Washington, D.C. U.S. Environmental Protection Agency. NTIS PB85-211944. EPA 440/4-81-021.

Wong, A.A., Cosby, D.G. 1981. Photodecomposition of pentachlorophenol in water. J. Agric. Food Chem. 29:130-135.

Young, H.C., Carroll, J.C. 1951. The decomposition of pentachlorophenol when applied as a residual pre-emergence pesticide. Agron. 43:504-507. 


\section{THIS PAGE LEFT INTENTIONALLY BLANK}




\section{POLYCHLORINATED BIPHENYLS - AROCLOR 1254, AROCLOR 1260 \\ CAS Nos.: $\quad 11097-69-1,11096-82-5$ \\ Major Synonym(s) PCB, Chlorodiphenyls}

Polychlorinated biphenyls (PCBs) are anthropogenic in origin. They were widely used as coolants and lubricators in transformers, capacitors, and other electrical equipment. PCBs have also been used in fluorescent lighting fixtures, and as plasticizers for celluloses, vinyl resins, and crude rubbers. PCBs have not been manufactured in the United States since 1977 because of their harmful effects on human health and the environment. Unfortunately, because of their presence in landfills and equipment produced prior to the cessation of their production, PCBs still find their way into the environment.

There are 209 individual compounds which comprise the family of PCBs. Some of the PCB mixtures which were available commercially are known by the trade name Aroclor. These compounds are colorless to pale yellow viscous liquids which solidify below $50^{\circ} \mathrm{F}$. They have a mild hydrocarbon odor and are non-flammable. Boiling points range from $689-734^{\circ} \mathrm{F}$ and vapor pressure is $.00006 \mathrm{~mm} \mathrm{Hg}$ at $20^{\circ} \mathrm{C}$.

\section{FATE AND TRANSPORT}

PCBs were disposed of in landfills prior to the recognition of their harmful effects. Adsorption of PCBs to sediments is strong, particularly in organic soils. The atmospheric concentration of PCBs near Superfund hazardous waste sites is elevated above background concentrations, suggesting that volatilization is a transport mechanism from soil to air (Hermanson and Hites 1989). Degradation of PCBs in soil is slow and limited to biodegradation (ATSDR 1991). Storm water runoff may transport PCBs from sediments to surface waters.

In water, PCBS will adsorb to sediments or other organic matter (Eisenreich et al. 1983). While adsorbed to aquatic sediment, PCBs are sequestered from the water column, however, these sediments act as a sink and may release PCBs slowly for long periods of time (Baker et al. 1985). Photolysis, biodegradation, and evaporation are the pathways by which PCBs are degraded or removed from the water column. Photolysis of PCBs proceeds slowly but can be enhanced by the addition of sodium borohydride (Epling et al. 1988). Biodegradation of PCBs occurs in both aerobic and anaerobic conditions.

In the atmosphere, $\mathrm{PCBs}$ react with hydroxyl radicals and are transformed. The rate of transformation varies with the number of chlorines present in the molecule (Atkinson 1987). Photolytic degradation of PCBs has been observed under laboratory conditions and may also contribute to PCB degradation in air (Hutzinger et al. 1972).

\section{HUMAN HEALTH EFFECTS}

Workers exposed to Arochlor vapors developed chloracne, eczematous rashes, hyperpigmentation, eye irritation, respiratory irritation, gastrointestinal symptoms, neurological symptoms and liver damage (Meigs et al. 1954; Ouw et al. 1976; Fischbein et al. 1979). 
Two additional occupational studies have been reported an association between PCB exposure and increased risk of death from all neoplasms (Bertazzi et al. 1981) and incidence of malignant melanomas and pancreatic carcinomas (Bahn et al. 1976). Although there were methodological limitations to these studies, the IARC (1978) felt the study by Bahn et al. (1976) suggested evidence of human carcinogenicity.

Humans exposed to an average total oral dose of $500-800 \mathrm{mg}$ PCBs (in contaminated food) showed dermatological symptoms, eye problems, chronic bronchitis, some neurologic and muscular disorders and hepatotoxicity (Reggiani and Bruppacher 1985; Kuratsune et al. 1972). Exposure also causes suppression of the immune system, with resultant increased susceptibility to infections (Shigematsu et al. 1978; Chang et al. 1981; Chang et al. 1982; Lu and $\mathrm{Wu}$ 1985).

Subchronic and chronic PCB exposure in animals causes skin lesions, immune system effects and alterations to the liver (Zinki 1977; Barsotti et al. 1976; Allen et al. 1972; Kimbrough et al. 1975). Hepatic enzyme induction has been observed following exposure through the diet (Bruckner et al. 1974).

Many women exposed through a food contamination incident reported irregular menstrual cycles (Reggiani and Bruppacher 1985). Studies showing reduced birth weights, slow weight gain, reduced gestational ages, and behavioral deficits in infants following maternal ingestion of PCB-contaminated fish (Fein et al. 1984a,b; Jacobson et al. 1983; Jacobson et al. 1984) should be viewed with caution because of possible confounding factors (Kimbrough 1987).

In a follow-up of patients exposed to PCB-contaminated food, $35 \%$ of the deceased died from malignancies of various organs (Urabe et al. 1979). Further details on this study are lacking.

The U.S. EPA considers PCBs to be carcinogens from the oral route of exposure (weight of evidence B2), as reported in IRIS. The oral slope factor established for these PCBs is $7.7(\mathrm{mg} / \mathrm{kg} / \text { day })^{-1}$. This value was derived from a study by Norback and Weltman (1985).

Workers exposed to PCBs through the skin developed skin diseases and hepatomegaly (Marconi et al. 1981). Dermal application has caused hepatic enzyme induction in laboratory animals (Bickers et al. 1975).

\section{ECOLOGICAL EFFECTS}

Aquatic species such as fish, shrimp, and oyster bioaccumulate PCBs many orders of magnitude higher than ambient water concentrations (Leifer et al. 1983). There is a correlation between the amount of bioconcentrated by a species and the water zone in which they predominantly reside and the fate of PCBs. For example, bottom dwelling species concentrate greater amounts of PCBs than species which reside in the median water zones. Likewise, the concentration of PCBs in the bottom sediments is several orders of magnitude higher than the concentration of PCBs within the water column (ATSDR 1991). 
Terrestrial plants tend to accumulate PCBs, primarily from transfer of vapor phase PCBs from the atmosphere to aerial portions of plants. Uptake of PCBs from soil through the roots of plants is minimal based upon calculation of the bioconcentration factor (Concentration of $\mathrm{PCB}$ in the organism divided by concentration of $\mathrm{PCB}$ in the ambient medium) (O'Connor et al. 1990).

\section{U.S. ENVIRONMENTAL PROTECTION AGENCY HUMAN HEALTH CRITERIA} (As measured for PCB-1254 and PCB-1260)

Oral Slope Factor

Inhalation Slope Factor

Chronic Oral RfD

Chronic Inhalation RfD
$7.7 \mathrm{E}+0 \mathrm{~kg} / \mathrm{mg} /$ day $^{-1}$

NA

$7 \mathrm{E}-5 \mathrm{mg} / \mathrm{kg} /$ day

NA

\section{DRINKING WATER CRITERIA AND STANDARDS}

U.S. Environmental Protection Agency

Maximum Concentration Limit

Maximum Concentration Limit Goal

$0.0005 \mathrm{~m} / \mathrm{L}$ (All PCBs)

$0.0 \mathrm{mg} / \mathrm{L}$ (All PCBs)

\section{AMBIENT WATER QUALITY CRITERIA}

U.S. Environmental Protection Agency Ambient Water Quality Criteria

Human Health - Water and Fish Ingestion

Human Health - Fish Ingestion Only

Aquatic Organisms - FW Acute

Aquatic Organisms - FW Chronic

Aquatic Organisms - Marine Acute

Aquatic Organisms - Marine Chronic
$4.4 \mathrm{E}-05 \mu \mathrm{g} / \mathrm{L}$

$4.4 \mathrm{E}-05 \mu \mathrm{g} / \mathrm{L}$

$2.0 \mu \mathrm{g} / \mathrm{L}$

$0.014 \mu \mathrm{g} / \mathrm{L}$

$10.0 \mu \mathrm{g} / \mathrm{L}$

$0.03 \mu \mathrm{g} / \mathrm{L}$

South Carolina Department of Health and Environmental Control Water Quality Criteria

Human Health Criteria

Fish and Aquatic Life - Continuous

Fish and Aquatic Life - Maximum
4.5E-04 $\mu \mathrm{g} / \mathrm{L}$ (All PCBs) $0.014 \mu \mathrm{g} / \mathrm{L}$ (All PCBs)

NA $=$ Not Available

\section{REFERENCES:}

Agency for Toxic Substances and Disease Registry. 1991. Toxicology Profile for Polychlorinated Biphenals. Prepared by Clement International Corporation, contract no. 205-88-0608. 
Allen, J.R. and D.A. Barsotti. 1976. The effects of transplacental and mammary movement of PCBs on infant rhesus monkeys. Toxicology 6:331-340.

Allen, J.R., L.A. Carstens and D.A. Barsotti. 1974a. Residual effects of short-term, lowlevel exposure of nonhuman primates to polychlorinated biphenyls. Toxicol. Appl. Pharmacol. 30:440-451.

Allen, J.R., D.H. Norback and I.C. Hsu. 1974b. Tissue modifications in monkeys as related to absorption, distribution and excretion of polychlorinated biphenyls. Arch. Environ. Contam. Toxicol. 2:86-95,

Atkinson, R. 1987. Estimation of $\mathrm{OH}$ radical reaction rate constants and atmospheric lifetimes for polychlorobiphenyls, dibenzo-p-dioxins, and dibenzofurans. Environ. Sci. Technol. 21:305-307.

Bahn, A.K, I. Rosenwaike, N. Herrman, P. Grover, J. Stellman and K. O'Leary. 1976. Melanoma after exposure to PCBs. New Engl. J. Med. 295-450.

Baker, J.E., Eisenreich, S.J., Johnson, T.C. et al. 1985. Chlorinated hydrocarbon cycling in the benthic nepheloid layer of Lake Superior. Environ. Sci. Technol. 19:854-861.

Barsotti, D.A., R.J. Marlar and J.R. Allen. 1976. Reproductive dysfunction in rhesus monkeys exposed to low levels of polychlorinated biphenyls. Food Cosmet. Toxicol. 14:99-103.

Bertazzi, P.A., C. Zocchetti, S. Guercilena, M.D. Folia, A. Pesatori and L. Ribaldi. 1981. Mortality study of male and female workers exposed to PCBs. Paper presented at the International Symposium on Prevention of Occupational Cancer, Helsinki, Finland, April 1981.

Bruckner, J.V., KL. Khamma and H.H. Cornish. 1974. Effect of prolonged ingestion of polychlorinated biphenyls on the rat. Food Cosmet. Toxicol. 12:323-330.

Chang, KJ., K. Hsieh, T. Lee, S. Tang and T. Tung. 1981. Immunologic evaluation of patients with polychlorinated biphenyls poisoning: Determination of lymphocyte subpopulations. Toxicol. Appl. Pharmacol. 61:58-63.

Chang, KJ., K. Hsieh, S. Tang and T.C. Tung. 1982. Immunologic evaluation of patients with polychlorinated biphenyls poisoning: Evaluation of delayed-type skin hypersensitive response and its relation to clinical studies. J. Toxicol. Environ. Health. 9:217-223.

Eisenreich, S.J., Capel, P.D., Looney, B.B. 1983. PCB dynamics in Lake Superior water. In: Mackay D., ed. Physical behavior of PCBs in the Great Lakes. Ann Arbor, MI: Ann Arbor Science Press, 181-211. 
Epling, G.A., McVicar, W.M., Kumar, A. 1988. Borohydride-enhanced photodegradation of Aroclor 1232, 1242, 1254, and 1260. Chemiosphere 17: 1355-1362.

Fein, G.G., J.L. Jacobson, S.W. Jacobson, P.M. Schwartz and J.K. Dowler. 1984a. Prenatal exposure to polychlorinated biphenyls. Effects on birth size and gestational age. J. Pediatr. 102:315-320.

Fein, G.G., J.L. Jacobson, S.W. Jacobson and P.M. Schwartz. 1984b. Intrauterine Exposure of Humans to PCBs: Newborn Effects. NTIS BP84-188887. U.S. EPA 600/384-060.

Fischbein, A., M.S. Wolff, R. Lilas, J. Thornton and I.J. Selikoff. 1979. Clinical findings among PCB-exposed capacitor manufacturing workers. Ann. N.Y. Acad. Sci. 320:702-715.

Hermanson, M.H., Hites, R.A. 1989 . Long-term measurements of atmospheric polychlorinated biphenyls in the vicinity of Superfund dumps. Environ. Sci. Technol. 23:1253-1258.

Hutzinger, O., Safe, S., Zitko, V. 1972. Photochemical degradation of chlorobiphenyls (PCBs). Environ. Health Perspect. 1:15-20.

IARC (International Agency for Research on Cancer). 1978. IARC Monographs on the Evaluation of the Carcinogenic Risk of Chemicals to Humans. Polychlorinated Biphenyls and Polybrominated Biphenyls. 18:43-103.

Jacobson, J.L., G.G. Fein, S.W. Jacobsen, P.M. Schwartz and J.K. Dowler. 1984. The transfer of polychlorinated biphenyls (PCBs) and polychlorinated biphenyls (PBBs) across the human placenta and into maternal milk. Am. J. Pub. Health. 74:378-379.

Jacobson, S.W., J.L. Jacobson, P.M. Schwartz and G.G. Fein. 1983. Intrauterine exposure of human newborns to PCBs: Human and Environmental Hazards. Boston, Butterworth Publishers, pp. 311-343.

Kimbrough, R.D., R.A. Squire, R.E. Linder, J.D. Strandberg, R.J. Montali, and V.W. Burse. 1975. Induction of liver tumors in Sherman strain rats by polychlorinated biphenyl Aroclor 1260. J. Natl. Cancer Inst. 55:1453-1459.

Kimbrough, R.D. -1987. Human health effect of polychlorinated biphenyls (PCBs) and polybominated biphenyls (PBBs) Ann Rev. Pharmacol. Toxicol. 27:87-111.

Kuratsune, M., T. Yoshimura, J. Matsuzaka and A. Yamaguchi. 1972. Epidemiologic study on Yusho, a poisoning caused by ingestion of rice oil contaminated with a commercial brand of polychlorinated biphenyls. Environ. Health Perspect. 1:119-128. 
Leifer, A., Brink, R.H., Thom, G.C., et al. 1983. Environmental transport and transformation of polychlorinated biphenyls. EPA-560/5-83-025. Washington, DC: Office of Pesticides and Toxic Substances, U.S. Environmental Protection Agency, NTIS PB84-142579.

Lu, Y. and Y. Wu. 1985. Clinical findings and immunological abnormalities in Yu-Cheng patients. Environ. Health Perspect. 59:17-29.

Maroni, J., T. Colombit, S. Carboni, E. Ferioli and V. Foa. 1981. Occupational exposure to polychlorinated biphenyls in electrical workers. Br. J. Ind. Med. 38:40-60.

McConnell, E.E. 1980. Acute and chronic toxicity, carcinogenesis, reproduction, teratogenesis and mutagenesis in animals. In: Kimbrough, R. D., ed. Halogenated Biphenyls, Terphenyls, Naphthalenes, Dibenzodioxins and Related Products. New York, Elsevier/North Holland Biomedical Press, pp. 109-150.

Meigs, J.W., Albom, J.J., Kartin, B.L. 1954. Chloracne from an unusual exposure to Aroclor. J. Am. Med. Assoc. 154:1417-1418.

Norback, D.H. and R.H. Weltman. 1985. Polychlorinated biphenyl induction of hepatocellular carcinoma in the Sprague-Dawley rat. Environ. Health Perspect. 60:97-105.

O'Connor, G.A., Kiehl, D, Eiceman, GA, et al. 1990. Plant uptake of sludge-borne PCBs. J. Environ Qual 19:113-118.

Ouw, H.K., Simpson, G.R., and Siyali, D.S. 1976. Use and health effects of Arochlor 1242, a polychlorinated biphenyl in an electrical industry. Arch. Environ. Health. 31:189-194.

Reggiani, G. and R. Bruppacher. 1985. Symptoms, signs and findings in humans exposed to PCBs and their derivatives. Environ. Health Perspect. 59:225-232.

Shigematsu, T., S. Ishimaru, R. Saito, T. Ikeda, K. Matsuba, K. Sugiyama and Y. Masuda. 1978. Respiratory involvement in PCB poisoning. Environ. Res. 16:92-100.

U.S. EPA (U.S. Environmental Protection Agency). 1984. Health effects assessment for polychlorinated biphenyls. Environmental Criteria and Assessment Office, Cincinnati, $\mathrm{OH}$. EPA 540/1-86-004.

Zinki, J.G. 1977. Skin and liver lesions in rats fed a polychlorinated biphenyl mixture. Arch. Environ. Contamin. Toxicol. 5:217-288. 


\section{POLYCYLIC AROMATIC HYDROCARBONS (PAH)}

CAS Nos:

$82-32-9,208-96-8,120-12-7,56-55-3,50-32-8,205-99-2$, 207-08-9, 191-24-2, 218-01-9, 53-70-3, 206-44-0, 86-73-7, $193-39-5,85-01-8,129-00-0$

Major Synonym(s) Acenaphthene, Acenapthylene, Anthracine, Benzo(a)anthracene, Benzo(a)pyrene, Benzo(b)fluoranthene, Benzo(g,h,i) Perylene, Benzo(k) fluoranthene, Chrysene, Dibenzo(a,h)anthracine, Fluoranthene, Fluorene, indeno(1,2,3-cd)pyrene, Phenanthrene, Pyrene

Polycyclic Aromatic Hydrocarbons (PAH) are a group of chemicals produced from combustion from automobiles and other gas-burning engines, wood-burning stoves and furnaces, tobacco smoke, industrial smoke, and char-broiled foods (IARC 1983). PAHs occur in nature in crude oil and shale oil and are released during forest fires and volcanic eruptions (HSDB 1988). Anthracene, acenaphthene, fluorene, phenanthrene and fluoranthene are produced commercially for industrial use (Hawley 1987; Windholz 1983; HSDB 1988; NRC 1983).

As a group of similar chemical compounds, PAHs typically exist as colorless, white, or pale yellow-green solids. They tend to occur as mixtures of two or more PAHs. Molecular weights range from $158.2-278.35$. Most are insoluble in water, yet soluble in organics.

\section{FATE AND TRANSPORT}

PAHs are further classified according to molecular weight and the members within a group behave similarly in the environment (ATSDR 1989). They are grouped as follows:

-. Low molecular weight PAHs (153-178 g/mol): acenapthene, acenapthylene, anthracene, fluorene, and phenanthrene

- Medium molecular weight PAHs $(202 \mathrm{~g} / \mathrm{mol})$ : fluoranthene and pyrene

- High molecular weight PAHs (228-278 $\mathrm{g} / \mathrm{mol}$ ): benz(a)anthracene, benzo(b)fluoranthene, benzo(k)fluoranthene, benzo(g,h,i) perylene, benzo(a)pyrene, chrysene, dibenzo(a,h) anthracine, and indeno(1,2,3-cd)pyrene

The bulk of PAH released to the environment is to the air, where they occur as vapor or as solids adsorbed to particulate matter. PAHs sorbed to particulate matter are transported and deposited according to the size of the particles. PAHs may be photo-oxidized in the air or may interact with other atmospheric pollutants (NRC 1983). Some of the products of these reactions are also toxic (Gibson et. al. 1978)

PAH contamination of surface waters arises primarily from atmospheric deposition (Santodonata 1981), however, industrial effluents and runoff, municipal and urban runoff and oil spills also contribute (Barric 1982; Snider and Manning 1982; Giger and Blumer 
1974; Mackenzie and Hunter 1979). A certain amount of PAH will volatilize from water to the atmosphere, particularly low molecular weight compounds (Readman et al. 1982). Microbes will biodegrade low molecular weight compounds, whereas medium and high molecular weight compounds may sorb to particles in the water column and remain suspended or settle to the bottom (Readman et al. 1982).

PAHs are also present in soil, where they may be found adsorbed to soil and sediment. Their occurrence in soil may result from atmospheric deposition, automotive exhaust, use of compost and fertilizer, and sludge from sewage treatment plants (Perwak et al. 1982, Santodonato 1981). Most degradation of PAH in soil occurs through microbial degradation (Sims and Overcash 1983).

\section{HUMAN HEALTH EFFECTS}

Generally, the toxicity of PAHs (other than carcinogenicity) has not been of concern in exposed human populations. Several studies have shown increased incidence of lung cancer in workers exposed to coke oven emissions and coal gas (Lloyd 1972; Doll 1952; Kennaway and Kennaway 1936).

Thyssen et al. (1981) observed upper respiratory tract tumors in hamsters exposed to benzo(a)pyrene [B(a)P] by inhalation. Respiratory tract tumors have also followed intratracheal instillation of benzo(a)pyrene, dibenzo(a,i)pyrene and benzo(b)fluoranthene . in various laboratory animals (Feron and Kruysse 1978; Feron et al. 1973; Stenback and Sellakumar 1974; Deutsch-Wenzel et al. 1983).

According to the U.S. EPA Region IV staff, some PAHs are considered to be carcinogenic by the inhalation route of exposure (weight of evidence B2). For these PAHs, an inhalation slope factor of $6.1(\mathrm{mg} / \mathrm{kg} / \text { day })^{-1}$ has been used in this assessment, even though it has recently been removed from IRIS (U.S. EPA 1992b).

Oral administration of various PAHs has caused weight loss, aplastic anemia and mild liver and kidney damage in laboratory animals (U.W. EPA 1984; Robinson et al. 1975; Nebert et al. 1977, 1980).

Forestomach, esophageal and mammary tumors developed in rats and mice exposed orally to B(a)P (Rigdon and Neal 1966; Neal and Rigdon 1967; Rigdon and Neal 1969; Fedorenko and Yanysheva 1966; Huggins and Yang 1962). Similar types of tumors, along with hepatomas and lung tumors, have been seen upon oral administration of dibenzo(a,h)anthracene (Larinow and Soboleva 1938; Snell and Stewart 1962; Biancifiori and Caschera 1962).

When rats were fed benzo(a)pyrene during pregnancy (Rigdon and Rennels 1964), only one dam out of seven treated animals carried viable fetuses to term. In mice, offspring exposed in utero to benzo(a)pyrene had reduced fertility and reproductive capacity; almost complete sterility was noted in both sexes of offspring exposed in utero to $40 \mathrm{mg} / \mathrm{kg} /$ day (Mackenzie and Angevine 1981). 
The U.S. EPA (1992a) has verified an oral slope factor of $5.8(\mathrm{mg} / \mathrm{kg} / \mathrm{day})^{-1}$ for benzo(a)pyrene (weight of evidence B2). For the other carcinogenic PAHs, slope factors are calculated from that for B(a)P using toxicity equivalency factors (U.S. EPA 1992a); see Section 4.2.3.

Workers dermally exposed to PAH-containing mixtures (coal, tar, mineral oil, petroleum waxes) have developed chronic dermatitis and hyperkeratosis (Santadonato et al. 1981).

Many experiments with repeated dermal applications of $\mathrm{B}(\mathrm{a}) \mathrm{P}$ on mice have shown that $\mathrm{B}(\mathrm{a}) \mathrm{P}$ produces skin tumors. The studies indicate that the threshold dose is affected by the strain of mouse and the solvent chosen (Wynder et al. 1957; Poel 1963; Poel 1959; Bingham and Falk 1969; Hoffman and Wynder 1966). Many other PAHs have produced skin papillomas and carcinomas in mice following skin application (Hoffman and Wynder 1966; Badger et al. 1949; Masuda and Kagawa 1972; Pai and Ranadive 1965; Barry et al. 1935; Wynder and Hoffman 1964; Wynder and Hoffman 1959).

\section{ECOLOGICAL EFFECTS}

Aquatic organisms may bioaccumulate PAH through contact with contaminated water, sediments, and food. The higher molecular weight compounds are bioconcentrated to a greater degree than the lower molecular weight compounds (ATSDR 1989). Biomagnification, however, has not been reported at higher trophic levels, perhaps because many organisms metabolize and/or eliminate these compounds at a rapid rate (Eisler 1987). Metabolites of PAH have been associated with the development of tumors and mutagenesis in fish (Eisler 1987).

PAHs may accumulate in terrestrial organisms and at least one species, the gray-tailed vole, bioconcentrated PAHs above the soil concentration. This particular species was unable to metabolize PAHs, at least under the experimental conditions.

\section{U.S. ENVIRONMENTAL PROTECTION AGENCY HUMAN HEALTH CRITERIA}

Oral Slope Factor

Inhalation Slope Factor

Chronic Oral RfD

Chronic Inhalation RfD
$7.3 \mathrm{E}+0 \mathrm{~kg} / \mathrm{mg} / \mathrm{day}^{-1} *$

$6.1 \mathrm{E}+0 \mathrm{~kg} / \mathrm{mg} /$ day $^{-1}$ *

$4 \mathrm{E}-2$ (fluoranthene, fluorene)

3E-2 (Pyrene) $\mathrm{mg} / \mathrm{kg} /$ day

NA

\section{DRINKING WATER CRITERIA AND STANDARDS}

U.S. Environmental Protection Agency

Maximum Concentration Limit

Maximum Concentration Limit Goal
$0.0001-0.0002 \mathrm{mg} / \mathrm{L}$

0 


\section{AMBIENT WATER QUALITY CRITERIA}

U.S. Environmental Protection Agency Ambient Water Quality Criteria
Human Health - Water and Fish Ingestion
Human Health - Fish Ingestion Only
$20 \mu \mathrm{g} / \mathrm{L}$ (Acenapthene)
Aquatic Organisms - FW Acute
NA
Aquatic Organisms - FW Chronic
$1700 \mu \mathrm{g} / \mathrm{L}$ (Acenapthene)
Aquatic Organisms - Marine Acute
$520 \mu \mathrm{g} / \mathrm{L}$ (Acenapthene)
Aquatic Organisms - Marine Chronic
$970 \mu \mathrm{g} / \mathrm{L}$ (Acenapthene)
$500 \mu \mathrm{g} / \mathrm{l}$ (Acenapthene)

South Carolina Department of Health and Environmental Control Water Quality Criteria

Human Health Criteria

Fish and Aquatic Life - Continuous

Fish and Aquatic Life - Maximum
$0.311 \mu \mathrm{g} / \mathrm{L}^{*}$

NA

NA

* for all compounds calculated

NA $=$ Not Available

\section{REFERENCES}

Agency for Toxic Substances and Disease Registry. 1989. Toxicological Profile for Polycyclic Aromatic Hydrocarbons. Prepared by Clement International Corp., contract no. 205-88-0608.

Badger, G. M., J. W. Cook, C. L. Hewett, E. L. Kennaway, N. M. Kennaway, R. H. Martin and A. M. Robinson. 1940. The production of cancer by pure hydrocarbons. V. Proc. R. Soc. London, Ser. B. 129:439-467.

Barrick, RC. 1982. Flux of aliphatic and polycyclic aromatic hydrocarbons to central Puget sound from Seattle (Westpoint) primary sewage effluent. Environ Sci Technol 16:682-692.

Barry, G., J. W. Cook, G. A. D. Haslewood, C. L. Hewett, I. Hieger and E. L. Kennaway. 1935. The production of cancer by pure hydrocarbons. III. Proc. R. Soc. London, Ser. B 117:318-351.

Biancifiori, C. and F. Cashchera. 1962. The relation between pseudopregnancy and the chemical induction by four carcinogens of mammary and ovarian tumors in BALB/c mice. Br. J. Cancer 16:722-730.

Bingham, E. and H. L. Falk. 1969. Environmental carcinogens. The modifying effect of cocarcinogens on the threshold response. Arch. Environ. Health 19:779-783. 
Deutsch-Wenzel, R. P., H. Burne and G. Grimmer. 1983. Experimental studies on the carcinogenicity of five nitrogen-containing polycyclic aromatic compounds directly injected into rat lungs. Cancer Lett. 20:97-101.

Doll, R. 1952. The causes of death among gas workers with special reference to cancer of the lung. Br. J. Ind. Med. 9:180-187.

Eisler, R. 1987. Polycyclic aromatic hydrocarbons hazards to fish, wildlife, and intertebrates: A synoptic review. Laureal, MD: US Fish and Wildlife Service Patuxent wildlife Research Center.

EPA. 1984. Health effects assessment for polynuclear aromatic hydrocarbons (PAH).

Fedorenko, Z. P. and N. Y. Yanysheva. 1966. Hyg. and Sanit. 31:168-173. (Cited in U.S. EPA, 1982).

Feron, J. J., D. DeJong and P. Emmelot. 1973. Dose-response correlation for the induction of respiratory-tract tumors in Syrian golden hamsters by intratracheal installations of benzo(a)pyrene. Eruop. J. Cancer 9:387-390.

Feron, J. J. and A. Kruysse. 1978. Effects of exposure to furfural vapor in hamsters simultaneously treated with benzo(a)pyrene or diethylnitrosamine. - Toxicology11:127-144.

Gibson, TL, Smart VB, Smith LL. 1978. Non-enzymatic activation of polycyclic aromatic hydrocarbons as mutagents. Mutat Res 49:153-161.

Giger, W, Blumer M. 1974. Polycyclic aromatic hydrocarbons in the environment: Isolation and characterization by chromatography visible, ultraviolet, and mass spectrometry. Anal Chem 46:1163.

Hawley, GG. 1987. The condensed chemical dictionary. New York: Van Nostrand Reinhold Company.

Hoffman, D. and E. L. Wynder. 1966. Contribution to the carcinogenic properties of dibenzopyrenes. (Ger.) Z. Krebsforsch. 68:137-149.

HSDB. 1988. Hazardous Substance Data Bank. National Library of Medicine, National Toxicology Program, Bethesda, MD. December 5, 1988.

Huggins, C. and N. C. Yang. 1962. Induction and extinction of mammary cancer. Science 137:257-262.

IARC. 1983. Monographs on the evaluation of the carcinogenic risk of chemicals to man. Vol. 32, Lyon, France: International Agency for Research on Cancer, 303. 
Kennaway, M. and E. R. Kennaway. 1936. A study of the incidence of cancer of the lung and larynx. J. Hyg. 36:236-267.

Larinow, L. F. and N. G. Soboleva. 1938. Gastric tumors experimentally produced in mice by means of benzopyrene and dibenzanthracene. Vestn. Rentogenol. Radio. 20:276.

Lloyd, JW. 1971. Long-term mortality study of steelworkers. V. Respiratory cancer in coke plant workers. J Occup Med 13:53-68.

Lloyd, J. W. 1972. Long-term mortality study of steel workers. V. Respiratory cancer in coke plant workers. J. Occup. Med. 13:53-68.

Mackenzie, K. M. and D. M. Angevine. 1981. Infertility in mice exposed in utero to benzo(a)pyrene. Biol. Reprod. 24:183-191.

Mackenzie, M. J., Hunter, J.V. 1979. Sources and fates of aromatic compounds in urban stormwater runoff. Environ Sci Technol 13:179-183.

Masuda, Y. and R. Kagawa. 1972. A novel synthesis and carcinogenicity of dibenzo(a,l)pyrene. Chem. Pharm. Bull. 29:2736-2737.

Nebert, D. W., R. C. Levitt, N.M. Jensen, G. H. Lambert and J. S. Felton. 1977. Birth defects and aplastic anemia: Differences in polycyclic hydrocarbon toxicity associated with the Ah locus. Arch. Toxicol. 39:109-132.

NRC. 1983. Polycyclic aromatic hydrocarbons: Evaluation of sources and effects. Washington, D.C.: National Research Council. National Academy Press, ES/1-ES/7.

Pai, S. R. and K. J. Ranadive. 1965. Biological testing of analogues of 3,4-benzpyrene. Indian J. Med. Res. 53:638-644.

Perwak, J., Byrne M., Coons S., et al. 1982. An exposure and risk assessment for benzo[a]pyrene and other polycyclic aromatic hydrocarbons. Volume IV. Benzo[a]pyrene, acenaphthylene, benz[a] anthracene, benzo[b]fluoranthene, benzo[k]fluoranthene, benzo[g,h,i]perylene, chrysene, dibenz[a,h]anthracene, and indeno[1,2,3-c,d]pyrene. Washington, D.C.: US Environmental Protection Agency, Office of Water Regulations and Standards. EPA 440/4-85-020-V4.

Poel, W. E. 1959. -.Effect of carcinogenic dosage and duration of exposure on skin tumor induction in mice. J. Natl. Cancer Inst. 22:19-35.

Poel, W. E. 1963. Skin as a test site for the bioassay of carcinogens and carcinogen precursors. Natl. Cancer Inst. Mono. 10:611. 
Readman, J.W., Mantourar, R.F.C., Rhead M.M., et al. 1982. Aquatic distribution and heterotrophic degradation and polycyclic aromatic hydrocarbons in the Tamar Estuary, England, UK. Estuarine Coastal Shelf Sci 14:369-389.

Rigdon, R. H. and J. Neal. 1966. Gastric carcinomas and pulmonary adenomas in mice given benzo(a)pyrene. Tex. Rep. Biol. Med. 24:195-207.

Rigdon, R. H. and J. Neal. 1969. Relationship of leukemia to lung and stomach tumors in mice fed benzo(a)pyrene. Proc. Soc. Exp. Biol. Med. 130:146-148.

Rigdon, R. H. and E. G. Rennels. 1964. Effect of feeding benzpyrene on reproduction in the rat. Experiential 20:224-226.

Robinson, J. R., J. S. Felton, R. C. Levitt, S. S. Thorgeirsson and D. W. Nebert. 1975. Relationship between "aromatic hydrocarbons: and the survival times in mice treated with various drugs and environmental compounds. Mol. Pharmacol. 11:850-865.

Santodonato, J., P. Howard and D. Basu. 1981. Health and ecological assessment of polynuclear aromatic hydrocarbons. J. Environ. Pathol. Toxicol. 5:1-172.

Sims, R.C., Overcash, M.R. 1983. Fate of polynuclear aromatic compounds (PNAs) in soilplant systems. Res Rev 88:1-68.

Snell, K C. and H. L. Steward. 1962. Pulmonary adenomatosis induced in DBA/2 mice by oral administration of dibenz(a,h)anthracene. J. Natl. Cancer inst. 28:1043-1051.

Snider, E.H., Manning, F.S. 1982. A survey of pollutant emission levels in waste waters and residuals from the petroleum refining industry. Environment International 7:237-258.

Stenback, F. and A. Sellakumar. 1974. Lung tumor induction by dibenz(a,i)pyrene in Syrian golden hamster. Z. Krebsforsch. 82:175-182.

Thyssen, J., J. Althoff, G. Kimmerle and U. Mohr. 1981. Inhalation studies with benzo(a)pyrene in Syrian golden hamsters. J. Natl. Cancer Inst. 66:565-577.

U.S. EPA (U.S. Environmental Protection Agency). 1984. Health effects assessment for polychlorinated aromatic hydrocarbons (PAHs). Office of Research and Development, Environmental Criteria and Assessment Office, Cincinnati, $\mathrm{OH}$ EPA/540/1-86/013.

Windholz, M. 1983. The Merck index. 10th ed. Rahway, NJ: Merck and Co.

Wynder, E. L., L. Fritz and N. Furthe. 1957. Effect of concentration of benzo(a)pyrene in skin carcinogenesis. J. Natl. Cancer Inst. 19:361-370. 
Wynder, E. L and D. Hoffman. 1959. The carcinogenicity of benzofluoranthenes. Cancer 12:1194-1199.

Wynder, E. L. and D. Hoffman. 1964. Experimental tobacco carcinogenesis. Adv. Cancer Res. ${ }^{*}: 249-253$. 
SILVER

CAS No.: $\quad 7440-22-4$

Synonym(s) argentum

Silver is a naturally occurring soft metal used to make jewelry, silverware, electronic equipment, and dental fillings. It is mined concurrently with copper, lead, zinc, and gold (Grayson 1978). Because it is a rare metal, much of it is recycled. Silver may occur in nature in association with other elements such as nitrate (silver nitrate), oxygen (silver oxide), sulfur (silver sulfide), and chlorine (silver chloride). Silver compounds are used in photographic materials, which account for the bulk of silver emissions to the environment. Natural releases of silver occur through erosion (Scow et al. 1981).

Silver, in its pure form, is a lustrous, white solid that is insoluble in water, but soluble in nitric acid. Its melting point is $961^{\circ} \mathrm{C}$ and molecular weight is 107.868 . Silver compounds are white or charcoal colored and are either crystalline or powder. They melt at lower temperatures than pure silver, usually in the range of $200-400^{\circ} \mathrm{C}$ (ATSDR 1989).

\section{FATE AND TRANSPORT}

Silver released to the atmosphere occurs as an aerosol, and consists primarily of metallic silver, silver sulfide, silver carbonate, and silver halides (Smith and Carson 1977). Small particles in the aerosol may be transported by air currents, whereas larger particles may be deposited on the earth's surface through wet deposition or gravitational settling (Scow et al. 1981; Davidson et al. 1985).

The major forms of silver in water are silver sulfate, silver bicarbonate, and silver sulfate salts which are complexed with other ions (Boyle 1968). Most of the silver compounds which reach the water adsorb to particles and are deposited in aquatic sediments (Callahan et al. 1979).

Silver tends to complex with particles and organic matter in soils (Smith and Carson 1977), however, variables such as $\mathrm{pH}$, clay concentration, particle size, and drainage affect the mobility of soil-bound silver and silver compounds.

\section{HUMAN HEALTH EFFECTS}

Inhalation of silver dusts can occur from occupational exposure and produces symptoms of respiratory, eye, gastrointestinal, and skin irritation (Moss et al. 1979; Konradova 1968; Rosenman et al. 1979).

Silver ingestion may produce discoloration of the skin (Bleehen et al. 1981). Gastrointestinal discomfort has also been reported to result from ingestion of silver (Aaseth et al. 1981). Several reports describe deposition of silver-containing granules in the brains of patients who apparently ingested silver over a duration of time (Landas et al. 1985). The clinical manifestation of this CNS deposition in humans is unknown, however Rungby and 
Danscher (1984) report decreased physical activity in mice fed silver nitrate in drinking water.

\section{ECOLOGICAL EFFECTS}

Silver is toxic to soil microorganisms (Domsch 1984), however it apparently bioaccumulates in marine algae (Fisher et al. 1984). Studies of bottom-dwelling species such as clams, oysters, and scallops indicate that these species also bioaccumulate silver (Thomson et al. 1984; Pesch et al. 1977). Bioconcentration within the food chain has not been demonstrated, but may potentially occur.

\section{U.S. ENVIRONMENTAL PROTECTION AGENCY HUMAN HEALTH CRITERIA}

Oral Slope Factor

Inhalation Slope Factor

Chronic Oral RfD

Chronic Inhalation RfD
NA

NA

$5 \mathrm{E}-3 \mathrm{mg} / \mathrm{kg} /$ day

NA

\section{DRINKING WATER CRITERIA AND STANDARDS}

U.S. Environmental Protection Agency

Maximum Concentration Limit

Maximum Concentration Limit Goal

NA

NA

\section{AMBIENT WATER QUALITY CRITERIA}

U.S. Environmental Protection Agency Ambient Water Quality Criteria

Human Heaith - Water and Fish Ingestion

Human Health - Fish Ingestion Only

Aquatic Organisms - FW Acute

Aquatic Organisms - FW Chronic

Aquatic Organisms - Marine Acute

Aquatic Organisms - Marine Chronic
$50 \mu \mathrm{g} / \mathrm{L}$

NA

$4.1 \mu \mathrm{g} / \mathrm{L}$

$0.12 \mu \mathrm{g} / \mathrm{L}$

$2.3 \mu \mathrm{g} / \mathrm{L}$

NA

South Carolina Department of Health and Environmental Control Water Quality Criteria

Human Health Criteria

Fish and Aquatic Life - Continuous

Fish and Aquatic Life - Maximum
$50 \mu \mathrm{g} / \mathrm{L}$

$\mathrm{NA}$

$1.23 \mu \mathrm{g} / \mathrm{L}$

$\mathrm{NA}=$ Not Available 


\section{REFERENCES}

Aaseth, J., Olsen, A., Halse, J., et al. 1981. Argyria-tsudeposn of silver as selenide. Scand J. Clin. Lab Invest 41: 247-251.

Agency for Toxic Substances and Disease Registry (ATSDR). 1989. Toxicological Profile for Silver. Prepared by Clement International Corp., contract no. 205-88-0608.

Bleehen, S.S., Gould, D.J., Harrington, C.I. et al. 1981. The effect of $110 \mathrm{mAg}$ on ceruloplasmin oxidase activity in rats. Journal Hyg. Epidemiol Microbiol Immunol. 104:19-26.

Boyle, R.W. 1968. Geochem. of silver and its deposit: Notes on geochem prospecting for the elements. Geol. Survey of Can., Ottawa, Ont: Canada, Dept. Energy, Mines, and Resources 160., 1-96.

Callahan, M.A., Slimak, M.W., Gabel, N.W., et al. 1979. Water-related env. fate of 129 priority pollutants. EPA-440/4-79-029a,b., 17-1-17-11.

Davidson, C.I., Goold, W.D., Mathison, T.P., et al. 1985. Airborne trace elements in Great Smokey Mtns, Olympic, and Glacier National Parks. Environ. Sci. Technol. 19:27-35.

Domsch, K.H. 1984. Effects of pesticides and heavy metals on biological processes in soil. Plant \& Soil 76:367-378.

Fisher, N.S., Bohe, M., Teyssie, J.L. 1984. Accumulation and toxicity of $\mathrm{Cd}, \mathrm{Zn}, \mathrm{Ag}, \& \mathrm{Hg}$ in four marine phytoplankters. Mar Ecol Prog Ser 18:201-213.

Grayson, M., ed. 1978, Silver \& silver alloys; Silver and silver compounds. Kirk-Othmer Encyclopedia of Chemical Technology. Vol.21, 3rd ed. 1-32.

Konradova, V. 1968. The ultrastructure of the tracheal epithelium in rabbits following the inhalation of aerosols of colloidal solutions of heavy metals. 1. Changes in the ultrastructure of the tracheal epithelium after 2-hour inhalation of colloidal solutions of iron and silver. Folia Morphol (Praha) 16: 258-264.

Landas, S., Fischer, J., Wilkin, L.D., et al. 1985. Demonstration of regional blood-brain barrier permeability in human brain. Neurosci. Leh. 57:251-256.

Moss, A.P., Sugar, A., Hargett, N.A., et al. 1979. The ocular manifestations and functional effects of occupational argyrosis. Arch. Opthalmol. 97:906-908.

Pesch, G., Reynolds, B., Rogerson, P. 1977. Trace metals in scallops from within and around two ocean disposal sites. Marine Pollution Bulletin 8:224-228. 
Rosenman, K.D., Moss, A., Kon, S. 1979. Argyria: Clinical implications of exposure to silver nitrate and silver oxide. Journal of Occupational Medicine 21:430-435.

Rungby, J., Danscher, G. 1984. Hypoactivity in silver exposed mice. Acta Pharmacol et Tosicol 55:398-401.

Scow, K., Goyer, M., Nelken, L., et al. 1981 Exposure \& risk assessment for silver. Report to US EPA, office of water regs \& standards, Washington, DC, by Arthur D. Little, Inc., Cambridge, MA. PB85-211993.

Smith, I.C., Carson, B.L. 1977. Trace metals in the environment. Vol: 2. Silver. Ann Arbor, MI: Ann Arbor Science Publishers, Inc.

Thompson, E.A., Luoma, S.N., Johansson, C.E., et al. 1984. Comparison of sediments and org in identifying sources of biol. available trace metal contaminations. Water Research 18:755-766. 
TETRACHLOROETHYLENE

$\begin{array}{ll}\text { CAS No(s).: } & 79-01-6 \\ \text { Synonym(s) } & \text { PCE, perchloroethylene, carbon dichloride }\end{array}$

Tetrachloroethylene is widely used as a dry cleaning agent. It is also used for textile processing, as a degreaser, a spot remover, a rug and upholstery cleaner, and in the production of CFC 113.

Tetrachloroethylene is a colorless, viscous liquid which is relatively insoluble in water but miscible in organic solvents and oils. It is volatile, but nonflammable. Tetrachloroethylene has a molecular weight of 165.84 , boiling point of $121.2^{\circ} \mathrm{C}$ and melting point of $-22^{\circ} \mathrm{C}$ (Sax and Lewis 1987; Lide 1990).

\section{FATE AND TRANSPORT}

Most releases of tetrachloroethylene are to air as a result of the dry cleaning process. Releases to outdoor air occur in the vicinity of dry cleaning establishments, however, indoor air releases can produce high levels in homes from freshly dry cleaned clothes, draperies, and upholstery. Airborne tetrachloroethylene typically exists in the vapor phase and very little binding to particulate matter occurs. Transport can occur to regions remote from the source of contamination and can be deposited to surfaces by rain. Degradation of tetrachloroethylene in air occurs through interaction with hydroxyl radicals (Singh et al. 1982).

Tetrachloroethylene is highly volatile and large portions of surficial tetrachloroethylene evaporate rapidly from water (Zytner et al. 1989). There is conflicting data concerning biodegradation of tetrachloroethylene. Certain studies have shown tetrachloroethylene to be resistant to biodegradation (Bouwer and McCarty 1982) whereas others provide evidence that tetrachloroethylene can be completely biodegraded to ethylene (Freedman and Gossett 1989).

Tetrachloroethylene, which leaches into ground water, is resistant to degradation, and does not evaporate. Drinking water tested in several urban areas contained tetrachloroethylene in excess of EPA standards, probably resulting from contact with tetrachloroethylene-treated pipes (Harte et al. 1991).

Tetrachloroethylene is resistant to volatilization from soils; however, volatilization rates have been positively correlated with surface to volume ratio (Chodola et al. 1989) and in some studies to tetrachloroethylene concentration (Zytner et al. 1989). It is unclear whether or not microorganisms can biodegrade tetrachloroethylene in soils. There is evidence that some degradation occurs in anaerobic soils (Freedman and Gossett 1989), however others have demonstrated that tetrachloroethylene is toxic to soil microorganisms (Kanazawa and Filip 1987). 


\section{HUMAN HEALTH EFFECTS}

Inhalation of tetrachloroethylene vapors represents a significant risk to workers exposed in the workplace. Acute exposure to tetrachloroethylene in humans produces eye, nose, and throat irritation, dizziness, and confusion. Prolonged exposure or absorption of very high quantities has produced CNS depression, convulsions, respiratory depression and ventricular fibrillation (Klaasen 1980, Harte et al. 1991). Doses sufficient to cause lightheadedness in humans may also cause temporary liver damage (Harte et al. 1991). Irreversible liver damage is a potential sign of prolonged and/or chronic exposure. Chronic tetrachloroethylene is also nephrotoxic, causing permanent kidney damage in mice (Klaasen and Plaa 1966).

Tetrachloroethylene has been classified as a $\mathrm{B}_{1}$ carcinogen (probable human carcinogen) by the EPA. Tests of mutagenicity have, for the most part, yielded negative results in animal studies (Harte et al. 1991).

\section{ECOLOGICAL EFFECTS}

Tetrachloroethylene has been detected in a variety of food products including commercially packaged dairy products, oils and fats, beverages, as well as in fruits and vegetables. There is evidence to suggest that, in at least some cases, contamination occurred after packaging (Entz and Daichenko 1988). There is no experimental basis to suggest that tetrachloroethylene bioconcentrates in the food chain. Likewise the calculated bioconcentrate potential for tetrachloroethylene in aquatic animals is quite low (Kawasaki 1980).

\section{U.S. ENVIRONMENTAL PROTECTION AGENCY HUMAN HEALTH CRITERIA}

Oral Slope Factor

Inhalation Slope Factor

Chronic Oral RfD

Chronic Inhalation RfD .
$5.2 \mathrm{E}-2 \mathrm{~kg} / \mathrm{mg} / \mathrm{day}^{-1}$

$2.0 \mathrm{E}-3 \mathrm{~kg} / \mathrm{mg} /$ day $^{-1}$

$1.0 \mathrm{E}-2 \mathrm{mg} / \mathrm{kg} /$ day

NA

\section{DRINKING WATER CRITERIA AND STANDARDS}

U.S. Environmental Protection Agency

Maximum Concentration Limit

Maximum Concentration Limit Goal

$0.005 \mathrm{mg} / \mathrm{L}$

$0.0 \mathrm{mg} / \mathrm{L}$

\section{AMBIENT WATER QUALITY CRITERIA}

U.S. Environmental Protection Agency Ambient Water Quality Criteria

Human Health - Water and Fish Ingestion

Human Health - Fish Ingestion Only

Aquatic Organisms - FW Acute

$0.8 \mu \mathrm{g} / \mathrm{L}$

$8.85 \mu \mathrm{g} / \mathrm{L}$

$5,280 \mu \mathrm{g} / \mathrm{L}$ 
Aquatic Organisms - FW Chronic

Aquatic Organisms - Marine Acute

Aquatic Organisms - Marine Chronic
$840 \mu \mathrm{g} / \mathrm{L}$

$10,200 \mu \mathrm{g} / \mathrm{L}$

$450 \mu \mathrm{g} / \mathrm{L}$

South Carolina Department of Health and Environmental.Control Water Quality Criteria

Human Health Criteria

Fish and Aquatic Life - Continuous

Fish and Aquatic Life - Maximum
$88.5 \mu \mathrm{g} / \mathrm{L}$

NA

NA

$\mathrm{NA}=$ Not Available

\section{REFERENCES}

Agency for Toxic Substances and Disease Registry. 1991. Toxicological Profile for Tetrachloroethylene. Prepared by Clement International Corp., contract no. 205-880608.

Bouwer, E.J., McCarty, P.L. 1982. Removal of trace chlorinated compounds by activated carbon and fixed film bacteria. Environ. Sci. Technol. 16:836-843.

Chodola, G.R., Biswas, N., Bewtra, J.K, et al. 1989: - Fate of selected volatile organic substances in aqueous environment. Water Pollut. Res. J. Can. 24-119-142.

Entz, R.C., Diachenko, G.W. 1988. Residues of volatile halocarbons in margarines. Food Addit. Contam. 5:267-276.

Freedman, D.L., Gossett, J.M. 1989. Biological reductive dechlorination of tetrachloroethylene and trichloroethylene to ethylene under methanogenic conditions. Appl. Environ. Microbiol. 55:2144-2151.

Kanazawa, S., Filip, Z. 1987. Effects of trichloroethylene, tetrachloroethylene, and dichloromethane on soil biomass and microbial counts. Zentralbl Bakteriol Hyg. [B] 184:24-33.

Kawasaki, M. 1980. Experiences with the test scheme under the chemical control law of Japan: An approach to structure-activity correlations. Ecotoxicol Environ. Safety $4: 444-454$.

Klaasen, C.D., Plaa, G.L. 1966. Relative effect of various chlorinated hydrocarbons on liver and kidney function in mice. Toxicol. Appl. Pharmacol. 9:139-151.

Klaasen, C.D. 1980. Nonmetallic environmental toxicants: air pollutants, solvents and vapors, and pesticides in Goodman and Gilman's, The Pharmacological Basis of Therap. Seventh edition, Gilman, A.G., Goodman, L.S., Gilman, A. (eds.). NY. Macmillan Publishing Co., Inc. 
Lide, D.R., ed. 1990. CRC Handbook of Chemistry and Physics. 71st ed. Ann Arbor, MI: CRC Press, Inc., 3-242.

Sax, N.I., Lewis, R.J., eds. 1987. Hawley's condensed chemical dictionary. New York, NY: Van Nostrand Reinhold, 11:886.

Singh, H.B., Salas, L.J., Stiles, R.E. 1982. Distribution of selected gaseous organic mutagens and suspect carcinogens in ambient air. Environ. Sci. Technol. 16:872-880.

Zytner, R.G., Biswas, N., Bewtra, J.K. 1989b. Volatilization of perchloroethylene from stagnant water and soil. In:-Bell, J.M., ed., Proceedings of the 43rd Industrial Waste Conference, Purdue University, May 10-12, 1988. Chelsea, MI: Lewis Publishers, Inc. 101-108. 
TOLUENE

CAS No.: $\quad$ 108-88-3

Major Synonym(s) Tolulol, Methylbenzene, Phenylmethane

Toluene is primarily used as a gasoline additive to improve octane ratings (EPA 1990a), but also finds usage in the production of benzene, as a solvent in many household products, in the production of polymers, pharmaceuticals, cosmetics, and Trinitrotoluene (TNT). Toluene is a natural component of crude oil.

Toluene is a colorless liquid with a sweet, pungent benzene-like odor. It is miscible in water, alcohol, chloroform, ether, acetone, glacial acetic acid, and carbon disulfide. Toluene has a boiling point of $232^{\circ} \mathrm{F}$ and a vapor pressure of $22 \mathrm{~mm} \mathrm{Hg}$ at $20^{\circ} \mathrm{C}$.

\section{FATE AND.TRANSPORT}

The potential for exposure of the general population to toluene is high due to the large quantities released to the environment each year. The most frequent releases of toluene occur as a result of the production, transport, and use of gasoline (Verschueren 1977).

Most toluene enters the environment via direct release to the air, and that which is released in liquid form to soil or surface water quickly volatilizes. In the atmosphere, toluene is degraded through reaction with hydroxyl radicals to yield simple hydrocarbons as a final product (Davis et al. 1979). The half-life of toluene based on the rate constant for this process is estimated at approximately 13 hours. Toluene may also react with nitrogen dioxide, oxygen, and ozone to a lesser degree (Altshuller et al. 1971).

Toluene released to the soil can be biodegraded by bacteria, in both aerobic and anaerobic conditions (Fewson 1981; Dolfing et al. 1990). The half-life of toluene in the soil can range from 1-7 days (ERT 1984).

Degradation of toluene in surface and shallow ground water occurs primarily through microbial biodegradation. The half-life of toluene in water can be less than one day if temperature and other environmental parameters are favorable (Wakeham et al. 1983).

\section{HUMAN HEALTH EFFECTS}

Subjective complaints of fatigue and mild headache result from single, short-term exposure to toluene at $50-100 \mathrm{ppm}$. As the concentration and duration of exposure progressively increase, irritation of mucous membranes, tearing, dizziness, impaired reaction time, incoordination, nausea, confusion, nervousness and weakness occur. Narcosis and death result from exposure to extremely concentrated vapors (10,000-30,000 ppm) (IPCS 1985).

Irritation of the nose and throat, headaches, dizziness, and decreased test performance were noted in buman volunteers exposed to $375 \mathrm{mg} / \mathrm{M}^{3}$ toluene for six hours/day for four days (Andersen et al. 1983). 
Initial stages of toluene abuse (sniffing) are characterized by drunkenness, euphoria, nausea and vomiting, and sometimes visual and auditory hallucinations (Streicher et al. 1981). With longer exposure, subjects experience confusion and disorientation, blurred vision, drowsiness, ataxia and depressed reflexes. In extreme cases, seizures may occur with acute exposure (King et al. 1981). Chronic abuse of toluene can lead to permanent neurological impairment (for a review see IPCS 1985).

Several studies have suggested an association between toluene and spontaneous abortion, growth retardation, and congenital abnormalities in humans. In some cases, the evidence is confounded by simultaneous exposure to other industrial solvents. Various congenital defects were reported in children born to mothers who inhaled large quantities of pure toluene throughout pregnancy (Hersh et al. 1985).

An excess of chromosomal aberrations has been observed in rotogravure workers exposed to toluene (Bauchinger et al. 1982; Pelclova et al. 1990). Evaluation of chromosomal damage secondary to occupational exposure to toluene is complicated by the fact that workers are also exposed to other toxic chemicals (IPCS 1985).

\section{ECOLOGICAL EFFECTS}

Toluene appears to be extremely bioavailable in the environment, with inhalation, oral, and dermal adsorption all potential exposure pathways. Although toluene is highly lipid soluble, it does not appear to bioaccumulate, probably because it is rapidly metabolized to more polar metabolites (ATSDR 1992).

\section{U.S. ENVIRONMENTAL PROTECTION AGENCY HUMAN HEALTH CRITERIA}

Oral Slope Factor

Inhalation Slope Factor

Chronic Oral RfD

Chronic Inhalation RfD
NA

NA

$2 \mathrm{E}-01 \mathrm{mg} / \mathrm{kg} / \mathrm{day}$

$4.0 \mathrm{E}-01 \mathrm{mg} / \mathrm{m}^{3}$

\section{DRINKING WATER CRITERIA AND STANDARDS}

\section{U.S. Environmental Protection Agency}

Maximum Concentration Limit

Maximum Concentration Limit Goal

$1.0 \mathrm{mg} / \mathrm{L}$

$1.0 \mathrm{mg} / \mathrm{L}$

\section{AMBIENT WATER QUALITY CRITERIA}

U.S. Environmental Protection Agency Ambient Water Quality Criteria

Human Health - Water and Fish Ingestion

Human Health - Fish Ingestion Only

Aquatic Organisms - FW Acute
$14.3 \mathrm{mg} / \mathrm{L}$

$424 \mathrm{mg} / \mathrm{L}$

$17 ; 500 \mu \mathrm{g} / \mathrm{L}$ 
Aquatic Organisms - FW Chronic

Aquatic Organisms - Marine Acute

Aquatic Organisms - Marine Chronic
NA

$6,300 \mu \mathrm{g} / \mathrm{L}$

$5,000 \mu \mathrm{g} / \mathrm{L}$

South Carolina Department of Health and Environmental Control Water Quality Criteria

Human Health Criteria

Fish and Aquatic Life - Continuous

Fish and Aquatic Life - Maximum
$302 \mathrm{mg} / \mathrm{L}$

NA

NA

NA $=$ Not Available

\section{REFERENCES}

Agency for Toxic Substances and Disease Registry. 1992. Toxicological Profile for Toluene. Prepared by Clement International Corp., contract no. 205-88-0608.

Altshuller, AP, Lonneman WA, Sutterfield FD, et al. 1971. Hydrocarbon composition of the atmosphere of the Los Angeles Basin-1967. Environ Sci Technol 5:1009-1016.

Andersen, 1., G.R. Lundquist, L. Molhave, O.F. Pedersen, D.F. Proctor, M. Vaeth and D.P. Wyon. 1983. Human response to controlled levels of toluene in six-hour exposures. Scan. J. Work Environ. Health 9:405-418.

Bauchinger, M., E. Schmid, J. Dresp, J. Kolin-Gerresheim, R. Hauf and E. Suhr. 1982. Chromosome changes in lymphocytes after occupational exposure to toluene. Mutat. Res. 102:439-445.

Bruckner, J.V. and R.G. Peterson. 1981a. Evaluation of toluene and acetone inhalant abuse. I. Pharmacology and pharmacodynamics. Toxicol. Appl. Pharmacol. 61:27-38.

Bruckner, J.V. and R.G. Peterson. 1981b. Evaluation of toluene and acetone inhalant abuse. II. Model development and toxicology. Toxicol. Appl. Pharmacol. 61:302-312.

Davis, DD, Heaps W, Philen D, et al. 1979. Boundary layer measurements of the $\mathrm{OH}$ radical in the vicinity of an isolated power plant plume: $\mathrm{SO}_{2}$ and $\mathrm{NO}_{2}$ chemical conversion times. Atmos Environ 13:1197-1203.

Dolfing J, Zeyer J, Binder-Eicher P, et al. 1990. Isolation and characterization of a bacterium that mineralizes toluene in the absence of molecular oxygen. Arch Microbiol 154:336-341.

EPA. 1990a. Drinking water criteria document for toluene. Cincinnati, OH: U.S. Environmental Protection Agency, Office of Health and Environmental Assessment, Research and Development, ECAO-CIN-408. 
ERT. 1984. The land treatability of Appendix VIII constituents present in petroleum industry wastes. Environmental Research \& Technology, Inc. Washington, DC. B974-200.

Fewson, CA. 1981. Biodegradation of aromatics with industrial relevance. Fems Symp 12:141-179.

Gibson, J.E. and J.F. Hardisty. 1983. Chronic toxicity and oncogenicity bioassay of inhaled toluene in Fischer-344 rats. Fundam. Appl. Toxicol. 3:315-319.

Hersh, J.H., P.E. Podruch, G. Rogers and B. Weisskopf. 1985. Toluene embryopathy. J. Ped. 106:922-927.

Hudak, A. and G. Ungvary. 1978. Embryotoxic effects of benzene and its methyl derivatives: Toluene and xylene. Toxicology. 11:55-63.

IPCS (International Programme on Chemical Safety). 1985. Environmental Health Criteria 52: Toluene. World Health Organization, Geneva.

King, M.D., R.E. Day, J.S. Oliver, M. Lush and J.M. Watson. 1981. Solvent encephalopathy. Br. Med. J. 283:663-665.

Lyapkalo, A. 1973. Genetic activity of benzene and toluene. Gig. Tr. Prof. Zabol. 17:24-28. (Russian). (Cited in IPCS, 1985).

Nawrot, P.S. and R.E. Staples. 1979. Embryo-fetal toxicity and teratogenicity of benzene and toluene in the mouse. Teratology 19:41A. (Abstract).

NTP (National Toxicology Program). 1989. Toxicology and carcinogenesis studies of toluene in F344/N rats and B6C3F1 mice. Technical Report Series No. 371. Research Triangle Park, N.C.

Pelclova, D., P. Rossner and J. Pickova. 1990. Chromosome aberrations in rotogravure printing plant workers. Mut. Res. 245:299-303.

Smyth, H.F., Jr., C.P. Carpenter, C.S. Weil, U.C. Pozzani, J.A. Striegel and J.S. Nycum. 1969. Range-finding toxicity data lists. VII. Am. Ind. Hyg. Assoc. J. 30(5):470-476.

Streicher, H.Z., P.A. Gabow, A.H. Moss, D. Kono and W.D. Kaehny. 1981. Syndromes of toluene sniffing in adults. Ann. Intern. Med. 94:758-762.

Ungvary, G., E. Manyai, E. Tatrai, S.Z. Szebereny, R.J. Cseh, J. Molnar and G. Folly. 1980. Effect of toluene inhalation on the liver of rats - dependence on sex, dose and exposure time. J. Hyg. Epidemiol. Microbiol. Immunol. 24:242-252. 
Ungvary, G.Y., E. Tatrai, G.Y. Barcaz and G.Y. Krasznai. 1979. Acute poisoning effects of toluene, o-, m-, and p-xylene and their mixtures. Munkaveldelem. 25(7-9):37-38. (Hungarian). (Cited in IPCS, 1985).

U.S. EPA (U.S. Environmental Protection Agency). 1991a. Integrated Risk Information System (IRIS). Online. Office of Health and Environmental Assessment, Environmental Criteria and Assessment Office, Washington, D.C.

U.S. EPA (U.S. Environmental Protection Agency). 1991b. Health Effects Assessment Summary Tables (HEAST). Office of Research and Development, Office of Emergency and Remedial Response, Washington, D.C. Wolf, M.A., V.K. Rowe, D.D. McCollister, R.I. Hollingworth and F. Oyer. 1956. Toxicological studies of certain alkylated benzenes and benzene. Arch. Ind. Health 14:387-398.

Verschueren K. 1977. Handbook of environmental data on organic chemicals. 1st ed. New York, NY: Van Nostrand Reinhold Company.

Wakeham SG, Davis AC, Karas JL. 1983. Mesocosm experiments to determine the fate and persistence of volatile organic compounds in coastal seawater. Environ Sci Technol 17:611-617. 
THIS PAGE LEFT INTENTIONALLY BLANK 
TRICHLOROETHANES - 1,1,2-TRICHLOROETHANE; 1,1,1-TRICHLOROETHANE

CAS Nos.:

Synonym(s)
$79-00-5 ; 71-55-6$

TCA, methylchloroform; vinyl chloride

Trichloroethane (TCA) is a commonly used solvent for fats, resins, spot removers, printing inks, etc., and for organic synthesis. It is widely used as a solvent in industry, primarily because it is thought to be the least toxic of the chlorinated solvents. For the purposes of this profile, 1,1,2-trichloroethane and 1,1,1-trichloroethane will be used synonymously, except where differences merit their separate treatment.

1,1,1-TCA has a molecular weight of 133.41 whereas 1,1,2-TCA has a molecular weight of 133.42. The specific gravity of $1,1,1-\mathrm{TCA}$ is 1.31 , while that of $1,1,2-\mathrm{TCA}$ is 1.44 . Both are colorless, nonflammable liquids with a sweet odor.

\section{FATE AND TRANSPORT}

Trichloroethane does not occur naturally, therefore all releases to the environment are from anthropogenic origins. Trichloroethane is a volatile compound that readily enters the vapor phase and becomes distributed in the atmosphere. Photochemically produced hydroxyl radicals in the air may react with trichloroethane and degrade it (Butler et al. 1978). These types of reactions are estimated to remove only about $1 \%$ of the total atmospheric concentration of trichloroethane in 177 days in a typical January and 11 days in a typical July in the midwestern United States (Altshuller 1979). Due to the prolonged life-time of trichloroethane in the atmosphere, it has been suggested that this compound will migrate to the stratosphere, where it is degraded and may contribute to ozone depletion (Crutzen et al 1983).

Trichloroethane is degraded by anaerobic bacteria in ground water (Vargas and Ahlert 1987), however there is no evidence to of biodegradation in aerobic surface waters. Hydrolysis may occur at a slow rate, with the half-life of trichloroethane in water estimated at 4.8 years (Ellenrieder and Reinhard 1988).

Trichloroethane is predicted to be highly mobile in soil, readily leaching into groundwater (ATSDR 1989), and then finding that groundwater contamination occurs near known surface contamination sources supports this contention (Henson et al. 1988).

\section{HUMAN HEALTH EFFECTS}

Trichloroethane is rapidly absorbed by the lungs, gastrointestinal tract, and the skin. Most is eliminated unchanged at a rapid rate, probably accounting for the low toxicity of these compounds. At high concentrations, both compounds produce irritation of the eyes and mucous membranes (Hardie 1964). Prolonged exposure to high doses causes CNS depression, cardiovascular effects, loss of balance, and pulmonary edema. In animals, 1,1,2trichloroethane appears to be more hepatotoxic than 1,1,1-trichloroethane but both are less toxic than chloroform (Gehring 1968). Additionally, 1,1,2-trichloroethane is strongly nephrotoxic in rats (Plaa and Larson 1965). 
1,1,2-Trichloroethane, administered long-term to mice and rats, resulted in an increased incidence of hepatic carcinomas and adrenal phaeochromocytoma (NCI 1978). Mutagenesis studies of chronic 1,1,2-trichloroethane administration in mice of both sexes were negative (White et al. 1985). 1,1,1-trichloroethane has not been shown to have either mutagenic or carcinogenic properties, however it bears caution in dismissing these properties since other related compounds have been shown to be carcinogenic.

\section{ECOLOGICAL EFFECTS}

Trichloroethane is not believed to bioconcentrate to any substantial degree in fish and aquatic organisms, therefore it should not biomagnify in the food chain. However, trichloroethane has been detected in packaged fish, shrimp, clams, and oysters (Young et al. 1983; Ferrario et al. 1985). It was speculated that the contamination in these food products may have occurred during processing and/or packaging (ATSDR 1989).

US ENVIRONMENTAL PROTECTION AGENCY HUMAN HEALTH CRITERIA

Oral Slope Factor

Inhalation Slope Factor

Chronic Oral RfD

Chronic Inhalation RfD
$5.7 \mathrm{E}-2 \mathrm{~kg} / \mathrm{mg} / \mathrm{day}^{-1}$

$5.7 \mathrm{E}-2 \mathrm{~kg} / \mathrm{mg} / \mathrm{day}^{-1}$

$4 \mathrm{E}-3 \mathrm{mg} / \mathrm{kg} /$ day

$\mathrm{NA} \mathrm{mg} / \mathrm{kg} /$ day

\section{DRINKING WATER CRITERIA AND STANDARDS}

U.S. Environmental Protection Agency

Maximum Concentration Limit

Maximum Concentration Limit Goal

$0.005 \mathrm{mg} / \mathrm{L}$

$0.003 \mathrm{mg} / \mathrm{L}$

\section{AMBIENT WATER QUALITY CRITERIA}

U.S. Environmental Protection Agency Ambient Water Quality Criteria

Human Health-Water and Fish Ingestion

Human Health-Fish Ingestion Only

Aquatic Organisms-FW Acute

Aquatic Organisms-FW Chronic

Aquatic Organisms-Marine Acute

Aquatic Organisms-Marine Chronic
$0.6 \mu \mathrm{g} / \mathrm{L}$ $41.8 \mu \mathrm{g} / \mathrm{L}$ $18000 \mu \mathrm{g} / \mathrm{L}$ $9400 \mu \mathrm{g} / \mathrm{L}$ $31200 \mu \mathrm{g} / \mathrm{L}$ NA 
South Carolina Department of Health and Environmental control Water Quality Criteria

Human Health Criteria

Fish and Aquatic Life-Continuous

Fish and Aquatic Life-Maximum
$419.9 \mu \mathrm{g} / \mathrm{L}$

NA

NA

$\mathrm{NA}=$ Not Available

\section{REFERENCES}

Agency for Toxic Substances and Disease Registry. 1989. Toxicological Profile for Trichloroethanes. Prepared by Clement International Corp., contract no. 205-880608.

Altshuller, AP. 1979. Lifetimes of organic molecules in the troposphere and lower stratosphere. Adv Environ Sci Technol 10:181-219.

Butler, R., Solomon, I.J., Snelson, A. 1979. Rate constants for the reaction of OH with halocarbons in the presence of O2 + N2. J Air Pollut Contr Fed 28:1131-1133.

Crutzen, PH, Gidel, LT. 1983. A two-dimensional photochemical model of the atmosphere. 2: The tropospheric budgets of the anthropogenic chlorocarbons, carbon monoxide, methane, chloromethane and the effect of various nitrogen oxides sources on the tropospheric ozone. J Geophys Res.

Ellenrieder, E., Reinhard, M. 1988. Athias - an information system for abiotic transformations of halogenated hydrocarbons in aqueous solution. Chemosphere 17:331-344.

Ferrario, JB, Lawler, GC, DeLeon, IR, et al. 1985. Volatile organic pollutants in biota and sediments of Lake Pontchartrain. Bull Environ Contam Toxicol 34:246-255.

Gehring, P.J. 1968. Hepatotoxicity potency of various chlorinated hydrocarbon vapors relative to their narcotic and lethal potencies in mice. Toxicol. Appl. Pharmacol. $13: 287-298$.

Hardie, D.W.F. 1964. Chlorocarbons and chlorohydrocarbons. 1,1,2-trichloroethane In: Encyclopedia of Chemical Technology 2nd Ed. Vot 5, New York, John Wiley and sons. In: LARC Monographs of the Carcinogenic Risk of Chemicals to Humans, Vol.20. Hydrocarbons and Chlorohydrocarbons.

Henson, JM, Yates, MV, Cochran, JW, et al. 1988. Microbial removal of halogenated methanes, ethanes, and ethylenes in an aerobic soil exposed to methane. Fems Microbial Ecology 53:193-201. 
NCI. 1978. National Cancer Institute. Bioassay of 1,1,2-trichloroethane for possible carcinogenicity (Technical Report Series No. 74), DHEW Publication No. (NIH) 781324, Washington,D.C. US Dept. Health, Education and Welfare.

i iaa, G.L., Larson, R.E. 1965. Relative nephrotoxic properties of chlorinated methane, ethane and ethylene derivatives in mice. Toxicol. Appl. Pharmacol. 7:37-44.

Vargas, C, Ahlert, RC. 1987. Anaerobic degradation of chlorinated solvents. Water Pollut Contr Fed J 59:964-968.

Young, DR, Gossett, RW, Baird, RB, et al. 1983. Wastewater inputs and marine bioaccumulation of priority pollutant organics off Southern California. In: Water chlorination: Environ Impact Health Effects, 871-884. 
TRICHLOROETHYLENE

$\begin{array}{ll}\text { CAS No.: } & 79-01-6 \\ \text { Major Synonym(s) } & \text { Acetylene trichloride, ethinyl trichloride, ethylene trichloride, TCE }\end{array}$

Trichloroethylene, or TCE, is used extensively by the automotive and metals industries as a vapor degreaser. The textile industry employs trichloroethylene as a solvent in waterless dying and to scour cotton, wool, and other fabrics (CMR 1983; IARC 1979; McNeill 1979). Trichloroethylene is also used as a solvent for adhesives, lubricants, paint-related products, pesticides, and cold metal cleaners (Hawley 1981; IARC 1979; Mannsville 1985; McNeill 1979; Windholz 1983). Additionally, trichloroethylene is an intermediate in the production of other chemicals such as pharmaceuticals and flame retardant materials (Mannsville 1985; Windholz 1983).

Trichloroethylene is a colorless liquid at ambient temperature with a sweet, chloroform-like odor. It has a molecular weight of 131.40 and a density of 1.465 at $20^{\circ} \mathrm{C}$. Trichloroethylene meits at $-87.1^{\circ} \mathrm{C}$ (HSDB 1987; McNeil 1979).

\section{FATE AND TRANSPORT}

The primary source of trichloroethylene release to the atmosphere is through degreasing, however, its production, its use in the manufacture of other chemicals, and evaporation from consumer products constitute minor sources of atmospheric emissions (Pandullo et al. 1985). Due to its high vapor pressure, trichloroethylene will exist in the vapor phase rather than adsorbing to particulate matter (Eisenreich et al. 1981). Transport may occur from air to land and water via precipitation, however, the majority is removed by wet deposition and will be recycled back to the atmosphere (ATSDR 1991). -

Hydroxyl radicals, produced by photolysis, react with trichloroethylene in the atmosphere, with an estimated half-life of 6.8 days (Atkinson 1985; ATSDR 1991). Long distance transport through air should not be significant due to the short atmospheric half-life of trichloroethylene, however, continual release may keep atmospheric levels elevated.

The majority of trichloroethylene which is released to soil or surface water will evaporate (Dilling et al. 1975). Trichloroethylene is highly mobile in soil and leaching into groundwater is a potential human exposure pathway, manifested through consumption of drinking water (ATSDR 1991). Aerobic biodegradation is a significant method of decomposition of trichloroethylene in seawater, with $80 \%$ degraded in 8 days under experimental conditions (Jensen and Rosenberg 1975). -

\section{HUMAN HEALTH EFFECTS}

At trichloroethylene concentrations of $50-100 \mathrm{ppm}$ (commonly found in the workplace), some common complaints are headache, fatigue, dulling of the senses, nausea, vomiting, insomnia and burning eyes (WHO 1985). There is a prevalence of subjective symptoms such as lightheadedness, forgetfulness, and dry mouth at lower concentrations. In a group of $\mathbf{3 0}$ workers exposed to trichloroethylene for one to five years, long-lasting symptoms such as 
headache, dizziness, sleepiness, nausea, vomiting and conjunctivitis were reported (El Ghawabi et al. 1973). Chronic exposures to relatively high levels of trichloroethylene also produce swelling of the eyes, face and hands, excessive sleepiness and loss of appetite (Milby 1968). Trichloroethylene is addictive for some people and has proven fatal in some instances (James 1963).

At this time there is good evidence that trichloroethylene vapor is a hepatocarcinogen in some strains of mice.(NCI 1976; Maltoni and Maioli 1977), and that it induces adenoma of the lung in ICR and Swiss mice (Fukuda et al. 1983; Maltoni et al. 1979). In a cohort study of men exposed to trichloroethylene vapor in the workplace, the major finding was a significant excess of urogenital tract cancer and hematolymphatic malignancies (Achelson et al. 1984). Trichloroethylene vapor exposure induced unscheduled DNA synthesis both with and without metabolic activation in human lymphocytes (Percocco and Prodi 1981).

Based on the studies by Fukuda et al. (1983) and Maltoni et al. (1979), the U.S. EPA has classified trichloroethylene as a B2 carcinogen - sufficient evidence of carcinogenicity in animals with inadequate or lack of evidence in humans, by the inhalation route of exposure, with an inhalation slope factor of $1.7 \cdot \mathrm{E}-02 \mathrm{mg} / \mathrm{kg} /$ day $^{-1}$ (U.S. EPA 1991b).

Trichloroethylene is a mild skin irritant, but can cause rash and blistering if present on the skin for a substantial length of time (WHO 1985). It can act as both a primary irritant and as a sensitizer, and produces generalized dermatitis (Bauer and Rabens 1974).

\section{ECOLOGICAL EFFECTS}

Biomagnification of trichloroethylene in the aquatic food chain occurs to a small degree. In a study by Person and McConnell (1975), marine species from significant trophic levels were examined for tissue concentrations of trichloroethylene. Top predators exhibited a maximum concentration of less than 100 fold above water concentration.

There is a dearth of information regarding trichloroethylene bioconcentration in terrestrial food chains. At least one study indicated that fruits and vegetables can take up and assimilate trichloroethylene, suggesting a potential for bioaccumulation (McConnell et al. 1975).

\section{U.S. ENVIRONMENTAL PROTECTION AGENCY HUMAN HEALTH CRITERIA}

Oral Slope Factor

Inhalation Slope Factor

Chronic Oral RfD

Chronic Inhalation RfD
$1.1 \mathrm{E}-2 \mathrm{~kg} / \mathrm{mg} /$ day $^{-1}$ $6 \mathrm{E}-4 \mathrm{~kg} / \mathrm{mg} /$ day $^{-1}$

NA

NA 
DRINKING WATER CRITERIA AND STANDARDS

U.S. Environmental Protection Agency

Maximum Concentration Limit

Maximum Concentration Limit Goal

$5 \mathrm{E}-04 \mathrm{mg} / \mathrm{L}$

$0.0 \mathrm{mg} / \mathrm{L}$

AMBIENT WATER QUALITY CRITERIA

U.S. Environmental Protection Agency Ambient Water Quality Criteria

Human Health - Water and Fish Ingestion

Human Health - Fish Ingestion Only

Aquatic Organisms - FW Acute

Aquatic Organisms - FW Chronic

Aquatic Organisms - Marine Acute

Aquatic Organisms - Marine Chronic

$2.7 \mu \mathrm{g} / \mathrm{L}$

$80.7 \mu \mathrm{g} / \mathrm{L}$

$4.5 \mathrm{E}+4 \mu \mathrm{g} / \mathrm{L}$

$2.2 \mathrm{E}+4 \mu \mathrm{g} / \mathrm{L}$

$2 \mathrm{E}+3 \mu \mathrm{g} / \mathrm{L}$

NA

South Carolina Department of Health and Environmental Control Water Quality Criteria

Human Health Criteria

Fish and Aquatic Life - Continuous

Fish and Aquatic Life - Maximum

$5 \mu \mathrm{g} / \mathrm{L}$

NA

NA

$\mathrm{NA}=$ Not Available

\section{REFERENCES}

Achelson, O., Andersson, K., Selden, A., and Hogstedt, C. 1984. In: ICOST (International Conference on Organic Solvent Toxicity). Stockholm 15-17 November, 1984. (Abstract in Arbeteoch Haelsa 29:126). (Cited in Achelson 1986).

Achelson, O. 1986. Epidemiological studies of workers with exposure to tri- and tetrachloroethylene. In: Chambers, P.L., P. Gehring and F. Ceca, Ed. new Concepts and Developments in Toxicology. Elsevier Science Publishers, B.V., pp. 223-230.

Agency for Toxic Substances and Disease Registry. 1991. Toxicological Profile for Trichloroethylene. Prepared by Clement International Corp., contract no. 205-880608.

Atkinson, R. 1985. Kinetics and mechanisms of the gas-phase reactions of hydroxyl radical with organic compounds under atmospheric conditions: Chem Rev 85:69-201.

Bauer, M. and S.F. Rabens. 1974. Cutaneous manifestations of trichloroethylene toxicity. Arch. Dermatol. 110:886-890. 
Beliles, R.P., D.J. Brusick and F.J. Mecler. 1980. Teratogenic-mutagenic risk of workplace contaminants: Trichloroethylene, perchloroethylene and carbon disulfide. Washington, D.C., U.S. Department of Health, Education and Welfare, pp. 234. (Report for U.S. DHEW Contract No. 210-77-0047).

CMR. 1983. Chemical Profile-Trichloroethylene. Chemical Marketing Reporter, February $14,1983$.

Dorfmueller, M.A., S.P. Henne, R.G. York, R.L. Bornschein and J.M. Manson. 1979. Evaluation of teratogenicity and behavioral toxicity with inhalation exposure of maternal rats to trichloroethylene. Toxicology. 14:153-166.

Dilling, W.L, Tefertiller N.B., Kallos G.J. 1975. Evaporation rates and reactivities of methylene chloride, chloroform, 1,1,1-trichloroethane, trichloroethylene, tetrachloroethylene, and other chlorinated compounds in dilute aqueous solutions. Environ Sci Technol 9:833-838.

Eisenreich, SJ, Looney BB, Thornton JD. 1981. Airborne organic contaminants in the Great Lakes ecosystem. Environ Sci Technol 15:30-38.

El Ghawabi, S., M. Namsoor, M. El Gamel, A. El Saharti and F. El Enany. 1973. Chronic trichloroethylene exposure. J. Egypt. Med. Assoc. 56(11-12):715-724.

Fukuda, K., K. Takemoto and $\mathrm{H}$. Tsurata. 1983. Inhalation carcinogenicity of trichloroethylene in mice and rats. Ind. Health. 21:243-254.

Hawley, GG. 1981. The Condensed Chemical Dictionary. 10th ed. New York: Van Nostrand Reinhold Co., 1041-1042.

HSDB. 1990. Hazardous Substances Data Bank. National Library of Medicine, National Toxicology Information Program, Bethesda, MD. August 1990.

IARC. 1987. Monographs on the Evaluation of the Carcinogenic Risk of Chemicals to Humans. Some Halogenated Hydrocarbons. Lyons, France: World Health Organization.

James, W.R.L. 1963. Fatal addiction to trichloroethylene. Br. J. Ind. Med. 20:47-49.

Jensen, S, Rosenberg R. 1975. Degradability of some chlorinated aliphatic hydrocarbons in sea water and sterilized water. Water Res. 9:659-61.

Leong, B.K.J., B.A. Schwetz and P.J. Gearing. 1975. Embryo- and fetotoxicity of inhaled trichloroethylene, perchloroethylene, methyl chloroform and methylene chloride in mice and rats. Toxicol. Appl. Pharmacol. 33:136. 
Maltoni, C., G. Lefmine and G. Cotti. 1979. Experimental research on trichloroethylene carcinogenesis. In: Maltoni, C. and M.A. Mehlman, Ed. Archives of Research on Industrial Carcinogenesis, Vol. 5. (Cited in WHO 1985).

Maltoni, I.C. and P. Maioli. 1977. [Long-term bioassay of carcinogenicity of trichloroethylene. Preliminary results.] Osp. Vita. 4:108-110. (Italian). (Cited in WHO 1985).

Mannsville. 1985. Chemical product synopsis: trichloroethylene. Courtland, NY: Mannsville Chemical Products Corp.

McConnell, G, Ferguson DM, Pearson CR. 1975. Chlorinated hydrocarbons and the environment. Endeavour 34:13-8.

McNeill, WC Jr. 1979. Trichloroethylene. In: Grayson M, Eckroth P, ed., Kirk-Othmer encyclopedia of chemical technology. 3rd ed. Vol. 5. New York, NY: John Wiley and Sons, Inc., 745-753.

Milby, T.H. 1968. Chronic trichloroethylene intoxication. J. Occup. Med. 10:252-254.

NCI (National Cancer Institute). 1976. Carcinogenesis bioassay of trichloroethylene. CAS No. 79-01-6, DHEW (NCI) Publication No. 76-802. U.S Department of Health, Education and Welfare, Washington, D.C.

NTP (National Toxicology Program). 1983. NTP Technical Report on the Carcinogenesis of Trichloroethylene (without epichlorohydrin) in F344/N rats and B6C3F1 mice (gavage studies). NTP Tech Rep. Ser. No. 243, NIH 83-1799.

Pandullo, RF, Shareef SA, Kincaid LE, Murphy PV. 1985. Survey of trichloroethylene emission sources. EPA-450/3-85-021. Washington, DC: EPA, 1-1 to 2-8, 2-12.

Pearson, CR, McConnell G. 1975. Chlorinated C1 and C2 hydrocarbons in the marine environment. Proc R Soc Lond B 189:305-332.

Perocco, P. and G. Prodi. 1981. DNA damage by haloalkanes in human lymphocytes cultured in vitro. Cancer Lett. 13:213-218.

Schwetz, B.A., B.K.J. Leong and P.J. Gearing. 1975. The effect of maternally-inhaled trichloroethylene, perchloroethylene, methyl chloroform and methylene chloride on embryonal and fetal development in mice and rats. Toxicol: Appl. Pharmacol. 32:84-96.

Tucker, A.N., V.M. Sanders, P.W. Barnes, T.J. Bradshaw, K.L. White, Jr., L.E. Sain, J.F. Borzellaca and A.E. Munson. 1982. Toxicology of trichloroethylene in the mouse. Toxicol. Appl. Pharmacol. 62:351-357. 
U.S. EPA (U.S. Environmental Protection Agency). 1991a. Intègrated Risk Information System (IRIS). Online. Office of Health and Environmental Assessment, Environmental Criteria and Assessment Office, Washington, D.C.

U.S. EPA (U.S. Environmental Protection Agency). 1991b. Health Effects Assessment Summary Tables (HEAST), FY-1991 Annual. Office of Research and Development, Office of Emergency and Remedial Response, Washington, D.C.

Von Oettingen, W.F. 1955. The Halogenated Aliphatic, Olifinic, Cyclic, Aromatic and Aliphatic-Aromatic Hydrocarbons, Including the Halogenated Insecticides: Their Toxicity and Potential Dangers. U.S. Government Printing Office, Washington, D.C. (Cited in WHO 1985).

WHO (World Health Organization). 1985. Environmental Health Criteria 50: Trichloroethylene. ISBN 92-4-154190-3.

Windholz, M. 1983. The Merck Index. 10th ed. Rahway, New Jersey: Merck and Co., Inc. 1378. 
TRICHLOROFLUOROMETHANE

$\begin{array}{ll}\text { CAS No.: } & 75-69-4 \\ \text { Synonym(s) } & \text { CFC, Freon-11 }\end{array}$

The freons are a family of compounds containing carbon and fluorine. Many contain $\mathrm{Cl}, \mathrm{Br}$, or $\mathrm{H}$ atoms. The physical and chemical properties of these compounds render them very useful as refrigerants and propellants. In general, these products are non-flammable, have exceptional thermal and chemical stability, low viscosity, and low surface tension (Dupont).

Trichlorofluoromethane, or Freon-11, has a molecular weight of $137.4^{\circ} \mathrm{C}$ and a boiling point of $23.8^{\circ} \mathrm{C}$. It has low solubility in water but is miscible with lubricating oils. This compound has relatively good solvent properties for dissolving non-polar compounds (Dupont; EPA 1989).

\section{FATE AND TRANSPORT}

Freons were widely found in aerosol sprays until their use for this purpose was withdrawn. These compounds continue to reach the atmosphere, however, because they volatilize during production and use. Once in the atmosphere, Freon-11 is very persistent due to its chemical stability. This product does not absorb at wavelengths of uv radiation present in the troposphere (Hubrich et al. 1980), and therefore is not subject to photolysis at tropospheric altitudes. It does not react with hydroxyl radicals (Hampson 1980), nor does it degrade in photochemical smog (HSDB 1993). Because of its persistence (half-life $=52-207$ years in the troposphere), freon-11 reaches the stratosphere, where shorter wavelength uv radiation causes degradation via photolysis (HSDB 1993).

Most freon-11 released to surface waters will be lost by evaporation in a matter of hours (HSDB 1993). Although the octanol/water partition coefficient indicates that freon-11 can adsorb onto organic particles, this does not seem to occur in nature to any appreciable degree (EPA 1989). Biodegradation and hydrolysis are not substantial degradation pathways for freon-11 in water (HSDB 1993; EPA 1989).

Freon-11 is mobile in soils. Most of it will rapidly evaporate, however leaching will occur at a somewhat slower rate, where product may pass through soil and reach groundwater sources (HSDB 1988).

\section{HUMAN HEALTH EFFECTS}

There appear to be few reports of freon-11 toxicity following ingestion by humans and experimental animals. GI absorption following oral ingestion is relatively low (Ellenhorn 1988). Dermal exposure can cause chilling or freezing of tissue (EPA 1989). Additionally, Freon-11 can dissolve the protective fat from skin and lead to irritation, especially with prolonged or repeated exposures (Dupont).

Inhalation exposure to Freon-11 presents the highest risk of clinical toxicity. Deliberate concentration and inhalation of vapors from aerosol products was a significant abuse 
problem in the past and lead to numerous deaths. The primary effects of inhalation are respiratory irritation, CNS depression, and cardiovascular excitation (HSDB 1993; Dupont). CNS effects produce an initial feeling of intoxication followed by loss of coordination and loss of consciousness. In the cardiovascular system, Freon-11 sensitizes myocardium to the effects of the naturally circulating catecholamines, epinephrine and norepinephrine. Ventricular fibrillation is the most significant and serious clinical effect and may result in death (Dupont; HSDB 1993)

\section{ECOLOGICAL EFFECTS}

Freon-11 that is degraded by photolysis in the stratosphere releases a significant amount of chlorine atoms which destroy atmospheric ozone. This, of course, leads to an increase in the amount of UV radiation able to reach the earth's surface. UV radiation can damage juvenile aquatic species of animals and plants. There is also evidence that UV-B can modify the internal structure and plant associations of terrestrial ecosystems (HSDB1993). The lipophilic properties of Freon-11 render it likely to bioaccumulate in organisms. One report suggested that Freon-11 was bioaccumulated to some degree in various organs of fish and mollusks, however additional information is needed to determine the extent of bioconcentration and biomagnification within the food chain (EPA 1989).

\section{US ENVIRONMENTAL PROTECTION AGENCY HUMAN HEALTH CRITERIA}

Oral Slope Factor

Inhalation Slope Factor

Chronic Oral RfD

Chronic Inhalation RfD
$3 \mathrm{E}-1 \mathrm{mg} / \mathrm{kg} / \mathrm{day}^{-1}$

NA

NA

NA

\section{DRINKING WATER CRITERIA AND STANDARDS}

U.S. Environmental Protection Agency

Maximum Concentration Limit Maximum Concentration Limit Goal

NA

NA

\section{AMBIENT WATER QUALITY CRITERIA}

U.S. Environmental Protection Agency Ambient Water Quality Criteria

Human Health-Water and Fish Ingestion

Human Health = Fish Ingestion Only

Aquatic Organisms-FW Acute

Aquatic Organisms-FW Chronic

Aquatic Organisms-Marine Acute

Aquatic Organisms-Marine Chronic
NA

NA

NA

NA

NA

NA 
South Carolina Department of Health and Environmental Control

Human Health Criteria

Fish and Aquatic Life-Continuous

Fish and Aquatic Life-Maximum
NA

NA

NA

NA. $=$ Not Available

\section{REFERENCES}

Dupont. "Freon" fluorocarbons: Properties and applications. FREON Product Information Guide.

Ellenhorn. 1988. Medical Toxicology. p.884. (as cited in HSB 1993).

EPA 1989. Trichlorofluoromethane. Drinking Water Health Advisory. Office of Water. US Environmental Protection Agency.

Hampton, R.F. 1980. Chemical, Kinetic and Photochemical Data Sheets for Atmospheric Reactions. FAA-EE-80-17. (as cited in HSDB 1993).

Hubrich, C., Stuhl, F. 1980. J. Photochem. 12:93-107. (as cited in HSDB 1993).

HSDB. 1993. Hazardous Substance Data Bank. NLM. On-Line Computer Data Base. September 13, 1994. 


\section{THIS PAGE LEFT INTENTIONALLY BLANK}




\section{VANADIUM}

$\begin{array}{ll}\text { CAS·No.: } & 7440-62-2 \\ \text { Synonym(s) } & \text { None }\end{array}$

Vanadium occurs naturally in more than 65 different minerals and in oxidized form, usually as metal vanadate. Trace amounts are found in fossil fuels (National Research Council 1977). Vanadium is usually a light-grey or white lustrous powder, but can be found in fused hard lumps. It is relatively insoluble in water, hydrochloric acid, and alkali solutions (Merck Index 1983). It is soluble in aqua regia, sulfuric acid, nitric acid, and hydrogen fluoride (Weást 1979). Vanadium has a molecular weight of $50.94 \mathrm{~g} / \mathrm{mole}$, melts at $1,917^{\circ} \mathrm{C}$ (at one atmosphere), boils at $3,380^{\circ} \mathrm{C}$ (at one atmosphere), and has a density of 6.11 at $18.7^{\circ} \mathrm{C}$.

\section{FATE AND TRANSPORT}

Vanadium is ubiquitous, entering the environment from mining operations, refineries, and the use of petroleum products, as well as via plants that absorb vanadium from naturally occurring sources. It can form covalent bonds with organic molecules to create organometal compounds (National Research Council 1977). Vanadium has been measured in urban air in the United States as a result of the use of fossil fuels in these areas. Marine invertebrates, such as the ascidians, and plants tend to accumulate vanadium, which is deposited in sediment upon their death. Among terrestrial mammals, inhaled soluble salts of vanadium are readily absorbed, while there is little absorption of salts in the gastrointestinal tract and only minor amounts of absorption through the skin (World Health Organization 1988).

\section{HUMAN HEALTH EFFECTS}

Vanadium is considered nontoxic as a metal when ingested (Hawley 1977). Humans do not store or accumulate vanadium in toxic amounts. Most ingested vanadium remains unabsorbed in the gastrointestinal tract and is excreted (National Research Council 1977 and 1980). The only reported biological effect of ingested vanadium is the possible alteration of sulfur metabolism of the liver and an increased turnover of protein sulfur (Clayton and Clayton 1981-1982). Dermatitis was reported in workers exposed to $6.5 \mu \mathrm{g}$ vanadium pentoxide per cubic meter in air (World Health Organization 1988). Short-term exposure to vanadium symptoms include respiratory irritation, including coughing, wheezing, breathing difficulties, bronchitis, and chest pains; eye irritation; possible skin irritation; and greenish black discoloration of the tongue (National Institute for Occupational Safety and Health 1977; National Academy of Sciences 1974). Among persons who recover from acute vanadium exposure, persistent bronchitis resembling asthma, as well as labored breathing, can occur (National Institute for Occupational Safety and Health 1977). There is no evidence of carcinogenicity for vanadium and the U.S. Environmental Protection Agency has classed it as a Class D carcinogen.

\section{ECOLOGICAL EFFECTS}

Vanadium has been shown to reduce growth levels in rainbow trout (Hilton and Bettger 1988). At high levels of dietary vanadium (greater than $493 \mathrm{mg} / \mathrm{kg}$ ), feed avoidance and 
increased mortality were apparent. The dietary vanadium appeared to bioaccumulate in the fish as opposed to waterborne vanadium, which does not bioaccumulate. Giles et al. (1979) reported a $96-$ hour $\mathrm{LC}_{50}$ of 6.43 to $21.75 \mathrm{mg} / \mathrm{L}$ for rainbow trout, while Sprague et al. (1978) reported a 7-day $\mathrm{LC}_{50}$ of 2.4 to $5.6 \mathrm{mg} / \mathrm{L}$ for rainbows. Hudson (1964) reported lethal doses of various vanadium salts and compounds of 1 to $20 \mathrm{mg} / \mathrm{kg}$ body weight for rabbits, 1 to $50 \mathrm{mg} / \mathrm{kg}$ for guinea pigs, 10 to $190 \mathrm{mg} / \mathrm{kg}$ for rats, and 25 to $150 \mathrm{mg} / \mathrm{kg}$ for mice. In general, sodiumhexavanate and vanadyl sulfate showed the least toxic effects and oral routs of exposure resulted in low toxicity, inhalation resulted in moderate toxicity, and injection resulted in higher toxicity. Vanadium is not an essential element in plants (World Health Organization 1988). Soil levels of 10 to $1,258 \mathrm{mg} / \mathrm{kg}$.are toxic (Hopkins et al. 1977), and soluble vanadium concentrations of 10 to $20 \mathrm{mg} / \mathrm{L}$ are detrimental to most plants (Cannon 1963).

\section{U.S. ENVIRONMENTAL PROTECTION AGENCY HUMAN HEALTH CRITERIA}

Oral Slope Factor

Inhalation Slope Factor

Chronic Oral RfD

Chronic Inhalation RfD
NA

NA

$7.00 \mathrm{E}-03(\mathrm{mg} / \mathrm{kg} /$ day $)$

NA

\section{DRINKING WATER CRITERIA AND STANDARDS}

U.S. Environmental Protection Agency

Maximum Concentration Limit Maximum Concentration Limit Goal

NA

NA

\section{AMBIENT WATER QUALITY CRITERIA}

U.S. Environmental Protection Agency Ambient Water Quality Criteria

$\begin{array}{ll}\text { Human Health - Water \& Fish Ingestion } & \text { NA } \\ \text { Human Health - Fish Ingestion Only } & \text { NA } \\ \text { Aquatic Organisms - FW Acute } & \text { NA } \\ \text { Aquatic Organisms - FW Chronic } & \text { NA } \\ \text { Aquatic Organisms - Marine Acute } & \text { NA } \\ \text { Aquatic Organisms - Marine Chronic } & \text { NA }\end{array}$

South Carolina Department of Health and Environmental Control

Human Health Criteria

Fish and Aquatic Life - Continuous

Fish and Aquatic Life - Maximum
NA

NA

NA

NA $=$ Not Available 


\section{REFERENCES}

Agency for Toxic Substances and Disease Registry. 1990. Toxicological Profile for Vanadium. Prepared by Clement Inernational Corp., contract no. 205-88-0608.

Cannon, H.L. 1963. The Biogeochemistry of Vanadium. Soil Sci., 96(3):196-204.

Clayton, G.D. and F.E. Clayton (eds.). 1981-1982. Patty's Industrial Hygiene and Toxicology, 3rd Ed., Vols. 2A, 2B, 2C. John Wiley and Sons, New York, NY.

Giles, M.A., J.F. Klaverkamp, and S.G. Lawrence. 1979. The Acute Toxicity of Saline Groundwater and of Vanadium to Fish and Aquatic Invertebrates. Environment Alberta, Edmonton, Alberta, Canada.

Hawley, G.G. 1977. The Condensed Chemical Dictionary, 9th Ed. Van Nostrand Reinhold Co., New York, NY.

Hilton, J.W. and W.J. Bettger. 1988. Aquat Toxicol 12:63-72.

Hopkins, L.L., H.L. Cannon, A.T. Musch, R.M. Welch, and F.H. Nielsen. 1977. Vanadium. Geochem. Environ., 2:93-107.

Hudson, T.G.F. 1964. Vanadium: Toxicology and Biological Significance. Elsevier Science Publishers, New York, NY.

Merck Index, 10th Ed. 1983. Merck and Co., Rahway, NJ.

National Academy of Sciences. 1974. Vanadium. Committee of Biologic Effects of Atmospheric Pollutants, Division of Medical Sciences, National Research Council, Washington, DC.

National Institute for Occupational Safety and Health. 1977. Criteria for a Recommended Standard--Occupational Exposure to Vanadium, NIOSH Pub. No. 77-22. National Institute for Occupational Safety and Health, Washington, DC.

National Research Council. 1977. Drinking Water and Health, Vol. I. National Academy Press, Washington, DC.

National Research.Council. 1980. Drinking Water and Health, Vol. 3. National Academy Sprague, J.B., D.A. Holdway, and D.H. Stendahl. 1978. Acute and Chronic Toxicity of Vanadium to Fish. Environment Alberta, Edmonton, Alberta, Canada. 
Weast, R.C. (ed.). 1979. Handbook of Chemistry and Physics, 60th Ed. CRC Press Inc., Boca Raton, FL.

World Health Organization. 1988. Vanadium. Environmental Health Criteria 81. Geneva, Switzerland. 


\section{XYLENE, META-XYLENE, ORTHO-XYLENE, PARA-XYLENE}

CAS Nos.: $\quad 1330-20-7,108-38-3,95-47-6,106-42-3$

Synonym(s) Methyl toluene, m-xylene, o-xylene, p-xylene

Most xylenes are produced anthropogenically as solvents for paints and coatings, as a blending agent in gasoline, or as an intermediate in the chemical production of ethyl benzene or other compounds. There are 3 isomers of xylene: meta-xylene (m-isomer), ortho-xylene (o-isomer), and para-xylene (p-isomer). These are produced together and collectively are called mixed xylene. The ratio of the isomers in mixed xylene varies, depending on the method of synthesis (ATSDR 1989).

Xylene, or mixed xylene, and each of the isomers have a molecular weight of 106.16. The chemical make-up of each isomer is the same, however structurally, placement of the methyl group varies in its position on the ring. All of the isomers have a boiling point around $138^{\circ} \mathrm{C}$, but melting points range from $-45^{\circ} \mathrm{C}$ to $-13^{\circ} \mathrm{C}$. They are all colorless liquids with a sweet odor. All are completely or nearly insoluble in water, but soluble in organic solvents (Windholz 1983; Sax and Lewis 1989).

\section{FATE AND TRANSPORT}

Xylenes occur in many types of media and have been detected in air, rain, soil, surface water, ground water, aquatic biota, human blood, urine, and expired air (ATSDR 1989). Most releases of xylene occur from industrial uses, as a component of fuel, and during production and handling of product (Fishbein 1985). Natural emissions, only a minor source of emissions, can occur in smoke from forest fires or through seepage of petroleum from underground formations into the oceans (Merian and Zander 1982).

Xylenes are highly volatile and most emissions eventually partition into the atmosphere (Jori et al. 1986). Photo-oxidation is the primary pathway for transformation of xylenes, and the estimated half-life for the photo-oxidation of xylene is 24.1 hrs (Jori et al. 1986). Reactions with hydroxyl radicals also occur and ultimately lead to the formation of 2 products: carbon dioxide and water (Guisti et al. 1974).

Xylene spilled on land is mobile and will either leach into the ground or evaporate. Retention time may be increased in organic or hydric soils (Green et al. 1981; Amoozegar et al. 1986). Photo-oxidation may transform xylene on the soil surface at a rate similar to photo-oxidation in the atmosphere (Jori et al. 1986), however the importance of this degradation pathway is unclear, based on the rate of mobility of xylene in soils. In subsurface soils, biodegradation is a slow; but-significant, process. Apparently, specific bacteria are capable of biodegrading specific isomeric forms of xylene (Davis et al. 1968). These degradation processes occur under both aerobic and anaerobic conditions (Barker 1987).

Water-borne xylene is subject to evaporation from surface waters and to microbial biodegradation. Oxidation and hydrolysis are not likely degradation pathways (Mill 1980; Bridie et al. 1979). 


\section{HUMAN HEALTH EFFECTS}

Acute inhalation exposure produces cyanosis of the extremities, neurologic impairment consisting of amnesia and temporary confusion, and alterations in balance (Morley et al. 1970) and respiratory dysfunction manifested as dyspnea, irritation, and lung congestion (Morley et al. 1970; Goldie 1960). Gastrointestinal symptoms such as nausea, vomiting, and irritation (Goldie 1960) as well as renal impairment (Morley et al. 1970) have also been reported.

Chronic xylene exposure reportedly produced labored breathing and impaired pulmonary function in industrial workers (Hipolito 1980). Additionally, heart palpitations, severe chest pain, and abnormal ECG were associated with chronic occupational exposure to xylene (Hipolito 1980). Regarding changes in renal function, blood urea concentrations were increased, creatinine clearance was decreased, and excess urinary excretion of albumin, erythrocytes, and leukocytes were all noted in subjects occupationally exposed to xylene (Askergien 1981).

EPA has classified mixed xylene as a Group D agent (Iris 1989). This classification is reserved for chemicals for which insufficient evidence of carcinogenicity in animals exists.

Ethanol and aspirin are contraindicated in persons occupationally exposed to xylenes. Both agents apparently interfere with xylene metabolism through competition for different steps within the metabolic pathway (Romer et al. 1986; Campbell et al. 1988). Pregnant women, their fetuses, and small children may be at special risk with regard to xylene exposure. Animal studies have suggested that developmental problems may result from xylene exposure (Marks et al. 1982). Young children, without mature hepatic function, may be less able to detoxify xylene (Calabrese 1978).

\section{ECOLOGICAL EFFECTS}

Xylene is ubiquitous in the environment, occurring in all media types. In spite of its availability and its lipophilic nature, higher animals tend to metabolize xylene more rapidly than it can be assimilated (NRC 980). Lower animals, such as shrimp, clams, and eels reportedly bioconcentrate xylene (Sanborn and Malins 1980; Nunes and Benville 1979; Ogata and Miyake 1978). However, for reasons mentioned above, xylenes do not appreciably accumulate at the higher trophic levels.

\section{U.S. ENVIRONMENTAL PROTECTION AGENCY HUMAN HEALTH CRITERIA}

Oral Slope Factor

Inhalation Slope Factor

Chronic Oral RfD

Chronic Inhalation RfD
NA

NA

$2.0 \mathrm{mg} / \mathrm{kg} /$ day

NA 


\section{DRINKING WATER .CRITERIA AND STANDARDS}

U.S. Environmental Protection Agency

Maximum Concentration Limit

Maximum Concentration Limit Goal

$10 \mathrm{mg} / \mathrm{L}$

$10 \mathrm{mg} / \mathrm{L}$

\section{AMBIENT WATER QUALITY CRITERIA}

U.S. Environmental Protection Agency Ambient Water Quality Criteria

Human Health - Water and Fish Ingestion NA

Human Health - Fish Ingestion Only NA

Aquatic Organisms - FW Acute NA

Aquatic Organisms - FW Chronic NA

Aquatic Organisms - Marine Acute NA

Aquatic Organisms - Marine Chronic NA

South Carolina Department of Health and Environmental Control Water Quality Criteria

Human Health Criteria NA

Fish and Aquatic Life - Continuous NA

Fish and Aquatic Life - Maximum NA

$\mathrm{NA}=$ Not Available

\section{REFERENCES}

Agency for Toxic Substances and Disease Registry (ATSDR). 1989. Toxicological Profile for Total Xylenes. Prepared by Clement International Corp., contract no. 205-880608.

Amoozegar, A., Warrick, A.W., Fuller, W.H. 1986. Movement of selected organic liquids into dry soils. Hazardous Waste and Hazardous Materials 3:29-41.

Askergien, A. 1981. Studies on kidney function in subjects exposed to organic solvents: III. Excretion of cells in the urine. Acta. Med. Scand. 210:103-106.

Barker, J.F. 1987. Volatile aromatic and chlorinated organic contaminants in ground water at six Ontario landfills. Water Pollut. Res. J. Can. 22:33-48.

Bridie, A.L., Wolf, C.J.M., Winter, M. 1979. BOD and COD of some petrochemicals. Water Res. 13:627-630.

Calabrese, A.J. 1978. Pollutants and high risk groups. NY, NY. John Wiley \& Sons, Inc. 4-8. 
Campbell, L., Wilson, H.K., Samuel, A.M., et al. 1988. Interactions of m-xylene and aspirin metabolism in man. Br. J. Ind. Med. 45:127-132.

Davis, R.S., Hossler, F.E., Stone, R.W. 1968. Metabolism of p-and m-xylene by species of pseudomonas. Can. J. Microbial 14: 1005-1009.

Fishbein, L. 1985. An overview of env. and toxicological aspects of aromatic hydrocarbons III: Xylene, Sci. Total Environ. 43:165-183.

Goldie, I. 1960. Can xylene (xylol) provoke convulsive seizures. Ind. Med. Surg. 29:33-35.

Guisti, D.M., Conway, R.A., Lawson, C.T. 1974. Activated Carbon Adsorption of Petrohemicals. Journal Water Pollution Control Fed. 53:13477-1354.

Hipolito, R.N. 1980. Xylene poisoning in laboratory workers: Case reports and discussion. Lab Med. II: 593-595.

Jori, A., Calamari, D., Di Domenico, A, et al. 1986. Ecotoxicity profile of xylenes: working party on ecotoxicity profiles of chemicals. Ecotoxicol Environ. Safety 11:44-80.

Marks, T.A., Ledoux, T.A., Moore, J.A. 1982. Teratogenicity of a commercial xylene mixture in the mouse. J. Toxicol. Environ. Health 9:97-105.

Merian, E., Zander, M. 1982. Volatile aromatics. In: Hutzinger, G., ed. Handbook of Env. Chem. Vol. 3 (P+B), Berlin: Springer, 117-161.

Mill, T. 1980. Chem and photo-oxidation. In: Hutzinger, O. ed. Handbook of Env. Chem. Vol..2, Springer-Verlag, Berlin. 77-105.

Morley, R., Eccleston, D.W., Douglas, C.P., et al. 1970. Xylene poisoning: A report on one fatal case and two cases of recovery after prolonged unconsciousness. $\mathrm{Br}$. J. Med. 3:442-443.

NRC. 1980. Drinking water and health. Vol. 3. Washington, DC. Natl. Acad. Press, 178$181 ; 231-261$.

Nunes, P., Benville, P.J. 1979. Uptake and depuration of petroleum hydrocarbons in the manila clam, tapes semidecussata reeve. Bull. Env. Contam. Toxicol. 21:719-726.

Ogata, M., Miyake, Y. 1978. Disappearance of aromatic hydrocarbons and organic sulfur compounds from fish reared in crude oil suspension. Water Res. 12:1041-1044.

Romer, K.G., Federsel, R.J., Freundt, K.J. 1986. Rise of inhaled toluene, ethyl benzene, m-xylene, or mesixylene in rat blood after treatment with ethanol. Bull. Environ. Contam. Toxicol. 37:874-876. 
Sanborn, H.R., Malins, D.C. 1980. The disposition of aromatic HCs in adult spot shrimp (Pandulus Piatyceros) and the formation of metabolites of naphthalene in adult and larval spot shrimp. Xenobiotica. 10: 193-200.

Sax, N.I., Lewis, R.J., Sr. 1989. Rapid guide to haz. chems. in the workplace. NY, NY. Van Nostrand Reinhold Co. 178-179.

Windholz, M., ed. 1983. The Merck Index. 10th ed. Rahway, NJ: Merck \& Co., Inc. 14471448. 


\section{THIS PAGE LEFT INTENTIONALLY BLANK}


ZINC

CAS No: $\quad$ 7440-66-6

Synonym(s) None

Zinc is found in nature as ores of sulfide (sphalerite), oxide (franklinite), and carbonate (smithsonite). It is never found free and therefore metallic zinc must be obtained by extraction from ore. References to the use of zinc ores dates back to biblical times (BC), however it was not recognized as a distinct element for ages. Zinc is used in the production of brass, alloys, die castings, and electrical conductors. Zinc oxide is used in the production of a variety of common items, including paint, rubber products, cosmetics, pharmaceuticals, soap, textiles, etc. (Weast 1985; Sittig 1980).

Zinc is a bluish-white metal which is brittle at ambient temperatures, but malleable at 100$150^{\circ} \mathrm{C}$. It has a molecular weight of 65.38 , a melting point of $419.58^{\circ} \mathrm{C}$, and a boiling point of $907^{\circ} \mathrm{C}$. Its specific gravity is 7.133 (Weast 1985).

\section{FATE AND TRANSPORT}

As with most metals, processing may cause aerosols to be released into the air as dust. Burning of coal tar may also release zinc to the atmosphere. These particles eventually settle out by gravitational forces or through deposition by precipitation. Zinc may be slowly oxidized in air; a process which is accelerated by moisture (Bowen 1979; Sittig 1980).

Zinc may form organic complexes in fresh water and settle in aquatic sediments, however sedimentation is slower than for some metals. In polluted estuaries zinc becomes desorbed from suspended matter, whereas in pristine estuaries zinc may remain sorbed to particles and become trapped in sediments (Bowen 1979).

\section{HUMAN HEALTH EFFECTS}

Zinc is an essential nutrient and is taken in by humans through ingestion of food and water at an average daily intake of $12-30 \mathrm{mg} /$ day. It is relatively non-toxic to humans, even at intakes as high as $150 \mathrm{mg} /$ day for up to 6 months (Greaves and Sillen 1970). Zinc poisoning has been attributed to ingestion of extremely high amounts in food, ranging from 1000-2000 ppm (Brown et al. 1964).

Persons occupationally exposed to zinc fumes have, in some cases, developed a syndrome known as oxide shakes or zinc chills (Weast 1985). Additionally, high intakes of zinc may interfere with copper and other minerals, and may cause reversible effects in persons with copper deficiency. Zinc sulfate used therapeutically precipitated copper deficiency in persons with sickle cell anemia and coaliac disease (Sittig 1980).

\section{ECOLOGICAL EFFECTS}

Zinc is acutely toxic to fresh water organisms in concentrations as low as $26 \mu \mathrm{g} / \mathrm{L}$ (Spehar 1976). In marine systems, organisms exposed to higher concentrations of zinc $(141 \mu \mathrm{g} / \mathrm{L}$ - 
$220 \mu \mathrm{g} / \mathrm{L}$ ), both acutely and chronically, developed toxic reactions (Reish et al. 1976). The ability of organisms to bioconcentrate zinc is not known, however it should be noted that zinc is an essential element, taken up by organisms in small amounts under normal conditions.

\section{US ENVIRONMENTAL PROTECTION AGENCY HUMAN HEALTH CRITERIA}

Oral Slope Factor

Inhalation Slope Factor

Chronic Oral RfD

Chronic Inhalation RfD
NA

NA

$3 \mathrm{E}-1 \mathrm{mg} / \mathrm{kg} /$ day

NA

\section{DRINKING WATER CRITERIA AND STANDARDS}

U.S. Environmental Protection Agency

Maximum Concentration Limit

NA

Maximum Concentration Limit Goal . NA

\section{AMBIENT WATER QUALITY CRITERIA}

U.S. Environmental Protection Agency Ambient Water Quality Criteria

Human Health-Water and Fish Ingestion

NA

Human Health =Fish Ingestion Only

NA

Aquatic Organisms-FW Acute

$320 \mu \mathrm{g} / \mathrm{L}$

Aquatic Organisms-FW Chronic

Aquatic Organisms-Marine Acute

Aquatic Organisms-Marine Chronic

$47 \mu \mathrm{g} / \mathrm{L}$

$170 \mu \mathrm{g} / \mathrm{L}$

$58 \mu \mathrm{g} / \mathrm{L}$

South Carolina Department of Health and Environmental Control

Human Health Criteria

Fish and Aquatic Life-Continuous

Fish and Aquatic Life-Maximum
NA

$58.91 \mu \mathrm{g} / \mathrm{L}$

$65.04 \mu \mathrm{g} / \mathrm{L}$

$\mathrm{NA}=$ Not Available

\section{REFERENCES}

Bowen. 1979. Environmental chemistry of the elements. New York,NY. Academy Press.

Brown, M.A. et al. 1964. Food poisoning involving zinc contaminants. Arch. Environ. Health 8:657-658. 
Reish, D.J. et al. 1976. The effect of heavy metals on laboratory populations of two - polycheates with comparisons to the water quality conditions and standards in southern California marine waters. Water Res. 10:299.

Sittig, M. (ed) 1980. Priority toxic pollutants:Health impacts and allowable limits. Environmental Health Review No.1., Park Ridge, NJ. Noyes Data Corp.

Spehar, R.L. 1976. Cadmium and zinc toxicity to flagfish, Jordanella floridae. J. Fish Res. Board Can. 33:1939.

Weast, R.C. 1985. CRC Handbook of Chemistry and Physics. 66th edition. Boca Raton, FL. CRC Press. 
THIS PAGE LEFT INTENTIONALLY BLANK 


\section{TOXICITY PROFILES FOR RADIONUCLIDES}

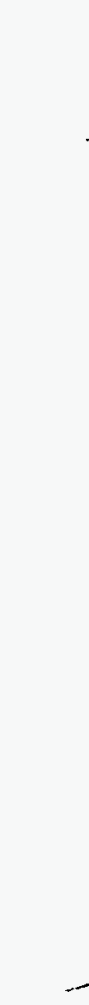


THIS PAGE LEFT INTENTIONALLY BLANK 


\section{CESIUM}

Cas No: $\quad 7440-46-2$

Synonym: None

Cesium is an alkali metal which occurs naturally in the earth's crust at approximately $1 \mathrm{ppm}$. Cesium is silver-white, ductile, and oxidizes rapidly in air (MERK, 1983). Cesium-133 is a naturally occurring isotope. Other isotopes include cesium-123, -125-132, and -134-144. The most abundant anthropogenic isotope of cesium is cesium-137, which is produced as a byproduct of fission reactions. Cesium-137 has been released to the atmosphere in large quantities through weapons testing.

Cesium-137 is used for encapsulated energy sources for purposes of food irradiation and medical uses. The radioactive decay of cesium-137 occurs through beta particle emission. However, emissions from the decay of cesium- 137 includes gamma ray emission through its equilibrium with barium-137m. The half-life of cesium-137 is 30 years.

\section{FATE AND TRANSPORT}

As stated earlier, large quantities of cesium-137 have been released to the atmosphere through weapons testing. Cesium-137 has a tendency to adsorb to particulate matter, therefore, deposition from the atmosphere to surface soils, surface waters, and plants would be the primary removal mechanism. The partitioning coefficient of cesium-137 is approximately $270 \mathrm{ml} / \mathrm{g}$ (NUREG/CR-5512, 1992). In surface soils and in surface waters and groundwaters, the tendency to adsorb to particulate matter causes particulate bound cesium to be relatively immobile. In surface waters, sediment bound cesium would settle to the bottom of water bodies.

\section{HUMAN HEALTH EFFECTS}

Chemically, animal studies have indicated a low toxicity; however, cesium can act as an analog to potassium and thus be potentially harmful (MERK, 1983). The primary danger from cesium-137 is through its release of gamma rays and beta particles. Gamma rays are fully penetrating energies. Beta particles are only partially penetrating. Chronic exposure to radiation has be shown to cause cancer.

\section{ECOLOGICAL EFFECTS}

The bioaccumulation factor in fish for cesium- 137 uptake is relatively high. Literature indicates that the bioaccumulation factor is approximately 2000 (NUREG/CR-5512, 1992). Soil-to-plant concentration factors ( $\mathrm{pCi} / \mathrm{kg}$ dry weight per $\mathrm{pCi} / \mathrm{kg}$ soil) for cesium-137 in leafy vegetables, root vegetables, fruit, and grain are $0.13,0.05,0.22$, and 0.03 , respectively. Animal product transfer factors (wet-weight basis) for beef, poultry, milk, and eggs are 0.02 $\mathrm{d} / \mathrm{kg}, 4.4 \mathrm{~d} / \mathrm{kg}, 0.007 \mathrm{~d} / \mathrm{l}$, and $0.5 \mathrm{~d} / \mathrm{kg}$, respectively (NUREG/CR-5512). In consideration of these factors, cesium-137 does have the potential to bioaccumulate through parts of the food chain. 
US ENVIRONMENTAL PROTECTION AGENCY HUMAN HEALTH CRITERIA

\begin{tabular}{||l|l|l||}
\hline Slope Factors & Cs -137 & Cs-137+D \\
\hline $\begin{array}{l}\text { Oral } \\
\text { (Risk/pCi) }\end{array}$ & $2.8 \mathrm{E}-11$ & $2.8 \mathrm{E}-11$ \\
\hline $\begin{array}{l}\text { Inhalation } \\
\text { (Risk/pCi) }\end{array}$ & $1.9 \mathrm{E}-11$ & $1.9 \mathrm{E}-11$ \\
\hline $\begin{array}{l}\text { External Exposure } \\
\text { (Risk/yr per pCi/g) }\end{array}$ & $0.0 \mathrm{E}+00$ & $2.0 \mathrm{E}-06$ \\
\hline
\end{tabular}

$+\mathrm{D}=$ Includes Daughter Products

\section{DRINKING WATER STANDARDS}

U.S. Environmental Protection Agency

$\mathrm{MCL}=$ Maximum Contaminant Level Cs-137

Proposed, EPA, 1991

\section{AMBIENT WATER QUALITY CRITERIA}

U.S. Environmental Protection Agency Ambient Water Quality Criteria

NA

South Carolina Department of Health and Environmental Control

NA

NA $=$ Not Available

REFERENCES

DHEW, U.S. Department of Health, Education, and Welfare, Radiological Health Handbook, Revised Edition, January 1970.

MERK, The MERK Index, An Encyclopedia of Chemicals, Drugs, and Biologicals, Tenth Edition, 1983.

NUREG/CR-5512, U.S. Nuclear Regulatory Commission, Residual Radioactive Contamination from Decommissioning, Technical Basis for Translating Contamination Levels to Annual Total Effective Dose Equivalent, NUREG/CR-5512, PNL-7994, Vol. 1, October 1992. 


\section{PLUTONIUM}

Cas No: 7440-07-5

Synonyms: None

Plutonium is a silvery-white radioactive metal. Minute quantities of plutonium occur in nature; however, large amounts are produced by man in nuclear reactors. Plutonium-238 is used as a heat source; as radioisotope thermoelectric generator; in radionuclide batteries for pacemakers; and with beryllium as a neutron source. Plutonium-239 is used in nuclear weapons and in power reactors.

Plutonium is produced by the bombardment of uranium with neutrons. Plutonium-239 is produced in large quantities in nuclear reactors. Plutonium-240, -241, and -242 are produced from successive absorption of neutrons by plutonium-239 atoms.

As of 1980 , the worlds nuclear power reactors were producing more than $20,000 \mathrm{~kg}$ of plutonium per year (ATSDR, 1990). In addition, the United States Department of Energy (DOE) historically produced plutonium for the nation's defense program. Currently, DOE does not produce plutonium.

The radioactive half-lives of plutonium-238, $-239,-240,-241$, and -242 are 86.4 years, 24,390 years, 6,580 years, 13.2 years, and $3.8 \times 10^{5}$ years, respectively (DHEW, 1970). With the exception of plutonium-241, the plutonium isotopes undergo radioactive decay by alpha emission. Plutonium-241 decays through beta emission. Regardless of the type of decay, the decay of the plutonium isotopes results in uranium $\mathrm{x}$-ray emission.

\section{FATE AND TRANSPORT}

Anthropogenic releases of plutonium are the primary sources of plutonium to the atmosphere. Weapons testing, accidental releases, and routine releases from processing activities are sources of plutonium in the environment. Particulate bound plutonium in the atmosphere will deposit on surface soils, plants, and in surface waters.

The transport and partitioning of plutonium in soils depends on the form of the compound. The solubility of plutonium depends on the properties of the soil, the presence of organic and inorganic complexing agents, the form of plutonium that enters the environment, and the presence of soil microorganisms (ATSDR,.1990).

Plutonium will migrate in soils as the hydrolyzed ion or as a complex, formed with organic or inorganic acids. Microorganisms present in soils have been found to enhance the solubility of plutonium by initiating a change in the oxidation state of plutonium.

The behavior of plutonium in surface waters is dependent upon the oxidation state and the nature of suspended solids and sediments in the water column. The oxidized forms of plutonium are found in natural waters when the concentrations of dissolved organic matter or dissolved solids are low (ATSDR, 1990). The formation of plutonium complexes with organic carbon causes plutonium to remain in solution as a complex. The partitioning of 
plutonium form surface waters to sediments depends in the equilibrium between plutonium IV and plutonium V. However, sorption to clays has been found to be irreversible, therefore, the particulate bound plutonium would settle to bottom sediments.

Transformation of plutonium will occur primarily as a result of radioactive decay. In the environment, oxidation/reduction reactions transform plutonium from one oxidation state to another.

\section{HUMAN HEALTH EFFECTS}

Plutonium poses a radiation hazard as well as a chemical hazard. Exposure to plutonium can occur through ingestion, inhalation, and external exposure to radiation. Radiation exposures pose risks of developing cancer.

Plutonium tends to concentrate in bones (MERK, 1983) if ingested. Inhalation studies of plutonium in animals indicate an increased risk of pneumonitis, alveolar edema, fibrosis, and pulmonary hyperplasia and metaplasia (ATSDR, 1990).

\section{ECOLOGICAL EFFECTS}

Plutonium has been found to bioaccumulate in aquatic organisms, primarily at the lower end of the food chain. The bioconcentration factors have been estimated to be 1000 for mollusks and algae, 100 for crustacea, and 10 for fish (ATSDR, 1990). Plutonium in soils has been shown to be taken up by plants. The highest concentrations of plutonium in plants are found in the roots where plutonium is present as a surface-absorbed plutonium complex, a stabilized complex, or as a soluble plutonium complex (ATSDR, 1990). Studies have shown that no evidence of bioconcentration through the food chain from soil to plants to animals occur (ATSDR, 1990).

US ENVIRONMENTAL PROTECTION AGENCY HUMAN HEALTH CRITERIA

\begin{tabular}{||l|l|l|l|l|l||}
\hline Slope Factors & Pu-238 & Pu-239 & Pu-240 & Pu-241 & Pu-242 \\
\hline $\begin{array}{l}\text { Oral } \\
\text { (Risk/pCi) }\end{array}$ & $2.2 \mathrm{E}-10$ & $2.3 \mathrm{E}-10$ & $2.3 \mathrm{E}-10$ & $3.6 \mathrm{E}-12$ & $2.2 \mathrm{E}-10$ \\
\hline $\begin{array}{l}\text { Inhalation } \\
\text { (Risk/pCi) }\end{array}$ & $3.9 \mathrm{E}-08$ & $3.8 \mathrm{E}-08$ & $3.8 \mathrm{E}-08$ & $2.3 \mathrm{E}-10$ & $3.6 \mathrm{E}-08$ \\
\hline $\begin{array}{l}\text { External Exposure } \\
\text { (Risk/yr per pCi/g) }\end{array}$ & $2.8 \mathrm{E}-11$ & $1.7 \mathrm{E}-11$ & $2.7 \mathrm{E}-11$ & $0.0 \mathrm{E}+00$ & $2.3 \mathrm{E}-11$ \\
\hline
\end{tabular}




\section{DRINKING WATER STANDARDS}

U.S. Environmental Protection Agency

\begin{tabular}{|l|c|c|c|c|}
\hline & Pu-238 & Pu-239/240 & Pu-241 & Pu-242 \\
\hline MCL (pCi/1) & 7.02 & 62.1 & 62.6 & 65.4 \\
\hline
\end{tabular}

MCL = Maximum Contaminant Level

Proposed, EPA, 1991

\section{AMBIENT WATER QUALITY CRITERIA}

U.S. Environmental Protection Agency Ambient Water Quality Criteria

NA

South Carolina Department of Health and Environmental Control

NA

NA $=$ Not Available

REFERENCES

ATSDR, Agency for Toxic Substances and Disease Registry, Toxicological Profile for Plutonium, February 16, 1990.

DHEW, U.S. Department of Health, Education, and Welfare, Radiological Health Handbook, Revised Edition, January 1970.

MERK, The MERK Index, An Encyclopedia of Chemicals, Drugs, and Biologicals, Tenth Edition, 1983. 
THIS PAGE LEFT INTENTIONALLY BLANK 
POTASSIUM (K)

Cas.No: $\quad 7473$

Synonyms: None

Potassium is a soft, silvery-white metal that tarnishes upon exposure to air. Of all the naturally occurring potassium isotopes, Potassium- 40 (i.e., $\mathrm{K}-40$ ) is the only isotope that is radioactive. It makes up about 0.12 percent of naturally occuring potassium, and has a halflife greater than $10^{9}$ years. Because it is naturally occurring, $\mathrm{K}-40$ is a contributor to natural background radiation. In addition, because potassium is an essential element to the body, a small percentage of potassium in the human body is $\mathrm{K}-40$.

Potassium exists in the +1 valence state. Because it shares a similar atomic structure in its outer electron shells with sodium ( $\mathrm{Na})$, rubidium $(\mathrm{Rb})$, cesium $(\mathrm{Cs})$, and francium $(\mathrm{Fr})$, it may substitute for any of these elements.

\section{FATE AND TRANSPORT}

$\mathrm{K}-40$ is ubiquitous in nature. It is present in most soils, and is an important element in the lattice of expandable clays. It initially enters the biosphere through weathering of rocks and soils. Because it is very soluble, $\mathrm{K}-40$ is mobilized in surface or groundwaters and is taken up and assimilated by plants and animals through the food chain. $\mathrm{K}-40$ is eliminated from the body, therefore, it does not biomagnify as do some other elements.

Physicochemical factors such as oxidation-reduction potential and $\mathrm{pH}$ do not have a great effect on the mobility of $\mathrm{K}-40$ in natural waters. Because of its monovalent charge, other . elements outcompete $\mathrm{K}-40$ for binding sites on organic materials or inorganic compounds in the soil or groundwaters.

\section{HUMAN HEALTH EFFECTS}

$\mathrm{K}-40$ emits both a beta particle and a positron. Both are equal to an electron's mass, but of opposite charge. The beta particle from $\mathrm{K}-40$ can travel less than a quarter of an inch in the body. While it can penetrate the skin and cause an external dose, because potassium is an essential element particularly in the muscular system and in the circulatory system, it is more likely to contribute to an individual's internal dose.

$\mathrm{K}-40$ also emits gamma rays which can penetrate the skin. The energy of these photons is is sufficient to cause an external dose, but not one that is significant in terms of risk to human health.

Because $\mathrm{K}-40$ is a component of natural background and is found in very small quantitities in the food that we eat, there is a very small risk that each of us accepts on a daily basis. 


\section{ECOLOGICAL EFFECTS}

$\mathrm{K}-40$ is an essential nutrient to life. Because it is ingested, assimilated, and eliminated from organisms at nearly the same rate, there is no biomagnification of $\mathrm{K}-40$ in organisms.

\section{US ENVIRONMENTAL PROTECTION AGENCY HUMAN HEALTH CRITERIA}

The oral, inhalation, and external slope factors of potassium- 40 are $1.1 \mathrm{E}-11$ risk $/ \mathrm{pCi}, 7.6 \mathrm{E}-$ 12 risk/pCi, and $5.4 \mathrm{E}-07$ risk/yr per $\mathrm{pCi} / \mathrm{g}$, respectively.

\section{DRINKING WATER STANDARDS}

U.S. Environmental Protection Agency

MCL = Maximum Contaminant Level Proposed, EPA, 1991

$\mathrm{K}-40$ 300

\section{AMBIENT WATER QUALITY CRITERIA}

U.S. Environmental Protection Agency Ambient Water Quality Criteria

NA

South Carolina Department of Health and Environmental Control

NA

NA $=$ Not Available

\section{REFERENCES}

DHEW, U.S. Department of Health, Education, and Welfare, Radiological Health Handbook, Revised Edition, January 1970.

MERK, The MERK Index, An Encyclopedia of Chemicals, Drugs, and Biologicals, Tenth Edition, 1983. 


\section{RADIUM}

Cas No: $\quad 7440-14-4$

Synonyms:

Radium is a naturally occurring silvery-white metal that exists as several radioactive isotopes. Radium is formed when uranium and thorium decay in the environment. Radium exists at low levels in soil, rocks, water, coal, plants, and certain foods.

Radium has been used as a radiation source for treating neoplastic diseases, as a radon source, in radiography of metals, and as a neutron source for research. Until the 1960s, radium was a component of luminous paints used for watch and clock dials, instrument panels in airplanes, military instruments, and compasses (ATSDR, 1990). Radium isotopes are not commercially produced in the United States.

Radium has 16 known isotopes; however, only 4 occur naturally (radium-223, $-224,-226$, and -228). The half-life of radium-226 is 1,620 years. Thea half-lives of radium-228, radium-224, and radium-223 are 5.8 years, 3.6 days, and 11.4 days, respectively (DHEW, 1970).

\section{FATE AND TRANSPORT}

The combustion of coal and the mining of uranium have been important mechanism for the release of radium to the environment. Radium is transported in air by particulate matter. The particulate matter is subject to atmospheric dispersion, gravitational settling, and washout by rain (ATSDR, 1990).

Radium has been released to surface waters primarily as a result of uranium mining. Radium in water exists as a divalent ion $(+2)$. The solubility of radium salts in water generally increase with increased $\mathrm{pH}$ levels. Radium nitrate, chloride, and iodate are very soluble in water and thus very mobile. Radium sulfate and carbonate have very low solubilities and thus are less mobile. Radium also has a tendency to coprecipitate with barite. Field data and distribution coefficient studies indicate that radium adsorbs to particulate matter which generally retards its mobility. This tendency to sorb to particulates characterizes the mobility of radium in soils and groundwater as limited.

Radium does not degrade in the environment by any other means than by radioactive decay. Radium-226 and radium-228 have the longest half-lives of the four naturally occurring isotopes. Radium-226 decays by alpha particle emission and radium-228 decays by beta particle emission. Each isotope yields progeny which produce gamma rays that can be detrimental to human health.

\section{HUMAN HEALTH EFFECTS}

Adverse effects of radium isotopes are believed to be the consequence of the radiation emitted from the elements themselves and their progeny rather than the chemical properties of radium. Chronic exposures to high levels of radium may result in harmful effects including anemia, cataracts, fractured teeth, cancer (especially bone cancer), and death. 
Exposure to radium can occur through ingestion, inhalation, dermal contact, and external exposure to radiation from the progeny.

\section{ECOLOGICAL EFFECTS}

Existing information indicates that radium may be transferred through the food chain from lower trophic levels to humans. Bioconcentration factors for freshwater fish have ranged from 1 to 60 for flesh portions, and from 40 to 1,800 in bone samples. Radium in soils have been shown to readily adsorbed by certain plants and depending on the type of soil. Additionally, there is a potential for human exposure to radium by the consumption of fish and beef and milk derived from cattle which graze on forage containing radium (ATSDR, 1990).

US ENVIRONMENTAL PROTECTION AGENCY HUMAN HEALTH CRITERIA

\begin{tabular}{||l|l|l|l|l|l|l||}
\hline \hline Slope Factors & Ra-223 & Ra-224 & Ra-226 & Ra-226+D & Ra-228 & Ra-228+D \\
\hline $\begin{array}{l}\text { Oral } \\
\text { (Risk/pCi) }\end{array}$ & $6.4 \mathrm{E}-11$ & $3.8 \mathrm{E}-11$ & $1.2 \mathrm{E}-10$ & $1.2 \mathrm{E}-10$ & $1.0 \mathrm{E}-10$ & $1.0 \mathrm{E}-10$ \\
\hline $\begin{array}{l}\text { Inhalation } \\
\text { (Risk/pCi) }\end{array}$ & $3.9 \mathrm{E}-09$ & $1.2 \mathrm{E}-09$ & $3.0 \mathrm{E}-09$ & $3.0 \mathrm{E}-09$ & $6.6 \mathrm{E}-10$ & $6.9 \mathrm{E}-10$ \\
\hline $\begin{array}{l}\text { External } \\
\text { Exposure } \\
\text { (Risk/yr per } \\
\text { pCi/g) }\end{array}$ & $2.3 \mathrm{e}-07$ & $2.3 \mathrm{E}-08$ & $1.2 \mathrm{E}-08$ & $6.0 \mathrm{E}-06$ & $0.0 \mathrm{E}+00$ & $2.9 \mathrm{E}-06$ \\
\hline
\end{tabular}

$+\mathrm{D}=$ Includes Daughter Products

\section{DRINKING WATER STANDARDS}

U.S. Environmental Protection Agency

Maximum Contaminant Level

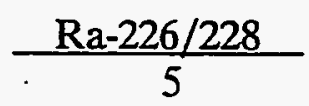

(pCi/l) - Final CFR, 1991

\section{AMBIENT WATER QUALITY CRITERIA}

U.S. Environmental Protection Agency Ambient Water Quality Criteria

NA

South Carolina Department of Health and Environmental Control 
NA

$\mathrm{NA}=$ Not Available

REFERENCES

ATSDR, Agency for Toxic Substances and Disease Registry, Toxicological Profile for Radium, February 16, 1990.

DHEW, U.S. Department of Health, Education, and Welfare, Radiological Health Handbook, Revised Edition, January 1970.

MERK, The MERK Index, An Encyclopedia of Chemicals, Drugs, and Biologicals, Tenth Edition, 1983. 
THIS PAGE LEFT INTENTIONALLY BLANK 
STRONTIUM (Sr)

Cas No.: $\quad 8699$

Synonym(s) None

Strontium (Sr) is an alkaline earth metal that is found in small quantities associated with calcium or barium minerals. Because the atomic structure in the outer shells is similar to magnesium, calcium, barium, and radium, and shares some of the same chemical properties, it substitutes for those metals. Sr-90 is one of 15 radioactive isotopes of $\mathrm{Sr}$, and it has a half-life of 28 years. Sr-90 is a product of the nuclear fissioning process, and is a component of atmospheric fallout from above ground weapons testing conducted during the 1960s.

\section{FATE AND TRANSPORT}

Because Sr-90 is a component of atmospheric fallout, it is found in natural systems. Sr-90 occurs in the +2 valence state, and is soluble in soils and surface and groundwaters. Like other divalent cations, it generally displaces monovalent cations like Cs- 137 and $\mathrm{K}-40$ on soil particles and organic matter.

\section{HUMAN HEALTH EFFECTS}

Sr-90 is a beta particle emitter. Its range in soft tissue is approximately $1 / 16^{\text {th }}$ inch, therefore, it releases all of its energy in a very short range when it is deposited internally. There is not a significant human health risk from external exposure to Sr-90. A more important consideration is that, because of its similar chemical structure to calcium, $\mathrm{Sr}-90$ behaves like calcium. As a result, when it is assimilated, Sr-90 can be a potential . contributor to the skeletal dose of an individual.

\section{ECOLOGICAL EFFECTS}

Sr-90 can also be of concern because it can be incorporated into the skeletal structure of terrestrial and aquatic organisms . However, the dose is only significant when the concentrations of Sr-90 are higher by several orders pf magnitude when compared to the natural background concentrations from atmospheric fallout. Sr-90 can also bioaccumulate in plants that concentrate calcium and when there is a calcium deficiency in soils. 
THIS PAGE LEFT INTENTIONALLY BLANK 


\section{TRITIUM}

Cas No: $\quad 10028-17-8$.

Synonyms: Titerium

Tritium is a naturally occurring radioactive isotope of hydrogen. Tritium is found in the environment as form of hydrogen gas and more commonly associated with water molecules. Since tritium readily exchanges with hydrogen, it largely follows the water cycle, both inside the body and in the environment. Tritium is a major component of the off-streams of nuclear reactors, and even more so of reprocessing plants (Eicholz, 1985).

Tritium is produced in reactors through the slow neutron bombardment of lithium-6 flouride. Tritium is used in fusion-based thermonuclear weapons, as a radioactive tracer in chemical, biochemical, biological, and environmental studies (MERK, 1983). The United States Department of Energy historically produced tritium for the national defense program.

Tritium decays through the release of a low-energy beta particle. The half-life of tritium is 12.3 years (DHEW, 1970).

\section{FATE AND TRANSPORT}

Tritium in the atmosphere converts to tritiated water vapor. As stated above, tritium associated with water molecules generally follows the water cycle. No reconcentration would be expected with respect to ordinary hydrogen, therefore, most of the tritium will ultimately end up in the oceans (Eicholz, 1985).

\section{HUMAN HEALTH EFFECTS}

Water within the body has a residence time of approximately 8.6 days (Eicholz, 1985). Since tritium only releases a weak energy beta particle, health impacts should be minimal. Owing to the low energy of the beta particles emitted and the rapid dilution and elimination of the tritium, its maximum permissible concentration in drinking water is the highest for any radioisotope, $20,000 \mathrm{pCi} / 1$.

\section{ECOLOGICAL EFFECTS}

Ecological effects of tritium would be similart to that of human health effects. Due to the rapid elimination of tritium associated with water in ecological receptors, impacts would be considered minimal.

\section{US ENVIRONMENTAL PROTECTION AGENCY HUMAN HEALTH CRITERIA}

The oral, inhalation, and external slope factors of tritium are $5.4 \mathrm{E}-14$ risk/pCi, $7.8 \mathrm{E}-14$ risk $/ \mathrm{pCi}$, and $0.0 \mathrm{E}+00$ risk/yr per $\mathrm{pCi} / \mathrm{g}$, respectively.

\section{DRINKING WATER STANDARDS}


U.S. Environmental Protection Agency

MCL $=$ Maximum Contaminant Level

$\mathrm{H}-3$

20,000

Final, CFR, 1991

\section{AMBIENT WATER QUALITY CRITERIA}

U.S. Environmental Protection Agency Ambient Water Quality Criteria

NA

South Carolina Department of Health and Environmental Control

NA

$\mathrm{NA}=$ Not Ávailable

REFERENCES

DHEW, U.S. Department of Health, Education, and Welfare, Radiological Health Handbook, Revised Edition, January 1970.

Eicholz, Geoffrey G.; Environmental Aspects of Nuclear Power, Lewis Publishers, 1985.

MERK, The MERK Index, An Encyclopedia of Chemicals, Drugs, and Biologicals, Tenth Edition, 1983.

NUREG/CR-5512, U.S. Nuclear Regulatory Commission, Residual Radioactive Contamination from Decommissioning, Technical Basis for Translating Contamination Levels to Annual Total Effective Dose Equivalent, NUREG/CR-5512, PNL-7994, Vol. 1, October 1992. 


\section{URANIUM}

Cas No: $\quad$ 7440-61-1

Synonyms: None

Natural uranium is a sliver-colored metal that is radioactive. Small amounts of uranium are present in rocks, soil, water, groundwater, plants and animals, and contribute to weak background radiation from such substances (ATSDR, 1990). Natural uranium occurs primarily as three isotopes including uranium-234, uranium-235, and uranium-238. The amount of uranium-238 in natural uranium ore is more than $99 \%$. Uranium-235 and uranium-234 occur at natural abundance levels of $0.72 \%$ and $0.006 \%$, respectively. The radioactive half-lives of the uranium isotopes range from $10^{5}$ years (uranium-235) to $10^{9}$ years (uranium-238).

Uranium is used primarily in nuclear power reactors and nuclear weapons. Uranium ore is mined in the United States for these purposes. Uranium-235 is enriched to approximately $96 \%$ for use in nuclear reactors and nuclear weapons. Depleted uranium is used to manufacture armor-piercing ammunition for the military, in inertial guidance devices and gyro compasses, as a counterweight for missile reentry vehicles, as radiation shielding material, and x-ray targets (ATSDR, 1990). Uranium also is present in phosphate fertilizers used frequently in the United States.

Uranium exists in four oxidation states, $+3,+4,+5$, and +6 ; however, the +4 and +6 oxidations states are the most stable to be of practical importance (ASTDR, 1990). The atomic mass of uranium-238, uranium-235, and uranium-234 are 238.050, 235.045, and 234.043, respectively (DHEW, 1970). Uranium is silver-white, malleable, and ductile (MERK, 1983).

\section{FATE AND TRANSPORT}

Uranium is released to the environment by both anthropogenic and natural processes. Wind-blown soil is a natural source and contributes to background uranium in the air and surface soils. Man-made activities which release uranium to the environment include uranium mining, milling, handling, processing, and use of phosphate fertilizers. Previous weapons testing conducted in the United States has released uranium isotopes to the atmosphere.

Particulate uranium is removed from the atmosphere to surface water, plant surfaces, and surface soils by dry and wet deposition (ATSDR, 1990). Resuspension of particulates on these media intermittently occurs. In surface water, the ultimate fate of uranium adsorbed to particulate matter is to bottom sediments. The four most important factors controlling the mobility of uranium in natural waters are: oxidation-reduction potential, $\mathrm{pH}$, characteristics of complexing agents or ligands present, and the nature of sorbing materials in water (ATSDR, 1990). Inorganic ions or organic ligands that can form soluble complexes with uranium will increase its mobility. The stability of these complexes, however, are $\mathrm{pH}$ dependent. Uranium is more likely to be in solution as carbonate complexes with high alkalinities, but in water with $\mathrm{pH}$ less than 6 uranium will be in solution as soluble organic 
complexes. An oxidation state of +4 (supported by anoxic waters) reduces the concentration of soluble uranium complexes.

The behavior of uranium in soil is dependent on similar factors as that of uranium in water. The sorption of uranium in most soil is such that the uranium would not leach readily from the soil matrix, particularly in soils containing clay and iron oxide (ATSDR, 1990). Sorption in most soils attains a maximum when the neutral hydroxy complex of uranium is maximal. However, at $\mathrm{pH}$ above 6 and in the presence of high carbonate or hydroxide concentrations, uranium may form anionic species which have greater mobilities (ATSDR, 1990).

Radioactive uranium decays naturally to progeny radionuclides. The progeny eventually decay to stable lead.

\section{HUMAN HEALTH EFFECTS}

Uranium emits low energy alpha particles which cannot penetrate the skin, but can travel short distances in the body if particulates containing uranium are inhaled or ingested. Radiation hazards do exist from the inhalation of fine particles of 1 micron.

Natural uranium emits very small quantities of gamma rays, which can penetrate the skin; however, the levels of gamma radiation would be insignificant in terms of danger to human health.

The primary health risk from uranium is due to its chemical effects and not is radiological properties (ASTDR, 1990). Uranium and its salts are highly toxic. Dermatitis, renal . damage, acute necrotic arterial lesions, and death may occur from exposure to uranium (MERK, 1983). Exposure to uranium can occur through inhalation, ingestion, and dermal contact.

\section{ECOLOGICAL EFFECTS}

The measured bioconcentration factors for natural uranium in algae and plankton are 1576 and 459, respectively (ATSDR, 1990). However, the levels of bioconcentration factors decline with successive trophic level of aquatic species. Therefore, minimal to no biomagnification of uranium from the aquatic food chain is expected to occur. 
US ENVIRONMENTAL PROTECTION AGENCY HUMAN HEALTH CRITERIA

\begin{tabular}{||l|l|l|l|l|l||}
\hline \hline Slope Factors & $\mathrm{U}-234$ & $\mathrm{U}-235$ & $\mathrm{U}-235+\mathrm{D}$ & $\mathrm{U}-238$ & $\mathrm{U} 238+\mathrm{D}$ \\
\hline $\begin{array}{l}\text { Oral } \\
\text { (Risk/pCi) }\end{array}$ & $1.6 \mathrm{E}-11$ & $1.6 \mathrm{E}-11$ & $1.6 \mathrm{E}-11$ & $2.8 \mathrm{E}-11$ & $2.8 \mathrm{E}-11$ \\
\hline $\begin{array}{l}\text { Inhalation } \\
\text { (Risk/pCi) }\end{array}$ & $2.6 \mathrm{E}-08$ & $2.5 \mathrm{E}-08$ & $2.5 \mathrm{E}-08$ & $2.4 \mathrm{E}-08$ & $5.2 \mathrm{E}-08$ \\
\hline $\begin{array}{l}\text { External Exposure } \\
\text { (Risk/yr per pCi/g) }\end{array}$ & $3.0 \mathrm{E}-11$ & $2.4 \mathrm{E}-07$ & $2.4 \mathrm{E}-07$ & $3.6 \mathrm{E}-08$ & $3.6 \mathrm{E}-08$ \\
\hline
\end{tabular}

$+\mathrm{D}=$ Includes Daughter Products

DRINKING WATER STANDARDS

U.S. Environmental Protection Agency

Maximum Contaminant Level $\quad \frac{\mathrm{U}-234}{1.39 \mathrm{E}+01} \quad \frac{\mathrm{U}-235}{1.45 \mathrm{E}+01} \quad \frac{\mathrm{U}-238}{1.45 \mathrm{E}+01}$

(pCi/l) - Proposed 1991

\section{AMBIENT WATER QUALITY CRITERIA}

U.S. Environmental Protection Agency Ambient Water Quality Criteria

NA

South Carolina Department of Health and Environmental Control

NA

NA $=$ Not Available

\section{REFERENCES}

ATSDR, Agency for Toxic Substances and Disease Registry, Toxicological Profile for Uranium, February 16, 1990.

DHEW, U.S. Department of Health, Education, and Welfare, Radiological Health Handbook, Revised Edition, January 1970.

MERK, The MERK Index, An Encyclopedia of Chemicals, Drugs, and Biologicals, Tenth Edition, 1983. 
THIS PAGE LEFT INTENTIONALLY BLANK 
HUMAN HEALTH AND ECOLOGICAL 


\section{THIS PAGE LEFT INTENTIONALLY BLANK}




\section{APPENDIX F}

\section{CALCULATION OF REMEDIAL GOAL OPTIONS (RGOS)}

\section{RGO Calculation Method}

Recent guidelines from USEPA Region IV (November, 1995, Supplemental Guidance to RAGS: Region 4 Bulletin No. 5, Development of Risk-Based Remedial Options) specify that RGOs may be calculated in a simplified manner using the ratio of the calculated risk to the target risk as a multiplier for the exposure point concentration, or in a more comprehensive manner where the risk equations are rearranged and substituted with target risk levels to allow the backcalculation of a target concentration. Because the comprehensive approach provides a more thorough consideration of media and pathway specific contributions to risk, this method was chosen for calculating the RGOs for the F-Area Burning/Rubble Pits and Rubble Pit BRA.

The comprehensive approach makes use of 1) site-specific exposure factors used in the BRA (e.g., intake rates, exposure frequencies), 2) standard USEPA toxicity values -- slope factors and reference doses, and 3) target cancer and non-cancer risk levels recommended by USEPÁ Region IV. This collection of parameters provides all of the variables needed to calculate RGOs.

Since risk estimates indicated the need to calculate RGOs for residential and worker use scenarios, separate calculations were made incorporating each set of exposure factors. In the residential scenarios, exposure factors for a combined adult/child receptor were used in calculating the RGOs based on cancer risk. For non-cancer based RGOs, child exposure factors were used since the child is generally the more sensitive receptor for non-cancer effects. In the worker scenarios, an adult worker was used for all RGO calculations. The exposure factors used in calculating RGOs were the same as those used in the BRA and presented in Section 3 and Appendix D.

Separate calculations are made for each of three target risk levels for both cancer and non-cancer concerns, in accordance with Region IV recommendations. The target excess cancer risk levels were $1 \times 10^{-4}, 1 \times 10^{-5}$, and $1 \times 10^{-6}$. The target hazard quotients (non-cancer) were 3,1 , and 0.1. These target levels are specified by USEPA Region IV (November, 1995).

The rearranged risk equations used in this BRA are equivalent to those presented in the recent Region IV guidance. These equations represent an extension of the approach used in RAGS, Part 
B: Development of Risk-Based Preliminary Remediation Goals (USEPA, OERR, 9285.7-01B, 1991) to include consideration of site-specific exposure factors and the exposure potentially received through inhalation pathways. The specific equations used in calculating RGOs for soil and groundwater will be presented below.

\section{RGO Calculation Equations}

\section{Soil RGOs}

RGOs calculated for soil account for intake from ingestion, dermal, and inhalation pathways of exposure for both residential and worker receptors

For cancer-risk-based soil RGOs:

1. $\mathrm{RGO}=$

$$
T R \times \frac{B W \times A T C \times C F 1}{(E F \times E D) \times\left((S F o \times I R o)+(S F d \times S A \times A F \times A B S)+\left(S F i \times I R i \times\left(\frac{1}{V F}+\frac{1}{P E F}\right)\right)\right)^{W}}
$$
here:

$$
\begin{aligned}
& \text { TR }=\text { Target Risk Level }-1 \times 10^{-4}, 1 \times 10^{-5}, \text { or } 1 \times 10^{-6} \\
& \text { BW }=\text { Body Weight }- \text { receptor specific } \\
& \text { ATC }=\text { Averaging Time }- \text { Cancer }-25550 \text { days } \\
& \text { CF1 }=\text { Conversion Factor }=1 \times 10^{6} \mathrm{mg} / \mathrm{kg} \\
& \text { EF }=\text { Exposure Frequency }-- \text { receptor specific } \\
& \text { ED }=\text { Exposure Duration }-- \text { receptor specific } \\
& \text { SFo }=\text { Oral Slope Factor }-- \text { chemical specific } \\
& \text { IRo }=\text { Oral Intake Rate }- \text { receptor specific } \\
& \text { SFd }=\text { Dermal Slope Factor }- \text { chemical specific } \\
& \text { SA }=\text { Surface Area -- receptor specific }
\end{aligned}
$$


$\mathrm{AF}=$ Adherence Factor -0.2

$\mathrm{ABS}=$ Dermal Absorbance Factor -- chemical specific

$\mathrm{SFi}=$ Inhalation Slope Factor - chemical specific

$\mathrm{IRi}=$ Inhalation Rate - receptor specific

VF $=$ Volatilization Factor - - chemical specific

PEF = Particulate Emissions Factor -- chemical specific

Values for receptor-specific parameters are found in Section 3 and Appendix D - Table D.1.

Values for chemical-specific parameters are found in Section 3 and the relevant risk tables in Appendix D.

For those chemicals where toxicity values (e.g. inhalation slope factors) were not available from USEPA, the relevant portion of the equation was omitted.

For non-cancer-risk-based soil RGOs:

2. $\mathrm{RGO}=$

$$
T H I \times \frac{B W \times A T N \times C F 1}{(E F \times E D) \times\left(\left(\frac{I R o}{R f D o}\right)+\left(\frac{S A \times A F \times A B S}{R f D d}\right)+\left(\frac{I R i \times\left(\frac{1}{V F}+\frac{1}{P E F}\right)}{R f D i}\right)\right)}
$$

Where:

THI $=$ Target Hazard Index $(3,1$, or 0.1$)$

BW = Body Weight -- receptor specific

ATN $=$ Averaging Time - Noncancer - receptor specific

$\mathrm{CF} 1=$ Conversion Factor $=1 \times 10^{6} \mathrm{mg} / \mathrm{kg}$ 


$$
\begin{aligned}
& \mathrm{EF}=\text { Exposure Frequency }- \text { receptor specific } \\
& \mathrm{ED}=\text { Exposure Duration }- \text { receptor specific } \\
& \mathrm{IRo}=\text { Oral Intake Rate }- \text { receptor specific } \\
& \mathrm{RfD}=\text { Oral Reference Dose }- \text { chemical specific } \\
& \mathrm{SA}=\text { Surface Area }- \text { receptor specific } \\
& \mathrm{AF}=\text { Adherence Factor }-0.2 \\
& \mathrm{ABS}=\text { Dermal Absorbance Factor }- \text { chemical specific } \\
& \mathrm{RfDd}=\text { Dermal Reference Dose }- \text { chemical specific } \\
& \mathrm{IRi}=\text { Inhalation Rate }- \text { receptor specific } \\
& \mathrm{VF}=\text { Volatilization Factor }- \text { chemical specific } \\
& \mathrm{PEF}=\text { Particulate Emissions Factor }- \text { chemical specific } \\
& \mathrm{RfDi}=\text { Inhalation Reference Dose }- \text { chemical specific }
\end{aligned}
$$

Values for receptor-specific parameters are found in Section 3 and Appendix D - Table D.1.

Values for chemical-specific parameters are found in Section 3 and the relevant risk tables in Appendix D.

For those chemicals where toxicity values (e.g. inhalation slope factors) were not available from USEPA, the relevant portion of the equation was omitted.

\section{Groundwater RGOs}

For the Residential Receptor, ingestion and inhalation exposure were accounted for in the calculation of RGOs. Since inhalation exposure was estimated to be equivalent to ingestion exposure for this BRA, in accordance with Region IV guidance, the same simplifying assumption was made in calculating RGOs for groundwater. That is, total intake was set equal to $2 \mathrm{X}$ the intake via ingestion. 
For the Worker Receptor, intake via ingestion alone was considered in the calculation of RGOs since this was the only completed groundwater pathway for workers identified in the BRA.

For cancer-risk-based groundwater RGOs:

Residential Receptor

$$
\mathrm{RGO}=T R \times \frac{B W \times A T C}{(E F \times E D) \times(S F o \times(2 \times I R o))}
$$

Worker Receptor

$$
\mathrm{RGO}=T R \times \frac{B W \times A T C}{(E F \times E D) \times(S F o \times I R o)}
$$

Where:

$$
\begin{aligned}
& \text { TR }=\text { Target Risk Level }-1 \times 10^{-4}, 1 \times 10^{-5}, \text { or } 1 \times 10^{-6} \\
& \mathrm{BW}=\text { Body Weight }-- \text { receptor specific } \\
& \mathrm{ATC}=\text { Averaging Time }- \text { Cancer }-25550 \text { days } \\
& \mathrm{EF}=\text { Exposure Frequency }- \text { receptor specific } \\
& \mathrm{ED}=\text { Exposure Duration }-- \text { receptor specific } \\
& \mathrm{SFo}=\text { Oral Slope Factor }- \text { chemical specific } \\
& \text { IRo }=\text { Oral Intake Rate }- \text { receptor specific }
\end{aligned}
$$

Values for receptor-specific parameters are found in Section 3 and Appendix D - Table D.1.

Values for chemical-specific parameters are found in Section 3 and the relevant risk tables in Appendix D. 
For non-cancer-risk-based groundwater RGOs:

Residential Receptor

$$
\mathrm{RGO}=T H I \times \frac{B W \times A T N}{(E F \times E D) \times\left(\frac{2 \times I R o}{R f D o}\right)}
$$

Worker Receptor

$$
\mathrm{RGO}=T H I \times \frac{B W \times A T N}{(E F \times E D) \times\left(\frac{I R o}{R f D o}\right)}
$$

Where:

$$
\begin{aligned}
& \text { THI }=\text { Target Hazard Index }(3,1, \text { or } 0.1) \\
& \text { BW }=\text { Body Weight }- \text { - receptor specific } \\
& \text { ATN }=\text { Averaging Time }- \text { Noncancer }- \text { receptor specific } \\
& \text { EF }=\text { Exposure Frequency }- \text { - receptor specific } \\
& \text { ED }=\text { Exposure Duration }- \text { receptor specific } \\
& \text { IRo }=\text { Oral Intake Rate }- \text { receptor specific } \\
& \text { RfDo }=\text { Oral Reference Dose }- \text { chemical specific }
\end{aligned}
$$

Values for receptor-specific parameters are found in Section 3 and Appendix D - Table D.1.

Values for chemical-specific parameters are found in Section 3 and the relevant risk tables in Appendix D.

\section{Radionuclide RGOS}

For radionuclides, RGOs were derived using the following equation:

$$
\mathrm{RGO}=\mathrm{EPC}_{\text {chemical } i} \times \text { Target Risk } / \text { Calculated Risk } \text { chemicali }
$$

